MAR 251996

OSTI

\title{
Site Observational Work Plan For THE UMTRA PROJECT SITE At Monument Valley, Arizona
}

March 1996

\section{DISCLAIMER}

This report was prepared as an account of work sponsored by an agency of the United States Government. Neither the United States Government nor any agency thereof, nor any of their employees, makes any warranty, express or implied, or assumes any legal liability or responsibility for the accuracy, completeness, or usefulness of any information, apparatus, product, or process disclosed, or represents that its use would not infringe privately owned rights. Reference herein to any specific commercial product, process, or service by trade name, trademark, manufacturer, or otherwise does not necessarily constitute or imply its endorsement, recommendation, or favoring by the United States Government or any agency thereof. The views and opinions of authors expressed herein do not necessarily state or reflect those of the United States Government or any agency thereof. 


\section{INTENDED FOR PUBLIC RELEASE}

This report has been reproduced from the best available copy. Available in paper copy and microfiche.

Number of pages in this report: 629

DOE and DOE contractors can obtain copies of this report from:

Office of Scientific and Technical Information

P.O. Box 62

Oak Ridge, TN 37831

(615) 576-8401

This report is publicly available from:

National Technical Information Service

Department of Commerce

5285 Port Royal Road

Springfield, VA 22161

(703) 487-4650 


\section{SITE OBSERVATIONAL WORK PLAN \\ FOR THE UMTRA PROJECT SITE \\ AT MONUMENT VALLEY, ARIZONA}

\section{March 1996}

Work performed under DOE Contract No. DE-AC04-91AL62350

Prepared for

U.S. Department of Energy Grand Junction Projects Office

Prepared by Jacobs Engineering Group Inc.

Albuquerque, New Mexico 



\section{EXECUTIVE SUMMARY}

The site observational work plan (SOWP) for the Monument Valley, Arizona, U.S. Department of Energy (DOE) Uranium Mill Tailings Remedial Action (UMTRA) Project site is one of the first site-specific documents developed to achieve ground water compliance at the site. This SOWP applies information about the Monument Valley site to a regulatory compliance framework that identifies strategies that could be used to meet ground water compliance. The compliance framework was developed in the UMTRA Ground Water programmatic environmental impact statement (DOE, 1996).

The DOE's goal is to implement a cost-effective site strategy that complies with the U.S. Environmental Protection Agency (EPA) ground water standards and protects human health and the environment. The compliance strategy that emerges in the final version of the SOWP will be evaluated in the site-specific environmental assessment to determine potential environmental impacts and provide stakeholders a forum for review and comment. When the compliance strategy is acceptable, it will be detailed in a remedial action plan that will be subject to review by the state and/or tribe and concurrence by the U.S. Nuclear Regulatory Commission (NRC).

Information for the preparation of this SOWP indicates active remediation is the most likely compliance strategy for the Monument Valley site. Additional data are needed to determine the most effective remediation technology. These data will help define the horizontal and vertical extent of ground water contamination, characterize aquifer properties, and define biological and geochemical process in the aquifer. These data then will be used to determine the effectiveness of a site-specific compliance strategy and the feasibility of remediation technology. 


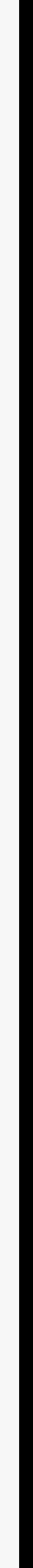




\section{TABLE OF CONTENTS}

\section{Section}

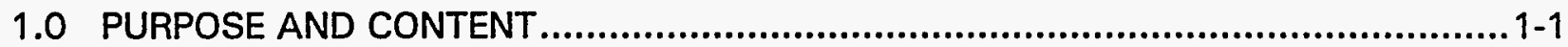

1.1 ground water compliance strategy .................................................... 1-2

1.2 Relationship to programmatic UMTRA Project ground water documents ............1-2

1.3 Relationship to site-specific documents ................................................. $1-2$

2.0 REGULATORY FRAMEWORK ....................................................................

2.1 Uranium mill tailings radiation control act ......................................................2-1

2.1.1 Environmental Protection Agency ground water standards ...................2-1

2.1.2 Cooperative agreements ............................................................... 2-4

2.2 National environmental policy act .................................................... $2-4$

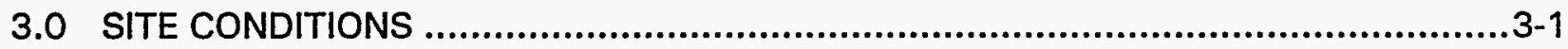

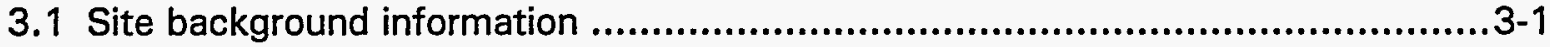

3.1.1 Data sources .........................................................................

3.1.2 Site history...........................................................................

3.1.3 Sources of ground water contamination ...................................... $3-5$

3.1.4 Quantities of waste water and processing chemicals ......................... $3-6$

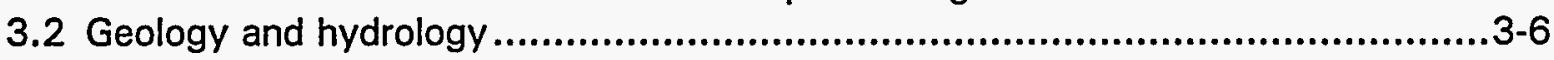

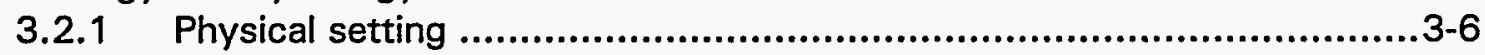

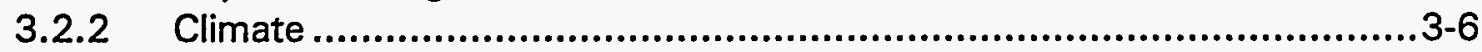

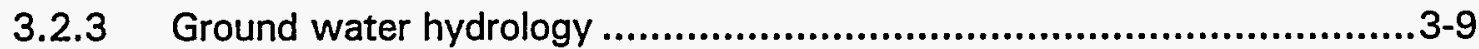

3.2.4 Surface water hydrology......................................................... $3-25$

3.2.5 Geochemical data for ground water, tailings solutions, surface water, and sediments .............................................................3-26

3.2.6 Background ground water quality.......................................... 3-27

3.2.7 Ground water flow from the De Chelly Sandstone to the alluvial aquifer ....................................................................3-28

3.2.8 Nature and extent of contamination .........................................3-36

3.2.9 Ground water contamination in the De Chelly Sandstone ..................3-52

3.2.10 Contaminant fate and transport mechanisms .................................. $3-55$

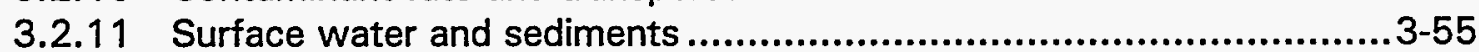

3.3 Human health risk summary ............................................................... 3-60

3.3.1 Contaminants of potential concern.......................................... 3-61

3.3.2 Potential public health impacts .............................................. $3-64$

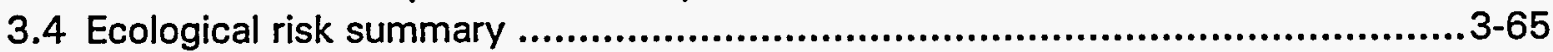

3.4.1 Potential ecological receptors and effects .......................................

3.4.2 Potential livestock and agricultural effects ................................... $3-66$

3.5 Assessment of preliminary data needs .................................................

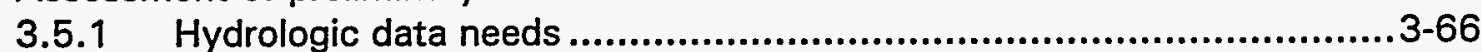

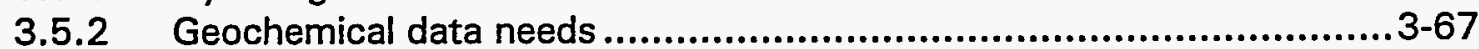

3.5.3 Risk assessment data needs...................................................... 3-68

3.6 Evaluation of interim remedial action.................................................... 3-68 
TABLE OF CONTENTS (Concluded)

Section

Page

4.0 GROUND WATER COMPLIANCE STRATEGY SELECTION

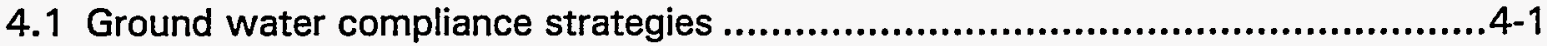

4.2 Site-specific compliance strategy selection ........................................... $4-1$

4.3 Remediation technologies ...............................................................4-3

4.4 Deviations, contingencies, and decision rules...........................................4-7

5.0 DATA COLLECTION AND ASSESSMENT ...................................................

5.1 Data collection objectives .............................................................5-1

5.2 Data collection activities ................................................................ 5-2

5.2.1 Monitor well installation..........................................................5-2

5.2.2 Water level and barometric pressure monitoring .............................5-3

5.2 .3 Aquifer testing................................................................. 5-3

5.2.4 Infiltration testing................................................................ 5-3

5.2.5 Geophysical surveys...........................................................5-3

5.2.6 Determination of background surface water and soil chemistry............ 5-4

5.2.7 Desorption and dissolution of contaminants from the aquifer matrix......5-4

5.3 Data quality objectives .................................................................5-5

5.3.1 Monitor well and well point installation...................................... 5-5

5.3.2 Water level and barometric pressure monitoring ...............................5-5

5.3.3 Aquifer testing.................................................................. 5-6

5.3.4 Infiltration testing............................................................... 5-6

5.3.5 Geophysical surveys............................................................ 5-7

5.3.6 Background surface water chemistry ........................................... 5-7

5.3.7 Background soil chemistry .................................................... 5-8

5.3.8 Contaminant desorption and dissolution from the aquifer matrix ...........5-9

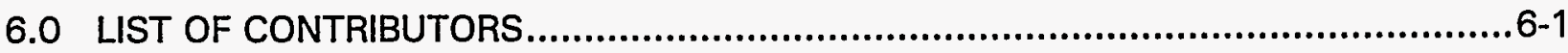

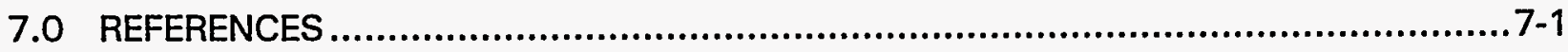

APPENDIX A - WELL LOGS (LITHOLOGIC LOGS), WELL COMPLETION LOGS ADDERIDIX R - MATERLFVFIS ALI WELLS 


\subsection{PURPOSE AND CONTENT}

The Monument Valley, Arizona, site observational work plan (SOWP) will be used to develop an approach for achieving compliance with the U.S. Environmental Protection Agency (EPA) ground water standards (40 CFR Part 192). The purpose of this SOWP is to document the observational approach, to recommend additional data collection efforts, and to assist in selecting an appropriate proposed ground water compliance strategy.

A SOWP summarizes site conditions, presents a site conceptual model based on existing characterization data, identifies the most likely site-specific compliance strategy, and defines data collection activities to address uncertainties. A site conceptual model describes the source of contaminants of potential concern and defines current site conditions and potential environmental and human health risks. This SOWP identifies data gaps in the site conceptual model and presents defensible data collection objectives (DCO) and appropriate data quality objectives (DOO) for conducting additional fieldwork. This SOWP will be reviewed to incorporate the results of suggested data collection efforts.

Section 2.0 describes the regulatory framework that guides the selection of a ground water compliance strategy for Uranium Mill Tailings Remedial Action (UMTRA) Project sites. Section 3.0 defines the current conditions at the Monument Valley site and presents the site conceptual model that includes potential environmental and human health risks supporting the ground water compliance strategy proposed at this time. Section 4.0 provides the decision-making framework used to arrive at the proposed ground water compliance strategy. Section 5.0 presents additional data collections and activities needed to refine the site conceptual model and to select a remediation technology. Section 5.0 also addresses uncertainties regarding remediation technologies.

The Monument Valley SOWP is a multi-year process of sequenced document preparation and field collection activities. Up to three versions could be prepared: Revision 0 (initial submittal), Revision 1, and Revision 2 (final revision).

The initial SOWP includes all previous information about the site, proposes a compliance strategy, describes additional data to be collected and how this data will assist in refining the most likely compliance strategy, and/or evaluates possible remediation technology. Following stakeholder review and resolution of comments, fieldwork will be conducted to collected additional data.

Revision 1 of this SOWP will include all existing site-related ground water data, evaluate the additional data collected and the DQOs used, address changes in the site conceptual model and the most likely compliance strategy as a result of new information, and summarize the results related to the DCOs. Revision 1 also will propose a pilot field program and/or laboratory program for evaluating the effectiveness of the recommended remediation technology. Following stakeholder review and comment resolution of Revision 1 , the pilot programs may be implemented.

The final revision (Revision 2) will present the most likely compliance strategy and the recommended remediation technology and will formally document the data collection 
activities and applicable modeling results. The final revision will be prepared after review by affected stakeholders and comment resolution.

\subsection{GROUND WATER COMPLIANCE STRATEGY}

The current ground water compliance strategy proposed for the Monument Valley site is active remediation. This most likely compliance strategy is based on the information presented in Section 3.0. Ground water beneath the Monument Valley site was contaminated by water infiltrating from uranium ore processing activities. The aquifer contains five potential contaminants of concern: ammonium, nitrate, manganese, sulfate, and uranium. An active remediation strategy is proposed to attain the EPA maximum concentration limits (MCL) for the UMTRA Project, alternate concentration limits $(A C L)$, or background ground water concentration levels.

\subsection{RELATIONSHIP TO PROGRAMMATIC UMTRA PROJECT GROUND WATER} DOCUMENTS

Programmatic documents that provide guidance for the SOWP include the UMTRA Ground Water Project Plan (DOE, 1993a), the programmatic environmental impact statement (PEIS) (DOE, 1996) for the UMTRA Ground Water Project, and the Technical Approach to Ground Water Restoration (TAGR) (DOE, 1993b). The UMTRA Ground Water Project Plan states the mission, need, and objectives for the UMTRA Ground Water Project and provides an overall technical plan and management approach for conducting the UMTRA Ground Water Project. The PEIS provides an objective programmatic decision-making framework for conducting the UMTRA Ground Water Project, assesses the potential programmatic impacts of conducting the Project, provides a method for determining the site-specific ground water compliance strategies, and provides data and information that can be used to analyze site-specific environmental impacts more efficiently. The TAGR provides general technical guidance for conducting the UMTRA Ground Water Project.

\section{$1.3 \quad$ RELATIONSHIP TO SITE-SPECIFIC DOCUMENTS}

A site-specific National Environmental Policy Act (NEPA) document (e.g., an environmental assessment [EA]) will be prepared to determine any potential impacts of implementing this compliance strategy.

The surface remedial action plan (RAP) provides site characterization information (DOE, 1993c). Updated, this information is used in this SOWP to formulate the site conceptual model. If the ground water compliance strategy for this site requires remedial action, a ground water RAP or surface RAP modification is prepared.

A baseline risk assessment identifies potential public health and environmental risks at the site (DOE, 1994a). This SOWP considers these potential risks and site-specific data interpreted after the risk assessment was completed, ensuring 
SITE OBSERVATIONAL WORK PLAN FOR THE

the most likely compliance strategy is protective of human health and the environment. 



\subsection{REGULATORY FRAMEWORK}

This SOWP recommends a ground water compliance strategy for the Monument Valley site so as to achieve compliance with the EPA ground water standards applicable to Title I UMTRA Project processing sites (40 CFR Part 192). The relationship of the Uranium Mill Tailings Radiation Control Act (UMTRCA), the EPA standards, the cooperative agreements, and the NEPA to the UMTRA Ground Water Project are identified below.

\subsection{URANIUM MILL TAILINGS RADIATION CONTROL ACT}

The U.S. Congress passed the UMTRCA (42 USC $\$ 7901$ et seq.) in 1978 in response to public concerns about the potential health hazards from exposure to uranium mill tailings. The UMTRCA authorized the U.S. Department of Energy (DOE) to stabilize, dispose of, and control uranium mill tailings and other contaminated materials at uranium mill processing sites.

Three UMTRCA titles apply to uranium processing sites. Title I designates 24 inactive processing sites that will undergo remediation; directs the EPA to promulgate standards; mandates remedial action in accordance with standards prescribed by the EPA; directs the remedial action to be selected and performed with the concurrence of the U.S. Nuclear Regulatory Commission (NRC) and in consultation with states and Indian tribes; directs the NRC to license the disposal sites for long-term care; and directs the DOE to enter into cooperative agreements with the affected states and Indian tribes. Title II applies to active uranium mills. Title III applies to certain uranium mills in New Mexico. The UMTRA Project is responsible for administering only Title I of the UMTRCA.

In 1988 Congress amended the UMTRCA Amendments Act, authorizing the DOE to extend without limitation the time needed to complete ground water remediation activities at the processing sites.

\subsubsection{Environmental Protection Agency ground water standards}

The UMTRCA requires the EPA to promulgate standards for protecting human health and the environment from hazardous constituents associated with uranium processing and the resulting residual radioactive materials (RRM). On 5 January 1983, the EPA published standards (40 CFR Part 192) for RRM disposal and cleanup. The standards were revised as $60 \mathrm{FR} 2854,11$ January 1995, and published in July 1995 (40 CFR Part 192).

The standards address two ground water contamination scenarios. The first addresses ground water protection after disposal cell construction; the second addresses the cleanup of residual contamination that occurred before disposal of the tailings piles (40 CFR Part 192). The UMTRA Surface Project addresses future protection of the ground water at the disposal sites, the design attributes of disposal cells and long-term surveillance plans. The UMTRA Ground Water Project addresses the residual contamination that occurred at the processing 
sites before the surface remedial action was completed and is regulated by Subparts $B$ and $C$ of the EPA standards.

Subpart B, "Standards for Cleanup of Land and Buildings Contaminated With Residual Radioactive Materials from Inactive Uranium Processing Sites" (40 CFR Part 192) requires that remedial action at processing sites is conducted to ensure the amounts of RRM in ground water meet any one of three specified criteria:

- Background level: constituent concentration in the uppermost aquifer that was not affected by processing activities.

- MCL: the EPA's maximum limit for the UMTRA Project for concentrations of certain hazardous constituents in ground water. Table 2.1 gives the MCLs for inorganic constituents that apply to UMTRA Project sites.

- $\quad$ ACL: alternate limit for a hazardous constituent that does not pose a substantial present or potential future hazard to human health or the environment, as long as the limit is not exceeded. An ACL may be applied after considering options to achieve background levels or MCLs.

Under certain specific conditions, the DOE may apply supplemental standards to contaminated ground water in lieu of background levels, MCLs, or ACLs (40 CFR Part 192). Supplemental standards may be applied if any one of the following conditions is met:

- Remedial action necessary to implement Subpart A or B would pose a significant risk to workers or the public.

- Remedial action to meet the standards would directly produce environmental harm that is clearly excessive, compared to the health benefits of remediation, to persons living on or near the sites, now or in the future.

- The estimated cost of remedial action is unreasonably high relative to the long-term benefits, and the RRM does not pose a clear present or future hazard.

- There is no known remedial action.

- The remediation of ground water quality at any processing site is technically impractical from an engineering standpoint.

- The ground water is classified as limited use ground water. Subpart B of the EPA standards defines limited use ground water as ground water that is not a current or potential source of drinking water because total dissolved solids (TDS) exceed 10,000 milligrams per liter $(\mathrm{mg} / \mathrm{L})$; there is widespread 
Table 2.1 Maximum concentration limits of inorganic constituents for ground water protection at UMTRA Project sites

\begin{tabular}{lc}
\hline \multicolumn{1}{c}{ Constituent } & $\mathrm{MCL}^{\mathrm{a}}$ \\
\hline Arsenic & 0.05 \\
Barium & 1.0 \\
Cadmium & 0.01 \\
Chromium & 0.05 \\
Lead & 0.05 \\
Mercury & 0.002 \\
Molybdenum & 0.1 \\
Nitrate (as $\mathrm{NO}_{3}$ ) & $10.0^{\mathrm{b}}$ \\
Selenium & 0.01 \\
Silver & 0.05 \\
Combined radium-226 and radium-228 & $5 \mathrm{pCi} / \mathrm{L}$ \\
Combined uranium-234 and uranium-238 & $30 \mathrm{pCi} / \mathrm{L}^{\mathrm{c}}$ \\
Gross alpha-particle activity & $15 \mathrm{pCi} / \mathrm{L}$ \\
(excluding radon and uranium) & \\
\hline Note: Information based on 40 CFR $\$ 264.94$ and & $\mathrm{CFR}$ \\
\hline
\end{tabular}

Note: Information based on 40 CFR $\$ 264.94$ and 40 CFR

Part 192.

${ }^{a}$ Milligrams per liter (mg/L) unless otherwise noted.

${ }^{\mathrm{b}}$ Equivalent to $44 \mathrm{mg} / \mathrm{L}$ nitrate as nitrate $\left(\mathrm{NO}_{3}\right)$.

${ }^{c}$ Equivalent to $0.044 \mathrm{mg} / \mathrm{L}$, assuming secular equilibrium between uranium-234 and -238 .

$\mathrm{pCi} / \mathrm{L}$ - picocuries per liter.

ambient contamination that cannot be cleaned up using treatment methods reasonably employed in public water supply systems; or the quantity of water available to a well is less than 150 gallons (gal) (570 liters [L]) per day (40 CFR Part 192). When limited use ground water applies, supplemental standards ensure that current and reasonably projected uses of the ground water are preserved (40 CFR Part 192).

- Radiation from radionuclides other than radium-226 and its decay products (e.g., thorium-230) is present in sufficient quantity and concentration to constitute a significant radiation hazard from RRM.

Subpart B allows natural flushing as a way of meeting the EPA standards. Natural flushing lets natural processes reduce the ground water contamination to EPA standards (background levels, MCLs, or ACLs), which must be met within 100 years. In addition, ground water must not be a current or projected source 
of drinking water during the natural flushing period. Institutional controls (measures that restrict access to contamination, protect human health, and satisfy beneficial uses of ground water) must be established and maintained during the natural flushing period.

Subpart C, "Implementation," provides guidance for implementing methods and procedures that will reasonably assure the provisions of Subpart B are satisfied. Subpart $C$ requires meeting the conditions of Subpart $B$ on a site-specific basis, using information gathered from site characterization and monitoring. Subpart $C$ also requires that the plan meet the conditions of Subpart $B$, as stated in the compliance strategy plan or RAP that contains the compliance strategy; a demonstration of effectiveness; and a monitoring program, if required.

\subsubsection{Cooperative agreements}

The UMTRCA requires that remedial action includes full participation of the affected states and Indian tribes on whose lands the uranium mill tailings are located. The UMTRCA also directs the DOE to enter into cooperative agreements with these states and Indian tribes.

\section{$2.2 \quad$ NATIONAL ENVIRONMENTAL POLIICY ACT}

Implementation of the UMTRCA represents a major federal action subject to the requirements of the NEPA of 1969 (42 USC $\$ 4321$ et seq.). The Council on Environmental Quality's regulations that implement the NEPA are codified in 40 CFR Parts 1500-1508. These regulations require each federal agency to develop its own implementing procedures (40 CFR Part 1500). The DOE NEPA regulations are contained in the "National Environmental Policy Act Implementing Procedures" (10 CFR Part 1021). DOE guidance is provided in Recommendations for the Preparation of Environmental Assessments and Environmental Impact Statements (DOE, 1993d).

Pursuant to the NEPA, the DOE drafted an UMTRA Ground Water Project PEIS to analyze the potential impacts of implementing four programmatic alternatives for ground water compliance at the UMTRA Project processing sites (DOE, 1996). The DOE will select the preferred alternative, which will be published in a record of decision. All subsequent Ground Water Project activity must comply with this record of decision.

The environmental impacts from implementing the proposed compliance strategies presented in the final Monument Valley SOWP will be addressed in a site-specific NEPA document that will meet NEPA requirements. 


\subsection{SITE CONDITIONS}

The Monument Valley site is on the Navajo Reservation in northern Arizona (Figure 3.1). It is accessed by Bureau of Indian Affairs (BIA) Navajo Service Road 6440, approximately 5 miles (mi) (8 kilometers [km]) south of the Arizona-Utah border and $14 \mathrm{mi}(22 \mathrm{~km})$ south of the Mexican Hat UMTRA Project disposal site. The Monument Valley site is not actually within Monument Valley, but in the adjacent Cane Valley to the east separated by Yazzie Mesa. The site is the location of a uranium mill that operated from 1955 through 1968 (Figure 3.2).

\subsection{SITE BACKGROUND INFORMATION}

\subsubsection{Data sources}

This SOWP includes observations from site visits and review of data and documentation available at the UMTRA Project Document Control Center in Albuquerque, New Mexico. Merritt (1971) provides detailed descriptions of the uranium concentration process, mill byproducts, and process waste streams. Geologic and hydrologic conditions for the area are reported in Witkind and Thaden (1963), Cooley et al. (1969), Irwin et al. (1971), and James (1973). Site-specific hydrogeologic conditions are described in the engineering assessment (DOE, 1981), the EA (DOE, 1989), the RAP (DOE, 1993c), the baseline risk assessment (DOE, 1994a), and the water sampling and analysis plan (DOE, 1994b).

Additional site-specific information was obtained by examining monitor well completion reports and ground water chemistry data contained in the DOE's Software Program for Environmental Analysis and Reporting (SPEAR) (DOE, 1993 e). The SPEAR data include the results of periodic water quality and static water level data monitoring at the site from 1980 to 1995.

\subsubsection{Site history}

Mining in the Monument Valley site vicinity began in 1942 at Monument No. 2 Mine, approximately $1 \mathrm{mi}(1.6 \mathrm{~km})$ west of the site (Figure 3.3). Monument No. 2 Mine operated as a strip mine from 1942 through 1949 (Witkind and Thaden, 1963). Underground and strip mining were conducted from 1949 until the mill closed in 1968.

Before 1955, there was no mill at the site and all ore was trucked to uranium mills in Durango and Naturita, Colorado. Mill operations began at the Monument Valley site in 1955 . From 1955 through 1964 the mill was a mechanical separator. In this operation, ore was crushed and sorted by grain size. The finer-grained material, which was higher in uranium content, was shipped off-site for chemical concentration to the Durango and Naturita, Colorado, mills (FBDU, 1981). No chemicals were used, other than minor amounts of flocculants. 
Figure 3.1

Site Location Map

Monument Valley, Arizona

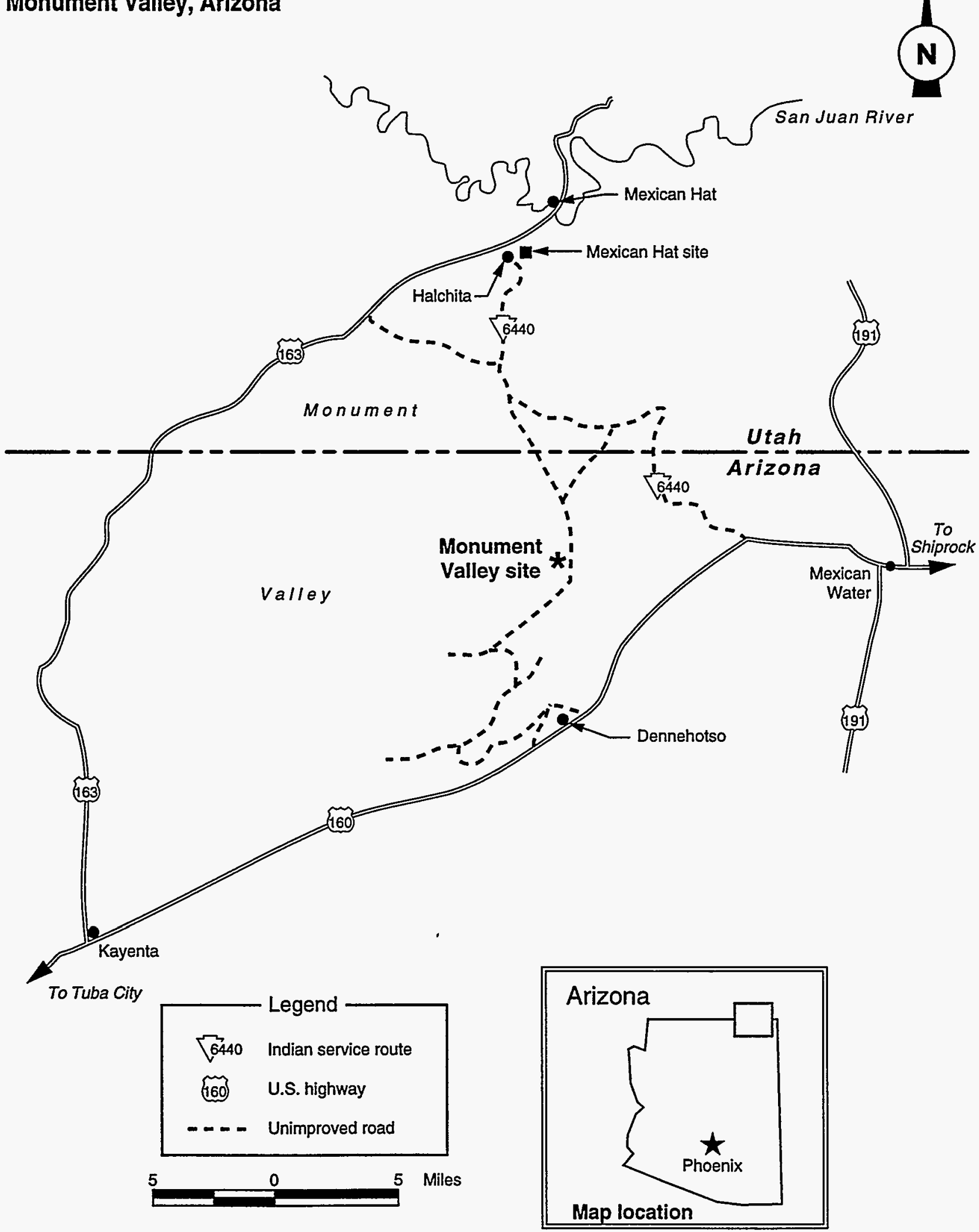

MAC: STEMONSOWPISITELOC 


\section{Figure 3.2}

\section{Location of Uranium Mill and Monitor Wells}

Monument Valley, Arizona, Site
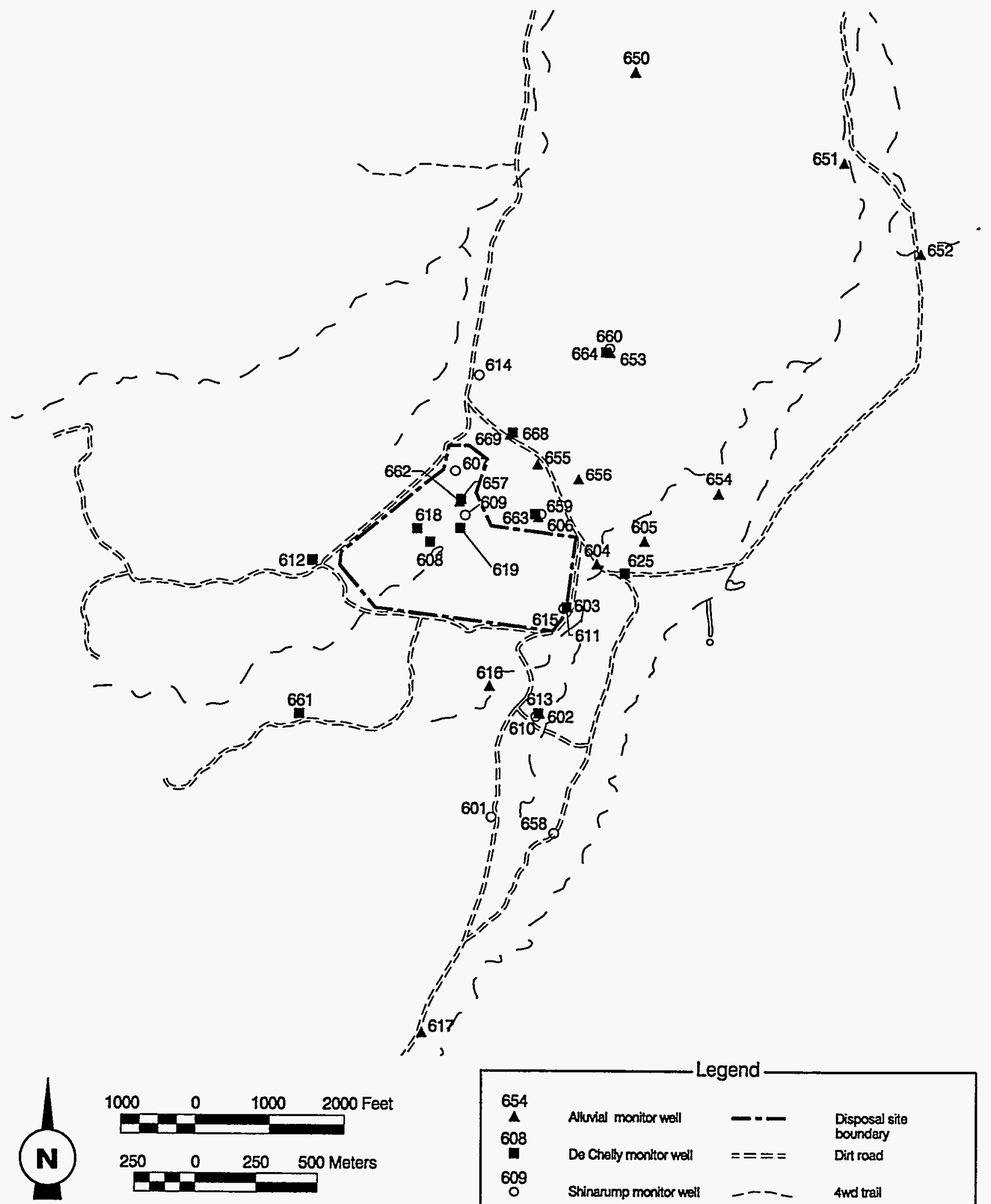

Legend

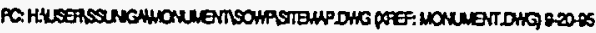

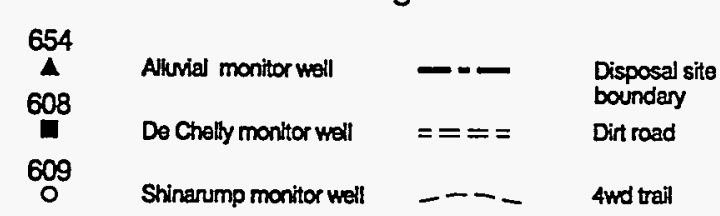


Figure 3.3

Former Mill Operating Area, Monument Valley, Arizona, Site

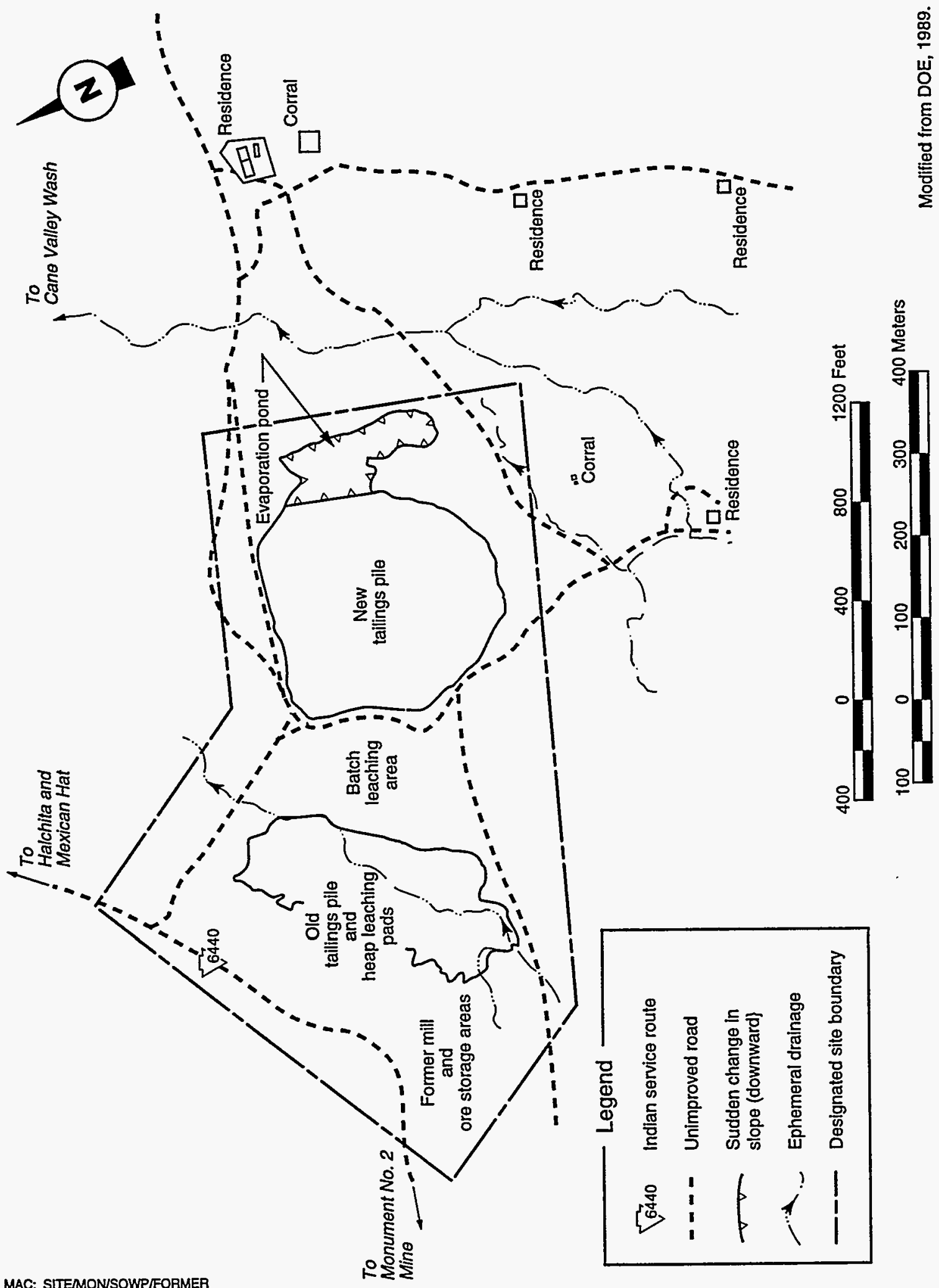


However, large amounts of water were used to separate the fine- and coarsegrained materials. The coarser-grained material remained on the site and was piled in the areas identified as former mill and old tailings (Figure 3.3). The water came from on-site wells in the De Chelly Formation.

Phase II of the mill operation commenced in 1964 after installation of batch leaching equipment. This process continued for approximately 3 years, during which approximately $1,000,000$ tons of sandy tailings were processed 1925 tons per day). A separate heap leaching operation was used on an additional 100,000 tons of low-grade ore. Both operations required large quantities of chemicals to remove the uranium and vanadium from the former tailings and low-grade ore (Merritt, 1971).

The mill buildings and milling equipment were removed after 1968 . Beginning in 1992 , the tailings piles, windblown tailings, contaminated materials, concrete foundations, and debris were removed from the site and placed in the Mexican . Hat UMTRA Project disposal cell. Relocation of these materials was completed in January 1994.

The mill site was leased from the Navajo Nation until 1968, when the mill closed and the lease expired. Control of the site, structures, and materials reverted to the Navajo Nation at that time (DOE, 1989).

\subsubsection{Sources of ground water contamination}

Some ground water contamination probably occurred during the mechanical processing period (1955 to 1964) as a result of water draining from stockpiles of the finer-grained material and, to a lesser extent, from the sandy tailings that were placed as a slurry. The primary contaminants would have been the relatively soluble components of the ore: uranium, calcium, and sulfate (the source of calcium and sulfate would have been gypsum, which was part of the ore body). Infiltration of the contaminated water would have occurred at the areas designated former mill and old tailings (Figure 3.3). The amount of infiltrated water and concentrations of contaminants is unknown. Section 3.2 presents further information on the chemistry of this possible source of contaminants.

Both the batch and heap leaching operations used sulfuric acid to leach out uranium and molybdenum. The process used to remove the uranium and vanadium from the batch leaching solution is not known, but an ion exchange process probably was used. The ion exchange resin probably was regenerated with a nitrate solution. After the uranium and vanadium were extracted from this regenerant liquid, the remaining spent liquid probably was discharged to the new tailings pile along with the tailings. Merritt (1971) states that the sulfuric acid heap leaching solution was neutralized to $\mathrm{pH} 4$ with ammonia; then lime (calcium oxide) was added to raise the $\mathrm{pH}$ to 7 to produce a bulk precipitate, which was trucked to the Shiprock, New Mexico, where the uranium and 
Figure 3.5

East-West Cross Section

Monument Valley, Arizona, Site Area

Feet (msl)

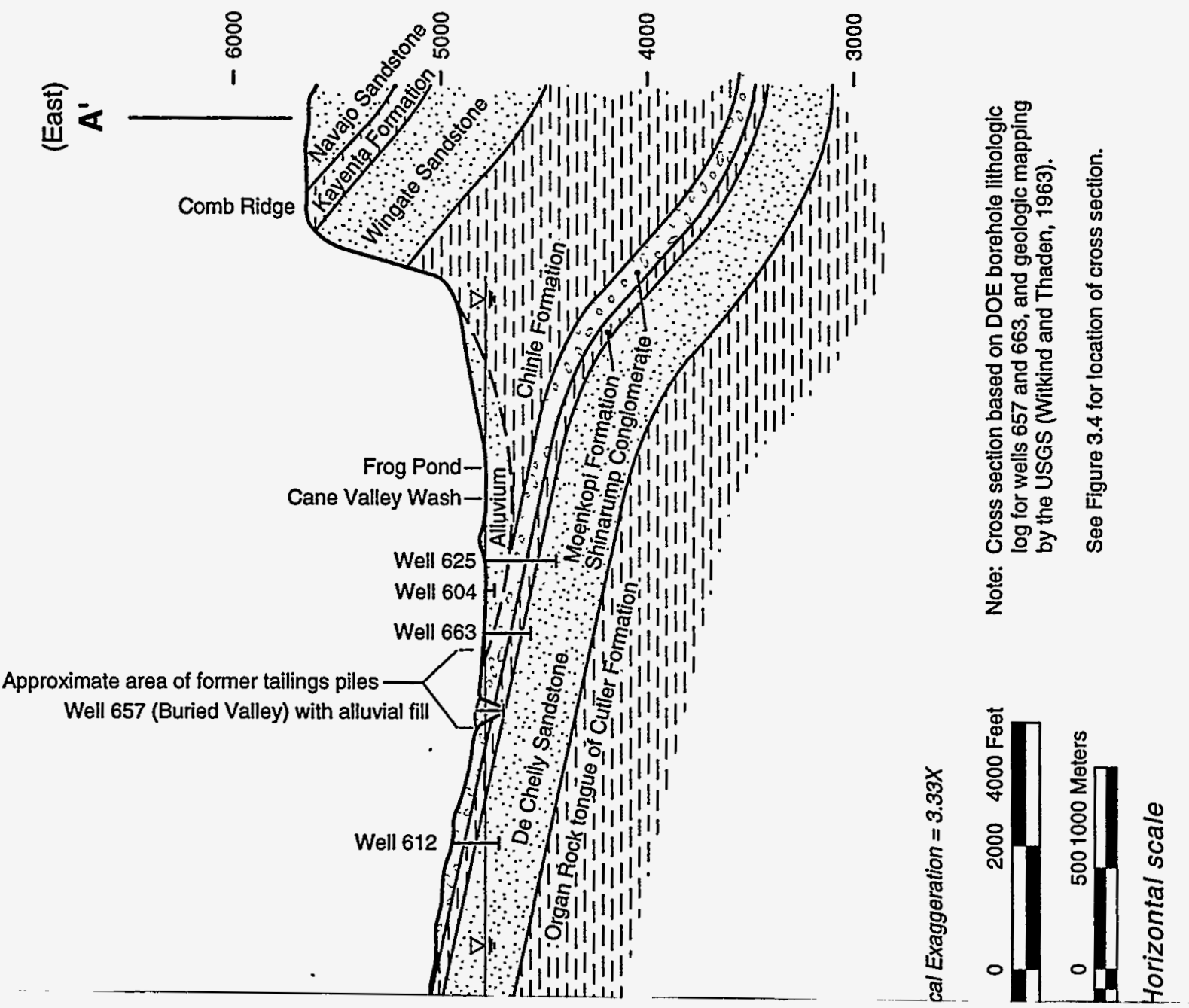


Formation, and 4) the De Chelly Sandstone of the Cutler Formation (Witkind and Thaden, 1963). Figure 3.6 shows monitor well locations at the site.

Well logs prepared when monitor wells were installed at the site were used to prepare the cross sections. The geologic units (e.g., Moenkopi Formation, De Chelly Sandstone) were identified by comparing the descriptions of Witkind and Thaden (1963) to the lithologic descriptions on the well logs and observations of geologic outcrops at and near the site. Copies of the well logs and well completion details are presented in Appendix $A$ and are summarized in Table 3.2. The table includes total well depth, screen and sand pack interval, elevation at the top of the well casing, and the hydrologic unit monitored by each well. Table 3.3 summarizes the hydrologic properties of each geologic unit.

\section{Alluvial_aquifer}

Geology. The alluvial aquifer consists of windblown deposits of fine- to medium: grained sands and water-transported sand, gravel, and bedrock fragments. Because the alluvial aquifer is derived in part from the nearby uranium-bearing rock units, it naturally contains uranium.

The sediments that comprise the alluvial aquifer are limited in areal extent and vary in thickness. The unit is more than $100 \mathrm{ft}(30 \mathrm{~m})$ thick in the center of Cane Valley, but thins toward each edge of the valley and is not present at the bedrock exposures along the eastern and western sides of the valley. The alluvium is thickest $(120 \mathrm{ft}[37 \mathrm{~m}])$ at well 657 . (Note: All well locations at this site are numbered MON-01-0xxx. This report uses only the last three numbers to identify well location.)

Figure 3.7 shows the thickness and extent of the alluvium in the site vicinity. The areal extent of the alluvium was estimated from aerial photographs of the site and from Witkind and Thaden (1963). The alluvium thickness was estimated from well logs (Appendix A).

The above information (i.e., well logs, aerial photographs, and the geologic report) and field observations of the surficial geology and rock outcrops strongly indicate there are buried channels covered by alluvial and windblown sands near the center of Cane Valley and under a portion of the UMTRA site. For example, the alluvium is thickest at well 657 where it is reported to be $120 \mathrm{ft}(37 \mathrm{~m})$ and neither the Shinarump Conglomerate or Moenkopi Formation are present at that location (Figure 3.5).

The buried channels were created before or during the early periods of alluvial deposition and eroded through the Shinarump Conglomerate and portions (or, in some places, all) of the Moenkopi Formation. The channels result in thicker areas of the alluvium and a more direct connection (hydraulically) between the alluvial aquifer and the De Chelly Sandstone. 
Figure 3.6

\section{Monitor Well and Surface Sampling Locations} Monument Valley, Arizona, Site

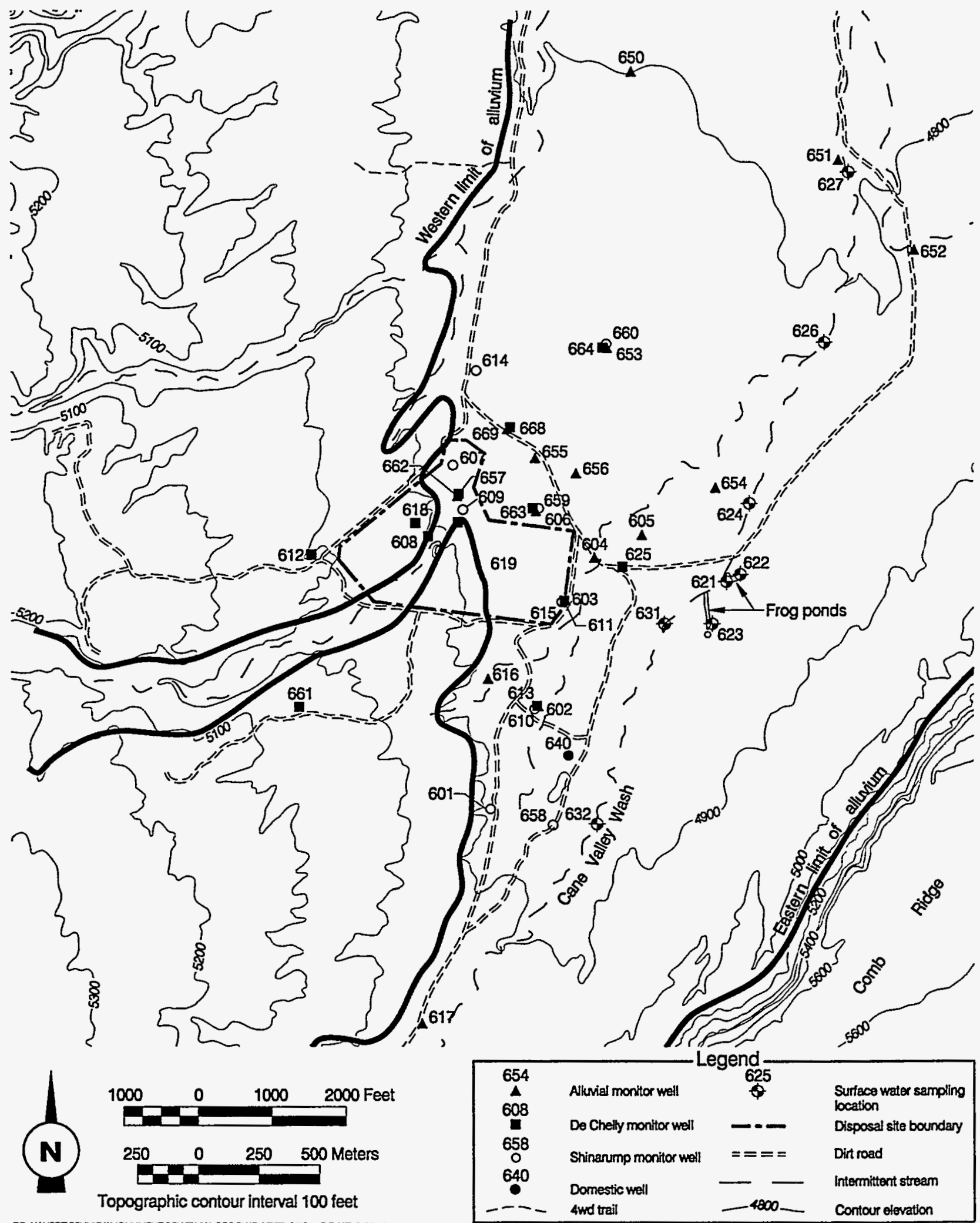


Table 3.2 Well construction summary, Monument Valley, Arizona, site

REPORT DATE: 02/26/96

\begin{tabular}{|c|c|c|c|c|c|c|c|c|c|c|c|c|}
\hline$\underset{\text { ID }}{\text { LOCATIOH }}$ & $\begin{array}{l}\text { MONTH } \\
\text { COONDIMATE } \\
\text { (FT) }\end{array}$ & $\begin{array}{c}\text { EAST } \\
\text { coopdimte } \\
\text { (FT) }\end{array}$ & $\begin{array}{c}\text { EROUM } \\
\text { ELEVATION } \\
\text { (FT HSLS }\end{array}$ & $\begin{array}{l}\text { COREHOLE } \\
\text { DEPTH } \\
\text { (FT) }\end{array}$ & $\begin{array}{l}\text { COREHOLE } \\
\text { OINETER } \\
\text { (INCHES) }\end{array}$ & $\begin{array}{l}\text { CASING } \\
\text { ELEVATION } \\
\text { (FT NSL) }\end{array}$ & $\begin{array}{l}\text { CASING } \\
\text { LENGTH } \\
\text { (FT) }\end{array}$ & $\begin{array}{l}\text { CASING } \\
\text { OINWETER } \\
\text { (INCHES) }\end{array}$ & $\begin{array}{c}\text { SCREEN } \\
\text { BEGIMNING } \\
\text { DEPTH (FT) }\end{array}$ & $\begin{array}{l}\text { SCREEN } \\
\text { LENGTH } \\
\text { (FT) }\end{array}$ & $\begin{array}{l}\text { FLOU } \\
\text { CODE }\end{array}$ & $\begin{array}{c}\text { FORMTION } \\
\text { OF } \\
\text { COMPLETION }\end{array}$ \\
\hline $\begin{array}{l}0601 \\
0602 \\
0603 \\
0604 \\
0605 \\
0606 \\
0607 \\
0608 \\
0609 \\
0610 \\
0611 \\
0612 \\
0613 \\
0614 \\
0615 \\
0616 \\
0617 \\
0618 \\
0619 \\
0625 \\
0640 \\
0650 \\
0651 \\
0652 \\
0653 \\
0654 \\
0655 \\
0656 \\
0657 \\
0658 \\
0659 \\
0660 \\
0661 \\
0662 \\
0663 \\
0664 \\
0668 \\
0669\end{array}$ & $\begin{array}{l}54980.4 \\
56377.4 \\
57812.7 \\
58397.0 \\
58707.6 \\
59034.2 \\
59657.2 \\
58685.7 \\
59052.1 \\
56338.8 \\
57811.0 \\
58437.2 \\
56377.8 \\
60940.5 \\
57794.4 \\
56747.3 \\
52090.6 \\
58868.3 \\
58876.5 \\
58272.5 \\
55815 \\
64970.2 \\
63788.9 \\
62581.5 \\
61249.3 \\
59351.4 \\
59754.6 \\
59545.2 \\
59266.0 \\
54763.4 \\
59069.8 \\
61303.4 \\
56366.1 \\
59236.5 \\
59059.6 \\
61255.9 \\
60171.4 \\
60146.3\end{array}$ & $\begin{array}{l}88017.7 \\
80661.2 \\
89036.9 \\
89424.6 \\
90066.7 \\
88634.0 \\
87519.2 \\
87189.4 \\
87652.9 \\
88611.6 \\
89017.5 \\
85614.8 \\
88643.7 \\
87832.6 \\
88940.7 \\
87967.6 \\
87094.4 \\
87016.1 \\
87587.9 \\
8903.1 \\
89010 \\
89923.8 \\
92734.3 \\
93759.3 \\
89596.3 \\
91063.4 \\
88624.1 \\
89175.1 \\
87596.5 \\
88857.0 \\
88670.3 \\
89584.3 \\
85447.7 \\
87577.5 \\
88593.5 \\
89537.0 \\
88287.0 \\
88247.8\end{array}$ & $\begin{array}{l}4881.51 \\
4861.53 \\
4846.64 \\
4837.30 \\
4831.42 \\
4860.44 \\
4869.41 \\
4901.08 \\
4878.30 \\
4860.83 \\
4846.35 \\
5000.09 \\
4860.81 \\
4854.28 \\
4847.50 \\
4869.00 \\
4907.96 \\
4920.00 \\
4886.52 \\
4838.80 \\
4875 \\
4790.45 \\
4781.46 \\
4800.69 \\
4832.14 \\
4824.41 \\
4857.73 \\
4852.07 \\
4881.18 \\
4876.19 \\
4860.84 \\
4831.38 \\
5057.34 \\
4877.07 \\
4861.56 \\
4832.84 \\
4864.27 \\
4863.19\end{array}$ & $\begin{array}{r}24.00 \\
35.00 \\
55.00 \\
30.00 \\
32.00 \\
47.00 \\
30.00 \\
120.00 \\
15.00 \\
130.5 \\
185.00 \\
215.00 \\
160.00 \\
84.50 \\
110.00 \\
- \\
5 \\
153.00 \\
154.00 \\
89.00 \\
. \\
99.50 \\
82.00 \\
56.00 \\
76.00 \\
79.00 \\
60.00 \\
60.00 \\
140.00 \\
165.00 \\
110.00 \\
155.00 \\
218.00 \\
70.00 \\
217.00 \\
233.00 \\
218.00 \\
56.00\end{array}$ & $\begin{array}{l}6.625 \\
6.625 \\
6.625 \\
6.625 \\
6.625 \\
6.625 \\
6.625 \\
6.625 \\
6.625 \\
6.625 \\
6.625 \\
6.625 \\
6.625 \\
8.0 \\
6.625 \\
- \\
- \\
12.000 \\
12.000 \\
12.000 \\
. \\
7.875 \\
7.875 \\
7.875 \\
7.875 \\
7.875 \\
7.875 \\
7.875 \\
7.875 \\
7.875 \\
7.875 \\
7.875 \\
7.875 \\
7.875 \\
7.875 \\
7.875 \\
7.875 \\
7.875\end{array}$ & $\begin{array}{r}4884.43 \\
4864.02 \\
4848.56 \\
4839.09 \\
4833.96 \\
4863.31 \\
4871.26 \\
4903.11 \\
4880.17 \\
4861.81 \\
4848.35 \\
5001.74 \\
4862.82 \\
4855.68 \\
4849.29 \\
4869.92 \\
4908.96 \\
4921.02 \\
4887.42 \\
4840.09 \\
4-5 \\
4793.29 \\
4784.51 \\
4803.84 \\
4834.94 \\
4827.01 \\
4860.39 \\
4854.61 \\
4883.21 \\
4878.82 \\
4863.63 \\
4834.16 \\
5059.99 \\
4879.81 \\
4864.41 \\
4835.76 \\
4866.71 \\
4865.78\end{array}$ & $\begin{array}{r}24.00 \\
31.50 \\
55.00 \\
30.00 \\
31.00 \\
47.00 \\
27.50 \\
120.00 \\
14.0 \\
85.00 \\
185.00 \\
215.00 \\
160.00 \\
70.00 \\
90.00 \\
8.00 \\
153.00 \\
154.00 \\
89.00 \\
0 \\
99.50 \\
82.00 \\
58.00 \\
78.00 \\
79.00 \\
60.00 \\
60.00 \\
138.00 \\
157.00 \\
109.00 \\
155.00 \\
212.00 \\
69.50 \\
217.00 \\
233.00 \\
215.00 \\
56.00\end{array}$ & $\begin{array}{r}2.000 \\
2.000 \\
2.000 \\
2.000 \\
2.000 \\
2.000 \\
2.000 \\
2.000 \\
2.000 \\
2.000 \\
2.000 \\
2.000 \\
2.000 \\
2.000 \\
2.000 \\
4 \\
4 \\
12.000 \\
12.000 \\
12.000 \\
4 \\
4.000 \\
4.000 \\
4.000 \\
4.000 \\
4.000 \\
4.000 \\
4.000 \\
4.000 \\
4.000 \\
4.000 \\
4.000 \\
4.000 \\
4.000 \\
4.000 \\
4.000 \\
4.000 \\
4.000\end{array}$ & $\begin{array}{r}12.00 \\
19.50 \\
43.00 \\
13.00 \\
14.00 \\
32.00 \\
12.50 \\
90.00 \\
7.00 \\
63.00 \\
163.00 \\
175.00 \\
138.00 \\
48.00 \\
68.00 \\
- \\
- \\
- \\
- \\
- \\
2 \\
77.50 \\
20.00 \\
34.00 \\
56.00 \\
57.00 \\
38.00 \\
38.00 \\
121.00 \\
135.00 \\
87.00 \\
133.00 \\
190.00 \\
37.50 \\
175.00 \\
211.00 \\
180.00 \\
34.00\end{array}$ & $\begin{array}{l}10.0 \\
10.0 \\
10.0 \\
15.0 \\
15.0 \\
10.0 \\
10.0 \\
20.0 \\
5.0 \\
20.0 \\
20.0 \\
20.0 \\
20.0 \\
20.0 \\
20.0 \\
- \\
. \\
- \\
- \\
- \\
20 \\
20.0 \\
60.0 \\
20.0 \\
20.0 \\
20.0 \\
20.0 \\
20.0 \\
15.0 \\
20.0 \\
20.0 \\
20.0 \\
20.0 \\
30.0 \\
40.0 \\
20.0 \\
20.0 \\
20.0\end{array}$ & 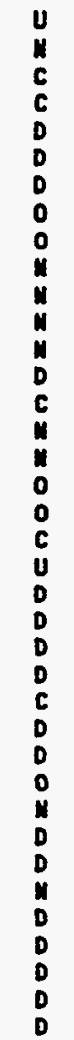 & $\begin{array}{l}S R \\
A L \\
A L \\
A L \\
A L \\
A L \\
S R \\
D C \\
S R \\
S R \\
D C \\
D C \\
D C \\
S R \\
S R \\
A L \\
A L \\
D C \\
D C \\
D C \\
N R \\
A L \\
A L \\
A L \\
A L \\
A L \\
A L \\
A L \\
D C \\
S R \\
S R \\
S R \\
D C \\
A L \\
D C \\
D C \\
D C \\
A L\end{array}$ \\
\hline QHTIO & $\mathrm{co}$ & COE: & . & & & & & & \multicolumn{4}{|c|}{ 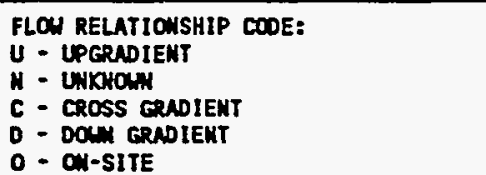 } \\
\hline
\end{tabular}

DATA FILE: DARTVONOTUMI 10002.0AT

FIELS DISPLAYED WITH A DASH INOICATE THE DATA IS WUAVILABLE

Note: Wells 618, 619, and 625 are former Vanadium Corporation of America water wells. Wells 616,617 , and 640 are private wells. All other wells are DOE monitoring wells. 


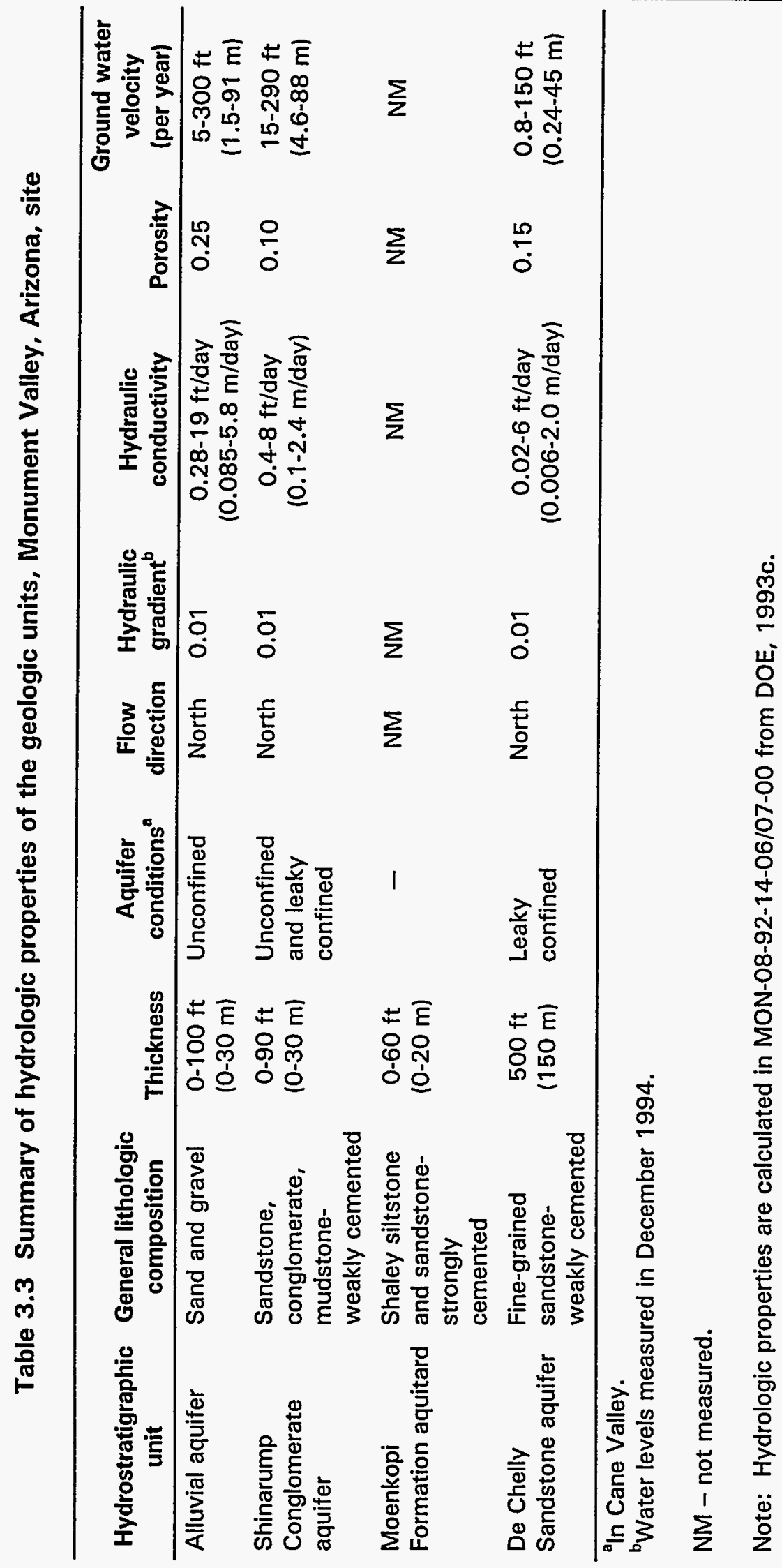




\section{Figure 3.7}

\section{Thickness of Alluvium}

\section{Monument Valley, Arizona, Site}

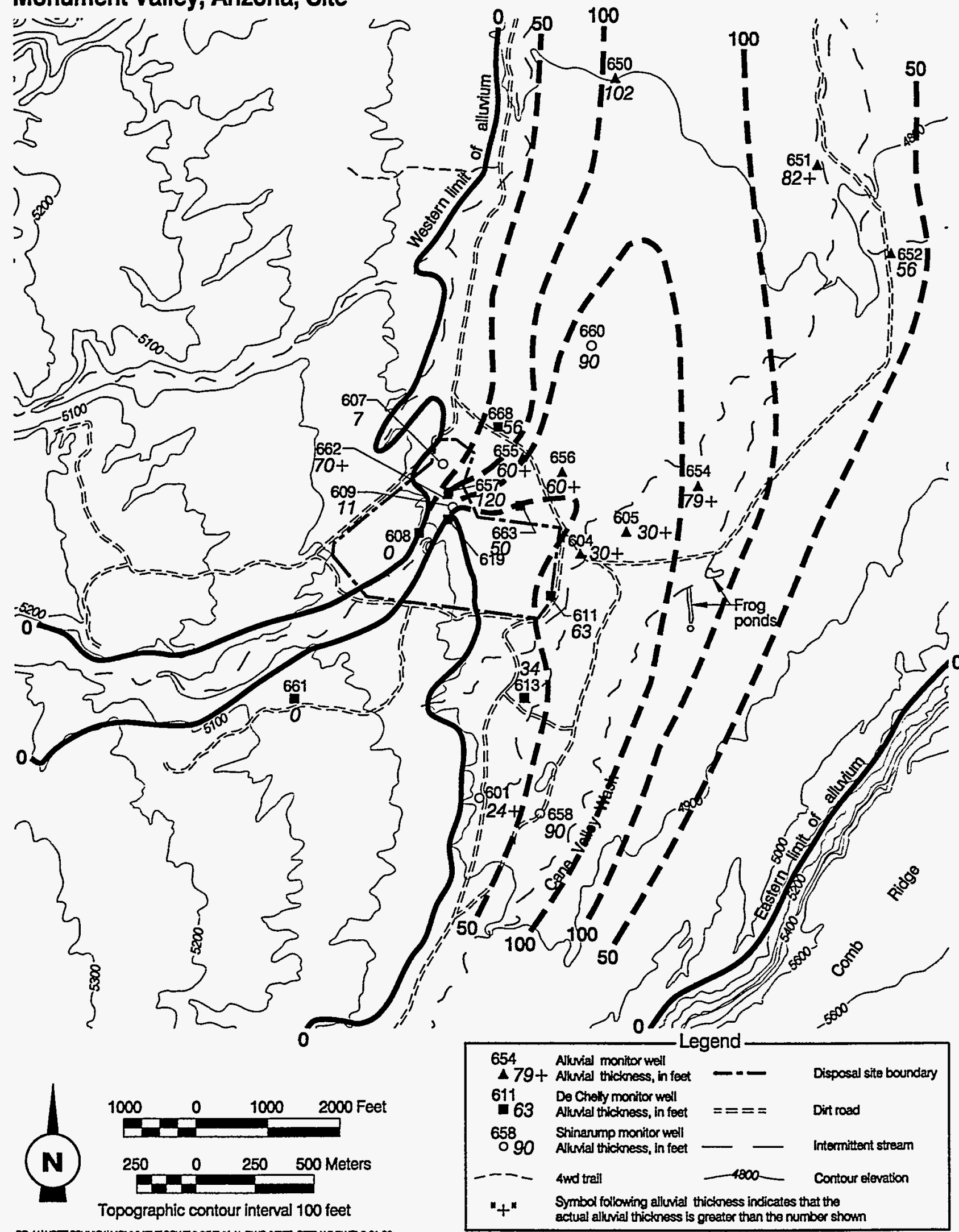

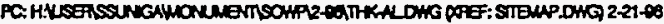


Hydrology. Ground water occurring within the alluvial aquifer is generally unconfined, however, deeper alluvial deposits show water levels that indicate either leaky or confined conditions. For example, well 654, which is completed near the base of the alluvium, yields water levels exceeding the ground surface. Depths to ground water range from the land surface near the center of Cane Valley Wash to slightly more than $30 \mathrm{ft}(10 \mathrm{~m})$ below grade near the site and the approximate center of Cane Valley.

A comparison of water-level measurements (Appendix B) shows slight differences in water-level elevations over time. However, the water table contour map (Figure 3.8) is representative of the water-table configuration and can be used to determine ground water flow direction and gradient.

Overall, ground water flows north in the alluvial aquifer in the site vicinity, with some localized variations. For example, water levels in well 654 are anomalously high and water-table contours near the Frog Ponds may indicate leakage to the alluvial aquifer from the De Chelly Sandstone. Because the potentiometric surface of the De Chelly Sandstone is at or above the elevation of the water table and land surface in that area, water will flow at the ground surface from the De Chelly Sandstone. Geochemical data supporting recharge of the alluvial aquifer from the De Chelly Sandstone are presented in Section 3.2.7.

Upward leakage of ground water from the De Chelly Sandstone to the Frog Ponds also may have influenced the configuration of the contaminant plume. Vertical ground water flow may have prevented contaminants from migrating downward into the Shinarump Conglomerate. Section 3.2.8 details the extent of site-related contamination.

Water-level measurements taken during December 1994 (Figure 3.8) show the hydraulic gradient in the alluvial aquifer is about 0.01 in the site vicinity.

Hydraulic conductivity computed from aquifer pumping test data resulted in values ranging from 0.28 to $19 \mathrm{ft}(01$ to $6 \mathrm{~m})$ per day for the alluvial aquifer. Appendix F of the Monument Valley and Mexican Hat RAP includes the hydraulic conductivity calculations (DOE, 1993c).

The porosity of the alluvial aquifer has not been measured. However, most of the aquifer materials are fine- to medium-grained sand and gravel. The total porosity of sand ranges from 0.25 to 0.50 and the total porosity of gravel ranges from 0.25 to 0.40 . Because the alluvial materials are poorly sorted (i.e., the aquifer materials are a wide range of grain sizes), the porosity of the alluvial aquifer likely is at the low end of the sand and gravel porosity ranges (Freeze and Cherry, 1979). Because effective porosity (the pore spaces through which water readily travels) is lower than total porosity, the effective porosity of the alluvial aquifer is approximately 0.25. 
Figure 3.8

Alluvial Water Table Contours, 7-8 December 1994

Monument Valley, Arizona, Site

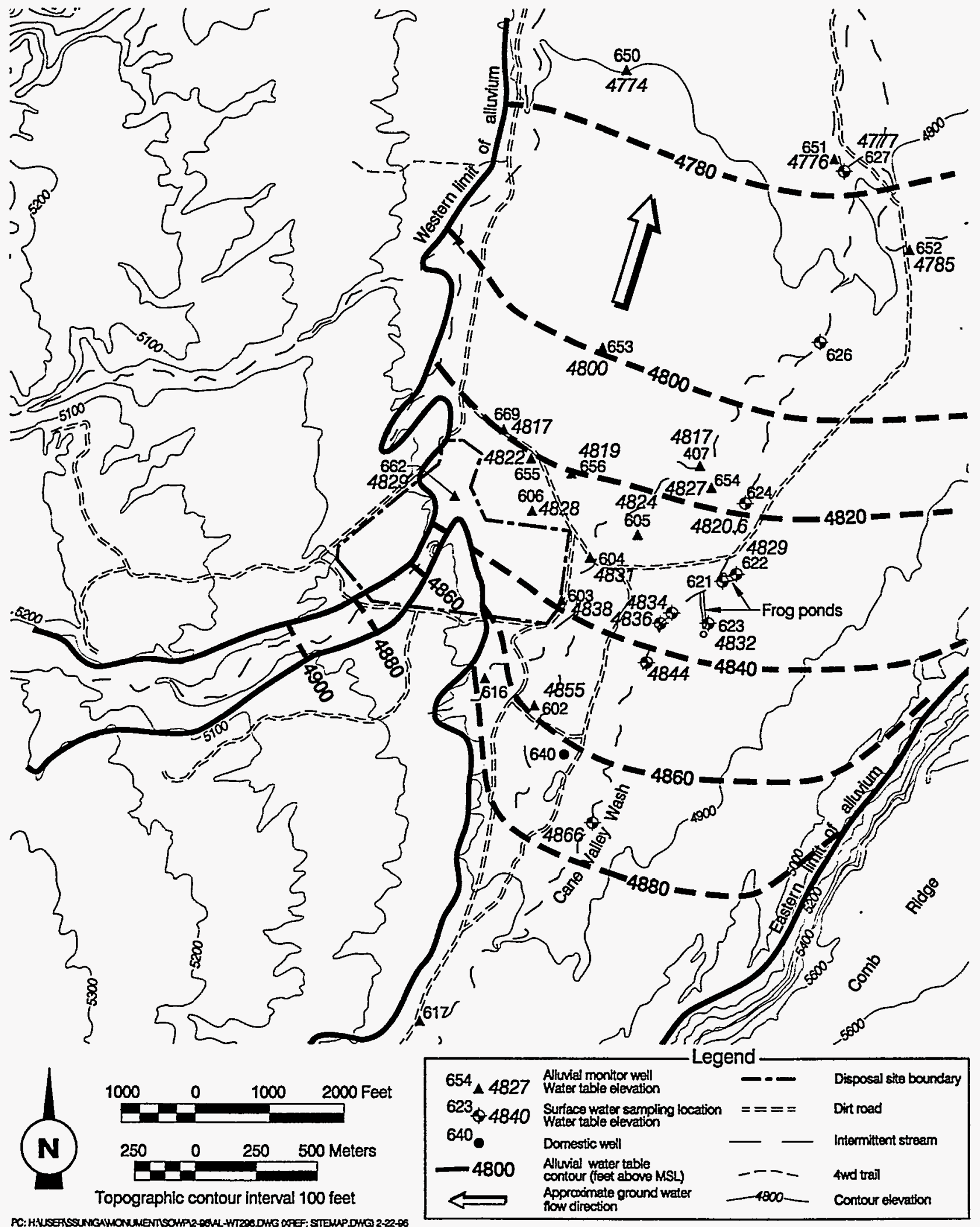


Ground water velocity is calculated from equation 5.18 a from Driscoll (1986):

$$
V_{a}=\frac{K I}{n}
$$

Where: $V_{a}$ = average ground water flow velocity

$\mathrm{K}=$ hydraulic conductivity

I = hydraulic gradient

$\mathrm{n}$ = porosity

Using these hydraulic conductivity, hydraulic gradient, and porosity data, the range of velocity values was calculated to be 5 to $300 \mathrm{ft}(1.5$ to $91 \mathrm{~m})$ per year.

A comparison can be made between the actual extent of site-related contaminants (based on water quality results from monitor wells) and the estimated time of plume migration (based on the above velocity calculation and the period of mill operation). Approximately 35 years have elapsed since the start of mill operation, yielding an estimated plume travel distance ranging from 175 to $10,500 \mathrm{ft}(53$ to $3200 \mathrm{~m})$. To calculate plume travel, multiply the ground water velocity $(300 \mathrm{ft}[90 \mathrm{~m}]$ per year) by the number of years contaminants could have been in the ground water system ( 35 years).

Based on water quality results of late 1994, the sulfate plume is at least $3000 \mathrm{ft}$ $(1000 \mathrm{~m})$ downgradient from the former mill site (i.e., it is present at well 653) but it appears to be less than about $6500 \mathrm{ft}(2000 \mathrm{~m})$ downgradient from the former mill site (i.e., it is not present at well 650 , although this well is screened in a deeper portion of the aquifer and may not intercept shallow ground water contamination). This equates to an approximate ground water velocity of about 90 to $200 \mathrm{ft}(25$ to $60 \mathrm{~m}$ ) per year, calculated by dividing the length of the plume (e.g., $3000 \mathrm{ft}[1000 \mathrm{~m}]$ ) by the number of years it was present in the ground water system (i.e., 35 years). The water quality results are consistent with the estimated plume travel distance.

Six factors could account for the differences between calculated and observed plume extent. Four relate to uncertainties in velocity equation input: 1) the estimate of the time the plume was present in the ground water system; 2) the estimate of hydraulic gradient; 3) the estimate of porosity; and 4) the estimated dispersion scale of heterogeneity changes in hydraulic gradient over time. The fifth factor relates to possible effects of the aquifer matrix on contaminant migration. The sixth is the possibility that contaminated ground water is not uniformly distributed vertically in the aquifer and therefore not intercepted by well 650. In keeping with the observational approach for site assessment and cleanup, further investigations to evaluate factors 1, 3, and 5 will be initiated only if necessary, and will be presented in a later version of this document.

Recharge to the alluvial aquifer is from precipitation and from upward leakage from the aquifers below. Recharge is also provided by discharge from artesian monitor wells 611,613 , and 615 and former production well 625 , which are 
completed in the De Chelly Sandstone and occasionally flow at ground surface. There also may be recharge to the aquifer from the De Chelly Sandstone in the area of well 654. Discharge from the alluvial aquifer is through evapotranspiration, evaporation where the water table is at or near land surface, and occasional discharges to Cane Valley Wash when and where the water table intersects the ground surface. Pumping from the alluvial aquifer is limited, accounting for very minor discharge compared to natural aquifer discharge. Pumping of the alluvial aquifer does not occur downgradient from the site.

\section{Shinarump Conglomerate}

Geology. The Shinarump Conglomerate is a heterogeneous combination of lenticular, cross-bedded formations of sandstone and conglomerate with occasional thin mudstone layers. Large quantities of fossil plant matter (as coal) and silicified wood are associated with the coarser-grained materials. The coarser-grained materials (i.e., conglomerate) are generally at the base of the unit, grading upward into finer-grained materials (i.e., sandstone and some mudstone). The most probable method of deposition for the unit was as alluvial fans (Witkind and Thaden, 1963). The Shinarump Conglomerate west of the site at the location of the Monument No. 2 Mine (Figure 3.4) was the source of vanadium and uranium ore though some mineralization was also reported to be in the upper De Chelly Sandstone (Witkind and Thaden, 1963).

The Shinarump Conglomerate is present at land surface at the site and forms the bedrock slope west of site. The unit strikes approximately due north and dips at about 4 degrees to the east (Witkind and Thaden, 1963). On portions of the site and farther east, the Shinarump is under the alluvial aquifer. It is up to up to $90 \mathrm{ft}(30 \mathrm{~m})$ thick at well 664 (near the center of Cane Valley); but it is not present at well 657, which is near the north boundary of the site. Well 657 probably is in the area of a buried channel, which (based on the well logs) appears to have eroded the unit (Figure 3.5). The Shinarump Conglomerate was replaced by alluvium at that location.

Hydrology. Ground water within the Shinarump Conglomerate occurs under both unconfined and leaky confined conditions. Water is unconfined where it is present in the unit and where outcrops of the Shinarump Conglomerate are present at land surface. In the site vicinity, unconfined portions of the Shinarump Conglomerate are not common because water usually is not present in the unit where it outcrops (i.e., the depth to water is usually greater than the depth to the bottom of the unit where it outcrops). Most water within the Shinarump Conglomerate is under semiconfined conditions. The upper portions of the unit are finer-grained and act as the semiconfining bed.

Water level measurements at the site indicate that ground water generally flows north-northeast (Figure 3.9). Using the equation presented in Section 3.2.3, the hydraulic gradient is 0.01 for the Shinarump Conglomerate between potentiometric contours of 4860 and $4800 \mathrm{ft}$. The distance between the two contours is approximately $5500 \mathrm{ft}(1700 \mathrm{~m})$. 


\section{Figure 3.9}

\section{Shinarump Conglomerate Plezometric Surface, 7-8 December 1994 Monument Valley, Arizona, Site}

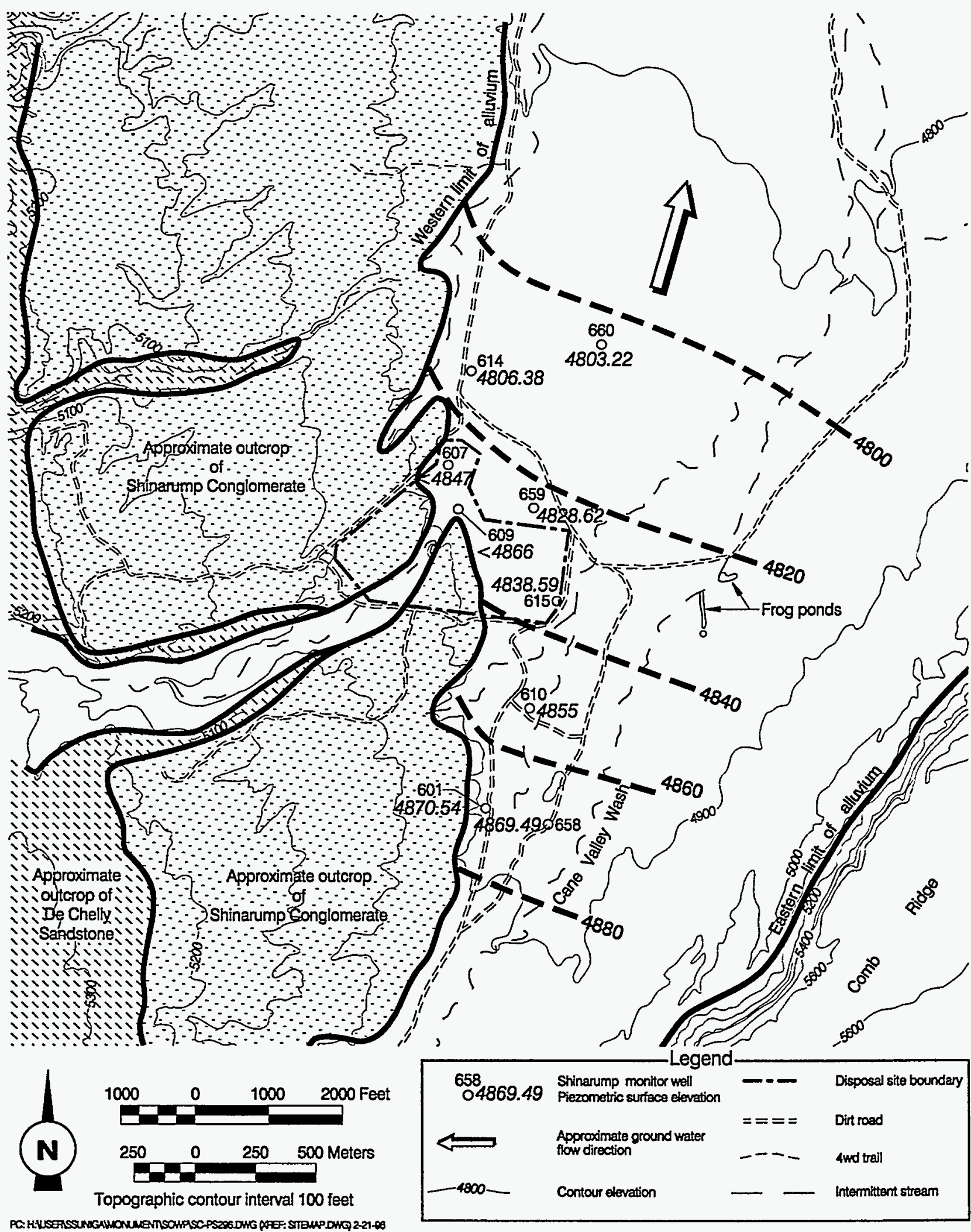


Hydraulic conductivity (computed from four slug tests in the Shinarump Conglomerate) varies from 0.4 to $8 \mathrm{ft}(0.1$ to $2.4 \mathrm{~m})$ per day. Hydraulic conductivity calculations are included in Appendix $F$ of the Monument Valley and Mexican Hat RAP (DOE, 1993c). The range of hydraulic conductivity values may be due to the heterogeneous composition of the sediments that comprise the Shinarump Conglomerate and the uncertainties associated with the results of the slug tests.

Porosity measurements of the Shinarump Conglomerate have not been made; however, porosity can be estimated from Table 2.4 of Freeze and Cherry (1979). The unit consists primarily of conglomerate (not listed in the table) and sandstone (which has a porosity range of 0.05 to 0.30 ). The conglomerate portions of the Shinarump Conglomerate probably are similar in porosity to a mixture of sand and gravel (based on the descriptions in the well logs); therefore, a good estimate of the effective porosity is about 0.25 . The amount of cementing materials is relatively minor in the sandstone (Witkind and Thaden, 1963); therefore, the effective porosity (about 0.25 ) of the sandstone portions of the Shinarump Conglomerate probably is closer to the upper bound of porosity on the Freeze and Cherry (1979) table.

Ground water velocity in the Shinarump Conglomerate can be estimated from the velocity equation. Using a gradient of 0.01 , a hydraulic conductivity of $0.4 \mathrm{ft}$ $(0.1 \mathrm{~m})$ per day, and a porosity of 0.25 , the velocity is $6 \mathrm{ft}(2 \mathrm{~m})$ per year. Using the same gradient and porosity, but the upper estimate of hydraulic conductivity (i.e., $8 \mathrm{ft}[2 \mathrm{~m}]$ per day), the ground water velocity is $100 \mathrm{ft}(30 \mathrm{~m})$ per year.

Recharge to the Shinarump Conglomerate is from precipitation and possibly from upward leakage from the De Chelly Sandstone in some areas, and infiltration of water from the alluvial aquifer in other areas. Discharge from the Shinarump Conglomerate probably is limited to the alluvial aquifer. There are no known water supply wells in the Shinarump Conglomerate in the site vicinity.

\section{Moenkopi Formation}

Geology. The Moenkopi Formation is a dark- and reddish-brown shaley siltstone and sandstone that underlies the Shinarump Conglomerate (Table 3.1). The color of the Moenkopi Formation contrasts greatly with the light gray of the Shinarump Conglomerate and the light tan of the De Chelly Sandstone. The unit likely was deposited in a near-shore mud flat type of environment, where lagoons, playas, and deltas formed (Witkind and Thaden, 1963).

Where present, the Moenkopi Formation is about 15 to $60 \mathrm{ft}(5$ to $20 \mathrm{~m})$ thick in the site vicinity, based on logs of boreholes that have fully penetrated the unit. Variations in thickness probably are a result of erosion during the deposition of the Shinarump Conglomerate. The Moenkopi Formation is not present near the northern site boundary (well 657), where a buried channel has completely eroded the unit (Figure 3.5). 
Hydrology. The Moenkopi Formation acts as a confining unit due to the finegrained nature of the sediments that comprise it. Calcium carbonate, silica, and iron oxide are present as intergranular cement (Witkind and Thaden, 1963), adding to the confining nature of the unit by decreasing the hydraulic conductivity and porosity.

The degree to which the Moenkopi Formation acts as a confining unit depends on its low-hydraulic conductivity and thickness. Because the thickness of the unit varies, its effectiveness as a confining unit also varies. For example, the unit is absent at well 657 (Figure 3.5). Therefore, the alluvial aquifer and De Chelly Sandstone are in hydraulic connection in that area. Where the Moenkopi Formation is thin or absent, water can exchange between the De Chelly Sandstone and the Shinarump Conglomerate and/or the alluvial aquifer.

Generally, there is an upward hydraulic gradient between the De Chelly Sandstone and the overlying aquifers and water can flow from the De Chelly to the upper units. The gradient has reversed at times (during pumping of former mill supply wells); however, the wells are no longer pumped and the upward gradient has reestablished.

Because the Moenkopi Formation acts as a confining unit (wells completed in the unit would not produce water), monitor and water-supply wells have not been completed in it. Therefore, piezometric surface maps have not been made, and hydraulic gradient, hydraulic conductivity, and ground water velocities have not been estimated.

\section{De Chelly Sandstone}

Geology. The De Chelly Sandstone is a grayish-yellow-to-tan, fine-grained sandstone. Although none of the UMTRA Project wells fully penetrated the unit, Witkind and Thaden (1963) report that it is approximately $500 \mathrm{ft}(150 \mathrm{~m})$ thick in the area of the site. It is the lowest aquifer of concern beneath the Monument Valley tailings site and is underlain by the low-permeability Organ Rock tongue (Table 3.1). The sand grains consists almost entirely of quartz, and the unit is weakly cemented with silica, calcium carbonate, and iron oxide. The iron oxide gives the De Chelly Sandstone unit its color (Witkind and Thaden, 1963).

Hydrology. Ground water within the De Chelly Sandstone generally is confined by the overlying Moenkopi Formation. It probably is unconfined, or only partially confined in the vicinity of the buried channel near the northern site boundary.

The piezometric surface of the De Chelly Sandstone within Cane Valley is slightly above ground surface to about $160 \mathrm{ft}(48 \mathrm{~m})$ below ground surface, due to variations in ground surface elevations across the valley (Figure 3.5; see Appendix B for water-level measurements). Wells completed in the De Chelly Sandstone will flow where the piezometric surface is above ground surface. This generally occurs in the topographically lower portions of Cane Valley le.g., at wells 611,613, and 
625). Areas west of the site are topographically higher, and the depth to the piezometric surface is greater.

Figure 3.10 is the piezometric surface map for the De Chelly Sandstone for December 1994. Ground water flows generally north. Using the equations presented in Section 3.2.3, the distance between wells 663 and $664(2400 \mathrm{ft}$ [730 m]), and the 8 December 1994 water-level elevations for wells 663 and 664 (4831.84 and $4806.56 \mathrm{ft}$ [1472.76 and $1465.06 \mathrm{~m}$ ], respectively), the horizontal hydraulic gradient is 0.01 .

Table 3.4 shows the difference in hydraulic head between the De Chelly Sandstone and the alluvial aquifers for December 1994. The table was prepared by comparing the wells completed in the alluvial aquifer with adjacent wells completed in the De Chelly Sandstone aquifer. December 1994 is representative of low water use and essentially nonpumping conditions for both the alluvial aquifer and the De Chelly Sandstone; the only water use would have been relatively minor withdrawals for limited domestic use. An upward gradient from the De Chelly Sandstone connects it to the alluvial aquifer. Water levels in the De Chelly Sandstone are about 4 to $25 \mathrm{ft}(1$ to $8 \mathrm{~m})$ higher than water levels in the alluvial aquifer. The upward gradient prevents downward migration of ground water from the alluvium to the De Chelly.

Water levels are inconsistent in the vicinity of wells 608,618 , and 657 with the normal ground water flow pattern in that well 618 shows a lower ground water level than nearby wells. Although ground water pumpage could cause such a water level response, none was reported during the time period of water level measurement.

The hydraulic conductivity computed from an aquifer pumping test in the De Chelly Sandstone is $6 \mathrm{ft}(2 \mathrm{~m})$ per day. Hydraulic conductivity calculations are included in Appendix $F$ of the Monument Valley and Mexican Hat RAP (DOE, 1993c).

The porosity of the De Chelly Sandstone has not been measured; however, porosity can be estimated from Table 2.4 of Freeze and Cherry (1979). The unit consists of sandstone (with porosity of 0.05 to 0.30 ). Because the sandstone is fine-grained and contains some cementing materials, a reasonable estimate of the effective porosity is near the lower portion of the range listed, and is likely about 0.15 .

Ground water velocity in the De Chelly Sandstone can be estimated from the velocity equation. Using a gradient of 0.01 , a hydraulic conductivity of $6 \mathrm{ft}(2 \mathrm{~m})$ per day, and a porosity of 0.15 , the velocity is $150 \mathrm{ft}(45 \mathrm{~m})$ per year.

Recharge to the De Chelly Sandstone is from rainfall runoff and snowmelt. Although precipitation is limited, the De Chelly Sandstone has a relatively large outcrop area along Cane Valley, west and south of the site. Much of the bedrock outcrop is buried beneath alluvial and windblown sands and water may infiltrate 
Figure 3.10

De Chelly Piezometric Surface, 7-8 December 1994

Monument Valley, Arizona, Site

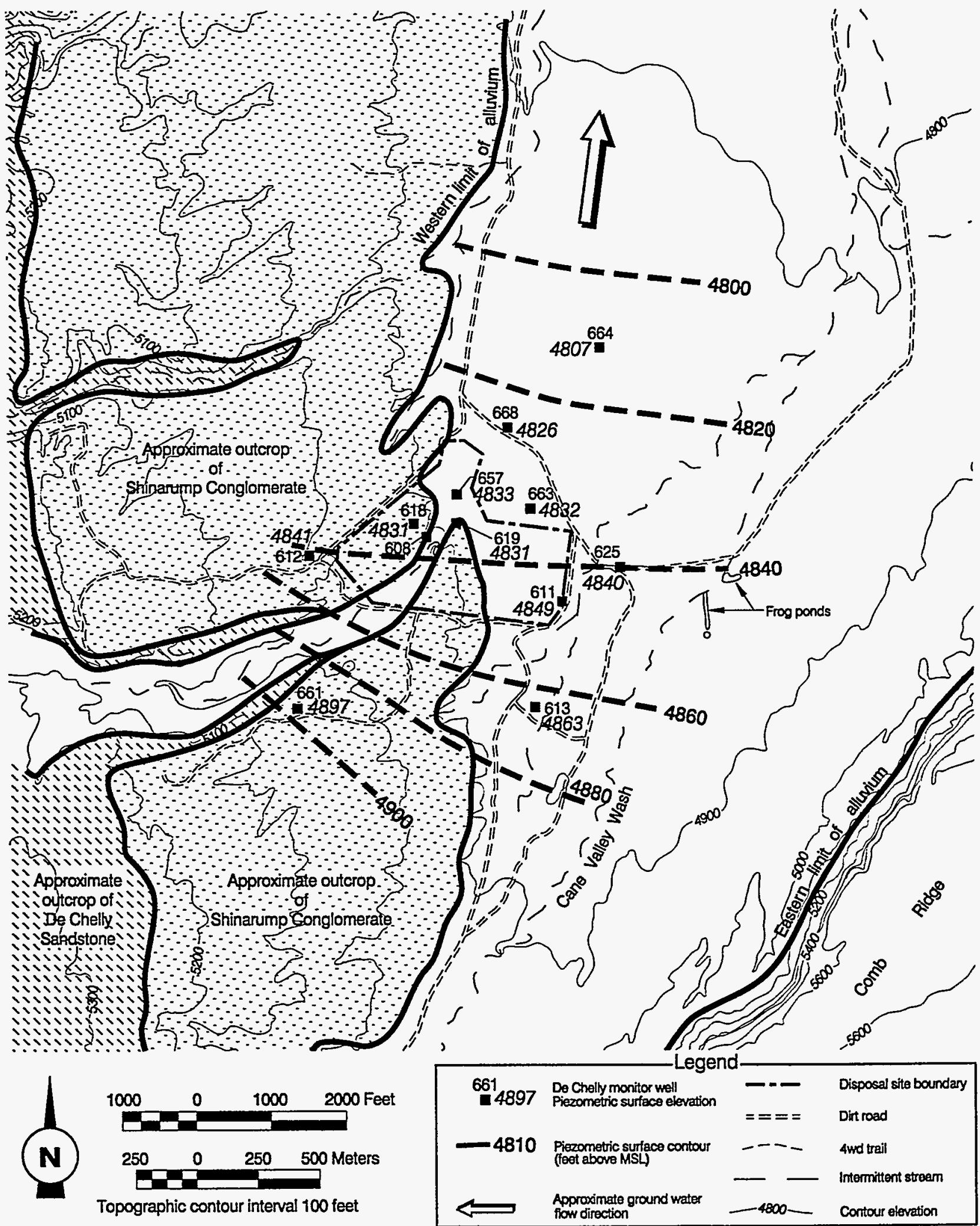

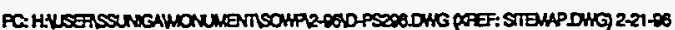


Table 3.4 Hydraulic head difference between De Chelly Sandstone and alluvial aquifers in adjacent wells

\begin{tabular}{|c|c|c|c|c|c|}
\hline \multicolumn{2}{|c|}{ Well designation } & \multirow{2}{*}{$\begin{array}{l}\text { Approximate } \\
\text { ground surface } \\
\text { elevation } \\
\text { (ft NGVD) }\end{array}$} & \multicolumn{2}{|c|}{ Ground water elevation } & \multirow{2}{*}{$\begin{array}{c}\text { Head } \\
\text { difference } \\
\text { (ft) }\end{array}$} \\
\hline Alluvium & De Chelly & & $\begin{array}{l}\text { Alluvium } \\
\text { (ft NGVD) }\end{array}$ & $\begin{array}{l}\text { De Chelly } \\
\text { (ft NGVD) }\end{array}$ & \\
\hline 602 & 613 & 4861 & 4855 & 4863 & 25 \\
\hline 603 & 611 & 4847 & 4838 & 4849 & 11 \\
\hline 606 & 663 & 4860 & 4828 & 4832 & 4 \\
\hline 669 & 668 & 4864 & 4817 & 4826 & 9 \\
\hline 653 & 664 & 4832 & 4800 & 4807 & 7 \\
\hline
\end{tabular}

Note: Measured in December 1994.

NGVD - National Geodetic Vertical Datum.

rapidly through these sands and into the De Chelly Sandstone. Loss of recharge to plants is limited, due to the sparse vegetation where the sandstone is exposed at land surface. Discharge is by vertical leakage into overlying units and by pumpage or flow from water-supply wells for domestic and stock use.

\subsubsection{Surface water hydrology}

Surface water features in the vicinity of the Monument Valley site are Cane Valley Wash, several small drainage channels (arroyos), and several ponds known locally as the Frog Ponds (Figure 3.6). Cane Valley Wash flows roughly south to north, with a drainage area of approximately 90 square miles $\left(\mathrm{mi}^{2}\right)$ $\left(230 \mathrm{~km}^{2}\right)(57,000$ acres [ac] [23,000 hectares (ha)] south of the UMTRA site (DOE, 1993c).

Surface water flow in the small drainage channels in the site vicinity is intermittent (i.e., occurring for a short period of time following rainfall or snowmelt), as is flow along the length of Cane Valley Wash. However, small pools within the wash have standing water for prolonged periods (several weeks or longer). Water in these pools is a result of the water table intersecting land surface. As the ground water table declines in response to evaporation and transpiration, the pools get smaller and many go dry. The exact locations of all of the pools, their size, and duration are not known, but the pools begin in the wash east of monitor well 658 and occur downstream (north) for several miles.

The most obvious surface water features in the site vicinity are the Frog Ponds. They contain water all year as a result of discharge from the alluvial aquifer. Water flows from these ponds to Cane Valley Wash. 


\subsubsection{Geochemical data for ground water, tailings solutions, surface water, and} sediments

The DOE collected validated ground water quality data from the processing site and vicinity from April 1985 through December 1994. These data and associated statistical reports are available through the UMTRA Project Team (DOE, 1993e). A total of 38 wells were sampled, including 32 DOE monitor wells, 3 former Vanadium Corporation of America water wells used for processing uranium and vanadium ores, and 3 private wells. These wells were completed in different hydrogeologic units, including the windblown and alluvial surficial deposits (alluvial aquifer), the Shinarump Conglomerate, and the De Chelly Sandstone. Table 3.2 lists these wells by hydrogeologic zone of completion and location (e.g., upgradient, crossgradient, or downgradient).

The most recent information available (ground water and surface water quality data obtained since 1988) were used to assess ground water and surface water quality, due to the improved analytical detection limits and quality control procedures for this period. Data from one to eight sampling rounds were incorporated from each well. Data collected during 1985 through 1987 were used to document trends in ground water quality over time and to characterize the tailings solutions.

From April 1988 through December 1994, most ground water samples were filtered prior to analysis. However, unfiltered samples are also available. One round of unfiltered analyses for all constituents was collected from two wells (614 and 616). Additionally, unfiltered samples were collected and analyzed from 29 wells (at least once and up to 8 times) for most constituents, including major elements and ammonium, arsenic, barium, cadmium, chromium, manganese, iron, molybdenum, radium-226, radium-228, selenium, strontium, uranium, vanadium, and zinc. Analyses from unfiltered ground water data generally are not available for aluminum, beryllium, boron, bromide, cobalt, copper, mercury, nickel, silver, and tin. An analysis of paired filtered and unfiltered data indicates that, for most constituents, there are no notable differences. The exceptions are iron, lead-210, manganese, and zinc, which show higher concentrations in the unfiltered samples, because a portion of these metals are apparently being adsorbed onto suspended particles in ground water.

Organic solvents were probably not used in the milling process. Nonetheless, in 1990, ground water from the processing site was screened (Hill, 1989; DOE, 1989) for organic constituents listed in Appendix IX of 40 CFR Part 264 of the Resource Conservation and Recovery Act. This screening included one tailings solution sample and one ground water sample. Common laboratory-derived contaminants such as phthalates were detected in either the tailings or ground water samples at levels near the laboratory method detection limit (MDL). Methyl iodide was reported in the ground water below the MDL; however it is unlikely that this compound, if truly present in ground water, is related to site activities. The herbicide 2,4,5-T was detected in the tailings solution near the 
MDL, but not in ground water. No other organic constituents were detected in this screening.

Tailings solutions were sampled in October 1985 and April 1986. Samples were collected at the new tailings pile and evaporation pond using suction lysimeters. At each location, lysimeters were installed at different depths. At the new tailings pile, lysimeters were installed at $5,10,15$, and $20 \mathrm{ft}\{1.5,3,4.5$, and $6.1 \mathrm{~m}$ ) below the tailings surface (lysimeters 814, 815, 816, and 817, respectively). At the evaporation pond area east of the new tailings pile, lysimeters were installed at 15 and $20 \mathrm{ft}(4.5$ and $6.1 \mathrm{~m})$ below the surface (lysimeters 805 and 804 , respectively).

Samples of tailings solution were not collected from the old tailings pile. However, two analyses of water leachate are available for samples from this pile.

Surface water and near-surface water samples were collected from the Frog Ponds east of the site, and from locations in Cane Valley Wash. Surface water samples were collected from the Frog Ponds from June 1982 through December 1994. These pond samples were collected at three separate locations (621, 622, and 623). Surface water samples were collected in December 1993 and 1994 from a shallow pool about $1200 \mathrm{ft}(370 \mathrm{~m})$ downstream of the Frog Ponds in Cane Valley Wash (624), and in December 1993 from a second shallow pool about $3400 \mathrm{ft}(1030 \mathrm{~m})$ downstream of the Frog Ponds. A near-surface water sample was collected about $1 \mathrm{mi}(1.6 \mathrm{~km})$ downstream by digging a 2 -ft $(0.6 \mathrm{~m})$ deep hole in the bed of the wash and allowing it to fill with water seeping from the alluvium (location 627). Three rounds of data are available from this location. Both filtered and unfiltered data are available for surface and nearsurface water samples. One analysis is available from a sample collected in 1993 from a spring west of Cane Valley Wash (620).

Sediment samples were collected in June 1993 from the Frog Ponds and Cane Valley Wash at surface water locations $623,620,624,626$, and near-surface water location 627.

Appendix $C$ provides all surface water, ground water, sediment, and lysimeter analytical data used in this report. To illustrate the general quality of various ground waters, median concentrations of constituents are used in summary tables within this section. In most cases, these median values are representative of water quality.

\subsubsection{Background ground water quality}

\section{Alluvial aquifer}

Background ground water quality is defined as the quality the water would have if uranium milling activities had not taken place. Background ground water quality data for the alluvial aquifer near the processing site are available from 
upgradient monitor wells 602 and 603 and from upgradient private wells 616 and 617 (Figure 3.11).

Water quality is similar in wells $602,603,616$, and 617 (Table 3.5). TDS results range from about 400 to $450 \mathrm{mg} / \mathrm{L}$. The predominant anion is bicarbonate (reported as alkalinity in Table 3.5) with lesser amounts of sulfate and chloride (Figure 3.12). The predominant cation is sodium with lesser amounts of magnesium, calcium, potassium, and strontium. Nitrate is present over a range of less than 0.04 to $44 \mathrm{mg} / \mathrm{L}$ (expressed as $\mathrm{NO}_{3}$ ), with a median concentration of about $5 \mathrm{mg} / \mathrm{L}$. Commonly detected trace constituents include barium, bromide, fluoride, iron, lead-210, manganese, radium-226, radium-228, selenium, uranium, and zinc. The water $\mathrm{pH}$ is above neutral $\mathrm{lpH}$ of about 7.8 to 8.2) and the redox condition is oxidizing (oxidation-reduction potential of about 400 to 450 millivolts [mV]).

\section{Shinarump aquifer}

Background ground water quality data for the Shinarump aquifer are available from upgradient monitor wells 601 and 615 (Figure 3.6). Ground water in the Shinarump is a sodium bicarbonate type, similar in composition to that in the alluvial aquifer (Table 3.6 and Figure 3.12). TDS range from about 300 to 430 $\mathrm{mg} / \mathrm{L}$. The water $\mathrm{pH}$ is above neutral $(\mathrm{pH}$ of about 7.8$)$ and the redox condition is oxidizing (oxidation-reduction potential of about 250 to $440 \mathrm{mV}$ ). Trace constituents are similar to those in the alluvial aquifer and include antimony, cadmium, molybdenum, selenium, and uranium. Nitrate is present with a median concentration of about $4 \mathrm{mg} / \mathrm{L}$ as nitrate. Particulate (filterable) concentrations of iron, manganese, and zinc are high in the background wells, with concentrations of iron in unfiltered samples ranging up to $107 \mathrm{mg} / \mathrm{L}$ (Table 3.6).

\section{De Chelly Sandstone}

Background ground water quality data for De Chelly Sandstone are available from upgradient monitor wells 612 and 613 (Figure 3.6). Ground water in the De Chelly is a calcium-magnesium-bicarbonate type (Figure 3.12). The water is chemically similar to that in the alluvial and Shinarump aquifers, but has somewhat less sodium and is more dilute (Table 3.6). TDS ranges from about 200 to $300 \mathrm{mg} / \mathrm{L}$. The water $\mathrm{pH}$ is above neutral $(\mathrm{pH}$ of about 7.6 to 8.0$)$ and the redox condition is oxidizing (oxidation-reduction potential of about $450 \mathrm{mV}$ ). Trace constituents include cadmium, molybdenum, uranium, and vanadium. Nitrate is present with median concentrations ranging from 4.3 to $8 \mathrm{mg} / \mathrm{L}$ as nitrate.

\subsubsection{Ground water flow from the De Chelly Sandstone to the alluvial aquifer}

Vertical head distributions indicate the potential for upward ground water flow from the De Chelly Sandstone into the alluvial aquifer. Ground water levels and ground water quality data from well 654 suggest that this flow may be occurring. 


\section{Figure 3.11}

\section{Location of Monitoring Wells Completed in the Alluvial} Aquifer and Surface Water Sampling Locations

Monument Valley, Arizona, Site

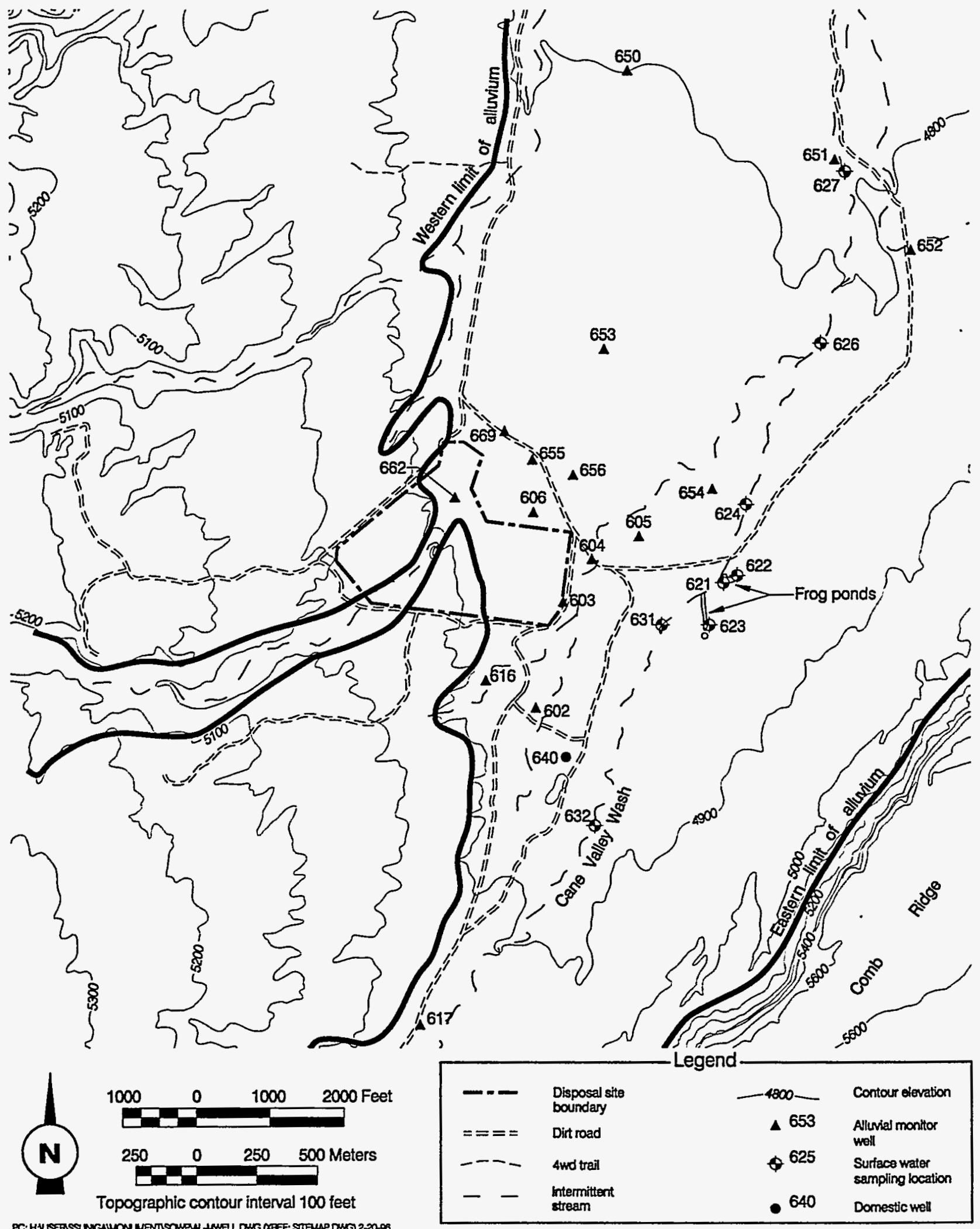


Table 3.5 Background ground water quality in the alluvial aquifer, Monument Valley, Arizona, site

\begin{tabular}{|c|c|c|c|c|}
\hline \multirow[b]{2}{*}{ Constituent } & \multicolumn{4}{|c|}{ Well location } \\
\hline & MON-01-0602 & MON-01-0603 & MON-01-0616 & MON-01-0617 \\
\hline Alkalinity & 217 & 200 & 242 & 270 \\
\hline Aluminum & $<0.05$ & $<0.05$ & $<0.05$ & 0.06 \\
\hline Ammonium & $<0.1$ & 0.3 & $<0.1$ & $<0.01$ \\
\hline Arsenic & 0.005 & 0.005 & $<0.01$ & $<0.01$ \\
\hline Barium & 0.05 & 0.05 & 0.11 & 0.08 \\
\hline Bromide & 0.2 & 0.1 & $<0.1$ & NA \\
\hline Cadmium & 0.0005 & 0.0005 & $<0.001$ & 0.0015 \\
\hline Calcium & 28 & 19 & 36 & 23 \\
\hline Chloride & 15 & 12 & 12 & 23 \\
\hline Fluoride & 0.2 & 0.15 & 0.3 & NA \\
\hline Iron & $<0.03$ & $<0.03$ & $<0.03$ & $<0.1$ \\
\hline Iron (unfiltered) & 0.15 & 0.12 & 0.14 & 0.23 \\
\hline Lead-210 & $<2.0$ & $<2.0$ & NA & NA \\
\hline Lead-210 (unfiltered) & $<2.0$ & $<2.0$ & 5.3 & 5.7 \\
\hline Magnesium & 19 & 14 & 30 & 42 \\
\hline Manganese & $<0.01$ & $<0.01$ & $<0.01$ & $<0.01$ \\
\hline Manganese (unfiltered) & 0.003 & 0.005 & 0.005 & 0.005 \\
\hline Mercury & $<0.0002$ & 0.0002 & $<0.0002$ & $<0.0002$ \\
\hline Molybdenum & 0.005 & 0.005 & $<0.007$ & 0.01 \\
\hline Nitrate & 4.1 & 4.5 & 5.8 & 13.8 \\
\hline $\mathrm{pH}$ & 7.8 & 7.8 & 8.2 & 8.2 \\
\hline Phosphate & 0.2 & 0.3 & $<0.1$ & 0.1 \\
\hline Potassium & 1.9 & 2.5 & 1.1 & 0.6 \\
\hline Radium-226 & 0.7 & 0.4 & $<0.5$ & 2.9 \\
\hline Radium-228 & 0.8 & 1.2 & $<0.3$ & $<0.3$ \\
\hline Redox potential & 441 & 450 & NA & 407 \\
\hline Selenium & 0.004 & 0.003 & $<0.005$ & $<0.005$ \\
\hline Silica & 14 & 12 & 26 & 31 \\
\hline Sodium & 100 & 100 & 72 & 111 \\
\hline Strontium & 0.28 & 0.24 & 0.47 & 0.52 \\
\hline Sulfate & 130 & 115 & 134 & 123 \\
\hline Sulfide & 0.28 & 0.28 & $<1$ & NA \\
\hline
\end{tabular}


Table 3.5 Background ground water quality in the alluvial aquifer, Monument Valley, Arizona, site (Concluded)

\begin{tabular}{lcccc}
\hline & \multicolumn{4}{c}{ Well location } \\
\cline { 2 - 5 } \multicolumn{1}{c}{ Constituent } & MON-01-0602 & MON-01-0603 & MON-01-0616 & MON-01-0617 \\
\hline Thorium-230 & 1 & $<1$ & $<1$ & NA \\
Total dissolved solids & 441 & 396 & 420 & 450 \\
Uranium & 0.004 & 0.003 & 0.004 & 0.007 \\
Vanadium & $<0.005$ & $<0.005$ & $<0.01$ & 0.01 \\
Zinc & $<0.005$ & 0.013 & $<0.01$ & $<0.02$ \\
Zinc (unfiltered) & 0.015 & 0.007 & 0.039 & 0.043 \\
\hline
\end{tabular}

Notes: 1. Data listed are median concentrations for filtered samples (except as noted) collected from 1988 through 1994.

2. All data in milligrams per liter (mg/L) except for the following: lead-210, radium-226, radium-228, thorium-230 in picocuries per liter; $\mathrm{pH}$ in standard units; redox potential in millivolts; alkalinity as $\mathrm{mg} / \mathrm{L}$ calcium carbonate equivalent.

3. The following were never detected in either filtered or unfiltered background ground water (method detection limit is given in parentheses): antimony $(0.03 \mathrm{mg} / \mathrm{L})$, beryllium $(0.005 \mathrm{mg} / \mathrm{L})$, boron $(0.005 \mathrm{mg} / \mathrm{L})$, chromium $(0.005 \mathrm{mg} / \mathrm{L})$, cobalt $(0.03 \mathrm{mg} / \mathrm{L})$, copper $(0.01 \mathrm{mg} / \mathrm{L})$, lead $(0.005 \mathrm{mg} / \mathrm{L})$, nickel $(0.04 \mathrm{mg} / \mathrm{L})$, silver $(0.01 \mathrm{mg} / \mathrm{L})$, thallium $(0.01 \mathrm{mg} / \mathrm{L})$, and tin (0.005 mg/L).

$<-$ indicates less than the given value.

NA- not analyzed. 
Figure 3.12

\section{Trilinear Plots of Water Chemistry, Monument Valley, Arizona, Site}
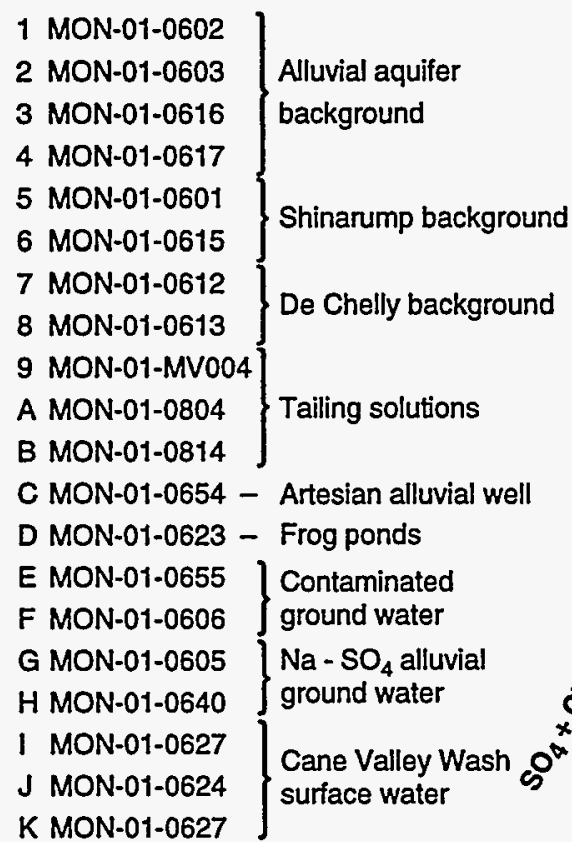

Shinarump background

De Chelly background

Tailing solutions

Artesian alluvial well

Frog ponds

Contaminated ground water $\mathrm{Na}-\mathrm{SO}_{4}$ alluvial ground water

Cane Valley Wash surface water

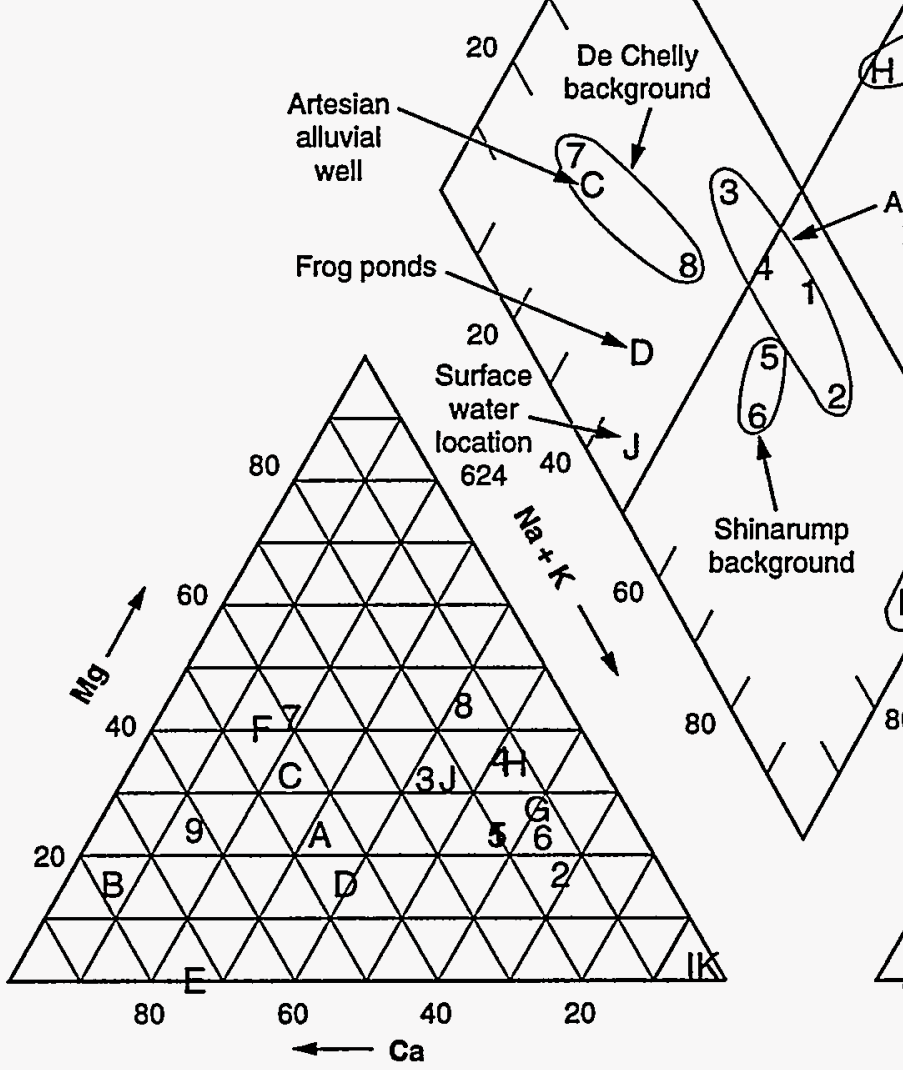

Cations
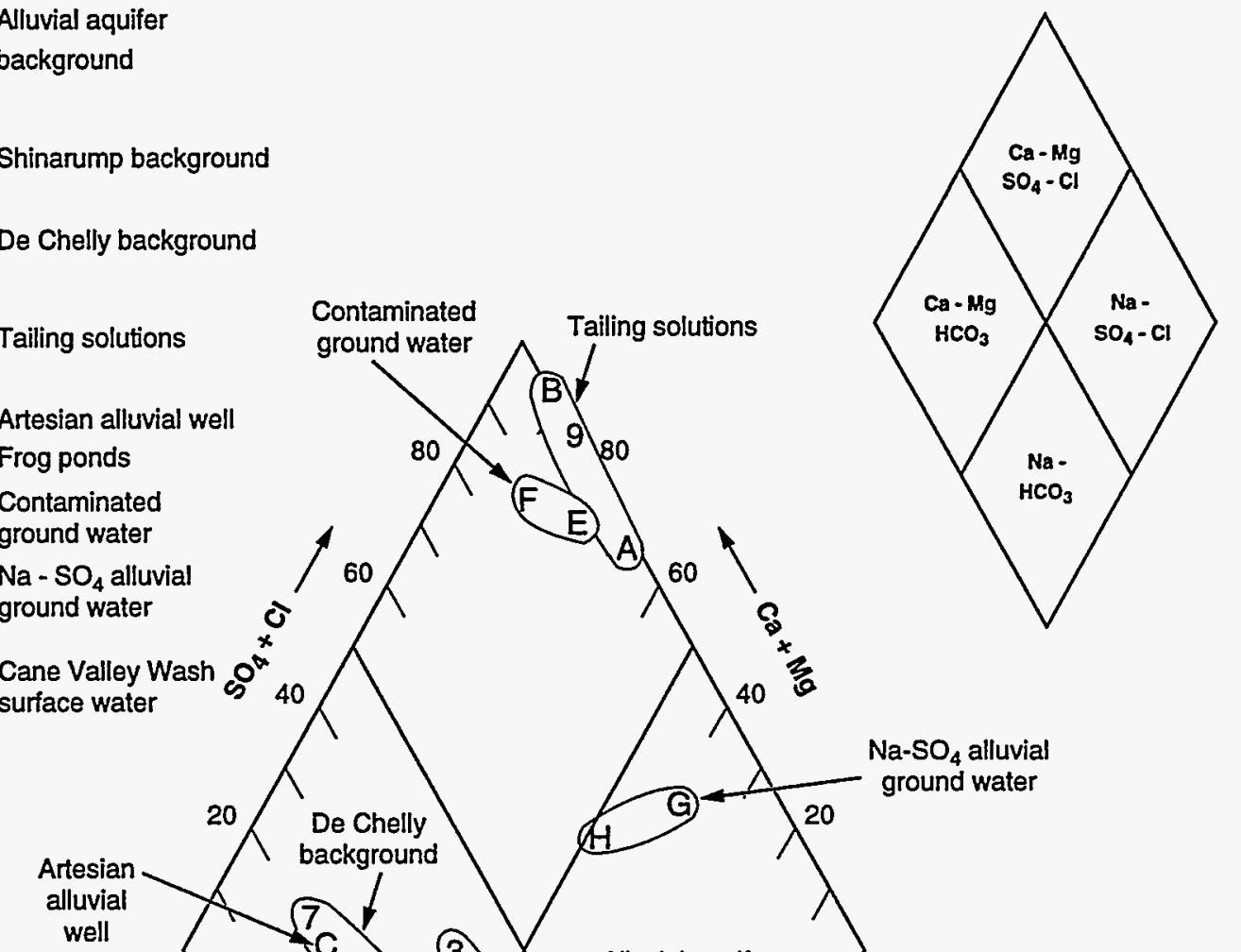
backgrounde surface water at location 627
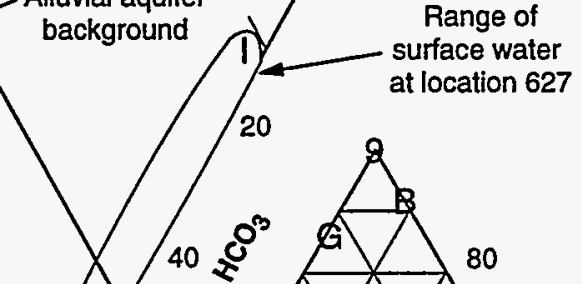
Table 3.6 Background ground water quality in the Shinarump and De Chelly Sandstones, Monument Valley, Arizona, site

\begin{tabular}{|c|c|c|c|c|}
\hline \multirow[b]{2}{*}{ Constituent } & \multicolumn{2}{|c|}{ Shinarump } & \multicolumn{2}{|c|}{ De Chelly } \\
\hline & MON-01-0601 & MON-01-0615 & MON-01-0612 & MON-01-0613 \\
\hline Alkalinity & 280 & 226 & 121 & 185 \\
\hline Aluminum & 0.07 & $<0.1$ & NA & $<0.1$ \\
\hline Ammonium & 0.13 & $<0.1$ & $<0.1$ & $<0.1$ \\
\hline Antimony & 0.004 & $<0.003$ & $<0.003$ & $<0.003$ \\
\hline Arsenic & $<0.005$ & 0.003 & $<0.01$ & $<0.005$ \\
\hline Barium & 0.05 & 0.05 & NA & 0.05 \\
\hline Cadmium & 0.0004 & 0.0003 & 0.004 & 0.0005 \\
\hline Calcium & 28 & 18 & 28 & 16 \\
\hline Chloride & 15 & 10 & 6 & 8 \\
\hline Fluoride & 0.2 & 0.4 & NA & 0.3 \\
\hline Iron & $<0.03$ & 0.14 & NA & $<0.03$ \\
\hline Iron (unfiltered) & 107 & 2.95 & NA & 0.04 \\
\hline Lead-210 & $<2$ & $<7.3$ & NA & $<2.0$ \\
\hline Lead-210 (unfiltered) & $<2$ & 7.2 & NA & 5.8 \\
\hline Magnesium & 18 & 16 & 18 & 29 \\
\hline $\begin{array}{l}\text { Manganese } \\
\text { (unfiltered) }\end{array}$ & 1.46 & 0.35 & NA & 0.005 \\
\hline Molybdenum & 0.005 & $<0.005$ & 0.01 & 0.005 \\
\hline Nitrate & 4.3 & 4.3 & 8.0 & 4.3 \\
\hline $\mathrm{pH}$ & 7.8 & 7.8 & 7.6 & 8.0 \\
\hline Phosphate (unfiltered) & 2.6 & $<0.1$ & NA & $<0.1$ \\
\hline Potassium & 1.9 & 3.1 & 5.3 & 2.8 \\
\hline Radium-226 & $<0.3$ & 1.2 & NA & $<0.3$ \\
\hline Radium-228 & 1.35 & 2.7 & NA & $<1.0$ \\
\hline Redox Potential & 442 & 248 & NA & 446 \\
\hline Selenium & 0.005 & $<0.002$ & $<0.005$ & $<0.005$ \\
\hline Silica & 14 & 9 & NA & 11 \\
\hline Sodium & 99 & 88 & 13 & 50 \\
\hline Strontium & 0.29 & 0.63 & NA & 0.35 \\
\hline Sulfate & 125 & 63 & 20 & 60 \\
\hline Total dissolved solids & 429 & 327 & 313 & 195 \\
\hline Uranium & 0.005 & 0.001 & 0.010 & 0.004 \\
\hline
\end{tabular}


Table 3.6 Background ground water quality in the Shinarump and De Chelly Sandstones, Monument Valley, Arizona, site (Concluded)

\begin{tabular}{lcccccc}
\hline & \multicolumn{2}{c}{ Shinarump } & & \multicolumn{2}{c}{ De Chelly } \\
\cline { 2 - 3 } \cline { 6 - 6 } \multicolumn{1}{c}{ Constituent } & MON-01-0601 & MON-01-0615 & & MON-01-0612 & MON-01-0613 \\
\hline Vanadium & $<0.005$ & $<0.005$ & & NA & 0.005 \\
\hline Zinc & $<0.005$ & 0.013 & & NA & $<0.005$ \\
Zinc (unfiltered) & 0.15 & 0.038 & & NA & 0.005 \\
\hline
\end{tabular}

Notes: 1. Data listed are median concentrations for filtered samples (except as noted) collected from 1988 through 1994. Only one analysis is available for well MON-01-0612.

2. All data in milligrams per liter (mg/L) except for the following: lead-210, radium-226, radium-228, thorium-230 in $\mathrm{pCi} / \mathrm{L} ; \mathrm{pH}$ in standard units; redox potential in millivolts; alkalinity as $\mathrm{mg} / \mathrm{L}$ calcium carbonate equivalent.

3. The following were never detected in either filtered or unfiltered background ground water (method detection limit is given in parentheses): beryllium $(0.005$ $\mathrm{mg} / \mathrm{L})$, bromide $(0.1 \mathrm{mg} / \mathrm{L})$, chromium $(0.005 \mathrm{mg} / \mathrm{L})$, cobalt $(0.005 \mathrm{mg} / \mathrm{L})$, copper $(0.02 \mathrm{mg} / \mathrm{L})$, mercury $(0.0002 \mathrm{mg} / \mathrm{L})$, nickel $(0.04 \mathrm{mg} / \mathrm{L})$, silver $(0.01$ $\mathrm{mg} / \mathrm{L})$, sulfide $(0.01 \mathrm{mg} / \mathrm{L})$, thallium $(0.01 \mathrm{mg} / \mathrm{L})$, and thorium-230 (1.0 pCi/L).

$<-$ indicates less than the given value.

NA - not analyzed. 
Ground water levels in monitor well 654 are above the land surface, so the well flows at the surface. The boring log and well completion records indicate that well 654 is screened in the lower portion of the alluvial aquifer (from 51 to $79 \mathrm{ft}$ [15.5 to $24.0 \mathrm{~m}$ ]) below the surface (Appendix A). The fact that the well is flowing suggests that the alluvial aquifer may be receiving recharge from the De Chelly Sandstone at this location.

Water quality data further indicate that the source of water in the alluvium in the vicinity of monitor well 654 is the De Chelly Sandstone rather than the horizontal flow in the alluvial aquifer. In general, background water quality in the De Chelly Sandstone has lower concentrations of major elements than those of the alluvial aquifer (Table 3.7). By contrast, higher concentrations of major elements in the alluvial aquifer reflect the influence of near-surface evaporation on ground water quality. The chemistry of ground water from well 654 is like that of the De Chelly Sandstone when it is compared to background water quality in both the De Chelly Sandstone and alluvial aquifers (Table 3.7 and Figure 3.12). Water from the Frog Ponds (at location 623, near the source surface water flow in the Frog Ponds) is also similar to De Chelly water quality, especially the concentrations of chloride and sulfate (Table 3.7 and Figure 3.12). Higher concentrations of sodium and alkalinity reflect dissolution of surface evaporite salts, which form on the banks of Frog Ponds and then are transported into the surface water by rain, wind, and livestock trampling the banks. Thus, both water level data and geochemical data indicate that, at least locally, there may be recharge of the alluvial aquifer from the De Chelly Sandstone.

Table 3.7 Water quality data from alluvial well 654 and Frog Ponds compared to the De Chelly Sandstone and alluvial aquifer data, Monument Valley, Arizona, site

\begin{tabular}{lcccc}
\hline \multicolumn{1}{c}{ Constituent } & MON-01-0654 & $\begin{array}{c}\text { Frog Ponds } \\
\text { (MON-01-0623) }\end{array}$ & $\begin{array}{c}\text { De Chelly Sondstone } \\
\text { (background wells } \\
\text { MON-01-0612 and } \\
-0613)\end{array}$ & $\begin{array}{c}\text { Alluvial aquifer } \\
\text { (background wells } \\
\text { MON-01-0602, -0603, } \\
\text {-0616, and -0617) }\end{array}$ \\
\hline Alkalinity & 176 & 276 & $121-185$ & $200-270$ \\
Calcium & 42 & 46 & $16-28$ & $19-36$ \\
Chloride & 6 & 9 & $6-8$ & $12-23$ \\
Magnesium & 18 & 29 & $18-29$ & $14-42$ \\
Nitrate & 0.5 & $<1.0$ & $4-8$ & $4-14$ \\
Potassium & 2.5 & 2.9 & $2.8-5.3$ & $0.6-2.5$ \\
Sodium & 24 & 48 & $13-50$ & $72-111$ \\
Sulfate & 42 & 36 & $20-60$ & $115-134$ \\
Total dissolved solids & 242 & 350 & $313-195$ & $396-450$ \\
Uranium & 0.001 & 0.003 & 0.0100 .004 & $0.003-0.007$ \\
\hline
\end{tabular}

Notes: 1. Data expressed as milligrams per liter. Alkalinity expressed as milligrams per liter calcium carbonate equivalent.

2. Data listed are median values, or the range in median values for background wells.

$<-$ indicates less than the given value. 


\subsubsection{Nature and extent of contamination}

\section{Sources of ground water contamination}

There were two major sources of ground water contamination at the site: the old tailings pile/heap leach pad and the new tailings pile. The old tailings pile was composed of the sand tailings that were a residual product of the mechanical upgrading of ore. The upgrading process used water that contained a minor amount of flocculents but no other processing chemicals. Thus, tailings solutions in the old pile basically were water-equilibrated to minerals in the ore. Heap leaching of these old tailings occurred in the area where they were stored. Old tailings were placed on the heap leach pad and sulfuric acid was added to the tailings. Heap leach pads were lined to collect the leachate which contained sulfuric acid. By contrast, the new tailings pile contained sands tailings and processing solutions. The processing solutions contained sulfate, nitrate, and ammonium from the processing chemicals.

Table 3.8 summarizes results of analyses of solutions in or derived from the old and new tailings piles. There are no direct analyses of tailings solutions from the old pile. However, leaching experiments on tailings collected from the old pile (DOE, 1983) demonstrate that water in contact with the old tailings would contain primarily calcium and sulfate and several trace constituents including arsenic, cadmium, chromium, copper, lead, uranium, and vanadium. These constituents probably are derived from the dissolution of ore-associated minerals in the tailings, including gypsum (calcium sulfate), uranyl vanadates, and minor amounts of copper-bearing minerals (Witkind and Thaden, 1963). The dissolution of the mineral gypsum (hydrous calcium sulfate) may explain the predominance of both calcium and sulfate in the old tailings leachates.

Direct analysis of tailings solutions (collected using suction lysimeters) are available from the new tailings pile (Table 3.8). In general, the concentrations of these solutions increased with increasing depth in the pile, reflecting seepage of the solutions from the base of the pile, and infiltration of precipitation into the top of the pile. The tailings solutions were acid ( $\mathrm{pH}$ as low as 4.3). The solutions are ammonium-nitrate and calcium-sulfate solutions, reflecting the presence of gypsum in the ores, the dissolution of other calcium-bearing minerals in the ores, and the addition of sulfuric acid and ammonium nitrate to the processing solutions. Chloride levels are notably low in these solutions and about the same as in background ground waters. Metals and trace elements include aluminum, iron, manganese, antimony, barium, chromium, cobalt, copper, molybdenum, nickel, strontium, uranium, vanadium, and zinc. These elements were derived from the dissolution of the ores.

Solutions beneath the evaporation pond area differ from those in the tailings piles. Notably, the acidity of the solutions has been reduced by reactions with carbonate minerals in the subsoil. Also, the ammonium and sulfate concentrations decreased, and sodium concentrations increased. These changes reflect reactions of the tailings solutions with the subsoil. The resulting 
Table 3.8 Chemistry of tailings solutions and leachates, Monument Valley, Arizona, site

\begin{tabular}{|c|c|c|c|}
\hline Constituent & $\begin{array}{c}\text { Leachate } \\
\text { (old tailings pile) }\end{array}$ & $\begin{array}{l}\text { Tailings solution } \\
\text { (new tailings pile) }\end{array}$ & $\begin{array}{c}\text { Tailings solution } \\
\text { (evaporation pond subsoil) }\end{array}$ \\
\hline Alkalinity & NA & 2 & 44 \\
\hline Aluminum & 1.8 & 5.5 & 0.9 \\
\hline Ammonium & NA & 1200 & 47 \\
\hline Antimony & NA & 0.003 & $<0.003$ \\
\hline Arsenic & 0.31 & $<0.01$ & $<0.01$ \\
\hline Barium & 0.14 & 0.3 & 0.5 \\
\hline Cadmium & 0.115 & $<0.001$ & $<0.001$ \\
\hline Calcium & 446 & 626 & 425 \\
\hline Chloride & 6.1 & 20 & 45 \\
\hline Chromium & 0.24 & 0.02 & 0.03 \\
\hline Cobalt & NA & 1.41 & 0.11 \\
\hline Copper & 0.55 & 0.11 & 0.04 \\
\hline Fluoride & NA & NA & 1.7 \\
\hline Iron & 1.2 & 1.86 & 0.04 \\
\hline Lead & 0.43 & $<0.01$ & $<0.01$ \\
\hline Magnesium & 97 & 78.3 & 135 \\
\hline Manganese & 35 & 3.94 & 0.77 \\
\hline Molybdenum & $<0.06$ & 0.32 & 0.24 \\
\hline Nickel & NA & 1.1 & 0.15 \\
\hline Nitrate & NA & 530 & 570 \\
\hline $\mathrm{pH}$ & 6.3 & 4.3 & 7.2 \\
\hline Phosphate & NA & NA & NA \\
\hline Potassium & 48 & 21.9 & 5.2 \\
\hline Radium-226 & NA & 13 & 4.7 \\
\hline Radium-228 & NA & NA & NA \\
\hline Redox Potential & 535 & NA & NA \\
\hline Selenium & $<0.06$ & $<0.005$ & $<0.005$ \\
\hline Silica & 6.6 & NA & NA \\
\hline Silver & NA & $<0.01$ & $<0.01$ \\
\hline Sodium & 97 & 70.7 & 362 \\
\hline Strontium & NA & 0.07 & 1.6 \\
\hline Sulfate & 1450 & 4510 & 1610 \\
\hline Total dissolved solids & NA & 6850 & 2900 \\
\hline
\end{tabular}


Table 3.8 Chemistry of tailings solutions and leachates, Monument Valley, Arizona, site (Concluded)

\begin{tabular}{lccc}
\hline \multicolumn{1}{c}{ Constituent } & $\begin{array}{c}\text { Leachate } \\
\text { (old tailings pile) }\end{array}$ & $\begin{array}{c}\text { Tailings solution } \\
\text { (new tailings pile) }\end{array}$ & $\begin{array}{c}\text { Tailings solution } \\
\text { (evaporation pond subsoil) }\end{array}$ \\
\hline Uranium & 0.74 & 0.075 & 1.08 \\
Vanadium & 6.2 & 1.08 & 0.91 \\
Zinc & NA & 3.9 & 0.13 \\
\hline
\end{tabular}

Notes: 1. Leachate data in milligrams per kilogram $(\mathrm{mg} / \mathrm{kg}$ ) except for $\mathrm{pH}$ (in standard units) and redox potential (in millivolts).

2. Data listed are maximum measured concentrations in filtered samples.

3. Leachate data are from samples MV004-01 and MV004-02 (DOE, 1983).

4. Tailings solution samples collected suction lysimeters at the evaporation ponds (lysimeter locations 0804 and 0805) and at the new tailings piles (locations 0814 through 0817).

5. Tailings solution data in milligrams per liter ( $\mathrm{mg} / \mathrm{L}$ ) except for the following: radium-226 in picocuries per liter, $\mathrm{pH}$ in standard units, redox potential in millivolts; alkalinity expressed as $\mathrm{mg} / \mathrm{L}$ calcium carbonate equivalent.

6. The following were not detected (method detection limit is given in parentheses): boron $(0.1 \mathrm{mg} / \mathrm{L})$, mercury $(0.0002 \mathrm{mg} / \mathrm{L})$, silver $(0.01 \mathrm{mg} / \mathrm{L})$, sulfide $(0.01 \mathrm{mg} / \mathrm{L})$, and tin $(0.005)$.

$<-$ indicates less than the given value.

NA - not analyzed. 
solution is a calcium-sodium-nitrate-sulfate solution. As with the tailings solutions, chloride concentrations are notably low (45 mg/L), only slightly exceeding background concentrations (up to $23 \mathrm{mg} / \mathrm{L}$ ). All metals and trace elements (except antimony) present in the tailings solutions are also present in the evaporation pond area.

In general, the tailings solutions from all identified sources contain much greater proportions of calcium, magnesium, and sulfate than are present in background ground waters (Figure 3.12).

\section{Ground water contamination in the alluvial aquifer}

The surficial alluvial aquifer is the aquifer most affected by the ground water contamination. Four constituents associated with the processing solutions serve as indicators for delineating the extent of ground water contamination: calcium, sulfate, nitrate, and uranium. All four occur in background ground water, but at lower levels than in the tailings solutions and the high proportion of calcium, magnesium, and sulfate help distinguish contaminated ground water from background ground water (Figure 3.12). Also, all four tend to be mobile in the ground water under the conditions of the site.

Maps showing the distribution of sulfate, nitrate, and uranium indicate there may be two separate sources of ground water contamination at the site (Figures $3.13,3.14$, and 3.15). The calcium distribution is not shown as a figure but it closely reflects the sulfate distribution. Contamination from one source appears to be about $1000 \mathrm{ft}(305 \mathrm{~m})$ or more north of the site while contamination from a second source is further south and appears to be close to the new tailings area of the site. Table 3.9 compares constituent concentrations in water from tailings solutions and a background well to median values for the two wells displaying the greatest contamination levels in the northern and southern plumes (wells 606 and 655, respectively). The northern plume may be associated with the old tailings pile and heap leach pad area, which were located on windblown sands overlying a buried channel. This plume is characterized by several constituents that occur at high levels above background, including sulfate, calcium, magnesium, strontium, and uranium. The northern plume also contains nitrate. However, compared to background concentrations, elevated sulfate concentrations extend beyond the limits of elevated nitrate.

By contrast, the southern area plume is smaller than the northern plume, and is characterized by the highest observed concentrations of nitrate and ammonium in ground water at the site. Other site-related constituents (sulfate and uranium) are present, but at lower levels than those observed to the north.

Contaminated ground water from both sources appears to converge in the area of monitor well 655. The most contaminated ground water is the ammoniumnitrate calcium-sulfate water in the southern plume, and the calcium-nitratesulfate water in area of well 655. 
Figure 3.13

Distribution of Sulfate in the Alluvial Aquifer

Monument Valley, Arizona, Site

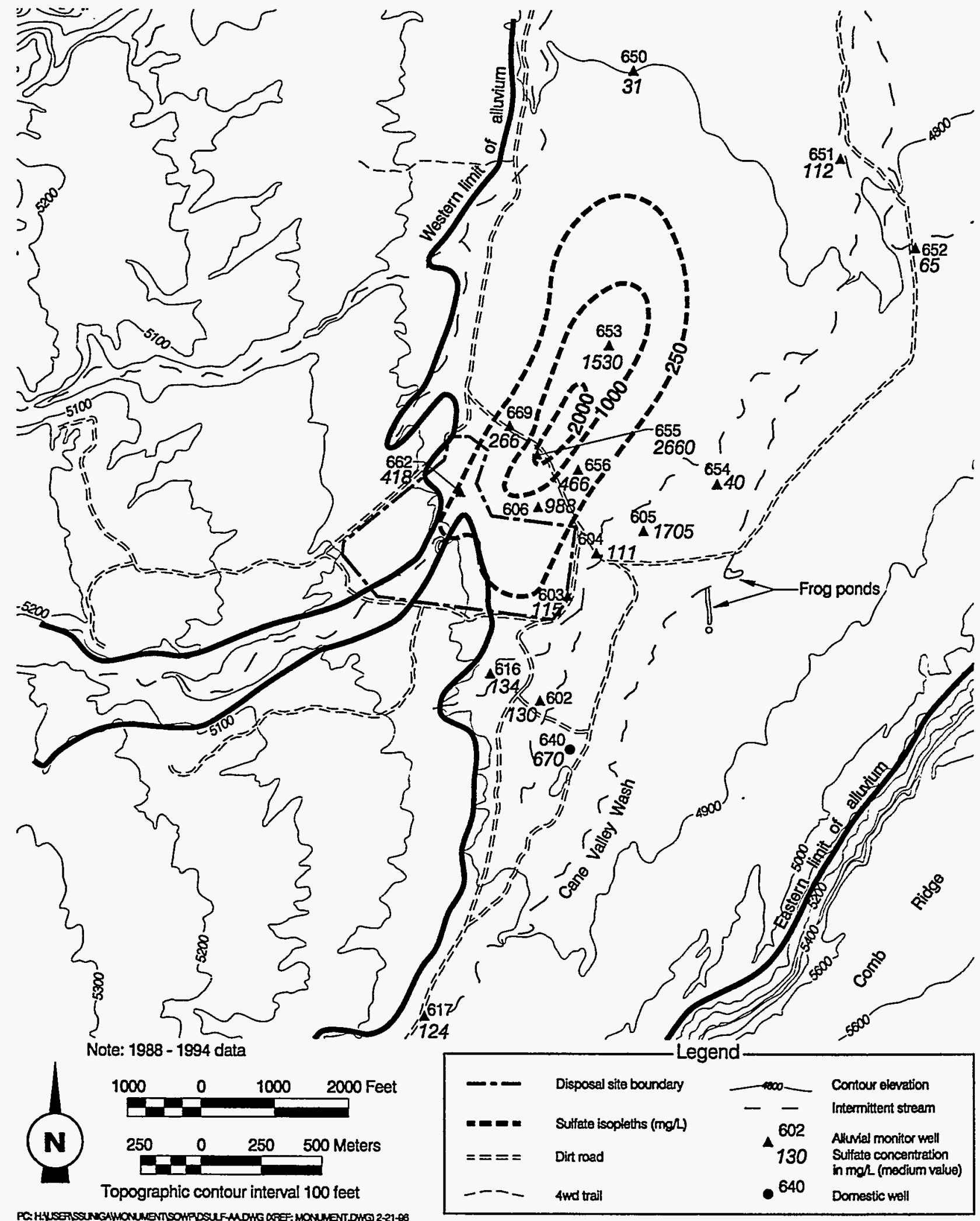




\section{Figure 3.14}

\section{Distribution of Nitrate in the Alluvial Aquifer Monument Valley, Arizona, Site}

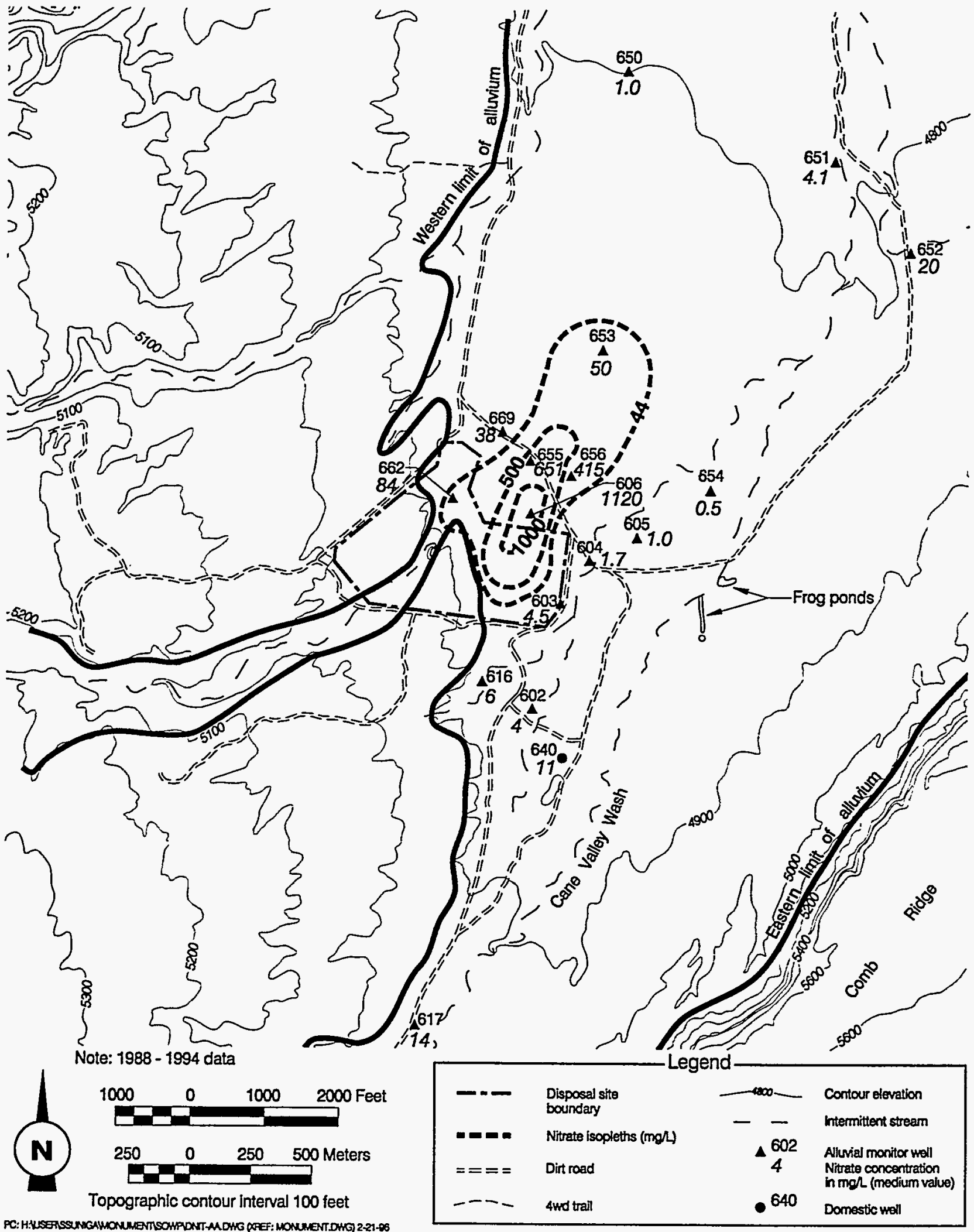


Figure 3.15

Distribution of Uranium in the Alluvial Aquifer

Monument Valley, Arizona, Site

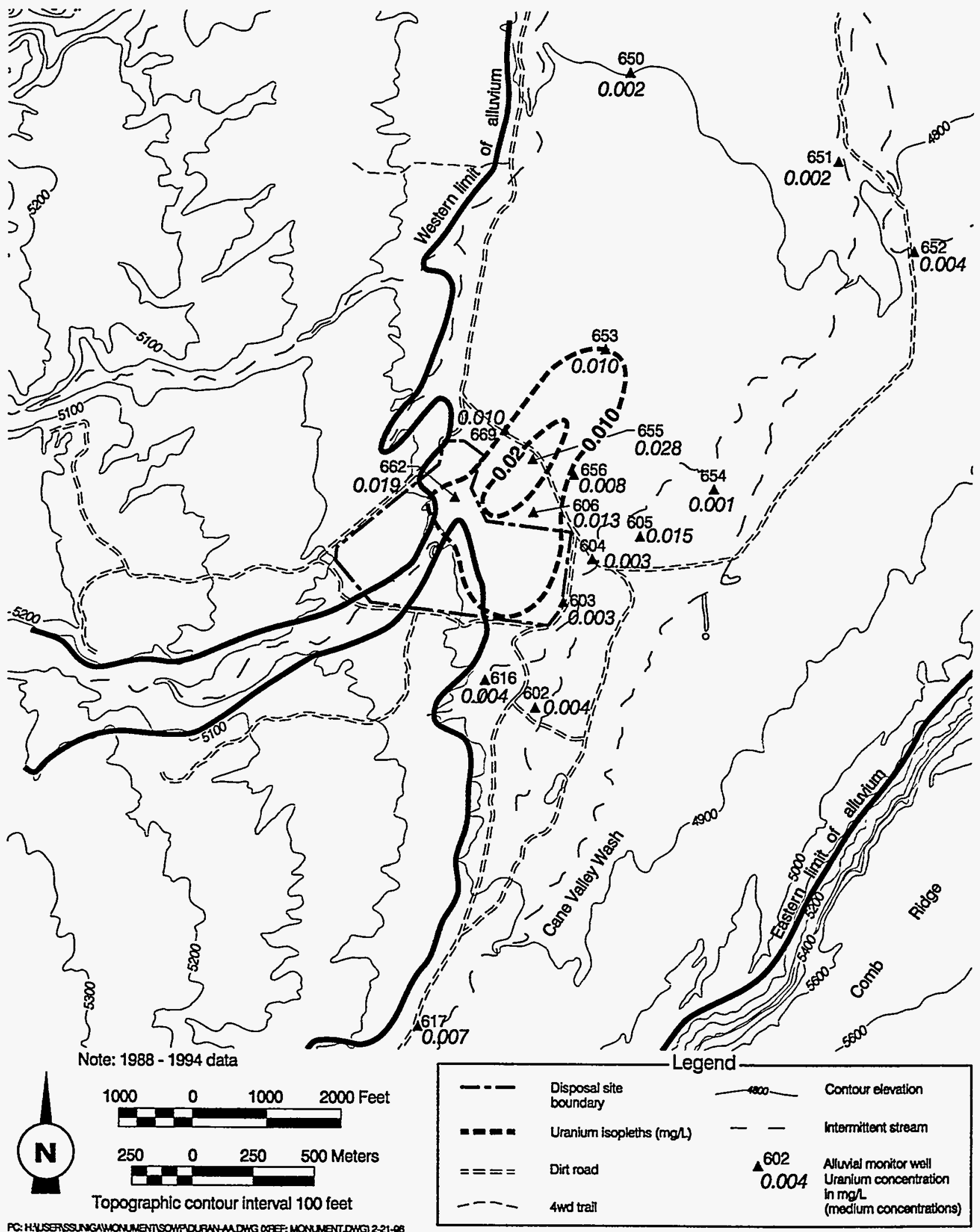


Table 3.9 Comparison of contaminated ground water to tailings solution and background ground water quality data in the alluvial aquifer, Monument Valley, Arizona, site

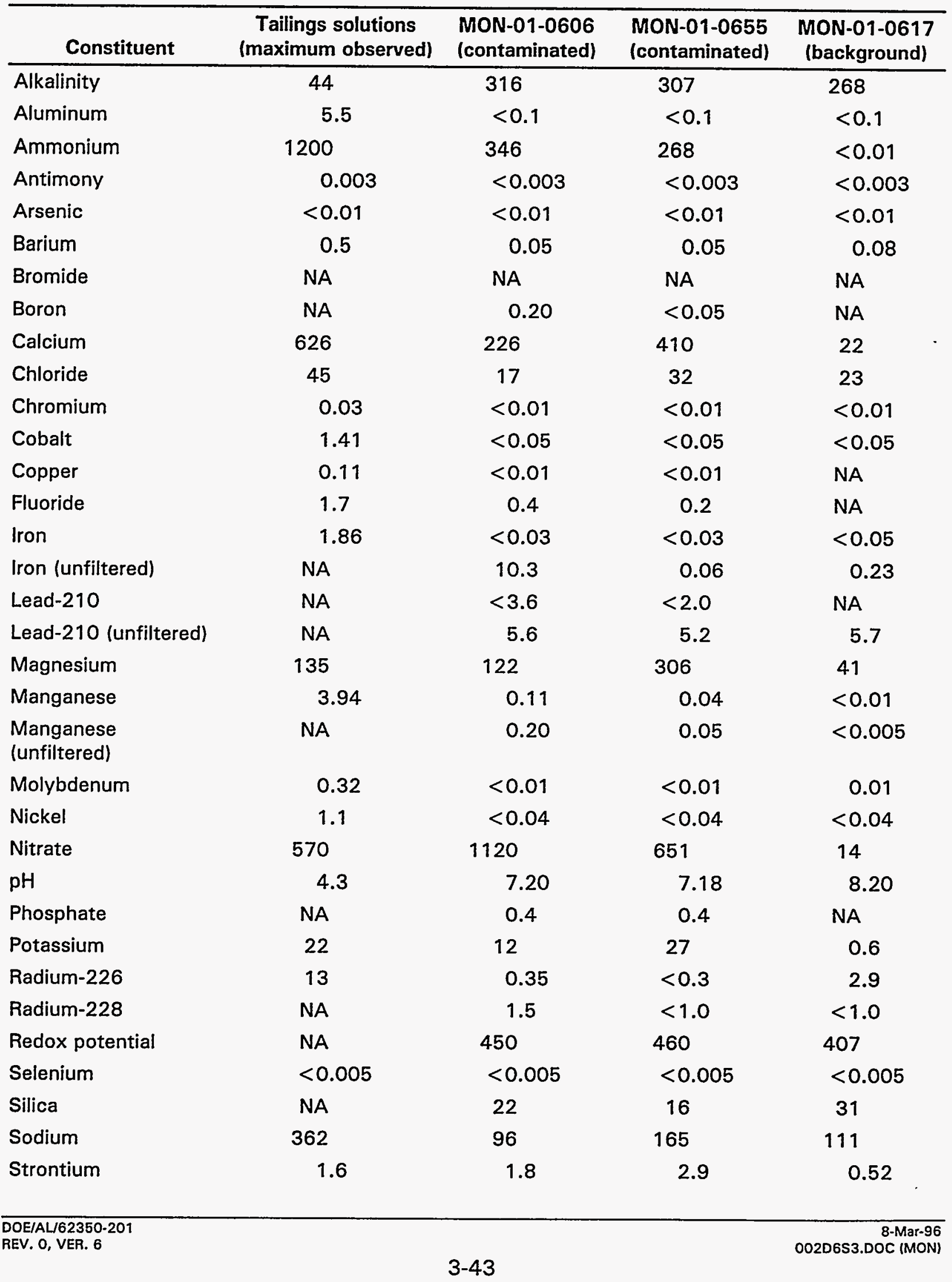


Table 3.9 Comparison of contaminated ground water to tailings solution and background ground water quality data in the alluvial aquifer, Monument Valley, Arizona, site (Concluded)

\begin{tabular}{lcccc}
\hline \multicolumn{1}{c}{ Constituent } & $\begin{array}{c}\text { Tailings solutions } \\
\text { (maximum observed) }\end{array}$ & $\begin{array}{c}\text { MON-01-0606 } \\
\text { (contaminated) }\end{array}$ & $\begin{array}{c}\text { MON-01-0655 } \\
\text { (contaminated) }\end{array}$ & $\begin{array}{c}\text { MON-01-0617 } \\
\text { (background) }\end{array}$ \\
\hline Sulfate & 4510 & 902 & 2270 & 103 \\
\hline Tin & NA & $<0.005$ & $<0.005$ & NA \\
Thorium-230 & NA & $<1.0$ & $<1.0$ & NA \\
Total dissolved solids & 6850 & 2084 & 4165 & 450 \\
Uranium & 1.08 & 0.013 & 0.028 & 0.007 \\
Vanadium & 1.08 & $<0.01$ & $<0.01$ & 0.018 \\
Zinc & 3.9 & 0.010 & 0.003 & $<0.005$ \\
Zinc (unfiltered) & NA & 0.029 & 0.019 & 0.043 \\
\hline
\end{tabular}

Notes: 1. Tailings solution data are the maximum observed values for filtered samples collected from lysimeters.

2. Ground water data listed are median values for filtered samples (except as noted) collected from 1988 through 1994.

3. All data in milligrams per liter ( $\mathrm{mg} / \mathrm{L}$ ) except for the following: lead-210, radium-226, radium-228, thorium-230 in picocuries per liter; $\mathrm{pH}$ in standard units; redox potential in millivolts; alkalinity as $\mathrm{mg} / \mathrm{L}$ calcium carbonate equivalent.

4. The following were not detected in samples of tailings solutions and ground water (method detection limit is given in parentheses): beryllium $(0.005 \mathrm{mg} / \mathrm{L}$ ), cadmium $(<0.001)$, lead $(0.01 \mathrm{mg} / \mathrm{L})$, mercury $(0.0002)$, silver $(0.01 \mathrm{mg} / \mathrm{L})$, and thallium $(0.01 \mathrm{mg} / \mathrm{L})$.

$<-$ indicates less than the given value.

NA - not analyzed. 
Ground water sampled from monitor wells 606 and 655 represent the most contaminated ground water observed in monitor wells. However, both wells are downgradient of (rather than beneath) the former tailings piles. Lysimeter data, though old (1983), suggest that beneath the new tailings pile and evaporation pond area, the magnitude of contamination may be greater than that observed in downgradient monitor wells. This is supported by the fact that nitrate (Figure 3.16) and ammonium concentrations near the new tailings pile (well 606) have not significantly decreased since 1985 .

In contrast to nitrate, concentrations of sulfate (Figure 3.17), TDS, calcium, magnesium, and uranium have decreased in alluvial wells within about $1000 \mathrm{ft}$ $(300 \mathrm{~m}$ ) of the site (monitor wells 606,655 and 669$)$, while concentrations have increased in well 653 located about $2800 \mathrm{ft}(850 \mathrm{~m})$ from the site. These trends are consistent with inference that there is not a single source of contaminants, as was discussed earlier. It appears that the area of greatest ground water contamination associated with one source is presently located north of the site (between wells 653 and 655) and continues to move north at a detectable rate. However, the area of greatest contamination associated with the second source, an ammonium and nitrate-rich source, appears to be or closer to the new tailings pile area and is moving at a slower rate. Thus, relatively high levels of contaminants may still exist beneath the site. More data are needed to confirm these suppositions.

One monitoring well east (crossgradient) of the site has relatively high sulfate concentrations (well 605). Also, a private well (no longer operable) upgradient of the site has relatively high sulfate concentrations compared to the ground water in other background wells. Uranium concentrations are also higher than in the other background samples. However, ground water in these wells is not thought to be influenced by contaminated ground water flow, but by ground water 1) with salts concentrated by evaporation, or 2) in contact with naturally occurring soluble salts in the alluvial aquifer. This is indicated by 1) the fact that private well 640 is located upgradient of the site and therefore cannot be affected by contaminated ground water flow; 2) the lack of nitrate (above background levels) in these two wells; and 3) the presence of relatively high concentrations of chloride. As noted, chloride concentrations in the tailings pore fluids are low.

The elevated concentrations of chloride, calcium, magnesium, sodium, potassium, alkalinity, and uranium are consistent with evaporative concentration of natural ground water. During evaporation, concentrations of major elements tend to remain at constant proportions. Thus, while sulfate and chloride concentrations increase, their relative proportions remain the same. In background ground water, the sulfate-to-chloride ratio is about 11 to 1 or less (Figure 3.18). By contrast, in the tailings solutions the sulfate-to-chloride ratio was about 137 to 1 or greater (based on data from lysimeters within the new tailings pile) because during ore processing, sulfate was added to the processing solutions while chloride was not. In contaminated ground water, the proportion 
Figure 3.16

Trends in Nitrate Concentrations over Time in Downgradiant Wells within the Alluvial Aquifer Monument Valley, Arizona, Site Area

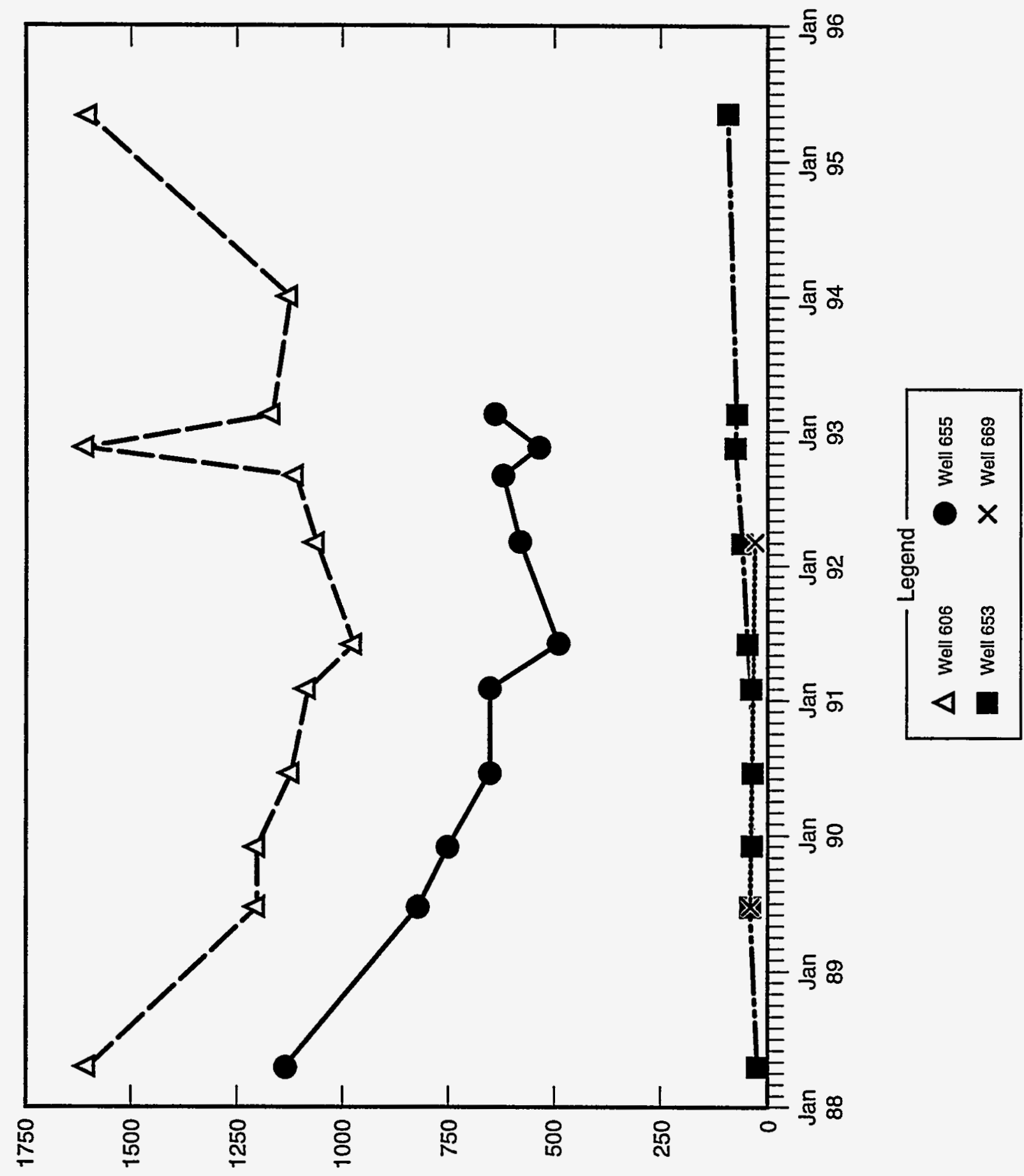

(7/6w) ә린 
Figure 3.17

Trends in Sulfate Concentrations over Time in Downgradiant Wells within the Alluvial Aquifer

Monument Valley, Arizona, Site Area

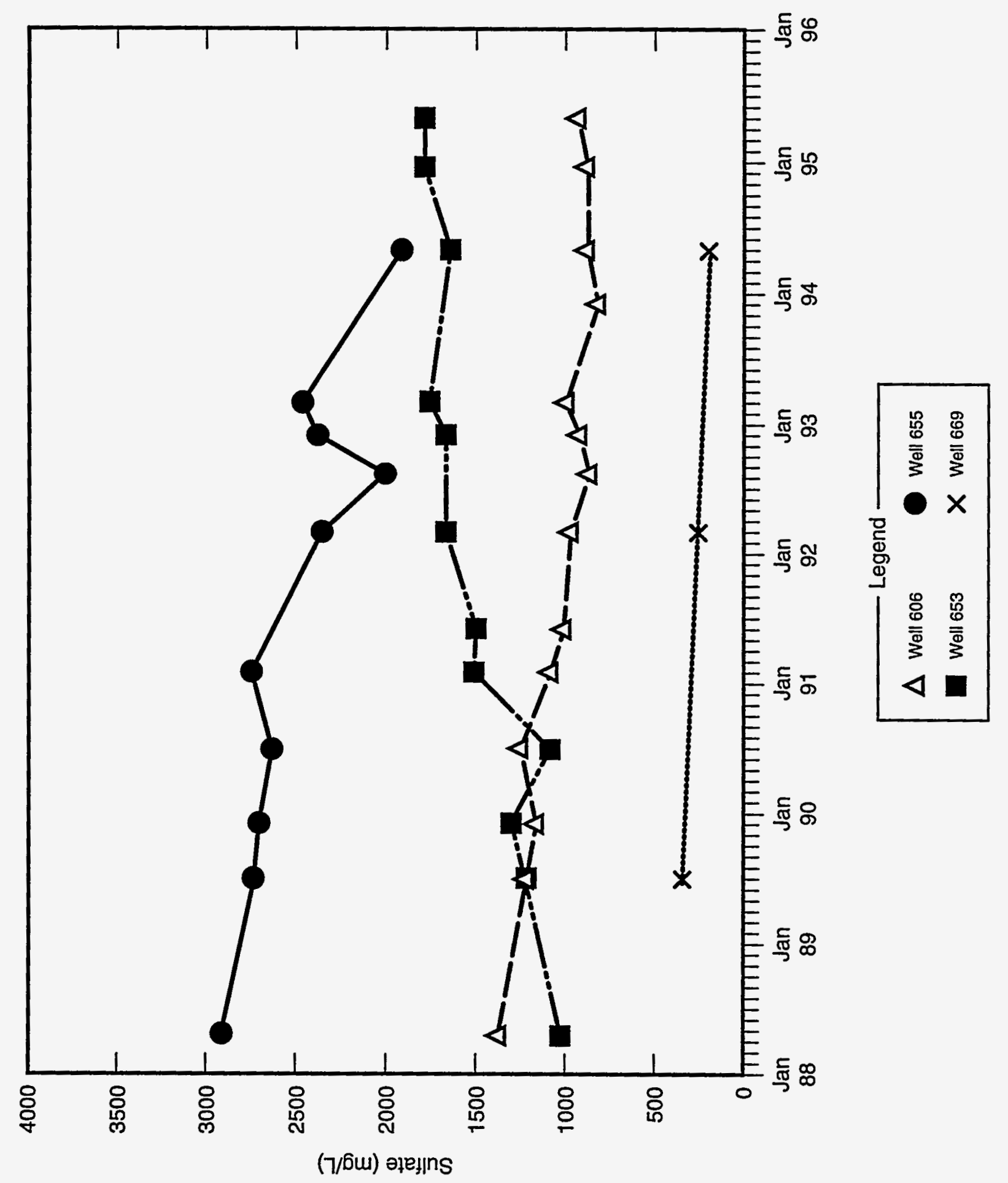


Figure 3.18

Distribution of Sulfate to Chloride Ratios in the

Alluvial Aquifer and Surface Waters

Monument Valley, Arizona, Site

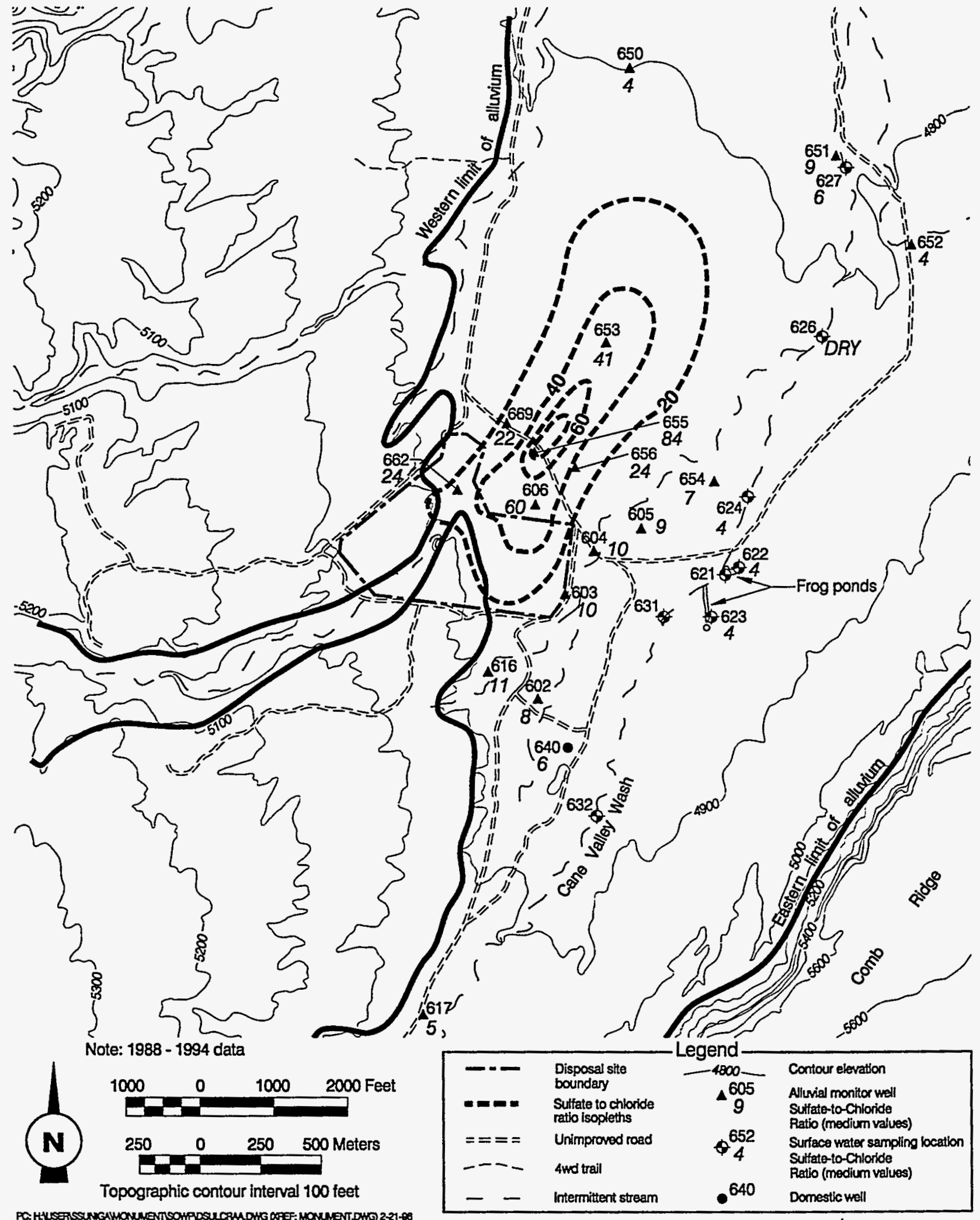

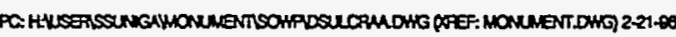


of sulfate to chloride is greater than 11 to 1 , ranging from about 60 to 1 (Figure 3.18).

Ground water in wells 640 and 605 have sulfate-to-chloride ratios in the range of background ( 6 to 1 and 9 to 1 , respectively) (Figure 3.18).

Based on all indicators of ground water contamination (sulfate, nitrate, uranium, and sulfate-to-chloride ratios), the extent of contamination appears to be within an area delineated by monitor wells $603,604,605,654,652,651$, and 650 east and north of the site; several of these downgradient uncontaminated wells are completed in the deeper portion of the alluvial aquifer, however, shallow ground water contamination could extend farther downgradient than is shown in Figure 3.12 (in the area of wells 650,651,652, and 654). The hydraulic gradient prevents contamination from extending south of the site, and the depositional limit of the alluvial aquifer corresponds to the western limit of possible contamination in the aquifer (Figures 3.13 through 3.16).

The vertical distribution of ground water contamination is not fully known. The saturated zone in the alluvial aquifer is from 0 to at least $60 \mathrm{ft}(18 \mathrm{~m})$ thick in the area of ground water contamination. In a few wells near the site, such as wells 603 and 606, the alluvial aquifer is thin and almost the entire saturated thickness of the alluvial aquifer is screened. Farther from the site, where the aquifer is thicker (wells 655, 656, and 653), only the upper 25 to $40 \mathrm{ft}$ ( 8 to $12 \mathrm{~m}$ ) of the saturated thickness of the alluvial aquifer is screened. Still farther from the site, in wells 650 and 654 , only the lower 50 to $84 \mathrm{ft}(15$ to $26 \mathrm{~m})$ of the saturated thickness of the aquifer is screened. Thus, the lateral and vertical distribution of contamination is not fully characterized in those areas. Northeast of the site, near wells 651 and 652, most of the saturated thickness is screened. However, the upper 10 to $15 \mathrm{ft}(3$ to $4.5 \mathrm{~m})$ of the saturated thickness remain to be sampled for ground water contamination.

\section{Ground water contamination in the Shinarump aquifer}

Ground water data collected since 1988 are available from five wells completed in the Shinarump aquifer: 601,614,615, 659, and 660. Wells 601 and 615 are the background wells, while the remainder are downgradient of the site. Of these, only well 614 displays evidence of site-related ground water contamination. In this well, the indicator parameters (calcium, sulfate, and nitrate) are elevated about 2 to 5 times those of background. Also, sulfate-tochloride ratios exceed 11 to 1 , suggesting contamination. Uranium is about 6 times background, but the level (median of $0.032 \mathrm{mg} / \mathrm{L}$ ) is still below the UMTRA uranium MCL. Table 3.10 compares the median values from this well to that in background.

Ground water contamination in the Shinarump aquifer at well 614 may be due to cross-formational flow of contaminated ground water from the alluvial aquifer into the upper portion of the Shinarump aquifer. The magnitude of contamination in well 614 , compared to the distribution of contamination in the 
Table 3.10 Comparison of contaminated ground water to background ground water quality data in the Shinarump aquifer, Monument Valley, Arizona, site

\begin{tabular}{|c|c|c|c|}
\hline Constituent & $\begin{array}{c}\text { MON-01-0601 } \\
\text { (background) }\end{array}$ & $\begin{array}{l}\text { MON-01-0615 } \\
\text { (background) }\end{array}$ & $\begin{array}{l}\text { MON-01-0614 } \\
\text { (contaminated) }\end{array}$ \\
\hline Alkalinity & 280 & 226 & 189 \\
\hline Aluminum & 0.07 & $<0.1$ & $<0.1$ \\
\hline Ammonium & 0.125 & $<0.1$ & $<0.1$ \\
\hline Antimony & 0.004 & $<0.003$ & $<0.003$ \\
\hline Arsenic & 0.005 & 0.003 & 0.005 \\
\hline Barium & 0.05 & 0.05 & 0.1 \\
\hline Boron & 0.05 & $<0.1$ & $<0.1$ \\
\hline Bromide & $<0.1$ & NA & $<0.1$ \\
\hline Cadmium & $<0.005$ & $<0.001$ & $<0.001$ \\
\hline Calcium & 28.4 & 17.5 & 88.0 \\
\hline Chloride & 15.1 & 9.9 & 20.0 \\
\hline Fluoride & 0.2 & 0.4 & 0.2 \\
\hline Iron & $<0.03$ & 0.13 & $<0.03$ \\
\hline Iron (unfiltered) & 107 & 2.95 & 3.06 \\
\hline Lead & $<0.005$ & $<0.01$ & $<0.01$ \\
\hline Lead 210 & $<2.0$ & $<2.0$ & NA \\
\hline Lead-210 (unfiltered)) & $<2.0$ & 7.2 & NA \\
\hline Magnesium & 18 & 16 & 57 \\
\hline Manganese & 0.005 & 0.005 & $<0.01$ \\
\hline manganese (unfiltered) & 1.46 & 0.35 & 0.11 \\
\hline Molybdenum & 0.005 & $<0.005$ & $<0.007$ \\
\hline Nitrate & 4.25 & 4.3 & 25.2 \\
\hline $\mathrm{pH}$ & 7.79 & 7.84 & 7.59 \\
\hline phosphate (unfiltered) & 2.6 & $<0.1$ & 1.3 \\
\hline Potassium & 1.9 & 3.1 & 1.8 \\
\hline Radium-226 & 0.3 & 1.2 & 0.5 \\
\hline Radium-228 & 1.4 & 2.7 & 1.0 \\
\hline Redox potential & 442 & 248 & 456 \\
\hline Selenium & 0.005 & $<0.005$ & 0.005 \\
\hline Silica & 14 & 9 & 14 \\
\hline Sodium & 99 & 88 & 31 \\
\hline Strontium & 0.29 & 0.63 & 0.69 \\
\hline $\begin{array}{l}\text { OEE/AL/62350-201 } \\
\text { EEV. 0, VER. } 6\end{array}$ & & & $\begin{array}{r}8-M a \\
002 D 653 . D O C ~\end{array}$ \\
\hline
\end{tabular}


Table 3.10 Comparison of contaminated ground water to background ground water quality data in the Shinarump aquifer, Monument Valley, Arizona, site (Concluded)

\begin{tabular}{lccc}
\hline \multicolumn{1}{c}{ Constituent } & $\begin{array}{c}\text { MON-01-0601 } \\
\text { (background) }\end{array}$ & $\begin{array}{c}\text { MON-01-0615 } \\
\text { (background) }\end{array}$ & $\begin{array}{c}\text { MON-01-0614 } \\
\text { (contaminated) }\end{array}$ \\
\hline Sulfate & 125 & 63 & 255 \\
\hline Thorium-230 & $<1.0$ & $\mathrm{NA}$ & $<1.0$ \\
Total dissolved solids & 429 & 327 & 663 \\
Uranium & 0.005 & 0.001 & 0.032 \\
Vanadium & $<0.025$ & $<0.005$ & $<0.01$ \\
Zinc & 0.0025 & 0.0130 & 0.005 \\
Zinc (unfiltered) & 0.143 & 0.038 & 0.050 \\
\hline
\end{tabular}

Notes: 1. Data listed are median values for filtered samples (except as noted) collected from 1988 through 1994.

2. All data in milligrams per liter (mg/L) except for the following: lead-210, radium-226, radium-228, thorium-230 in picocuries per liter; $\mathrm{pH}$ in standard units; redox potential in millivolts; alkalinity as $\mathrm{mg} / \mathrm{L}$ calcium carbonate equivalent.

3. The following were not detected in filtered ground water samples (method detection limit is given in parentheses): beryllium $(0.005 \mathrm{mg} / \mathrm{L})$, cadmium (0.001), chromium (0.005), cobalt (0.05), copper $(0.02 \mathrm{mg} / \mathrm{L})$, mercury $(0.0002)$, nickel $(0.04 \mathrm{mg} / \mathrm{L})$, silver $(0.01 \mathrm{mg} / \mathrm{L})$, thallium $(0.01 \mathrm{mg} / \mathrm{L})$, and tin $(0.01 \mathrm{mg} / \mathrm{L})$.

$<-$ indicates less than the given value.

NA - not analyzed. 
alluvial aquifer, is consistent with this model. In well 614, the ground water table is essentially at the contact between the alluvial and Shinarump aquifers. Therefore, the contaminated ground water would have to flow laterally (from the southeast) rather than vertically into the Shinarump at this point.

The two Shinarump wells (614 and 659) immediately beneath the contaminated plume within the alluvial aquifer do not show evidence of ground water contamination.

\subsubsection{Ground water contamination in the De Chelly Sandstone}

Two wells within the De Chelly Sandstone (619 and 657) show the influence of site-related contamination. In both wells, located within a few hundred feet of each other, concentrations of calcium, nitrate, sulfate, and uranium exceed background concentrations (Table 3.11). Also, sulfate-to-chloride ratios exceed 11 to 1 , consistent with site-related contamination. Both wells are in the area of the site once occupied by the old tailings pile and heap leach pads. This area is underlain by a 100-ft (30-m)-deep buried channel, in bedrock filled with watertransported and windblown sands. The boring log for well 657 indicates the channel has eroded through the Shinarump and Moenkopi such that the alluvial aquifer rests directly upon the De Chelly Sandstone in this area. Also, well 619 was used to provide processing water during milling operations, and water for the tailings removal operations. Pumping this well appears to have drawn contaminated ground water from the alluvial aquifer into the De Chelly Sandstone in this area. No other wells in the De Chelly Sandstone show evidence of site-related ground water contamination.

\section{Constituents above background}

Constituents identified as above background in the alluvial aquifer are ammonium, calcium, chloride, iron, magnesium, manganese, nitrate, potassium, silica, sodium, strontium, sulfate, uranium, vanadium, and zinc (DOE, 1994a). Several of these (chloride, silica, sodium, vanadium, and zinc) occur at levels only slightly elevated above background (less than 3 times background) and are poor indicators of contamination. Those that occur at moderate levels above background ( 3 to 10 times background) are iron, magnesium, strontium, and uranium. Constituents that provide the greatest contrast to background (more than 10 times background) are ammonium, calcium, nitrate, and sulfate.

While calcium, nitrate, sulfate, and uranium in De Chelly wells 619 and 657 suggest site-related impacts to De Chelly ground water, concentrations of uranium in these wells suggest there may be additional sources for uranium. Uranium levels in wells 619 and 657 are relatively high while other site-related show only minor elevations from background. Thus the uranium concentrations at depth (Table 3.11) are much greater than expected when compared to other site-related constituents (calcium, nitrate and sulfate). Because there is no geochemical explanation for preferential migration of uranium from the tailings 
Table 3.11 Comparison of contaminated ground water to background ground water quality data in the De Chelly Sandstone, Monument Valley, Arizona, site

\begin{tabular}{|c|c|c|c|c|}
\hline Constituent & $\begin{array}{c}\text { MON-01-0612 } \\
\text { (background) }\end{array}$ & $\begin{array}{l}\text { MON-01-0613 } \\
\text { (background) }\end{array}$ & $\begin{array}{l}\text { MON-01-0619 } \\
\text { (contaminated) }\end{array}$ & $\begin{array}{l}\text { MON-01-0657 } \\
\text { (contaminated) }\end{array}$ \\
\hline Alkalinity & 121 & 185 & 189 & 193 \\
\hline Aluminum & NA & $<0.1$ & NA & NA \\
\hline Ammonium & $<0.1$ & $<0.1$ & $<0.1$ & $<0.1$ \\
\hline Antimony & NA & $<0.003$ & $<0.003$ & $<0.003$ \\
\hline Arsenic & 0.01 & $<0.005$ & $<0.005$ & 0.005 \\
\hline Barium & NA & 0.05 & $<0.1$ & 0.20 \\
\hline Boron & NA & $<0.05$ & NA & $<0.1$ \\
\hline Bromide & NA & $<0.1$ & NA & $<0.1$ \\
\hline Cadmium & 0.004 & $<0.005$ & NA & 0.001 \\
\hline Calcium & 28 & 16 & 56 & 64 \\
\hline Chloride & 6 & 8 & 7 & 8 \\
\hline Fluoride & NA & 0.3 & NA & 0.2 \\
\hline Iron & NA & $<0.03$ & $<0.03$ & $<0.03$ \\
\hline Iron (unfiltered) & NA & 0.04 & $<0.03$ & 0.06 \\
\hline Lead 210 & NA & $<2.0$ & $<2.0$ & $<2.0$ \\
\hline Lead-210 (unfiltered)) & NA & 5.8 & NA & NA \\
\hline Magnesium & 18 & 29 & 32 & 41 \\
\hline Manganese & NA & $<0.005$ & $<0.01$ & $<0.01$ \\
\hline manganese (unfiltered) & NA & $<0.005$ & $<0.01$ & $<0.01$ \\
\hline Molybdenum & 0.01 & 0.005 & 0.03 & $<0.01$ \\
\hline Nitrate & 8.0 & 4.3 & 20 & 20 \\
\hline $\mathrm{pH}$ & 7.64 & 8.01 & 7.20 & 7.39 \\
\hline phosphate (unfiltered) & NA & $<0.1$ & $<0.1$ & $<0.1$ \\
\hline Potassium & 5.3 & 2.8 & 2.0 & 1.8 \\
\hline Radium-226 & NA & 0.3 & NA & $<1.0$ \\
\hline Radium-228 & NA & $<1.0$ & NA & $<1.0$ \\
\hline Redox potential & NA & 447 & 447 & 431 \\
\hline Selenium & $<0.005$ & 0.0025 & NA & $<0.005$ \\
\hline Silica & NA & 11 & 13 & 14 \\
\hline Sodium & 13 & 50 & 22 & 15 \\
\hline Strontium & NA & 0.35 & 0.45 & 0.51 \\
\hline Sulfate & 20 & 61 & 73 & 127 \\
\hline
\end{tabular}


Table 3.11 Comparison of contaminated ground water to background ground water quality data in the De Chelly Sandstone, Monument Valley, Arizona, site (Concluded)

\begin{tabular}{lcccc}
\hline \multicolumn{1}{c}{ Constituent } & $\begin{array}{c}\text { MON-01-0612 } \\
\text { (background) }\end{array}$ & $\begin{array}{c}\text { MON-01-0613 } \\
\text { (background) }\end{array}$ & $\begin{array}{c}\text { MON-01-0619 } \\
\text { (contaminated) }\end{array}$ & $\begin{array}{c}\text { MON-01-0657 } \\
\text { (contaminated) }\end{array}$ \\
\hline Thorium-230 & NA & $<1.0$ & NA & $<1.0$ \\
Total dissolved solids & 195 & 289 & 320 & 335 \\
Uranium & 0.010 & 0.004 & 0.134 & 0.064 \\
Vanadium & NA & 0.005 & 0.02 & 0.06 \\
Zinc & NA & 0.003 & 0.105 & $<0.005$ \\
Zinc (unfiltered) & NA & 0.005 & 0.035 & 0.197 \\
\hline
\end{tabular}

Notes: 1. Data listed are median values for samples collected from 1988 through 1994.

2. All data in milligrams per liter ( $\mathrm{mg} / \mathrm{L}$ ) except for the following: lead-210, radium-226, radium-228, thorium-230 in picocuries per liter; $\mathrm{pH}$ in standard units; redox potential in millivolts; alkalinity as $\mathrm{mg} / \mathrm{L}$ calcium carbonate equivalent.

3. The following were not detected in filtered ground water samples (method detection limit is given in parentheses): beryllium $(0.005 \mathrm{mg} / \mathrm{L})$, cadmium (0.001), chromium (0.005), cobalt (0.05), copper $(0.02 \mathrm{mg} / \mathrm{L})$, lead (0.005), mercury $(0.0002)$, nickel $(0.04 \mathrm{mg} / \mathrm{L})$, silver $(0.01 \mathrm{mg} / \mathrm{L})$, thallium $(0.01 \mathrm{mg} / \mathrm{L})$, and tin $(0.01 \mathrm{mg} / \mathrm{L})$.

$<-$ indicates less than the given value.

NA - not analyzed. 
piles through ground water, the relatively high levels of uranium may reflect contribution from a nearby uranium ore body in addition to site-related effects. This possibility is supported by reports of vanadium mineralization (presumably with associated uranium) in the De Chelly Sandstone at the Monument No. 2 Mine (Witkind and Thaden, 1963).

\subsubsection{Contaminant fate and transport mechanisms}

Site-related contaminants in ground water will be subject to dispersion, radioactive decay, and various chemical reactions, including oxidation/reduction reactions, precipitation and coprecipitation, adsorption onto the aquifer mineral surfaces, cation exchange, and biologically controlled oxidation/reduction reactions.

Ground water quality data provide insight into the fate and transport of siterelated contaminants in ground water at the site. Several constituents identified in the tailings solutions are absent or occur at much lower levels in the contaminated ground water, due to reactions of these contaminants with the aquifer matrix. Contaminants present in tailings solutions, but absent (below detection) in ground water at the site, are trace metals, including antimony, chromium, cobalt, copper, molybdenum, and nickel. Contaminants in tailings solutions that are greatly attenuated, but detectable above background, are ammonium, radium-226, uranium, and vanadium. Contaminants that are at levels in ground water similar to those in the tailings solutions are major cations and anions (calcium, nitrate, magnesium, chloride, potassium, strontium, and sulfate) (Table 3.9 ).

Dispersion and precipitation reactions control concentrations of the major cations and anions such as calcium, iron, magnesium, nitrate, potassium, silica, sodium, strontium, and sulfate. Precipitation/dissolution reactions will occur in the portion of the plumes closest to the former tailings piles. Precipitation reactions currently are active because the shallow ground water in this zone is oversaturated with gypsum. With the tailings piles removed, the ground water sulfate concentrations will decrease, allowing gypsum to redissolve. Dissolution will buffer sulfate and calcium concentrations until the gypsum is exhausted. At this point, dispersion with background water will lower sulfate and calcium concentrations.

Cation exchange reactions with clays and oxidation to nitrate perhaps, mediated by bacterial action, will decrease ammonium concentrations. Dispersion and adsorption mainly will decrease manganese, uranium, vanadium, and zinc concentrations.

\subsubsection{Surface water and sediments}

Field observations along Cane Valley Wash are important in interpreting the results of sediment and surface water sampling. In the area of the Frog Ponds and Cane Valley Wash north of monitor well 658 (upgradient of the site), the 
ground water table in the alluvial aquifer is commonly at or within a few feet of the surface. Capillary action keeps the sediments in the bottom of the wash wet, and evaporation and transpiration by plants of the capillary water results in the precipitation and accumulation of a 1- to 3-mm-thick crust of salts over most of the surface of the wash. In some areas downstream of the Frog Ponds, the area covered by salts is more than $300 \mathrm{ft}(100 \mathrm{~m})$ wide. Examination of these salts indicates that they are predominantly sodium carbonate and very soluble. Wind transport of the salts has been observed, inferring that wind ablation and dissolution of the salts during rains precludes the formation of thick salt deposits in the wash.

Natural scour pools in the wash are common and often intersect the ground water table. Except after rains, water in these surface pools is derived from the near-surface ground water affected by evaporation and transpiration. Thus, the water in the pools along the wash tend to have high TDS as determined by electrical conductivity measurements in the field.

\section{Sediment results}

Table 3.12 presents chemical results for sediments collected in 1993. Sediment collected from the northernmost Frog Pond (location 623) is upgradient from the site and was not identified as a vicinity property (VP). Therefore, this location provides sediment background samples. Levels of arsenic, strontium, uranium, vanadium, and zinc are low compared to average concentrations in soils and sediments (Shacklette and Boerngen, 1984), possibly reflecting the sandy, well sorted nature of the sediments derived from windblown sands. Sulfate concentrations are notable, and may reflect the accumulation of natural sulfate salts in the sediments due to evaporation and transpiration.

Comparison of sediments in Cane Valley Wash $(624,626$, and 627) indicates no notable differences in sediment chemistry from those in the Frog Ponds area. Zinc at location 627 and arsenic at location 620 are elevated when compared to the Frog Pond sample; however, more data would be required to determine if these levels of arsenic and zinc are beyond the range of ambient values.

\section{Surface water results}

Table 3.13 summarizes surface water sampling results. Location 623 serves as a background sample. Locations 621 and 622 are downstream (north) of 623, in a second frog pond fed by water in the southern frog pond. This northern pond was a VP. The banks were stabilized with uranium mineralized rock. The rock was removed during surface remediation. Because water samples from locations 621 and 622 were collected before remediation, some constituents in surface water at these locations could be elevated by the ore rock. However, the hydraulic gradient precludes contamination of the Frog Ponds by ground water discharge. 
Table 3.12 Summary of sediment chemistry data, Monument Valley, Arizona, site

\begin{tabular}{lccccc}
\hline Constituent & $\begin{array}{c}\text { MON-01-0623 } \\
\text { (background) }\end{array}$ & MON-01-0624 & MON-01-0626 & MON-01-0627 & MON-01-0620 \\
\hline Arsenic & 0.9 & 0.6 & 0.8 & 0.9 & 4 \\
Nitrate & 1.8 & 3.8 & 0.4 & 0.9 & 0.9 \\
Strontium & 116 & 46 & 26 & 54 & 28 \\
Sulfate & 771 & 935 & 42 & 35 & 48 \\
Uranium & $<1.0$ & $<1.0$ & $<1.0$ & $<1.0$ & 1.0 \\
Vanadium & 6 & 3 & 5 & 9 & 9.0 \\
Zinc & 10.7 & 10.8 & 6.8 & 29.2 & 18.7 \\
\hline
\end{tabular}

Notes: 1. Samples collected June 1993.

2. All data in milligrams per kilogram.

$<-$ indicates less than the given value.

Variations in alkalinity, chloride, magnesium, sodium, sulfate, and TDS at sampling locations 621,622 , and 623 are due to differences in evaporation and transpiration rates caused by climatic conditions at the time of sampling. For example, chloride concentrations vary from 5 to $172 \mathrm{mg} / \mathrm{L}$ while sulfate varies from 10 to $452 \mathrm{mg} / \mathrm{L}$ in the same samples. The sulfate-to-chloride ratio tends to remain the same during evaporative concentration (provided saturation with sulfate salts is not reached, as is the case with the sampled surface waters). In the background surface water samples, the sulfate-to-chloride ratio is about 4 to 1 . Likewise, in samples from locations 621 and 622 , the sulfate-to-chloride ratios also are about 4 to 1 , regardless of the sulfate concentration. Thus, variations in most constituents in these samples are due to varying amounts of evaporative concentration. This also may be true of uranium and molybdenum. However, the former presence of ore rock in the southern pond is another possible source of ore uranium and molybdenum. Sampling the southern pond after remediation will help determine if past observed uranium is natural or a result of site-related contamination.

Surface and near-surface water quality data from Cane Wash also indicate evaporation of ground water discharge to the wash is affecting water quality at locations 624,626, and 627. Samples collected from location 627 best demonstrate this. Water collected at this location is sodium-carbonate water, with dissolved carbonate concentrations of up to $15,100 \mathrm{mg} / \mathrm{L}$. This high carbonate concentration is the expected result of evaporation of sodium bicarbonate water, such as characterizes background water in the area. By contrast, the contaminated ground water does not have elevated concentrations of bicarbonate or carbonate. Further, it can be demonstrated that evaporation of a calcium sulfate water, such as the contaminated ground water at the site, 


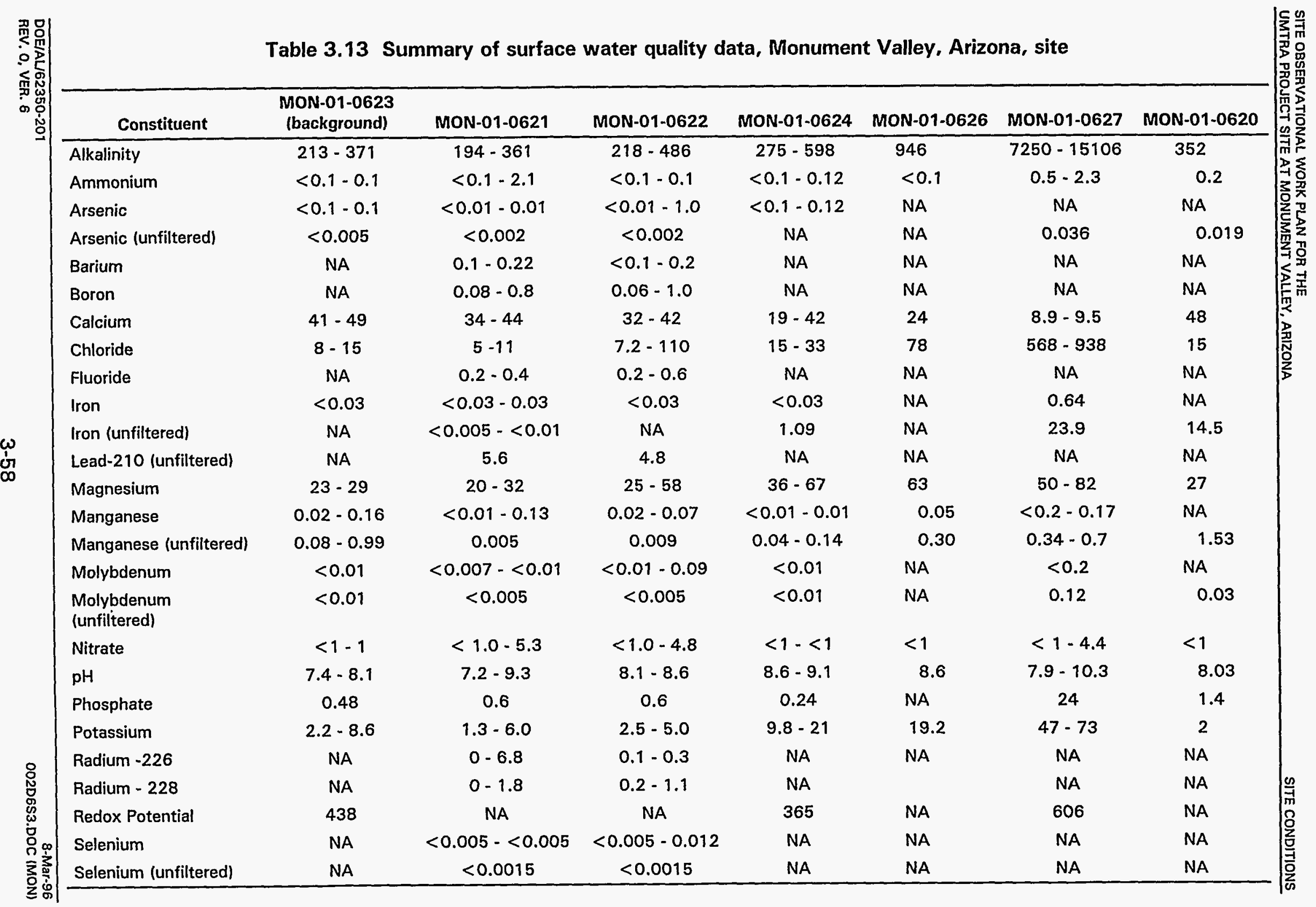




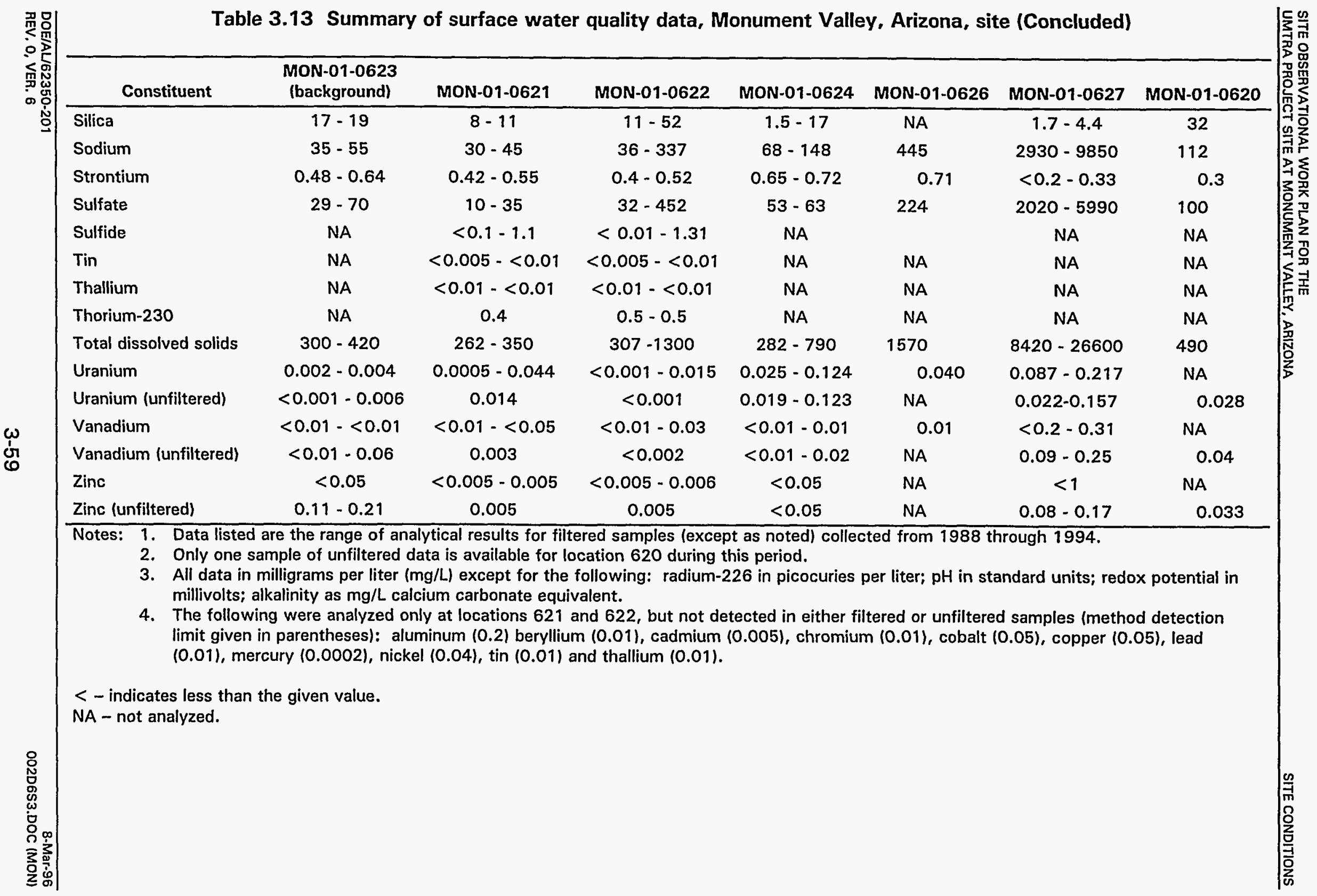


would lead to a saline water containing little or no carbonate (Drever, 1982). Thus, the high TDS near surface water is not related to contaminated ground water discharge to Cane Valley Wash. The conditions listed below support this conclusion.

- Chloride concentrations much higher than those found in contaminated ground water.

- Sulfate to chloride ratios of about 5 to 1 , consistent with an evaporative concentration of natural ground water discharge rather than discharge of contaminated ground water.

- The relatively low concentration indicators of ground water contamination other than sulfate, including calcium, nitrate, and ammonium.

Thus, it is clear that the variations in most, if not all, constituent concentrations in Cane Valley Wash are due to varying rates of evaporation and ground water discharge. Chloride concentrations suggest that evaporation may concentrate natural constituents in near-surface waters by a factor of 60 or more. Given that the background ground water discharging to the wash contain up to $0.006 \mathrm{mg} / \mathrm{L}$ uranium, it is reasonable to expect uranium concentrations of more than $0.36 \mathrm{mg} / \mathrm{L}$ in evaporated surface water. This is especially true because uranium is very soluble and stable in the presence of dissolved carbonate. Nonetheless, the available data do not completely rule out the possibility that at least some uranium, molybdenum, and vanadium in the wash are derived from ore rock and tailings used to construct haul-road crossings over the wash and its tributaries. This possibility, however, is not supported by the available sediment data discussed above.

Surface water data were collected once since 1988, from a spring $2 \mathrm{mi}(3.2 \mathrm{~km})$ downgradient of the site. This spring (location 620) is on a tributary to Cane Valley Wash. Water quality data are similar to those of the Frog Ponds and do not show evidence of contaminated ground water discharge. Uranium, vanadium, and molybdenum are at elevated levels when compared to location 623; because of the VP (haul-road crossing) upstream of the sampling location, it cannot be determined if these three ore-related elements occur naturally or are due to uranium ore rock washed down the stream.

\subsection{HUMAN HEALTH RISK SUMMARY}

The 1994 baseline risk assessment evaluated the potential public health and environmental risks of ground water contamination caused by activities at the former Monument Valley processing site (DOE, 1994a). Site-specific data collected after the risk assessment was completed are used in this document to help determine a probable compliance strategy for the site. Section 3.4 summarizes the ecological risk. 
Domestic ground water use is defined in this document as water used for drinking, bathing, and other household purposes such as cooking and garden irrigation. Ground water is the sole source of domestic water in the Monument Valley area. Domestic well users upgradient of the site and several domestic wells downgradient of the site have not been impacted by the contaminant plume (Figures 3.13 through 3.15). The depth to ground water is shallow in some areas near the site (i.e., Cane Valley Wash). In the areas where ground water reaches the surface, ponds form that are accessed by people and animals. However, these areas have not been impacted by the contaminated ground water; therefore, complete exposure pathways to contaminated ground water discharging to the surface water and sediment do not exist.

Currently, no one uses the contaminated ground water and, therefore, no human health risks have resulted from the use of the water. However, future use of the contaminated ground water is possible. Evaluation of potential future use indicates certain health risks could occur if the contaminated ground water (i.e., ground water within the most contaminated portion of the plume) were ingested as drinking water.

\subsubsection{Contaminants of potential concern}

Contaminants of potential concern are contaminants that could cause adverse health effects if taken into the body. Contaminants of potential concern were selected for the Monument Valley site using the following procedure.

Initially, if a constituent statistically exceeded background concentrations, it was placed on a screening list that included the following constituents: ammonium, calcium, chloride, iron, magnesium, manganese, nitrate, potassium, silica, sodium, strontium, sulfate, uranium, vanadium, and zinc (Table 3.14).

If the maximum detected concentration of a constituent on the screening list fell into acceptable nutritional requirement levels that would not be exceeded with exposure, it was not retained as a contaminant of potential concern. The constituents that fell into this category were chloride, iron, potassium, and zinc.

If the maximum detected concentration of a constituent on the screening list fell into the high end of expected dietary ranges but was of low toxicity, it was not retained as a contaminant of potential concern. The constituents that fell into this category were ammonium, calcium, magnesium, silica, and sodium.

All remaining constituents on the list were considered contaminants of potential concern because of their potential toxic effects if ingested. These contaminants were evaluated quantitatively in the risk assessment: nitrate, sulfate, manganese, strontium, vanadium, and uranium. Of these contaminants, uranium is the only carcinogen.

Five exposure routes were evaluated for their potential adverse health effects: ground water ingestion and dermal contact, the ingestion of garden produce 


\begin{tabular}{|c|c|c|c|c|c|c|}
\hline \multirow{4}{*}{ 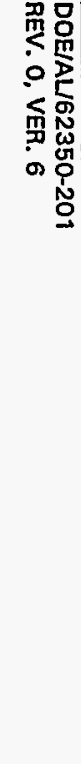 } & Table 3.14 & \multicolumn{5}{|c|}{$\begin{array}{l}\text { Constituents detected above background and evaluated in the } 1994 \text { risk assessment, Monument Valley, } \\
\text { Arizona, site }\end{array}$} \\
\hline & Ammonium & $6-13$ & 0.02 & NA & NA & $\begin{array}{l}\text { Ammonium is not a dietary component but is produced in the body } \\
\text { at levels greater than } 4000 \mathrm{mg} / \text { day }^{\mathrm{d}} \text {. If the concentrations in the } \\
\text { water combine with what is created in the body, toxicity is not } \\
\text { expected to occur. Ammonium can convert into nitrate. }\end{array}$ \\
\hline & Calcium & $11-19$ & 2 & 23 & $20-37$ & $\begin{array}{l}\text { Detected concentrations fall within nutritional requirements. If } \\
\text { concentrations in the water are added to the dietary intake range, } \\
\text { exposure falls above the high end of the dietary intake range but } \\
\text { adverse effects would not be expected. }\end{array}$ \\
\hline & Chloride & $0.8-1$ & 2 & $5-21$ & 170 & $\begin{array}{l}\text { Concentrations detected in the water fall below daily nutritional } \\
\text { requirements; detected concentrations are considered to be at safe } \\
\text { levels. }\end{array}$ \\
\hline & Manganese & $0.001-0.03$ & 0.0003 & $0.03-0.09$ & $0.03-0.08$ & $\begin{array}{l}\text { Concentrations fall below nutritional requirement and dietary } \\
\text { range; manganese is more bioavailable in water than food, risk } \\
\text { assessment determined highest exposure levels have the potential } \\
\text { to exhibit mild toxic effects. }\end{array}$ \\
\hline & Nitrate & $28-46$ & 1 & NA & NA & $\begin{array}{l}\text { Detected in concentrations determined toxic by the risk } \\
\text { assessments (especially to infants); detected above the } \mathrm{MCL} \text {. The } \\
\text { conversion of ammonium into nitrate would increase the nitrate } \\
\text { concentration. }\end{array}$ \\
\hline 总 & Potassium & $0.7-1$ & 0.07 & $0.4-0.6$ & 46 & $\begin{array}{l}\text { Detected concentrations fall below nutritional requirements; if } \\
\text { added to the dietary range, concentrations are considered to be } \\
\text { safe. }\end{array}$ \\
\hline
\end{tabular}




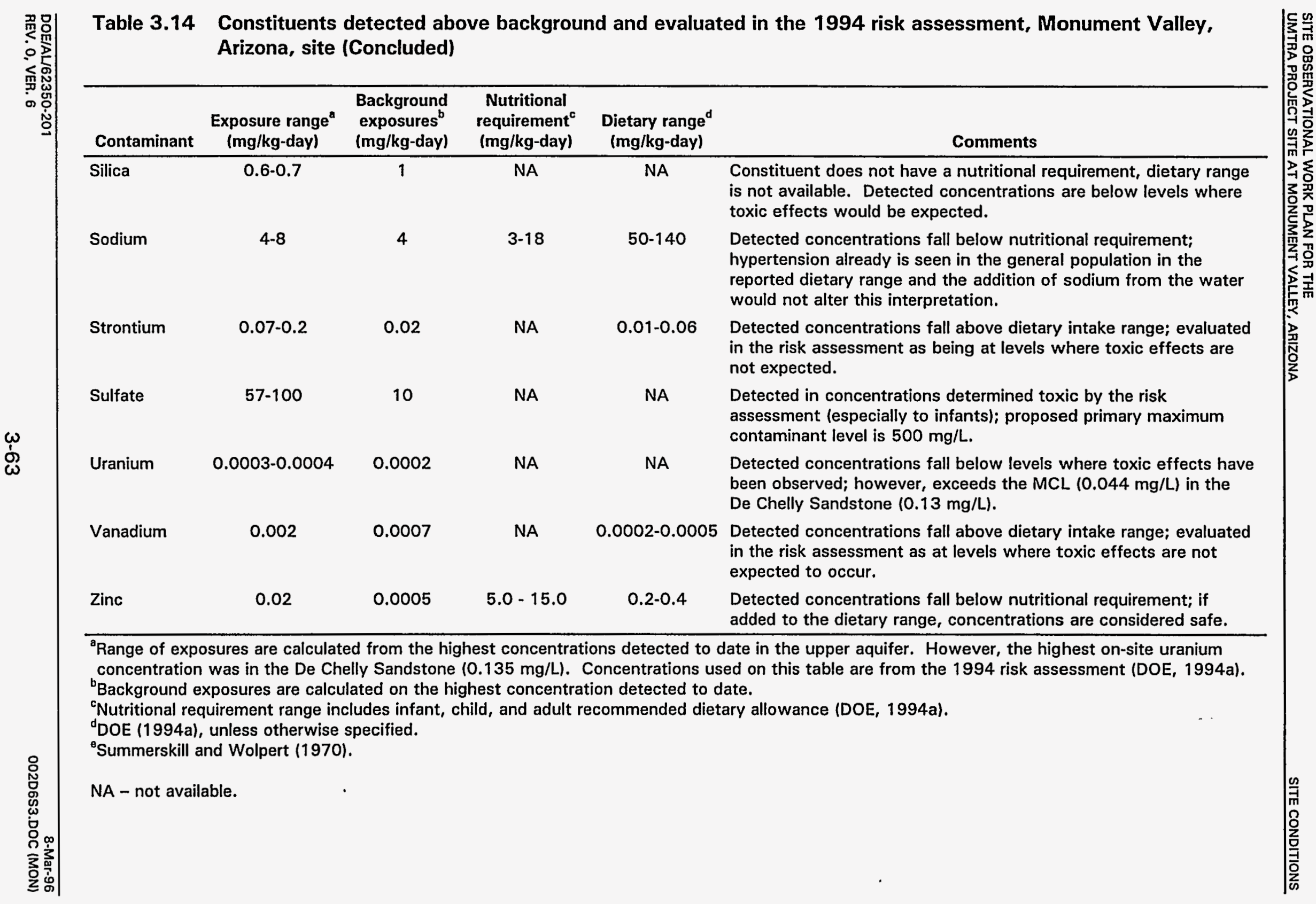


irrigated with contaminated ground water, and the ingestion of milk and meat from livestock watered with ground water from the contaminated aquifer. Toxicity due to exposure from these exposure routes was evaluated by calculating the exposure dose, using the upper 95 percent confidence limit of the mean concentration in ground water. The main exposure route risk contributors were identified by calculating a ratio of these exposure doses to the exposure dose from ground water ingestion (as drinking water). Ground water used as a sole source of drinking water was determined to contribute the greatest potential risk relative to all the exposure pathways screened. Therefore, the use of ground water as drinking water was further evaluated quantitatively.

Probability distributions for contaminant concentrations and exposure variables were integrated to estimate the range of contaminant exposure doses people could ingest from a hypothetical well constructed in the most contaminated portion of the plume. Filtered water quality data from 1988 to 1993 from DOE monitor well 655 were used to evaluate on-site levels of contaminants in the alluvial aquifer. The estimated amounts of contaminants a child could ingest through drinking water were compared to toxic effects anticipated for each contaminant at these amounts. Children (1 to 10 years) were evaluated for these exposure scenarios because children consume relatively more water than adults and, consequently ingest a higher contaminant dose than adults. However, when a subpopulation was identified as more sensitive to exposure to certain contaminants, that population was evaluated. For the Monument Valley site, infants have been identified as the population most sensitive to sulfate and nitrate. Adults were evaluated for the carcinogen uranium, because carcinogens are evaluated over a lifetime of 70 years.

\subsubsection{Potential public health impacts}

The most serious noncarcinogenic health risks from ingesting ground water at this site would result from nitrate. Some degree of methemoglobinemia (the interference of the oxygen-carrying capacity of the blood, could occur with any infant consumption of nitrate in ground water at this site. Severe diarrhea in infants could be expected from sulfate exposure. Manganese levels could cause mild neurological symptoms such as irritability and speech disturbances. More than 99 percent of the strontium and vanadium exposures fell below any levels where any noncarcinogenic toxic effects have been observed in humans. The entire range of uranium exposures fell below any level where noncarcinogenic toxic effects have been observed in humans.

Since the completion of the risk assessment the carcinogenic toxicity factor for uranium has changed. Based on this change, the increased individual lifetime cancer risk for uranium in the alluvial aquifer is estimated to be $2 \mathrm{E}-05$, or 2 chances in 100,000 of developing cancer. For the maximum concentration of uranium detected in the De Chelly Sandstone $10.13 \mathrm{mg} / \mathrm{L})$, the estimated risk level is $1 \mathrm{E}-04$, or 1 chance in 10,000 of developing cancer. These estimated risk levels fall into the acceptable EPA risk range for carcinogens of $1 \mathrm{E}-04$ to 1E-06 (1 chance in 10,000 to 1 chance in $1,000,000$ of developing cancer). 
It should be noted that the results of the risk assessment are based on the most contaminated portion of the plume characterized to date. Higher contaminant levels could be detected in the ground water because the entire plume has not. been characterized. Therefore, the potential public health risk could be underestimated at this site.

\subsection{ECOLOGICAL RISK SUMMARY}

This section assesses the potential adverse effects of site-related contaminants on the existing biological communities and grazing and other agricultural practices in the site area; incorporates data collected since the preparation of the baseline risk assessment; and reevaluates some analyses in the baseline risk assessment, based on recent hydrologic and geochemical analysis.

Currently, the EPA has no guidance for quantifying potential ecological impacts of the release of hazardous constituents; however, the EPA has developed a qualitative approach for use in ecological risk assessments (EPA, 1989). The ecological risk assessment for the Monument Valley site is consistent with this approach.

\subsubsection{Potential ecological receptors and effects}

The ecological resources at the site that could be exposed to site-related contaminants are Cane Valley Wash, approximately $1500 \mathrm{ft}(460 \mathrm{~m})$ east of the former lower tailings pile, and the Frog Ponds, 1500 to $2000 \mathrm{ft}(460$ to $610 \mathrm{~m})$ east-northeast of the former lower tailings pile (Figure 3.6). Another potential pathway into the biological communities is plant root uptake of contaminated ground water. The area over the plume is sandy soil, dominated by desert shrub. In general, the plants are widely spaced by bare sandy soil.

The contaminant plume is moving north roughly parallel with Cane Valley Wash and the contaminated ground water is not discharging into the wash or any other location, including the Frog Ponds. The risk assessment evaluated several ground water plume constituents in near-surface water (sample locations 624 and 627) (Figure 3.6). Geochemical analysis of this water shows that the elevated constituent concentrations are from evaporation and are not siterelated. Therefore, it is believed that the contaminated ground water from the Monument Valley site is not discharging into the environment and does not pose ecological risk to the aquatic or terrestrial biological communities.

As indicated in Section 3.3, the contaminated ground water at the site is $\mathbf{3 0}$ to $35 \mathrm{ft}(9$ to $11 \mathrm{~m})$ below land surface. Few if any of the plants growing in the sparse plant community over the contaminant plume send roots this deep into the soil. The rooting depths of some species growing over the plume are salt bush roots, up to $4 \mathrm{ft}(1.2 \mathrm{~m})$; black greasewood, up to $4.5 \mathrm{ft}(1.3 \mathrm{~m})$; and Russian thistle, up to $6 \mathrm{ft}(1.8 \mathrm{~m})$ (Branson et al., 1976; Wiese, n.d.). Species such as black greasewood can root much deeper. Plant roots were noted in 
$30 \mathrm{ft}(9 \mathrm{~m})$ excavations into water-filled paleochannels at the Grand Junction, Colorado, disposal cell; black greasewood was the most common species growing over these paleochannels and these roots may have been from this species. Therefore, the potential risk associated with plants contacting contaminated ground water is considered slight to nonexistent.

\subsubsection{Potential livestock and agricultural effects}

The contaminated ground water is not being used to water livestock or irrigate crops, or for any other agricultural purpose. However, if the ground water in the contaminated portion of the plume were accessed for domestic use, it could be used to water livestock or irrigate plants. Nitrate and sulfate concentrations in the plume preclude using ground water to water livestock. However, the degree to which various constituents in ground water will accumulate in plant tissue and pose a hazard is not known. The DOE and the University of Arizona are studying this phenomenon.

\subsection{ASSESSMENT OF PRELIMINARY DATA NEEDS}

Data gaps for the Monument Valley site have been identified in site hydrology, geochemistry, and risk assessment. Additional data will be used to better evaluate the recommended ground water cleanup technologies. Preliminary data needs for each category are summarized below.

\subsubsection{Hydrologic data needs}

Define alluvial aquifer ground water flow conditions. Additional alluvial aquifer monitor wells are needed north and west of the site to better define the direction and magnitude of ground water flow. Data gaps exist between wells 655 and 660 , wells 660 and 650 , and west of the site in the vicinity of Cane Valley Wash and the Frog Ponds. Defining the ground water flow direction and gradient will help determine plume extent and velocity and pumping effectiveness. Long-term water-level monitoring (with pressure transducers and data loggers) will be used to evaluate trends in water-level fluctuations; each aquifer, surface water body, and barometric pressure must be monitored to evaluate the interaction between the different components of the hydrologic system.

Define thickness variations in the alluvial aquifer. Geophysical surveys, such as a shallow seismic geophysical survey, will aid in determining the thickness of the alluvial aquifer. Air photos, bedrock outcrops, and existing borings indicate the alluvial aquifer overlies a bedrock surface of considerable topographic relief. The buried topographic surface is known to include at least one 120-ft $(37-\mathrm{m})$-deep canyon on the site. Further data are needed to characterize the extent of this and perhaps other canyons buried beneath the alluvial aquifer. Areas where the canyons cut through the confining layer (Moenkopi Formation) into the De Chelly Sandstone may be areas of upward ground water flow and recharge to the alluvial aquifer. Thus, the buried bedrock topography will affect ground water flow, 
contaminant transport, vertical and horizontal distribution of contaminated ground water, mass distribution of contaminants to any one location, and aquifer response to pumping at any one location.

Determine hydraulic conductivity in the alluvial aquifer. Testing the alluvial aquifer will provide additional information on the hydraulic conductivity of the unit. This information will allow a better evaluation of the effectiveness of ground water pumping. During testing, the lower units' response to pumping should be monitored to determine the amount of water that travels from the lower units to the alluvial aquifer. The tests also will provide preliminary information on aquifer characteristics, including long-term aquifer yield. Testing also will indicate the potential impacts of aquifer pumping the hydrologic system, including surface water bodies such as the Frog Ponds and ponded areas within the wash.

Determine soil infiltration. The capacity of the upper portion of the soils to accept water must be determined to evaluate technologies that rely on returning some or all of the pumped water to the alluvial aquifer.

Modeling of the hydrologic system. Although not specifically a data need, ground water flow and contaminant transport computer modeling of the hydrologic system will be required to predict long-term contaminant distribution in the site vicinity. After data collected as a result of this version of the SOWP are reviewed, an appropriate model will be selected.

\subsubsection{Geochemical data needs}

The following hydrogeochemical data are needed to define the geochemical conditions in ground water, surface water, and soils to evaluate the most appropriate cleanup technologies.

Magnitude and extent of ground water contamination in the alluvial aquifer. A geophysical survey of ground conductivity will better define the lateral extent and relative magnitude of contamination. The results, in conjunction with a shallow seismic survey, will be used to determine additional monitor well locations in the center and periphery of ground water contamination. Well clusters or multiport wells can be used at each of these locations to further define the vertical distribution of contamination and local hydraulic gradients. Current data gaps pertaining to the magnitude and extent of contamination are beneath and downgradient of the site. Both the limits and vertical distribution of contamination are uncertain. Also, shallow well points along Cane Valley Wash (Appendix D) will further characterize the near-surface water geochemistry, providing information on ground water discharge from evaporation and transpiration.

Background soil chemistry. More information about the existing soils is needed to evaluate the possible effects of land application of treated ground water. Soil type and chemistry must be analyzed to establish preremediation conditions and 
to evaluate the most appropriate cleanup technologies. For example, soils and surface water at the site appear to be alkaline, and applying calcium sulfate can benefit alkaline soils. In addition, existing surface water quality data suggest that naturally occurring uranium and other metals may be concentrated in alkaline soils on the site, but background data are required to confirm this.

Background surface water. Further analysis of surface waters in Cane Valley Wash, especially in areas unaffected by VP material (redistributed ore-rock and tailings), are needed to establish the natural levels of uranium and other orerelated metals in saline surface and near-surface waters.

Contaminant desorption and dissolution from the aquifer matrix. Water chemistry suggests that on and immediately adjacent to the site, the alluvial aquifer contains gypsum derived from processing solutions. This gypsum may release calcium and sulfate to ground water during remediation, increasing the total volume of contaminated ground water to be treated. Other contaminants, such as uranium, also could be released from the aquifer matrix. Leaching experiments using core from upgradient and beneath the site will provide information on the mass of contaminants available for dissolution and desorption.

\subsubsection{Risk assessment data needs}

To protect human health and the ecological environment from adverse effects of ground water contamination, the following data gaps need to be addressed so all exposure pathways and ground water contaminants are identified.

The lateral extent and relative magnitude of contamination have not been characterized totally. Therefore, the magnitude of contamination must be better defined (by installing additional well points) to verify that the baseline risk assessment did not underestimate the risk to human health at the Monument Valley site. The plant community growing over the contaminant plume should be assessed in terms of species composition, community structure, and the rooting depth of selected plants. In addition, when the University of Arizona plant study is complete, the results should be used to evaluate the potential contaminant uptake from ground water by irrigated crops.

Although the use of organics during processing activities has not been reported, data are insufficient to verify that they were not used. Therefore, the ground water should be analyzed for organic constituents so the absence or presence of organics at this site can be verified and all potential human health and environmental risks can be identified.

\subsection{EVALUATION OF INTERIM REMEDIAL ACTION}

The human health and ecological risk evaluations for the site indicate no immediate threats to human health or the environment. Therefore, interim remedial action is not appropriate at this time. 


\subsection{GROUND WATER COMPLIANCE STRATEGY SELECTION}

This section identifies and defines the strategy available for meeting compliance with EPA ground water standards; applies site-specific data to this compliance framework; presents remediation technologies; and analyzes deviations, contingencies, and decisions that affect the selection of a compliance strategy and a remediation technology.

\subsection{GROUND WATER COMPLIANCE STRATEGIES}

The Ground Water Project PEIS (DOE, 1996) presents a selection framework (Figure 4.1) for determining the appropriate strategy for achieving compliance with the EPA standards. The following three available compliance strategies comprise the framework:

No remediation. Application of the no remediation strategy would mean compliance with the EPA ground water standards would be met without altering or cleaning up the ground water. This strategy could be applied at sites where contamination does not exceed MCLs or background levels, or at sites where contamination is above MCLs or background levels, but these sites qualify for supplemental standards or ACLs (see Section 2.1.1). For example, at the Monument Valley site, ground water contains constituents above background levels that may qualify for the application of ACLs based on acceptable human health and environmental risks.

Natural flushing. The system that allows natural ground water movement and geochemical processes to decrease, within a given time period, the contaminant concentrations to levels within regulatory limits is referred to as natural flushing. At the Monument Valley site active remediation, where effective monitoring and institutional controls could be maintained and the ground water is not currently, nor is projected to be used in the future as a source of drinking water, natural flushing would achieve compliance within 100 years.

Active ground water remediation. Active ground water remediation would require the application of engineered ground water remediation technologies such as ground water extraction, treatment, and land application, and bio-remediation to achieve compliance with EPA ground water standards. This strategy could be applied at the Monument Valley site in combination with natural flushing to reduce contaminant levels in the ground water.

\subsection{SITE-SPECIFIC COMPLIANCE STRATEGY SELECTION}

Ground water constituents at the Monument Valley site have been screened for potential risks, and for compliance purposes, divided into two groups (based on potential risks). The proposed ground water compliance strategy is no remediation for constituents that do not pose a potential risk and do not exceed EPA ground water standards. For the constituents that pose a potential risk and/or exceed the EPA ground water standards, the proposed ground water 
Figure 4.1

\section{Compliance Selection Framework, Monument Valley, Arizona, Site}

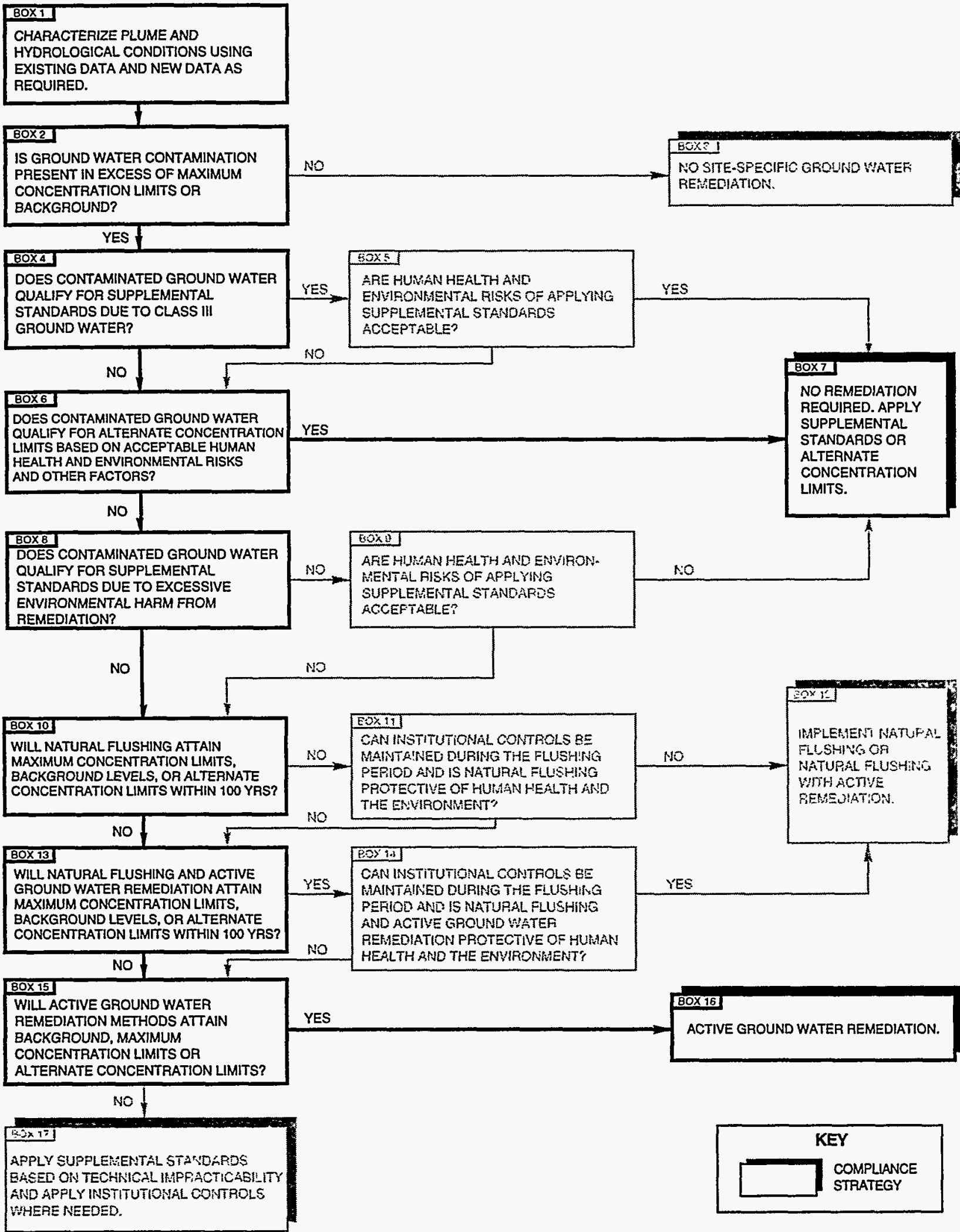

MAC: SITEMONSOWP/FLWCHT 
compliance strategy is active remediation. Justification follows for the recommendation of the compliance strategies for the Monument Valley site.

Analysis of ground water quality has revealed that 15 constituents in ground water under and downgradient from the site exceed background levels. These constituents are ammonium, calcium, chloride, iron, magnesium, manganese, nitrate, potassium, sodium, silica, strontium, sulfate, uranium, vanadium, and zinc. The elevated levels of these constituents appear to be a direct result of past uranium milling operations (Figure 4.1, box 2). Two of these constituents, nitrate (in the alluvial aquifer) and uranium (in the De Chelly Sandstone), exceed MCLs.

Ground water at the Monument Valley site is not classified as limited use (Figure 4.1, box 4); therefore, it does not qualify for supplemental standards (see Section 2.1.11.

Of the 15 constituents at the Monument Valley site, 10 may be considered for ACLs, due to acceptable human health and environmental risks (Figure 4.1, box 6). These constituents are calcium, chloride, iron, magnesium, potassium, silica, sodium, strontium, vanadium, and zinc. The constituents that may require remediation in the alluvial ground water are sulfate, manganese, nitrate, and ammonium. Although ammonium is not present in toxic concentrations, ammonium can convert to nitrate, thereby increasing nitrate concentrations in the aquifer. Additionally, uranium may need remediation in the De Chelly Sandstone because it exceeds the MCL.

The next step in the framework (Figure 4.1 , box 10) requires an evaluation of the effectiveness of natural flushing. The regulatory time limit for natural flushing is 100 years. Based on the length of time the plume has been present and the relatively constant levels of contaminants, natural flushing within the most concentrated portion of the plume does not appear to be a viable option.

Because with natural flushing ground water quality apparently will not reach MCLs (or background levels for constituents without MCLs) within 100 years, the compliance strategy will be active remediation.

The next compliance strategy is active ground water remediation (Figure 4.1, box 15). The conceptual model suggests that because of the characteristics of the aquifer and its ability to supply water, active ground water remediation may reduce contamination to background levels or MCLs.

\subsection{REMEDIATION TECHNOLOGIES}

Several remediation technologies are considered viable options for achieving compliance with ground water standards at the Monument Valley site. Analytical results show that elevated concentrations of sulfate, nitrate, ammonia, and uranium have been detected. However, uranium has been detected above the UMTRA MCL in only two wells in the De Chelly Sandstone in 
a specific area of the site, and the uranium concentration in the extracted ground water in the alluvial aquifer is not expected to exceed the UMTRA MCL. Elevated magnesium and calcium ions, while not threatening human health or the environment, often have to be removed from the ground water where aboveground mechanical water treatment processes are used to remove sulfate, nitrate, ammonia, and uranium. Six remediation technologies are under consideration:

- Extraction and evaporation.

- Extraction and land application.

- Extraction, treatment, and land application.

- Extraction, treatment, and infiltration.

- In situ bioremediation.

These technologies could also be used in combination.

The advantages and disadvantages of each remedial technology are discussed below.

\section{Extraction and evaporation}

The first technology involves the removal and solar/spray evaporation of contaminated ground water. Its advantages are the simple treatment design and low cost of operation. The primary disadvantages are that the treated water is not recovered and the salt residue that is produced will require special handling and disposal when remediation is completed.

\section{Extraction and land application}

The advantages to this remediation technology are that no residuals exist after treatment that would require special handling and disposal, land application may prove useful to growing vegetation, and a portion of the treated ground water is returned to the aquifer. The primary disadvantage is that treating the contaminated ground water would require a longer time period, because this technology can be employed only during the growing season. Also, the volume of water to be treated may require a large surface area for proper land application, and there is concern that salt buildup over time may limit irrigation. In addition, some of the treated water would not be returned to the aquifer because of evaporation and plant transpiration. However, the amount of water lost through evaporation and transpiration will vary with site conditions and cannot be determined without additional data. An additional issue at the Monument Valley site is the degree to which the constituents would accumulate in plant tissue, threatening livestock or humans who consume the vegetation. The DOE and the University of Arizona are studying this issue. 


\section{Extraction, treatment, and land application}

The primary advantage of this remediation technology is that treating contaminated ground water after extraction reduces high concentrations of specific contaminants that would otherwise limit land application, thereby reducing the area required. Furthermore, a portion of the ground water would be returned to the aquifer.

The disadvantage to this remediation technology is the likely residues from the treatment system would require disposal, thereby increasing operation and maintenance requirements. Additionally, as with the preceding option, land application would be limited to the growing season, and a significant portion of the extracted water would be lost to evaporation and plant transportation.

\section{Extraction, treatment, and infiltration}

The primary advantage to this technology is that most of the treated water would be returned to the aquifer through shallow infiltration trenches, which also may facilitate the remediation process by naturally flushing contamination from the aquifer. This technology also is viable throughout the year. The disadvantages are that there may be a net loss through the evaporation/removal of residues as a result of the treatment process. Disposal of residue and maintenance of this technology would be costly.

\section{In situ bioremediation}

The primary advantages of in situ bioremediation consist of minimal ground water loss from the aquifer and year-round operation of the system. Moreover, this is the mechanically simplest option of the remediation technologies under consideration.

The primary disadvantage is that in situ bioremediation has not been accepted as an industry standard to reduce nitrate contamination and high sulfate levels in ground water at former uranium processing sites. Another disadvantage is the availability of a viable food source to facilitate the bioremediation process.

Table 4.1 lists the treatment processes that can be used to remove specific contaminants.

\section{Water recovery}

Water recovery (i.e., the amount of water that remains after treatment) will be a factor in selecting the optimum remediation technology. Table 4.2 summarizes the approximate anticipated water recovery associated with some of the treatment options listed above. 
Table 4.1 Treatment processes

\begin{tabular}{|c|c|}
\hline Contaminant & Processes \\
\hline Nitrate & $\begin{array}{l}\text { Denitrification in ponds. } \\
\text { Membrane separation. } \\
\text { In situ denitrification. } \\
\text { Plant uptake (land irrigation). }\end{array}$ \\
\hline Ammonia & $\begin{array}{l}\text { Nitrify in ponds. } \\
\text { Membrane separation. } \\
\text { Plant uptake (land irrigation). }\end{array}$ \\
\hline Uranium & $\begin{array}{l}\text { Reverse osmosis. } \\
\text { lon exchange. }\end{array}$ \\
\hline Sulfate & Membrane separation. \\
\hline $\begin{array}{l}\text { Magnesium and } \\
\text { calcium }\end{array}$ & $\begin{array}{l}\text { Lime softening. } \\
\text { Membrane separation. }\end{array}$ \\
\hline Total dissolved solids & $\begin{array}{l}\text { Membrane separation. } \\
\text { Distillation. }\end{array}$ \\
\hline
\end{tabular}

a'Membrane separation includes reverse osmosis, nanofiltration, and electrodialysis reversal.

Table 4.2 Water recovery

\begin{tabular}{lc}
\hline Treatment options & $\begin{array}{c}\text { Recovery } \\
(\%)\end{array}$ \\
\hline Denitrification (in ponds) & 99 \\
Lime softening & 95 \\
Nanofiltration & 75 \\
Land treatment & $10-50$ \\
Reverse osmosis & 65 \\
lon exchange & 95 \\
In situ bioremediation & 100 \\
\hline
\end{tabular}


A successful remediation technology may not only reduce the concentration levels of the contaminants of potential concern, but may also have the added advantage of reducing the concentration levels for constituents that fall within dietary ranges but above background levels. Data collection activities described in Section 5.0 will produce site-specific aquifer and soil data that will be used to evaluate the five remedial technologies under consideration. This information will be used initially to determine if any of these technologies are not suitable, or are impractical for this site. After the data obtained from these studies are reviewed, some remedial technologies may be dropped from consideration in this revision of the SOWP.

\subsection{DEVIATIONS, CONTINGENCIES, AND DECISION RULES}

Possible deviations from the conceptual site model are the plume is smaller or larger than current information indicates and hydrologic characteristics of the alluvial aquifer are different than current data indicate. The work proposed in Section 5.0 will provide additional information regarding both possible deviations.

The proposed ground water compliance strategy apparently will not be affected by these possible deviations. If the extent of the plume is larger than current understanding, extraction will remain a viable alternative; the area covered by the extraction system must be expanded. Aquifer testing will provide information about pumping rates and extraction well spacing. Ground water extraction has been performed at sites with similar hydrologic conditions; therefore, the most likely compliance strategy would still be valid.

The infiltration tests (Section 5.0) will assist in formulating decisions for land application, extent of water treatment, and infiltration details. For example, if results from the infiltration testing show that application of untreated plume water adversely affects the environment, the decision would be to treat the extracted ground water prior to land application or infiltration. 
. 


\subsection{DATA COLLECTION AND ASSESSMENT}

In the ground water compliance strategy selection process presented in Section 4.0, active remediation is proposed to restore ground water at the site. Active restoration methods under consideration are extraction and evaporation; extraction, and land application; extraction, treatment, and land application; extraction, treatment, and infiltration; and in situ bioremediation. The following sections define the data collection activities, objectives, and quality requirements to select the appropriate technology or combination of technologies for ground water remediation.

\section{$5.1 \quad$ DATA COLLECTION OBJECTIVES}

The following objectives were determined for the data collection activities of this investigation:

- Refine ground water flow conditions in the alluvial aquifer by installing additional shallow monitor wells and data loggers.

- Refine aquifer characteristics for the alluvial aquifer through short- and longterm aquifer tests. The deeper aquifers also will be monitored to evaluate the effects of pumping the upper unit. Among other uses, this information will be used to evaluate the effects of pump and evaporation technology on the aquifer.

- Test the infiltration capacity of the shallow soils in areas that most likely will receive treated and/or untreated ground water. The effect on the water table configuration and soil/ground water geochemistry will be monitored during the tests. This information will be used to evaluate treatment and reinfiltration and land application technologies.

- Refine the extent and thickness of the alluvial aquifer through a shallow seismic survey of areas on and downgradient of the site.

- Refine the lateral extent, vertical extent, and relative magnitude of ground water contamination in the alluvial aquifer through a geophysical survey of ground conductivity and the installation of well clusters or multiport wells.

- Determine background soil chemistry. Through analyses of soil type and chemistry, establish preremediation conditions and evaluate the most appropriate cleanup technologies (e.g., the effects of land application of treated ground water on soils).

- Determine background surface water quality. By sampling and analyzing surface water in Cane Valley Wash, distinguish the sources of uranium, molybdenum, and vanadium (natural or VP materials). 
- Measure contaminant desorption and dissolution from the aquifer matrix by sampling and analyzing alluvial aquifer matrix cores. Analyses of leachates form core material collected upgradient, beneath, and downgradient of the site will provide information on the mass of contaminants available for dissolution and desorption. This information will be used to evaluate the five technologies under consideration and to determine the volume of water that must be pumped from or flushed through the aquifer to meet the goals of the remediation strategy.

\subsection{DATA COLLECTION ACTIVITIES}

To achieve the DCOs of this investigation, the activities described below must be implemented.

\subsubsection{Monitor well installation}

The installation of additional monitor wells will help to refine data on the ground water flow conditions in the alluvial aquifer and the extent and magnitude of the plume. Two types of wells will be installed: shallow, hand driven well points in the Cane Valley Wash vicinity and deeper wells installed with a drilling rig near the center of Cane Valley.

It is suggested that up to 16 well points be installed by manually driving them just below the water table along Cane Valley Wash (Figure 3.6). They will be installed upgradient, downgradient, and crossgradient from the known location of the plume. The wells will consist of 2-inch $(0.05-\mathrm{m})$ well casing and well points/well screen. Manual installation was selected because it will minimize disturbance to the surrounding areas and because the depth to ground water is within about $6 \mathrm{ft}(2 \mathrm{~m})$ of ground surface. The depth to water and sandy soils allow the method to be feasible along the wash.

Water-level elevations and water-quality will be determined from each well. UMTRA Project standard operating procedures (SOP) (JEG, n.d.) will be followed during the installation, sampling, and water-level measurements for the well points. Concrete pads and steel protective casings will be installed at each well point to protect the integrity and increase the longevity of the well points.

Up to six alluvial aquifer well locations are suggested in the area between wells 653 and 650 to further define the extent and magnitude of the plume and ground water flow conditions in the alluvial aquifer. The wells will be installed using a drilling rig and hollow stem augers due to the greater depth to water (about $30 \mathrm{ft}[10 \mathrm{~m}]$ or greater). Well clusters may be installed at each location to assess plume concentrations with depth where the thickness of the alluvial aquifer exceeds about $50 \mathrm{ft}(15 \mathrm{~m})$. UMTRA Project SOPs (JEG, n.d.) will be followed during the installation, sampling, and water-level measurements for the monitor wells. 


\subsubsection{Water level and barometric pressure monitoring}

Electronic data loggers and pressure transducers will be used to collect water level information from monitor wells in the different aquifers, water level information from the Frog Ponds, and barometric pressure readings. After the measurements are collected four times each day for 4 weeks, the data will be evaluated to determine the most appropriate frequency. Data loggers will be installed in at least two well clusters within the plume, one or more well points near the Frog Ponds, and one or more upgradient monitor wells. Barometric pressure readings are needed from one location only. Additionally, at least one stilling well will be needed in one of the Frog Ponds to obtain surface water level measurements.

\subsubsection{Aquifer testing}

Specific capacity testing of all newly installed well points and monitor wells is suggested for short-term aquifer testing. Additionally, the discharge water in the field will be measured for specific conductance, $\mathrm{pH}$, and temperature.

Depending on results from the newly installed wells and well points, additional specific-capacity tests may be performed in the other alluvial aquifer wells. For example, if the results from specific-capacity testing on the newly installed wells vary greatly between locations and/or depths, additional testing for other wells is recommended. UMTRA Project SOPs will be followed during well testing (JEG, n.d.).

Long-term aquifer tests will be performed for 15 days or more to define aquifer characteristics and to evaluate the effects of delayed yield. The tests should be performed at two well clusters within the plume, possibly the 606, 659, 663 well cluster and the $653,660,664$ well cluster. Water levels will be monitored routinely in all nearby wells, in addition to periodic field analyses of specific conductance, $\mathrm{pH}$, and temperature, and laboratory analyses of plume constituents.

\subsubsection{Infiltration testing}

Locations upgradient, downgradient, and within the plume will be selected for infiltration testing. UMTRA Project SOPs and/or commonly accepted practices will be employed for the testing methods and evaluation of results (JEG, n.d.).

\subsubsection{Geophysical survevs}

A shallow (low-energy) refraction seismic survey will be performed on and downgradient of the site to delineate subsurface bedrock topography beneath the alluvial aquifer, following industry standards. The survey should be of sufficient resolution to identify and trace the buried canyon crossing the site.

A ground conductivity survey will be performed on and downgradient of the site to delineate the lateral and relative magnitude of ground water contamination in 
the alluvial aquifer. Because the electrical conductivity of water is largely determined by the TDS concentration, measurements of terrain conductivity can be used to broadly delineate the outline of ground water contamination in the alluvial aquifer. The survey will use a EM34 terrain conductivity meter (DOE, 1994c). This instrument will be used in the field to quickly outline the approximate downgradient extent of ground water contamination. This information will be used to locate additional monitor wells in strategic locations.

\subsubsection{Determination of background surface water and soil chemistry}

Background surface and near-surface water chemistry data will be collected from surface water pools in Cane Valley Wash. Near-surface water has been observed in the wash east of monitor well 658. This location is upgradient of the site and identified VPs. Surface or near-surface water will be collected from two or more locations in this area in conjunction with routine monitor well sampling at the site. Both filtered and unfiltered samples will be collected and analyzed for major and trace elements indicative of contamination, to determine the background concentrations of these constituents. Downstream samples also will be collected (from previously sampled locations) for comparison.

Soil samples will be collected from background and downgradient locations to determine the chemical characteristics of the soils prior to ground water remediation. It appears that soils near the site are alkaline. Soils will be tested for $\mathrm{pH}$, soluble salt chemistry, grain size, cation exchange capacity, and carbon content. Soil samples will be collected from Cane Valley Wash and from the windblown sands that characterize the soils downgradient of the site. Soils will be collected along east-west transects upgradient and downgradient of the site. Salt content and chemistry will be characterized as a function of depth. Standard American Society for Testing and Materials (ASTM), methods will be used (ASTM, 1991).

\subsubsection{Desorption and dissolution of contaminants from the aquifer matrix}

Core from newly drilled monitor wells will be analyzed to determine the mass of contaminants available to ground water via desorption and dissolution. Of particular importance is the mass of calcium and sulfate available from the dissolution of gypsum. Other coprecipitated or adsorbed constituents, such as uranium, strontium and magnesium, will also be analyzed. Core from the site will analyzed over a range of depths to determine the vertical distribution of the soluble contaminants. Core from downgradient locations will be analyzed to determine the lateral distribution of soluble contaminants. Upgradient core will be used as a control. The mass of available contaminants will be determined by 24-hour leaching experiments using background alluvial ground water as a lixiviant. One column experiment using the core with the greatest amount of gypsum will be used to determine the rate of release of the soluble contaminants. 


\section{$5.3 \quad$ DATA QUALITY OBJECTIVES}

All data will be generated, documented, and verified in conformance with the UMTRA Project Quality Assurance Implementation Plan (QAIP) (DOE, 1994d). When equipment such as data loggers and field water quality instruments are used, the manufacturer's specifications and SOPs will be followed. DQOs for the specific field tasks are described below.

\subsubsection{Monitor well and well point installation}

Additional monitor wells and well points will be installed to further define the extent of ground water contamination and the flow regime of the alluvial aquifer (Appendix D). The monitor wells will be 4-inch $(0.1-\mathrm{m})$-diameter polyvinyl chloride (PVC) and the well points will be 2-inch (0.05-m)-diameter PVC or steel. Installation, construction, and development of the wells will be in accordance with applicable UMTRA Project SOPs to ensure the wells are adequate for their intended purpose (JEG, n.d.). Lithologic descriptions will be compiled from borehole cuttings from the monitor wells; lithologic descriptions will not be possible for the well points because they will be driven and no cuttings will be produced.

\subsubsection{Water level and barometric pressure monitoring}

Water levels and barometric pressure are monitored by examining the contrast in barometric efficiency to determine long-term trends in water level fluctuations and if the shallow and deep portions of the aquifer have the same hydraulic conditions (e.g., both unconfined and confined). This study will help determine recharge and discharge events and also will help accurately evaluate long-term aquifer test results. Water level fluctuations not related to the actual tests can be applied to the data gathered during the test.

Ground water levels fluctuate in response to atmospheric pressure (Domenico and Schwartz, 1990). The barometric efficiency describes how closely water levels respond to changes in atmospheric pressure. Barometric efficiency is low to nonexistent in wells tapping alluvial aquifers. Thus, by comparing the barometric efficiency of a shallow well to that of a deep well, the type of flow regime can be determined for the area tapped by each well.

Collecting of these data will determine the proper conceptual model for the site. Sufficient sensitivity, accuracy, and temporal recording resolution will ensure that the collected data can be used for their intended purpose. Standard pressure transducers have a resolution of $0.004 \mathrm{ft}(0.001 \mathrm{~m})$ with an accuracy of $0.035 \mathrm{ft}(0.011 \mathrm{~m})$. The expected water level fluctuations from barometric pressure changes are on the order of tenths of feet; thus, standard equipment and procedures should be sufficiently accurate to compute barometric efficiency. Barometric pressure and water levels will be recorded every 6 hours for a period of 4 weeks. 


\subsubsection{Aquifer testing}

Short- and long-term aquifer test analyses are suggested to determine the spatial distribution of hydraulic conductivity and to determine how the aquifer responds to conditions similar to those associated with the remediation technologies under review. Standard data loggers and pressure transducers used in accordance with the manufacturers' instructions will produce reliable data for the analysis. Tests will be designed and analyzed according to various standards, such as ASTM Standard D4050-1991 (ASTM, 1991).

The short-term tests will examine how local heterogeneity can influence pumping well performance (a crucial factor in the selected remediation technology) and provide information on the distribution of hydraulic conductivity (such as the existence of internal aquifer barriers or preferential flow paths). The depositional process that created the alluvial aquifer tends to produce hydraulic conductivity that is higher along the bedding planes (anisotropy). This anisotropy will control the efficiency of the selected remediation technology and can be investigated with the long-term test. The test should be run long enough that the effects of delayed yield are evident and drawdown is observed in all observation wells.

A potential problem with analyzing the pumping tests is the possibility of obtaining multiple, equally good solutions (Horne, 1994). To help alleviate this problem, each test will be analyzed during both the drawdown and recovery phases to provide a cross check on the results.

Because of well inefficiency, the actual rate required for testing may be different than that estimated analytically. The estimation of specific capacity (from which transmissivity and hydraulic conductivity can be derived) will assist in determining the appropriate pumping rates for the long-term tests and well efficiency. Knowledge of these parameters will allow fine tuning of the longterm tests in the field to produce optimum results.

\subsubsection{Infiltration testing}

Infiltration testing is conducted to determine the rate at which the soils will accept water without generating surface runoff, the effect of infiltration on the configuration of the water table, and changes to soil and ground water chemistry as a result of water infiltration. Installation, construction, and testing of the infiltration areas will be in accordance with applicable UMTRA Project SOPs (JEG, n.d.), agricultural guidelines, and other applicable industry standards to ensure the tests are adequate. 


\subsubsection{Geophysical surveys}

Geophysical surveys are conducted to delineate the magnitude and extent of contamination in the alluvial aquifer, and subsurface bedrock topography beneath the alluvial aquifer and the attendant variations in thickness. It must be determined if variations in bedrock topography and thickness of the alluvial aquifer influence contaminant distribution and migration, and where monitor wells should be located to define both lateral and vertical variations in contamination. Information needed to support these decisions are the location of the highest levels of contamination; the lateral extent of the plume; the location of buried bedrock topographic features, especially the buried canyon that crosses the site and extends downgradient; and the locations of the thicker and thinner alluvial aquifer. The areas that must be investigated are the areas comprising the site; areas about $500 \mathrm{ft}(150 \mathrm{~m})$ south and east of the site; and the area north of the site bounded by the depositional edge of the alluvial aquifer to the west, monitor wells 650,651 , and 652 to the north and north east, and monitor wells 654 and 605 to the east. These areas encompass an area about $8000 \mathrm{ft}(2400 \mathrm{~m})$ long and from 4000 to $6000 \mathrm{ft}(1200$ to $1800 \mathrm{~m}$ ) wide. This large area is best investigated with surface geophysical techniques, such as terrain conductivity, used to determine the shape of the contaminant plume and areas of greatest contamination; and shallow refraction seismic surveys, used to determine the buried bedrock topography and thickness of the overlying alluvial aquifer.

The terrain conductivity survey should be of sufficient resolution to detect and delineate areas of maximum contamination of at least 500 by $500 \mathrm{ft}(150$ by $150 \mathrm{ml}$. The survey should be sensitive enough to detect variations in the salinity of the contaminated ground water of about $400 \mathrm{mg} / \mathrm{L}$ (about 10 percent of the maximum observed contamination, and similar to that of background). This will ensure locating major areas with contaminants at levels greater than 10 percent of the observed maximums. The shallow seismic survey should be of sufficient resolution to identify buried topographic features at least $200 \mathrm{ft}(61 \mathrm{~m})$ wide and $20 \mathrm{ft}(6 \mathrm{~m})$ deep; the methods used and space between measurements should keep uncertainties within these limits.

\subsubsection{Background surface water chemistry}

The source of uranium, molybdenum, and vanadium concentrations in surface and near-surface water must be determined as natural or related to the use of ore rock as road fill in the area (at VPs). Collecting background surface water samples will provide additional information on the natural concentrations of these site-related contaminants in surface waters effected by evaporation. The area to be investigated is along Cane Valley Wash, from a point immediately east of monitor well 658, downstream to the Frog Ponds. This area is known to have near-surface ground water and is upstream of identified VPs. At least two sampling locations will be identified and sampled for surface or near-surface water; the latter will be collected by digging a $2-\mathrm{ft}(0.61-\mathrm{m})$ hole into saturated alluvium. Samples will be tested for field parameters (alkalinity, $\mathrm{pH}$, electrical 
conductivity, temperature, redox potential, and turbidityl, for major elements (calcium, chloride, magnesium, potassium, sodium, and.sulfate), and for uranium, molybdenum, and vanadium. Other parameters may be added also. Samples will be collected during at least four sampling rounds to determine natural variations in the levels of these constituents.

\subsubsection{Background soil chemistry}

Soils near the site must be characterized. Preliminary study suggests that naturally sodic, alkaline soils occur in the in the area of the site (Section 5.2.6). One possible remediation method is the land application of treated or untreated ground water. Soil characteristics and the types of vegetation available for nitrate consumption from contaminated ground water will be important in determining the feasibility of land application. Also, sodic, alkaline soils can benefit from the application of calcium-sulfate ground water, depending on the clay content of the soil and its cation exchange capacity. Finally, soils containing a significant amount of soluble salts may, in fact, become a source of ground water contamination as percolating surface water dissolves those salts and then recharges the underlying ground water with saline waters. Thus, the characteristics of natural soils will be important in determining the feasibility of land application of treated ground water. Soil data also will provide information on the natural levels of site-related contaminants (such as uranium, nitrate, sulfate, and manganese) in soils near the site prior to ground water remediation. Parameters that will be measured are soil particle size distribution (using method ASTM D 422-63, reproved 1990) and cation exchange capacity (EPA method 9080 or 9081$)$. Soluble components will be measured by water leaching and $x$-ray powder diffraction to determine their mineralogy, if they exceed 1 percent of the total soil mass. The following soluble components will be measured: alkalinity, ammonium, calcium, chloride, iron, magnesium, manganese, molybdenum, nitrate, potassium, sodium, strontium, sulfate, uranium, vanadium, and zinc. These components are expected in natural soils and/or may be added to soils (as soluble salts) during ground water remediation.

A preliminary study will answer questions about the types of soils present and will verify field observations. The site ecologist and geochemist will select the sampling locations. Soil survey data, if available, will also be used to identify sampling locations. Three basic types of areas will be sampled. One is in the areas along Cane Valley Wash where evaporative ground water discharge maintains damp soils with salt efflorescence. Another is in low basins within areas covered by windblown sand where surface salts have been observed. Last are topographically higher areas within the areas covered by windblown sands. These higher areas also have been observed to be partially cemented by surface salts. Each of these three soil types will be sampled in at least four areas. At each location, a sample profile will include the top $1 \mathrm{~cm}$ of depth, the next 1 to $10 \mathrm{~cm}$ of depth, and the lower 10 to $30 \mathrm{~cm}$ of depth. Thus, a total of at least 27 samples will be collected. Samples will be collected both upgradient and upwind (south) of the site and downgradient and downwind (north). The 
samples to the south will demonstrate background conditions. Samples to the north are expected to be similar (unaffected by site-related contamination).

\subsubsection{Contaminant desorption and dissolution from the aquifer matrix}

Water chemistry suggests that on and immediately adjacent to the site the alluvial aquifer contains gypsum derived from processing solutions. In addition, it is possible that other contaminants, especially trace metals, could be sorbed onto the aquifer matrix. Previous studies (DOE, 1994e) at other sites have demonstrated that site-related ground water contaminants are often concentrated in the upper few feet of the aquifer, beneath tailings piles and evaporation ponds. Thus, this potential contaminant source may have been removed by surface remediation. Nonetheless, designing a remediation program will require accurate data on the amount of gypsum and adsorbed metals available to the aquifer as a potential source of ground water contamination.

An initial series of batch leach tests on core samples obtained from the former processing site will provide insight into this possible contaminant source. Data will be collected on the leachable concentrations of the contaminants of potential concern to human health (ammonium, manganese, nitrate, sulfate, and uranium) and calcium (to determine the influence of gypsum as a source of sulfate). Note that leachable sources of ammonium and nitrate are not expected but will be investigated. The analyte list will be expanded to include other potential contaminants, if they are identified.

Core samples also will be collected from the old tailings pile/heap leach pad area, the new tailings pile area, and the evaporation pond area. Also, at least one upgradient (background) core will be sampled for comparison. Samples will be taken from four intervals in each core: the land surface to $1 \mathrm{~m}$, the $1-\mathrm{m}$ interval midway between the water table and the surface, the interval from $1 \mathrm{~m}$ above the water table to the water table, and the interval $1 \mathrm{~m}$ below the water table.

Each sample will be subjected to a 24-hour batch leach test using background ground water as a lixiviant. Results will be used to determine if there is a problem with leachable contaminants that would require more detailed sampling and analysis. If there is no evidence for significant levels of leachable contaminants in the aquifer matrix, further study will not be required. 


\subsection{LIST OF CONTRIBUTORS}

The following individuals contributed to the preparation of this document.

\begin{tabular}{ll}
\hline Name & Contribution \\
\hline M. Bradshaw & Document coordinator \\
$\begin{array}{l}\text { C. Burt, G. Hartmann, T. Jackson, } \\
\text { J. Marshall, A. Miller, R. Neri Zagal }\end{array}$ & Authors \\
J. Gibb, A. Holm, J. McBee, R. Saar & Reviewers \\
L. Sanchez, WordCenter, Inc. & Text processing \\
K. DeGruyter, S. Suniga & Graphics \\
D. Kahl, D. Tamez, J. Torline & $\begin{array}{l}\text { Technical editor/Document production } \\
\text { coordinator }\end{array}$ \\
\hline
\end{tabular}




\subsection{REFERENCES}

ASTM (American Society for Testing and Materials), 1991. Standard Test Method (Field Procedure) for Withdrawal and Injection Well Tests for Determining Hydraulic Properties of Aquifer Systems, ASTM Designation: D 4050-1991, Annual Book of ASTM Standards, Vol. 04.08.

Branson et al., (F. A. Branson, R. F. Miller, and I. S. McQueen), 1976. “Moisture

Relationships in Twelve Northern Desert Shrub Communities Near Grand Junction, Colorado," Ecology, Volume 57, pp. 1104-1124.

Cooley et al., (M. E. Cooley, J. W. Harshbarger, J. P. Akers, and W. F. Hardt), 1969. Regional Hydrogeology of the Navajo and Hopi Indian Reservations, Arizona, New Mexico, and Utah, U.S. Geological Survey Professional Paper 521-A, Washington, DC.

DOE (U.S. Department of Energy), 1996. Programmatic Environmental Impact Statement for the Uranium Mill Tailings Remedial Action Ground Water Project, DOE/EIS-0198, January 1996, prepared by the U.S. Department of Energy, UMTRA Project Office, Albuquerque Operations Office, Albuquerque, New Mexico.

DOE (U.S. Department of Energy), 1994a. Baseline Risk Assessment of Ground Water Contamination at the Monument Valley Uranium Mill Tailings Site, Cane Valley Arizona, DOE/AL-62350-43, Rev. 1, August 1994, prepared by the U.S. Department of Energy, UMTRA Project Office, Albuquerque Operations Office, Albuquerque, New Mexico.

DOE (U.S. Department of Energy), 1994b. UMTRA Project Water Sampling and Analysis Plan Monument Valley, Arizona, DOE/AL-62350-123, March 1994, prepared for the U.S. Department of Energy, UMTRA Project Office, Albuquerque, New Mexico, by Jacobs Engineering Group Inc., Albuquerque, New Mexico.

DOE, (U.S. Department of Energy), 1994c. Geophysical Surveys at Tuba City, prepared for U.S. Department of Energy, UMTRA Project Office, Albuquerque, New Mexico, by Jacobs Engineering Group Inc., Albuquerque, New Mexico.

DOE (U.S. Department of Energy), 1994d. UMTRA Project Technical Assistance Contractor Quality Assurance Implementation Plan, DOE/AL 62350-72D, March 1994, prepared by the U.S. Department of Energy, UMTRA Project Office, Albuquerque Operations Office, Albuquerque, New Mexico.

DOE (U.S. Department of Energy), 1994e. Gunnison, Colorado, Subpile Study Report, DOE/AL-62350-110, prepared for U.S. Department of Energy , UMTRA Project Office, Albuquerque, New Mexico, by Jacobs Engineering Group Inc., Albuquerque, New Mexico. 
DOE (U.S. Department of Energy), 1993a. UMTRA Ground Water Project Plan, UMTRADOE/AL-62350-1, December 1993, prepared by the U.S. Department of Energy, UMTRA Project Office, Albuquerque Operations Office, Albuquerque, New Mexico.

DOE (U.S. Department of Energy), 1993b. Technical Approach to Ground Water Restoration, DOE/AL/62350-2OF, November 1993, prepared by the U.S. Department of Energy, UMTRA Project Office, Albuquerque Operations Office, Albuquerque, New Mexico.

DOE (U.S. Department of Energy), 1993c. Remedial Action Plan for the Codisposal and Stabilization of the Monument Valley and Mexican Hat Uranium Mill Tailings at Mexican Hat, Utah, UMTRA-DOE/AL-62350-050509, February 1993, prepared by the U.S. Department of Energy, UMTRA Project Office, Albuquerque Operations Office, Albuquerque, New Mexico.

DOE (U.S. Department of Energy), 1993d. Recommendations for the Preparation of Environmental Assessments and Environmental Impact Statements, Office of NEPA Oversight, U.S. Department of Energy, Washington, D.C.

DOE (U.S. Department of Energy), 1993e. Software Program for Environmental Analysis and Reporting, UMTRA Project Data Management System, UMTRA Project Office, Albuquerque Operations Office, Albuquerque, New Mexico.

DOE (U.S. Department of Energy), 1989. Environmental Assessment of Remedial Action at the Monument Valley Uranium Mill Tailings Site, Monument Valley, Arizona, final, UMTRA-DOE/AL 0368, prepared by U.S. Department of Energy, UMTRA Project Office, Albuquerque Operations Office, Albuquerque, New Mexico.

DOE (U.S. Department of Energy), 1983. Data for the Geochemical Investigation of UMTRAP Designated Site at Monument Valley, Arizona, UMTRA-DOE/AL-0247, prepared by the U.S. Department of Energy, UMTRA Project Office, Albuquerque, New Mexico.

DOE (U.S. Department of Energy), 1981. Engineering Assessment of Inactive Uranium Mill Tailings, Monument Valley Site, Monument Valley, Arizona, (October 1981), Prepared for U.S. Department of Energy, Albuquerque Office, UMTRA Project Office, Albuquerque, New Mexico, by Ford, Bacon \& Davis Utah, Inc.

Domenico, P. A., and F. W. Schwartz, 1990. Physical and Chemical Hydrogeology, John Wiley and Sons, Inc., New York, New York, 824p.

Drever, J. I., 1982. The Geochemistry of Natural Waters, Prentice-Hall, Inc., Englewood Cliffs, New Jersey, 388p.

Driscoll, F. G., 1986. Groundwater and Wells (2nd edition), Johnson Division, St. Paul, Minnesota 55112.

EPA (U.S. Environmental Protection Agency), 1989. Risk Assessment Guidance for Superfund Vol. I, Human Health Evaluation Manual, EPA/540/1-89/002, Office of Emergency and Remedial Response, Washington, D.C. 
FBDU (Ford, Bacon \& Davis Utah, Inc.), 1981. Engineering Assessment of Inactive Uranium Mill Tailings, Shiprock Site, Shiprock, New Mexico, UMTRA-DOE/UMT-0104, FBDU 360-02, Uc-70, prepared by FBDU, Salt Lake City, Utah, for the U.S. Department of Energy, UMTRA Project Office, Albuquerque Operations Office, Albuquerque, New Mexico.

Freeze, R. A. and J. A. Cherry, 1979. Groundwater, Prentice-Hall, Inc., Englewood Cliffs, New Jersey.

HEW (U.S. Department of Health, Education, and Welfare), 1962. Waste Guide for the Uranium Milling Industry, Technical Report W62-12 PB 226 362, U.S. Department of Health, Education, and Welfare, Public Health Service.

Hill, S. R., 1989. Appendix IX Special Study Screening, Contract No. DE-AC0482-AL14086, prepared for the U.S. Department of Energy by Jacobs Engineering Group, Albuquerque Operations Office, Albuquerque, New Mexico.

Horne, 1994. ASTM (American Society for Testing and Materials), 1991. Standard Test Method (Field Procedure) for Withdrawal and Injection Well Tests for Determining Hydraulic Properties of Aquifer Systems, ASTM Designation: D 4050-1991, Annual Book of ASTM Standards, Vol. 04.08.

Irwin, et al. (J. H. Irwin, P. R. Stevens, and M. E. Cooley), 1971. Geology of the Paleozoic Rocks, Navajo and Hopi Indian Reservations, Arizona, New Mexico, and Utah, U.S. Geological Survey Professional Paper 521-C.

James, H.L. (editor), 1973. Guidebook of Monument Valley and Vicinity; Arizona and Utah, New Mexico Geological Society.

JEG (Jacobs Engineering Group Inc.), n.d. Albuquerque Operations Manual, standard operating procedures, prepared by Jacobs Engineering Group Albuquerque, New Mexico, for the U.S. Department of Energy, UMTRA Project Office, Albuquerque Operations Office, Albuquerque, New Mexico.

Merritt, R. C., 1971. The Extractive Metallurgy of Uranium, Colorado School of Mines Research Institute, Library of Congress Catalog Card No. 71-157056.

Shacklette, H. T., and J. G. Boerngen, 1984. Element Concentrations in Soils and Other Surficial Materials of the Conterminous United States, U.S. Geological Survey Professional Paper 1270.

Summerskill, W. H. H., and E. Wolpert, 1970. "Ammonia Metabolism in the Gut," American Journal of Clinical Nutrition, Vol. 23, pp. 633-639.

Witkind, I. J., and R. E. Thaden, 1963. "Geology and Uranium-Vanadium Deposits of the Monument Valley Area, Apache and Navajo Counties, Arizona," in Geological Survey Bulletin 1103, Washington, D.C. 
Wiese, A. F., n.d., Russian Thistle--Salsola iberica Sennen and Pau, Texas Agriculture Experiment Station, Bushland, Texas.

\section{CODE OF FEDERAL REGULATIONS}

10 CFR Part 1021, National Environmental Policy Act; Implementing Procedures and Guidelines Revocation; Final Rule and Notice, U.S. Department of Energy.

40 CFR Part 192, Health and Environmental Protection Standard for Uranium and Thorium Mill Tailings, U.S. Environmental Protection Agency.

40 CFR Part 264, Standards for Owners and Operators of Hazardous Waste Treatment, Storage, and Disposal Facilities, U.S. Environmental Protection Agency. Administration, Washington, D.C.

40 CFR Parts 1500-1508, Regulations for Implementing the Procedural Provisions of the National Environmental Policy Act, Council on Environmental Quality.

\section{FEDERAL REGISTER}

60 FR 2854, Groundwater Standards for Remedial Actions at Inactive Uranium Processing Sites, Final Rule, 11 January 1995.

\section{UNITED STATES CODE}

42 USC $§ 4321$ et seq., National Environmental Policy Act, 1 January 1970.

42 USC $\S 7901$ et seq., Uranium Mill Tailings Radiation Control Act, 8 November 1978. 
APPENDIX A

WELL LOGS (LITHOLOGIC LOGS), WELL COMPLETION LOGS 


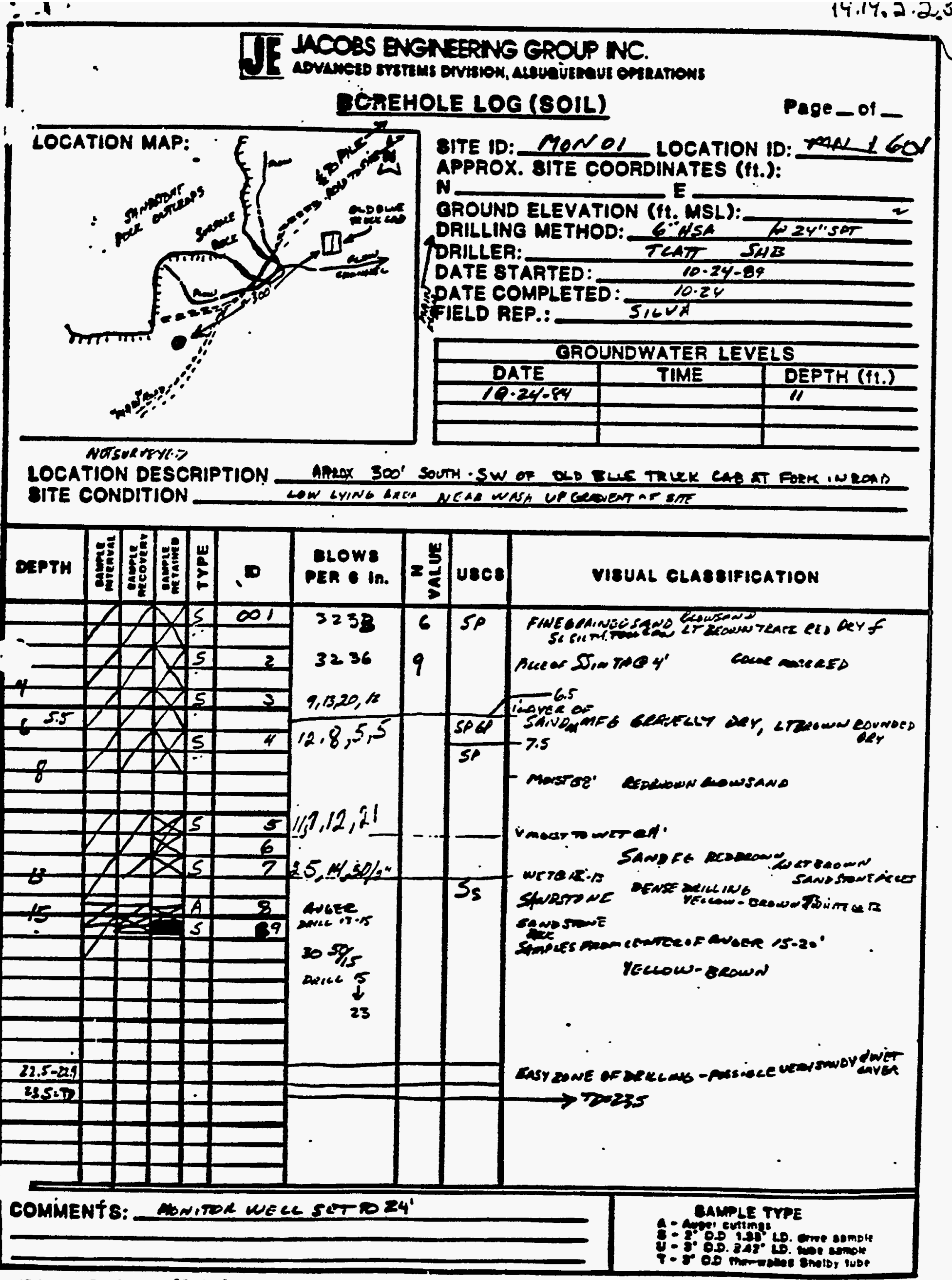




\section{TIE HOOBS ENGN EERTG GROUP NC.

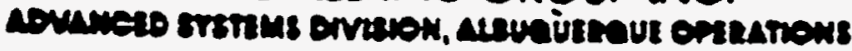

QOREHOLE LOG (8OIL)

Pagetor 3 603

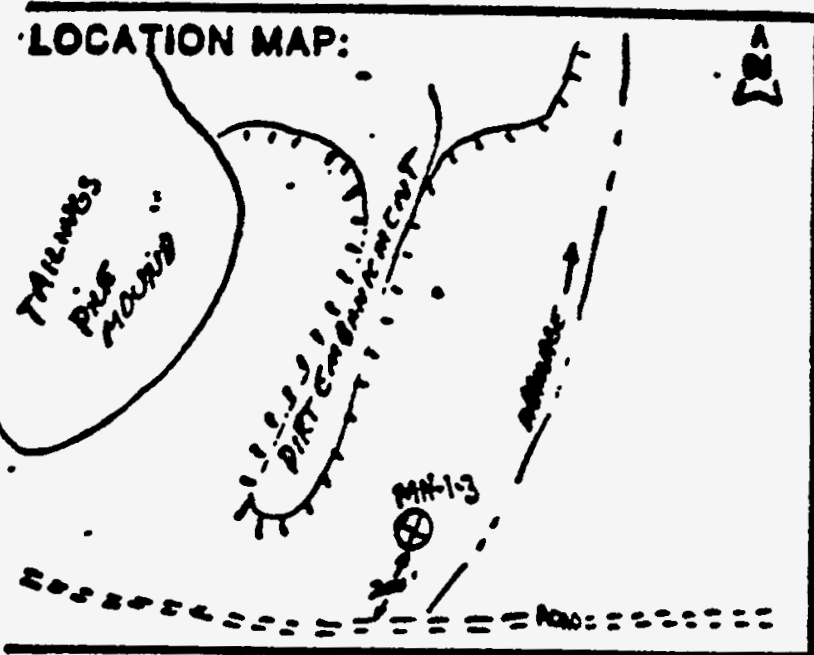

OITE ID: MONOL LOCATION ID: $1-3$ APPROX. BITE COORDINATES (fI.):

NDOLND ELENATION

OROUND ELEVATION (fI. MSL):

DRILLING METHOD: G" GSA CHESS

DRILLER: $S H B$ T

DATE 8TARTED: 10.25 .64

DATE COMPLETED:

FIELD REP.: SILVA

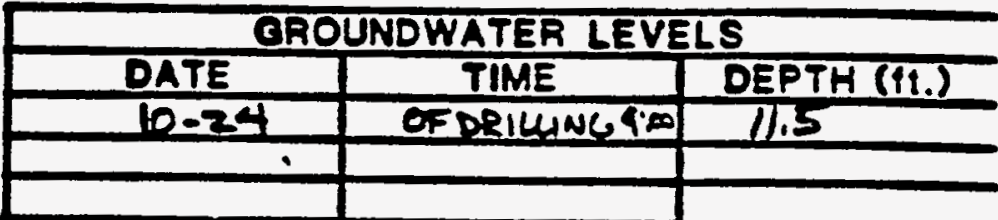

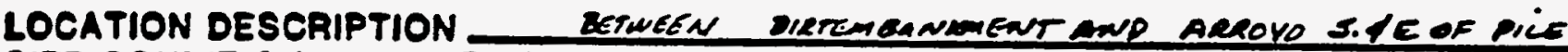
BITE CONDITION zOO NORTH of DIR L LAD

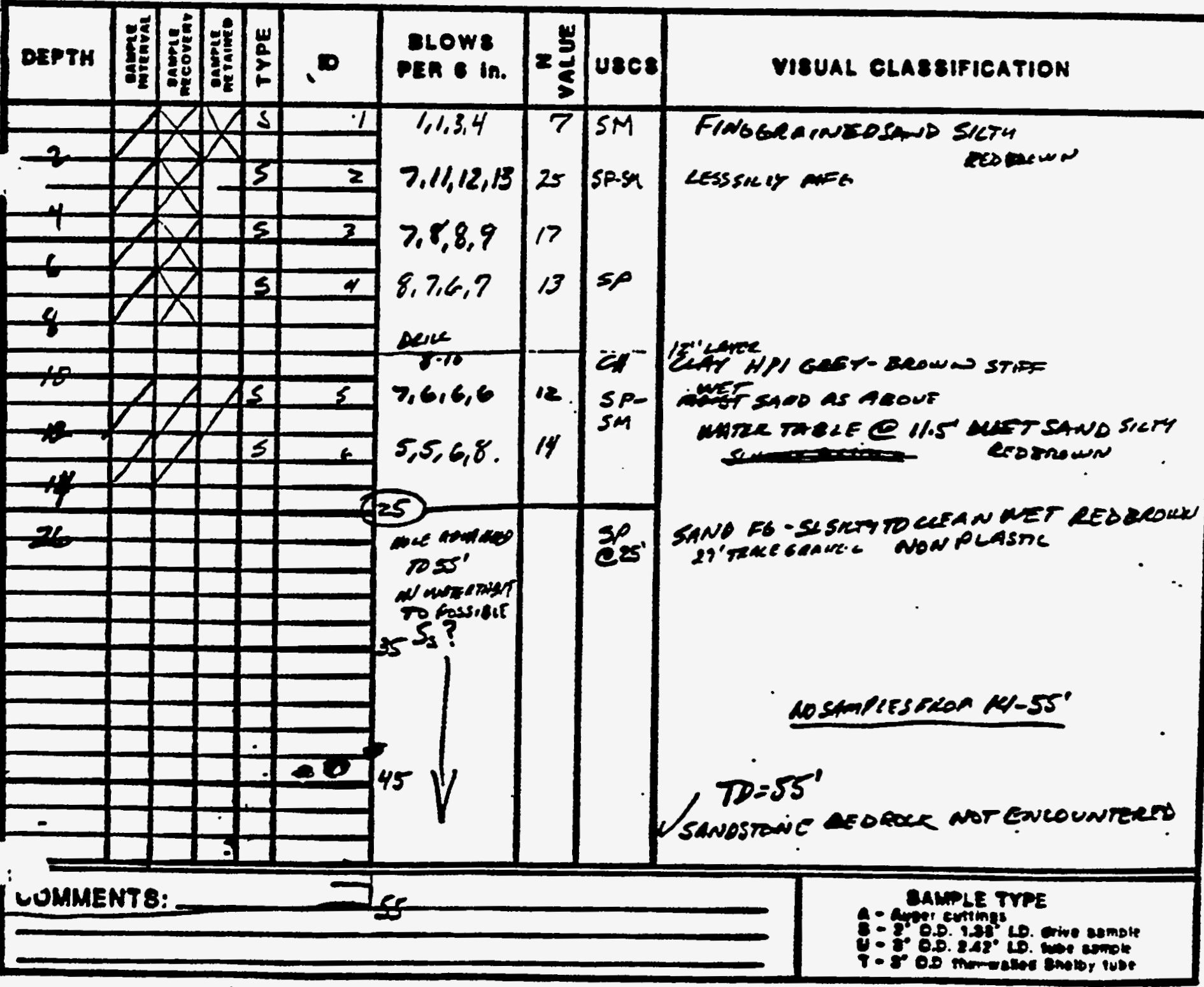




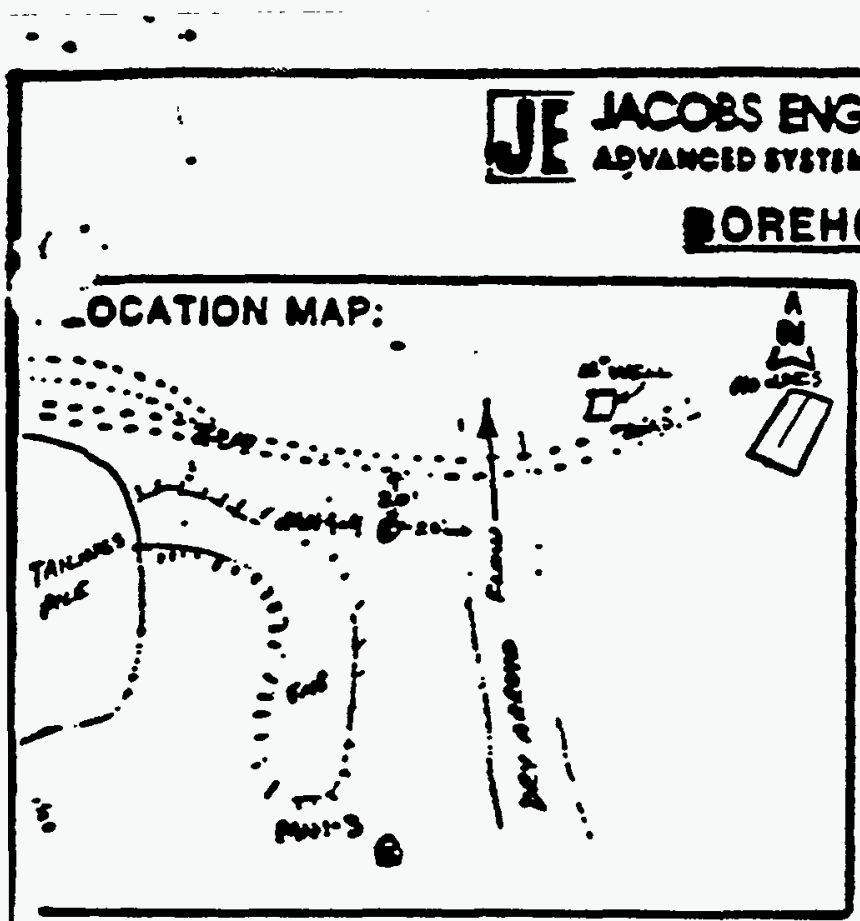

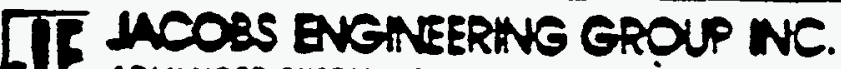

(

OREHOLE LOG (SOIL)

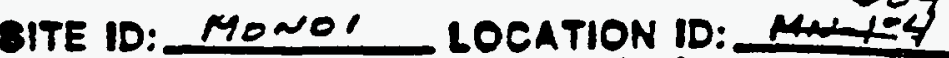
APPROX. SITE COORDINATES (ft.):

N NET SuRvere.2

CROUND ELEVATION (ft. MSL): Sman: Nereve

ORILLING METHOD: G" HSA

DRILLER: $\quad$ TeATS SNO

DATE STARTED: $10-25-84$

DATE COMPLETED:

FIELD REP.:

SILVA

\begin{tabular}{|c|c|c|}
\hline \multicolumn{3}{|c|}{ GROUNDWATER LEVELS } \\
\hline DATE & TIME & DEPTH (11.) \\
\hline 10.25 & 13.20 & 11.5 \\
\hline & & \\
\hline
\end{tabular}

LOCATION DESCRIPTION NOZNEAET OF PILE NEXTR DOAS CMCLVO

GITE CONDITION WEYT PDEY ALLOYO DLD

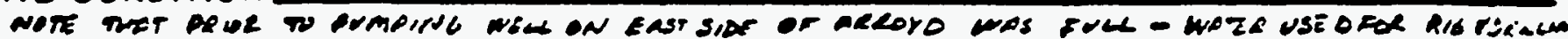

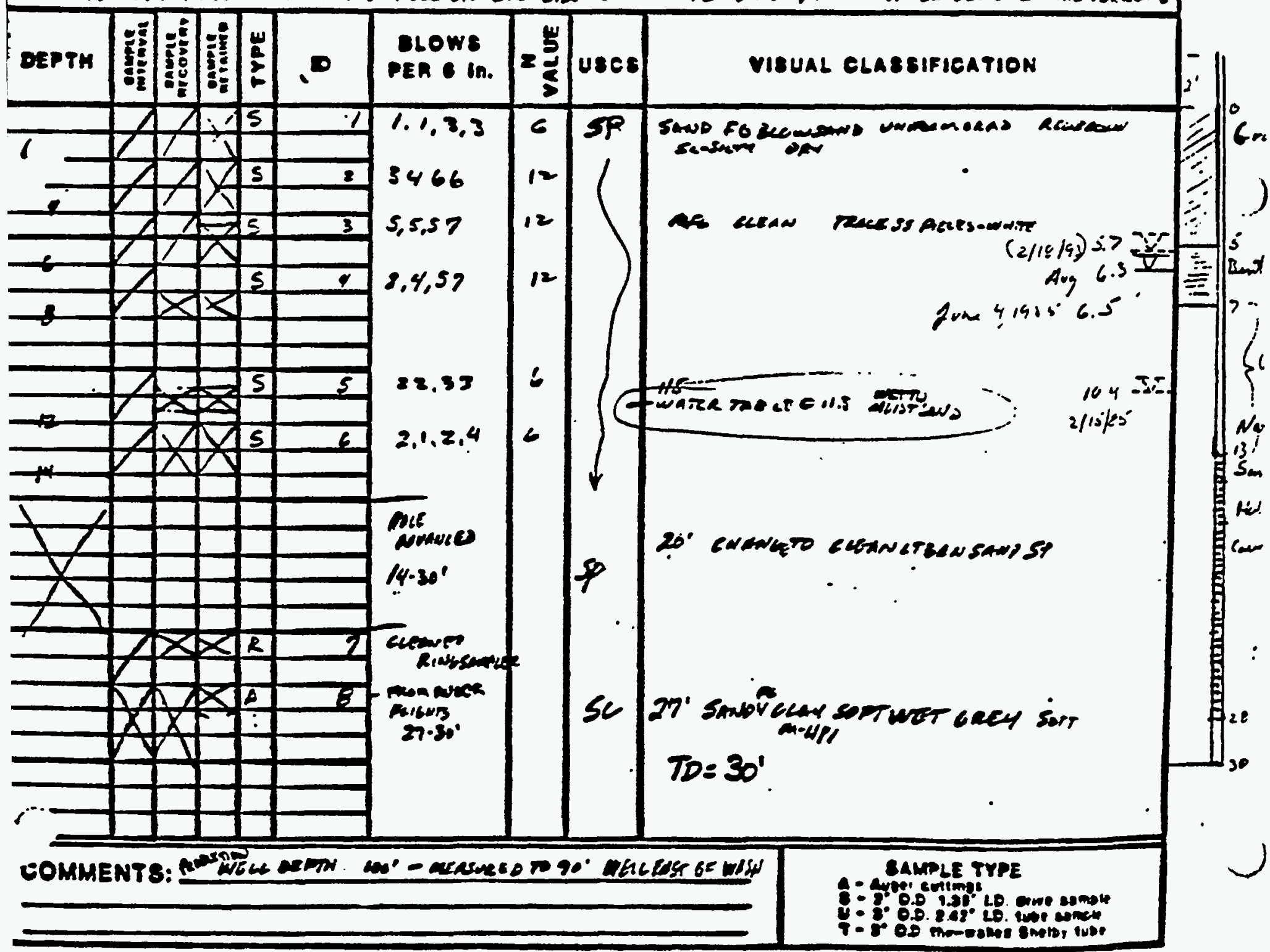

(EQ-Al-Ena-28. (2)

-... $\because$ 


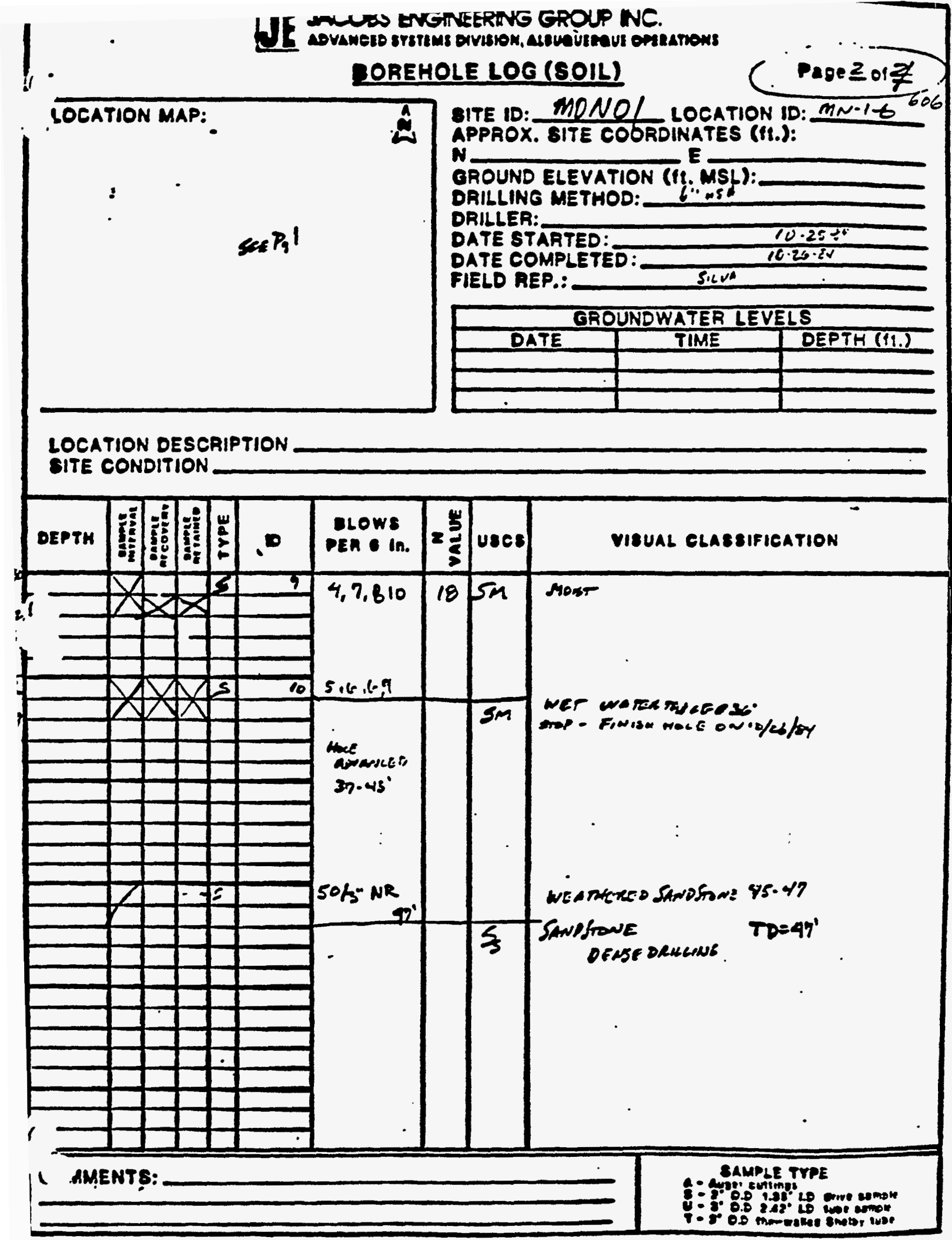

LOCATION DESCRIPTION SITE CONDITION 


\section{TF MCOBS ENENEERTE EROUP NC.

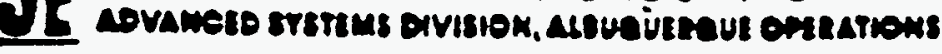

BOREHOLE LOG (SOIL)

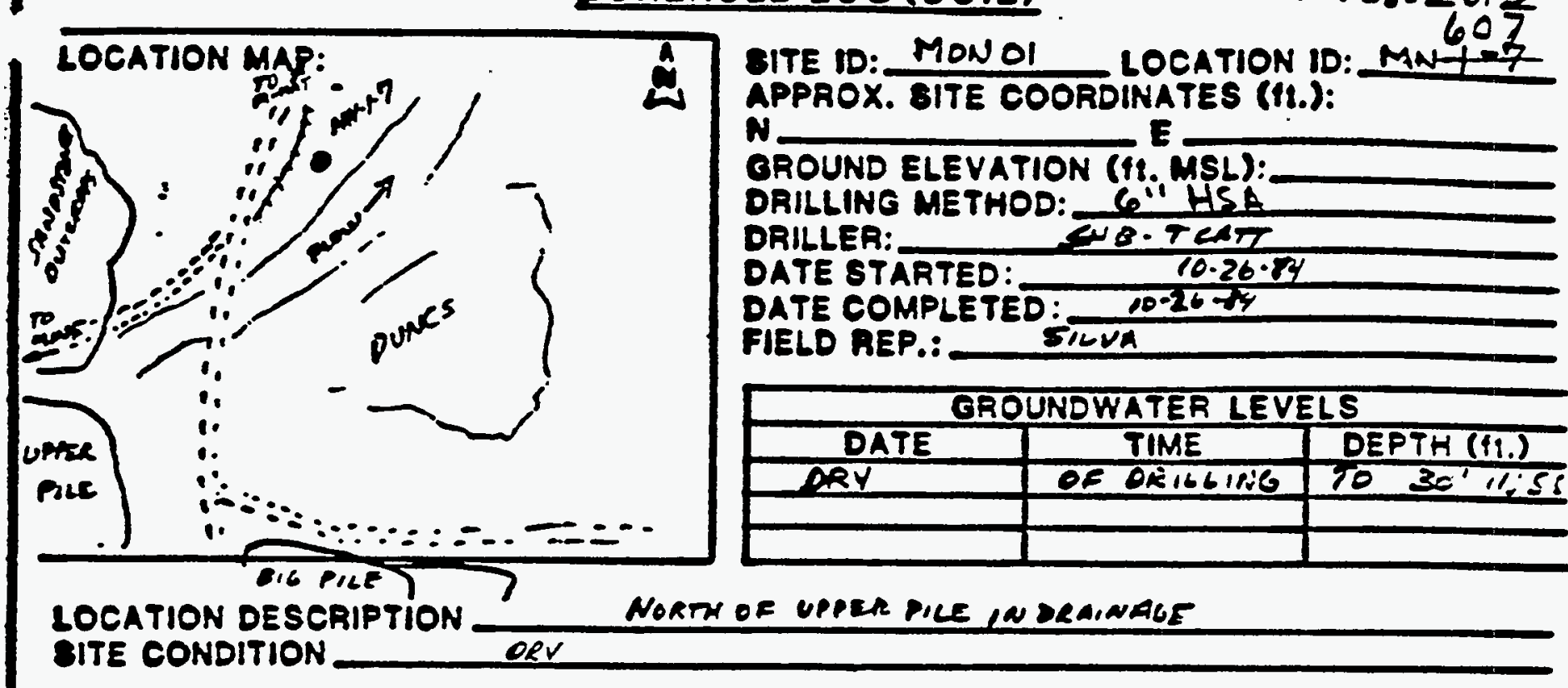

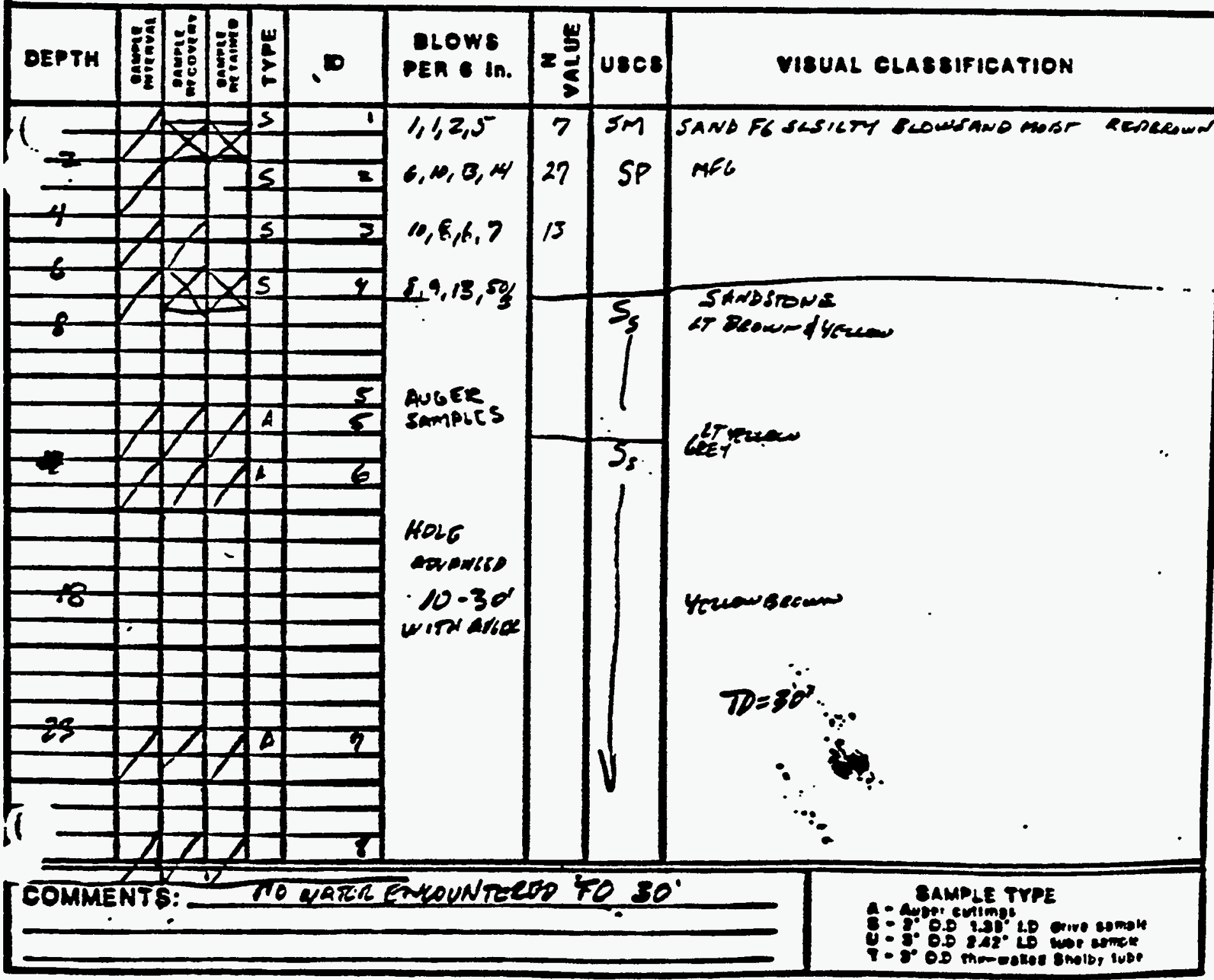




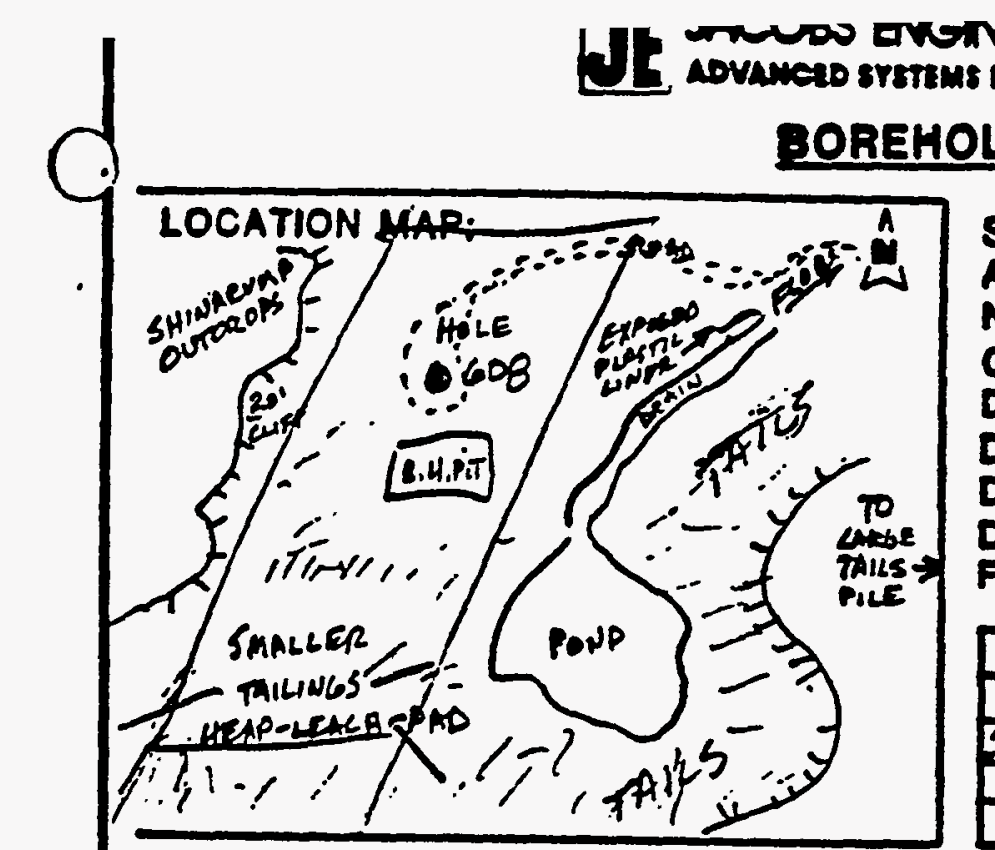

\section{BOREHOLE LOG (SOIL)I}

Page $L$ of 1

SITE D: MON O/ LOCATION ID: 608 APPROX. SITE COORDINATES (11.):

N 58685.713 E ETIB9. 364

GROUND ELEVATION (ft. MSL): 4901.08

DRILLING METHOD: 12 "HSA IO"PVE CASING

DRILLER: SIM CARTOR IFASTFODIE CMETS

DATE STARTED: $1-3-84$

DATE COMPLETED: $1-4-846200 T$ TE

FIELD REP.: S SILVA

\begin{tabular}{|c|c|c|}
\hline \multicolumn{3}{|c|}{ GROUNDWATER LEVELS } \\
\hline DATE & TIME & DEPTH (ft.) \\
\hline STOWPRY AT & TIMEOF COSIN & ENSTTU TON \\
\hline & & \\
\hline & & \\
\hline
\end{tabular}

LOCATION DESCRIPTION $10^{\prime}$ NORTH OF IMKKIEE PIT ON SMHLER HEAFLACH PAD SITE CONDITION ELAT TAILING MATERALL PONA TO S.E.

SEE 100 OF BACKHE TEST PIT ADTALNTTO THLOCOTON

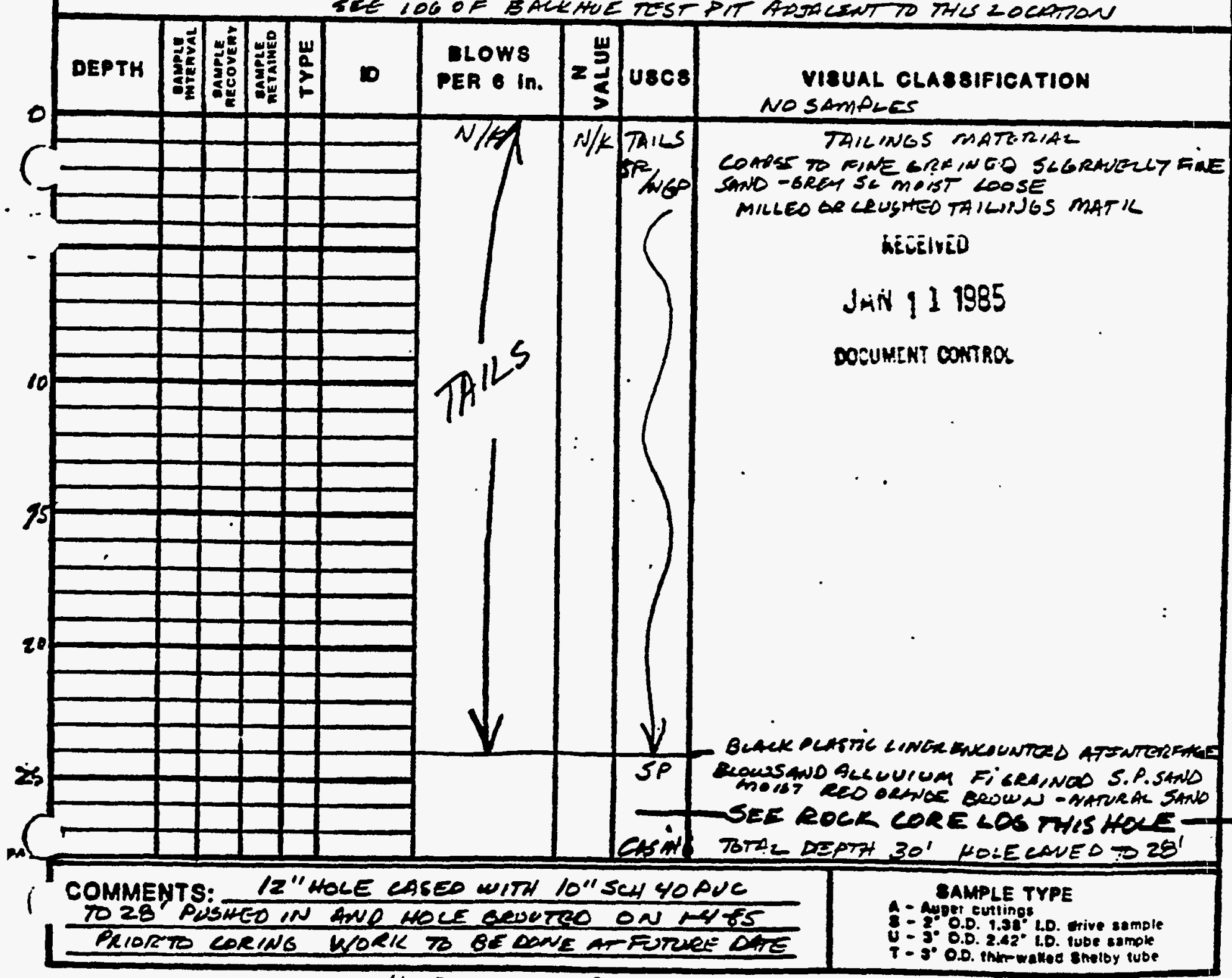

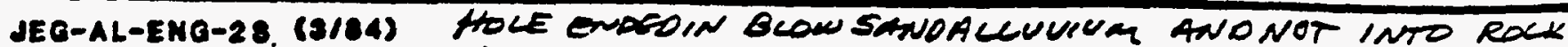
INTERACE 


\subsection{9 .4 .2}

T1 IACOBS QNGINEERING GROUP INC.

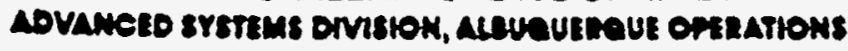

BOREHOLE LOG (ROCK)

Page $L$ of 7

LOCATION MAP:

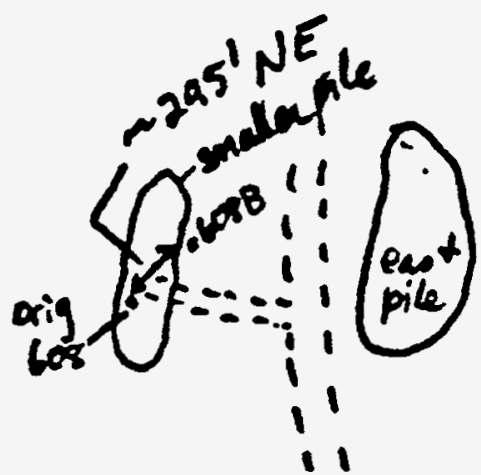

屯 SITE ID: MON-OI LOCATION ID: $608 \mathrm{~B}$

$N$

GROUND ELEVATION (fi. MSL):

DRILLING METHOD: NQ WIRE UNE

DRILLER: SHB /CARTER + PEREA /CME

DATE STARTED: 3115/85 TODAY 3/16/45

DATE COMPLETED: 3/10/55

FIELD REP.: $\mu_{1 L E R / M L E N E X Y}$

\begin{tabular}{|c|c|c|}
\hline \multicolumn{3}{|c|}{ GROUNDWATER LEVELS } \\
\hline DATE & TIME & DEPTH (ft.) \\
\hline & & \\
\hline & & \\
\hline
\end{tabular}

LOCATION DESCRIPTION APPCXX.295' NE of oriainal 608 location SITE CONDITION

\begin{tabular}{|c|c|c|c|c|c|c|c|c|c|c|}
\hline \multirow{4}{*}{ 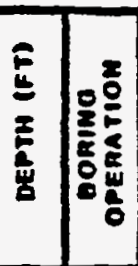 } & \multirow{4}{*}{\multicolumn{2}{|c|}{ 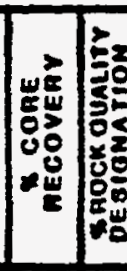 }} & \multirow{4}{*}{ 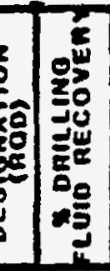 } & \multirow{4}{*}{ 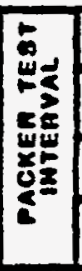 } & \multicolumn{3}{|c|}{ DISCONTINUITIES } & \multirow{4}{*}{ 可 } & \multirow{4}{*}{ 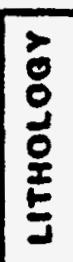 } & \multirow{4}{*}{$\begin{array}{r}\text { MOCK TYPE } \\
\text { REMARK } \\
\text { Aygered to } 9.5^{\circ}\end{array}$} \\
\hline & & & & & SPACING & ORIENTATION & 2 & & & \\
\hline & & & & & NIDE $=$ CLO8E & HORIZ. - VERT. & 量 & & & \\
\hline & & & & & 3 & $60^{\circ}$ & 8 & & & \\
\hline
\end{tabular}

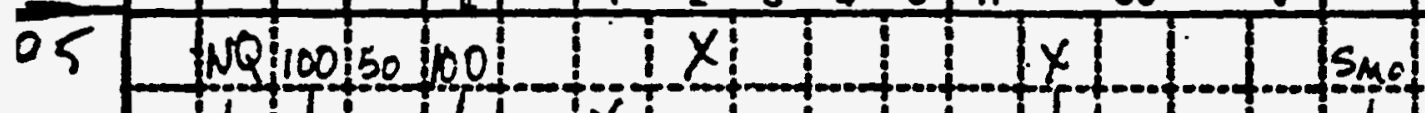

it

$\prod_{1.5}^{14 n}$ 20

$d$

im

Pat:

Cl ilignt gren (N7 Clay stone

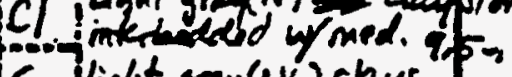
Ss fight groy (NG)clays" 10.3 - $5 S$ yellowish graytey sts med. to caarce gried, it 10015

IX

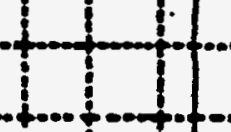
IX

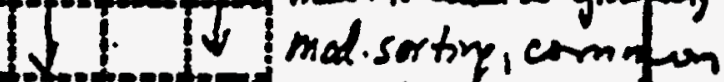
toritim of ear and 125' sw at mill prod. well 
SITE ID: MONOI LOCATION ID: $608 \mathrm{~B}$ N S (it.):

seepg. 1

GROUND ELEVATION ( $f$. MSL):

DRILLING METHOD: NQ WIREZINE

DRILLER: SHB CARTER + REREA

DATE STARTED: $3 / 15 / 85$ TOPAY आ/L/F5

DATE COMPLETED: $3 / 11 / X$

FIELD REP.: MULELIMCENERY

\begin{tabular}{|c|c|c|}
\hline \multicolumn{3}{|c|}{ GROUNDWATER LEVELS } \\
\hline DATE & TIME & DEPTH (f1.) \\
\hline & & \\
\hline & & \\
\hline & & \\
\hline
\end{tabular}

LOCATION DESCRIPTION

SITE CONDITION

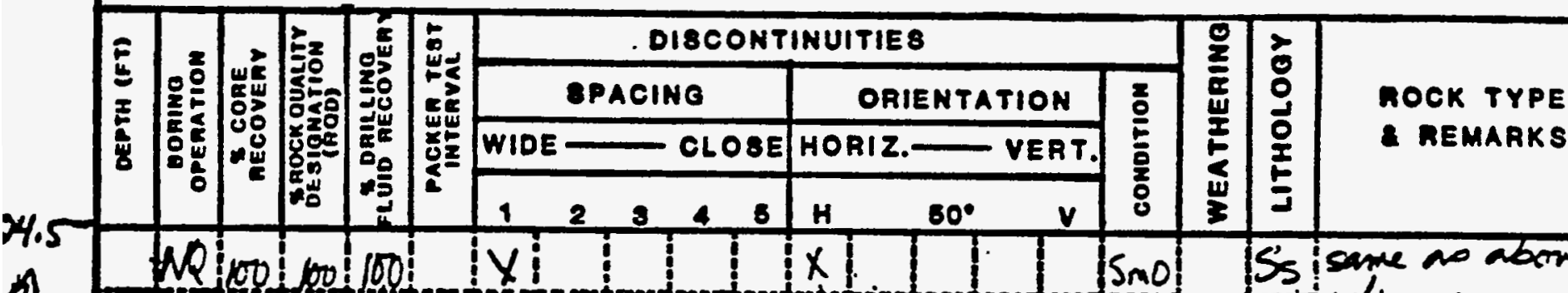

Ss isame no abore 1000!100!

\section{COMMENTS: lost cinc f drillip fluds at 2q'}

grayish orenge (orr $\geqslant 4$ ) samen siltstire gridiz into Aisht gran (Niv) at 34 's sme calcareuses intis. vem huivd, Top of Mopento, at $=20132.5$ $34 \div 39^{\prime} 7 \mathrm{iglt}$ gray (N7) sathy silfstix ur interbeded grauris ora. 


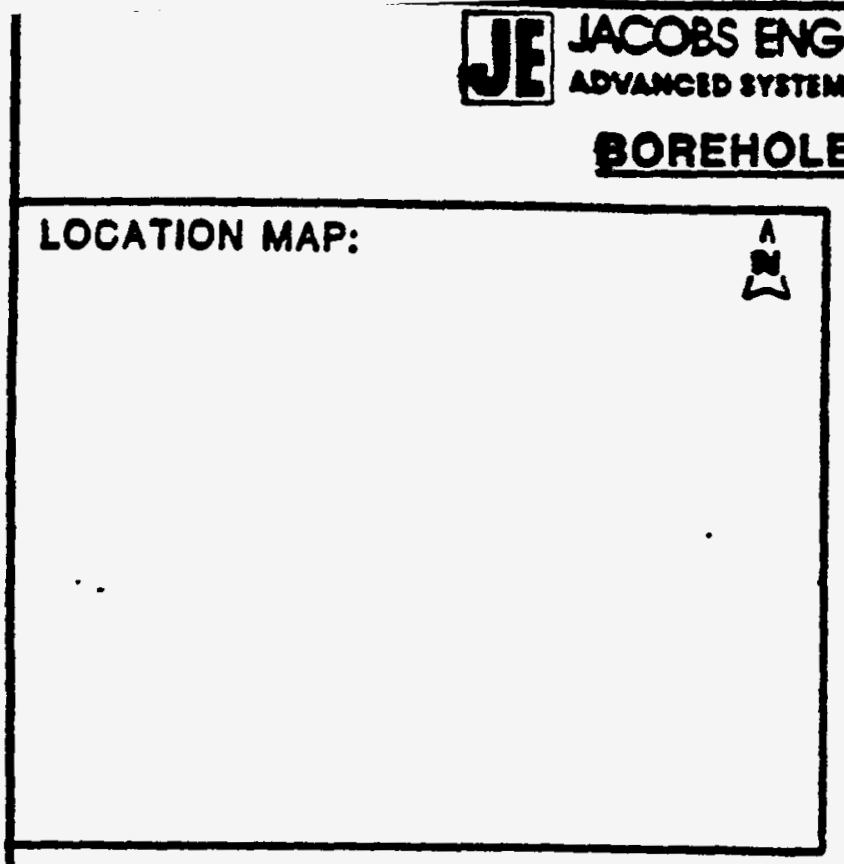

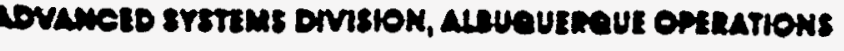

BOREHOLE LOG (ROCK)

Page of 7

SITE ID: MON QI LOCATION ID: 608 B APPROX. SITE COORDINATES (ft.):

$N$

GROUND ELEVATION (ft. MSL):

DRILLING METHOD: NQ WIRETNE

DRILLER: SHAB Carte + Perer

DATE STARTED: आ15/K5 TOPAY 3/18185

DATE COMPLETED: $3 / 11 / 5$

FIELD REP.: M EENERY

\begin{tabular}{|l|l|l|}
\hline \multicolumn{3}{|c|}{ GROUNDWATER LEVELS } \\
\hline DATE & TIME & DEPTH (I.). \\
\hline & & \\
\hline & & \\
\hline & & \\
\hline
\end{tabular}

LOCATION DESCRIPTION

SITE CONDITION

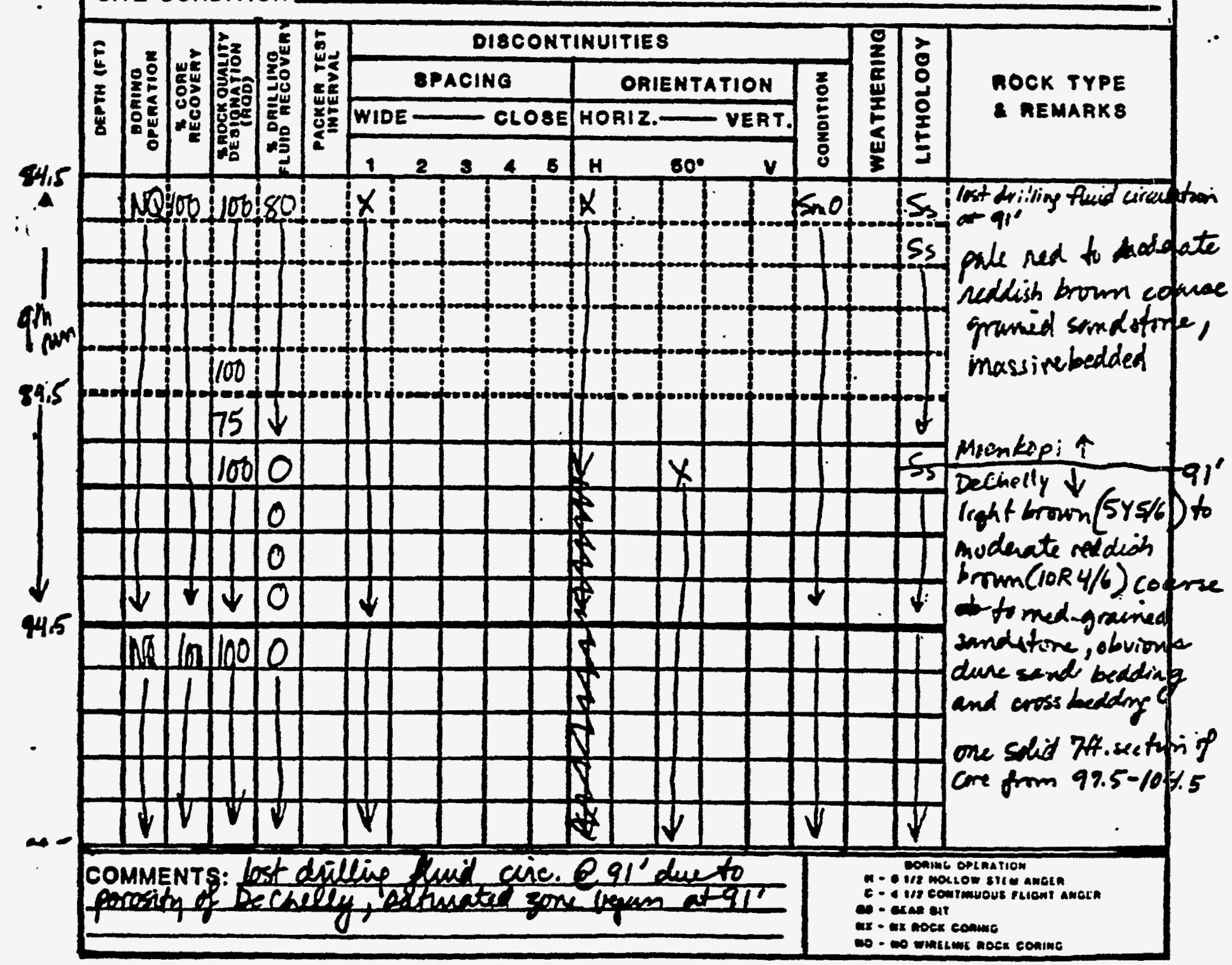

JEQ-AL-ENG-2A (3/84) 
1] JACOBS ENGHEERING GROUP HC.

AOVAMEED SYSTEMS DHVIOH, ALBUEUERAUE OPEATHONS

BOREHOLE LOG (ROCK)

Page 7 of 7

LOCATION MAP:

$\hat{\omega}$

SITE 1D: MONOI LOCATION ID: $608 B$ APPROX. SITE COORDINATES ( $\mathrm{ft}$.):

$N$ E

GROUND ELEVATION ( $f$. MSL):

DRILLING METHOD: NQ WIRELINE

DRILLER: $S H+B$ Castes Renea

DATE STARTED: $3 / 15 / 85$ TODAY $3 / 17 / 85$

DATE COMPLETED: $3 / 18 / 85$

FIELD REP.: MLEnery

\begin{tabular}{|c|c|c|}
\hline \multicolumn{3}{|c|}{ GROUNDWATER LEVELS } \\
\hline DATE & TIME & DEPTH (ft.) \\
\hline & & \\
\hline & & \\
\hline & \\
\hline
\end{tabular}

94.5

LOCATION DESCRIPTION

SITE CONDITION

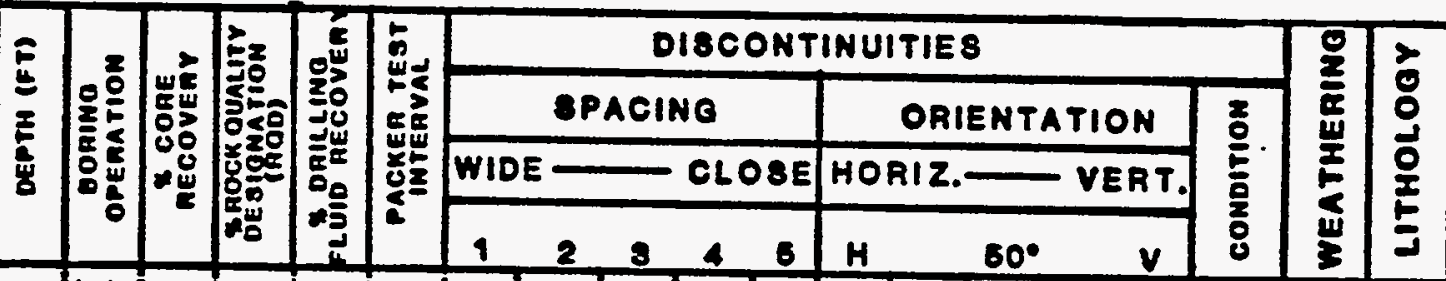

MOCK TYPE

a REMARKS

WQ $100 ! 100: 0: x$

$11+10$

NQ $1000100: 0$

mas

(Y)

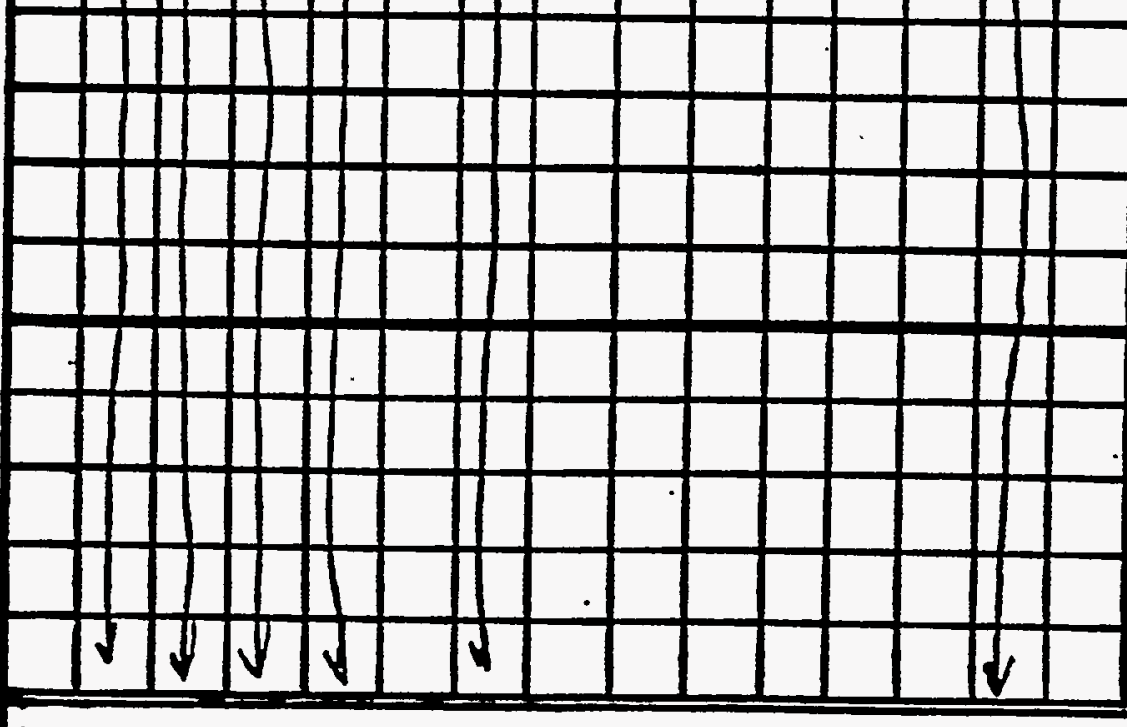

COMMENTS:
Smo Ss lithology continuour trom presisua decciption

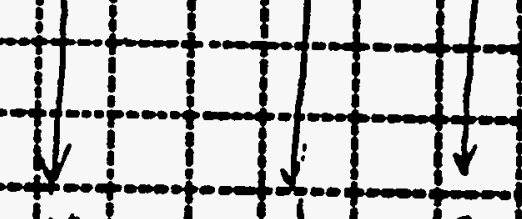

Ix 15 


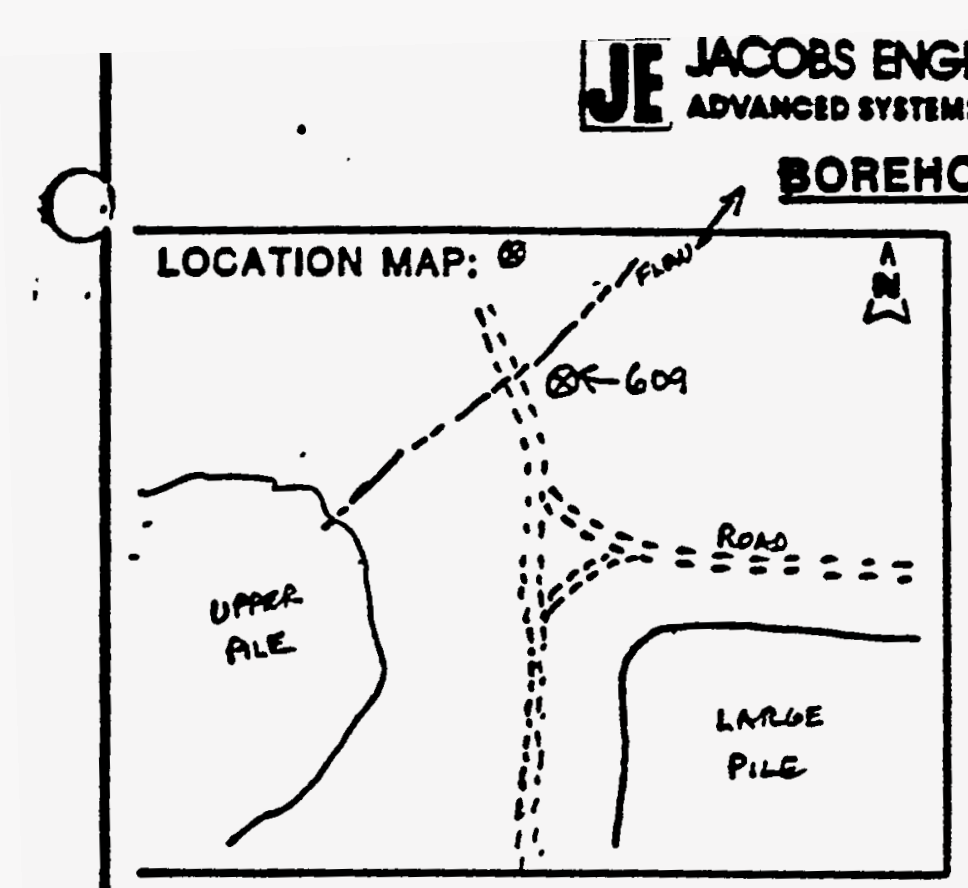

Page 1 of 1

SITE ID: MONOI

LOCATION 10: 609 -

APPROX. SITE COORDINATES (11.):

N 54052.132 E 62652.915

GROUND ELEVATION ( $f$ t. MSL): 4878.30

ORILLING METHOD: G" HSA

DRILLER: SHB bEAR MOBILE B-8O

DATE STARTED: $1-3-85$

DATE COMPLETED:

FIELD REP.: SILUA

\begin{tabular}{|c|c|c|}
\hline \multicolumn{3}{|c|}{ GROUNDWATER LEVELS } \\
\hline DATE & TIME & DEPTH (ft.) \\
\hline $1.3-85$ & OFORILL & OQY \\
\hline & & \\
\hline
\end{tabular}

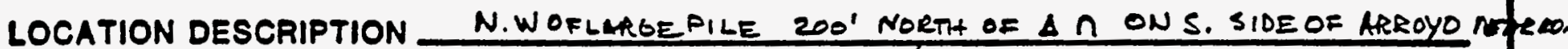

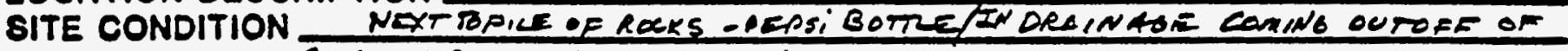
SMALWOX PILE - BRY SANOY SHACLOW ALLUUIUM WELC N.OF PILES

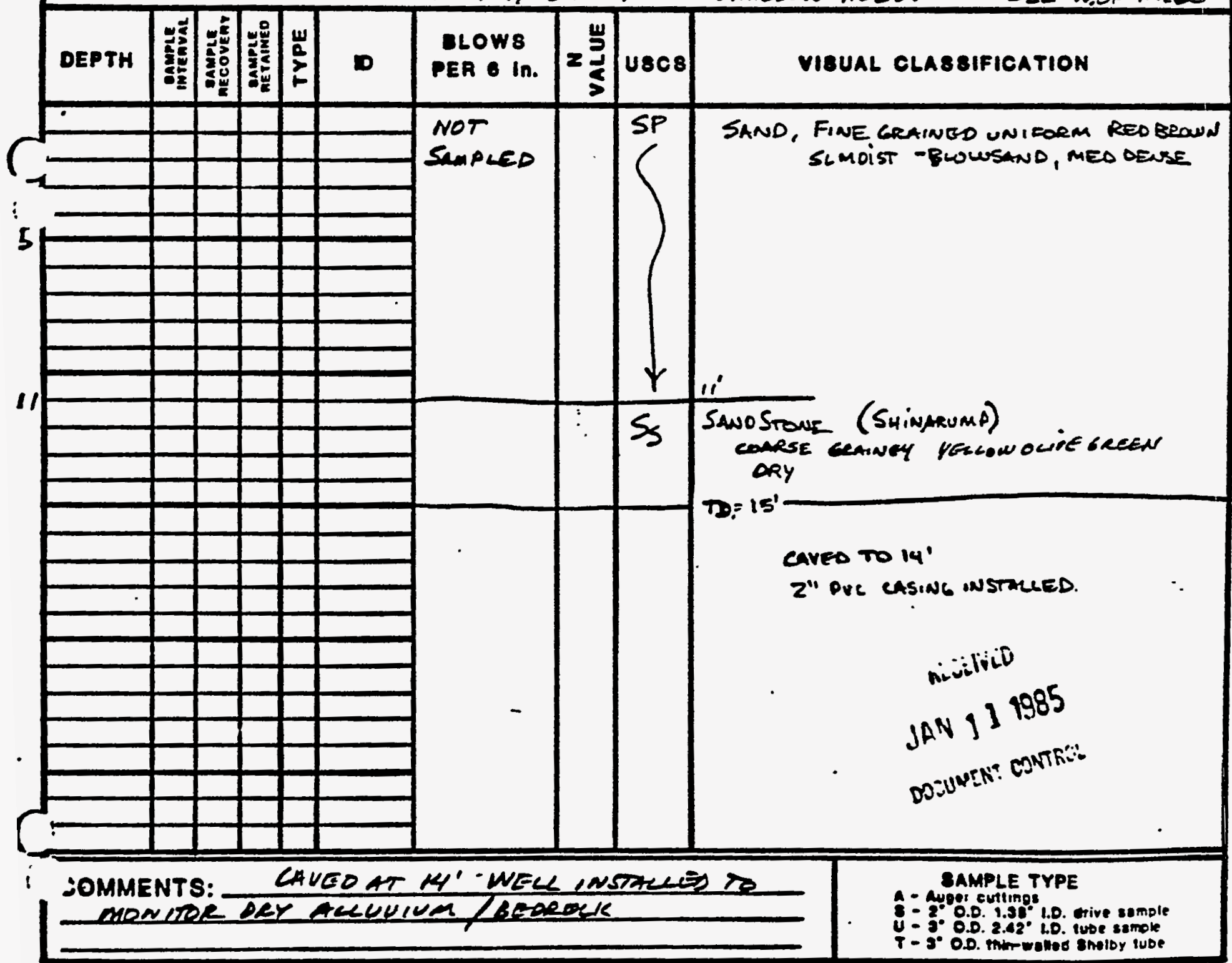

JEO-AL-ENO-28. (S/O4) 


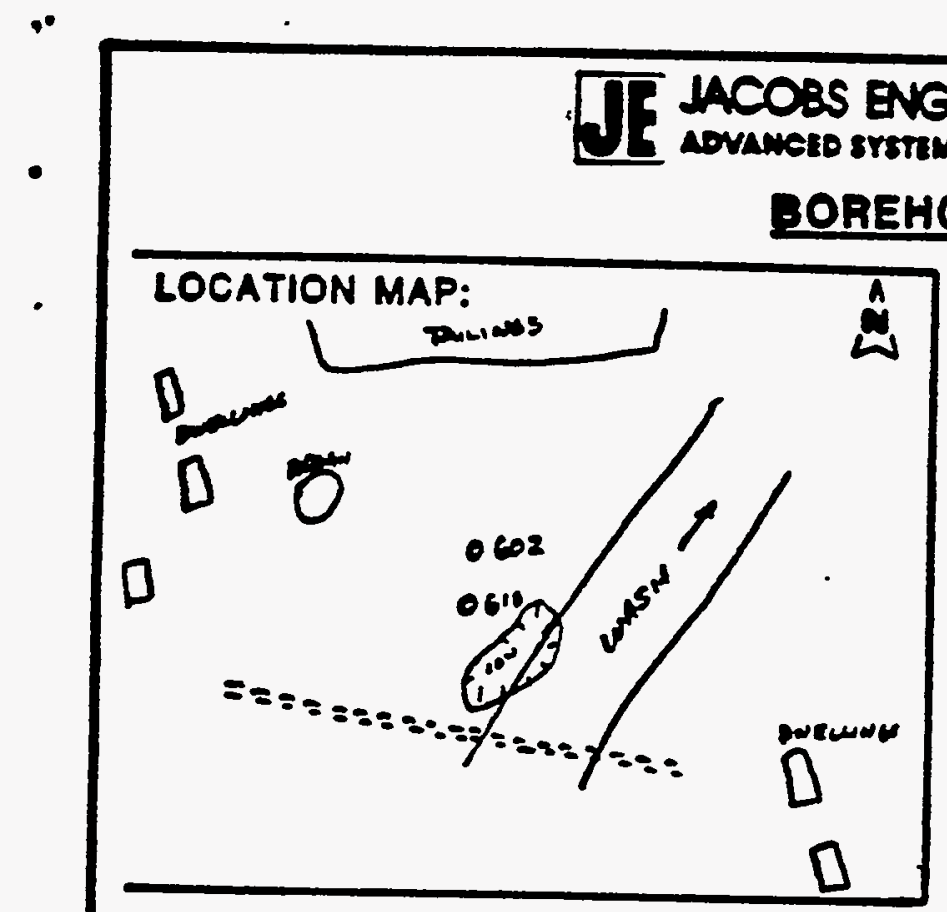

BOREHOLE LOG (SOIL)I

Page Lor

SITE ID: MONOI LOCATION ID: CIO. APPROX. SITE COORDINATES (ft.):

N

GROUND ELEVATION (ft. MSL):

DRILLING METHOD: 6 "HSA - NOWIEELWE

DRILLER:SHF BEAE

DATE STARTED: 12.3 .84

DATE COMPLETED: $\frac{12-4}{12-4}$

FIELD REP.:

SILPA

\begin{tabular}{|c|c|c|}
\hline \multicolumn{3}{|c|}{ GROUNDWATER LEVELS } \\
\hline DATE & TIME & DEPTH (11.) \\
\hline 12.3 .84 & $11.00 \mathrm{~cm}$ & $8^{\prime}$ \\
\hline & & \\
\hline & & \\
\hline
\end{tabular}

LOCATION DESCRIPTION

25 'Sovit of "602"

SOUTH OF TAILINGS PILE

SITE CONDITION

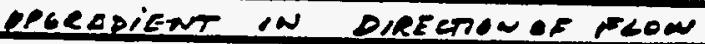

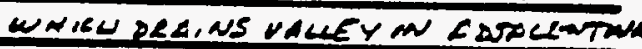

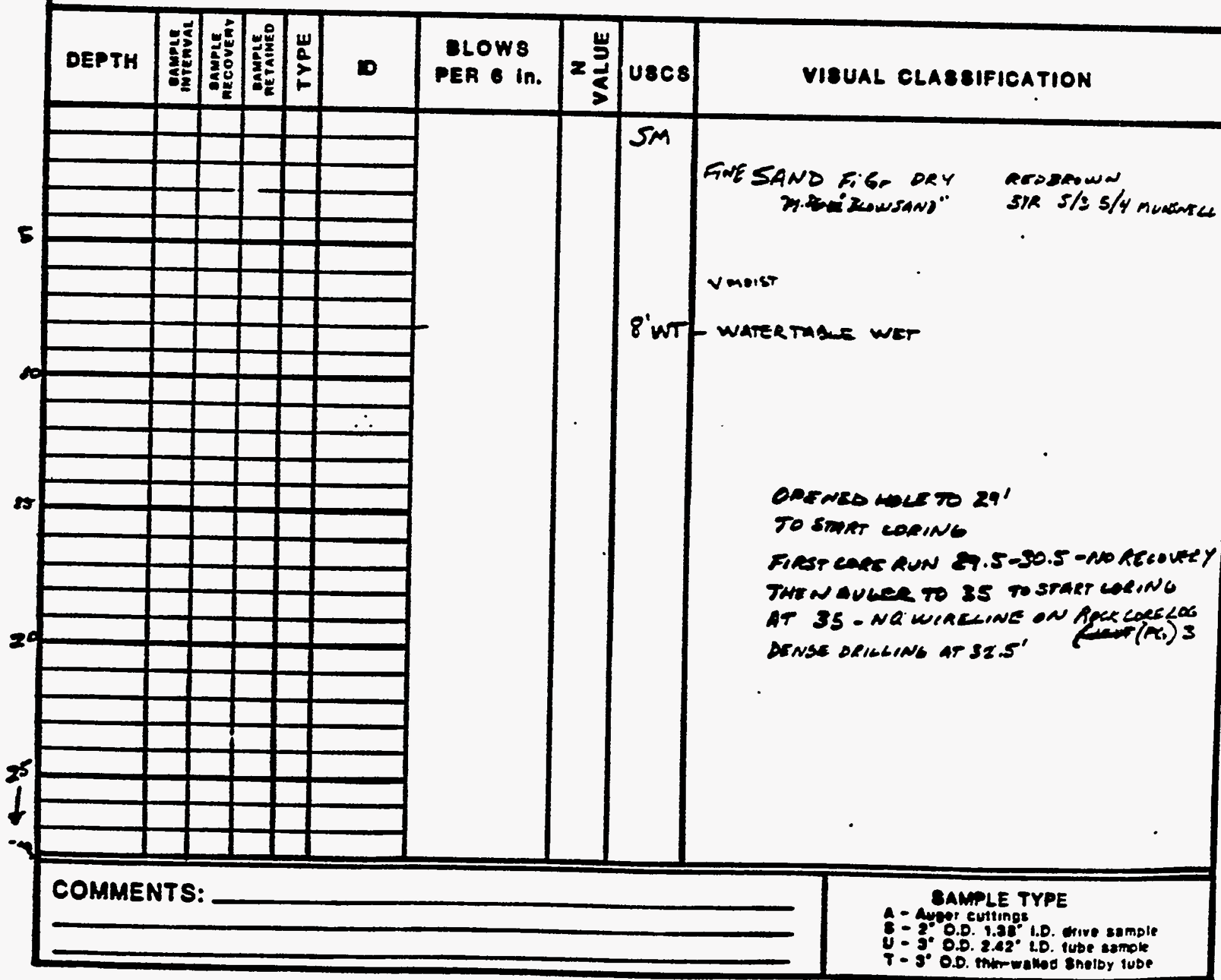

JEG-AL-EMG-28. (S/84) 
LIF ACOBS ENGNEERNG GROUP ANC.

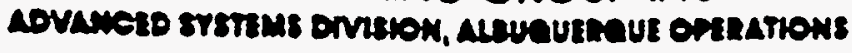

BOREHOLE LOG (SOIL))

Pagez of

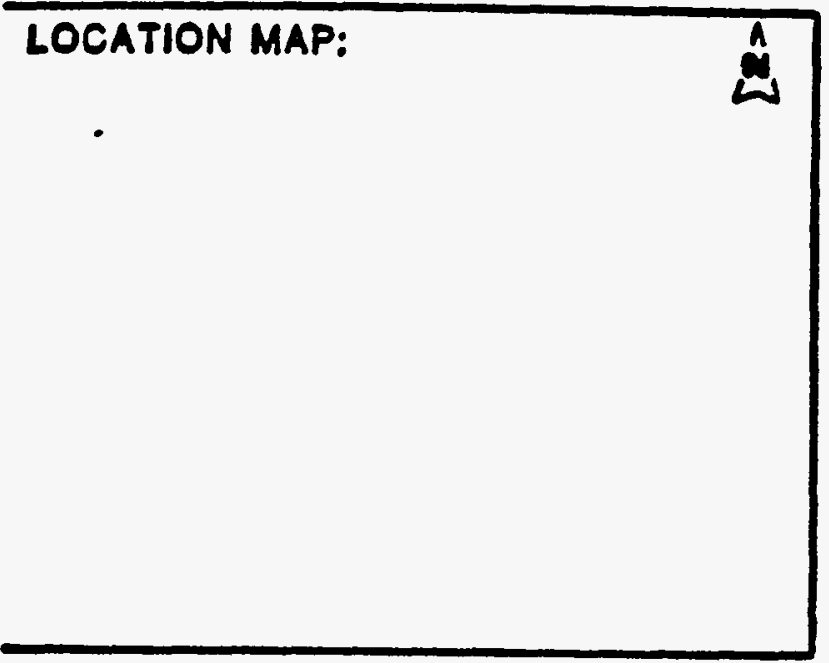

SITE ID: MONOI

APPROX. SITE COORDINATES (ft.):

N. E

GROUND ELEVATION (ff. MSL):

DRILLING METHOD: " "WA

DRILLER: SHB- SEAR

DATE STARTED: $\frac{12 \cdot 3 \cdot 14}{4}$

DATE COMPLETED:

FIELD REP.:

aler

\begin{tabular}{|c|c|c|}
\hline \multicolumn{3}{|c|}{ GAOUNDWATER LEVELS } \\
\hline DATE & TIME & DEPTH (ff.) \\
\hline & & \\
\hline & & \\
\hline & & \\
\hline
\end{tabular}

LOCATION DESCRIPTION SUTM OE GOZ SITE CONDITION

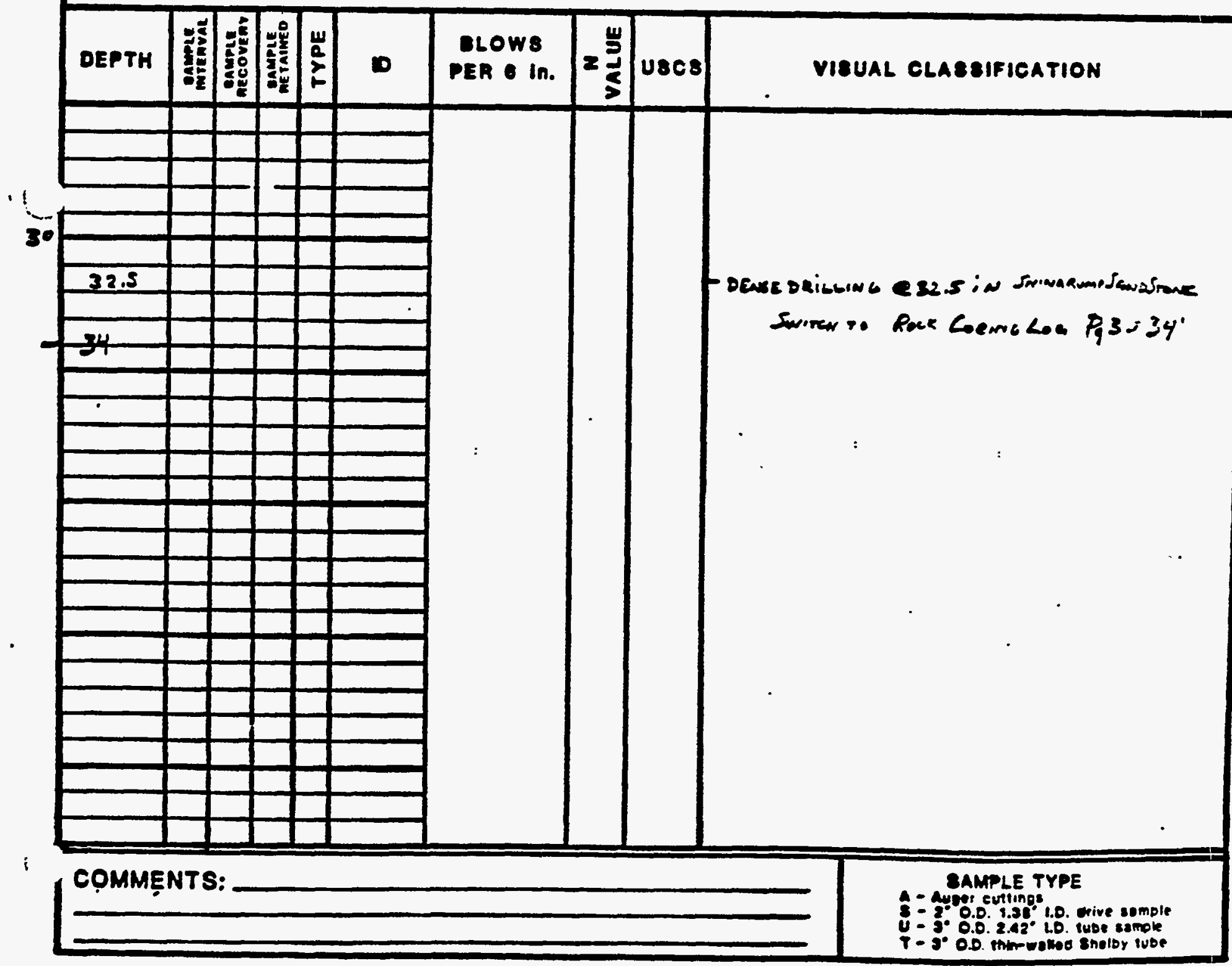

JEO-AL-ENB-28, (2/84) 


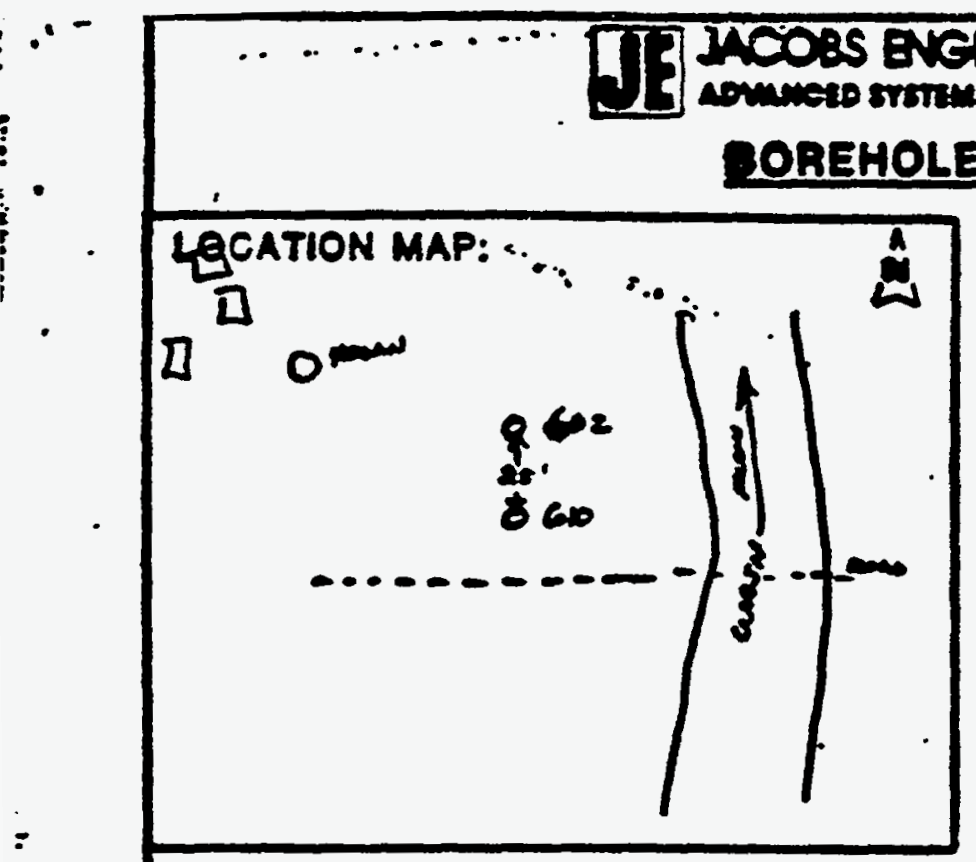

LOCATION DESCRIPTION

BITE CONDITION

SITE ID: MONO/ LOCATION ID: 610 APPROX. BITE COORDINATES (it.):

N.

E

QROUND ELEVATION (ft. MSL):

DRILLINO METHOD: Wa Whallane

DAILLER:EN DER

DATE 8TAATED: $2 J 4$

DATE COMPLETED:

FIELD REP.:

the

\begin{tabular}{|c|c|c|}
\hline \multicolumn{3}{|c|}{ OROUNDWATER LEVELS } \\
\hline DATE & TIME & DEPTH (14.) \\
\hline 12.8 .4 & 11.00 & 8 \\
\hline & & \\
\hline & & \\
\hline
\end{tabular}

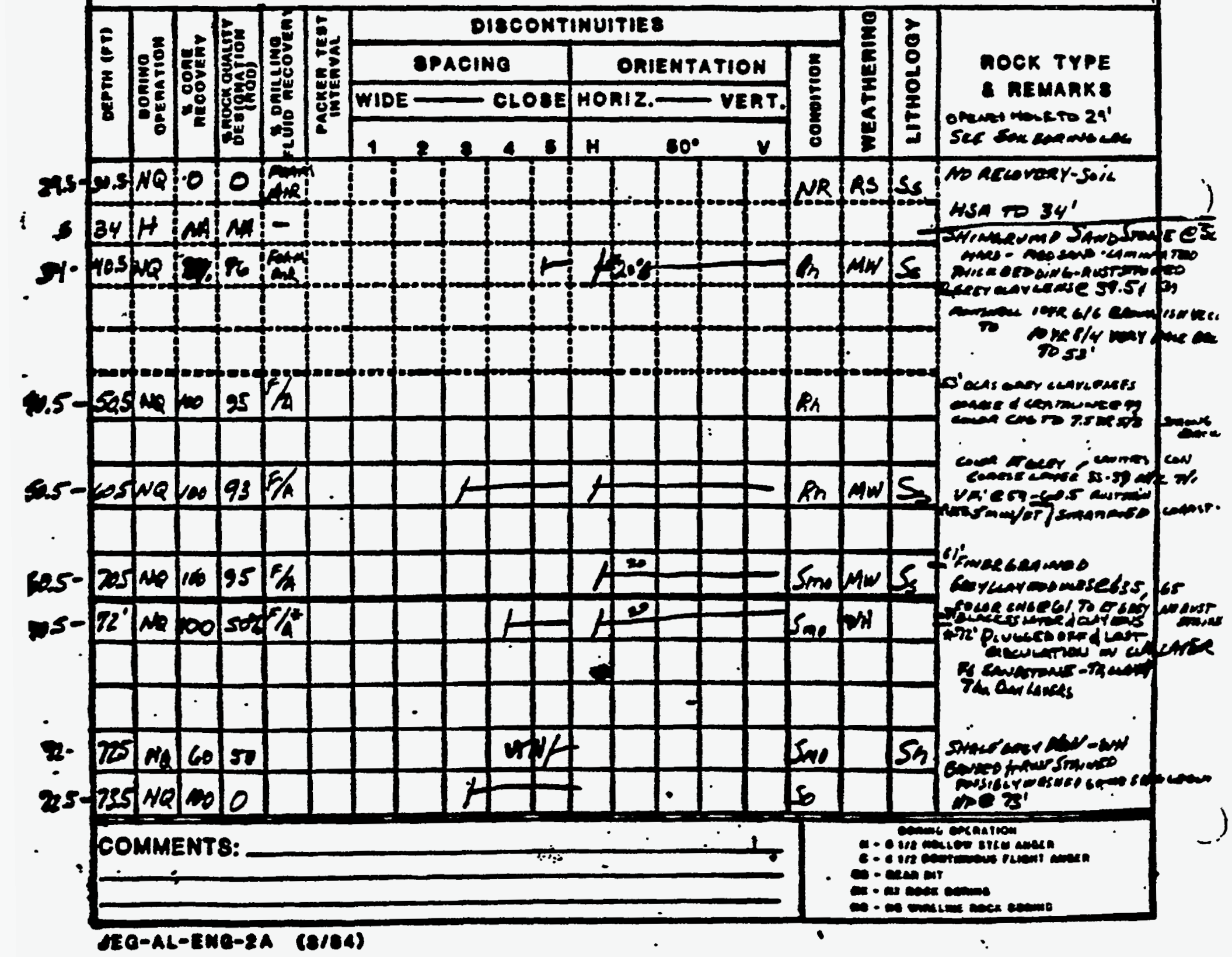




\section{BOREHOLE LOG (ROCK)}

LOCATION MAP:

$\operatorname{S\pi r} P_{\mathrm{g}} \operatorname{lon} 3$

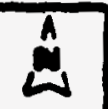

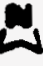

SITE ID: MON O

APPA

GROUND ELEVATION (ft. MSL):

DRILLING METHOD:

DRILLER:

Grane

DATE STARTED:

es-1tay

DATE COMPLETED:

FIELD REP.:

LOCATION DESCRIPTION

NEXT TO NN-1-2

SITE CONDITION

GROUNDWATER LEVELS

DATE TIME

ROCK TYPE

- REMARK 8

REE NITLED WAES

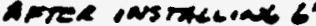
casine ro 34 '

usce verver $640^{\prime}$

smarco cor unte 75.5 IN SHALE

GREY SWALE

$S_{m e}=S h$ $33.5-75:$ NQ: $100: 15: 100: x$ 15 15 48 7 ezser $x$

\section{6}

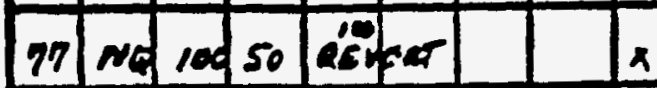

$\eta$ gastue coos ico revert $x$

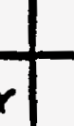

1 Se्se

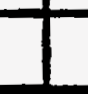

1

Smo

Smo $F$ Sh

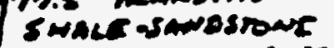
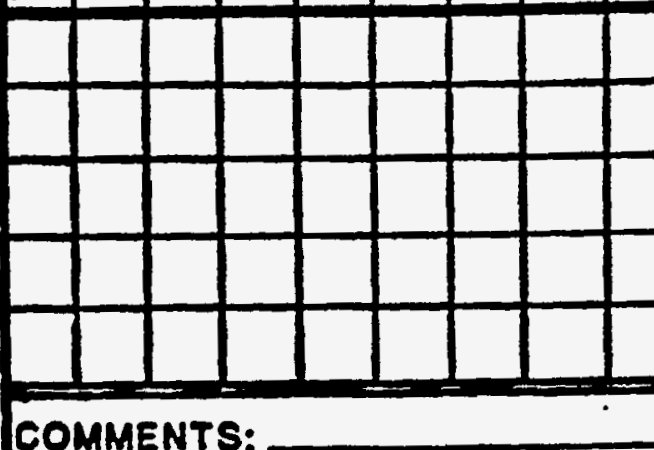

$\square$

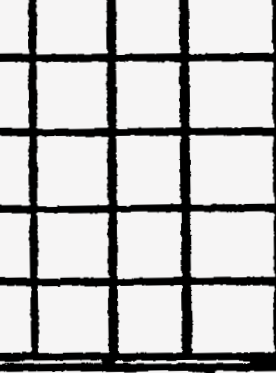

$\square$

COMMENTS:

JEQ-AL-ENO-2A (S/84)
75.5

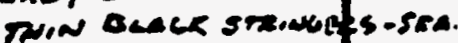

MASO . SANDY

Plugles arc at $75 \cdot \mathrm{m}$. ivuen sant 4

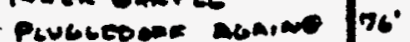

ia sor smale -camia arso

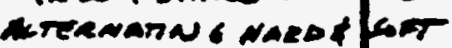
careas of gerrsinus

Fosues 97

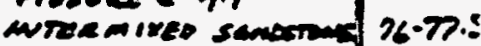

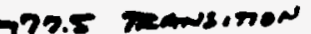

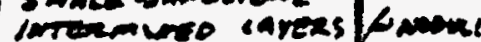
cresar

cer w waine hespormenco filn. Ss

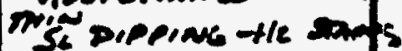
we reare raterwer $70.6-80.5$ 
LOCATION MAP:

$$
\operatorname{Sec} P_{7} 3
$$

$\hat{\omega}$

SITE ID: MONOI APPROX. SITE COORDINATES (ft.):

$N$ GROUND ELEVATION (ft. MSL):

DRILLING METHOD:

DRILLER: genR-ShB

DATE STARTED:

DATE COMPLETED:

FIELD REP.:

LOCATION DESCRIPTION

NEXT TO MN-1.2

SITE CONDITION

\begin{tabular}{|c|c|c|}
\hline \multicolumn{3}{|c|}{ OROUNDWATER LEVELS } \\
\hline DATE & TIME & DEPTH (fI.) \\
\hline & & \\
\hline & & \\
\hline & & \\
\hline
\end{tabular}

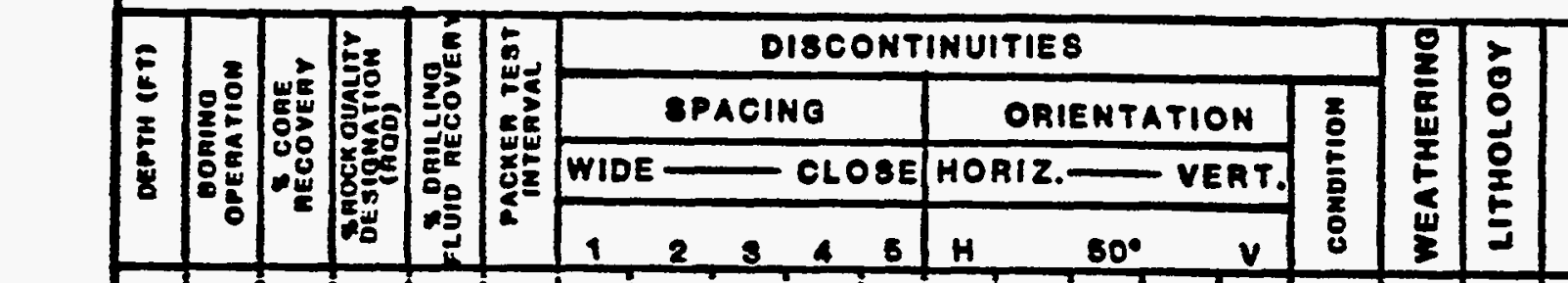

AOCK TYPE

( AEMARK 8

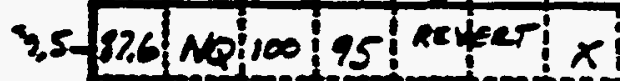

(1)

mentent

7.2 Ne no 95 Refloer

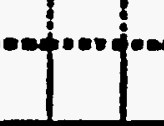

$-$

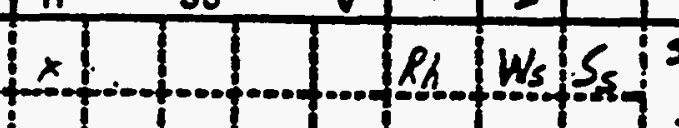

SANOSTONE

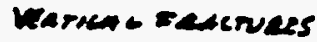

TNon Hz EAwDS of W ro or

Fi Gr Laveies

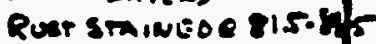

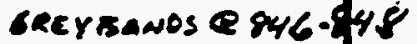

Tedec LeaYer in R.S. W W.G.

Reows ind $86 \cdot 86.7$

Docosese Geains d cen srume in fick Samo frowe WAS ROUNDEd 1/2"GRMnl 6

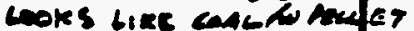
conchimenare towline 02.6 aconobur 'on Gervenysronf II Whi:

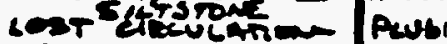
Ofe in 87.T in Sort Cay

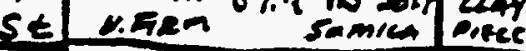
Ifr.5 Sat GuYstowe O4.5 himestowe QuF coes

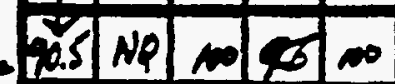

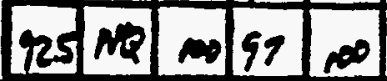
$\operatorname{Sim}_{\mathrm{s}} \boldsymbol{W}_{\mathrm{S}} \mathrm{Ct}_{\mathrm{t}}$

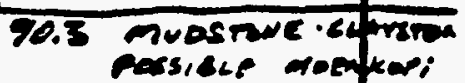
crosscodoes mins

2.5 maxer seown

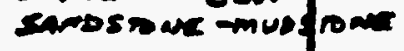
24.5 smatifico ch erren Sinosnowe in muest

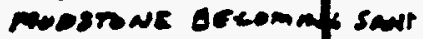
from $8.5-87.5$

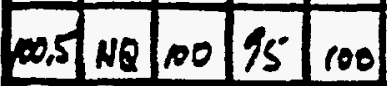

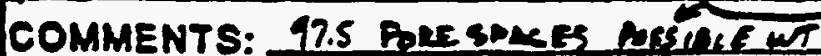

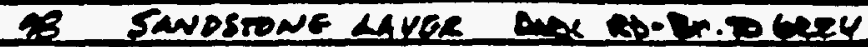

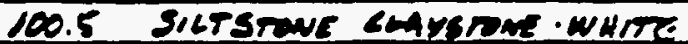


MONOI 610 HOLE SEQUENER:

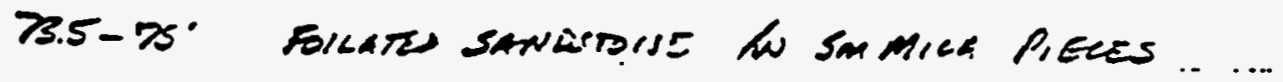

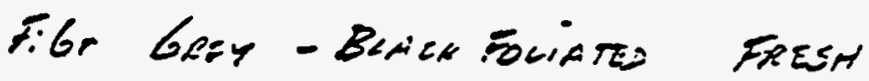

$73.76 \quad \because$ Tan sarjistine

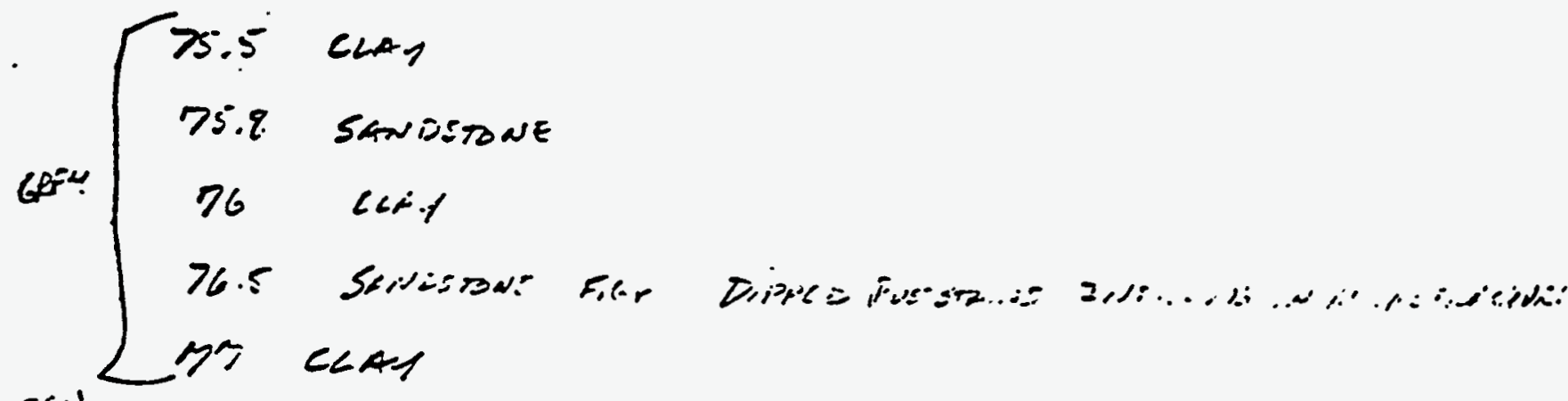

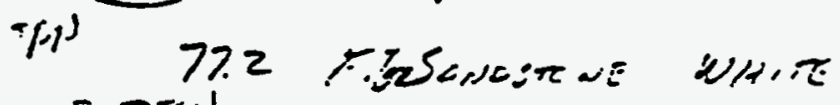

wi $=-20,07$

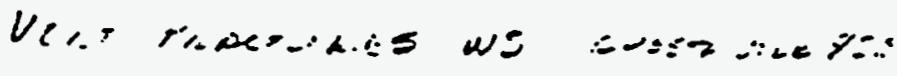

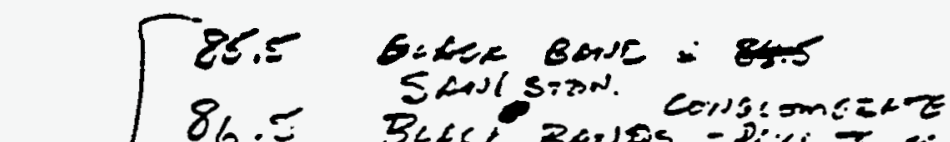

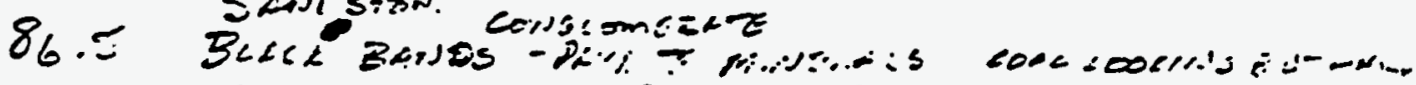

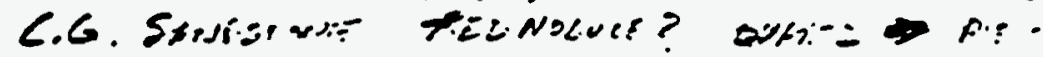

$$
\text { r. } \quad \therefore: \%
$$

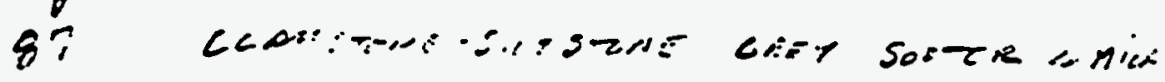

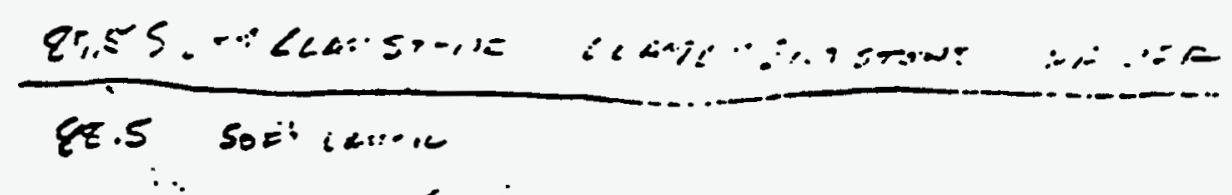

FT:S limestout ?

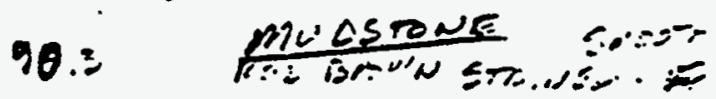

Cirs:-85.0.28: mase-

92.5 DATERBREN

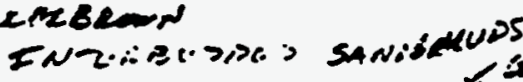

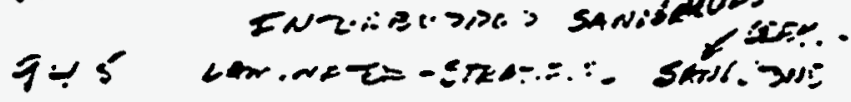

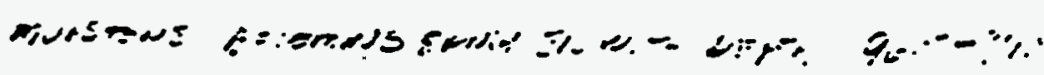

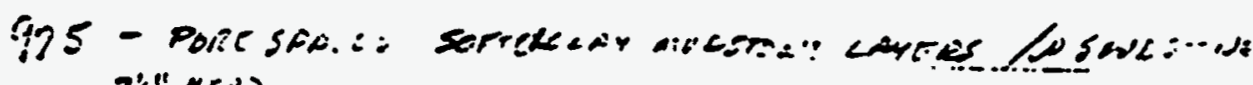

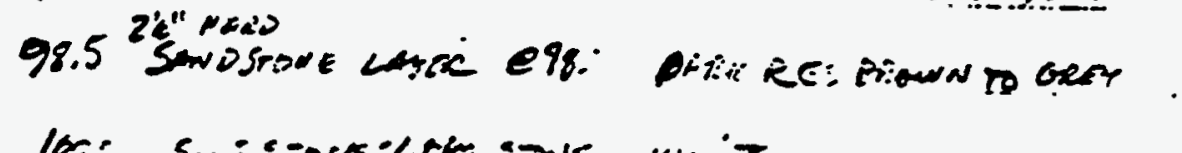

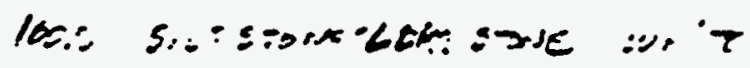




\section{MONOI HOLE GIO NOTES}

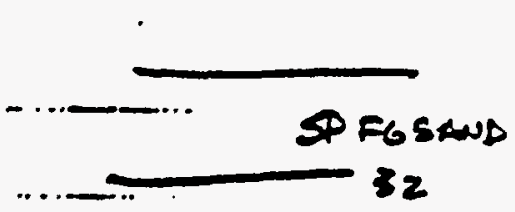

SH.1SĀ̃̂MPP
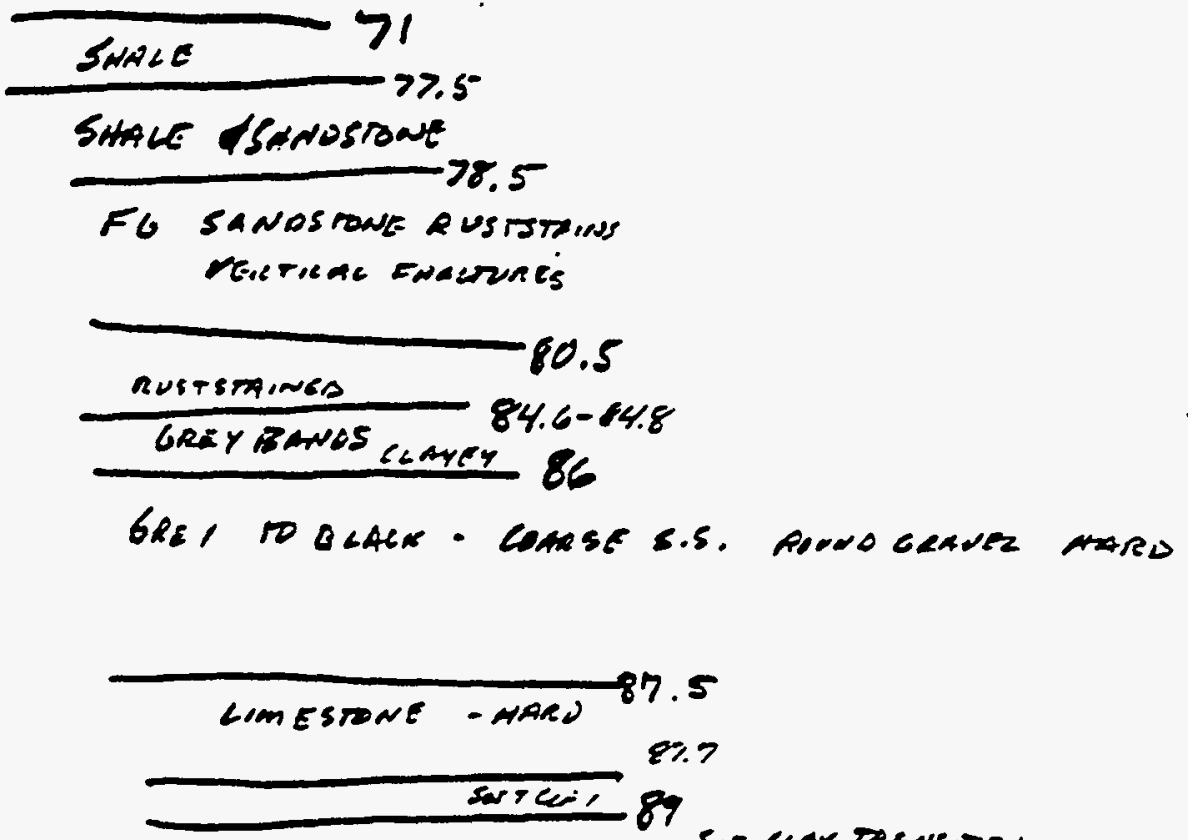

RGIBREWN CLASSTWE

greatifies ccaystonc= 
well cemented pedtesch brown.

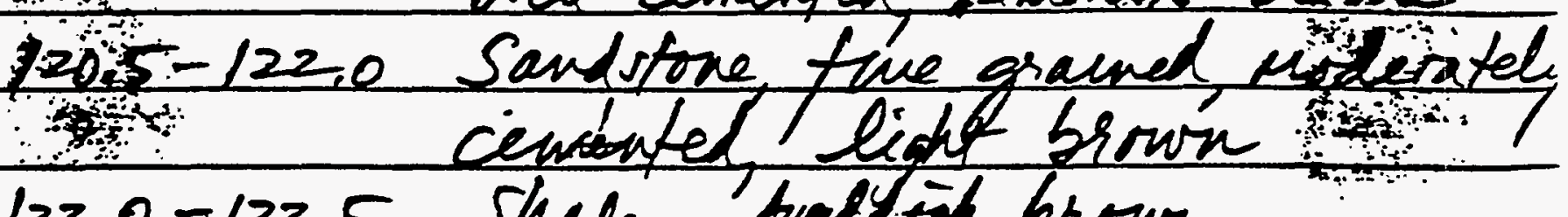

$122,0-122,5$ Shale, treating brown

$122,5-126.0$ Sand'stre, fur graved, well cemented reddest brown 126.0-130.5 sow stone, fuse grained, moderate cemented, reddish brown

To: Bill Mason

This is the description of the MoN 610 core as logged by Dar o the SHB Fine. Please transmit to Cary Milter Asap so de dean decide if the De Chellyanticill needs to be cored above 130 feet.

Thanks, OBetsill $3 / 1 / 85$ 


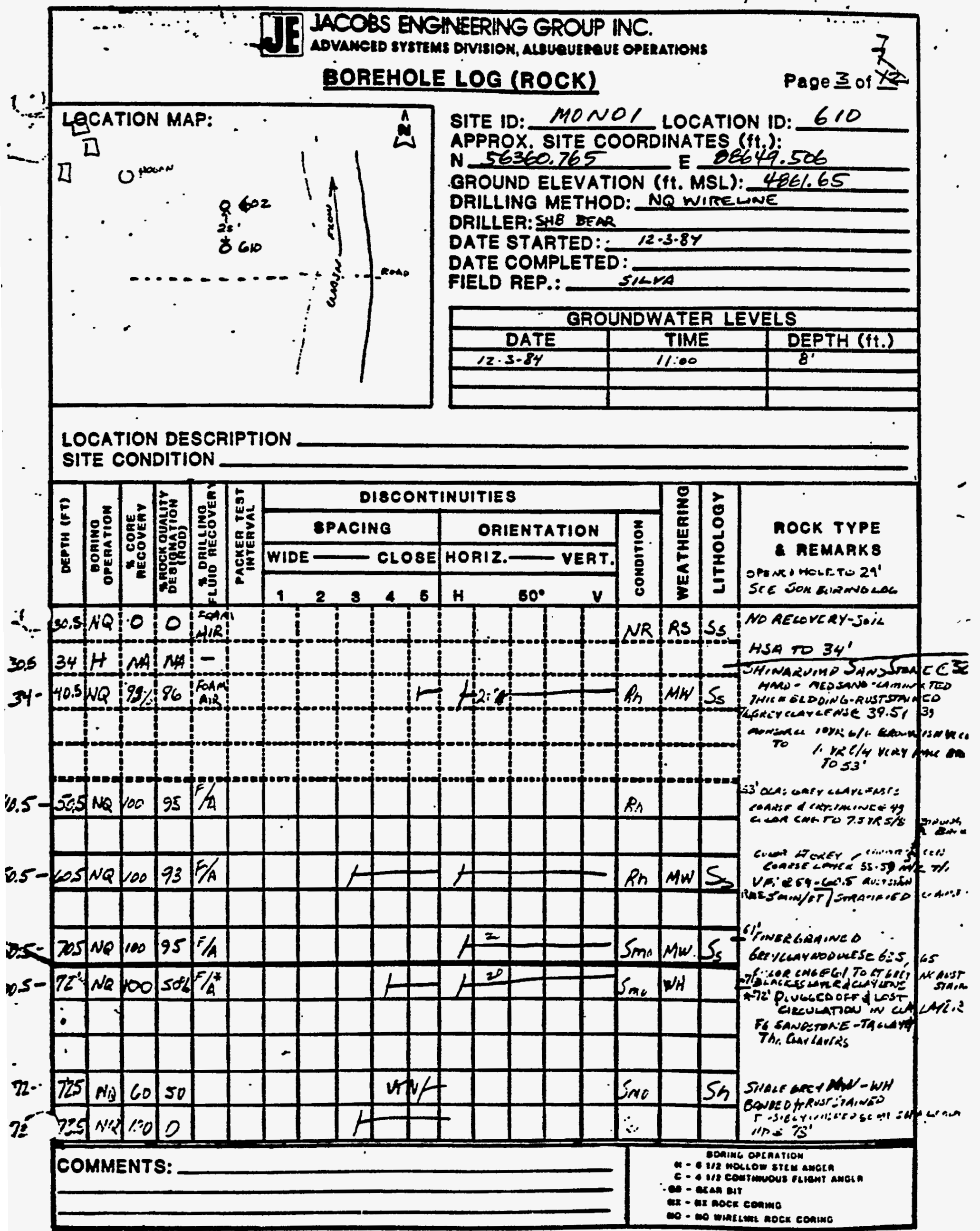



LOCATION MAP:

$\operatorname{SeE} P_{7} 3$

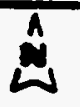

$\omega$

SITE ID: MIONOI

LOCATION ID: 610

APPROX, SITE COORDINATES ( $f t$ ):

$N$ S6360.765 E 38649.506

GROUND ELEVATION (ft. MSL): 436.65

DRILLING METHOD:

DRILLER:

DATE STARTED:

$\operatorname{SEn}-\operatorname{SHB}$

DATE COMPLETED:

FIELD REP.:

\begin{tabular}{|c|c|c|}
\hline \multicolumn{3}{|c|}{ GROUNDWATER LEVELS } \\
\hline DATE & TIME & DEPTH (11.) \\
\hline & & \\
\hline & & \\
\hline & & \\
\hline
\end{tabular}

LOCATION DESCRIPTION

NEXT TOMN-1.2

SITE CONDITION

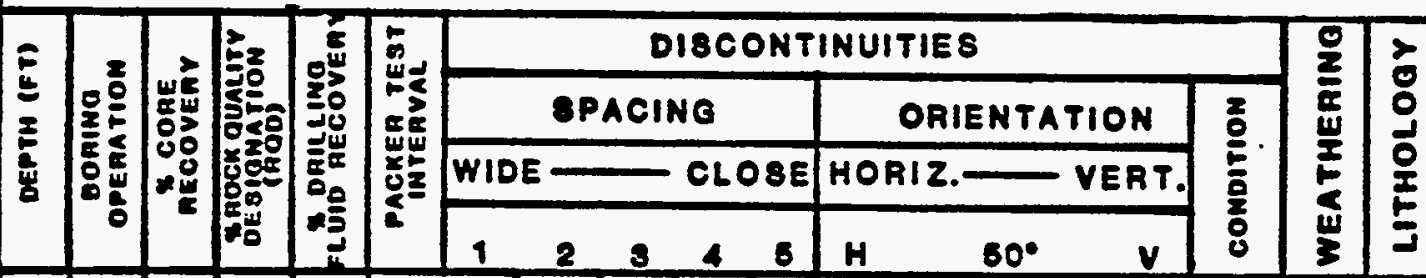

\section{if 87.6 NQ:100: 95 in acuer}

$+i$

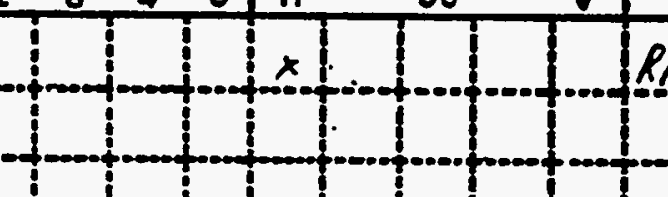

Rh Ws $W_{s}$

SANDSTONE

1

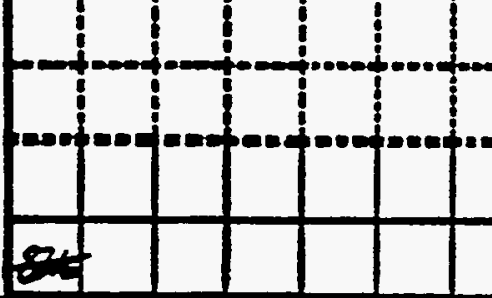

:

\begin{tabular}{ll|l|l|l}
7.4 & Ne & 100 & 95 & Reger
\end{tabular}

ind

;

:

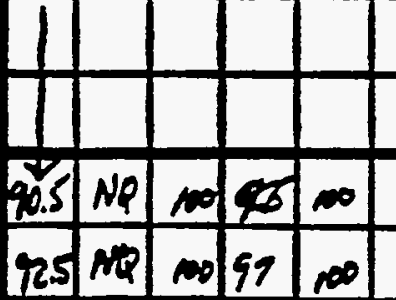

-

(1)

工

1

(1)

THIN HE EAWDS OF W TO OR.

Fi Gor Lavenes

Ruar STAINEDe 815.975

SRey sandos e s46-248

TRACE CLAYEY IN F:SA

TRens man $86-86.7$

To conese Gehine od centstaren

in FiGR Shmo srowe

ins rounded 1/2"Gemench

Looks bike canch Aruf $C$ T

conccomoxare dPar ire

02.6 RED woover, one

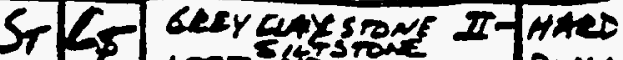

cost \&uts?intion Pube

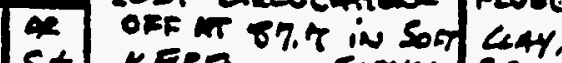

st vefiem samica piece

86.5 sent swaystowe

89.5 LIMESTONE Qu E CAEY

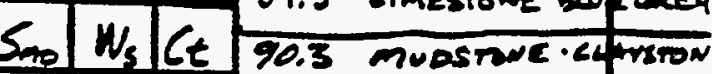
passisue moolkari CRosscedpes Anits

92.5 mexern geown sanos rowe mus rome

74.5 streatifico of Eyrsw SnNostone in muosn

\begin{tabular}{l|l|l|l|l|}
\hline 0,5 & NQ & 100 & 95 & 100 \\
\hline
\end{tabular}

muost ave GComm SAND

Fien $96.5 \cdot 97.5$

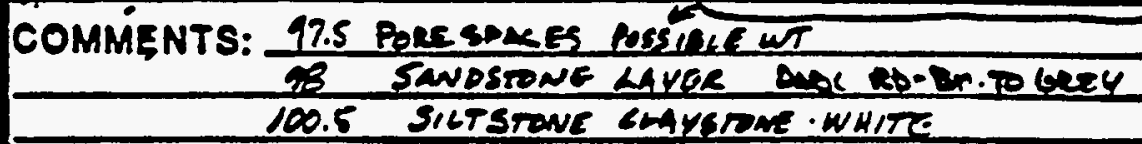

wo - Wo wialliml Mock comine 


\section{DEE ACOBS ENGINERRING GROUP INC.

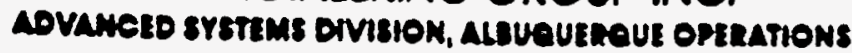

BOREHOLE LOG (ROCK)

LOCATION MAP: ش SITE ID: MONOI LOCATION ID: 610

OHOSAN

LOCATION DESCRIPTION NEXT TA NON GEZ

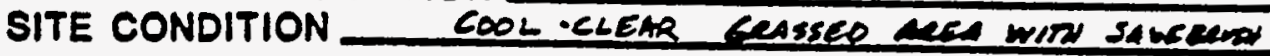

\begin{tabular}{|c|c|c|c|c|c|c|c|c|c|}
\hline \multirow{3}{*}{ 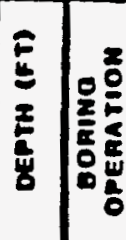 } & \multirow{3}{*}{ 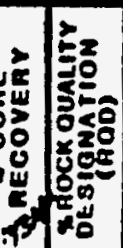 } & \multirow{3}{*}{ 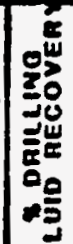 } & \multirow{3}{*}{ 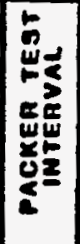 } & \multicolumn{3}{|c|}{ DISCONTINUITIES } & \multirow{3}{*}{\multicolumn{2}{|c|}{ 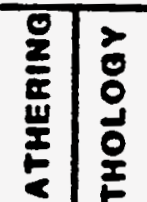 }} & \multirow{3}{*}{$\begin{array}{r}\text { ROCK TYPE } \\
\text { \& REMARK } \\
\text { UE OAMONOS OW }\end{array}$} \\
\hline & & & & BPACINC & ORIENTATION & $\underline{\underline{g}}$ & & & \\
\hline & & & & WIDE - CLOBE & HORIZ. $\longrightarrow$ VERT. & 5 & & & \\
\hline
\end{tabular}

1

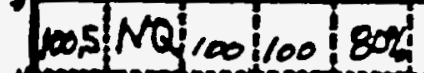
: $x: 2,3,5$

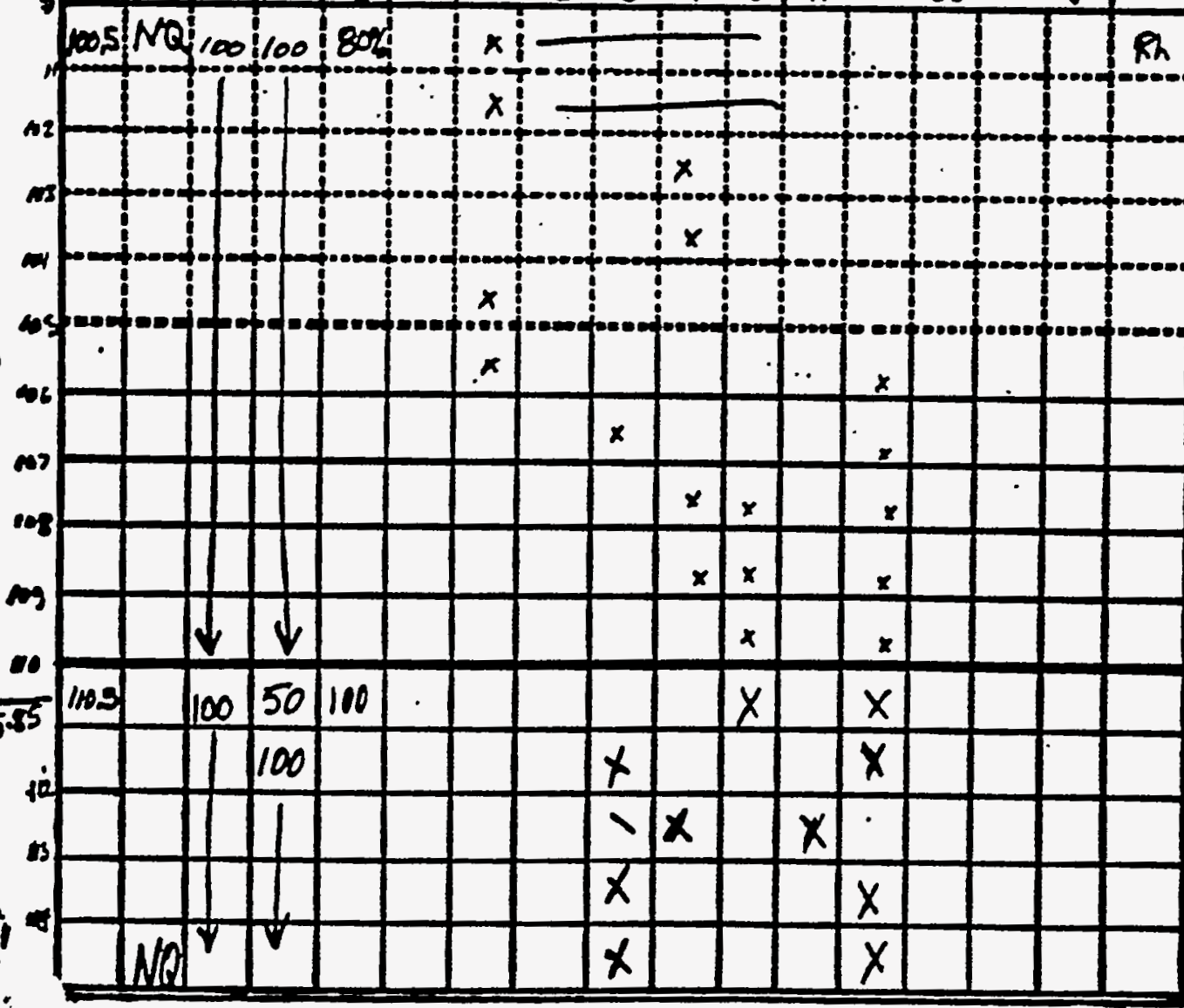

COMMENTS: 
T2- DE AACOBS ENGINEERRG GROUP INC.

BOREHOLE LOG (ROCK)

Page Iof

LOCATION MAP:

see $P 6$
SITE ID: MON OL LOCATION ID: 610 APPBOX. SITE COORDINATES ( $f t$.).

$N$ S6360.765 E 88649.506 GROUND ELEVATION (1T. MSL): 4861.65 DRILLING METHOD: DRILLER: SHB BEAR DATE STARTED: $\mid 2-11-84$ DATE COMPLETED: FIELD REP.: Ji toopes

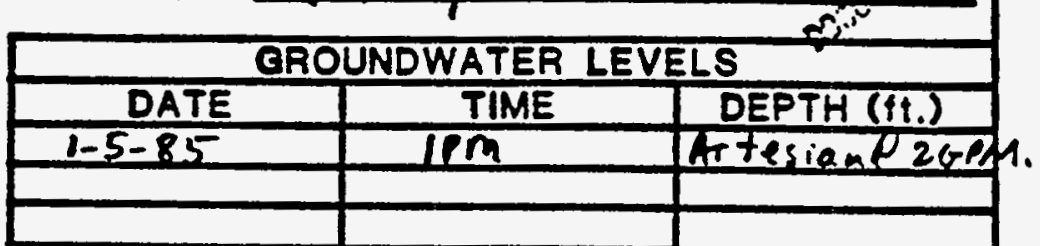

LOCATION DESCRIPTION NEXT to MON 602. SITE CONDITION $2 \%$ slope, gegehrush, savely spil.

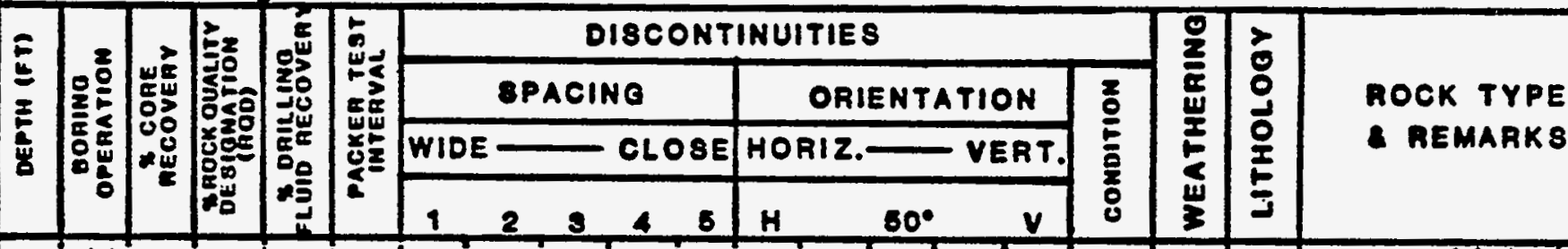

\section{SNQ:100:100!100!}

177 $+0+\cdots+1$

\section{0}

12

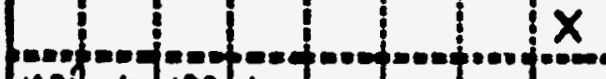

$x$

$1209 \mathrm{NQ} 100 \mathrm{O} 100$

(12)

$-10$

1

$x$

$x$

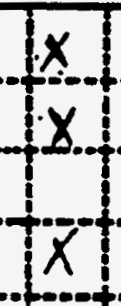

(1).....

WMI Ssi Pale reddish hrow

and light grey

bauded siltstorfe and sandstomef St believed to be Rico Formetion.

$$
\text { r }
$$

at $x$ $x$

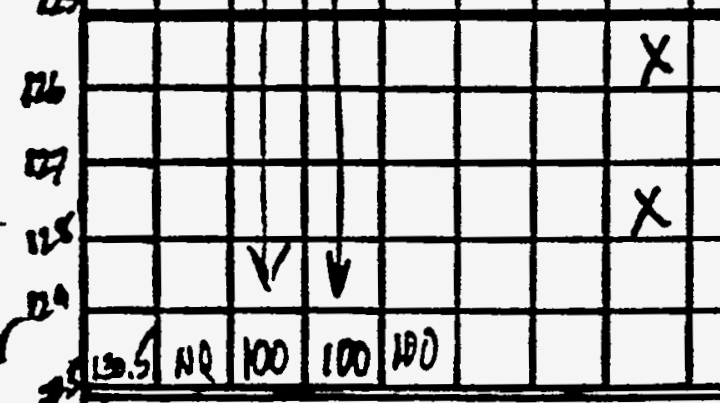
$x$ - $x$ commenss: $\frac{A l}{\text { caused but twe fractires ir core }}$ 


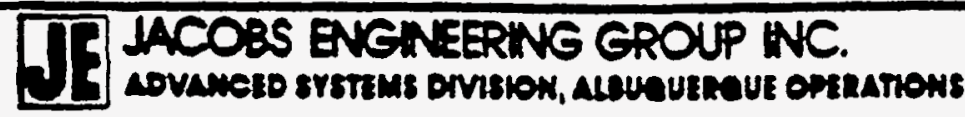

\section{POREHOLE LOG (ROCK)}

LOCATION MAP:

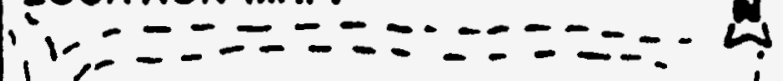

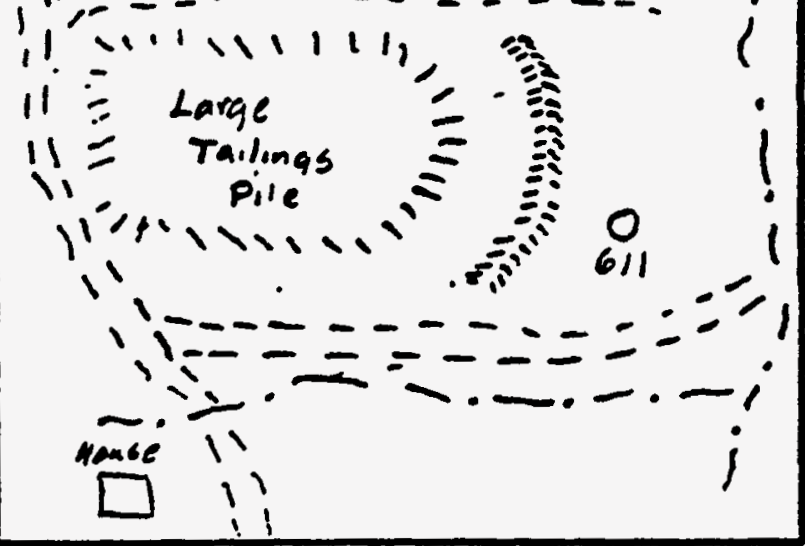

SITE ID: MON-OI LOCATION ID: $6 \%^{\circ}$ APPROX. SITE COORDINATES (ft.):

CROUND ELEVATION (ft. MSL):

DRILLING METHOD: Rotary to $65 \mathrm{ft}$ cove te ID DRILLER:

DATE STARTED:

DATE COMPLETED:

FIELD REP.:

R. Hollond

\begin{tabular}{|c|c|c|}
\hline \multicolumn{3}{|c|}{ GROUNDWATER LEVELS } \\
\hline DATE & TIME & DEPTH (ft.) \\
\hline $3 / 5 / 85^{-}$ & $1.30 \mathrm{em}$ & 6 \\
\hline & & \\
\hline
\end{tabular}

LOCATION DESCRIPTION East of Leirge Toulines pile. Near Dike. SITE CONDITION bondy weto four weng bait brush

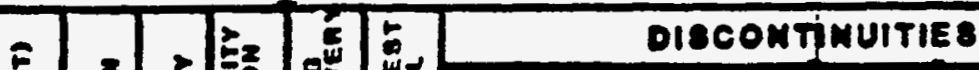

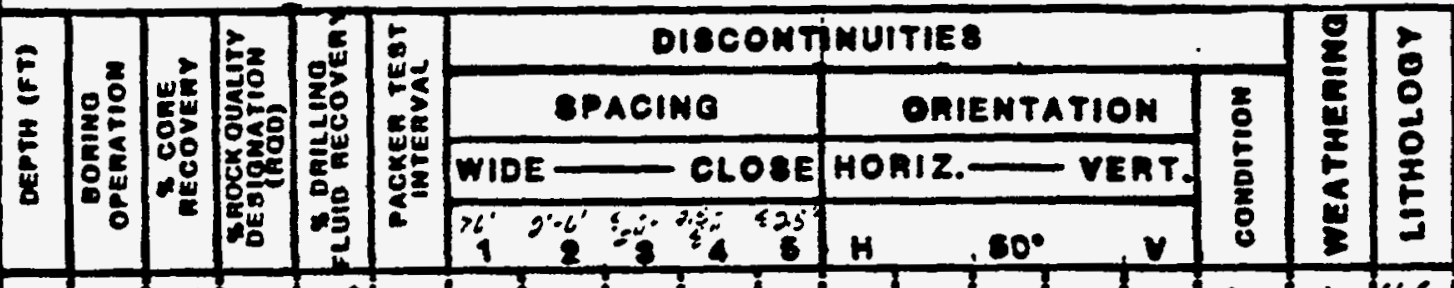

1

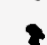

A

\section{T.NQ $100: 01509$}

61

68

69

90 mon 10.

4$$
79
$$

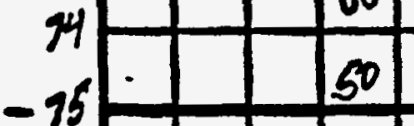

106

$\phi$

$10^{6}$

60$$
\text { - }
$$

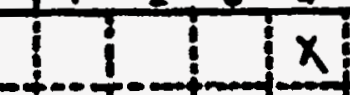

$+$

$x$

$x$

$x$

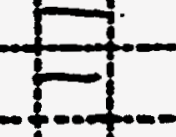

$x$

$x$

$=-20$

IR W stói Note: Hole prevosenty

ROCK TYPE

2 Remarks

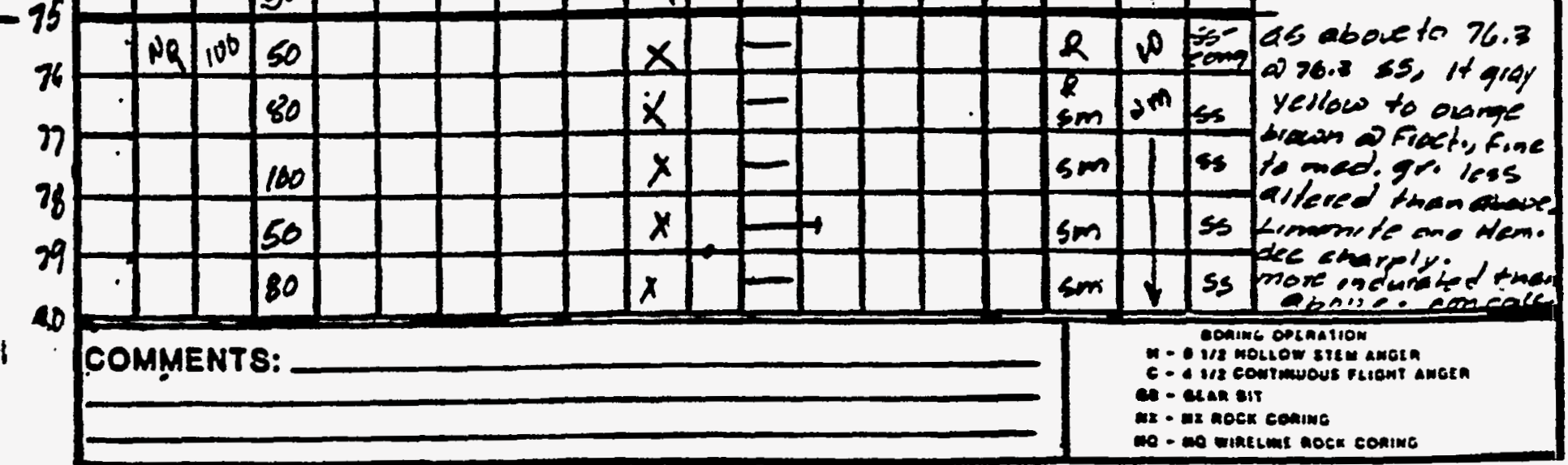




\section{1] LACOBS ENGENEERNG GROUP NC.}

BOREHOLE LOG (ROCK)

LOCATION MAP:

$\stackrel{\omega}{\omega}$

8ITE ID: MON - OI LOCATION ID: $6 / 1$. APPROX. SITE COORDINATES ( $f 1$.$) :$

$N$ E

GROUND ELEVATION (ft. MSL):

DRILLING METHOD:

DRILLER:

DATE STARTED:

DATE COMPLETED:

FIELD REP.:

$3 / 6 / 85$

Piflolland

\begin{tabular}{|c|c|c|}
\hline \multicolumn{3}{|c|}{ GROUNDWATER LEVELS } \\
\hline DATE & TIME & DEPTH (fi.) \\
\hline & & \\
\hline & & \\
\hline & & \\
\hline
\end{tabular}

LOCATION DESCRIPTION

SITE CONDITION

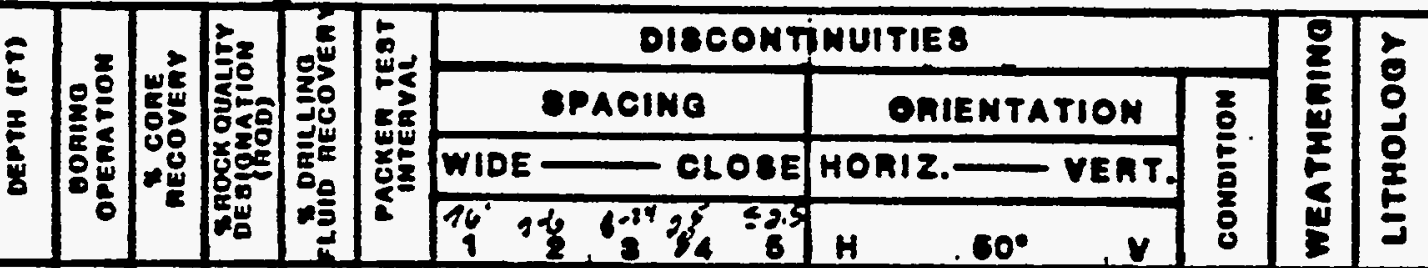

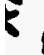

8

$-85$

4

i)

. N9:100: $05: 09 \%$

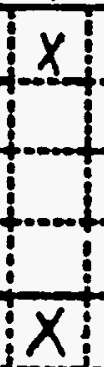

195:

$100 !$

88

\begin{tabular}{ll|l|l|}
\hline & 1 & 60 \\
\hline & & 40 \\
\hline
\end{tabular}

100

97

$1 \quad 10^{10}$

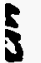

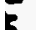

91

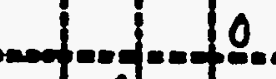

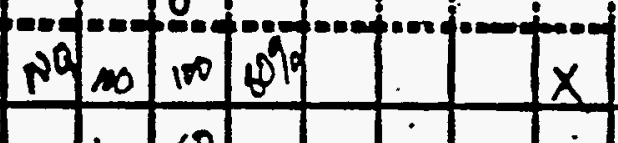

50
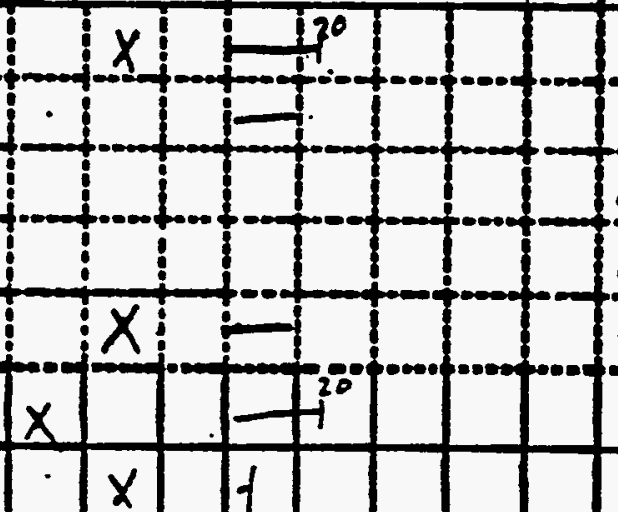

sm $1.0 \mathrm{~g}$.s

ROCK TYPE

- REMARKs

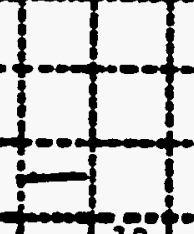

sm

100

$x$

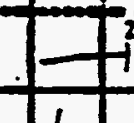

$1^{20}$

$x$

$\mathrm{gm}|\omega \mathrm{m}|$

sm vom

gm vin

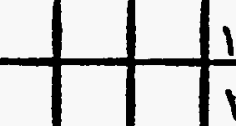

(16)

$x$

\begin{tabular}{l|l|l|l}
$\mathrm{bm}$ & $2 \mathrm{~m}$ & $\mathrm{bS}$ \\
\hline
\end{tabular}

Sandstone as

aboue. grain size

beecming insó. well uniferm a

fine to med. serieute micer mied aleng Fracture plaises. Limomite and hemifite stomian ine. a 91, 4र Gore is hard orientatian of Fraction is horie. to $20^{\circ}$ some carbon aceous material 100

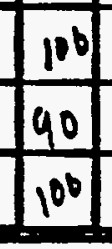

COMMENTS: conimc onthatiom

n - ils motion stl amoth

C. a is cemrmuous fliont amare

es - eran air

wx - ws meck cenike

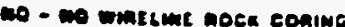


SITE ID: MON-OI LOCATION ID: $6 / 1$ APPROX. SITE COORDINATES (ft.):

GROUND ELEVATION (fi. MSL):

DRILLING METHOD:

DRILLER:

DATE STARTED:

DATE COMPLETED:

FIELD REP.: R.thellend

\begin{tabular}{|c|c|c|}
\hline \multicolumn{3}{|c|}{ GROUNDWATER LEVELS } \\
\hline DATE & TIME & DEPTH (\$.) \\
\hline & & \\
\hline & & \\
\hline & & \\
\hline
\end{tabular}

LOCATION DESCRIPTION SITE CONDITION

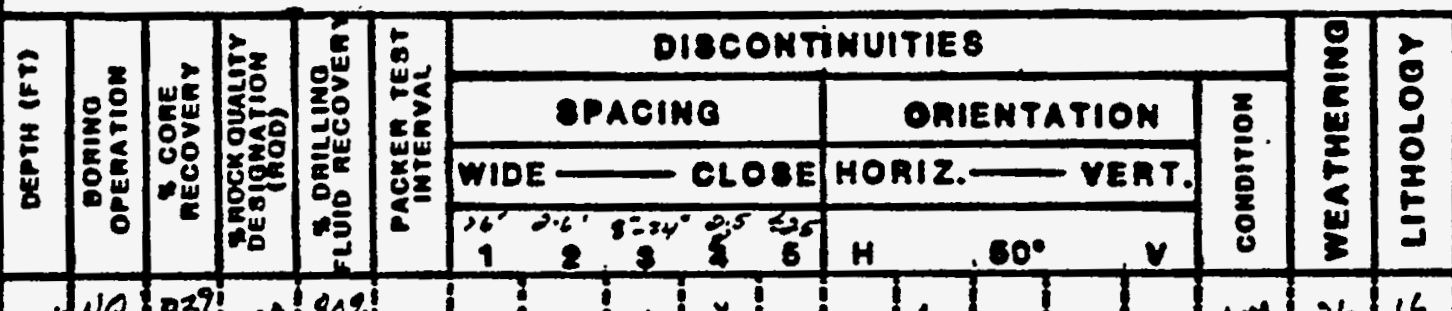
NQ $83 \% 100180 \%$

a $100.0 \mathrm{Ss}, 17$ greenush gray, very esarse are, quartoss

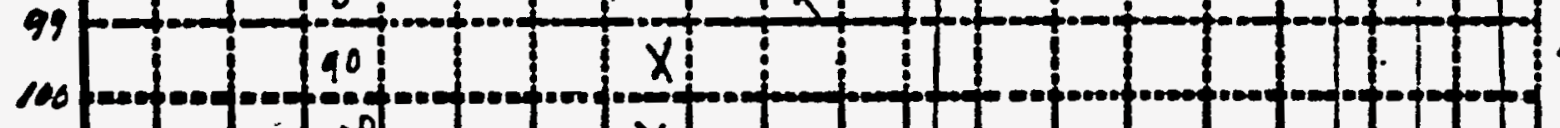
coorly consolidoted with pyrifle and coa/. Core is soft

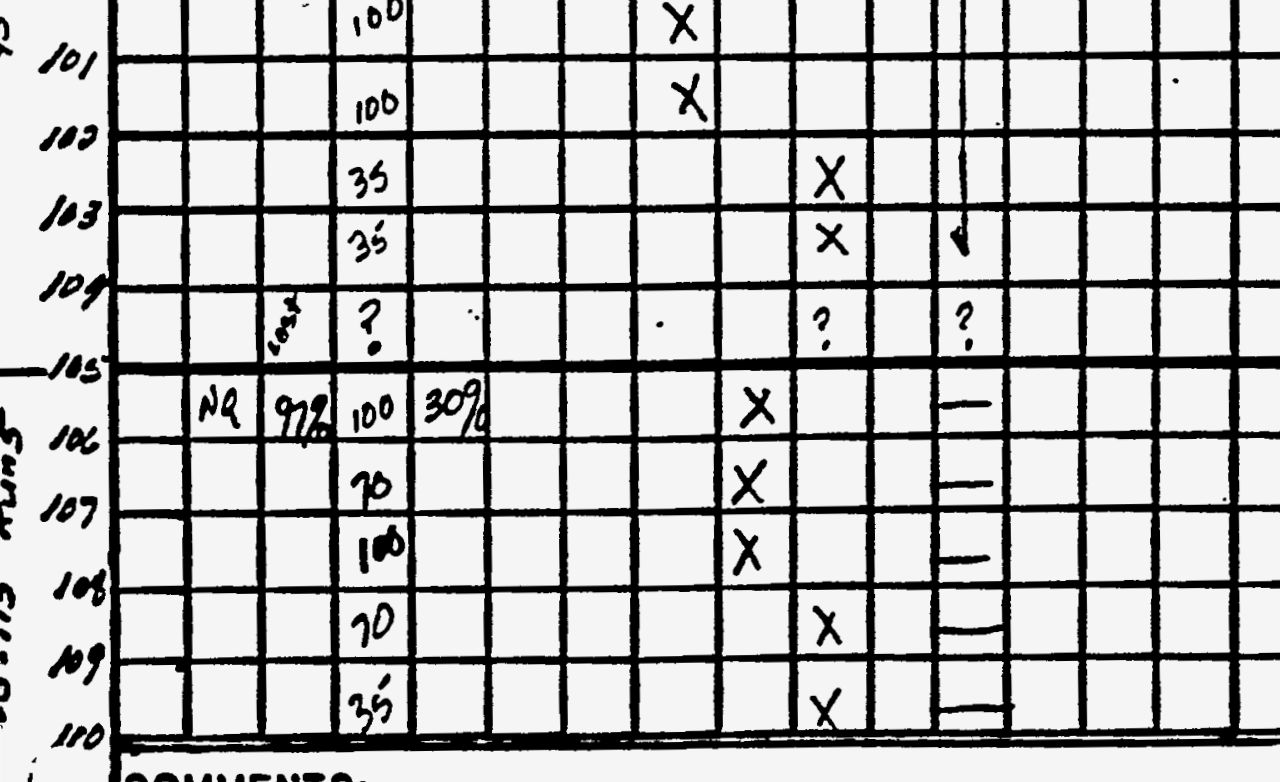

COMMENTS:

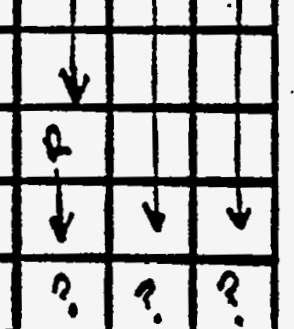

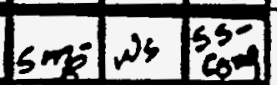
\begin{tabular}{|l|l|l|}
\hline 1 & we & 45 \\
\hline
\end{tabular} $\downarrow$ wes 5 ss R Wh $\mathrm{cons}$ Q
Fraet, Horiz to $20^{\circ}$

from 103.210 105.0 Lest

So as $A b$ with senc congromente it to to gresnisngray, pyife fomma son ealc. $\rightarrow$ 108 cong. peobly, woal fortion of Shin.s peobles are moetly cmert sub to well pounced.

- 07.6 Moentupi 
LOCATION MAP:

$\omega$

\section{LOCATION DESCRIPTION}

SITE CONDITION
SITE ID: MON-OI LOCATION ID: G/1APPROX. SITE COORDINATES (ft.):

$N$ E

GROUND ELEVATION (ft. MSL):

DRILLING METHOD:

ORILLER:

DATE STARTED:

DATE COMPLETED:

FIELD REP.:

\begin{tabular}{|c|c|c|}
\hline \multicolumn{3}{|c|}{ GROUNDWATER LEVELS } \\
\hline DATE & TIME & DEPTH (fi.) \\
\hline & & \\
\hline & & \\
\hline & & \\
\hline
\end{tabular}

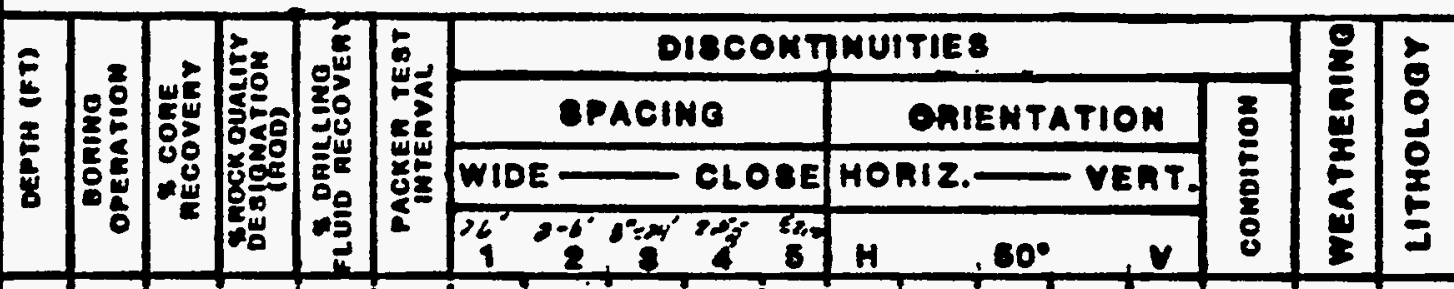

very clayey silf st, yanteodent 60

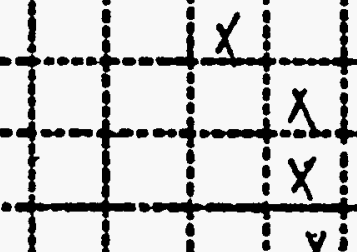

core mod. breken to 131.0

sofit to hard wote: 0 ise'penetration. rate: $8.5 \mathrm{~mm} / \mathrm{Ft}$ O131.0 sillohang red browe tery ergollacens, once unbrokien piece to $134.8 .4134 \leq 5$

\begin{tabular}{ll|l|l|l|l|l|}
\hline 35 & & & & & & \\
\hline
\end{tabular}
mottled red brown pter. ime pr. very aurd $\omega 135$ to 145 candstane, pinkints brown to green ast

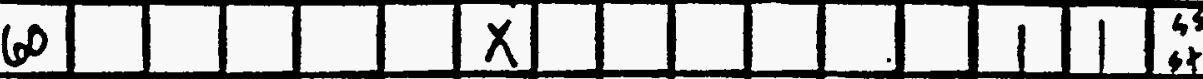

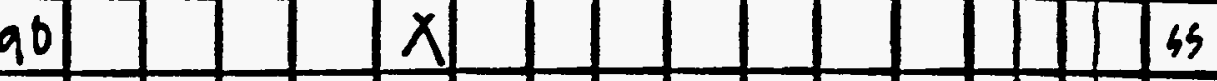
quy, f to med gri, andilaceous, cek to very cale. 


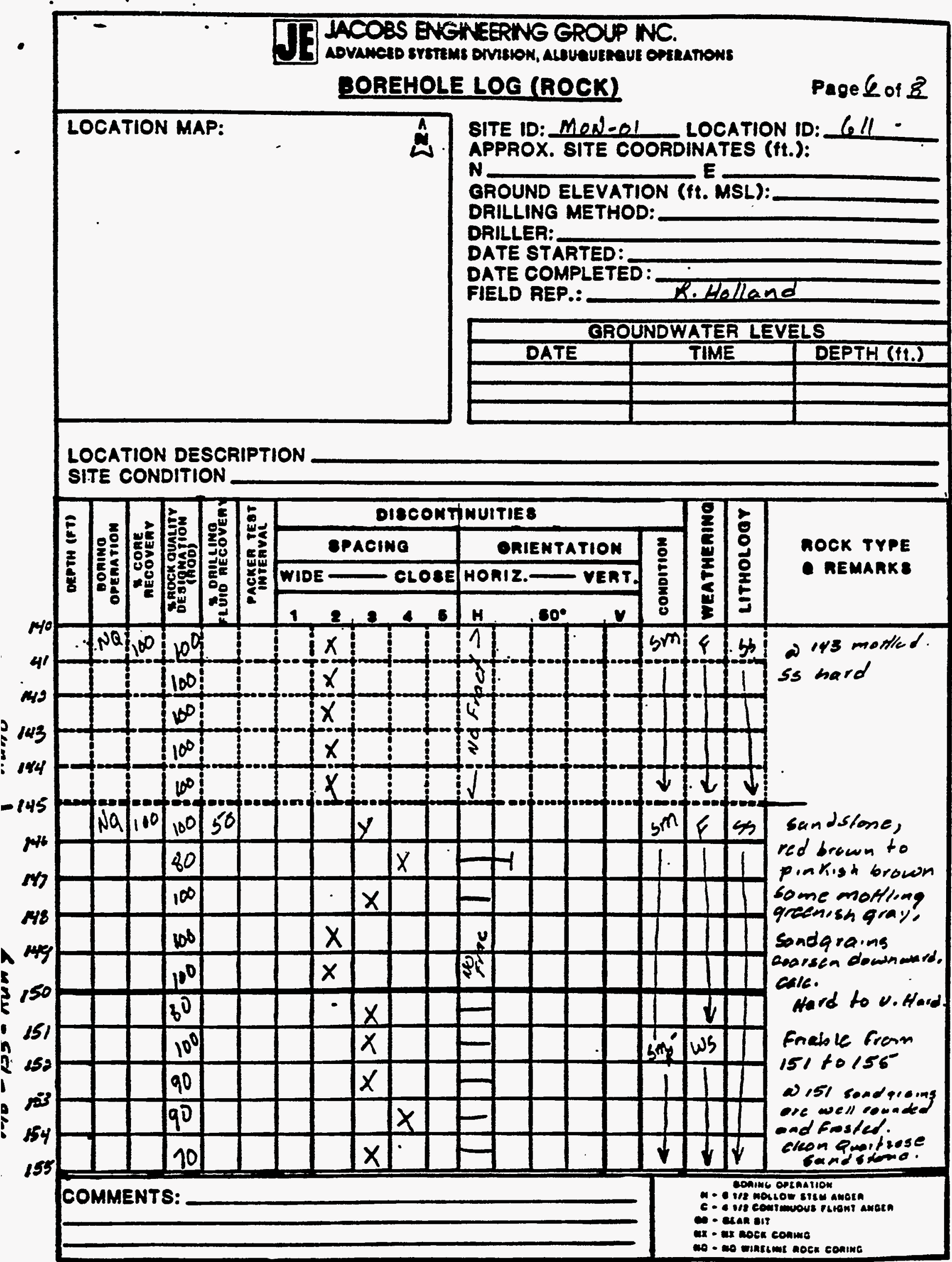


LOCATION MAP:

$\stackrel{a}{\omega}$

8ITE ID: MOAL - OI APPROX. BITE COORDINATES (ft.):

N

GROUND ELEVATION (fI. MSL):

DRILLING METHOD:

DRILLER:

DATE STARTED:

DATE COMPLETED:

FIELD REP.:

\begin{tabular}{|c|c|c|}
\hline \multicolumn{3}{|c|}{ GROUNDWATER LEVELS } \\
\hline DATE & TIME & DEPTH (ft.) \\
\hline & & \\
\hline & & \\
\hline & & \\
\hline
\end{tabular}

LOCATION DESCRIPTION

SITE CONDITION

\section{DISCONTINUITIES}

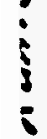

3

2

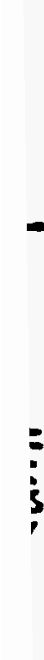
- .NQ? $100,100: 50$

(n)

159 -

vi:

Les

ces

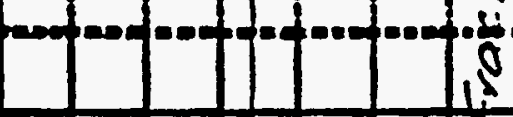

: $: 1$

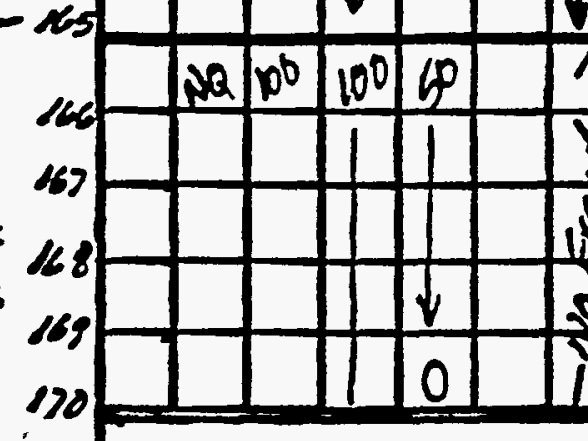

2

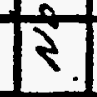

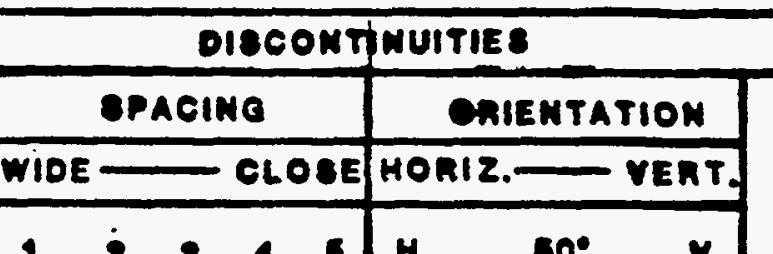

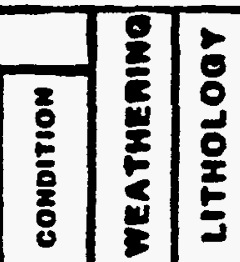

MOCK TYPE

- nemARK 8

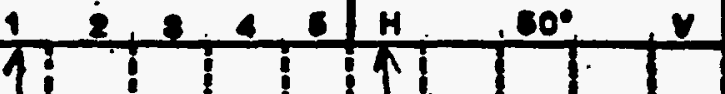
$5 m<5$

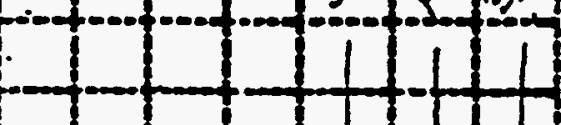

moenkojiglochelly contert b/56-2 0 sib as, reddianh brown to if ceddist biawn, fine grained, well eaunded frosted axestza grains, frisble on braxen surfeer.

well developed erows bedolim. riesa beds are 200 frem horiz. Croses lominac demoted by mongl? COMMENTS:

Gs ac ab: Cross bedding denoted by slight grein biele cheage 
LOCATION MAP:

SITE ID: MON-OI LOCATION ID: 611 APPROX. SITE COORDINATES (ft.):

$N$ E

GROUND ELEVATION (ft. MSL):

DRILLING METHOD:

DRILLER:

DATE STARTED:

DATE COMPLETED:

FIELD REP.: R. Holland.

\begin{tabular}{|c|c|c|}
\hline \multicolumn{3}{|c|}{ GROUNDWATER LEVELS } \\
\hline DATE & TIME & DEPTH (ft.) \\
\hline & & \\
\hline & & \\
\hline & & \\
\hline
\end{tabular}

LOCATION DESCRIPTION SITE CONDITION

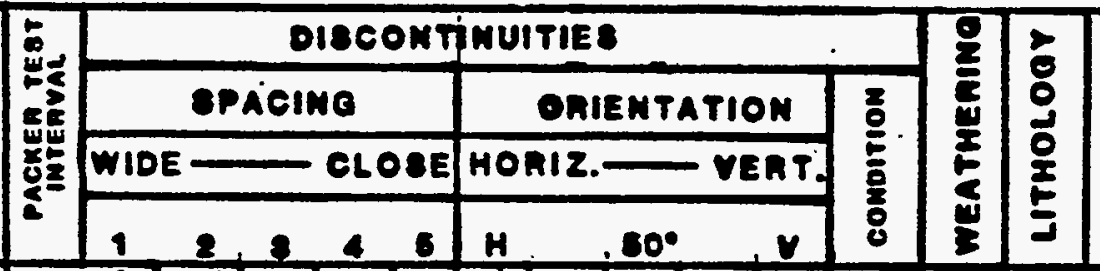

ROCK TYPE $+10010$ 11

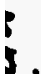
$|6|$

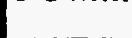




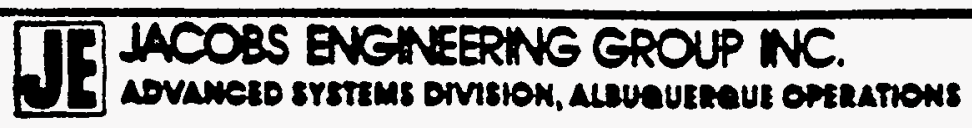

BOREHOLE LOG (ROCK)

LOCATION MAP:

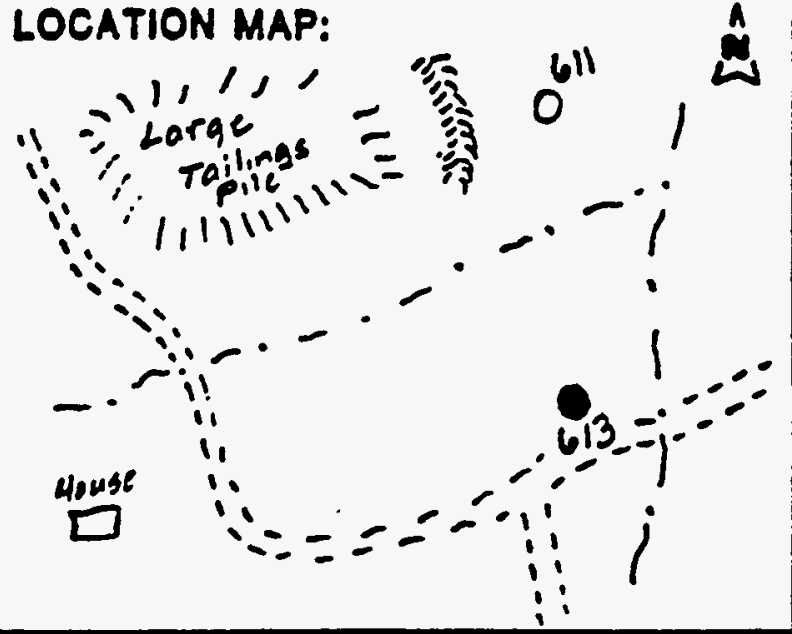

SITE ID: MBA - OI LOCATION ID: G13APPROX. SITE COORDINATES (ft.):

N GROUND ELEVATION (ft. MSL):

DRILLING METHOD: Rlug to $77.7^{\prime}$ then lere to 70 DRILLER: SHi 2 (1. Carter)

DATE STARTED: $3 / 3 / 85$

DATE COMPLETED: Raser sef 3/5/25

FIELD REP.: Loger Holland

\begin{tabular}{|c|c|c|}
\hline \multicolumn{3}{|c|}{ GROUNDWATER LEVELS } \\
\hline DATE & TIME & DEPTH (fI.) \\
\hline & & \\
\hline & & \\
\hline & & \\
\hline
\end{tabular}

LOCATION DESCRIPTION South Fest of baree Todues pile

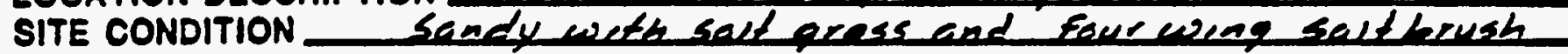

\begin{tabular}{|c|c|c|c|c|c|c|c|c|}
\hline \multirow{3}{*}{ 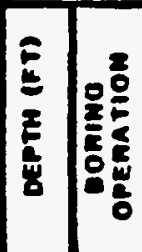 } & \multirow{3}{*}{ 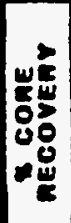 } & \multirow{3}{*}{ 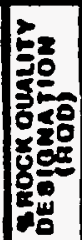 } & \multirow{3}{*}{ 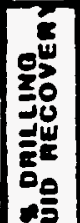 } & \multirow{3}{*}{ 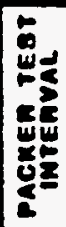 } & \multicolumn{2}{|c|}{ DISCONTINUITIES } & \multirow{3}{*}{$\begin{array}{l}\frac{\Sigma}{0} \\
\frac{0}{0} \\
\frac{0}{8} \\
8\end{array}$} & \multirow{3}{*}{ 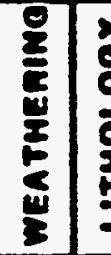 } \\
\hline & & & & & epacino & ORIENTATION & & \\
\hline & & & & & WIDE $\longrightarrow$ CLOSE & HOR12.- & & \\
\hline
\end{tabular}

$+1$

:

(1)

+...

$x$

\begin{tabular}{|c|c|c|c|}
\hline $\operatorname{sen}$ & 5 & 54 \\
\hline $5 n$ & 5 & 45 &
\end{tabular}

$x !$

ic:

$x$

sm if th

MOCK TYPE

a REMARK 8 en 5/3/85 ntary drolued form 34 to 77.5 cogan corom $077.7^{\circ}$ S5, very If. gray to yellow brown, $f$. to medigri, non calc., $x$ medded and inminiac bedeling nemitite and Lumonite common

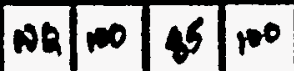

$x$

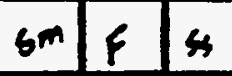
\begin{tabular}{|l|l|l|}
\hline $6 m$ & $f$ & $\&$ \\
\hline $\mathrm{sm}$ & $f$ & 4 \\
\hline
\end{tabular}

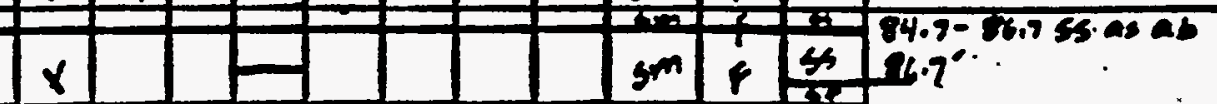
COMMENTS:

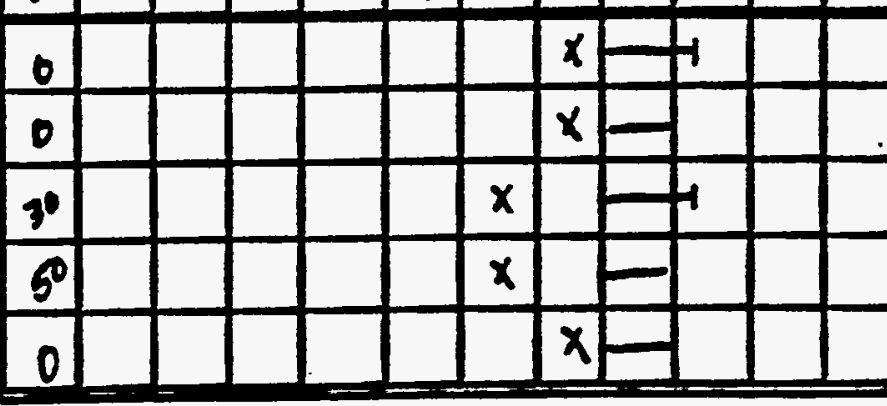

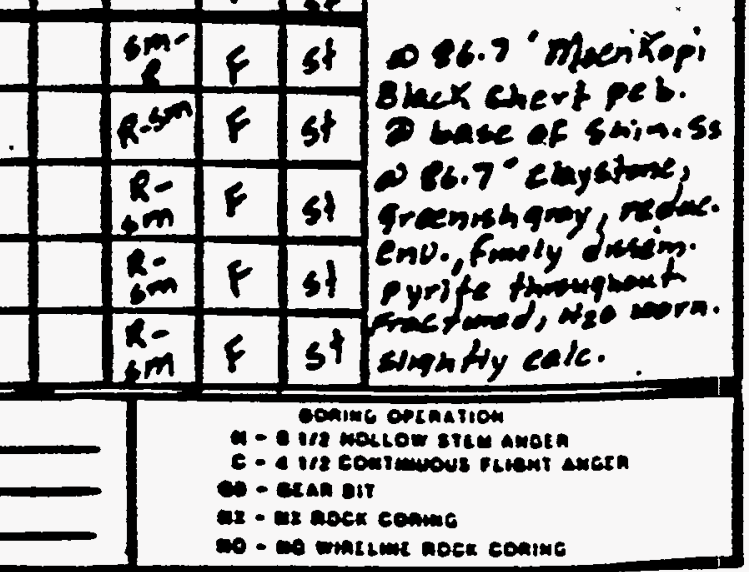




\section{J] IACOBS ENGINEERING GROUP INC.}

BOREHOLE LOG (ROCK)

LOCATION MAP:

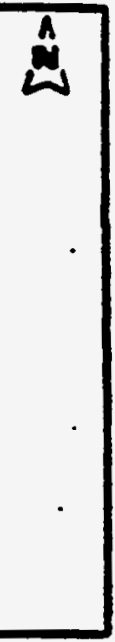

SITE ID: MON-OI

LOCATION ID: 613 .

APPROX. SITE COORDINATES (ft.):

$N$ E

GROUND ELEVATION (ft. MSL):

DRILLING METHOD:

DRILLER:

DATE STARTED:

DATE COMPLETED:

FIELD REP.:

R. Holland

\begin{tabular}{|c|c|c|}
\hline \multicolumn{3}{|c|}{ GROUNDWATER LEVELS } \\
\hline DATE & TIME & DEPTH (ft.) \\
\hline & & \\
\hline & & \\
\hline & & \\
\hline
\end{tabular}

LOCATION DESCRIPTION

SITE CONDITION

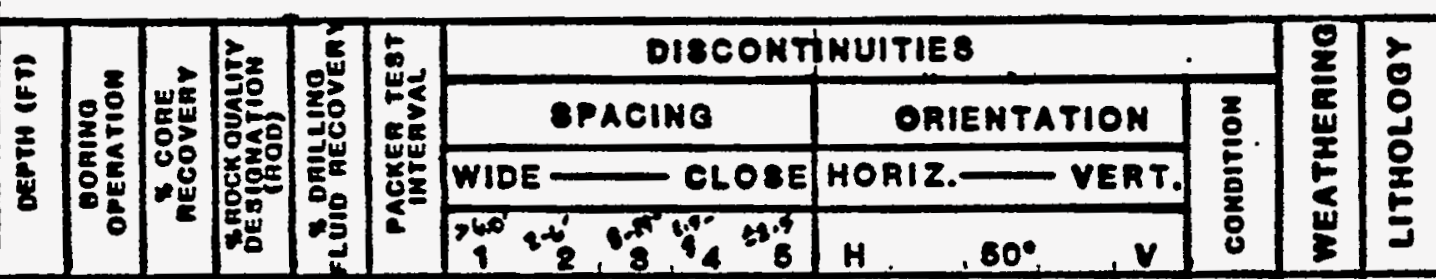

ROCK TYPE

Q REMARK
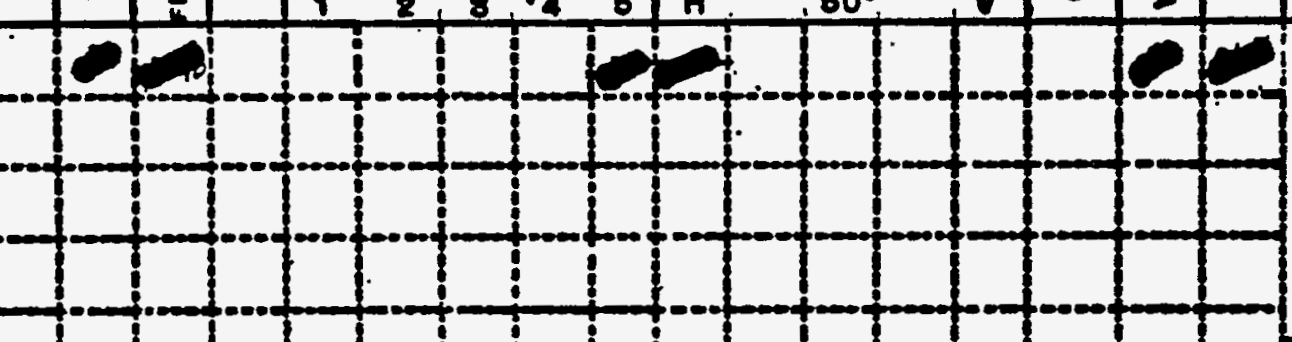

a regt: Claystone beenoming red brom aighly fract. slightily

to nom calc.

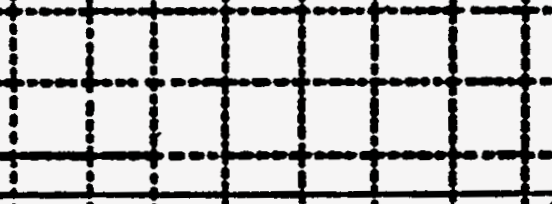
\begin{tabular}{l|l|l|}
\hline & 0 & \\
\hline
\end{tabular} $+\frac{1}{2}$

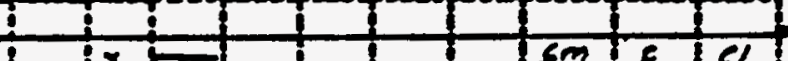

95

2

97

$\rightarrow$

9

tino

101

400

as

COMMENTS:

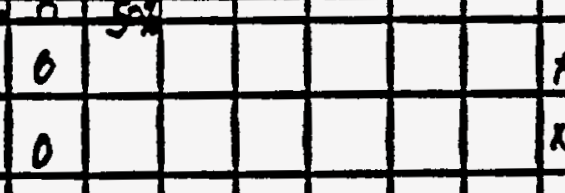

50

47

0

10 $x \times$

$x:$

$x \times$

\begin{tabular}{|l|l|l|}
\hline & $x$ & \\
$x$ & $x$ & \\
& $x$ & \\
& $x$ & \\
$x$ & 1 & \\
$x$ & $x$ & \\
$x$ & $x$ & \\
& $x$ & \\
& $x$ & \\
\hline & $x$ & \\
\hline
\end{tabular}

$=$

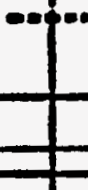

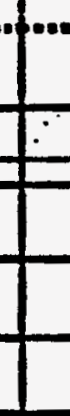

$\cos |f| c l$

sin $f$ CI

$\sin z=\mid \operatorname{sen}$

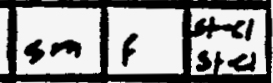

$\operatorname{sm} 6$ ptal

son ff sti

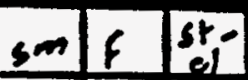

\begin{tabular}{l|l|l|l|}
\hline $\sin$ & $f$ & sit \\
\hline
\end{tabular}

sm- 6 st:

sig. 6 st-

\begin{tabular}{l|l|l|}
\hline ene & $f$ & sto \\
\hline
\end{tabular}
\$92.7. Claystene, daik crowa, micarecus, and calc. mapaly froce. weth momer broce pre cals. If reage meceweopes st. assio sutatim and cinyshome patertalld 4 remy to dr bram, enke, hapoly arnet. - pert exprancs srowen, corpin miacer inter bejts of silts tons and WPCD anclusions. o g guly Freatured. 0 108-a clarstome as al. whe: mo-10) (s.0 man/fl!) wed to carrse greant. candetorie. 


\section{J] LOCOBS ENGINEERING GROUP INC.}

BOREHOLE LOG (ROCK)

Page 3 of 5

LOCATION MAP:

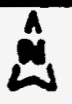

SITE ID: MON-OI

LOCATION ID: $6 / 3$.

APPROX. SITE COORDINATES (ft.):

$N$ E

GROUND ELEVATION (ft. MSL):

DRILLING METHOD:

DRILLER: SHAB (l.carter)

DATE STARTED: 3/2/85

DATE COMPLETED:

FIELD REP.:

P. Helland

\begin{tabular}{|c|c|c|}
\hline \multicolumn{2}{|c|}{ GROUNDWATER LEVELS } \\
\hline DATE & TIME & DEPTH (II.) \\
\hline & & \\
\hline & & \\
\hline & & \\
\hline
\end{tabular}

LOCATION DESCRIPTION SITE CONDITION

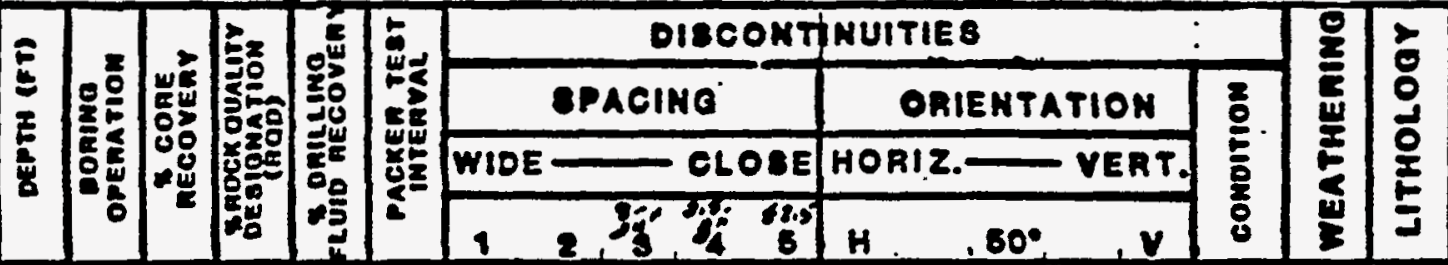

NOCK TYPE

- REMARK

m. Na lic 0 5\%

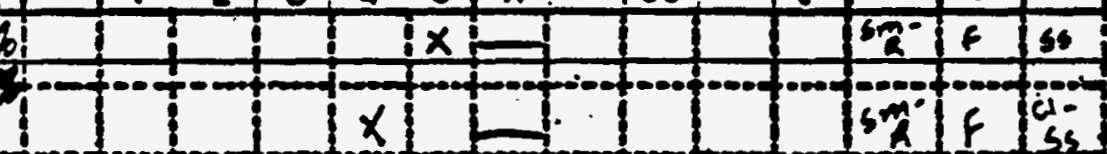

\&

27

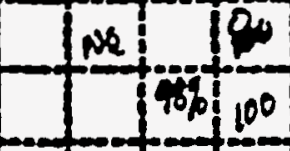

$+\frac{x}{x+\infty}$

80

195

$\sin$

$+\infty$

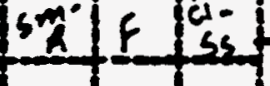

chaystorec andsu, dark brown al,

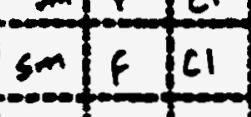

Atray so., ss is

G, eake. Chay frech.

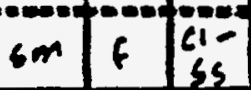

1es to 109 . Whes

ererstone - ss gino monst. Afentions sone

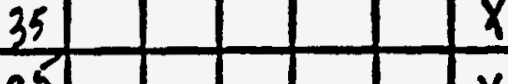

$\left.\begin{array}{ll|l}\hline \sin & f & 56 \\ C_{1}\end{array}\right]$

(rege append mecorked.

mote: $110-111(5.5 \mathrm{~mm} / \mathrm{a})$

from ico or to 112.5 core minlly

fractured.

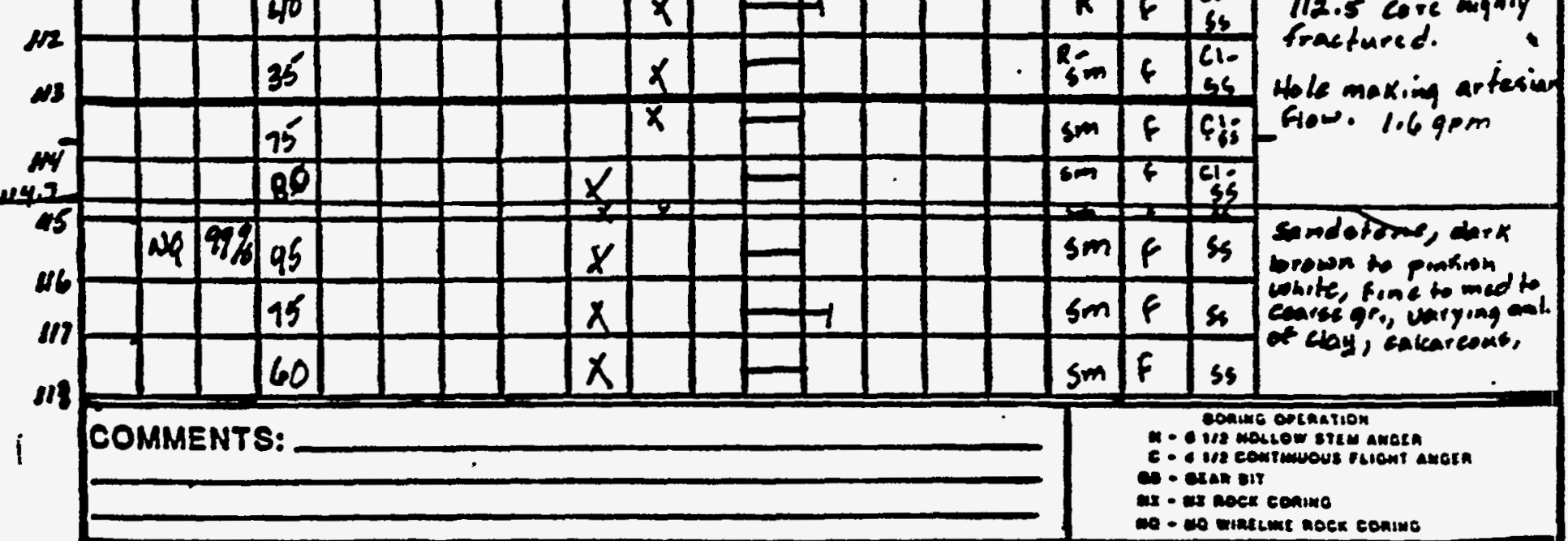

JEQ-AL-ENQ-2A (3/O4) 
BOREHOLE LOG (ROCK)

LOCATION MAP:

SITE ID: MON - DI

LOCATION ID: $6 / 3$

APPROX. SITE COORDINATES ( $f$ t.):

$N$

GROUND ELEVATION ( $f t, M S L)$ :

DRILLING METHOD:

DRILLER:

DATE STARTED:

DATE COMPLETED:

FIELD REP.: R.Hellane

\begin{tabular}{|c|c|c|}
\hline \multicolumn{3}{|c|}{ GROUNDWATER LEVELS } \\
\hline DATE & TIME & DEPTH (f1.) \\
\hline & & \\
\hline & & \\
\hline & & \\
\hline
\end{tabular}

LOCATION DESCRIPTION

SITE CONDITION

DISCONTINUITIE

$\therefore$. Wh 35

a

180

$80:-1: x$

SPACINC \begin{tabular}{|l|l}
\hline WIDE CLOSE HORIZ. & - YERT.
\end{tabular}

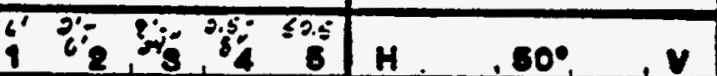

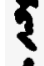

$\infty$

D3

pq

is

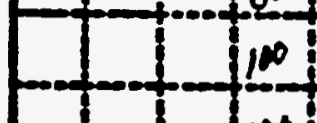

100

$=0=0$

$\{=0,100$

9

100

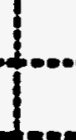

ix

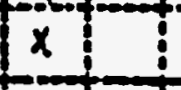

$x$

$x$

8

-oot-ont-

บ 8 :

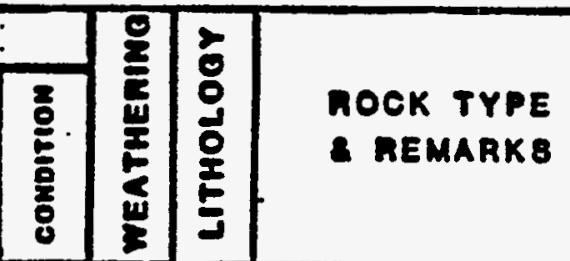

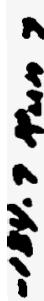

$\infty$

$=1005+100$

100

$f=\log \{x$

$x$

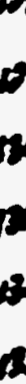

3

$1 \frac{100}{10}$

10

80

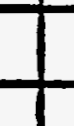

$+x^{-} \quad \frac{x}{x} y$

$x$

$x$

!

$\infty \infty 10 x$

$x$

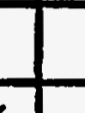

E

I

as

in

$x$

$x$

100

100

\begin{tabular}{l|l|l|l|}
\hline & & \\
\hline & & & \\
\hline & & & \\
\hline
\end{tabular}

COMMENTS: $\operatorname{sm} 6$ ss

$1 \mathrm{sm} F \mathrm{ss}$

sm $f$ S6 Dise so becoming

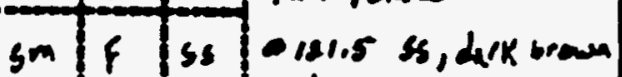

mont with vary.uy ant.

$\mathrm{sm}$. \& of of clay.

$\sin f \mid s e$

\begin{tabular}{|l|l|l|}
\hline $6 m$ & $F$ & 56 \\
\hline
\end{tabular}

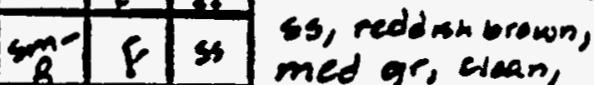

sm: $f$ is

rounded gro, fruble

ealearceus.
D.80 So, yellowing brown, fiene to of gro cress added. x lamance

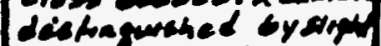
cuange en smin sure. and feor cementation. $x$ 


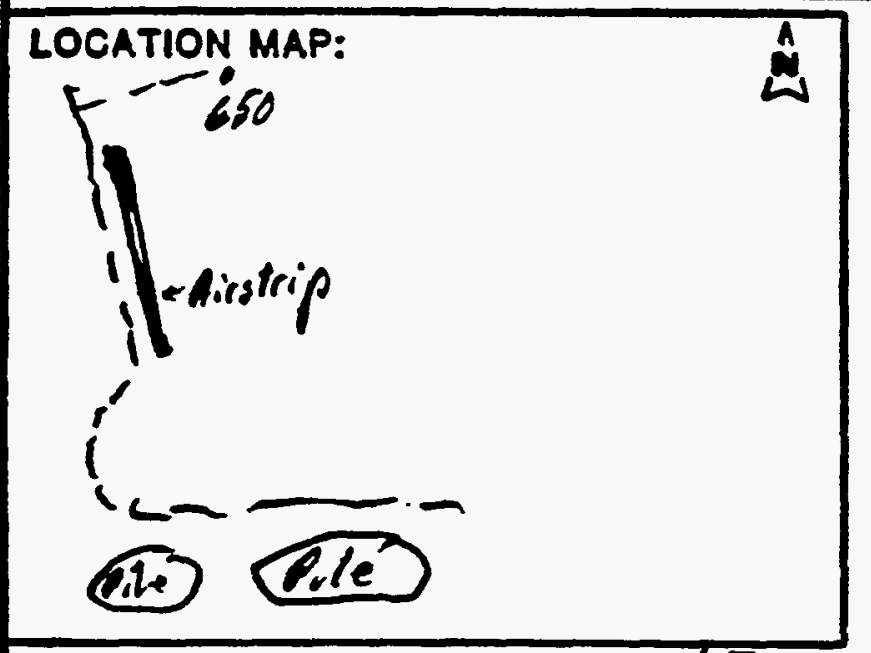

SITE ID: MeN -OL LOCATION ID:

SITE COORDINATES (ft.):

$N$ E GROUND ELEVATION (ft. MSL):

DRILLING METHOD: dotery

DRILLING CONTR.: Reenow Dellin Ge

DATE COMPLETED:

FIELD REP.:

\begin{tabular}{|c|c|c|}
\hline \multicolumn{3}{|c|}{ GROUNDWATER LEVELS } \\
\hline DATE & TIME & DEPTH (ft.) \\
\hline & & \\
\hline & & \\
\hline & & \\
\hline
\end{tabular}

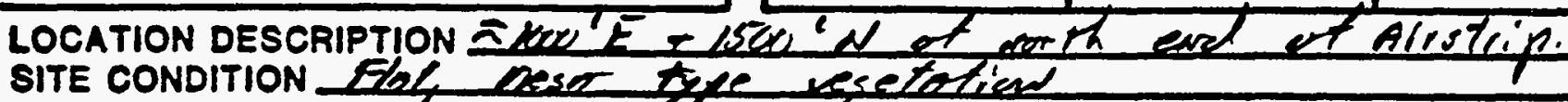
Elol aser

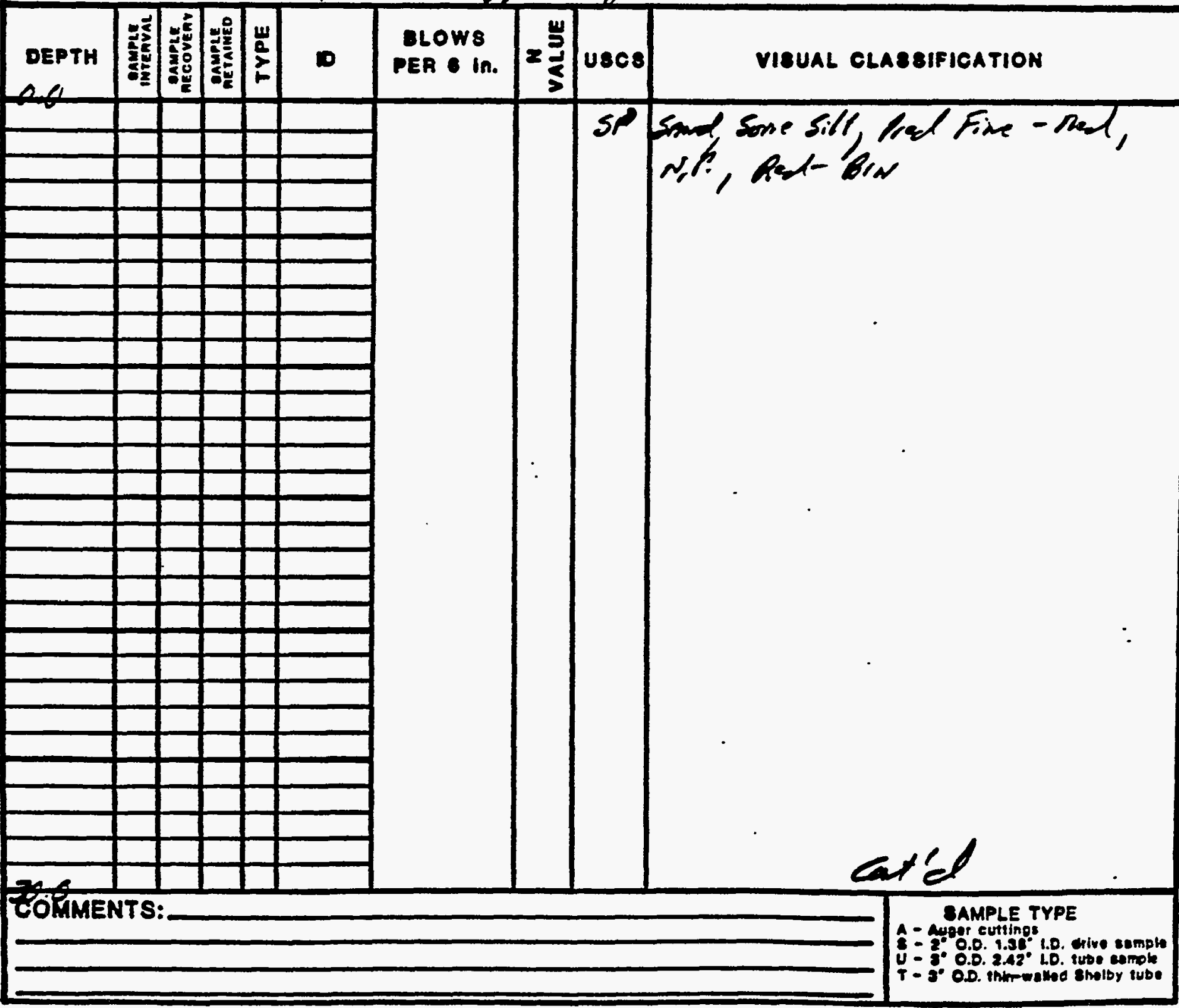


LOCATION MAP:

See of 1
$\stackrel{A}{\omega}$

SITE ID: Merc-61

SITE COORDINATES ( $t$ t.)

N.

GROUND ELEVATION ( $F$. MSL):

DRILLING METHOD: aty

DRILLING CONTR.:

DATE STARTED:

DATE COMPLETED:

FIELD REP.:

LOCATION DESCRIPTION

SITE CONDITION

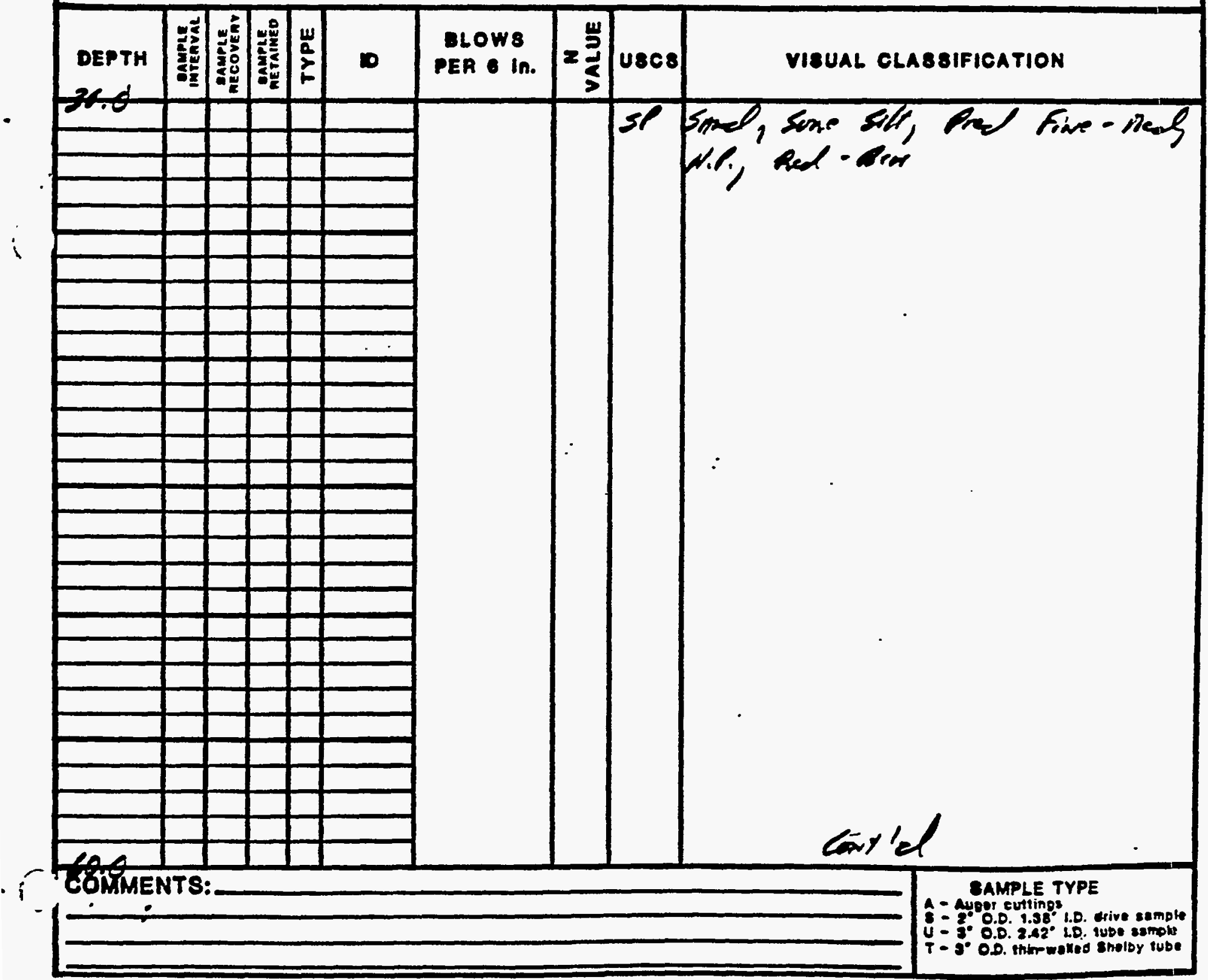


JACOBS ENGINEERING GROUP INC.

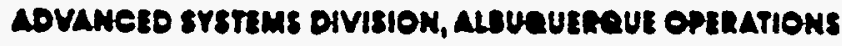

BOREHOLE LOG (SOIL)

Pagez of $y$

LOCATION MAP:

ڤ

SITE ID: Hax d

LOCATION 10: 650

SITE COORDINATES (ft.):

N E

GROUND ELEVATION (ft. MSL):

DRILLING METHOD: betecy

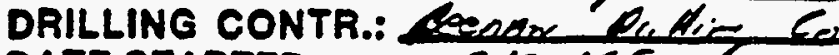

Se $\mathrm{g} l$

DATE STARTED:

9lseler

DATE COMPLETED:

FIELD REP.:

2/3 186

\begin{tabular}{|c|c|c}
\hline \multicolumn{3}{|c}{ GROUNDWATER LEVELS } \\
\hline DATE & TIME & DEPTH (ft.) \\
\hline & & \\
\hline & & \\
\hline & & \\
\hline
\end{tabular}

LOCATION DESCRIPTION

SITE CONDITION

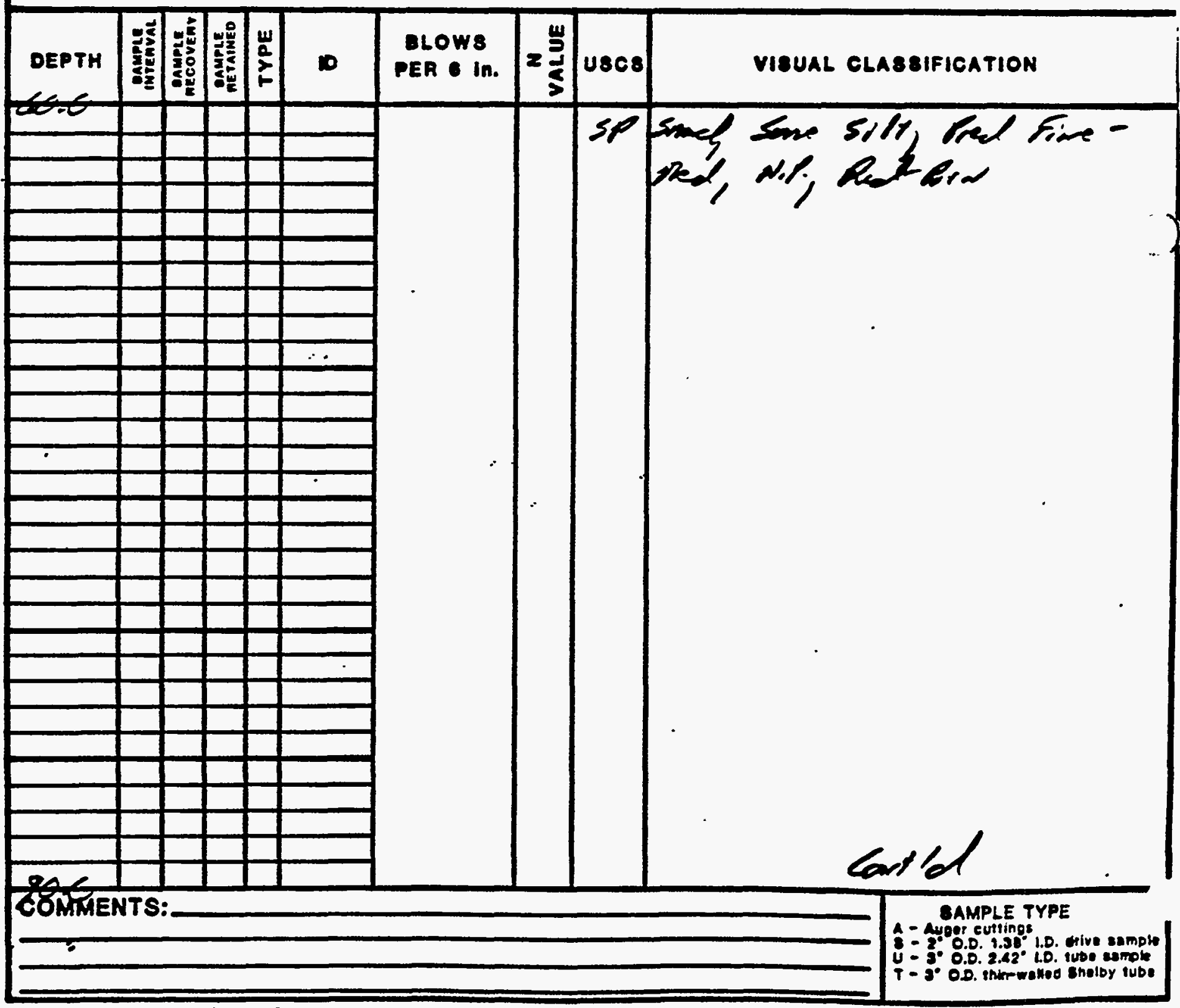


LOCATION MAP:

See of I

LOCATION DESCRIPTION

SITE CONDITION

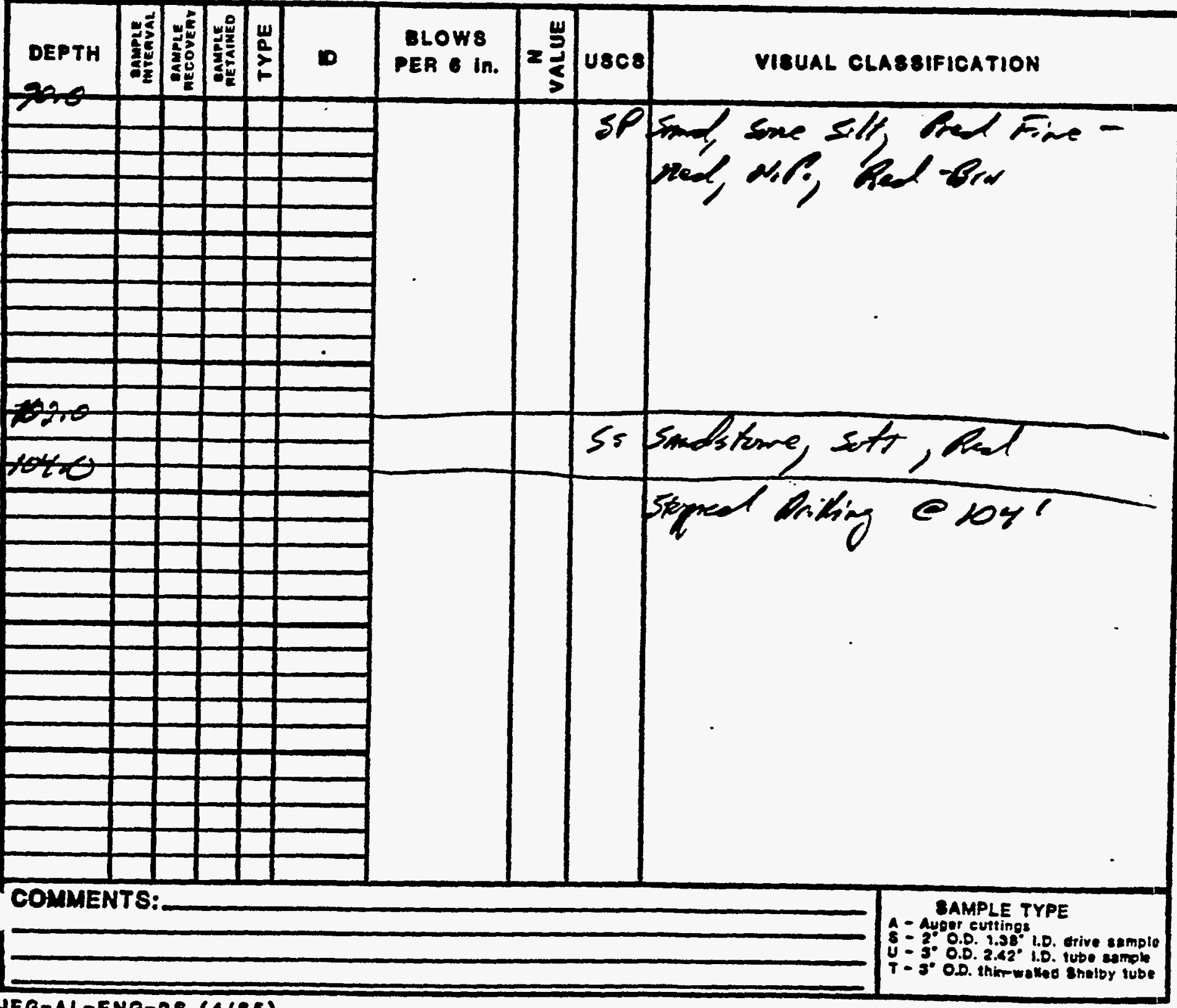
SITE COORDINATES ( $(t$.$) :$

N. GROUND ELEVATION (ft. MSL): DRILLING METHOD: DRILLING CONTR.:

DATE STARTED:

DATE COMPLETED:

FIELD REP.:

\begin{tabular}{|c|c|c|}
\hline \multicolumn{3}{|c|}{ GROUNDWATER LEVELS } \\
\hline DATE & TIME & DEPTH (4t.) \\
\hline & & \\
\hline & & \\
\hline & & \\
\hline
\end{tabular}

SITE ID: LEN-OI LOCATION ID: 100 


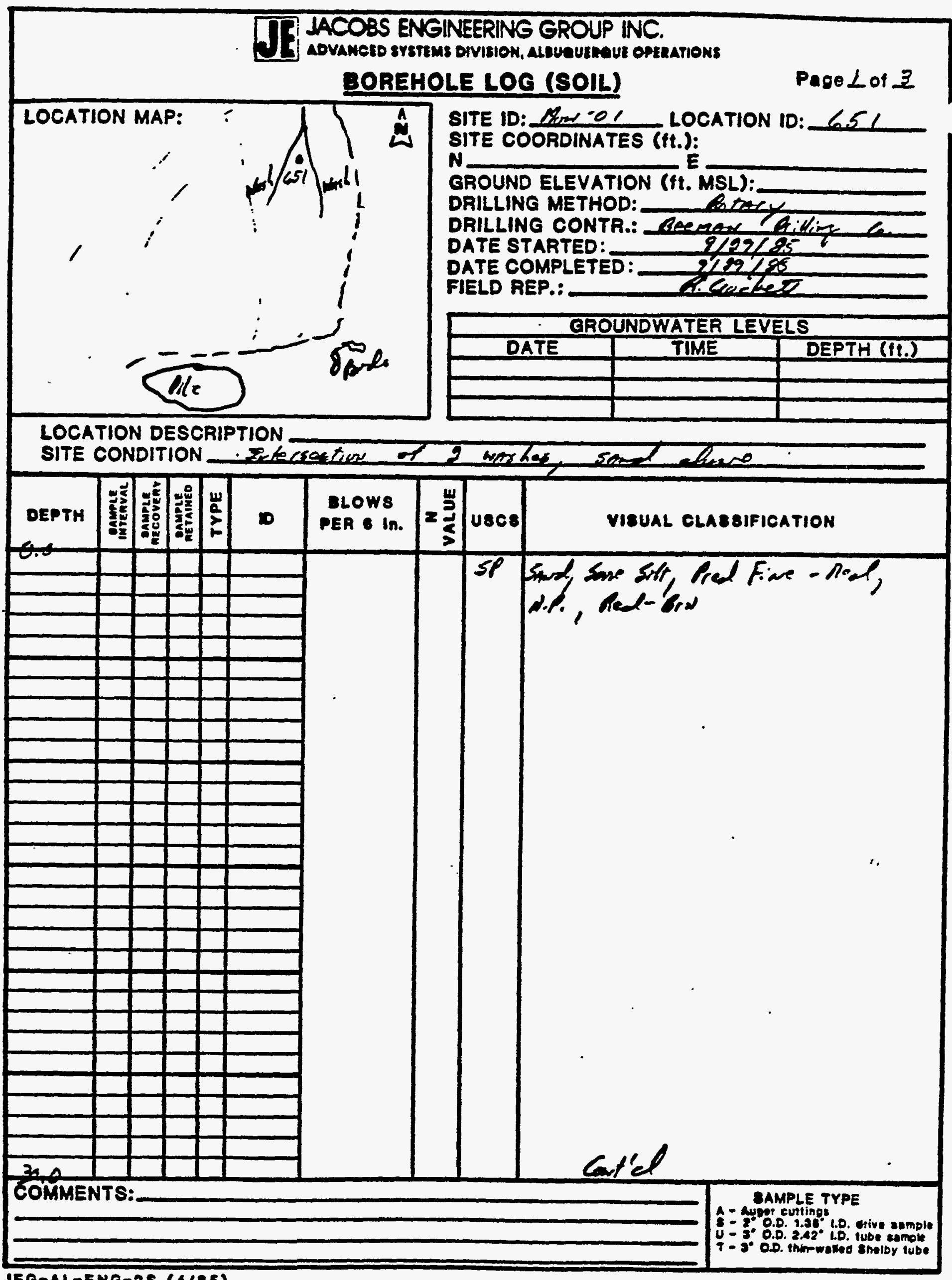


LOCATION MAP:

SITE ID: LOCATION ID: 65 ! SITE COORDINATES (ft.): $N$ - E GROUND ELEVATION (ft. MSL): DRILLING METHOD: DRILLING CONTR.: DATE STARTED:

See of 1 DATE COMPLETED:

FIELD REP.:

\begin{tabular}{|c|c|c|}
\hline \multicolumn{3}{|c|}{ GROUNDWATER LEVELS } \\
\hline DATE & TIME & DEPTH (ft.) \\
\hline & & \\
\hline & & \\
\hline & & \\
\hline
\end{tabular}

LOCATION DESCRIPTION SITE CONDITION

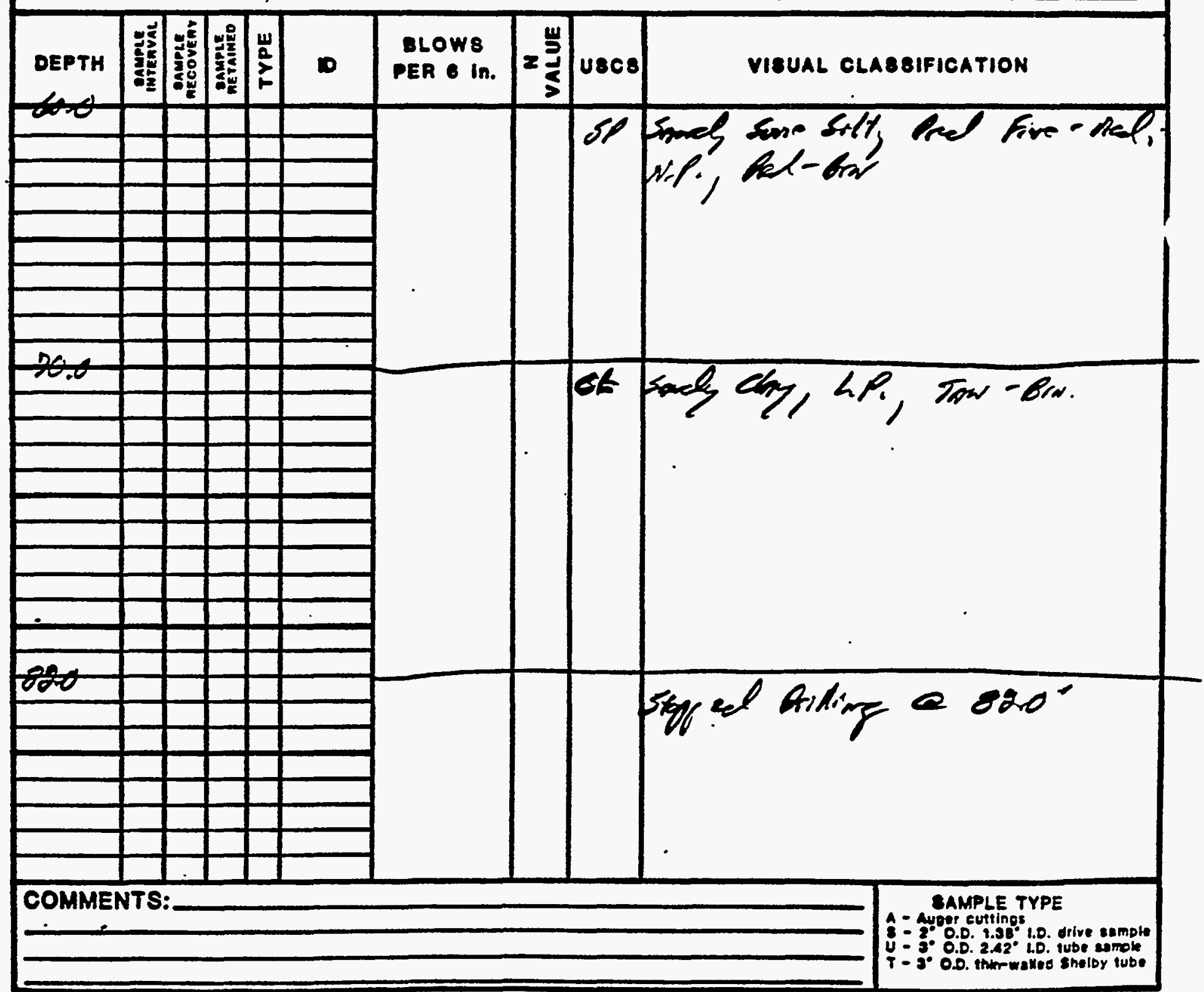




\section{BOREHOLE LOG (SOIL)}

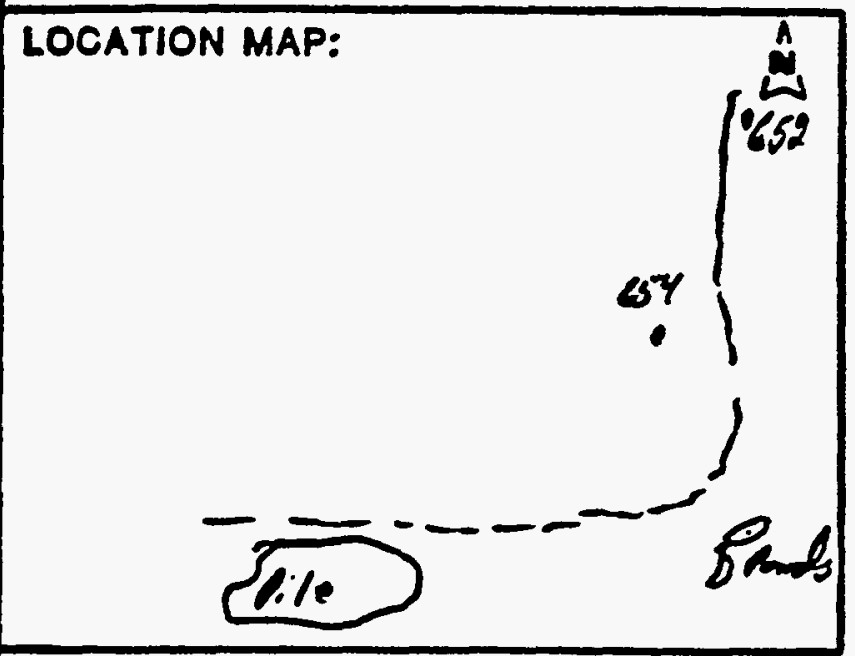

SITE ID: Mav-01

LOCATION ID:

SITE COORDINATES (II.):

N. E

GROUND ELEVATION (ft. MSL):

DRILLING METHOD: ChteCy

DRILLING CONTR.: Leenes

DATE STARTED: T/ee/ 85

DATE COMPLETED: $2128 / 15$

FIELD REP.:

\section{CROUNDWATER LEVELS}

LOCATION DESCRIPTION

ล 35000 f. SITE CONDITION Flot

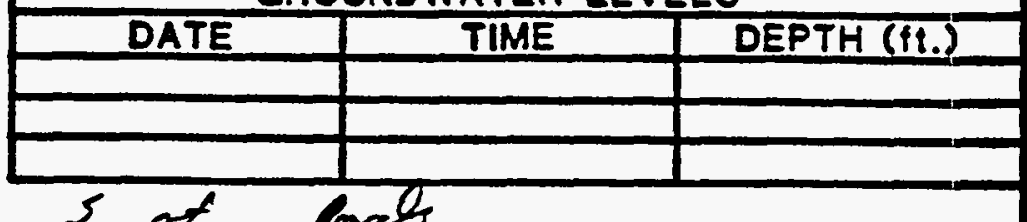
ese Ex

letetiese

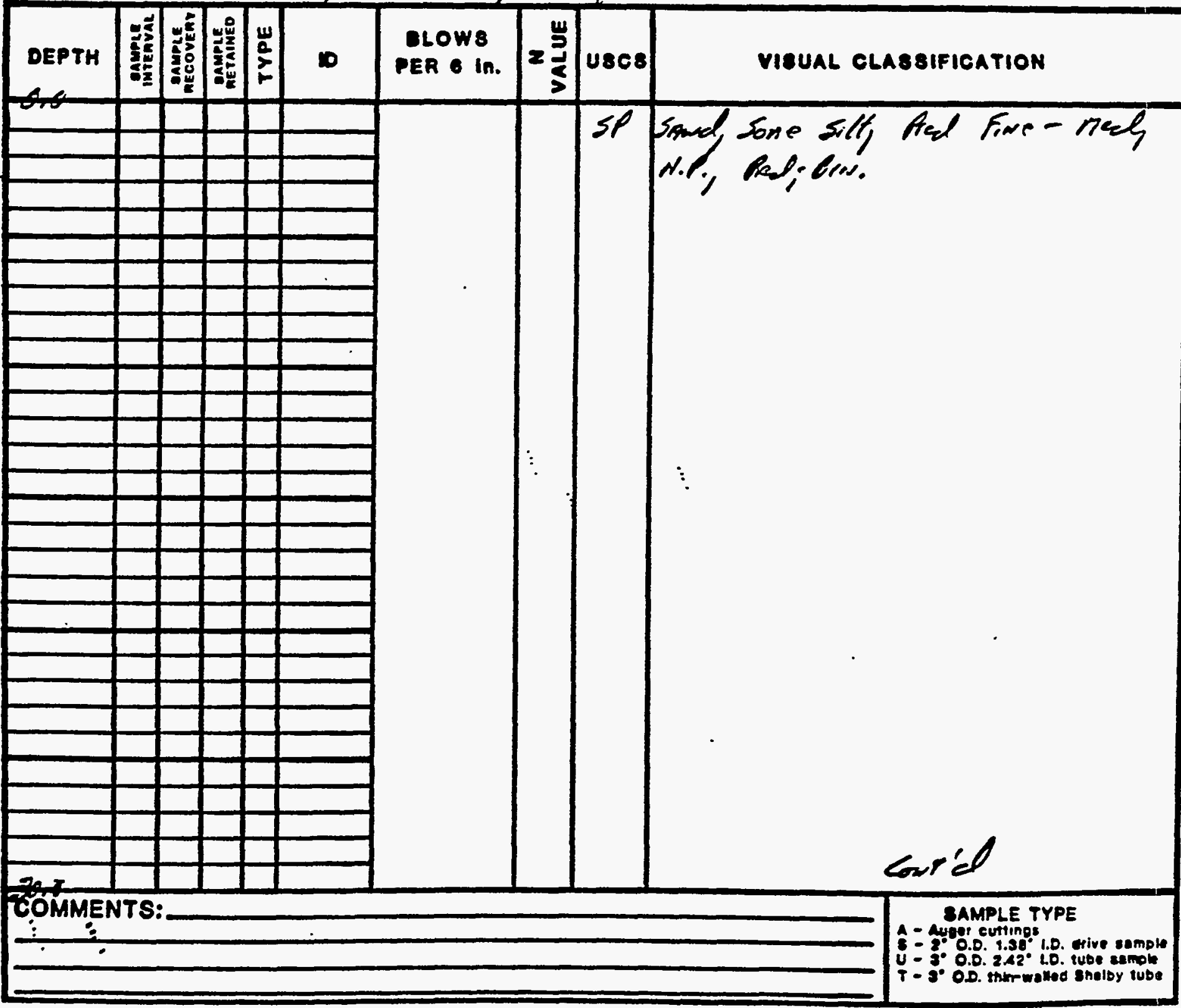




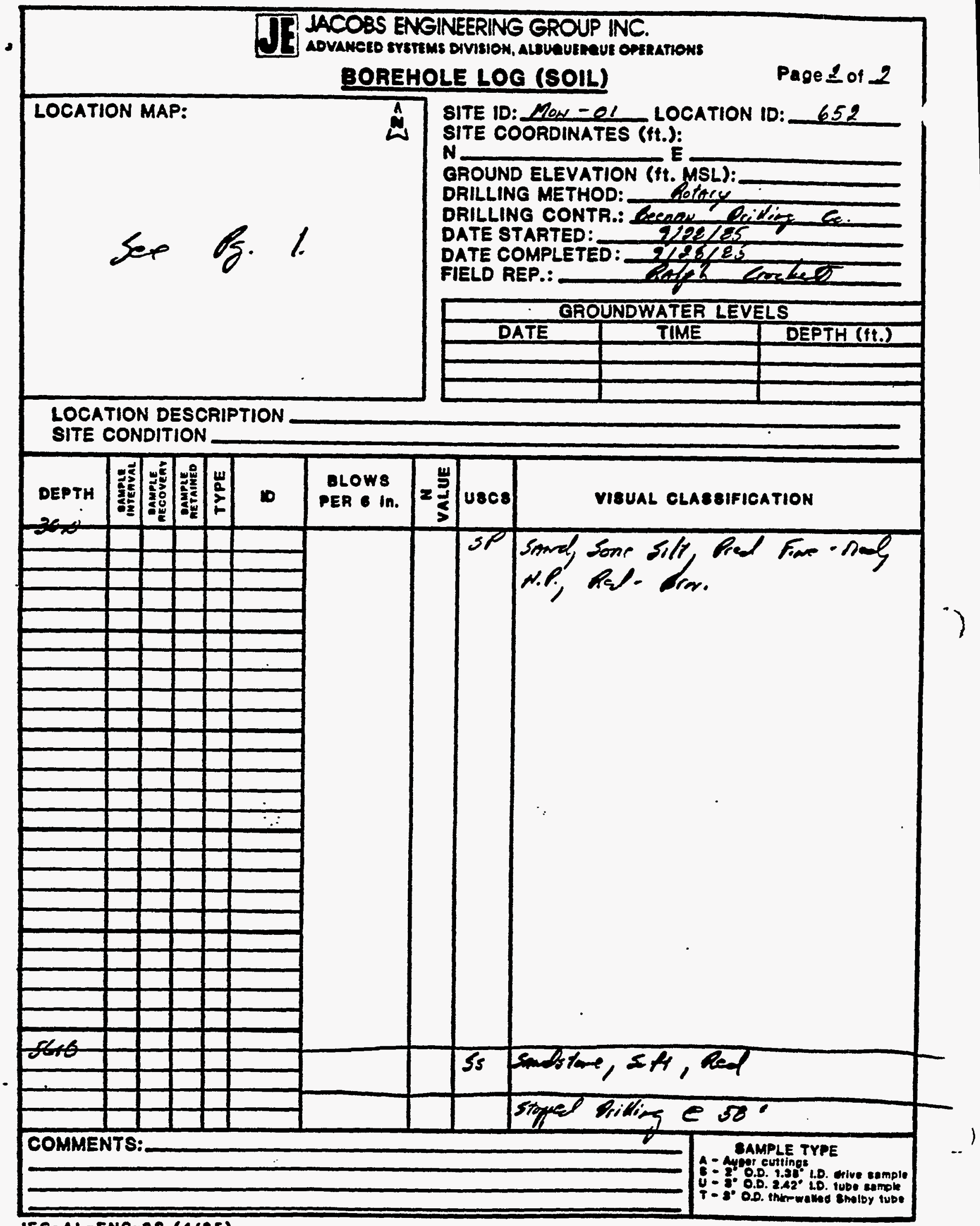




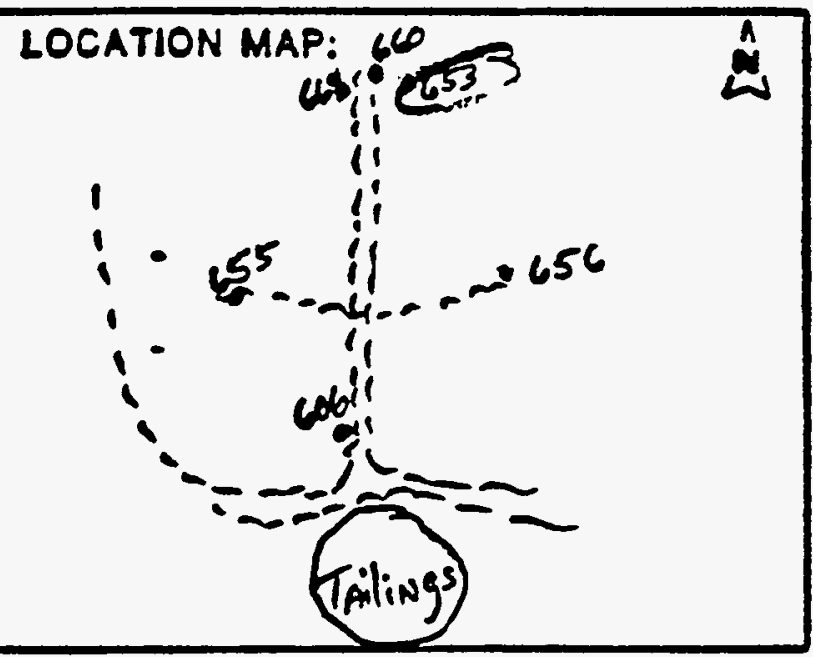

SITE ID: MON LOCATION ID: 653 SITE COORDINATES (1t.):

GROUND ELEVATION (ft. MSL):

GROUND ELEVATION (ft. MSL):

DRILLING CONTR.: Beb Beemań

DATE STARTED: Sept 25,85

DATE COMPLETED: $\frac{\text { SORt 25,85. }}{\text { FIELD REP.: }}$

\begin{tabular}{|c|c|c|}
\hline \multicolumn{3}{|c|}{ GROUNDWATER LEVELS } \\
\hline DATE & TIME & DEPTH (fl.) \\
\hline & & \\
\hline & & \\
\hline & & \\
\hline
\end{tabular}

LOCATION DESCRIPTION ApereX .7 miles Nerth of tailings pile SITE CONDITION Soft $\|$ Qd SAND

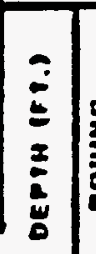

|

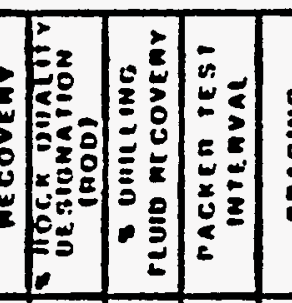

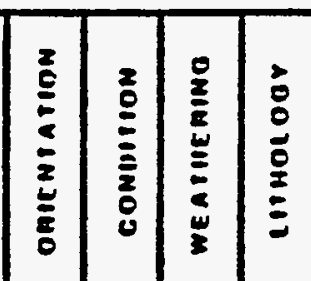

AOCK TYPE \& REMARKS

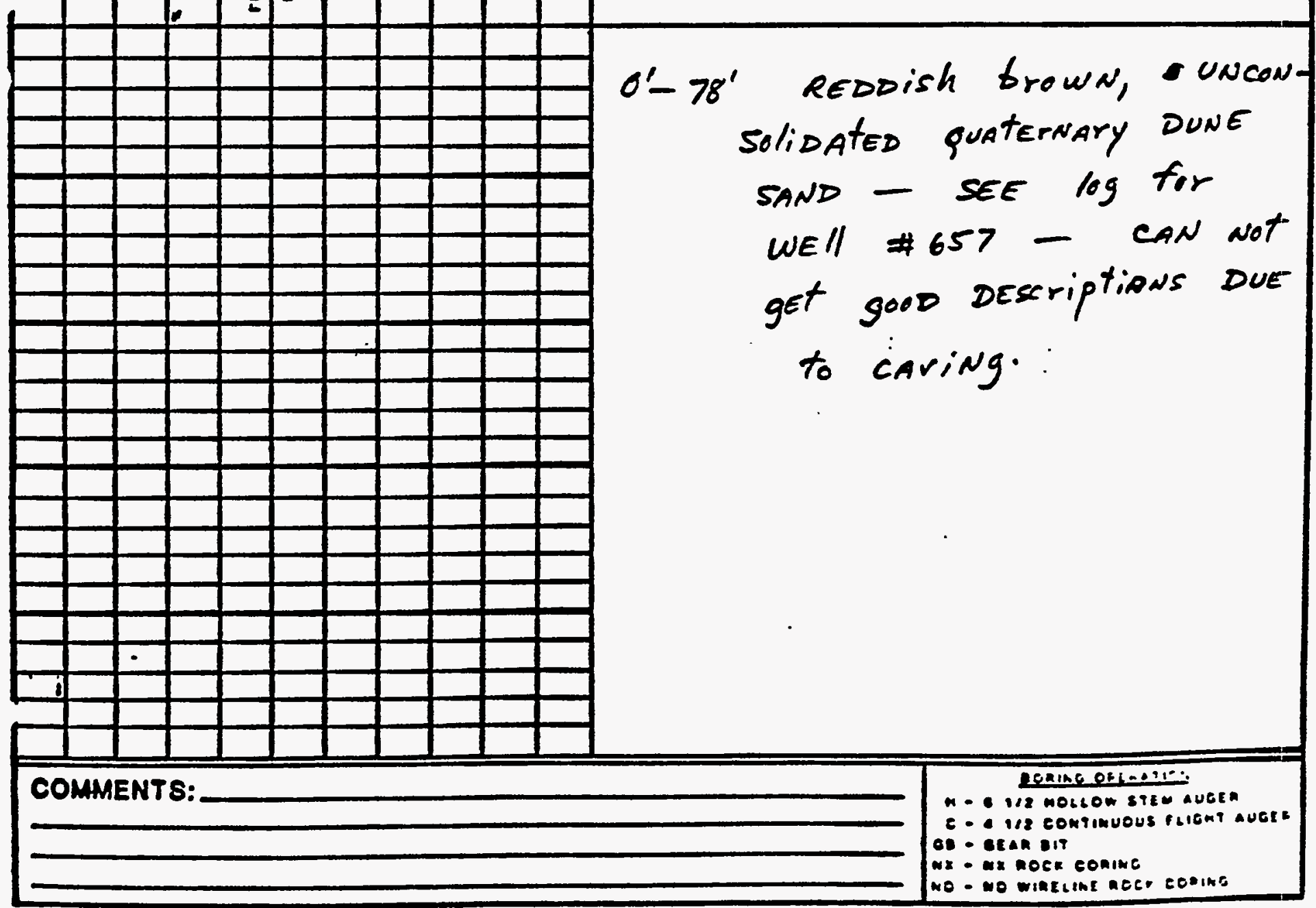




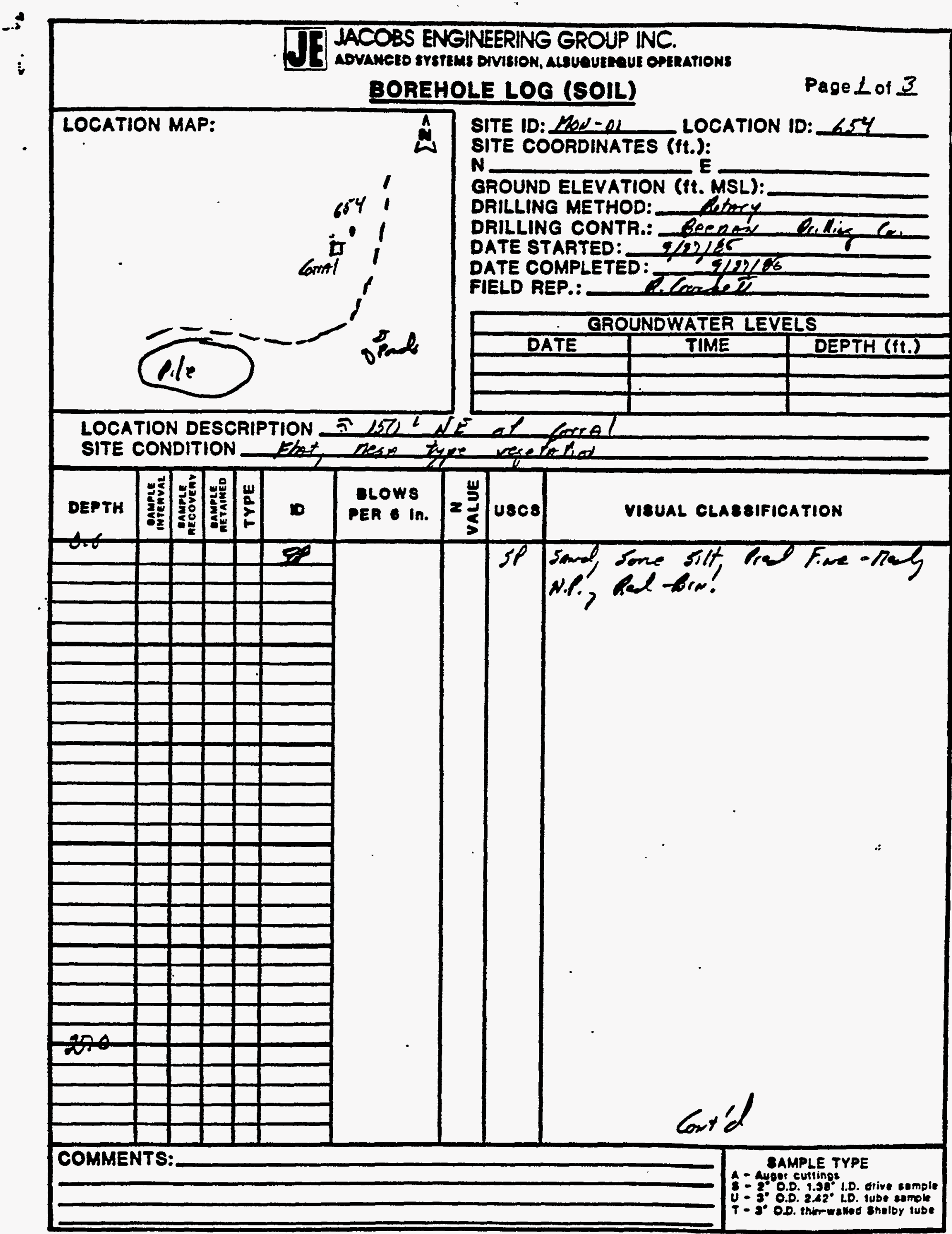




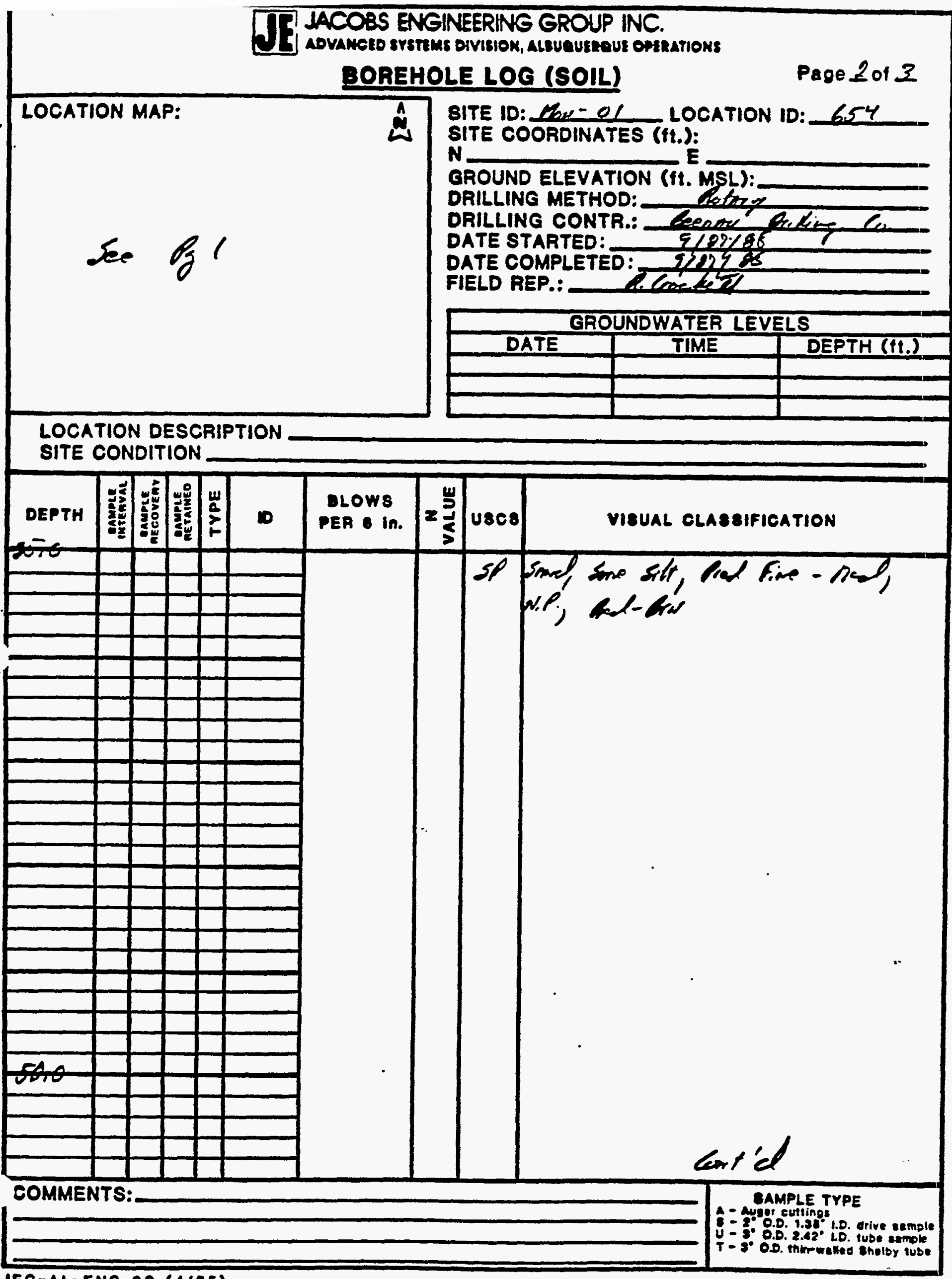

JEG-AL-ENG-2S (4/88) 


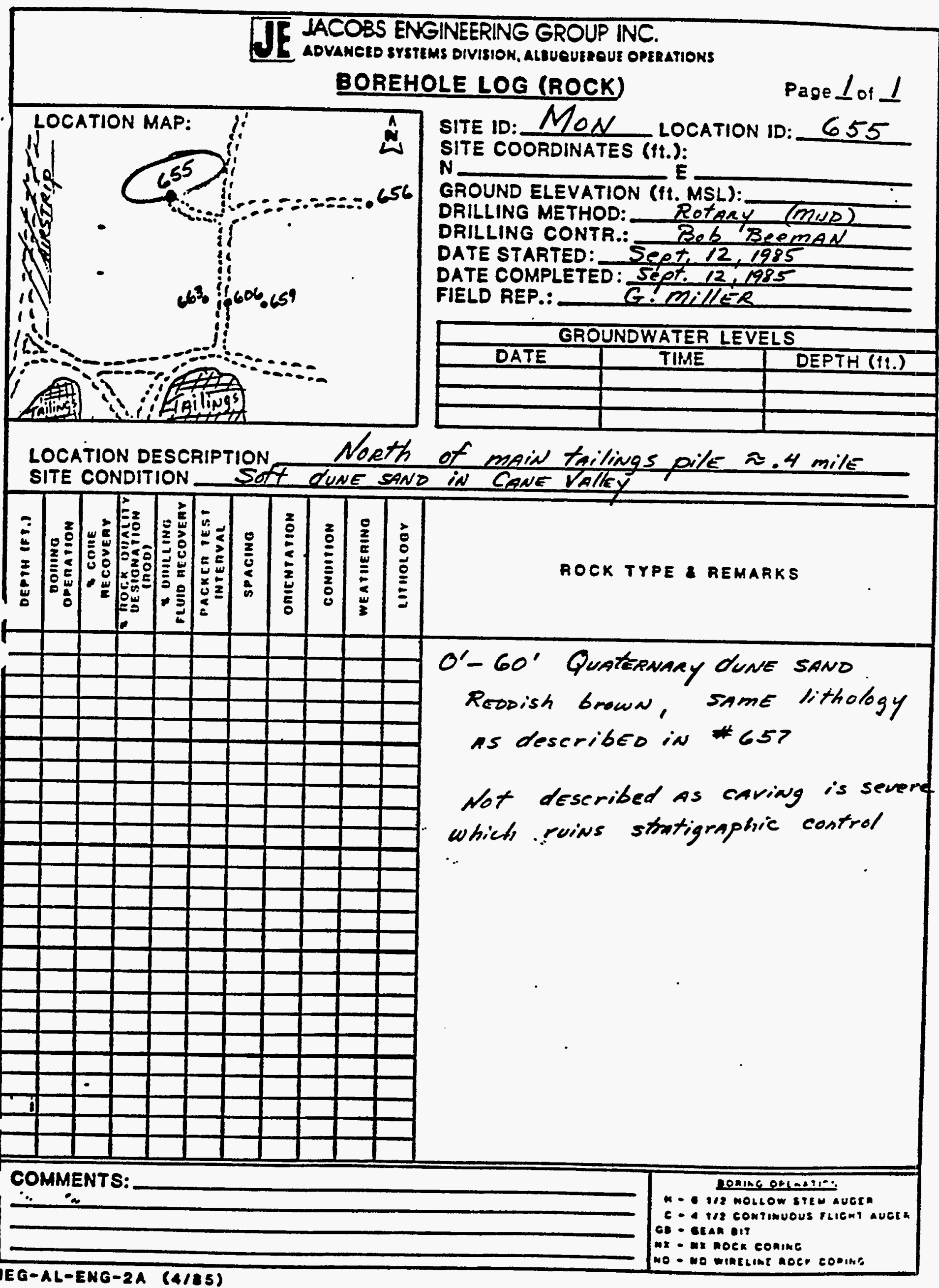




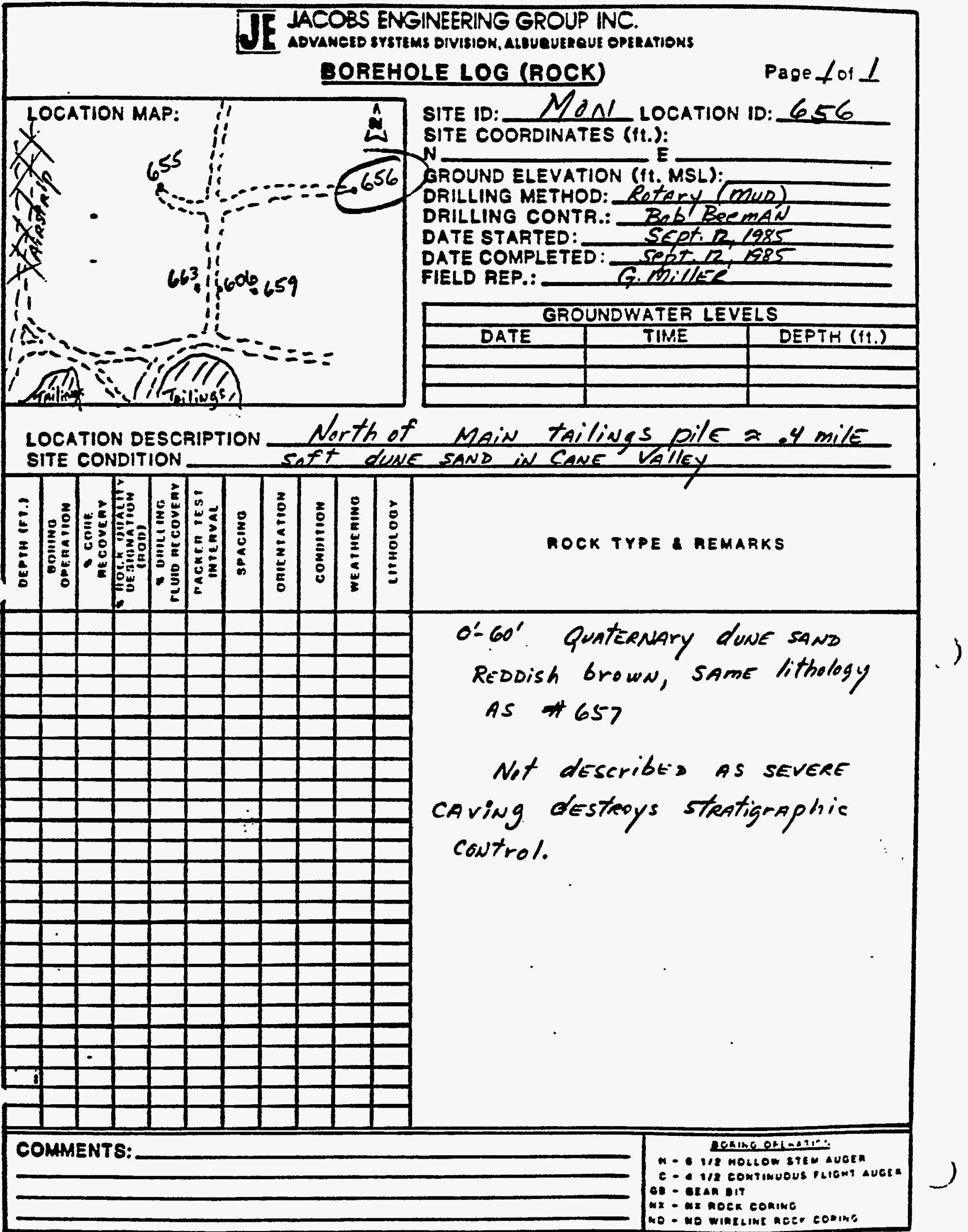


LACOBS ENGINEERING GROUP INC.

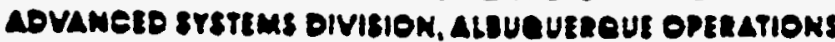

BOREHOLE LOG (ROCK) Page 2 of 3

LOCATION MAP:

离

SITE ID: MON

LOCATION ID: 657

SITE COORDINATES (tt.):

N. $E$

GROUND ELEVATION (fT. MSL):

DRILLING METHOD: Potary (mUD)

DRILLING CONTR.: Brb ReemaN

DATE STARTED:- Sopt. 4,1985

DATE COMPLETED: Sept 5,1985

FIELD REP.: G.MPller

\begin{tabular}{|c|c|c|}
\hline \multicolumn{3}{|c|}{ GROUNDWATER LEVELS } \\
\hline DATE & TIME & DEPTH (II.) \\
\hline & & \\
\hline & & \\
\hline & & \\
\hline
\end{tabular}

LOCATION DESCRIPTION SITE CONDITION

North of

old tailings pile

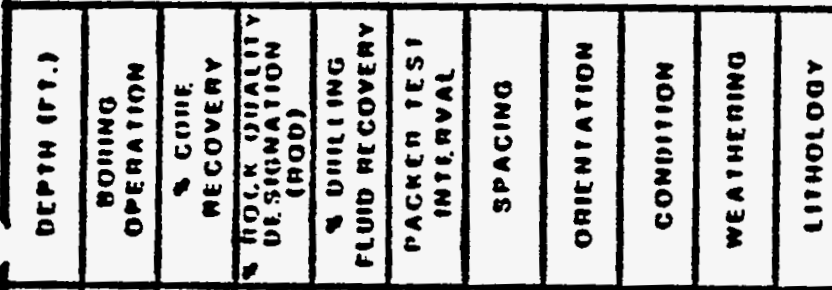

NOCK TYPE I REMARKS

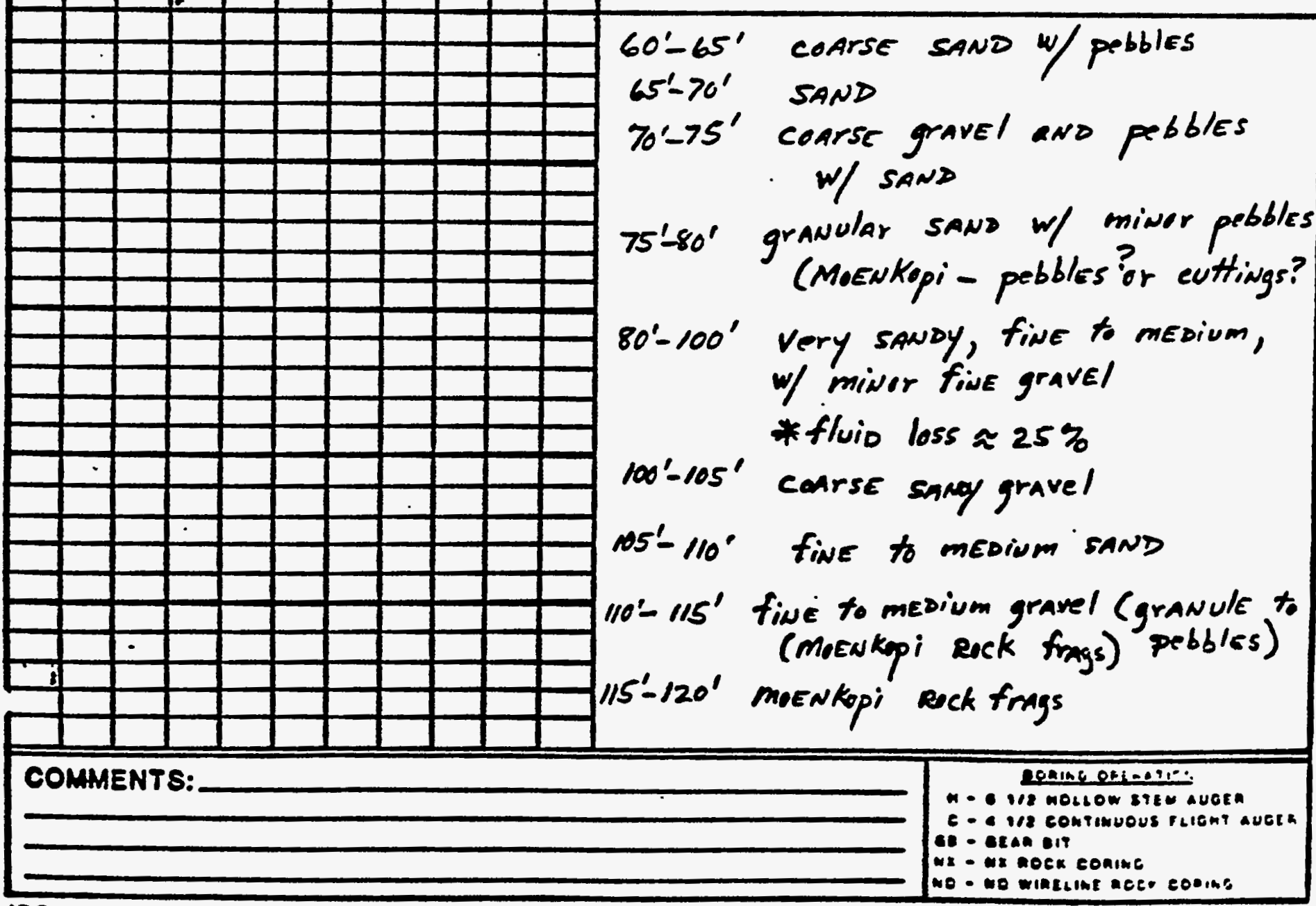




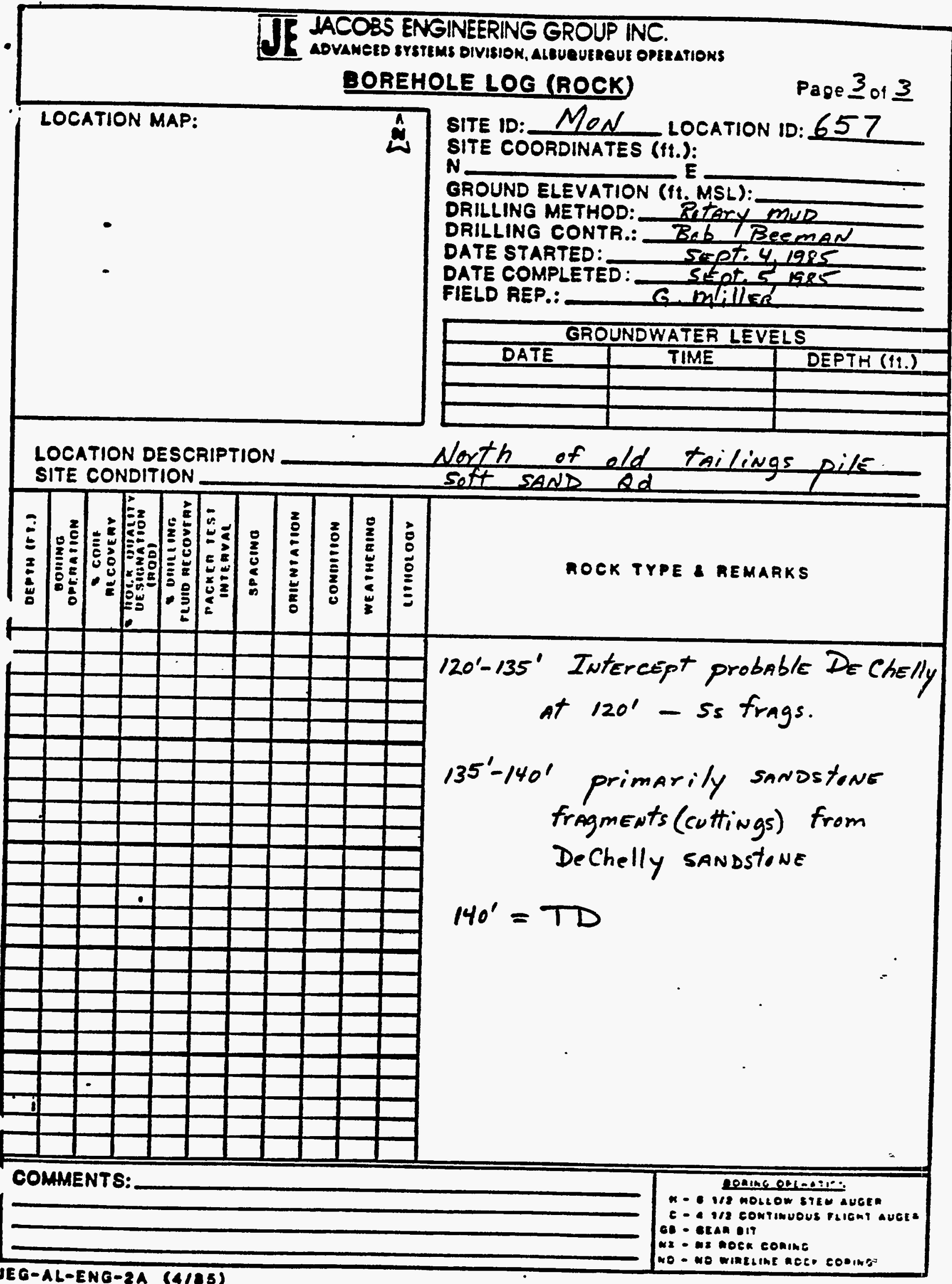




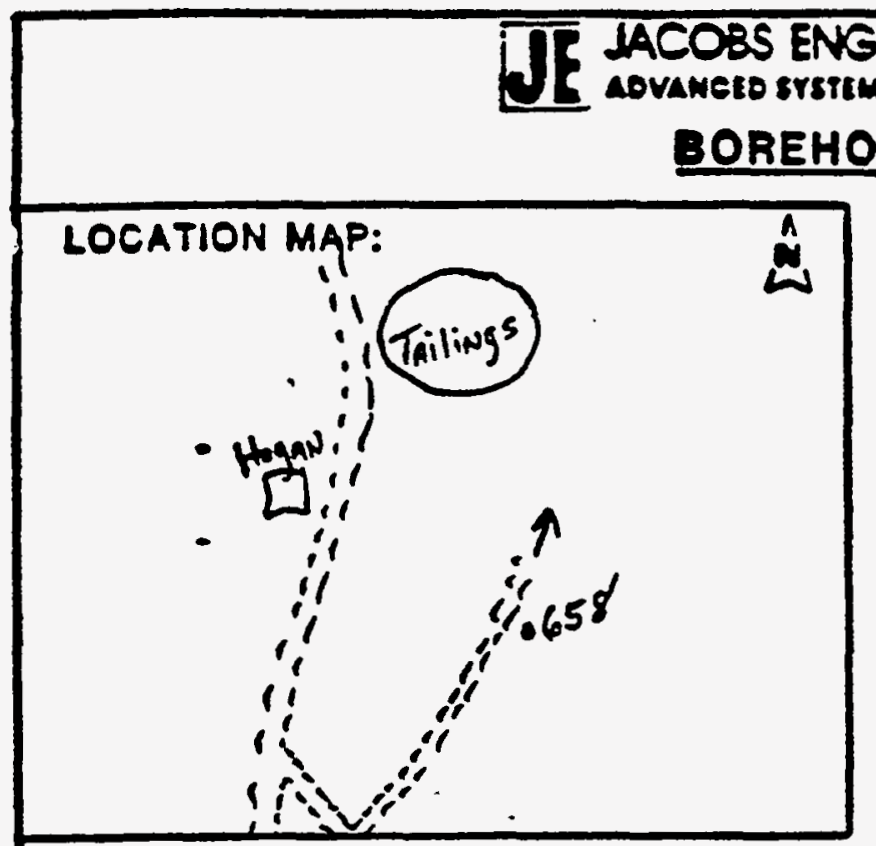

Page $\operatorname{Lot} L$

SITE ID: MON LOCATION ID: 658

SITE COORDINATES (1T.):

N

GROUND ELEVATION (1t. MSL):

DRILLING METHOD: RotACY (MUD)

DRILLING CONTR.: Bebl Beemań

DATE STARTED: SEPt. 25, 985

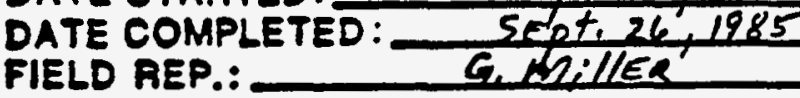

\begin{tabular}{|c|c|c|}
\hline \multicolumn{3}{|c|}{ GROUNDWATER LEVELS } \\
\hline DATE & TIME & DEPTH (II.) \\
\hline & & \\
\hline & & \\
\hline & & \\
\hline
\end{tabular}

LOCATION DESCRIPTION

South of tailings pile

SITE CONDITION

Soft SAND aND muD

\begin{tabular}{c|c}
\hline \\
$\vdots$ \\
$\vdots$ \\
$\vdots$ \\
$z$
\end{tabular}

\begin{tabular}{|c|}
\hline 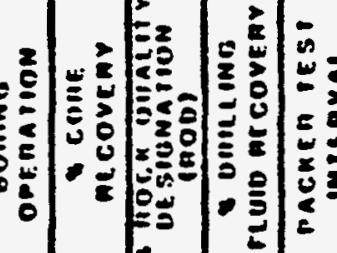 \\
\hline
\end{tabular}

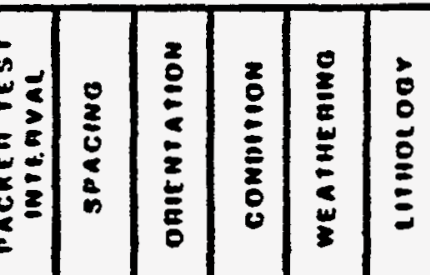

ROCK TYPE A REMARKS

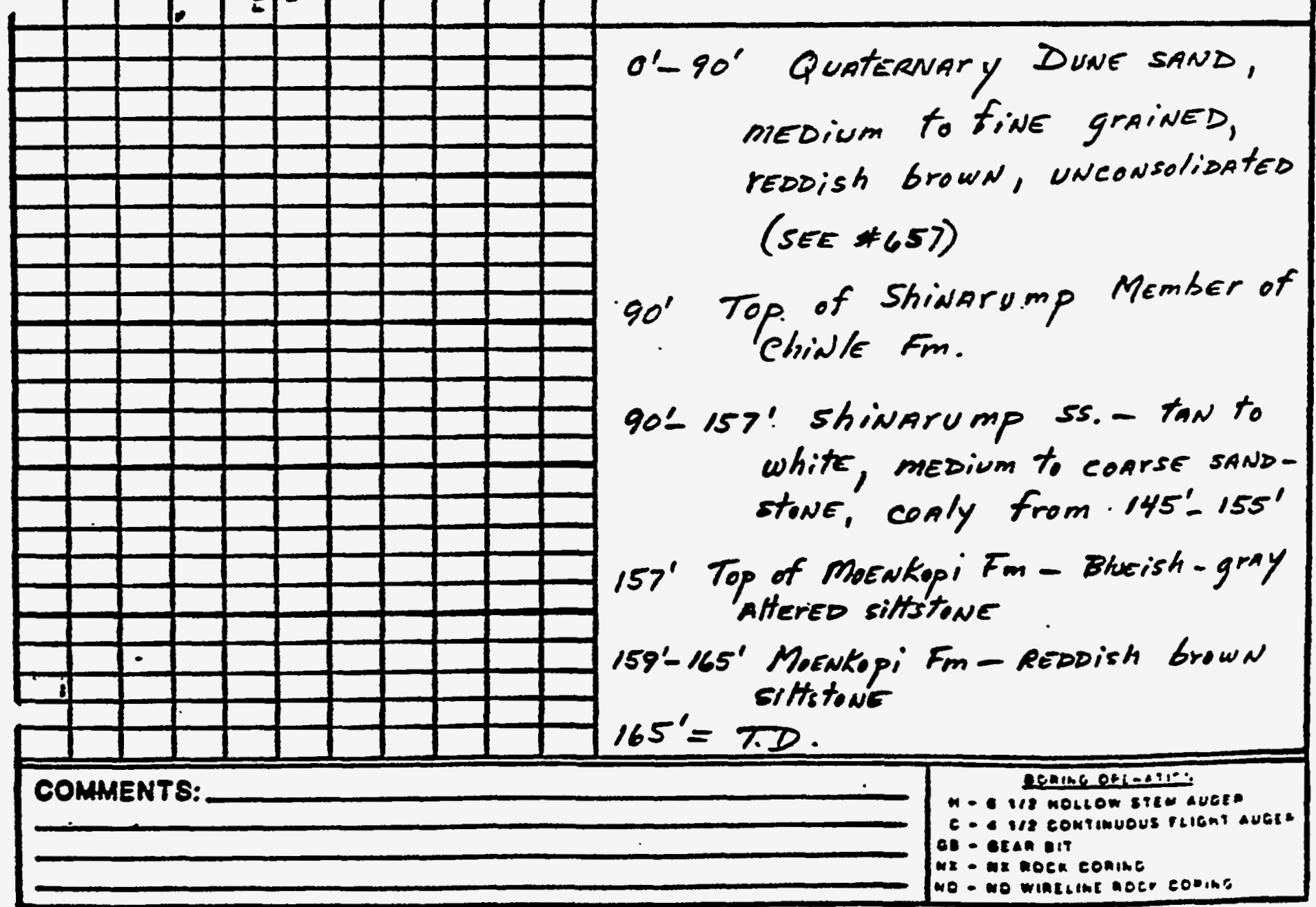


LACOSS ENGINEERING GROUP INC.

AOVAMELO ETSTEMS OIVISIOM, ALRUQUERQUE ORERATIONS

BOREHOLE LOG (ROCK)

Page 1 or 1

LOCATION MAP:

岕

SITE ID: Mod

LOCATION ID: 659

SITE COORDINATES (ft.):

$N$ E

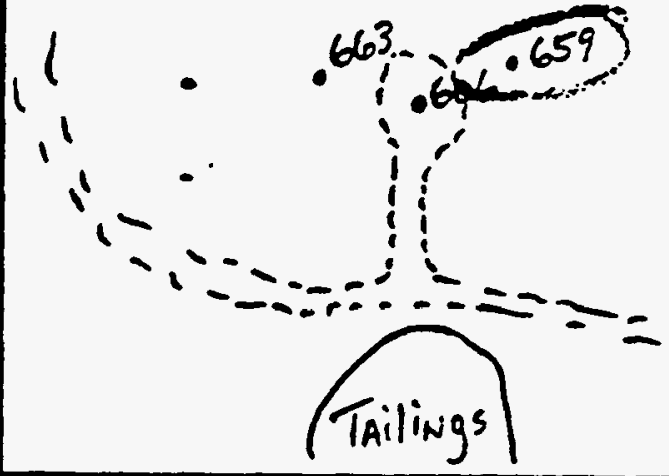

GROUND ELEVATION (ft. MSL):

DRILLING METHOD:

DRILLING CONTR.:

Ratory (mud)

DATE STARTED:

Bab Beeman

DATE COMPLETED: $\frac{\text { sEpt, 1P, } 1985}{G . M i l l E R}$

\begin{tabular}{|c|c|c|}
\hline \multicolumn{3}{|c|}{ GROUNDWATER LEVELS } \\
\hline DATE & TIME & DEPTH (fi.) \\
\hline & & \\
\hline & & \\
\hline & & \\
\hline
\end{tabular}

LOCATION DESCRIPTION

North of tailings <.l mile

SITE CONDITION

Seft $\operatorname{sen} \pi$ G.d

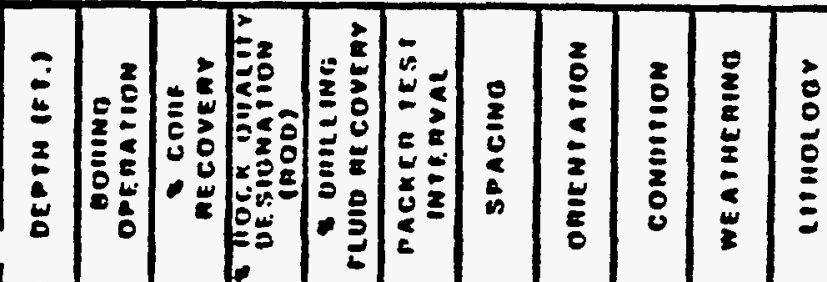

ROCK TYPE E REMARKS

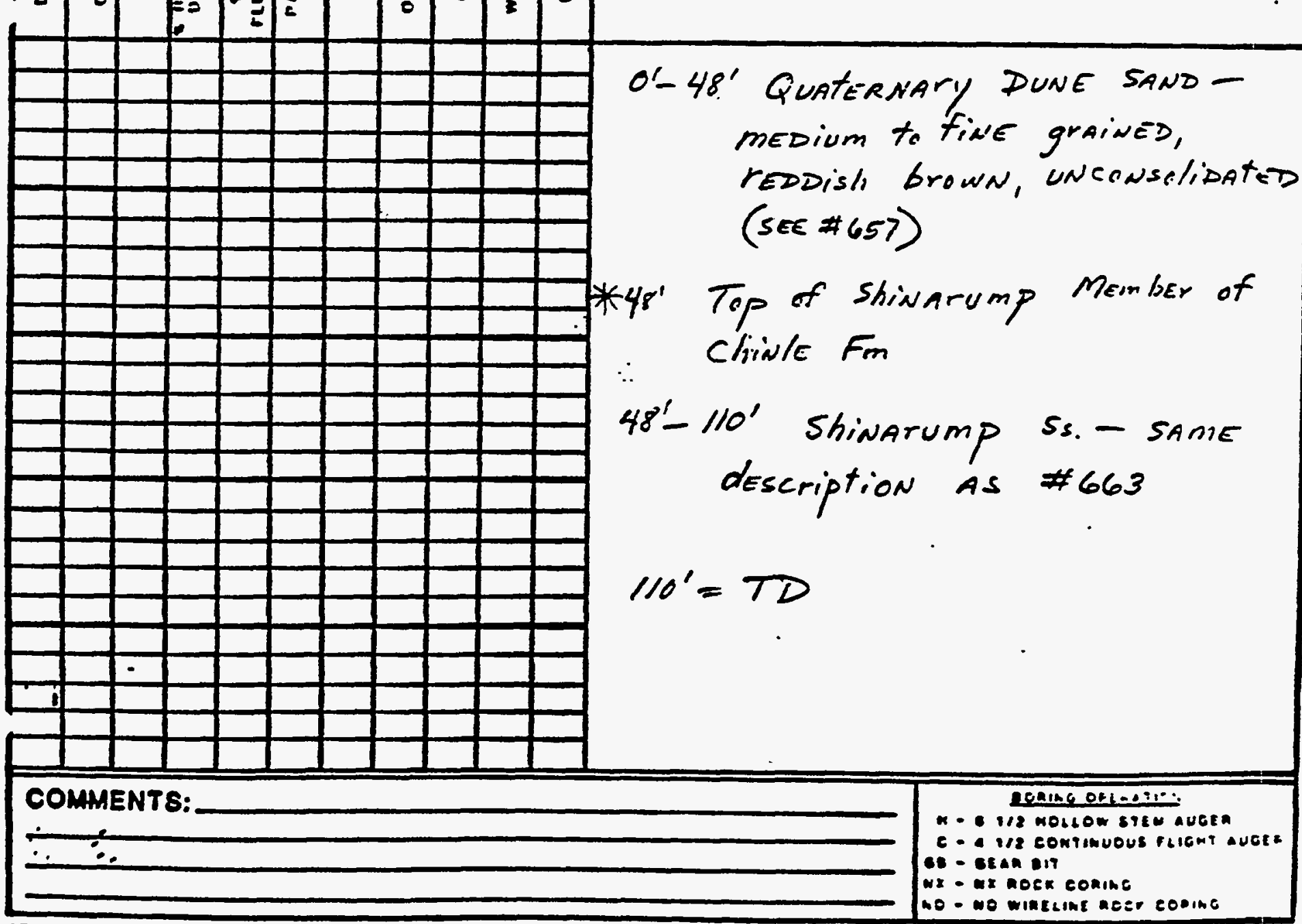

JEG-AL-ENG-2A (4/85) 


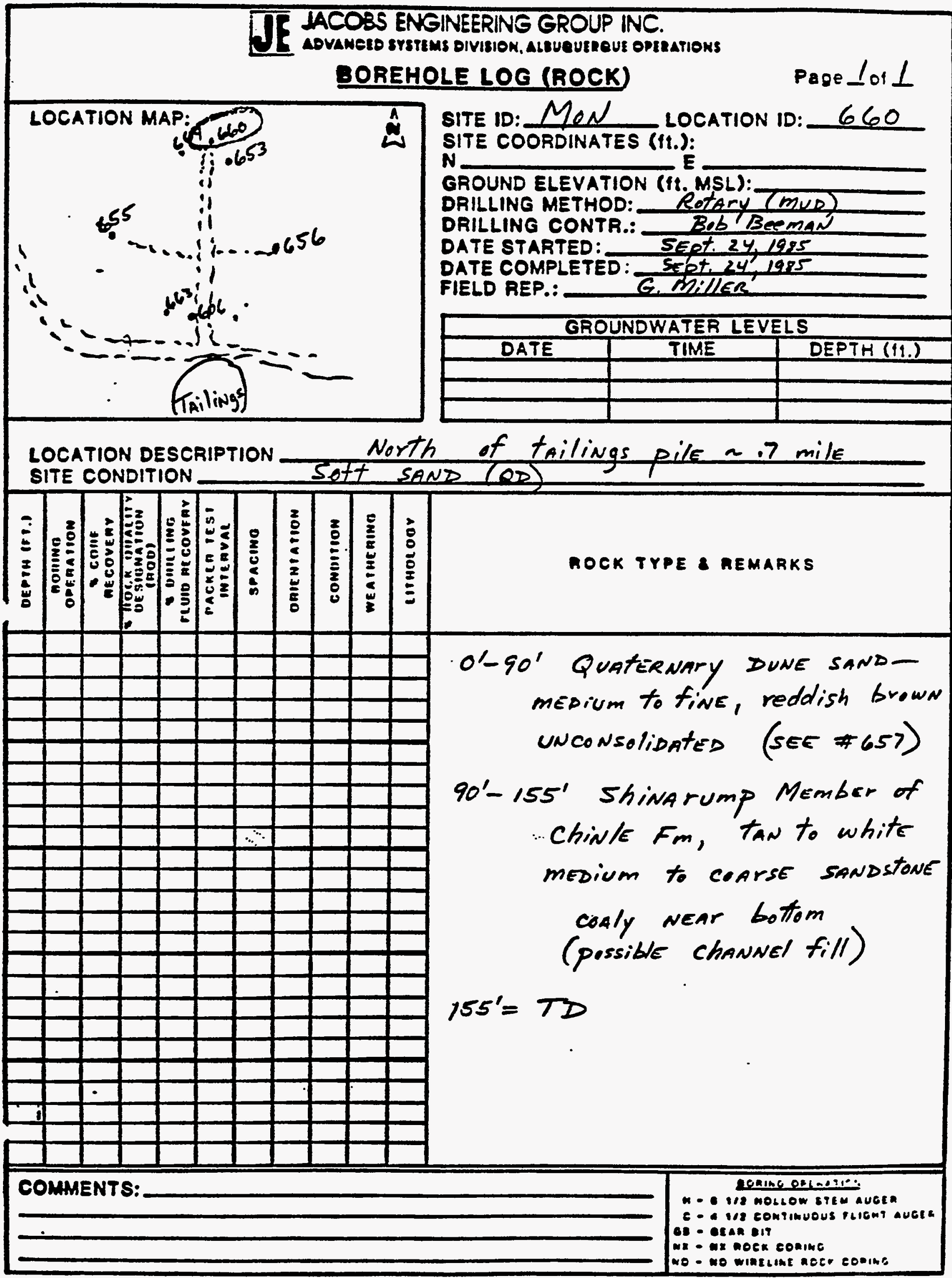


D] LACOBS ENGNEERNG GROUP NC.

DOREHOLE LOG (ROCK)

Page $\&$ of 2

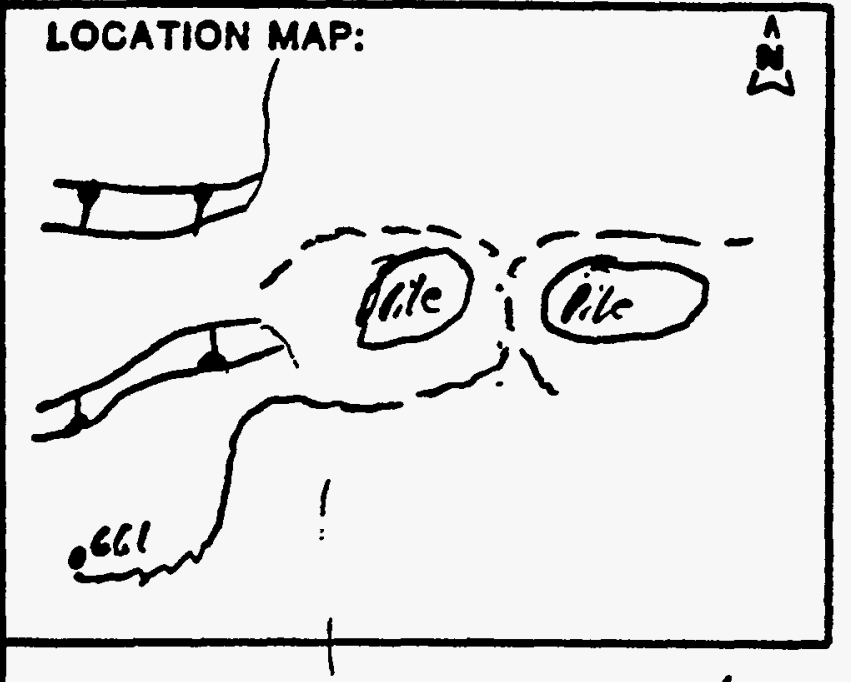

SITE ID: MOV OI LOCATION ID: 661
SITE COORDINATES (ft.):

N

GROUND ELEVATION (ft. MSL):

DRILLING METHOD: Petey

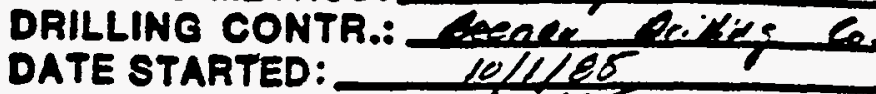

DATE COMPLETED:

FIELD REP.:

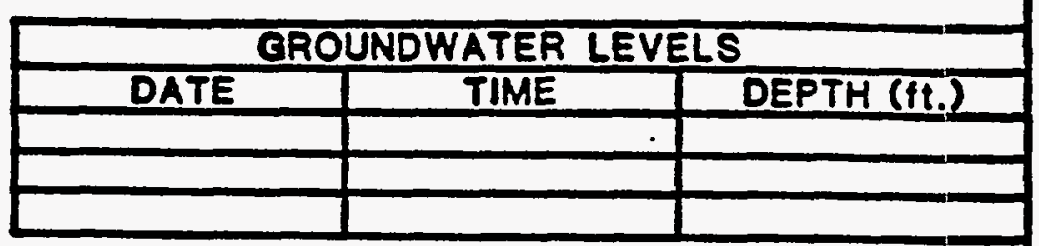

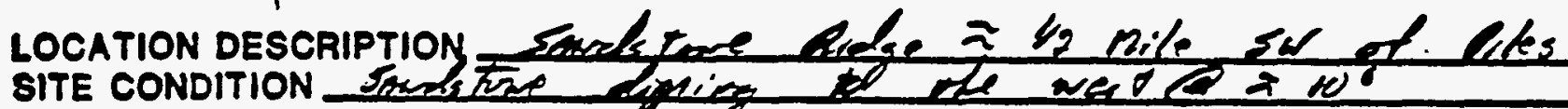

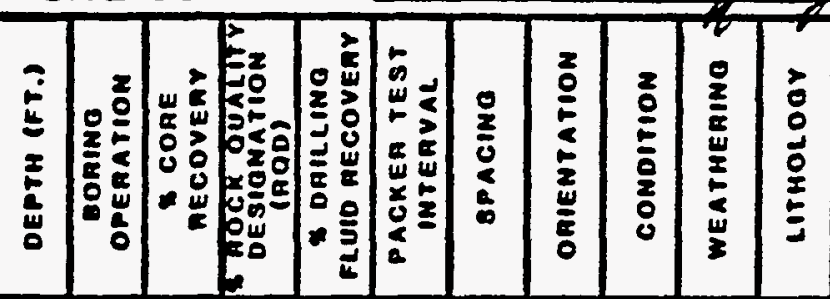

ROCK TYPE \& REMARKS

shiwarump Snotstome, five grained,

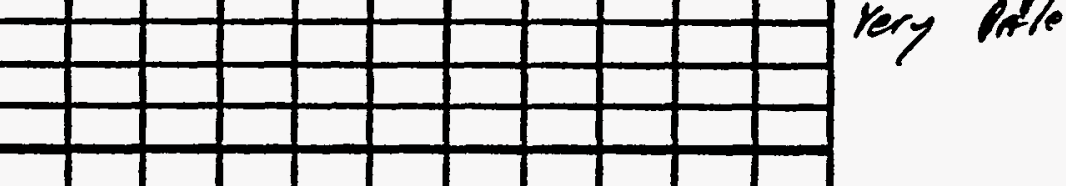

\begin{tabular}{|lllllllllll}
\hline & & & & & & & & & & \\
\hline & & & & & & & & & & \\
\hline & & & & & & & & & & \\
\hline & & & & & & & & & & \\
\hline & & & & & & & & & & \\
\hline & & & & & & & & & & \\
\hline & & & & & & & & & & \\
\hline & & & & & & & & & & \\
\hline & & & & & & & & & & \\
\hline & & & & & & & & & & \\
\hline & & & & & & & & & & \\
\hline
\end{tabular}

COMMENTS:

SoRing openation

M - 1/2 mollow eten auser

c - ile continuoue fllent auena

es - esan vit

$w x-\omega x$ mock conino

JEO-AL-ENG-2A (4/86) 


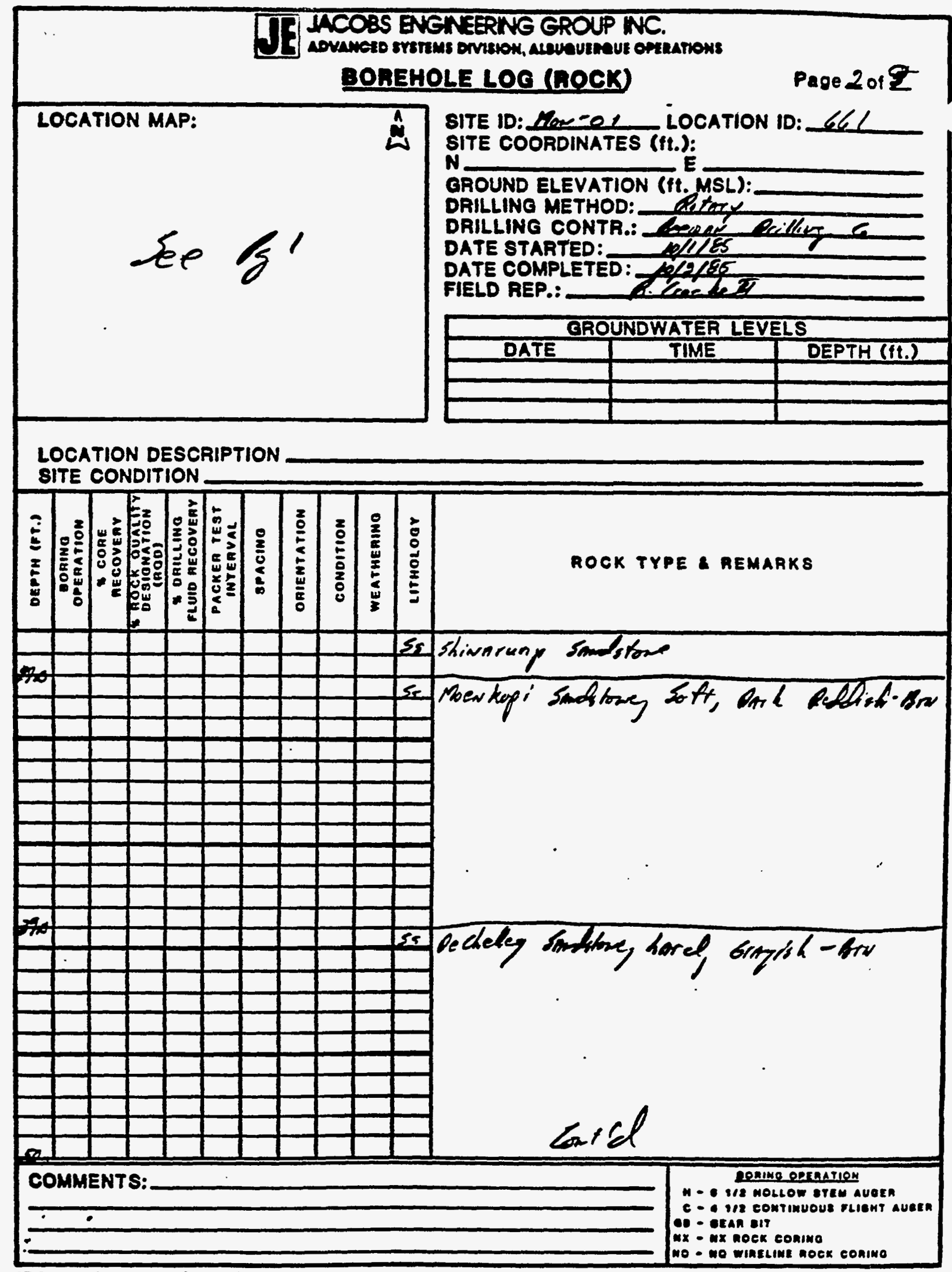

JEO-AL-ENG-2A (4/BS) 


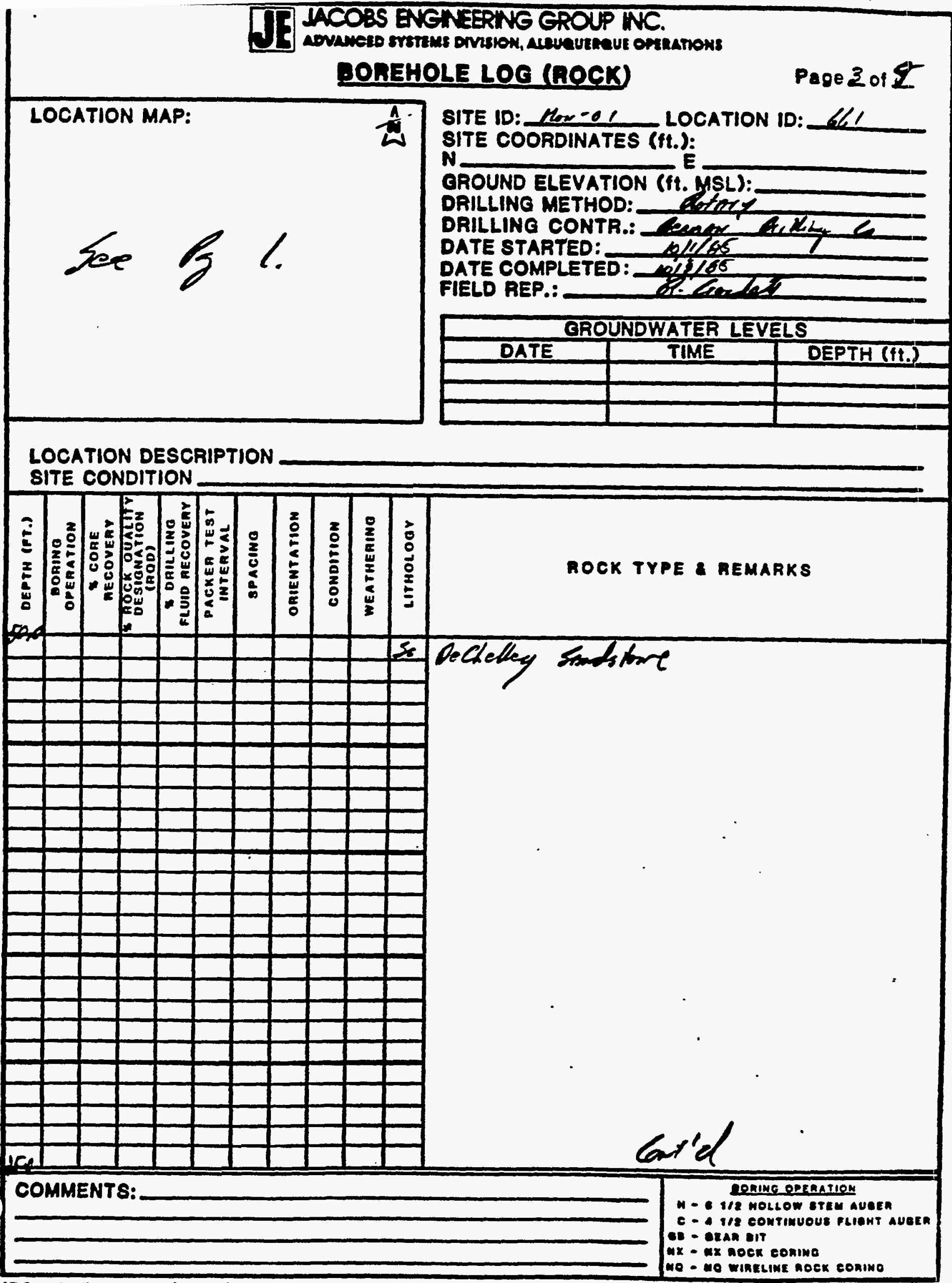



SITE COORDINATES (ft.):

N GROUND ELEVATION (ft. MSL):

DRILLING METHOD: DRILLING CONTR.:

DATE STARTED:

DATE COMPLETED:

FIELD REP.: E

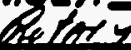

\begin{tabular}{|c|c|c|}
\hline \multicolumn{3}{|c|}{ QROUNDWATER LEVELS } \\
\hline DATE & TIME & DEPTH (fi.) \\
\hline & & \\
\hline & & \\
\hline
\end{tabular}

\section{LOCATION DESCRIPTION}

SITE CONDITION

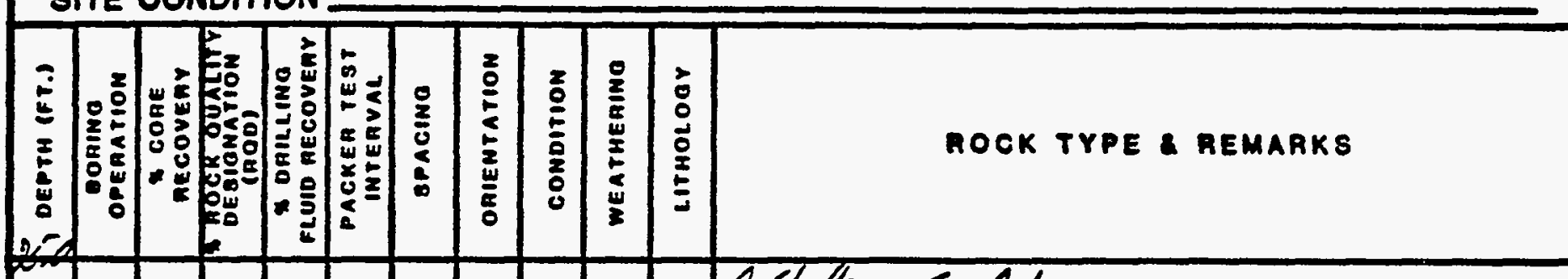

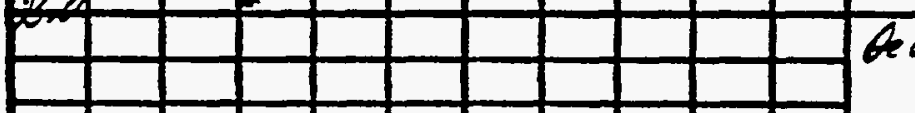

aldelley Sinditure

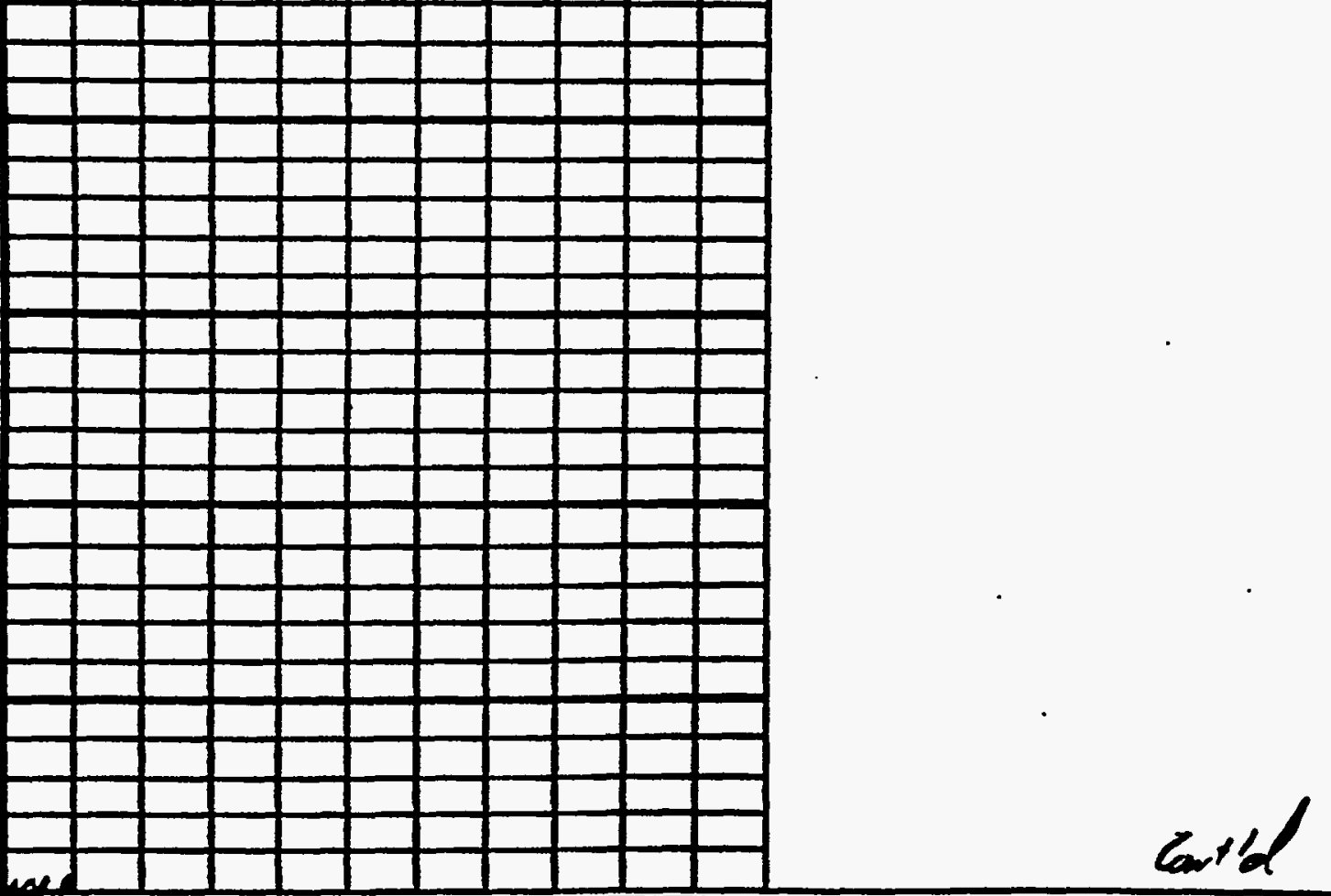

COMMENTS: 


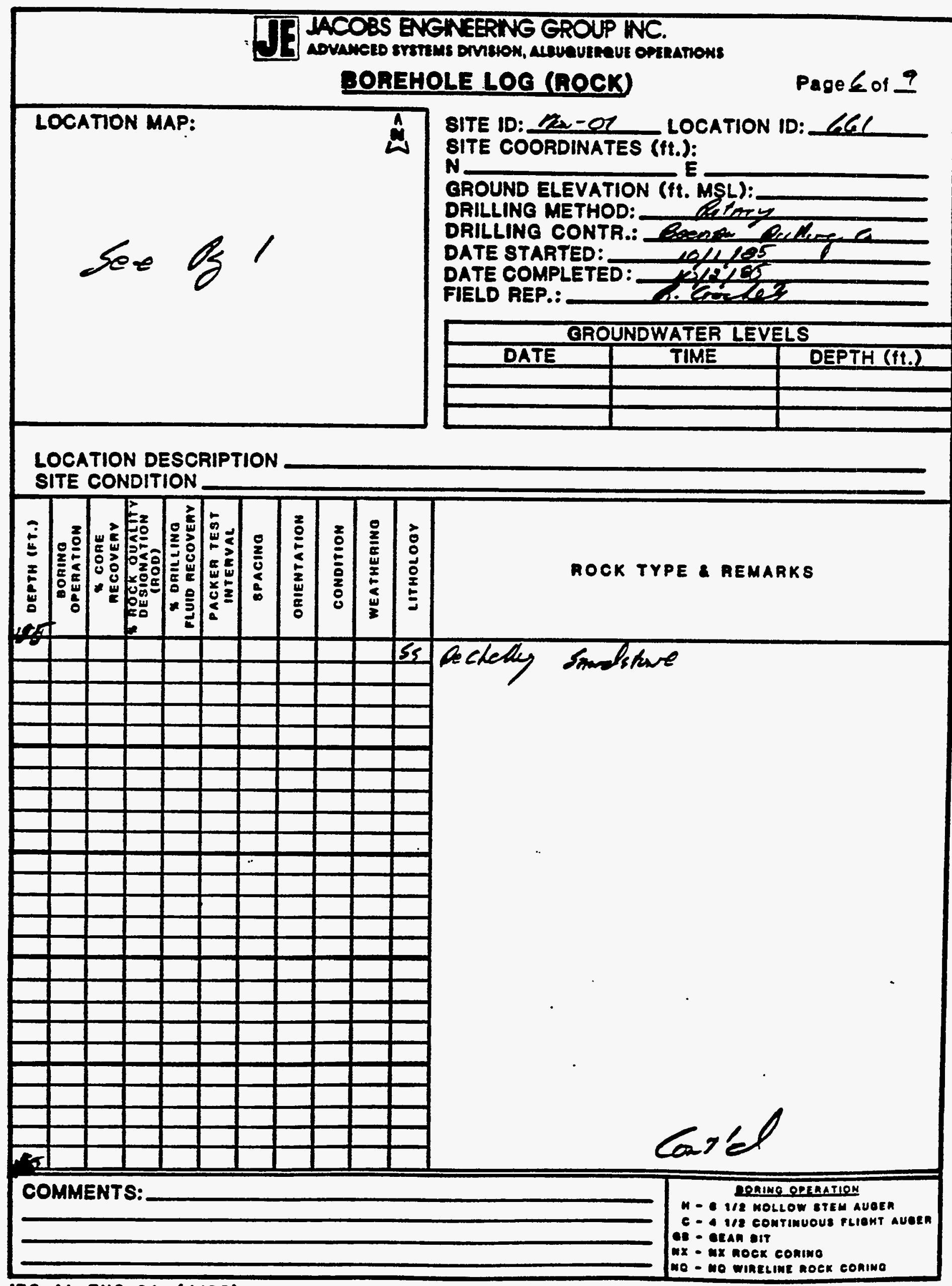

JEC-AL-ENO-2A (4/85) 
SITE ID: Aon-OT LOCATION ID:

LOCA

N

E

GROUND ELEVATION (ft. MSL):

DRILLING METHOD:

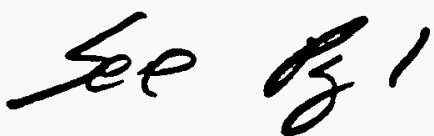

DRILLING CONTR:

DATE STARTED:

DATE COMPLETED:

FIELD REP.:

\begin{tabular}{|c|c|c|}
\hline \multicolumn{3}{|c|}{ GROUNDWATER LEVELS } \\
\hline DATE & TIME & DEPTH (ft.) \\
\hline & & \\
\hline & & \\
\hline & & \\
\hline
\end{tabular}

LOCATION DESCRIPTION

SITE CONDITION

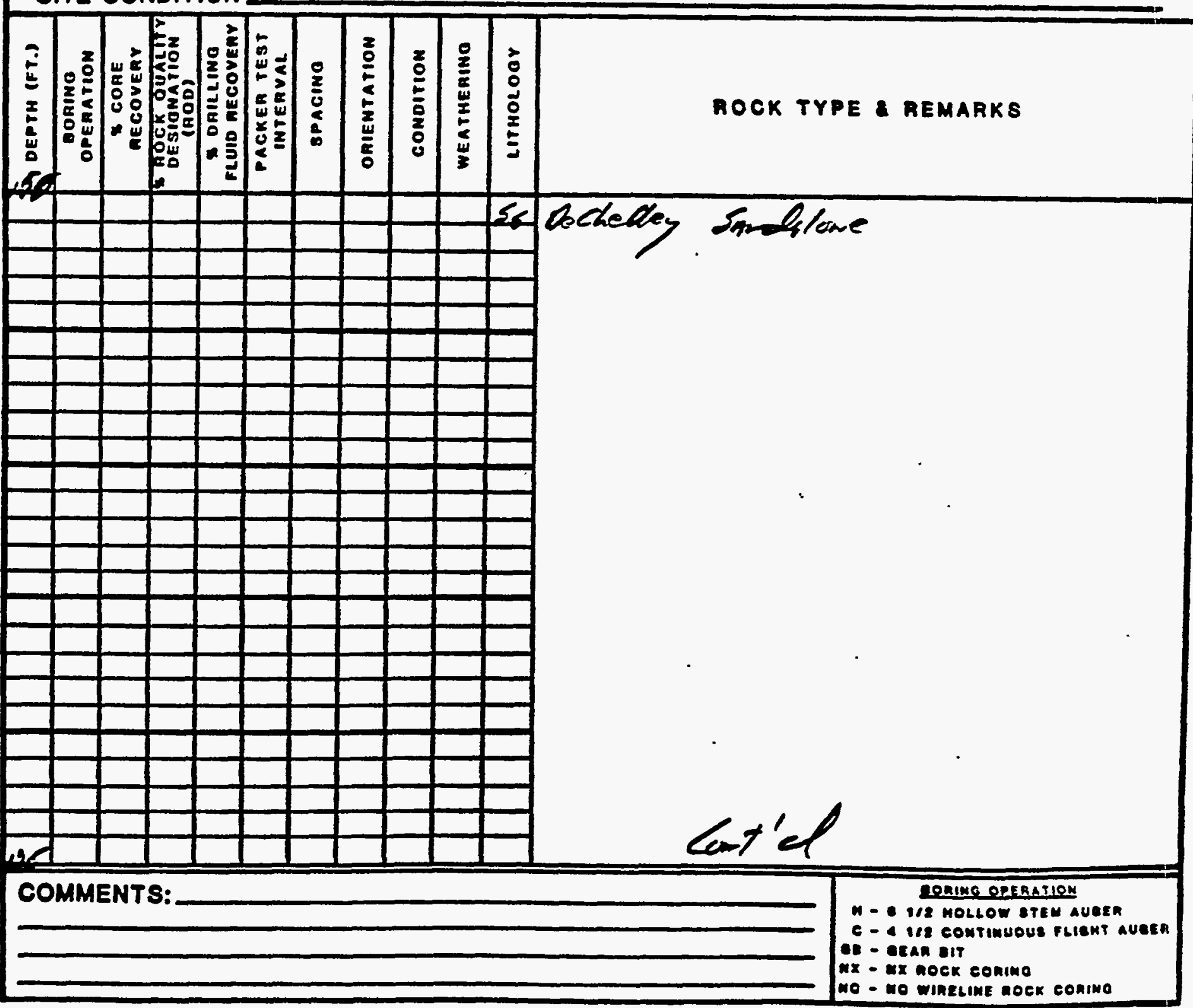

JEQ-AL-ENG-2A (4/85) 
IACOSS ENGNEERTH GROUP NC.

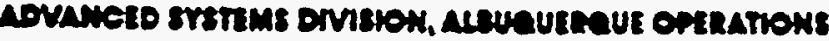

BOREHOLE LOQ (ROCK)

Page 2 of 9

LOCATION MAP: a

SITE ID: Mar-OI

LOCATION ID: G6I

SITE COORDINATES (ft.):

N.

E

GROUND ELEVATION (ft. MSL):

DRILLING METHOD:

DRILLING CONTR.:

DATE STARTED:

DATE COMPLETED:

FIELD REP.:

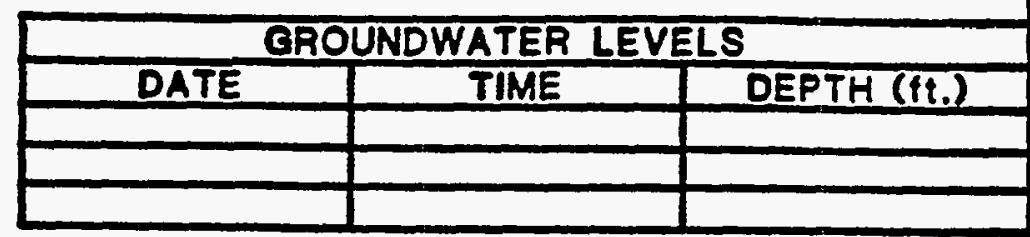

LOCATION DESCRIPTION

SITE CONDITION

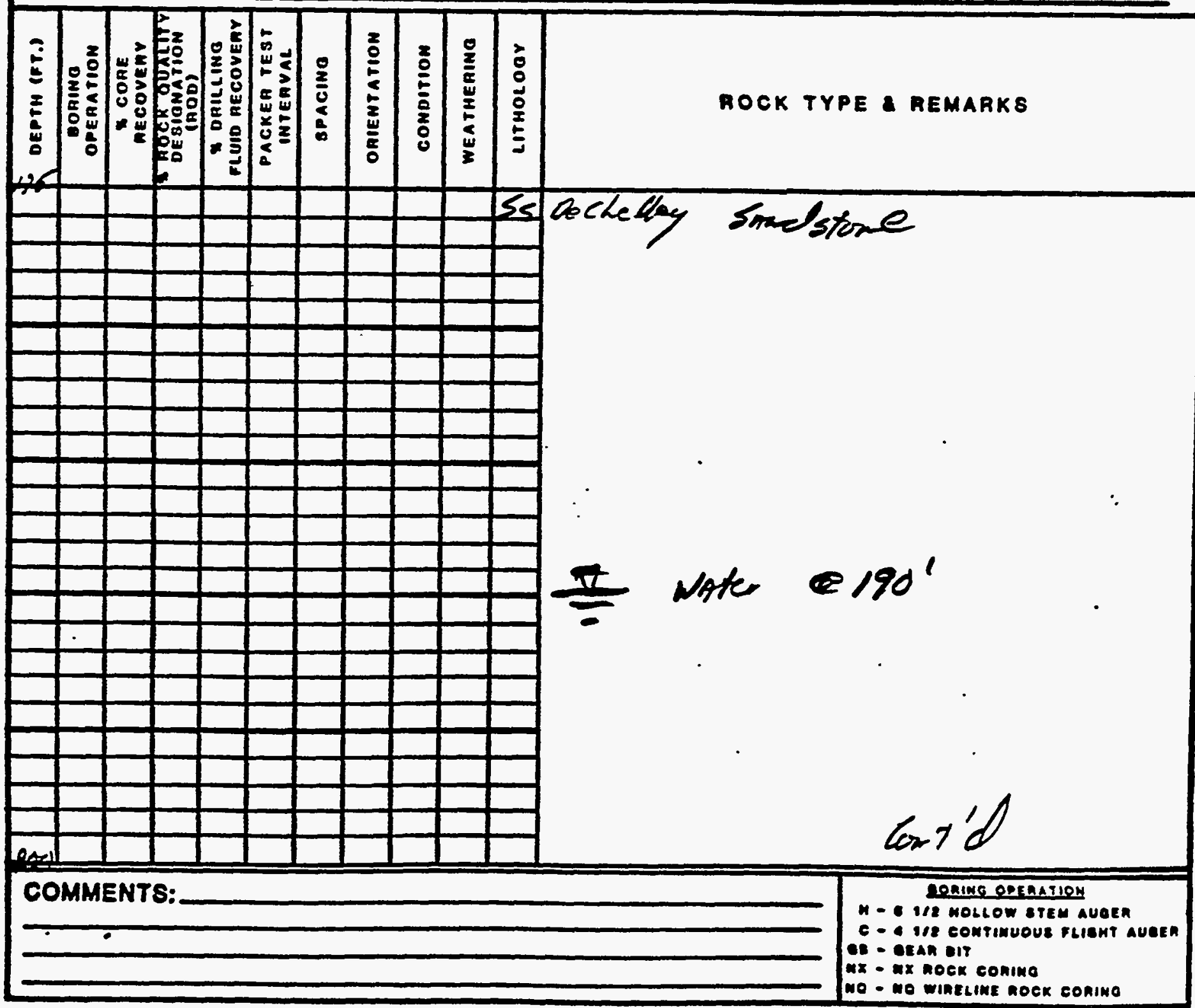

JEG-AL-ENG-2A (4/85) 
LACOBS ENCNEERING EROUP NAC.

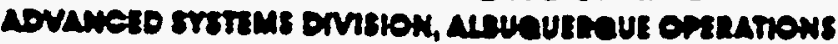

BOREHOLE LOQ (ROCK)

Page 2 of 9

LOCATION MAP:

$\operatorname{sog} 1$

LOCATION DESCRIPTION

SITE CONDITION

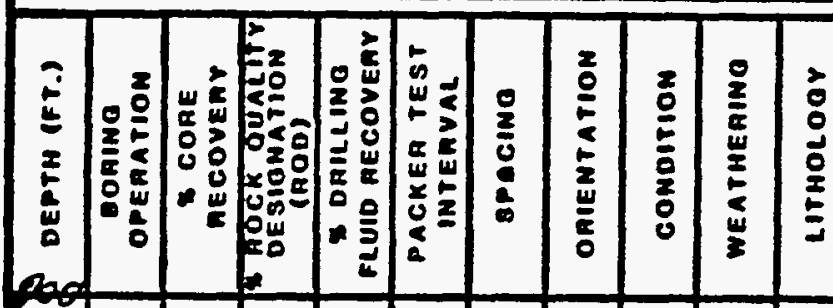

SITE ID: HM-OL LOCATION ID:

SITE COORDINATES ( $f$.):

$N$

GROUND ELEVATION (ft. MSL):

DRILLINO METHOD: tethey

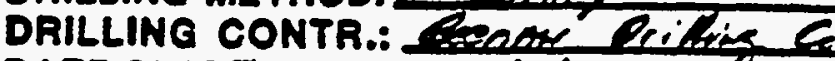

DATE STARTED:

DATE COMPLETED:

FIELD REP.:

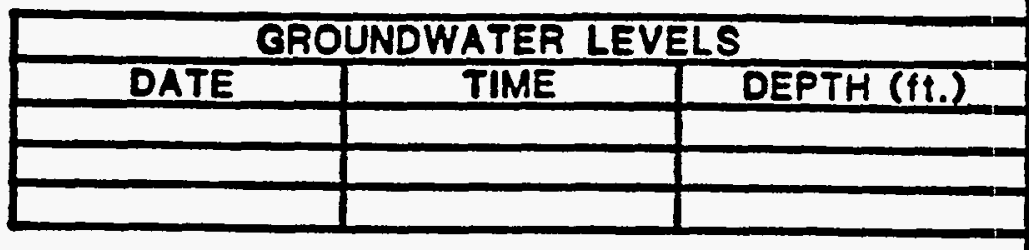

\section{F}

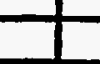

\section{Aochelley Srrudstere}

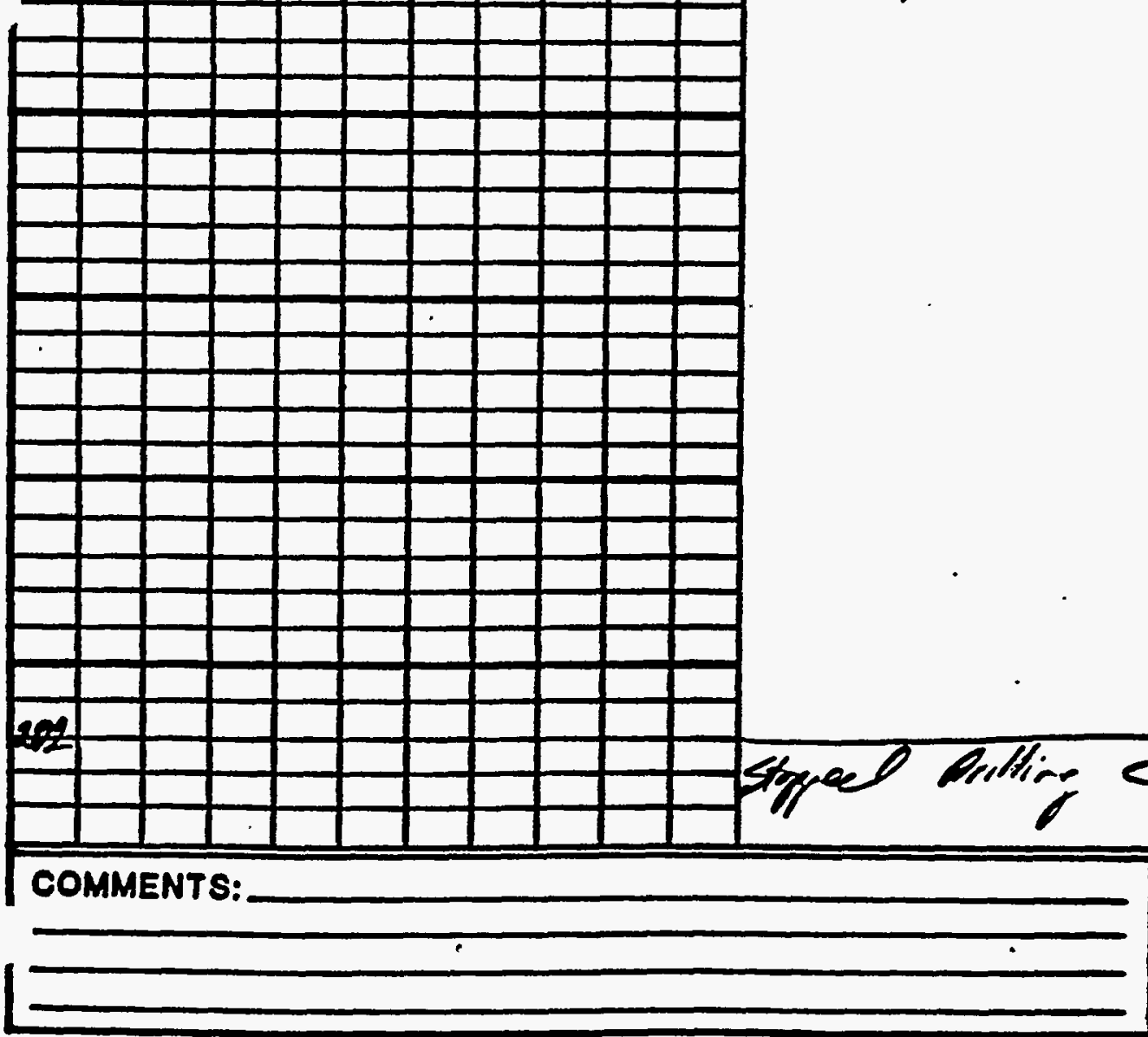


LOCATION MAP: SEE LOG OF HOLE 661. HOE G6IR $\approx 21^{\prime}$ EAST OF 661

w

SITE ID: MON. OI SITE COORDINATES ( $f t.):$

N

GROUND ELEVATION (ft. MSL):

DRILLING METHOD: ROTARY AIR $83 / 4 " 87 / 0^{\prime \prime}$

DRILLING CONTR:: BEEMAN DRILLING CO

DATE STARTED: 10-5-8S

DATE COMPLETED: $10-6-85$

FIELD REP.: IC. DONNELSON/SH2B GEOLOGST

LOCATION DESCRIPTION SOUTHWEST SIDE OF CANE WASH BASIN ON ROCK. SITE CONDITION EAST DIPPING SHINARUMP OUTCROP.

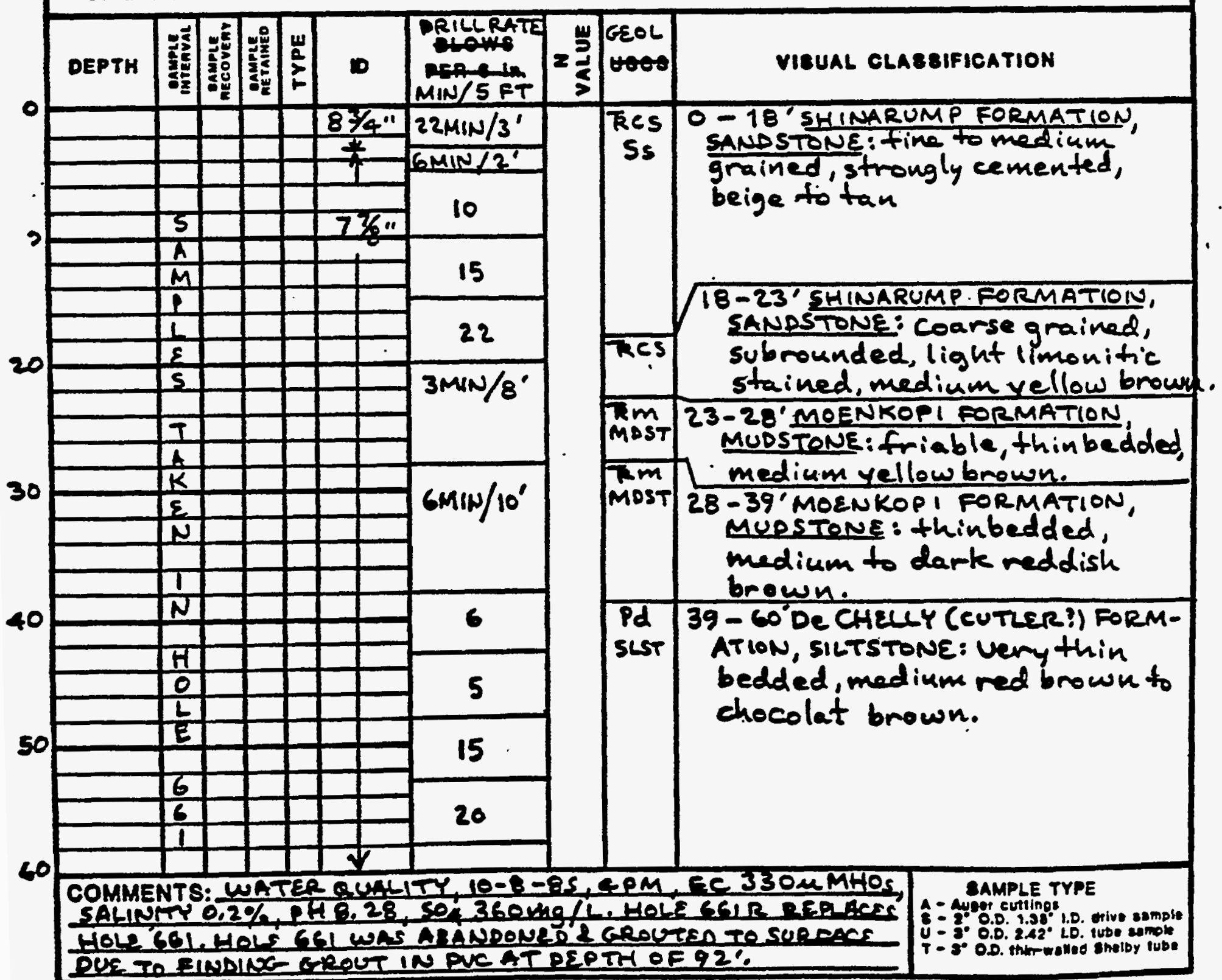




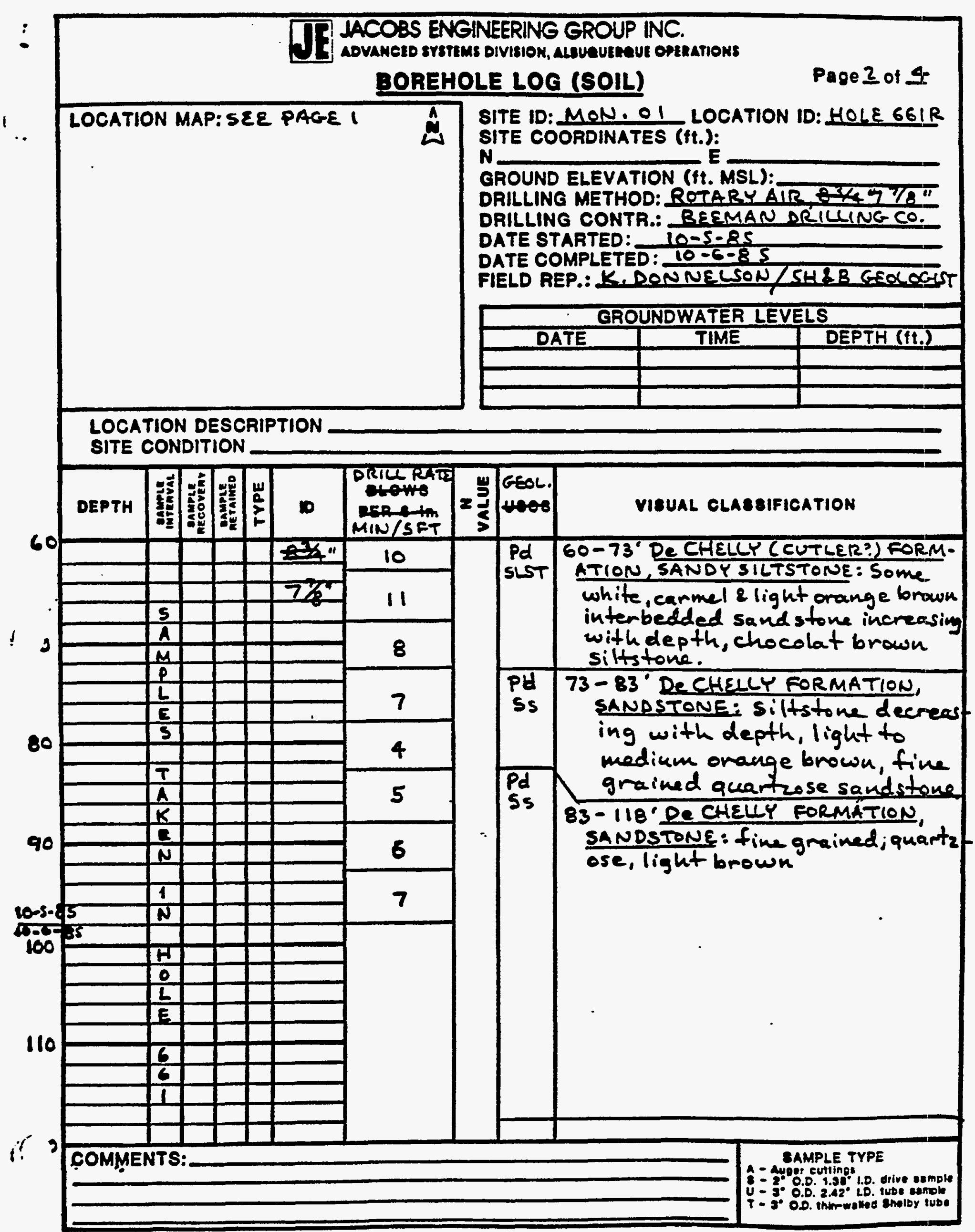




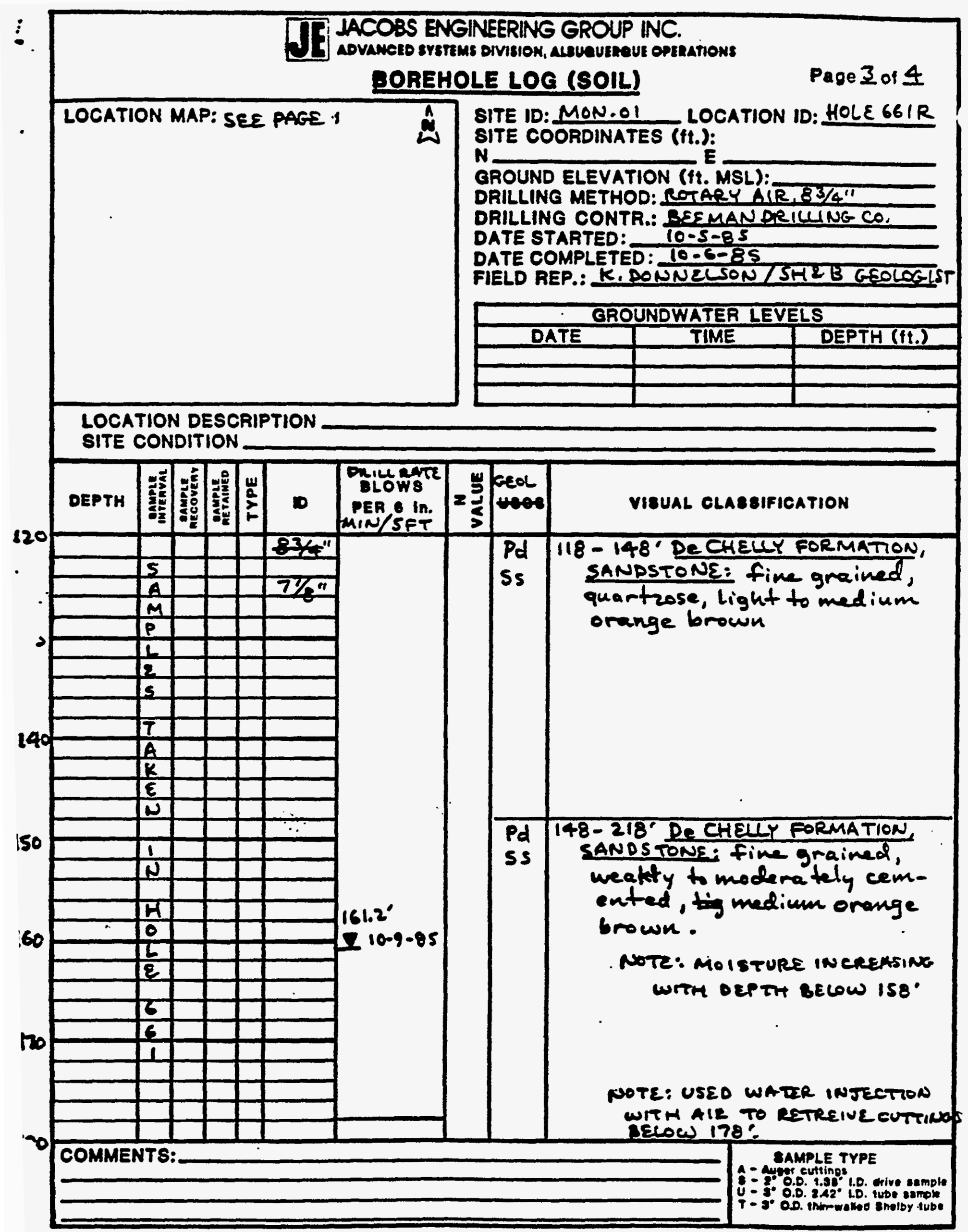


TI IACOBS ENGINEERING GROUP INC.

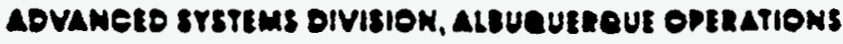

BOREHOLE LOG (ROCK)

Page lof 1

LOCATION MAP:

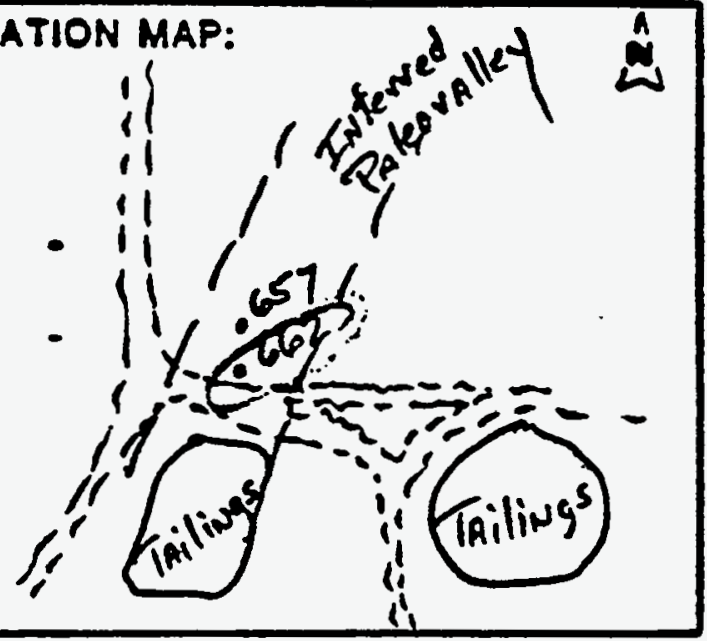

SITE ID: MON LOCATION ID: 662 SITE COORDINATES ( $f t$.$) :$

N E

OROUND ELEVATION (11. MSL):

DRILLING METHOD:_Rotary (mUD)

DRILLING CONTR.: Bab Breman

DATE STARTED:

Sept 58,85

DATE COMPLETED:

FIELD REP.: Sebt 585

G. mille?

\begin{tabular}{|c|c|c|}
\hline \multicolumn{3}{|c|}{ GROUNDWATER LEVELS } \\
\hline DATE & TIME & DEPTH (19.) \\
\hline & & \\
\hline & & \\
\hline & & \\
\hline
\end{tabular}

LOCATION DESCRIPTION

North

sot of old tailings pile

SITE CONDITION

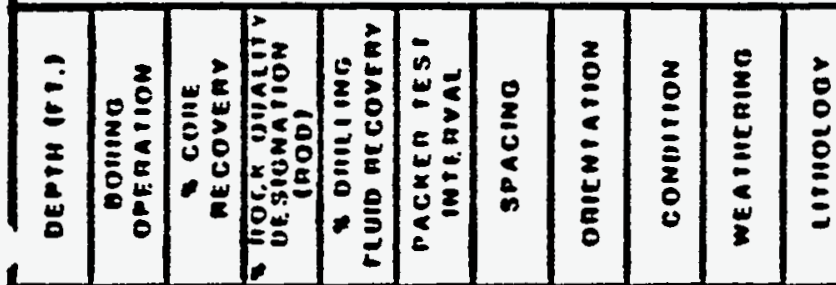

ROCK TYPE 8 REMARKS

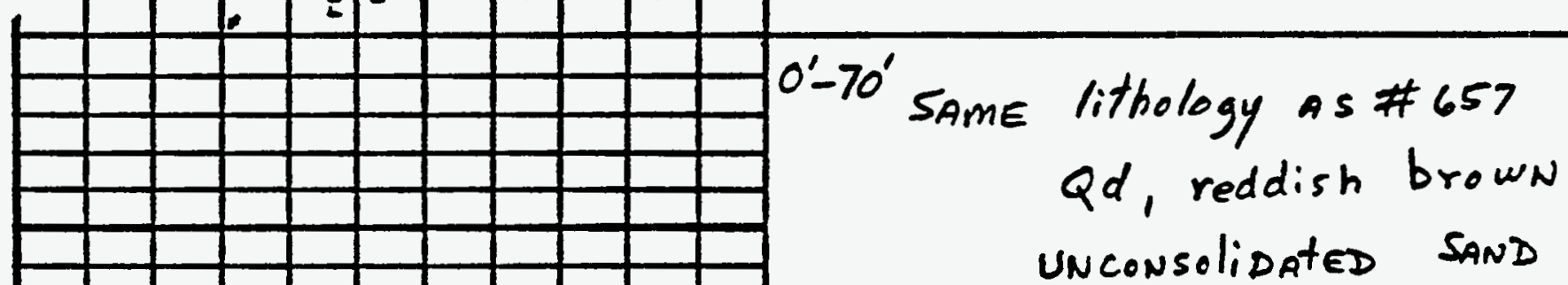

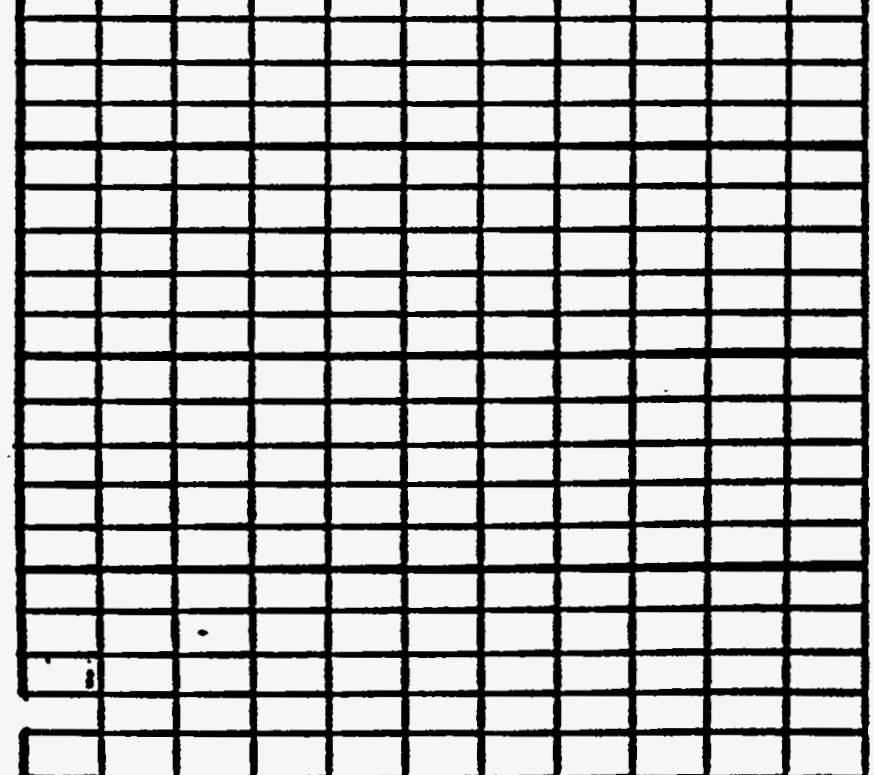

\section{COMAENTS:}




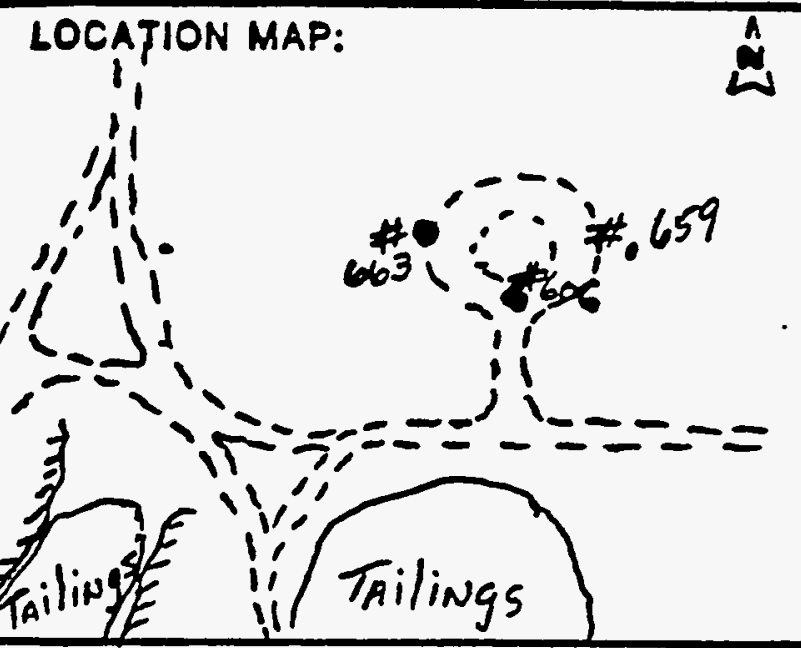

SITE ID: MON SITE COORDINATES (tt.):

LOCATION ID: \#663 N GROUND ELEVATION (\&H MSL):

DRILLING METHOD: RoTARY (muD) DRILLING CONTR.: $\frac{\beta 06 \text { Beeman; MaAb, UT, }}{9.6 .85}$ DATE STARTED:

DATE STARTED:

DATE COMPLETED: 9.10 .85

FIELD REP.:

\begin{tabular}{|c|c|c|}
\hline \multicolumn{3}{|c|}{ GROUNDWATER LEVELS } \\
\hline DATE & TIME & DEPTH (i1.) \\
\hline & & \\
\hline & & \\
\hline & & \\
\hline
\end{tabular}

\section{LOCATION DESCRIPTION}

SITE CONDITION

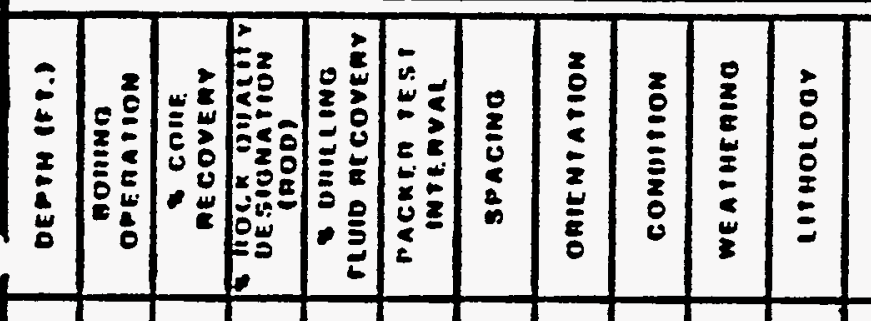

ROCK TYPE I REMARKS

Ol 50' Qunternary Dune sand and possible Allurium; ReDsish brown, fine to mevium geained quartz sand with minare fine gravel (mostly composeo of weathered out Shinarump pebbles aro miare Moentofi rock fangments)

*50' approximate top of Shinarump sariostawe, whitish. Tan to irow stained sandstowe cuttings, some cOARSE SAND AND bRAKEN pebbles, borehole begins making $\mathrm{H}_{2} \mathrm{O}$ at a stenoy Rute of $5 \mathrm{gpm}$ (estimate) feom about $50^{\prime}$ down for 5'-7; then conversely starteo using $\mathrm{H}_{2} \mathrm{O}$ feom

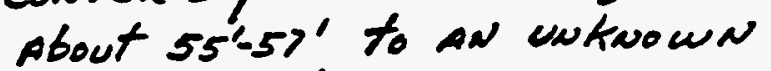
geenter atepth

50'- $115^{\prime}$ gm Shinareump sandstowe, taw, whitet ieew staived, etco, *107: 110' becomes very conese to couglomeentic with

COMMENTS: 


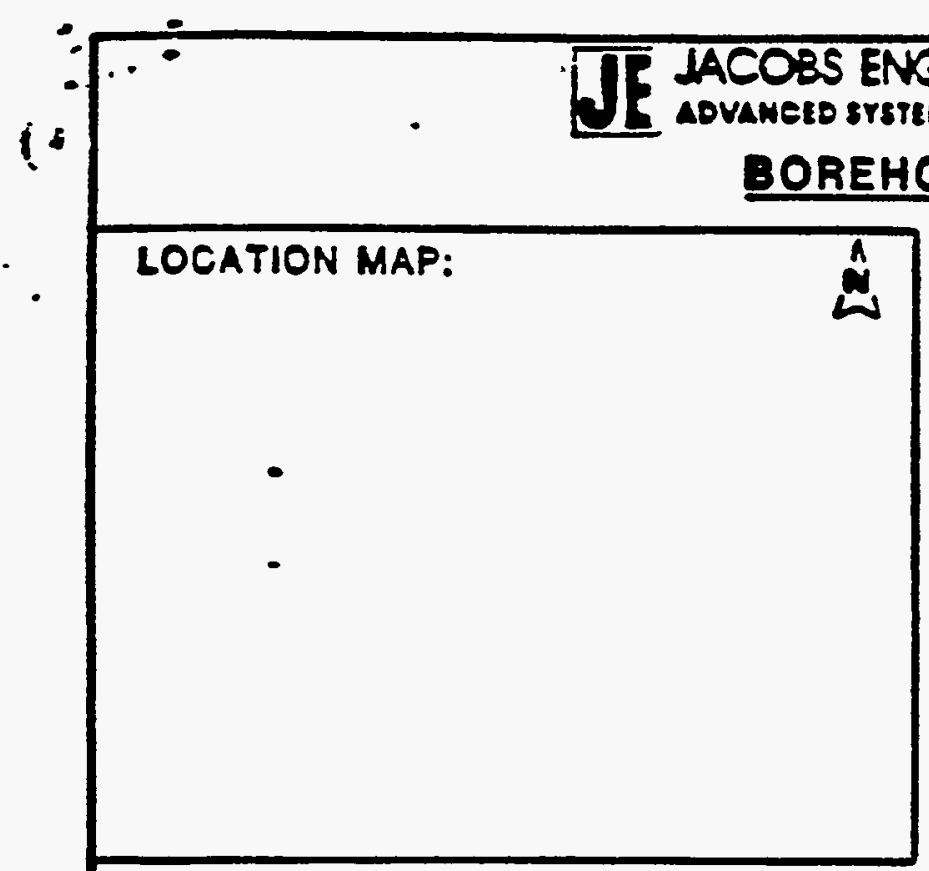

LOCATION MAP:

SITE ID: MON

$N$

GROUND ELEVATION (ft. MSL):

DRILLING METHOD: Petary (mUD)

DRILLING CONTR.: Bab Beeman

DATE STARTED:

Seet 5. 1985

DATE COMPLETED: $\frac{\text { sept. } 10,1585}{\text { Thille }}$

FIELD REP.:<smiles>[124IH]</smiles>
SITE COORDINATES (ft.)

LOCATION ID: \#663

\begin{tabular}{|c|c|c|}
\hline \multicolumn{3}{|c|}{ GROUNDWATER LEVELS } \\
\hline DATE & TIME & DEPTH (4t.) \\
\hline & & \\
\hline & & \\
\hline & & \\
\hline
\end{tabular}

LOCATION DESCRIPTION

SITE CONDITION

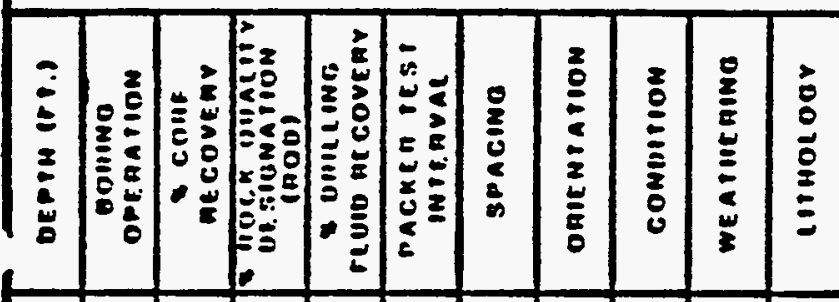

nOCK TYPE \& REMARKS

Shivarump (Cont) 107-110(cont)

with abuidant coaly material in the cuttings and on the mud pit

Borehole has intercepted prebable

major chaninel fill in Shiniarump.

* 1120' REDDish brou'e siltstone w/ blukish gray altered. (bleached) siltstone cuttings Top of Mönkagi Fm e 120 Approx. Top!

167' MoEnkopi Fm - reddish brown

sltstn. w/ minor sAnidstone '167'-217' Dechelly Ss. (c) 167' -

reddish to orangish, medium to fine grainED SANDSTONE

$217^{\prime}=T D$

COMMENTS: 


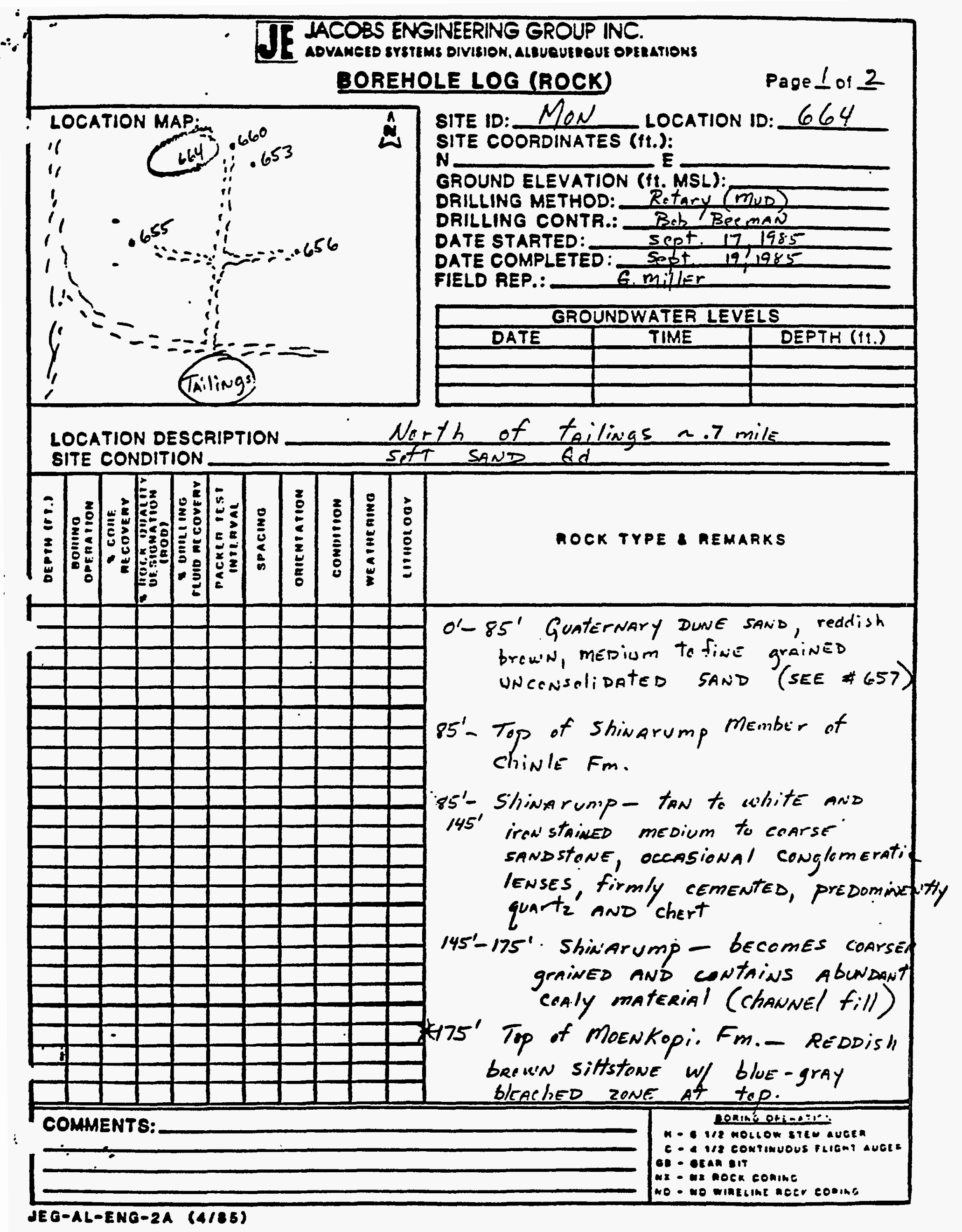


N. E

GROUND ELEVATION ( $t 4, M S L)$ :

DRILLING METHOD: $7 \%$ " ROTARY, MUDGEL

DRILLING CONTR.: BOB BEEMAN

DATE STARTED: $10-3-85$

DATE COMPLETED: $10-4-8 \delta$

FIELD REP.: $K . D$. DONNELSON / FHEB GEOLOAIT

\begin{tabular}{|c|c|c|}
\hline \multicolumn{3}{|c|}{ GROUNDWATER LEVELS } \\
\hline DATE & TIME & DEPTH (ft.) \\
\hline & & \\
\hline & & \\
\hline & & \\
\hline
\end{tabular}

LOCATION DESCRIPTION 26 MILES SOUTH OF MEXICAN HAT

SITE CONDITION ROLLING SAND DUNES, ORANGE, EINE (SP) SAND, SPAPSE VEG:

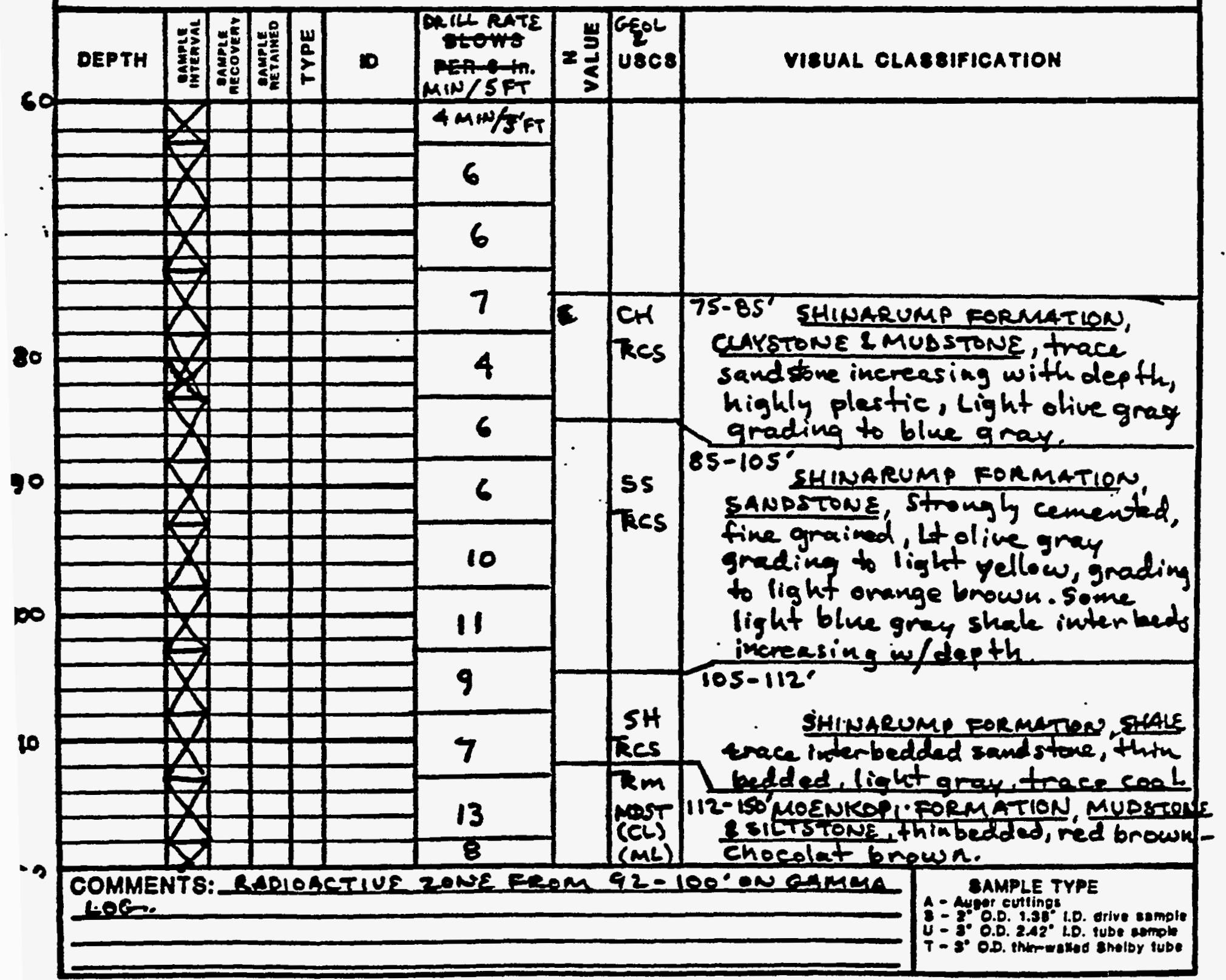

JEG-AL-ENG-28 (4/85) 


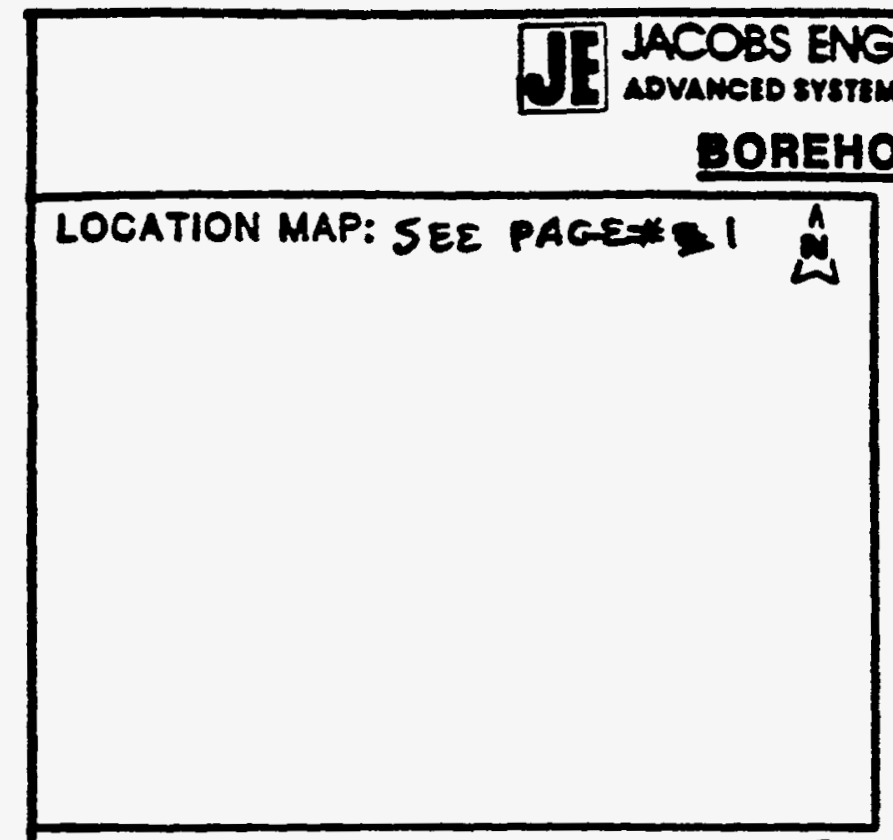

INEERING GROUP INC.

MS DIVISION, ALLUVUEREU OPERATIONS

BOREHOLE LOG (SOIL) Page 4 of 4

SITE ID: MON - OI LOCATION ID: HOLE 668

SITE COORDINATES (ft.):

N. E

GROUND ELEVATION ( $(1, M S L):$

DRILLING METHOD: 748 "ROTARY MUDGE

DRILLING CONTR.: B.OB BEEMAN

DATE STARTED: $10-3-35$

DATE COMPLETED: $\frac{10-4-65}{10}$

FIELD REP.: LE. DONNEISON/SHLBGSOLOGIST

\begin{tabular}{|c|c|c|}
\hline \multicolumn{3}{|c|}{ CROUNDWATER LEVELS } \\
\hline DATE & TIME & DEPTH (ft.) \\
\hline & & \\
\hline & & \\
\hline
\end{tabular}

LOCATION DESCRIPTION 26 MILES SOUTH OF MEKICAN HAT

SITE CONDITION ROLLUNE SAND DUNES, OPANCE FINE (SP) SAND, SPARE VEG.

80

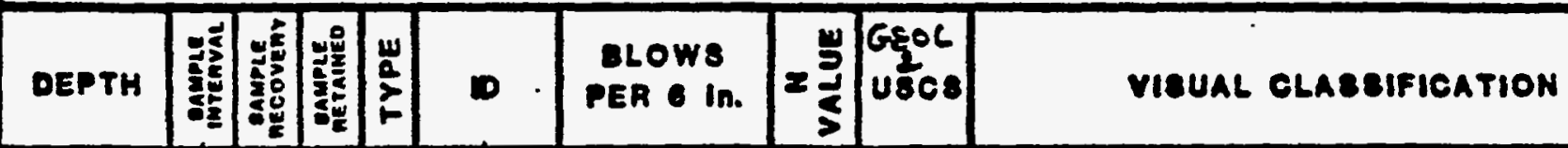

Do

10
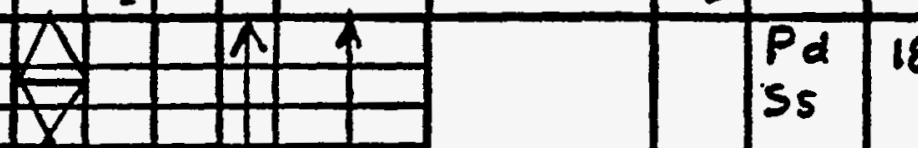

80-218' De CHELY EORMA TION,

SANOSTONE, fire grained medium red erange brown.

18

8

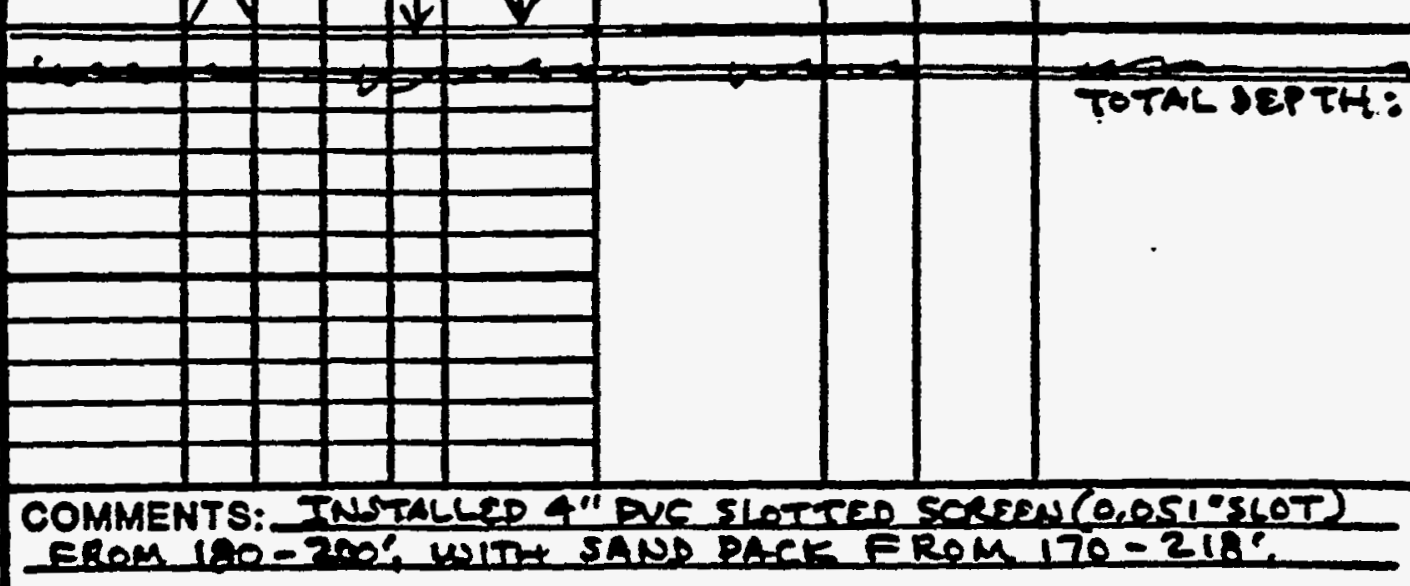

SAMPLE TYPE

A. - Ayeor cutting

-

4 - 30 0. $242^{\circ}$ LO tube semole

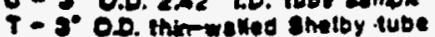

IEQ-AL-ENG-2S (4/8E) 
LOCATION MAP: See Location Map of Hole 668. ₹ $15^{\circ}$ 'sourt of 658

ఉ

8ITE ID: MON. OI BITE COORDINATES (ft.):

N. GROUND ELEVATION (It, MSL):

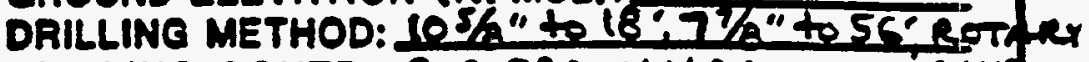
DRILLING CONTR.: BOB RERMAN DRILUWE MU. DATE STARTED: $\frac{10-5-85}{10-5-85}$

DATE COMPLETED: $10-5-85$

FIELD REP.: $1 C$. DONNELSON/SHB GEOLOGIST

LOCATION DESCRIPTION 26 MILES SOUTH OF MEXICAN HAT

BITE CONDITION ROLUNE SAND DUNES, DRANGE EINE(SP) SAND, SAARSE VECE

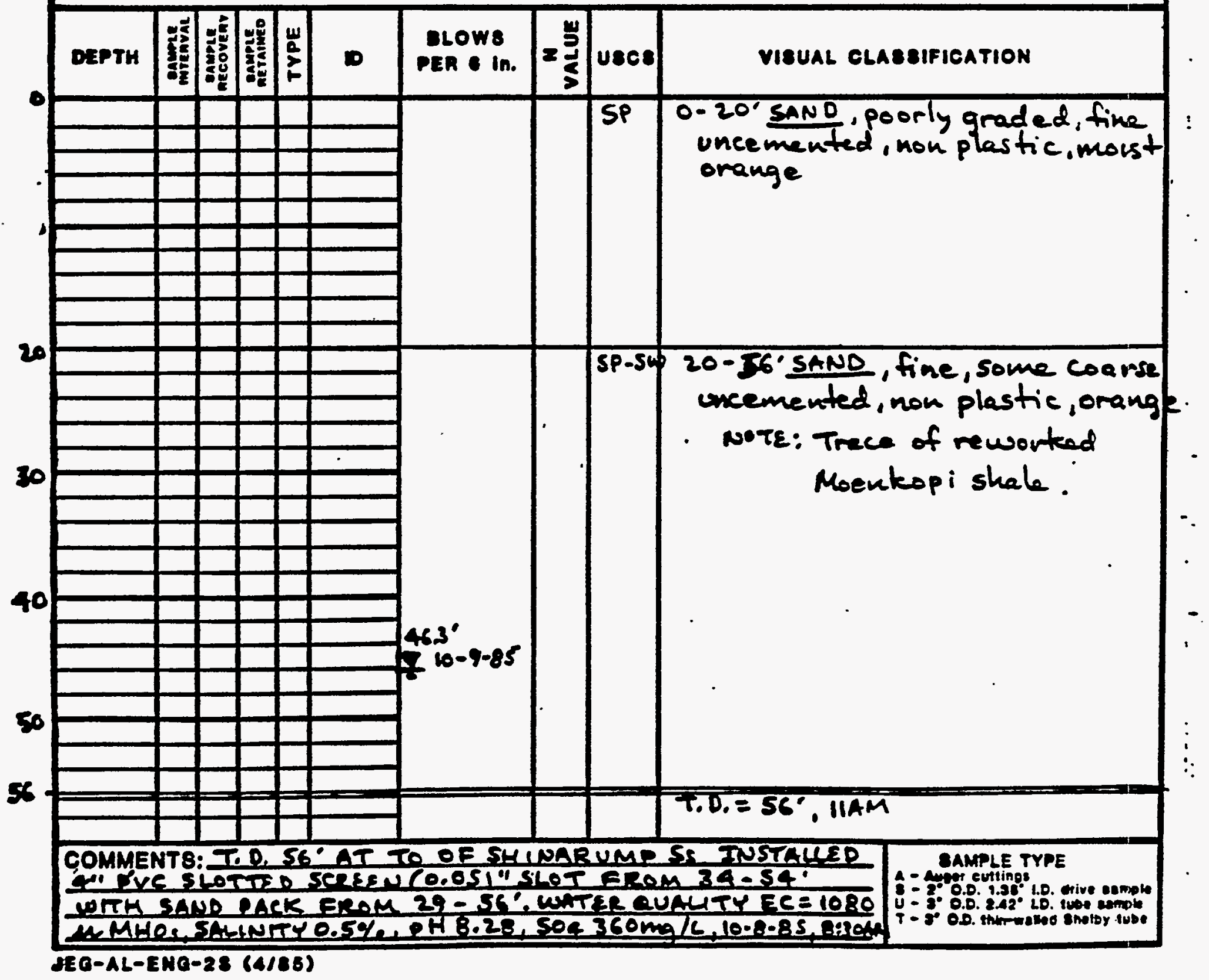




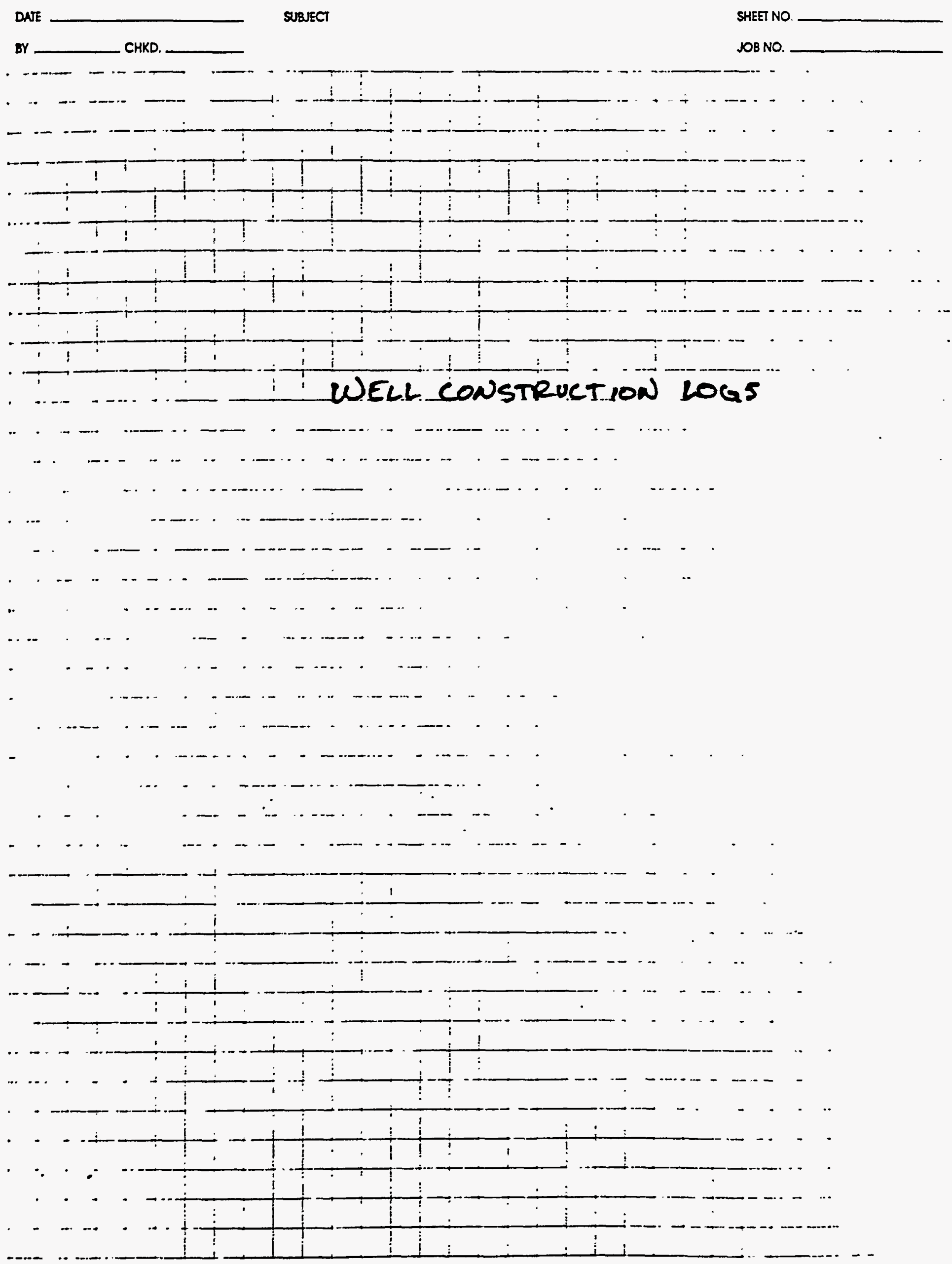

TI HCONS ANGNERANG GROUP NNC.

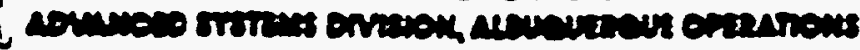

\section{MELL COMPLETION RECORD}

SITE 1D: MaNol LOCATION ID: DATE installed: $10-24-84$ APPROX. OITE COORDINATE8:(FT.) $\mathrm{N}$ OPEN AREA PER LINEAL FT. (IN2/FT.)

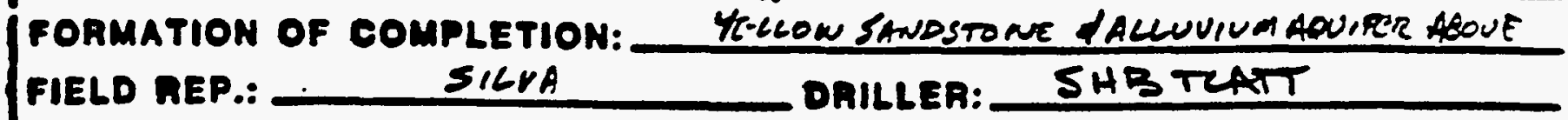

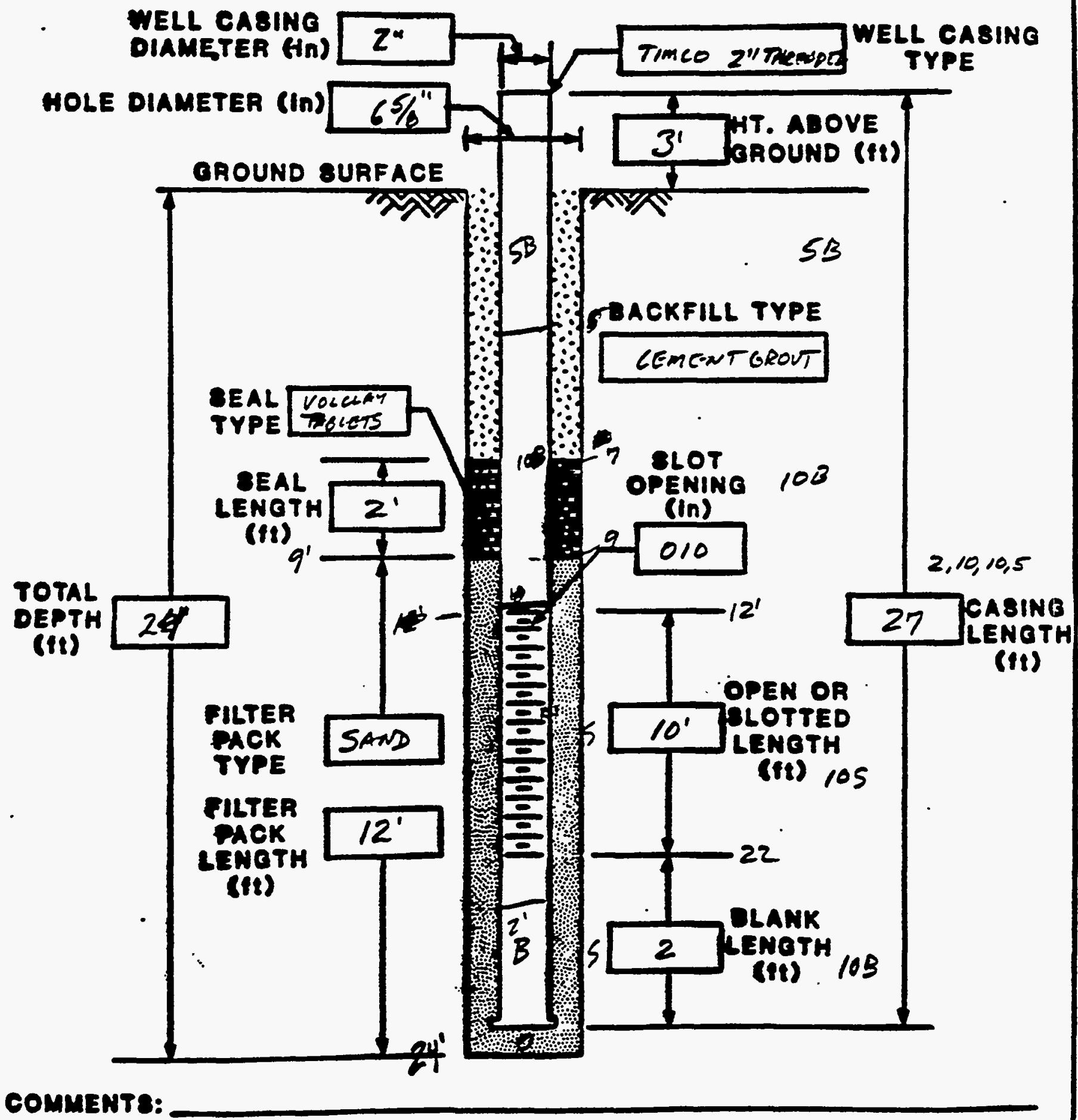

COMMENT8: 


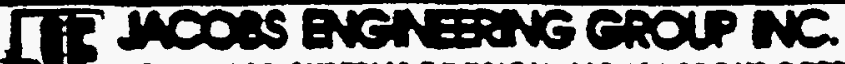 \\ - 15 comses ow \\ OOREHOLE}

OTE ID: M ONOL LOCATION ID:MN-1-2 FOLLD REP: SILVA APPAOX. OITE COORDINATES (FT.): N NOTSURVYYEP E CNOUnD ELEVATION (FT. MBL): COMPLETION DATE: $10 \cdot 24.8 \mathrm{U}^{\prime}$

DOREHOLE CMMMARY

OALLER: $S H B-T E D C A T T$

MO TrPE: CME 55

\begin{tabular}{|c|c|c|c|c|}
\hline on $m$ & AE $\begin{array}{l}\text { MPLE } \\
\text { OAA.; }\end{array}$ & gefor & & $\begin{array}{l}\text { UID } \\
\text { YPE }\end{array}$ \\
\hline HSA & $65 / 81$ & 35 & $N A_{T}$ & $\mathrm{H}=\mathrm{O}$ \\
\hline & & & & \\
\hline & & & & \\
\hline & & & & \\
\hline & casing & JMMARY & & \\
\hline CABING & DESCRIP & ION & pin. & DEPT \\
\hline & & & & \\
\hline $\bar{p}$ & $4^{\prime}$ STEEL LO & Kiake AP & $6^{\prime \prime}$ & $0-4$ \\
\hline$B$ & $z^{\prime} \quad B C A N$ & & $2^{\prime \prime}$ & $2-0$ \\
\hline 81 & $20^{\prime} \quad 5 \angle A$ & & $2^{11}$ & 2202 \\
\hline 5 & $10^{\prime}$ SCREE & ED & $2^{4}$ & $32-22$ \\
\hline$B$ & $Z^{\prime}$ BLANK & & $2^{11}$ & $34-32$ \\
\hline & & & & \\
\hline Pratioce & ethe D-loraen & $\operatorname{ang}$ & $m$ & manom \\
\hline SDeoth hr & con Tos at capher & & & \\
\hline & WELL CON & ThUetiol & & \\
\hline $\begin{array}{l}\text { TYPE } \\
\text { COOEE }\end{array}$ & sesen & PTION & & \\
\hline & & & & \\
\hline & & & & \\
\hline & 4010 & momen Besm & & \\
\hline $\bar{B}$ & $2^{1+3}$ cement 6 & $5 T A 4 \% B$ & Eminges & $3-0$ \\
\hline 5 & HOLCLAYRELL & 75254 & & $6-3$ \\
\hline $\bar{F}$ & Narreal cooce & WWSAND SP & & $35^{\prime}-6$ \\
\hline & & & & \\
\hline-8.1 & Dation & $2 \mathrm{eal}$ & $-\sqrt{n}$ & Pent \\
\hline - $8 \mathrm{sen}$ & 7 & & & \\
\hline
\end{tabular}

CONBTRUCTION TIME LOQ

\begin{tabular}{|c|c|c|c|}
\hline \multirow{2}{*}{ ACTIVITY } & \multicolumn{2}{|c|}{ otant } & \multirow{2}{*}{$\begin{array}{l}\text { END } \\
\text { TIME }\end{array}$} \\
\hline & DAte & TIME & \\
\hline DAILLING & $18-2484$ & $12: 00$ & $3: 0$ \\
\hline CaBing & & $3: 00$ & $3: 75$ \\
\hline FILTER PACK & & $3: 20$ & $5: 2$ \\
\hline BEAL & & $3: 25$ & $3 \cdot 3$ \\
\hline BACKFILL & & $3: 30$ & $3: 4$ \\
\hline $\begin{array}{c}\text { DEVELOPMENT } \\
\text { FLUSHING }\end{array}$ & & $3: 15$ & $3: 20$ \\
\hline $\begin{array}{l}\text { OTIER } \\
\text { CLEATUP }\end{array}$ & & $3: \$ 0$ & $4: 0$ \\
\hline
\end{tabular}

\section{NELL DEVELOPMENT}

PIPE FLUSHED WITH RIG WATRE FROM STNGUANRIVER

PHATURAZ SANO USED FOR FILTER PACX IECHUSE OF HOLE CAUING !

Commants: HoLE was caving CABING WAS PLAED INSIDE AUGER THEEN - AVGER WAS PULCEDOUT AFTER AUbEA WAS DUT HOLE AAD CAVEDQG'BEZOWSURFAC 
EITE ID: MON O I LOCATION ID: MN-1-3 DATE INSTALLED: 10_Z4.84 APPROX. BITE COORDINATE8:(FT.) N NOTSUFIR.NT:/ E OPEN AREA PER LIMEAL FT. (IN2/FT.) FORMATION OF COMPLETION:
FIELD REP.:

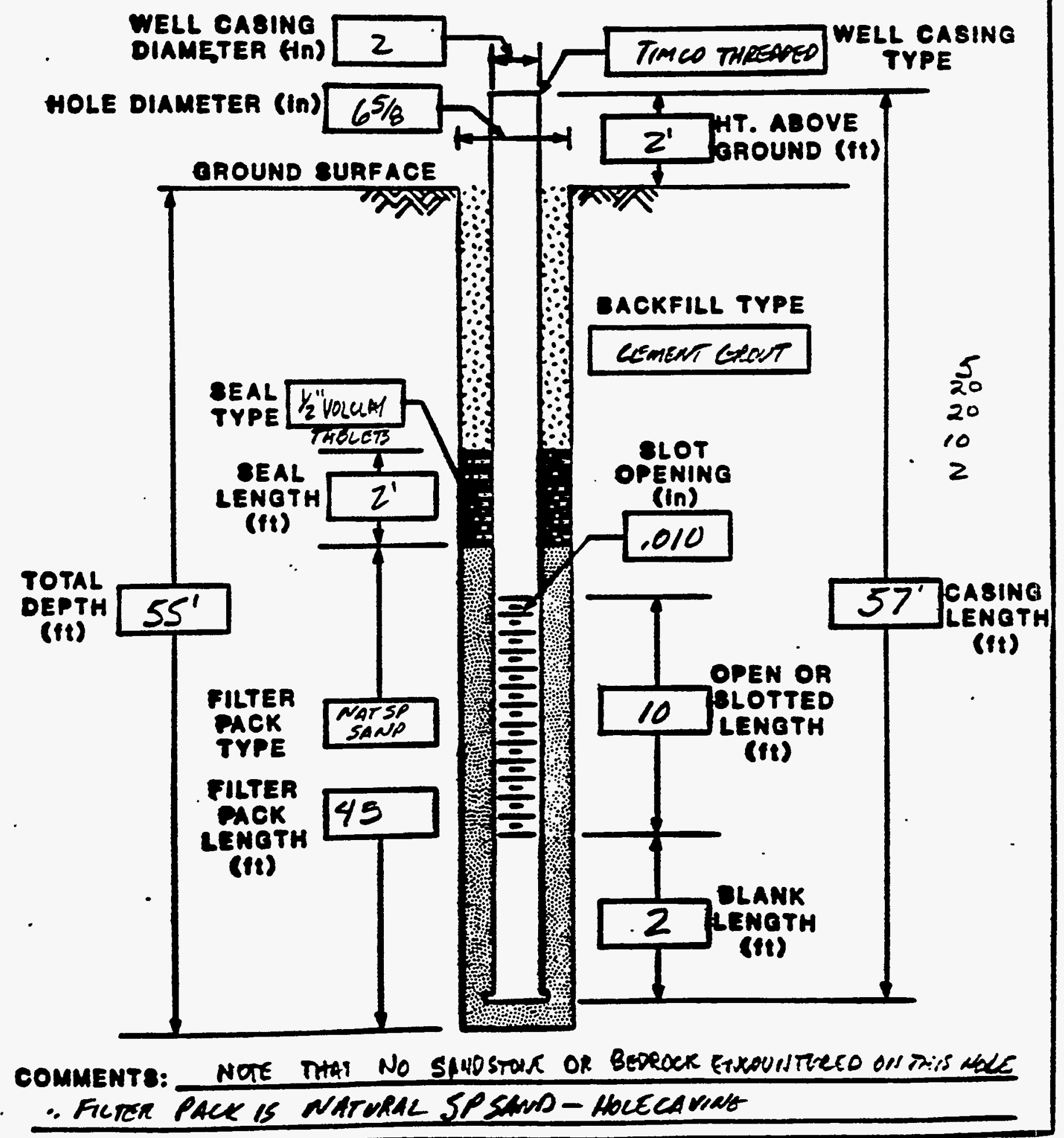




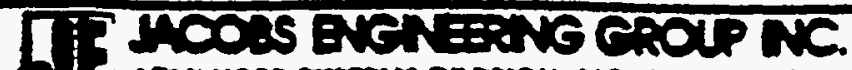

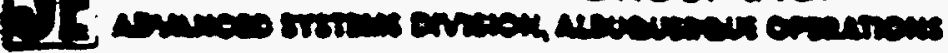 \\ DONEHOLENELL GONBTHUCTION LOG}

IITE ID: MONOI LOCATION 1D:ANL-1-3 GOJ PLLD REP: SIWA

APPROX. OITE COORDINATES (FT.): $N$ NOT SURUEYCD

CAOUND ELEVATION (FT. MBL):

COMPLETION DATE: $10-25 \cdot 84$

DOREHOLE CUMMARY

ONILLER: TUAT SHB

NIO TYPE: CME 55

\begin{tabular}{|c|c|c|c|}
\hline DIT TYPE & $\begin{array}{l}\text { POLE. } \\
\text { 9iA.) }\end{array}$ & ofyp & Trupp \\
\hline HSA Fivir & $65 / 3$ & $55^{\prime}$ & NAT $\mathrm{H}_{2} \mathrm{O}$ \\
\hline & & & \\
\hline & & & \\
\hline
\end{tabular}

CABING SUMMARY

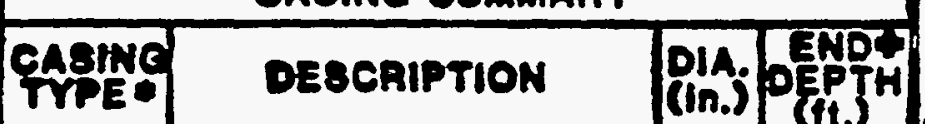

\begin{tabular}{|c|c|c|c|}
\hline$P 4^{\prime}$ & STECZ LAKING CAP & $6^{\prime \prime}$ & $0--2$ \\
\hline $85^{\circ}$ & BUANK & $2 " 1$ & -3 \\
\hline 820. & Eexth & $2^{\prime \prime}$ & 23 \\
\hline $120^{\circ}$ & BCANK & $2^{\prime \prime}$ & 13 \\
\hline $510^{\prime}$ & .010 slatres seeEeN & $2^{\circ}$ & 53 \\
\hline$B 2$ & $Z^{\prime}$ SLANK & $2^{*}$ & 55 \\
\hline
\end{tabular}

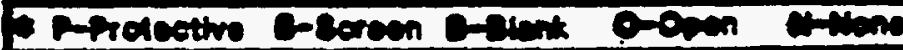
Someth wein Tce al coshe

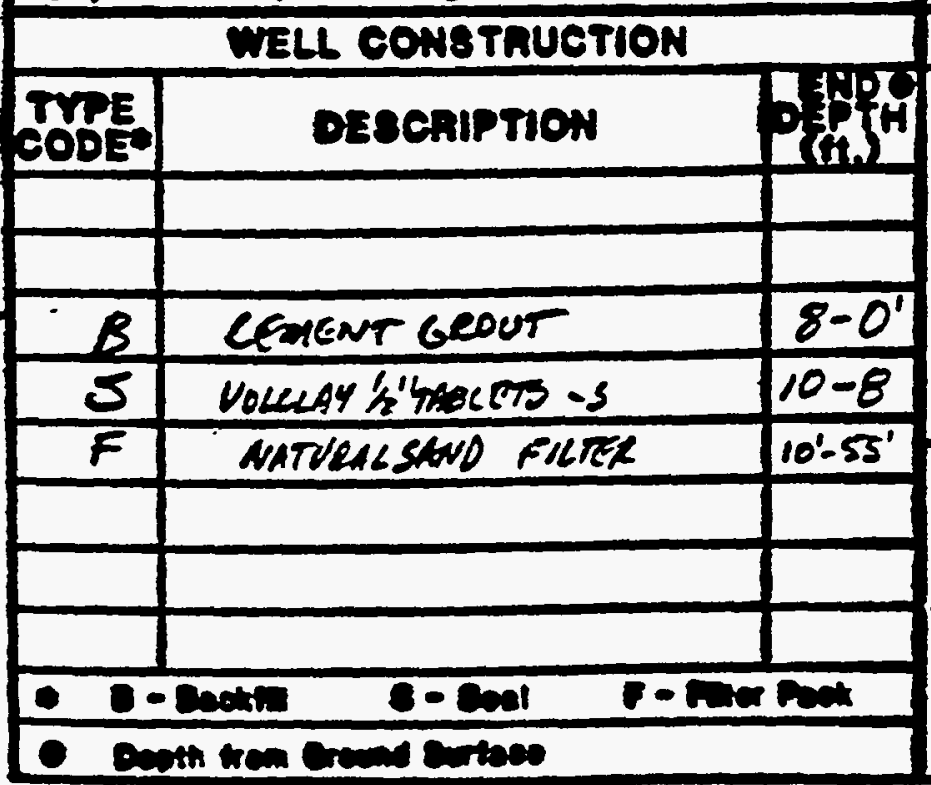

casing

FILTER PACK

SEAL

BACKFILL

DEVELOPMENT

JeT FLUSA PUW

FRomauber

OTHER 


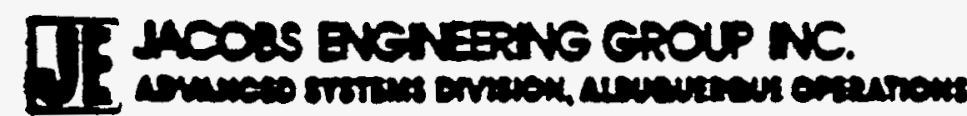

\section{MELL COMPLETION RECORD}

SITE ID:MOCJO1 LOCATION ID: MAT DATE INSTALLED: $10-25.84$ APPROX. OITE COORDINATES:(FT.) $\mathrm{N}$

OPEN AREA PER LINEAL FT. (IN2/FT.)

FORMATION OF COMPLETION:

AcLuviom

FIELD REP.:

$5, \angle V A$

DRILLER: SHB - 1C4:-

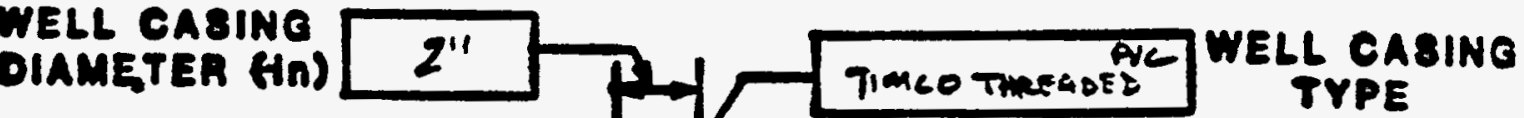

HOLE DIAMETER $(\ln ) 65 / 3$

OROUND SURFACE

TOTAL

DEPTH

(Ii)
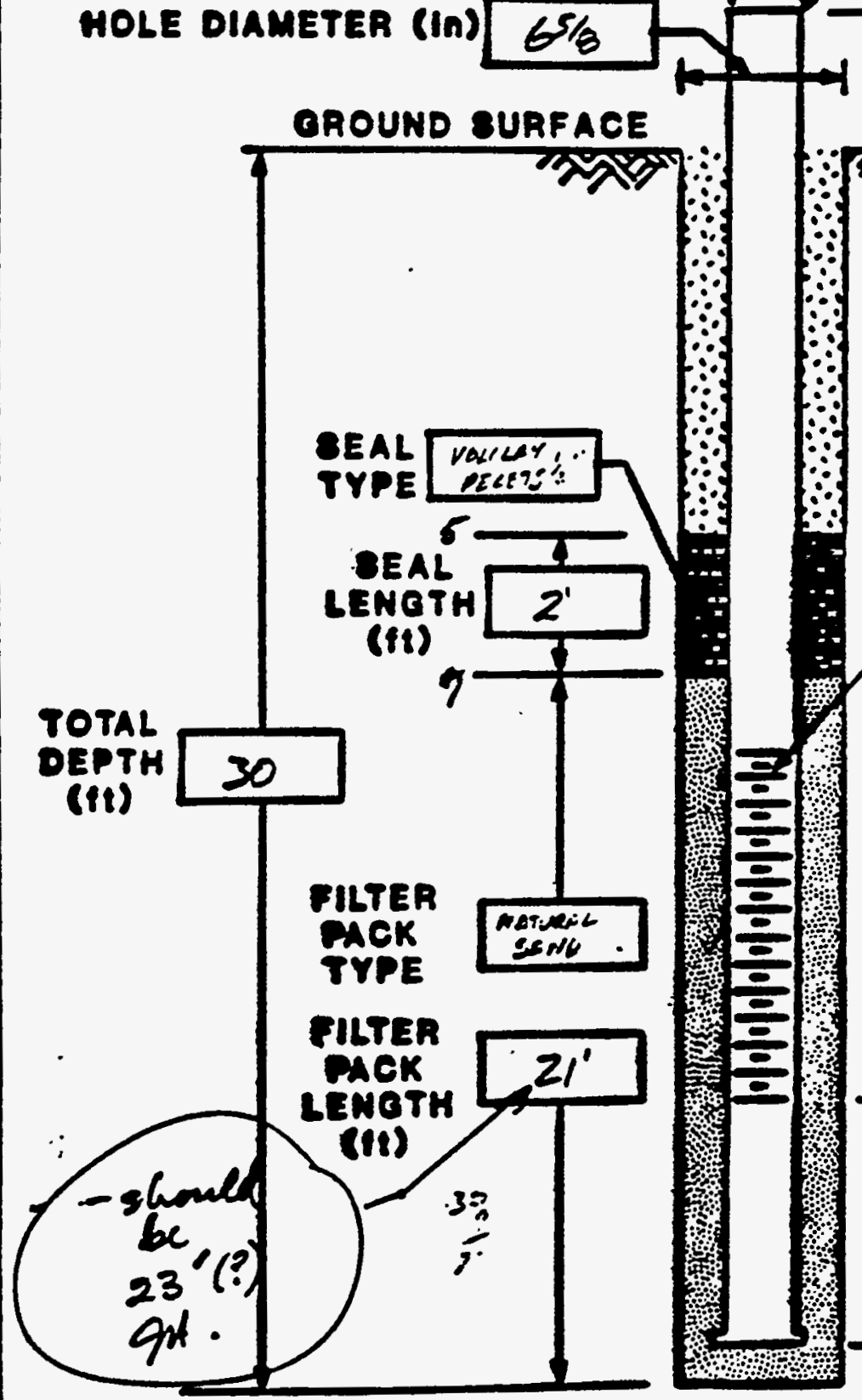

$\because$ आNE
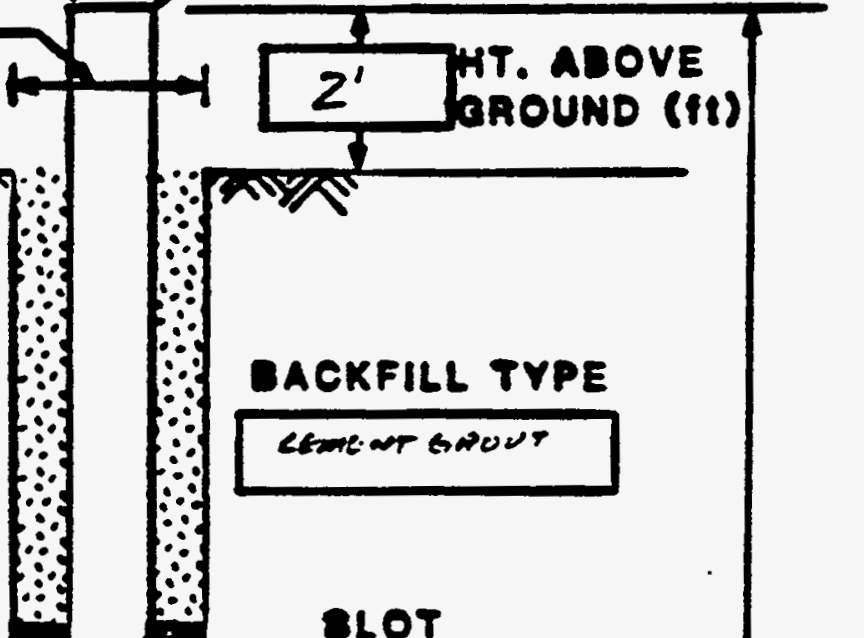

DACKFILL TYPE

Leatiar Gavor

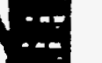

SLOT
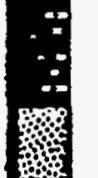

openine

(in)
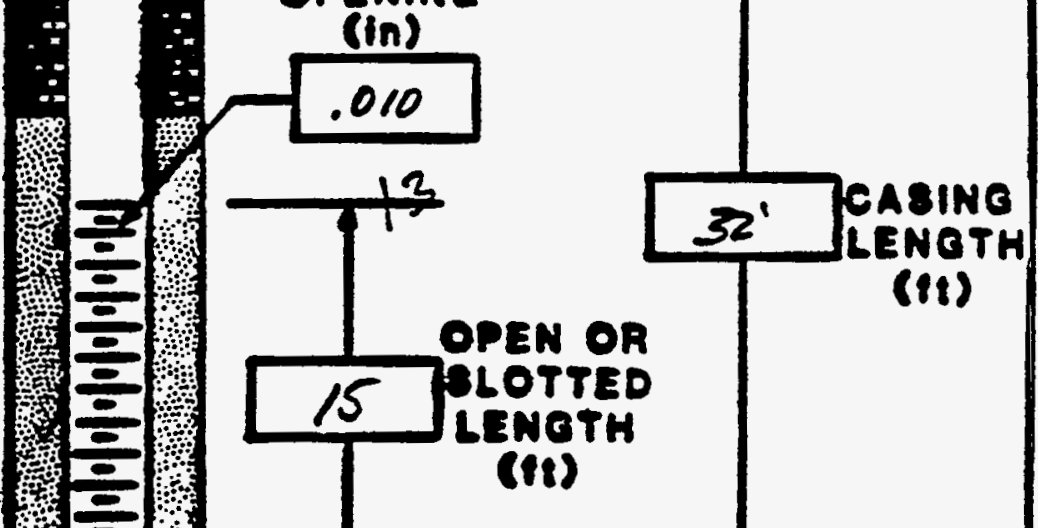

15

(11)

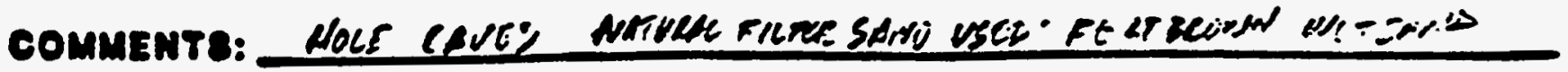

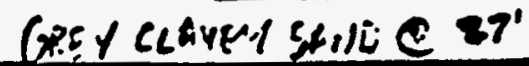




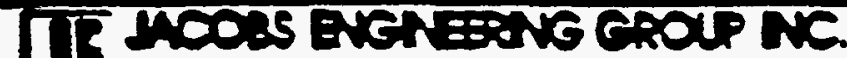

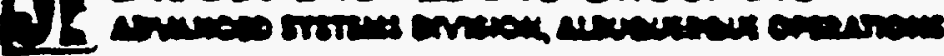

DOREHOLENELL CONSTAUETION LOG

OTTE DD: MONOL LOCATION 1D:MN-1-4 GOFELD REP: S/LVA

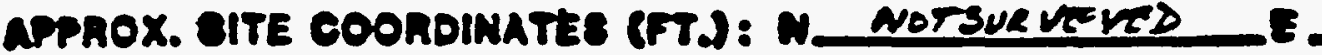

CROUND ELEVATION (FT. MBL):

COMPLETION OATE:

DOREHOLE GUMMARY

DALLER: SHB LCATI wiclinms

NO TME:

CME 55

\begin{tabular}{|c|c|c|c|}
\hline$m=$ & $\begin{array}{l}\text { GOLE } \\
\text { PiA. } \\
\text { (In.) }\end{array}$ & 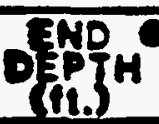 & $\begin{array}{l}\text { RUID } \\
\text { TYPE }\end{array}$ \\
\hline & $65 / 3$ & $30^{\prime}$ & MT NO \\
\hline
\end{tabular}

(1)

\begin{tabular}{|l|l|l|l|}
\hline & & & \\
\hline & & & \\
\hline & & \\
CASINO SUMHARY
\end{tabular}

GABING DEBCAIPTION DIA. ENNP TYPE - DESCAIPTION (IN.) PEPTH

\begin{tabular}{|c|c|c|c|}
\hline & $\cdot$ & 2 & \\
\hline $4 P$ & Phoreanve Loxk lip & $6^{11}$ & $0-4$ \\
\hline 58 & 5 schenk & $2^{\prime \prime}$ & $0-5$ \\
\hline 108 & $10^{\prime}$ Betare & - & $5-15$ \\
\hline 55 & 5's407t:0 e.010 & & $15-20$ \\
\hline 115 & 10.Senteso o. 010 & 7 & 2030 \\
\hline$Z^{\prime} B$ & $2^{\circ}$ EGONE & $\downarrow$ & $50-3$ \\
\hline & & & \\
\hline
\end{tabular}

\begin{tabular}{|c|c|c|}
\hline \multicolumn{3}{|c|}{ WELL CONETRUCTION } \\
\hline BE & \multicolumn{2}{|l|}{ PESERIPTION } \\
\hline & & \\
\hline & & \\
\hline & & \\
\hline 8 & crmont beart rams & $10-5$ \\
\hline 5 & 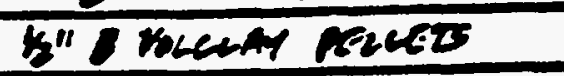 & $5 \cdot 7^{\prime}$ \\
\hline$F$ & 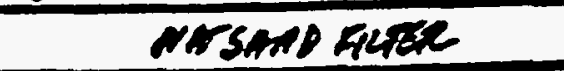 & $7-30^{\circ}$ \\
\hline- & & \\
\hline e. & e- 8001 & non \\
\hline 28 & 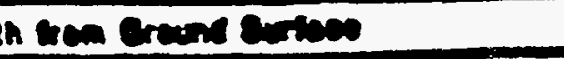 & \\
\hline
\end{tabular}

CABINO

FILTER PACK

GEAL

BACKFILL

DEVELOPMENT

OTHER

CONSTAUETION TIME LOQ

ACTIVTY

PRILLING
CABINO
FILTER PACK
EEAL
EACKFILL
DEVELOPMENT
OTHER

NELL DEVELOPMENT

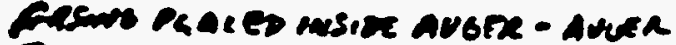

nereemonos -

Sine cares - $709^{\circ}$

86

SP sunos

(aor Izra)

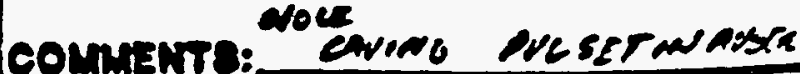

nuw evoce leweres
SND

TIME

dise

j.2e

$1: 22 \quad 1: 24$

$1: 24 \quad 1: 00$

130

1.50 


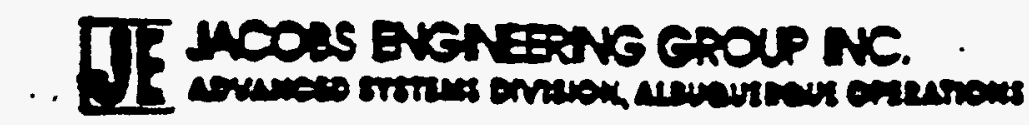

MELL GOMPLETION AECOND

OITE 10: MONOI LOCATION ID: MN-1-5 DATE MNTALLED: $10-2524$ APPROX. BITE COOORDINATES:(FT.) $N$ OPEN AREA PER LINEAL FT. CINZJFT.P

foRMATION OF COMPLETION:

FIELD REP. M MIKE SILUA DRILLER: TEATT-SHE

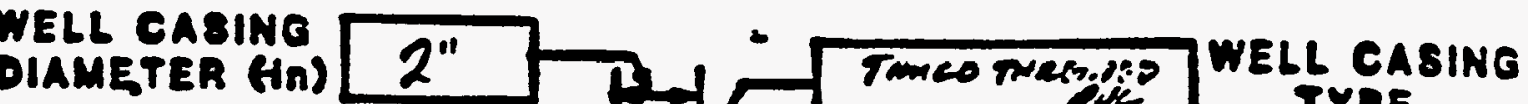

HOLE DIAMETER ins $65 / 3$ TYPE

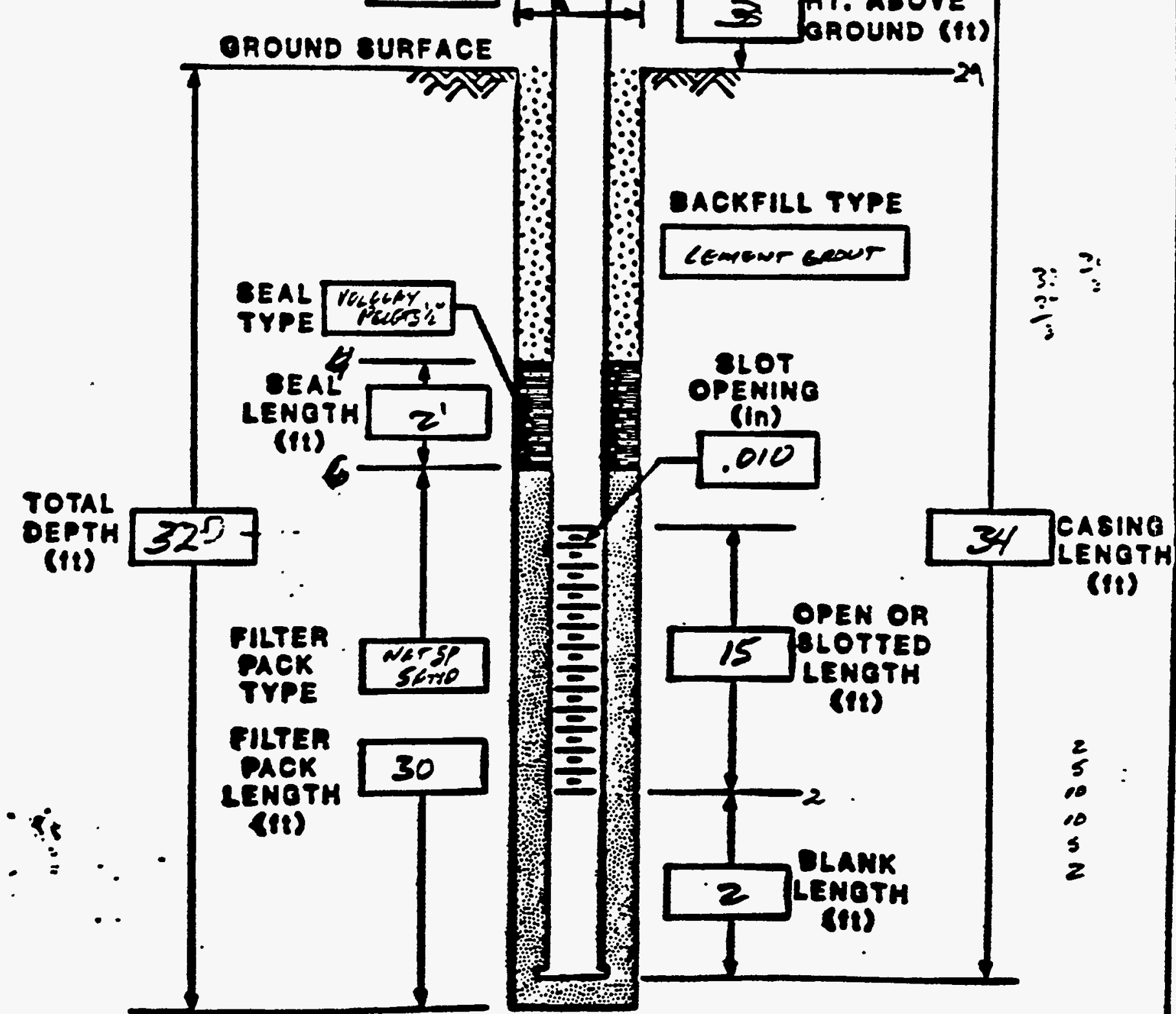

COMMENTS: 

DOREHOLE GUMMARY

อAILLER: TeAT SHT MO TYPE: CME. 55

\begin{tabular}{|c|c|c|c|}
\hline OTT TYPE & $\begin{array}{l}\text { GOLE } \\
\text { PIA. } \\
\text { (in.) }\end{array}$ & D) & Ruip \\
\hline 6"HSA & $6 \%$ & & \\
\hline & & & \\
\hline & & & \\
\hline & & & \\
\hline
\end{tabular}

CASING SUMMARY

\begin{tabular}{|c|c|c|c|}
\hline $\begin{array}{l}\text { GASINE } \\
\text { TrPE }\end{array}$ & DEECRIPTION & $\begin{array}{l}\text { pia } \\
\text { (in.) }\end{array}$ & \\
\hline $4 P$ & Srees Lakine Cap & $6^{*}$ & 0.4 \\
\hline$Z B$ & $2^{\prime}$ SLANSK & $2^{\prime \prime}$ & \\
\hline 58 & $5^{\prime}$ BLANK & & \\
\hline $10 B$ & $.10^{\circ}$ BLAnK & & 2 \\
\hline 55 & S'scortits & & $17 \cdot 8$ \\
\hline 105 & $10^{\circ}$ sLorts & & $22 \cdot 32$ \\
\hline 28 & $2^{\prime}$ Bchisk & $\boldsymbol{V}$ & $52-3$ \\
\hline \multicolumn{4}{|c|}{ 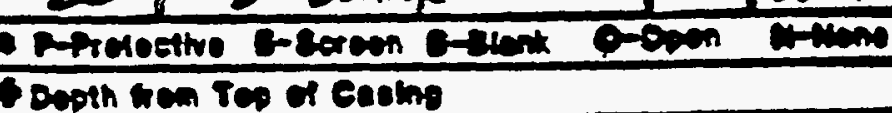 } \\
\hline
\end{tabular}

\begin{tabular}{|c|c|c|}
\hline \multicolumn{3}{|c|}{ WELL CONETRUCTION } \\
\hline TYPE & DEschiption & \\
\hline & & \\
\hline & & \\
\hline & & \\
\hline 8 & comont srout zorb & 0.4 \\
\hline 3 & PILUNSEA & 46 \\
\hline$F$ & 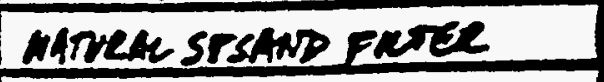 & 6.3 \\
\hline & & \\
\hline e & $p \cdot \sqrt{a n}$ & \\
\hline
\end{tabular}

\section{CAsING}

FILTER PACK

SEAL

BACKFILL

DEVELOPHENT

OTHER

canos CONSTRUETION TIME LOO

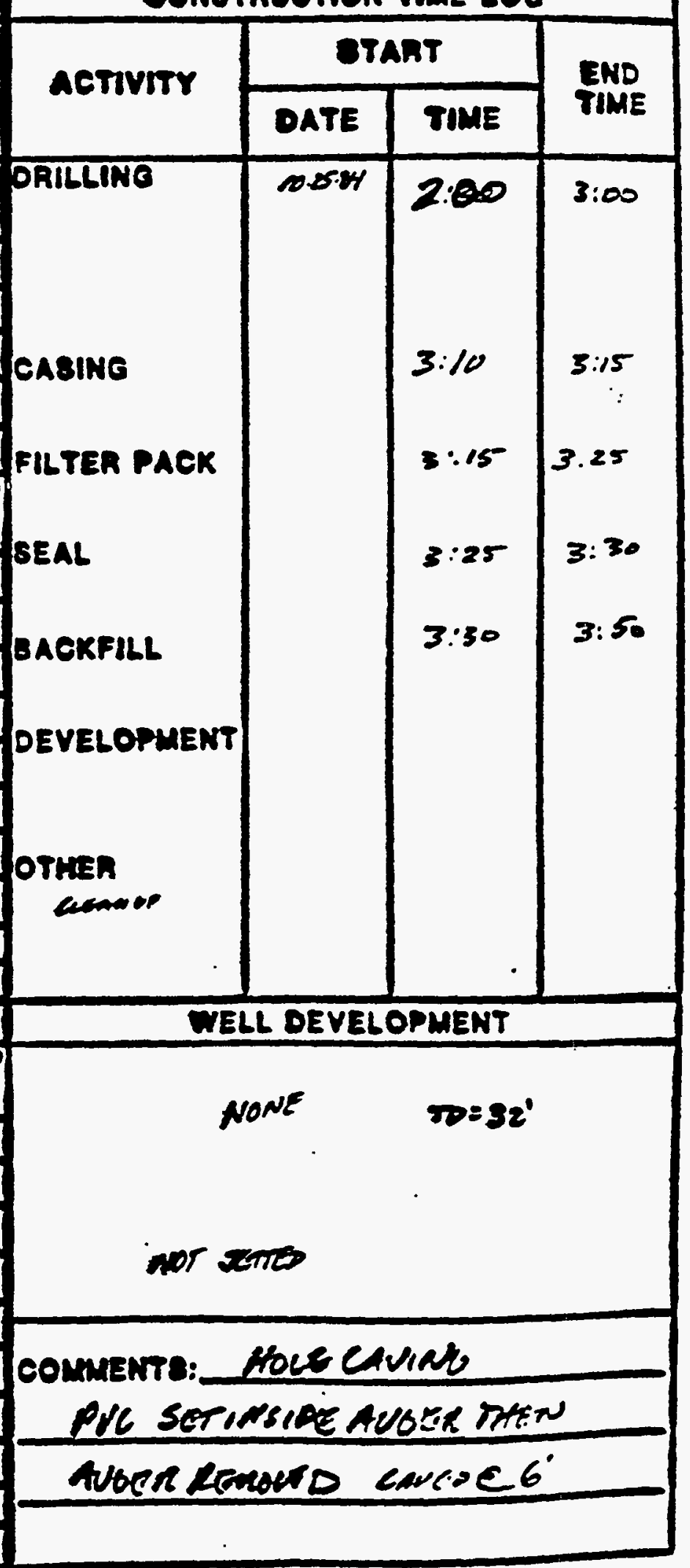




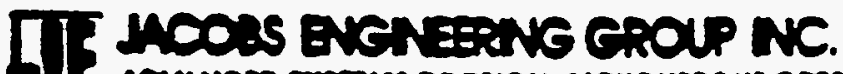

\section{MELL COMPLETION RECORD}

SITE DD: MONOI LOCATION ID: MM-t-6OLATE INSTALLED: $10-26-84$ APPROX. SITE COORDINATE8:(FT.) $N$ OPEN AREA PER LINEAL FT. (IN2/FT.)

IFORMATION OF COMPLETION: AGG UNIUN FIELD REP.: - SILUA. ORILLER: SHB - TCLT

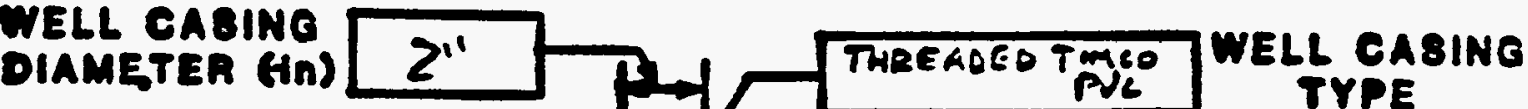

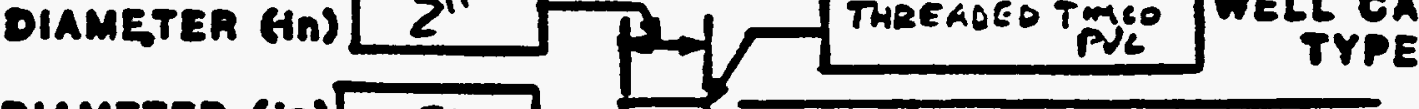
HOLE DIAMETER (in) $65 / 3$

GROUND SURFACE
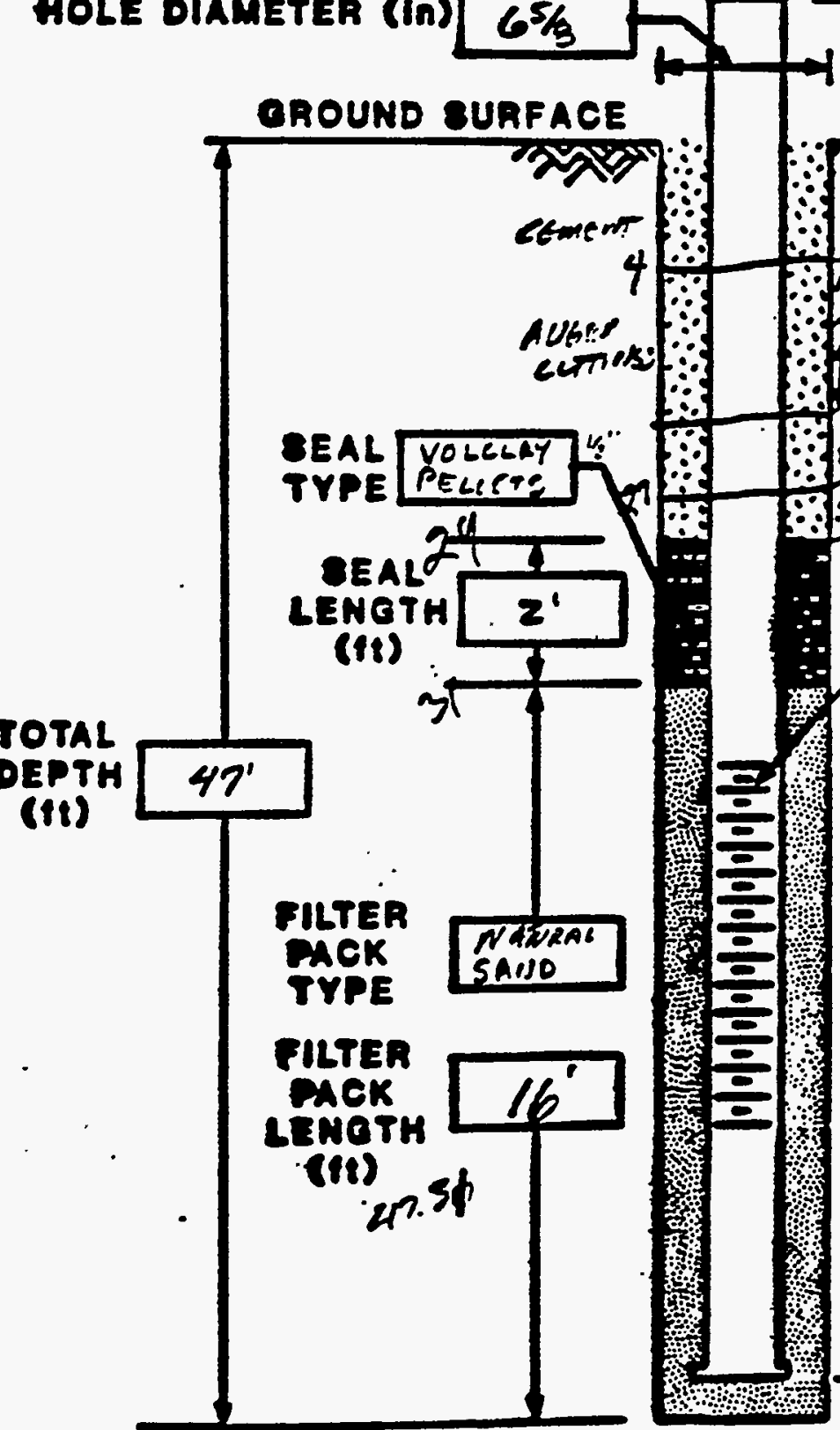

$\because$ SNY

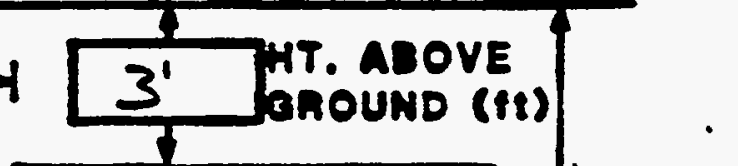

TOTAL DEPTH (It)

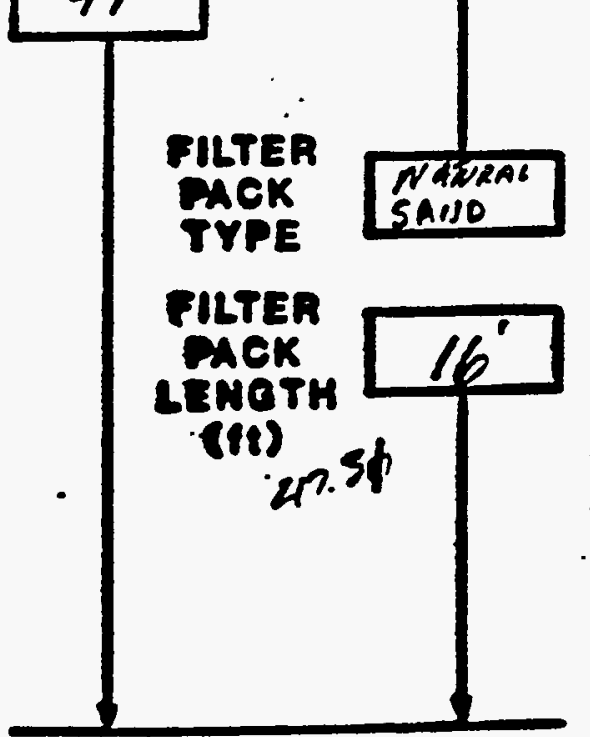

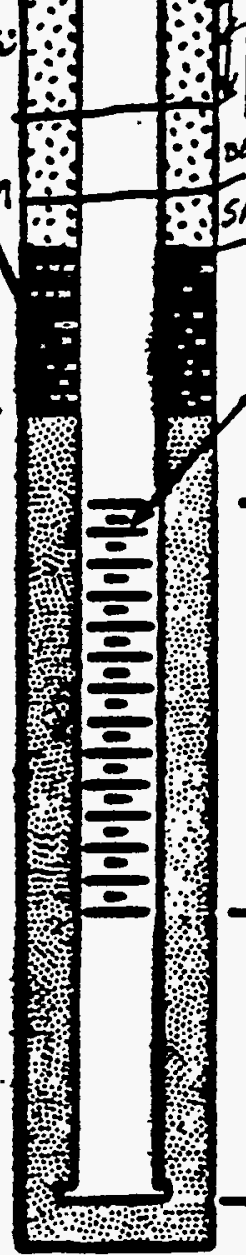
$\boldsymbol{E}$

COMMEnT: 


\section{MELL COMPLETION RECORD}

SITE ID: MONOI LOCATION ID: $M N-1-6$ DATE IN8TALLED: $10-26-84$ APPROX. SITE COORDINATES:(FT.) $N$ E OPEN AREA DER LUNEAL FT. (IN2/FT.)

FORMATION OF COMPLETION: $\frac{\text { AW WNIUN }}{\text { SILUA }}$
FIELD REP.:

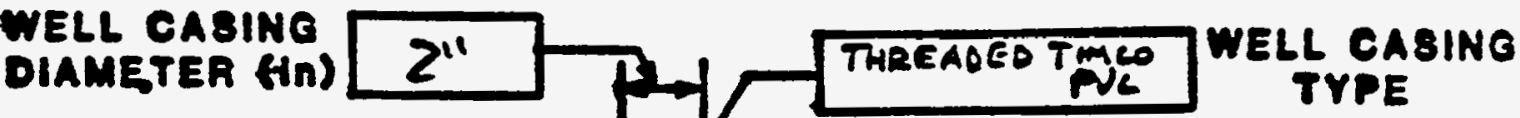
HOLE DIAMETER $(\ln ) 65 / 8$

GROUND SURFACE
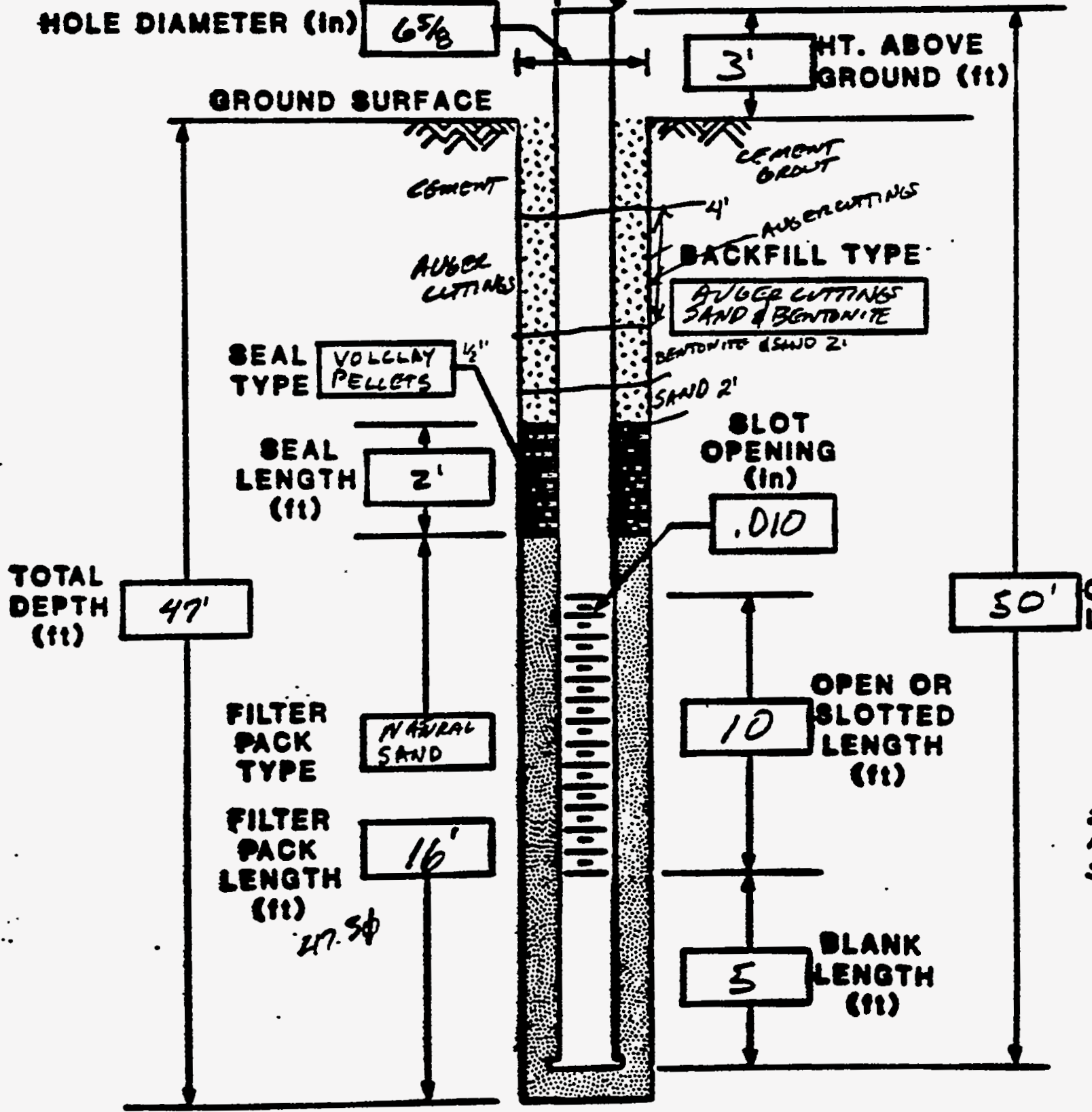

$\because 1$ ADGG LaTTNas

$\therefore$ SAND PCONTNITE

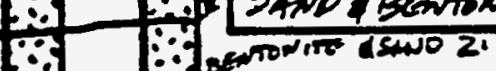
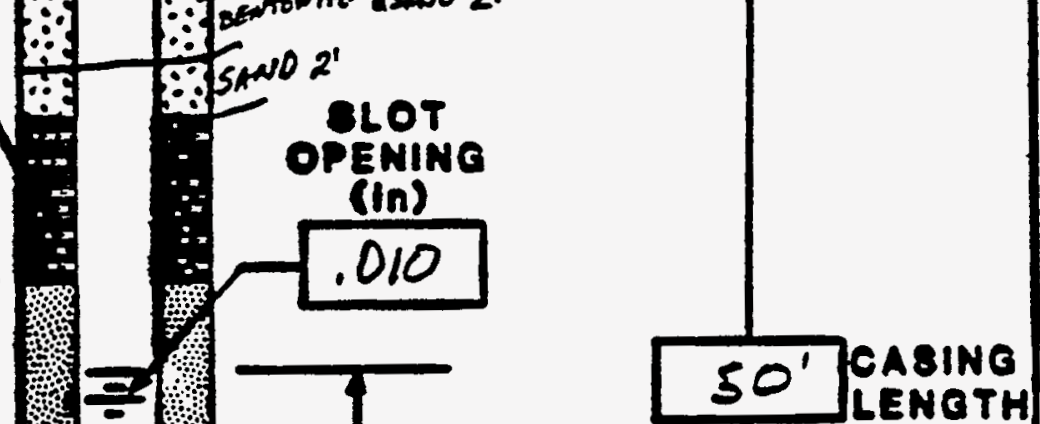


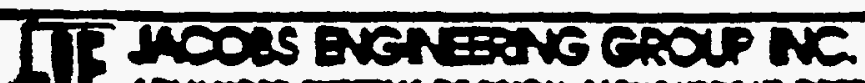

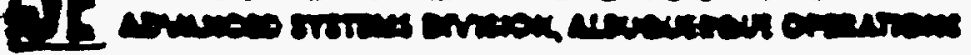 \\ DOREHOLEINELL CONSTAUETION LOG}

sif:

( I OTE DD: MONOI LOCATION DD:MN-1-6 COCLELO REP: SILVA APPhOx. OITE GOOADINATIS IFT. : N QHOUND ELEVATION (FT. CHEL): COMPLETION DATE: $10 \cdot 26-? Y^{\circ}$

DOREHOLE OUMMARY

vallen: SHB - 7 CRT

RIO MPE: CME 55

\begin{tabular}{|c|c|c|c|}
\hline DIT TYPE & $\begin{array}{l}\text { POLE } \\
\text { PIA } \\
\text { (in.) }\end{array}$ & ofpj & RUP \\
\hline $6^{\prime \prime} H S A$ & $6^{5 / 8}$ & $47^{\prime}$ & DRV \\
\hline & & & \\
\hline & & & \\
\hline & & & \\
\hline
\end{tabular}

Casino sumMARY

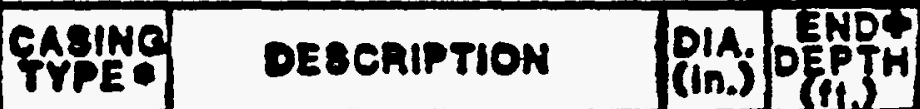

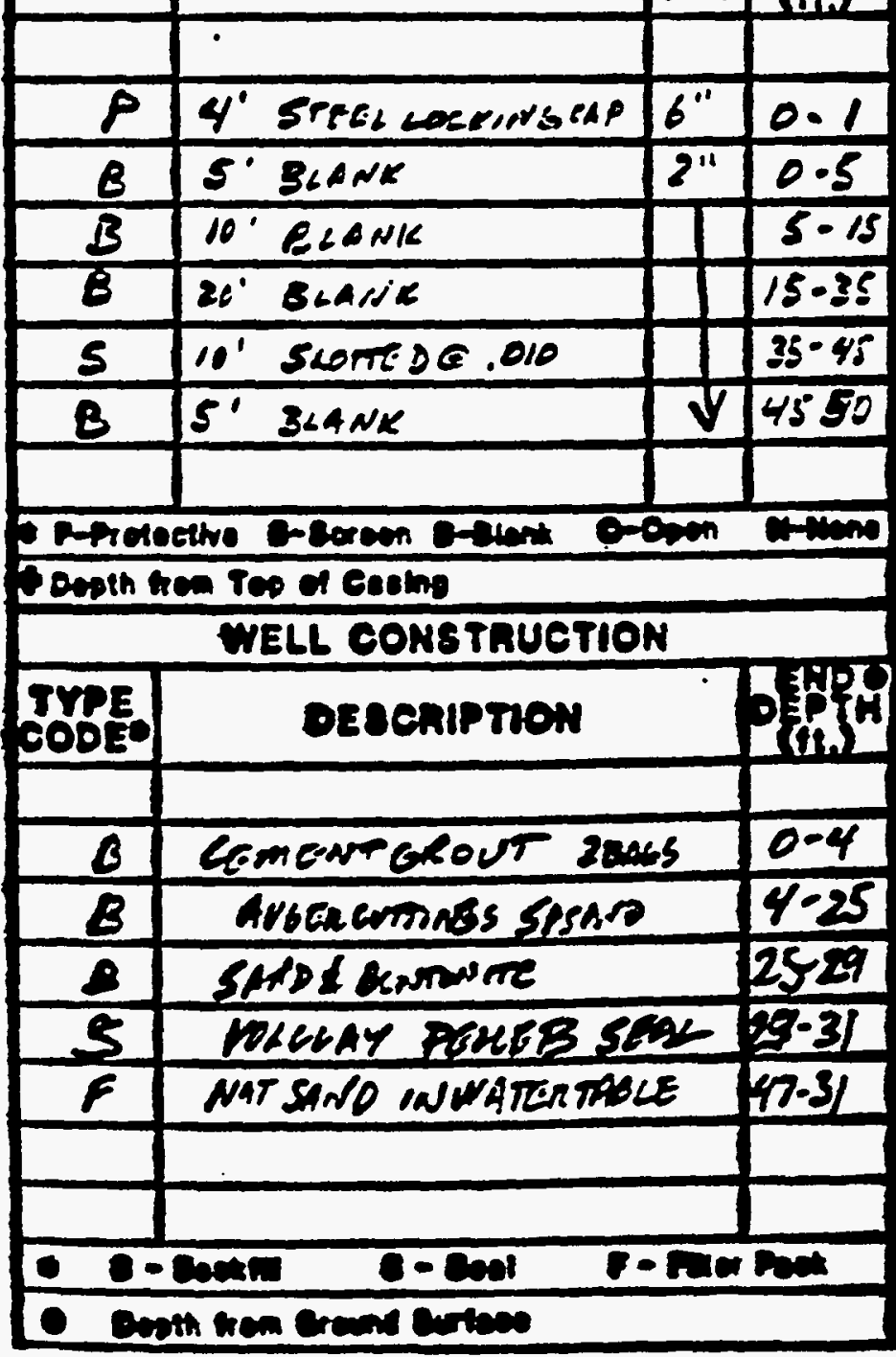

CONBTRUCTION TIME 100

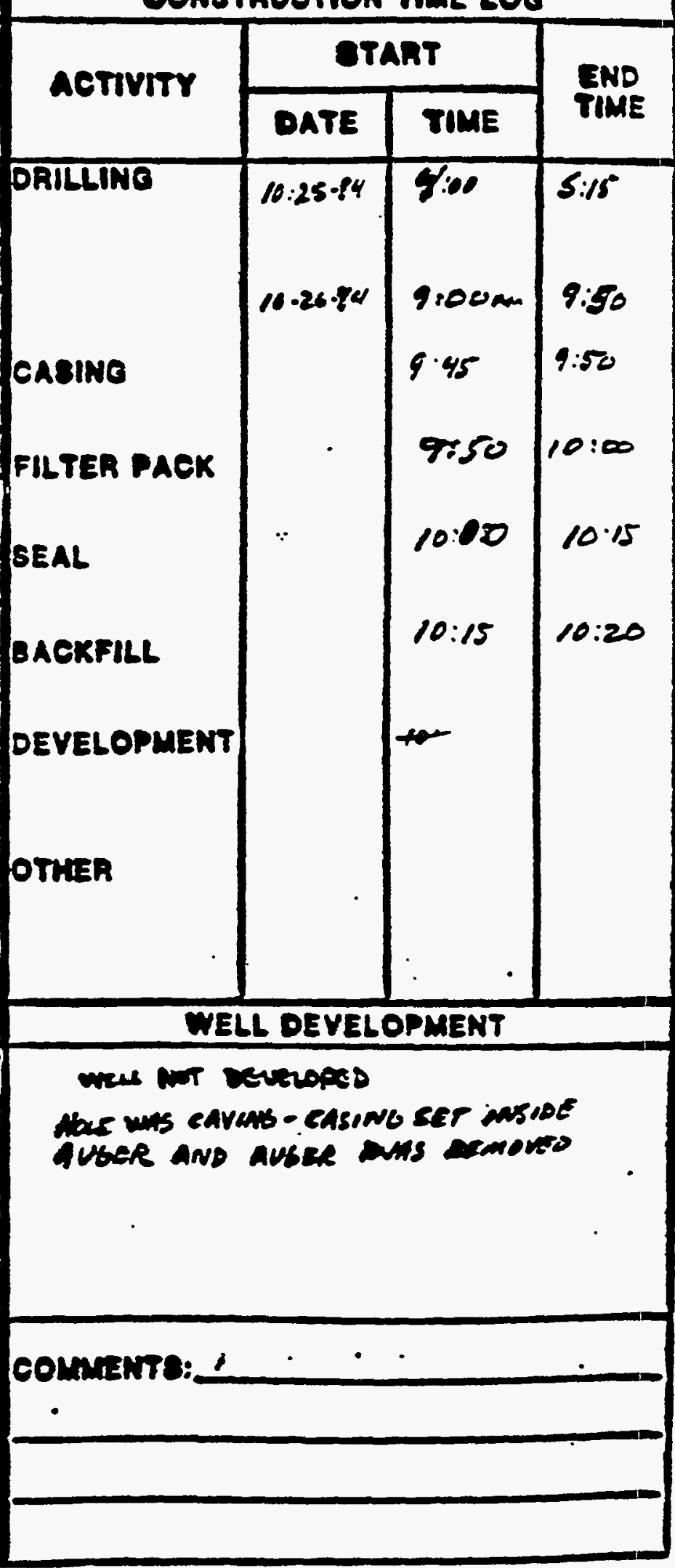




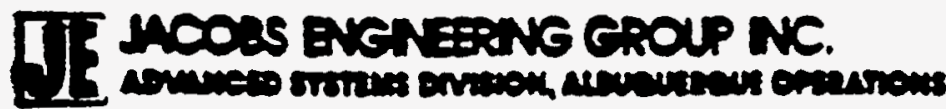

\section{MELL COMPLETION RECOND}

SITE ID: MONOI LOCATION ID: MN+7667 DATE IN8TALLED: 10-20-84 APPROX. SITE COONDINATE8:(FT.) $N$ $\boldsymbol{E}$

OPEN AREA DER LINEAL FT. (IN2/FT.)

FORMATION OF COMPLETION: SARASTONE - YEZ160N J2OUN

FIELD REP.: SILVA DAILLER: SHQ TCANT

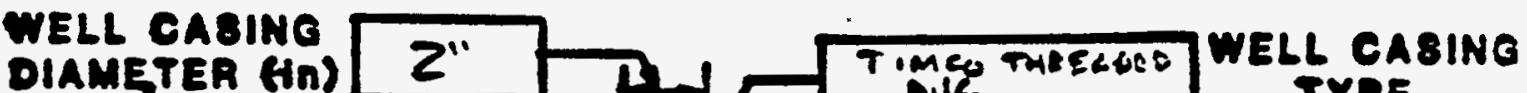

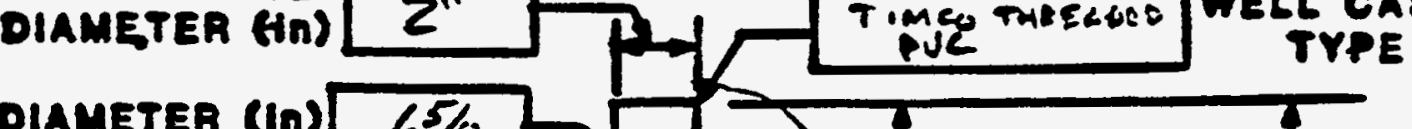
HOLE DIAMETER (In) $65 / 3$ OROUND SURFACE
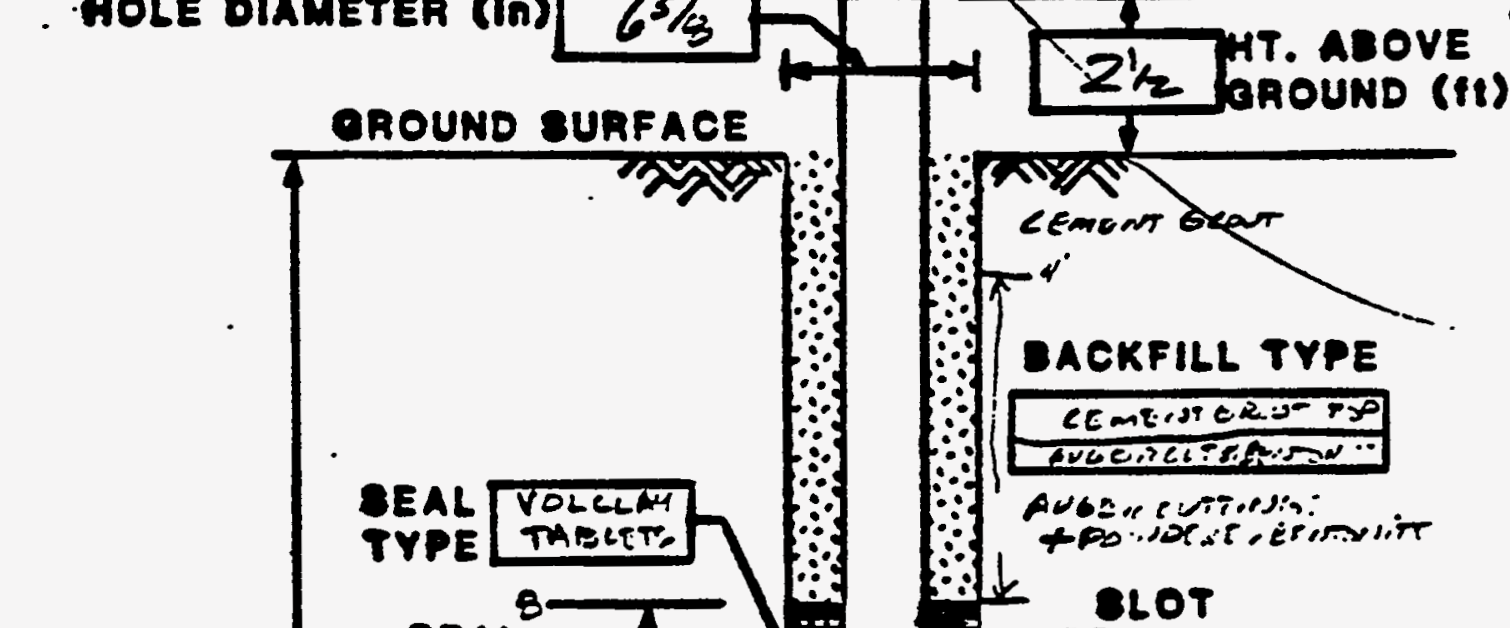

TOTAL OEPTH (81)
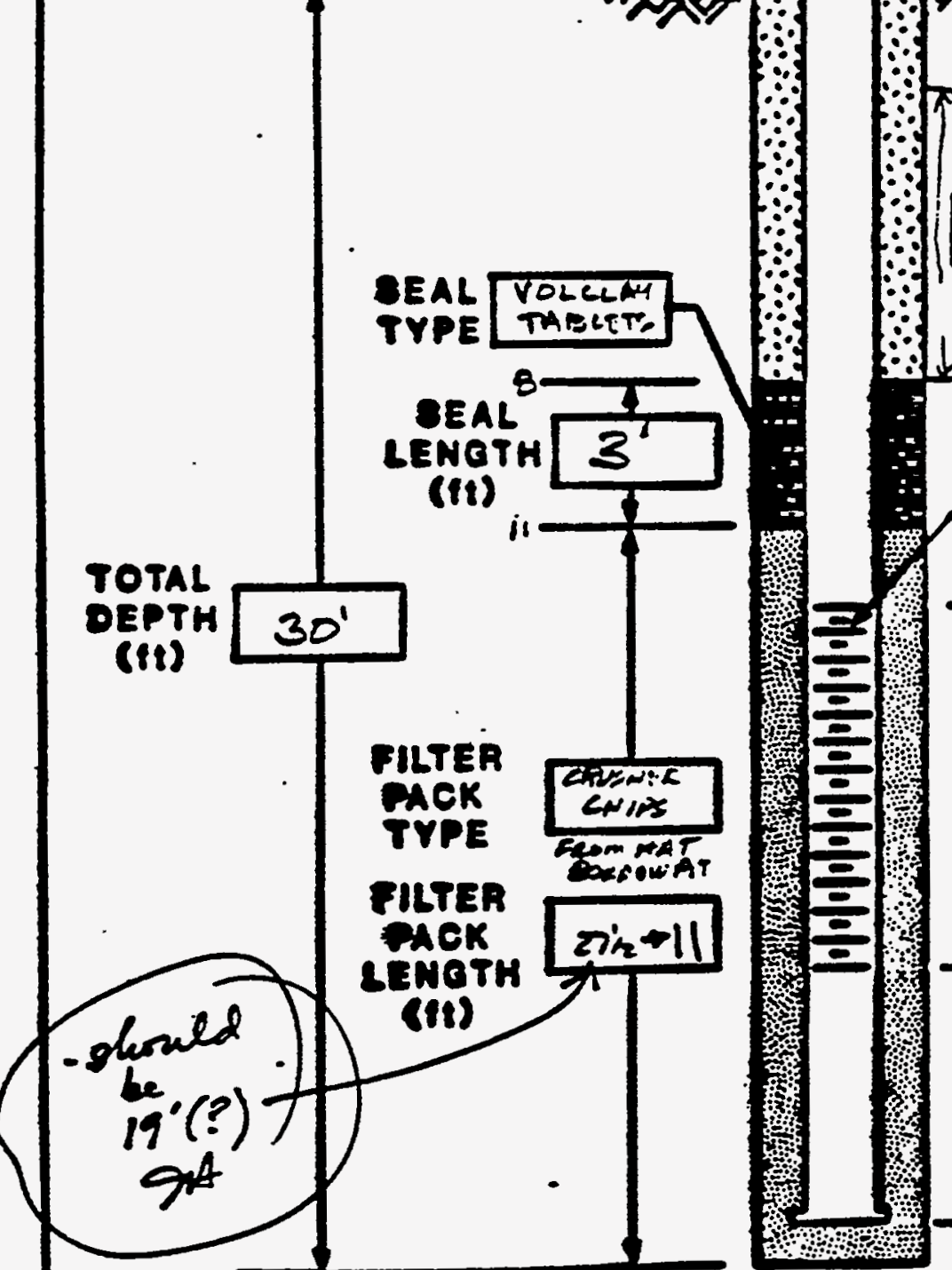
$-9$

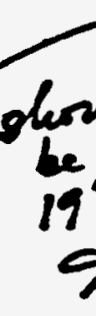

wed (?)

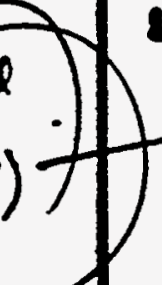

FILTER

MCK

TYPE is

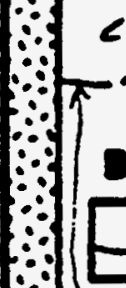

CEmont Deent

ACKFILL TVPE

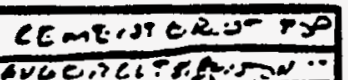

$\because$ AvGa.e cutioss:

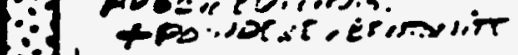

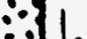

\section{BLOT}

opening

$\frac{.010}{155}$

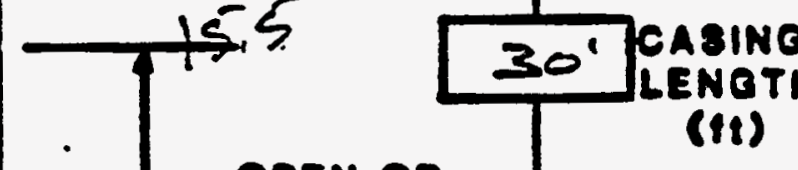

)

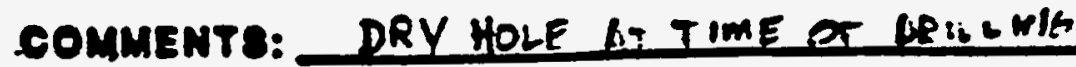


DI HCONS ANGN ERANG EROAP NC.

\section{WELL COMPLETION RECORD}

SITE DD: MONOI LOCATION ID: MN-H7 OATE IN8TALLED: $10-26-84$ APPROX. OITE COOORDIMATE8:(FT.) $N$

OPEN AREA DER LUNEAL FT. (IN2/FT.) E

FOAMATION DF COMPLETION: SANASTONE - YEZLON ZLOUN

FIELD REP.: SILVA DRILLER: GHE TCANT

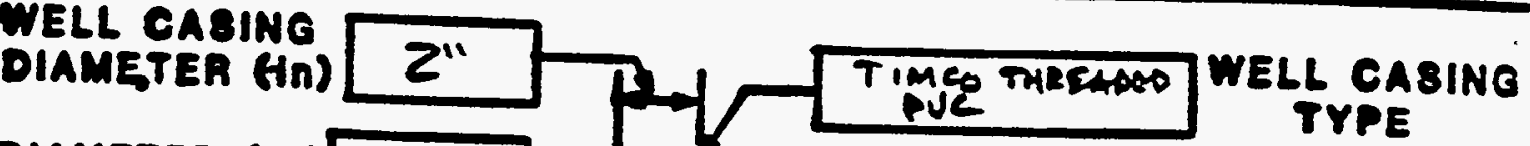

HOLE DIAMETER $(\ln ) 65 / 8$

BAOUND SURFACE

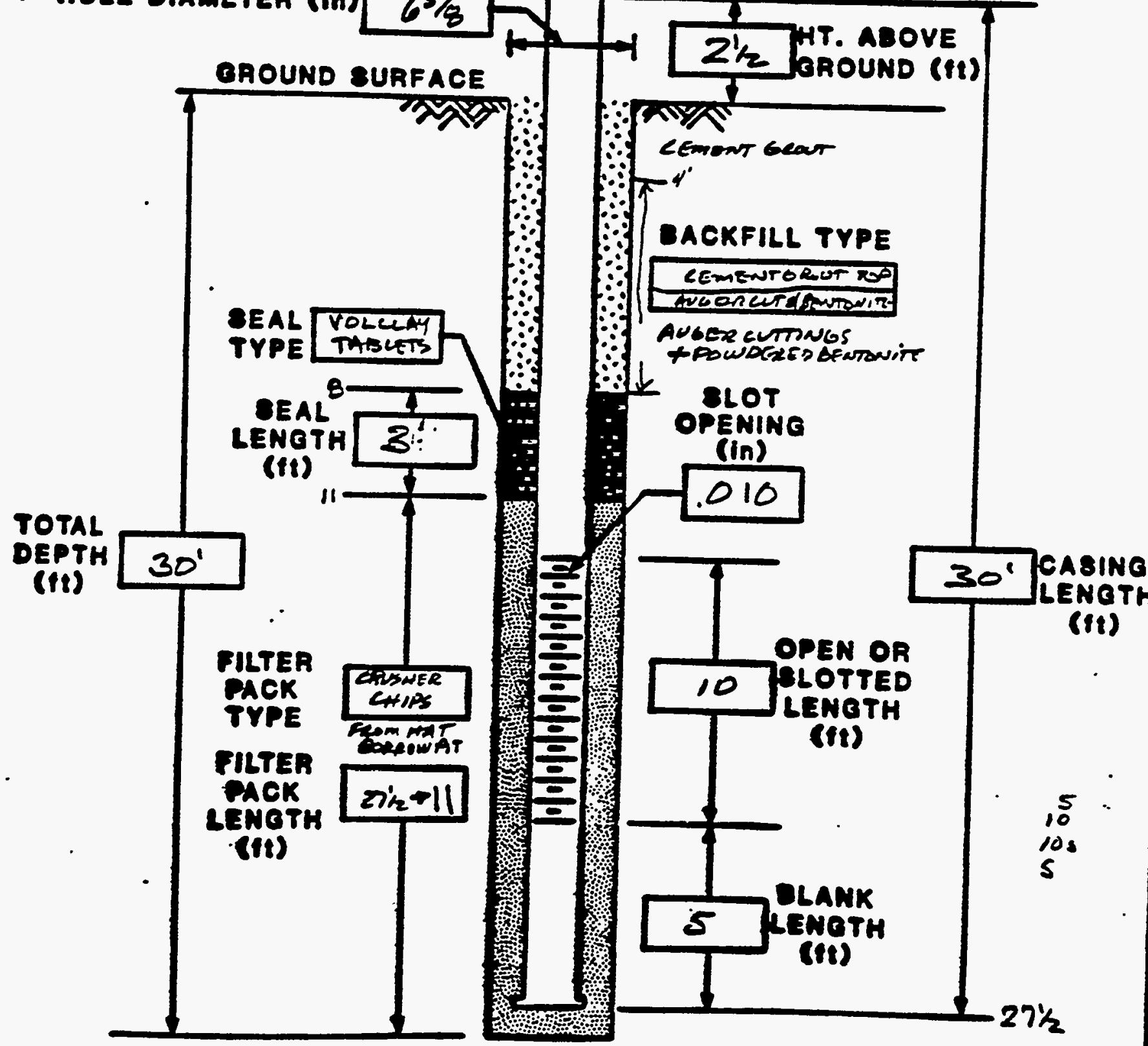

$7_{n}$

COMMENTO: DRY HOLE AT TIME OE PRULWW 


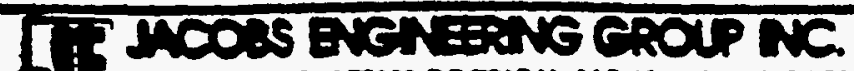

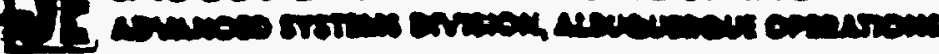 \\ DOREHOLEAMELL CONETRUCTION LOG}

C. OTTE DD: MONOI LOCATION ID:MN-F7 FIELO REP:SILVA

AFrnox. OITE COOADNATEB (FT.): $\mu$

enoun ELEVAToin (FT. MaL):

COMPLETION DATE: $10-26-84$

DOREHOLE CUMMARY

DAlLER: 5 HB TCATT

AO TME: CME 55

\begin{tabular}{|c|c|c|c|}
\hline & & Fipth & rup \\
\hline
\end{tabular}

\begin{tabular}{|c|c|c|c|}
\hline $6^{5 / 8 H S h}$ & $6^{5 / 8}$ & $30^{\prime}$ & DeY \\
\hline & & & \\
\hline & & & \\
\hline & & & \\
\hline
\end{tabular}

\begin{tabular}{|c|c|c|c|}
\hline \multicolumn{4}{|c|}{ CAEINE UUMMARY } \\
\hline CABING & DEBCRIPTION & (in.). & \\
\hline & & & \\
\hline & & & \\
\hline$\Psi^{\prime} P$ & Proctzose - Lockecte & 6 & $0-$ \\
\hline$S^{\prime} B$ & S'BLAnk & $2^{\prime \prime}$ & $0 \cdot 2.5$ \\
\hline 1013 & 1011 & & $25 \cdot 125$ \\
\hline $10 ' s$ & $10^{\prime} 5 \angle 0 \pi R \cdot 1$ & 7 & 125225 \\
\hline$S^{\prime} B$ & $5^{\prime} B$ BANK & I & $22.5-27,5$ \\
\hline & & & \\
\hline
\end{tabular}

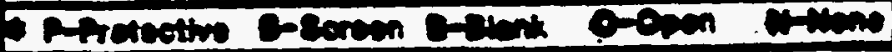

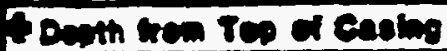

\begin{tabular}{|c|c|c|}
\hline \multicolumn{3}{|c|}{ MELL GONETRUCTION } \\
\hline TOOE & DEschiption & \\
\hline & & \\
\hline & & \\
\hline $\bar{B}$ & crment aesut 23Ns & \\
\hline 8 & 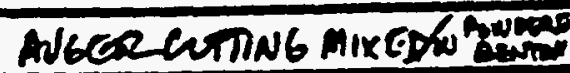 & \\
\hline 5 & rOLCLAY PCLCET3 25 LOS & $2-1$ \\
\hline$F$ & CAUShELR CAIISS - GILTER & $11-3$ \\
\hline & & \\
\hline 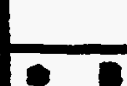 & 8-sen & \\
\hline 6 & 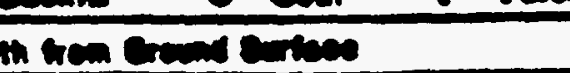 & \\
\hline
\end{tabular}

CONBTRUCTION TIME LOQ

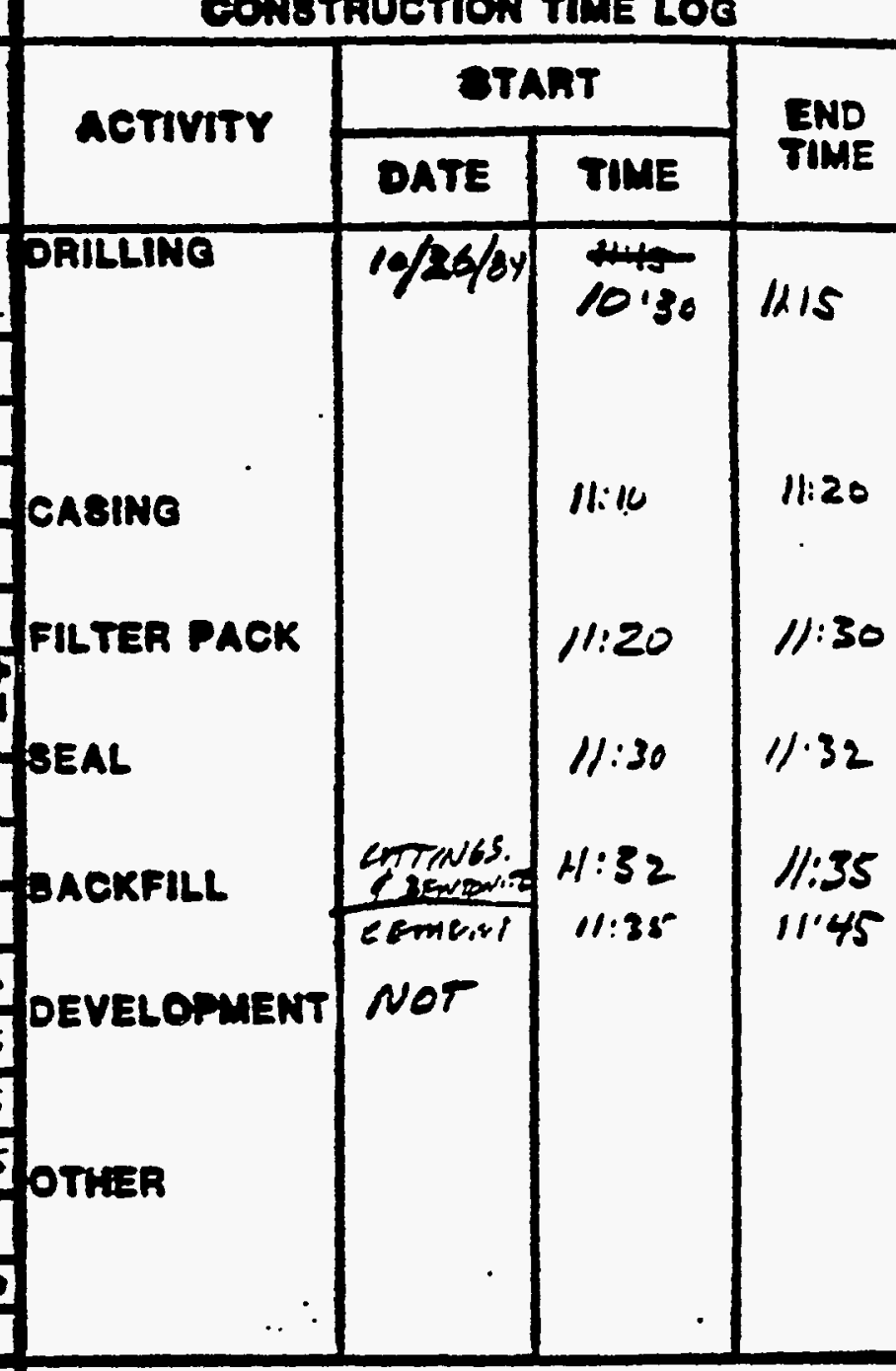

NELL DEVELOPMENT 


\section{YELL COMPLETION RECORD}

SITE DD: MON OI LOCATION ID: COOB 3 OATE INSTALLED: $=/ 19 /$ is APPROX. OITE COORDIMATES:(FT.) N 58720.0 $E=87390.0$ OPEN AREA PER LNEAL FT. (IN2/FT.) FORMATION OF COMPLETION: Deheley FIELD MEP.: MCENery ORILLER: SH + B

well casino DIAMETER (In)

HOLE DIAMETER SIIn)
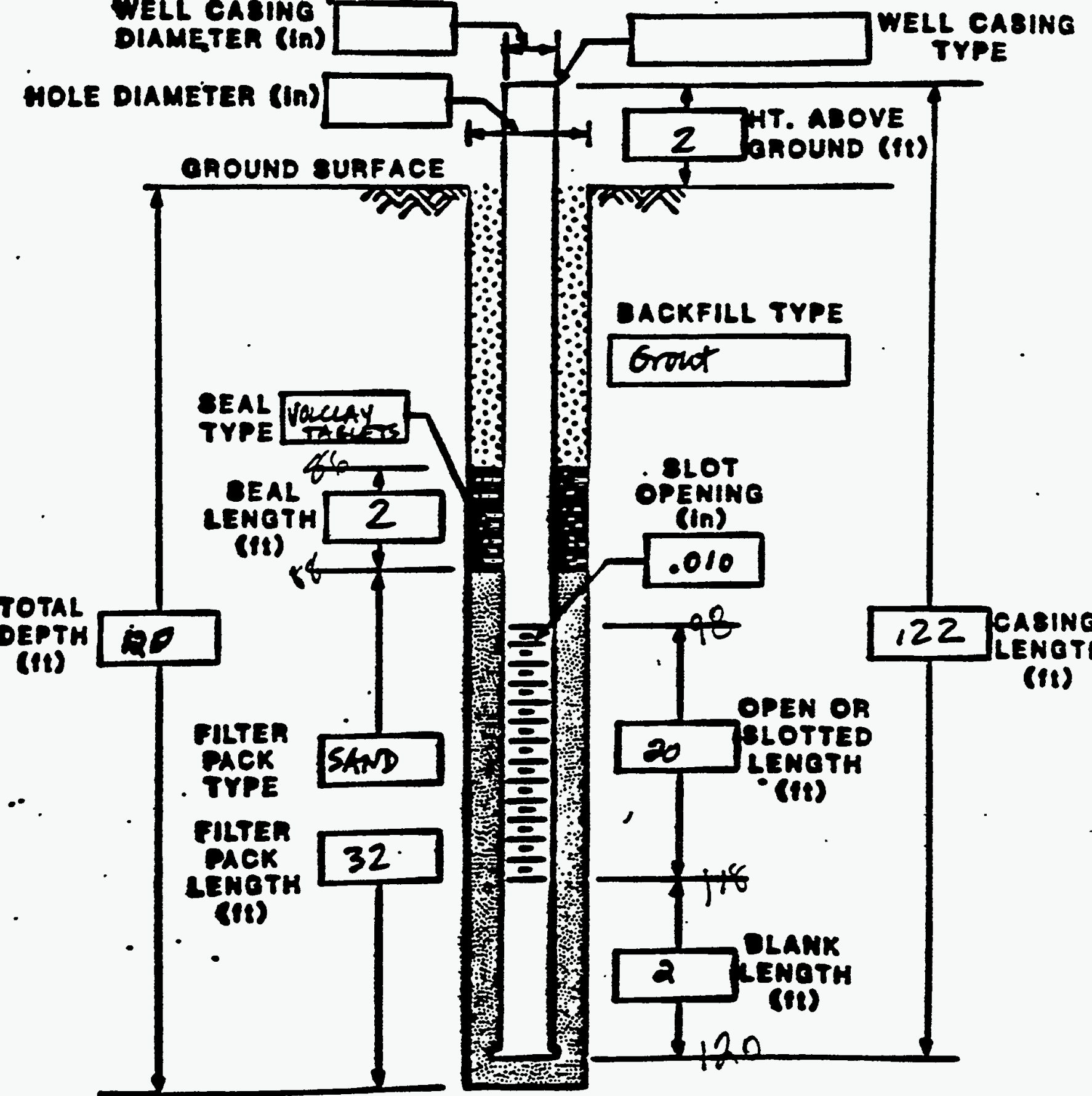

TMT. ADOVE

DACKFILL TYPE

Grout

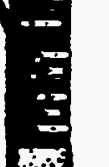

TOTAL DEPTH ino

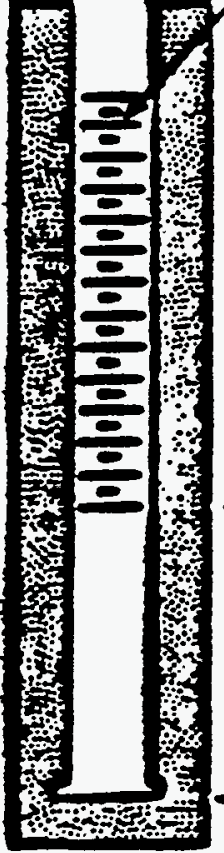
खॅ

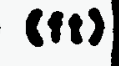




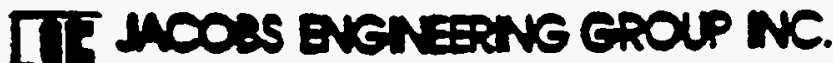

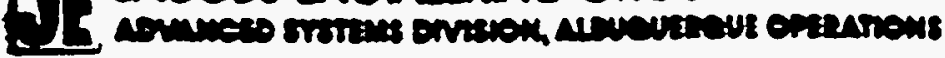

\section{MELL COMPLETION RECORD}

SITE ID: MON Ol LOCATION ID: COBB DATE INSTALLED: $3 / 19 / 85$ APPROX. SITE COORDINATE8:(FT.) $\mathrm{N}$ E OPEN AREA PER LINEAL FT. (IN2/FT.) fFORMATION OF COMPLETION: Dedielly FIELD REP.: MCSANEY DRILLER: SH+B

well casino DIAMETER (In)
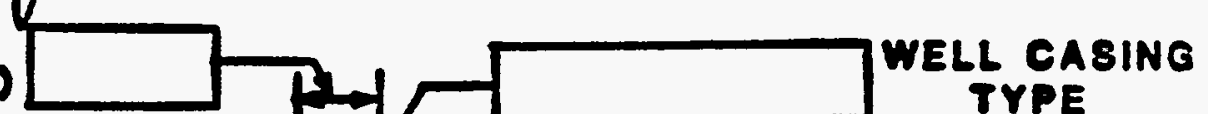
hole diameter (In)
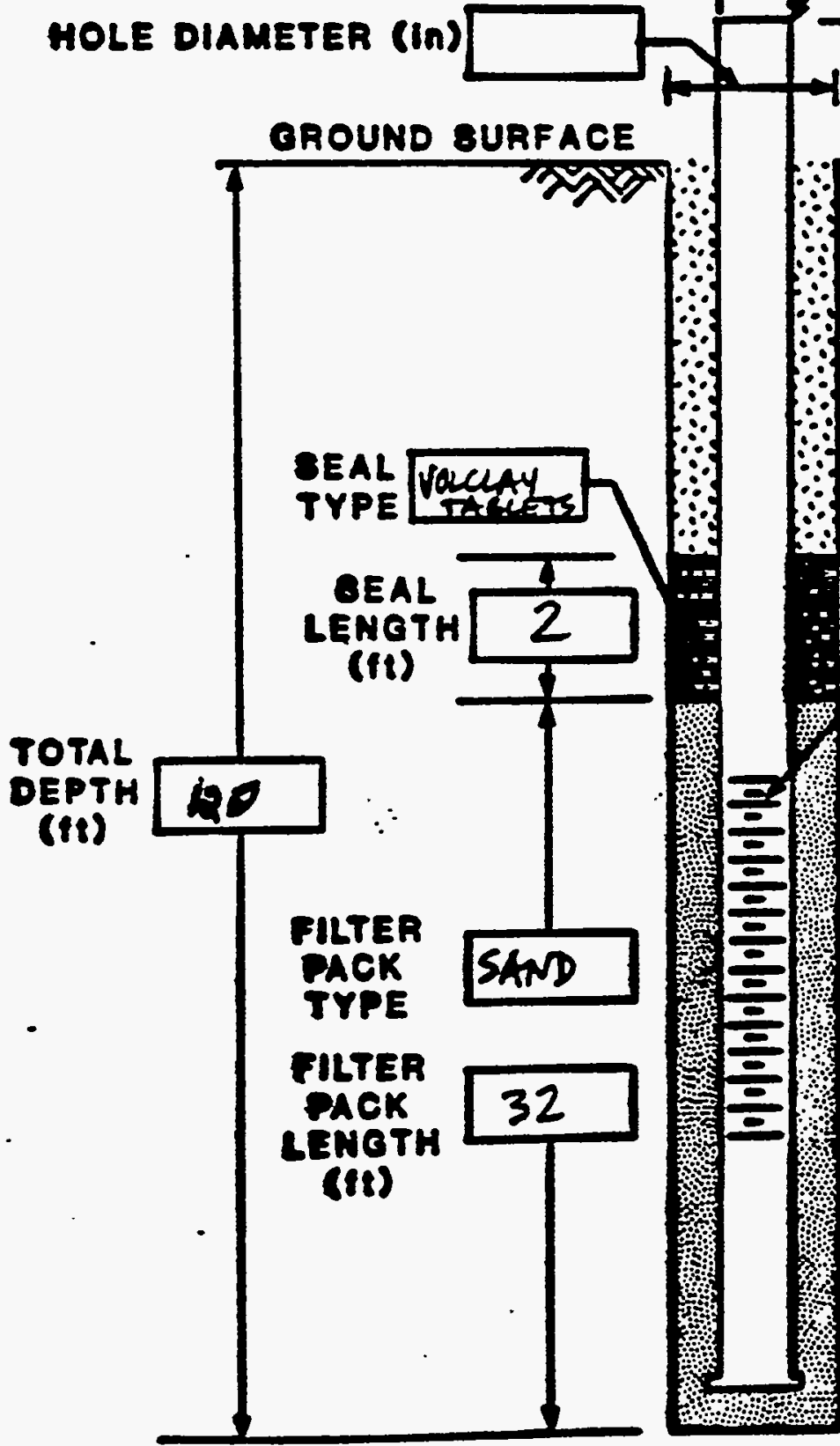
TYPE

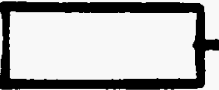




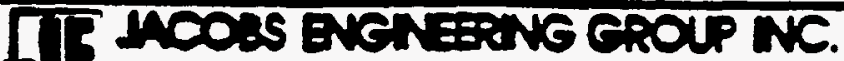

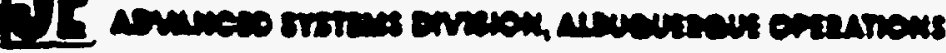 \\ BOREHOLE/WELL CONSTRUCTION LOG}

( : SITE ID: MON O L LOCATION ID:COSB FIELD REP: $M$ LENYY

APPROX. EITE COORDINATES (FT.): W

OROUND ELEVATION (FT. MBL):

COMPLETION DATE: 3/19/85

DOREHOLE SUMMARY

DAILLER:

AIG TYPE:

care

remin

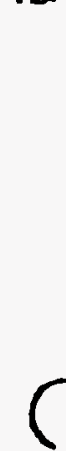

C

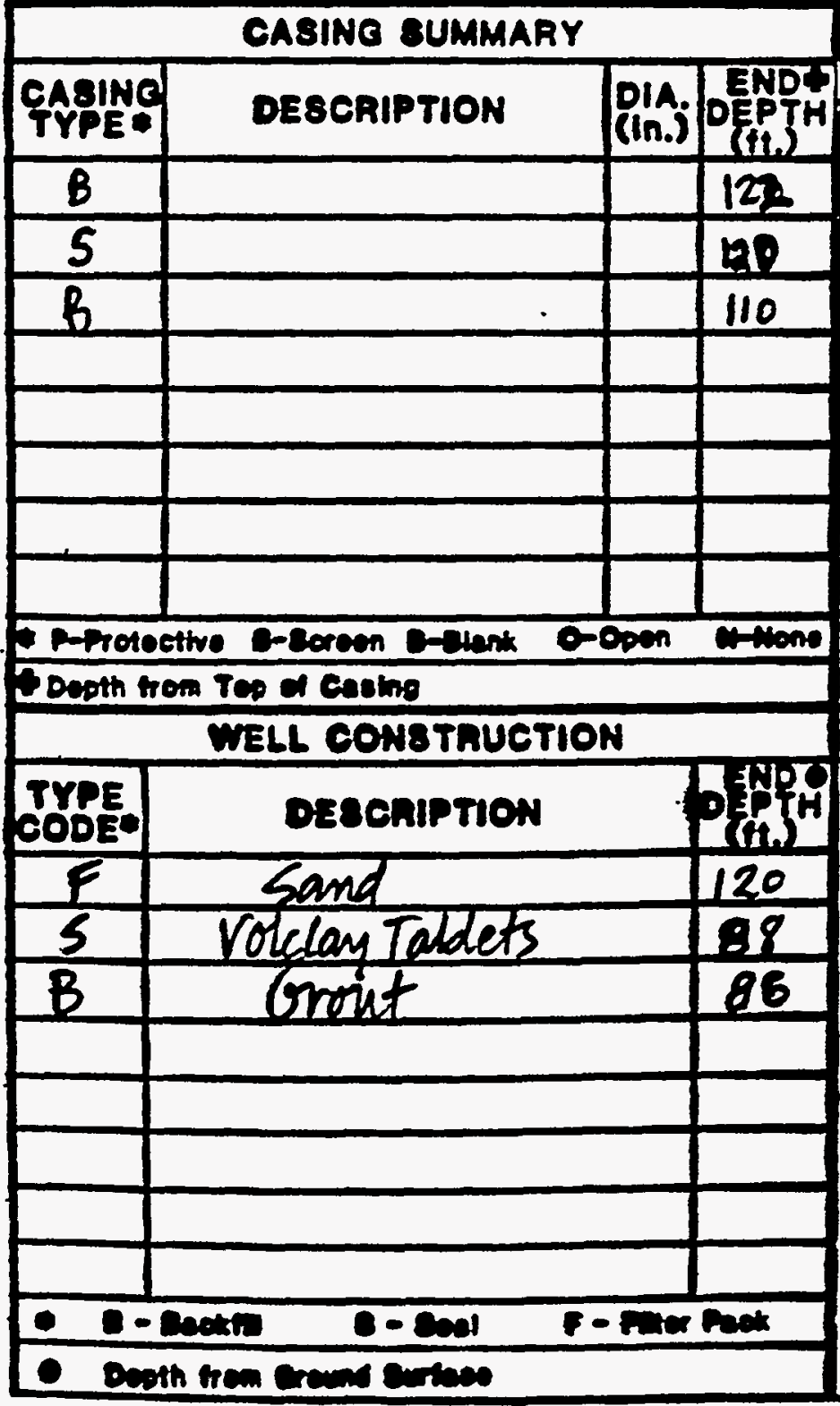

CONBTRUCTION TIME LOG

\begin{tabular}{|c|c|c|c|}
\hline \multirow{2}{*}{ ACTIVITY } & \multicolumn{2}{|c|}{ OTAAT } & \multirow{2}{*}{$\begin{array}{l}\text { END } \\
\text { TIME }\end{array}$} \\
\hline & DATE & TIME & \\
\hline DRILLING & & & \\
\hline CORING & $3 / 16 / 85$ & $900 \mathrm{~mm}$ & 314075 \\
\hline REATANA & $8 / 18 / 15$ & $2: 30 \mathrm{pm}$ & 6:00pm \\
\hline CABING & $3 / 19 / 85$ & $1: 30 \mathrm{~m}$ & 200 pri \\
\hline FILTER PACK & $3 / 19 / 155$ & $3: 00$ & $3: 15$ \\
\hline SEAL & $3 / 29 / 85$ & $3: 15$ & $3: 20$ \\
\hline BACKFILL & $3 / 4 / 15$ & $3: 20$ & $5: 30$ \\
\hline DEVELOPMENT & & & \\
\hline OTHER & & & \\
\hline & & & \\
\hline
\end{tabular}

WELL DEVELOPMENT

COMIENTS: 
A] jooss EveN zaing exoup NC.

\section{YELL COMPLETION RECORD}

SITE ID: MONOI LOCATION ID: 609 DATE INSTALLED: $1-375$ APPROX. SITE COOORDINATE8:(FT.) N 59052.132 E 07652,915 OPEN AREA PER LINEAL FT. (IN2/FT.) 0.440

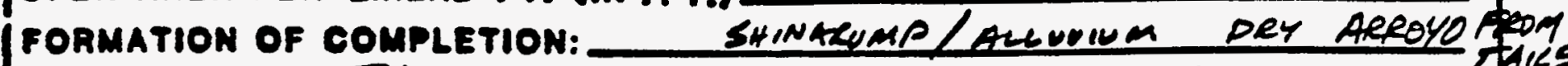
FIELD REP.: SILUA DRILLER: BCAR SHB DIAMETER $(\mathrm{fn})$ 2"

HOLE DIAMETER (in) 65/3"
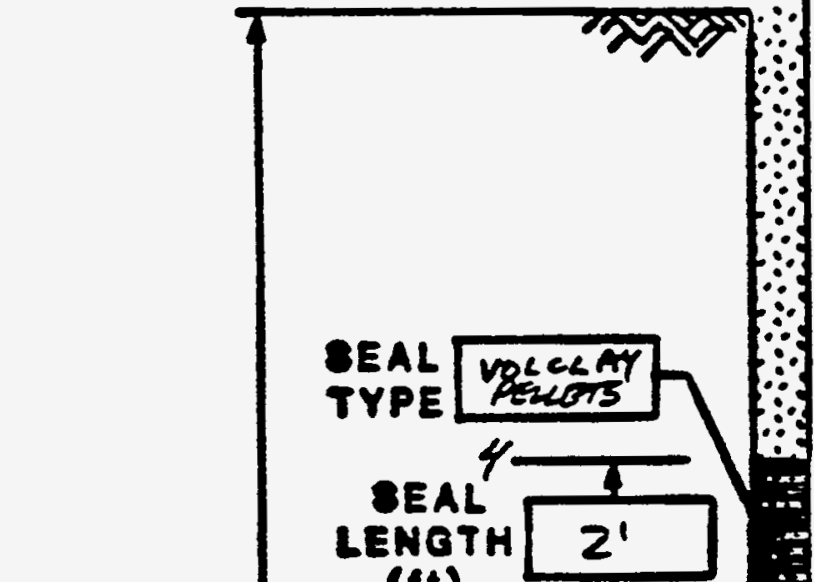

$\because$ अIN TYPE

TOTAL DEPTH (it)

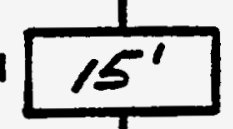

(ft)

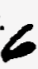

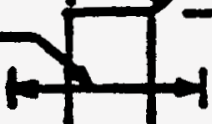

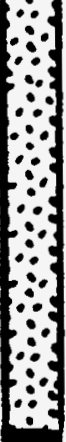

$\vdots$
$\vdots$
$\vdots$
$\vdots$
$\vdots$
$\vdots$
$\vdots$
$\vdots$

DACKFILL TYPE

Cemont bear
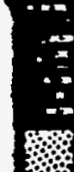

\section{.}

OPENINO

(In)

1T. ABOVE

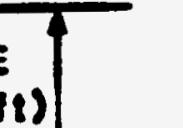




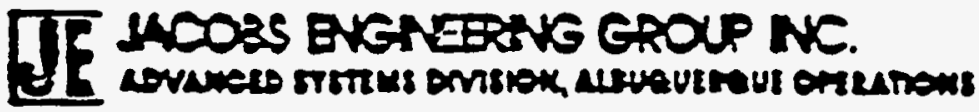

WELL COMPLETION RECORD

SITE ID: MONOI LOCATION ID: 609 DATE INSTALLED: 1.3 E'S APPROX. IITE COORDINATES:(FT.) N 59052.132 E 87652.915 OPEN AREA DER LINEAL FT. (IN2/FT.) 0,440

fFORMATION OF COMPLETION: SHINGEMP/ALUUIUM DEY ARROYO

FIELD REP.: SILUA DRILLER: DEAR SHO

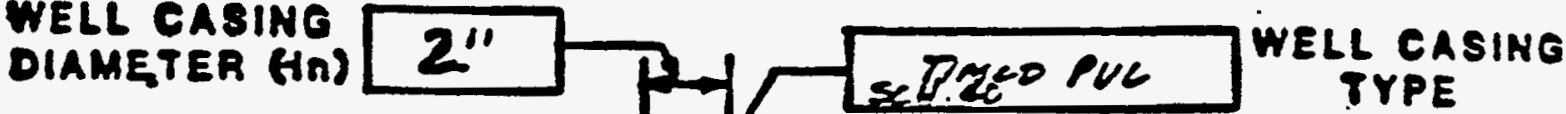

HOLE DIAMETER $(\ln ) 6^{5 / 3}$ oROUND SURFACE

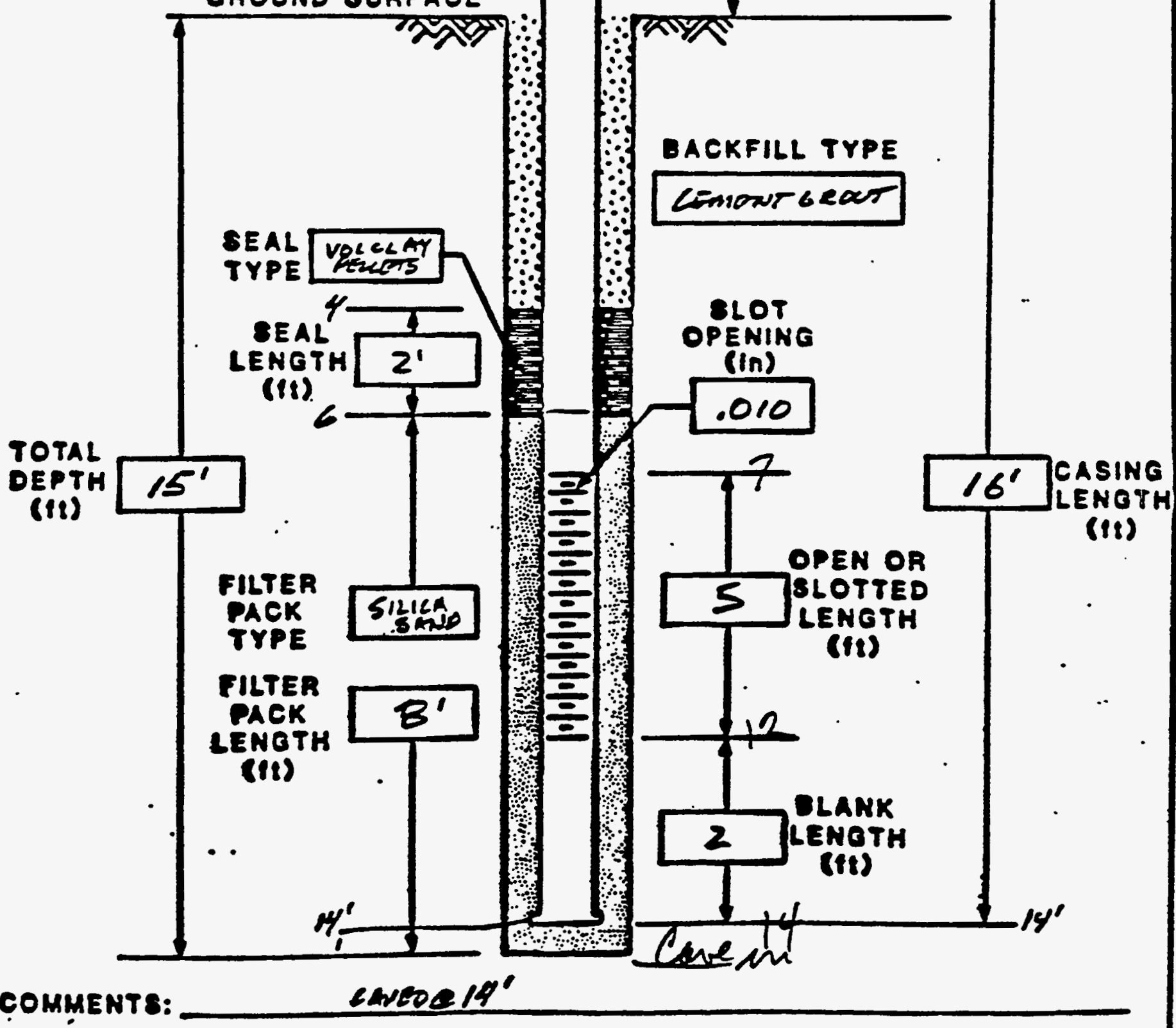

COOMMENTS:

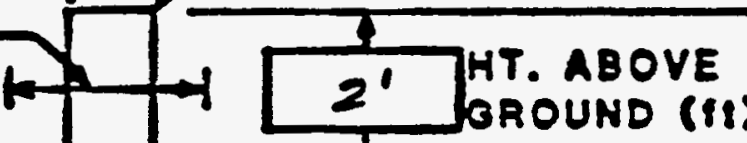




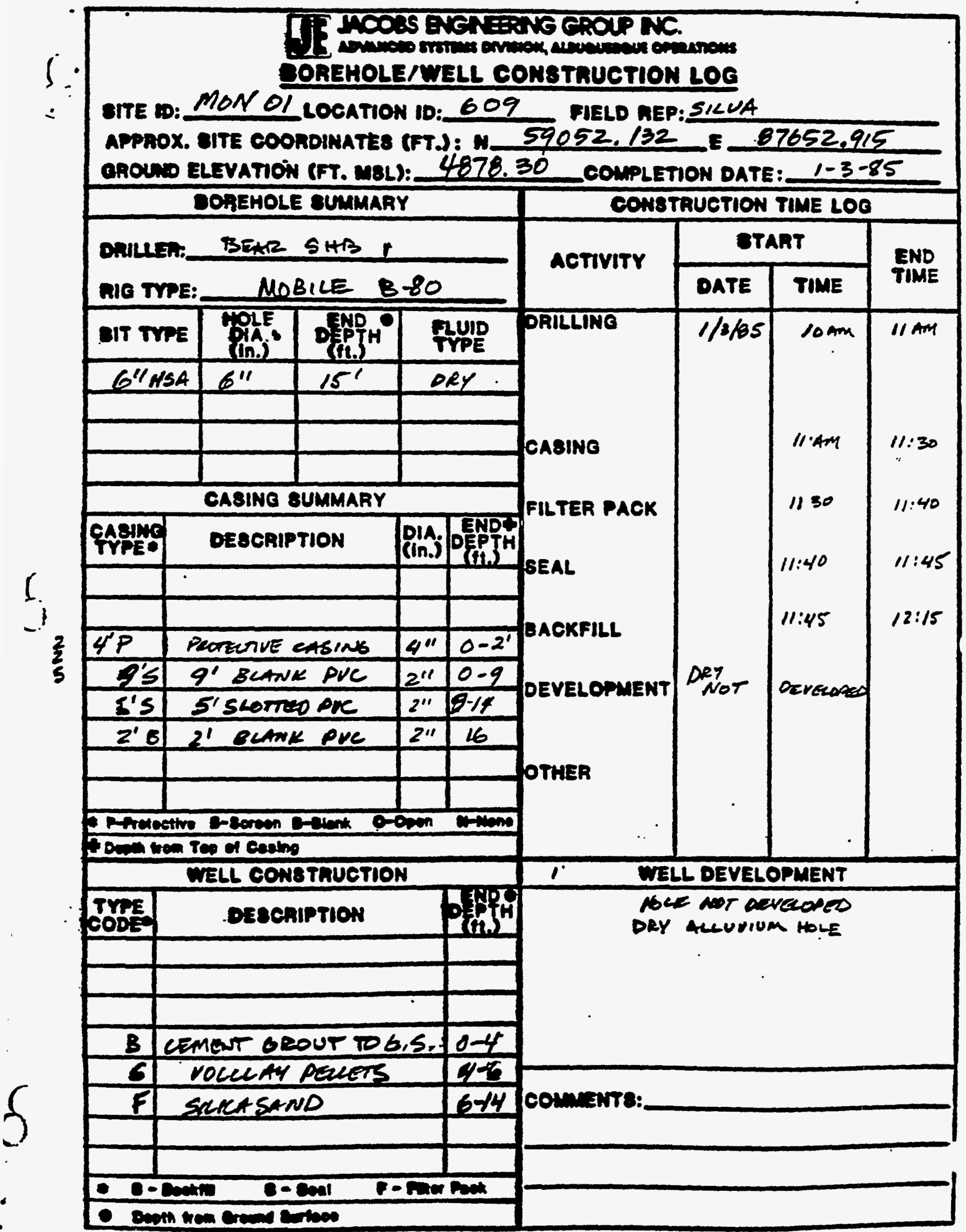


DI ACOSS ANGNANG EROUP NC. WELL COMPLETION RECORD

PAKKER INSTAKCTION

SITE ID: MONO L LOCATION 1D: 610

APPROX. SITE COORDINATES:(FT.) N 56360.765 E 08649.506 OPEN AREA DER LINEAL FT. (IN2/FT.) 0.440

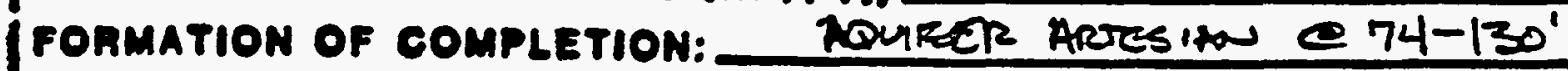

FIELD REP.: DRILLER: beARshB

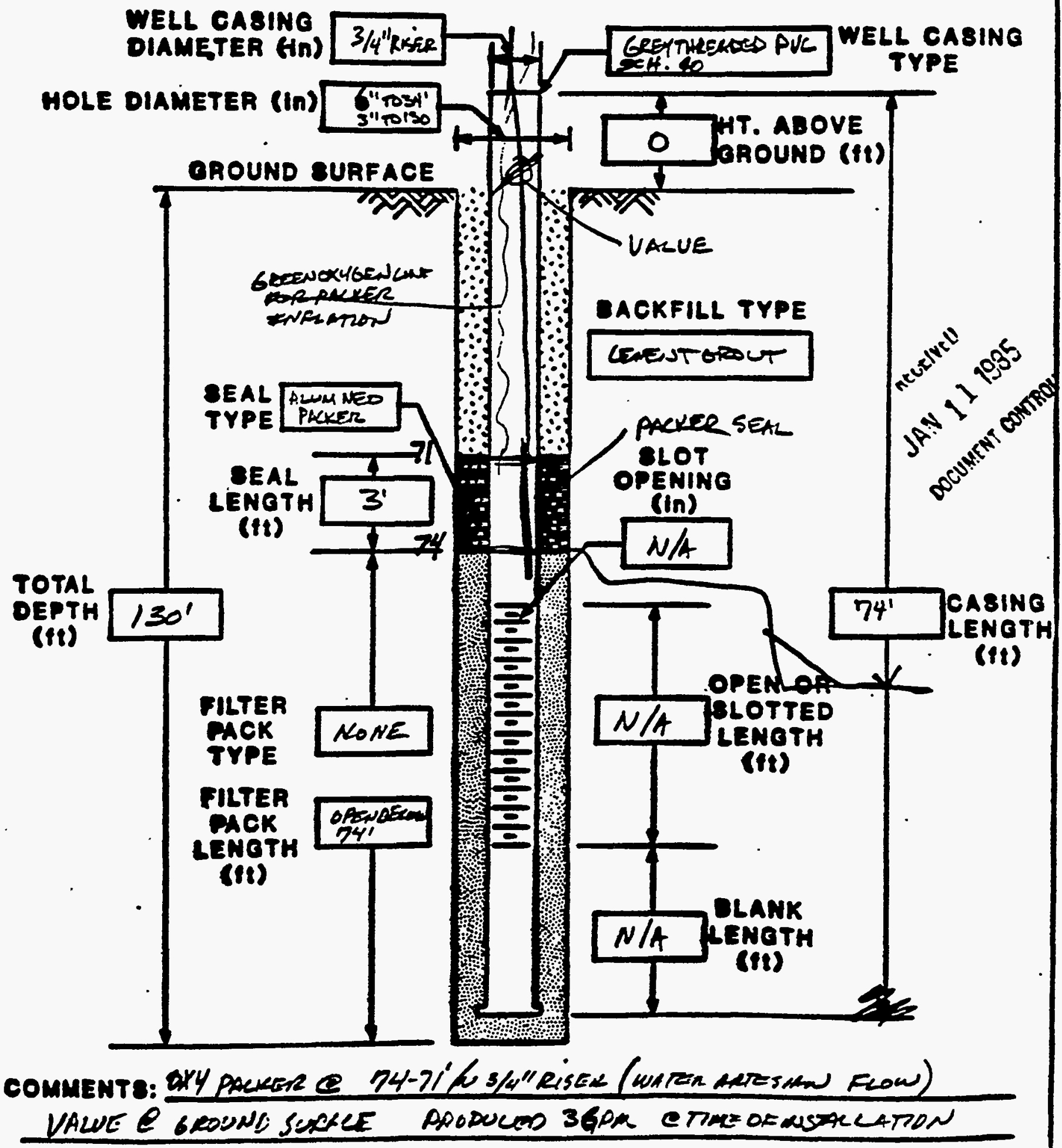

25a-Ab-swe-s (ara4) 


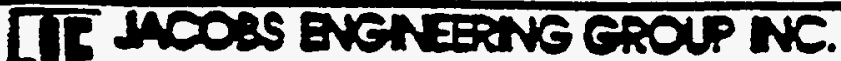

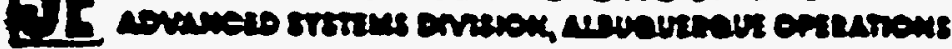 DOREHOLE/MELL CONSTRUCTION LOG}

ITE D:MON-O! LOCATION DD:hlo FIELD REP:_Roger Hallend. APPROX. OITE COORDINATES (FT.): N_56360.765 E B8649.56h QROUND ELEVATION (FT. MSL): 4641.45 COMPLETION DATE: 3/22/65 DOREMOLE SUMMARY

DAILLER: SHIB (Ed Adams) NIS TYPE:

\begin{tabular}{|c|c|c|c|}
\hline DIT TYPE & $\begin{array}{l}\text { HoLE } \\
\text { gix.: } \\
\text { (in.) }\end{array}$ & 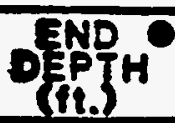 & $\begin{array}{l}\text { FUUID } \\
\text { TYPE }\end{array}$ \\
\hline zinger & 650 & 7 & clear uno \\
\hline Tricone & $4.5^{\circ}$ & $97^{\prime}$ & d) \\
\hline & & & \\
\hline & & & \\
\hline
\end{tabular}

\begin{tabular}{|c|c|c|c|}
\hline $\begin{array}{l}\text { CASING } \\
\text { TYPE }\end{array}$ & DESCAIPTION & pia. & DEPTH \\
\hline $8=$ & wht $=8 \cdot x=$ & 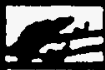 & \\
\hline$B$ & $P$ PUC & $2^{\prime \prime}$ & 83.5 \\
\hline 5 & Pue $\quad .010$ & 211 & $63.5^{\circ}$ \\
\hline$B$ & PVC & $2^{4}$ & $+105^{-1}$ \\
\hline & & & \\
\hline & & & \\
\hline & & & \\
\hline & & & \\
\hline
\end{tabular}

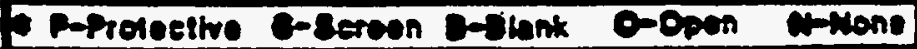
Soopin from roo of Casing

\begin{tabular}{|c|c|c|}
\hline \multicolumn{2}{|c|}{ WELL CONSTRUCTION } & \\
\hline $\begin{array}{l}\text { TYPE } \\
\text { CODE }\end{array}$ & DESCRIPTION & \\
\hline$\beta$ & cement Grout & 9 \\
\hline 5 & sentonite & $\mathrm{ag}^{\prime}$ \\
\hline$F$ & Ghencsand & 25,5 \\
\hline & & \\
\hline & & \\
\hline & & \\
\hline & & \\
\hline & & \\
\hline
\end{tabular}

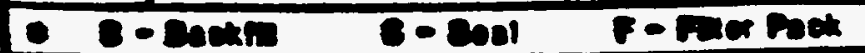

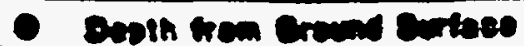

se-al-zue-s (olch).

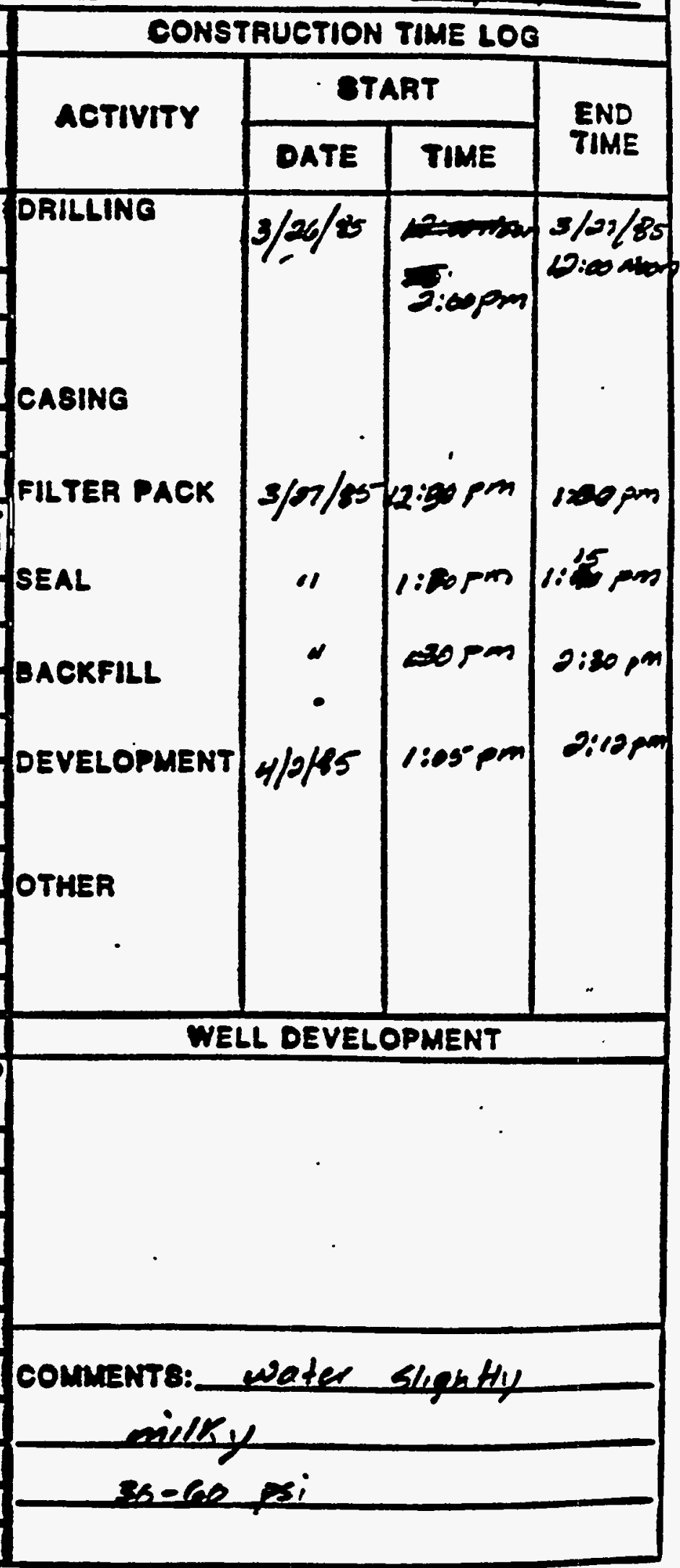


ITE ID: MON OI LOCATION ID:LO10 FIELD REP: SILVA

APPROX. QTTE COONDINATES (FT.): N 56360,765 E E8649.506 EROUNO ELEVATION (FT. MOL): 4861.65 COMPLETION DATE: $1-7-85$

DONEHOLE CUMMARY

OALLER: WHME TEVGENAGER

RIO THE: MOBILE $8-B 2$

\begin{tabular}{|c|c|c|c|}
\hline VPE & $\begin{array}{l}\text { POLE } \\
\text { PIA } \\
\text { (n.i) }\end{array}$ & 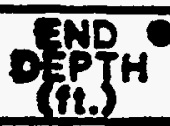 & $\begin{array}{l}\text { TuID } \\
\text { TYPE }\end{array}$ \\
\hline 6"NSA & $65 / 3$ & $34^{\prime}$ & MATLL \\
\hline 30104866 & $3^{11}$ & $130^{\prime}$ & Fla leen \\
\hline 4 waterenty & & & WATER \\
\hline
\end{tabular}

\section{GABING DESCRIPTION}

\begin{tabular}{|c|c|c|c|}
\hline GABING & DE8CAIPTION & $\begin{array}{l}\text { pia. } \\
\text { (in.) }\end{array}$ & DEPTH \\
\hline & 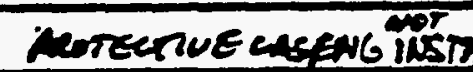 & 200 & \\
\hline & GREEN OXYY HOSE & $V_{4}^{\prime \prime}$ & \\
\hline$\Delta$ & KALVE & & 0 \\
\hline$B$ & 3/4" GLEY PUCRGER & $3 / 4$ & 71 \\
\hline SEALA & Aperer sorup & 3 & 74 \\
\hline & & & \\
\hline & & & \\
\hline & $\therefore$ & & \\
\hline DPfion & tho C-Soreen Calent o & $m$ & 0 anome \\
\hline Sopoth & Tes a caske & & \\
\hline & WELL CONBTRUCTIO & & \\
\hline $\begin{array}{l}\text { TYPE } \\
\text { CODE }\end{array}$ & DESCAIPTION & & \\
\hline & & & \\
\hline$B$ & ciment Geour exen & ife & \\
\hline 5 & encerresente & & $71-y^{\prime}$ \\
\hline & Not Schervas le & 23 & pre \\
\hline & & & \\
\hline & & & \\
\hline$\bullet$ & $2-c_{0 a 1}$ & Trex & \\
\hline$e$ & 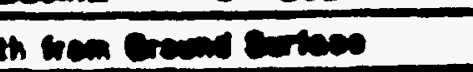 & & \\
\hline
\end{tabular}

CasineCONBTAUCTION TIME LOQ comimanto: ARTESINN whree Source From sWWDSTONES \& UARIOUS AOVIFER From $74-130^{\prime}$ 


\section{LT MCOSS ENEN ERING EROUP NK.

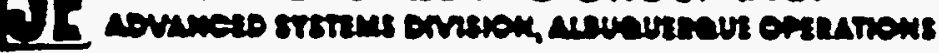 \\ DOREHOLE/WELL CONSTAUCTION LOG}

SITE DD:MON-OI LOCATION ID: LLO

APPROX. SITE COORDINATES (FT.): N

OROUND ELEVATION (FT. MSL):

DOREHOLE BUMMARY

DRILLER: SHIB (Ed Adams)

RIG TYPE:

\begin{tabular}{|c|c|c|c|}
\hline BIT TYPE & $\begin{array}{l}\text { HOLE } \\
\text { gik.: } \\
\text { (in.) }\end{array}$ & OFNPH & $\begin{array}{l}\text { FLUID } \\
\text { TYPE }\end{array}$ \\
\hline ruace & 65 & 372 & clear 400 \\
\hline Tricone & $4.5^{\circ}$ & $87^{\prime}$ & 11 \\
\hline & & & \\
\hline & & & \\
\hline
\end{tabular}

\begin{tabular}{|c|c|c|}
\hline & ING & \\
\hline CASING & DESCRIPTION & pia.jo \\
\hline
\end{tabular}

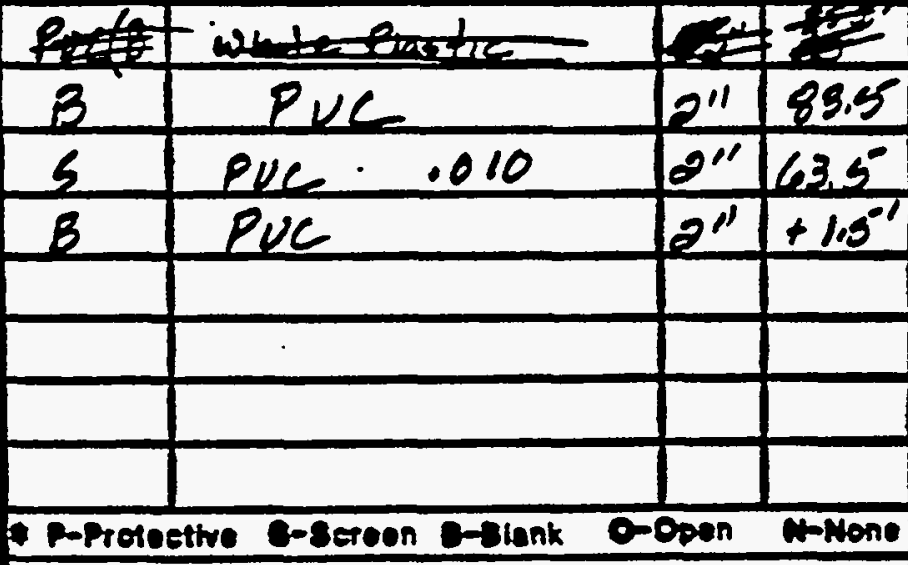

3 Dopsh from Toe of Ceolne

\begin{tabular}{|c|c|c|}
\hline \multicolumn{3}{|c|}{ WELL CONSTRUCTION } \\
\hline $\begin{array}{l}\text { TYPE } \\
\text { CODE }\end{array}$ & DESCAIPTION & PePph \\
\hline$B$ & cement crout & $59^{\prime}$ \\
\hline 5 & Bentonite & $63^{\prime}$ \\
\hline$F$ & Gepen.sand & $25,5^{\circ}$ \\
\hline & & \\
\hline & & \\
\hline & & \\
\hline & & \\
\hline & & \\
\hline 08 & e-cal & Pach \\
\hline
\end{tabular}

OTHER
E

COMPLETION DATE: $3 / 22 / 65$

CASING

FILTER PACK

SEAL

BACKFILL

DEVELOPMENT CONSTRUCTION TIME LOQ

ACTIVITY

DRILLING

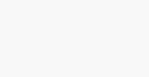

$$
\text { (1) }
$$

START

\begin{tabular}{|l|l|}
\hline DATE & TIME \\
\hline
\end{tabular}

END

TIME

\begin{tabular}{|c|c|c|}
\hline $3 / 26 / 25$ & 3:00pm & $\begin{array}{l}5 / 23 / 85 \\
10: 00 \mathrm{dtan}\end{array}$ \\
\hline
\end{tabular}

COMMENTE: water Shepty

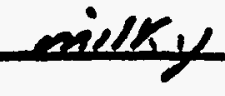

ss-re psi 

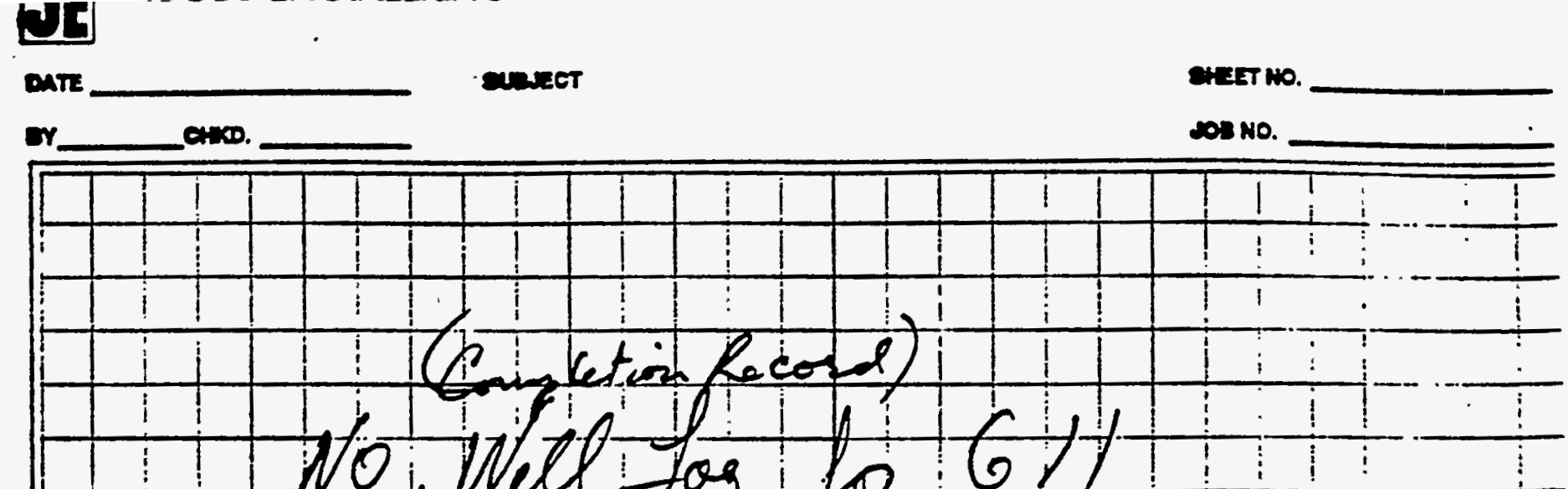

$1:$

No, Nell $\log$ fo $6 H$

Stet Casing set to 63 for coning to 185 then seamed.

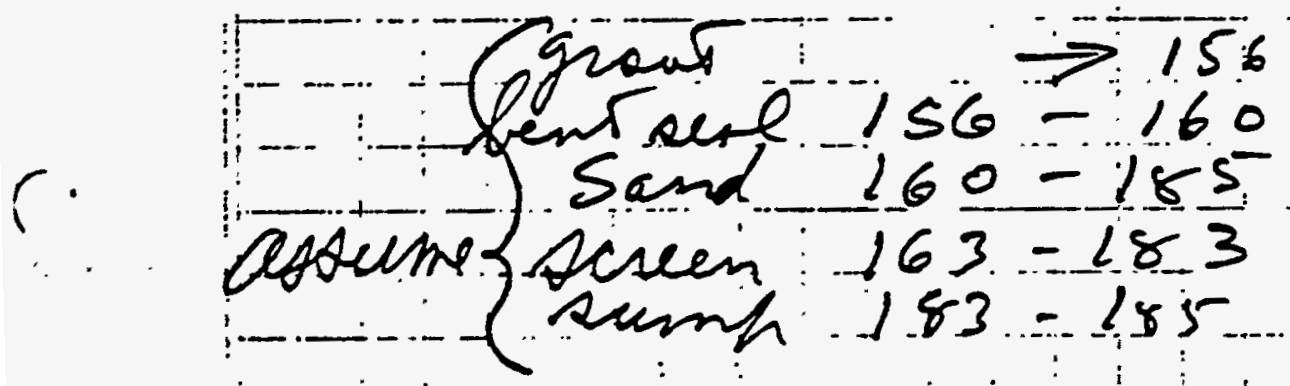




\section{IT MOOSS EVEN ZENG GROUP NC.

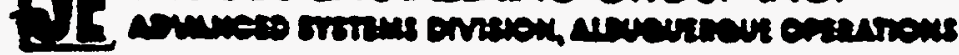

YELL COMPLETION AECORD (filled out by B.Williams):

SITE ID: MON-01 LOCATION ID: Lo/2 DATE INSTALLED: 3 -

APPAOX. SITE COOORDINATE8:(FT.) $N$

OPEN AREA DER LINEAL FT. SIN2/FT.)

fFORMATION OF COMPLETION: Dechelly

FIELD REP.: G.Miller

DRILLea: B, Williams

WELL Casine

DIAMETER (In)

$2^{\prime \prime}$

HOLE DIAMETER (ln)

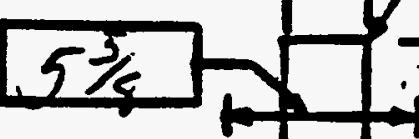

OROUND SUAFACE
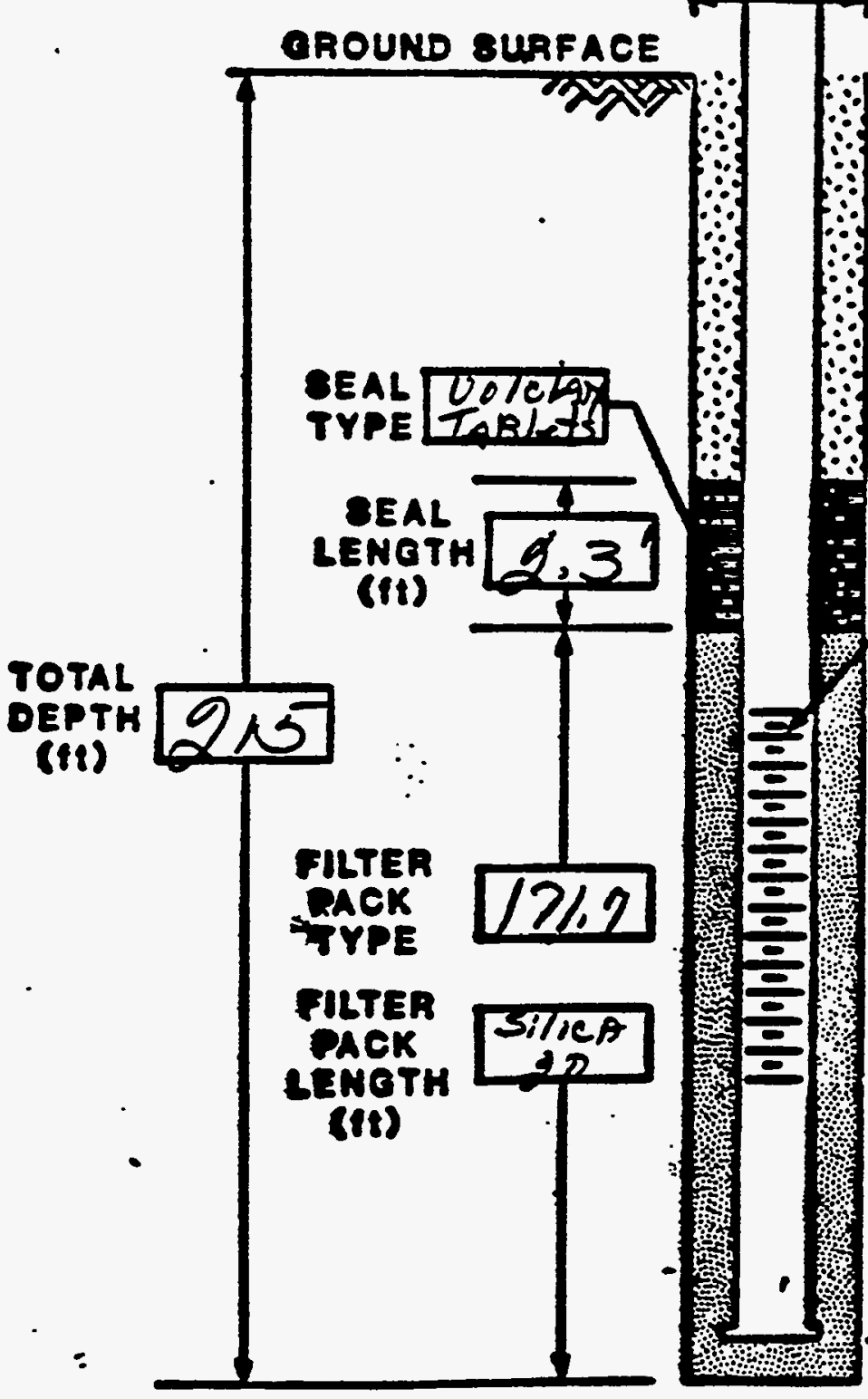
E

$42 \% \sqrt{\text { ta }}$

BACKFILL TYPE

AnouT
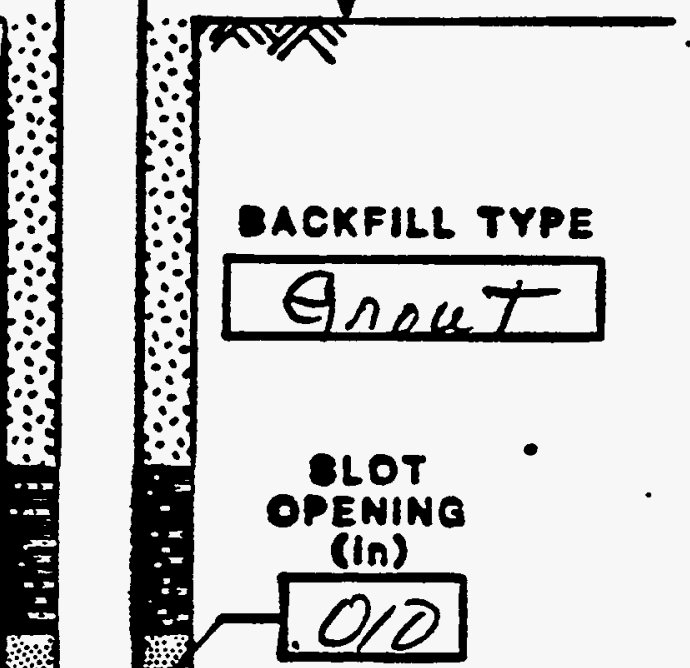

.

SLOT

OPENINO

(in)
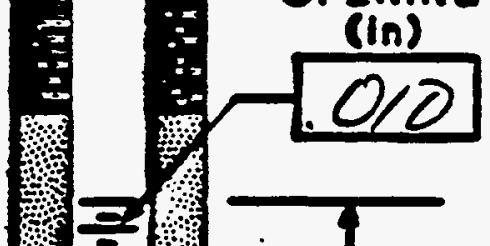

. 2/Rfonsing TYPE

OOMMENTS: 


\section{Tí meos avenszing exoup ne.

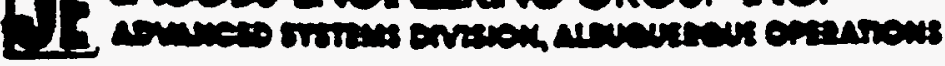

\section{- MELL COMPLETION RECOAD}

SITE ID: MON 07 LOCATION ID: $6 / 3$

APPROX. SITE COOORDINATE8:(FT.) N

DATE InstaLLED: $3 / 18 / 85$

OPEN AREA DER LWEAL FT. (IN2/FT.)

FORMATION OF COMPLETION: DeChelly

FIELO REP.: MEEAYY OAILLER: $5 H+B$

well casino DIAMETER (In)

hole dIAMETER (In)

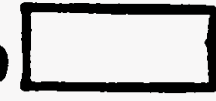

OROUND BURFACE

TOTAL Depth (ti)
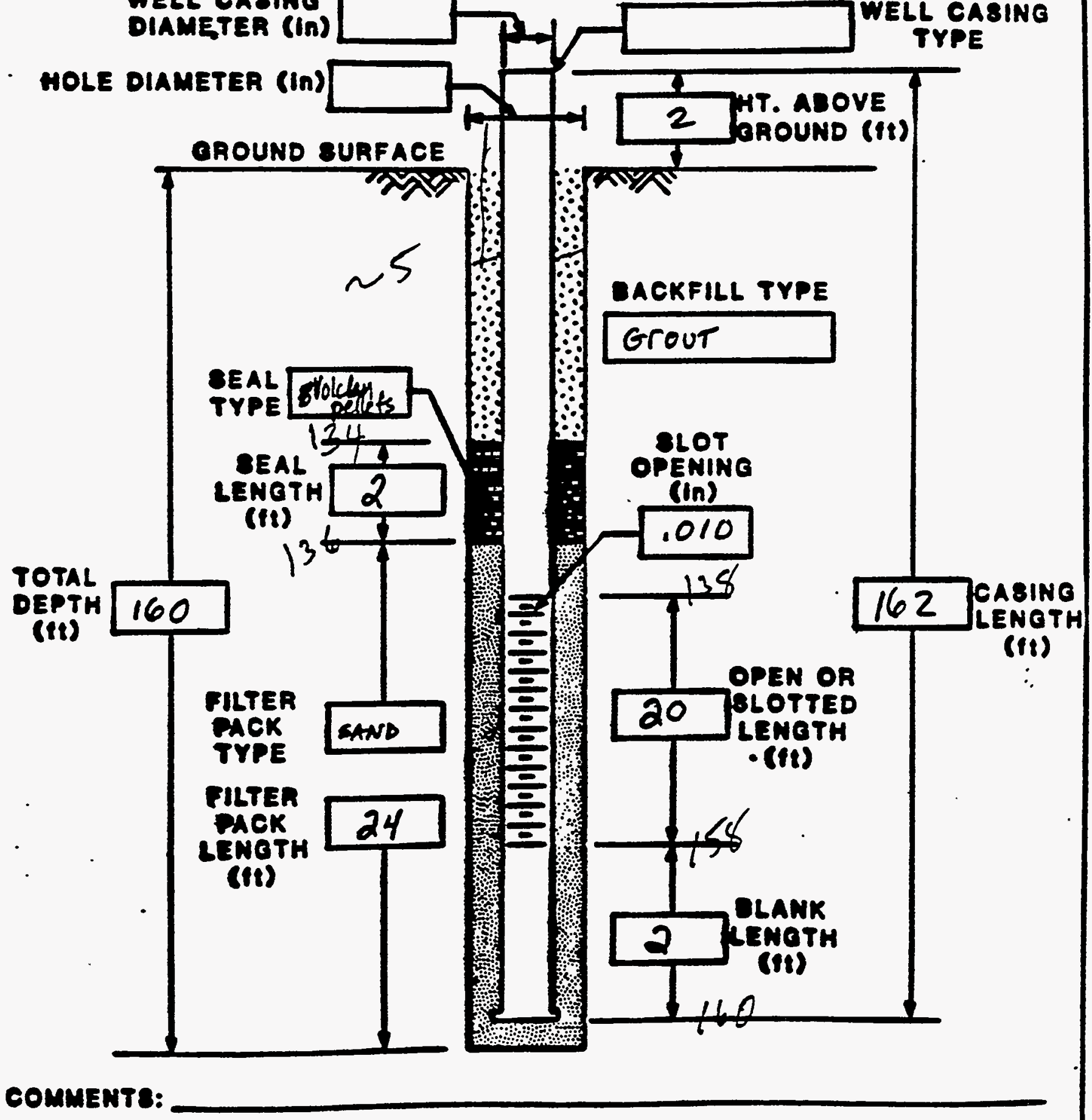

SEAL TYPE
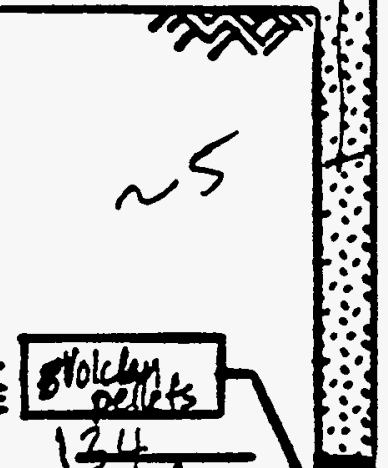

$\because: 3$

DACKFILL TYPE

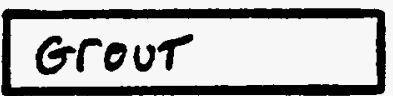

2 BROUND (it) TYPE 


\section{- YELL COMPLETION AECORD}

SITE ID: MON O7 LOCATION ID: 613 ATPROX. SITE COOADINATES:(FT.) N DATE INSTALLED: 3/18/85 OPEN AREA PER LINEAL FT. $\left(\right.$ IN $^{2} / F T$.) FORMATION OF COMPLETION: Dechelly FIELD MEP.: MEnETY DRILLER: $S H+B$ E

well casino DIAMETER (In)

HOLE DIAMETER (In) OAOUND SURFACE

TOTAL DEPTH (11)
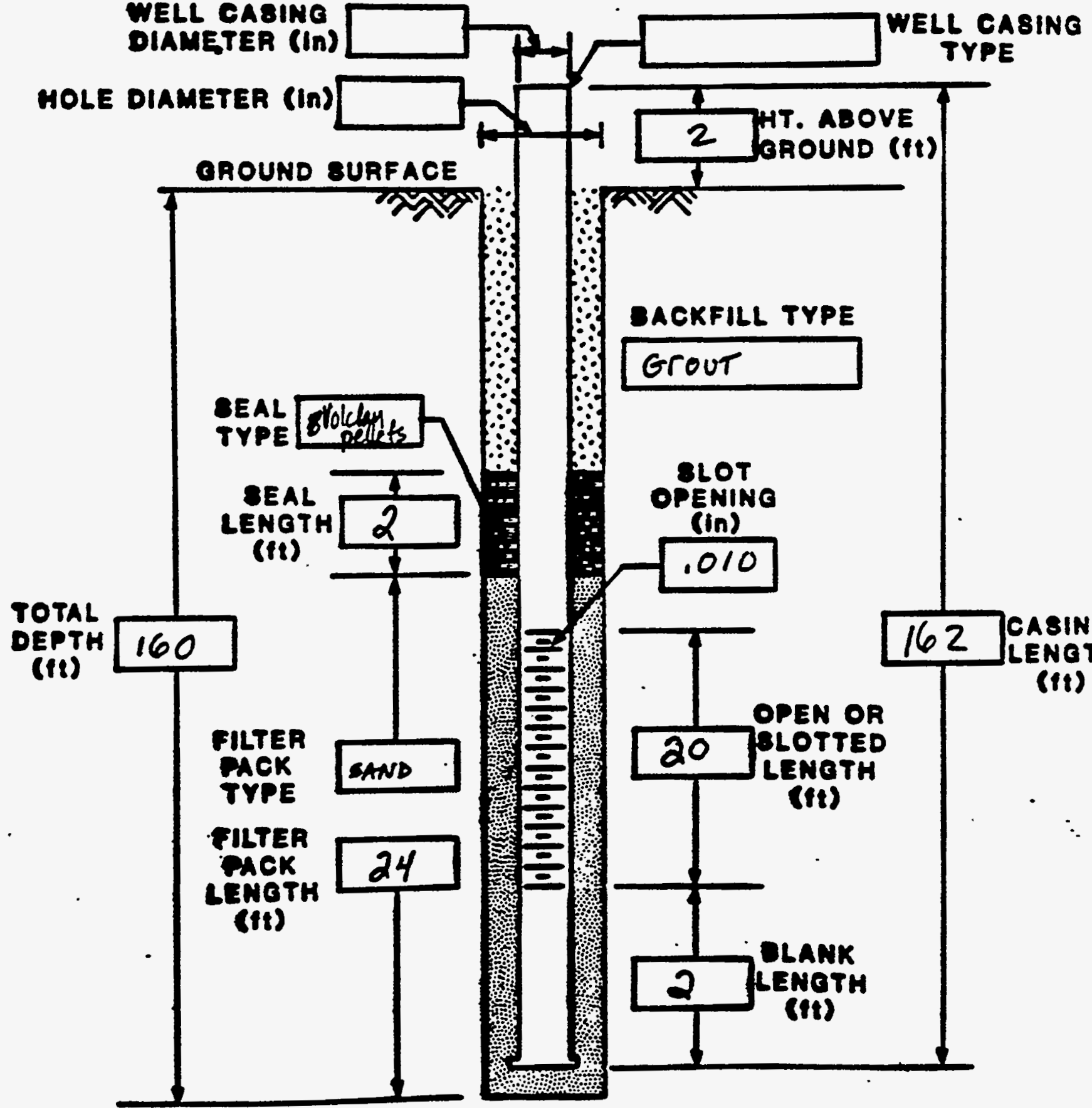

TROUND (tt)

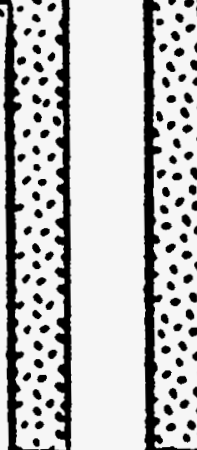

BACKFILL TYPE

Grout

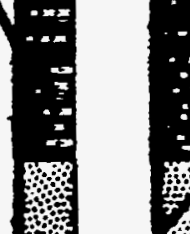

SLOT

openINO

(in)

$\frac{=}{=}$
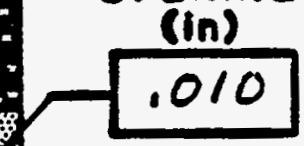

?

COMMENTS: 
MELL COMPLETION RECORD (filled ont by B.W.lliams)

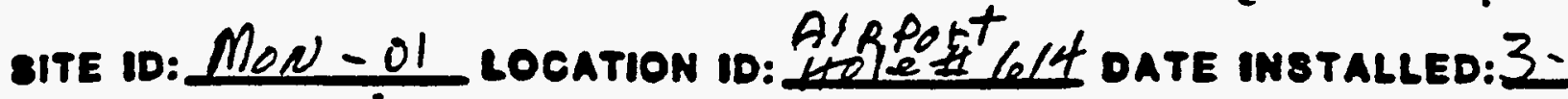
APPROX. SITE COORDINATE8:(FT.) N OPEN AREA PER LINEAL FT. $\left(N^{2} / F T.\right)$ fonmation of coMpletion: MoeN Jlopl FIELD NEP.: G Miller DRILLER: \& Wellions

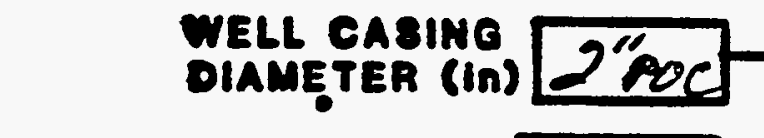

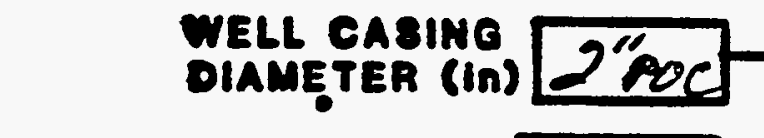

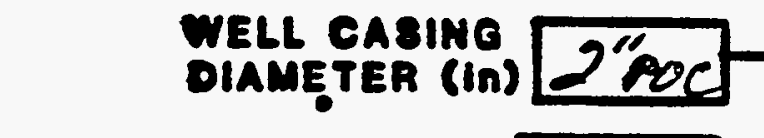
hole DIAMETER (In) $8 "$ fu 2 POC WELL CABING

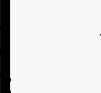

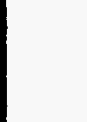

5

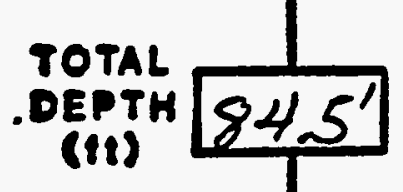

OROUND SURFACE phs

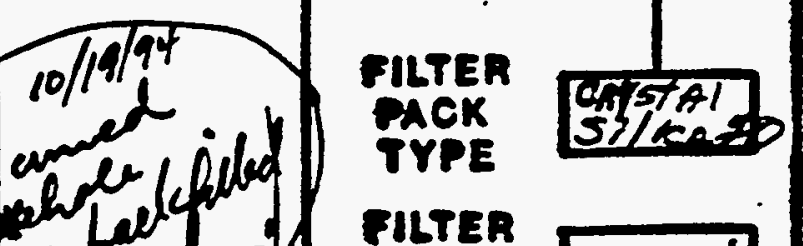
OEAL VOlc/ay
TYPE TARlets

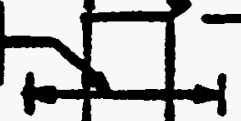
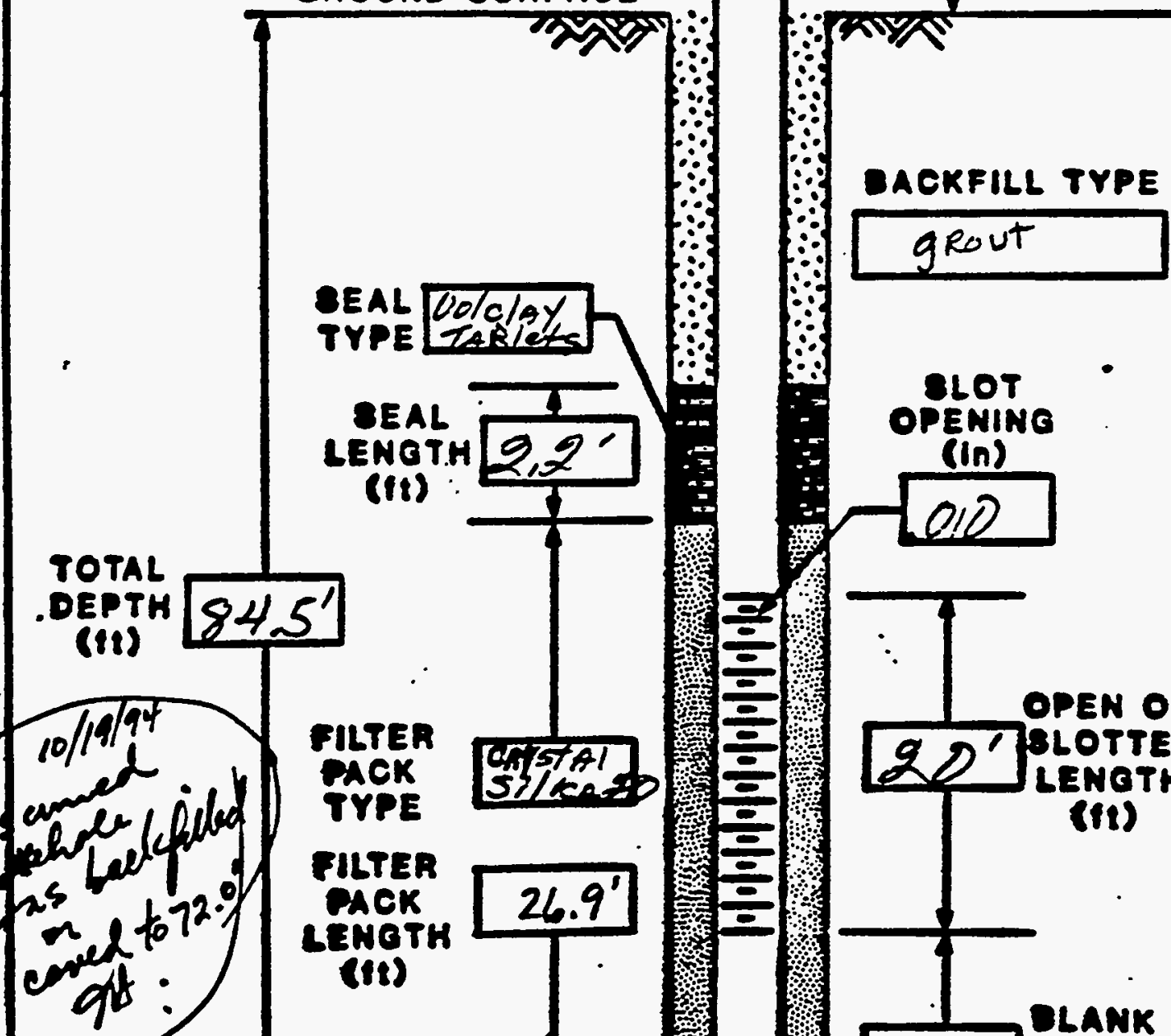

FILTER

BEAL
LENOTH $2, Z^{\prime}$
(II):
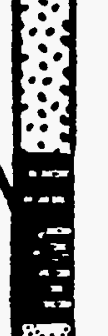
DI MCONS ANEN ENA GROUP NC.

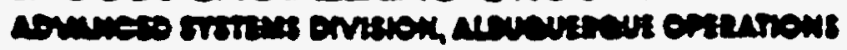

\section{MELL COMPLETION RECORD}

BITE ID: MON LOCATION ID: 615 DATE INSTALLED: $3 / 16-17 / 86$ APPROX. BITE COORDINATES:(FT.) N E

OPEN AREA PEA LINEAL FT. (IN2/FT.)

formation of completion: Shivarump Member of Chinle Fm,

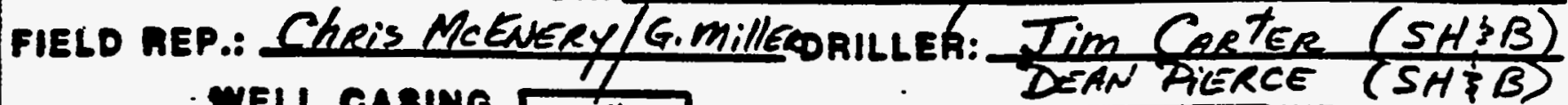

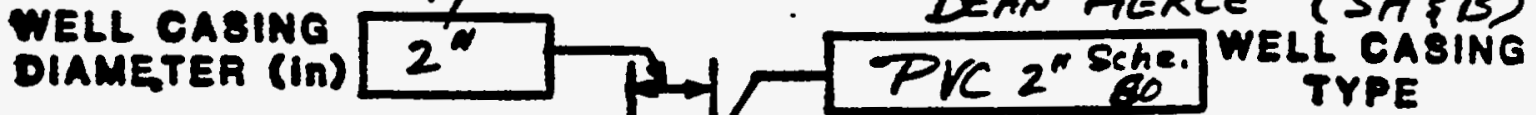

HOLE DIAMETER (in) $67 / 8$

BROUND SURFACE

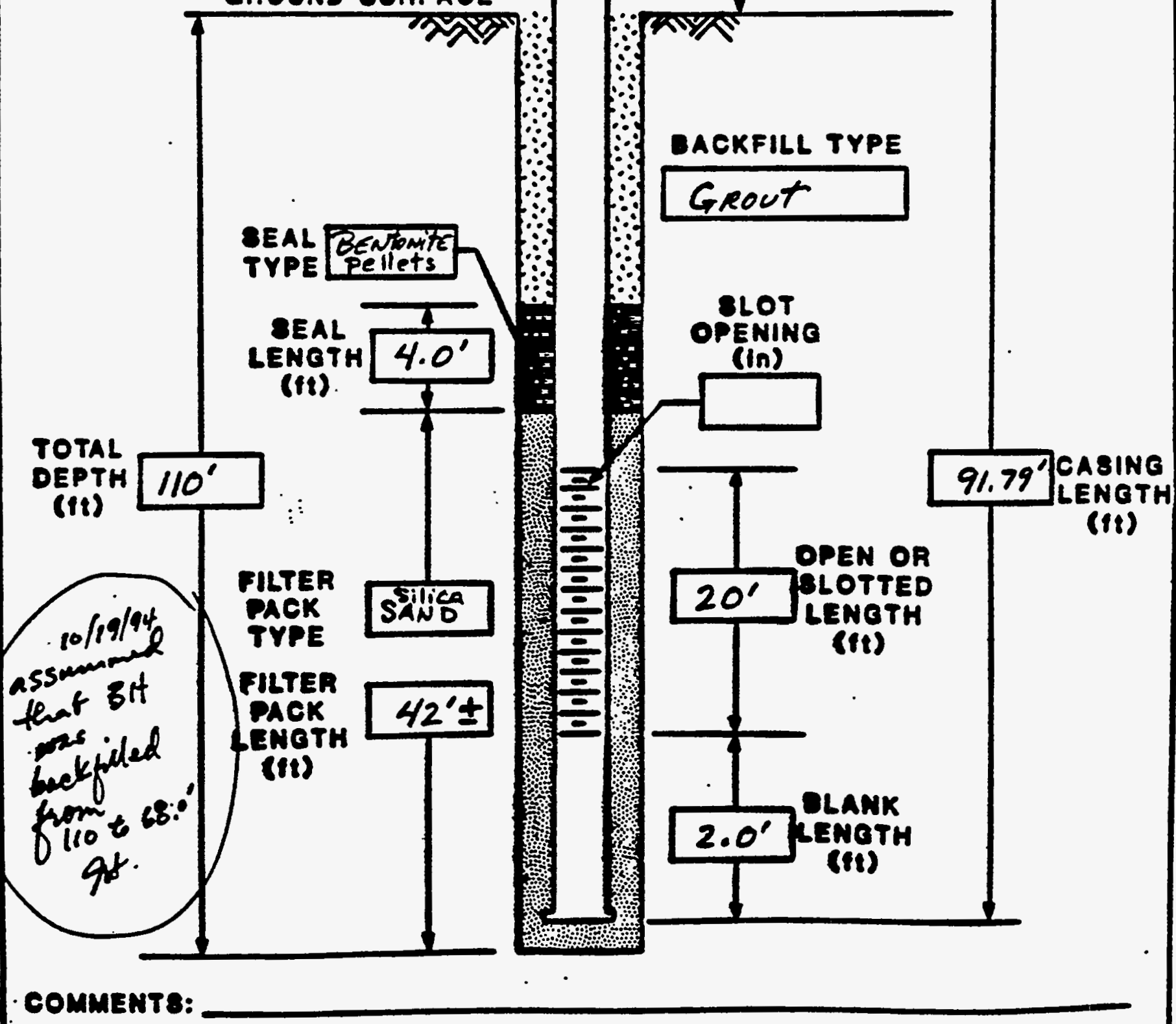




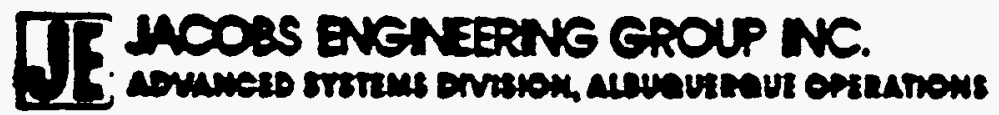

\section{WELL COMPLETION AECORD}

SITE DD: MEC-eL LOCATION ID: 650 DATE IN8TALLED: $2 / 30 / 86$ APPAOX. OITE COORDINATE8:(FT.) $N$ E

OPEN AREA PER LINEAL FT. (NN2/FT.)

FORMATION OF COMPLETION:

Pllueluen

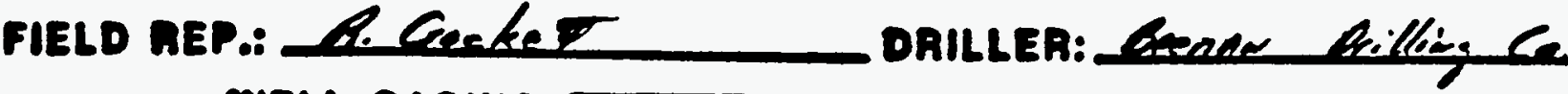

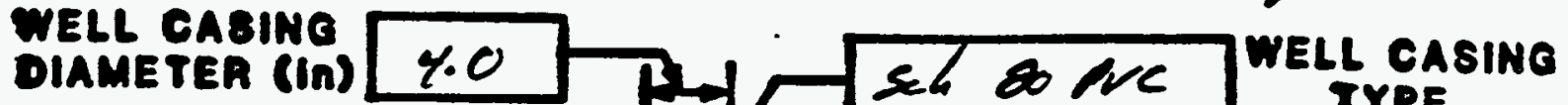

HOLE DIAMETER (In) $7 \%$

QROUND SURFACE

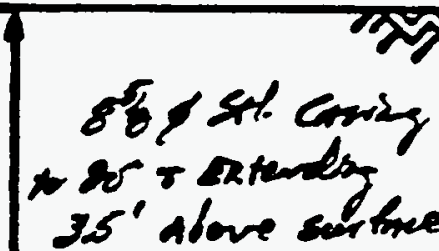

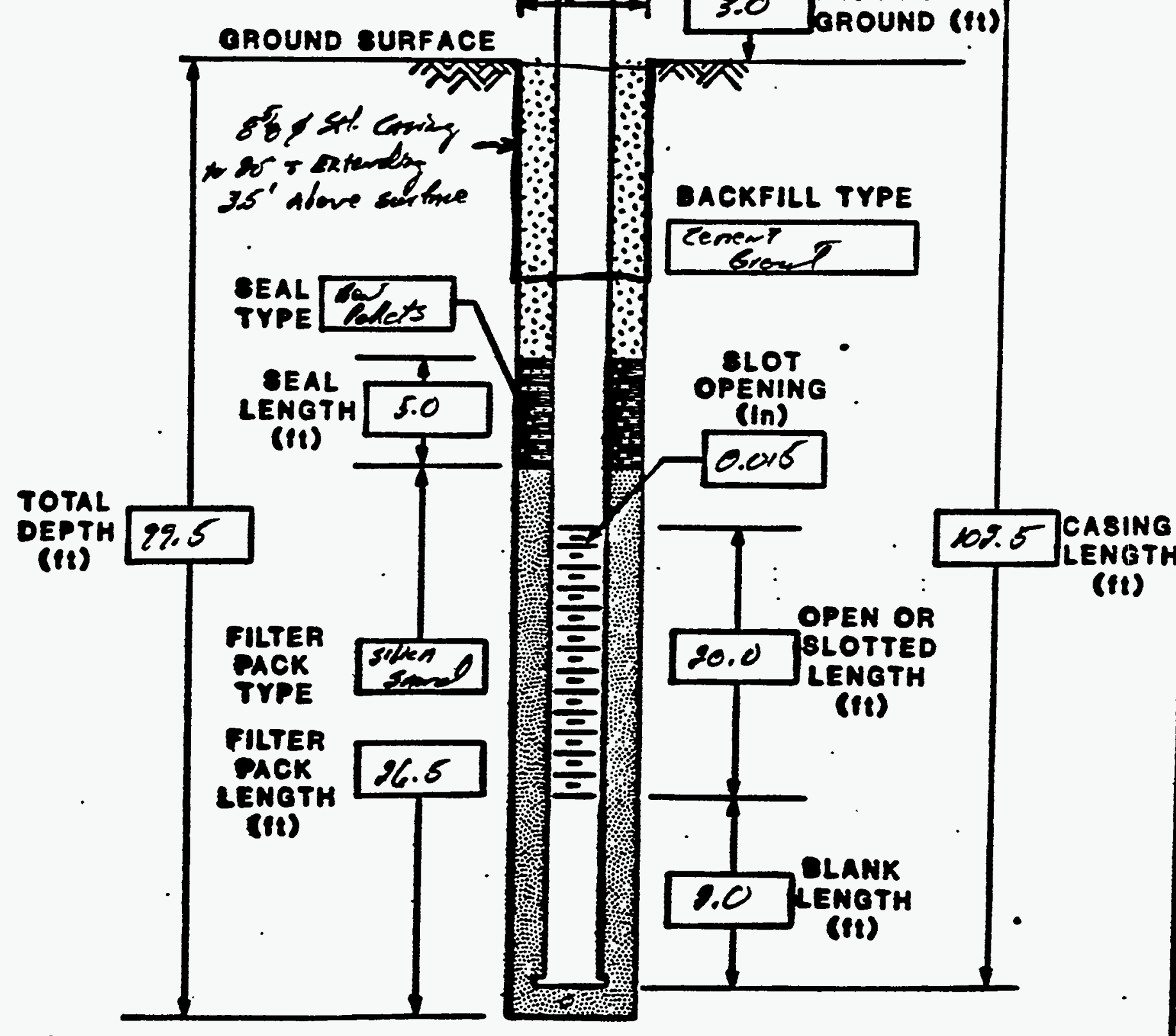

$\sqrt{-1}$ TYPE

COMMENTS: 


\section{IIE HODUS BNG ESONG CROUP NC

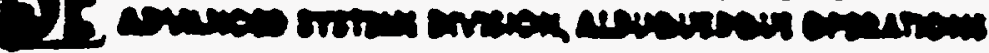 \\ DOREHOLEIMELL CONETAUCTION 200}

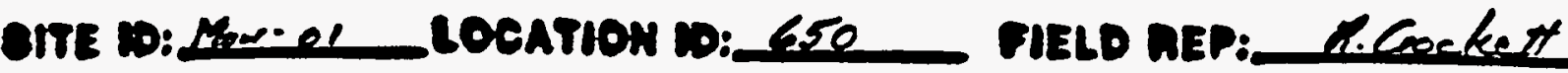

: APPnox. OITE COOADIMATES (FTd: N___

OAOUND ELEVATION (FT. MEL):

COMPLETION DATE: 2/3O/ES

OOREHOLE OUMMANY

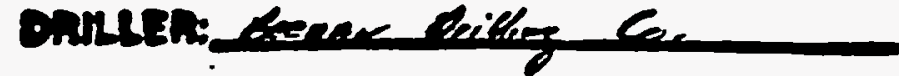

No Trm: Gacelower - daver

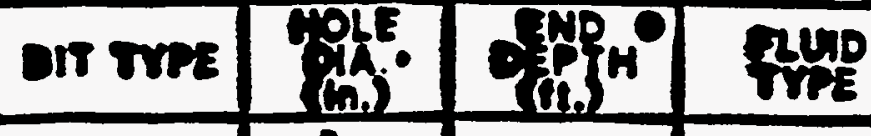

Teleme

iey 1 ent

\section{casino summany}

\begin{tabular}{|c|c|c|c|}
\hline cAYing & DESCAIPTION & $\begin{array}{l}\text { Pin. } \\
\text { (in.) }\end{array}$ & 霫 \\
\hline$B$ & Sted & 88 & \\
\hline a & sh so ave & 4 & 12 \\
\hline 5 & $n$ & 4 & 12 \\
\hline$B$ & $a$ & 4 & \\
\hline & & & \\
\hline & & & \\
\hline & & & \\
\hline & & & \\
\hline
\end{tabular}

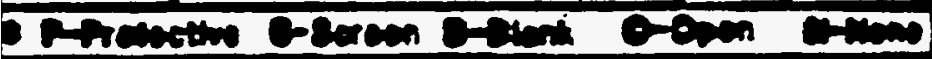
Sompon res ct Casm

WELL COMBTAUCTION

\begin{tabular}{|c|c|}
\hline & gesentp \\
\hline 6 & Gent \\
\hline 5 & solied a \\
\hline
\end{tabular}

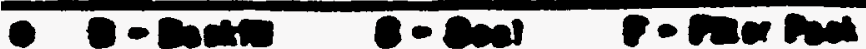

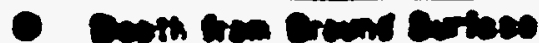

CONBTRUCTION TME LOO

\begin{tabular}{|c|c|c|c|}
\hline \multirow{2}{*}{ Activity } & \multicolumn{2}{|c|}{ otant } & \multirow{2}{*}{$\begin{array}{l}\text { END } \\
\text { TIME }\end{array}$} \\
\hline & Date & SIME & \\
\hline DRILLINO & T/xeler & 7.00 & 10000 \\
\hline AOINO & $9 / 30$ & 10.20 & א.3० \\
\hline FILTER PACK & $y / 30$ & $11: 20$ & 1.00 \\
\hline EAL & $1 / 30$ & 1.00 & 1830 \\
\hline BACKFILL & $2 / 86$ & 1.30 & $9 i 00$ \\
\hline DEVELOPMENT & $10 / 6$ & $8: 30$ & \\
\hline OTMER & & & \\
\hline & L DEV & DMENT & \\
\hline
\end{tabular}




\section{YELL COMPLETION RECORD}

SITE DD: Mar-O LOCATION DD: 651 Date installed: $z 2 g / 85$ APPAOX. SITE COORDINATE8:(FT.) $N$ E OPEN AREA PER LINEAL FT. (IN2/FT.) FORMATION OF COMPLETION:

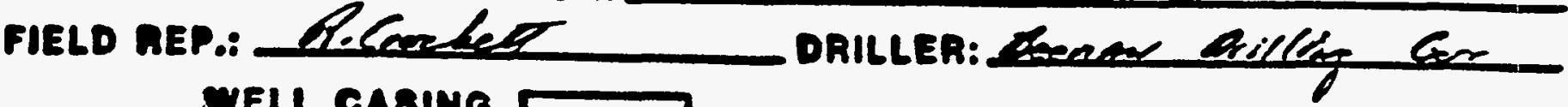

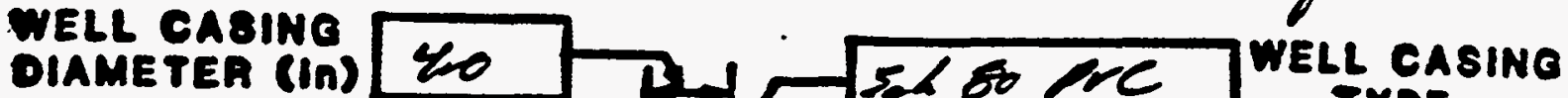
hole diameter (In) $2 / 8$
OROUND BURFACE

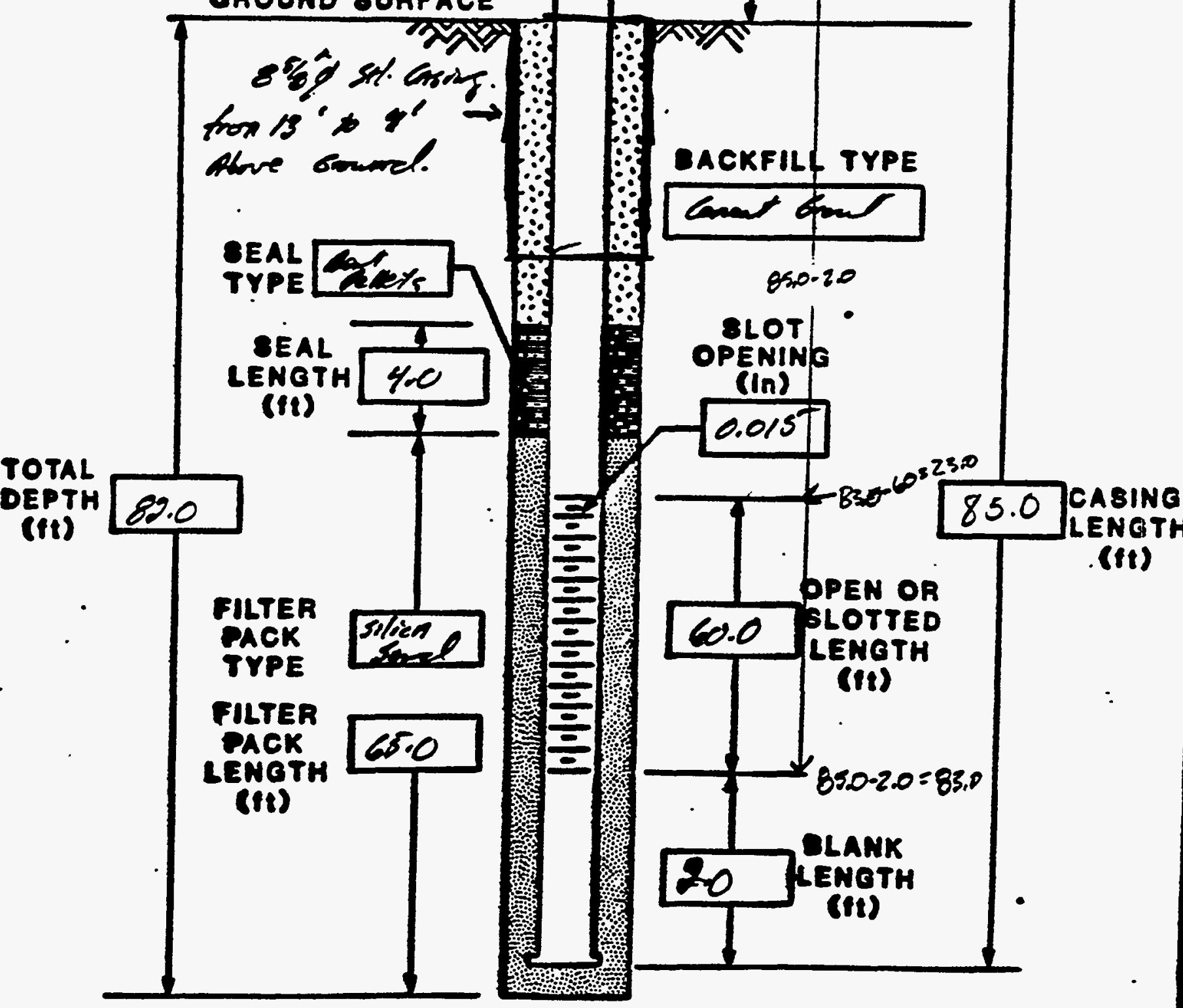
COMMENT 8: 


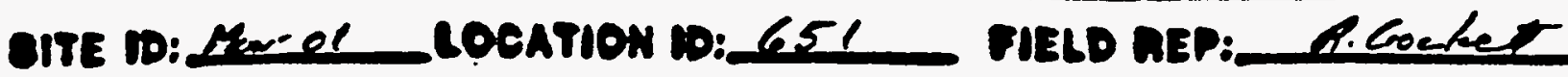

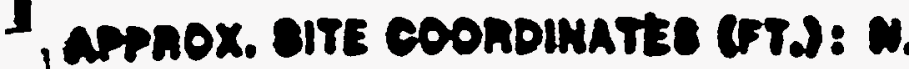
jOAOUND ELEVATION SFT. MOLY: DOAEHOLE quMMaRY

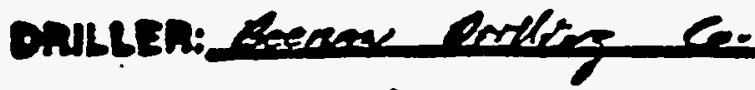
nis TrPE: Gerelos - Buer OIT MPE POLE Tingue (in.: $2 \%$

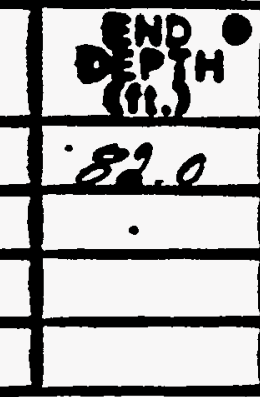

casine oumaARY

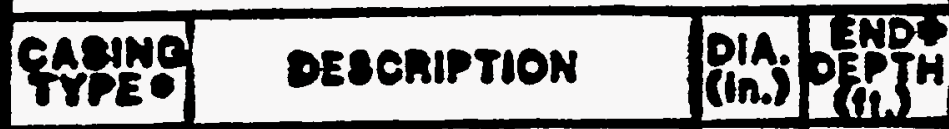

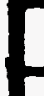
6 1

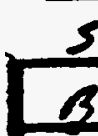
stel lasing sh ece \begin{tabular}{l|l}
5 & $n$
\end{tabular} 32

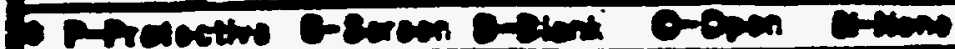
Sorm tron res el Capho

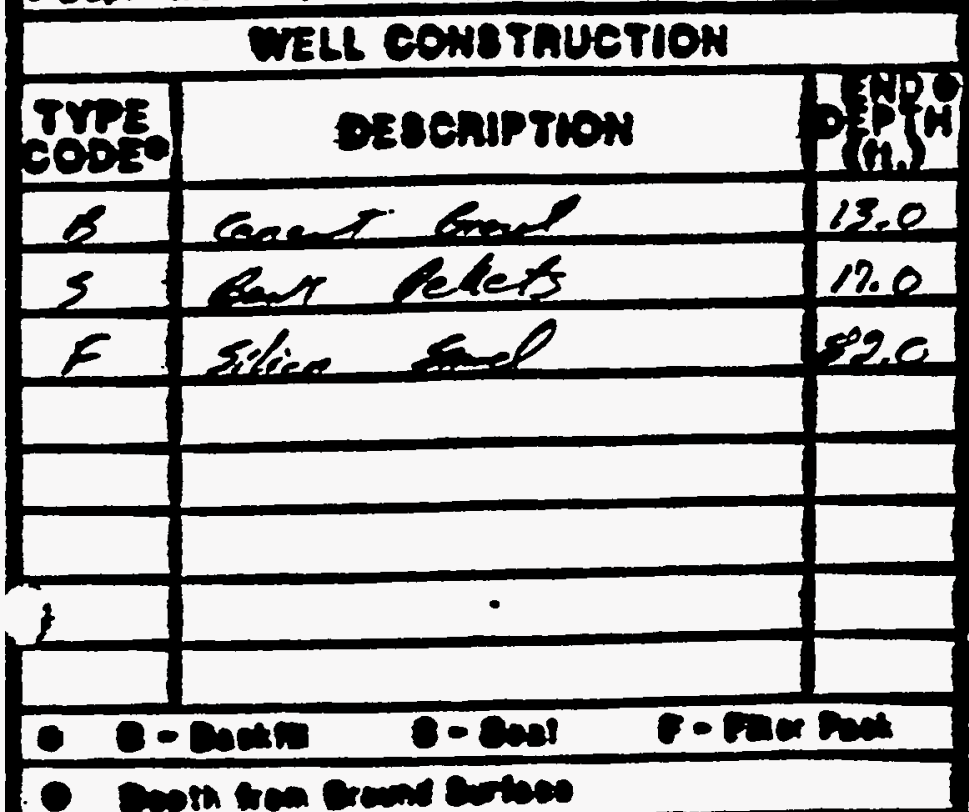

COMARENT: E.

Complethon Date: 2/22/86constauction THE 100

\begin{tabular}{|c|c|c|c|}
\hline \multirow{2}{*}{ AETIVITY } & \multicolumn{2}{|c|}{ DTART } & \multirow{2}{*}{$\begin{array}{l}\text { SND } \\
\text { TIME }\end{array}$} \\
\hline & DAtE & TUME & \\
\hline DRILLING & $9 / 89 / 15$ & 8.20 & T20 \\
\hline CABINO & 9199 & b.15 & 19.15 \\
\hline FILTEA PACK & $9 / 99$ & 12,15 & \\
\hline BEAL & $7 / 29$ & 3.00 & $3: 30$ \\
\hline AACKFILL & $9 / 29$ & 3.30 & 4.00 \\
\hline DEVELOPUENT & & & \\
\hline OTHER & & & \\
\hline & & & \\
\hline & DEYE & IENT & \\
\hline
\end{tabular}




\section{TT MCOSS ENeNERING EROUP NC.

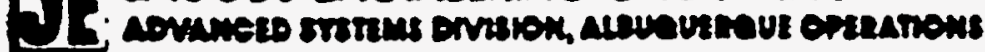

\section{WELL COMPLETION RECORD}

SITE 10: MON-OI LOCATION 10: 652 DATE INSTALLED: $2 / 2 Y / S^{\circ}$ APPROX. OITE COORDINATE8:(FT.) $\mathrm{N}$ OPEN AREA PER LINEAL FT. $\left(\mathrm{N}^{2} / \mathrm{FT}.\right)$ FORMATION OF COMPLETION: Allewerar

FIELD REP.: L Lacehet DRILLER: Geamear melling Eer

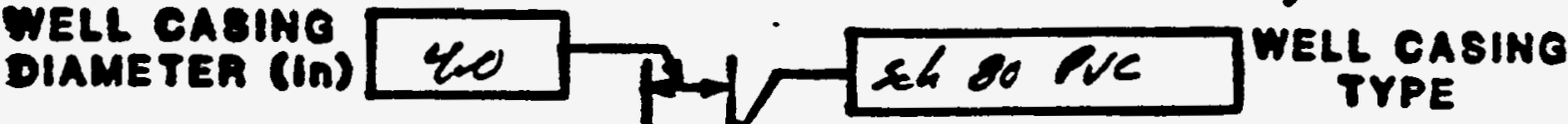
MOLE DIAMETER (In) $2 \% 8$ OAOUND SURFACE

TOTAL DEPTH (18)

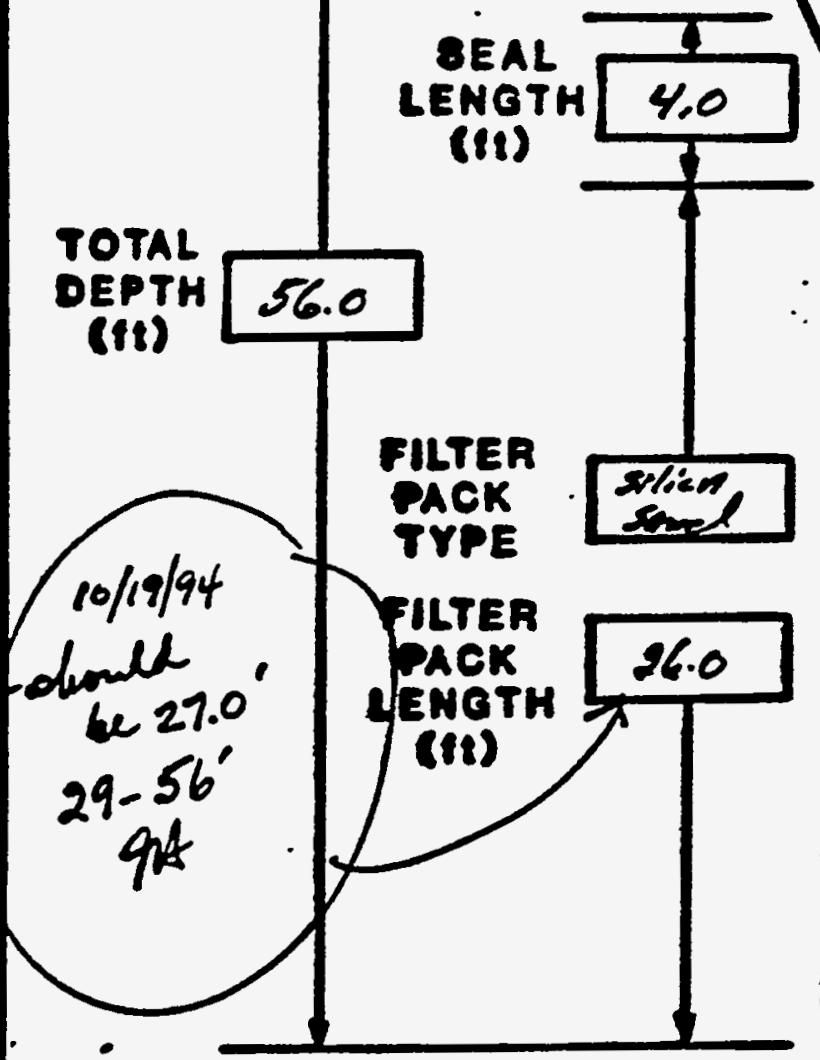

SEAL ZRT TYPE Gakers

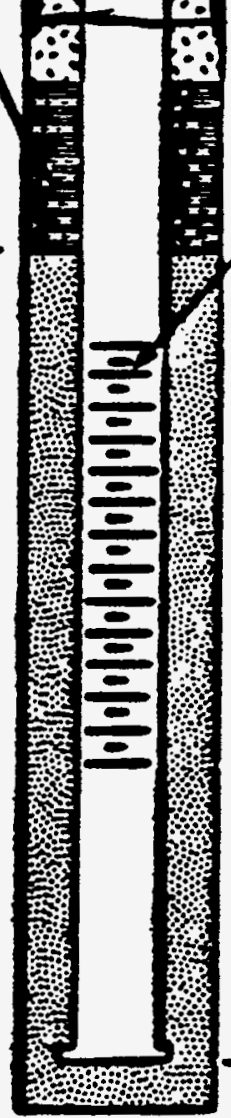

DACKFILL TYPE

Comar Grout

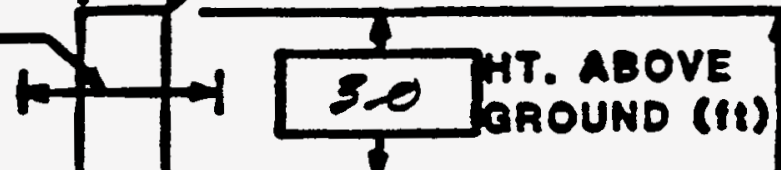




\section{WELL COMPLETION RECORD}

SITE ID: MON LOCATION ID: \#653

DATE INSTALLED: 9.25 .85 APPROX. SITE COORDINATES:(FT.) N E

OPEN AREA DER LINEAL FT. (IN2/FT.)

formátion of completion: Quaternary DUNE SAND $(Q d)$ FIELD REP.: G. Miller DRILLER: Bob BePMAN

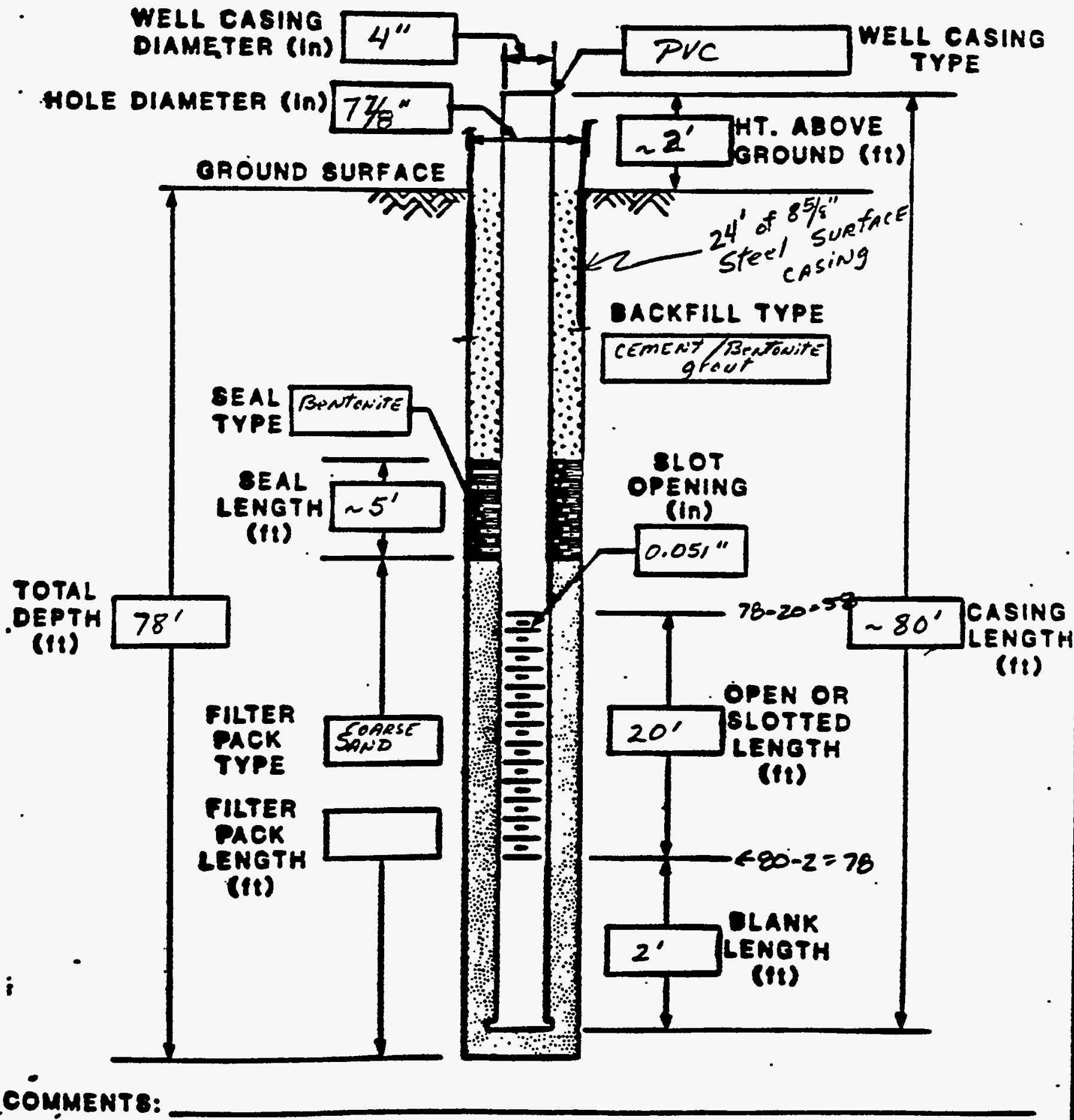

WELL CAEING 


\section{DT Mcoss ENeN JERNG CROUP NC.

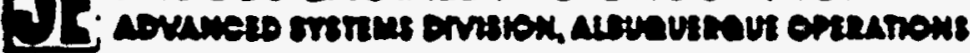

\section{WELL COMPLETION RECORD}

SITE DD: $\mathrm{Max}$ ol LOCATION ID: 654 Date installed: Zlag/es APPROX. BITE COORDINATE8:(FT.) $N$ OPEN AREA PER LINEAL FT. (IM2/FT.) FORMATION OF COMPLETION: Alkviun FIELD REP.:

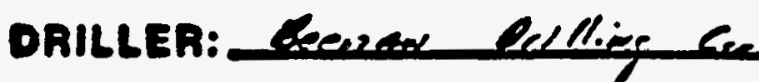

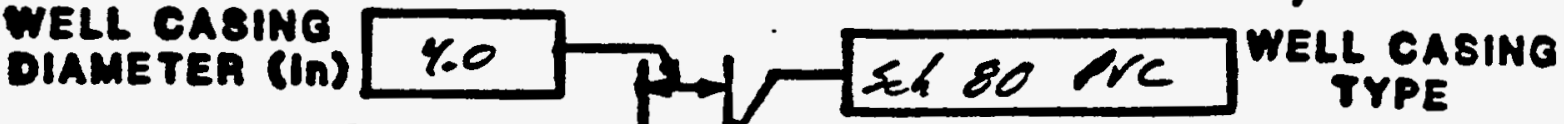
HOLE DIAMETER (in) $2 \frac{1}{8}$ OROUND BURFACE

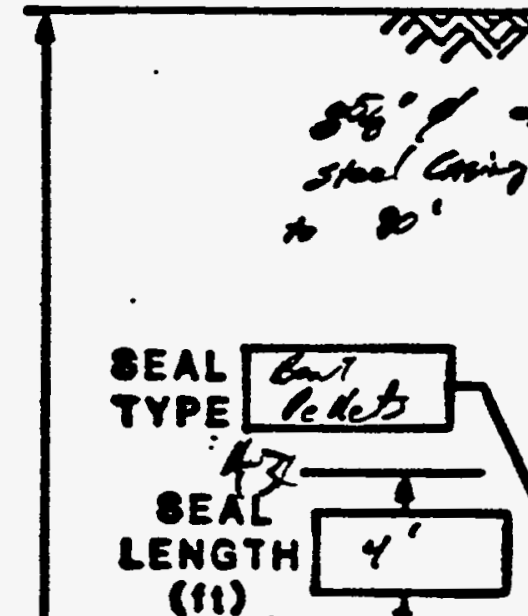

TOTAL DEPTH (I1)

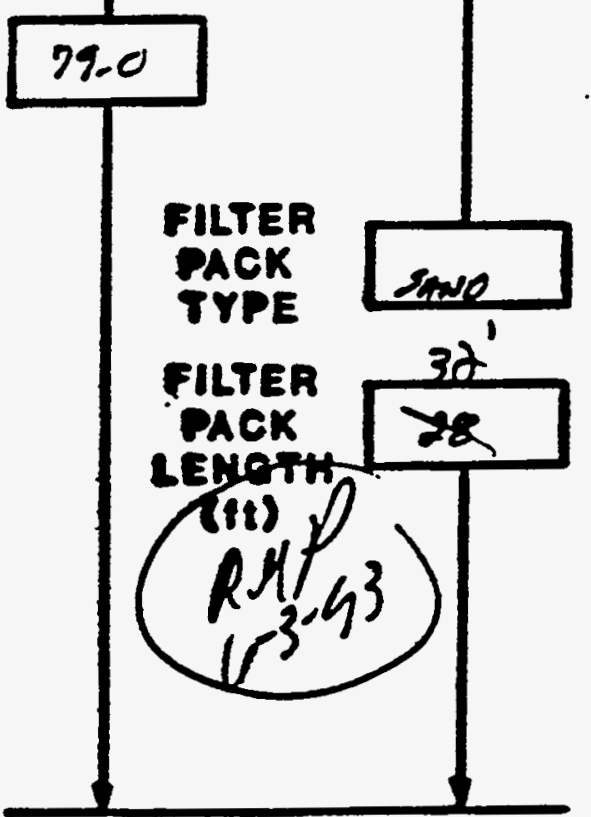

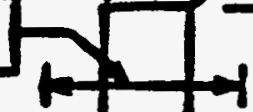<smiles>C1C2CC12</smiles><smiles></smiles>

BACKFILL TYPE
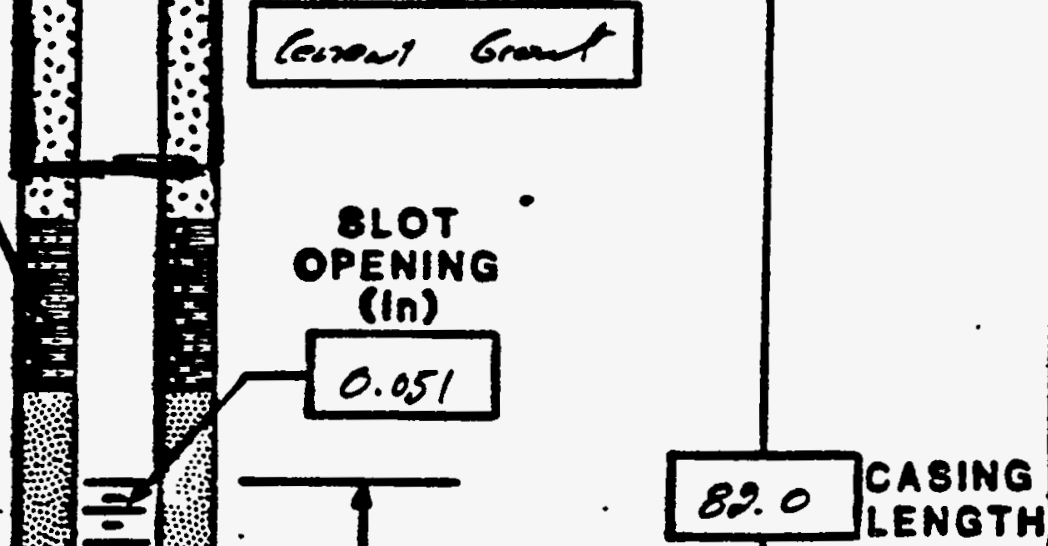

(ft)
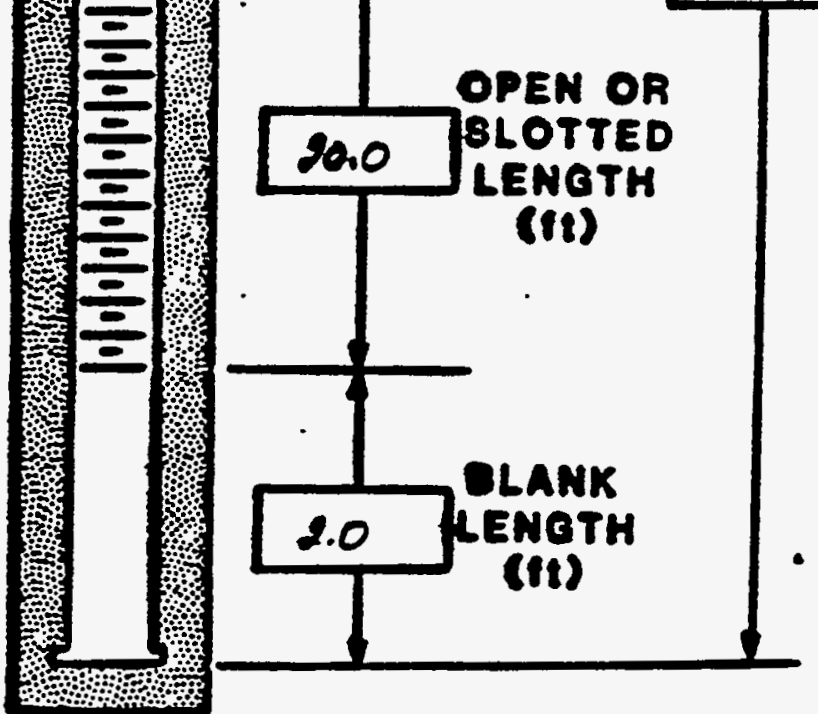

COMMENTE: 


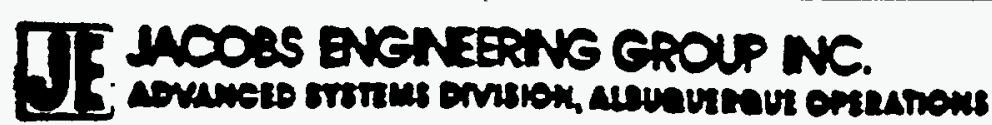

\section{MELL COMPLETION RECORD}

SITE DD: $\mathrm{kec}$ ol LOCATION DD: 654 DATE INSTALLED: 2/82/ES APPROX. OITE COOADINATE8:(FT.) $\mathrm{N}$ OPEN AREA PER LIMEAL FT. (IN2/FT.) FORMATION OF COMPLETION: Altuviun

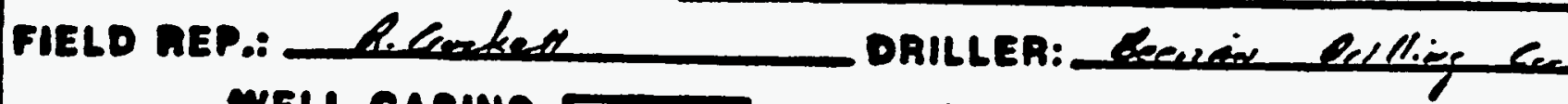

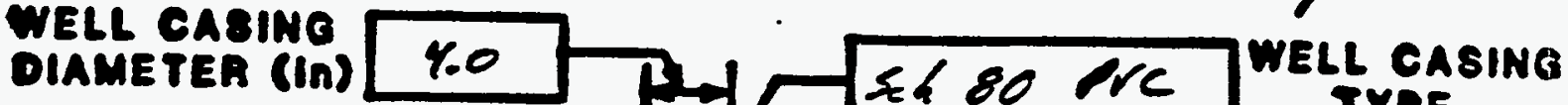

MOLE DIAMETER (In) $7 \frac{1}{8}$

OROUND SURFACE
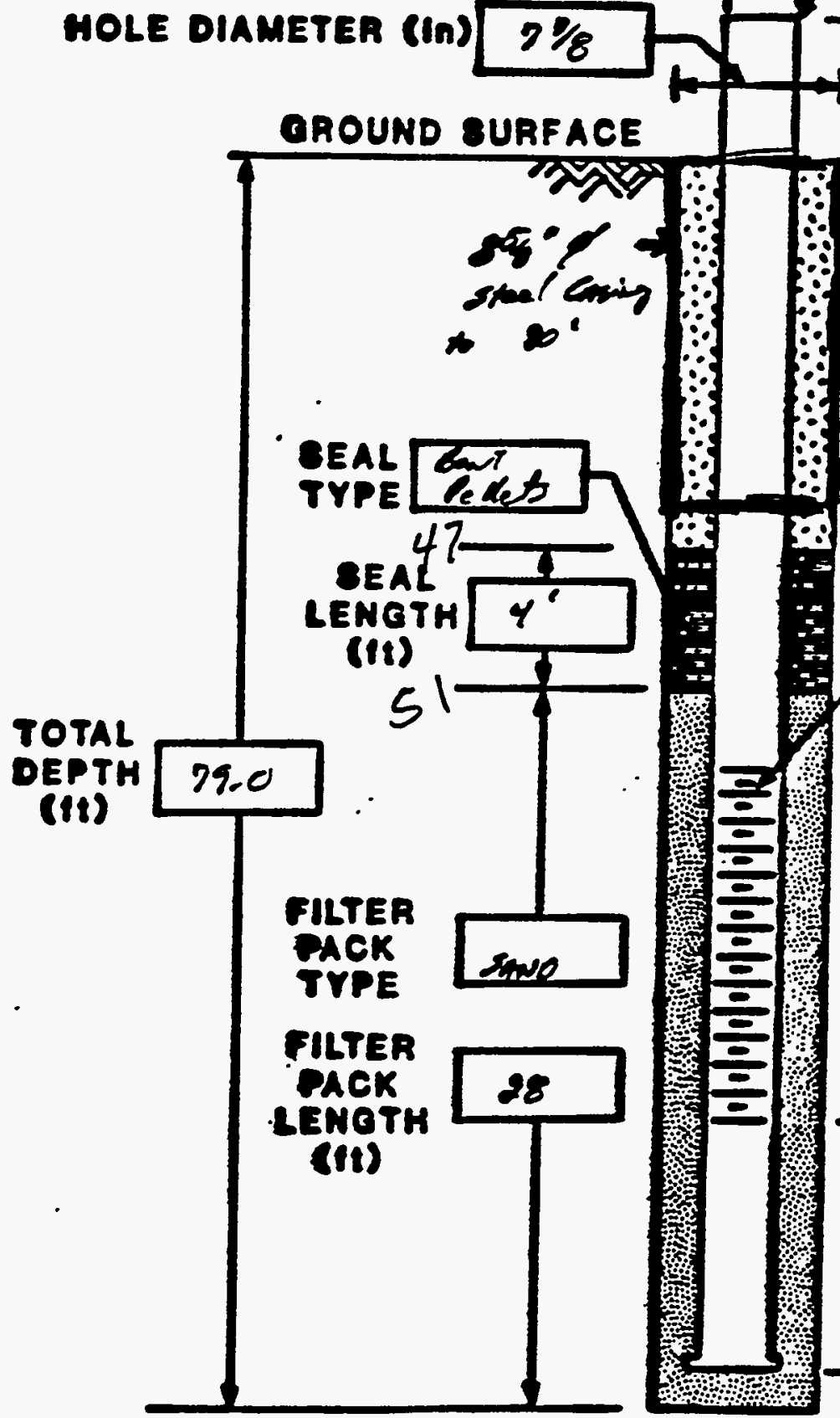
TYPE
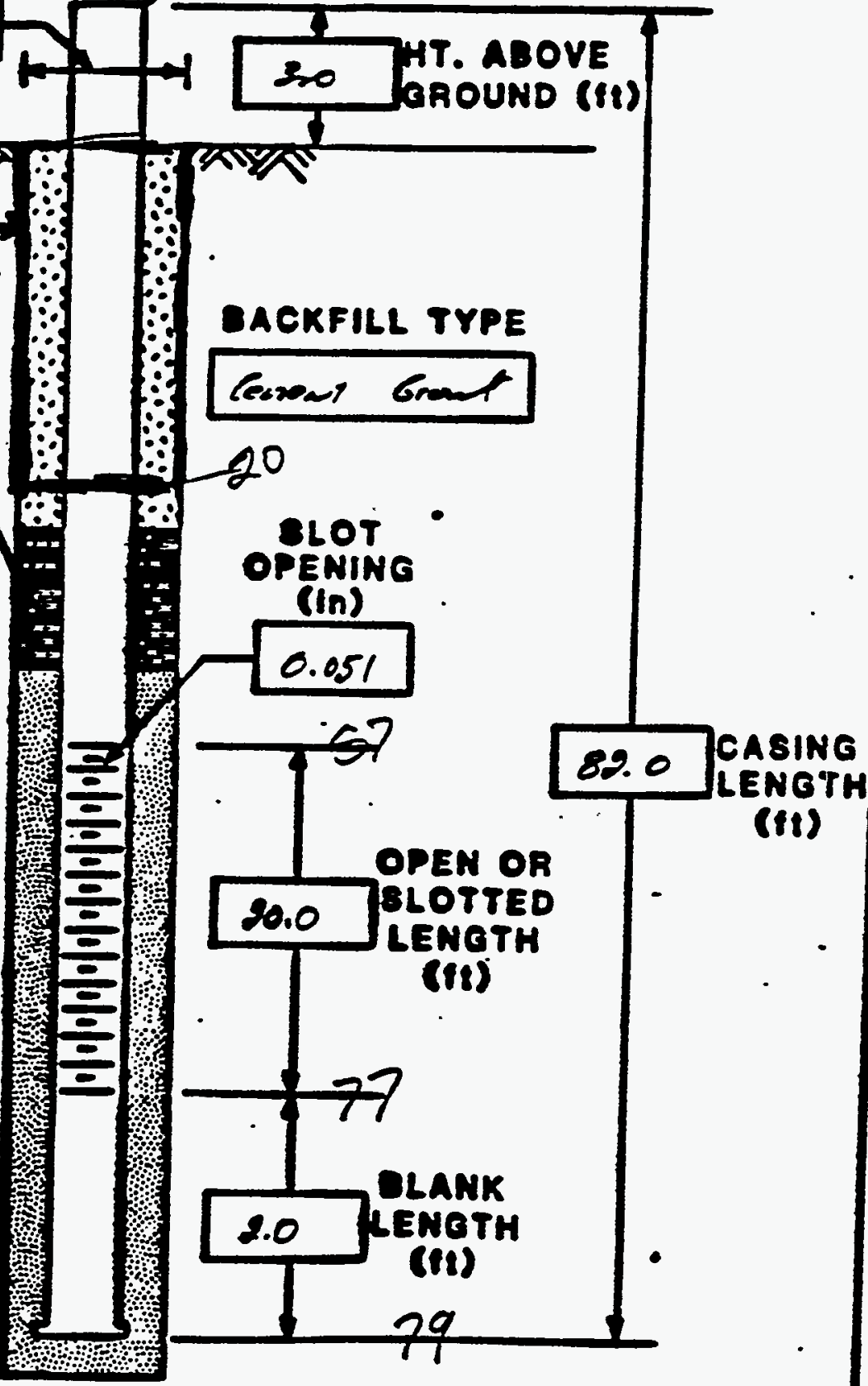

COMNENTE: 


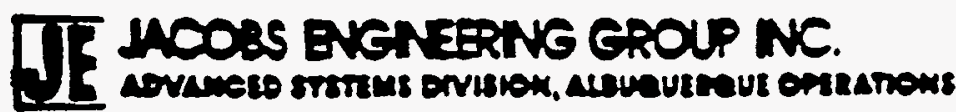

\section{WELL COMPLETION RECORD}

SITE ID: MON LOCATION ID: \# 655 DATE INSTALLED: 9.12 .85 APPAOX. SITE COORDINATES:(FT.) N E OPEN AREA PER LINEAL FT. $\left(I N^{2} / F T\right.$.)

FORMATION OF COMPLETION: QUATESNARY DUNE SAND FIELD NEP.: G. MillER DRILLER: B. BEEmAN

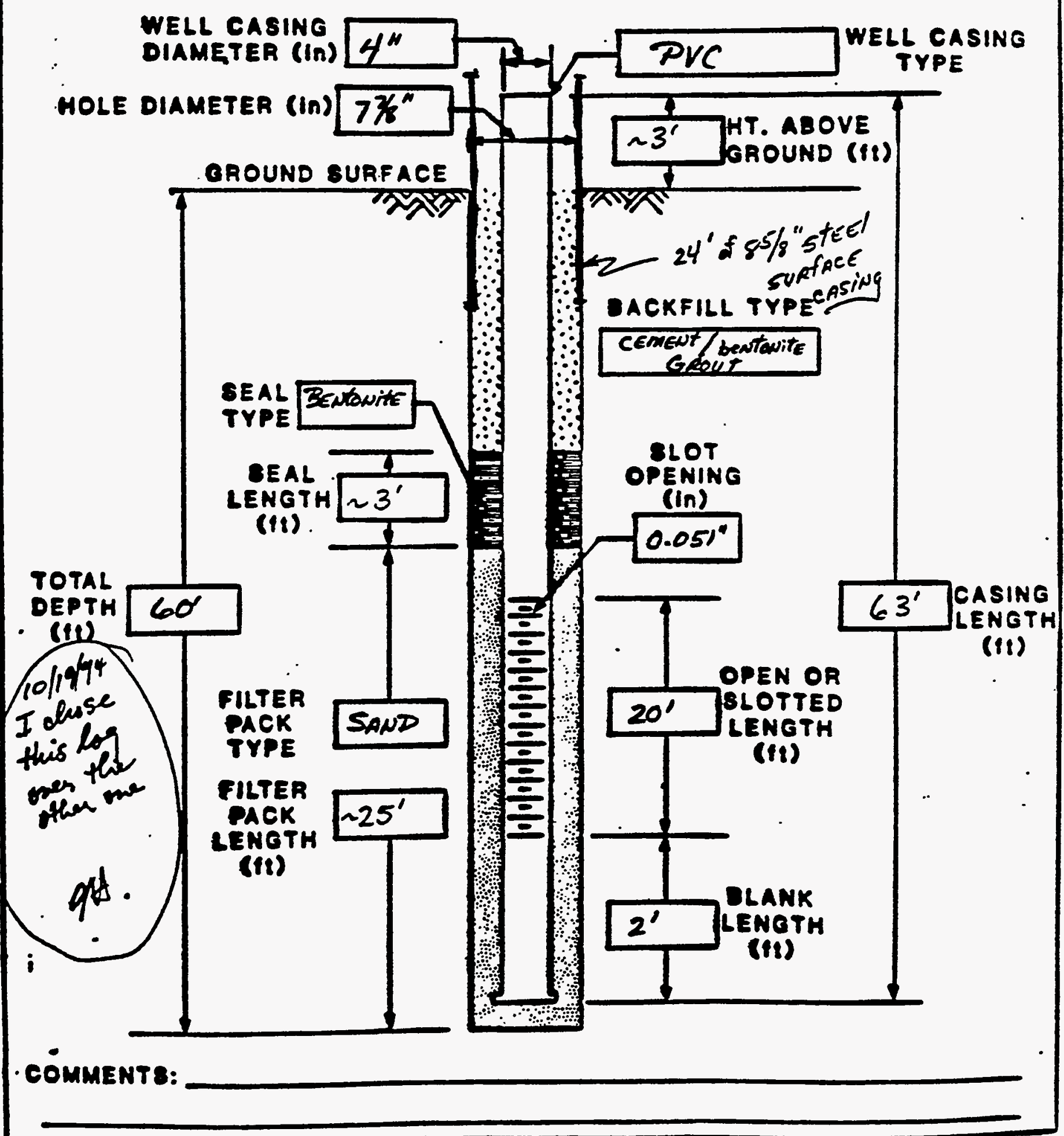


A[ WCOsS ENGN EERNG EROUP NC.

\section{WELL COMPLETION RECORD}

SITE ID:MON LOCATION DD: \#655 DATE INSTALLED: 9.12 .85 APPROX. 8ITE COORDINATES:(FT.) N E OPEN AREA PER LINEAL FT. (IN2/FT.) formation of cOMPLETION: Quateruary PUNE SAND fIELD MEP.: G. Millek ORILLER: B. BeEmAN

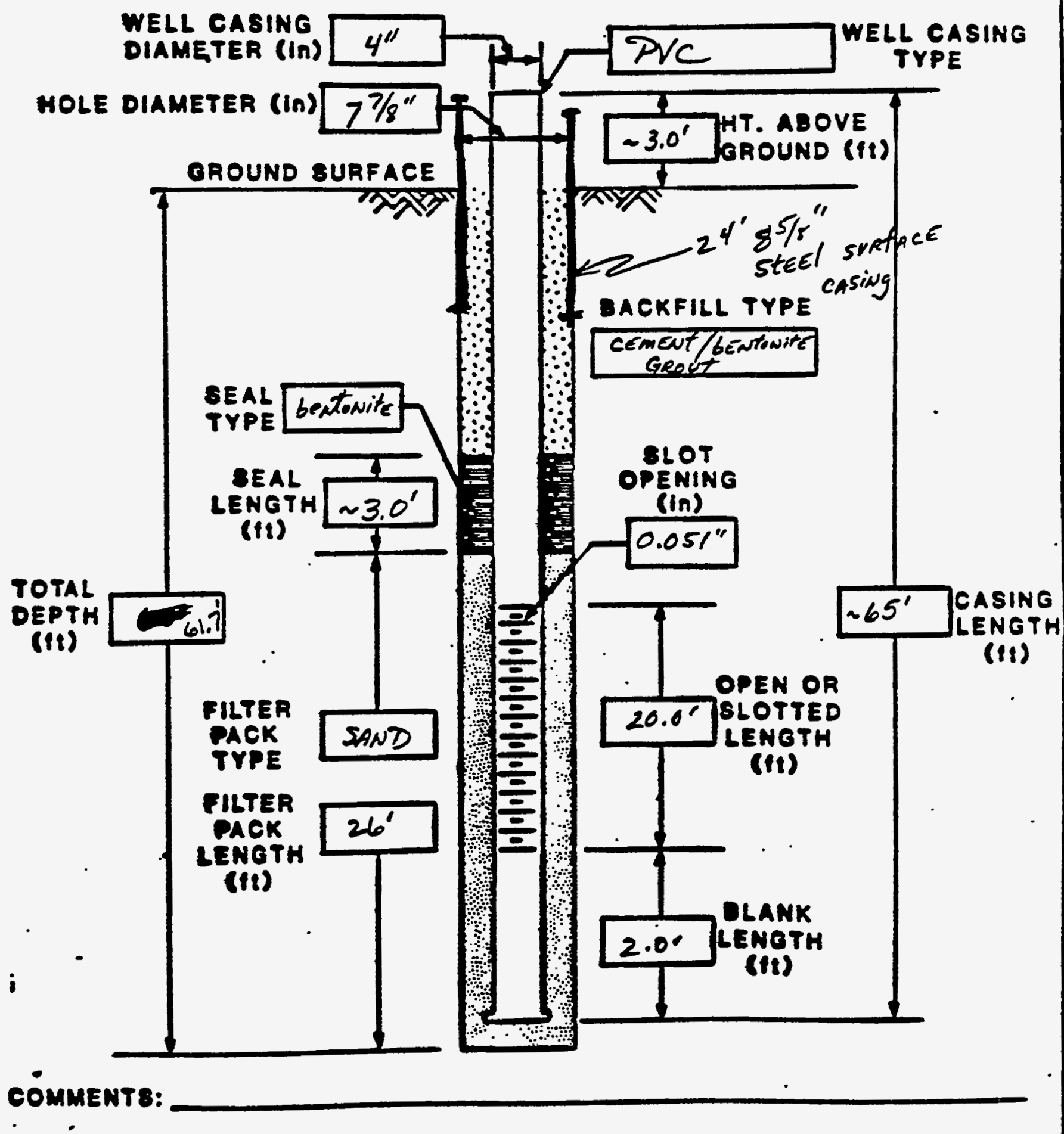




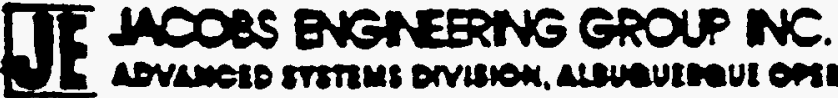

\section{WELL COMPLETION RECORD}

SITE ID: MON Location ID: \#656 Date installed: 9.12 .85 APPROX. SITE COORDINATES:(FT.) N OPEN AREA PER LINEAL FT. (IN2/FT.)

FORMÁtION OF COMPLETION: Guaternary Dune SAND FIELD AEP.: G. MilleR DRILLER: Bab BeemAN

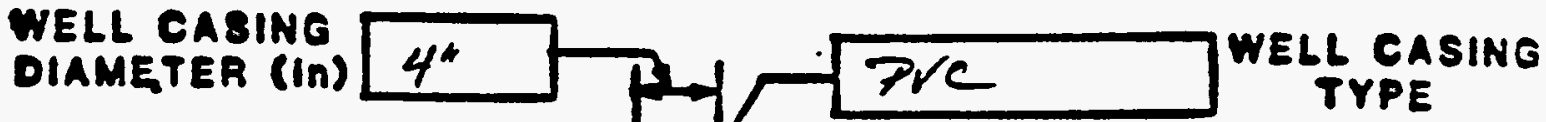

HOLE DIAMETER $(\ln ) 77 / 8^{\prime \prime}$

BROUND SURFACE

TOTAL

DEPTH भूर्रF

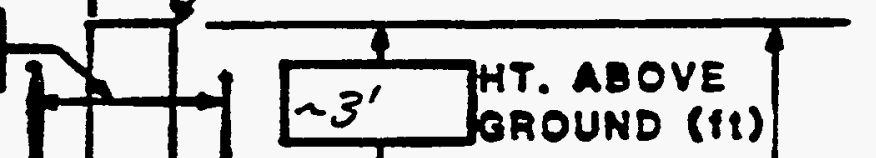

(ii)

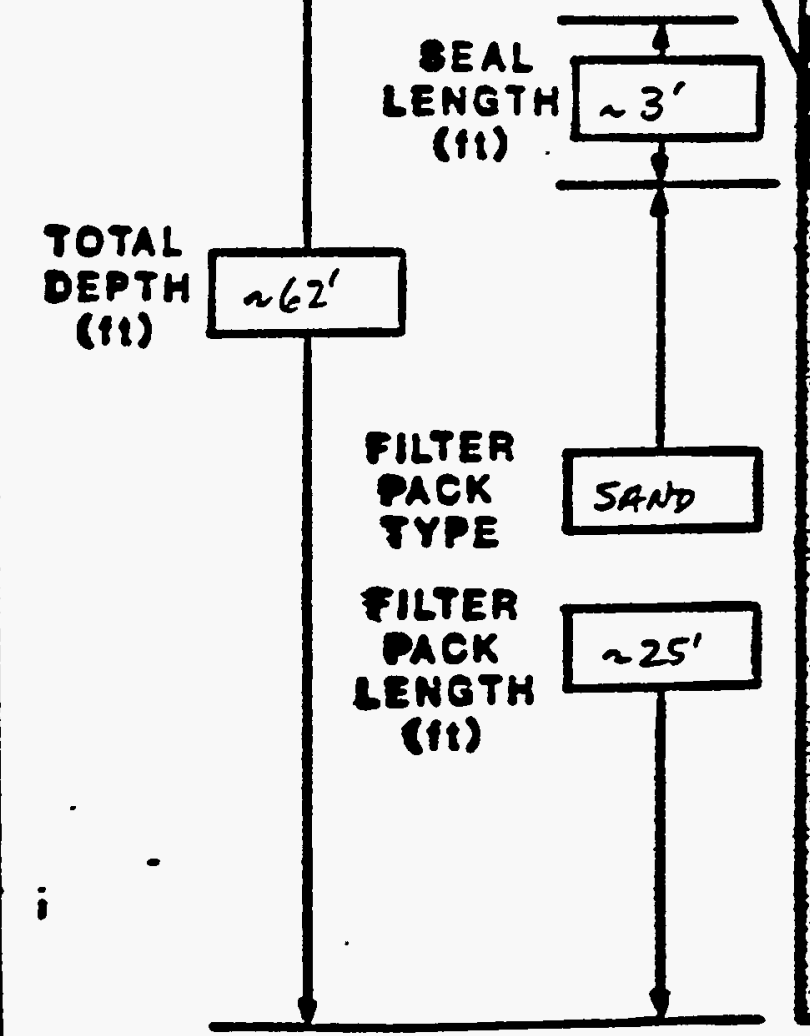

TYPE EENTENTE
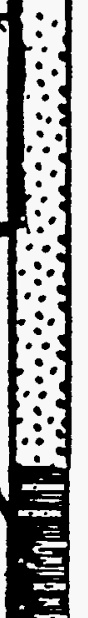

$\because$ ॠঙ GROUND (fi)

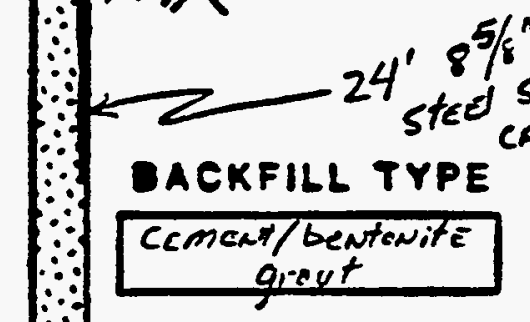

COMMENTS: 


\section{DE MCOBS BNGNEERNG EROUP NC.}

\section{WELL COMPLETION RECORD}

Site 1D: MON location 10: \#656 Date installed: 9.12 .85 APPROX. SITE COORDINATES:(FT.) N E

OPEN AREA PER LINEAL FT. (IN2/FT.)

FORMATION OF COMPLETION: QUATERNIARY DUNE SAND

FIELD AEP.: G. MilleR DRILLER:

Beb Beeman

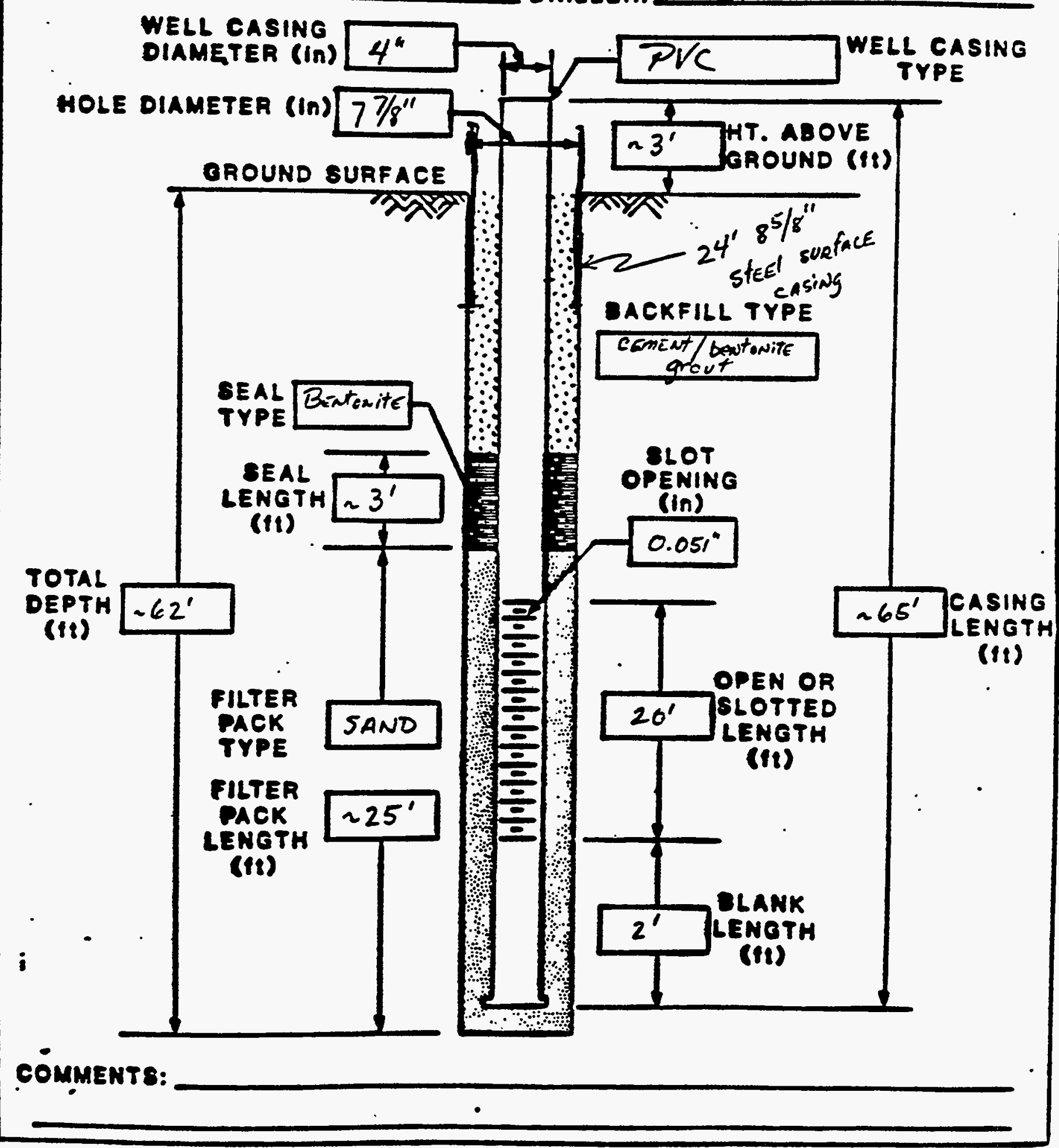

WELL CASING $4^{*}$
DIAMETER (In) 


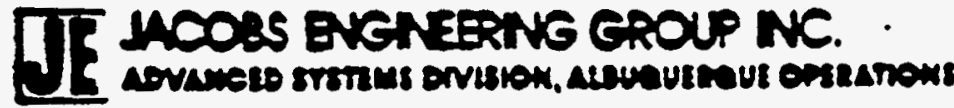

\section{WELL COMPLETION RECORD}

SITE ID: MON LOCATION ID: $\$ 657$ DATE INSTALLED: 9.5 .85 APPROX. SITE COORDINATES:(FT.) N E OPEN AREA PER LINEAL FT. (IN²/FT.)

IFORMÁTION OF COMPLETION: DeCkelly $\&$ Mbe. of CutlER Fm. FIELD MEP.: G. MilleR DRILLER: B. BeEmAN

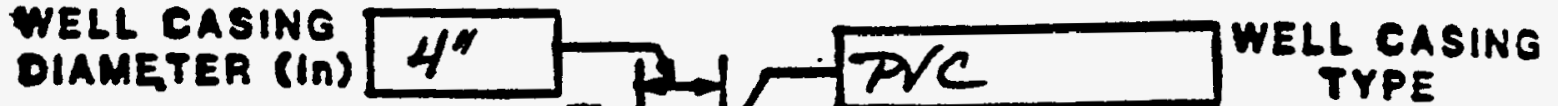

HOLE DIAMETER (In) $77 \% "$ OROUND SURFACE

TOTAL DEPTH (11) $138^{\prime}$

SEAL Eatowite
TYPE Eato Bत्रF
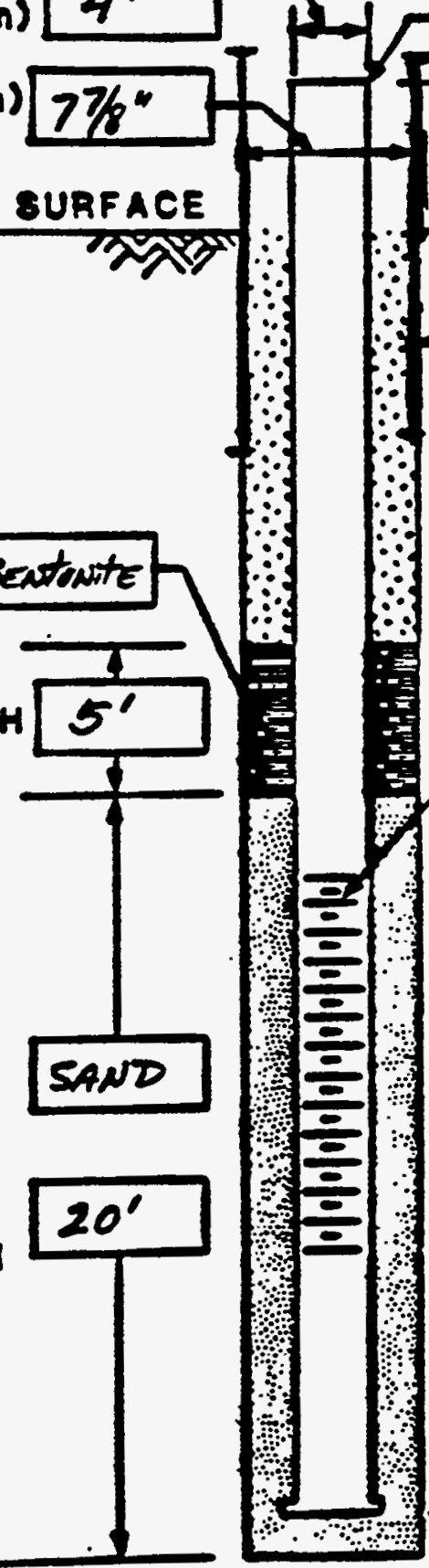

$\sim 3.0^{\prime}$ HT. ABOVE

$\therefore$ अप्र

$2-24^{\prime} 8^{5 / 8}$ "stEEl

$\because 2$

$\therefore$

DACKFILL TYPE Casing

Cement/dentavite

BEAL

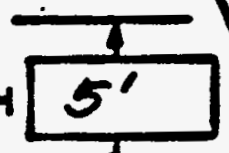

(11)

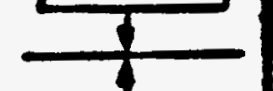

OLOT

OPENING

(in)

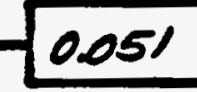

0.051

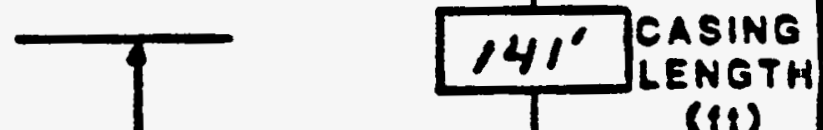

FILTER

PACK

TYPE

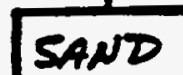

FILTER PACK

LENOTH

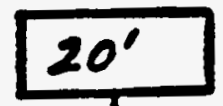

(II)

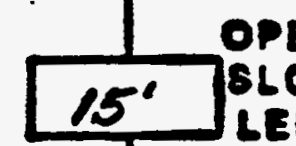

OPEN OR OTTED (it)
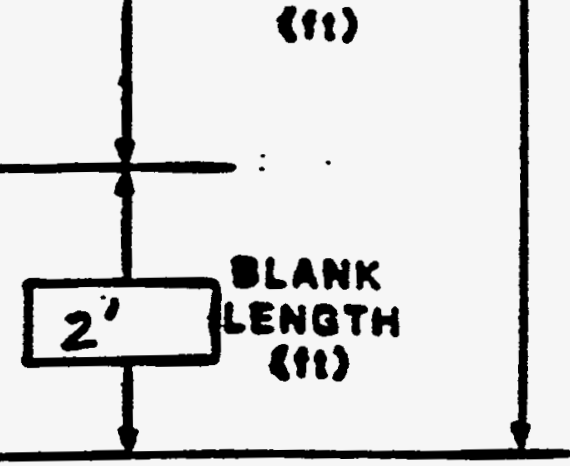
site id: Mon location id: \#658 oate installed: 9.26 .85 APPROX. SITE COORDINATES:(FT.) N E OPEN AREA DER LINEAL FT. (IN2/FT.) fFORMATION OF COMPLETION: ShINARUMP SS Mbr. of ChIN/E FM. DIAMETER (In) 4" PAC PVC WEL CASING HOLE DIAMETER $(\ln ) 7 \%$ \%"
OROUND SURFACE

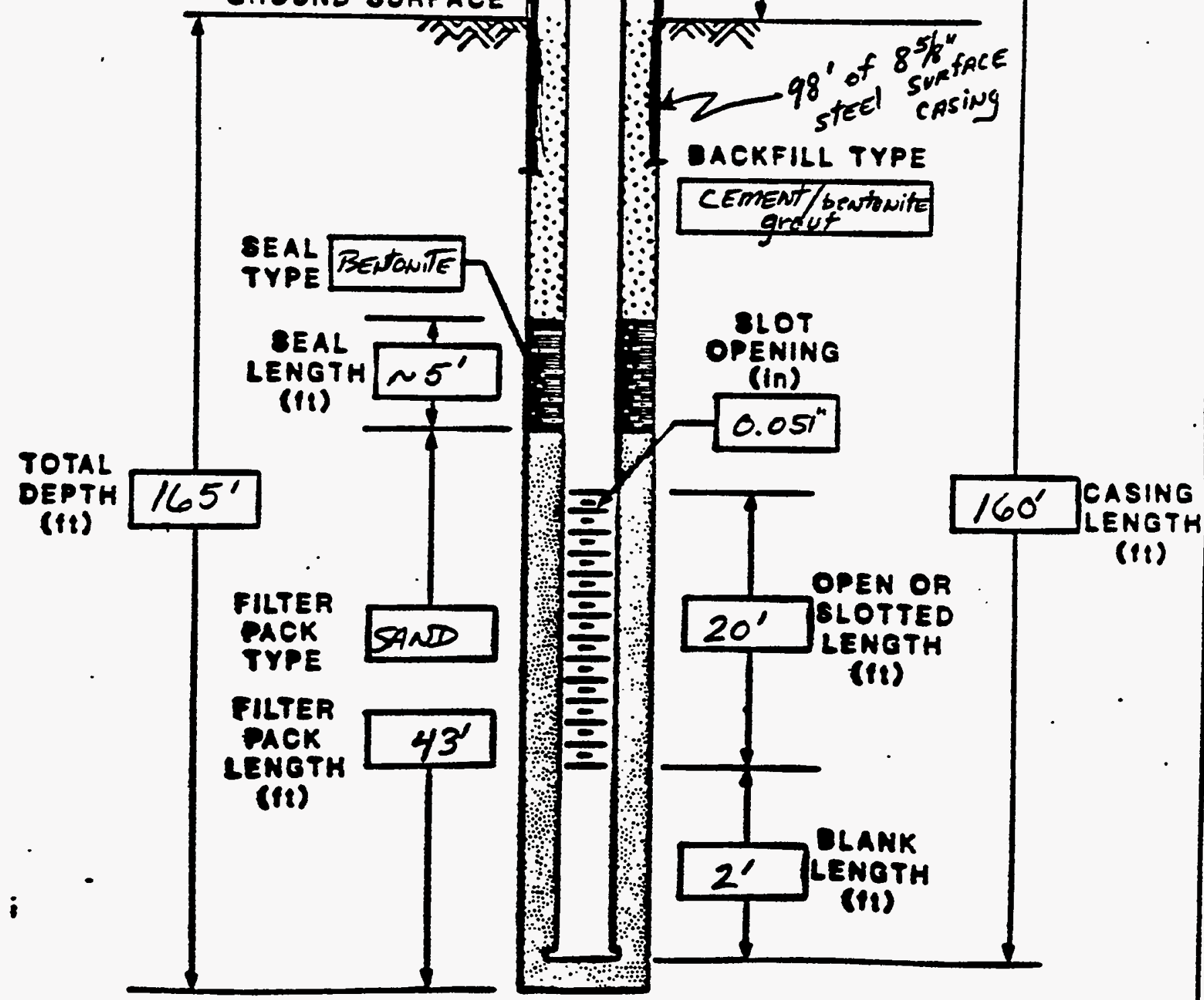
CÖMMENT8: 


\section{WELL COMPLETION RECORD}

Site ID: MoN Location ID: \#659 DATE Installed: 9.11 .85 APPROX. BITE COORDINATES:(FT.) $N$ OPEN AREA PER LINEAL FT. (IN2/FT.)

fFOAMATION OF COMPLETION: ShineRump Mbr. of Chinle Fm. FIELD AEP.: G. MIIlER DRILLER: 8. BEERAN

well casing DIAMETER (In) HOLE DIAMETER (in) $7 \%$. BROUND SURFACE

TOTAL DEPTH (It)

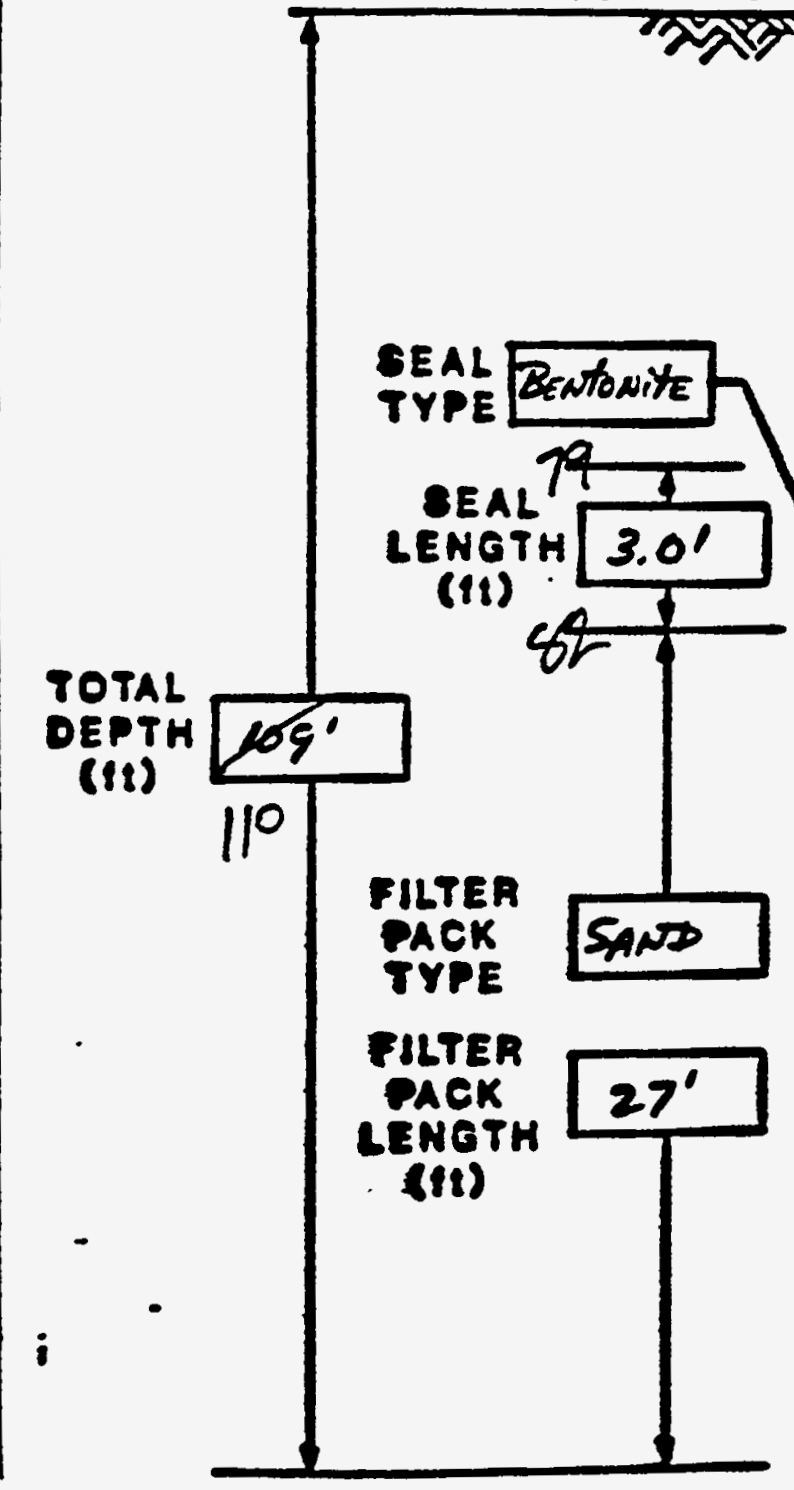

CÓMMENT8:
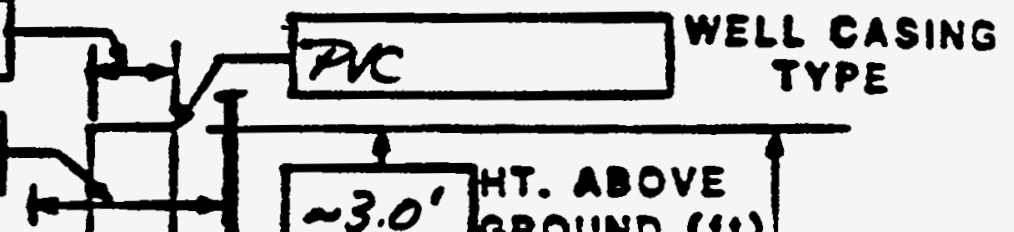

$\sim 3.0^{\circ}$ HT. ABOVE

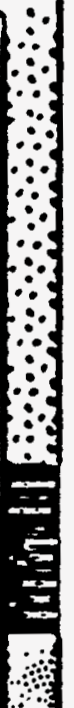

$\because$ सण्र

$\therefore \quad 54^{\prime}$ of

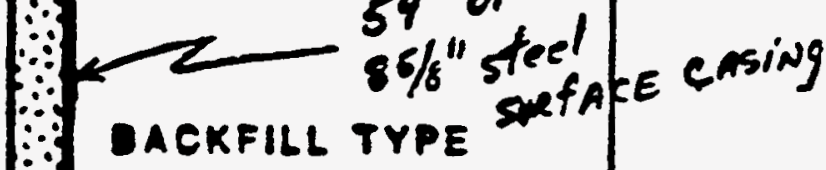

Cement/bentowits 51

SLOT

OPENING (in)

0.051

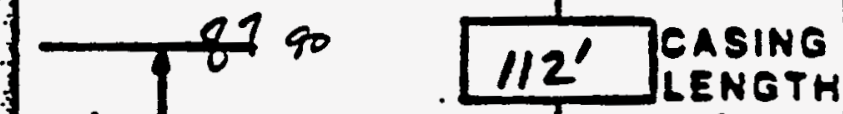

(Ii)

\&E-AL-8wO-S (a) 


\section{TIS Meoss Even zank eneup we.

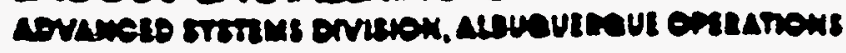

YELL COMPLETION RECORD

SITE ID: MON LOCATION ID: $\$ 659$ DATE INSTALLED: 9.11 .85 APPROX. SITE COOADINATES:(FT.) N $E$ OPEN AREA PER LINEAL FT. (IN2/FT.)

FORMATION OF COMPLETION: ShiNARUMP MbR. of Chinle Fm. FIELD REP.: G. Miller DRILLER: 8. BEEMAN

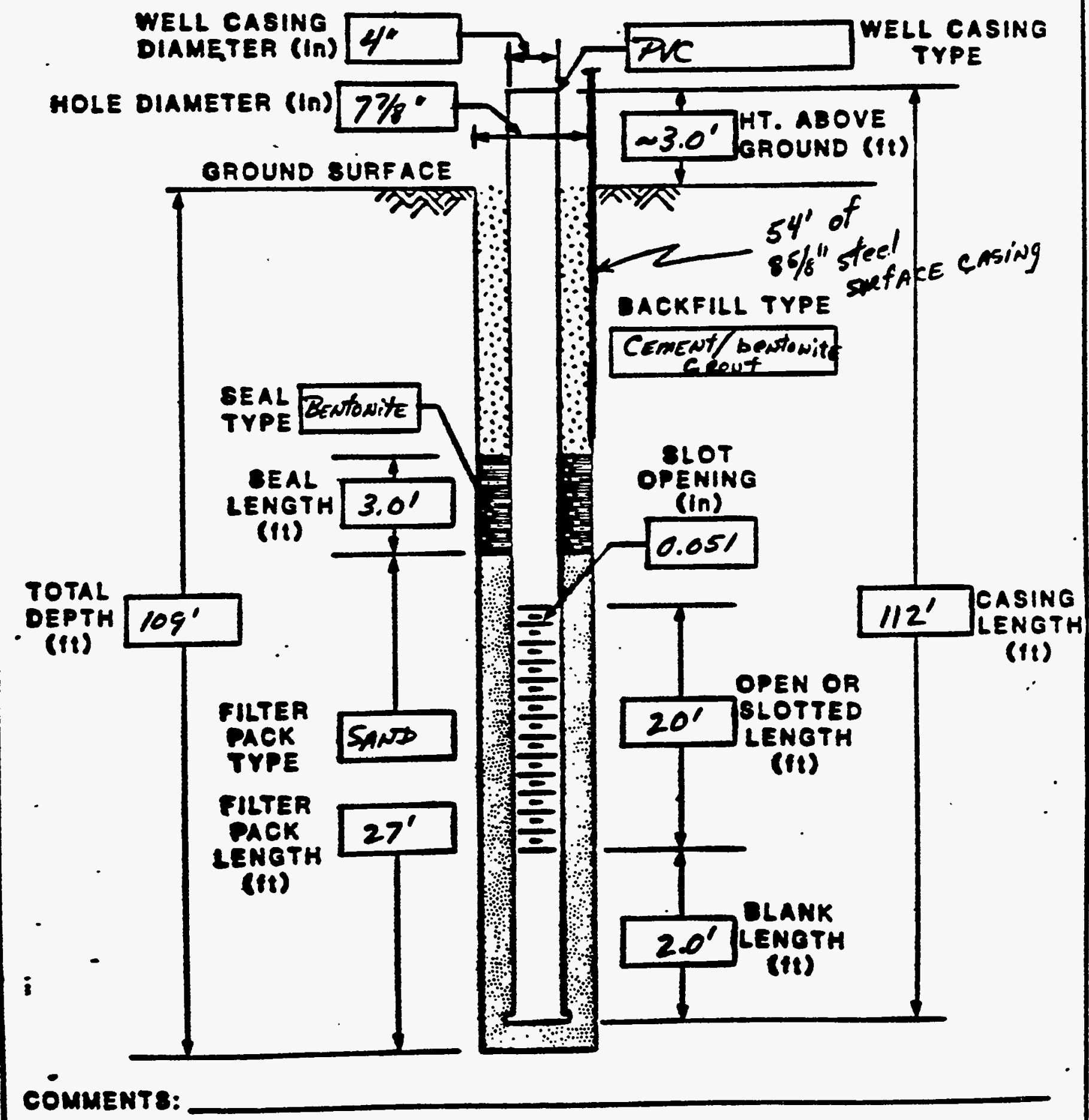




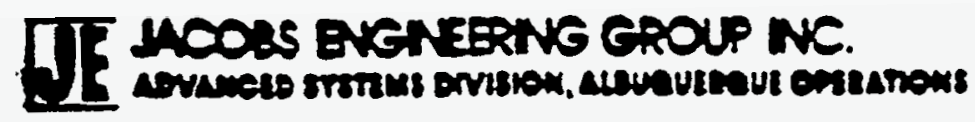

\section{MELL COMPLETION RECORD}

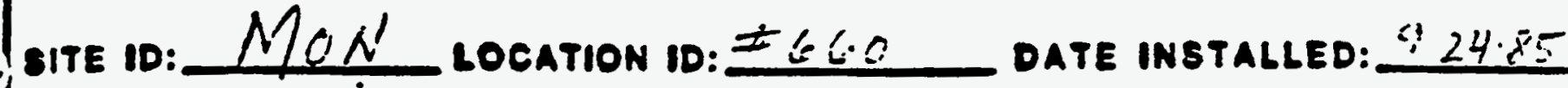
APPROX. OITE COORDINATES:(FT.) $\mathrm{N}$ E OPEN AREA PER LINEAL FT. (IN2/FT.)

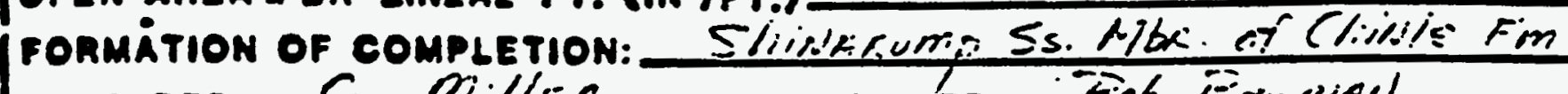

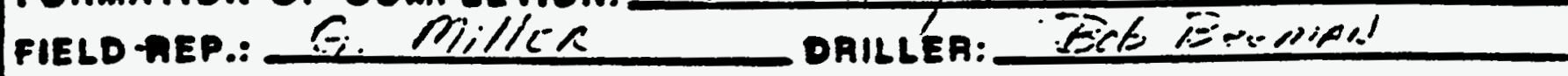
WELL CASING
DIAMETER (In) $j^{\prime \prime}$

HOLE DIAMETER (in) $77 / 8 "$

oROUND SURFACE

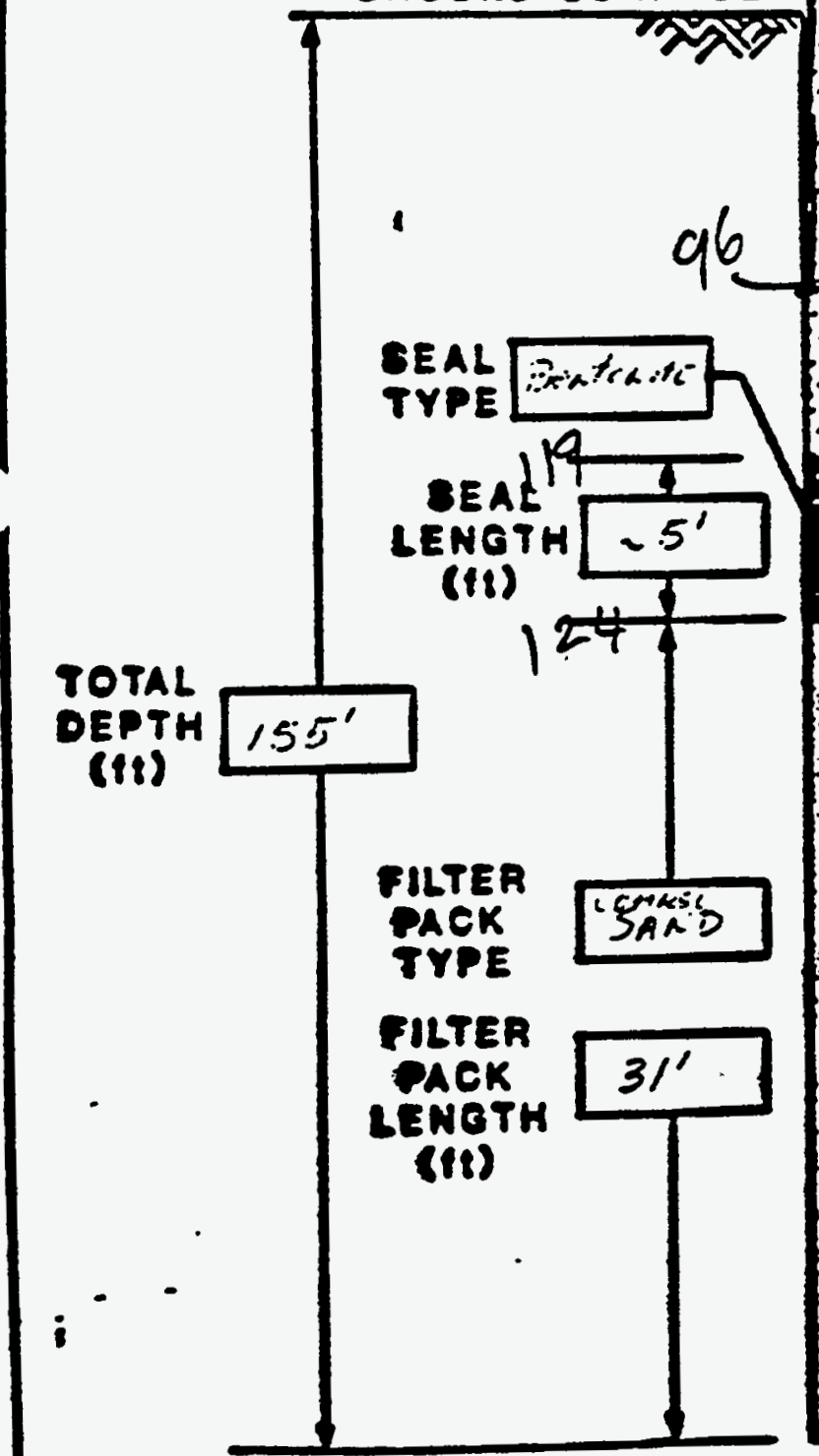

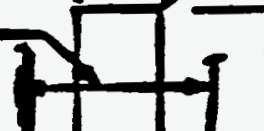

1
$23^{\prime}$ HT. ABOVE
GROUND (fi)

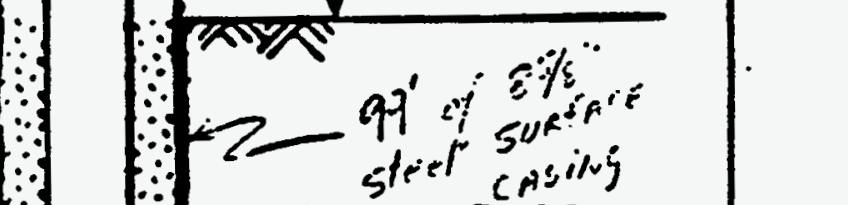




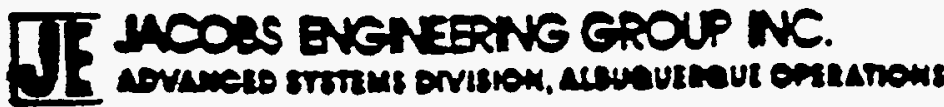

\section{YELL COMPLETION RECORD}

Site id: MON location ID: \#660 DAte Installed: 9.24 .85 APPROX. SITE COORDINATES:(FT.) N E

OPEN AREA PER LINEAL FT. (IN2/FT.)

formation OF cOMPLETION: ShinaRump 5 s. Mbe. of Chinle Fm FIELD AEP.: G. MillER DRILLER:

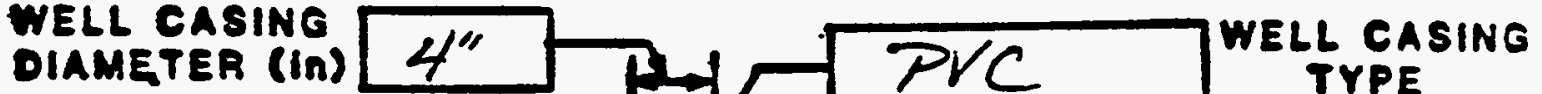

HOLE DIAMETER $(\ln ) 77 / 8^{\prime \prime}$ GROUND SURFACE

TOTAL DEPTH (11)

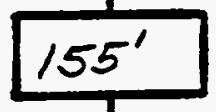

SEAL Bintadite TYPE

EAL LENGTH (it)
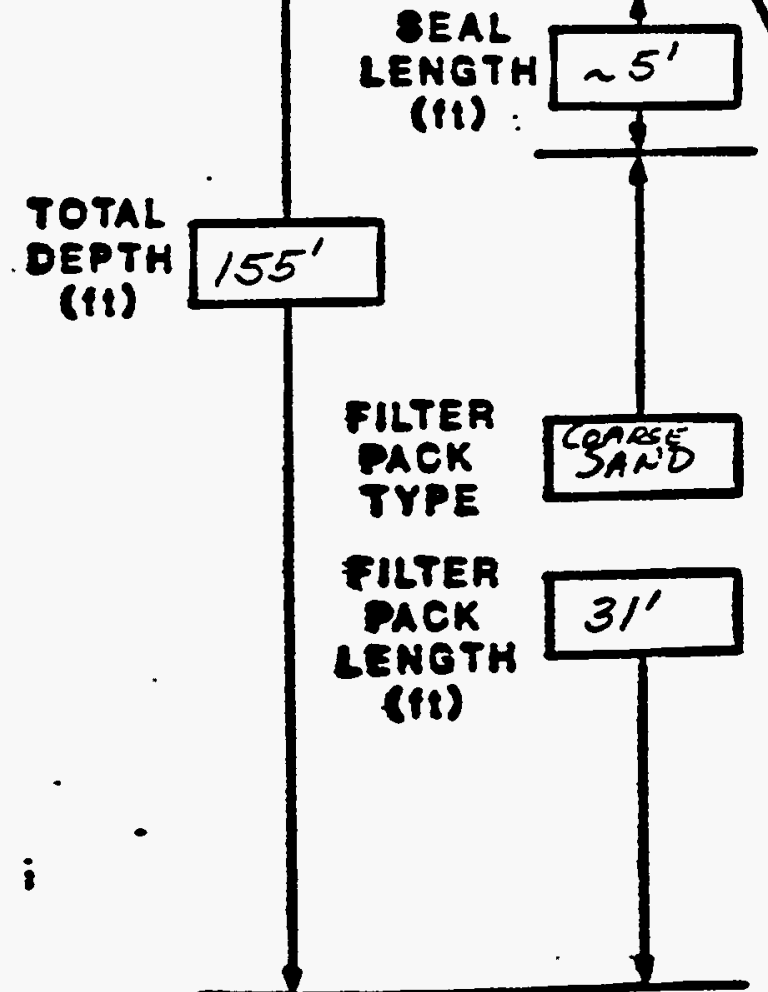
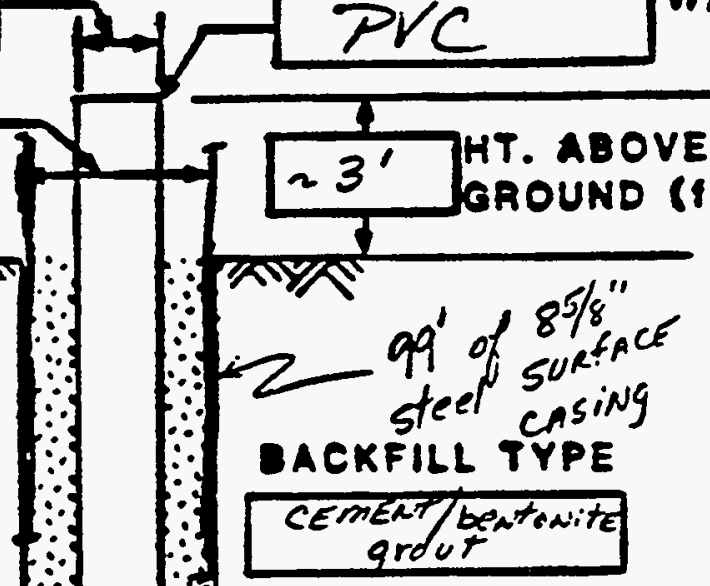
TYPE 


\section{YELL COMPLETION RECORD}

OITE. DD: RE-01 LOCATION ID: APPROX. OITE COORDINATES:(FT.) $\mathrm{N}$ Date installed: y/g/es OPEN AREA PER LINEAL FT. (IN2/FT.) FORMATION OF COMPLETION: FIELD AEP.: Rlowelet a clader rendice

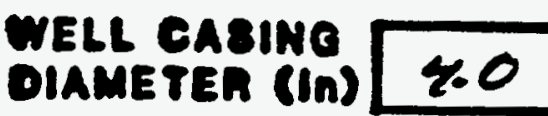
DRILLER: berren leciliug Ca

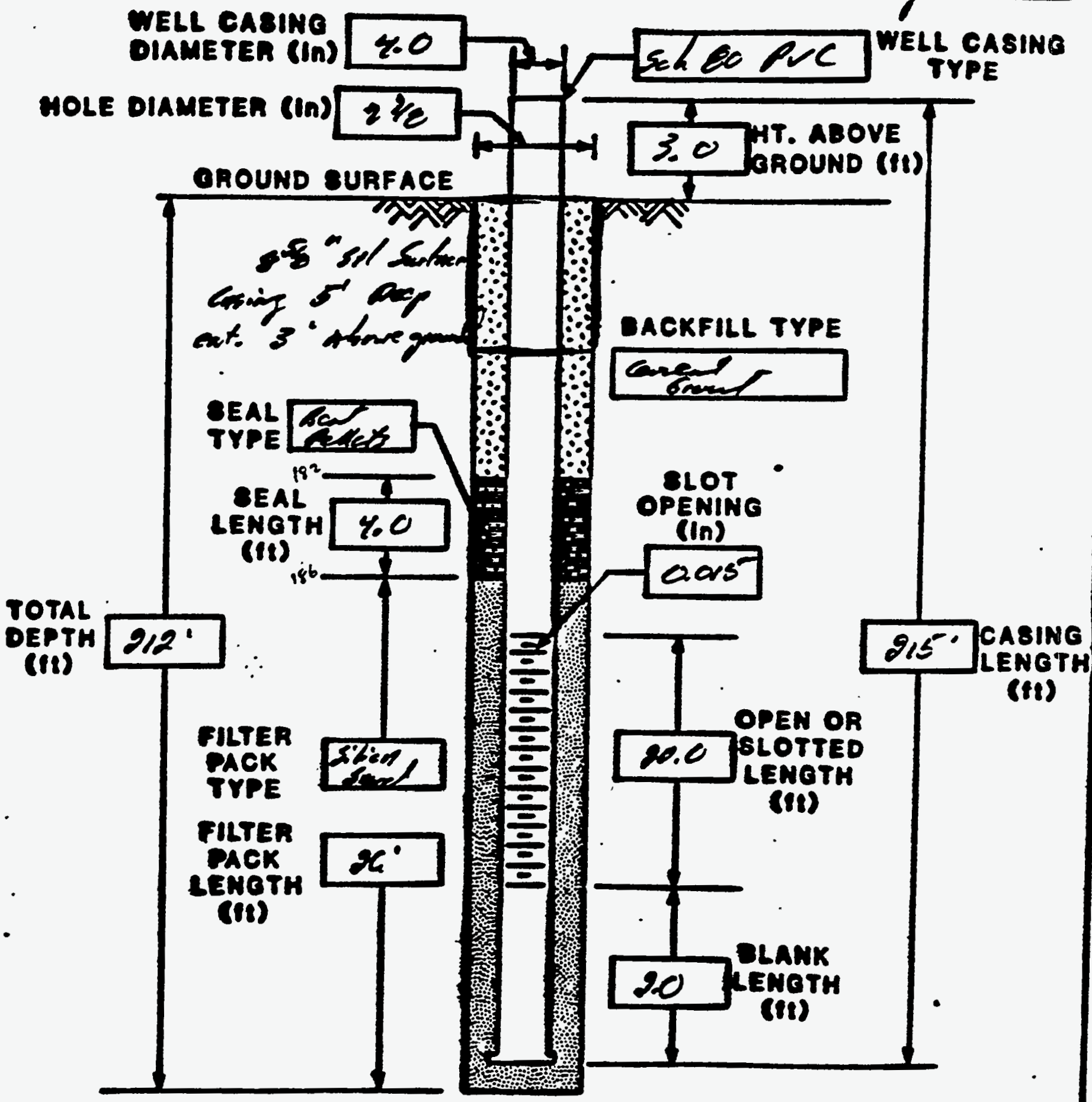

COMLEENTS: 
DE MOONS ANENERANG CROUP NC. YELL COMPLETION RECORD

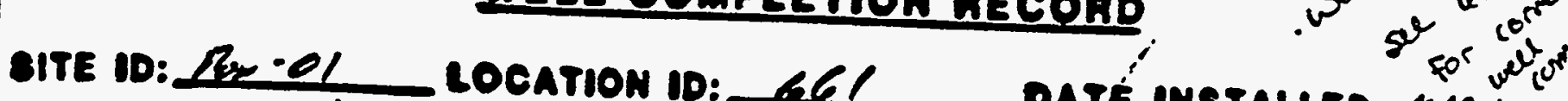
APPROX. EITE CODRDINATES:(FT.) N OPEN AREA PER LIMEAL FT. (IN2JFT.) FORMATION OF COMPAETION: FIELD AEP.: ReGeht

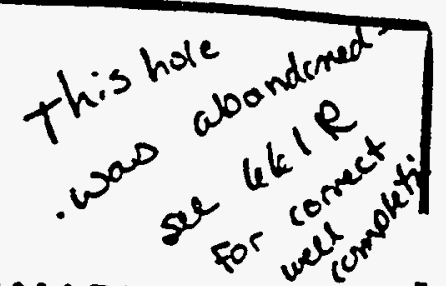
DATE IMBTALLED: WLELES E

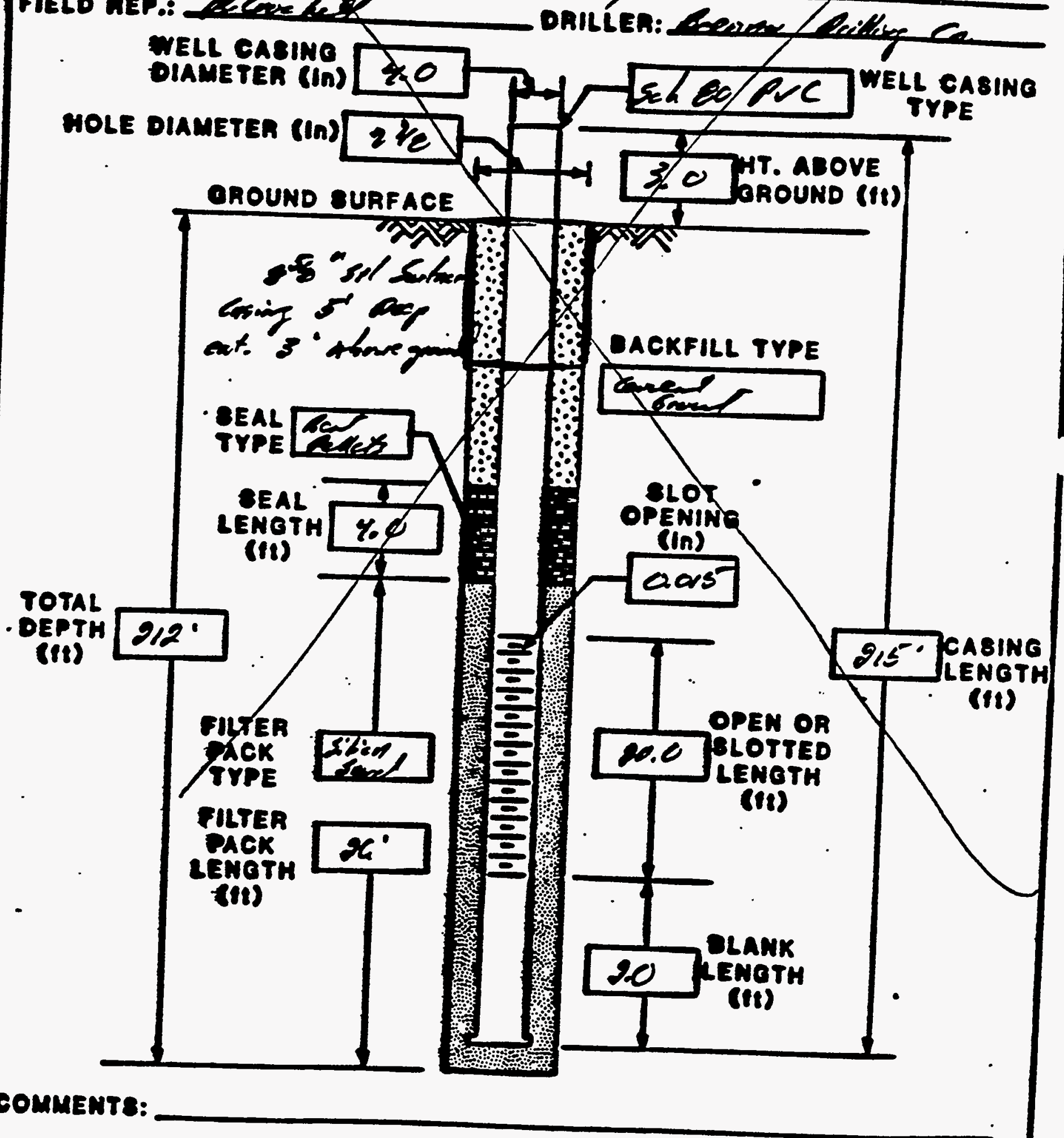




\section{IT MCOSS ENeN ERTE CROUP NC. \#661R

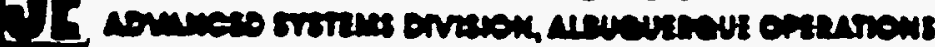

\section{YELL COMPLETION RECORD}

8ITE ID: MON.OI LOCATION ID: HOLE\# GSTR APPROX. OITE CÖOROINATE8:(FT.) $N$ DATE INSTALLED: $10-6-85$ OPEN AREA DER LUNEAL FT. (IN2/FT.)

f FOAMATION OF COMPLETION: DE CHELLY FORMATION SANDSTONE FIELO AEP.: K.DONNELSON/SHEB GELPRILLER: ROR BEEMAN DRILLINCCO.

WELL CABING DIAMETER (In)

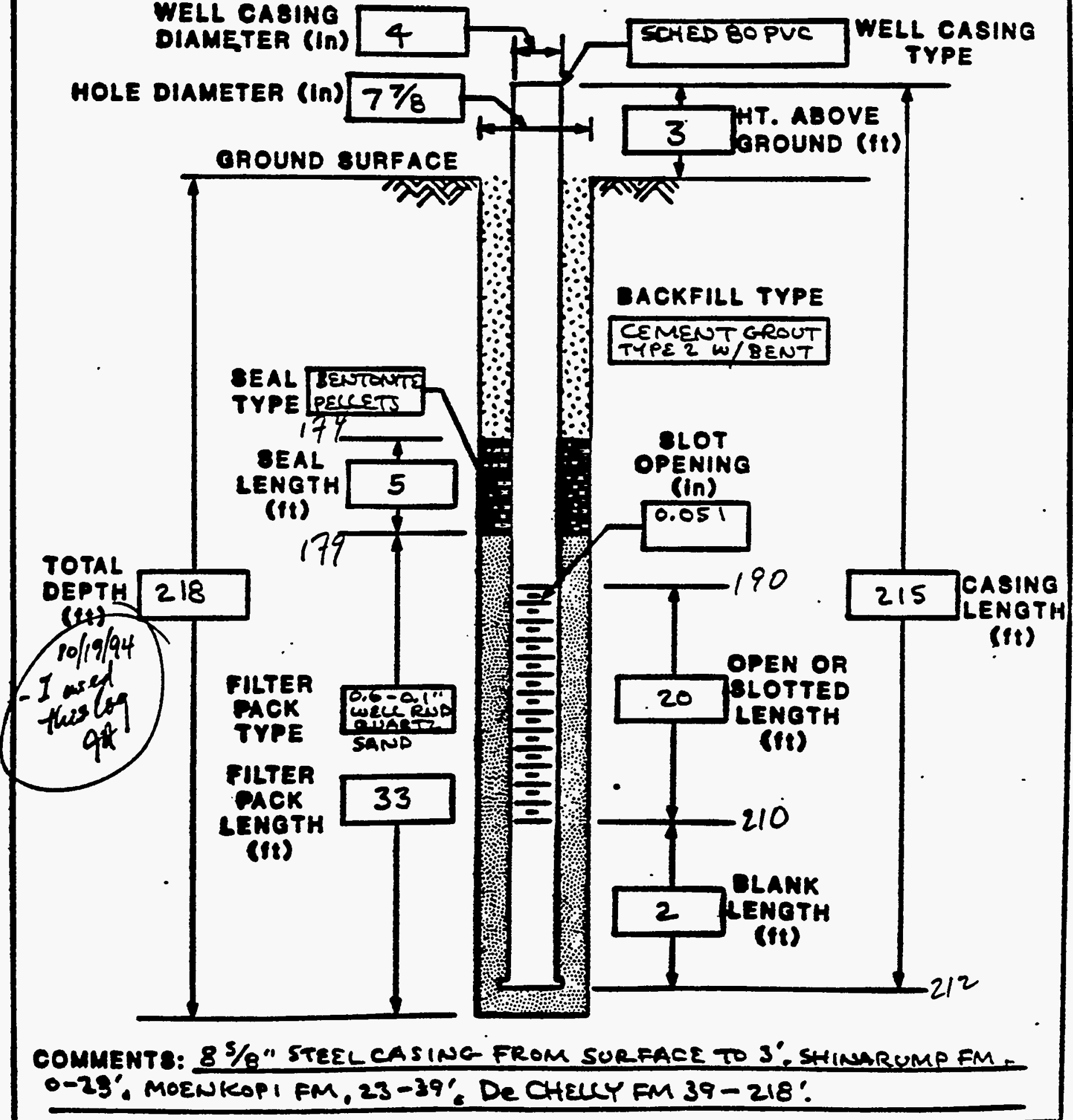

HOLE DIAMETER (In) $7 \%$
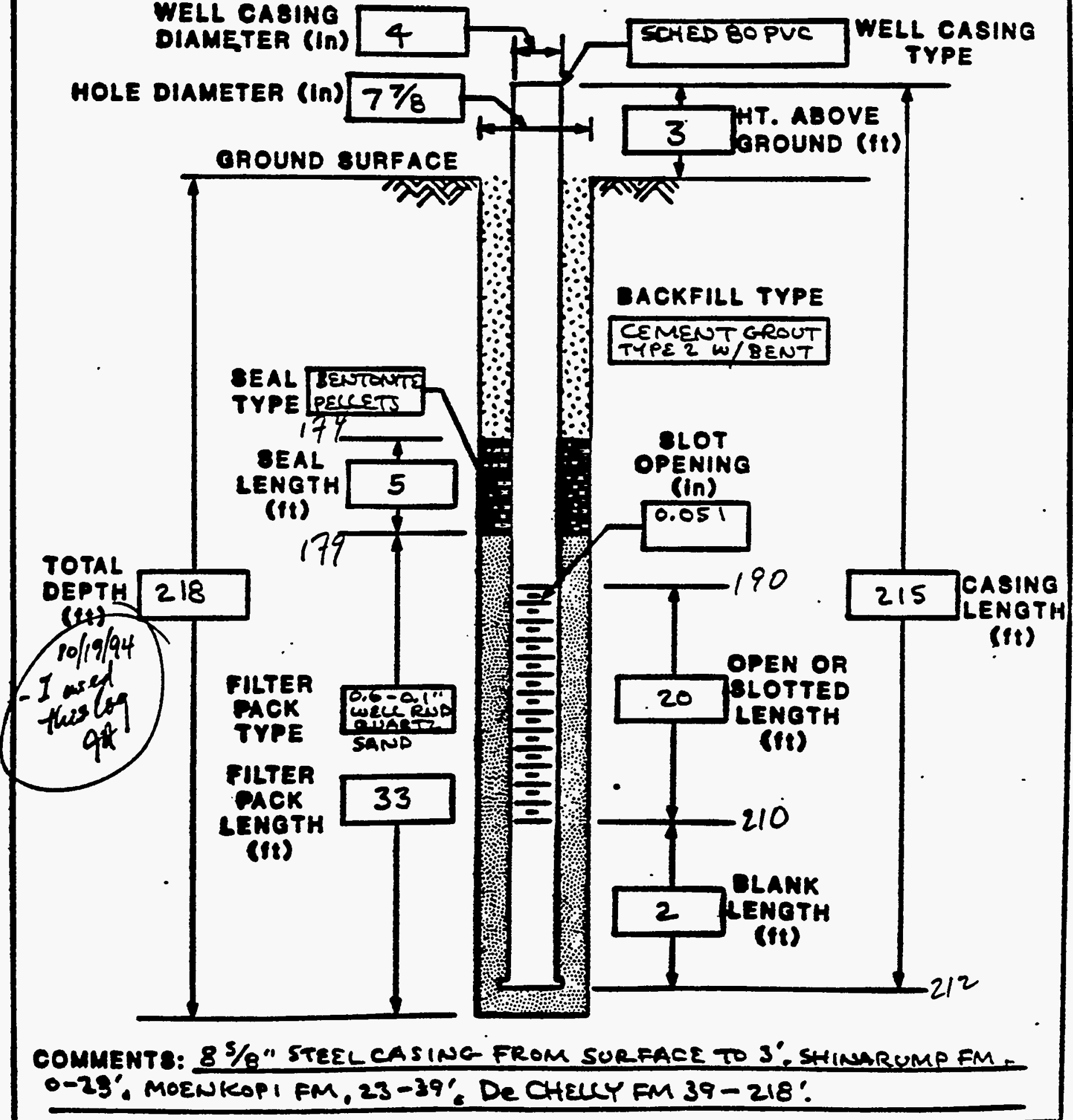

GROUND BURFACE

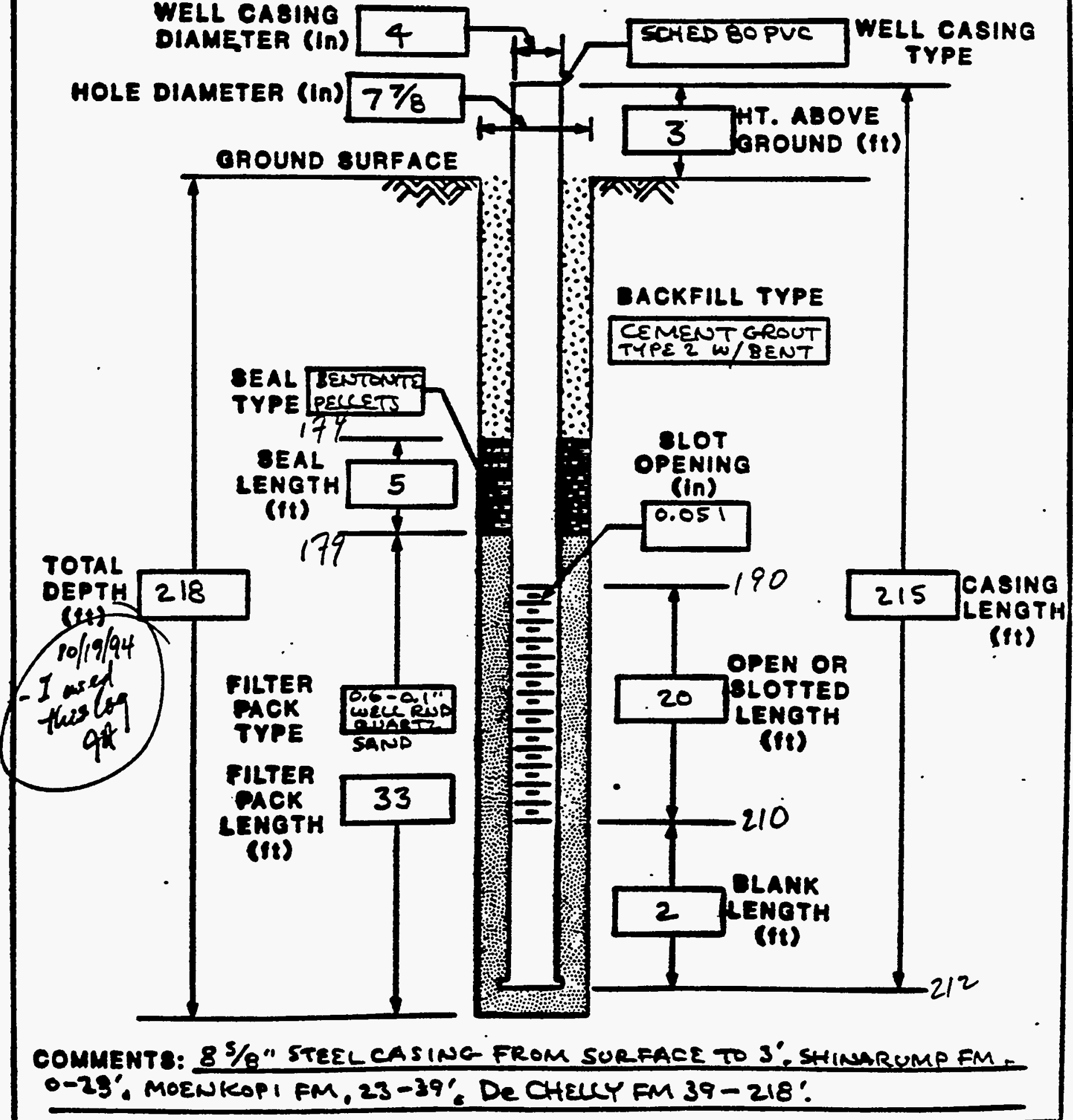

TOTAL 218

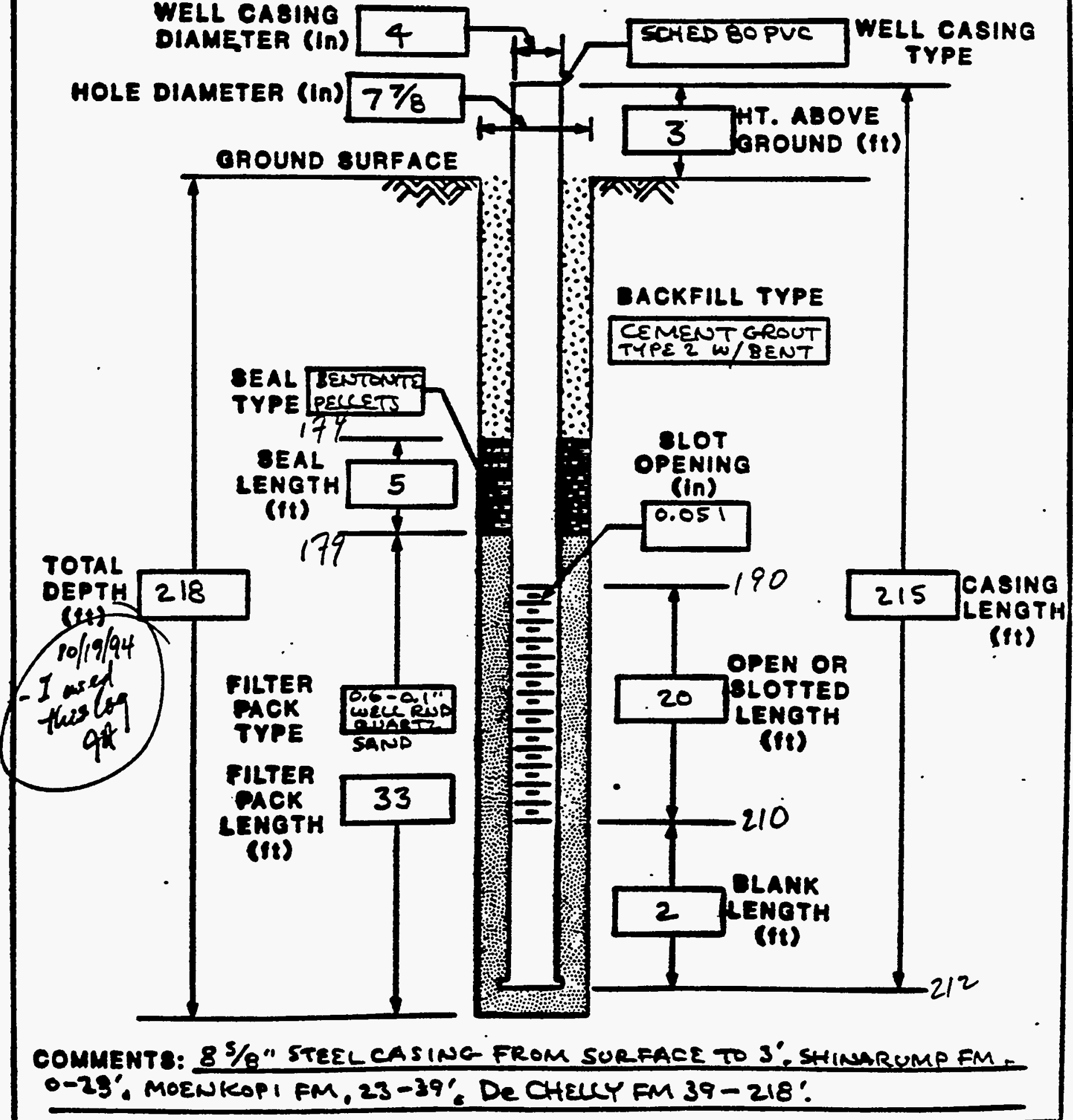
E 


\section{[T] MCONS ENGNERNG CROPP NC. \#661R

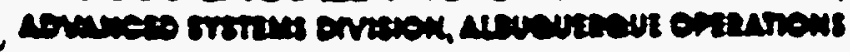

\section{VELL COMPLETION RECORD}

SITE ID: MON.O1 LOCATION ID: HOLE\# GGR DATE INSTALLED: 10-6-85 APPAOX. SITE COORDINATES:(FT.) N E OPEN AREA DER LINEAL FT. (IN2/FT.)

FORMATION OF COMPLETION: DE CHELLY FORMATION, SANDSTONE FIELD REP.: K.PONNELSON/SH BB GEQDDRILLER: BOR BEEMAN DRILLINCCO.

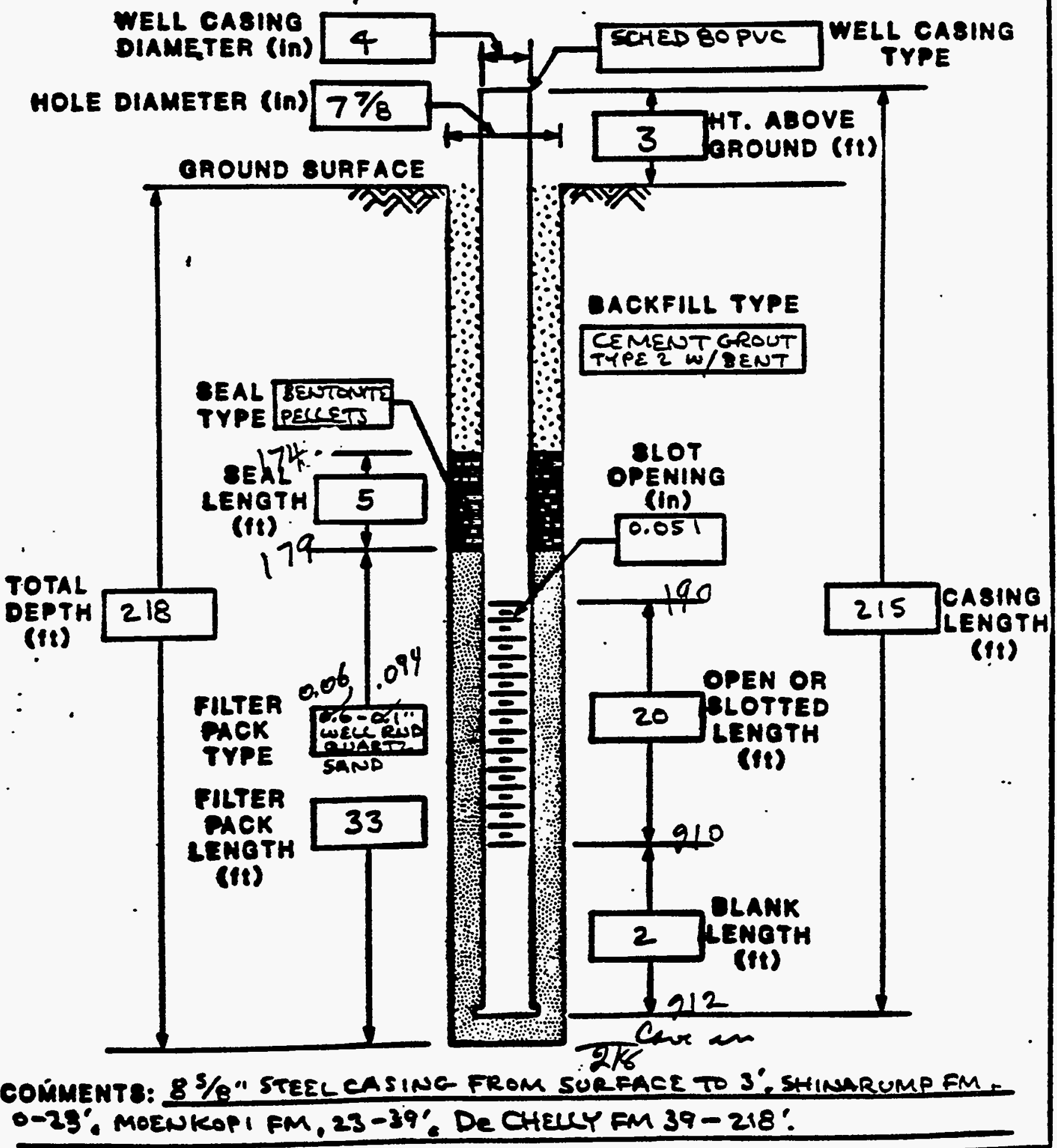




\section{OAREHOLE UWEL CONBTRUCTION LOO}

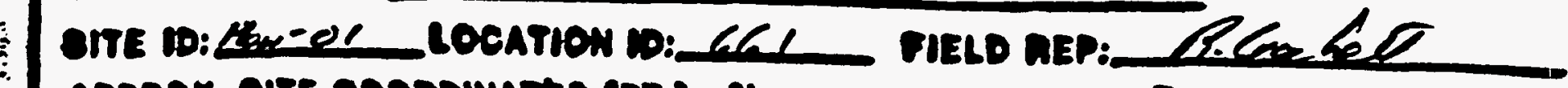

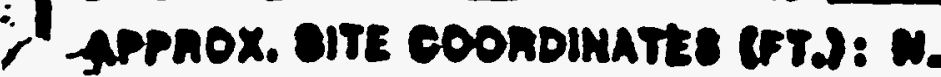

i Onound BLYATHOA CFT. MoLt:

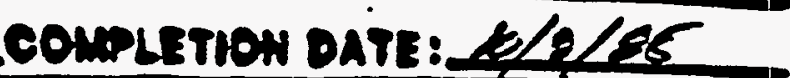

DONEHOLE OUMMARY

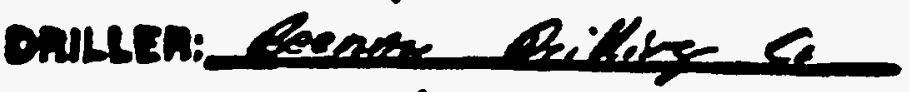

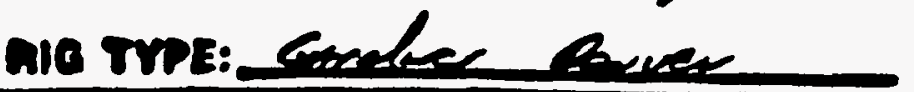

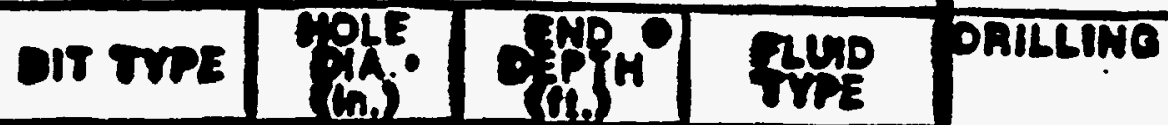

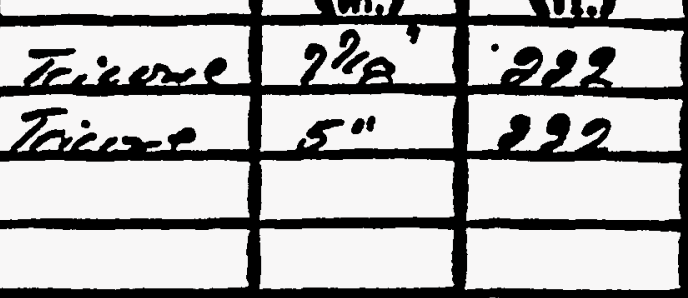

casino ovmmany

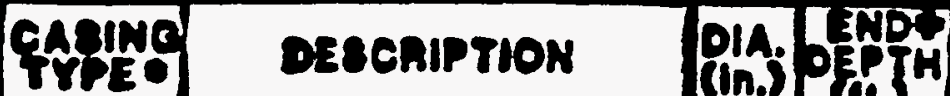

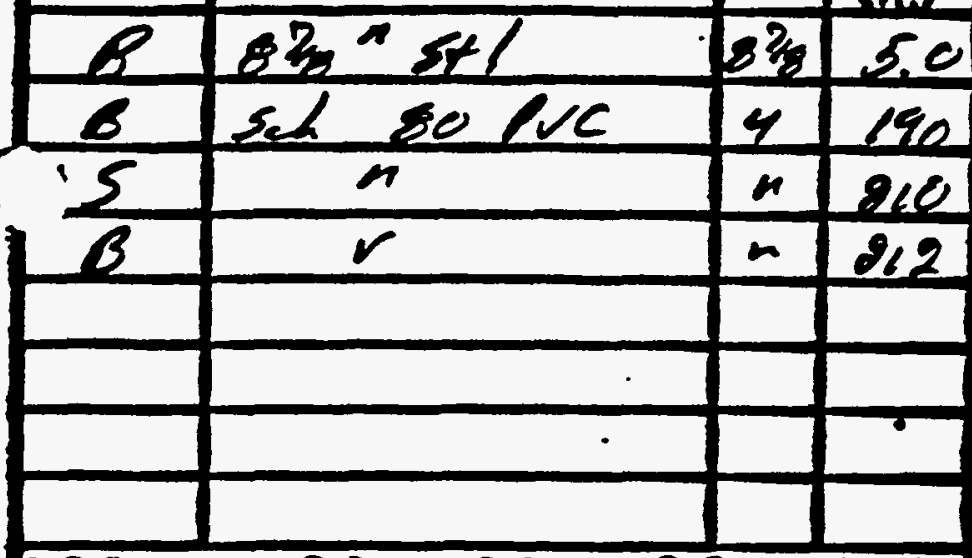

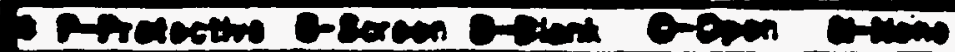

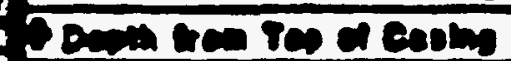

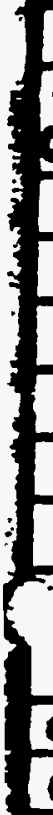

CELL CONBTAUCTION

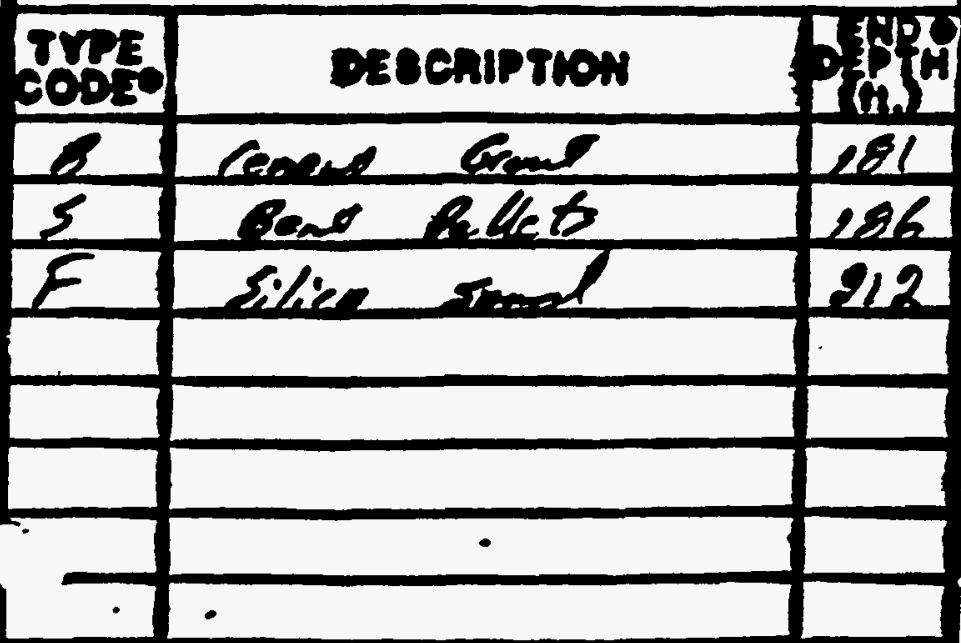

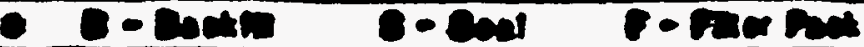

- Cosin tren Crems coneco

CoIMENTS:
constaLCTION TIME 100

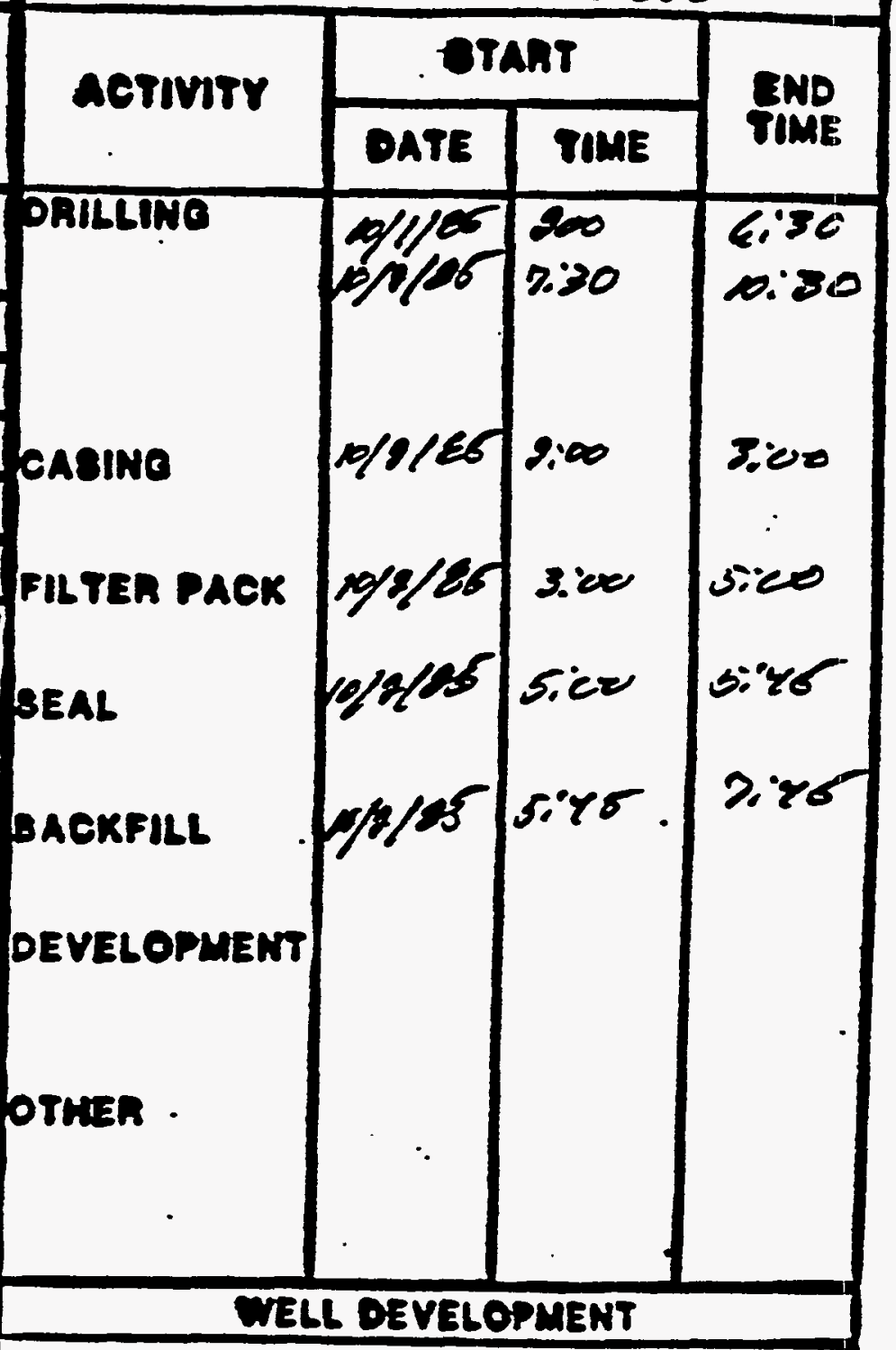




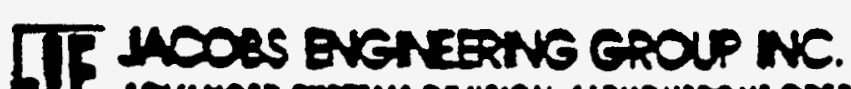

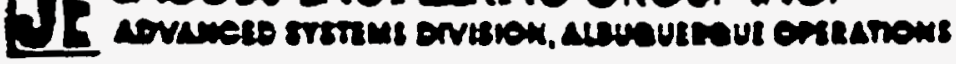 \\ WELL COMPLETION RECORD}

SITE ID: Mon LOCATION ID: \#662 Date installed: 9.5 .85 APPROX. SITE COORDINATES:(FT.) N OPEN AREA PER LINEAL FT. (IN2/FT.)

FORMATION OF COMPLETION: QuOETENARY DUNE SAND. FIELD AEP.: G. MillER DRILLER: Z. BEEMAN

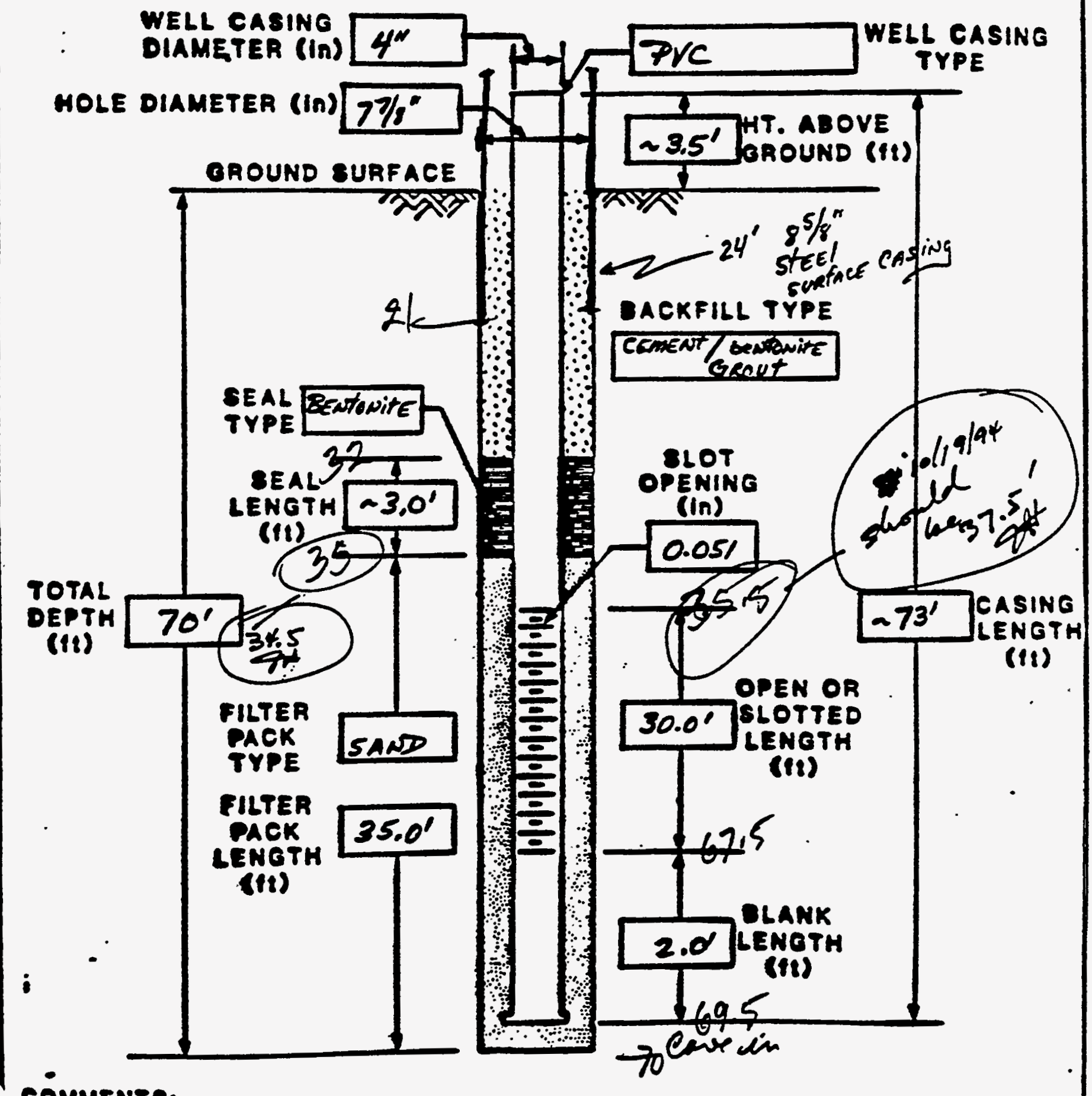

COMMENTS: 


\section{IT MCOSS ENENEERTG CROUP NC.}

\section{WELL COMPLETION RECORD}

SITE ID: MON LOCATION ID: \#662 DATE INSTALLED: 9.5 .85 APPROX. SITE COORDINATES:(FT.) N E

OPEN AREA PER LINEAL FT. (IN2/FT.)

fFORMATION OF COMPLETION: RuOtERNACY DNNE SAND.

FIELD REP.:

G. MilleR

DRILLER:

3. BeEmaN

WELL CASING

DIAMETER (in)

HOLE DIAMETER $(\ln ) 7 \%$

BROUND SURFACE
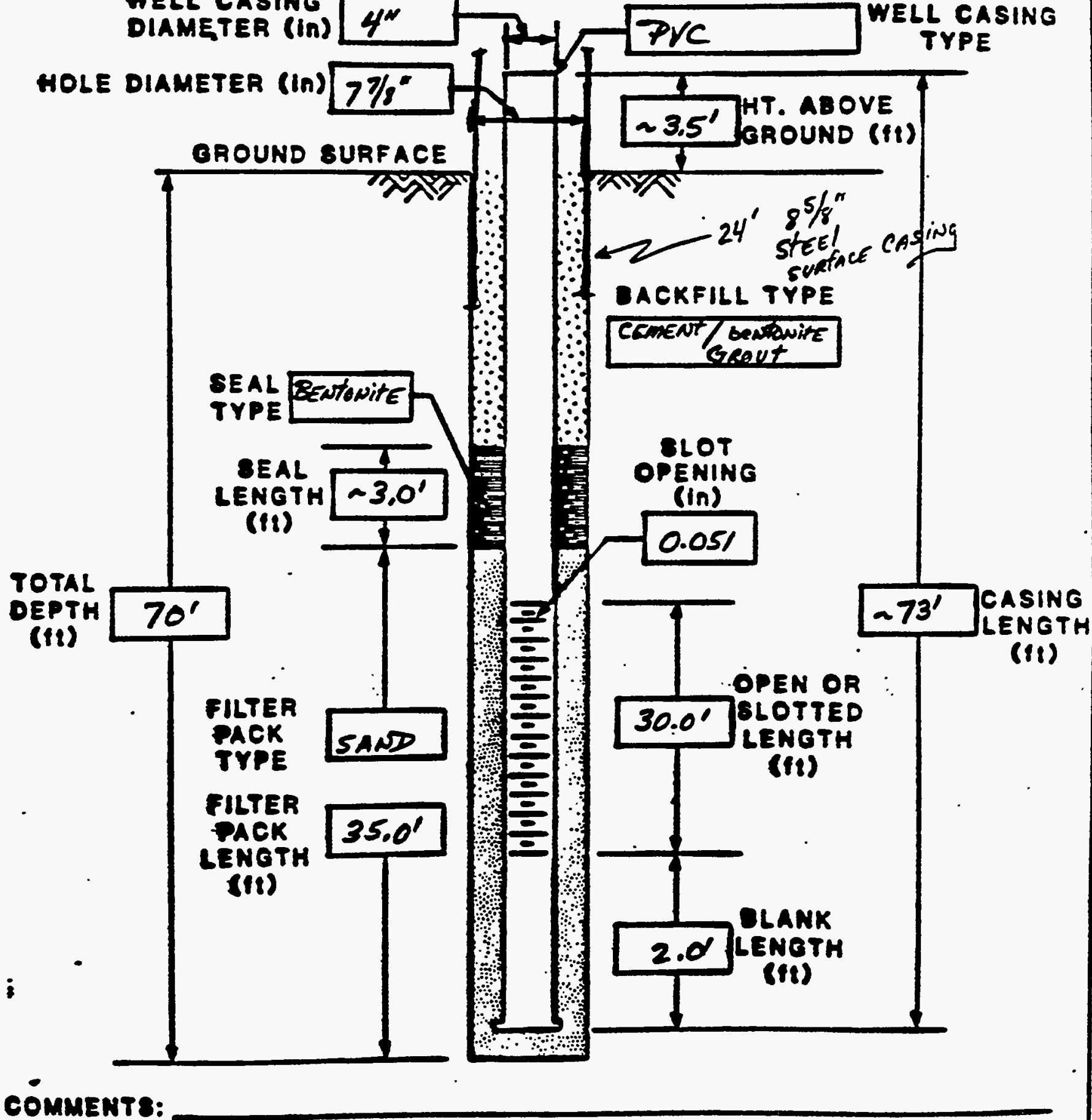

PrC

WELL CASING TYPE

COMMENTS: 


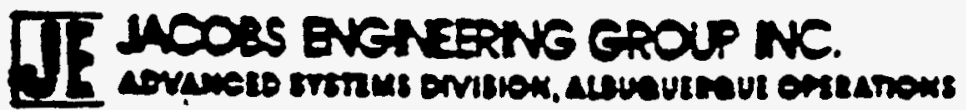

\section{MELL COMPLETION RECORD}

Site ID: MON location ID: \# 663 DATE installed: 9.10 .85 APPAOX. SITE COOADINATE8:(FT.) N $\boldsymbol{E}$

OPEN AREA DER LINEAL FT. (IN2/FT.) FORMATION OF COMPLETION: DeChelly 55. Mbr. of Cutlee Fm.
FIELD AEP.: G.MilleR

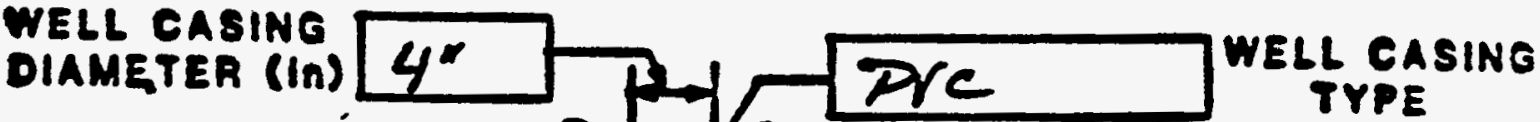

HOLE DIAMETER (In) 7\%/8"

BROUND SURFACE

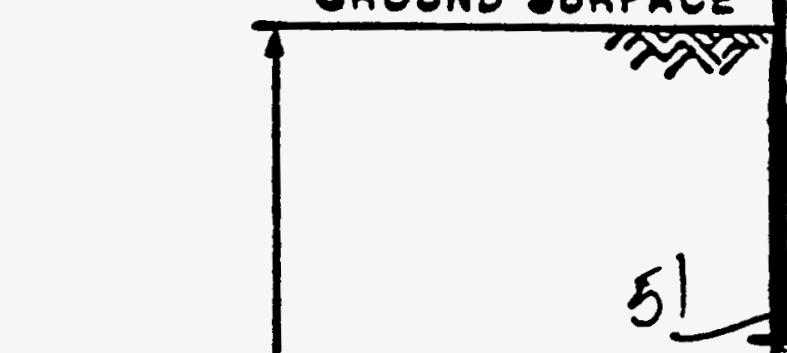

TOTAL

DEPTH (11)
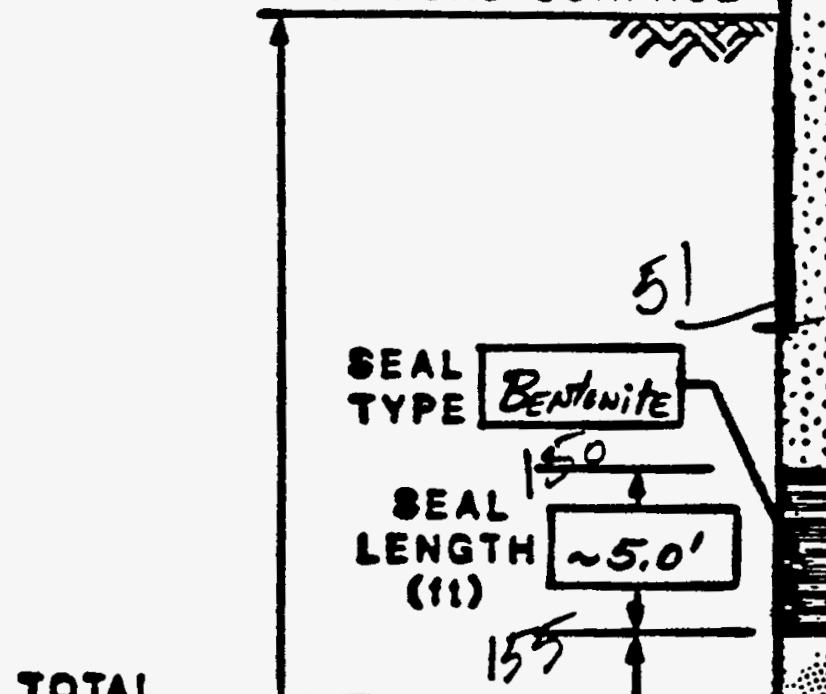

$217^{\prime}$
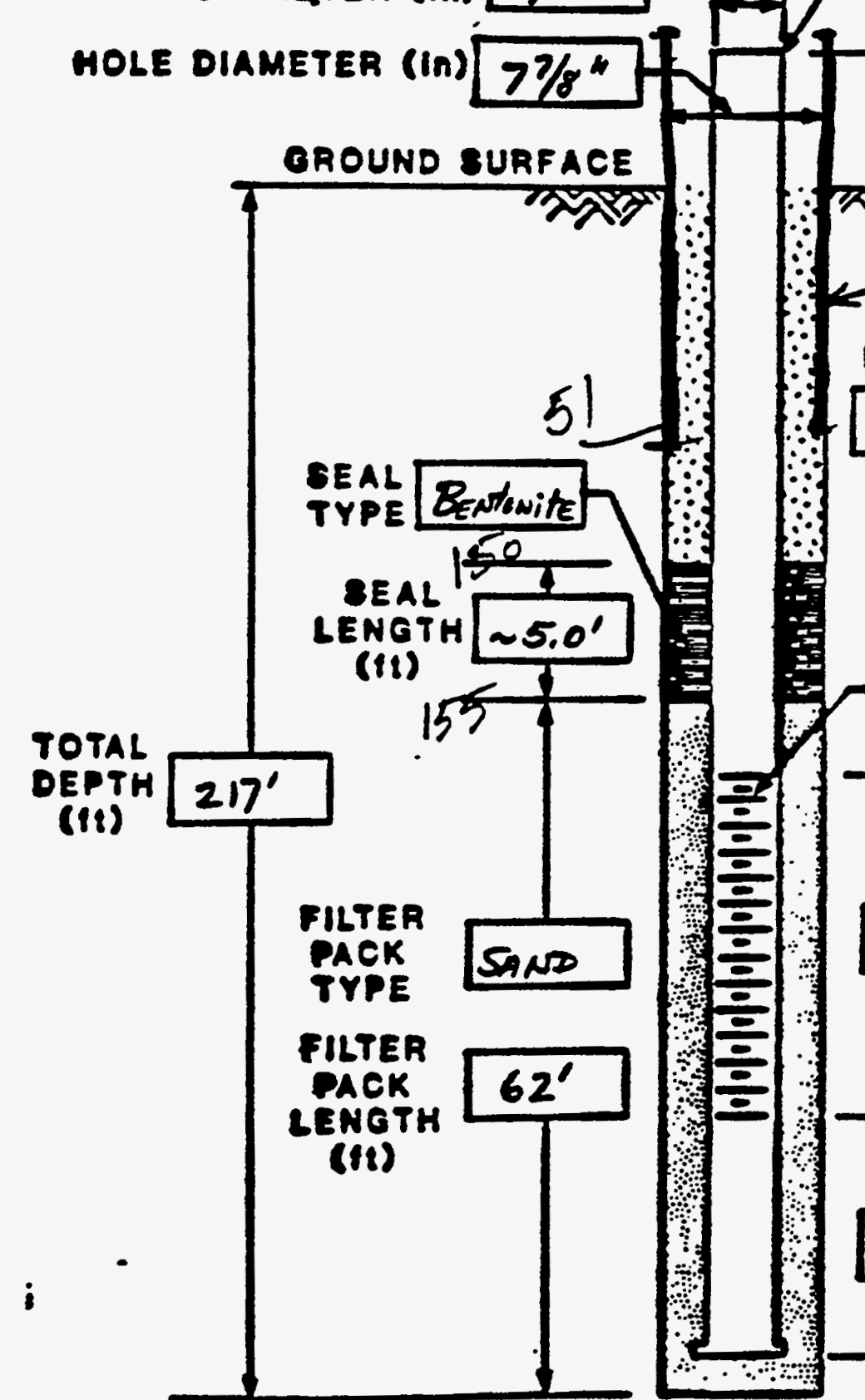

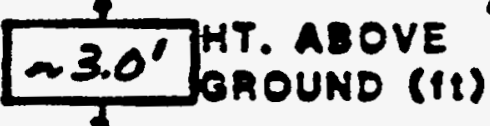
$\therefore$ खण्र

$54^{\prime}$ of - 85t" steel casing

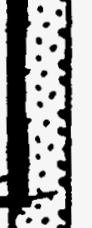

$\because$

DACKFILL TYPE

: Croment/butwites

Cerout

$\because$

$\therefore$
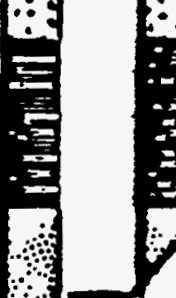

OLOT

OPENING
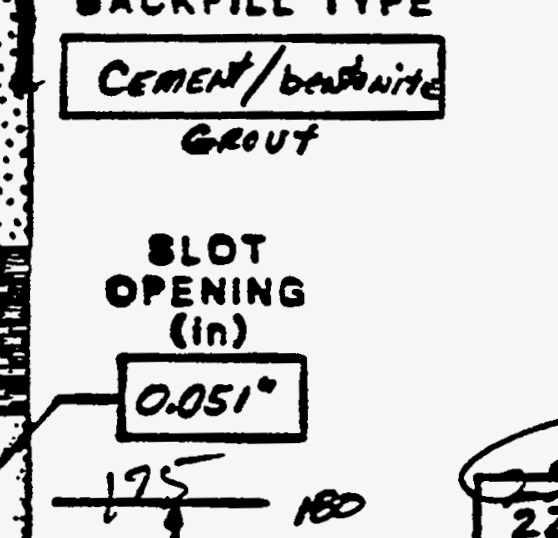
I[ MoOss ENeN serNG EROUP NC.

WELL COMPLETION RECORD

Site 10: MON Location ID: \#663 DATE installed: 9.10 .85 APPROX. SITE COORDINATES: (FT.) N E

OPEN AREA PER LINEAL FT. (IN2/FT.)

FORMATION OF COMPLETION: DeChelly 55 . Mbe. of Cutlee Fm. FIELD AEP.: G.MillER DRILLER: B. BEEmAN

WELL CASING

DIAMETER (in)

hole DIAMETER (in) $7 \%$

oROUND SURFACE

TOTAL

DEPTH

(18)
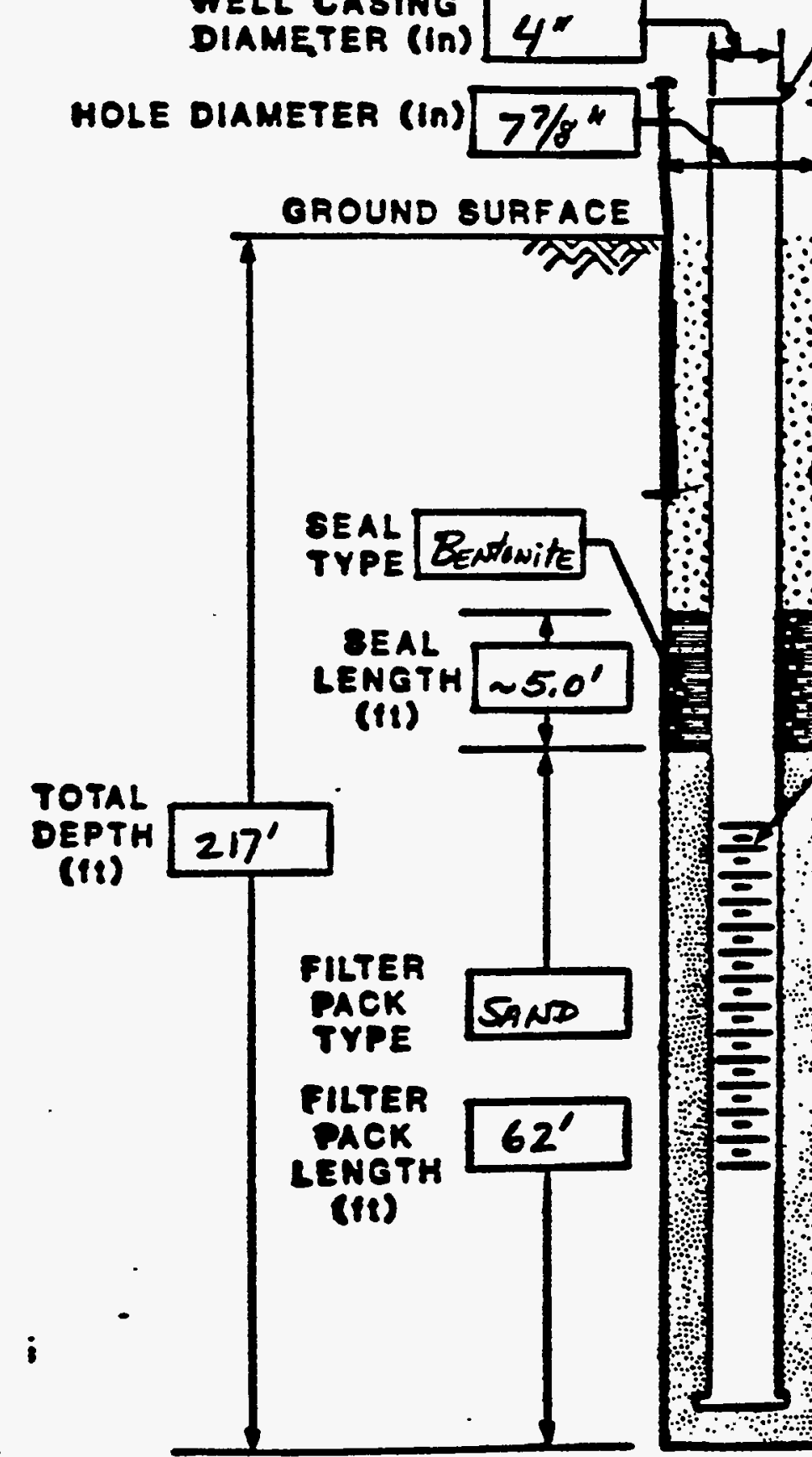

Pre

WELL CASING TYPE
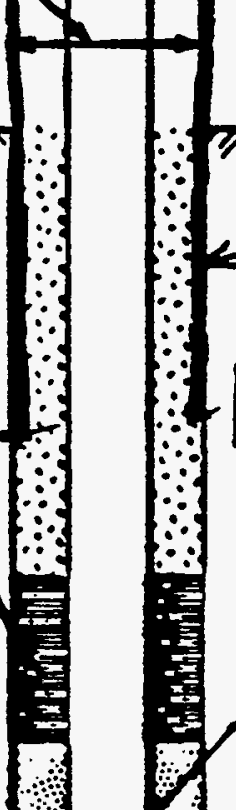

$23.0^{\prime}$

HT. ABOVE

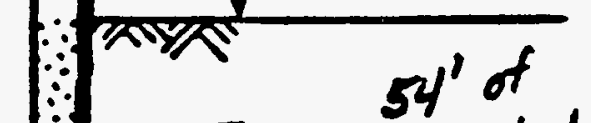

$\therefore 2$ 85\%" steel casing

$\because \because \mathrm{Ba}$

DACKFILL TYPE

Cenent/becturtes

$\because$ Creout

$\therefore$

$\because$

$\because$ SLOT

OPENING

(in)
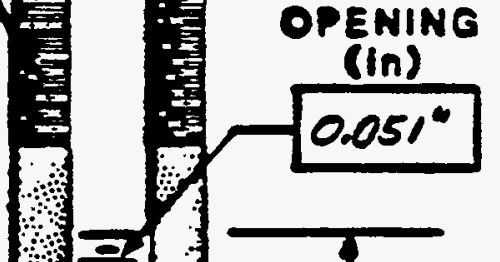

4

$222^{\prime}$

CASING LENGTH

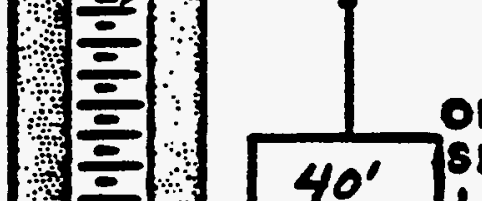

(ti)

-

COMMENTS: 


\section{WELL COMPLETION RECORD}

SITE ID: M/C.N' LOCATION ID: \#66.4 DATE INSTALLED: 9.1985 APPROX. SITE COOADINATES:(FT.) $N$ OPEN AREA PER LINEAL FT. (IN2/FT.) fFORMATION OF COMPLETION: $\frac{\text { De ChOlly SE. Mlor. af Cutlec Fm. }}{\text { FIELD DEP.: } G \text { M.llER }}$ DAILLER: B But Berman'

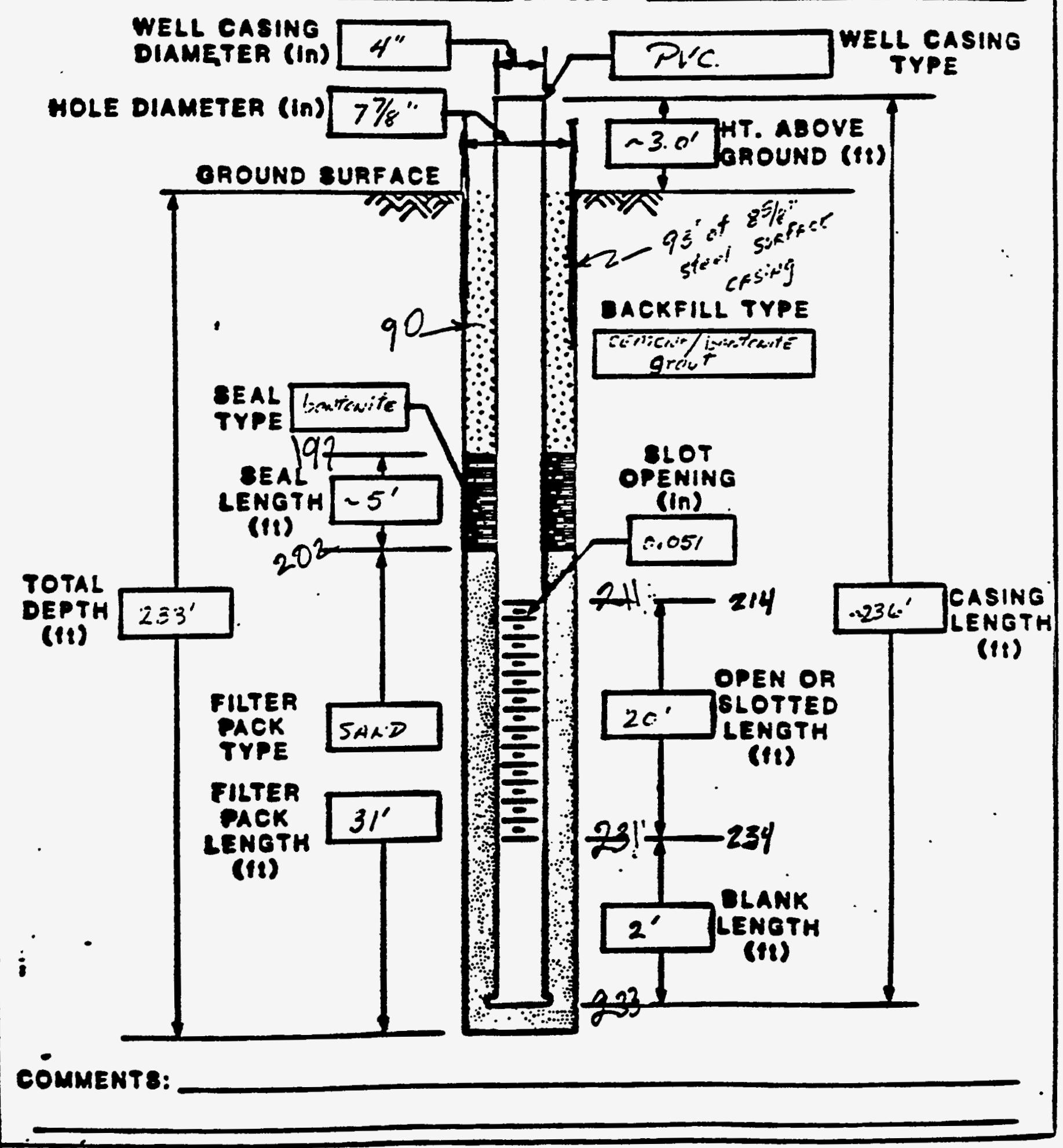




\section{LIT MOOSS ENENEERTG CROUP NK.

MELL COMPLETION RECORD

SITE ID: MlON LOCATION ID: \#664 DATE Installed: 9.19 .85 APPROX. SITE COORDINATES:(FT.) N $E$

OPEN AREA DER LINEAL FT. (IN2/FT.)

fORMÁtION OF COMPLETION: DeChelly $5_{5}$. Mbr. of Cutler Fm.

FIELD REP.: G. MIVIER DRILLER: Bob Beeman

WELL CABING

DIAMETER (In)

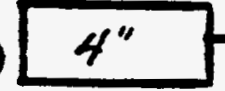

hOLE DIAMETER (in) 7\%/8"

GROUND SURFACE
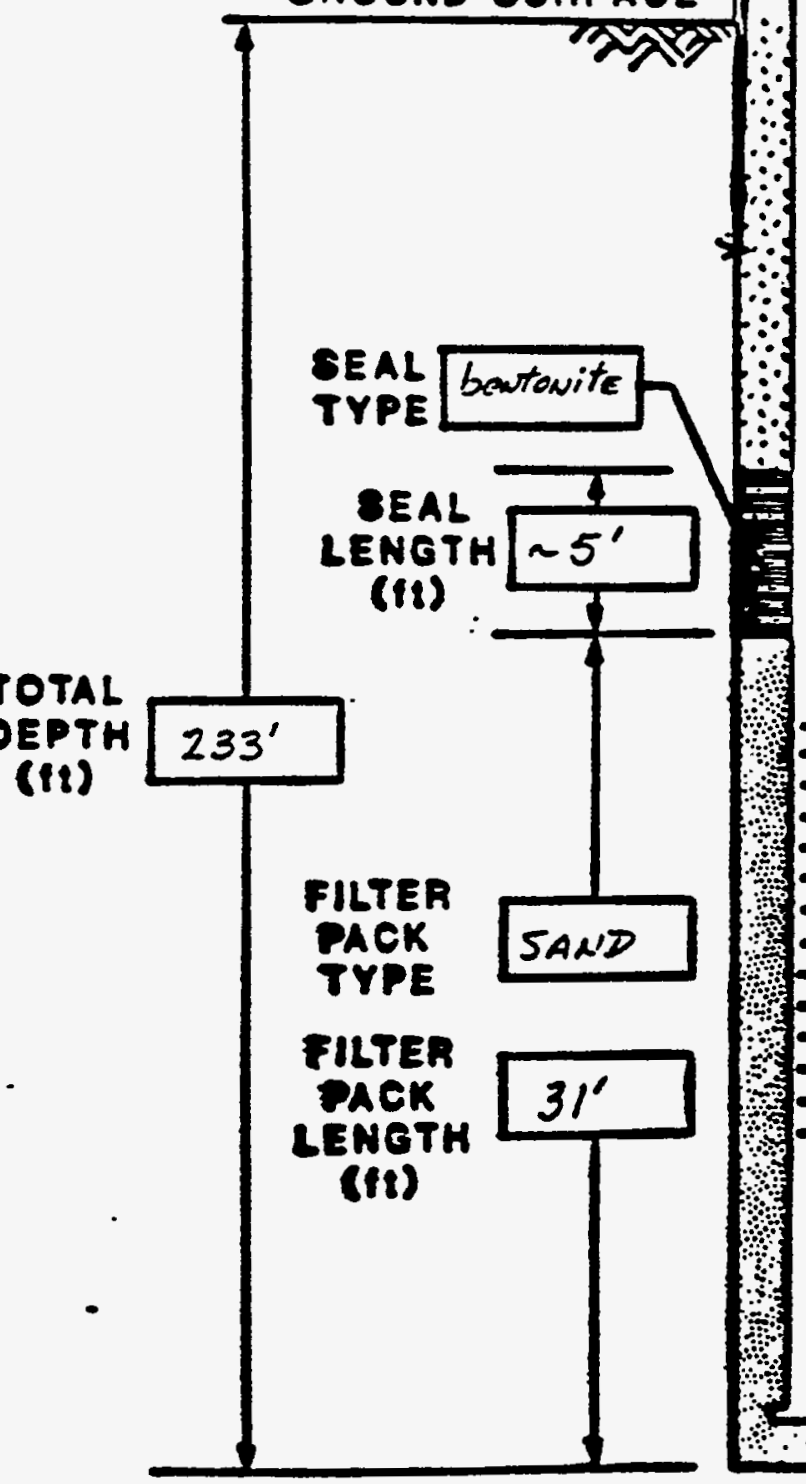
PVC TYPLE TASING
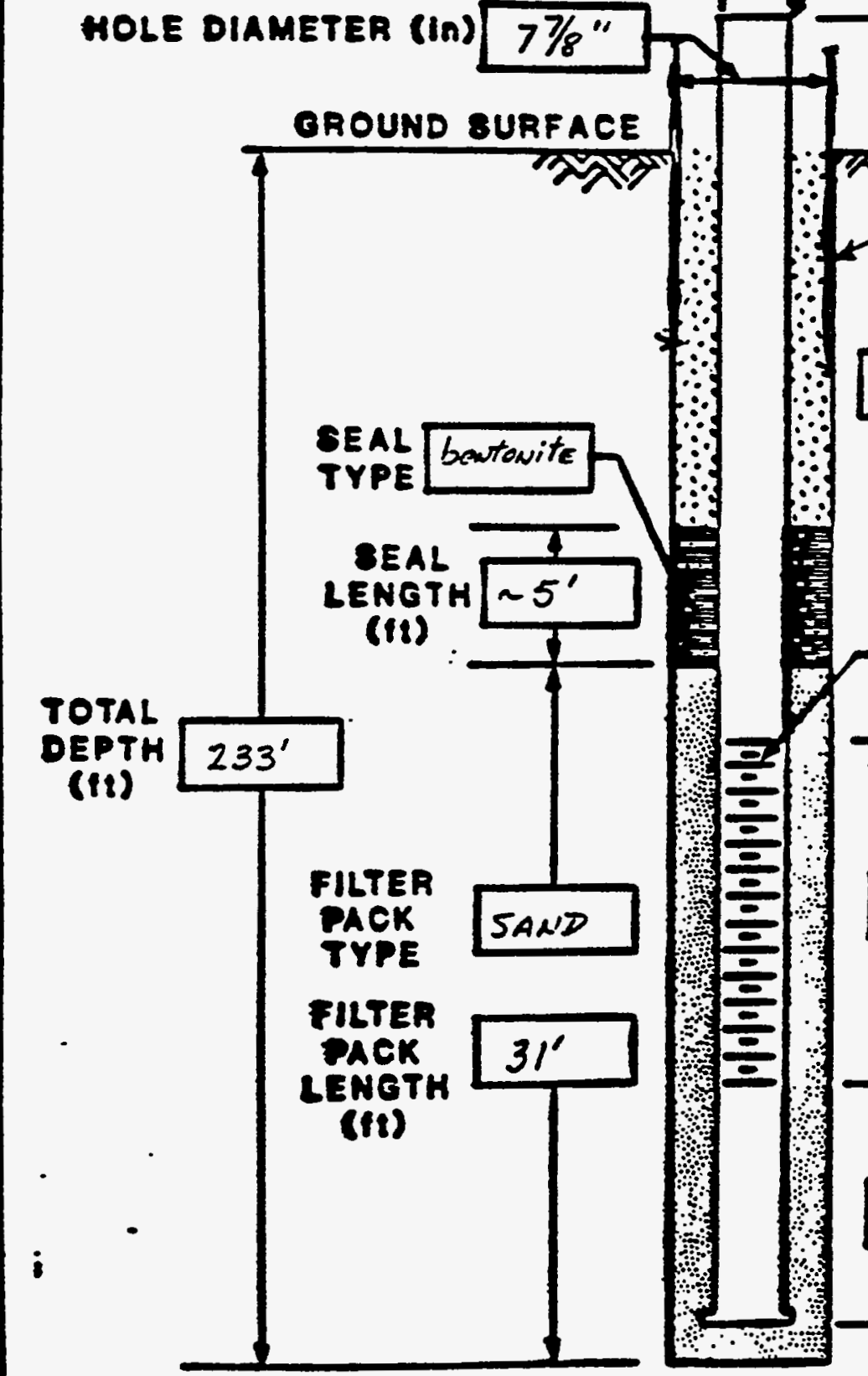

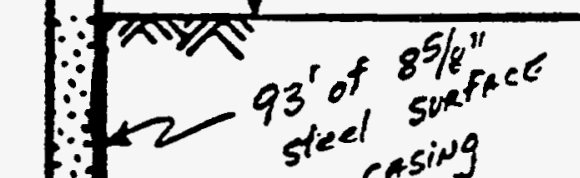




\section{AI mooss Even zang eRoup NC.

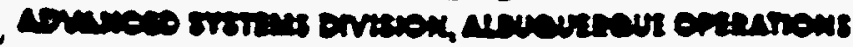

\section{YELL COMPLETION RECORD}

8ITE ID: MON. OI LOCATION ID: HOLEEGS8 OATE INBTALLED: 10-4-ES APPAOX. SITE COORDINATES:(FT.) N

OPEN AREA PER LNEAL FT. (IN2/FT.)

FORMATION OF COMPLETION: DE CHELLY FORMATION

FIELD REP.: K.PONNELSON/SHEB GEOL DAILLER: SOB BESMNN DRILLNK CO

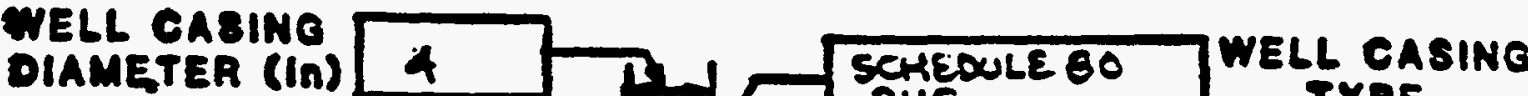
TYPE

HOLE DIAMETEA $(\ln ) 7 \%$

GROUND SURFACE
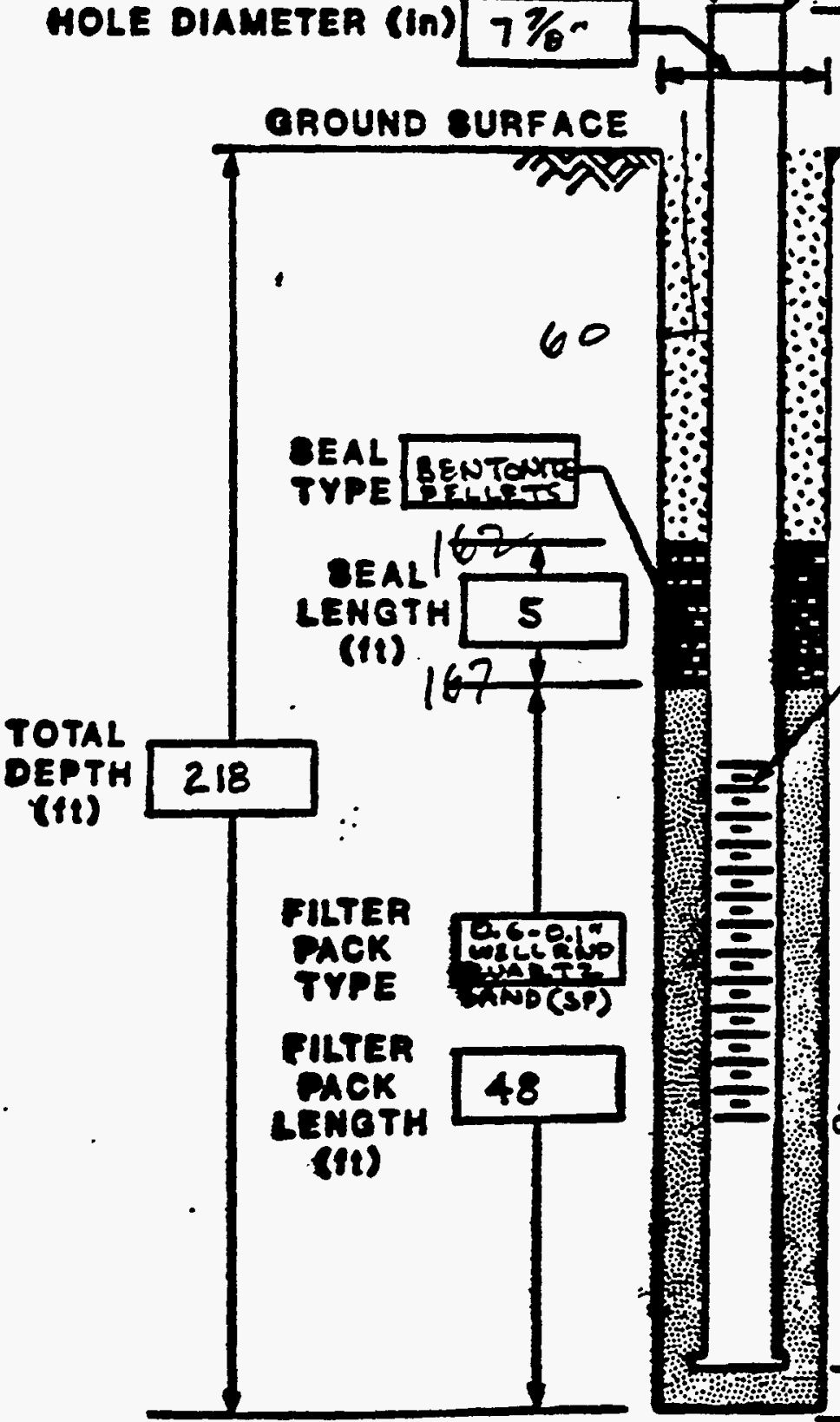

2.5 GR. AQOVE

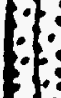

$\because$ खNY

(fi)

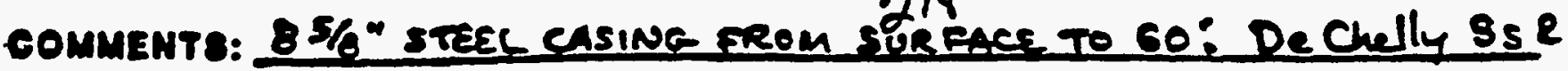

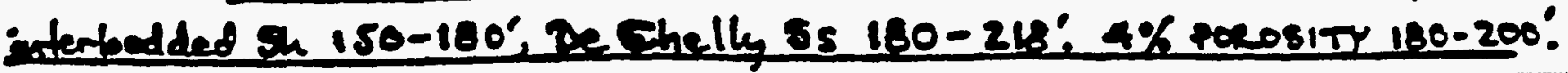




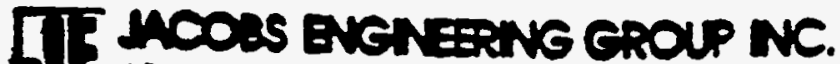

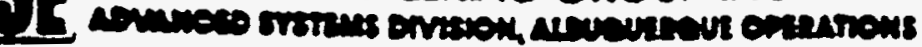

\section{MELL COMPLETION RECORD}

SITE 10: MON. OI LOCATION 10: HOLEZ668 DATE INBTALLED: 10-9-85 APPROX. OITE COORDINATES:(FT.) $N$ $\boldsymbol{E}$ OPEN AREA.PER LUNEAL FT. (INR/FT.)

FORMATION OF COMPLETION: DE CHELLY FORIMATON

FIELD REP.: K. PONNELSON/SHEB GSOL DRILLER: BAB BESMWN PRILUNG CO

well Casino

DIAMETER (In)

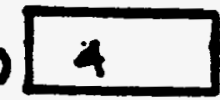

HOLE DIAMETER (in) $7 \%$.

OROUND SURFACE

TOTAL DEPTH (18)

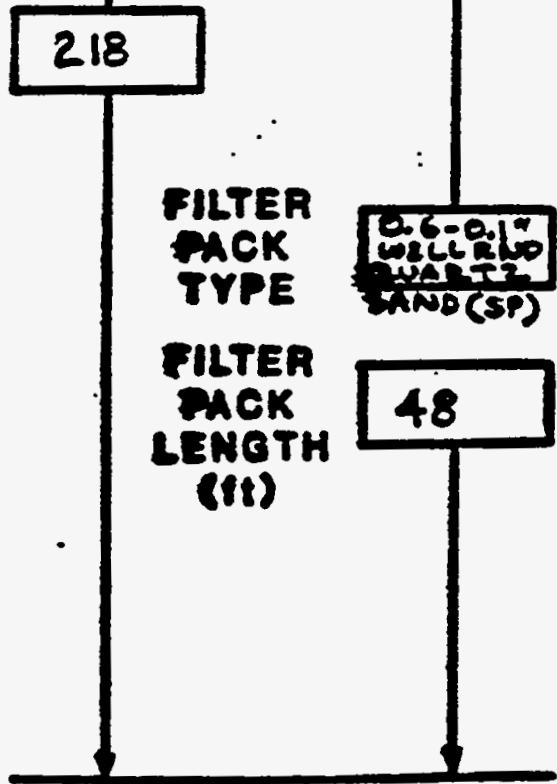

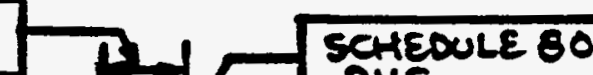
Suc

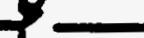
TYPE

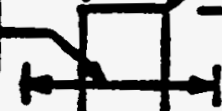

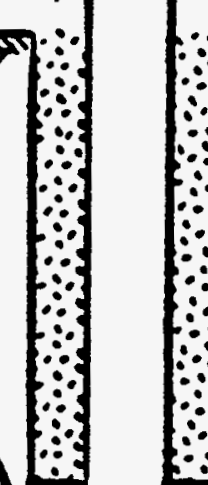

DACKFILL TYPE

CEMENT GROUT

OEAL BENTOWRE TIPE 2 WRBENT

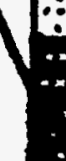

$\because 3$ 2.5 JT. ABOVE सחश

SEAL LENOTH (II)
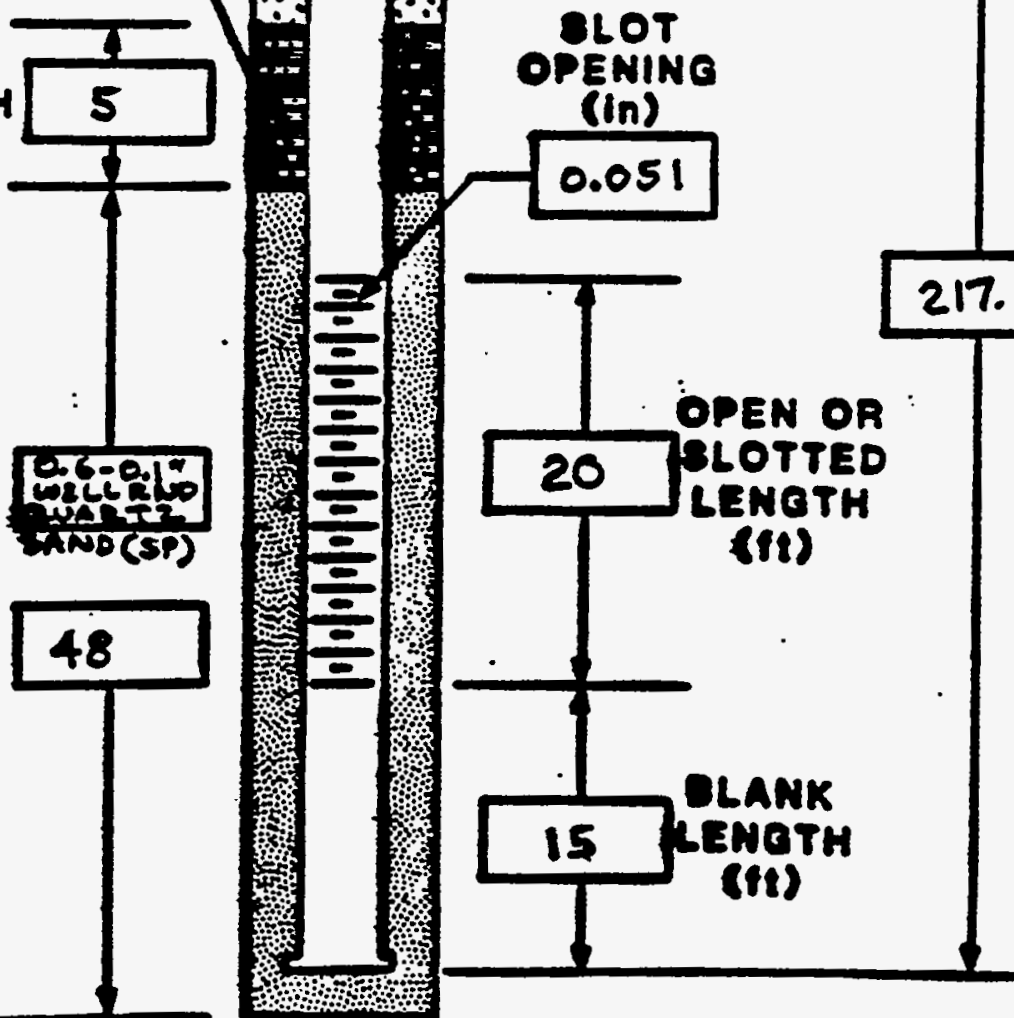

CABINO LENOTH (It)

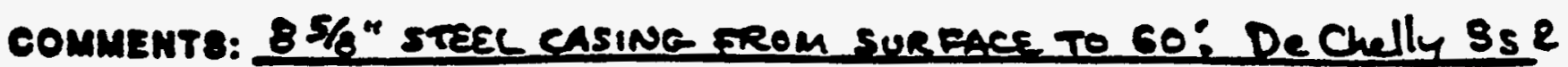

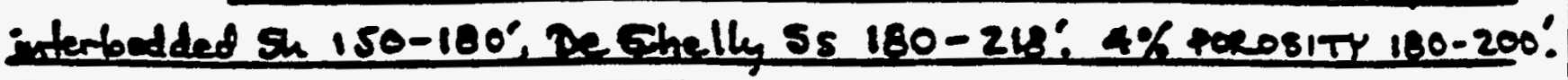




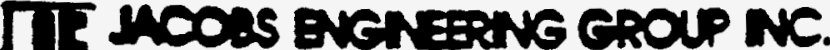

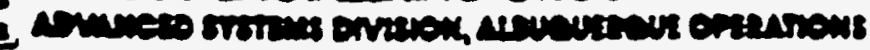

\section{WELL COMPLETION RECOAD}

BITE ID: MON. OI LOCATION ID:HOLE \# G9 OATE IN8TALLED: 10-5-8S APPAOX. OITE COORDINATE8:(FT.) $N$ E OPEN AREA PER LNEAL FT. (INZJFT.)

(FONMATION OF COMPLETION: ALLUIUM (SP-SW) ABOUE SHINARUMP FIELO AEP.: K. DONNELSON/GL GE DRILLER: BOB EESMAN DRILLINECO.

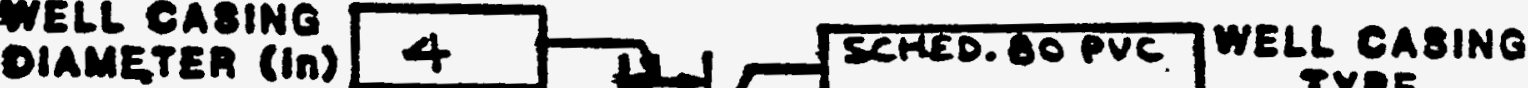

MOLE DIAMETER (In)
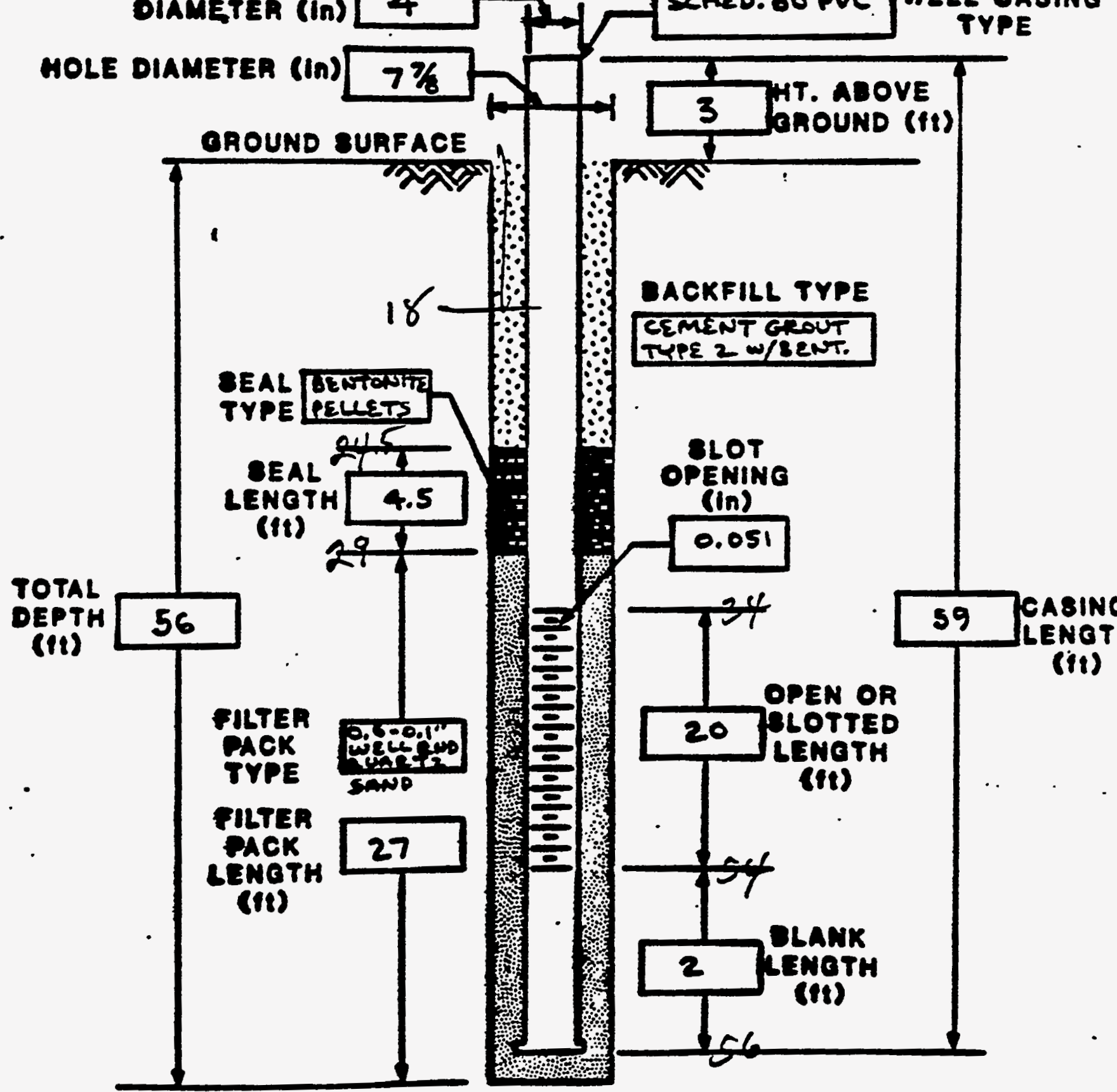

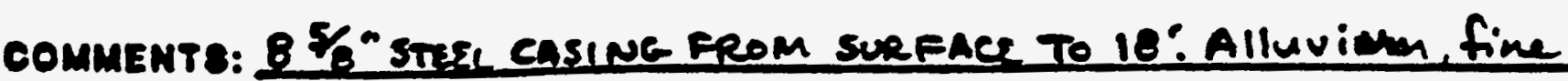
se sind $0-20^{\circ}$, fine-coarse SP-w sand 20-56: Top ShinarumeC $56^{\circ}$ 


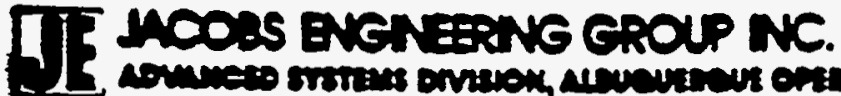

\section{MELL COMPLETION RECORD}

SITE ID: MON. OI LOCATION ID: HOLE\# 669 DATE INSTALLED: 10-5-85 APPROX. BITE COORDINATES:(FT.) $N$ $\boldsymbol{E}$

OPEN AREA PER LINEAL FT. (H'/FT.)

FORMATION OF COMPLETION: ALWUIUM (SP-SW) ABOUE SHINARUMP

FIELD REP.: K. DONNELSON/SHLB GEO DAILLER: BOB BEEMAN DRILLINTC CO.

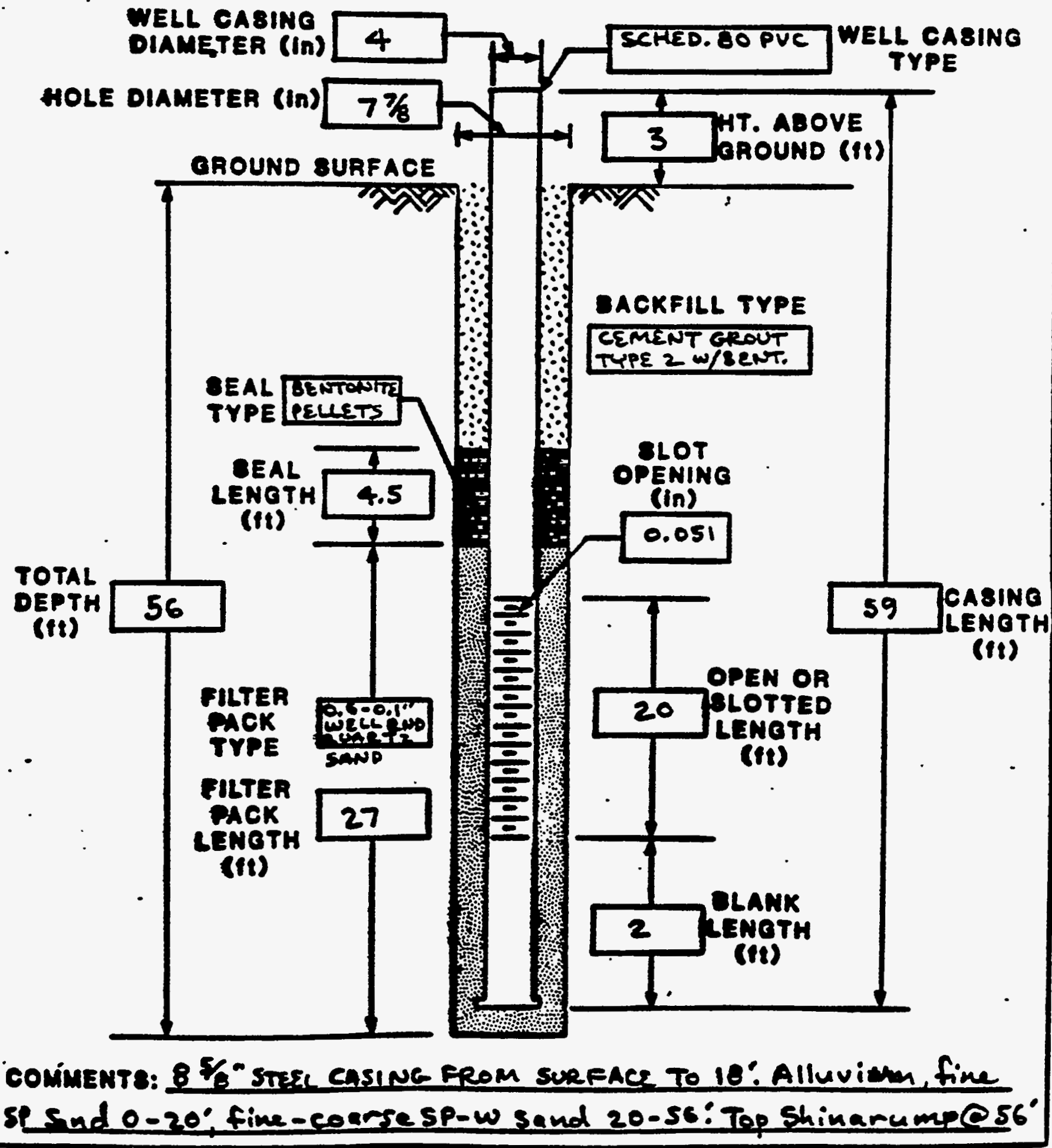


APPENDIX B

WATER LEVELS, ALL WELLS 


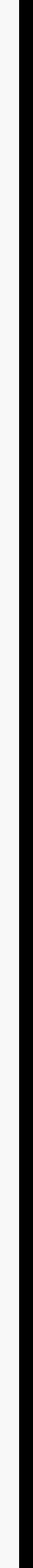


Mонхип - $M$

INaIOYyad - n

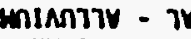

: 3000 dIHSMOLIV734 mor

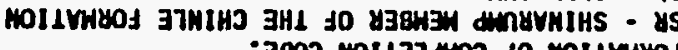

\begin{tabular}{|c|c|c|c|c|c|c|c|c|c|c|c|}
\hline 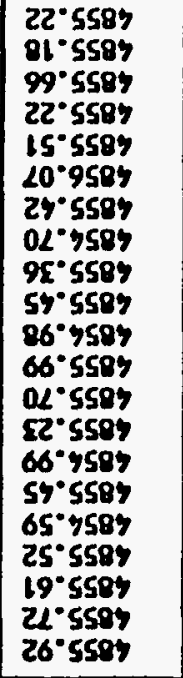 & 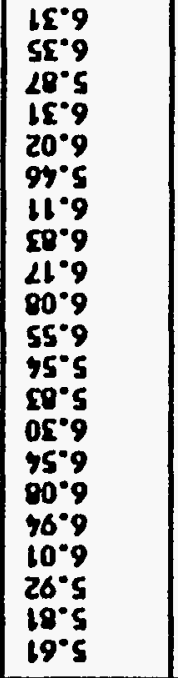 & 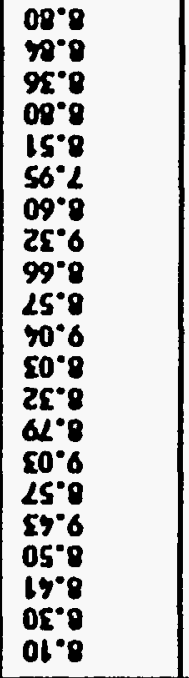 & $\begin{array}{l}17: 01 \\
2 y:=11 \\
51: 01 \\
62: y b \\
20: 91 \\
67: 60 \\
02: 11 \\
80: 91 \\
01: 51 \\
07: 51 \\
75: 11 \\
05: 21 \\
01: 21 \\
00: 21 \\
50: 51 \\
50: 21 \\
07: 91 \\
71: 21 \\
22: 90 \\
00: 91 \\
00: 21\end{array}$ & $\begin{array}{l}76 / 71 / 21 \\
76 / 20 / 21 \\
76 / 02 / 90 \\
56 / 60 / 21 \\
56 / 62 / 90 \\
56 / 81 / 20 \\
26 / 22 / 11 \\
26 / 01 / 90 \\
26 / 52 / 20 \\
16 / 05 / 50 \\
69 / 90 / 90 \\
80 / 12 / 40 \\
29 / 80 / 50 \\
29 / 92 / 50 \\
99 / 60 / 01 \\
98 / 90 / 40 \\
59 / 81 / 80 \\
58 / 90 / 90 \\
58 / 52 / 40 \\
59 / 21 / 50 \\
58 / 20 / 50\end{array}$ & $25^{\circ} 1987$ & $20 * 7987$ & N & 78 & $z^{-19900}$ & T०LE95 & 2090 \\
\hline 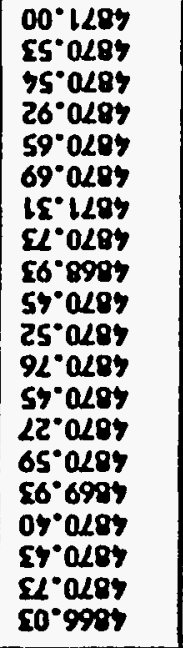 & 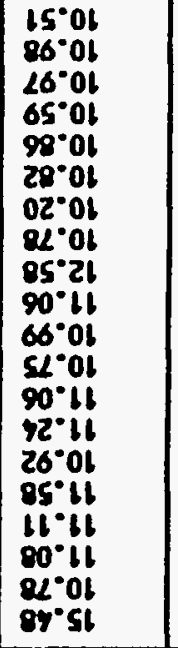 & 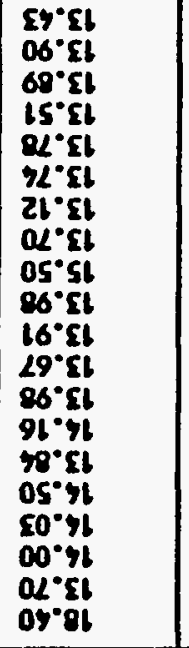 & $\begin{array}{l}20: 11 \\
77: 01 \\
05: 71 \\
27: 11 \\
65: 71 \\
52: 01 \\
62: 60 \\
07: 60 \\
25: 21 \\
00: 91 \\
50: 01 \\
5 y: 21 \\
00: 21 \\
00: 51 \\
01: 51 \\
80: 21 \\
00: 21 \\
00: 91 \\
00: 21 \\
00: 21\end{array}$ & $\begin{array}{l}56 / 61 / 70 \\
76 / 41 / 21 \\
76 / 20 / 21 \\
76 / 12 / 90 \\
56 / 60 / 21 \\
56 / 62 / 90 \\
56 / 81 / 20 \\
26 / 22 / 16 \\
26 / 16 / 80 \\
26 / 52 / 20 \\
16 / 15 / 50 \\
29 / 80 / 50 \\
29 / 92 / 50 \\
99 / 60 / 01 \\
99 / 20 / 90 \\
58 / 51 / 80 \\
59 / 70 / 90 \\
50 / 21 / 50 \\
58 / 20 / 50 \\
58 / 51 / 20\end{array}$ & $15^{\circ} 1087$ & 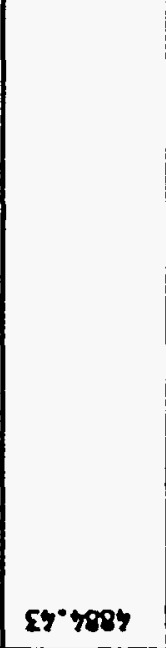 & n & ys & . & roosys & 1090 \\
\hline 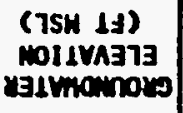 & 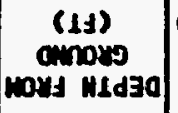 & 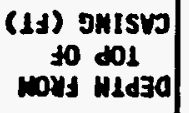 & $\begin{array}{r}3411 \\
907\end{array}$ & בLVa 901 & $\begin{array}{c}\text { (7SH 1f) } \\
\text { molivaj73 } \\
\text { ompors }\end{array}$ & 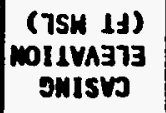 & $\begin{array}{l}3000 \\
\text { nots }\end{array}$ & 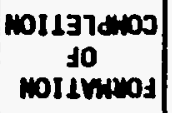 & $\begin{array}{c}\text { (Ld) } \\
\text { 3LYmiavo00 } \\
\text { LSV3 }\end{array}$ & 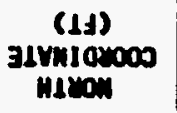 & at \\
\hline
\end{tabular}


- 
STATIC GROUMOUATER LEVELS

SITE: MONO1 MOMUENT VALLEY

REPORT DATE: 06/21/95

\begin{tabular}{|c|c|c|c|c|c|c|c|c|c|c|c|}
\hline$\underset{10}{\text { LOCATION }}$ & $\begin{array}{l}\text { month } \\
\text { coonoIKATE } \\
\text { (FT) }\end{array}$ & $\begin{array}{l}\text { EAST } \\
\text { COORDIMATE } \\
\text { (FT) }\end{array}$ & $\begin{array}{c}\text { FORMATION } \\
\text { OF } \\
\text { COMPLETION }\end{array}$ & $\begin{array}{l}\text { FLOW } \\
\text { CODE }\end{array}$ & $\begin{array}{l}\text { CASING } \\
\text { ELEVATION } \\
\text { (FT MSL) }\end{array}$ & $\begin{array}{l}\text { GROMW } \\
\text { ELEVATION } \\
\text { (FT HSL) }\end{array}$ & LOG DATE & $\begin{array}{l}\text { LOG } \\
\text { TIME }\end{array}$ & $\begin{array}{l}\text { DEPTH FROA } \\
\text { TOP OF } \\
\text { CASING (FT) }\end{array}$ & $\begin{array}{l}\text { DEPTH FRON } \\
\text { GROUID } \\
\text { (FT) }\end{array}$ & $\begin{array}{l}\text { GROUMDUATER } \\
\text { ELEVATION } \\
\text { (FT HSL) }\end{array}$ \\
\hline 0602 & 56377.4 & 88661.2 & $\mathbf{A L}$ & N & 4864.02 & 4861.53 & $04 / 19 / 95$ & $11: 03$ & 8.28 & 5.79 & 4855.74 \\
\hline 0603 & 57812.7 & 89036.9 & AL & C & 4848.56 & 4846.64 & $\begin{array}{l}02 / 15 / 85 \\
03 / 07 / 85 \\
03 / 12 / 85 \\
04 / 23 / 85 \\
06 / 04 / 85 \\
08 / 15 / 85 \\
04 / 06 / 86 \\
10 / 09 / 86 \\
03 / 26 / 87 \\
05 / 08 / 87 \\
04 / 22 / 88 \\
06 / 04 / 89 \\
05 / 31 / 91 \\
02 / 22 / 92 \\
08 / 10 / 92 \\
11 / 21 / 92 \\
02 / 18 / 93 \\
06 / 29 / 93 \\
12 / 09 / 93 \\
04 / 20 / 94 \\
12 / 07 / 94 \\
12 / 14 / 94 \\
04 / 19 / 95\end{array}$ & $\begin{array}{l}12: 00 \\
12: 00 \\
16: 00 \\
17: 46 \\
11: 45 \\
11: 26 \\
11: 46 \\
12: 50 \\
12: 00 \\
11: 35 \\
15: 00 \\
09: 24 \\
12: 26 \\
15: 00 \\
14: 51 \\
09: 50 \\
09: 53 \\
09: 07 \\
14: 24 \\
10: 03 \\
15: 54 \\
10: 37 \\
10: 52\end{array}$ & $\begin{array}{c}13.90 \\
10.20 \\
14.40 \\
10.25 \\
10.31 \\
10.88 \\
10.33 \\
10.37 \\
10.40 \\
9.96 \\
9.75 \\
10.38 \\
10.25 \\
10.46 \\
10.56 \\
10.05 \\
9.70 \\
10.13 \\
10.58 \\
10.03 \\
10.33 \\
10.35 \\
9.90\end{array}$ & $\begin{array}{r}11.98 \\
8.28 \\
12.48 \\
8.33 \\
8.39 \\
8.98 \\
8.41 \\
8.45 \\
8.48 \\
8.04 \\
7.83 \\
8.46 \\
8.33 \\
8.54 \\
8.64 \\
8.13 \\
7.78 \\
8.21 \\
8.66 \\
8.11 \\
8.61 \\
8.43 \\
7.98\end{array}$ & $\begin{array}{l}4834.66 \\
4838.36 \\
4834.16 \\
4838.31 \\
4838.25 \\
4837.68 \\
4838.23 \\
4838.19 \\
4838.16 \\
4838.60 \\
4838.81 \\
4838.18 \\
4838.31 \\
4838.10 \\
4838.00 \\
4838.51 \\
4838.86 \\
4838.43 \\
4837.98 \\
4838.53 \\
4838.23 \\
4838.21 \\
4838.66\end{array}$ \\
\hline 0604 & 58397.0 & 89424.6 & AL & c & 4839.09 & 4837.30 & $\begin{array}{l}02 / 15 / 85 \\
03 / 07 / 85 \\
03 / 12 / 85 \\
04 / 23 / 85 \\
06 / 04 / 85 \\
08 / 18 / 85 \\
04 / 06 / 86 \\
10 / 09 / 86 \\
03 / 26 / 87 \\
05 / 08 / 87 \\
06 / 05 / 89 \\
05 / 31 / 91 \\
02 / 22 / 92 \\
08 / 11 / 92 \\
11 / 19 / 92 \\
02 / 18 / 93\end{array}$ & $\begin{array}{l}12: 00 \\
12: 00 \\
16: 00 \\
11: 50 \\
11: 30 \\
17: 25 \\
10: 44 \\
11: 45 \\
12: 00 \\
13: 05 \\
10: 17 \\
13: 58 \\
16: 08 \\
16: 51 \\
14: 25 \\
10: 00\end{array}$ & $\begin{array}{r}12.20 \\
8.10 \\
8.20 \\
8.11 \\
8.26 \\
8.89 \\
8.09 \\
8.23 \\
7.80 \\
7.71 \\
8.19 \\
7.99 \\
8.09 \\
8.30 \\
7.75 \\
7.45\end{array}$ & $\begin{array}{c}10.41 \\
6.31 \\
6.41 \\
6.32 \\
6.47 \\
7.10 \\
6.30 \\
6.44 \\
6.01 \\
5.92 \\
6.40 \\
6.20 \\
6.30 \\
6.51 \\
5.96 \\
5.66\end{array}$ & $\begin{array}{l}4826.89 \\
4830.99 \\
4830.89 \\
4830.96 \\
4830.83 \\
4830.20 \\
4831.00 \\
4830.86 \\
4831.29 \\
4831.38 \\
4830.90 \\
4831.10 \\
4831.00 \\
4830.79 \\
4831.34 \\
4831.64\end{array}$ \\
\hline
\end{tabular}

FORHATIOH OF COMPLETION COOE

AL - ALLUVIUH
FLON RELATIONSHIP COOE

H - UHKHOWN

C - CROSS GRADIENT 
STATIC GROMDUATER LEVELS

SITE: MONO1 MONLNENT VAL

\begin{tabular}{|c|c|c|c|c|c|c|c|c|c|c|c|}
\hline$\underset{10}{\text { LOCATIOA }}$ & $\begin{array}{l}\text { MORTH } \\
\text { COORDIMATE } \\
\text { (FT) }\end{array}$ & $\begin{array}{l}\text { EAST } \\
\text { COORDINATE } \\
\text { (FT) }\end{array}$ & $\begin{array}{c}\text { FORMATION } \\
\text { OF } \\
\text { COMPLETION }\end{array}$ & $\begin{array}{l}\text { FLON } \\
\text { CODE }\end{array}$ & $\begin{array}{l}\text { CASIHG } \\
\text { ELEVATION } \\
\text { (FT MSL) }\end{array}$ & $\begin{array}{l}\text { GROUND } \\
\text { ELEVATION } \\
\text { (FT MSL) }\end{array}$ & LOG DATE & $\begin{array}{l}\text { LOS } \\
\text { TIME }\end{array}$ & $\begin{array}{l}\text { DEPTH FRON } \\
\text { TOP OF } \\
\text { CASIHG (FT) }\end{array}$ & $\begin{array}{l}\text { DEPTH FROM } \\
\text { GROUIND } \\
\text { (FT) }\end{array}$ & $\begin{array}{c}\text { GROUWDWATER } \\
\text { ELEVATION } \\
\text { (FT MSL) }\end{array}$ \\
\hline 0604 & 58397.0 & 89424.6 & AL. & C & 4839.09 & 4837.30 & $\begin{array}{l}06 / 29 / 93 \\
12 / 10 / 93 \\
04 / 20 / 94 \\
12 / 07 / 94 \\
12 / 14 / 94 \\
04 / 19 / 95\end{array}$ & $\begin{array}{l}16: 35 \\
09: 53 \\
09: 51 \\
14: 09 \\
10: 10 \\
10: 04\end{array}$ & $\begin{array}{l}7.67 \\
8.20 \\
7.72 \\
7.95 \\
7.94 \\
7.62\end{array}$ & $\begin{array}{l}5.88 \\
6.41 \\
5.93 \\
6.16 \\
6.15 \\
5.83\end{array}$ & $\begin{array}{l}4831.42 \\
4830.89 \\
4831.37 \\
4831.14 \\
4831.15 \\
4831.47\end{array}$ \\
\hline 0605 & 58707.6 & 90066.7 & $\dot{\mathbf{A L}}$ & D & 4833.96 & 4831.42 & $\begin{array}{l}02 / 15 / 85 \\
03 / 07 / 85 \\
03 / 12 / 85 \\
04 / 23 / 85 \\
06 / 04 / 85 \\
08 / 20 / 85 \\
04 / 05 / 86 \\
10 / 09 / 86 \\
03 / 26 / 87 \\
05 / 08 / 87 \\
04 / 21 / 88 \\
06 / 05 / 89 \\
12 / 04 / 89 \\
06 / 19 / 90 \\
01 / 25 / 91 \\
05 / 31 / 91 \\
08 / 15 / 92 \\
11 / 22 / 92 \\
02 / 18 / 93 \\
06 / 29 / 93 \\
12 / 10 / 93 \\
04 / 20 / 94 \\
12 / 07 / 94 \\
12 / 14 / 94 \\
04 / 19 / 95\end{array}$ & $\begin{array}{l}12: 00 \\
12: 00 \\
16: 00 \\
16: 45 \\
11: 35 \\
11: 30 \\
12: 55 \\
12: 05 \\
12: 00 \\
13: 10 \\
13: 30 \\
15: 36 \\
16: 00 \\
15: 30 \\
14: 05 \\
15: 30 \\
13: 50 \\
14: 15 \\
10: 30 \\
16: 41 \\
10: 02 \\
11: 29 \\
16: 10 \\
11: 12 \\
10: 15\end{array}$ & $\begin{array}{r}15.20 \\
9.70 \\
9.50 \\
9.63 \\
9.68 \\
10.53 \\
9.67 \\
10.12 \\
9.53 \\
9.35 \\
9.27 \\
9.91 \\
10.76 \\
10.30 \\
9.98 \\
9.80 \\
10.40 \\
9.65 \\
9.24 \\
9.10 \\
9.60 \\
9.18 \\
7.91 \\
9.76 \\
9.17\end{array}$ & $\begin{array}{r}12.66 \\
7.16 \\
6.96 \\
7.09 \\
7.14 \\
7.99 \\
7.13 \\
7.58 \\
6.99 \\
6.81 \\
6.73 \\
7.37 \\
8.22 \\
7.76 \\
7.44 \\
7.26 \\
7.86 \\
7.11 \\
6.70 \\
6.56 \\
7.06 \\
6.64 \\
5.37 \\
7.22 \\
6.63\end{array}$ & $\begin{array}{l}4818.76 \\
4824.26 \\
4824.46 \\
4824.33 \\
4824.28 \\
4823.43 \\
4824.29 \\
4823.84 \\
4824.43 \\
4824.61 \\
4824.69 \\
4824.05 \\
4823.20 \\
4823.66 \\
4823.98 \\
4824.16 \\
4823.56 \\
4824.31 \\
4824.72 \\
4824.86 \\
4824.36 \\
4824.78 \\
4826.05 \\
4824.20 \\
4824.79\end{array}$ \\
\hline 0606 & 59034.2 & 88634.0 & AL & D & 4863.31 & 4860.44 & $\begin{array}{l}03 / 07 / 85 \\
03 / 12 / 85 \\
04 / 23 / 85 \\
06 / 04 / 85 \\
04 / 05 / 86 \\
10 / 09 / 86 \\
03 / 26 / 87 \\
05 / 08 / 87 \\
04 / 22 / 88\end{array}$ & $\begin{array}{l}12: 00 \\
16: 00 \\
16: 36 \\
11: 20 \\
15: 15 \\
17: 00 \\
12: 00 \\
10: 50 \\
13: 45\end{array}$ & $\begin{array}{l}35.20 \\
35.40 \\
35.37 \\
35.39 \\
34.95 \\
35.18 \\
34.89 \\
34.82 \\
34.61\end{array}$ & $\begin{array}{l}32.33 \\
32.53 \\
32.50 \\
32.52 \\
32.08 \\
32.31 \\
32.02 \\
31.95 \\
31.74\end{array}$ & $\begin{array}{l}4828.11 \\
4827.91 \\
4827.94 \\
4827.92 \\
4828.36 \\
4828.13 \\
4828.42 \\
4828.49 \\
4828.70\end{array}$ \\
\hline
\end{tabular}

FORMATION OF COMPLETION COOE: AL - ALLUVIUA
FLON RELATIOHSHIP CODE:

C - CROSS GRADIENT 
STATIC GROMNOUATER LEVELS

SITE: MONO1 MOMUNENT VALLEY

REPORT DATE: 06/21/95

\begin{tabular}{|c|c|c|c|c|c|c|c|c|c|c|c|}
\hline$\underset{\text { ID }}{\text { Location }}$ & $\begin{array}{l}\text { MORTH } \\
\text { COORDINATE } \\
\text { (FT) }\end{array}$ & $\begin{array}{c}\text { EAST } \\
\text { COORDINATE } \\
\text { (FT) }\end{array}$ & $\begin{array}{c}\text { Fonintion } \\
\text { of } \\
\text { COnPLETION }\end{array}$ & $\begin{array}{l}\text { FLON } \\
\text { CODE }\end{array}$ & $\begin{array}{l}\text { CASING } \\
\text { ELEVATION } \\
\text { (FT MSL) }\end{array}$ & $\begin{array}{l}\text { gRoum } \\
\text { ELEVATION } \\
\text { (FT MSL) }\end{array}$ & LOG DATE & $\begin{array}{l}\text { LOG } \\
\text { TIME }\end{array}$ & $\begin{array}{l}\text { OEPTH FROH } \\
\text { TOP OF } \\
\text { CASING (FT) }\end{array}$ & $\begin{array}{c}\text { DEPTH FROH } \\
\text { GROUWD } \\
\text { (FT) }\end{array}$ & $\begin{array}{l}\text { GROUDHATER } \\
\text { ELEYATION } \\
\text { (FT MSL.) }\end{array}$ \\
\hline 0606 & 59034.2 & 88634.0 & AL & D & 4863.31 & 4860.44 & $\begin{array}{l}06 / 07 / 89 \\
12 / 01 / 89 \\
06 / 23 / 90 \\
01 / 27 / 91 \\
06 / 01 / 91 \\
02 / 21 / 92 \\
08 / 11 / 92 \\
11 / 24 / 92 \\
02 / 18 / 93 \\
06 / 29 / 93 \\
12 / 09 / 93 \\
04 / 20 / 94 \\
12 / 08 / 94 \\
12 / 14 / 94 \\
04 / 19 / 95\end{array}$ & $\begin{array}{l}15: 55 \\
15: 10 \\
12: 25 \\
13: 15 \\
09: 26 \\
08: 20 \\
15: 07 \\
09: 00 \\
12: 20 \\
17: 31 \\
14: 13 \\
09: 43 \\
15: 09 \\
10: 27 \\
11: 56\end{array}$ & $\begin{array}{l}34.08 \\
36.20 \\
35.11 \\
34.74 \\
34.96 \\
35.06 \\
35.0 \\
34.62 \\
34.73 \\
34.50 \\
35.12 \\
34.76 \\
34.81 \\
34.80 \\
34.62\end{array}$ & $\begin{array}{l}31.21 \\
33.33 \\
32.24 \\
31.87 \\
32.09 \\
32.19 \\
32.13 \\
31.75 \\
31.86 \\
31.63 \\
32.25 \\
31.89 \\
31.94 \\
31.93 \\
31.75\end{array}$ & $\begin{array}{l}4829.23 \\
4827.11 \\
4828.20 \\
4828.57 \\
4828.35 \\
4828.25 \\
4828.31 \\
4828.69 \\
4828.58 \\
4828.81 \\
4828.19 \\
4828.55 \\
4828.50 \\
4828.51 \\
4828.69\end{array}$ \\
\hline 0607 & 59657.2 & 87519.2 & SR & D & 4871.26 & 4869.41 & $\begin{array}{l}02 / 15 / 85 \\
03 / 07 / 85 \\
03 / 12 / 85 \\
04 / 23 / 85 \\
06 / 04 / 85 \\
04 / 06 / 86 \\
03 / 26 / 87 \\
05 / 08 / 87 \\
06 / 06 / 89 \\
11 / 23 / 92 \\
06 / 29 / 93 \\
12 / 09 / 93 \\
04 / 20 / 94 \\
12 / 07 / 94 \\
12 / 14 / 94 \\
04 / 19 / 95\end{array}$ & $\begin{array}{l}12: 00 \\
12: 00 \\
16: 00 \\
16: 08 \\
09: 30 \\
12: 30 \\
12: 00 \\
09: 30 \\
13: 00 \\
09: 25 \\
09: 54 \\
12: 08 \\
08: 21 \\
12: 48 \\
11: 50 \\
12: 21\end{array}$ & $\begin{array}{l}\text { DRY } \\
\text { DRY } \\
\text { ORY } \\
\text { DRY } \\
\text { DRY } \\
\text { DRY } \\
\text { DRY } \\
\text { DRY } \\
\text { DRY } \\
\text { ORY } \\
\text { DRY } \\
\text { ORY } \\
\text { ORY } \\
\text { DRY } \\
\text { DRY } \\
\text { DRY }\end{array}$ & $\begin{array}{l}\text { DRY } \\
\text { DRY } \\
\text { ORY } \\
\text { DRY } \\
\text { ORY } \\
\text { ORY } \\
\text { DRY } \\
\text { ORY } \\
\text { DRY } \\
\text { DRY } \\
\text { ORY } \\
\text { DRY } \\
\text { DRY } \\
\text { DRY } \\
\text { DRY } \\
\text { DRY }\end{array}$ & $\begin{array}{l}: \\
: \\
: \\
: \\
: \\
: \\
: \\
: \\
:\end{array}$ \\
\hline 0608 & 58685.7 & 87189.4 & DC & 0 & 4903.11 & 4901.08 & $\begin{array}{l}04 / 23 / 85 \\
06 / 04 / 85 \\
08 / 16 / 85 \\
04 / 07 / 86 \\
10 / 09 / 86 \\
03 / 26 / 87 \\
05 / 08 / 87\end{array}$ & $\begin{array}{l}16: 31 \\
09: 45 \\
18: 30 \\
15: 00 \\
14: 55 \\
12: 00 \\
10: 05\end{array}$ & $\begin{array}{l}58.38 \\
57.80 \\
58.00 \\
57.62 \\
57.25 \\
57.64 \\
57.44\end{array}$ & $\begin{array}{l}56.35 \\
55.77 \\
55.97 \\
55.59 \\
55.22 \\
55.61 \\
55.41\end{array}$ & $\begin{array}{l}4844.73 \\
4845.31 \\
4845.11 \\
4845.49 \\
4845.86 \\
4845.47 \\
4845.67\end{array}$ \\
\hline 0609 & 59052.1 & 87652.9 & SR & 0 & 4880.17 & 4878.30 & $02 / 15 / 85$ & $12: 00$ & DRY & DRY & - \\
\hline
\end{tabular}

FORMATION OF COAPLETION CODE:

AL - ALLUVIUN
SR - SHIHARUHP MEMBER OF THE CHIHLE FORMATIOH

FLOW RELATIONSHIP COOE:

DC - DECHELLEY MEMBER OF THE CUTLER FORMATION 
STATIC GROUMOUATER LEVELS

SITE: MOHO1 MOMUEEUT VALLY

REPORT DATE: 06/21/95

\begin{tabular}{|c|c|c|c|c|c|c|c|c|c|c|c|}
\hline$\underset{10}{\text { Location }}$ & $\begin{array}{c}\text { MORTH } \\
\text { COORDIMATE } \\
\text { (FT) }\end{array}$ & $\begin{array}{l}\text { EAST } \\
\text { COORD IMATE } \\
\text { (FT) }\end{array}$ & $\mid \begin{array}{c}\text { FORMATION } \\
\text { OF } \\
\text { COMPLETION }\end{array}$ & $\begin{array}{l}\text { FLON } \\
\text { COOE }\end{array}$ & $\begin{array}{l}\text { CASING } \\
\text { ELEVATION } \\
\text { (FT MSL) }\end{array}$ & $\begin{array}{l}\text { GROUmD } \\
\text { ELEVATION } \\
\text { (FT MSL) }\end{array}$ & LOG DATE & $\begin{array}{l}\text { LOG } \\
\text { TIME }\end{array}$ & $\begin{array}{c}\text { DEPTH FROA } \\
\text { TOP OF } \\
\text { CASING (FT) }\end{array}$ & \begin{tabular}{|} 
DEPTH FROM \\
GROMND \\
(FT)
\end{tabular} & $\begin{array}{l}\text { GROUMDUATER } \\
\text { ELEVATION } \\
\text { (FT MSL) }\end{array}$ \\
\hline 0609 & 59052.1 & 87652.9 & SR & 0 & 4880.17 & 4878.30 & $\begin{array}{l}03 / 07 / 85 \\
03 / 12 / 85 \\
04 / 23 / 85 \\
06 / 04 / 85 \\
04 / 06 / 86 \\
03 / 26 / 87 \\
05 / 08 / 87 \\
06 / 06 / 89 \\
06 / 29 / 93 \\
12 / 09 / 93 \\
04 / 20 / 94 \\
12 / 07 / 94 \\
12 / 14 / 94 \\
04 / 19 / 95\end{array}$ & $\begin{array}{l}12: 00 \\
16: 00 \\
16: 14 \\
09: 40 \\
12: 40 \\
12: 00 \\
10: 00 \\
13: 30 \\
10: 06 \\
11: 07 \\
08: 40 \\
13: 02 \\
11: 57 \\
12: 35\end{array}$ & $\begin{array}{l}\text { DRY } \\
\text { ORY } \\
\text { DRY } \\
\text { DRY } \\
\text { ORY } \\
\text { DRY } \\
\text { DRY } \\
\text { ORY } \\
\text { DRY } \\
\text { DRY } \\
\text { DRY } \\
\text { DRY } \\
\text { ORY } \\
\text { ORY }\end{array}$ & $\begin{array}{l}\text { DRY } \\
\text { DRY } \\
\text { DRY } \\
\text { DRY } \\
\text { DRY } \\
\text { DRY } \\
\text { DRY } \\
\text { DRY } \\
\text { ORY } \\
\text { DRY } \\
\text { ORY } \\
\text { DRY } \\
\text { DRY } \\
\text { DRY }\end{array}$ & $\begin{array}{l}: \\
: \\
: \\
: \\
: \\
: \\
: \\
:\end{array}$ \\
\hline 0610 & 56338.8 & 88611.6 & SR & N & 4861.81 & 4860.83 & $\begin{array}{l}02 / 15 / 85 \\
03 / 07 / 85 \\
03 / 12 / 85 \\
04 / 23 / 85 \\
06 / 04 / 85 \\
08 / 18 / 85 \\
04 / 06 / 86 \\
10 / 09 / 86 \\
02 / 18 / 93 \\
04 / 20 / 94 \\
12 / 07 / 94 \\
12 / 14 / 94 \\
04 / 19 / 95\end{array}$ & $\begin{array}{l}12: 00 \\
12: 00 \\
16: 00 \\
08: 30 \\
12: 12 \\
17: 04 \\
11: 59 \\
12: 55 \\
09: 45 \\
10: 12 \\
14: 40 \\
10: 40 \\
11: 01\end{array}$ & $\begin{array}{l}\text { DRY } \\
\text { DRY } \\
\text { ORY } \\
5.39 \\
5.52 \\
6.32 \\
5.40 \\
5.87 \\
\text { DRY } \\
\text { DRY } \\
\text { DRY } \\
\text { DRY } \\
\text { DRY }\end{array}$ & $\begin{array}{l}\text { DRY } \\
\text { DRY } \\
\text { DRY } \\
4.41 \\
4.54 \\
5.34 \\
4.42 \\
4.89 \\
\text { DRY } \\
\text { DRY } \\
\text { DRY } \\
\text { DRY } \\
\text { DRY }\end{array}$ & $\begin{array}{c}: \\
4856.42 \\
4856.29 \\
4855.49 \\
4856.41 \\
4855.94 \\
- \\
- \\
- \\
-\end{array}$ \\
\hline 0611 & 57811.0 & 89017.5 & DC & $N$ & 4848.35 & 4846.35 & $\begin{array}{l}03 / 07 / 85 \\
03 / 12 / 85 \\
04 / 23 / 85 \\
06 / 04 / 85 \\
03 / 26 / 87 \\
05 / 08 / 87 \\
06 / 07 / 89 \\
08 / 15 / 92 \\
11 / 23 / 92 \\
06 / 29 / 93 \\
12 / 09 / 93 \\
04 / 20 / 94 \\
12 / 07 / 94\end{array}$ & $\begin{array}{l}12: 00 \\
16: 00 \\
17: 50 \\
12: 20 \\
12: 00 \\
11: 35 \\
12: 45 \\
10: 56 \\
12: 50 \\
06: 38 \\
10: 37 \\
10: 05 \\
15: 56\end{array}$ & $\begin{array}{l}\text { FLOWING } \\
\text { FLONING } \\
\text { FLONING } \\
\text { FLOWING } \\
\text { FLOWING } \\
\text { FLOHING } \\
10.71 \\
10.98 \\
10.40 \\
10.62 \\
10.98 \\
10.44 \\
10.70\end{array}$ & $\begin{array}{r}\text { FLONING } \\
\text { FLONING } \\
\text { FLONING } \\
\text { FLOWING } \\
\text { FLONING } \\
\text { FLOWING } \\
8.71 \\
8.98 \\
8.40 \\
8.62 \\
8.98 \\
8.44 \\
8.70\end{array}$ & $\begin{array}{c}- \\
: \\
: \\
: \\
4837.64 \\
4837.37 \\
4837.95 \\
4837.73 \\
4837.37 \\
4837.91 \\
4837.65\end{array}$ \\
\hline
\end{tabular}

FORMATION OF COMPLETION COOE:

SR - SHINARUMP MENBER OF THE CHIHLE FORMATIO

DC - DECHELLEY MEMBER OF THE CUTLER FORMATION
FLOU RELATIOHSHIP COOE:

o - ON-SITE 


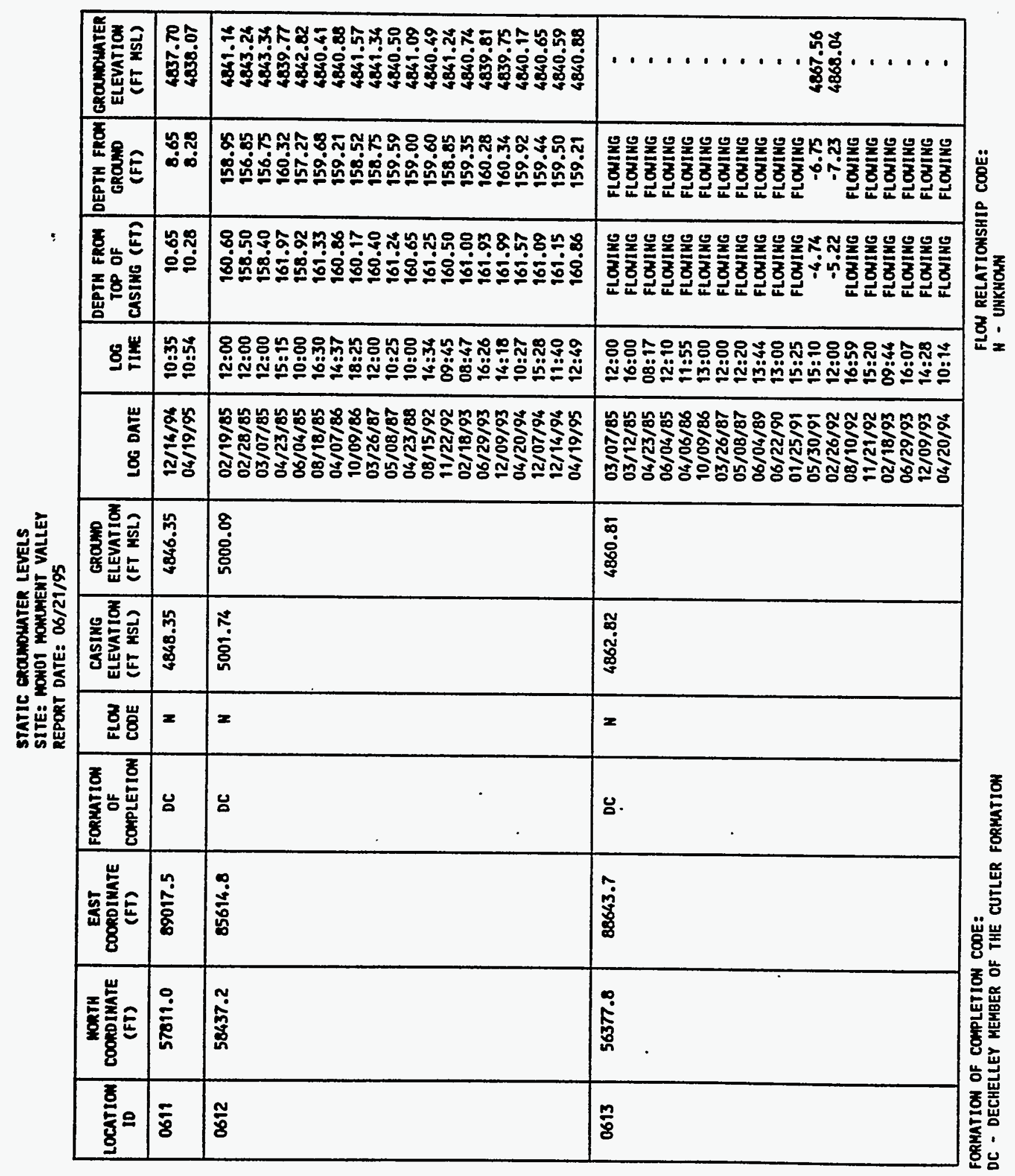


STATIC GRONDUATER LEVELS

SITE: HONOI MOMUNENT VALLEY

REPORT DATE: 06/21/95

\begin{tabular}{|c|c|c|c|c|c|c|c|c|c|c|c|}
\hline$\underset{\text { ID }}{\text { LOcation }}$ & $\begin{array}{l}\text { MORTH } \\
\text { COORDIHATE } \\
\text { (FT) }\end{array}$ & $\begin{array}{l}\text { EAST } \\
\text { COORDIMATE } \\
\text { (FT) }\end{array}$ & $\begin{array}{l}\text { FORMATION } \\
\text { OF } \\
\text { COMPLETION }\end{array}$ & $\begin{array}{l}\text { FLON } \\
\text { COOE }\end{array}$ & $\begin{array}{l}\text { CASING } \\
\text { ELEVATION } \\
\text { (FT HSL) }\end{array}$ & $\begin{array}{l}\text { GROUMD } \\
\text { ELEVATION } \\
\text { (FT RSL) }\end{array}$ & LOG DATE & $\begin{array}{l}\text { LOG } \\
\text { TIME }\end{array}$ & $\begin{array}{l}\text { DEPTH FROM } \\
\text { TOP. OF } \\
\text { CASIMG (FT) }\end{array}$ & $\begin{array}{c}\text { DEPTH FRON } \\
\text { GROUND } \\
\text { (FT) }\end{array}$ & $\begin{array}{l}\text { GROQWDUMATER } \\
\text { ELEVATION } \\
\text { (FT MSL) }\end{array}$ \\
\hline 0613 & 56377.8 & 88643.7 & OC & N & 4862.82 & 4860.81 & $\begin{array}{l}12 / 07 / 94 \\
12 / 14 / 94 \\
04 / 19 / 95\end{array}$ & $\begin{array}{l}16: 41 \\
10: 34 \\
11: 00\end{array}$ & $\begin{array}{l}\text { FLOWIMG } \\
\text { FLOWING } \\
\text { FLOWIMG }\end{array}$ & $\begin{array}{l}\text { FLOUING } \\
\text { FLOWING } \\
\text { FLONING }\end{array}$ & 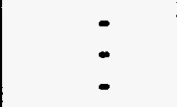 \\
\hline 0614 & 60940.5 & 87832.6 & SR & D & 4855.66 & 4854.28 & $\begin{array}{l}03 / 07 / 85 \\
03 / 12 / 85 \\
04 / 23 / 85 \\
06 / 04 / 85 \\
08 / 19 / 85 \\
04 / 05 / 86 \\
10 / 09 / 86 \\
03 / 26 / 87 \\
05 / 08 / 87 \\
04 / 19 / 88 \\
06 / 07 / 89 \\
12 / 02 / 89 \\
06 / 23 / 90 \\
01 / 28 / 91 \\
06 / 01 / 91 \\
08 / 10 / 92 \\
11 / 24 / 92 \\
02 / 18 / 93 \\
06 / 29 / 93 \\
12 / 09 / 93 \\
04 / 20 / 94 \\
12 / 07 / 94 \\
12 / 14 / 94 \\
04 / 19 / 95\end{array}$ & $\begin{array}{l}12: 00 \\
16: 00 \\
15: 01 \\
09: 15 \\
12: 50 \\
10: 15 \\
16: 00 \\
12: 00 \\
13: 55 \\
15: 20 \\
10: 00 \\
16: 00 \\
15: 00 \\
14: 15 \\
12: 13 \\
12: 54 \\
12: 40 \\
11: 44 \\
17: 53 \\
13: 48 \\
09: 16 \\
15: 43 \\
09: 50 \\
09: 21\end{array}$ & $\begin{array}{l}48.30 \\
48.50 \\
49.90 \\
49.61 \\
49.59 \\
49.67 \\
50.08 \\
50.24 \\
49.64 \\
49.35 \\
49.40 \\
50.16 \\
49.58 \\
49.26 \\
49.68 \\
49.70 \\
49.43 \\
48.88 \\
48.40 \\
48.89 \\
49.07 \\
49.28 \\
49.34 \\
49.26\end{array}$ & $\begin{array}{l}46.92 \\
47.12 \\
48.52 \\
48.23 \\
48.21 \\
48.29 \\
48.70 \\
48.86 \\
48.26 \\
47.97 \\
48.02 \\
48.78 \\
48.20 \\
47.88 \\
48.30 \\
48.32 \\
48.05 \\
47.50 \\
47.02 \\
47.51 \\
47.69 \\
47.90 \\
47.96 \\
47.88\end{array}$ & $\begin{array}{l}4807.36 \\
4807.16 \\
4805.76 \\
4806.05 \\
4806.07 \\
4805.99 \\
4805.58 \\
4805.42 \\
4806.02 \\
4806.31 \\
4806.26 \\
4805.50 \\
4806.08 \\
4806.40 \\
4805.98 \\
4805.96 \\
4806.23 \\
4806.78 \\
4807.26 \\
4806.77 \\
4806.59 \\
4806.38 \\
4806.32 \\
4806.40\end{array}$ \\
\hline 0615 & 57794.4 & 88980.7 & SR & C & 4849.29 & 4847.50 & $\begin{array}{l}04 / 23 / 85 \\
06 / 04 / 85 \\
08 / 15 / 85 \\
04 / 06 / 86 \\
10 / 09 / 86 \\
03 / 26 / 87 \\
05 / 08 / 87 \\
06 / 08 / 89 \\
08 / 15 / 92 \\
11 / 23 / 92 \\
06 / 29 / 93 \\
12 / 09 / 93\end{array}$ & $\begin{array}{l}17: 55 \\
11: 50 \\
16: 30 \\
11: 38 \\
12: 45 \\
12: 00 \\
11: 40 \\
09: 20 \\
11: 06 \\
12: 25 \\
07: 55 \\
10: 41\end{array}$ & $\begin{array}{r}10.67 \\
10.71 \\
11.25 \\
10.71 \\
10.76 \\
11.06 \\
10.33 \\
\text { FLONING } \\
\text { FLOUING } \\
\text { FLOUIHG } \\
\text { FLOWING } \\
\text { FLONING }\end{array}$ & $\begin{array}{r}8.88 \\
8.92 \\
9.46 \\
8.92 \\
8.97 \\
9.27 \\
8.54 \\
\text { FLOUING } \\
\text { FLOUING } \\
\text { FLOUING } \\
\text { FLOWING } \\
\text { FLOUING }\end{array}$ & $\begin{array}{c}4838.62 \\
4838.58 \\
4838.04 \\
4838.58 \\
4838.53 \\
4838.23 \\
4838.96 \\
- \\
- \\
- \\
-\end{array}$ \\
\hline
\end{tabular}

FORMATION OF COMPLETION COOE:

DC - DECHELLEY KEMBER OF THE CUTLER FORMATION

SR - SHIMARUMP MEMBER OF THE CHIMLE FORMATION
FLOU RELATIONSHIP COOE:

N - UNKHONH

D - DOWN GRADIENT

C - CROSS GRADIENT 
STATIC GHONDMATER LEVELS

SITE: MOHOI MOMUNENT VALLEY

REPORT DATE: 06/21/9S

\begin{tabular}{|c|c|c|c|c|c|c|c|c|c|c|c|}
\hline$\underset{\text { ID }}{\text { LOCATION }}$ & $\begin{array}{l}\text { Month } \\
\text { coorotuAte } \\
\text { (FT) }\end{array}$ & $\begin{array}{l}\text { EAST } \\
\text { COORDIKATE } \\
\text { (FT) }\end{array}$ & $\begin{array}{l}\text { FORHATION } \\
\text { OF } \\
\text { COAPLETION }\end{array}$ & $\begin{array}{l}\text { FLOA } \\
\text { CODE }\end{array}$ & $\begin{array}{l}\text { CASING } \\
\text { ELEVATION } \\
\text { (FT MSL) }\end{array}$ & $\begin{array}{l}\text { GROUWD } \\
\text { ELEVATION } \\
\text { (FT MSL) }\end{array}$ & LOS DATE & $\begin{array}{l}\text { LOG } \\
\text { TIME }\end{array}$ & $\begin{array}{l}\text { DEPTH FROM } \\
\text { TOP OF } \\
\text { CASING (FT) }\end{array}$ & $\begin{array}{c}\text { DEPTH FRON } \\
\text { GROWID } \\
\text { (FT) }\end{array}$ & $\begin{array}{l}\text { CROWWOHATER } \\
\text { ELEVATION } \\
\text { (FT MSL) }\end{array}$ \\
\hline 0615 & 57794.4 & 88980.7 & SR & C & 4849.29 & 4847.50 & $\begin{array}{l}04 / 20 / 94 \\
12 / 07 / 94 \\
12 / 14 / 96 \\
04 / 19 / 95\end{array}$ & $\begin{array}{l}10: 01 \\
15: 57 \\
10: 33 \\
10: 56\end{array}$ & $\begin{array}{l}\text { FLOUING } \\
\text { FLOWING } \\
\text { FLONING } \\
\text { FLOHING }\end{array}$ & $\begin{array}{l}\text { FLONING } \\
\text { FLONING } \\
\text { FLONIHG } \\
\text { FLOUING }\end{array}$ & $\begin{array}{l}- \\
- \\
-\end{array}$ \\
\hline 0616 & 56747.3 & 87987.6 & $\mathbf{A L}$ & $N$ & 4869.92 & 4869.00 & $04 / 19 / 95$ & $11: 45$ & 8.65 & 7.73 & 4861.27 \\
\hline 0618 & 58868.3 & 87016.1 & DC & 0 & 4921.02 & 4920.00 & $\begin{array}{l}04 / 23 / 85 \\
06 / 04 / 85 \\
04 / 07 / 86 \\
10 / 09 / 86 \\
03 / 26 / 87 \\
12 / 09 / 93 \\
04 / 20 / 94 \\
12 / 07 / 94\end{array}$ & $\begin{array}{l}15: 55 \\
11: 10 \\
14: 27 \\
18: 15 \\
12: 00 \\
11: 30 \\
08: 49 \\
13: 30\end{array}$ & $\begin{array}{l}60.21 \\
89.56 \\
88.15 \\
89.14 \\
93.39 \\
91.0 \\
90.10 \\
89.63\end{array}$ & $\begin{array}{l}59.19 \\
88.54 \\
87.13 \\
88.12 \\
92.37 \\
89.98 \\
89.08 \\
88.61\end{array}$ & $\begin{array}{l}4860.81 \\
4031.46 \\
4032.87 \\
4831.86 \\
4027.63 \\
4830.02 \\
4030.92 \\
4031.39\end{array}$ \\
\hline 0619 & 58876.5 & 87587.9 & DC & 0 & 4887.42 & 4886.52 & $\begin{array}{l}02 / 15 / 85 \\
03 / 07 / 85 \\
03 / 12 / 85 \\
06 / 04 / 85 \\
04 / 07 / 86 \\
10 / 09 / 86 \\
03 / 26 / 87 \\
05 / 08 / 87 \\
04 / 20 / 94 \\
12 / 07 / 94 \\
04 / 26 / 95\end{array}$ & $\begin{array}{l}12: 00 \\
12: 00 \\
16: 00 \\
09: 43 \\
14: 58 \\
14: 00 \\
12: 00 \\
14: 35 \\
08: 45 \\
13: 14 \\
14: 00\end{array}$ & $\begin{array}{l}57.90 \\
55.40 \\
55.60 \\
55.49 \\
55.24 \\
54.90 \\
55.29 \\
54.20 \\
54.6 \\
56.54 \\
56.46\end{array}$ & $\begin{array}{l}57.00 \\
54.50 \\
54.70 \\
54.59 \\
54.34 \\
54.00 \\
54.39 \\
53.30 \\
53.70 \\
55.64 \\
55.56\end{array}$ & $\begin{array}{l}4829.52 \\
4832.02 \\
4831.82 \\
4831.93 \\
4832.18 \\
4832.52 \\
4832.13 \\
4833.22 \\
4832.82 \\
4830.88 \\
4830.96\end{array}$ \\
\hline 0625 & 58272.5 & 89803.1 & DC & C & 4840.09 & 4838.80 & $\begin{array}{l}02 / 15 / 85 \\
03 / 07 / 85 \\
03 / 12 / 85 \\
04 / 23 / 85 \\
06 / 04 / 85 \\
04 / 23 / 86 \\
10 / 09 / 86 \\
03 / 26 / 87 \\
05 / 08 / 87 \\
02 / 26 / 92 \\
08 / 15 / 92 \\
11 / 21 / 92 \\
02 / 18 / 93\end{array}$ & $\begin{array}{l}12: 00 \\
12: 00 \\
16: 00 \\
16: 58 \\
11: 35 \\
10: 30 \\
12: 00 \\
12: 00 \\
14: 26 \\
13: 15 \\
13: 45 \\
14: 10 \\
10: 15\end{array}$ & $\begin{array}{r}\text { FLONING } \\
\text { FLONING } \\
\text { FLONING } \\
0.62 \\
0.63 \\
0.54 \\
1.37 \\
0.59 \\
0.62 \\
\text { FLOHING } \\
1.35 \\
\text { FLONING } \\
\text { FLOMING }\end{array}$ & $\begin{array}{r}\text { FLOUING } \\
\text { FLOWIHG } \\
\text { FLOWING } \\
-0.67 \\
-0.66 \\
-0.75 \\
0.08 \\
-0.70 \\
-0.67 \\
\text { FLOWING } \\
0.06 \\
\text { FLOUING } \\
\text { FLOWING }\end{array}$ & $\begin{array}{c}- \\
- \\
4839.47 \\
4839.46 \\
4839.55 \\
4838.72 \\
4839.50 \\
4839.47 \\
4838.74 \\
- \\
-\end{array}$ \\
\hline
\end{tabular}

FORMATION OF COMPLETION COOE:

SR - SHIMARUMP MEMBER OF THE CHIMLE FORMATIOH

AL. - ALLUVIUM

DC - DECHELLEY MEMBER OF tHE CUTLER fORMATION
FLOU RELATIONSHIP CODE:

C - CROSS GRADIENT

H - UNKHOWN
O - OH-SITE 
STATIC GROUMDHATER LEVELS

SITE: MONO1 MOWUWENT VALLEY

REPORT DATE: 06/21/95

\begin{tabular}{|c|c|c|c|c|c|c|c|c|c|c|c|}
\hline$\underset{10}{\operatorname{Lochrion}}$ & $\begin{array}{l}\text { MONTH } \\
\text { COORDIMATE } \\
\text { (FT) }\end{array}$ & $\begin{array}{c}\text { EAst } \\
\text { CoonoINATE } \\
\text { (FT) }\end{array}$ & $\left|\begin{array}{c}\text { FOARATION } \\
\text { OF } \\
\text { COMPLETION }\end{array}\right|$ & $\begin{array}{l}\text { FLON } \\
\text { CODE }\end{array}$ & $\begin{array}{l}\text { CASING } \\
\text { ELEVATION } \\
\text { (FT MSL) }\end{array}$ & $\begin{array}{l}\text { GROUND } \\
\text { ELEVATION } \\
\text { (FT MSL) }\end{array}$ & LOG DATE & $\begin{array}{l}\text { LOG } \\
\text { TIME }\end{array}$ & $\begin{array}{l}\text { DEPTH FROA } \\
\text { TOP OF } \\
\text { CASING (FT) }\end{array}$ & \begin{tabular}{|} 
DEPTH FRON \\
GROUND \\
(FT)
\end{tabular} & $\begin{array}{l}\text { GRONWDWATER } \\
\text { ELEVATION } \\
\text { (FT MSL) }\end{array}$ \\
\hline 0625 & 58272.5 & 89803.1 & DC & c & 4840.09 & 4838.80 & $04 / 20 / 94$ & $09: 57$ & FLOUIMG & flonIng & - \\
\hline 0650 & 64970.2 & 89923.8 & AL & D & 4793.29 & 4790.15 & $\begin{array}{l}10 / 19 / 85 \\
04 / 29 / 86 \\
03 / 26 / 87 \\
05 / 08 / 87 \\
04 / 25 / 88 \\
06 / 05 / 89 \\
06 / 03 / 91 \\
02 / 25 / 92 \\
08 / 13 / 92 \\
11 / 21 / 92 \\
02 / 18 / 93 \\
12 / 10 / 93 \\
04 / 20 / 94 \\
12 / 14 / 94 \\
04 / 19 / 95\end{array}$ & $\begin{array}{l}12: 00 \\
09: 00 \\
12: 00 \\
13: 50 \\
08: 30 \\
08: 56 \\
20: 13 \\
12: 10 \\
13: 49 \\
16: 45 \\
11: 34 \\
09: 08 \\
18: 21 \\
09: 39 \\
09: 07\end{array}$ & $\begin{array}{l}19.34 \\
19.15 \\
19.87 \\
19.05 \\
18.91 \\
19.30 \\
19.26 \\
19.43 \\
19.57 \\
19.37 \\
19.10 \\
18.82 \\
18.75 \\
19.16 \\
18.92\end{array}$ & $\begin{array}{l}16.20 \\
16.01 \\
16.73 \\
15.91 \\
15.77 \\
16.16 \\
16.12 \\
16.29 \\
16.43 \\
16.23 \\
15.96 \\
15.68 \\
15.61 \\
16.02 \\
15.78\end{array}$ & $\begin{array}{l}4773.95 \\
4774.14 \\
4773.42 \\
4774.24 \\
4774.38 \\
4773.99 \\
4774.03 \\
4773.86 \\
4773.72 \\
4773.92 \\
4774.19 \\
4774.47 \\
4774.54 \\
4774.13 \\
4774.37\end{array}$ \\
\hline 0651 & 63788.9 & 92734.3 & AL & D & 4784.51 & 4781.46 & $\begin{array}{l}10 / 19 / 85 \\
04 / 29 / 86 \\
10 / 09 / 86 \\
03 / 26 / 87 \\
05 / 08 / 87 \\
04 / 19 / 88 \\
06 / 03 / 89 \\
12 / 03 / 89 \\
06 / 21 / 90 \\
01 / 26 / 91 \\
06 / 02 / 91 \\
02 / 25 / 92 \\
08 / 13 / 92 \\
11 / 21 / 92 \\
02 / 18 / 93 \\
06 / 29 / 93 \\
12 / 10 / 93 \\
04 / 20 / 94 \\
12 / 14 / 94 \\
04 / 19 / 95\end{array}$ & $\begin{array}{l}12: 00 \\
13: 00 \\
17: 45 \\
12: 00 \\
13: 35 \\
12: 00 \\
08: 30 \\
09: 15 \\
15: 44 \\
14: 15 \\
09: 01 \\
12: 35 \\
09: 23 \\
09: 25 \\
10: 54 \\
17: 09 \\
11: 08 \\
11: 58 \\
09: 01 \\
10: 37\end{array}$ & $\begin{array}{l}5.70 \\
8.11 \\
8.62 \\
8.87 \\
7.98 \\
7.54 \\
8.30 \\
8.81 \\
5.50 \\
8.10 \\
8.14 \\
8.05 \\
8.80 \\
8.36 \\
7.44 \\
8.19 \\
8.14 \\
7.77 \\
8.17 \\
7.79\end{array}$ & $\begin{array}{l}2.65 \\
5.06 \\
5.57 \\
5.82 \\
4.93 \\
4.49 \\
5.25 \\
5.76 \\
2.45 \\
5.05 \\
5.09 \\
5.00 \\
5.75 \\
5.31 \\
4.39 \\
5.14 \\
5.09 \\
4.72 \\
5.12 \\
4.74\end{array}$ & $\begin{array}{l}4776.81 \\
4776.40 \\
4775.89 \\
4775.64 \\
4776.53 \\
4776.97 \\
4776.21 \\
4775.70 \\
4779.01 \\
4776.41 \\
4776.37 \\
4776.46 \\
4775.71 \\
4776.15 \\
4777.07 \\
4776.32 \\
4776.37 \\
4776.74 \\
4776.34 \\
4776.72\end{array}$ \\
\hline 0652 & 62581.5 & 93759.3 & AL & D & 4803.84 & 4800.69 & $\begin{array}{l}10 / 19 / 85 \\
04 / 29 / 86 \\
10 / 09 / 86\end{array}$ & $\begin{array}{l}12: 00 \\
12: 30 \\
17: 40\end{array}$ & $\begin{array}{l}18.96 \\
18.58 \\
19.00\end{array}$ & $\begin{array}{l}15.81 \\
15.43 \\
15.85\end{array}$ & $\begin{array}{l}4784.88 \\
4785.26 \\
4784.84\end{array}$ \\
\hline
\end{tabular}

FORHATION OF COMPLETION CODE:

DC - DECHELLEY MEMBER OF THE CUTLER FORMATIO

FLOU RELATIONSHIP COOE:

AL - DECHELLEY

FLON RELATIONSHIP COOE

D - DOWH GRADIENT 
STATIC EROMNOMTER LEVELS

SITE: MONOI MOWUEEMT VALLEY

REPORT DATE: 06/21/95

\begin{tabular}{|c|c|c|c|c|c|c|c|c|c|c|c|}
\hline $\begin{array}{c}\text { LOCATION } \\
\text { 10 }\end{array}$ & $\begin{array}{l}\text { month } \\
\text { cooroinate } \\
\text { (FT) }\end{array}$ & $\begin{array}{l}\text { EAST } \\
\text { COORDIMATE } \\
\text { (FT) }\end{array}$ & $\mid \begin{array}{c}\text { FORMATION } \\
\text { OF } \\
\text { COMPLETION }\end{array}$ & $\begin{array}{l}\text { FLON } \\
\text { COOE }\end{array}$ & $\begin{array}{l}\text { CASING } \\
\text { ELEVATION } \\
\text { (FT MSL) }\end{array}$ & $\begin{array}{l}\text { GROUnid } \\
\text { ELEVATION } \\
\text { (FT MSL) }\end{array}$ & LOG DATE & $\begin{array}{l}\text { LOS } \\
\text { TIME }\end{array}$ & $\begin{array}{l}\text { DEPTH FROA } \\
\text { TOP OF } \\
\text { CASING (FT) }\end{array}$ & $\begin{array}{l}\text { OEPTH FROA } \\
\text { GROWWD } \\
\text { (FT) }\end{array}$ & $\begin{array}{l}\text { GROUNOWATER } \\
\text { ELEVRTION } \\
\text { (FT HSL) }\end{array}$ \\
\hline 0652 & 62581.5 & 93759.3 & AL & D & 4803.84 & 4800.69 & $\begin{array}{l}03 / 26 / 87 \\
05 / 08 / 87 \\
06 / 01 / 91 \\
02 / 23 / 92 \\
08 / 12 / 92 \\
11 / 21 / 92 \\
02 / 18 / 93 \\
06 / 29 / 93 \\
12 / 10 / 93 \\
04 / 20 / 94 \\
12 / 08 / 94 \\
12 / 14 / 94 \\
04 / 19 / 95\end{array}$ & $\begin{array}{l}12: 00 \\
13: 30 \\
16: 59 \\
12: 30 \\
15: 48 \\
14: 14 \\
10: 44 \\
17: 05 \\
10: 57 \\
11: 50 \\
08: 44 \\
08: 55 \\
10: 32\end{array}$ & $\begin{array}{l}19.55 \\
18.42 \\
18.60 \\
18.67 \\
19.08 \\
18.92 \\
18.46 \\
18.51 \\
18.58 \\
18.29 \\
18.64 \\
18.62 \\
18.29\end{array}$ & $\begin{array}{l}16.40 \\
15.27 \\
15.45 \\
15.52 \\
15.93 \\
15.77 \\
15.31 \\
15.36 \\
15.43 \\
15.14 \\
15.49 \\
15.47 \\
15.14\end{array}$ & $\begin{array}{l}4704.29 \\
4785.42 \\
4785.24 \\
4785.17 \\
4784.76 \\
4784.92 \\
4785.38 \\
4785.33 \\
4755.26 \\
4785.55 \\
4785.20 \\
4785.22 \\
4785.55\end{array}$ \\
\hline 0653 & 61249.3 & 89596.3 &. & D & 4834.94 & 4832.14 & $\begin{array}{l}10 / 19 / 85 \\
04 / 28 / 86 \\
10 / 09 / 86 \\
03 / 26 / 87 \\
05 / 08 / 87 \\
04 / 21 / 88 \\
06 / 22 / 89 \\
12 / 01 / 89 \\
06 / 19 / 90 \\
01 / 24 / 91 \\
06 / 04 / 91 \\
02 / 25 / 92 \\
08 / 13 / 92 \\
11 / 19 / 92 \\
02 / 18 / 93 \\
06 / 29 / 93 \\
12 / 09 / 93 \\
04 / 20 / 94 \\
12 / 08 / 94 \\
12 / 14 / 94 \\
04 / 19 / 95\end{array}$ & $\begin{array}{l}12: 00 \\
15: 00 \\
16: 45 \\
12: 00 \\
11: 15 \\
09: 10 \\
12: 30 \\
13: 00 \\
09: 30 \\
14: 30 \\
19: 10 \\
09: 40 \\
18: 45 \\
09: 14 \\
11: 19 \\
17: 47 \\
17: 01 \\
11: 17 \\
13: 04 \\
10: 04 \\
09: 45\end{array}$ & $\begin{array}{l}35.55 \\
35.06 \\
35.25 \\
36.07 \\
34.99 \\
34.93 \\
35.00 \\
33.50 \\
35.05 \\
34.98 \\
35.14 \\
35.28 \\
35.20 \\
35.18 \\
35.12 \\
34.75 \\
34.67 \\
34.63 \\
34.74 \\
34.76 \\
34.72\end{array}$ & $\begin{array}{l}32.75 \\
32.26 \\
32.45 \\
33.27 \\
32.19 \\
32.13 \\
32.20 \\
30.70 \\
32.25 \\
32.18 \\
32.34 \\
32.48 \\
32.40 \\
32.38 \\
32.32 \\
31.95 \\
31.87 \\
31.83 \\
31.94 \\
31.96 \\
31.92\end{array}$ & $\begin{array}{l}4799.39 \\
4799.88 \\
4799.69 \\
4798.87 \\
4799.95 \\
4800.01 \\
4799.94 \\
4801.44 \\
4799.89 \\
4799.96 \\
4799.80 \\
4799.66 \\
4799.74 \\
4799.76 \\
4799.82 \\
4800.19 \\
4800.27 \\
4800.31 \\
4800.20 \\
4800.18 \\
4800.22\end{array}$ \\
\hline 0654 & 59351.4 & 91063.4 & AL. & C & 4827.01 & 4824.41 & $\begin{array}{l}10 / 19 / 85 \\
04 / 27 / 86 \\
10 / 09 / 86 \\
03 / 26 / 87 \\
05 / 08 / 87 \\
04 / 23 / 88\end{array}$ & $\begin{array}{l}12: 00 \\
10: 28 \\
12: 15 \\
12: 00 \\
13: 20 \\
14: 15\end{array}$ & $\begin{array}{l}1.72 \\
1.23 \\
1.23 \\
2.18 \\
1.15 \\
0.78\end{array}$ & $\begin{array}{l}-0.88 \\
-1.37 \\
-0.77 \\
-0.42 \\
-1.45 \\
-1.82\end{array}$ & $\begin{array}{l}4825.29 \\
4825.78 \\
4825.18 \\
4824.83 \\
4825.86 \\
4826.23\end{array}$ \\
\hline
\end{tabular}

FORHATION OF COMPLETIOH COOE:

FLOU RELATIONSHIP COOE:

AL - AlLUVIUH

D - DOWN GRADIENT

C - CROSS GRADIENT 
STATIC GROUMDATER LEVELS

SITE: MONOT MONUMENT VALLEY

REPORT DATE: 06/21/95

\begin{tabular}{|c|c|c|c|c|c|c|c|c|c|c|c|}
\hline$\underset{\text { ID }}{\text { LOCATION }}$ & $\begin{array}{l}\text { MOnTH } \\
\text { COORDIHATE } \\
\text { (FT) }\end{array}$ & $\begin{array}{c}\text { EAST } \\
\text { COORDINATE } \\
\text { (FT) }\end{array}$ & $\begin{array}{l}\text { FORMATION } \\
\text { OF } \\
\text { COMPLETION }\end{array}$ & $\begin{array}{l}\text { FLOW } \\
\text { COOE }\end{array}$ & $\begin{array}{l}\text { CASING } \\
\text { ELEVATION } \\
\text { (FT HSL) }\end{array}$ & $\begin{array}{l}\text { GROUHD } \\
\text { ELEVATION } \\
\text { (FT MSL) }\end{array}$ & LOG DATE & $\begin{array}{l}\text { LOG } \\
\text { TIRE }\end{array}$ & $\begin{array}{l}\text { OEPTH FROM } \\
\text { TOP OF } \\
\text { CASING (FT) }\end{array}$ & $\begin{array}{l}\text { DEPTH FROM } \\
\text { GROUMD } \\
\text { (FT) }\end{array}$ & $\begin{array}{l}\text { GROUMDWATER } \\
\text { ELEVATION } \\
\text { (FT HSL) }\end{array}$ \\
\hline 0654 & 59351.4 & 91063.4 & AL. & C & 4827.01 & 4824.41 & $\begin{array}{l}06 / 06 / 89 \\
12 / 03 / 89 \\
06 / 19 / 90 \\
01 / 27 / 91 \\
06 / 02 / 91 \\
08 / 12 / 92 \\
11 / 22 / 92 \\
02 / 18 / 93 \\
06 / 29 / 93 \\
12 / 10 / 93 \\
04 / 20 / 94 \\
12 / 14 / 94 \\
04 / 19 / 95\end{array}$ & $\begin{array}{l}12: 55 \\
11: 20 \\
14: 14 \\
09: 30 \\
12: 40 \\
13: 17 \\
10: 30 \\
10: 36 \\
16: 49 \\
10: 10 \\
11: 34 \\
10: 16 \\
10: 22\end{array}$ & $\begin{array}{r}1.42 \\
2.04 \\
1.52 \\
1.31 \\
1.28 \\
1.75 \\
1.42 \\
.95 \\
1.37 \\
1.33 \\
0.91 \\
\text { FLOUING } \\
0.90\end{array}$ & $\begin{array}{r}-1.18 \\
-0.56 \\
-1.08 \\
-1.29 \\
-1.32 \\
-0.85 \\
-1.18 \\
-1.65 \\
-1.23 \\
-1.27 \\
-1.69 \\
\text { FLONING } \\
-1.70\end{array}$ & $\begin{array}{r}4825.59 \\
4824.97 \\
4825.49 \\
4825.70 \\
4825.73 \\
4825.26 \\
4825.59 \\
4826.06 \\
4825.64 \\
4825.68 \\
4826.10 \\
- \\
4826.11\end{array}$ \\
\hline 0655 & 59754.6 & 88624.1 & AL & D & 4860.39 & 4857.73 & $\begin{array}{l}10 / 19 / 85 \\
04 / 29 / 86 \\
10 / 09 / 86 \\
03 / 26 / 87 \\
05 / 08 / 87 \\
04 / 20 / 88 \\
06 / 08 / 89 \\
12 / 03 / 89 \\
06 / 22 / 90 \\
01 / 23 / 91 \\
06 / 02 / 91 \\
02 / 22 / 92 \\
08 / 13 / 92 \\
11 / 19 / 92 \\
02 / 18 / 93 \\
06 / 29 / 93 \\
12 / 09 / 93 \\
04 / 20 / 94 \\
12 / 07 / 94 \\
12 / 14 / 94 \\
04 / 19 / 95\end{array}$ & $\begin{array}{l}12: 00 \\
09: 45 \\
16: 35 \\
12: 00 \\
11: 10 \\
10: 20 \\
11: 15 \\
13: 30 \\
14: 20 \\
13: 30 \\
15: 09 \\
12: 35 \\
15: 50 \\
12: 05 \\
12: 07 \\
17: 36 \\
14: 06 \\
09: 32 \\
13: 53 \\
10: 21 \\
11: 50\end{array}$ & $\begin{array}{l}39.23 \\
38.80 \\
38.87 \\
39.47 \\
38.65 \\
38.55 \\
33.60 \\
39.47 \\
38.86 \\
38.65 \\
38.80 \\
38.83 \\
38.80 \\
38.80 \\
38.60 \\
38.65 \\
39.09 \\
38.78 \\
38.67 \\
38.60 \\
38.50\end{array}$ & $\begin{array}{l}36.57 \\
36.14 \\
36.21 \\
36.81 \\
35.99 \\
35.89 \\
30.94 \\
36.81 \\
36.20 \\
35.99 \\
36.14 \\
36.17 \\
36.14 \\
36.14 \\
35.94 \\
35.99 \\
36.43 \\
36.12 \\
36.01 \\
35.94 \\
35.84\end{array}$ & $\begin{array}{l}4821.16 \\
4821.59 \\
4821.52 \\
4820.92 \\
4821.74 \\
4821.84 \\
4826.79 \\
4820.92 \\
4821.53 \\
4821.74 \\
4821.59 \\
4821.56 \\
4821.59 \\
4821.59 \\
4821.79 \\
4821.74 \\
4821.30 \\
4821.61 \\
4821.72 \\
4821.79 \\
4821.89\end{array}$ \\
\hline 0656 & 59545.2 & 89175.1 & $\mathbf{A L}$ & D & 4854.61 & 4852.07 & $\begin{array}{l}10 / 19 / 85 \\
04 / 29 / 86 \\
10 / 09 / 86 \\
03 / 26 / 87 \\
05 / 08 / 87 \\
06 / 23 / 89\end{array}$ & $\begin{array}{l}12: 00 \\
12: 00 \\
16: 40 \\
12: 00 \\
14: 20 \\
10: 15\end{array}$ & $\begin{array}{l}35.98 \\
35.47 \\
35.79 \\
37.08 \\
35.30 \\
35.25\end{array}$ & $\begin{array}{l}33.44 \\
32.93 \\
33.25 \\
34.54 \\
32.76 \\
32.71\end{array}$ & $\begin{array}{l}4818.63 \\
4819.14 \\
4818.82 \\
4817.53 \\
4819.31 \\
4819.36\end{array}$ \\
\hline
\end{tabular}

FORMATION OF COMPLETIOH COOE:

AL - ALLUVIUM
FLOH RELATIONSHIP CODE:

C - CROSS GRADIENT 
STATIC CROWMUATER LEVELS

SITE: MOWOI MOMUNENT VALLEY

REPORT DATE: 06/21/95

\begin{tabular}{|c|c|c|c|c|c|c|c|c|c|c|c|}
\hline$\underset{10}{\text { Location }}$ & $\begin{array}{l}\text { MORTH } \\
\text { COORDIKATE } \\
\text { (FT) }\end{array}$ & $\begin{array}{l}\text { EAST } \\
\text { COORDINATE } \\
\text { (FT) }\end{array}$ & $\begin{array}{c}\text { FORMATION } \\
\text { OF } \\
\text { COMPLETION }\end{array}$ & $\begin{array}{l}\text { FLON } \\
\text { COOE }\end{array}$ & $\begin{array}{l}\text { CASIMG } \\
\text { ELEVATION } \\
\text { (FT MSL) }\end{array}$ & $\begin{array}{l}\text { GRONMD } \\
\text { ELEVATIONA } \\
\text { (FT NSL) }\end{array}$ & LOG DATE & $\begin{array}{l}\text { LOG } \\
\text { TIME }\end{array}$ & $\begin{array}{l}\text { DEPTH FROA } \\
\text { TOP OF } \\
\text { CASIME (FT) }\end{array}$ & \begin{tabular}{|} 
DEPTH FROM \\
GROUMD \\
(FT)
\end{tabular} & $\begin{array}{l}\text { GROUNOWATER } \\
\text { ELEVATION } \\
\text { (FT MSL) }\end{array}$ \\
\hline 0656 & 59545.2 & 89175.1 & AL. & D & 4854.61 & 4852.07 & $\begin{array}{l}08 / 13 / 92 \\
11 / 22 / 92 \\
02 / 18 / 93 \\
08 / 29 / 93 \\
12 / 10 / 93 \\
12 / 07 / 94 \\
04 / 19 / 95\end{array}$ & $\begin{array}{l}18: 32 \\
15: 15 \\
12: 33 \\
12: 06 \\
09: 43 \\
14: 00 \\
09: 56\end{array}$ & $\begin{array}{l}35.50 \\
35.36 \\
35.18 \\
34.95 \\
35.35 \\
35.16 \\
35.00\end{array}$ & $\begin{array}{l}32.96 \\
32.80 \\
32.64 \\
32.41 \\
32.81 \\
32.62 \\
32.46\end{array}$ & $\begin{array}{l}4819.11 \\
4819.27 \\
4819.43 \\
4819.66 \\
4819.26 \\
4819.45 \\
4819.61\end{array}$ \\
\hline 0657 & 59266.0 & 87596.5 & $D C$ & 0 & 4883.21 & 4881.18 & $\begin{array}{l}10 / 19 / 85 \\
04 / 28 / 86 \\
10 / 09 / 86 \\
03 / 26 / 87 \\
05 / 08 / 87 \\
04 / 22 / 88 \\
06 / 21 / 89 \\
12 / 04 / 89 \\
06 / 21 / 90 \\
01 / 24 / 91 \\
11 / 23 / 92 \\
06 / 29 / 93 \\
12 / 09 / 93 \\
04 / 20 / 94 \\
12 / 07 / 94 \\
12 / 14 / 94 \\
04 / 19 / 95\end{array}$ & $\begin{array}{l}12: 00 \\
11: 35 \\
16: 25 \\
12: 00 \\
09: 50 \\
09: 00 \\
10: 30 \\
09: 30 \\
11: 30 \\
09: 30 \\
10: 00 \\
12: 35 \\
10: 56 \\
08: 27 \\
12: 52 \\
11: 55 \\
12: 25\end{array}$ & $\begin{array}{l}50.20 \\
49.90 \\
50.75 \\
51.10 \\
49.89 \\
49.66 \\
49.80 \\
50.58 \\
50.31 \\
49.73 \\
48.70 \\
52.36 \\
51.81 \\
50.60 \\
49.95 \\
50.00 \\
49.82\end{array}$ & $\begin{array}{l}48.17 \\
47.87 \\
48.72 \\
49.07 \\
47.86 \\
47.63 \\
47.77 \\
48.55 \\
48.28 \\
47.70 \\
46.67 \\
50.33 \\
49.78 \\
48.57 \\
47.92 \\
47.97 \\
47.79\end{array}$ & $\begin{array}{l}4833.01 \\
4833.31 \\
4832.46 \\
4832.11 \\
4833.31 \\
4833.55 \\
4833.41 \\
4832.63 \\
4832.90 \\
4833.48 \\
4834.51 \\
4830.85 \\
4831.40 \\
4832.61 \\
4833.26 \\
4833.21 \\
4833.39\end{array}$ \\
\hline 0658 & .54763 .4 & 88857.0 & SR & H & 4878.82 & 4876.19 & $\begin{array}{l}10 / 19 / 85 \\
04 / 29 / 86 \\
10 / 09 / 86 \\
03 / 26 / 87 \\
05 / 08 / 87 \\
08 / 14 / 92 \\
11 / 24 / 92 \\
02 / 18 / 93 \\
06 / 29 / 93 \\
12 / 09 / 93 \\
04 / 21 / 94 \\
12 / 07 / 94 \\
04 / 19 / 95\end{array}$ & $\begin{array}{l}12: 00 \\
11: 00 \\
13: 20 \\
12: 00 \\
12: 55 \\
18: 05 \\
10: 50 \\
09: 35 \\
16: 00 \\
14: 54 \\
13: 22 \\
14: 58 \\
11: 33\end{array}$ & $\begin{array}{r}9.61 \\
9.27 \\
9.60 \\
10.64 \\
9.15 \\
9.83 \\
9.45 \\
8.82 \\
9.26 \\
9.31 \\
8.99 \\
9.33 \\
8.94\end{array}$ & $\begin{array}{l}6.98 \\
6.64 \\
6.97 \\
8.01 \\
6.52 \\
7.20 \\
6.82 \\
6.19 \\
6.63 \\
6.68 \\
6.36 \\
6.70 \\
6.31\end{array}$ & $\begin{array}{l}4869.21 \\
4869.55 \\
4869.22 \\
4868.18 \\
4869.67 \\
4868.99 \\
4869.37 \\
4870.00 \\
4869.56 \\
4869.51 \\
4869.83 \\
4869.49 \\
4869.88\end{array}$ \\
\hline 0659 & 59069.0 & 88670.3 & SR & D & 4863.63 & 4860.84 & $10 / 19 / 85$ & $12: 00$ & 36.45 & 33.66 & 4827.18 \\
\hline
\end{tabular}

FORMATION OF COMPLETION CODE:

AL - ALLUVIUH

FLON RELATIOHSHIP COOE:

MELEY MER OF THE CUTLER FORMATION

SR - SHINARUMP MEMBER OF THE CHIKLE FORMATIOH 
STATIC GROUNOWATER LEVELS

SITE: MONO1 MOWUNENT VALLEY

REPORT DATE: 06/21/95

\begin{tabular}{|c|c|c|c|c|c|c|c|c|c|c|c|}
\hline$\underset{10}{\text { LOCATION }}$ & $\begin{array}{l}\text { MORTH } \\
\text { COORDINATE } \\
\text { (FT) }\end{array}$ & $\begin{array}{l}\text { EAST } \\
\text { COORDIMATE } \\
\text { (FT) }\end{array}$ & $\begin{array}{l}\text { Formation } \\
\text { OF } \\
\text { COMPLETION }\end{array}$ & $\begin{array}{l}\text { FLOW } \\
\text { CODE }\end{array}$ & $\begin{array}{l}\text { CASIMG } \\
\text { ELEVATION } \\
\text { (FT MSL) }\end{array}$ & $\begin{array}{l}\text { GROWID } \\
\text { ELEVATION } \\
\text { (FT MSL) }\end{array}$ & LOG DATE & $\begin{array}{l}\text { LOG } \\
\text { TIME }\end{array}$ & $\mid \begin{array}{c}\text { OEPTH FROM } \\
\text { TOP OF } \\
\text { CASIHG (FT) }\end{array}$ & $\mid \begin{array}{c}\text { DEPTH FROM } \\
\text { GROUND } \\
\text { (FT) }\end{array}$ & $\begin{array}{l}\text { GROUIDUATER } \\
\text { ELEVATION } \\
\text { (FT MSL) }\end{array}$ \\
\hline 0659 & 59069.8 & 80670.3 & SR & D & 4863.63 & 4860.84 & $\begin{array}{l}04 / 27 / 86 \\
10 / 09 / 86 \\
03 / 26 / 87 \\
05 / 08 / 87 \\
04 / 25 / 88 \\
06 / 22 / 89 \\
12 / 02 / 89 \\
06 / 20 / 90 \\
01 / 28 / 91 \\
06 / 04 / 91 \\
02 / 20 / 92 \\
08 / 13 / 92 \\
11 / 18 / 92 \\
11 / 24 / 92 \\
02 / 18 / 93 \\
06 / 29 / 93 \\
12 / 09 / 93 \\
04 / 20 / 94 \\
12 / 08 / 94 \\
12 / 14 / 94 \\
04 / 19 / 95\end{array}$ & $\begin{array}{l}15: 35 \\
17: 15 \\
12: 00 \\
11: 05 \\
11: 30 \\
16: 30 \\
14: 00 \\
14: 00 \\
10: 30 \\
17: 11 \\
14: 35 \\
17: 23 \\
10: 17 \\
09: 30 \\
12: 24 \\
17: 33 \\
14: 16 \\
09: 45 \\
15: 12 \\
10: 29 \\
11: 59\end{array}$ & $\begin{array}{l}35.33 \\
35.43 \\
35.71 \\
34.96 \\
34.83 \\
35.00 \\
36.18 \\
36.30 \\
35.10 \\
35.20 \\
35.28 \\
35.22 \\
35.20 \\
34.73 \\
34.93 \\
34.74 \\
35.32 \\
34.95 \\
35.01 \\
35.30 \\
34.89\end{array}$ & $\begin{array}{l}32.54 \\
32.64 \\
32.92 \\
32.17 \\
32.04 \\
32.21 \\
33.39 \\
33.51 \\
32.31 \\
32.41 \\
32.49 \\
32.43 \\
32.41 \\
31.94 \\
32.14 \\
31.95 \\
32.53 \\
32.16 \\
32.22 \\
32.51 \\
32.10\end{array}$ & $\begin{array}{l}4828.30 \\
4828.20 \\
4827.92 \\
4828.67 \\
4828.80 \\
4828.63 \\
4827.45 \\
4827.33 \\
4828.53 \\
4828.43 \\
4828.35 \\
4828.41 \\
4828.43 \\
4828.90 \\
4828.70 \\
4828.89 \\
4828.31 \\
4828.68 \\
4828.62 \\
4828.33 \\
4828.74\end{array}$ \\
\hline 0660 & 61303.4 & 89584.3 & SR & D & 4834.16 & 4831.38 & $\begin{array}{l}10 / 19 / 85 \\
04 / 28 / 86 \\
10 / 09 / 86 \\
03 / 26 / 87 \\
05 / 08 / 87 \\
04 / 20 / 88 \\
06 / 22 / 89 \\
12 / 01 / 89 \\
06 / 23 / 90 \\
01 / 26 / 91 \\
08 / 13 / 92 \\
11 / 19 / 92 \\
02 / 18 / 93 \\
06 / 29 / 93 \\
12 / 09 / 93 \\
04 / 20 / 94 \\
12 / 08 / 94 \\
12 / 14 / 94 \\
04 / 19 / 95\end{array}$ & $\begin{array}{l}12: 00 \\
14: 25 \\
16: 45 \\
12: 00 \\
11: 20 \\
13: 45 \\
10: 30 \\
09: 30 \\
09: 30 \\
09: 30 \\
16: 48 \\
10: 43 \\
11: 22 \\
17: 45 \\
17: 04 \\
11: 15 \\
13: 00 \\
10: 02 \\
09: 44\end{array}$ & $\begin{array}{l}33.25 \\
31.35 \\
31.54 \\
32.28 \\
31.30 \\
31.04 \\
31.15 \\
32.09 \\
31.30 \\
31.17 \\
31.43 \\
31.40 \\
31.30 \\
31.26 \\
31.20 \\
30.83 \\
30.94 \\
31.30 \\
30.93\end{array}$ & $\begin{array}{l}30.47 \\
28.57 \\
28.76 \\
29.50 \\
28.52 \\
28.26 \\
28.37 \\
29.31 \\
28.52 \\
28.39 \\
28.65 \\
28.62 \\
28.52 \\
28.48 \\
28.42 \\
28.05 \\
28.16 \\
28.52 \\
28.15\end{array}$ & $\begin{array}{l}4800.91 \\
4802.81 \\
4802.62 \\
4801.88 \\
4802.86 \\
4803.12 \\
4803.01 \\
4802.07 \\
4802.86 \\
4802.99 \\
4802.73 \\
4802.76 \\
4802.86 \\
4802.90 \\
4802.96 \\
4803.33 \\
4803.22 \\
4802.86 \\
4803.23\end{array}$ \\
\hline 0661 & 56366.1 & 85447.7 & DC & $N$ & 5059.99 & 5057.34 & $10 / 19 / 85$ & $12: 00$ & 164.20 & 161.55 & 4895.79 \\
\hline
\end{tabular}

FOOMATIOH OF COMPLETIOH COOE:

SR - SHIMARUMP MEMBER OF THE CHIMLE FORMATIOU

FLOW RELATIOHSHIP COOE:

DC - DECHELLEY MEMBER OF THE CUTLER FORMATIOW

D - DONA GRADIENT

H - UNKNONA 
STATIC GROWWOWATER LEVELS

SITE: MOHO1 MOMUAENT VALLEY

REPORT DATE: 06/21/95

\begin{tabular}{|c|c|c|c|c|c|c|c|c|c|c|c|}
\hline$\underset{10}{\text { Locarion }}$ & $\begin{array}{l}\text { MORTH } \\
\text { COORDIMATE } \\
\text { (FT) }\end{array}$ & $\begin{array}{l}\text { EAST } \\
\text { COORDIMATE } \\
\text { (FT) }\end{array}$ & $\begin{array}{c}\text { Formation } \\
\text { OF } \\
\text { Completion }\end{array}$ & $\begin{array}{l}\text { FLOW } \\
\text { CODE }\end{array}$ & $\begin{array}{l}\text { CASING } \\
\text { ELEVATION } \\
\text { (FT HSL) }\end{array}$ & $\begin{array}{l}\text { GROWHD } \\
\text { ELEVATION } \\
\text { (FT MSL) }\end{array}$ & LOS DATE & $\begin{array}{l}\text { LOG } \\
\text { TIME }\end{array}$ & $\begin{array}{l}\text { DEPTH FROA } \\
\text { TOP OF } \\
\text { CASING (FT) }\end{array}$ & \begin{tabular}{|} 
DEPTH FROA \\
GROUMD \\
(FT)
\end{tabular} & $\begin{array}{l}\text { GROWHOUATER } \\
\text { ELEVATION } \\
\text { (FT MSL) }\end{array}$ \\
\hline 0661 & 56366.1 & 85447.7 & DC & n & 5059.99 & 5057.34 & $\begin{array}{l}04 / 29 / 86 \\
10 / 09 / 86 \\
03 / 26 / 87 \\
05 / 08 / 87 \\
02 / 18 / 93 \\
12 / 09 / 93 \\
04 / 20 / 94 \\
12 / 12 / 94 \\
12 / 14 / 94 \\
04 / 19 / 95\end{array}$ & $\begin{array}{l}14: 00 \\
14: 40 \\
12: 00 \\
14: 40 \\
09: 04 \\
13: 51 \\
10: 50 \\
16: 17 \\
11: 28 \\
13: 06\end{array}$ & $\begin{array}{l}162.75 \\
162.18 \\
163.67 \\
163.57 \\
163.74 \\
163.31 \\
163.33 \\
164.14 \\
163.31 \\
163.20\end{array}$ & $\begin{array}{l}160.10 \\
159.53 \\
161.02 \\
160.92 \\
161.09 \\
160.66 \\
160.66 \\
161.49 \\
160.66 \\
160.55\end{array}$ & $\begin{array}{l}4897.24 \\
4897.81 \\
4896.32 \\
4896.42 \\
4896.25 \\
4896.60 \\
4896.66 \\
4895.85 \\
4896.68 \\
4896.79\end{array}$ \\
\hline 0662 & 59236.5 & 87577.5 & AL & 0 & 4879.81 & 4877.07 & $\begin{array}{l}10 / 19 / 85 \\
04 / 28 / 86 \\
10 / 09 / 86 \\
03 / 26 / 87 \\
05 / 08 / 87 \\
06 / 21 / 89 \\
06 / 05 / 91 \\
02 / 26 / 92 \\
11 / 23 / 92 \\
06 / 29 / 93 \\
12 / 09 / 93 \\
04 / 20 / 94 \\
12 / 07 / 94 \\
12 / 14 / 94 \\
04 / 19 / 95\end{array}$ & $\begin{array}{l}12: 00 \\
11: 35 \\
16: 25 \\
12: 00 \\
09: 55 \\
14: 00 \\
15: 36 \\
09: 30 \\
09: 35 \\
09: 30 \\
10: 51 \\
08: 33 \\
12: 56 \\
11: 53 \\
12: 28\end{array}$ & $\begin{array}{l}51.50 \\
50.97 \\
50.52 \\
51.75 \\
50.82 \\
50.75 \\
51.00 \\
50.85 \\
40.57 \\
53.28 \\
52.75 \\
51.55 \\
50.88 \\
50.93 \\
50.72\end{array}$ & $\begin{array}{l}48.76 \\
48.23 \\
47.76 \\
49.01 \\
48.08 \\
48.01 \\
48.26 \\
48.11 \\
37.83 \\
50.54 \\
50.01 \\
48.81 \\
48.14 \\
48.19 \\
47.98\end{array}$ & $\begin{array}{l}4828.31 \\
4828.84 \\
4829.29 \\
4828.06 \\
4828.99 \\
4829.06 \\
4828.81 \\
4828.96 \\
4839.24 \\
4826.53 \\
4827.06 \\
4828.26 \\
4828.93 \\
4828.88 \\
4829.09\end{array}$ \\
\hline 0663 & 59069.6 & 88593.5 & OC & D & 4864.41 & 4861.56 & $\begin{array}{l}10 / 19 / 85 \\
04 / 27 / 86 \\
10 / 09 / 86 \\
03 / 26 / 87 \\
05 / 08 / 87 \\
04 / 24 / 88 \\
06 / 23 / 89 \\
12 / 02 / 89 \\
06 / 22 / 90 \\
01 / 25 / 91 \\
06 / 04 / 91 \\
02 / 20 / 92 \\
08 / 15 / 92 \\
11 / 20 / 92 \\
02 / 18 / 93\end{array}$ & $\begin{array}{l}12: 00 \\
11: 49 \\
17: 08 \\
12: 00 \\
11: 00 \\
08: 45 \\
13: 30 \\
08: 30 \\
09: 15 \\
09: 30 \\
13: 19 \\
10: 15 \\
09: 55 \\
10: 08 \\
12: 14\end{array}$ & $\begin{array}{l}33.15 \\
32.56 \\
32.03 \\
32.70 \\
32.18 \\
31.93 \\
32.10 \\
33.33 \\
32.80 \\
32.20 \\
32.58 \\
32.32 \\
32.42 \\
31.37 \\
32.78\end{array}$ & $\begin{array}{l}30.30 \\
29.71 \\
29.18 \\
29.85 \\
29.33 \\
29.08 \\
29.25 \\
30.48 \\
29.95 \\
29.35 \\
29.73 \\
29.47 \\
29.57 \\
28.52 \\
29.93\end{array}$ & $\begin{array}{l}4031.26 \\
4031.85 \\
4832.38 \\
4831.71 \\
4832.23 \\
4832.48 \\
4832.31 \\
4831.08 \\
4831.61 \\
4832.21 \\
4831.83 \\
4832.09 \\
4831.99 \\
4833.04 \\
4831.63\end{array}$ \\
\hline
\end{tabular}

FORMATION OF COMPLETION COOE:

DC - DECHELLEY MEMBER OF THE CUTLER FORMATIOA

AL. - Alluvion
FLOU REL.ATIOHSHIP COOE:

D. DOM GRadent 


\begin{tabular}{|c|c|c|c|c|}
\hline 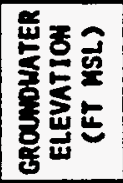 & 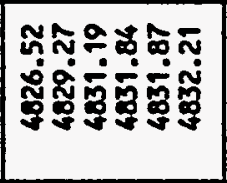 & 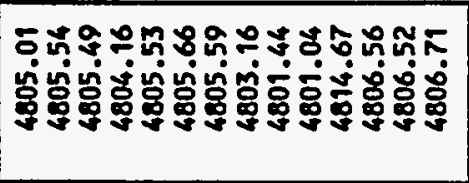 & 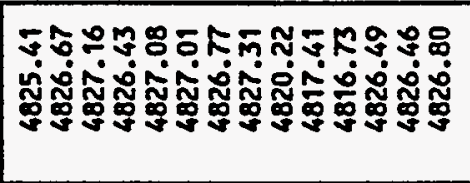 & 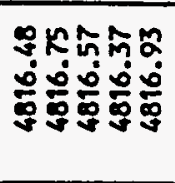 \\
\hline 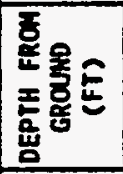 & 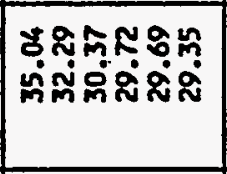 & 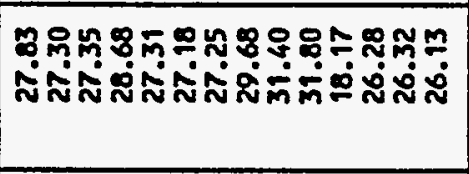 & 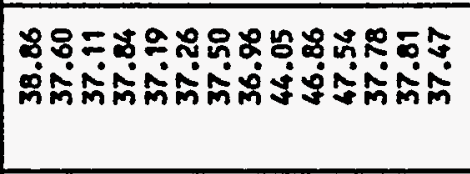 & 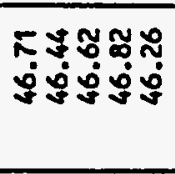 \\
\hline 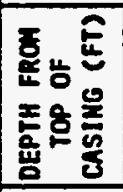 & 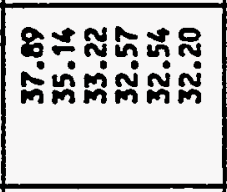 & 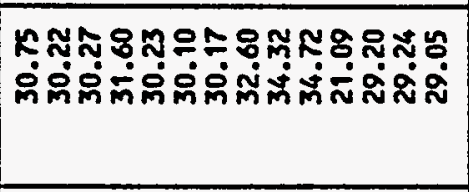 & 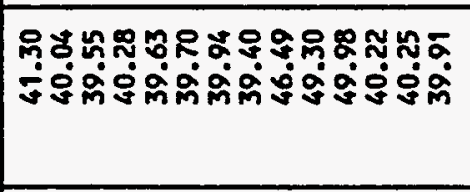 & 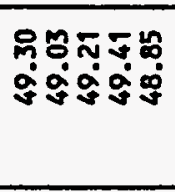 \\
\hline 岁望 & 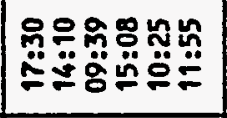 & 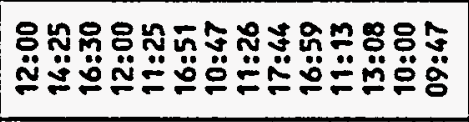 & 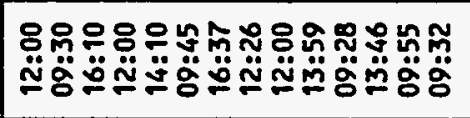 & 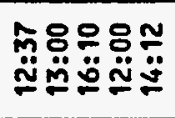 \\
\hline 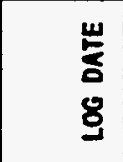 & 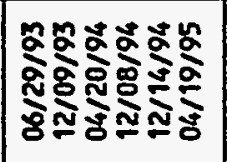 & 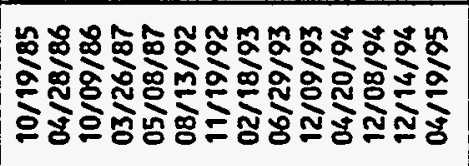 & 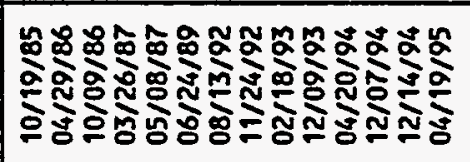 & 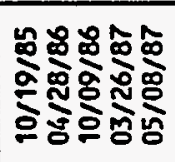 \\
\hline 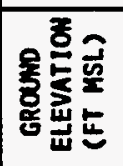 & 旁 & 营 & 草 & $\frac{a}{0.0}$ \\
\hline 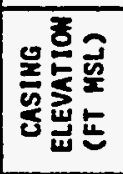 & 范 & م & $\mid \begin{array}{l}\overline{6} \\
0 \\
0 \\
0\end{array}$ & 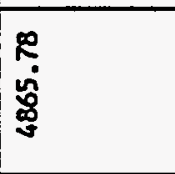 \\
\hline 훈 & 0 & 0 & 0 & 0 \\
\hline 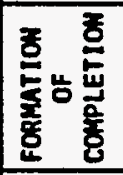 & \& & : & $\ddot{\Delta}$ & z \\
\hline 宸 & 商 & 量 & 品 & 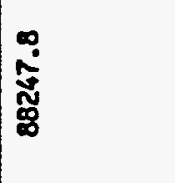 \\
\hline 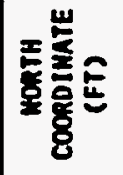 & 葛 & : & $\frac{2}{5}$ & mo \\
\hline 홍잉 & 管 & 营 & 8 & 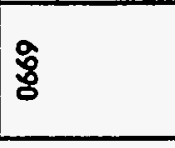 \\
\hline
\end{tabular}


STATIC CAOUNDMTER LEVELS

SITE: MOWOI MOMUNEWT VALLEY

REPORT DATE: 06/21/95

\begin{tabular}{|c|c|c|c|c|c|c|c|c|c|c|c|}
\hline$\underset{10}{\text { Locntion }}$ & $\begin{array}{l}\text { Month } \\
\text { Coonoluhte } \\
\text { (fT) }\end{array}$ & $\begin{array}{l}\text { EAAST } \\
\text { CoOMDIMATE } \\
\text { (FT) }\end{array}$ & $\begin{array}{l}\text { Fonmution } \\
\text { Of } \\
\text { Completion }\end{array}$ & $\begin{array}{l}\text { FLON } \\
\text { COOE }\end{array}$ & $\begin{array}{l}\text { CASING } \\
\text { ELEVATION } \\
\text { (FT HSL) }\end{array}$ & $\begin{array}{l}\text { ERoumd } \\
\text { ELEVATION } \\
\text { (FT MSL) }\end{array}$ & LOS DATE & $\begin{array}{l}\text { Lon } \\
\text { TIRE }\end{array}$ & $\begin{array}{l}\text { DEPTH FROA } \\
\text { TOP OF } \\
\text { CAsING (FT) }\end{array}$ & $\begin{array}{l}\text { DEPTH FROM } \\
\text { GRONivo } \\
\text { (FT) }\end{array}$ & $\begin{array}{l}\text { GRODDAMTER } \\
\text { ELEVATION } \\
\text { (FT MSL) }\end{array}$ \\
\hline 0669 & 60146.3 & 80267.8 & AL & D & 4865.78 & 4863.19 & $\begin{array}{l}06 / 24 / 99 \\
02 / 24 / 92 \\
08 / 13 / 92 \\
11 / 24 / 92 \\
02 / 18 / 93 \\
06 / 29 / 93 \\
12 / 09 / 93 \\
04 / 20 / 94 \\
12 / 07 / 94 \\
12 / 14 / 94 \\
04 / 19 / 95\end{array}$ & $\begin{array}{l}11: 00 \\
08: 50 \\
16: 33 \\
12: 24 \\
11: 52 \\
14: 08 \\
13: 56 \\
09: 23 \\
13: 45 \\
09: 57 \\
09: 34\end{array}$ & $\begin{array}{l}48.80 \\
49.07 \\
49.10 \\
40.89 \\
48.67 \\
48.90 \\
49.39 \\
49.08 \\
48.04 \\
48.86 \\
48.76\end{array}$ & $\begin{array}{l}46.21 \\
46.48 \\
46.51 \\
46.30 \\
46.08 \\
46.31 \\
46.80 \\
46.49 \\
46.25 \\
46.27 \\
46.17\end{array}$ & $\begin{array}{l}4816.98 \\
4816.71 \\
4816.68 \\
4816.89 \\
4817.11 \\
4816.88 \\
4816.39 \\
4816.70 \\
4816.94 \\
4816.92 \\
4817.02\end{array}$ \\
\hline
\end{tabular}

FORMATION OF COMPLETION COOE:

FLON RELATIONSHIP COOE:

AL. - Alluvion

D - DOW GRADIEMT

DATA FILE: IDARTVMONOTIGL1OOOS.DAT

Fields displayed uIth a dash imotcate the data is umavailable 

APPENDIX C

WATER QUALITY AND SEDIMENT CHEMISTRY 


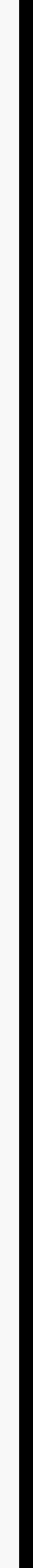




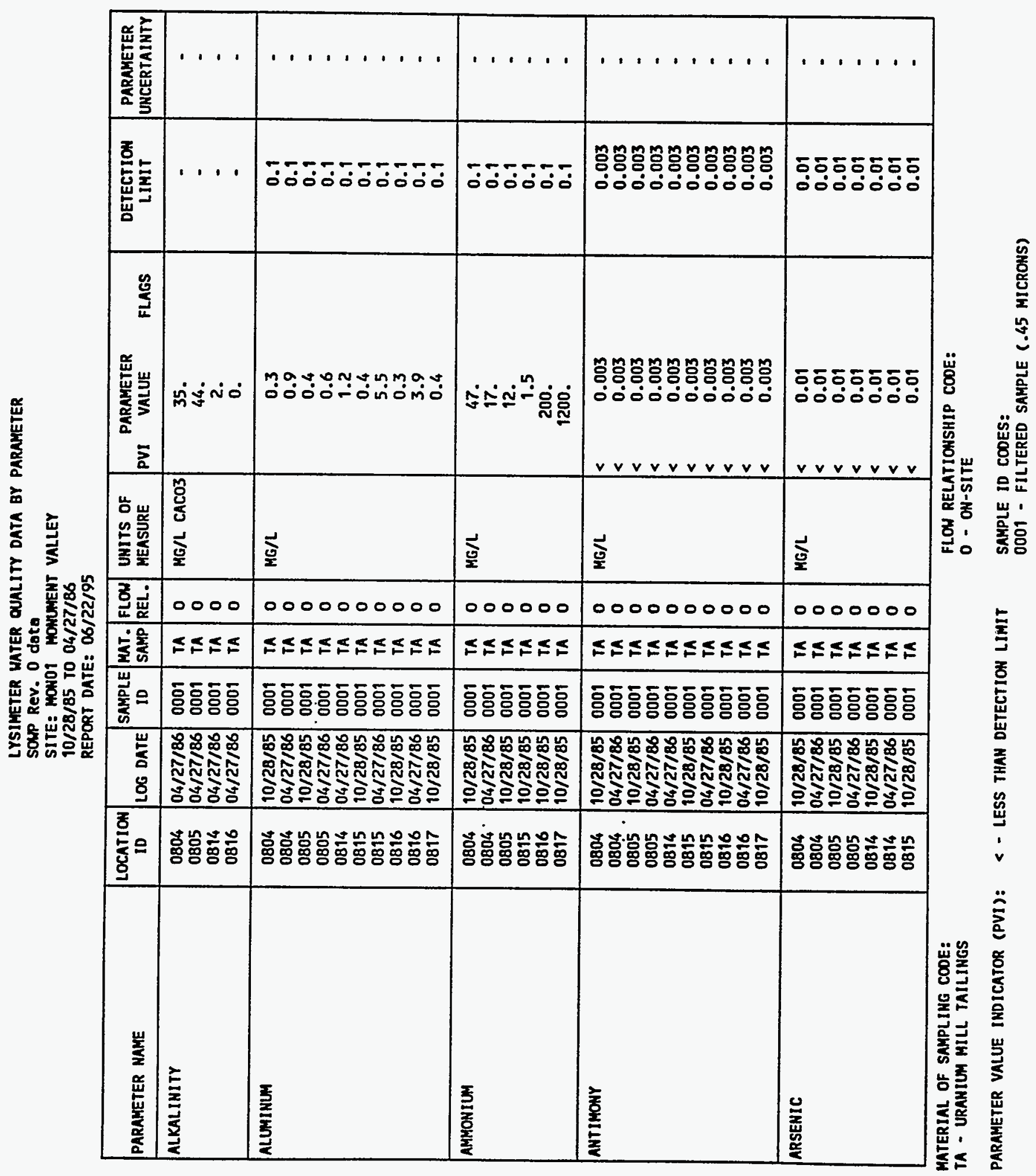


LYSIMETER MATER QUALITY DATA BY PARAMETER

SOWP ReV. 0 date

SITE: MONOI MONUMENT VALLEY

10/28/85 TO 04/27/86

REPORT DATE: 06/22/95

\begin{tabular}{|c|c|c|c|c|c|c|c|c|c|c|}
\hline PARAMETER NAME & $\underset{\text { ID }}{\text { LOCATION }}$ & LOG DATE & $\underset{\text { SAMPLE }}{\text { ID }}$ & $\begin{array}{l}\text { MAT. } \\
\text { SAMP }\end{array}$ & $\begin{array}{l}\text { FLOW } \\
\text { REL. }\end{array}$ & $\begin{array}{l}\text { UNITS OF } \\
\text { MEASURE }\end{array}$ & PVI & $\begin{array}{l}\text { PARAMETER } \\
\text { VALUE }\end{array}$ & $\begin{array}{l}\text { DETECTION } \\
\text { LIMIT }\end{array}$ & $\begin{array}{c}\text { PARAMETER } \\
\text { UNCERTAINTY }\end{array}$ \\
\hline ARSENIC & $\begin{array}{l}0815 \\
0816 \\
0816 \\
0817\end{array}$ & $\begin{array}{l}04 / 27 / 86 \\
10 / 28 / 85 \\
04 / 27 / 86 \\
10 / 28 / 85\end{array}$ & $\begin{array}{l}0001 \\
0001 \\
0001 \\
0001\end{array}$ & $\begin{array}{l}\text { TA } \\
\text { TA } \\
\text { TA } \\
\text { TA }\end{array}$ & $\begin{array}{l}0 \\
0 \\
0 \\
0\end{array}$ & $M G / L$ & $\begin{array}{l}< \\
< \\
< \\
<\end{array}$ & $\begin{array}{l}0.01 \\
0.01 \\
0.01 \\
0.01\end{array}$ & $\begin{array}{l}0.01 \\
0.01 \\
0.01 \\
0.01\end{array}$ & $\begin{array}{l}- \\
- \\
-\end{array}$ \\
\hline BARIUH & $\begin{array}{l}0804 \\
0804 \\
0805 \\
0805 \\
0814 \\
0815 \\
0815 \\
0816 \\
0816 \\
0817\end{array}$ & \begin{tabular}{|l|}
$10 / 28 / 85$ \\
$04 / 27 / 86$ \\
$10 / 28 / 85$ \\
$04 / 27 / 86$ \\
$04 / 27 / 86$ \\
$10 / 28 / 85$ \\
$04 / 27 / 86$ \\
$10 / 28 / 85$ \\
$04 / 27 / 86$ \\
$10 / 28 / 85$
\end{tabular} & $\begin{array}{l}0001 \\
0001 \\
0001 \\
0001 \\
0001 \\
0001 \\
0001 \\
0001 \\
0001 \\
0001\end{array}$ & $\begin{array}{l}\text { TA } \\
\text { TA } \\
\text { TA } \\
\text { TA } \\
\text { TA } \\
\text { TA } \\
\text { TA } \\
\text { TA } \\
\text { TA } \\
\text { TA }\end{array}$ & $\begin{array}{l}0 \\
0 \\
0 \\
0 \\
0 \\
0 \\
0 \\
0 \\
0 \\
0\end{array}$ & MG/L & $1<$ & $\begin{array}{l}0.3 \\
0.1 \\
0.5 \\
0.2 \\
0.1 \\
0.3 \\
0.1 \\
0.3 \\
0.1 \\
0.3\end{array}$ & $\begin{array}{l}0.1 \\
0.1 \\
0.1 \\
0.1 \\
0.1 \\
0.1 \\
0.1 \\
0.1 \\
0.1 \\
0.1\end{array}$ & $\begin{array}{l}- \\
- \\
- \\
- \\
- \\
- \\
-\end{array}$ \\
\hline BORON & $\begin{array}{l}0804 \\
0805 \\
0814 \\
0815 \\
0816\end{array}$ & $\mid \begin{array}{l}04 / 27 / 86 \\
04 / 27 / 86 \\
04 / 27 / 86 \\
04 / 27 / 86 \\
04 / 27 / 86\end{array}$ & $\begin{array}{l}0001 \\
0001 \\
0001 \\
0001 \\
0001\end{array}$ & $\begin{array}{l}\text { TA } \\
\text { TA } \\
\text { TA } \\
\text { TA } \\
\text { TA }\end{array}$ & $\begin{array}{l}0 \\
0 \\
0 \\
0 \\
0\end{array}$ & $M G / L$ & $\mid \begin{array}{l}< \\
< \\
< \\
< \\
<\end{array}$ & $\begin{array}{l}0.1 \\
0.1 \\
0.1 \\
0.1 \\
0.1\end{array}$ & $\begin{array}{l}0.1 \\
0.1 \\
0.1 \\
0.1 \\
0.1\end{array}$ & $\begin{array}{l}- \\
- \\
-\end{array}$ \\
\hline CADMIUH & $\begin{array}{l}0804 \\
0804 \\
0805 \\
0805 \\
0814 \\
0815 \\
0815 \\
0816 \\
0816 \\
0817\end{array}$ & $\begin{array}{l}10 / 28 / 85 \\
04 / 27 / 86 \\
10 / 28 / 85 \\
04 / 27 / 86 \\
04 / 27 / 86 \\
10 / 28 / 85 \\
04 / 27 / 86 \\
10 / 28 / 85 \\
04 / 27 / 86 \\
10 / 28 / 85\end{array}$ & $\begin{array}{l}0001 \\
0001 \\
0001 \\
0001 \\
0001 \\
0001 \\
0001 \\
0001 \\
0001 \\
0001\end{array}$ & $\begin{array}{l}T A \\
T A \\
T A \\
T A \\
T A \\
T A \\
T A \\
T A \\
T A \\
T A\end{array}$ & $\begin{array}{l}0 \\
0 \\
0 \\
0 \\
0 \\
0 \\
0 \\
0 \\
0 \\
0\end{array}$ & MG/L. & $\begin{array}{l}< \\
< \\
< \\
< \\
< \\
< \\
< \\
< \\
< \\
< \\
k\end{array}$ & $\begin{array}{l}0.001 \\
0.001 \\
0.001 \\
0.001 \\
0.001 \\
0.001 \\
0.001 \\
0.001 \\
0.001 \\
0.001\end{array}$ & $\begin{array}{l}0.001 \\
0.001 \\
0.001 \\
0.001 \\
0.001 \\
0.001 \\
0.001 \\
0.001 \\
0.001 \\
0.001\end{array}$ & $\begin{array}{l}- \\
= \\
- \\
- \\
- \\
-\end{array}$ \\
\hline CALCIUH & $\begin{array}{l}0804 \\
0804 \\
0805 \\
0805 \\
0814 \\
0814 \\
0815 \\
0815\end{array}$ & $\begin{array}{l}10 / 28 / 85 \\
04 / 27 / 86 \\
10 / 28 / 85 \\
04 / 27 / 86 \\
10 / 28 / 85 \\
04 / 27 / 86 \\
10 / 28 / 85 \\
04 / 27 / 86\end{array}$ & $\begin{array}{l}0001 \\
0001 \\
0001 \\
0001 \\
0001 \\
0001 \\
0001 \\
0001\end{array}$ & $\begin{array}{l}\text { TA } \\
T A \\
T A \\
T A \\
T A \\
T A \\
\text { TA } \\
\text { TA }\end{array}$ & $\begin{array}{l}0 \\
0 \\
0 \\
0 \\
0 \\
0 \\
0 \\
0\end{array}$ & HG/L & & $\begin{array}{l}36.9 \\
39.4 \\
425 . \\
198 . \\
585 . \\
534 . \\
544 . \\
510 .\end{array}$ & $\begin{array}{l}0.01 \\
0.01 \\
0.01 \\
0.01 \\
0.01 \\
0.01 \\
0.01 \\
0.01\end{array}$ & $\begin{array}{l}- \\
- \\
- \\
-\end{array}$ \\
\hline
\end{tabular}

MATERIAL OF SAMPLING COOE:

TA - URANIUN MILL TAILINGS

PARAMETER VALUE INDICATOR (PVI): < : LESS THAN DETECTION LIMIT
FLON RELATIONSHIP COOE:

O - ON-SITE

SAMPLE IO COOES:

0001 - FILTERED SAMPLE (.45 MICRONS) 
LYSIMETER WATER QUALITY DATA BY PARAMETER

SOMP REV. 0 dato

SITE: MONO1 MONUHENT VALLEY

10/2B/B5 TO 04/27/86

REPORT DATE: 06/22/95

\begin{tabular}{|c|c|c|c|c|c|c|c|c|c|c|c|}
\hline PARAMETER NAHE & $\underset{\text { ID }}{\text { LOCATION }}$ & LOG OATE & $\underset{10}{\text { SAMPLE }}$ & $\begin{array}{l}\text { MAT. } \\
\text { SAMPP }\end{array}$ & $\begin{array}{l}\text { FLOW } \\
\text { REL. }\end{array}$ & $\begin{array}{l}\text { UNITS OF } \\
\text { MEASURE }\end{array}$ & PVI & $\begin{array}{l}\text { ARAMETER } \\
\text { VALUE }\end{array}$ & FLAGS & $\begin{array}{l}\text { DETECTION } \\
\text { LIMIT }\end{array}$ & $\begin{array}{l}\text { PARAMETER } \\
\text { UHCERTAINTY }\end{array}$ \\
\hline CALCIUH & $\begin{array}{l}0816 \\
0816 \\
0817\end{array}$ & $\left|\begin{array}{l}10 / 28 / 85 \\
04 / 27 / 86 \\
10 / 28 / 85\end{array}\right|$ & $\begin{array}{l}0001 \\
0001 \\
0001\end{array}$ & $\begin{array}{l}\text { TA } \\
\text { TA } \\
\text { TA }\end{array}$ & $\begin{array}{l}0 \\
0 \\
0\end{array}$ & MG/L & & $\begin{array}{l}626 . \\
457 . \\
555 .\end{array}$ & & $\begin{array}{l}0.01 \\
0.01 \\
0.01\end{array}$ & - \\
\hline CHLORIDE & $\begin{array}{l}0804 \\
0804 \\
0805 \\
0805 \\
0814 \\
0815 \\
0816 \\
0817\end{array}$ & $\begin{array}{l}10 / 28 / 85 \\
04 / 27 / 86 \\
10 / 28 / 85 \\
04 / 27 / 86 \\
10 / 28 / 85 \\
10 / 28 / 85 \\
10 / 28 / 85 \\
10 / 28 / 85\end{array}$ & $\begin{array}{l}0001 \\
0001 \\
0001 \\
0001 \\
0001 \\
0001 \\
0001 \\
0001\end{array}$ & $\begin{array}{l}\text { TA } \\
\text { TA } \\
\text { TA } \\
\text { TA } \\
\text { TA } \\
\text { TA } \\
\text { TA } \\
\text { TA }\end{array}$ & $\begin{array}{l}0 \\
0 \\
0 \\
0 \\
0 \\
0 \\
0 \\
0\end{array}$ & MG/L & & $\begin{array}{l}26 . \\
33 . \\
41 . \\
45 \\
5 . \\
7 . \\
15 \\
20\end{array}$ & $\mathbf{R}$ & $\begin{array}{l}1 . \\
1 . \\
1 . \\
1 . \\
1 . \\
1 .\end{array}$ & $\begin{array}{l}- \\
- \\
- \\
- \\
-\end{array}$ \\
\hline CHROMIUM & $\begin{array}{l}0804 \\
0804 \\
0805 \\
0805 \\
0814 \\
0815 \\
0815 \\
0816 \\
0816 \\
0817\end{array}$ & $\mid \begin{array}{l}10 / 28 / 85 \\
04 / 27 / 86 \\
10 / 28 / 85 \\
04 / 27 / 86 \\
04 / 27 / 86 \\
10 / 28 / 85 \\
04 / 27 / 86 \\
10 / 28 / 85 \\
04 / 27 / 86 \\
10 / 28 / 85\end{array}$ & $\begin{array}{l}0001 \\
0001 \\
0001 \\
0001 \\
0001 \\
0001 \\
0001 \\
0001 \\
0001 \\
0001\end{array}$ & $\begin{array}{l}\text { TA } \\
\text { TA } \\
\text { TA } \\
\text { TA } \\
\text { TA } \\
\text { TA } \\
\text { TA } \\
\text { TA } \\
\text { TA } \\
\text { TA }\end{array}$ & $\begin{array}{l}0 \\
0 \\
0 \\
0 \\
0 \\
0 \\
0 \\
0 \\
0 \\
0\end{array}$ & MG/L & $\mid<$ & $\begin{array}{l}0.02 \\
0.01 \\
0.03 \\
0.03 \\
0.03 \\
0.02 \\
0.05 \\
0.04 \\
0.05 \\
0.02\end{array}$ & & $\begin{array}{l}0.01 \\
0.01 \\
0.01 \\
0.01 \\
0.01 \\
0.01 \\
0.01 \\
0.01 \\
0.01 \\
0.01\end{array}$ & $\begin{array}{l}- \\
: \\
: \\
: \\
- \\
-\end{array}$ \\
\hline $\cos A L T$ & $\begin{array}{l}0804 \\
0804 \\
0805 \\
0805 \\
0814 \\
0815 \\
0815 \\
0816 \\
0816 \\
0817\end{array}$ & \begin{tabular}{|l|}
$10 / 28 / 85$ \\
$04 / 27 / 86$ \\
$10 / 28 / 85$ \\
$04 / 27 / 86$ \\
$04 / 27 / 86$ \\
$10 / 28 / 85$ \\
$04 / 27 / 86$ \\
$10 / 28 / 85$ \\
$04 / 27 / 86$ \\
$10 / 28 / 85$
\end{tabular} & $\begin{array}{l}0001 \\
0001 \\
0001 \\
0001 \\
0001 \\
0001 \\
0001 \\
0001 \\
0001 \\
0001\end{array}$ & $\begin{array}{l}T A \\
T A \\
T A \\
T A \\
T A \\
T A \\
T A \\
T A \\
T A \\
T A\end{array}$ & $\begin{array}{l}0 \\
0 \\
0 \\
0 \\
0 \\
0 \\
0 \\
0 \\
0 \\
0\end{array}$ & $M G / L$ & $<$ & $\begin{array}{l}0.05 \\
0.07 \\
0.05 \\
0.11 \\
0.34 \\
0.61 \\
0.87 \\
0.65 \\
1.41 \\
0.99\end{array}$ & & $\begin{array}{l}0.05 \\
0.05 \\
0.05 \\
0.05 \\
0.05 \\
0.05 \\
0.05 \\
0.05 \\
0.05 \\
0.05\end{array}$ & $\begin{array}{l}- \\
- \\
- \\
- \\
- \\
-\end{array}$ \\
\hline COPPER & $\begin{array}{l}0804 \\
0804 \\
0805 \\
0805 \\
0814\end{array}$ & $\begin{array}{l}10 / 28 / 85 \\
04 / 27 / 86 \\
10 / 28 / 85 \\
04 / 27 / 86 \\
04 / 27 / 86\end{array}$ & $\begin{array}{l}0001 \\
0001 \\
0001 \\
0001 \\
0001\end{array}$ & $\begin{array}{l}\text { TA } \\
\text { TA } \\
T A \\
T A \\
\text { TA }\end{array}$ & $\begin{array}{l}\mathbf{0} \\
0 \\
0 \\
0 \\
0\end{array}$ & MG/L & $<$ & $\begin{array}{l}0.02 \\
0.03 \\
0.04 \\
0.04 \\
0.04\end{array}$ & & $\begin{array}{l}0.02 \\
0.02 \\
0.02 \\
0.02 \\
0.02\end{array}$ & $\begin{array}{l}- \\
- \\
-\end{array}$ \\
\hline
\end{tabular}

MATERIAL OF SAMPLING COOE:

TA - URAMIUM MILL TAILIHGS

PARAMETER VALUE IHDICATOR (PVI): < - LESS THAN DETECTION LIMIT
FLON RELATIONSHIP COOE:

O- OH-SITE

SAMPLE ID CODES:
0001 - FILTERED SAMPLE (.45 MICRONS)

OTHER PARAMETER VALUE FLAGS:

R - UHUSABLE DATA POIHT 
LYSIMETER WATER QUALITY DATA GY PARAMETER

SOUP REV 0 data

SITE: MONO1 MONUMENT VALLEY

10/28/85 TO 04/27/86

REPORT DATE: 06/22/95

\begin{tabular}{|c|c|c|c|c|c|c|c|c|c|c|}
\hline PARAMETER NAME & $\underset{\text { ID }}{\text { LOCATION }}$ & LOG DATE & $\begin{array}{c}\text { SAMPLE } \\
\text { ID }\end{array}$ & $\begin{array}{l}\text { MAT. } \\
\text { SAMP }\end{array}$ & $\begin{array}{l}\text { FLOW } \\
\text { REL. }\end{array}$ & $\begin{array}{l}\text { UNITS OF } \\
\text { MEASURE }\end{array}$ & PVI & $\begin{array}{l}\text { PARAMETER } \\
\text { VALUE }\end{array}$ & $\begin{array}{l}\text { DETECTION } \\
\text { LIMIT }\end{array}$ & $\begin{array}{l}\text { PARAMETER } \\
\text { UNCERTAINTY }\end{array}$ \\
\hline COPPER & $\begin{array}{l}0815 \\
0815 \\
0816 \\
0816 \\
0817\end{array}$ & $\begin{array}{l}10 / 28 / 85 \\
04 / 27 / 86 \\
10 / 28 / 85 \\
04 / 27 / 86 \\
10 / 28 / 85\end{array}$ & $\begin{array}{l}0001 \\
0001 \\
0001 \\
0001 \\
0001\end{array}$ & $\begin{array}{l}\text { TA } \\
\text { TA } \\
\text { TA } \\
\text { TA } \\
\text { TA }\end{array}$ & $\begin{array}{l}0 \\
0 \\
0 \\
0 \\
0\end{array}$ & MG/L & & $\begin{array}{l}0.04 \\
0.11 \\
0.03 \\
0.14 \\
0.04\end{array}$ & $\begin{array}{l}0.02 \\
0.02 \\
0.02 \\
0.02 \\
0.02\end{array}$ & $\begin{array}{l}- \\
- \\
- \\
-\end{array}$ \\
\hline FLUORIDE & $0804^{\circ}$ & $04 / 27 / 86$ & 0001 & TA & 0 & $M G / L$ & & 1.7 & 0.1 & - \\
\hline IRON & $\begin{array}{l}0804 \\
0804 \\
0805 \\
0805 \\
0814 \\
0815 \\
0815 \\
0816 \\
0816 \\
0817\end{array}$ & $\begin{array}{l}10 / 28 / 85 \\
04 / 27 / 86 \\
10 / 28 / 85 \\
04 / 27 / 86 \\
04 / 27 / 86 \\
10 / 28 / 85 \\
04 / 27 / 86 \\
10 / 28 / 85 \\
04 / 27 / 86 \\
10 / 28 / 85\end{array}$ & $\begin{array}{l}0001 \\
0001 \\
0001 \\
0001 \\
0001 \\
0001 \\
0001 \\
0001 \\
0001 \\
0001\end{array}$ & $\begin{array}{l}\text { TA } \\
\text { TA } \\
\text { TA } \\
\text { TA } \\
\text { TA } \\
\text { TA } \\
\text { TA } \\
\text { TA } \\
\text { TA } \\
\text { TA }\end{array}$ & $\begin{array}{l}0 \\
0 \\
0 \\
0 \\
0 \\
0 \\
0 \\
0 \\
0 \\
0\end{array}$ & MG/L & $\mid<$ & $\begin{array}{l}0.03 \\
0.03 \\
0.04 \\
0.04 \\
0.1 \\
0.03 \\
0.11 \\
0.29 \\
0.16 \\
1.86\end{array}$ & $\begin{array}{l}0.03 \\
0.03 \\
0.03 \\
0.03 \\
0.03 \\
0.03 \\
0.03 \\
0.03 \\
0.03 \\
0.03\end{array}$ & $\begin{array}{l}- \\
- \\
- \\
- \\
- \\
- \\
-\end{array}$ \\
\hline LEAD & $\begin{array}{l}0804 \\
0805 \\
0814 \\
0815 \\
0816\end{array}$ & $\begin{array}{l}04 / 27 / 86 \\
04 / 27 / 86 \\
04 / 27 / 86 \\
04 / 27 / 86 \\
04 / 27 / 86\end{array}$ & $\begin{array}{l}0001 \\
0001 \\
0001 \\
0001 \\
0001\end{array}$ & $\begin{array}{l}\text { TA } \\
\text { TA } \\
\text { TA } \\
\text { TA } \\
\text { TA }\end{array}$ & $\begin{array}{l}\mathbf{0} \\
\mathbf{0} \\
\mathbf{0} \\
\mathbf{0} \\
\mathbf{0}\end{array}$ & $M G / L$ & $\begin{array}{l}< \\
< \\
< \\
< \\
<\end{array}$ & $\begin{array}{l}0.01 \\
0.01 \\
0.01 \\
0.01 \\
0.01\end{array}$ & $\begin{array}{l}0.01 \\
0.01 \\
0.01 \\
0.01 \\
0.01\end{array}$ & $\begin{array}{l}- \\
- \\
- \\
-\end{array}$ \\
\hline MAGNESIUH & $\begin{array}{l}0804 \\
0804 \\
0805 \\
0805 \\
0814 \\
0814 \\
0815 \\
0815 \\
0816 \\
0816 \\
0817\end{array}$ & $\begin{array}{l}10 / 28 / 85 \\
04 / 27 / 86 \\
10 / 28 / 85 \\
04 / 27 / 86 \\
10 / 28 / 85 \\
04 / 27 / 86 \\
10 / 28 / 85 \\
04 / 27 / 86 \\
10 / 28 / 85 \\
04 / 27 / 86 \\
10 / 28 / 85\end{array}$ & $\begin{array}{l}0001 \\
0001 \\
0001 \\
0001 \\
0001 \\
0001 \\
0001 \\
0001 \\
0001 \\
0001 \\
0001\end{array}$ & $\begin{array}{l}\text { TA } \\
\text { TA } \\
\text { TA } \\
\text { TA } \\
\text { TA } \\
\text { TA } \\
\text { TA } \\
\text { TA } \\
\text { TA } \\
\text { TA } \\
\text { TA }\end{array}$ & $\begin{array}{l}\mathbf{0} \\
0 \\
0 \\
0 \\
0 \\
0 \\
0 \\
0 \\
0 \\
0 \\
0\end{array}$ & MG/L & & $\begin{array}{c}18.3 \\
17.6 \\
135 . \\
90 . \\
3.94 \\
3.02 \\
6.3 \\
5.26 \\
31.8 \\
35 . \\
78.3\end{array}$ & $\begin{array}{l}0.001 \\
0.001 \\
0.001 \\
0.001 \\
0.001 \\
0.001 \\
0.001 \\
0.001 \\
0.001 \\
0.001 \\
0.001\end{array}$ & $\begin{array}{l}- \\
- \\
- \\
- \\
- \\
- \\
- \\
-\end{array}$ \\
\hline MANGANESE & $\begin{array}{l}0804 \\
0804 \\
0805 \\
0805\end{array}$ & $\begin{array}{l}10 / 28 / 85 \\
04 / 27 / 86 \\
10 / 28 / 85 \\
04 / 27 / 86\end{array}$ & $\begin{array}{l}0001 \\
0001 \\
0001 \\
0001\end{array}$ & $\begin{array}{l}\text { TA } \\
\text { TA } \\
\text { TA } \\
\text { TA }\end{array}$ & $\begin{array}{l}\mathbf{0} \\
0 \\
0 \\
0\end{array}$ & $M G / L$ & & $\begin{array}{l}0.37 \\
0.17 \\
0.67 \\
0.77\end{array}$ & $\begin{array}{l}0.01 \\
0.01 \\
0.01 \\
0.01\end{array}$ & $\begin{array}{l}- \\
- \\
-\end{array}$ \\
\hline
\end{tabular}

MATERIAL OF SAMPLING COOE:

TA - URANIUN MILL TAILINGS

FLOW RELATIONSHIP COOE:

PARAMETER VALUE INDICATOR (PVI): < - LESS THAN DETECTION LIHIT

SAMPLE ID COOES:

0001 - FILTERED SAMPLE (.45 MICROHS) 


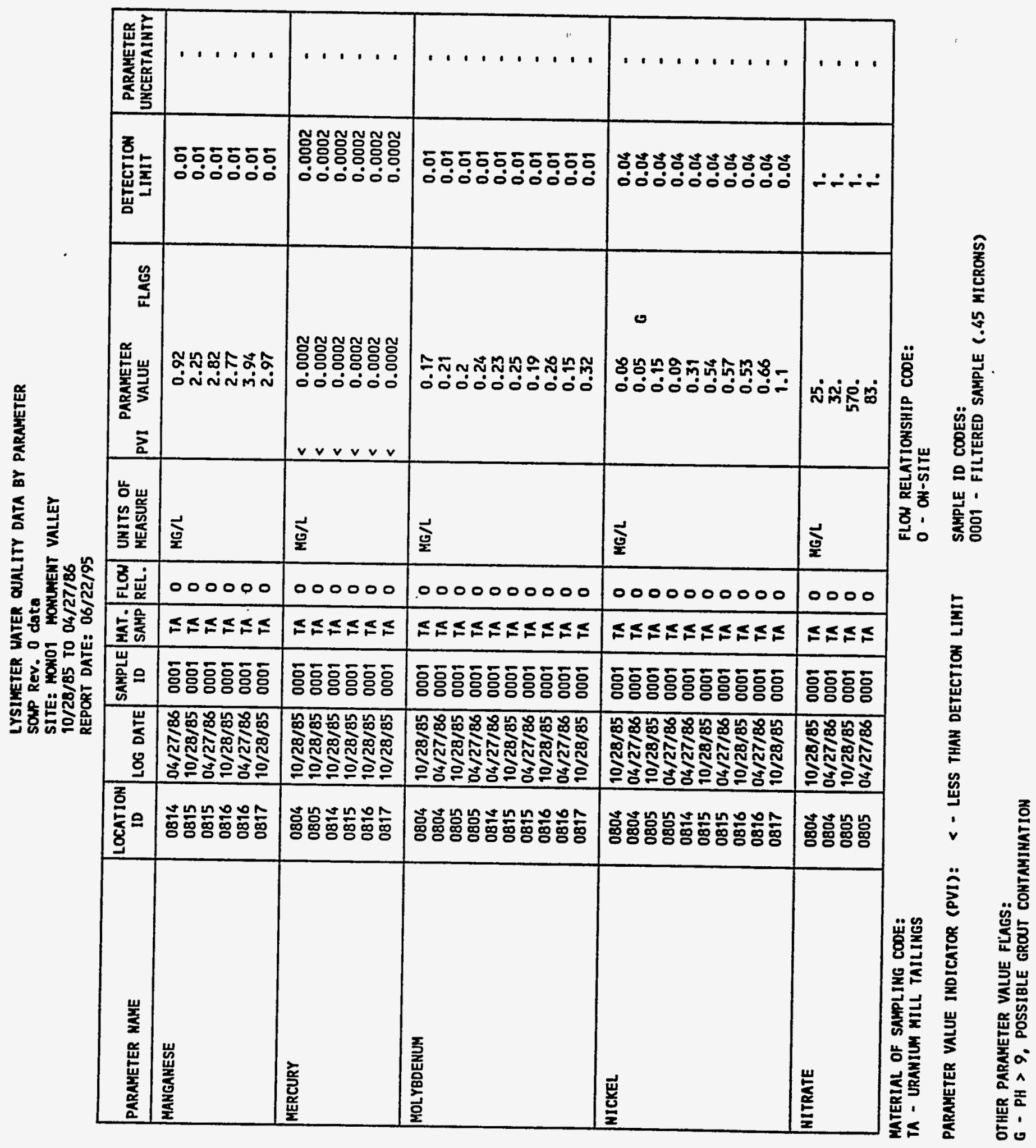


LYSIMETER WATER QUALITY DATA BY PARAMETER

SOWP Rev. 0 dato

SITE: MONO1 MOHUNENT VALLEY

$10 / 28 / 85$ TO $04 / 27 / 86$

REPORT DATE: $06 / 22 / 95$

\begin{tabular}{|c|c|c|c|c|c|c|c|c|c|c|}
\hline PARAMETER NAME & $\underset{\text { ID }}{\text { LOCATION }}$ & LOG DATE & $\begin{array}{c}\text { SAMPLE } \\
\text { ID }\end{array}$ & MAT. & $\begin{array}{l}\text { FLOW } \\
\text { REL. }\end{array}$ & $\begin{array}{l}\text { UHITS OF } \\
\text { MEASURE }\end{array}$ & PVI & $\begin{array}{l}\text { ARAMETER } \\
\text { VALUE }\end{array}$ & $\begin{array}{l}\text { DETECTION } \\
\text { LIMIT }\end{array}$ & $\begin{array}{l}\text { PARANETER } \\
\text { UNCERTAINTY }\end{array}$ \\
\hline NITRATE & $\begin{array}{l}\text { OB14 } \\
0815 \\
0816 \\
0817\end{array}$ & $\begin{array}{l}10 / 28 / 85 \\
10 / 28 / 85 \\
10 / 28 / 85 \\
10 / 28 / 85\end{array}$ & $\begin{array}{l}0001 \\
0001 \\
0001 \\
0001\end{array}$ & $\begin{array}{l}\text { TA } \\
\text { TA } \\
\text { TA } \\
\text { TA }\end{array}$ & $\begin{array}{l}0 \\
0 \\
0 \\
0\end{array}$ & MG/L & & $\begin{array}{l}14 . \\
18 \\
27 \\
530\end{array}$ & $\begin{array}{l}1 . \\
1 . \\
1 .\end{array}$ & 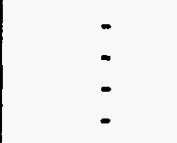 \\
\hline $\mathrm{PH}$ & $\begin{array}{l}0804 \\
0804 \\
0805 \\
0805 \\
0814 \\
0814 \\
0815 \\
0815 \\
0816 \\
0816 \\
0817\end{array}$ & $\begin{array}{l}10 / 28 / 85 \\
04 / 27 / 86 \\
10 / 28 / 85 \\
04 / 27 / 86 \\
10 / 28 / 85 \\
04 / 27 / 86 \\
10 / 28 / 85 \\
04 / 27 / 86 \\
10 / 28 / 85 \\
04 / 27 / 86 \\
10 / 28 / 85\end{array}$ & $\begin{array}{l}0001 \\
0001 \\
0001 \\
0001 \\
0001 \\
0001 \\
0001 \\
0001 \\
0001 \\
0001 \\
0001\end{array}$ & $\begin{array}{l}\text { TA } \\
\text { TA } \\
\text { TA } \\
\text { TA } \\
\text { TA } \\
\text { TA } \\
\text { TA } \\
\text { TA } \\
\text { TA } \\
\text { TA } \\
\text { TA }\end{array}$ & $\begin{array}{l}0 \\
0 \\
0 \\
0 \\
0 \\
0 \\
0 \\
0 \\
0 \\
0 \\
0\end{array}$ & SU & & $\begin{array}{l}7.63 \\
6.72 \\
7.24 \\
7.19 \\
6.64 \\
5.82 \\
6.23 \\
4.56 \\
6.32 \\
4.3 \\
6.16\end{array}$ & $\begin{array}{l}- \\
- \\
- \\
- \\
- \\
- \\
-\end{array}$ & $\begin{array}{l}- \\
- \\
- \\
- \\
- \\
- \\
- \\
-\end{array}$ \\
\hline POTASSIUH & $\begin{array}{l}0804 \\
0804 \\
0805 \\
0805 \\
0814 \\
0814 \\
0815 \\
0815 \\
0816 \\
0816 \\
0817\end{array}$ & $\begin{array}{l}10 / 28 / 85 \\
04 / 27 / 86 \\
10 / 28 / 85 \\
04 / 27 / 86 \\
10 / 28 / 85 \\
04 / 27 / 86 \\
10 / 28 / 85 \\
04 / 27 / 86 \\
10 / 28 / 85 \\
04 / 27 / 86 \\
10 / 28 / 85\end{array}$ & $\begin{array}{l}0001 \\
0001 \\
0001 \\
0001 \\
0001 \\
0001 \\
0001 \\
0001 \\
0001 \\
0001 \\
0001\end{array}$ & $\begin{array}{l}\text { TA } \\
T A \\
T A \\
T A \\
T A \\
T A \\
T A \\
T A \\
T A \\
T A \\
T A\end{array}$ & $\begin{array}{l}0 \\
0 \\
0 \\
0 \\
0 \\
0 \\
0 \\
0 \\
0 \\
0 \\
0\end{array}$ & $M G / L$ & & $\begin{array}{l}4.81 \\
3.27 \\
5.21 \\
3.25 \\
3.79 \\
2.24 \\
3.83 \\
3.13 \\
8.91 \\
6.07 \\
21.9\end{array}$ & $\begin{array}{l}0.01 \\
0.01 \\
0.01 \\
0.01 \\
0.01 \\
0.01 \\
0.01 \\
0.01 \\
0.01 \\
0.01 \\
0.01\end{array}$ & $\begin{array}{l}- \\
- \\
- \\
- \\
- \\
- \\
- \\
-\end{array}$ \\
\hline RADIUN-226 & $\begin{array}{l}0804 \\
0805 \\
0815 \\
0816 \\
0817\end{array}$ & \begin{tabular}{|l|}
$10 / 28 / 85$ \\
$10 / 28 / 85$ \\
$10 / 28 / 85$ \\
$10 / 28 / 85$ \\
$10 / 28 / 85$
\end{tabular} & $\begin{array}{l}0001 \\
0001 \\
0001 \\
0001 \\
0001\end{array}$ & $\begin{array}{l}\text { TA } \\
T A \\
T A \\
T A \\
T A\end{array}$ & $\begin{array}{l}0 \\
0 \\
0 \\
0 \\
0\end{array}$ & $\mathrm{PCI} / \mathrm{L}$ & & $\begin{array}{c}2.9 \\
4.7 \\
8.1 \\
13 . \\
3.8\end{array}$ & $\begin{array}{l}1 . \\
1 . \\
1 . \\
1 . \\
1 .\end{array}$ & $\begin{array}{l}2.1 \\
2.1 \\
3.3 \\
4.3 \\
2.3\end{array}$ \\
\hline SELENIUM & $\begin{array}{l}0804 \\
0804 \\
0805 \\
0805 \\
0814 \\
0814\end{array}$ & $\begin{array}{l}10 / 28 / 85 \\
04 / 27 / 86 \\
10 / 28 / 85 \\
04 / 27 / 86 \\
10 / 28 / 85 \\
04 / 27 / 86\end{array}$ & $\begin{array}{l}0001 \\
0001 \\
0001 \\
0001 \\
0001 \\
0001\end{array}$ & $\begin{array}{l}T A \\
T A \\
T A \\
T A \\
T A \\
T A\end{array}$ & $\begin{array}{l}0 \\
0 \\
0 \\
0 \\
0 \\
0\end{array}$ & MG/L & $\mid \begin{array}{l}< \\
< \\
< \\
< \\
< \\
<\end{array}$ & $\begin{array}{l}0.005 \\
0.005 \\
0.005 \\
0.005 \\
0.005 \\
0.005\end{array}$ & $\begin{array}{l}0.005 \\
0.005 \\
0.005 \\
0.005 \\
0.005 \\
0.005\end{array}$ & $\begin{array}{l}- \\
- \\
- \\
- \\
-\end{array}$ \\
\hline
\end{tabular}

MATERIAL OF SAMPLING COOE:

PARAMETER VALUE IHDICATOR (PVI): < - LESS THAK DETECTIOH LIMIT
FLOU RELATIONSHIP COOE:

O - OH-SITE

SAMPLE ID COOES:

0001 - FILTERED SAMPLE (.45 MICRONS) 
LYSIMETER WATER QUALITY DATA BY PARAMETER

SOWP Rev. 0 data

SITE: MOHO1 MOHUMENT VALLEY

10/28/85 T0 04/27/86

REPORT DATE: 06/22/95

\begin{tabular}{|c|c|c|c|c|c|c|c|c|c|c|}
\hline PARAMETER NAME & $\underbrace{\text { LOCATION }}_{\text {ID }}$ & LOG DATE & $\begin{array}{c}\text { SAMPLE } \\
\text { ID }\end{array}$ & $\begin{array}{l}\text { MAT. } \\
\text { SAMP }\end{array}$ & $\begin{array}{l}\text { FLON } \\
\text { REL. }\end{array}$ & $\begin{array}{l}\text { UHITS OF } \\
\text { MEASURE }\end{array}$ & PVI & $\begin{array}{l}\text { PARAMETER } \\
\text { VALUE }\end{array}$ & $\begin{array}{l}\text { DETECTION } \\
\text { LIMIT }\end{array}$ & $\begin{array}{l}\text { PARAHETER } \\
\text { UHCERTAINTY }\end{array}$ \\
\hline SELENIUM & $\begin{array}{l}0815 \\
0815 \\
0816 \\
0816 \\
0817\end{array}$ & $\begin{array}{l}10 / 28 / 85 \\
04 / 27 / 86 \\
10 / 28 / 85 \\
04 / 27 / 86 \\
10 / 28 / 85\end{array}$ & $\begin{array}{l}0001 \\
0001 \\
0001 \\
0001 \\
0001\end{array}$ & $\begin{array}{l}\text { TA } \\
\text { TA } \\
\text { TA } \\
\text { TA } \\
\text { TA }\end{array}$ & $\begin{array}{l}\mathbf{0} \\
\mathbf{0} \\
\mathbf{0} \\
\mathbf{0} \\
\mathbf{0}\end{array}$ & MG/L & $\mid \begin{array}{l}k \\
< \\
k \\
k \\
k\end{array}$ & $\begin{array}{l}0.005 \\
0.005 \\
0.005 \\
0.005 \\
0.005\end{array}$ & $\begin{array}{l}0.005 \\
0.005 \\
0.005 \\
0.005 \\
0.005\end{array}$ & $\begin{array}{l}- \\
- \\
-\end{array}$ \\
\hline SILVER & $\begin{array}{l}0804 \\
0804 \\
0805 \\
0805 \\
0814 \\
0815 \\
0815 \\
0816 \\
0816 \\
0817\end{array}$ & $\begin{array}{l}10 / 28 / 85 \\
04 / 27 / 86 \\
10 / 28 / 85 \\
04 / 27 / 86 \\
04 / 27 / 86 \\
10 / 28 / 85 \\
04 / 27 / 86 \\
10 / 28 / 85 \\
04 / 27 / 86 \\
10 / 28 / 85\end{array}$ & $\begin{array}{l}0001 \\
0001 \\
0001 \\
0001 \\
0001 \\
0001 \\
0001 \\
0001 \\
0001 \\
0001\end{array}$ & $\begin{array}{l}\text { TA } \\
\text { TA } \\
\text { TA } \\
\text { TA } \\
\text { TA } \\
\text { TA } \\
\text { TA } \\
\text { TA } \\
\text { TA } \\
\text { TA }\end{array}$ & $\begin{array}{l}0 \\
0 \\
0 \\
0 \\
0 \\
0 \\
0 \\
0 \\
0 \\
0\end{array}$ & $M G / L$ & $\mid \begin{array}{l}< \\
< \\
< \\
< \\
< \\
< \\
< \\
< \\
< \\
<\end{array}$ & $\begin{array}{l}0.01 \\
0.01 \\
0.01 \\
0.01 \\
0.01 \\
0.01 \\
0.01 \\
0.01 \\
0.01 \\
0.01\end{array}$ & $\begin{array}{l}0.01 \\
0.01 \\
0.01 \\
0.01 \\
0.01 \\
0.01 \\
0.01 \\
0.01 \\
0.01 \\
0.01\end{array}$ & $\begin{array}{l}- \\
- \\
- \\
- \\
- \\
- \\
-\end{array}$ \\
\hline SODIUM & $\begin{array}{l}0804 \\
0804 \\
0805 \\
0805 \\
0814 \\
0814 \\
0815 \\
0815 \\
0816 \\
0816 \\
0817\end{array}$ & $\begin{array}{l}10 / 28 / 85 \\
04 / 27 / 86 \\
10 / 28 / 85 \\
04 / 27 / 86 \\
10 / 28 / 85 \\
04 / 27 / 86 \\
10 / 28 / 85 \\
04 / 27 / 86 \\
10 / 28 / 85 \\
04 / 27 / 86 \\
10 / 28 / 85\end{array}$ & $\begin{array}{l}0001 \\
0001 \\
0001 \\
0001 \\
0001 \\
0001 \\
0001 \\
0001 \\
0001 \\
0001 \\
0001\end{array}$ & $\begin{array}{l}\text { TA } \\
\text { TA } \\
\text { TA } \\
\text { TA } \\
\text { TA } \\
\text { TA } \\
\text { TA } \\
\text { TA } \\
\text { TA } \\
\text { TA } \\
\text { TA }\end{array}$ & $\begin{array}{l}0 \\
0 \\
0 \\
0 \\
0 \\
0 \\
0 \\
0 \\
0 \\
0 \\
0\end{array}$ & HG/L & & $\begin{array}{c}107 . \\
171 . \\
362 . \\
232 . \\
18.1 \\
3.75 \\
9.51 \\
3.57 \\
22.6 \\
8.36 \\
70.7\end{array}$ & $\begin{array}{l}0.002 \\
0.002 \\
0.002 \\
0.002 \\
0.002 \\
0.002 \\
0.002 \\
0.002 \\
0.002 \\
0.002 \\
0.002\end{array}$ & $\begin{array}{l}- \\
- \\
- \\
- \\
- \\
- \\
- \\
-\end{array}$ \\
\hline SPECIFIC CONDUCTANCE & $\begin{array}{l}0804 \\
0805 \\
0814 \\
0815 \\
0816\end{array}$ & $\begin{array}{l}04 / 27 / 86 \\
04 / 27 / 86 \\
04 / 27 / 86 \\
04 / 27 / 86 \\
04 / 27 / 86\end{array}$ & $\begin{array}{l}0001 \\
0001 \\
0001 \\
0001 \\
0001\end{array}$ & $\begin{array}{l}\text { TA } \\
\text { TA } \\
\text { TA } \\
T A \\
\text { TA }\end{array}$ & $\begin{array}{l}0 \\
0 \\
0 \\
0 \\
0\end{array}$ & UMHO/CM & & $\begin{array}{r}435 . \\
850 . \\
800 . \\
850 . \\
1200 .\end{array}$ & $\begin{array}{l}- \\
\dot{-} \\
\dot{-}\end{array}$ & $\begin{array}{l}- \\
- \\
-\end{array}$ \\
\hline STROHTIUN & $\begin{array}{l}0804 \\
0804 \\
0805 \\
0805 \\
0814 \\
0815\end{array}$ & $\begin{array}{l}10 / 28 / 85 \\
04 / 27 / 86 \\
10 / 28 / 85 \\
04 / 27 / 86 \\
04 / 27 / 86 \\
10 / 28 / 85\end{array}$ & $\begin{array}{l}0001 \\
0001 \\
0001 \\
0001 \\
0001 \\
0001\end{array}$ & $\begin{array}{l}\text { TA } \\
\text { TA } \\
\text { TA } \\
\text { TA } \\
\text { TA } \\
\text { TA }\end{array}$ & $\begin{array}{l}\mathbf{0} \\
\mathbf{0} \\
\mathbf{0} \\
\mathbf{0} \\
\mathbf{0} \\
\mathbf{0}\end{array}$ & MG/L & & $\begin{array}{l}0.2 \\
0.1 \\
1.6 \\
1.3 \\
0.7 \\
0.7\end{array}$ & $\begin{array}{l}0.1 \\
0.1 \\
0.1 \\
0.1 \\
0.1 \\
0.1\end{array}$ & :- \\
\hline
\end{tabular}

MATERIAL OF SAMPLING COOE:

FLOW RELATIONSHIP COOE:

TA - URANIUH MILL TAILIHGS

O - ON-SITE

PARAMETER VALUE IHDICATOR (PVI): < = LESS THAN DETECTION LIMIT

SAMPLE ID CODES:

0001 - FILTERED SAMPLE (.45 MICRONS) 
LYSIMETER WATER QUALITY DATA BY PARAMETER

SOWP REV. 0 data

SITE: MOHO1 MONUMEHT VALLEY

10/28/85 TO 04/27/86

REPORT DATE: 06/22/95

\begin{tabular}{|c|c|c|c|c|c|c|c|c|c|c|}
\hline PARAMETER HAME & $\underset{\text { ID }}{\text { LOCATION }}$ & LOG DATE & $\begin{array}{c}\text { SAMPLE } \\
\text { ID }\end{array}$ & $\begin{array}{l}\text { MAT. } \\
\text { SAMP }\end{array}$ & $\begin{array}{l}\text { FL.OW } \\
\text { REL. }\end{array}$ & $\begin{array}{l}\text { UNITS OF } \\
\text { MEASURE }\end{array}$ & PVI & $\begin{array}{l}\text { PARAMETER } \\
\text { VALUE }\end{array}$ & $\begin{array}{l}\text { DETECTION } \\
\text { LIMIT }\end{array}$ & $\begin{array}{l}\text { PARAMETER } \\
\text { UNCERTAINTY }\end{array}$ \\
\hline STRONTIUM & $\begin{array}{l}0815 \\
0816 \\
0816 \\
0817\end{array}$ & $\begin{array}{l}04 / 27 / 86 \\
10 / 28 / 85 \\
04 / 27 / 86 \\
10 / 28 / 85\end{array}$ & \begin{tabular}{l|}
0001 \\
0001 \\
0001 \\
0001
\end{tabular} & $\begin{array}{l}\text { TA } \\
T A \\
T A \\
T A\end{array}$ & $\begin{array}{l}0 \\
0 \\
0 \\
0\end{array}$ & $M G / L$ & & $\begin{array}{l}0.7 \\
0.7 \\
0.7 \\
0.7\end{array}$ & $\begin{array}{l}0.1 \\
0.1 \\
0.1 \\
0.1\end{array}$ & $\begin{array}{l}- \\
-\end{array}$ \\
\hline SULFATE & $\begin{array}{l}0804 \\
0804 \\
0805 \\
0805 \\
0814 \\
0815 \\
0816 \\
0817\end{array}$ & \begin{tabular}{|l|}
$10 / 28 / 85$ \\
$04 / 27 / 86$ \\
$10 / 28 / 85$ \\
$04 / 27 / 86$ \\
$10 / 28 / 85$ \\
$10 / 28 / 85$ \\
$10 / 28 / 85$ \\
$10 / 28 / 85$ \\
\end{tabular} & $\begin{array}{l}0001 \\
0001 \\
0001 \\
0001 \\
0001 \\
0001 \\
0001 \\
0001\end{array}$ & $\begin{array}{l}\text { TA } \\
\text { TA } \\
\text { TA } \\
\text { TA } \\
\text { TA } \\
\text { TA } \\
\text { TA } \\
\text { TA }\end{array}$ & $\begin{array}{l}0 \\
0 \\
0 \\
0 \\
0 \\
0 \\
0 \\
0\end{array}$ & MG/L & & $\begin{array}{c}212 . \\
286 . \\
1610 . \\
738 . \\
1420 . \\
1310 . \\
2050 . \\
4510 .\end{array}$ & $\begin{array}{l}0.1 \\
0.1 \\
0.1 \\
0.1 \\
0.1 \\
0.1 \\
0.1 \\
0.1\end{array}$ & $\begin{array}{l}: \\
: \\
: \\
: \\
:\end{array}$ \\
\hline TEMPERATURE & $\begin{array}{l}0804 \\
0805 \\
0814 \\
0815 \\
0816\end{array}$ & $\begin{array}{l}04 / 27 / 86 \\
04 / 27 / 86 \\
04 / 27 / 86 \\
04 / 27 / 86 \\
04 / 27 / 86\end{array}$ & $\begin{array}{l}0001 \\
0001 \\
0001 \\
0001 \\
0001\end{array}$ & $\begin{array}{l}\text { TA } \\
\text { TA } \\
\text { TA } \\
\text { TA } \\
\text { TA }\end{array}$ & $\begin{array}{l}0 \\
0 \\
0 \\
0 \\
0\end{array}$ & C - DEGREE & & $\begin{array}{l}16 . \\
15.5 \\
16 . \\
16 . \\
16 .\end{array}$ & $\begin{array}{l}- \\
- \\
-\end{array}$ & $\begin{array}{l}- \\
- \\
-\end{array}$ \\
\hline TIH & $\begin{array}{l}0804 \\
0804 \\
0805 \\
0805 \\
0814 \\
0815 \\
0815 \\
0816 \\
0816 \\
0817\end{array}$ & $\begin{array}{l}10 / 28 / 85 \\
04 / 27 / 86 \\
10 / 28 / 85 \\
04 / 27 / 86 \\
04 / 27 / 86 \\
10 / 28 / 85 \\
04 / 27 / 86 \\
10 / 28 / 85 \\
04 / 27 / 86 \\
10 / 28 / 85\end{array}$ & $\begin{array}{l}0001 \\
0001 \\
0001 \\
0001 \\
0001 \\
0001 \\
0001 \\
0001 \\
0001 \\
0001\end{array}$ & $\begin{array}{l}\text { TA } \\
\text { TA } \\
\text { TA } \\
\text { TA } \\
\text { TA } \\
\text { TA } \\
\text { TA } \\
\text { TA } \\
\text { TA } \\
\text { TA }\end{array}$ & $\begin{array}{l}0 \\
0 \\
0 \\
0 \\
0 \\
0 \\
0 \\
0 \\
0 \\
0\end{array}$ & MG/L & $\begin{array}{l}< \\
< \\
< \\
< \\
< \\
< \\
< \\
< \\
< \\
< \\
<\end{array}$ & $\begin{array}{l}0.005 \\
0.005 \\
0.005 \\
0.005 \\
0.005 \\
0.005 \\
0.005 \\
0.005 \\
0.005 \\
0.005\end{array}$ & $\begin{array}{l}0.005 \\
0.005 \\
0.005 \\
0.005 \\
0.005 \\
0.005 \\
0.005 \\
0.005 \\
0.005 \\
0.005\end{array}$ & $\begin{array}{l}- \\
- \\
- \\
- \\
- \\
-\end{array}$ \\
\hline TOTAL DISSOLVED SOLIDS & $\begin{array}{l}0804 \\
0805 \\
0814 \\
0815 \\
0816 \\
0817\end{array}$ & $\mid \begin{array}{l}10 / 28 / 85 \\
10 / 28 / 85 \\
10 / 28 / 85 \\
10 / 28 / 85 \\
10 / 28 / 85 \\
10 / 28 / 85\end{array}$ & $\begin{array}{l}0001 \\
0001 \\
0001 \\
0001 \\
0001 \\
0001\end{array}$ & $\begin{array}{l}\text { TA } \\
\text { TA } \\
\text { TA } \\
\text { TA } \\
\text { TA } \\
\text { TA }\end{array}$ & $\begin{array}{l}\mathbf{0} \\
\mathbf{0} \\
\mathbf{0} \\
\mathbf{0} \\
\mathbf{0} \\
\mathbf{0}\end{array}$ & MG/L & & $\begin{array}{l}703 . \\
2900 . \\
2220 . \\
2080 . \\
3140 . \\
6850 .\end{array}$ & $\begin{array}{l}10 . \\
10 . \\
10 . \\
10 . \\
10 . \\
10 .\end{array}$ & $\begin{array}{l}- \\
- \\
-\end{array}$ \\
\hline URANIUM & $\begin{array}{l}0804 \\
0804 \\
0805\end{array}$ & \begin{tabular}{|l|}
$10 / 28 / 85$ \\
$04 / 27 / 86$ \\
$10 / 28 / 85$
\end{tabular} & $\begin{array}{l}0001 \\
0001 \\
0001\end{array}$ & $\begin{array}{l}\text { TA } \\
\text { TA } \\
\text { TA }\end{array}$ & $\begin{array}{l}0 \\
0 \\
0\end{array}$ & MG/L & & $\begin{array}{l}0.0201 \\
0.0205 \\
0.78\end{array}$ & $\begin{array}{l}0.003 \\
0.003 \\
0.003\end{array}$ & - \\
\hline
\end{tabular}

MATERIAL OF SAMPLING COOE:

TA - URANIUH MILL TAILIHGS

FLON RELATIONSHIP CODE:

PARAMETER VALUE INDICATOR (PVI): < - LESS THAH DETECTION LIMIT

SNMPLE ID COOES:

0001 - FILTERED SAMPLE (.45 MICRONS) 
LYSIMETER MATER OUALITY DATA BY PARNMETER

SOWP Rev. 0 doto

SITE: MONO1 MONUNENT VALLEY

10/28/85 TO 04/27/86

REPORT DATE: 06/22/95

\begin{tabular}{|c|c|c|c|c|c|c|c|c|c|c|c|}
\hline PARAMETER MAME & $\underset{\text { ID }}{\text { LOCATION }}$ & LOG DATE & $\begin{array}{c}\text { SAMPLE } \\
\text { ID }\end{array}$ & $\begin{array}{l}\text { MAT. } \\
\text { SAMP }\end{array}$ & $\begin{array}{l}\text { FLOW } \\
\text { REL. }\end{array}$ & $\begin{array}{l}\text { UHITS OF } \\
\text { MEASURE }\end{array}$ & PVI & $\begin{array}{l}\text { PARAMETER } \\
\text { VALUE }\end{array}$ & FLAGS & $\begin{array}{l}\text { DETECTION } \\
\text { LIMIT }\end{array}$ & $\begin{array}{c}\text { PARAMETER } \\
\text { UNCERTAIHTY }\end{array}$ \\
\hline URANIUN & $\begin{array}{l}0805 \\
0814 \\
0814 \\
0815 \\
0815 \\
0816 \\
0816 \\
0817\end{array}$ & $\begin{array}{l}04 / 27 / 86 \\
10 / 28 / 85 \\
04 / 27 / 86 \\
10 / 28 / 85 \\
04 / 27 / 86 \\
10 / 28 / 85 \\
04 / 27 / 86 \\
10 / 28 / 85\end{array}$ & $\begin{array}{l}0001 \\
0001 \\
0001 \\
0001 \\
0001 \\
0001 \\
0001 \\
0001\end{array}$ & $\begin{array}{l}\text { TA } \\
T A \\
T A \\
T A \\
T A \\
T A \\
T A \\
T A\end{array}$ & $\begin{array}{l}0 \\
0 \\
0 \\
0 \\
0 \\
0 \\
0 \\
0\end{array}$ & $M G / L$ & & $\begin{array}{l}1.08 \\
0.0033 \\
0.0006 \\
0.036 \\
0.0064 \\
0.0753 \\
0.054 \\
0.0008\end{array}$ & $\begin{array}{l}\mathbf{J} \\
\mathbf{J}\end{array}$ & $\begin{array}{l}0.003 \\
0.003 \\
0.003 \\
0.003 \\
0.003 \\
0.003 \\
0.003 \\
0.003\end{array}$ & $\begin{array}{l}- \\
: \\
: \\
- \\
-\end{array}$ \\
\hline VANADIUM & $\begin{array}{l}0804 \\
0804 \\
0805 \\
0805 \\
0814 \\
0815 \\
0815 \\
0816 \\
0816 \\
0817\end{array}$ & $\begin{array}{l}10 / 28 / 85 \\
04 / 27 / 86 \\
10 / 28 / 85 \\
04 / 27 / 86 \\
04 / 27 / 86 \\
10 / 28 / 85 \\
04 / 27 / 86 \\
10 / 28 / 85 \\
04 / 27 / 86 \\
10 / 28 / 85\end{array}$ & $\begin{array}{l}0001 \\
0001 \\
0001 \\
0001 \\
0001 \\
0001 \\
0001 \\
0001 \\
0001 \\
0001\end{array}$ & $\begin{array}{l}\text { TA } \\
\text { TA } \\
\text { TA } \\
\text { TA } \\
\text { TA } \\
\text { TA } \\
\text { TA } \\
\text { TA } \\
\text { IA } \\
\text { TA }\end{array}$ & $\begin{array}{l}0 \\
0 \\
0 \\
0 \\
0 \\
0 \\
0 \\
0 \\
0 \\
0\end{array}$ & $M G / L$ & & $\begin{array}{l}0.3 \\
0.69 \\
0.62 \\
0.91 \\
1.08 \\
0.48 \\
0.89 \\
0.26 \\
0.97 \\
0.25\end{array}$ & & $\begin{array}{l}0.01 \\
0.01 \\
0.01 \\
0.01 \\
0.01 \\
0.01 \\
0.01 \\
0.01 \\
0.01 \\
0.01\end{array}$ & $\begin{array}{l}- \\
: \\
: \\
- \\
- \\
- \\
-\end{array}$ \\
\hline ZINC & $\begin{array}{l}0804 \\
0804 \\
0805 \\
0805 \\
0814 \\
0815 \\
0815 \\
0816 \\
0816 \\
0817\end{array}$ & $\begin{array}{l}10 / 28 / 85 \\
04 / 27 / 86 \\
10 / 28 / 85 \\
04 / 27 / 86 \\
04 / 27 / 86 \\
10 / 28 / 85 \\
04 / 27 / 86 \\
10 / 28 / 85 \\
04 / 27 / 86 \\
10 / 28 / 85\end{array}$ & $\begin{array}{l}0001 \\
0001 \\
0001 \\
0001 \\
0001 \\
0001 \\
0001 \\
0001 \\
0001 \\
0001\end{array}$ & $\begin{array}{l}T A \\
T A \\
T A \\
T A \\
T A \\
T A \\
T A \\
T A \\
T A \\
T A\end{array}$ & $\begin{array}{l}0 \\
0 \\
0 \\
0 \\
0 \\
0 \\
0 \\
0 \\
0 \\
0\end{array}$ & $M G / L$ & & $\begin{array}{l}0.06 \\
0.129 \\
0.129 \\
0.107 \\
1.41 \\
1.82 \\
0.912 \\
3.86 \\
0.86 \\
2.09\end{array}$ & & $\begin{array}{l}0.005 \\
0.005 \\
0.005 \\
0.005 \\
0.005 \\
0.005 \\
0.005 \\
0.005 \\
0.005 \\
0.005\end{array}$ & $\begin{array}{l}- \\
: \\
- \\
- \\
- \\
-\end{array}$ \\
\hline
\end{tabular}

MATERIAL OF SAMPLING CODE:

FLON RELATIONSHIP COOE:

TA - URANIUM MILL TAILINGS

O - OH-SITE

PARAMETER VALUE IMDICATOR (PVI): < - LESS THAH DETECTION LIMIT

SAMPLE ID COOES:

0001 - FILTERED SAMPLE (.45 MICRONS)

OTHER PARAHETER VALUE FLAGS:

J - ESTIMATEd VALUE

DATA FILE HAME: IOARTIMOHO1YLYS10000.DAT 
$\therefore$ 
SURFACE WATER QUALITY DATA BY LOCATIOH

SOWP ReV. 0 data

SITE: MOHO1 HONUMENT VALLEY

LOCATIOH: 0620

NORTH COORDIHATE: 70685.0 FT

EAST COORDINATE: 90918.8 FT

$04 / 11 / 88$ TO $12 / 14 / 94$

REPORT DATE: 06/22/95

\begin{tabular}{|c|c|c|c|c|c|c|c|c|}
\hline PARAMETER NAME & LOG DATE & $\begin{array}{c}\text { SAMPLE } \\
\text { ID }\end{array}$ & $\begin{array}{l}\text { UNITS OF } \\
\text { HEASURE }\end{array}$ & PVI & $\begin{array}{l}\text { PARAMETER } \\
\text { VALUE }\end{array}$ & FLAGS & $\begin{array}{l}\text { DETECTION } \\
\text { LIMIT }\end{array}$ & $\begin{array}{l}\text { PARAMETER } \\
\text { UMCERTAINTY }\end{array}$ \\
\hline ALKALIHITY & $06 / 27 / 93$ & NOOI & $\mathrm{MG} / \mathrm{L} \mathrm{CACO}$ & \multicolumn{3}{|c|}{352} & - & - \\
\hline AmONIUM (TOTAL) & $06 / 27 / 93$ & NoOI & MG/L & \multicolumn{3}{|c|}{0.2} & 0.1 & $\cdot$ \\
\hline ARSENIC (TOTAL) & $06 / 27 / 93$ & NOOI & MG/L & \multicolumn{3}{|c|}{0.019} & 0.005 & - \\
\hline BARIUN (TOTAL) & $06 / 27 / 93$ & NOOI & $M G / L$ & \multicolumn{3}{|c|}{0.4} & 0.1 & - \\
\hline CALCIUA (TOTAL) & $06 / 27 / 93$ & NoOI & MG/L & \multicolumn{3}{|c|}{46.7} & 0.5 & - \\
\hline CHLORIDE (TOTAL) & $06 / 27 / 93$ & NOOI & MG/L & \multicolumn{3}{|c|}{14.5} & 0.5 & $\cdot$ \\
\hline CROSS ALPHA (TOTAL) & $06 / 27 / 93$ & H0O1 & $\mathrm{PCI} / \mathrm{L}$ & \multicolumn{3}{|c|}{39} & 5 & 13 \\
\hline GROSS BETA (TOTAL) & $06 / 27 / 93$ & HOOI & $\mathrm{PCI} / \mathrm{L}$ & \multicolumn{3}{|c|}{28} & 5 & 6 \\
\hline IROH (TOTAL) & $06 / 27 / 93$ & N001 & $M G / L$ & \multicolumn{3}{|c|}{14.5} & 0.03 & - \\
\hline MAGHESIUM (TOTAL) & $06 / 27 / 93$ & N001 & $M G / L$ & \multicolumn{3}{|c|}{27.2} & 0.9 & - \\
\hline MAMGAKESE (TOTAL) & $06 / 27 / 93$ & N001 & $M G / L$ & \multicolumn{3}{|c|}{1.53} & 0.01 & $\cdot$ \\
\hline MOLYBDENUH (TOTAL) & $06 / 27 / 93$ & No01 & MG/L & \multicolumn{3}{|c|}{0.03} & 0.01 & - \\
\hline NET GROSS ALPHA (TOTAL) ** & $06 / 27 / 93$ & N001 & $\mathrm{PCI} / \mathrm{L}$ & \multicolumn{3}{|c|}{19.79} & - & - \\
\hline MITRATE (TOTAL) & $06 / 27 / 93$ & N001 & $M G / L$ & \multicolumn{3}{|c|}{1} & 1 & - \\
\hline $\mathrm{PH}$ & $06 / 27 / 93$ & HOO1 & SU & \multicolumn{3}{|c|}{8.03} & - & - \\
\hline PHOSPHATE (TOTAL) & $06 / 27 / 93$ & N001 & $M G / L$ & \multicolumn{3}{|c|}{1.4} & 0.1 & - \\
\hline POTASSIUM (TOTAL) & $06 / 27 / 93$ & NOOI & $M G / L$ & \multicolumn{3}{|c|}{7.3} & 0.1 & - \\
\hline SILICA - SIO2 (TOTAL) & $06 / 27 / 93$ & NOOI & MG/L & \multicolumn{3}{|c|}{$32.1 \quad H$} & 0.5 & - \\
\hline SOOIUH (TOTAL) & $06 / 27 / 93$ & N001 & $M G / L$ & \multicolumn{3}{|c|}{112} & 1 & - \\
\hline SPECIFIC CONDUCTANCE & $06 / 27 / 93$ & N001 & UHHO/CH & \multicolumn{3}{|c|}{831} & - & - \\
\hline STRONTIUM (TOTAL) & $06 / 27 / 93$ & N001 & $M G / L$ & \multicolumn{3}{|c|}{0.42} & 0.01 & - \\
\hline SULFATE (TOTAL) & $06 / 27 / 93$ & HOO1 & $M G / L$ & \multicolumn{3}{|c|}{84} & 1 & - \\
\hline TEMPERATURE & $06 / 27 / 93$ & NOO1 & $C$ - DEGREE & \multicolumn{3}{|c|}{27.0} & - & - \\
\hline TOTAL DISSOLVED SOLIDS (TOTAL) & $06 / 27 / 93$ & N001 & $M G / L$ & \multicolumn{3}{|c|}{490} & 10 & $\cdot$ \\
\hline URAMIUN (TOTAL) & $06 / 27 / 93$ & N001 & MG/L & \multicolumn{3}{|c|}{0.028} & 0.001 & - \\
\hline VAHADIUH (TOTAL) & $06 / 27 / 93$ & N001 & $M G / L$ & \multicolumn{3}{|c|}{0.04} & 0.01 & $\cdot$ \\
\hline ZIKC (TOTAL) & $06 / 27 / 93$ & N001 & $M G / L$ & & 0.033 & $\mathbf{J}$ & 0.005 & - \\
\hline
\end{tabular}

* NET GROSS ALPHA (TOTAL)

(TOTAL GROSS ALPHA - TOTAL URAHIUH)

PARAKETER VALUE IMDICATOR (PVI): < - LESS THAN DETECTION LIMIT

OTHER PARAMETER VALUE FLAGS:

H - HOLD TIME EXPIRED, VALUE SUSPECT

J. Estimated value 
SURFACE WRTER QUALITY DATA BY LOCATION

SOWP ReV. 0 data

SITE: MONO1 MONUMENT VALLEY

LOCATION: 0620

NORTH COORDINATE: 70685.0 FT

EAST COORDINATE: 90918.8 FT

$04 / 11 / 88$ TO 12/14/94

REPORT DATE: $06 / 22 / 95$

\begin{tabular}{|c|c|c|c|c|c|c|c|c|}
\hline PARAHETER NAME & LOG DATE & $\underset{10}{\text { SAMPLE }}$ & $\begin{array}{l}\text { UNITS OF } \\
\text { MEASURE }\end{array}$ & PVI & $\begin{array}{l}\text { PARANETER } \\
\text { VALUE }\end{array}$ & FLAGS & $\begin{array}{l}\text { DETECTION } \\
\text { LIMIT }\end{array}$ & $\begin{array}{l}\text { PARAMETER } \\
\text { UNCERTAIHTY }\end{array}$ \\
\hline
\end{tabular}

PARAMETER VALUE IHDICATOR (PVI): < - LESS THAN DETECTION LIMIT

SAMPLE ID COOES: 
SURFACE WATER QUALITY DATA BY LOCATION

SOWP ReV. 0 data

SITE: MONOI MONUMENT VALLEY

LOCATION: 0621

NORTH COORDIHATE: 57935.9 FT

EAST COORDINATE: 90924.4 FT

$04 / 19 / 88$ TO $12 / 14 / 94$

REPORT DATE: 06/22/95

\begin{tabular}{|c|c|c|c|c|c|c|c|c|}
\hline PARAMETER MAME & LOG DATE & $\begin{array}{c}\text { SAMPLE } \\
\text { ID }\end{array}$ & $\begin{array}{l}\text { UHITS OF } \\
\text { MEASURE }\end{array}$ & PVI & $\begin{array}{l}\text { PARAMETER } \\
\text { VALUE }\end{array}$ & FLAGS & $\begin{array}{l}\text { DETECTION } \\
\text { LIMIT }\end{array}$ & $\begin{array}{l}\text { PARAMETER } \\
\text { UNCERTAINTY }\end{array}$ \\
\hline ALKALIKITY & $\begin{array}{l}06 / 09 / 89 \\
06 / 01 / 91 \\
02 / 21 / 92 \\
08 / 14 / 92 \\
11 / 22 / 92\end{array}$ & $\begin{array}{l}0001 \\
0001 \\
0001 \\
0001 \\
H 001\end{array}$ & MG/L CACO3 & & $\begin{array}{l}201 . \\
237 \\
278 \\
194 \\
361\end{array}$ & J & $\begin{array}{l}- \\
: \\
-\end{array}$ & $\begin{array}{l}- \\
- \\
- \\
.\end{array}$ \\
\hline ALUHINUA & $\begin{array}{l}06 / 09 / 89 \\
06 / 01 / 91 \\
02 / 21 / 92 \\
08 / 14 / 92\end{array}$ & $\begin{array}{l}0001 \\
0001 \\
0001 \\
0001\end{array}$ & MG/L & $\begin{array}{l}< \\
< \\
<\end{array}$ & $\begin{array}{l}0.1 \\
0.05 \\
0.05 \\
0.2\end{array}$ & $J$ & $\begin{array}{l}0.1 \\
0.05 \\
0.05 \\
0.2\end{array}$ & i- \\
\hline AmHONIUA & $\begin{array}{l}06 / 09 / 89 \\
06 / 01 / 91 \\
02 / 29 / 92\end{array}$ & $\begin{array}{l}0001 \\
0001 \\
0001\end{array}$ & MG/L & $<$ & $\begin{array}{l}0.1 \\
2.1 \\
0.1\end{array}$ & $J$ & $\begin{array}{l}0.1 \\
0.1 \\
0.1\end{array}$ & : \\
\hline AKTIMONY & $\begin{array}{l}06 / 09 / 89 \\
06 / 01 / 91 \\
02 / 21 / 92 \\
08 / 14 / 92\end{array}$ & $\begin{array}{l}0001 \\
0001 \\
0001 \\
0001\end{array}$ & $M G / L$ & $\begin{array}{l}< \\
< \\
<\end{array}$ & $\begin{array}{l}0.003 \\
0.003 \\
0.003 \\
0.06\end{array}$ & $\begin{array}{l}J \\
H\end{array}$ & $\begin{array}{l}0.003 \\
0.003 \\
0.003 \\
0.06\end{array}$ & : \\
\hline ARSEHIC & $\begin{array}{l}06 / 09 / 89 \\
06 / 01 / 91 \\
02 / 21 / 92 \\
08 / 14 / 92\end{array}$ & $\begin{array}{l}0001 \\
0001 \\
0001 \\
0001\end{array}$ & MG/L & $<$ & $\begin{array}{l}0.01 \\
0.01 \\
0.01 \\
0.01\end{array}$ & J & $\begin{array}{l}0.01 \\
0.01 \\
0.01 \\
0.01\end{array}$ & $\begin{array}{l}- \\
- \\
-\end{array}$ \\
\hline ARSENIC (TOTAL) & $11 / 22 / 92$ & NOOI & MG/L & $<$ & 0.0015 & & 0.0015 & - \\
\hline BARIUA & $\begin{array}{l}06 / 09 / 89 \\
06 / 01 / 91 \\
02 / 21 / 92 \\
08 / 14 / 92\end{array}$ & $\begin{array}{l}0001 \\
0001 \\
0001 \\
0001\end{array}$ & MG/L & & $\begin{array}{l}0.2 \\
0.18 \\
0.19 \\
0.22\end{array}$ & 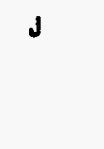 & $\begin{array}{l}0.1 \\
0.09 \\
0.01 \\
0.2\end{array}$ & :- \\
\hline BARIUH (TOTAL) & $11 / 22 / 92$ & H0O1 & MG/L & & 0.15 & & 0.0015 & - \\
\hline BERYLLIUM & $\begin{array}{l}06 / 09 / 89 \\
06 / 01 / 91 \\
08 / 14 / 92\end{array}$ & $\begin{array}{l}0001 \\
0001 \\
0001\end{array}$ & $M G / L$ & $\begin{array}{l}< \\
< \\
<\end{array}$ & $\begin{array}{l}0.01 \\
0.005 \\
0.005\end{array}$ & J & $\begin{array}{l}0.01 \\
0.005 \\
0.005\end{array}$ & : \\
\hline BOROH & $\begin{array}{l}06 / 09 / 89 \\
06 / 01 / 91\end{array}$ & $\begin{array}{l}0009 \\
0001\end{array}$ & MG/L & $<$ & $\begin{array}{l}0.1 \\
0.08\end{array}$ & $J$ & $\begin{array}{l}0.1 \\
0.05\end{array}$ & - \\
\hline BROMIDE & $06 / 01 / 91$ & 0001 & MG/L & $<$ & 0.1 & & 0.1 & - \\
\hline CADHIU: & $\begin{array}{l}06 / 09 / 89 \\
06 / 01 / 91 \\
02 / 21 / 92 \\
08 / 14 / 92\end{array}$ & $\begin{array}{l}0001 \\
0001 \\
0001 \\
0001\end{array}$ & MG/L & $\begin{array}{l}< \\
< \\
< \\
<\end{array}$ & $\begin{array}{l}0.001 \\
0.001 \\
0.0005 \\
0.005\end{array}$ & $\begin{array}{l}\text { J } \\
\text { E }\end{array}$ & $\begin{array}{l}0.001 \\
0.001 \\
0.0005 \\
0.005\end{array}$ & $\begin{array}{l}- \\
- \\
-\end{array}$ \\
\hline CADMIUM (TOTAL) & $11 / 22 / 92$ & N001 & $M G / L$ & $<$ & 0.00013 & 3 HH & 0.00013 & - \\
\hline CALCIUM & $\begin{array}{l}06 / 09 / 89 \\
06 / 01 / 91 \\
02 / 21 / 92\end{array}$ & $\begin{array}{l}0001 \\
0001 \\
0001\end{array}$ & $M G / L$ & & $\begin{array}{l}43.7 \\
33.5 \\
39.7\end{array}$ & J & $\begin{array}{l}0.01 \\
0.5 \\
0.5\end{array}$ & $:$ \\
\hline
\end{tabular}

PARAMETER VALUE IMDICATOR (PVI): < - LESS THAN DETECTION LIMIT

OTHER PARAMETER VALUE FLAGS:

E - ESTIMATED VALUE bECAUSE OF IHTERFERENCE, SEE CASE MARRATIVE

J - ESTIMATED VALUE

- SPIKE SAMPLE RECOVERY NOT HITHIN CONTROL LIMITS

$W$ - POST-DIGEST SPIKE OUT OF CNTR LIM WHILE SAMP ABS < 50\% SPIKE
SNYPLE IO COOES:

O001 - FILTERED SAMLE (.45 MICRONS)

NOO1 - UMFILTERED SAMPLE 
SURFACE WATER QUALITY DATA BY LOCATION

SOWP ReV. 0 data

SITE: MONO1 MONUMENT VALLEY

LOCATION: 0621

NORTH COORDINATE: 57935.9 FT

EAST COORDINATE: 90924.4 FT

$04 / 91 / 88$ TO $12 / 14 / 94$

REPORT DATE: 06/22/95

\begin{tabular}{|c|c|c|c|c|c|c|c|c|}
\hline PARAMETER RAME & LOG DATE & $\underset{\text { ID }}{\text { SAMPLE }}$ & $\begin{array}{l}\text { UNITS OF } \\
\text { MEASURE }\end{array}$ & PVI & $\begin{array}{l}\text { PARAMETER } \\
\text { VALUE }\end{array}$ & FLAGS & $\begin{array}{l}\text { DETECTIOH } \\
\text { LIMIT }\end{array}$ & $\begin{array}{l}\text { PARAMETER } \\
\text { UHCERTAINTY }\end{array}$ \\
\hline CALCIUH & $08 / 14 / 92$ & 0009 & $M G / L$ & \multicolumn{3}{|c|}{38} & 5 & - \\
\hline CHLORIDE & $\begin{array}{l}06 / 09 / 89 \\
06 / 01 / 91 \\
02 / 21 / 92 \\
08 / 14 / 92\end{array}$ & $\begin{array}{l}0001 \\
0001 \\
0001 \\
0001\end{array}$ & $M G / L$ & & $\begin{array}{r}4.9 \\
8.0 \\
11.1 \\
5.3\end{array}$ & J & $\begin{array}{l}1.1 \\
0.5 \\
0.5 \\
0.016\end{array}$ & $:$ \\
\hline CHROMIUY & $\begin{array}{l}06 / 09 / 89 \\
06 / 01 / 91 \\
02 / 21 / 92 \\
08 / 14 / 92\end{array}$ & $\begin{array}{l}0001 \\
0001 \\
0001 \\
0001\end{array}$ & MG/L & $\begin{array}{l}< \\
< \\
<\end{array}$ & $\begin{array}{l}0.01 \\
0.01 \\
0.01 \\
0.01\end{array}$ & J & $\begin{array}{l}0.01 \\
0.01 \\
0.01 \\
0.01\end{array}$ & $:$ \\
\hline CHROHIUN (TOTAL) & $19 / 22 / 92$ & HOO1 & $M G / L$ & $<$ & 0.0053 & & 0.0053 & - \\
\hline COBALT & $\begin{array}{l}06 / 09 / 89 \\
06 / 01 / 91\end{array}$ & $\begin{array}{l}0001 \\
0001\end{array}$ & $M G / L$ & $<$ & $\begin{array}{l}0.05 \\
0.03\end{array}$ & $\mathrm{~J}$ & $\begin{array}{l}0.05 \\
0.03\end{array}$ & $\overline{-}$ \\
\hline COPPER & $\begin{array}{l}06 / 09 / 89 \\
06 / 01 / 91\end{array}$ & $\begin{array}{l}0001 \\
0001\end{array}$ & $M G / L$ & $<$ & $\begin{array}{l}0.02 \\
0.01\end{array}$ & J & $\begin{array}{l}0.02 \\
0.01\end{array}$ & : \\
\hline CYANIDE & $06 / 09 / 89$ & 0009 & MG/L & $<$ & 0.01 & $\mathrm{~J}$ & 0.01 & - \\
\hline FLUORIDE & $\begin{array}{l}06 / 09 / 89 \\
06 / 01 / 91\end{array}$ & $\begin{array}{l}0001 \\
0001\end{array}$ & $M G / L$ & & $\begin{array}{l}0.4 \\
0.2\end{array}$ & J & $\begin{array}{l}0.1 \\
0.1\end{array}$ & : \\
\hline GROSS ALPHA & $\begin{array}{l}06 / 01 / 91 \\
02 / 21 / 92 \\
08 / 14 / 92\end{array}$ & $\begin{array}{l}0001 \\
0001 \\
0001\end{array}$ & PCI/L & & $\begin{array}{l}41.6 \\
19.7 \\
13\end{array}$ & & 9.0 & $\begin{array}{l}8.4 \\
6.7 \\
5\end{array}$ \\
\hline GROSS ALPHA (TOTAL) & $19 / 22 / 92$ & N001 & PCI/L & & 2.1 & N & 8.8 & 5.5 \\
\hline GROSS BETA & $\begin{array}{l}06 / 01 / 91 \\
02 / 21 / 92 \\
08 / 14 / 92\end{array}$ & $\begin{array}{l}0009 \\
0001 \\
0009\end{array}$ & $\mathrm{PCI} / \mathrm{L}$ & & $\begin{array}{r}17.6 \\
13.8 \\
7.4\end{array}$ & & $\begin{array}{l}0.5 \\
0.5 \\
4.2\end{array}$ & $\begin{array}{l}3.6 \\
3.3 \\
3.0\end{array}$ \\
\hline GROSS BETA (TOTAL) & $11 / 22 / 92$ & N009 & PCI/L & & 8.4 & N & 6.5 & 4.3 \\
\hline IRON & $\begin{array}{l}06 / 09 / 89 \\
06 / 01 / 91 \\
02 / 21 / 92 \\
08 / 14 / 92\end{array}$ & $\begin{array}{l}0001 \\
0001 \\
0001 \\
0001\end{array}$ & MG/L & $<$ & $\begin{array}{l}0.03 \\
0.03 \\
0.03 \\
0.1\end{array}$ & J & $\begin{array}{l}0.03 \\
0.03 \\
0.03 \\
0.1\end{array}$ & $:$ \\
\hline LEAD & $\begin{array}{l}06 / 09 / 89 \\
06 / 01 / 99\end{array}$ & $\begin{array}{l}0001 \\
0001\end{array}$ & MG/L & $<$ & $\begin{array}{l}0.01 \\
0.005\end{array}$ & J & $\begin{array}{l}0.01 \\
0.005\end{array}$ & $:$ \\
\hline LEAD (TOTAL) & $19 / 22 / 92$ & HOO1 & MG/L & $<$ & 0.0015 & & 0.0015 & - \\
\hline LEAD-210 (TOTAL) & $11 / 22 / 92$ & N001 & PCI/L & & 5.6 & & 0.3 & 0.9 \\
\hline MAGNESIUM & $\begin{array}{l}06 / 09 / 89 \\
06 / 01 / 91 \\
02 / 21 / 92 \\
08 / 14 / 92\end{array}$ & $\begin{array}{l}0009 \\
0001 \\
0009 \\
0009\end{array}$ & $M G / L$ & & $\begin{array}{l}30.3 \\
20.4 \\
31.7 \\
20\end{array}$ & J & $\begin{array}{l}0.001 \\
0.1 \\
0.1 \\
5\end{array}$ & : \\
\hline MAHGANESE & $06 / 09 / 89$ & 0009 & MG/L & & 0.06 & J & 0.01 & - \\
\hline
\end{tabular}

PARAMETER VALUE IMDICATOR (PVI): < - LESS THAN DETECTIOH LIMIT

SAMPLE ID COOES:

OOO - FILTERED SAMPLE (.45 MICROHS)

OTHER PARAMETER VALUE FLAGS:

NOO1 - UNFILTERED SAMPLE

$J$ - ESTIMATED VALUE

N - SPIKE SAMPLE RECOVERY HOT HITHIN CONTROL LIMITS 
SURFACE WATER QUALITY DATA BY LOCATION

SOWP ReV. $O$ data

SITE: MONO 1 MONUMENT VALLEY

LOCATION: 0621

MORTH COORDINATE: $\quad 57935.9$ FT

EAST COORDIHATE: 90924.4 FT

$04 / 11 / 88$ TO $12 / 14 / 94$

REPORT DATE: 06/22/95

\begin{tabular}{|c|c|c|c|c|c|c|c|c|}
\hline PARAMETER NAME & LOG DATE & $\begin{array}{c}\text { SAMPLE } \\
\text { ID }\end{array}$ & $\begin{array}{l}\text { UHITS OF } \\
\text { MEASURE }\end{array}$ & PVI & $\begin{array}{l}\text { PARAMETER } \\
\text { VALUE }\end{array}$ & FLAGS & $\begin{array}{l}\text { OETECTIOH } \\
\text { LIMIT }\end{array}$ & $\begin{array}{l}\text { PARAMETER } \\
\text { UMCERTAINTY }\end{array}$ \\
\hline MAMGAHESE & $\begin{array}{l}06 / 01 / 91 \\
02 / 21 / 92 \\
08 / 14 / 92\end{array}$ & $\begin{array}{l}0001 \\
0001 \\
0001\end{array}$ & MG/L & $<$ & $\begin{array}{l}0.13 \\
0.01 \\
0.11\end{array}$ & & $\begin{array}{l}0.01 \\
0.01 \\
0.01\end{array}$ & $:$ \\
\hline MANGAKESE (TOTAL) & $11 / 22 / 92$ & HoO1 & MG/L & & 0.0050 & & 0.0015 & - \\
\hline MERCURY & $06 / 09 / 89$ & 0001 & $M G / L$ & $<$ & 0.0002 & J & 0.0002 & - \\
\hline MOLYADEKUM & $\begin{array}{l}06 / 09 / 89 \\
06 / 01 / 91 \\
02 / 21 / 92 \\
08 / 94 / 92\end{array}$ & $\begin{array}{l}0001 \\
0001 \\
0001 \\
0001\end{array}$ & MG/L & $\begin{array}{l}< \\
< \\
<\end{array}$ & $\begin{array}{l}0.01 \\
0.01 \\
0.01 \\
0.007\end{array}$ & J & $\begin{array}{l}0.01 \\
0.01 \\
0.01 \\
0.007\end{array}$ & $\dot{-}$ \\
\hline MOLYBDEHUM (TOTAL) & $11 / 22 / 92$ & HOOI & MG/L & $<$ & 0.0049 & H & 0.0049 & - \\
\hline MET GROSS ALPHA * & $\begin{array}{l}06 / 01 / 91 \\
02 / 21 / 92 \\
08 / 14 / 92\end{array}$ & $\begin{array}{l}0001 \\
0001 \\
0001\end{array}$ & $\mathrm{PCI} / \mathrm{L}$ & & $\begin{array}{r}11.42 \\
-2.94 \\
6.83\end{array}$ & & - & - \\
\hline HET GROSS ALPHA (TOTAL) ** & $11 / 22 / 92$ & NOOI & PCI/L & & -7.50 & & - & - \\
\hline HICKEL & $\begin{array}{l}06 / 09 / 89 \\
06 / 01 / 91\end{array}$ & $\begin{array}{l}0001 \\
0001\end{array}$ & $M G / L$ & $<$ & $\begin{array}{l}0.04 \\
0.04\end{array}$ & s & $\begin{array}{r}0.04 \\
.0 .04\end{array}$ & - \\
\hline NITRATE & $\begin{array}{l}06 / 09 / 89 \\
06 / 01 / 91 \\
02 / 21 / 92 \\
08 / 14 / 92 \\
11 / 22 / 92\end{array}$ & $\begin{array}{l}0001 \\
0001 \\
0001 \\
0001 \\
\text { N001 }\end{array}$ & MG/L & $<$ & $\begin{array}{l}5.3 \\
2.9 \\
1.0 \\
4.3 \\
0.071\end{array}$ & $j$ & $\begin{array}{l}1 . \\
1.0 \\
1.0 \\
0.075 \\
0.044\end{array}$ & $\begin{array}{l}- \\
- \\
- \\
-\end{array}$ \\
\hline WITRITE AND NITRATE & $06 / 01 / 91$ & 0001 & MG/L & & 0.65 & & 0.05 & - \\
\hline PH & $\begin{array}{l}06 / 09 / 89 \\
06 / 01 / 91 \\
02 / 21 / 92 \\
08 / 14 / 92 \\
11 / 22 / 92\end{array}$ & $\begin{array}{l}0001 \\
0001 \\
0001 \\
0001 \\
N 001\end{array}$ & SU & & $\begin{array}{l}8.86 \\
7.90 \\
8.47 \\
7.54 \\
9.25\end{array}$ & & $\begin{array}{l}- \\
\dot{-} \\
\dot{-} \\
.\end{array}$ & $\begin{array}{l}- \\
\dot{-} \\
. \\
.\end{array}$ \\
\hline PHOSPHATE & $06 / 09 / 89$ & 0001 & $M G / L$ & & 0.6 & J & 0.1 & - \\
\hline POTASSIUH & $\begin{array}{l}06 / 09 / 89 \\
06 / 01 / 91 \\
02 / 21 / 92 \\
08 / 14 / 92\end{array}$ & $\begin{array}{l}0001 \\
0001 \\
0001 \\
0001\end{array}$ & $M G / L$ & & $\begin{array}{l}1.3 \\
5.4 \\
6.04 \\
5.1\end{array}$ & J & $\begin{array}{l}0.01 \\
0.01 \\
0.01 \\
0.575\end{array}$ & 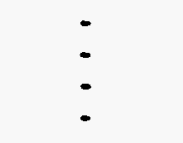 \\
\hline $\begin{array}{c}\text { RADIUH-226 } \\
:\end{array}$ & $\begin{array}{l}06 / 09 / 89 \\
06 / 01 / 91 \\
02 / 21 / 92 \\
08 / 14 / 92\end{array}$ & $\begin{array}{l}0001 \\
0001 \\
0001 \\
0001\end{array}$ & PCI/L & & $\begin{array}{l}0.0 \\
6.8 \\
3.8 \\
0.0\end{array}$ & J & $\begin{array}{l}1 . \\
1.0 \\
1.0 \\
0.4\end{array}$ & $\begin{array}{l}0.1 \\
1.1 \\
0.9 \\
0.1\end{array}$ \\
\hline RADIUH-226 (TOTAL) & $11 / 22 / 92$ & HOO1 & $\mathrm{PCI} / \mathrm{L}$ & & 0.2 & & 0.3 & 0.2 \\
\hline
\end{tabular}

- MET GROSS ALPHA (GROSS ALPHA - URANIUM) HITH 1 MG URAHIUM = 686 PCI

* HET GROSS ALPHA (TOTAL) (TOTAL GROSS ALPHA - TOTAL URANIUM)

PARAMETER VALUE IhDICATOR (PVI): < - LESS THAN DETECTION LIMIT

OTHER PARAMETER VALUE FLAGS:

SAMPLE ID COOES:

DOD1 - FILTERED SAMPLE (.45 MICRONS)

J - ESTIMATED VALUE

NOO1 - UHFILTERED SAMPLE

- SPIKE SAMPLE RECOVERY HOT WITHIN CONTROL LIMITS 
SURFACE WATER QUALITY DATA BY LOCATION

SOWP REV. 0 data

SITE: MONO1 MONUMENT VALLEY

LOCATION: 0621

NORTH COORDINATE: $\quad 57935.9$ FT

EAST COORDINATE: 90924.4 FT

04/11/88 TO 12/14/94

REPORT DATE: $06 / 22 / 95$

\begin{tabular}{|c|c|c|c|c|c|c|c|c|}
\hline PARAHETER NAME & LOG DATE & $\begin{array}{c}\text { SAMPLE } \\
\text { ID }\end{array}$ & $\begin{array}{l}\text { UNITS OF } \\
\text { MEASURE }\end{array}$ & PVI & $\begin{array}{l}\text { PARAMETER } \\
\text { VALUE }\end{array}$ & FLAGS & $\begin{array}{c}\text { DETECTION } \\
\text { LIMIT }\end{array}$ & $\begin{array}{l}\text { PARAMETER } \\
\text { UNCERTAINTY }\end{array}$ \\
\hline RADIUN-226 + RADIUH-228 & $\begin{array}{l}06 / 09 / 89 \\
06 / 01 / 91 \\
02 / 21 / 92 \\
08 / 14 / 92\end{array}$ & $\begin{array}{l}0001 \\
0001 \\
0001 \\
0001\end{array}$ & $\mathrm{PCI} / \mathrm{L}$ & & $\begin{array}{l}0.00 \\
8.60 \\
3.80 \\
0.00\end{array}$ & & - & $\begin{array}{l}- \\
- \\
-\end{array}$ \\
\hline RADIUA-226 + RADIUM-228 (TOTAL) & $11 / 22 / 92$ & No01 & PCI/L & & 3.00 & & - & - \\
\hline RADIUH-228 & $\begin{array}{l}06 / 09 / 89 \\
06 / 01 / 91 \\
02 / 21 / 92 \\
08 / 14 / 92\end{array}$ & $\begin{array}{l}0001 \\
0001 \\
0001 \\
0001\end{array}$ & PCI/L & & $\begin{array}{l}0.0 \\
1.8 \\
0.0 \\
0\end{array}$ & $J$ & $\begin{array}{l}1 . \\
1.0 \\
1.0\end{array}$ & $\begin{array}{l}0.9 \\
1.5 \\
1.5 \\
1\end{array}$ \\
\hline RADIUN-228 (TOTAL) & $11 / 22 / 92$ & No01 & PCI/L & & 2.8 & & 1.9 & 1.4 \\
\hline SELENIUM & $\begin{array}{l}06 / 09 / 89 \\
06 / 01 / 91 \\
02 / 21 / 92 \\
08 / 14 / 92\end{array}$ & $\begin{array}{l}0001 \\
0001 \\
0001 \\
0001\end{array}$ & $M G / L$ & $\begin{array}{l}< \\
< \\
< \\
<\end{array}$ & $\begin{array}{l}0.005 \\
0.005 \\
0.005 \\
0.005\end{array}$ & $\begin{array}{l}\mathfrak{J} \\
\mathbf{U}\end{array}$ & $\begin{array}{l}0.005 \\
0.005 \\
0.005 \\
0.005\end{array}$ & $\begin{array}{l}- \\
- \\
-\end{array}$ \\
\hline SELENIUH (TOTAL) & $11 / 22 / 92$ & No01 & $M G / L$ & $<$ & 0.0015 & $\boldsymbol{U}$ & 0.0015 & - \\
\hline SILICA - SIO2 & $\begin{array}{l}06 / 09 / 89 \\
06 / 01 / 91\end{array}$ & $\begin{array}{l}0001 \\
0001\end{array}$ & $M G / L$ & & $\begin{array}{r}11 . \\
8.2\end{array}$ & \rfloor & $\begin{array}{l}2 . \\
0.1\end{array}$ & - \\
\hline SILVER & $\begin{array}{l}06 / 09 / 89 \\
06 / 01 / 91\end{array}$ & $\begin{array}{l}0001 \\
0001\end{array}$ & $M G / L$ & $\begin{array}{l}< \\
<\end{array}$ & $\begin{array}{l}0.01 \\
0.01\end{array}$ & $J$ & $\begin{array}{l}0.01 \\
0.01\end{array}$ & - \\
\hline SOOIUH & $\begin{array}{l}06 / 09 / 89 \\
06 / 01 / 91 \\
02 / 21 / 92 \\
08 / 14 / 92\end{array}$ & $\begin{array}{l}0001 \\
0001 \\
0001 \\
0001\end{array}$ & $M G / L$ & & $\begin{array}{l}44.7 \\
41 . \\
43 . \\
30\end{array}$ & $J$ & $\begin{array}{l}0.002 \\
1 . \\
1 . \\
5\end{array}$ & $\begin{array}{l}- \\
\text { - } \\
\text { - }\end{array}$ \\
\hline SPECIFIC CONDUCTANCE & $\begin{array}{l}06 / 09 / 89 \\
06 / 01 / 91 \\
02 / 21 / 92 \\
08 / 14 / 92 \\
11 / 22 / 92\end{array}$ & $\begin{array}{l}0001 \\
0001 \\
0001 \\
0001 \\
N 001\end{array}$ & UMHO/CH & & $\begin{array}{l}377 . \\
460 \\
402 \\
422 \\
570\end{array}$ & & $\begin{array}{l}- \\
. \\
.\end{array}$ & - \\
\hline STRONTIUH & $\begin{array}{l}06 / 01 / 91 \\
02 / 21 / 92 \\
08 / 14 / 92\end{array}$ & $\begin{array}{l}0001 \\
0001 \\
0001\end{array}$ & MG/L & & $\begin{array}{l}0.42 \\
0.55 \\
0.49\end{array}$ & & $\begin{array}{l}0.01 \\
0.01 \\
0.2\end{array}$ & $\because$ \\
\hline STRONTIUM (TOTAL) & $11 / 22 / 92$ & NoOI & $M G / L$ & & 0.55 & + & 0.00035 & - \\
\hline SULFATE & $\begin{array}{l}06 / 09 / 89 \\
06 / 01 / 91 \\
02 / 21 / 92 \\
08 / 14 / 92\end{array}$ & $\begin{array}{l}0001 \\
0001 \\
0001 \\
0001\end{array}$ & MG/L & & $\begin{array}{l}35 . \\
14 . \\
32 . \\
10\end{array}$ & $d$ & $\begin{array}{c}0.1 \\
0.1 \\
10 . \\
0.059\end{array}$ & $\begin{array}{l}- \\
- \\
-\end{array}$ \\
\hline SULFATE (TOTAL) & $11 / 22 / 92$ & No01 & $M G / L$ & & 50 & & 1.0 & - \\
\hline SULFIDE & $\begin{array}{l}06 / 09 / 89 \\
06 / 01 / 91\end{array}$ & $\begin{array}{l}0001 \\
0001\end{array}$ & $M G / L$ & $<$ & $\begin{array}{l}0.1 \\
1.10\end{array}$ & J & $\begin{array}{l}0.1 \\
1 .\end{array}$ & - \\
\hline TEMPERATURE & $06 / 09 / 89$ & 0001 & C - DEGREE & & 25.5 & & - & - \\
\hline
\end{tabular}

PARAMETER VALUE INDICATOR (PVI): < - LESS THAN DETECTION LIMIT

SAMPLE ID COOES:

OTHER PARAMETER VALUE FLAGS:

OOO - FILTERED SNMPLE (.45 MICROHS)

+ CORRELATION COEFFICIENT FOR HSA < 0.995

NOO1 - UNFILTERED SNMPLE

J - ESTIMATED VALUE

4 - POST-DIGEST SPIKE OUT OF CNTR LIM WHILE SAMP ABS < 50\% SPIKE 
SURFACE HATER QUALITY DATA BY LOCATION

SOWP REV. 0 data

SITE: MONO1 MONUMENT VALLEY

LOCATION: 0621

MORTH COORDIHATE: 57935.9 FT

EAST COORDINATE: 90924.4 FT

$04 / 11 / 88$ TO $12 / 14 / 94$

REPORT DATE: 06/22/95

\begin{tabular}{|c|c|c|c|c|c|c|c|c|}
\hline PARAMETER NAME & LOG DATE & $\begin{array}{c}\text { SAMPLE } \\
\text { ID }\end{array}$ & $\begin{array}{l}\text { UNITS OF } \\
\text { MEASURE }\end{array}$ & PVI & $\begin{array}{l}\text { PARAMETER } \\
\text { VALUE }\end{array}$ & FLAGS & $\begin{array}{l}\text { DETECTIOH } \\
\text { LIMIT }\end{array}$ & $\begin{array}{l}\text { PARAMETER } \\
\text { UNCERTAIHTY }\end{array}$ \\
\hline TEMPERATURE & $\begin{array}{l}06 / 01 / 91 \\
02 / 21 / 92 \\
08 / 14 / 92 \\
11 / 22 / 92\end{array}$ & $\begin{array}{l}0001 \\
0001 \\
0001 \\
N 001\end{array}$ & C - DEGREE & & $\begin{array}{r}14.4 \\
9.6 \\
20.1 \\
12.3\end{array}$ & & $\begin{array}{l}- \\
- \\
-\end{array}$ & $\begin{array}{l}- \\
- \\
.\end{array}$ \\
\hline THALLIUM & $\begin{array}{l}06 / 09 / 89 \\
06 / 01 / 91\end{array}$ & $\begin{array}{l}0001 \\
0001\end{array}$ & $M G / L$ & $<$ & $\begin{array}{l}0.01 \\
0.01\end{array}$ & $\mathbf{J}$ & $\begin{array}{l}0.01 \\
0.01\end{array}$ & - \\
\hline THORIUM-230 & $06 / 01 / 91$ & 0001 & $\mathrm{PCI} / \mathrm{L}$ & & 0.4 & & 1. & 0.6 \\
\hline TIN & $\begin{array}{l}06 / 09 / 89 \\
06 / 01 / 91\end{array}$ & $\begin{array}{l}0001 \\
0001\end{array}$ & MG/L & $<$ & $\begin{array}{l}0.005 \\
0.01\end{array}$ & 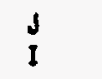 & $\begin{array}{l}0.005 \\
0.01\end{array}$ & - \\
\hline TOTAL DISSOLVED SOLIDS & $\begin{array}{l}06 / 09 / 89 \\
06 / 01 / 91 \\
02 / 21 / 92 \\
08 / 14 / 92\end{array}$ & $\begin{array}{l}0001 \\
0001 \\
0001 \\
0001\end{array}$ & $M G / L$ & & $\begin{array}{l}297 . \\
262 . \\
350 \\
270\end{array}$ & $\mathbf{d}$ & $\begin{array}{l}10 . \\
10 . \\
10 \\
42\end{array}$ & $\because$ \\
\hline TOTAL ORGANIC CARBON & $\begin{array}{l}06 / 01 / 91 \\
02 / 21 / 92 \\
08 / 14 / 92\end{array}$ & $\begin{array}{l}0001 \\
0001 \\
0001\end{array}$ & $M G / L$ & & $\begin{array}{l}5 . \\
4 . \\
5.1\end{array}$ & & $\begin{array}{l}1 . \\
1.0\end{array}$ & - \\
\hline URAKIUM & $\begin{array}{l}06 / 09 / 89 \\
06 / 01 / 91 \\
02 / 21 / 92 \\
08 / 14 / 92\end{array}$ & $\begin{array}{l}0001 \\
0001 \\
0001 \\
0001\end{array}$ & $M G / L$ & & $\begin{array}{l}0.0005 \\
0.044 \\
0.033 \\
0.009\end{array}$ & 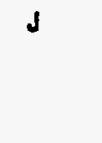 & $\begin{array}{l}0.003 \\
0.001 \\
0.001 \\
0.001\end{array}$ & $\begin{array}{l}- \\
- \\
-\end{array}$ \\
\hline URANIUM (TOTAL) & $11 / 22 / 92$ & No01 & $M G / L$ & & 0.014 & & 0.001 & $\bullet$ \\
\hline VAHADIUM & $\begin{array}{l}06 / 09 / 89 \\
06 / 01 / 91 \\
02 / 21 / 92 \\
08 / 14 / 92\end{array}$ & $\begin{array}{l}0001 \\
0001 \\
0001 \\
0001\end{array}$ & $M G / L$ & $\begin{array}{l}< \\
< \\
< \\
<\end{array}$ & $\begin{array}{l}0.01 \\
0.01 \\
0.01 \\
0.05\end{array}$ & $\mathbf{J}$ & $\begin{array}{l}0.01 \\
0.01 \\
0.01 \\
0.05\end{array}$ & - \\
\hline VAKADIUM (TOTAL) & $11 / 22 / 92$ & No01 & $M G / L$ & & 0.0030 & & 0.0019 & - \\
\hline ZIKC & $\begin{array}{l}06 / 09 / 89 \\
06 / 01 / 91 \\
02 / 21 / 92 \\
08 / 14 / 92\end{array}$ & $\begin{array}{l}0001 \\
0001 \\
0001 \\
0001\end{array}$ & $M G / L$ & $\begin{array}{l}< \\
<\end{array}$ & $\begin{array}{l}0.005 \\
0.005 \\
0.005 \\
0.02\end{array}$ & $J$ & $\begin{array}{l}0.005 \\
0.005 \\
0.005 \\
0.02\end{array}$ & $\begin{array}{l}- \\
-\end{array}$ \\
\hline ZIMC (TOTAL) & $11 / 22 / 92$ & No01 & $M G / L$ & & 0.0050 & & 0.0010 & - \\
\hline
\end{tabular}

PARAMETER VALUE IHDICATOR (PVI): < - LESS THAN DETECTION LIMIT

SAMPLE ID COOES:

OTHER PARAMETER VALUE FLAGS:

0001 - FILTERED SAMPLE (.45 MICROHS)

- DUPLICATE ANALYSIS NOT HITHIN CONTROL LIHITS

NOO1 - UNFILTERED SUMPLE

1 - INCREASED DETECTIOH LIHIT DUE TO REOUIRED DILUTIOH

- estimated VALUE 
SURFACE WATER QUALITY DATA BY LOCATION

SOWP ReV. 0 data

SITE: MONO1 MONUMENT VALLEY

LOCATION: 0622

NORTH COORDINATE: $\quad 56576.1$ FT

EAST COORDINATE: 89945.2 FT

$04 / 11 / 88$ TO $12 / 14 / 94$

REPORT DATE: $06 / 22 / 95$

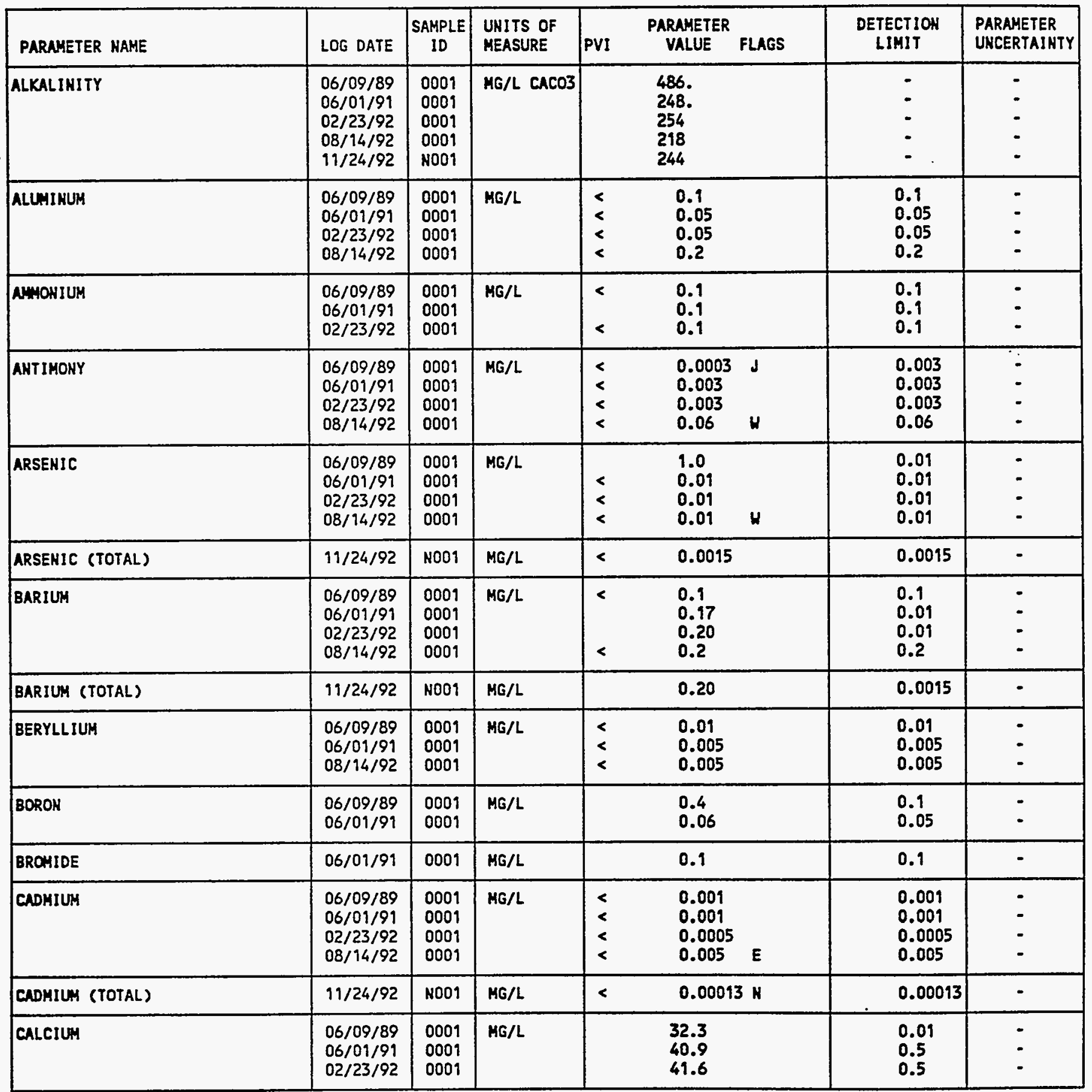

PARAMETER VALUE IMDICATOR (PVI): < - LESS THAN DETECTION LIHIT

OTHER PARAMETER VALUE fLAGS:

E - ESTIMATED VALUE BECAUSE OF INTERFERENCE, SEE CASE MARRATIVE

J - ESTIMATED VALUE

N - SPIKE SAMPLE RECOVERY HOT HITHIN CONTROL LIHITS

$H$ - POST-DIGEST SPIKE OUT OF CHTR LIM WHILE SAMP ABS < 50\% SPIKE
SAMPLE ID COOES:

0001 - FILTERED SAMPLE (.45 MICRONS)

NOO1 - UNFILTERED SAMPLE 
SURFACE WATER QUALITY DATA BY LOCATIOH

SOWP REV. 0 data

SITE: MONO1 MONUMENT VALLEY

LOCATION: 0622

NORTH COORDINATE: 56576.1 FT

EAST COORDINATE: 89945.2 FT

$04 / 19 / 88$ TO $12 / 14 / 94$

REPORT DATE: $06 / 22 / 95$

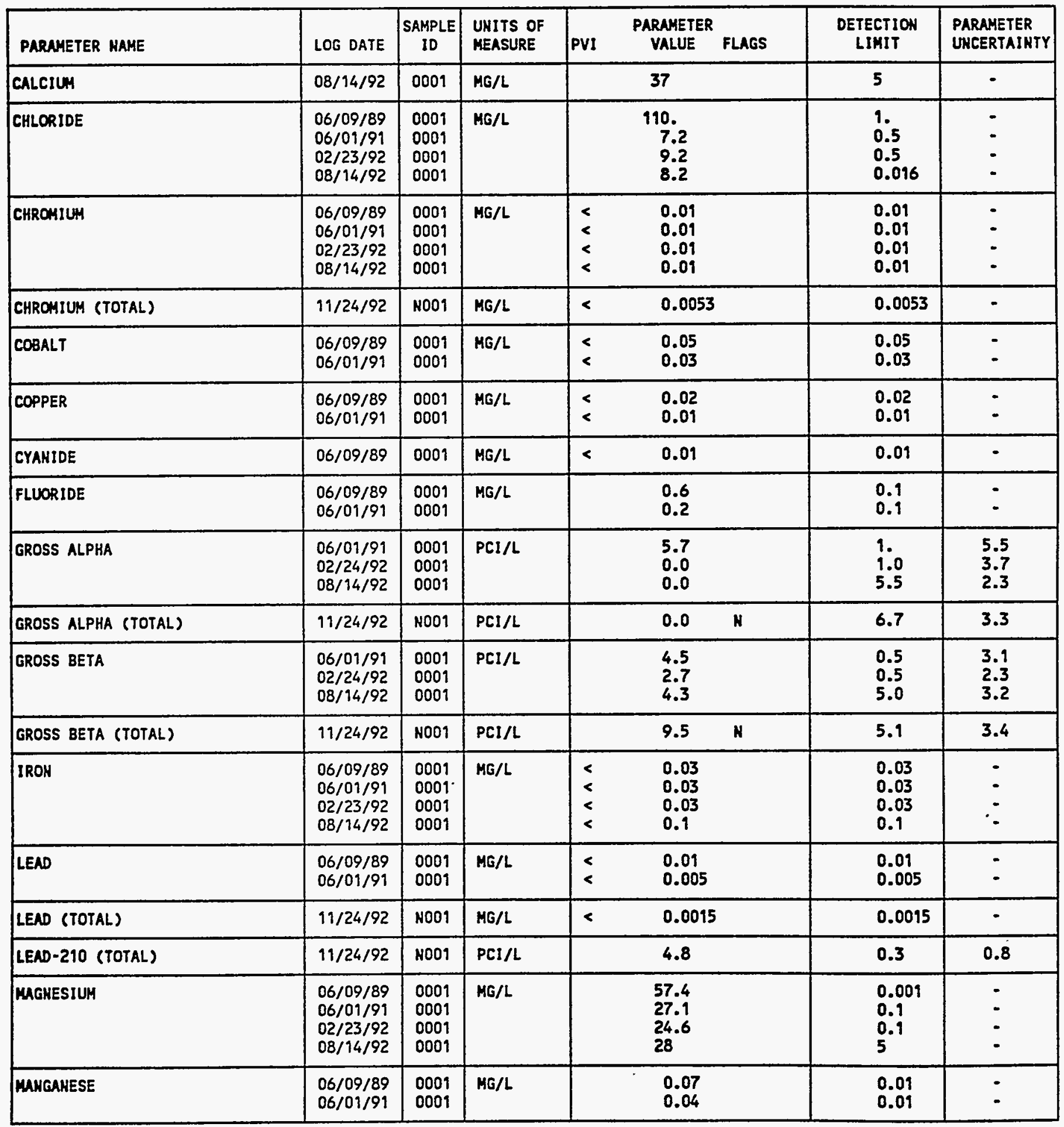

PARAMETER VALUE INDICATOR (PVI): < - LESS THAN DETECTION LIMIT

OTHER PARAMETER VALUE FLAGS:

H - SPIKE SAMPLE RECOVERY MOT HITHIN CONTROL LIMITS
SAMPLE ID COOES:

OOO1 - FILTERED SAMPLE (.45 MICRONS)

NOD1 - UNFILTERED SAMPLE 
SURFACE WATER QUALITY DATA BY LOCATION

SOWF REV. O data

SITE: MON01 MONUHENT VALLEY

LOCATION: 0622

NORTH COORDINATE: 56576.1 FT

EAST COORDINATE: 89945.2 FT

$04 / 11 / 88$ TO $12 / 14 / 94$

REPORT DATE: $06 / 22 / 95$

\begin{tabular}{|c|c|c|c|c|c|c|c|}
\hline PARAMETER NAME & LOG DATE & $\mid \begin{array}{c}\text { SAMPLE } \\
\text { ID }\end{array}$ & $\begin{array}{l}\text { UHITS OF } \\
\text { MEASURE }\end{array}$ & PVI & $\begin{array}{l}\text { PARAMETER } \\
\text { VALUE FLAGS }\end{array}$ & $\begin{array}{l}\text { DETECTION } \\
\text { LIMIT }\end{array}$ & $\begin{array}{l}\text { PARAMETER } \\
\text { UHCERTAINTY }\end{array}$ \\
\hline MUHGANESE & $\begin{array}{l}02 / 23 / 92 \\
08 / 14 / 92\end{array}$ & $\begin{array}{l}0001 \\
0001\end{array}$ & MG/L & & $\begin{array}{l}0.02 \\
0.048\end{array}$ & $\begin{array}{l}0.01 \\
0.01\end{array}$ & - \\
\hline MANGAHESE (TOTAL) & $11 / 24 / 92$ & NoO1 & MG/L & & 0.0090 & 0.0015 & - \\
\hline MERCURY & $06 / 09 / 89$ & 0001 & MG/L & $<$ & 0.0002 & 0.0002 & - \\
\hline IOLYYBDENUM & $\begin{array}{l}06 / 09 / 89 \\
06 / 01 / 91 \\
02 / 23 / 92 \\
08 / 14 / 92\end{array}$ & $\begin{array}{l}0001 \\
0001 \\
0001 \\
0001\end{array}$ & MG/L & $<$ & $\begin{array}{l}0.09 \\
0.01 \\
0.01 \\
0.007\end{array}$ & $\begin{array}{l}0.01 \\
0.01 \\
0.01 \\
0.007\end{array}$ & : \\
\hline MOLYBDENUM (TOTAL) & $11 / 24 / 92$ & N001 & MG/L & $<$ & $0.0049 \mathrm{~N}$ & 0.0049 & - \\
\hline HET GROSS ALPHA * & $\begin{array}{l}06 / 01 / 91 \\
02 / 24 / 92 \\
08 / 14 / 92\end{array}$ & $\begin{array}{l}0001 \\
0001 \\
0001\end{array}$ & $\mathrm{PCI} / \mathrm{L}$ & & $\begin{array}{r}5.36 \\
-2.06 \\
-0.34\end{array}$ & : & - \\
\hline HET GROSS ALPHA (TOTAL) ** & $11 / 24 / 92$ & No01 & PCI/L & & -0.34 & - & - \\
\hline MICKEL & $\begin{array}{l}06 / 09 / 89 \\
06 / 01 / 91\end{array}$ & $\begin{array}{l}0001 \\
0001\end{array}$ & $M G / L$ & $<$ & $\begin{array}{l}0.04 \\
0.04\end{array}$ & $\begin{array}{l}0.04 \\
0.04\end{array}$ & :- \\
\hline MITRATE & $\begin{array}{l}06 / 09 / 89 \\
06 / 01 / 91 \\
02 / 23 / 92 \\
08 / 14 / 92 \\
11 / 24 / 92\end{array}$ & $\begin{array}{l}0001 \\
0001 \\
0001 \\
0001 \\
\text { N001 }\end{array}$ & $M G / L$ & $<$ & $\begin{array}{l}4.8 \\
2.7 \\
1.0 \\
2.3 \\
0.48\end{array}$ & $\begin{array}{l}9 . \\
1.0 \\
1.0 \\
0.075 \\
0.044\end{array}$ & $\begin{array}{l}- \\
\dot{-} \\
\dot{-}\end{array}$ \\
\hline MITRITE AND NITRATE & $06 / 01 / 91$ & 0001 & $M G / L$ & & 0.61 & 0.05 & - \\
\hline PH & $\begin{array}{l}06 / 09 / 89 \\
06 / 01 / 91 \\
02 / 23 / 92 \\
08 / 14 / 92 \\
11 / 24 / 92\end{array}$ & $\begin{array}{l}0001 \\
0001 \\
0001 \\
0001 \\
N 001\end{array}$ & SU & & $\begin{array}{l}8.61 \\
8.25 \\
8.19 \\
8.32 \\
8.14\end{array}$ & $\begin{array}{l}- \\
: \\
-\end{array}$ & $\begin{array}{l}- \\
- \\
- \\
-\end{array}$ \\
\hline PHOSPHATE & $06 / 09 / 89$ & 0001 & $M G / L$ & & 0.6 & 0.1 & - \\
\hline POTASSIUH & $\begin{array}{l}06 / 09 / 89 \\
06 / 01 / 91 \\
02 / 23 / 92 \\
08 / 14 / 92\end{array}$ & $\begin{array}{l}0001 \\
0001 \\
0001 \\
0001\end{array}$ & MG/L & $\cdot$ & $\begin{array}{l}5.0 \\
2.5 \\
4.30 \\
3.7\end{array}$ & $\begin{array}{l}0.01 \\
0.01 \\
0.01 \\
0.575\end{array}$ & $\begin{array}{l}- \\
- \\
-\end{array}$ \\
\hline RADIUA-226 & $\begin{array}{l}06 / 09 / 89 \\
06 / 01 / 91 \\
02 / 24 / 92 \\
08 / 14 / 92\end{array}$ & $\begin{array}{l}0001 \\
0001 \\
0001 \\
0001\end{array}$ & $\mathrm{PCI} / \mathrm{L}$ & & $\begin{array}{l}0.1 \\
0.3 \\
0.1 \\
0.2\end{array}$ & $\begin{array}{l}1 . \\
1.0 \\
1.0 \\
0.6\end{array}$ & $\begin{array}{l}0.2 \\
0.3 \\
0.3 \\
0.1\end{array}$ \\
\hline RADIUA-226 (TOTAL) & $11 / 24 / 92$ & NoO1 & $\mathrm{PCl} / \mathrm{L}$ & & 2.1 & 0.3 & 0.5 \\
\hline RADIUH-226 + RADIUM-228 & $06 / 09 / 89$ & 0001 & $\mathrm{PCI} / \mathrm{L}$ & & 0.50 & - & - \\
\hline
\end{tabular}

- MET GROSS ALPHA (GROSS ALPHA - URANIUM) WITH I MG URANIUH = 686 PCI

* NET GROSS ALPHA (TOTAL) (TOTAL GROSS ALPHA - TOTAL URAKIUH)

PARAHETER VALUE INDICATOR (PVI): < - LESS THAN DETECTION LIMIT

OTHER PARAMETER VALUE FLAGS:

N - SPIKE SAMPLE RECOVERY NOT WITHIN CONTROL LIMITS
SNMPLE IO COOES:

OOO1 - FILTERED SAMPLE (.45 HICRONS)

NOOI - UNFILTERED SNMPLE 
SURFACE WATER QUALITY DATA BY LOCATIOH

SOWP ReV. $O$ data

SITE: MONO1 MONUMENT VALLEY

LOCATION: 0622

NORTH COORDINATE: 56576.9 FT

EAST COORDINATE: 89945.2 FT

04/11/88 TO $12 / 14 / 94$

REPORT DATE: 06/22/95

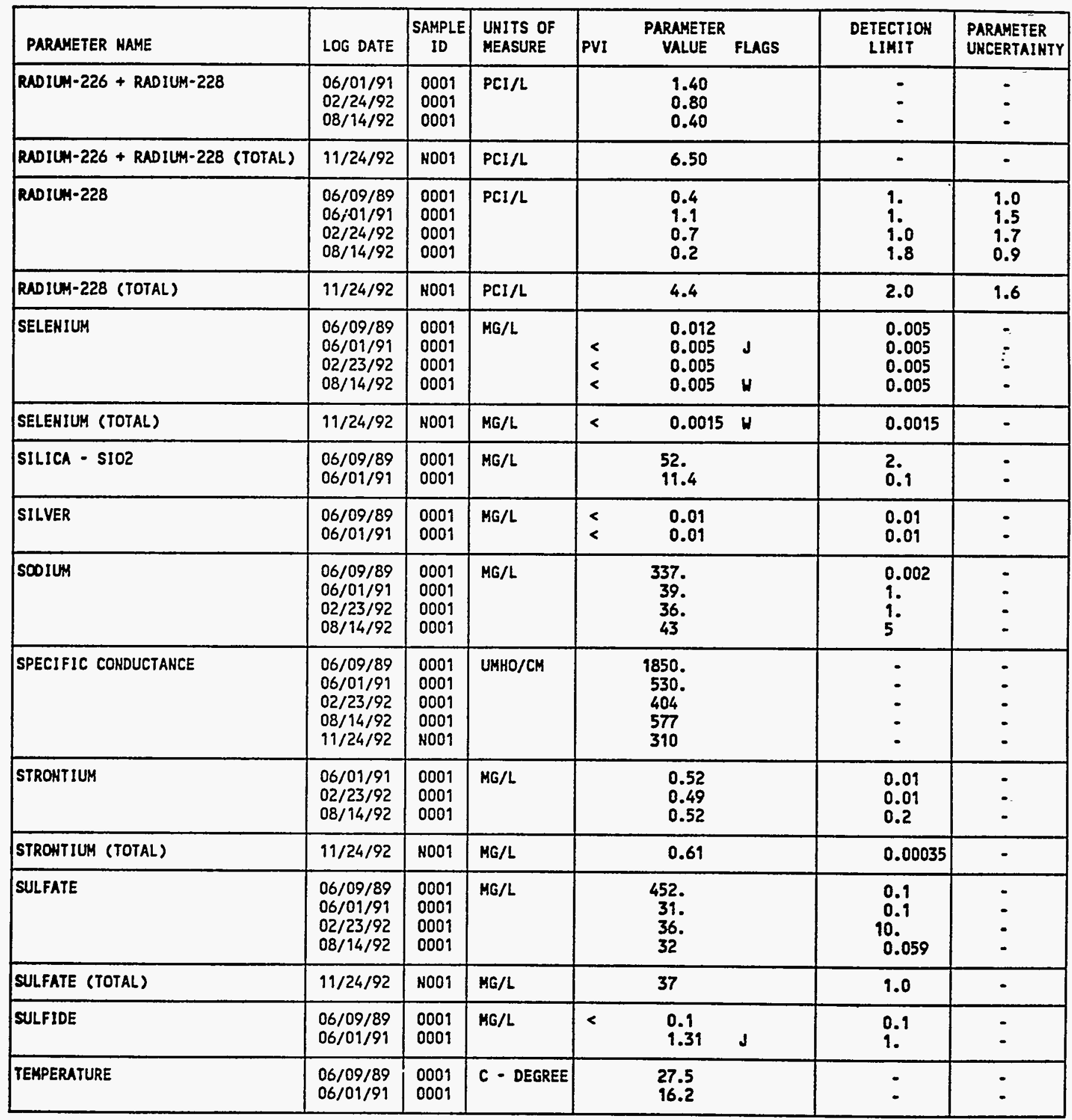

PARAMETER VALUE INDICATOR (PVI): < - LESS THAN DETECTION LIMIT

SAMPLE ID COOES:

OTHER PARAMETER VALUE FLAGS:

0001 - FILTERED SAMPLE (.45 MICRONS)

$J$ - ESTIMATED VALUE

NOO - UNFILTERED SAMPLE

W - POST-DIGEST SPIKE OUT OF CNTR LIM WHILE SAMP ABS < 50\% SPIKE 
SURFACE WATER QUALITY DATA BY LOCATION

SOWP ReV. 0 data

SITE: MONO1 MONUMENT VALLEY

LOCATION: 0622

NORTH COORDINATE: 56576.1 FT

EAST COORDINATE: 89945.2 FT

$04 / 11 / 88$ TO $12 / 14 / 94$

REPORT DATE: $06 / 22 / 95$

\begin{tabular}{|c|c|c|c|c|c|c|c|}
\hline PARAMETER NAME & LOG DATE & $\begin{array}{l}\text { SAMPLE } \\
\text { ID }\end{array}$ & $\begin{array}{l}\text { UNITS OF } \\
\text { MEASURE }\end{array}$ & PVI & $\begin{array}{l}\text { PARAMETER } \\
\text { VALUE FLAGS }\end{array}$ & $\begin{array}{l}\text { DETECTION } \\
\text { LIMIT }\end{array}$ & $\begin{array}{l}\text { PARAMETER } \\
\text { UHCERTAINTY }\end{array}$ \\
\hline TEHPERATURE & $\begin{array}{l}02 / 23 / 92 \\
08 / 14 / 92 \\
11 / 24 / 92\end{array}$ & $\begin{array}{l}0001 \\
0001 \\
N 001\end{array}$ & C - DEGREE & & $\begin{array}{r}12.3 \\
30.2 \\
5.6\end{array}$ & : & : \\
\hline THALLIUM & $\begin{array}{l}06 / 09 / 89 \\
06 / 01 / 91\end{array}$ & $\begin{array}{l}0001 \\
0001\end{array}$ & $M G / L$ & $<$ & $\begin{array}{l}0.01 \\
0.01\end{array}$ & $\begin{array}{l}0.01 \\
0.01\end{array}$ & : \\
\hline THORIUH-230 & $06 / 01 / 91$ & 0001 & $\mathrm{PCI} / \mathrm{L}$ & & 0.5 & 1. & 0.4 \\
\hline TIN & $\begin{array}{l}06 / 09 / 89 \\
06 / 01 / 91\end{array}$ & $\begin{array}{l}0001 \\
0001\end{array}$ & MG/L & $<$ & $\begin{array}{l}0.005 \\
0.01\end{array}$ & $\begin{array}{l}0.005 \\
0.01\end{array}$ & - \\
\hline TOTAL DISSOLVED SOLIDS & $\begin{array}{l}06 / 09 / 89 \\
06 / 01 / 91 \\
02 / 23 / 92 \\
08 / 14 / 92\end{array}$ & $\begin{array}{l}0001 \\
0001 \\
0001 \\
0001\end{array}$ & MG/L & & $\begin{array}{l}1300 . \\
307 . \\
311 . \\
310\end{array}$ & $\begin{array}{l}10 . \\
10 . \\
10 . \\
42\end{array}$ & $\begin{array}{l}- \\
- \\
-\end{array}$ \\
\hline TOTAL ORGANIC CARBON & $\begin{array}{l}06 / 01 / 91 \\
02 / 23 / 92 \\
08 / 14 / 92\end{array}$ & $\begin{array}{l}0001 \\
0001 \\
0001\end{array}$ & $M G / L$ & & $\begin{array}{l}4 . \\
3 . \\
8.3\end{array}$ & $\begin{array}{l}1 . \\
1.0\end{array}$ & $\dot{-}$ \\
\hline URANIUM & $\begin{array}{l}06 / 09 / 89 \\
06 / 01 / 91 \\
02 / 24 / 92 \\
08 / 14 / 92\end{array}$ & $\begin{array}{l}0001 \\
0001 \\
0001 \\
0001\end{array}$ & $M G / L$ & $<$ & $\begin{array}{l}0.015 \\
0.001 \\
0.003 \\
0.001\end{array}$ & $\begin{array}{l}0.003 \\
0.001 \\
0.001 \\
0.001\end{array}$ & : \\
\hline URANIUM (TOTAL) & $11 / 24 / 92$ & N001 & $M G / L$ & $<$ & 0.001 & 0.001 & - \\
\hline VAKADIUM & $\begin{array}{l}06 / 09 / 89 \\
06 / 01 / 91 \\
02 / 23 / 92 \\
08 / 14 / 92\end{array}$ & $\begin{array}{l}0001 \\
0001 \\
0001 \\
0001\end{array}$ & $M G / L$ & $<$ & $\begin{array}{l}0.03 \\
0.01 \\
0.01 \\
0.05\end{array}$ & $\begin{array}{l}0.01 \\
0.01 \\
0.01 \\
0.05\end{array}$ & : \\
\hline VAKADIUM (TOTAL) & $11 / 24 / 92$ & N001 & HG/L & $<$ & 0.0019 & 0.0019 & - \\
\hline ZIHC & $\begin{array}{l}06 / 09 / 89 \\
06 / 01 / 91 \\
02 / 23 / 92 \\
08 / 14 / 92\end{array}$ & $\begin{array}{l}0001 \\
0001 \\
0001 \\
0001\end{array}$ & $M G / L$ & $<$ & $\begin{array}{l}0.006 \\
0.005 \\
0.005 \\
0.02\end{array}$ & $\begin{array}{l}0.005 \\
0.005 \\
0.005 \\
0.02\end{array}$ & $\begin{array}{l}- \\
-\end{array}$ \\
\hline ZINC (TOTAL) & $11 / 24 / 92$ & N001 & MG/L & & 0.0050 & 0.0010 & $\cdot$ \\
\hline
\end{tabular}

PARAMETER VALUE IHDICATOR (PVI): < - LESS THAN DETECTION LIHIT

SAMPLE ID COOES:

OTHER PARAMETER VALUE FLAGS:

0001 - FILTERED SAMPLE (.45 MICRONS)

* DUPLICATE ANALYSIS hOT WITHIN CONTROL LIMITS

NOO1 - UNFILTERED SAMPLE

I - INCREASED DETECTION LIMIT DUE TO REQUIRED DILUTION 
SURFACE HATER QUALITY DATA BY LOCATION

SOWP REV. 0 data

SITE: HONO1 MONUMENT VALLEY

LOCATION: O623 RESERVED FOR RPAPUSCH

NORTH COORDINATE: UHKNOWN

EAST COORDINATE: UHKHOWH

04/11/88 TO $12 / 14 / 94$

REPORT DATE: $06 / 22 / 95$

\begin{tabular}{|c|c|c|c|c|c|c|c|c|}
\hline PARAMETER NAME & LOG DATE & $\underset{\text { SAMPLE }}{\text { ID }}$ & $\begin{array}{l}\text { UNITS OF } \\
\text { MEASURE }\end{array}$ & PVI & $\begin{array}{l}\text { PARAMETER } \\
\text { VALUE }\end{array}$ & FLAGS & $\begin{array}{l}\text { DETECTIOH } \\
\text { LIMIT }\end{array}$ & $\begin{array}{l}\text { PARAMETER } \\
\text { UHCERTAIHTY }\end{array}$ \\
\hline ALKALINITY & $\begin{array}{l}06 / 28 / 93 \\
12 / 15 / 93 \\
04 / 21 / 94 \\
12 / 14 / 94\end{array}$ & $\begin{array}{l}\text { NOO1 } \\
\text { NO01 } \\
\text { NO01 } \\
\text { N001 }\end{array}$ & MG/L CACO3 & & $\begin{array}{l}284 \\
213 \\
268 \\
371\end{array}$ & $\begin{array}{l}R X \\
R X \\
R X \\
R X\end{array}$ & $10^{-}$ & $\dot{-}$ \\
\hline AMYHIUH & $12 / 15 / 93$ & 0001 & MG/L & $<$ & 0.1 & RX & 0.1 & - \\
\hline AMONIUH (TOTAL) & $\begin{array}{l}06 / 28 / 93 \\
04 / 21 / 94 \\
12 / 14 / 94\end{array}$ & $\begin{array}{l}\text { No01 } \\
\text { N001 } \\
\text { N001 }\end{array}$ & MG/L & $<$ & $\begin{array}{l}0.1 \\
0.1 \\
0.1\end{array}$ & $\begin{array}{l}R X \\
R X \\
R X\end{array}$ & $\begin{array}{l}0.1 \\
0.1 \\
0.1\end{array}$ & - \\
\hline ARSEHIC (TOTAL) & $06 / 28 / 93$ & NOOI & $M G / L$ & $<$ & 0.005 & RX & 0.005 & - \\
\hline CALCIUA & $\begin{array}{l}12 / 15 / 93 \\
04 / 21 / 94 \\
12 / 14 / 94\end{array}$ & $\begin{array}{l}0001 \\
0001 \\
0001\end{array}$ & $M G / L$ & & $\begin{array}{l}41.3 \\
49.4 \\
45.7\end{array}$ & $\begin{array}{l}R X \\
R X \\
R X\end{array}$ & $\begin{array}{l}0.5 \\
0.5 \\
0.5\end{array}$ & - \\
\hline CALCIUM (TOTAL) & $\begin{array}{l}12 / 15 / 93 \\
04 / 21 / 94 \\
12 / 14 / 94\end{array}$ & $\begin{array}{l}\text { NOOI } \\
\text { NOOI } \\
\text { NOOI }\end{array}$ & MG/L & & $\begin{array}{c}43.9 \\
51.8 \\
171\end{array}$ & $\begin{array}{l}R X \\
R X \\
R X\end{array}$ & $\begin{array}{l}0.5 \\
0.5 \\
0.5\end{array}$ & - \\
\hline CHLORIDE & $\begin{array}{l}12 / 15 / 93 \\
04 / 21 / 94\end{array}$ & $\begin{array}{l}0001 \\
0001\end{array}$ & $M G / L$ & & $\begin{array}{r}8.0 \\
15.0\end{array}$ & $\begin{array}{l}R X \\
R X\end{array}$ & $\begin{array}{l}0.5 \\
0.5\end{array}$ & - \\
\hline CHLORIDE (TOTAL) & $12 / 14 / 94$ & NOOI & $M G / L$ & & 9 & RX & 0.5 & - \\
\hline IRON & $12 / 14 / 94$ & 0001 & $M G / L$ & $<$ & 0.03 & $R X$ & 0.03 & - \\
\hline IRON (TOTAL) & $12 / 14 / 94$ & NoO1 & MG/L & & 33.1 & $\mathbf{R X}$ & 0.03 & - \\
\hline MAGMESIUM & $\begin{array}{l}12 / 15 / 93 \\
04 / 21 / 94 \\
12 / 14 / 94\end{array}$ & $\begin{array}{l}0001 \\
0001 \\
0001\end{array}$ & $H G / L$ & & $\begin{array}{l}22.6 \\
28.8 \\
28.7\end{array}$ & $\begin{array}{l}R X \\
R X \\
R X\end{array}$ & $\begin{array}{l}0.1 \\
0.1 \\
0.1\end{array}$ & - \\
\hline MAGKESIUM (TOTAL) & $\begin{array}{l}12 / 15 / 93 \\
04 / 21 / 94 \\
12 / 14 / 94\end{array}$ & $\begin{array}{l}\text { NOO1 } \\
\text { NOO1 } \\
\text { NOO1 }\end{array}$ & $M G / L$ & & $\begin{array}{l}22.8 \\
28.0 \\
76.1\end{array}$ & $\begin{array}{l}R X \\
R X \\
R X\end{array}$ & $\begin{array}{l}0.1 \\
0.1 \\
0.1\end{array}$ & $\begin{array}{l}- \\
-\end{array}$ \\
\hline MAHGAHESE & $\begin{array}{l}12 / 15 / 93 \\
04 / 21 / 94 \\
12 / 14 / 94\end{array}$ & $\begin{array}{l}0001 \\
0001 \\
0001\end{array}$ & MG/L & - & $\begin{array}{l}0.07 \\
0.16 \\
0.02\end{array}$ & $\begin{array}{l}R X \\
R X \\
R X\end{array}$ & $\begin{array}{l}0.01 \\
0.01 \\
0.01\end{array}$ & : \\
\hline MAKGAKESE (TOTAL) & $\begin{array}{l}12 / 15 / 93 \\
04 / 21 / 94 \\
12 / 14 / 94\end{array}$ & $\begin{array}{l}\text { No01 } \\
\text { N001 } \\
\text { N001 }\end{array}$ & HG/L & & $\begin{array}{l}0.08 \\
0.17 \\
0.99\end{array}$ & $\begin{array}{l}R X \\
R X \\
R X\end{array}$ & $\begin{array}{l}0.01 \\
0.01 \\
0.01\end{array}$ & - \\
\hline MOLYBDENUH & $12 / 14 / 94$ & 0001 & $M G / L$ & $<$ & 0.01 & $\mathbf{R X}$ & 0.09 & - \\
\hline MLLYBDEKLM (TOTAL) & $12 / 14 / 94$ & NOO1 & $M G / L$ & $<$ & 0.09 & RX & 0.01 & - \\
\hline HITRATE & $12 / 15 / 93$ & 0001 & MG/L & $<$ & 1 & $R X$ & 1 & - \\
\hline MITRATE (TOTAL) & $\begin{array}{l}06 / 28 / 93 \\
04 / 21 / 94 \\
12 / 14 / 94\end{array}$ & $\begin{array}{l}\text { NoO1 } \\
\text { No01 } \\
\text { HOO1 }\end{array}$ & $M G / L$ & $<$ & $\begin{array}{l}1 \\
1 \\
1\end{array}$ & $\begin{array}{l}R X \\
R X \\
R X\end{array}$ & $\begin{array}{l}1 \\
1 \\
1\end{array}$ & - \\
\hline PH & $06 / 28 / 93$ & HOO1 & SU & & 7.51 & $R X$ & - & - \\
\hline
\end{tabular}

PARAMETER VALUE IHDICATOR (PVI): < - LESS THAN DETECTION LIMIT

OTHER PARAMETER VALUE FLAGS:

$R$ - UNUSABLE DATA POINT

$X$ - LOCATION UHDEFIHED
SAMPLE ID COOES:

0001 - FILTERED SAMPLE (.45 MICRONS)

NOO1 - UNFILTERED SAMPLE 
SURFACE HATER QUALITY DATA BY LOCATION

SOWP ReV. 0 data

SITE: MONOY MONUMENT VALLEY

LOCATION: 0623 RESERVED FOR RPAPUSCH

NORTH COORDINATE: UNKHOWN

EAST COORDINATE: UNKNOWN

04/11/88 TO $12 / 14 / 94$

REPORT DATE: 06/22/95

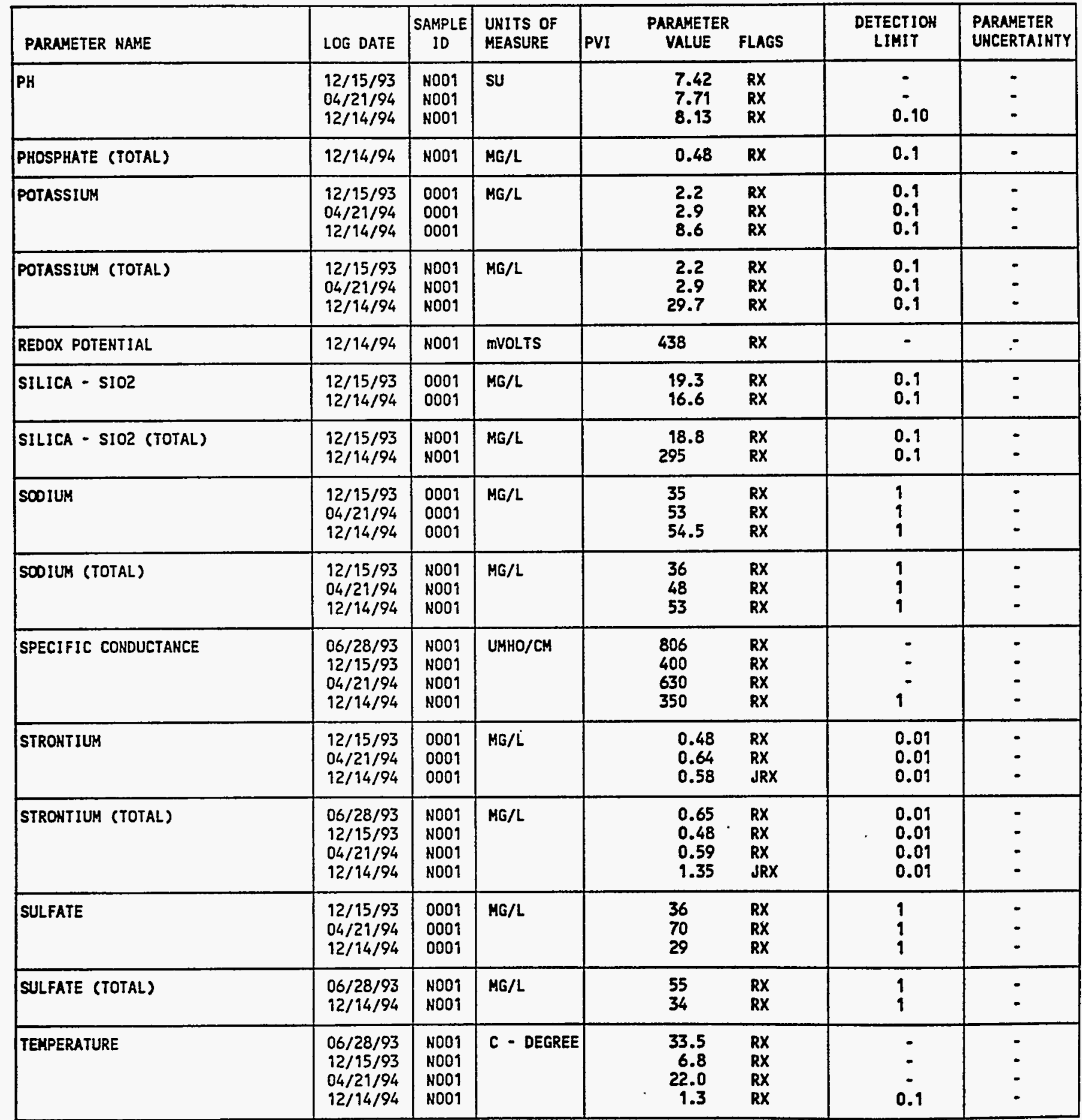

PARAMETER VALUE INDICATOR (PVI): < - LESS THAN DETECTION LIMIT

SAMPLE ID COOES

0001 - FILTERED SAMPLE (.45 MICRONS)

OTHER PARAMETER VALUE FLAGS:

NO01 - UMFILTERED SAMPLE

J- ESTIMATED VALUE

R - UNUSABLE DATA POINT

$\bar{X}$ - LOCATION UNDEFIHED 
SURFACE WATER QUALITY DATA BY LOCATION

SOWP REV. 0 data

SITE: MONOT MONUMENT VALLEY

LOCATION: 0623 RESERVED FOR RPAPUSCH

NORTH COORDINATE: UHKHOWN

EAST COORDINATE: UHKHOWN

04/11/88 TO $12 / 14 / 94$

REPORT DATE: 06/22/95

\begin{tabular}{|c|c|c|c|c|c|c|c|c|}
\hline PARAMETER HAME & LOG DATE & $\begin{array}{c}\text { SAMPLE } \\
\text { ID }\end{array}$ & $\begin{array}{l}\text { UNITS OF } \\
\text { MEASURE }\end{array}$ & PVI & $\begin{array}{l}\text { PARAMETER } \\
\text { VALUE }\end{array}$ & FLAGS & $\begin{array}{l}\text { DETECTIOH } \\
\text { LIMIT }\end{array}$ & $\begin{array}{l}\text { PARAMETER } \\
\text { UNCERTAINTY }\end{array}$ \\
\hline TOTAL DISSOLVED SOLIDS & $\begin{array}{l}12 / 15 / 93 \\
04 / 21 / 94 \\
12 / 14 / 94\end{array}$ & $\begin{array}{l}0001 \\
0001 \\
0001\end{array}$ & MG/L & & $\begin{array}{l}300 \\
420 \\
348\end{array}$ & $\begin{array}{l}R X \\
R X \\
R X\end{array}$ & $\begin{array}{l}10 \\
10 \\
10\end{array}$ & : \\
\hline TOTAL DISSOLVED SOLIDS (TOTAL) & $06 / 28 / 93$ & N001 & MG/L & & 400 & HRX & 10 & - \\
\hline URAKIUH & $\begin{array}{l}12 / 15 / 93 \\
04 / 21 / 94\end{array}$ & $\begin{array}{l}0001 \\
0001\end{array}$ & ME/L & & $\begin{array}{l}0.004 \\
0.002\end{array}$ & $\begin{array}{l}\text { RX } \\
\text { RX }\end{array}$ & $\begin{array}{l}0.001 \\
0.001\end{array}$ & : \\
\hline LRANIUM (TOTAL) & $\begin{array}{l}06 / 28 / 93 \\
12 / 15 / 93 \\
04 / 21 / 94 \\
12 / 14 / 94\end{array}$ & $\begin{array}{l}\text { N001 } \\
\text { N001 } \\
\text { N001 } \\
\text { N001 }\end{array}$ & HG/L & $<$ & $\begin{array}{l}0.001 \\
0.004 \\
0.002 \\
0.006\end{array}$ & $\begin{array}{l}\text { RX } \\
\text { RX } \\
\text { RX } \\
\text { RX }\end{array}$ & $\begin{array}{l}0.001 \\
0.001 \\
0.001 \\
0.001\end{array}$ & $\dot{-}$ \\
\hline VAKADIUM & $\begin{array}{l}12 / 15 / 93 \\
04 / 21 / 94 \\
12 / 14 / 94\end{array}$ & $\begin{array}{l}0001 \\
0001 \\
0001\end{array}$ & MG/L & $\begin{array}{l}< \\
< \\
<\end{array}$ & $\begin{array}{l}0.01 \\
0.01 \\
0.01\end{array}$ & $\begin{array}{l}\text { RX } \\
\text { RX } \\
\text { RX }\end{array}$ & $\begin{array}{l}0.01 \\
0.01 \\
0.01\end{array}$ & 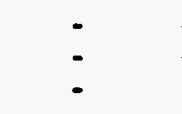 \\
\hline VAHADIUM (TOTAL) & $\begin{array}{l}06 / 28 / 93 \\
12 / 15 / 93 \\
04 / 21 / 94 \\
12 / 14 / 94\end{array}$ & $\begin{array}{l}\text { NOO1 } \\
\text { NO01 } \\
\text { N001 } \\
\text { NO01 }\end{array}$ & MG/L & $\begin{array}{l}< \\
<\end{array}$ & $\begin{array}{l}0.01 \\
0.01 \\
0.01 \\
0.06\end{array}$ & $\begin{array}{l}R X \\
R X \\
R X \\
R X\end{array}$ & $\begin{array}{l}0.01 \\
0.01 \\
0.01 \\
0.01\end{array}$ & $\begin{array}{l}- \\
-\end{array}$ \\
\hline ZIHC & $12 / 14 / 94$ & 0001 & $M G / L$ & $<$ & 0.05 & RX & 0.05 & - \\
\hline ZINC (TOTAL) & $\begin{array}{l}06 / 28 / 93 \\
12 / 14 / 94\end{array}$ & $\begin{array}{l}\text { HOOI } \\
\text { NOOI }\end{array}$ & MG/L & & $\begin{array}{l}0.213 \\
0.11\end{array}$ & $\begin{array}{l}\text { JRX } \\
\text { RX }\end{array}$ & $\begin{array}{l}0.005 \\
0.05\end{array}$ & - \\
\hline
\end{tabular}

PARAMETER VALUE INDICATOR (PVI):

SAMPLE ID COOES

OTHER PARAMETER VALUE FLAGS:

OOD1 - FILTERED SAMPLE (.45 MICRONS)

H - HOLD TIME EXPIRED, VALUE SUSPECT

NOO1 - UMFILTERED SAMPLE

$J$ - ESTIMATED VALUE

$R$ - UHUSABLE DATA POINT

$X$ - LOCATION UNDEFINED 
SURFACE WATER QUALITY DATA BY LOCATIOH

SOWP Rev. O data

SITE: MONO1 MONUMENT VALLEY

LOCATION: 0624 RESERVED FOR RPAPUSCH

NORTH COORDINATE: UNKNOWN

EAST COORDINATE: UNKHOWN

$04 / 11 / 88$ TO $12 / 14 / 94$

REPORT DATE: $06 / 22 / 95$

\begin{tabular}{|c|c|c|c|c|c|c|c|c|}
\hline PARAMETER NAME & LOG DATE & $\begin{array}{c}\text { SAMPLE } \\
\text { ID }\end{array}$ & $\begin{array}{l}\text { UNITS OF } \\
\text { MEASURE }\end{array}$ & PVI & $\begin{array}{l}\text { PARAMETER } \\
\text { VALUE }\end{array}$ & FLAGS & $\begin{array}{l}\text { DETECTIOH } \\
\text { LIMIT }\end{array}$ & $\begin{array}{l}\text { PARAMETER } \\
\text { UHCERTAINTY }\end{array}$ \\
\hline ALKALINITY & $\begin{array}{l}12 / 15 / 93 \\
04 / 21 / 94 \\
12 / 13 / 94\end{array}$ & $\begin{array}{l}\text { NO01 } \\
\text { NO01 } \\
\text { N001 }\end{array}$ & MG/L CACO3 & & $\begin{array}{l}598 \\
275 \\
349\end{array}$ & $\begin{array}{l}R X \\
R X \\
R X\end{array}$ & 10 & $\dot{-}$ \\
\hline AMONIUN & $12 / 15 / 93$ & 0001 & $M G / L$ & $<$ & 0.1 & RX & 0.1 & - \\
\hline NMIONIUN (TOTAL) & $\begin{array}{l}04 / 21 / 94 \\
12 / 13 / 94\end{array}$ & $\begin{array}{l}\text { NOO1 } \\
\text { N001 }\end{array}$ & MG/L & $<$ & $\begin{array}{l}0.1 \\
0.12\end{array}$ & $\begin{array}{l}\text { RX } \\
\text { RX }\end{array}$ & $\begin{array}{l}0.1 \\
0.1\end{array}$ & - \\
\hline CALCIUM & $\begin{array}{l}12 / 15 / 93 \\
04 / 21 / 94 \\
12 / 13 / 94\end{array}$ & $\begin{array}{l}0001 \\
0001 \\
0001\end{array}$ & MG/L & & $\begin{array}{l}29.3 \\
19.3 \\
41.5\end{array}$ & $\begin{array}{l}R X \\
R X \\
R X\end{array}$ & $\begin{array}{l}0.5 \\
0.5 \\
0.5\end{array}$ & $\dot{-}$ \\
\hline CALCIUN (TOTAL) & $\begin{array}{l}12 / 15 / 93 \\
04 / 21 / 94 \\
12 / 13 / 94\end{array}$ & $\begin{array}{l}\text { N001 } \\
\text { N001 } \\
\text { N001 }\end{array}$ & $M G / L$ & & $\begin{array}{l}38.3 \\
23.1 \\
43.4\end{array}$ & $\begin{array}{l}\text { RX } \\
\text { RX } \\
\text { RX }\end{array}$ & $\begin{array}{l}0.5 \\
0.5 \\
0.5\end{array}$ & $\begin{array}{l}- \\
-\end{array}$ \\
\hline CHLORIDE & $\begin{array}{l}12 / 15 / 93 \\
04 / 21 / 94\end{array}$ & $\begin{array}{l}0001 \\
0001\end{array}$ & MG/L & & $\begin{array}{l}33.3 \\
15.0\end{array}$ & $\begin{array}{l}\text { RX } \\
\text { RX }\end{array}$ & $\begin{array}{l}0.5 \\
0.5\end{array}$ & : \\
\hline CHLORIDE (TOTAL) & $12 / 13 / 94$ & N001 & MG/L & & 17 & RX & 0.5 & - \\
\hline IRON & $12 / 13 / 94$ & 0001 & MG/L & $<$ & 0.03 & RX & 0.03 & - \\
\hline IRON (TOTAL) & $12 / 13 / 94$ & N001 & $M G / L$ & & 1.09 & RX & 0.03 & - \\
\hline MAGNESIUM & $\begin{array}{l}12 / 15 / 93 \\
04 / 21 / 94 \\
12 / 13 / 94\end{array}$ & $\begin{array}{l}0001 \\
0001 \\
0001\end{array}$ & $M G / L$ & & $\begin{array}{l}67.1 \\
35.6 \\
38.2\end{array}$ & $\begin{array}{l}R X \\
R X \\
R X\end{array}$ & $\begin{array}{l}0.1 \\
0.1 \\
0.1\end{array}$ & - \\
\hline MAGHESIUM (TOTAL) & $\begin{array}{l}12 / 15 / 93 \\
04 / 21 / 94 \\
12 / 13 / 94\end{array}$ & $\begin{array}{l}\text { NOO1 } \\
\text { NOO1 } \\
\text { NOO1 }\end{array}$ & $M G / L$ & & $\begin{array}{l}72.0 \\
36.0 \\
38.1\end{array}$ & $\begin{array}{l}R X \\
R X \\
R X\end{array}$ & $\begin{array}{l}0.1 \\
0.1 \\
0.1\end{array}$ & $\begin{array}{l}- \\
-\end{array}$ \\
\hline MANGAHESE & $\begin{array}{l}12 / 15 / 93 \\
04 / 21 / 94 \\
12 / 13 / 94\end{array}$ & $\begin{array}{l}0001 \\
0001 \\
0001\end{array}$ & MG/L & $<$ & $\begin{array}{l}0.01 \\
0.01 \\
0.01\end{array}$ & $\begin{array}{l}R X \\
R X \\
R X\end{array}$ & $\begin{array}{l}0.01 \\
0.01 \\
0.01\end{array}$ & - \\
\hline MANGAMESE (TOTAL) & $\begin{array}{l}12 / 15 / 93 \\
04 / 21 / 94 \\
12 / 13 / 94\end{array}$ & $\begin{array}{l}\text { N001 } \\
\text { NO01 } \\
\text { N001 }\end{array}$ & $H G / L$ & & $\begin{array}{l}0.14 \\
0.05 \\
0.04\end{array}$ & $\begin{array}{l}R X \\
R X \\
R X\end{array}$ & $\begin{array}{l}0.01 \\
0.01 \\
0.01\end{array}$ & : \\
\hline HOLYBDENUM & $12 / 13 / 94$ & 0001 & MG/L & $<$ & 0.01 & $R X$ & 0.01 & - \\
\hline MOLYBDENUM (TOTAL) & $12 / 13 / 94$ & NOO1 & MG/L & $<$ & 0.01 & $R X$ & 0.01 & - \\
\hline NITRATE & $12 / 15 / 93$ & 0001 & MG/L & $<$ & 1 & RX & 1 & - \\
\hline MITRATE (TOTAL) & $\begin{array}{l}04 / 29 / 94 \\
12 / 13 / 94\end{array}$ & $\begin{array}{l}\text { N001 } \\
\text { N001 }\end{array}$ & $M G / L$ & $<$ & 9 & $\begin{array}{l}R X \\
R X\end{array}$ & $\begin{array}{l}1 \\
1\end{array}$ & $\dot{-}$ \\
\hline PH & $\begin{array}{l}12 / 15 / 93 \\
04 / 21 / 94 \\
12 / 13 / 94\end{array}$ & $\begin{array}{l}\text { NOO1 } \\
\text { HOOI } \\
\text { HOOI }\end{array}$ & SU & & $\begin{array}{l}8.63 \\
9.10 \\
8.92\end{array}$ & $\begin{array}{l}R X \\
R X \\
R X\end{array}$ & $\begin{array}{l}- \\
0.10\end{array}$ & $\dot{-}$ \\
\hline PHOSPHATE (TOTAL) & $12 / 13 / 94$ & NoOT & MG/L & & 0.24 & $R X$ & 0.1 & - \\
\hline
\end{tabular}

PARAMETER VALUE INDICATOR (PVI): < - LESS THAN DETECTION LIMIT

OTHER PARAMETER VALUE FLAGS:

$R$ - UMUSABLE DATA POINT

$X$ - LOCATION UNDEFINED
SAMPLE ID COOES:

0001 - FILTERED SAMPLE (.45 MICRONS)

NOO1 - UMFILTERED SAMPLE 
SURFACE WATER QUALITY DATA BY LOCATION

SOWP REV. $O$ data

SITE: MONO1 MOHUMENT VALLEY

LOCATION: 0624 RESERVED FOR RPAPUSCH

NORTH COORDINATE: UNKNOWN

EAST COORDINATE: UHKMOWN

O4/11/88 TO $12 / 14 / 94$

REPORT DATE: 06/22/95

\begin{tabular}{|c|c|c|c|c|c|c|c|c|}
\hline PARAMETER NAME & LOG DATE & $\begin{array}{c}\text { SAMPLE } \\
\text { ID }\end{array}$ & $\begin{array}{l}\text { UNITS OF } \\
\text { MEASURE }\end{array}$ & PVI & $\begin{array}{l}\text { PARAMETER } \\
\text { VALUE }\end{array}$ & FLAGS & $\begin{array}{l}\text { DETECTION } \\
\text { LIHIT }\end{array}$ & $\begin{array}{l}\text { PARAMETER } \\
\text { UNCERTAINTY }\end{array}$ \\
\hline POTASSIUM & $\begin{array}{l}12 / 15 / 93 \\
04 / 21 / 94 \\
12 / 13 / 94\end{array}$ & $\begin{array}{l}0001 \\
0001 \\
0001\end{array}$ & $M G / L$ & & $\begin{array}{r}21.4 \\
9.7 \\
13.6\end{array}$ & $\begin{array}{l}R X \\
R X \\
R X\end{array}$ & $\begin{array}{l}0.1 \\
0.1 \\
0.1\end{array}$ & $\dot{-}$ \\
\hline POTASSIUK (TOTAL) & $\begin{array}{l}12 / 15 / 93 \\
04 / 21 / 94 \\
12 / 13 / 94\end{array}$ & $\begin{array}{l}\text { N001 } \\
\text { NO01 } \\
\text { N001 }\end{array}$ & $M G / L$ & & $\begin{array}{l}23.7 \\
10.3 \\
13.6\end{array}$ & $\begin{array}{l}R X \\
R X \\
R X\end{array}$ & $\begin{array}{l}0.1 \\
0.1 \\
0.1\end{array}$ & - \\
\hline REDOX POTENTIAL & $12 / 13 / 94$ & NO01 & mVOLTS & & 365 & $R X$ & - & - \\
\hline SILICA - SIOL & $\begin{array}{l}12 / 15 / 93 \\
12 / 13 / 94\end{array}$ & $\begin{array}{l}0001 \\
0001\end{array}$ & MG/L & & $\begin{array}{r}9.5 \\
16.6\end{array}$ & $\begin{array}{l}\text { RX } \\
\text { RX }\end{array}$ & $\begin{array}{l}0.1 \\
0.1\end{array}$ & 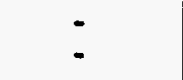 \\
\hline SILICA - SIO2 (TOTAL) & $\begin{array}{l}12 / 15 / 93 \\
12 / 13 / 94\end{array}$ & $\begin{array}{l}\text { N001 } \\
\text { N001 }\end{array}$ & HG/L & & $\begin{array}{l}27.8 \\
28.7\end{array}$ & $\begin{array}{l}R X \\
R X\end{array}$ & $\begin{array}{l}0.1 \\
0.1\end{array}$ & : \\
\hline SOOIUM & $\begin{array}{l}12 / 15 / 93 \\
04 / 21 / 94 \\
12 / 13 / 94\end{array}$ & $\begin{array}{l}0001 \\
0001 \\
0001\end{array}$ & HG/L & & $\begin{array}{l}148 \\
68 \\
92.5\end{array}$ & $\begin{array}{l}\text { RX } \\
\text { RX } \\
\text { RX }\end{array}$ & $\begin{array}{l}1 \\
1 \\
1\end{array}$ & $\dot{-}$ \\
\hline SOOIUH (TOTAL) & $\begin{array}{l}12 / 15 / 93 \\
04 / 21 / 94 \\
12 / 13 / 94\end{array}$ & $\begin{array}{l}\text { No01 } \\
\text { N001 } \\
\text { NO01 }\end{array}$ & $M G / L$ & & $\begin{array}{l}154 \\
61 \\
89.1\end{array}$ & $\begin{array}{l}\text { RX } \\
\text { RX } \\
\text { RX }\end{array}$ & $\begin{array}{l}1 \\
1 \\
1\end{array}$ & : \\
\hline SPECIFIC CONDUCTANCE & $\begin{array}{l}12 / 15 / 93 \\
04 / 21 / 94 \\
12 / 13 / 94\end{array}$ & $\begin{array}{l}\text { NO01 } \\
\text { NO01 } \\
\text { NO01 }\end{array}$ & UMHO/CM & & $\begin{array}{l}714 \\
647 \\
496\end{array}$ & $\begin{array}{l}\text { RX } \\
\text { RX } \\
\text { RX }\end{array}$ & 1 & - \\
\hline STRONTIUM & $\begin{array}{l}12 / 15 / 93 \\
04 / 21 / 94 \\
12 / 13 / 94\end{array}$ & $\begin{array}{l}0001 \\
0001 \\
0001\end{array}$ & $M G / L$ & & $\begin{array}{l}0.72 \\
0.55 \\
0.65\end{array}$ & $\begin{array}{l}\text { RX } \\
\text { RX } \\
\text { JRX }\end{array}$ & $\begin{array}{l}0.01 \\
0.01 \\
0.01\end{array}$ & - \\
\hline STRONTIUM (TOTAL) & $\begin{array}{l}12 / 15 / 93 \\
04 / 21 / 94 \\
12 / 13 / 94\end{array}$ & $\begin{array}{l}\text { No01 } \\
\text { NO01 } \\
\text { NO01 }\end{array}$ & MG/L & & $\begin{array}{l}0.78 \\
0.53 \\
0.6\end{array}$ & $\begin{array}{l}\text { RX } \\
\text { RX } \\
\text { JRX }\end{array}$ & $\begin{array}{l}0.01 \\
0.01 \\
0.01\end{array}$ & - \\
\hline SULFATE & $\begin{array}{l}12 / 15 / 93 \\
04 / 21 / 94 \\
12 / 13 / 94\end{array}$ & $\begin{array}{l}0001 \\
0001 \\
0001\end{array}$ & MG/L & & $\begin{array}{l}63 \\
53 \\
58\end{array}$ & $\begin{array}{l}R X \\
R X \\
R X\end{array}$ & $\begin{array}{l}1 \\
1 \\
1\end{array}$ & $\dot{-}$ \\
\hline SULFATE (TOTAL) & $12 / 13 / 94$ & No01 & MG/L & & 63 & RX & 1 & - \\
\hline TEMPERATURE & $\begin{array}{l}12 / 15 / 93 \\
04 / 21 / 94 \\
12 / 13 / 94\end{array}$ & $\begin{array}{l}\text { N001 } \\
\text { N001 } \\
\text { N001 }\end{array}$ & C - DEGREE & & $\begin{array}{r}1.9 \\
26.7 \\
4.7\end{array}$ & $\begin{array}{l}R X \\
R X \\
R X\end{array}$ & $\therefore$ & 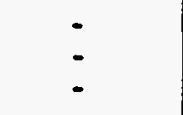 \\
\hline TOTAL DISSOLVED SOLIDS & $\begin{array}{l}12 / 15 / 93 \\
04 / 2 i / 94 \\
12 / 13 / 94\end{array}$ & $\begin{array}{l}0001 \\
0001 \\
0001\end{array}$ & $M G / L$ & & $\begin{array}{l}790 \\
400 \\
282\end{array}$ & $\begin{array}{l}\text { RX } \\
R X \\
R X\end{array}$ & $\begin{array}{l}10 \\
10 \\
10\end{array}$ & 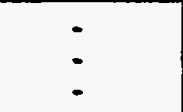 \\
\hline URANIUM & $\begin{array}{l}12 / 95 / 93 \\
04 / 21 / 94\end{array}$ & $\begin{array}{l}0001 \\
0001\end{array}$ & $M G / L$ & & $\begin{array}{l}0.124 \\
0.025\end{array}$ & $\begin{array}{l}\text { RX } \\
R X\end{array}$ & $\begin{array}{l}0.001 \\
0.001\end{array}$ & - \\
\hline URAHIUM (TOTAL) & $\begin{array}{l}12 / 15 / 93 \\
04 / 21 / 94\end{array}$ & $\begin{array}{l}\text { NOO1 } \\
\text { NOO1 }\end{array}$ & MG/L & & $\begin{array}{l}0.123 \\
0.025\end{array}$ & $\begin{array}{l}\text { RX } \\
\text { RX }\end{array}$ & $\begin{array}{l}0.001 \\
0.001\end{array}$ & - \\
\hline
\end{tabular}

PARAMETER VALUE INDICATOR (PVI): < - LESS THAN DETECTION LIMIT

SNYPLE ID COOES:

OTHER PARAMETER VALUE FLAGS:

DOD1 - FILTERED SMPLE (.45 MICRONS)

$\downarrow$ - estimated VALUE

NOO1 - UHFILTERED SAMPLE

$R$ - UAUSABLE DATA POINT

$X$ - LOCATION UHDEFIHED 
SURFACE WATER QUALITY DATA BY LOCATION

SOWP REV. $O$ data

SITE: MONO1 MONUMENT VALLEY

LOCATION: 0624 RESERVED FOR RPAPUSCH

NORTH COORDINATE: UHKHOWN

EAST COORDINATE: UNKNOWH

04/11/88 TO $12 / 14 / 94$

REPORT DATE: $06 / 22 / 95$

\begin{tabular}{|c|c|c|c|c|c|c|c|c|}
\hline PARAMETER NAME & LOG DATE & $\begin{array}{c}\text { SAMPLE } \\
\text { ID }\end{array}$ & $\begin{array}{l}\text { UNITS OF } \\
\text { MEASURE }\end{array}$ & PVI & $\begin{array}{l}\text { PARAMETER } \\
\text { VALUE }\end{array}$ & FLAGS & $\begin{array}{l}\text { DETECTION } \\
\text { LIMIT }\end{array}$ & $\begin{array}{l}\text { PARAMETER } \\
\text { UHCERTAIHTY }\end{array}$ \\
\hline URANIUM (TOTAL) & $12 / 13 / 94$ & NOO1 & $M G / L$ & & 0.019 & $R X$ & 0.001 & - \\
\hline VAKADIUH & $\begin{array}{l}12 / 15 / 93 \\
04 / 21 / 94 \\
12 / 13 / 94\end{array}$ & $\begin{array}{l}0001 \\
0001 \\
0001\end{array}$ & $M G / L$ & $<$ & $\begin{array}{l}0.01 \\
0.01 \\
0.01\end{array}$ & $\begin{array}{l}R X \\
R X \\
R X\end{array}$ & $\begin{array}{l}0.01 \\
0.01 \\
0.01\end{array}$ & : \\
\hline VANADIUM (TOTAL) & $\begin{array}{l}12 / 15 / 93 \\
04 / 21 / 94 \\
12 / 13 / 94\end{array}$ & $\begin{array}{l}\text { N001 } \\
\text { N001 } \\
\text { N001 }\end{array}$ & HG/L & $<$ & $\begin{array}{l}0.02 \\
0.02 \\
0.01\end{array}$ & $\begin{array}{l}\text { RX } \\
\text { RX } \\
\text { RX }\end{array}$ & $\begin{array}{l}0.01 \\
0.01 \\
0.01\end{array}$ & $:$ \\
\hline ZINC & $12 / 13 / 94$ & 0001 & MG/L & $<$ & 0.05 & RX & 0.05 & - \\
\hline ZINC (TOTAL) & $12 / 13 / 94$ & N001 & $M G / L$ & $<$ & 0.05 & RX & 0.05 & - \\
\hline
\end{tabular}

PARAMETER VALUE INDICATOR (PVI):

< - LESS THAN DETECTION LIMIT

SAMPLE ID CODES:

0009 - FILTERED SAMPLE (.45 MICRONS)

OTHER PARAMETER VALUE FLAGS:

NOO 9 - UNFILTERED SAMLE

$R$ - UNUSABLE DATA POINT

$X$ - LOCATION UNDEFIHED 
SURFACE WATER QUALITY DATA BY LOCATION

SOWP REV. 0 data

SITE: MONO1 MONUMENT YALLEY

LOCATION: 0626 RESERVED FOR RPAPUSCH

NORTH COORDINATE: UHKNOWH

EAST COORDINATE: UNKHOWH

04/19/88 TO $12 / 14 / 94$

REPORT DATE: $06 / 22 / 95$

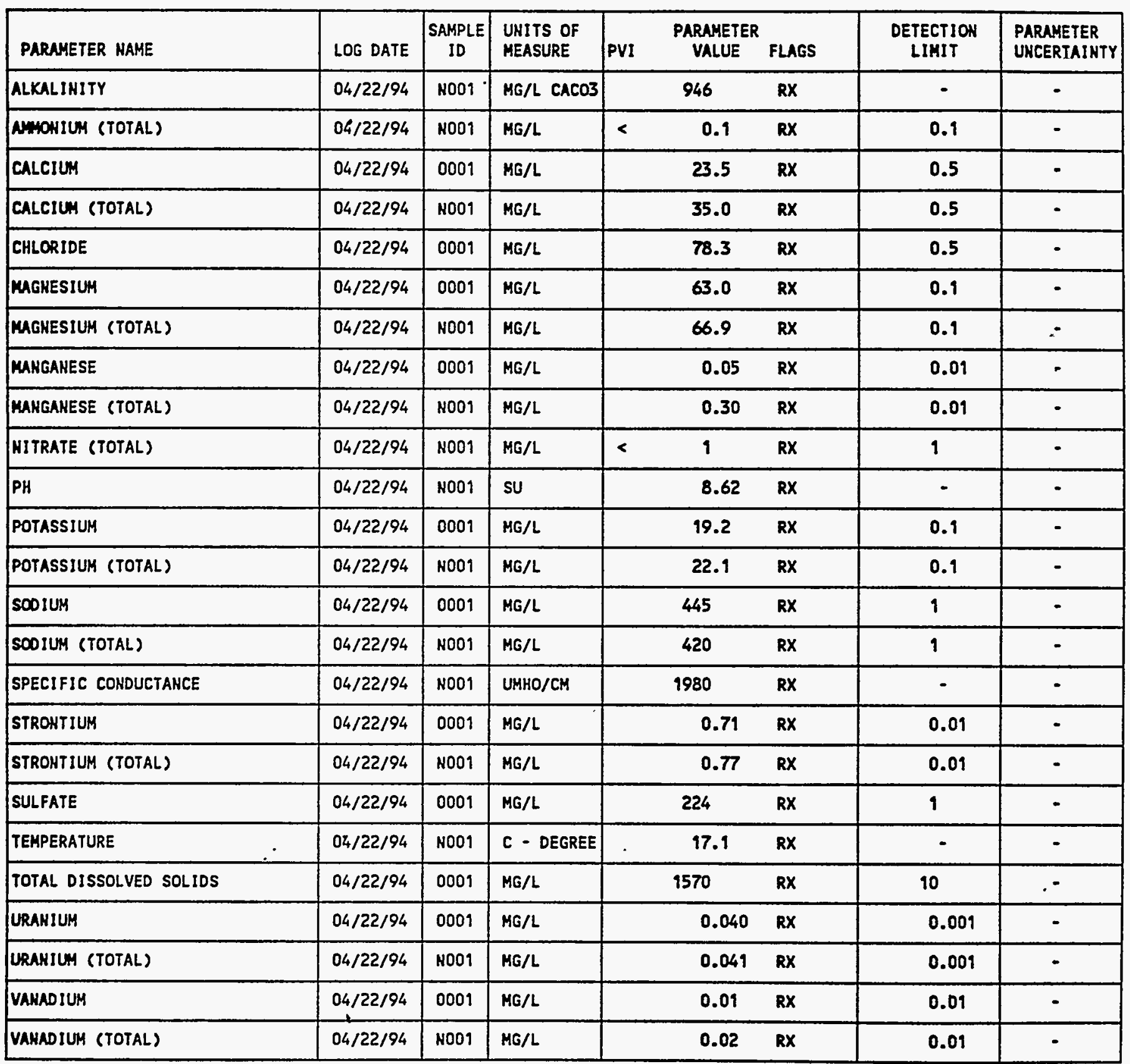

PARAMETER VALUE IMDICATOR (PVI): < - LESS THAN DETECTION LIMIT

SUMPLE ID COOES:

O009 - FILTERED SAMPLE (.45 MICROHS)

OTHER PARAMETER VALUE FLAGS:

NOOI - UNFILTERED SNMPLE

R - UHUSABLE DATA POINT

$X$ - LOCATION UHDEFIMED 
SURFACE WATER QUALITY DATA BY LOCATION

SOWP REV. 0 data

SITE: MONO1 MONUMENT VALLEY

LOCATION: 0627 RESERVED FOR RPAPUSCH

NORTH COORDINATE: UNKNOWN

EAST COORDINATE: UNKNOWN

$04 / 11 / 88$ TO $12 / 14 / 94$

REPORT DATE : 06/22/95

\begin{tabular}{|c|c|c|c|c|c|c|c|c|}
\hline PARAMETER NAME & LOG DATE & $\begin{array}{c}\text { SAMPLE } \\
\text { ID }\end{array}$ & $\begin{array}{l}\text { UNITS OF } \\
\text { MEASURE }\end{array}$ & PVI & $\begin{array}{l}\text { PARAMETER } \\
\text { VALUE }\end{array}$ & FLAGS & $\begin{array}{l}\text { DETECT ION } \\
\text { LIMIT }\end{array}$ & $\begin{array}{l}\text { PARAMETER } \\
\text { UNCERTAINTY }\end{array}$ \\
\hline ALKALINITY & $\begin{array}{l}07 / 01 / 93 \\
12 / 14 / 93 \\
04 / 22 / 94 \\
12 / 11 / 94\end{array}$ & $\begin{array}{l}\text { N001 } \\
\text { N001 } \\
\text { N001 } \\
\text { N001 }\end{array}$ & MG/L CACO3 & & $\begin{array}{r}3520 \\
15106 \\
3450 \\
725\end{array}$ & $\begin{array}{l}R X \\
R X \\
R X \\
\text { JRX }\end{array}$ & $10^{-}$ & $\begin{array}{l}- \\
- \\
-\end{array}$ \\
\hline AmSONIUM & $12 / 27 / 93$ & 0001 & $M G / L$ & & 1.6 & $\mathbf{R X}$ & 0.1 & - \\
\hline AmPONIUH (TOTAL) & $\begin{array}{l}07 / 01 / 93 \\
04 / 22 / 94 \\
12 / 11 / 94\end{array}$ & $\begin{array}{l}\text { NO01 } \\
\text { N001 } \\
\text { N001 }\end{array}$ & $M G / L$ & & $\begin{array}{l}0.5 \\
1.0 \\
2.3\end{array}$ & $\begin{array}{l}R X \\
R X \\
R X\end{array}$ & $\begin{array}{l}0.1 \\
0.1 \\
0.1\end{array}$ & - \\
\hline ARSENIC (TOTAL) & $07 / 01 / 93$ & No01 & $M G / L$ & & 0.036 & RSX & 0.005 & - \\
\hline CALCIUH & $\begin{array}{l}12 / 14 / 93 \\
04 / 22 / 94 \\
12 / 11 / 94\end{array}$ & $\begin{array}{l}0001 \\
0001 \\
0001\end{array}$ & $M G / L$ & $<$ & $\begin{array}{l}9.5 \\
8.9 \\
10\end{array}$ & $\begin{array}{l}\text { RX } \\
\text { RX } \\
\text { IRX }\end{array}$ & $\begin{array}{l}0.5 \\
0.5 \\
10\end{array}$ & - \\
\hline CALCIUH (TOTAL) & $\begin{array}{l}12 / 14 / 93 \\
04 / 22 / 94 \\
12 / 11 / 94\end{array}$ & $\begin{array}{l}\text { N001 } \\
\text { No01 } \\
\text { N001 }\end{array}$ & $H G / L$ & & $\begin{array}{l}30.5 \\
22.6 \\
45.8\end{array}$ & $\begin{array}{l}R X \\
R X \\
\text { IRX }\end{array}$ & $\begin{array}{l}0.5 \\
0.5 \\
5\end{array}$ & - \\
\hline CHLORIDE & $\begin{array}{l}12 / 27 / 93 \\
04 / 22 / 94\end{array}$ & $\begin{array}{l}0001 \\
0001\end{array}$ & $M G / L$ & & $\begin{array}{l}938 \\
568\end{array}$ & $\begin{array}{l}\mathbf{R X} \\
\mathbf{R X}\end{array}$ & $\begin{array}{l}0.5 \\
0.5\end{array}$ & - \\
\hline CHLORIDE (TOTAL) & $12 / 11 / 94$ & N001 & MG/L & & 746 & $\mathbf{R X}$ & 0.5 & - \\
\hline IRON & $12 / 11 / 94$ & 0001 & $M G / L$ & & 0.64 & IRX & 0.2 & - \\
\hline IRON (TOTAL) & $12 / 11 / 94$ & No01 & $M G / L$ & & 23.9 & IRX & 0.1 & - \\
\hline MAGNESIUM & $\begin{array}{l}12 / 14 / 93 \\
04 / 22 / 94 \\
12 / 11 / 94\end{array}$ & $\begin{array}{l}0001 \\
0001 \\
0001\end{array}$ & $M G / L$ & & $\begin{array}{l}82.0 \\
58.2 \\
50\end{array}$ & $\begin{array}{l}\text { RX } \\
\text { RX } \\
\text { IRX }\end{array}$ & $\begin{array}{l}0.1 \\
0.1 \\
4\end{array}$ & $\begin{array}{l}- \\
-\end{array}$ \\
\hline MAGHESIUM (TOTAL) & $\begin{array}{l}12 / 14 / 93 \\
04 / 22 / 94 \\
12 / 11 / 94\end{array}$ & $\begin{array}{l}\text { N001 } \\
\text { NC01 } \\
\text { NO01 }\end{array}$ & $M G / L$ & & $\begin{array}{l}70.4 \\
63.4 \\
76\end{array}$ & $\begin{array}{l}\text { RX } \\
\text { RX } \\
\text { IRX }\end{array}$ & $\begin{array}{l}0.1 \\
0.1 \\
2\end{array}$ & $\begin{array}{l}- \\
-\end{array}$ \\
\hline MANGANESE & $\begin{array}{l}12 / 14 / 93 \\
04 / 22 / 94 \\
12 / 11 / 94\end{array}$ & $\begin{array}{l}0001 \\
0001 \\
0001\end{array}$ & $M G / L$ & $<$ & $\begin{array}{l}0.17 \\
0.06 \\
0.2\end{array}$ & $\begin{array}{l}\mathbf{R X} \\
\mathbf{R X} \\
\mathbf{I R X}\end{array}$ & $\begin{array}{l}0.01 \\
0.01 \\
0.2\end{array}$ & $\begin{array}{l}- \\
-\end{array}$ \\
\hline MAKGANESE (TOTAL) & $\begin{array}{l}12 / 14 / 93 \\
04 / 22 / 94 \\
12 / 11 / 94\end{array}$ & $\begin{array}{l}\text { NOO1 } \\
\text { NO01 } \\
\text { NO01 }\end{array}$ & $M G / L$ & & $\begin{array}{l}0.62 \\
0.34 \\
0.7\end{array}$ & $\begin{array}{l}R X \\
R X \\
I R X\end{array}$ & $\begin{array}{l}0.01 \\
0.01 \\
0.01\end{array}$ & - \\
\hline MOLYBDENUM & $12 / 11 / 94$ & 0001 & $M G / L$ & $<$ & 0.2 & IRX & 0.2 & - \\
\hline MLLYBDENUM (TOTAL) & $12 / 11 / 94$ & N001 & HG/L & & 0.12 & IRX & 0.1 & - \\
\hline NITRATE & $12 / 27 / 93$ & 0001 & $M G / L$ & $<$ & 1 & $\mathbf{R X}$ & 1 & - \\
\hline NITRATE (TOTAL) & $07 / 01 / 93$ & No01 & MG/L & $<$ & 1 & $\mathbf{R X}$ & 1 & - \\
\hline
\end{tabular}

PARAMETER VALUE INDICATOR (PVI): < - LESS THAH DETECTION LIMIT

OTHER PARANETER VALUE FLAGS:

SAMPLE ID COOES:

0001 - FILTERED SAMPLE (.45 MICROHS)

I - INCREASED DETECTION LIMIT DUE TO REOUIRED DILUTION

NOO1 - UNFILTERED SAMPLE

J - ESTIMATED VALUE

$R$ - UNUSABLE DATA POINT

S - REPORTED VALUE DETERMIHED USING METHOD OF STD ADDITION (MSA)

X - LOCATION UHDEFIHED 
SURFACE WATER OUALITY DATA BY LOCATION

SOWP ReV. $O$ data

SITE: MONO1 MONUMENT VALLEY

LOCATION: 0627 RESERVED FOR RPAPUSCH

MORTH COORDINATE: UNKNOWN

EAST COORDINATE: UNKNOWH

$04 / 11 / 88$ TO $12 / 14 / 94$

REPORT DATE: 06/22/95

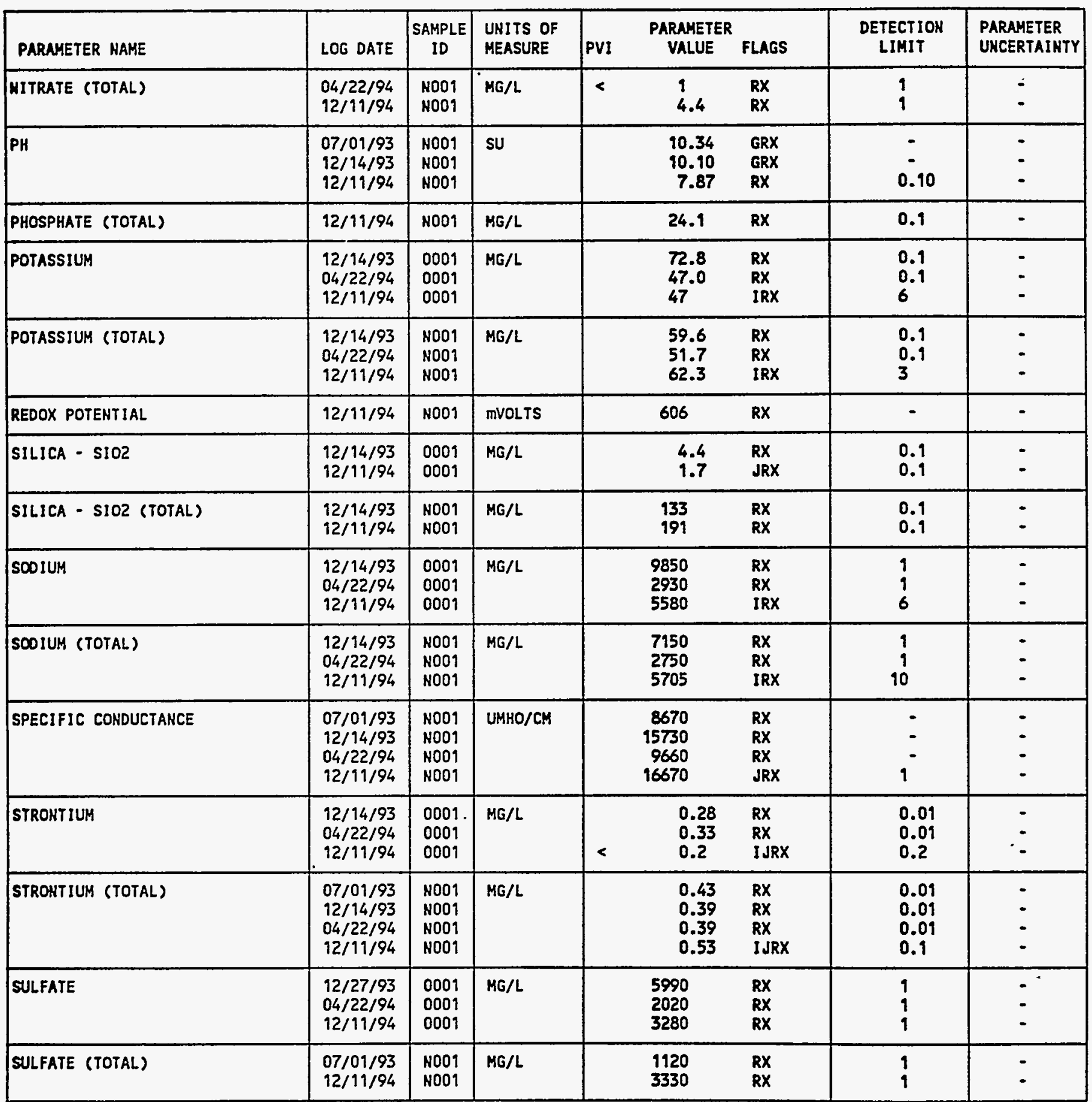

PARAMETER VALUE IHDICATOR (PVI): < - LESS THAN DETECTION LIMIT

SAMPLE ID COOES:

OOO1 - FILTERED SAMPLE (.45 MICRONS)

OTHER PARAMETER VALUE FLAGS:

MOO1 - UAFILTERED SAMPLE

G - PH > 9, POSSIBLE GROUT CONTAMINATION

I - IMCREASED DETECTION LIMIT DUE TO REQUIRED DILUTION

J - ESTIMATED VALUE

$R$ - UHUSABLE DATA POINT

$X$ - LOCATION UNDEFINED 
SURFACE WATER QUALITY DATA BY LOCATION

SOWP Rev. $O$ data

SITE: MONO1 MONUMENT VALLEY

LOCATION: 0627 RESERVED FOR RPAPUSCH

NORTH COORDINATE: UHKNOWN

EAST COORDINATE: UNKNOWH

04/11/88 TO $12 / 14 / 94$

REPORT DATE: $06 / 22 / 95$

\begin{tabular}{|c|c|c|c|c|c|c|c|c|}
\hline PARAMETER NAME & LOG DATE & $\begin{array}{c}\text { SAMPLE } \\
10\end{array}$ & $\begin{array}{l}\text { UNITS OF } \\
\text { MEASURE }\end{array}$ & PVI & $\begin{array}{l}\text { PARAMETER } \\
\text { VALUE }\end{array}$ & FLAGS & $\begin{array}{l}\text { DETECTIOH } \\
\text { LIMIT }\end{array}$ & $\begin{array}{l}\text { PARAMETER } \\
\text { UNCERTAIHTY }\end{array}$ \\
\hline TEMPERATURE & $\begin{array}{l}07 / 01 / 93 \\
12 / 14 / 93 \\
04 / 22 / 94 \\
12 / 11 / 94\end{array}$ & $\begin{array}{l}\text { N001 } \\
\text { NO01 } \\
\text { N001 } \\
\text { N001 }\end{array}$ & C - DEGREE & & $\begin{array}{r}26.7 \\
2.8 \\
18.9 \\
6.3\end{array}$ & $\begin{array}{l}\text { RX } \\
\text { RX } \\
\text { RX } \\
\text { RX }\end{array}$ & $\begin{array}{c}- \\
- \\
0.1\end{array}$ & - \\
\hline TOTAL DISSOLVED SOLIDS & $\begin{array}{l}12 / 27 / 93 \\
04 / 22 / 94 \\
12 / 11 / 94\end{array}$ & $\begin{array}{l}0001 \\
0001 \\
0001\end{array}$ & $M G / L$ & & $\begin{array}{r}26600 \\
8420 \\
16480\end{array}$ & $\begin{array}{l}R X \\
R X \\
R X\end{array}$ & $\begin{array}{l}10 \\
10 \\
10\end{array}$ & - \\
\hline TOTAL DISSOLVED SOLIDS (TOTAL) & $07 / 01 / 93$ & N001 & $M G / L$ & & 5670 & $R X$ & 10 & - \\
\hline URANIUH & $\begin{array}{l}12 / 14 / 93 \\
04 / 22 / 94\end{array}$ & $\begin{array}{l}0001 \\
0001\end{array}$ & MG/L & & $\begin{array}{l}0.217 \\
0.087\end{array}$ & $\begin{array}{l}R X \\
R X\end{array}$ & $\begin{array}{l}0.001 \\
0.001\end{array}$ & - \\
\hline URAKIUM (TOTAL) & $\begin{array}{l}07 / 01 / 93 \\
12 / 14 / 93 \\
04 / 22 / 94 \\
12 / 11 / 94\end{array}$ & $\begin{array}{l}\text { NOO1 } \\
\text { NOO1 } \\
\text { N001 } \\
\text { NOO1 }\end{array}$ & MG/L & & $\begin{array}{l}0.043 \\
0.157 \\
0.095 \\
0.022\end{array}$ & $\begin{array}{l}R X \\
R X \\
R X \\
R X\end{array}$ & $\begin{array}{l}0.001 \\
0.001 \\
0.001 \\
0.001\end{array}$ & - \\
\hline VANADIUM & $\begin{array}{l}12 / 14 / 93 \\
04 / 22 / 94 \\
12 / 11 / 94\end{array}$ & $\begin{array}{l}0001 \\
0001 \\
0001\end{array}$ & $M G / L$ & $<$ & $\begin{array}{l}0.31 \\
0.07 \\
0.2\end{array}$ & $\begin{array}{l}R X \\
R X \\
I R X\end{array}$ & $\begin{array}{l}0.01 \\
0.01 \\
0.2\end{array}$ & - \\
\hline VANADIUM (TOTAL) & $\begin{array}{l}07 / 01 / 93 \\
12 / 14 / 93 \\
04 / 22 / 94 \\
12 / 11 / 94\end{array}$ & $\begin{array}{l}\text { NOO9 } \\
\text { NOOY } \\
\text { NOOI } \\
\text { NOOI }\end{array}$ & MG/L & & $\begin{array}{l}0.10 \\
0.25 \\
0.09 \\
0.17\end{array}$ & $\begin{array}{l}R X \\
R X \\
R X \\
\text { IRX }\end{array}$ & $\begin{array}{l}0.01 \\
0.01 \\
0.01 \\
0.1\end{array}$ & - \\
\hline ZINC & $12 / 11 / 94$ & 0001 & $M G / L$ & $<$ & 9 & IRX & 1 & - \\
\hline ZINC (TOTAL) & $\begin{array}{l}07 / 01 / 93 \\
12 / 11 / 94\end{array}$ & $\begin{array}{l}\text { N001 } \\
\text { N001 }\end{array}$ & $M G / L$ & & $\begin{array}{l}0.081 \\
0.17\end{array}$ & $\begin{array}{l}\text { JRX } \\
\text { IRX }\end{array}$ & $\begin{array}{l}0.005 \\
0.1\end{array}$ & . \\
\hline
\end{tabular}

PARAMETER VALUE INDICATOR (PVI): < - LESS THAN DETECTION LIMIT

SAMPLE ID COOES:

0009 - FILTERED SAMPLE (.45 MICRONS)

OTHER PARAMETER VALUE FLAGS:

NOOI - UHFILTERED SAMPLE

1 - INCREASED DETECTION LIMIT DUE TO REQUIRED DILUTION

J - ESTIMATED VALUE

$R$ - UNUSABLE DATA POINT

$X$ - LOCATION UNDEFINED 
SURFACE WATER QUALITY DATA BY LOCATION

SOWP REV. $O$ data

SITE: MONOI MONUMENT VALLEY

LOCATION: 0976 HQD KNOWNS

NORTH COORDINATE: UNKHOUN

EAST COORDINATE: UNKHOWN

$04 / 11 / 88$ TO 12/14/94

REPORT DATE: $06 / 22 / 95$

\begin{tabular}{|c|c|c|c|c|c|c|c|c|}
\hline PARAMETER HAME & LOG DATE & $\begin{array}{c}\text { SAMPLE } \\
10\end{array}$ & $\begin{array}{l}\text { UNITS OF } \\
\text { MEASURE }\end{array}$ & PVI & $\begin{array}{l}\text { PARAMETER } \\
\text { VALUE }\end{array}$ & FLAGS & $\begin{array}{l}\text { DETECTION } \\
\text { LIMIT }\end{array}$ & $\begin{array}{l}\text { PARAMETER } \\
\text { UNCERTAINTY }\end{array}$ \\
\hline ALUAIHUM & $\begin{array}{l}05 / 22 / 99 \\
05 / 22 / 99 \\
05 / 22 / 91\end{array}$ & $\begin{array}{l}\text { OOAK } \\
\text { OODK } \\
\text { OOTK }\end{array}$ & MG/L & & $\begin{array}{l}0.72 \\
0.72 \\
0.700\end{array}$ & $\begin{array}{l}\text { RX } \\
\text { RX } \\
\text { RX }\end{array}$ & $\begin{array}{l}0.05 \\
0.05 \\
?\end{array}$ & : \\
\hline ARMONIUH & $\begin{array}{l}05 / 22 / 91 \\
05 / 22 / 91 \\
05 / 22 / 91\end{array}$ & $\begin{array}{l}\text { OOAK } \\
\text { OODK } \\
\text { OOTK }\end{array}$ & $M G / L$ & & $\begin{array}{l}0.6 \\
0.6 \\
0.580\end{array}$ & $\begin{array}{l}\text { RX } \\
R X \\
R X\end{array}$ & $\begin{array}{c}0.1 \\
0.1 \\
.\end{array}$ & : \\
\hline ANTIHONY & $\begin{array}{l}05 / 22 / 91 \\
05 / 22 / 91 \\
05 / 22 / 91\end{array}$ & $\begin{array}{l}\text { OOAK } \\
\text { OODK } \\
\text { OOTK }\end{array}$ & MG/L & & $\begin{array}{l}0.036 \\
0.043 \\
0.054\end{array}$ & $\begin{array}{l}R X \\
R X \\
R X\end{array}$ & $\begin{array}{l}0.003 \\
0.003 \\
-\end{array}$ & : \\
\hline ARSENIC & $\begin{array}{l}05 / 22 / 91 \\
05 / 22 / 91 \\
05 / 22 / 91\end{array}$ & $\begin{array}{l}\text { OOAK } \\
\text { OODK } \\
\text { OOTK }\end{array}$ & $M G / L$ & & $\begin{array}{l}0.14 \\
0.15 \\
0.175\end{array}$ & $\begin{array}{l}\text { RX } \\
\text { RX } \\
\text { RX }\end{array}$ & $\begin{array}{l}0.01 \\
0.01 \\
-\end{array}$ & $\begin{array}{l}- \\
:\end{array}$ \\
\hline BARIUM & $\begin{array}{l}05 / 22 / 91 \\
05 / 22 / 91 \\
05 / 22 / 91\end{array}$ & $\begin{array}{l}\text { OOAK } \\
\text { OODK } \\
\text { OOTK }\end{array}$ & $M G / L$ & & $\begin{array}{l}0.67 \\
0.68 \\
0.700\end{array}$ & $\begin{array}{l}R X \\
R X \\
R X\end{array}$ & $\begin{array}{c}0.01 \\
0.01 \\
-\end{array}$ & : \\
\hline BERYLLIUN & $\begin{array}{l}05 / 22 / 91 \\
05 / 22 / 91 \\
05 / 22 / 91\end{array}$ & $\begin{array}{l}\text { OOAK } \\
\text { OODK } \\
\text { OOTK }\end{array}$ & MG/L & & $\begin{array}{l}0.179 \\
0.181 \\
0.175\end{array}$ & $\begin{array}{l}R X \\
R X \\
R X\end{array}$ & $\begin{array}{l}0.005 \\
0.005 \\
?\end{array}$ & - \\
\hline BORON & $\begin{array}{l}05 / 22 / 91 \\
05 / 22 / 91 \\
05 / 22 / 91\end{array}$ & $\begin{array}{l}\text { OOAK } \\
\text { OODK } \\
\text { OOTK }\end{array}$ & $M G / L$ & & $\begin{array}{l}0.57 \\
0.57 \\
0.600\end{array}$ & $\begin{array}{l}R X \\
R X \\
R X\end{array}$ & $\begin{array}{l}0.05 \\
0.05 \\
-\end{array}$ & : \\
\hline BROHIDE & $\begin{array}{l}05 / 22 / 91 \\
05 / 22 / 91 \\
05 / 22 / 91\end{array}$ & $\begin{array}{l}\text { OOAK } \\
\text { OODK } \\
\text { OOTK }\end{array}$ & $M G / L$ & & $\begin{array}{l}1.1 \\
1.1 \\
1.10\end{array}$ & $\begin{array}{l}\text { RX } \\
\text { RX } \\
\text { RX }\end{array}$ & $\begin{array}{c}0.1 \\
0.1 \\
-\end{array}$ & - \\
\hline CADMIUM & $\begin{array}{l}05 / 22 / 91 \\
05 / 22 / 91 \\
05 / 22 / 91\end{array}$ & $\begin{array}{l}\text { OOAK } \\
\text { OODK } \\
\text { OOTK }\end{array}$ & $M G / L$ & & $\begin{array}{l}0.171 \\
0.172 \\
0.175\end{array}$ & $\begin{array}{l}R X \\
R X \\
R X\end{array}$ & $\begin{array}{l}0.005 \\
0.005 \\
-\end{array}$ & - \\
\hline CALCIUH & $\begin{array}{l}05 / 22 / 91 \\
05 / 22 / 91 \\
05 / 22 / 91\end{array}$ & $\begin{array}{l}\text { OOAK } \\
\text { OODK } \\
\text { OOTK }\end{array}$ & $M G / L$ & & $\begin{array}{l}20.0 \\
21.6 \\
20.0\end{array}$ & $\begin{array}{l}R X \\
R X \\
R X\end{array}$ & $\begin{array}{c}0.1 \\
0.5 \\
-\end{array}$ & 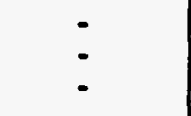 \\
\hline CHLORIDE & $\begin{array}{l}05 / 22 / 91 \\
05 / 22 / 91 \\
05 / 22 / 91\end{array}$ & $\begin{array}{l}\text { DOAK } \\
\text { OODK } \\
\text { OOTK }\end{array}$ & $M G / L$ & & $\begin{array}{l}12.0 \\
11.9 \\
11.3\end{array}$ & $\begin{array}{l}R X \\
R X \\
R X\end{array}$ & $\begin{array}{c}0.5 \\
0.5 \\
-\end{array}$ & $\dot{-}$ \\
\hline CHRONIUM & $\begin{array}{l}85 / 22 / 91 \\
05 / 22 / 91 \\
05 / 22 / 91\end{array}$ & $\begin{array}{l}\text { OOAK } \\
\text { OODK } \\
\text { OOTK }\end{array}$ & MG/L & & $\begin{array}{l}0.34 \\
0.35 \\
0.350\end{array}$ & $\begin{array}{l}\mathbf{R X} \\
\mathbf{R X} \\
\mathbf{R X}\end{array}$ & $\begin{array}{c}0.01 \\
0.01 \\
.\end{array}$ & $\dot{-}$ \\
\hline COBALT & $\begin{array}{l}05 / 22 / 91 \\
05 / 22 / 91 \\
05 / 22 / 91\end{array}$ & $\begin{array}{l}\text { OOAK } \\
\text { OODK } \\
\text { OOTK }\end{array}$ & HG/L & & $\begin{array}{l}0.31 \\
0.32 \\
0.315\end{array}$ & $\begin{array}{l}R X \\
R X \\
R X\end{array}$ & $\begin{array}{c}0.03 \\
0.03 \\
-\end{array}$ & - \\
\hline COPPER & $\begin{array}{l}05 / 22 / 91 \\
05 / 22 / 91 \\
05 / 22 / 91\end{array}$ & $\begin{array}{l}\text { OOAK } \\
\text { OODK } \\
\text { OOTK }\end{array}$ & $H G / L$ & & $\begin{array}{l}0.21 \\
0.21 \\
0.210\end{array}$ & $\begin{array}{l}R X \\
R X \\
R X\end{array}$ & $\begin{array}{c}0.01 \\
0.01 \\
.\end{array}$ & $\dot{-}$ \\
\hline DISSOLVED ORGANIC CARBOH & $05 / 22 / 91$ & 00TK & MG/L & & 4.50 & RX & - & - \\
\hline
\end{tabular}

PARAMETER VALUE IMDICATOR (PVI): < - LESS THAN DETECTION LIMIT

OTHER PARAMETER VALUE FLAGS:

$R$ - UHUSABLE DATA POINT

$X$ - LOCATION UNDEFINED
SAMPLE ID COOES:

OOAK - AHALYTICAL KMOWN SAMPLE

ODOK - DUPLICATE KHOWN SAMPLE

OOTK - TKEORETICAL KNON SAMPLE 
SURFACE WATER QUALITY DATA BY LOCATION

SOWP ReV. 0 data

SITE: MONO1 MONUMENT VALLEY

LOCATION: 0976 WOD KNOWNS

NORTH COORDINATE: UNKNOWN

EAST COORDINATE: UNKNOUN

04/11/88 TO 12/14/94

REPORT DATE: $06 / 22 / 95$

\begin{tabular}{|c|c|c|c|c|c|c|c|c|}
\hline PARAMETER NAME & LOG DATE & $\begin{array}{c}\text { SAMPLE } \\
\text { ID }\end{array}$ & $\begin{array}{l}\text { UNITS OF } \\
\text { MEASURE }\end{array}$ & PVI & $\begin{array}{l}\text { PARANETER } \\
\text { VALUE }\end{array}$ & FLAGS & $\begin{array}{l}\text { DETECTION } \\
\text { LIMIT }\end{array}$ & $\begin{array}{l}\text { PARAMETER } \\
\text { UNCERTAIHTY }\end{array}$ \\
\hline FLUORIDE & $\begin{array}{l}05 / 22 / 91 \\
05 / 22 / 91 \\
05 / 22 / 91\end{array}$ & $\begin{array}{l}\text { OOAK } \\
\text { OODK } \\
\text { OOTK }\end{array}$ & MG/L & & $\begin{array}{l}5.6 \\
6.2 \\
5.73\end{array}$ & $\begin{array}{l}R X \\
R X \\
R X\end{array}$ & $\begin{array}{c}0.1 \\
0.1 \\
.\end{array}$ & - \\
\hline GROSS ALPHA & $\begin{array}{l}05 / 22 / 91 \\
05 / 22 / 91 \\
05 / 22 / 91\end{array}$ & $\begin{array}{l}\text { OOAK } \\
\text { OODK } \\
\text { OOTK }\end{array}$ & $\mathrm{PCI} / \mathrm{L}$ & & $\begin{array}{l}32.5 \\
27.8 \\
20.3\end{array}$ & $\begin{array}{l}R X \\
R X \\
R X\end{array}$ & $\begin{array}{l}1 . \\
1 .\end{array}$ & $\begin{array}{c}6.7 \\
6.3 \\
.\end{array}$ \\
\hline GROSS BETA & $\begin{array}{l}05 / 22 / 91 \\
05 / 22 / 91 \\
05 / 22 / 91\end{array}$ & $\begin{array}{l}\text { OOAK } \\
\text { DODK } \\
\text { OOTK }\end{array}$ & $\mathrm{PCl} / \mathrm{L}$ & & $\begin{array}{c}13.5 \\
4.51 \\
16.2\end{array}$ & $\begin{array}{l}R X \\
R X \\
R X\end{array}$ & $\begin{array}{c}0.5 \\
0.5 \\
.\end{array}$ & $\begin{array}{c}2.6 \\
2.6\end{array}$ \\
\hline IRON & $\begin{array}{l}05 / 22 / 91 \\
05 / 22 / 91 \\
05 / 22 / 91\end{array}$ & $\begin{array}{l}\text { OOAK } \\
\text { OODK } \\
\text { OOTK }\end{array}$ & $M G / L$ & & $\begin{array}{l}0.53 \\
0.54 \\
0.525\end{array}$ & $\begin{array}{l}R X \\
R X \\
R X\end{array}$ & $\begin{array}{l}0.03 \\
0.03\end{array}$ & $\begin{array}{l}- \\
-\end{array}$ \\
\hline LEAD & $\begin{array}{l}05 / 22 / 91 \\
05 / 22 / 91 \\
05 / 22 / 91\end{array}$ & $\begin{array}{l}\text { DOAK } \\
\text { OODK } \\
\text { OOTK }\end{array}$ & $H G / L$ & & $\begin{array}{l}0.16 \\
0.16 \\
0.175\end{array}$ & $\begin{array}{l}R X \\
R X \\
R X\end{array}$ & $\begin{array}{c}0.005 \\
0.005 \\
-\end{array}$ & $\begin{array}{l}- \\
-\end{array}$ \\
\hline MAGNESIUM & $\begin{array}{l}05 / 22 / 91 \\
05 / 22 / 91 \\
05 / 22 / 91\end{array}$ & $\begin{array}{l}\text { OOAK } \\
\text { OODK } \\
\text { OOTK }\end{array}$ & MG/L & & $\begin{array}{l}20.2 \\
20.4 \\
20.0\end{array}$ & $\begin{array}{l}R X \\
R X \\
R X\end{array}$ & $\begin{array}{c}0.1 \\
0.1 \\
.\end{array}$ & - \\
\hline MAKGANESE & $\begin{array}{l}05 / 22 / 91 \\
05 / 22 / 91 \\
05 / 22 / 91\end{array}$ & $\begin{array}{l}\text { OOAK } \\
\text { OODK } \\
\text { OOTK }\end{array}$ & MG/L & & $\begin{array}{l}0.34 \\
0.35 \\
0.350\end{array}$ & $\begin{array}{l}R X \\
R X \\
R X\end{array}$ & $\begin{array}{l}0.01 \\
0.01 \\
-\end{array}$ & $\begin{array}{l}- \\
- \\
-\end{array}$ \\
\hline MOL YBDENUM & $\begin{array}{l}05 / 22 / 91 \\
05 / 22 / 91 \\
05 / 22 / 91\end{array}$ & $\begin{array}{l}\text { OOAK } \\
\text { OODK } \\
\text { OOTK }\end{array}$ & $M G / L$ & & $\begin{array}{l}0.16 \\
0.15 \\
0.153\end{array}$ & $\begin{array}{l}\text { RX } \\
\text { RX } \\
R X\end{array}$ & $\begin{array}{l}0.01 \\
0.01 \\
.\end{array}$ & $\begin{array}{l}- \\
\text { - }\end{array}$ \\
\hline NET GROSS ALPHA * & $\begin{array}{l}05 / 22 / 91 \\
05 / 22 / 91 \\
05 / 22 / 91\end{array}$ & $\begin{array}{l}\text { OOAK } \\
\text { OODK } \\
\text { OOTK }\end{array}$ & $\mathrm{PCI} / \mathrm{L}$ & & $\begin{array}{l}-95.78 \\
-97.05 \\
-99.75\end{array}$ & & - & $\begin{array}{l}- \\
-\end{array}$ \\
\hline HICKEL & $\begin{array}{l}05 / 22 / 91 \\
05 / 22 / 91 \\
05 / 22 / 91\end{array}$ & $\begin{array}{l}\text { OOAK } \\
\text { OODK } \\
\text { OOTK }\end{array}$ & MG/L & $\cdot$ & $\begin{array}{l}0.28 \\
0.28 \\
0.280\end{array}$ & $\begin{array}{l}\text { RX } \\
\text { RX } \\
\text { RX }\end{array}$ & $\begin{array}{c}0.04 \\
0.04 \\
-\end{array}$ & - \\
\hline NITRATE & $\begin{array}{l}05 / 22 / 91 \\
05 / 22 / 91 \\
05 / 22 / 91\end{array}$ & $\begin{array}{l}\text { OOAK } \\
\text { ODDK } \\
\text { OOTK }\end{array}$ & $M G / L$ & & $\begin{array}{l}3.6 \\
3.5 \\
4.21\end{array}$ & $\begin{array}{l}R X \\
R X \\
R X\end{array}$ & $\begin{array}{l}1 . \\
1 .\end{array}$ & $\begin{array}{l}- \\
-\end{array}$ \\
\hline WITRITE AHD MITRATE & $\begin{array}{l}05 / 22 / 91 \\
05 / 22 / 91 \\
05 / 22 / 91\end{array}$ & $\begin{array}{l}\text { OOAK } \\
\text { OODK } \\
\text { OOTK }\end{array}$ & $M G / L$ & & $\begin{array}{l}0.81 \\
0.80 \\
0.950\end{array}$ & $\begin{array}{l}\text { RX } \\
\text { RX } \\
\text { RX }\end{array}$ & $\begin{array}{l}0.05 \\
0.05 \\
.\end{array}$ & $\begin{array}{l}- \\
-\end{array}$ \\
\hline POTASSIUH & $\begin{array}{l}05 / 22 / 91 \\
05 / 22 / 91 \\
05 / 22 / 91\end{array}$ & $\begin{array}{l}\text { OOAK } \\
\text { OODK } \\
\text { OOTK }\end{array}$ & MG/L & & $\begin{array}{l}5.4 \\
5.4 \\
5.00\end{array}$ & $\begin{array}{l}\text { RX } \\
\text { RX } \\
\text { RX }\end{array}$ & $\begin{array}{l}0.01 \\
0.01 \\
.\end{array}$ & $\begin{array}{l}. \\
.\end{array}$ \\
\hline RADIUM-226 & $\begin{array}{l}05 / 22 / 91 \\
05 / 22 / 91\end{array}$ & $\begin{array}{l}\text { DOAK } \\
\text { OODK }\end{array}$ & PCI/L & & $\begin{array}{l}9.4 \\
9.3\end{array}$ & $\begin{array}{l}R X \\
R X\end{array}$ & 1. & $\begin{array}{l}0.2 \\
0.2\end{array}$ \\
\hline
\end{tabular}

- NET GROSS ALPHA (GROSS ALPHA - URANIUM) HITH 1 MG URAHIUN = 686 PCI

PARAMETER VALUE IHOICATOR (PVI): < - LESS THAN DETECTION LIHIT

OTHER PARAMETER VALUE FLAGS:

$R$ - UHUSABLE DATA POINT

$X$ - LOCATIOH UNDEFINED
SNMPLE ID CODES:

DOAK - AMALYTICAL KHOWN SAMPLE

OODK - DUPLICATE KMONA SAMPLE

DOTK - THEORETICAL KMOMN SAMPLE 
SURFACE WATER QUALITY DATA BY LOCATIOH

SOWP ReV. 0 data

SITE: MON01 MONUMENT VALLEY

LOCATION: 0976 WOD KNOWNS

HORTH COORDINATE: UNKHOWN

EAST COORDINATE: UHKHOWH

04/11/88 TO 12/14/94

REPORT DATE: 06/22/95

\begin{tabular}{|c|c|c|c|c|c|c|c|c|}
\hline PARAMETER HAME & LOG DATE & $\begin{array}{c}\text { SAMPLE } \\
\text { ID }\end{array}$ & $\begin{array}{l}\text { UNITS OF } \\
\text { MEASURE }\end{array}$ & PVI & $\begin{array}{l}\text { PARAMETER } \\
\text { VALUE }\end{array}$ & FLAGS & $\begin{array}{l}\text { DETECTIOH } \\
\text { LIMIT }\end{array}$ & $\begin{array}{l}\text { PARAMETER } \\
\text { UNCERTAINTY }\end{array}$ \\
\hline RADIUH-226 & $05 / 22 / 91$ & OOTK & $\mathrm{PCI} / \mathrm{L}$ & & 10.1 & RX & - & - \\
\hline RADIUM-226 + RADIUH-228 & $\begin{array}{l}05 / 22 / 91 \\
05 / 22 / 91 \\
05 / 22 / 91\end{array}$ & $\begin{array}{l}\text { OOAK } \\
\text { OODK } \\
\text { OOTK }\end{array}$ & $\mathrm{PCI} / \mathrm{L}$ & & $\begin{array}{l}13.20 \\
14.00 \\
15.23\end{array}$ & & - & : \\
\hline $\begin{array}{r}\text { RADIUHA-228 } \\
\text { : }\end{array}$ & $\begin{array}{l}05 / 22 / 99 \\
05 / 22 / 91 \\
05 / 22 / 91\end{array}$ & $\begin{array}{l}\text { OOAK } \\
\text { OOOK } \\
\text { DOTK }\end{array}$ & $\mathrm{PCI} / \mathrm{L}$ & & $\begin{array}{l}3.8 \\
4.7 \\
5.13\end{array}$ & $\begin{array}{l}\text { RX } \\
R X \\
R X\end{array}$ & 1. & $\begin{array}{l}2.8 \\
2.9 \\
.\end{array}$ \\
\hline SELENIUM & $\begin{array}{l}05 / 22 / 91 \\
05 / 22 / 91 \\
05 / 22 / 91\end{array}$ & $\begin{array}{l}\text { OOAK } \\
\text { OODK } \\
\text { OOTK }\end{array}$ & $M G / L$ & & $\begin{array}{l}0.210 \\
0.230 \\
0.350\end{array}$ & $\begin{array}{l}\text { JRX } \\
\text { JRX } \\
\text { JRX }\end{array}$ & $\begin{array}{l}0.005 \\
0.005 \\
-\end{array}$ & : \\
\hline SILICA - SIOZ & $\begin{array}{l}05 / 22 / 91 \\
05 / 22 / 91 \\
05 / 22 / 91\end{array}$ & $\begin{array}{l}\text { OOAK } \\
\text { OODK } \\
\text { OOTK }\end{array}$ & $M G / L$ & & $\begin{array}{l}2.4 \\
2.4 \\
2.35\end{array}$ & $\begin{array}{l}\text { RX } \\
\text { RX } \\
\text { RX }\end{array}$ & $\begin{array}{c}0.1 \\
0.1 \\
-\end{array}$ & : \\
\hline SILVER & $\begin{array}{l}05 / 22 / 91 \\
05 / 22 / 91 \\
05 / 22 / 91\end{array}$ & $\begin{array}{l}\text { OOAK } \\
\text { OODK } \\
\text { OOTK }\end{array}$ & MG/L & & $\begin{array}{l}0.17 \\
0.17 \\
0.175\end{array}$ & $\begin{array}{l}\text { RX } \\
\text { RX } \\
\text { RX }\end{array}$ & $\begin{array}{l}0.01 \\
0.01 \\
.\end{array}$ & - \\
\hline SOOIUM & $\begin{array}{l}05 / 22 / 91 \\
05 / 22 / 91 \\
05 / 22 / 91\end{array}$ & $\begin{array}{l}\text { OOAK } \\
\text { OODK } \\
\text { OOTK }\end{array}$ & $M G / L$ & & $\begin{array}{l}36 . \\
36 . \\
36.0\end{array}$ & $\begin{array}{l}\text { RX } \\
\text { RX } \\
\text { RX }\end{array}$ & 1. & : \\
\hline STRONTIUH & $\begin{array}{l}05 / 22 / 91 \\
05 / 22 / 91 \\
05 / 22 / 91\end{array}$ & $\begin{array}{l}\text { OOAK } \\
\text { OODK } \\
\text { OOTK }\end{array}$ & MG/L & & $\begin{array}{l}0.68 \\
0.69 \\
0.700\end{array}$ & $\begin{array}{l}\text { RX } \\
\text { RX } \\
\text { RX }\end{array}$ & $\begin{array}{l}0.01 \\
0.01 \\
-\end{array}$ & : \\
\hline SULFATE & $\begin{array}{l}05 / 22 / 91 \\
05 / 22 / 91 \\
05 / 22 / 91\end{array}$ & $\begin{array}{l}\text { OOAK } \\
\text { OODK } \\
\text { OOTK }\end{array}$ & $M G / L$ & & $\begin{array}{l}192 . \\
197 . \\
213 .\end{array}$ & $\begin{array}{l}\text { RX } \\
\text { RX } \\
\text { RX }\end{array}$ & $\begin{array}{l}0.1 \\
0.1 \\
-\end{array}$ & : \\
\hline SULFIDE & $\begin{array}{l}05 / 22 / 91 \\
05 / 22 / 91 \\
05 / 22 / 91\end{array}$ & $\begin{array}{l}\text { OOAK } \\
\text { OODK } \\
\text { OOTK }\end{array}$ & $M G / L$ & & $\begin{array}{l}6.9 \\
7.3 \\
5.28\end{array}$ & $\begin{array}{l}\text { JRX } \\
\text { JRX } \\
\text { JRX }\end{array}$ & 1. & $\dot{-}$ \\
\hline THALLIUM & $\begin{array}{l}05 / 22 / 91 \\
05 / 22 / 91 \\
05 / 22 / 91\end{array}$ & $\begin{array}{l}\text { OOAK } \\
\text { OODK } \\
\text { OOTK }\end{array}$ & $\begin{array}{r}\text { MG/L } \\
.\end{array}$ & & $\begin{array}{l}0.16 \\
0.16 \\
0.175\end{array}$ & $\begin{array}{l}\text { RX } \\
\text { RX } \\
\text { RX }\end{array}$ & $\begin{array}{l}0.01 \\
0.01 \\
-\end{array}$ & $\dot{-}$ \\
\hline THORIUM-230 & $\begin{array}{l}05 / 22 / 91 \\
05 / 22 / 91 \\
05 / 22 / 91\end{array}$ & $\begin{array}{l}\text { OOAK } \\
\text { OODK } \\
\text { OOTK }\end{array}$ & $\mathrm{PCI} / \mathrm{L}$ & & $\begin{array}{l}2.8 \\
3.0 \\
3.71\end{array}$ & $\begin{array}{l}\text { RX } \\
\text { RX } \\
\text { RX }\end{array}$ & 1. & $\begin{array}{l}0.7 \\
0.7 \\
-\end{array}$ \\
\hline TIH & $\begin{array}{l}05 / 22 / 91 \\
05 / 22 / 91 \\
05 / 22 / 91\end{array}$ & $\begin{array}{l}\text { OOAK } \\
\text { OODK } \\
\text { OOTK }\end{array}$ & $M G / L$ & & $\begin{array}{l}0.27 \\
0.24 \\
0.315\end{array}$ & $\begin{array}{l}\text { RX } \\
R X \\
R X\end{array}$ & $\begin{array}{l}0.01 \\
0.01 \\
-\end{array}$ & $:$ \\
\hline TOTAL DISSOLVED SOLIDS & $\begin{array}{l}05 / 22 / 91 \\
05 / 22 / 91 \\
05 / 22 / 91\end{array}$ & $\begin{array}{l}\text { OOAK } \\
\text { OODK } \\
\text { OOTK }\end{array}$ & HG/L & & $\begin{array}{l}418 . \\
420 . \\
424 .\end{array}$ & $\begin{array}{l}\text { RX } \\
\text { RX } \\
\text { RX }\end{array}$ & $\begin{array}{l}10 . \\
10 .\end{array}$ & : \\
\hline TOTAL ORGANIC CARBON & $05 / 22 / 91$ & OOAK & $M G / L$ & & 4. & RX & 9. & - \\
\hline
\end{tabular}

PARAMETER VALUE IMDICATOR (PVI): < - LESS THAN DETECTIOH LIMIT

OTHER PARAMETER VALUE FLAGS:

J - ESTIMATED VALUE

$R$ - UNUSABLE DATA POINT

I - LOCATION UNDEFINED

SAMPLE TO COOES

DOAK - AMALYTICAL KHON SAMPLE

OODK - DUPLICATE KNOWN SAMPLE

OOTK - THEORETICAL KMOWN SAMPLE 
SITE: MONOI MONUMENT VALLEY

LOCATION: 0976 WOD KNOWNS

NORTH COOROINATE: UNKHOWN

EAST COORDINATE: UNKHOWH

$04 / 11 / 88$ TO $12 / 14 / 94$

REPORT DATE: $06 / 22 / 95$

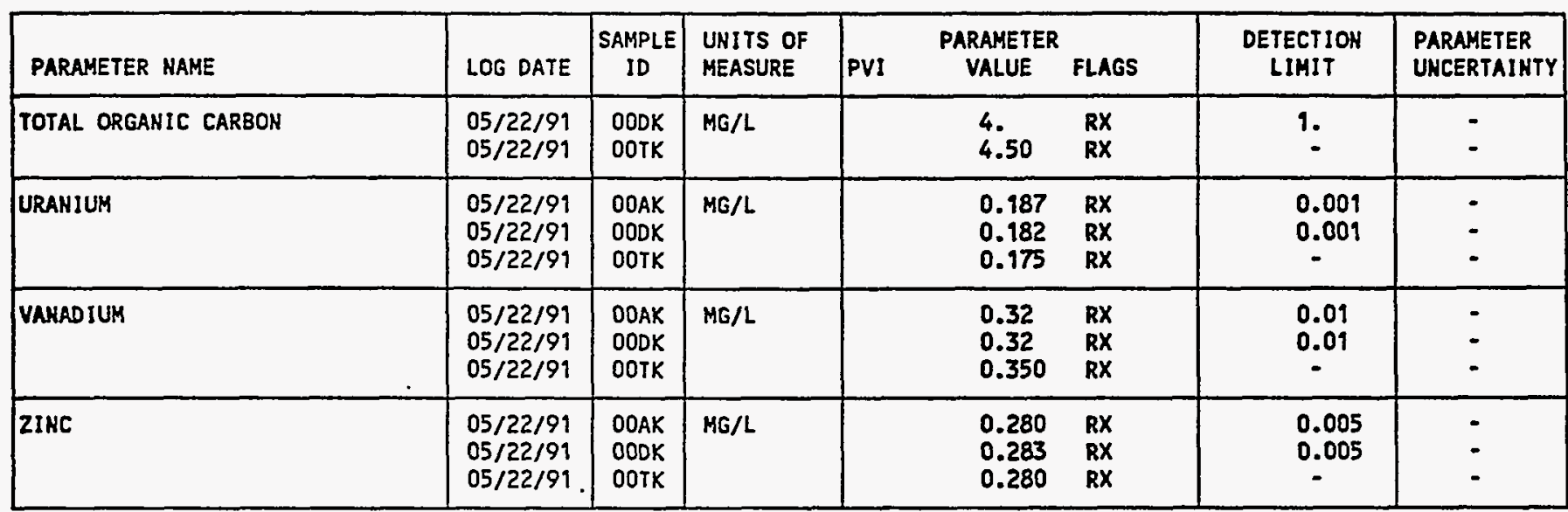

PARAMETER VALUE INDICATOR (PVI): < - LESS THAN DETECTION LIMIT

SAMPLE ID COOES:

OTHER PARAMETER VALUE FLAGS:

$R$ - UNUSABLE DATA POINT

DOAK - AHALYTICAL KNOWN SAMPLE

OODK - OUPLICATE KHOWH SAMPLE

$X$ - LOCATION UNDEFINED

OOTK - THEORETICAL KHONH SAMPLE 
SURFACE WATER QUALITY DATA BY LOCATION

SOWP REV. $O$ data

SITE: HONO 1 MONUMENT VALLEY

LOCATION: 0980 WOD KHOUNS

NORTH COORDINATE: UNKMONH

EAST COORDINATE: UNKNOWN

$04 / 11 / 88$ TO $12 / 14 / 94$

REPORT DATE: $06 / 22 / 95$

\begin{tabular}{|c|c|c|c|c|c|c|c|c|}
\hline PARAMETER NAHE & LOG DATE & $\begin{array}{c}\text { SAMPLE } \\
\text { ID }\end{array}$ & $\begin{array}{l}\text { UNITS OF } \\
\text { MEASURE }\end{array}$ & PVI & $\begin{array}{l}\text { PARAMETER } \\
\text { VALUE }\end{array}$ & FLAGS & $\begin{array}{l}\text { DETECTION } \\
\text { LIMIT }\end{array}$ & $\begin{array}{l}\text { PARAMETER } \\
\text { UNCERTAIHTY }\end{array}$ \\
\hline ALUMINUH & $\begin{array}{l}06 / 14 / 89 \\
06 / 15 / 89 \\
12 / 10 / 89 \\
12 / 11 / 89 \\
07 / 03 / 90 \\
07 / 05 / 90 \\
01 / 18 / 91 \\
01 / 21 / 91 \\
01 / 21 / 91\end{array}$ & $\begin{array}{l}\text { OOTK } \\
\text { OOAK } \\
\text { OOAK } \\
\text { OOTK } \\
\text { OOTK } \\
\text { OOAK } \\
\text { OOTK } \\
\text { OOAK } \\
\text { OODK }\end{array}$ & MG/L & & $\begin{array}{l}0.254 \\
0.3 \\
0.7 \\
0.700 \\
0.312 \\
0.42 \\
0.454 \\
0.50 \\
0.50\end{array}$ & & $\begin{array}{l}0.1 \\
0.1 \\
0.1 \\
0.1 \\
0.1 \\
0.1 \\
0.1 \\
0.1\end{array}$ & $\begin{array}{l}- \\
- \\
- \\
- \\
-\end{array}$ \\
\hline AYHONIUM & $\begin{array}{l}04 / 11 / 88 \\
04 / 11 / 88 \\
06 / 14 / 89 \\
06 / 15 / 89 \\
12 / 10 / 89 \\
12 / 11 / 89 \\
07 / 05 / 90 \\
01 / 18 / 91 \\
01 / 21 / 91 \\
01 / 21 / 91\end{array}$ & $\begin{array}{l}\text { OOAK } \\
\text { OOTK } \\
\text { OOTK } \\
\text { OOAK } \\
\text { OOAK } \\
\text { OOTK } \\
\text { OOAK } \\
\text { OOTK } \\
\text { OOAK } \\
\text { OODK }\end{array}$ & MG/L & $<$ & $\begin{array}{c}54 . \\
53.2 \\
0.644 \\
0.5 \\
12.7 \\
12.9 \\
0.1 \\
1.16 \\
1.3 \\
1.1\end{array}$ & & $\begin{array}{l}0.1 \\
0.1 \\
0.1 \\
0.1 \\
0.1 \\
0.1 \\
0.1 \\
0.1 \\
0.1 \\
0.1\end{array}$ & $\begin{array}{l}- \\
- \\
- \\
- \\
- \\
- \\
-\end{array}$ \\
\hline ANT IMONY & $\begin{array}{l}06 / 14 / 89 \\
06 / 15 / 89 \\
12 / 10 / 89 \\
12 / 11 / 89 \\
07 / 03 / 90 \\
07 / 05 / 90 \\
01 / 18 / 91 \\
01 / 21 / 91 \\
01 / 21 / 91\end{array}$ & $\begin{array}{l}\text { OOTK } \\
\text { OOAK } \\
\text { OOAK } \\
\text { OOTK } \\
\text { OOTK } \\
\text { OOAK } \\
\text { OOTK } \\
\text { OOAK } \\
\text { OODK }\end{array}$ & HG/L & & $\begin{array}{l}0.102 \\
0.365 \\
0.15 \\
0.192 \\
0.374 \\
0.405 \\
0.120 \\
0.125 \\
0.130\end{array}$ & J & $\begin{array}{l}0.003 \\
0.003 \\
0.003 \\
0.003 \\
0.003 \\
0.003 \\
- \\
0.003 \\
0.003\end{array}$ & $\begin{array}{l}- \\
- \\
- \\
- \\
- \\
-\end{array}$ \\
\hline ARSENIC & $\begin{array}{l}04 / 11 / 88 \\
04 / 11 / 88 \\
06 / 14 / 89 \\
06 / 15 / 89 \\
12 / 10 / 89 \\
12 / 11 / 89 \\
07 / 03 / 90 \\
07 / 05 / 90 \\
01 / 18 / 91 \\
01 / 21 / 91 \\
01 / 21 / 91\end{array}$ & $\begin{array}{l}\text { OOAK } \\
\text { OOTK } \\
\text { OOTK } \\
\text { OOAK } \\
\text { OOAK } \\
\text { OOTK } \\
\text { OOTK } \\
\text { OOAK } \\
\text { OOTK } \\
\text { OOAK } \\
\text { OODK }\end{array}$ & $M G / L$ & & $\begin{array}{l}0.881 \\
1.01 \\
0.215 \\
0.23 \\
0.30 \\
0.350 \\
0.156 \\
0.17 \\
0.227 \\
0.27 \\
0.27\end{array}$ & & $\begin{array}{l}0.01 \\
0.01 \\
0.01 \\
0.01 \\
0.01 \\
0.01 \\
0.01 \\
0.01 \\
- \\
0.01 \\
0.01\end{array}$ & $\begin{array}{l}- \\
: \\
: \\
\therefore \\
- \\
\therefore \\
-\end{array}$ \\
\hline BARIUM & $\begin{array}{l}06 / 14 / 89 \\
06 / 15 / 89 \\
12 / 10 / 89 \\
12 / 11 / 89 \\
07 / 03 / 90 \\
07 / 05 / 90 \\
01 / 18 / 91 \\
01 / 21 / 91 \\
01 / 21 / 91\end{array}$ & $\begin{array}{l}\text { OOTK } \\
\text { OOAK } \\
\text { OOAK } \\
\text { OOTK } \\
\text { OOTK } \\
\text { OOAK } \\
\text { OOTK } \\
\text { OOAK } \\
\text { OODK }\end{array}$ & MG/L & & $\begin{array}{l}0.153 \\
0.15 \\
0.7 \\
0.700 \\
0.312 \\
0.34 \\
0.454 \\
0.46 \\
0.47\end{array}$ & & $\begin{array}{l}0.1 \\
0.1 \\
0.1 \\
0.1 \\
0.1 \\
0.1 \\
-.1 \\
0.1 \\
0.1\end{array}$ & $\begin{array}{l}- \\
- \\
- \\
- \\
- \\
-\end{array}$ \\
\hline BERYLLIUH & $\begin{array}{l}06 / 14 / 89 \\
06 / 15 / 89 \\
12 / 10 / 89\end{array}$ & $\begin{array}{l}\text { OOTK } \\
\text { OOAK } \\
\text { OOAK }\end{array}$ & $M G / L$ & & $\begin{array}{l}0.070 \\
0.06 \\
0.183\end{array}$ & & $\begin{array}{l}0.01 \\
0.01 \\
0.005\end{array}$ & $\dot{-}$ \\
\hline
\end{tabular}

PARAMETER VALUE IHDICATOR (PVI): < - LESS THAN DETECTIOH LIMIT

SNMPLE ID COOES:

OTHER PARAMETER VALUE FLAGS:

DOAK - AMALYTICAL KNOWN SAMPLE

OODK - DUPLICATE KHOWN SAMPLE

$J$ - ESTIMATED VALUE

OOTK - THEORETICAL KHOWN SAMPLE 
SURFACE WATER OUALITY DATA BY LOCATION

SOWP ReV. 0 data

SITE: MON01 MONUMENT VALLEY

LOCATION: O98O WOD KHOWNS

NORTH COORDINATE: UNKNOWN

EAST COORDINATE: UNKNOWN

04/11/88 TO $12 / 14 / 94$

REPORT DATE: $06 / 22 / 95$

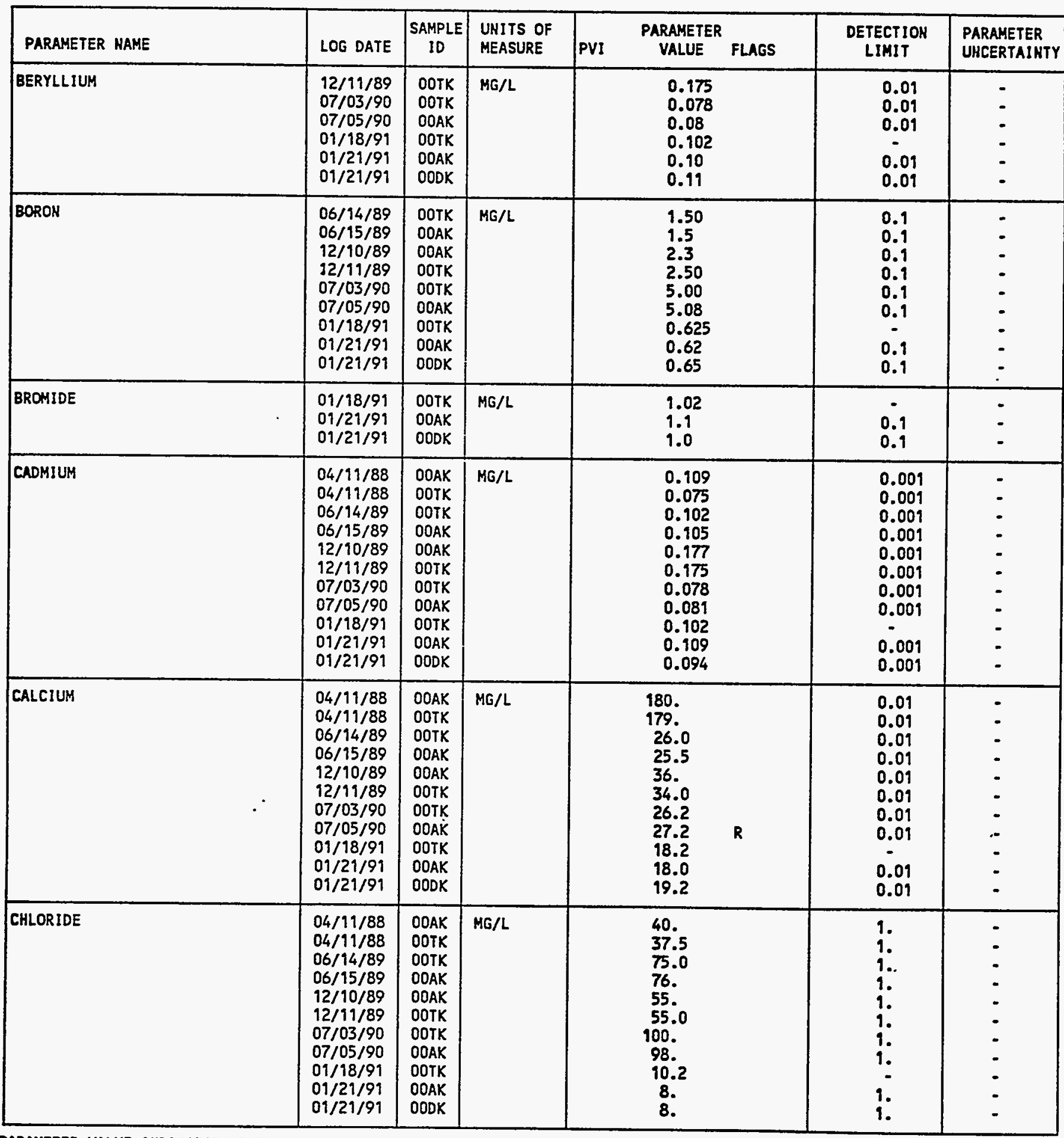

PARAMETER VALUE INDICATOR (PVI): < - LESS THAN DETECTION LIMIT

OTHER PARAMETER VALUE FLAGS:

$R$ - UNUSABLE DATA POINT
SAMPLE ID COOES:

OOAK - ANALYTICAL KNOWN SAMPLE

OODK - DUPLICATE KMONH SAMPLE

DOTK - THEORETICAL KMONH SAMPLE 
SURFACE WATER QUALITY DATA BY LOCATION

SOWP ReV. $O$ data

SITE: MONO1 MONUMENT VALLEY

LOCATION: 0980 WOD KHOWHS

NORTH COORDINATE: UNKNOWN

EAST COORDINATE: UNKHONH

04/11/88 TO $12 / 14 / 94$

REPORT DATE: 06/22/95

\begin{tabular}{|c|c|c|c|c|c|c|c|}
\hline PARAMETER HAME & LOG DATE & $\begin{array}{c}\text { SAMPLE } \\
\text { ID }\end{array}$ & $\begin{array}{l}\text { UNITS OF } \\
\text { MEASURE }\end{array}$ & PVI & $\begin{array}{l}\text { PARAMETER } \\
\text { VALUE FLAGS }\end{array}$ & $\begin{array}{l}\text { DETECTION } \\
\text { LIMIT }\end{array}$ & $\begin{array}{l}\text { PARAMETER } \\
\text { UNCERTAINTY }\end{array}$ \\
\hline CHROHIUM & $\begin{array}{l}06 / 14 / 89 \\
06 / 15 / 89 \\
12 / 10 / 89 \\
12 / 11 / 89 \\
07 / 03 / 90 \\
07 / 05 / 90 \\
01 / 18 / 91 \\
01 / 21 / 91 \\
01 / 21 / 91\end{array}$ & $\begin{array}{l}\text { OOTK } \\
\text { OOAK } \\
\text { OOAK } \\
\text { OOTK } \\
\text { OOTK } \\
\text { OOAK } \\
\text { OOTK } \\
\text { OOAK } \\
\text { OODK }\end{array}$ & $M G / L$ & & $\begin{array}{l}0.102 \\
0.10 \\
0.35 \\
0.350 \\
0.156 \\
0.16 \\
0.204 \\
0.20 \\
0.21\end{array}$ & $\begin{array}{l}0.01 \\
0.01 \\
0.01 \\
0.01 \\
0.01 \\
0.01 \\
\vdots \\
0.01 \\
0.01\end{array}$ & $\begin{array}{l}- \\
- \\
- \\
- \\
- \\
-\end{array}$ \\
\hline COBALT & $\begin{array}{l}06 / 14 / 89 \\
06 / 15 / 89 \\
12 / 10 / 89 \\
12 / 11 / 89 \\
07 / 03 / 90 \\
07 / 05 / 90 \\
01 / 18 / 91 \\
01 / 21 / 91 \\
01 / 21 / 91\end{array}$ & $\begin{array}{l}\text { OOTK } \\
\text { OOAK } \\
\text { OOAK } \\
\text { OOTK } \\
\text { OOTK } \\
\text { OOAK } \\
\text { OOTK } \\
\text { OOAK } \\
\text { OOOK }\end{array}$ & $M G / L$ & & $\begin{array}{l}0.136 \\
0.14 \\
0.33 \\
0.315 \\
0.140 \\
0.14 \\
0.184 \\
0.18 \\
0.19\end{array}$ & $\begin{array}{l}0.05 \\
0.05 \\
0.05 \\
0.05 \\
0.05 \\
0.05 \\
- \\
0.05 \\
0.05\end{array}$ & $\begin{array}{l}- \\
- \\
: \\
\vdots \\
: \\
-\end{array}$ \\
\hline COPPER & $\begin{array}{l}06 / 14 / 89 \\
06 / 15 / 89 \\
12 / 10 / 89 \\
12 / 11 / 89 \\
07 / 03 / 90 \\
07 / 05 / 90 \\
01 / 18 / 91 \\
01 / 21 / 91 \\
01 / 21 / 91\end{array}$ & $\begin{array}{l}\text { OOTK } \\
\text { OOAK } \\
\text { OOAK } \\
\text { DOTK } \\
\text { OOTK } \\
\text { OOAK } \\
\text { OOTK } \\
\text { OOAK } \\
\text { OODK }\end{array}$ & $M G / L$ & & $\begin{array}{l}0.136 \\
0.14 \\
0.30 \\
0.280 \\
0.125 \\
0.13 \\
0.163 \\
0.17 \\
0.17\end{array}$ & $\begin{array}{l}0.02 \\
0.02 \\
0.02 \\
0.02 \\
0.02 \\
0.02 \\
0 \\
0.02 \\
0.02\end{array}$ & $\begin{array}{l}- \\
- \\
- \\
- \\
- \\
-\end{array}$ \\
\hline CYANIDE & $\begin{array}{l}06 / 14 / 89 \\
06 / 15 / 89 \\
12 / 10 / 89 \\
12 / 11 / 89\end{array}$ & $\begin{array}{l}\text { OOTK } \\
\text { OOAK } \\
\text { OOAK } \\
\text { OOTK }\end{array}$ & $\mathrm{HG} / \mathrm{L}$ & & $\begin{array}{l}0.236 \\
0.21 \\
4.8 \\
4.72\end{array}$ & $\begin{array}{l}0.01 \\
0.01 \\
0.01 \\
0.01\end{array}$ & - \\
\hline DISSOLVED ORGANIC CARBOH & $01 / 18 / 91$ & DOTK & $M G / L$ & & 6.20 & - & - \\
\hline FLUORIDE & $\begin{array}{l}06 / 14 / 89 \\
06 / 15 / 89 \\
12 / 10 / 89 \\
12 / 11 / 89 \\
07 / 03 / 90 \\
07 / 05 / 90 \\
01 / 18 / 91 \\
01 / 21 / 91 \\
01 / 21 / 91\end{array}$ & $\begin{array}{l}\text { OOTK } \\
\text { OOAK } \\
\text { OOAK } \\
\text { OOTK } \\
\text { OOTK } \\
\text { OOAK } \\
\text { OOTK } \\
\text { OOAK } \\
\text { OODK }\end{array}$ & $M G / L$ & & $\begin{array}{c}6.06 \\
6.1 \\
10.0 \\
10.1 \\
20.1 \\
19.8 \\
6.11 \\
6.3 \\
6.5\end{array}$ & $\begin{array}{l}0.1 \\
0.1 \\
0.1 \\
0.1 \\
0.1 \\
0.1 \\
0.1 \\
0.1\end{array}$ & $\begin{array}{l}- \\
- \\
- \\
- \\
- \\
- \\
-\end{array}$ \\
\hline GROSS ALPHA & $\begin{array}{l}04 / 11 / 88 \\
04 / 11 / 88 \\
01 / 18 / 91 \\
01 / 21 / 91 \\
01 / 21 / 91\end{array}$ & $\begin{array}{l}\text { DOAK } \\
\text { OOTK } \\
\text { OOTK } \\
\text { OOAK } \\
\text { OODK }\end{array}$ & PCI/L & & $\begin{array}{l}240 . \\
232 . \\
19.3 \\
12 . \\
13 .\end{array}$ & $\begin{array}{l}0.2 \\
0.2 \\
- \\
1 . \\
1 .\end{array}$ & $\begin{array}{l}10 . \\
4 \\
4 .\end{array}$ \\
\hline GROSS BETA & $\begin{array}{l}04 / 11 / 88 \\
04 / 11 / 88 \\
01 / 18 / 91\end{array}$ & $\begin{array}{l}\text { OOAK } \\
\text { OOTK } \\
\text { OOTK }\end{array}$ & $\mathrm{PCl} / \mathrm{L}$ & & $\begin{array}{l}210 . \\
200 . \\
15.4\end{array}$ & 9. & 90. \\
\hline
\end{tabular}

PARAMETER VALUE IHDICATOR (PVI): < - LESS THAN DETECTION LIMIT

SAMPLE ID COOES:

OTHER PARAMETER VALUE FLAGS:

L - LESS THAH THREE BORE VOLUMES REMOVED BEFORE SAMPLING

DOAK - AHALYTICAL KHOWN SAHPLE

DOOK - DUPLICATE KNOWN SAMPLE

OOTK - THEORETICAL KHOWN SAMPLE 
SURFACE WATER QUALITY DATA BY LOCATION

SOWP REV. 0 data

SITE: MONO1 MONUMENT VALLEY

LOCATION: 0980 WOD KNOWNS

NORTH COORDINATE: UNKHOWN

EAST COORDINATE: UKKHOWN

04/11/88 TO $12 / 14 / 94$

REPORT DATE: $06 / 22 / 95$

\begin{tabular}{|c|c|c|c|c|c|c|c|}
\hline PARAMETER NAME & LOG DATE & $\begin{array}{c}\text { SAMPLE } \\
\text { ID }\end{array}$ & $\begin{array}{l}\text { UNITS OF } \\
\text { MEASURE }\end{array}$ & PVI & $\begin{array}{l}\text { PARAHETER } \\
\text { VALUE FLAGS }\end{array}$ & $\begin{array}{l}\text { DETECTIOH } \\
\text { LIMIT }\end{array}$ & $\begin{array}{l}\text { PARAMETER } \\
\text { UHCERTAINTY }\end{array}$ \\
\hline GROSS BETA & $\begin{array}{l}01 / 21 / 91 \\
01 / 21 / 91\end{array}$ & $\begin{array}{l}\text { OOAK } \\
\text { OODK }\end{array}$ & $\mathrm{PCI} / \mathrm{L}$ & & $\begin{array}{l}16 . \\
14 .\end{array}$ & $\begin{array}{l}0.5 \\
0.5\end{array}$ & $\begin{array}{l}3 . \\
3 .\end{array}$ \\
\hline $\begin{array}{l}\text { IRON } \\
:\end{array}$ & $\begin{array}{l}06 / 14 / 89 \\
06 / 15 / 89 \\
12 / 10 / 89 \\
12 / 11 / 89 \\
07 / 03 / 90 \\
07 / 05 / 90 \\
01 / 18 / 91 \\
01 / 21 / 91 \\
01 / 21 / 91\end{array}$ & $\begin{array}{l}\text { OOTK } \\
\text { OOAK } \\
\text { OOAK } \\
\text { OOTK } \\
\text { OOTK } \\
\text { OOAK } \\
\text { OOTK } \\
\text { OOAK } \\
\text { OODK }\end{array}$ & MG/L & & $\begin{array}{l}0.278 \\
0.28 \\
0.55 \\
0.525 \\
0.234 \\
0.26 \\
0.329 \\
0.34 \\
0.35\end{array}$ & $\begin{array}{l}0.03 \\
0.03 \\
0.03 \\
0.03 \\
0.03 \\
0.03 \\
0 \\
0.03 \\
0.03\end{array}$ & $\begin{array}{l}- \\
- \\
- \\
- \\
- \\
- \\
- \\
-\end{array}$ \\
\hline LEAD & $\begin{array}{l}06 / 14 / 89 \\
06 / 15 / 89 \\
12 / 10 / 89 \\
12 / 11 / 89 \\
07 / 03 / 90 \\
07 / 05 / 90 \\
01 / 18 / 91 \\
01 / 21 / 91 \\
01 / 21 / 91\end{array}$ & $\begin{array}{l}\text { OOTK } \\
\text { OOAK } \\
\text { OOAK } \\
\text { OOTK } \\
\text { OOTK } \\
\text { OOAK } \\
\text { OOTK } \\
\text { OOAK } \\
\text { OODK }\end{array}$ & MG/L & & $\begin{array}{l}0.119 \\
0.12 \\
0.16 \\
0.175 \\
0.078 \\
0.078 \\
0.102 \\
0.091 \\
0.090\end{array}$ & $\begin{array}{l}0.01 \\
0.01 \\
0.01 \\
0.01 \\
0.01 \\
0.01 \\
\vdots \\
0.01 \\
0.01\end{array}$ & $\begin{array}{l}- \\
- \\
- \\
- \\
- \\
- \\
- \\
-\end{array}$ \\
\hline MAGNESIUM & $\begin{array}{l}04 / 11 / 88 \\
04 / 11 / 88 \\
06 / 14 / 89 \\
06 / 15 / 89 \\
12 / 10 / 89 \\
12 / 11 / 89 \\
07 / 03 / 90 \\
07 / 05 / 90 \\
01 / 18 / 91 \\
01 / 21 / 91 \\
01 / 21 / 91\end{array}$ & $\begin{array}{l}\text { OOAK } \\
\text { OOTK } \\
\text { OOTK } \\
\text { OOAK } \\
\text { OCAK } \\
\text { OOTK } \\
\text { OOTK } \\
\text { DOAK } \\
\text { OOTK } \\
\text { OOAK } \\
\text { OODK }\end{array}$ & MG/L & & $\begin{array}{l}51.5 \\
53.6 \\
20.8 \\
21.2 \\
36 . \\
34.0 \\
26.2 \\
26.7 \\
18.2 \\
17.9 \\
21.8\end{array}$ & $\begin{array}{c}0.001 \\
0.001 \\
0.001 \\
0.001 \\
0.001 \\
0.001 \\
0.001 \\
0.001 \\
0.001 \\
0.001\end{array}$ & $\begin{array}{l}- \\
- \\
- \\
- \\
- \\
- \\
- \\
-\end{array}$ \\
\hline MANGANESE & $\begin{array}{l}06 / 14 / 89 \\
06 / 15 / 89 \\
12 / 10 / 89 \\
12 / 11 / 89 \\
07 / 03 / 90 \\
07 / 05 / 90 \\
01 / 18 / 91 \\
01 / 21 / 91 \\
01 / 21 / 91\end{array}$ & $\begin{array}{l}\text { OOTK } \\
\text { OOAK } \\
\text { OOAK } \\
\text { OOTK. } \\
\text { OOTK } \\
\text { OOAK } \\
\text { OOTK } \\
\text { OOAK } \\
\text { OODK }\end{array}$ & $M G / L$ & & $\begin{array}{l}0.290 \\
0.30 \\
0.37 \\
0.350 \\
0.156 \\
0.16 \\
0.227 \\
0.23 \\
0.23\end{array}$ & $\begin{array}{l}0.01 \\
0.01 \\
0.01 \\
0.01 \\
0.01 \\
0.01 \\
0 \\
0.01 \\
0.01\end{array}$ & $\begin{array}{l}- \\
- \\
- \\
- \\
- \\
- \\
-\end{array}$ \\
\hline MERCURY & $\begin{array}{l}06 / 14 / 89 \\
06 / 15 / 89 \\
12 / 10 / 89 \\
12 / 11 / 89 \\
07 / 03 / 90 \\
07 / 05 / 90\end{array}$ & $\begin{array}{l}\text { DOTK } \\
\text { OOAK } \\
\text { DOAK } \\
\text { OOTK } \\
\text { OOTK } \\
\text { OOAK }\end{array}$ & MG/L & & $\begin{array}{l}0.102 \\
0.091 \\
0.220 \\
0.210 \\
0.094 \\
0.103\end{array}$ & $\begin{array}{l}0.0002 \\
0.0002 \\
0.0002 \\
0.0002 \\
0.0002 \\
0.0002\end{array}$ & $\begin{array}{l}- \\
- \\
- \\
-\end{array}$ \\
\hline MOLYBDENUH & $\begin{array}{l}04 / 11 / 88 \\
04 / 11 / 88 \\
06 / 14 / 89 \\
06 / 15 / 89\end{array}$ & $\begin{array}{l}\text { DOAK } \\
\text { DOTK } \\
\text { DOTK } \\
\text { DOAK }\end{array}$ & $M G / L$ & & $\begin{array}{l}0.89 \\
0.900 \\
0.340 \\
0.31\end{array}$ & $\begin{array}{l}0.01 \\
0.01 \\
0.01 \\
0.01\end{array}$ & - \\
\hline
\end{tabular}

PARAMETER VALUE IHDICATOR (PVI): < - LESS THAN DETECTIOH LIMIT

OTHER PARAMETER VALUE FLAGS:

H - HOLD TIHE EXPIRED, VALUE SUSPECT

$J$ - ESTIMATED VALUE

$L$ - LESS THAH THREE BORE VOLUMES REMOVED BEFORE SAMPLING
SAMPLE ID CODES:

OOAK - ANALYTICAL KNOW SNPLE

OOOK - DUPLICATE KHOWN SAMPLE

ODTK - THEORETICAL KHOM SAMPIE 
SURFACE HATER OUALITY DATA BY LOCATION

SOWP ReV. 0 data

SITE: MONO1 MONUMENT VALLEY

LOCATION: 0980 WOD KHOUNS

HORTH COORDINATE: UNKHOWH

EAST COORDINATE: UNKNOWH

04/11/88 TO 12/14/94

REPORT DATE: $06 / 22 / 95$

\begin{tabular}{|c|c|c|c|c|c|c|c|}
\hline PARAMETER NAME & LOG DATE & $\begin{array}{c}\text { SAMPLE } \\
\text { ID }\end{array}$ & $\begin{array}{l}\text { UNITS OF } \\
\text { MEASURE }\end{array}$ & PVI & $\begin{array}{l}\text { PARAMETER } \\
\text { VALUE FLAGS }\end{array}$ & $\begin{array}{l}\text { DETECTIOH } \\
\text { LIMIT }\end{array}$ & $\begin{array}{l}\text { PARAMETER } \\
\text { UNCERTAINTY }\end{array}$ \\
\hline MOL YBDENUM & $\begin{array}{l}12 / 10 / 89 \\
12 / 11 / 89 \\
07 / 03 / 90 \\
07 / 05 / 90 \\
01 / 18 / 91 \\
01 / 21 / 91 \\
01 / 21 / 91\end{array}$ & $\begin{array}{l}\text { OOAK } \\
\text { OOTK } \\
\text { OOTK } \\
\text { OOAK } \\
\text { OOTK } \\
\text { OOAK } \\
\text { OODK }\end{array}$ & $M G / L$ & & $\begin{array}{l}0.63 \\
0.640 \\
1.25 \\
1.21 \\
0.400 \\
0.40 \\
0.39\end{array}$ & $\begin{array}{l}0.01 \\
0.01 \\
0.01 \\
0.01 \\
- \\
0.01 \\
0.01\end{array}$ & $\begin{array}{l}- \\
- \\
- \\
- \\
-\end{array}$ \\
\hline MET GROSS ALPHA * & $\begin{array}{l}04 / 11 / 88 \\
04 / 11 / 88 \\
01 / 18 / 91 \\
01 / 21 / 91 \\
01 / 21 / 91\end{array}$ & $\begin{array}{l}\text { OOAK } \\
\text { OOTK } \\
\text { OOTK } \\
\text { OOAK } \\
\text { OODK }\end{array}$ & PCI/L & & $\begin{array}{l}-433.65 \\
-444.40 \\
-184.44 \\
-199.29 \\
-203.78\end{array}$ & $\begin{array}{l}- \\
- \\
-\end{array}$ & $\begin{array}{l}- \\
- \\
-\end{array}$ \\
\hline HICKEL & $\begin{array}{l}06 / 14 / 89 \\
06 / 15 / 89 \\
12 / 10 / 89 \\
12 / 11 / 89 \\
07 / 03 / 90 \\
07 / 05 / 90 \\
01 / 18 / 91 \\
01 / 21 / 91 \\
01 / 21 / 91\end{array}$ & $\begin{array}{l}\text { OOTK } \\
\text { OOAK } \\
\text { OOAK } \\
\text { OOTK } \\
\text { OOTK } \\
\text { OOAK } \\
\text { OOTK } \\
\text { OOAK } \\
\text { OODK }\end{array}$ & HG/L & - & $\begin{array}{l}0.136 \\
0.14 \\
0.28 \\
0.280 \\
0.125 \\
0.13 \\
0.163 \\
0.17 \\
0.18\end{array}$ & $\begin{array}{l}0.04 \\
0.04 \\
0.04 \\
0.04 \\
0.04 \\
0.04 \\
0 \\
0.04 \\
0.04\end{array}$ & $\begin{array}{l}- \\
- \\
- \\
- \\
- \\
-\end{array}$ \\
\hline NITRATE & $\begin{array}{l}04 / 11 / 88 \\
04 / 11 / 88 \\
06 / 14 / 89 \\
06 / 15 / 89 \\
12 / 10 / 89 \\
12 / 11 / 89 \\
07 / 03 / 90 \\
07 / 05 / 90 \\
01 / 18 / 91 \\
01 / 21 / 91 \\
01 / 21 / 91\end{array}$ & $\begin{array}{l}\text { OOAK } \\
\text { OOTK } \\
\text { OOTK } \\
\text { OOAK } \\
\text { OOAK } \\
\text { OOTK } \\
\text { OOTK } \\
\text { OOAK } \\
\text { OOTK } \\
\text { OOAK } \\
\text { OODK }\end{array}$ & MG/L & & $\begin{array}{l}0.9 \\
1.11 \\
8.85 \\
8.6 \\
44.3 \\
44.3 \\
11.5 \\
12.0 \\
3.98 \\
4 . \\
4 .\end{array}$ & $\begin{array}{l}1 . \\
1 . \\
1 . \\
1 . \\
1 . \\
1 . \\
1 . \\
1 . \\
1 . \\
1 .\end{array}$ & $\begin{array}{l}- \\
- \\
: \\
- \\
- \\
- \\
-\end{array}$ \\
\hline POTASSIUH & $\begin{array}{l}04 / 11 / 88 \\
04 / 11 / 88 \\
06 / 14 / 89 \\
06 / 15 / 89 \\
12 / 10 / 89 \\
12 / 11 / 89 \\
07 / 03 / 90\end{array}$ & $\begin{array}{l}\text { OOAK } \\
\text { OOTK } \\
\text { OOTK } \\
\text { OOAK } \\
\text { OOAK } \\
\text { OOTK } \\
\text { OOTK }\end{array}$ & $M G / L$ & & $\begin{array}{l}9.13 \\
8.94 \\
5.20 \\
5.4 \\
7.9 \\
8.50 \\
6.56\end{array}$ & $\begin{array}{l}0.01 \\
0.01 \\
0.01 \\
0.01 \\
0.01 \\
0.01 \\
0.01\end{array}$ & $\begin{array}{l}- \\
- \\
- \\
-\end{array}$ \\
\hline
\end{tabular}

* het GROSS ALPHA (GROSS ALPHA - ÜRANIUM) HITH 1 MG URANIUM $=686$ PCI

PARAMETER VALUE IHDICATOR (PVI): < - LESS THAN DETECTION LIMIT

OTHER PARAMETER VALUE FLAGS:

J - ESTIMATED VALUE
SAMPLE ID COOES:

DOAK - AMALYTICAL KMOWN SAMPLE

OODK - DUPLICATE KHOMN SAMPLE

OOTK - THEORETICAL KMONN SAMPLE 
SURFACE WATER QUALITY DATA BY LOCATION

SOWP Rev. $O$ data

SITE: MONO1 MONUMENT VALLEY

LOCATION: 0980 WOD KNOWNS

MORTH COORDINATE: UNKNOWN

EAST COORDINATE: UNKNOWN

04/11/88 TO $12 / 14 / 94$

REPORT DATE: 06/22/95

\begin{tabular}{|c|c|c|c|c|c|c|c|}
\hline PARAMETER NAME & LOG DATE & $\begin{array}{c}\text { SAMPLE } \\
\text { ID }\end{array}$ & $\begin{array}{l}\text { UNITS OF } \\
\text { MEASURE }\end{array}$ & PVI & $\begin{array}{l}\text { PARAMETER } \\
\text { VALUE FLAGS }\end{array}$ & $\begin{array}{l}\text { DETECTION } \\
\text { LIMIT }\end{array}$ & $\begin{array}{l}\text { PARAMETER } \\
\text { UHCERTAINTY }\end{array}$ \\
\hline POTASSIUM & $\begin{array}{l}07 / 05 / 90 \\
01 / 18 / 91 \\
01 / 21 / 91 \\
01 / 21 / 91\end{array}$ & $\begin{array}{l}\text { OOAK } \\
\text { OOTK } \\
\text { OOAK } \\
\text { OODK }\end{array}$ & $M G / L$ & & $\begin{array}{l}6.9 \\
4.54 \\
4.3 \\
4.4\end{array}$ & $\begin{array}{l}0.01 \\
- \\
0.01 \\
0.01\end{array}$ & $\begin{array}{l}- \\
-\end{array}$ \\
\hline RAOIUH-226 & $\begin{array}{l}06 / 14 / 89 \\
06 / 15 / 89 \\
12 / 10 / 89 \\
12 / 11 / 89 \\
07 / 03 / 90 \\
07 / 05 / 90 \\
01 / 18 / 91 \\
01 / 21 / 91 \\
01 / 21 / 91\end{array}$ & $\begin{array}{l}\text { OOTK } \\
\text { OOAK } \\
\text { OOAK } \\
\text { OOTK } \\
\text { OOTK } \\
\text { OOAK } \\
\text { OOTK } \\
\text { OOAK } \\
\text { OODK }\end{array}$ & $\mathrm{PCI} / \mathrm{L}$ & & $\begin{array}{c}21.3 \\
23 . \\
51.9 \\
53.0 \\
33.2 \\
34 . \\
6.92 \\
13 . \\
7.5\end{array}$ & $\begin{array}{l}1 . \\
1 . \\
1 . \\
1 . \\
1 . \\
1 . \\
1 .\end{array}$ & $\begin{array}{c}- \\
3.5 \\
= \\
3 . \\
4 . \\
1.2\end{array}$ \\
\hline RADIUH-228 & $\begin{array}{l}06 / 14 / 89 \\
06 / 15 / 89 \\
12 / 10 / 89 \\
12 / 11 / 89 \\
07 / 03 / 90 \\
07 / 05 / 90 \\
01 / 18 / 91 \\
01 / 21 / 91 \\
01 / 21 / 91\end{array}$ & $\begin{array}{l}\text { OOTK } \\
\text { OOAK } \\
\text { OOAK } \\
\text { OOTK } \\
\text { OOTK } \\
\text { OOAK } \\
\text { OOTK } \\
\text { OOAK } \\
\text { OODK }\end{array}$ & PCI/L & & $\begin{array}{l}17.0 \\
18 . \\
11.9 \\
13.6 \\
16.0 \\
19 . \\
8.83 \\
8.5 \\
11 .\end{array}$ & $\begin{array}{l}1 . \\
1 . \\
1 . \\
1 . \\
1 . \\
1 . \\
1 .\end{array}$ & $\begin{array}{l}2 . \\
3.2 \\
= \\
2 . \\
1.5 \\
2 .\end{array}$ \\
\hline SILICA - SIO2 & $\begin{array}{l}06 / 14 / 89 \\
06 / 15 / 89 \\
12 / 10 / 89 \\
12 / 11 / 89 \\
07 / 03 / 90 \\
07 / 05 / 90 \\
01 / 18 / 91 \\
01 / 21 / 91 \\
01 / 21 / 91\end{array}$ & $\begin{array}{l}\text { OOTK } \\
\text { OOAK } \\
\text { OOAK } \\
\text { OOTK } \\
\text { OOTK } \\
\text { OOAK } \\
\text { OOTK } \\
\text { DOAK } \\
\text { OODK }\end{array}$ & $M G / L$ & & $\begin{array}{l}3.20 \\
3 . \\
5.3 \\
5.34 \\
10.7 \\
11.3 \\
3.25 \\
3.4 \\
3.5\end{array}$ & $\begin{array}{l}2 . \\
2 . \\
2 . \\
2 . \\
2 . \\
2 . \\
= \\
2 . \\
2 .\end{array}$ & $\begin{array}{l}- \\
: \\
: \\
: \\
-\end{array}$ \\
\hline SILVER & $06 / 14 / 89$ & OOTK & MG/L & & 0.102 & 0.01 & - \\
\hline
\end{tabular}

PARAMETER VALUE INDICATOR (PVI): < - LESS THAN DETECTION LIMIT

SAMPLE ID COOES:

OOAK - AKALYTICAL KNOWN SAMPLE

OODK - DUPLICATE KNOWN SAMPLE

OOTK - THEORETICAL KNOWN SAMPLE 
SURFACE WATER QUALITY DATA BY LOCATION

SOWP ReV. $O$ data

SITE: MONO1 MONUMENT VALLEY

LOCATION: 0980 WOD KHONHS

NORTH COORDINATE: UNKHOWH

EAST COORDINATE: UHKNOWH

04/11/88 TO $12 / 14 / 94$

REPORT DATE: $06 / 22 / 95$

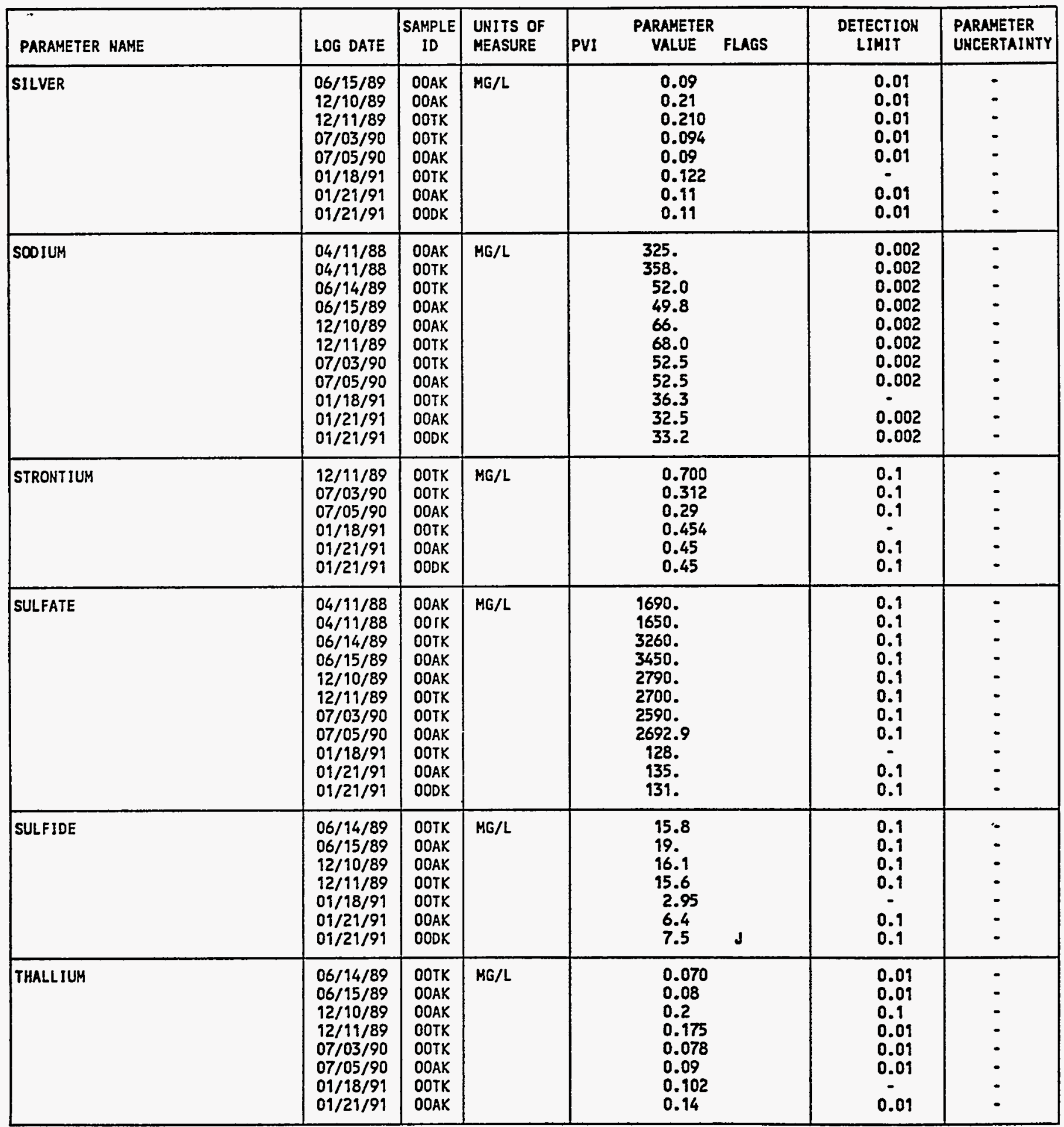

PARAMETER VALUE IHDICATOR (PVI): < - LESS THAN DETECTION LIMIT

SAMPLE ID COOES:

OTHER PARAMETER VALUE FLAGS:

DOAK - AHALYTICAL KHOWN SAMPLE

OODK - DUPLICATE KHOWN SAMPLE

$J$ - ESTIHATED VALUE

OOTK - THEORETICAL KNOWN SAMPLE 
SITE: MONO1 MONUMENT VALLEY

LOCATION: 0980 WQD KMOWNS

HORTH COORDINATE: UNKHOWN

EAST COORDINATE: UNKHOWN

04/11/88 TO 12/14/94

REPORT DATE: $06 / 22 / 95$

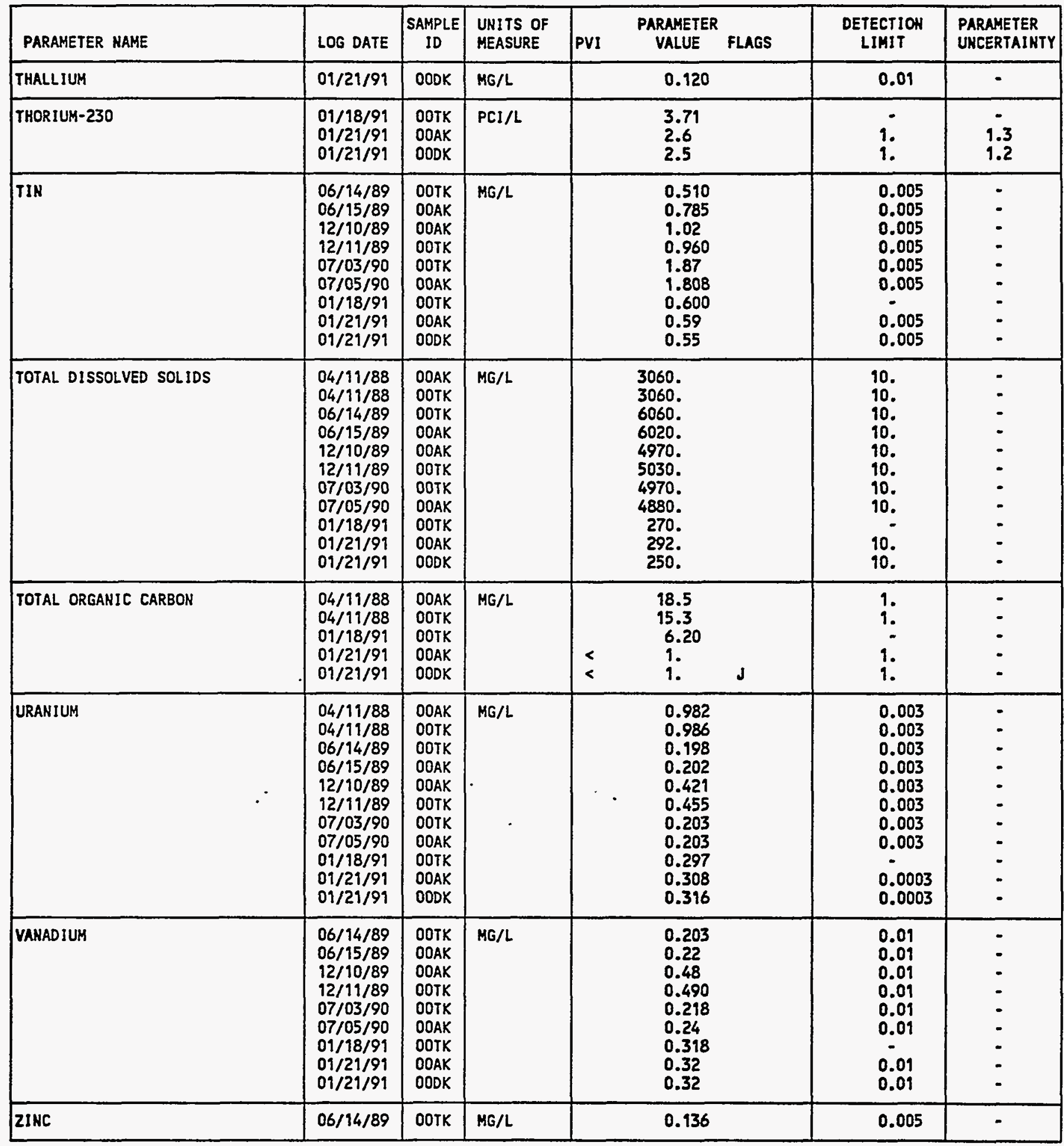

PARAMETER VALUE Indicator (PVI): ${ }^{\circ}$ < - LESS than DETECTION LIMIT

SAMPLE ID CODES:

OTHER PARAMETER VALUE FLAGS:

OOAK - AMALYTICAL KMOWN SAMPLE

J - ESTIMATED VALUE

OOOK - DUPLICATE KHOW SAMPLE

OOTK - THEORETICAL KNOWH SAMPLE 
SURFACE WATER QUALITY DATA BY LOCATIOH

SOWP REV. $O$ data

SITE: MONO1 MONUMENT VALLEY

- LOCATION: 0980 WOD KNOWNS

NORTH COORDINATE: UNKNOWN

EAST COORDINATE: UNKHOWN

04/11/88 TO 12/14/94

REPORT DATE: 06/22/95

\begin{tabular}{|c|c|c|c|c|c|c|c|}
\hline PARAMETER MAME & LOG DATE & $\begin{array}{c}\text { SAMPLE } \\
\text { ID }\end{array}$ & $\begin{array}{l}\text { UNITS OF } \\
\text { MEASURE }\end{array}$ & PVI & $\begin{array}{l}\text { PARAMETER } \\
\text { VALUE FLAGS }\end{array}$ & $\begin{array}{l}\text { DETECTIOW } \\
\text { LIMIT }\end{array}$ & $\begin{array}{l}\text { PARAMETER } \\
\text { UNCERTAINTY }\end{array}$ \\
\hline ZINC & $\begin{array}{l}06 / 15 / 89 \\
12 / 10 / 89 \\
12 / 11 / 89 \\
07 / 03 / 90 \\
07 / 05 / 90 \\
01 / 18 / 91 \\
01 / 21 / 91 \\
01 / 21 / 91\end{array}$ & $\begin{array}{l}\text { OOAK } \\
\text { OOAK } \\
\text { OOTK } \\
\text { OOTK } \\
\text { OOAK } \\
\text { OOTK } \\
\text { OOAK } \\
\text { OODK }\end{array}$ & $M G / L$ & & $\begin{array}{l}0.154 \\
0.29 \\
0.280 \\
0.125 \\
0.137 \\
0.163 \\
0.161 \\
0.162\end{array}$ & $\begin{array}{l}0.005 \\
0.005 \\
0.005 \\
0.005 \\
0.005 \\
0 \\
0.005 \\
0.005\end{array}$ & $\begin{array}{l}- \\
- \\
- \\
- \\
-\end{array}$ \\
\hline
\end{tabular}

PARAMETER VALUE IHDICATOR (PVI): < - LESS THAN DETECTION LIMIT

SAMPLE ID COOES:

OOAK - AKALYTICAL KHOWN SAMPLE

OOOK - DUPLICATE KNOWN SAMPLE

OOTK - THEORETICAL KNOWN SAMPLE 
SURFACE MATER QUALITY DATA BY LOCATION

SOUP ReV. $O$ data

SITE: HONO1 MONUMENT VALLEY

LOCATION: O981 WOD KNOWNS

HORTH COORDINATE: UHKHOWH

EAST COORDINATE: UNKNOWN

04/11/88 TO $12 / 14 / 94$

REPORT DATE: $06 / 22 / 95$

\begin{tabular}{|c|c|c|c|c|c|c|c|}
\hline PARAMETER NAME & LOG DATE & $\begin{array}{c}\text { SAMPLE } \\
\text { ID }\end{array}$ & $\begin{array}{l}\text { UNITS OF } \\
\text { MEASURE }\end{array}$ & PVI & $\begin{array}{l}\text { PARAMETER } \\
\text { VALUE FLAGS }\end{array}$ & $\begin{array}{l}\text { DETECTIOH } \\
\text { LIMIT }\end{array}$ & $\begin{array}{l}\text { PARAMETER } \\
\text { UKCERTAIHTY }\end{array}$ \\
\hline ALUMINUM & $\begin{array}{l}06 / 14 / 89 \\
06 / 15 / 89\end{array}$ & $\begin{array}{l}\text { OOTK } \\
\text { OOAK }\end{array}$ & MG/L & & $\begin{array}{l}0.520 \\
0.5\end{array}$ & $\begin{array}{l}0.1 \\
0.1\end{array}$ & - \\
\hline AMHONIUM & $\begin{array}{l}06 / 14 / 89 \\
06 / 15 / 89\end{array}$ & $\begin{array}{l}\text { OOTK } \\
\text { OOAK }\end{array}$ & MG/L & & $\begin{array}{l}2.58 \\
2.8\end{array}$ & $\begin{array}{l}0.1 \\
0.1\end{array}$ & $\dot{-}$ \\
\hline ANTIMONY & $\begin{array}{l}06 / 14 / 89 \\
06 / 15 / 89\end{array}$ & $\begin{array}{l}\text { OOTK } \\
\text { OOAK }\end{array}$ & $M G / L$ & & $\begin{array}{l}0.600 \\
0.610\end{array}$ & $\begin{array}{l}0.003 \\
0.003\end{array}$ & $\dot{-}$ \\
\hline ARSENIC & $\begin{array}{l}06 / 14 / 89 \\
06 / 15 / 89\end{array}$ & $\begin{array}{l}\text { OOTK } \\
\text { OOAK }\end{array}$ & MG/L & & $\begin{array}{l}0.490 \\
0.46\end{array}$ & $\begin{array}{l}0.01 \\
0.01\end{array}$ & $\dot{-}$ \\
\hline BARIUM & $\begin{array}{l}06 / 14 / 89 \\
06 / 15 / 89\end{array}$ & $\begin{array}{l}\text { OOTK } \\
\text { OOAK }\end{array}$ & $M G / L$ & & $\begin{array}{l}0.270 \\
0.3\end{array}$ & $\begin{array}{l}0.1 \\
0.1\end{array}$ & $\dot{-}$ \\
\hline BERYLLIUM & $\begin{array}{l}06 / 14 / 89 \\
06 / 15 / 89\end{array}$ & $\begin{array}{l}\text { DOTK } \\
\text { DOAK }\end{array}$ & MG/L & & $\begin{array}{l}0.250 \\
0.26\end{array}$ & $\begin{array}{l}0.01 \\
0.01\end{array}$ & 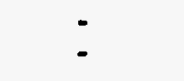 \\
\hline BORON & $\begin{array}{l}06 / 14 / 89 \\
06 / 15 / 89\end{array}$ & $\begin{array}{l}\text { OOTK } \\
\text { OOAK }\end{array}$ & $M G / L$ & & $\begin{array}{l}1.00 \\
1.0\end{array}$ & $\begin{array}{l}0.1 \\
0.1\end{array}$ & $\ddot{-}$ \\
\hline CADMIUM & $\begin{array}{l}06 / 14 / 89 \\
06 / 15 / 89\end{array}$ & $\begin{array}{l}\text { OOTK } \\
\text { OOAK }\end{array}$ & $M G / L$ & & $\begin{array}{l}0.165 \\
0.177\end{array}$ & $\begin{array}{l}0.001 \\
0.001\end{array}$ & $\dot{-}$ \\
\hline CALCIUM & $\begin{array}{l}06 / 14 / 89 \\
06 / 15 / 89\end{array}$ & $\begin{array}{l}\text { OOTK } \\
\text { OOAK }\end{array}$ & $M G / L$ & & $\begin{array}{l}62.5 \\
64.2\end{array}$ & $\begin{array}{l}0.01 \\
0.01\end{array}$ & $\dot{-}$ \\
\hline CHLORIDE & $\begin{array}{l}06 / 14 / 89 \\
06 / 15 / 89\end{array}$ & $\begin{array}{l}\text { DOTK } \\
\text { OOAK }\end{array}$ & $M G / L$ & & $\begin{array}{l}50.0 \\
51 .\end{array}$ & 1. & - \\
\hline CHROMIUM & $\begin{array}{l}06 / 14 / 89 \\
06 / 15 / 89\end{array}$ & $\begin{array}{l}\text { DOTK } \\
\text { OOAK }\end{array}$ & $M G / L$ & & $\begin{array}{l}0.180 \\
0.18\end{array}$ & $\begin{array}{l}0.01 \\
0.09\end{array}$ & $\because$ \\
\hline COBALT & $\begin{array}{l}06 / 14 / 89 \\
06 / 15 / 89\end{array}$ & $\begin{array}{l}\text { OOTK } \\
\text { OOAK }\end{array}$ & $M G / L$ & & $\begin{array}{l}0.220 \\
0.22\end{array}$ & $\begin{array}{l}0.05 \\
0.05\end{array}$ & - \\
\hline COPPER & $\begin{array}{l}06 / 14 / 89 \\
06 / 15 / 89\end{array}$ & $\begin{array}{l}\text { OOTK } \\
\text { OOAK }\end{array}$ & $M G / L$ & & $\begin{array}{l}0.220 \\
0.23\end{array}$ & $\begin{array}{l}0.02 \\
0.02\end{array}$ & - \\
\hline CYANIDE & $\begin{array}{l}06 / 14 / 89 \\
06 / 15 / 89\end{array}$ & $\begin{array}{l}\text { OOTK } \\
\text { OOAK }\end{array}$ & $M G / L$ & & $\begin{array}{l}2.48 \\
2.22\end{array}$ & $\begin{array}{l}0.09 \\
0.01\end{array}$ & . \\
\hline FLUORIDE & $\begin{array}{l}06 / 14 / 89 \\
06 / 15 / 89\end{array}$ & $\begin{array}{l}\text { OOTK } \\
\text { OOAK }\end{array}$ & $M G / L$ & & $\begin{array}{l}11.3 \\
11.4\end{array}$ & $\begin{array}{l}0.1 \\
0.1\end{array}$ & - \\
\hline IRON & $\begin{array}{l}06 / 14 / 89 \\
06 / 15 / 89\end{array}$ & $\begin{array}{l}\text { OOT̈̈ } \\
\text { OOAK }\end{array}$ & $M G / L$ & & $\begin{array}{l}0.590 \\
0.61\end{array}$ & $\begin{array}{l}0.03 \\
0.03\end{array}$ & - \\
\hline LEAD & $\begin{array}{l}06 / 14 / 89 \\
06 / 15 / 89\end{array}$ & $\begin{array}{l}\text { OOTK } \\
\text { OOAK }\end{array}$ & MG/L & & $\begin{array}{l}0.192 \\
0.20\end{array}$ & $\begin{array}{l}0.01 \\
0.01\end{array}$ & : \\
\hline MAGNESIUM & $\begin{array}{l}06 / 14 / 89 \\
06 / 15 / 89\end{array}$ & $\begin{array}{l}\text { DOTK } \\
\text { OOAK }\end{array}$ & $M G / L$ & & $\begin{array}{l}50.0 \\
51.9\end{array}$ & $\begin{array}{l}0.001 \\
0.001\end{array}$ & - \\
\hline HAMGANESE & $06 / 14 / 89$ & OOTK & $H G / L$ & & 0.625 & 0.01 & - \\
\hline
\end{tabular}

PARAMETER VALUE INDICATOR (PVI): < - LESS THAN DETECTION LIMIT

SAMPLE ID COOES: 
SURFACE HATER OUALITY DATA BY LOCATIOH

SOWP ReV. $O$ data

SITE: MONO1 MONUMENT VALLEY

LOCATION: 0981 WOD KHOWNS

NORTH COORDINATE: UNKNOWH

EAST COORDINATE: UNKNOWN

04/11/88 TO 12/14/94

REPORT DATE: 06/22/95

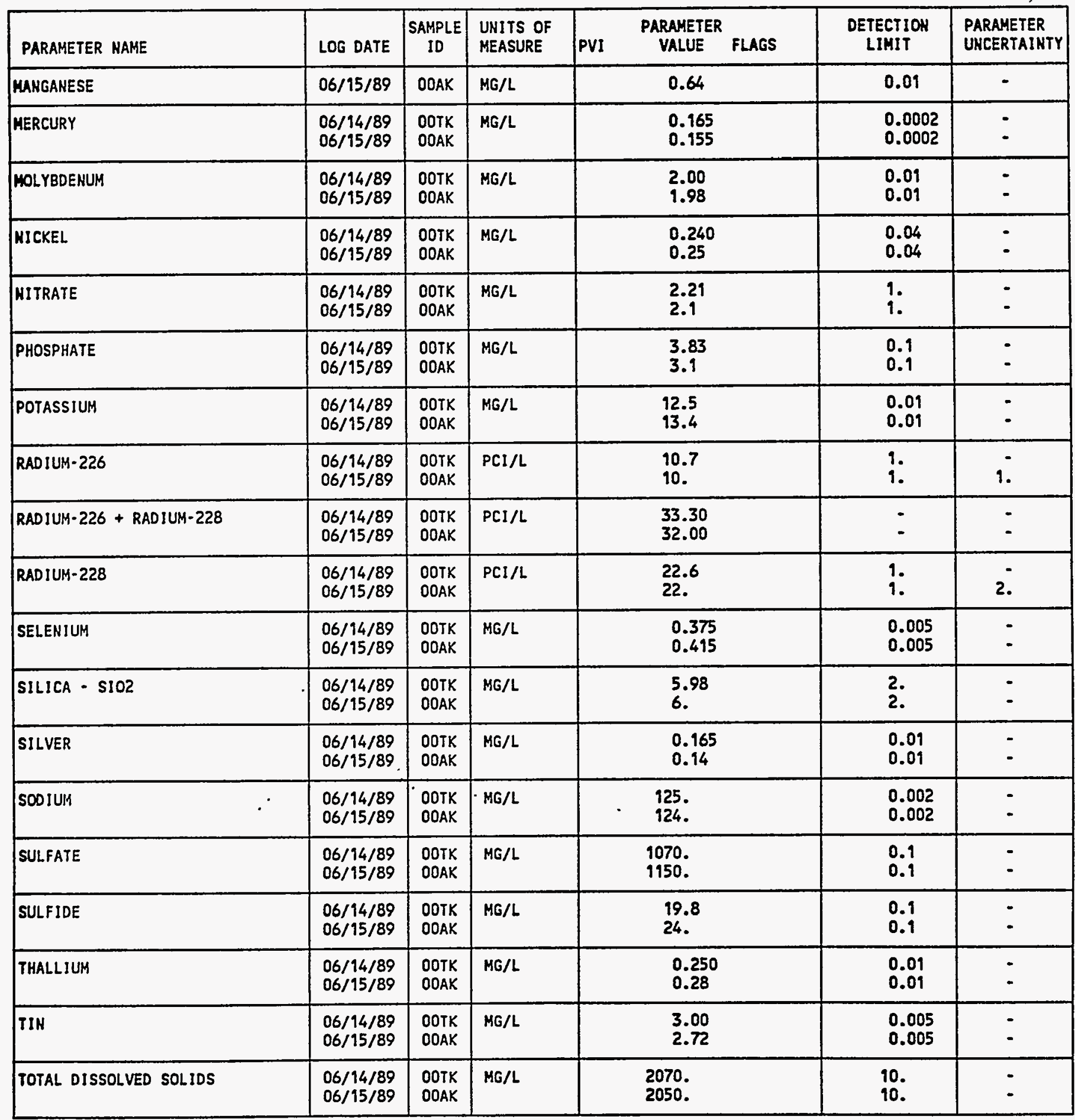

PARAMETER VALUE IHDICATOR (PVI): < - LESS THAN DETECTION LIMIT

SANPLE ID COOES:

DOAK - AMALYTICAL KMOWN SAMPLE

OOTK - THEORETICAL KNOWN SAMPLE 
SURFACE WATER QUALITY DATA BY LOCATION

SOWP REV. 0 data

SITE: MONO1 MONUMENT VALLEY

LOCATION: 0981 WOD KHOWNS

MORTH COORDINATE: UNKNOWN

EAST COORDINATE: UNKHOWH

04/11/88 TO 12/14/94

REPORT DATE: 06/22/95

\begin{tabular}{|c|c|c|c|c|c|c|c|}
\hline PARAMETER NAME & LOG DATE & $\underset{\text { ID }}{\text { SAMPLE }}$ & $\begin{array}{l}\text { UNITS OF } \\
\text { MEASURE }\end{array}$ & PVI & $\begin{array}{l}\text { PARAMETER } \\
\text { VALUE FLAGS }\end{array}$ & $\begin{array}{l}\text { DETECTION } \\
\text { LINIT }\end{array}$ & $\begin{array}{l}\text { PARAMETER } \\
\text { UHCERTAIHTY }\end{array}$ \\
\hline URANIUM & $\begin{array}{l}06 / 14 / 89 \\
06 / 15 / 89\end{array}$ & $\begin{array}{l}\text { OOTK } \\
\text { OOAK }\end{array}$ & $M G / L$ & & $\begin{array}{l}0.460 \\
0.522\end{array}$ & $\begin{array}{l}0.003 \\
0.003\end{array}$ & $\dot{-}$ \\
\hline VANADIUM & $\begin{array}{l}06 / 14 / 89 \\
06 / 15 / 89\end{array}$ & $\begin{array}{l}\text { OOTK } \\
\text { OOAK }\end{array}$ & MG/L & & $\begin{array}{l}0.455 \\
0.47\end{array}$ & $\begin{array}{l}0.01 \\
0.01\end{array}$ & $\dot{-}$ \\
\hline ZINC & $\begin{array}{l}06 / 14 / 89 \\
06 / 15 / 89\end{array}$ & $\begin{array}{l}\text { OOTK } \\
\text { OOAK }\end{array}$ & $M G / L$ & & $\begin{array}{l}0.240 \\
0.241\end{array}$ & $\begin{array}{l}0.005 \\
0.005\end{array}$ & $\dot{-}$ \\
\hline
\end{tabular}

PARAMETER VALUE INDICATOR (PVI): < - LESS THAN DETECTION LIMIT

SAMPLE ID CODES:

DOAK - AMALYTICAL KNOWN SAMPLE

DOTK - THEORETICAL KHOW SAMPLE 
SURFACE WATER OUALITY DATA BY LOCATION

SOWP Rev. 0 data

SITE: NONO 1 MONUMENT VALLEY

LOCATION: O990 WOD KHONNS

NORTH COORDINATE: UNKNOWN

EAST COORDINATE: UNKNOWN

04/11/88 TO 12/14/94

REPORT DATE: $06 / 22 / 95$

\begin{tabular}{|c|c|c|c|c|c|c|c|}
\hline PARAMETER NAME & LOG DATE & $\begin{array}{c}\text { SAMPLE } \\
\text { ID }\end{array}$ & $\begin{array}{l}\text { UNITS OF } \\
\text { MEASURE }\end{array}$ & PVI & $\begin{array}{l}\text { PARAMETER } \\
\text { VALUE FLAGS }\end{array}$ & $\begin{array}{l}\text { DETECTION } \\
\text { LIMIT }\end{array}$ & $\begin{array}{l}\text { PARAMETER } \\
\text { UNCERTAINTY }\end{array}$ \\
\hline ALUMINUM & $\begin{array}{l}06 / 14 / 89 \\
06 / 15 / 89 \\
12 / 10 / 89 \\
12 / 11 / 89 \\
07 / 03 / 90 \\
07 / 05 / 90\end{array}$ & $\begin{array}{l}\text { OOTK } \\
\text { OOAK } \\
\text { OOAK } \\
\text { OOTK } \\
\text { OOTK } \\
\text { OOAK }\end{array}$ & MG/L & & $\begin{array}{l}0.875 \\
0.9 \\
0.4 \\
0.375 \\
0.350 \\
0.41\end{array}$ & $\begin{array}{l}0.1 \\
0.1 \\
0.1 \\
0.1 \\
0.1 \\
0.1\end{array}$ & $\begin{array}{l}- \\
- \\
- \\
-\end{array}$ \\
\hline AMMONIUM & $\begin{array}{l}04 / 11 / 88 \\
04 / 11 / 88 \\
06 / 14 / 89 \\
06 / 15 / 89 \\
12 / 10 / 89 \\
12 / 11 / 89 \\
07 / 05 / 90\end{array}$ & $\begin{array}{l}\text { OOAK } \\
\text { OOTK } \\
\text { OOTK } \\
\text { OOAK } \\
\text { OOAK } \\
\text { OOTK } \\
\text { OOAK }\end{array}$ & $M G / L$ & . & $\begin{array}{l}15 . \\
14.6 \\
7.73 \\
7.8 \\
50.4 \\
51.5 \\
0.1\end{array}$ & $\begin{array}{l}0.1 \\
0.1 \\
0.1 \\
0.1 \\
0.1 \\
0.1 \\
0.1\end{array}$ & $\begin{array}{l}- \\
\dot{-} \\
\dot{-} \\
\dot{-}\end{array}$ \\
\hline AMTIMONY & $\begin{array}{l}06 / 14 / 89 \\
06 / 15 / 89 \\
12 / 10 / 89 \\
12 / 11 / 89 \\
07 / 03 / 90 \\
07 / 05 / 90\end{array}$ & $\begin{array}{l}\text { OOTK } \\
\text { OOAK } \\
\text { OOAK } \\
\text { OOTK } \\
\text { OOTK } \\
\text { OOAK }\end{array}$ & $M G / L$ & & $\begin{array}{l}0.048 \\
0.045 \\
0.045 \\
0.072 \\
0.120 \\
0.134\end{array}$ & $\begin{array}{l}0.003 \\
0.003 \\
0.003 \\
0.003 \\
0.003 \\
0.003\end{array}$ & $\begin{array}{l}- \\
: \\
:\end{array}$ \\
\hline ARSENIC & $\begin{array}{l}04 / 11 / 88 \\
04 / 11 / 88 \\
06 / 14 / 89 \\
06 / 15 / 89 \\
12 / 10 / 89 \\
12 / 11 / 89 \\
07 / 03 / 90 \\
07 / 05 / 90\end{array}$ & $\begin{array}{l}\text { OOAK } \\
\text { OOTK } \\
\text { OOTK } \\
\text { OOAK } \\
\text { OOAK } \\
\text { OOTK } \\
\text { OOTK } \\
\text { OQPAK }\end{array}$ & $M G / L$ & & $\begin{array}{l}0.675 \\
0.690 \\
0.750 \\
0.75 \\
0.23 \\
0.250 \\
0.203 \\
0.22\end{array}$ & $\begin{array}{l}0.01 \\
0.01 \\
0.01 \\
0.01 \\
0.01 \\
0.01 \\
0.01 \\
0.01\end{array}$ & $\begin{array}{l}- \\
- \\
- \\
- \\
-\end{array}$ \\
\hline BARIUM & $\begin{array}{l}06 / 14 / 89 \\
06 / 15 / 89 \\
12 / 10 / 89 \\
12 / 11 / 89 \\
07 / 03 / 90 \\
07 / 05 / 90\end{array}$ & $\begin{array}{l}\text { OOTK } \\
\text { OOAK } \\
\text { OOAK } \\
\text { OOTK } \\
\text { OOTK } \\
\text { OOAK }\end{array}$ & $M G / L$ & & $\begin{array}{l}0.450 \\
0.5 \\
0.5 \\
0.500 \\
0.406 \\
0.44\end{array}$ & $\begin{array}{l}0.1 \\
0.1 \\
0.1 \\
0.1 \\
0.1 \\
0.1\end{array}$ & :- \\
\hline BERYLLIUM & $\begin{array}{l}06 / 14 / 89 \\
06 / 15 / 89 \\
12 / 10 / 89 \\
12 / 11 / 89 \\
07 / 03 / 90 \\
07 / 05 / 90\end{array}$ & $\begin{array}{l}\text { OOTK } \\
\text { OOAK } \\
\text { OOAK } \\
\text { OOTK } \\
\text { OOTK } \\
\text { OOAK }\end{array}$ & $M G / L$ & & $\begin{array}{l}0.150 \\
0.15 \\
0.129 \\
0.125 \\
0.078 \\
0.09\end{array}$ & $\begin{array}{l}0.01 \\
0.001 \\
0.005 \\
0.01 \\
0.01 \\
0.01\end{array}$ & $\begin{array}{l}- \\
- \\
- \\
-\end{array}$ \\
\hline BORON & $\begin{array}{l}06 / 14 / 89 \\
06 / 15 / 89 \\
12 / 10 / 89 \\
12 / 11 / 89 \\
07 / 03 / 90 \\
07 / 05 / 90\end{array}$ & $\begin{array}{l}\text { OOTK } \\
\text { OOAK } \\
\text { OOAK } \\
\text { OOTK } \\
\text { OOTK } \\
\text { OOAK }\end{array}$ & MG/L & & $\begin{array}{l}2.80 \\
2.8 \\
0.3 \\
0.300 \\
0.400 \\
0.42\end{array}$ & $\begin{array}{l}0.1 \\
0.1 \\
0.1 \\
0.1 \\
0.1 \\
0.1\end{array}$ & $\begin{array}{l}- \\
- \\
- \\
-\end{array}$ \\
\hline CADMIUM & $\begin{array}{l}04 / 11 / 88 \\
04 / 11 / 88 \\
06 / 14 / 89\end{array}$ & $\begin{array}{l}\text { OOAK } \\
\text { OOTK } \\
\text { OOTK }\end{array}$ & $H G / L$ & & $\begin{array}{l}0.753 \\
0.690 \\
0.315\end{array}$ & $\begin{array}{l}0.001 \\
0.001 \\
0.001\end{array}$ & : \\
\hline
\end{tabular}

PARAMETER VALUE INDICATOR (PVI): < - LESS THAN DETECTION LIMIT SAMPLE ID COOES: 
SURFACE WATER QUALITY DATA BY LOCATION

SOWP ReV. D data

SITE: MONO1 MONUMENT VALLEY

LOCATION: 0990 WQD KHOWNS

HORTH COORDINATE: UNKHOWH

EAST COORDINATE: UHKNOWH

04/11/88 TO 12/14/94

REPORT DATE: 06/22/95

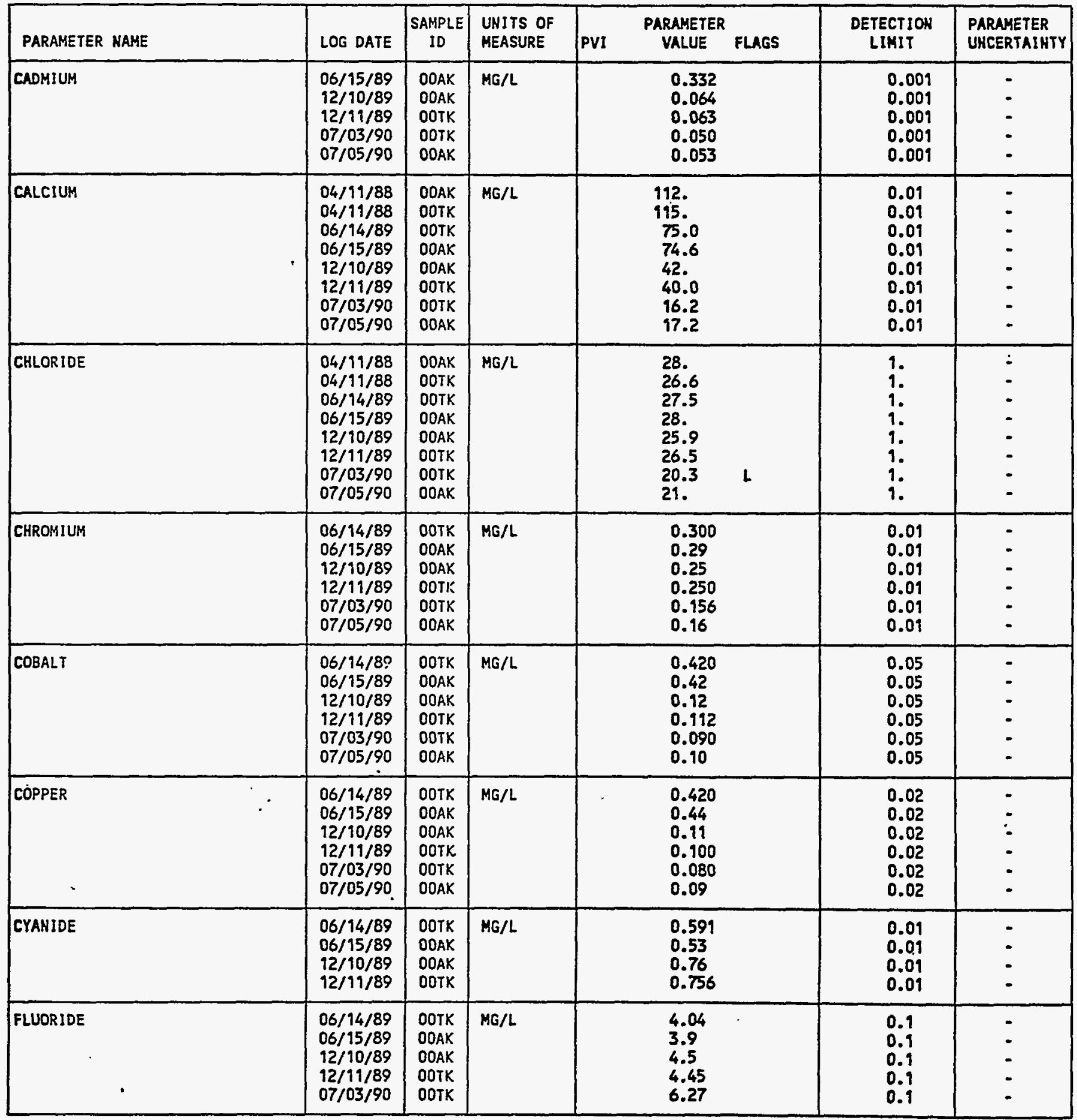

PARAMETER VALUE INDICATOR (PVI): < - LESS THAN DETECTIOH LIMIT

OTHER PARAMETER VALUE FLAGS:

1 - LESS THAN THREE BORE VOLUMES REMOVED BEFORE SAMPLING
SAMPLE ID COOES:

OOAK - AMALYTICAL KMOWN SMMPLE

OOTK - THEORETICAL KHOW SAMPLE 
SURFACE WATER QUALITY DATA BY LOCATIOH

SOHP REV. 0 data

SITE: MONO1 HONUMENT VALLEY

LOCATION: 0990 WOD KHOWHS

HORTH COORDINATE: UHKHOWH

EAST COORDINATE: UNKHOWH

$04 / 11 / 88$ TO $12 / 14 / 94$

REPORT DATE: $06 / 22 / 95$

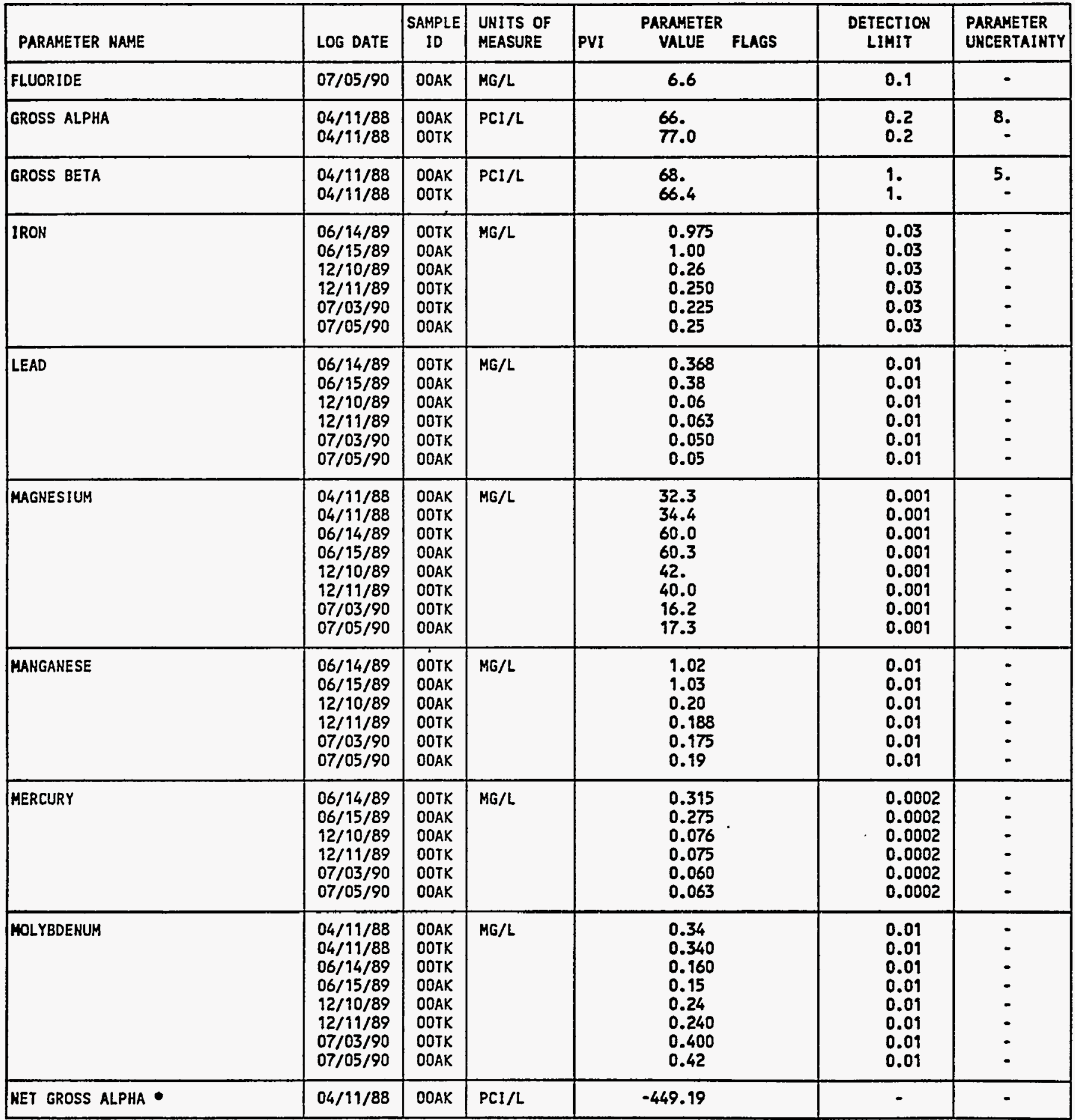

- NET GROSS ALPHA (GROSS ALPHA - URANIUM) HITH I HG URAHILM = 686 PCI

PARAMETER VALUE INDICATOR (PVI): < - LESS THAN DETECTION LIMIT
SAMPLE ID COOES:

DOAK - AMALYTICAL KNON SAMPLE

DOTK - THEORETICAL KNOWH SAMPLE 
SURFACE WATER QUALITY DATA BY LOCATION

SOWP ReV. $O$ data

SITE: MONO1 MONUMENT VALLEY

LOCATION: 0990 WOD KNOWNS

HORTH COORDINATE: UNKHOWN

EAST COORDINATE: UNKHOWN

$04 / 11 / 88$ TO $12 / 14 / 94$

REPORT DATE: $06 / 22 / 95$

\begin{tabular}{|c|c|c|c|c|c|c|c|}
\hline PARAMETER NAME & LOG DATE & $\begin{array}{c}\text { SAMPLE } \\
\text { ID }\end{array}$ & $\begin{array}{l}\text { UNITS OF } \\
\text { MEASURE }\end{array}$ & PVI & $\begin{array}{l}\text { PARAMETER } \\
\text { VALUE FLAGS }\end{array}$ & $\begin{array}{l}\text { DETECTION } \\
\text { LIMIT }\end{array}$ & $\begin{array}{l}\text { PARAMETER } \\
\text { UKCERTAINTY }\end{array}$ \\
\hline NET GROSS ALPHA * & $04 / 11 / 88$ & DOTK & PCI/L & & -372.33 & - & - \\
\hline HICKEL & $\begin{array}{l}06 / 14 / 89 \\
06 / 15 / 89 \\
12 / 10 / 89 \\
12 / 11 / 89 \\
07 / 03 / 90 \\
07 / 05 / 90\end{array}$ & $\begin{array}{l}\text { OOTK } \\
\text { DOAK } \\
\text { OOAK } \\
\text { OOTK } \\
\text { DOTK } \\
\text { DOAK }\end{array}$ & MG/L & & $\begin{array}{l}0.400 \\
0.40 \\
0.20 \\
0.200 \\
0.125 \\
0.14\end{array}$ & $\begin{array}{l}0.04 \\
0.04 \\
0.04 \\
0.04 \\
0.04 \\
0.04\end{array}$ & $\begin{array}{l}- \\
- \\
-\end{array}$ \\
\hline NITRATE & $\begin{array}{l}04 / 11 / 88 \\
04 / 11 / 88 \\
06 / 14 / 89 \\
06 / 15 / 89 \\
12 / 10 / 89 \\
12 / 11 / 89 \\
07 / 03 / 90 \\
07 / 05 / 90\end{array}$ & $\begin{array}{l}\text { DOAK } \\
\text { OOTK } \\
\text { OOTK } \\
\text { DOAK } \\
\text { DOAK } \\
\text { DOTK } \\
\text { DOTK } \\
\text { DOAK }\end{array}$ & $M G / L$ & & $\begin{array}{l}47 . \\
46.5 \\
26.6 \\
26 . \\
2.2 \\
2.21 \\
71.9 \\
79.0\end{array}$ & $\begin{array}{l}1 . \\
1 . \\
i . \\
1 . \\
1 . \\
1 . \\
1 .\end{array}$ & $\begin{array}{l}- \\
- \\
- \\
- \\
-\end{array}$ \\
\hline PHOSPHATE & $\begin{array}{l}06 / 14 / 89 \\
06 / 15 / 89 \\
12 / 10 / 89 \\
12 / 11 / 89 \\
07 / 03 / 90 \\
07 / 05 / 90\end{array}$ & $\begin{array}{l}\text { DOTK } \\
\text { OOAK } \\
\text { OOAK } \\
\text { OOTK } \\
\text { DOTK } \\
\text { DOAK }\end{array}$ & MG/L & & $\begin{array}{l}1.99 \\
1.9 \\
0.5 \\
0.460 \\
0.613 \\
0.8\end{array}$ & $\begin{array}{l}0.1 \\
0.1 \\
0.1 \\
0.1 \\
0.1 \\
0.1\end{array}$ & $\begin{array}{l}- \\
: \\
:\end{array}$ \\
\hline POTASSIUH & $\begin{array}{l}04 / 11 / 88 \\
04 / 11 / 88 \\
06 / 14 / 89 \\
06 / 15 / 89 \\
12 / 10 / 89 \\
12 / 11 / 89 \\
07 / 03 / 90 \\
07 / 05 / 90\end{array}$ & $\begin{array}{l}\text { OOAK } \\
\text { OOTK } \\
\text { OOTK } \\
\text { OOAK } \\
\text { OOAK } \\
\text { OOTK } \\
\text { OOTK } \\
\text { OOAK }\end{array}$ & $M G / L$ & & $\begin{array}{c}5.22 \\
5.73 \\
15.0 \\
15.5 \\
9.6 \\
10.0 \\
4.06 \\
4.2\end{array}$ & $\begin{array}{l}0.01 \\
0.01 \\
0.01 \\
0.01 \\
0.01 \\
0.01 \\
0.01 \\
0.01\end{array}$ & $\begin{array}{l}: \\
: \\
: \\
:\end{array}$ \\
\hline RADIUM-226 & $\begin{array}{l}06 / 14 / 89 \\
06 / 15 / 89 \\
12 / 10 / 89 \\
12 / 19 / 89 \\
07 / 03 / 90 \\
07 / 05 / 90\end{array}$ & $\begin{array}{l}\text { OOTK } \\
\text { DOAK } \\
\text { OOAK } \\
\text { ODTK } \\
\text { OOTK } \\
\text { OOAK }\end{array}$ & PCI/L & & $\begin{array}{l}53.0 \\
50 . \\
6.0 \\
12.8 \\
16.0 \\
17 .\end{array}$ & $\begin{array}{l}1 . \\
i . \\
i . \\
i .\end{array}$ & $\begin{array}{c}3 . \\
0.6 \\
\vdots \\
?\end{array}$ \\
\hline RADIUM-226 + RADIUM-228 & $\begin{array}{l}06 / 14 / 89 \\
06 / 15 / 89 \\
12 / 10 / 89 \\
12 / 11 / 89 \\
07 / 03 / 90 \\
07 / 05 / 90\end{array}$ & $\begin{array}{l}\text { DOTK } \\
\text { OOAK } \\
\text { OOAK } \\
\text { OOTK } \\
\text { OOTK } \\
\text { OOAK }\end{array}$ & $\mathrm{PCl} / \mathrm{L}$ & & $\begin{array}{r}104.00 \\
103.00 \\
18.70 \\
32.60 \\
49.40 \\
53.00\end{array}$ & $\begin{array}{l}- \\
\dot{-} \\
\dot{-}\end{array}$ & $\begin{array}{l}: \\
: \\
:\end{array}$ \\
\hline RADIUM-228 & $\begin{array}{l}06 / 14 / 89 \\
06 / 15 / 89 \\
12 / 10 / 89 \\
12 / 11 / 89 \\
07 / 03 / 90\end{array}$ & $\begin{array}{l}\text { OOTK } \\
\text { OOAK } \\
\text { OOAK } \\
\text { OOTK } \\
\text { OOTK }\end{array}$ & $\mathrm{PCI} / \mathrm{L}$ & & $\begin{array}{l}51.0 \\
53 . \\
12.7 \\
19.8 \\
33.4\end{array}$ & $\begin{array}{l}1 . \\
1 . \\
1 . \\
1 . \\
1 .\end{array}$ & $\begin{array}{l}- \\
3.7 \\
-\end{array}$ \\
\hline
\end{tabular}

- MET GROSS ALPHA (GROSS ALPHA - URANIUM) WITH 1 MG URANIUM = 686 PCI

PARAMETER VALUE INDICATOR (PVI): < - LESS THAN DETECTION LIMIT

SAMPLE ID COOES:

OOAK - ANALYTICAL KMON SAMPLE

OOTK - THEORETICAL KHOWN SAMPLE 
SURFACE MATER OUALITY OATA BY LOCATION

SOWP REV. $O$ data

SITE: MONO1 MONUMENT VALLEY

LOCATION: 0990 WAD KHONHS

HORTH COORDINATE: UHKHOWN

EAST COORDINATE: UNKNOWN

04/11/88 TO 12/14/94

REPORT DATE: 06/22/95

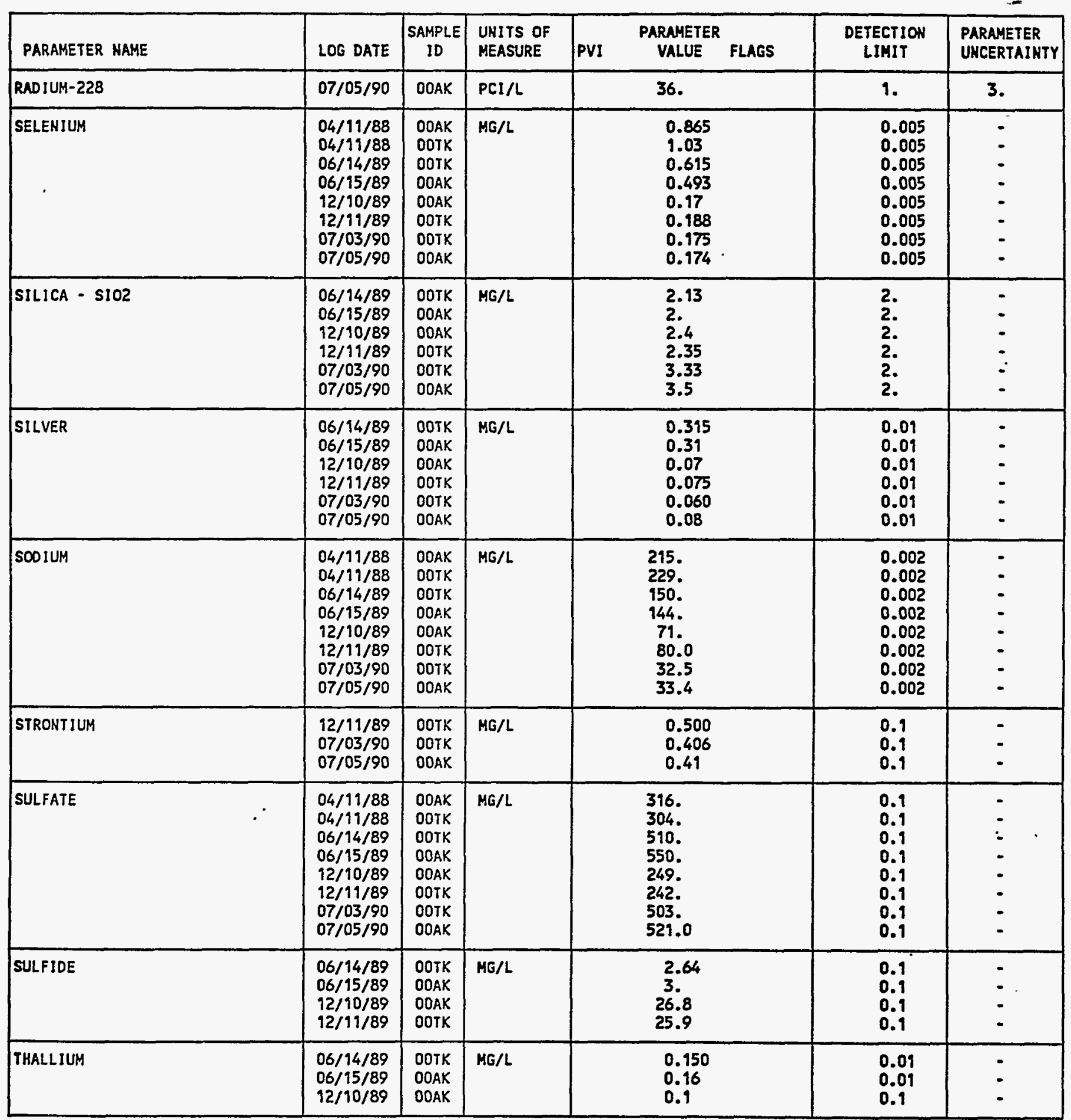

PARAMETER VALUE IHDICATOR (PVI): < - LESS THAN DETECTION LIMIT 
SURFACE WATER OUALITY DATA BY LOCATION

SOWP REV. $O$ data

SITE: MONO1 MONUMENT VALLEY

LOCATION: 0990 WQD KNOWNS

NORTH COORDINATE: UNKMOWN

EAST COORDINATE: UNKNOUN

04/11/88 TO 12/14/94

REPORT DATE: 06/22/95

\begin{tabular}{|c|c|c|c|c|c|c|c|}
\hline PARAMETER NAME & LOG DATE & SAMPLE & $\begin{array}{l}\text { UNITS OF } \\
\text { MEASURE }\end{array}$ & PVI & $\begin{array}{l}\text { PARAMETER } \\
\text { VALUE FLAGS }\end{array}$ & $\begin{array}{l}\text { DETECTION } \\
\text { LIMIT }\end{array}$ & $\begin{array}{l}\text { PARAMETER } \\
\text { UHCERTAIHTY }\end{array}$ \\
\hline THALLIUH & $\begin{array}{l}12 / 11 / 89 \\
07 / 03 / 90 \\
07 / 05 / 90\end{array}$ & $\begin{array}{l}\text { OOTK } \\
\text { OOTK } \\
\text { OOAK }\end{array}$ & $M G / L$ & & $\begin{array}{l}0.063 \\
0.050 \\
0.06\end{array}$ & $\begin{array}{l}0.01 \\
0.01 \\
0.01\end{array}$ & : \\
\hline TIN & $\begin{array}{l}06 / 14 / 89 \\
06 / 15 / 89 \\
12 / 10 / 89 \\
12 / 11 / 89 \\
07 / 03 / 90 \\
07 / 05 / 90\end{array}$ & $\begin{array}{l}\text { OOTK } \\
\text { OOAK } \\
\text { OOAK } \\
\text { OOTK } \\
\text { OOTK } \\
\text { OOAK }\end{array}$ & $M G / L$ & & $\begin{array}{l}0.240 \\
0.351 \\
0.37 \\
0.360 \\
0.600 \\
0.561\end{array}$ & $\begin{array}{l}0.005 \\
0.005 \\
0.005 \\
0.005 \\
0.005 \\
0.005\end{array}$ & $\begin{array}{l}\dot{-} \\
\dot{-} \\
\dot{-}\end{array}$ \\
\hline TOTAL DISSOLVED SOLIOS & $\begin{array}{l}04 / 11 / 88 \\
04 / 11 / 88 \\
06 / 14 / 89 \\
06 / 15 / 89 \\
12 / 10 / 89 \\
12 / 11 / 89 \\
07 / 03 / 90 \\
07 / 05 / 90\end{array}$ & $\begin{array}{l}\text { DOAK } \\
\text { OOTK } \\
\text { OOTK } \\
\text { OOAK } \\
\text { OOAK } \\
\text { OOTK } \\
\text { OOTK } \\
\text { OOAK }\end{array}$ & MG/L & & $\begin{array}{l}655 . \\
659 . \\
1000 . \\
979 . \\
482 . \\
498 . \\
967 . \\
968 .\end{array}$ & $\begin{array}{l}10 . \\
10 . \\
10 . \\
10 . \\
10 . \\
10 . \\
10 . \\
10 .\end{array}$ & $\begin{array}{l}\dot{-} \\
\dot{-} \\
\dot{-} \\
\dot{-} \\
-\end{array}$ \\
\hline TOTAL ORGANIC CARBON & $\begin{array}{l}04 / 11 / 88 \\
04 / 11 / 88\end{array}$ & $\begin{array}{l}\text { OOAK } \\
\text { OOTK }\end{array}$ & $M G / L$ & & $\begin{array}{l}3.0 \\
2.50\end{array}$ & 1. & - \\
\hline URANIUH & $\begin{array}{l}04 / 11 / 88 \\
04 / 11 / 88 \\
06 / 14 / 89 \\
06 / 15 / 89 \\
12 / 10 / 89 \\
12 / 11 / 89 \\
07 / 03 / 90 \\
07 / 05 / 90\end{array}$ & $\begin{array}{l}\text { OOAK } \\
\text { OOTK } \\
\text { OOTK } \\
\text { OOAK } \\
\text { OOAK } \\
\text { OOTK } \\
\text { OOTK } \\
\text { OOAK }\end{array}$ & MG/L & & $\begin{array}{l}0.751 \\
0.655 \\
0.700 \\
0.840 \\
0.299 \\
0.325 \\
0.269 \\
0.287\end{array}$ & $\begin{array}{l}0.003 \\
0.003 \\
0.003 \\
0.003 \\
0.003 \\
0.003 \\
0.003 \\
0.003\end{array}$ & $\begin{array}{l}: \\
: \\
: \\
: \\
-\end{array}$ \\
\hline VANADIUM & $\begin{array}{l}06 / 14 / 89 \\
06 / 15 / 89 \\
12 / 10 / 89 \\
12 / 11 / 89 \\
07 / 03 / 90 \\
07 / 05 / 90\end{array}$ & $\begin{array}{l}\text { OOTK } \\
\text { OOAK } \\
\text { OOAK } \\
\text { OOTK } \\
\text { OOTK } \\
\text { OOAK }\end{array}$ & $H G / L$ & & $\begin{array}{l}0.700 \\
0.70 \\
0.35 \\
0.350 \\
0.284 \\
0.30\end{array}$ & $\begin{array}{l}0.01 \\
0.01 \\
0.01 \\
0.01 \\
0.01 \\
0.01\end{array}$ & $\begin{array}{l}- \\
\dot{-} \\
\dot{-}\end{array}$ \\
\hline ZINC & $\begin{array}{l}06 / 14 / 89 \\
06 / 15 / 89 \\
12 / 10 / 89 \\
12 / 11 / 89 \\
07 / 03 / 90 \\
07 / 05 / 90\end{array}$ & $\begin{array}{l}\text { OOTK } \\
\text { OOAK } \\
\text { OOAK } \\
\text { OOTK } \\
\text { DOTK } \\
\text { OOAK }\end{array}$ & $M G / L$ & & $\begin{array}{l}0.400 \\
0.416 \\
0.21 \\
0.200 \\
0.125 \\
0.140\end{array}$ & $\begin{array}{l}0.005 \\
0.005 \\
0.005 \\
0.005 \\
0.005 \\
0.005\end{array}$ & $\begin{array}{l}- \\
- \\
- \\
-\end{array}$ \\
\hline
\end{tabular}

PARAMETER VALUE IHDICATOR (PVI): < - LESS THAN DETECTION LIHIT

SAMPLE ID COOES:

OOAK - ANALYTICAL KNONN SAMPLE

OOTK - THEORETICAL KHOW SAMPLE 
SURFACE HATER QUALITY DATA BY LOCATION

SOWP REV. 0 data

SITE: HONO1 MONUMENT VALLEY

LOCATION: 0999

NORTH COORDINATE: UNKNOWN

EAST COORDINATE: UNKNONH

04/11/88 TO 12/14/94

REPORT DATE: 06/22/95

\begin{tabular}{|c|c|c|c|c|c|c|c|}
\hline PARAMETER HAME & LOG DATE & $\begin{array}{c}\text { SAMPLE } \\
\text { ID }\end{array}$ & $\begin{array}{l}\text { UNITS OF } \\
\text { MEASURE }\end{array}$ & PVI & $\begin{array}{l}\text { PARAMETER } \\
\text { VALUE FLAGS }\end{array}$ & $\begin{array}{l}\text { DETECTION } \\
\text { LIHIT }\end{array}$ & $\begin{array}{l}\text { PARAYETER } \\
\text { UHCERTAINTY }\end{array}$ \\
\hline AMMONIUM (TOTAL) & $\begin{array}{l}06 / 30 / 93 \\
06 / 30 / 93 \\
06 / 30 / 93 \\
06 / 30 / 93 \\
12 / 12 / 93 \\
12 / 12 / 93 \\
04 / 24 / 94 \\
04 / 24 / 94 \\
04 / 29 / 94 \\
04 / 29 / 94 \\
12 / 11 / 94 \\
12 / 11 / 94 \\
12 / 13 / 94 \\
12 / 13 / 94\end{array}$ & $\begin{array}{l}\text { NOO1 } \\
\text { HOO2 } \\
\text { HOO3 } \\
\text { NOO4 } \\
\text { NOO1 } \\
\text { HOO2 } \\
\text { NOO1 } \\
\text { NOO2 } \\
\text { NOO3 } \\
\text { HOO4 } \\
\text { HOO1 } \\
\text { HOO2 } \\
\text { NOO3 } \\
\text { HOO4 }\end{array}$ & $M \dot{G} / L$ & $\begin{array}{l}< \\
< \\
< \\
< \\
< \\
< \\
< \\
< \\
< \\
< \\
< \\
< \\
< \\
<\end{array}$ & $\begin{array}{l}0.1 \\
0.1 \\
0.1 \\
0.1 \\
0.1 \\
0.1 \\
0.1 \\
0.1 \\
0.1 \\
0.1 \\
0.1 \\
0.1 \\
0.1 \\
0.1\end{array}$ & $\begin{array}{l}0.1 \\
0.1 \\
0.1 \\
0.1 \\
0.1 \\
0.1 \\
0.1 \\
0.1 \\
0.1 \\
0.1 \\
0.1 \\
0.1 \\
0.1 \\
0.1\end{array}$ & $\begin{array}{l}- \\
- \\
- \\
- \\
- \\
- \\
- \\
- \\
- \\
- \\
- \\
- \\
-\end{array}$ \\
\hline ARSENIC (TOTAL) & $\begin{array}{l}06 / 30 / 93 \\
06 / 30 / 93 \\
06 / 30 / 93 \\
06 / 30 / 93\end{array}$ & $\begin{array}{l}\text { HOO1 } \\
\text { NOO2 } \\
\text { HOO3 } \\
\text { NOO4 }\end{array}$ & $M G / L$ & $\begin{array}{l}< \\
< \\
< \\
<\end{array}$ & $\begin{array}{l}0.005 \\
0.005 \\
0.005 \\
0.005\end{array}$ & $\begin{array}{l}0.005 \\
0.005 \\
0.005 \\
0.005\end{array}$ & $\begin{array}{l}- \\
- \\
-\end{array}$ \\
\hline BARIUM (TOTAL) & $\begin{array}{l}06 / 30 / 93 \\
06 / 30 / 93 \\
06 / 30 / 93 \\
06 / 30 / 93\end{array}$ & $\begin{array}{l}\text { NoO1 } \\
\text { NOO2 } \\
\text { NOO3 } \\
\text { NOO4 }\end{array}$ & $M G / L$ & $\begin{array}{l}< \\
< \\
< \\
<\end{array}$ & $\begin{array}{l}0.1 \\
0.1 \\
0.1 \\
0.1\end{array}$ & $\begin{array}{l}0.1 \\
0.1 \\
0.1 \\
0.1\end{array}$ & $\begin{array}{l}- \\
- \\
-\end{array}$ \\
\hline CALCIUM & $\begin{array}{l}02 / 02 / 93 \\
02 / 02 / 93 \\
04 / 24 / 94 \\
04 / 24 / 94 \\
04 / 24 / 94 \\
04 / 24 / 94 \\
12 / 11 / 94 \\
12 / 11 / 94 \\
12 / 13 / 94 \\
12 / 13 / 94\end{array}$ & $\begin{array}{l}0001 \\
0002 \\
0001 \\
0002 \\
0003 \\
0004 \\
0001 \\
0002 \\
0003 \\
0004\end{array}$ & $M G / L$ & $\begin{array}{l}< \\
< \\
< \\
< \\
< \\
< \\
< \\
< \\
< \\
<\end{array}$ & $\begin{array}{l}0.5 \\
0.5 \\
0.5 \\
0.5 \\
0.5 \\
0.5 \\
0.5 \\
0.5 \\
0.5 \\
0.5\end{array}$ & $\begin{array}{l}0.5 \\
0.5 \\
0.5 \\
0.5 \\
0.5 \\
0.5 \\
0.5 \\
0.5 \\
0.5 \\
0.5\end{array}$ & $\begin{array}{l}- \\
- \\
- \\
- \\
- \\
- \\
- \\
-\end{array}$ \\
\hline CALCIUM (TOTAL) & $\begin{array}{l}06 / 30 / 93 \\
06 / 30 / 93 \\
06 / 30 / 93 \\
06 / 30 / 93 \\
12 / 12 / 93 \\
12 / 12 / 93\end{array}$ & $\begin{array}{l}\text { NOO1 } \\
\text { NCO2 } \\
\text { NOO3 } \\
\text { NOO4 } \\
\text { NOO1 } \\
\text { NOO2 }\end{array}$ & $M G / L$ & $\begin{array}{l}< \\
< \\
< \\
< \\
< \\
<\end{array}$ & $\begin{array}{l}0.5 \\
0.5 \\
0.5 \\
0.5 \\
0.5 \\
0.5\end{array}$ & $\begin{array}{l}0.5 \\
0.5 \\
0.5 \\
0.5 \\
0.5 \\
0.5\end{array}$ & $\begin{array}{l}- \\
- \\
- \\
-\end{array}$ \\
\hline CHLORIDE & $\begin{array}{l}02 / 02 / 93 \\
02 / 02 / 93 \\
04 / 24 / 94 \\
04 / 24 / 94 \\
04 / 24 / 94 \\
04 / 24 / 94\end{array}$ & $\begin{array}{l}0001 \\
0002 \\
0001 \\
0002 \\
0003 \\
0004\end{array}$ & $M G / L$ & $\begin{array}{l}< \\
< \\
< \\
< \\
< \\
<\end{array}$ & $\begin{array}{l}0.5 \\
0.5 \\
0.5 \\
0.5 \\
0.5 \\
0.5\end{array}$ & $\begin{array}{l}0.5 \\
0.5 \\
0.5 \\
0.5 \\
0.5 \\
0.5\end{array}$ & $\begin{array}{l}- \\
- \\
- \\
- \\
- \\
-\end{array}$ \\
\hline
\end{tabular}

PARAMETER VALUE IHDICATOR (PVI): < - LESS THAN DETECTION LIMIT

OTHER PARAMETER VALUE FLAGS:

H - HOLD TIME EXPIRED, VALUE SUSPECT
SAMPLE ID COOES:

0001 - FILTERED SAMPLE (.45 MICRONS)

0002 - FILTERED REPLICATE SAMPLE (.45 MICRONS)

O003 - FILTERED REPLICATE SNMPLE (.45 MICRONS)

0004 - FILTERED REPLICATE SAMPLE (.45 MICRONS)

HOO1 - UMFILTERED SNMPLE

MOO2 - UNFILTERED REPLICATE SAMPLE

MOO3 - UNFILTERED REPLICATE SAMPLE

MOO4 - UNFILTERED REPLICATE SAMPLE 
SURFACE WATER QUALITY DATA BY LOCATION

SOWP REV. $O$ data

SITE: MONO1 MONUNENT VALLEY

LOCATION: 0999

NORTH COORDINATE: UNKNOWH

EAST COORDINATE: UNKNOWH

04/11/88 TO $12 / 14 / 94$

REPORT DATE: 06/22/95

\begin{tabular}{|c|c|c|c|c|c|c|c|}
\hline PARAMETER NAME & LOG DATE & $\mid \begin{array}{c}\text { SAMPLE } \\
\text { ID }\end{array}$ & $\begin{array}{l}\text { UNITS OF } \\
\text { MEASURE }\end{array}$ & PVI & $\begin{array}{l}\text { PARAMETER } \\
\text { VALUE FLAGS }\end{array}$ & $\begin{array}{l}\text { DETECTIOH } \\
\text { LIMIT }\end{array}$ & $\begin{array}{l}\text { PARAMETER } \\
\text { UNCERTAINTY }\end{array}$ \\
\hline CHLORIDE & $\begin{array}{l}12 / 11 / 94 \\
12 / 19 / 94\end{array}$ & $\begin{array}{l}0001 \\
0002\end{array}$ & $M G / L$ & $<$ & $\begin{array}{l}0.5 \\
0.5\end{array}$ & $\begin{array}{l}0.5 \\
0.5\end{array}$ & $\dot{-}$ \\
\hline CHLORIDE (TOTAL) & $\begin{array}{l}06 / 30 / 93 \\
06 / 30 / 93 \\
06 / 30 / 93 \\
06 / 30 / 93 \\
12 / 12 / 93 \\
12 / 12 / 93\end{array}$ & $\begin{array}{l}\text { NOO1 } \\
\text { NOO2 } \\
\text { NOO3 } \\
\text { NOO4 } \\
\text { NOO1 } \\
\text { NOO2 }\end{array}$ & $M G / L$ & $\begin{array}{l}< \\
< \\
< \\
< \\
<\end{array}$ & $\begin{array}{l}0.5 \\
0.5 \\
0.5 \\
0.5 \\
0.5 \\
0.5\end{array}$ & $\begin{array}{l}0.5 \\
0.5 \\
0.5 \\
0.5 \\
0.5 \\
0.5\end{array}$ & $\begin{array}{l}\text { : } \\
\dot{-} \\
-\end{array}$ \\
\hline GROSS ALPHA & $\begin{array}{l}02 / 02 / 93 \\
02 / 02 / 93\end{array}$ & $\begin{array}{l}0001 \\
0002\end{array}$ & $\mathrm{PCI} / \mathrm{L}$ & & $\begin{array}{l}0 \\
0\end{array}$ & $\begin{array}{l}3 \\
3\end{array}$ & $\begin{array}{l}3 \\
2\end{array}$ \\
\hline GROSS ALPHA (TOTAL) & $\begin{array}{l}06 / 30 / 93 \\
06 / 30 / 93 \\
06 / 30 / 93 \\
06 / 30 / 93\end{array}$ & $\begin{array}{l}\text { NOO1 } \\
\text { NOOZ } \\
\text { NOO3 } \\
\text { NOO4 }\end{array}$ & PCI/L & & $\begin{array}{l}0 \\
0 \\
0 \\
0\end{array}$ & $\begin{array}{l}1 \\
1 \\
1 \\
1\end{array}$ & $\begin{array}{l}2 \\
1 \\
1 \\
1\end{array}$ \\
\hline GROSS BETA (TOTAL) & $\begin{array}{l}06 / 30 / 93 \\
06 / 30 / 93 \\
06 / 30 / 93 \\
06 / 30 / 93\end{array}$ & $\begin{array}{l}\text { NOO1 } \\
\text { NOO2 } \\
\text { NOO3 } \\
\text { N004 }\end{array}$ & $\mathrm{PCI} / \mathrm{L}$ & & $\begin{array}{l}0 \\
0 \\
0 \\
0\end{array}$ & $\begin{array}{l}4 \\
4 \\
4 \\
4\end{array}$ & $\begin{array}{l}4 \\
3 \\
3 \\
4\end{array}$ \\
\hline IRON & $\begin{array}{l}02 / 02 / 93 \\
02 / 02 / 93 \\
12 / 13 / 94 \\
12 / 13 / 94\end{array}$ & $\begin{array}{l}0001 \\
0002 \\
0003 \\
0004\end{array}$ & $M G / L$ & $\begin{array}{l}< \\
< \\
<\end{array}$ & $\begin{array}{l}0.03 \\
0.03 \\
0.03 \\
0.03\end{array}$ & $\begin{array}{l}0.03 \\
0.03 \\
0.03 \\
0.03\end{array}$ & : \\
\hline IRON (TOTAL) & $\begin{array}{l}06 / 30 / 93 \\
06 / 30 / 93 \\
06 / 30 / 93 \\
06 / 30 / 93\end{array}$ & $\begin{array}{l}\text { NOO1 } \\
\text { NOO2 } \\
\text { N003 } \\
\text { NOO4 }\end{array}$ & MG/L & $\begin{array}{l}< \\
<\end{array}$ & $\begin{array}{l}0.03 \\
0.03 \\
0.04 \\
0.03\end{array}$ & $\begin{array}{l}0.03 \\
0.03 \\
0.03 \\
0.03\end{array}$ & $\dot{-}$ \\
\hline MAGKESIUH (TOTAL) & $\begin{array}{l}06 / 30 / 93 \\
06 / 30 / 93\end{array}$ & $\begin{array}{l}\text { N001 } \\
\text { N002 }\end{array}$ & $M G / L$ & $<$ & $\begin{array}{l}0.1 \\
0.1\end{array}$ & $\begin{array}{l}0.1 \\
0.1\end{array}$ & : \\
\hline
\end{tabular}

PARAMETER VALUE INDICATOR (PVI): < - LESS THAN DETECTION LIMIT

SNMPLE ID COOES:

O001 - FILTERED SNMPLE (.45 MICRONS)

0002 - FILTERED REPLICATE SAMPLE (.45 MICROHS)

0003 - FILTERED REPLICATE SAMPLE (.45 MICROHS)

0004 - FILTERED REPLICATE SAMPLE (.45 HICRONS)

NOO1 - UNFILTERED SAMPLE

NOO2 - UNFILTERED REPLICATE SAMPLE

NOO3 - UHFILTERED REPLICATE SAMPLE

NOO4 - UAFILTERED REPLICATE SAMPLE 
SURFACE WATER QUALITY DATA BY LOCATION

SOWP REV. $O$ data

SITE: MONO1 MONUMENT VALLEY

LOCATION: 0999

NORTH COORDINATE: UNKMOWN

EAST COORDINATE: UNKNOWN

$04 / 11 / 88$ TO $12 / 14 / 94$

REPORT DATE : 06/22/95

\begin{tabular}{|c|c|c|c|c|c|c|c|}
\hline PARAMETER HAME & LOG DATE & $\begin{array}{c}\text { SAMPLE } \\
\text { ID }\end{array}$ & $\begin{array}{l}\text { UNITS OF } \\
\text { MEASURE }\end{array}$ & PVI & $\begin{array}{l}\text { PARAMETER } \\
\text { VALUE FLAGS }\end{array}$ & $\begin{array}{l}\text { DETECTION } \\
\text { LIHIT }\end{array}$ & $\begin{array}{l}\text { PARAMETER } \\
\text { UNCERTAIHTY }\end{array}$ \\
\hline MAGNESIUM (TOTAL) & $\begin{array}{l}06 / 30 / 93 \\
06 / 30 / 93 \\
12 / 12 / 93 \\
12 / 12 / 93\end{array}$ & $\begin{array}{l}\mathrm{NOO3} \\
\mathrm{NOO4} \\
\mathrm{NOO1} \\
\mathrm{NOO2}\end{array}$ & MG/L & $\begin{array}{l}< \\
< \\
<\end{array}$ & $\begin{array}{l}0.1 \\
0.1 \\
0.1 \\
0.1\end{array}$ & $\begin{array}{l}0.1 \\
0.1 \\
0.1 \\
0.1\end{array}$ & $\begin{array}{l}- \\
-\end{array}$ \\
\hline RANGANESE & $\begin{array}{l}02 / 02 / 93 \\
02 / 02 / 93 \\
04 / 24 / 94 \\
04 / 24 / 94 \\
04 / 24 / 94 \\
04 / 24 / 94 \\
12 / 11 / 94 \\
12 / 11 / 94 \\
12 / 13 / 94 \\
12 / 13 / 94\end{array}$ & $\begin{array}{l}0001 \\
0002 \\
6001 \\
0002 \\
0003 \\
0004 \\
0001 \\
0002 \\
0003 \\
0004\end{array}$ & $M G / L$ & $\begin{array}{l}< \\
< \\
< \\
< \\
< \\
< \\
< \\
< \\
<\end{array}$ & $\begin{array}{l}0.01 \\
0.09 \\
0.01 \\
0.01 \\
0.01 \\
0.01 \\
0.01 \\
0.01 \\
0.01 \\
0.01\end{array}$ & $\begin{array}{l}0.01 \\
0.01 \\
0.09 \\
0.01 \\
0.01 \\
0.01 \\
0.01 \\
0.01 \\
0.01 \\
0.01\end{array}$ & $\begin{array}{l}\dot{-} \\
\dot{-} \\
\dot{-} \\
\dot{-} \\
\dot{-} \\
\because\end{array}$ \\
\hline MANGANESE (TOTAL) & $\begin{array}{l}06 / 30 / 93 \\
06 / 30 / 93 \\
06 / 30 / 93 \\
06 / 30 / 93 \\
12 / 12 / 93 \\
12 / 12 / 93\end{array}$ & $\begin{array}{l}\text { NOO1 } \\
\text { NOO2 } \\
\text { NO03 } \\
\text { NOO4 } \\
\text { NOO1 } \\
\text { NOO2 }\end{array}$ & MG/L & $\begin{array}{l}< \\
< \\
< \\
< \\
<\end{array}$ & $\begin{array}{l}0.01 \\
0.01 \\
0.01 \\
0.01 \\
0.01 \\
0.01\end{array}$ & $\begin{array}{l}0.01 \\
0.01 \\
0.01 \\
0.01 \\
0.01 \\
0.01\end{array}$ & $\begin{array}{l}- \\
- \\
- \\
-\end{array}$ \\
\hline MOLYBDENUM & $\begin{array}{l}12 / 11 / 94 \\
12 / 19 / 94 \\
12 / 13 / 94 \\
12 / 13 / 94\end{array}$ & $\begin{array}{l}0001 \\
0002 \\
0003 \\
0004\end{array}$ & $M G / L$ & $\begin{array}{l}< \\
< \\
<\end{array}$ & $\begin{array}{l}0.01 \\
0.01 \\
0.01 \\
0.01\end{array}$ & $\begin{array}{l}0.01 \\
0.01 \\
0.01 \\
0.01\end{array}$ & : \\
\hline HOLYBDENUM (TOTAL) & $\begin{array}{l}06 / 30 / 93 \\
06 / 30 / 93 \\
06 / 30 / 93 \\
06 / 30 / 93\end{array}$ & $\begin{array}{l}\text { NOO1 } \\
\text { NOO2 } \\
\text { NOO3 } \\
\text { NOO4 }\end{array}$ & $M G / L$ & $\begin{array}{l}< \\
< \\
<\end{array}$ & $\begin{array}{l}0.01 \\
0.01 \\
0.01 \\
0.01\end{array}$ & $\begin{array}{l}0.01 \\
0.01 \\
0.01 \\
0.01\end{array}$ & $\begin{array}{l}- \\
- \\
-\end{array}$ \\
\hline HET GROSS ALPHA * & $\begin{array}{l}02 / 02 / 93 \\
02 / 02 / 93\end{array}$ & $\begin{array}{l}0001 \\
0002\end{array}$ & $\mathrm{PCI} / \mathrm{L}$ & & $\begin{array}{l}-0.34 \\
-0.34\end{array}$ & $\dot{-}$ & $\dot{-}$ \\
\hline HET GROSS ALPHA (TOTAL) ** & $\begin{array}{l}06 / 30 / 93 \\
06 / 30 / 93 \\
06 / 30 / 93 \\
06 / 30 / 93\end{array}$ & $\begin{array}{l}\text { NCO1 } \\
\text { NCO2 } \\
\text { NCO3 } \\
\text { NOO4 }\end{array}$ & PCI/L & & $\begin{array}{l}-0.34 \\
-0.34 \\
-0.34 \\
-0.34\end{array}$ & : & : \\
\hline HITRATE & $\begin{array}{l}02 / 02 / 93 \\
02 / 02 / 93\end{array}$ & $\begin{array}{l}0001 \\
0002\end{array}$ & $M G / L$ & $<$ & 1 & $\begin{array}{l}1 \\
1\end{array}$ & $\dot{-}$ \\
\hline MITRATE (TOTAL) & $06 / 30 / 93$ & NOO1 & MG/L & $<$ & 1 & 1 & - \\
\hline
\end{tabular}

- HET GROSS ALPHA (GROSS ALPHA - URANIUM) WITH 1 MG URAMIUH = 686 PCI

* HET GROSS ALPHA (TOTAL) (TOTAL GROSS ALPHA - TOTAL URANIUH)

PARAMETER VALUE INDICATOR (PVI): < - LESS THAN DETECTION LIMIT

SAMPLE ID COOES:

O001 - FILTERED SAMPLE (.45 MICRONS)

0002 - FILTERED REPLICATE SAMPLE (.45 MICRONS)

ODO3 - FILTERED REPLICATE SAMPLE (.45 MICRONS)

D004 - FILTERED REPLICATE SAMPLE (.45 MICRONS)

NOO1 - UNFILTERED SAMPLE

MOO2 - UAFILTERED REPLICATE SAMPLE

HOO3 - UNFILTERED REPLICATE SNMPLE

HOO4 - UMFILTERED REPLICATE SAMPLE 
SURFACE WATER QUALITY DATA BY LOCATION

SOWP ReV. 0 data

SITE: MONO1 MONUMENT VALLEY

LOCATION: 0999

NORTH COORDINATE: UNKNOWN

EAST COORDINATE: UNKNOWN

04/11/88 TO $12 / 14 / 94$

REPORT DATE: 06/22/95

\begin{tabular}{|c|c|c|c|c|c|c|c|c|}
\hline PARAMETER NAME & LOG DATE & $\begin{array}{c}\text { SAMPLE } \\
10\end{array}$ & $\begin{array}{l}\text { UNITS OF } \\
\text { MEASURE }\end{array}$ & PVI & $\begin{array}{l}\text { PARAMETER } \\
\text { VALUE }\end{array}$ & FLAGS & $\begin{array}{l}\text { DETECTION } \\
\text { LIMIT }\end{array}$ & $\begin{array}{l}\text { PARAMETER } \\
\text { UNCERTAIHTY }\end{array}$ \\
\hline $\begin{array}{c}\text { NITRATE (TOTAL) } \\
\text {. }\end{array}$ & $\begin{array}{l}06 / 30 / 93 \\
06 / 30 / 93 \\
06 / 30 / 93 \\
12 / 12 / 93 \\
12 / 12 / 93 \\
04 / 24 / 94 \\
04 / 24 / 94 \\
04 / 29 / 94 \\
04 / 29 / 94 \\
12 / 11 / 94 \\
12 / 11 / 94 \\
12 / 13 / 94 \\
12 / 13 / 94\end{array}$ & $\begin{array}{l}\text { NO02 } \\
\text { NOO3 } \\
\text { NOO4 } \\
\text { NO01 } \\
\text { NO02 } \\
\text { NO01 } \\
\text { NOO2 } \\
\text { NO03 } \\
\text { NOO4 } \\
\text { NO01 } \\
\text { NOO2 } \\
\text { NOO3 } \\
\text { NOO4 }\end{array}$ & $M G / L$ & $\begin{array}{l}< \\
< \\
< \\
< \\
< \\
< \\
< \\
< \\
< \\
< \\
< \\
< \\
<\end{array}$ & $\begin{array}{l}1 \\
1 \\
1 \\
1 \\
1 \\
1 \\
1 \\
1 \\
1 \\
1 \\
1 \\
1 \\
1\end{array}$ & 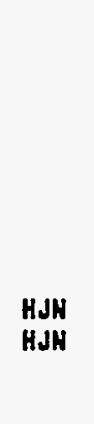 & $\begin{array}{l}1 \\
1 \\
1 \\
1 \\
1 \\
1 \\
1 \\
1 \\
1 \\
1 \\
1 \\
1 \\
1\end{array}$ & 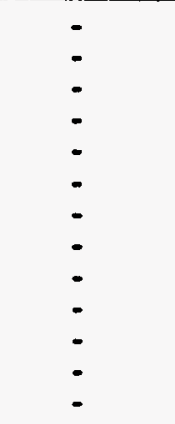 \\
\hline $\begin{array}{l}\text { PHOSPHATE (TOTAL) } \\
=\end{array}$ & $\begin{array}{l}06 / 30 / 93 \\
06 / 30 / 93 \\
06 / 30 / 93 \\
06 / 30 / 93 \\
12 / 13 / 94\end{array}$ & $\begin{array}{l}\text { NOO1 } \\
\text { NOO2 } \\
\text { NOO3 } \\
\text { NOO4 } \\
\text { NOO3 }\end{array}$ & HG/L & $\begin{array}{l}< \\
< \\
< \\
< \\
<\end{array}$ & $\begin{array}{l}0.1 \\
0.1 \\
0.1 \\
0.1 \\
0.1\end{array}$ & & $\begin{array}{l}0.1 \\
0.1 \\
0.1 \\
0.1 \\
0.1\end{array}$ & $\begin{array}{l}- \\
\dot{-} \\
\dot{-} \\
-\end{array}$ \\
\hline POTASSIUM & $\begin{array}{l}02 / 02 / 93 \\
02 / 02 / 93 \\
04 / 24 / 94 \\
04 / 24 / 94 \\
04 / 24 / 94 \\
04 / 24 / 94 \\
12 / 11 / 94 \\
12 / 11 / 94 \\
12 / 13 / 94 \\
12 / 13 / 94\end{array}$ & $\begin{array}{l}0001 \\
0002 \\
0001 \\
0002 \\
0003 \\
0004 \\
0001 \\
0002 \\
0003 \\
0004\end{array}$ & $M G / L$ & $\begin{array}{l}< \\
< \\
< \\
< \\
< \\
< \\
< \\
<\end{array}$ & $\begin{array}{l}0.1 \\
0.1 \\
0.1 \\
0.1 \\
0.1 \\
0.1 \\
0.1 \\
0.1 \\
0.1 \\
0.1\end{array}$ & & $\begin{array}{l}0.1 \\
0.1 \\
0.1 \\
0.1 \\
0.1 \\
0.1 \\
0.1 \\
0.1 \\
0.1 \\
0.1\end{array}$ & $\begin{array}{l}- \\
: \\
: \\
: \\
- \\
-\end{array}$ \\
\hline POTASSIUM (TOTAL) & $\begin{array}{l}06 / 30 / 93 \\
06 / 30 / 93 \\
06 / 30 / 93 \\
06 / 30 / 93 \\
12 / 12 / 93 \\
12 / 12 / 93\end{array}$ & $\begin{array}{l}\text { N001 } \\
\text { N002 } \\
\text { NO03 } \\
\text { NOO4 } \\
\text { NO01 } \\
\text { N002 }\end{array}$ & $M G / L$ & $\begin{array}{l}< \\
< \\
< \\
< \\
<\end{array}$ & $\begin{array}{l}0.1 \\
0.1 \\
0.1 \\
0.1 \\
0.1 \\
0.1\end{array}$ & & $\begin{array}{l}0.1 \\
0.1 \\
0.1 \\
0.1 \\
0.1 \\
0.1\end{array}$ & $\begin{array}{l}: \\
: \\
-\end{array}$ \\
\hline SILICA - SIOZ & $\begin{array}{l}12 / 11 / 94 \\
12 / 11 / 94 \\
12 / 13 / 94 \\
12 / 13 / 94\end{array}$ & $\begin{array}{l}0001 \\
0002 \\
0003 \\
0004\end{array}$ & $M G / L$ & $<$ & $\begin{array}{l}0.1 \\
0.2 \\
0.1 \\
0.1\end{array}$ & & $\begin{array}{l}0.1 \\
0.1 \\
0.1 \\
0.1\end{array}$ & $\therefore$ \\
\hline SILICA - SIO2 (TOTAL) & $\begin{array}{l}06 / 30 / 93 \\
06 / 30 / 93 \\
06 / 30 / 93 \\
06 / 30 / 93 \\
12 / 12 / 93 \\
12 / 12 / 93\end{array}$ & $\begin{array}{l}\text { NO01 } \\
\text { NO02 } \\
N 003 \\
N 004 \\
N 001 \\
N 002\end{array}$ & MG/L & $\begin{array}{l}< \\
< \\
< \\
< \\
<\end{array}$ & $\begin{array}{l}0.5 \\
0.5 \\
0.5 \\
0.5 \\
0.1 \\
0.1\end{array}$ & $\begin{array}{l}\mathbf{H} \\
\mathbf{H} \\
\mathbf{H} \\
\mathbf{H}\end{array}$ & $\begin{array}{l}0.5 \\
0.5 \\
0.5 \\
0.5 \\
0.1 \\
0.1\end{array}$ & $\begin{array}{l}: \\
: \\
: \\
-\end{array}$ \\
\hline
\end{tabular}

PARAMETER VALUE IMDICATOR (PVI): < - LESS THAN DETECTION LIMIT

SHMPLE ID COOES:

OTHER PARAMETER VALUE FLAGS:

ODO1 - FILTERED SNMPLE (.45 MICRONS)

0002 - FILTERED REPLICATE SAHPLE (.45 MICRONS)

0003 - FILTERED REPLICATE SAMPLE (.45 MICRONS)

0004 - FILTERED REPLICATE SAMPLE (.45 MICROHS)

$H$ - HOLD TIME EXPIRED
$J$ - ESTIMATED VALUE

H - SPIKE SAMPLE RECOVERY NOT WITHIN CONTROL LIMITS

NOOI - UMFILTERED SAMPLE

NOO2 - UNFILTERED REPLICATE SAMPLE

MOO3 - UAFILTERED REPLICATE SAMPLE

NOO4 - UNFILTERED REPLICATE SAMPLE 
SURFACE WATER QUALITY DATA BY LOCATIOH

SOWP REV. 0 data

SITE: HON01 MONUMENT VALLEY

LOCATIOH: 0999

NORTH COORDINATE: UNKNOWH

EAST COORDINATE: UNKNOWN

04/11/88 TO 12/14/94

REPORT DATE: $06 / 22 / 95$

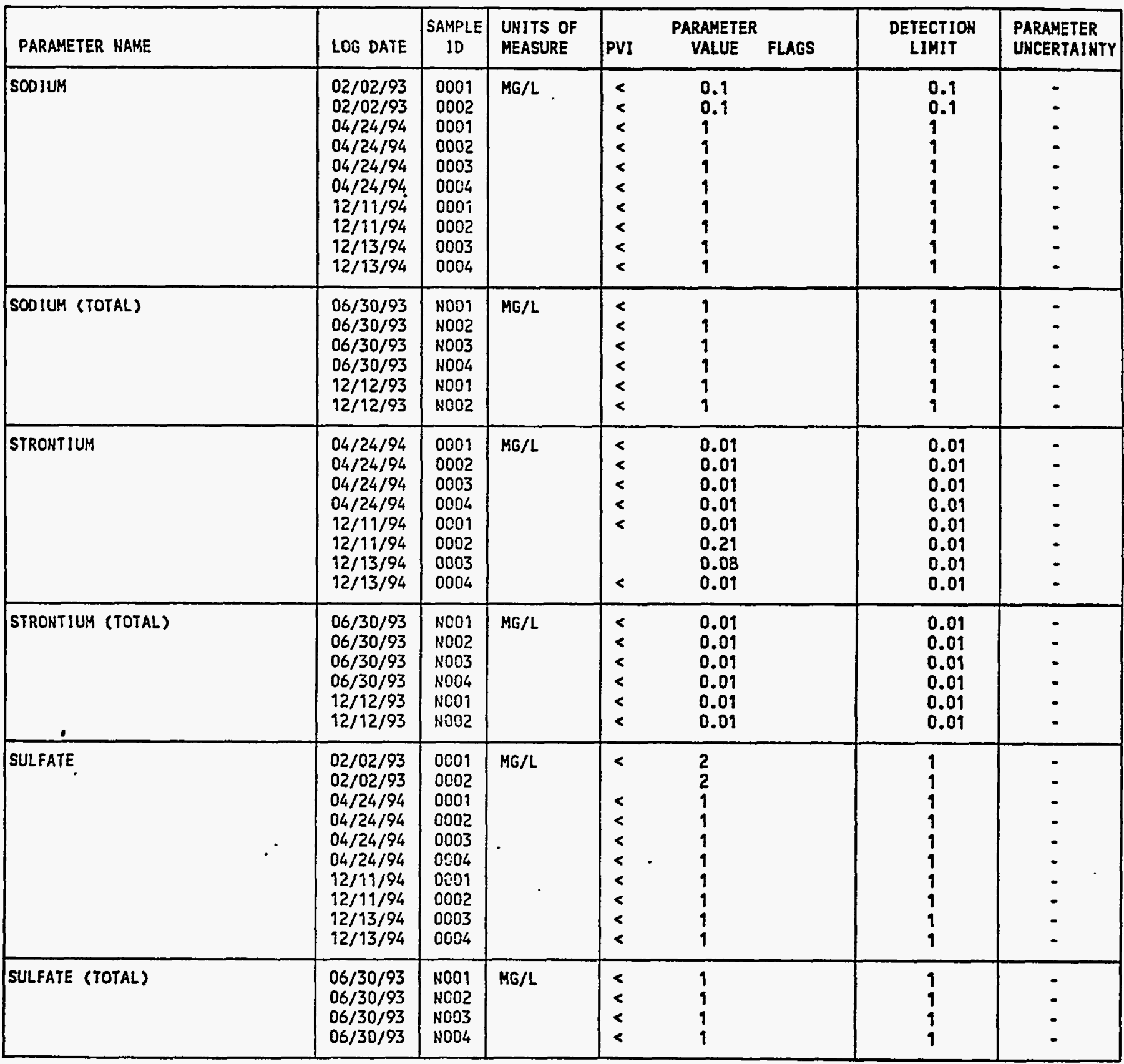

PARAMETER VALUE IHDICATOR (PVI): < - LESS THAN DETECTION LIMIT

SAMPLE 10 COOES:

0001 - FILTERED SAMPLE (.45 MICROHS)

0002 - FILTERED REPLICATE SAMPLE (.45 HICRONS)

0003 - FILTERED REPLICATE SNHPLE (.45 MICRONS)

0004 - FILTERED REPLICATE SAMPLE (.45 HICRONS)

NOD1 - UMFILTERED SNMPLE

NOO2 - UHFILTERED REPLICATE SAMPLE

NOO3 - UAFILTERED REPLICATE SAMPLE

NOO4 - UHFILTERED REPLICATE SAMPLE 
SURFACE WATER QUALITY DATA BY LOCATION

SOWP REV. 0 data

SITE: MONO1 MONUMENT VALLEY

LOCATION: 0999

NORTH COORDINATE: UNKNOWN

EAST COORDINATE: UNKNOWN

$04 / 11 / 88$ TO $12 / 14 / 94$

REPORT DATE: $06 / 22 / 95$

\begin{tabular}{|c|c|c|c|c|c|c|c|}
\hline PARAMETER NAME & LOG DATE & $\begin{array}{c}\text { SAMPLE } \\
\text { ID }\end{array}$ & $\begin{array}{l}\text { UNITS OF } \\
\text { MEASURE }\end{array}$ & PVI & $\begin{array}{l}\text { PARAMETER } \\
\text { VALUE FLAGS }\end{array}$ & $\begin{array}{l}\text { DETECTIOH } \\
\text { LIMIT }\end{array}$ & $\begin{array}{l}\text { PARAMETER } \\
\text { UHCERTAINTY }\end{array}$ \\
\hline SULFATE (TOTAL) & $\begin{array}{l}12 / 12 / 93 \\
12 / 12 / 93\end{array}$ & $\begin{array}{l}\text { N001 } \\
\text { N002 }\end{array}$ & $\mathrm{MG} / \mathrm{L}$ & $<$ & $\begin{array}{l}1 \\
1\end{array}$ & 1 & 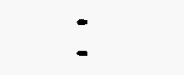 \\
\hline TOTAL DISSOLVED SOLIDS & $\begin{array}{l}02 / 02 / 93 \\
02 / 02 / 93 \\
04 / 24 / 94 \\
04 / 24 / 94 \\
04 / 24 / 94 \\
04 / 24 / 94 \\
12 / 11 / 94 \\
12 / 11 / 94 \\
12 / 13 / 94 \\
12 / 13 / 94\end{array}$ & $\begin{array}{l}0001 \\
0002 \\
0001 \\
0002 \\
0003 \\
0004 \\
0001 \\
0002 \\
0003 \\
0004\end{array}$ & MG/L & $\begin{array}{l}< \\
< \\
< \\
< \\
< \\
< \\
< \\
< \\
<\end{array}$ & $\begin{array}{l}10 \\
10 \\
10 \\
10 \\
10 \\
10 \\
10 \\
10 \\
10 \\
10\end{array}$ & $\begin{array}{l}10 \\
10 \\
10 \\
10 \\
10 \\
10 \\
10 \\
10 \\
10 \\
10\end{array}$ & $\begin{array}{l}: \\
: \\
: \\
: \\
- \\
- \\
-\end{array}$ \\
\hline TOTAL DISSOLVED SOLIDS (TOTAL) & $\begin{array}{l}06 / 30 / 93 \\
06 / 30 / 93 \\
06 / 30 / 93 \\
06 / 30 / 93 \\
12 / 12 / 93 \\
12 / 12 / 93\end{array}$ & $\begin{array}{l}\text { NOO1 } \\
\text { NOO2 } \\
\text { NCO3 } \\
\text { NOO4 } \\
\text { NO01 } \\
\text { NOC2 }\end{array}$ & $M G / L$ & $\begin{array}{l}< \\
< \\
< \\
< \\
<\end{array}$ & $\begin{array}{l}10 \\
10 \\
10 \\
10 \\
10 \\
10\end{array}$ & $\begin{array}{l}10 \\
10 \\
10 \\
10 \\
10 \\
10\end{array}$ & $\begin{array}{l}: \\
: \\
:\end{array}$ \\
\hline URANIUM & $\begin{array}{l}02 / 02 / 93 \\
02 / 02 / 93 \\
04 / 24 / 94 \\
04 / 24 / 94 \\
04 / 24 / 94 \\
04 / 24 / 94 \\
12 / 11 / 94 \\
.12 / 11 / 94\end{array}$ & $\begin{array}{l}0001 \\
0002 \\
0001 \\
0002 \\
0003 \\
0004 \\
0001 \\
0002\end{array}$ & MG/L & $\begin{array}{l}< \\
< \\
< \\
< \\
< \\
< \\
<\end{array}$ & $\begin{array}{l}0.001 \\
0.001 \\
0.001 \\
0.001 \\
0.001 \\
0.001 \\
0.001 \\
0.001\end{array}$ & $\begin{array}{l}0.001 \\
0.001 \\
0.001 \\
0.001 \\
0.001 \\
0.001 \\
0.001 \\
0.001\end{array}$ & $\begin{array}{l}: \\
: \\
: \\
: \\
-\end{array}$ \\
\hline URANIUM (TOTAL) & $\begin{array}{l}06 / 30 / 93 \\
06 / 30 / 93 \\
06 / 30 / 93 \\
06 / 30 / 93 \\
12 / 12 / 93 \\
12 / 12 / 93\end{array}$ & $\begin{array}{l}\text { NOO1 } \\
\text { NOC2 } \\
\text { NOO3 } \\
\text { NOO4 } \\
\text { NOO1 } \\
\text { NOO2 }\end{array}$ & MG/L & $\begin{array}{l}< \\
< \\
< \\
< \\
<\end{array}$ & $\begin{array}{l}0.001 \\
0.001 \\
0.001 \\
0.001 \\
0.001 \\
0.001\end{array}$ & $\begin{array}{l}0.001 \\
0.001 \\
0.001 \\
0.001 \\
0.001 \\
0.001\end{array}$ & $\begin{array}{l}: \\
: \\
: \\
-\end{array}$ \\
\hline VANADIUM & $\begin{array}{l}04 / 24 / 94 \\
04 / 24 / 94 \\
04 / 24 / 94 \\
04 / 24 / 94 \\
12 / 11 / 94 \\
12 / 11 / 94 \\
12 / 13 / 94 \\
12 / 13 / 94\end{array}$ & $\begin{array}{l}0001 \\
0002 \\
0003 \\
0004 \\
0001 \\
0002 \\
0003 \\
0004\end{array}$ & MG/L & $\begin{array}{l}< \\
< \\
< \\
< \\
< \\
< \\
< \\
<\end{array}$ & $\begin{array}{l}0.01 \\
0.01 \\
0.01 \\
0.01 \\
0.01 \\
0.01 \\
0.01 \\
0.01\end{array}$ & $\begin{array}{l}0.01 \\
0.01 \\
0.01 \\
0.01 \\
0.01 \\
0.09 \\
0.01 \\
0.01\end{array}$ & $\begin{array}{l}- \\
- \\
: \\
- \\
-\end{array}$ \\
\hline VANADIUM (TOTAL) & $\begin{array}{l}06 / 30 / 93 \\
06 / 30 / 93 \\
06 / 30 / 93\end{array}$ & $\begin{array}{l}\text { NOE1 } \\
\text { N002 } \\
\text { N003 }\end{array}$ & $M G / L$ & $<$ & $\begin{array}{l}0.01 \\
0.01 \\
0.01\end{array}$ & $\begin{array}{l}0.01 \\
0.01 \\
0.01\end{array}$ & - \\
\hline
\end{tabular}

PARAMETER VALUE INDICATOR (PVI): < - LESS THAN DETECTION LIMIT

OTHER PARAMETER VALUE FLAGS:

H - HOLD TIME EXPIRED, VALUE SUSPECT
SAMPLE ID COOES:

0001 - FILTERED SNMPLE (.45 MICRONS)

O0O2 - FILTERED REPLICATE SAMPLE (.45 MICRONS)

0003 - FILTERED REPLICATE SAMPLE (.45 MICROHS)

0004 - FILTERED REPLICATE SAMPLE (.45 MICROHS)

NDO1 - UHFILTERED SAMPLE

NOD2 - UNFILTERED REPLICATE SAMPLE

MO03 - UNFILTERED REPLICATE SAMPLE

NOO4 - UNFILTERED REPLICATE SAMPLE 
- SURFACE WATER QUALITY DATA BY LOCATION

SOWP REV. $O$ data

SITE: HONO1 MONUMENT VALLEY

LOCATION: 0999

NORTH COORDINATE: UNKNOWN

EAST COORDINATE: UNKNOWN

04/11 1/88 TO $12 / 14 / 94$

REPORT DATE: $06 / 22 / 95$

\begin{tabular}{|c|c|c|c|c|c|c|c|}
\hline PARAMETER MAME & LOG DATE & $\begin{array}{c}\text { SAMPLE } \\
\text { ID }\end{array}$ & $\begin{array}{l}\text { UNITS OF } \\
\text { MEASURE }\end{array}$ & PVI & $\begin{array}{l}\text { PARAMETER } \\
\text { VALUE FLAGS }\end{array}$ & $\begin{array}{l}\text { DETECTION } \\
\text { LIHIT }\end{array}$ & $\begin{array}{l}\text { PARAMETER } \\
\text { UNCERTAINTY }\end{array}$ \\
\hline VANADIUH (TOTAL) & $\begin{array}{l}06 / 30 / 93 \\
12 / 12 / 93 \\
12 / 12 / 93\end{array}$ & $\begin{array}{l}\text { HoO } \\
\text { NoO1 } \\
\text { HoO2 }\end{array}$ & MG/L & $\begin{array}{l}< \\
<\end{array}$ & $\begin{array}{l}0.01 \\
0.01 \\
0.01\end{array}$ & $\begin{array}{l}0.01 \\
0.01 \\
0.01\end{array}$ & - \\
\hline ZIHC & $\begin{array}{l}12 / 13 / 94 \\
12 / 13 / 94\end{array}$ & $\begin{array}{l}0003 \\
0004\end{array}$ & MG/L & $<$ & $\begin{array}{l}0.05 \\
0.05\end{array}$ & $\begin{array}{l}0.05 \\
0.05\end{array}$ & - \\
\hline ZINC (TOTAL) & $\begin{array}{l}06 / 30 / 93 \\
06 / 30 / 93 \\
06 / 30 / 93 \\
06 / 30 / 93\end{array}$ & $\begin{array}{l}\text { NoO1 } \\
N 002 \\
N 003 \\
N 004\end{array}$ & HG/L & & $\begin{array}{l}0.014 \\
0.010 \\
0.112 \\
0.022\end{array}$ & $\begin{array}{l}0.005 \\
0.005 \\
0.005 \\
0.005\end{array}$ & $\begin{array}{l}- \\
- \\
-\end{array}$ \\
\hline
\end{tabular}

PARAMETER VALUE IHDICATOR (PVI): < - LESS THAN DETECTION LIMIT

SAMPLE ID CODES:

DO03 - FILTERED REPLICATE SAMPLE (.45 MICRONS)

0004 - FILTERED REPLICATE SNMPLE (.45 MICRONS)

NOO1 - UHFILTERED SAMPLE

HOOZ - UNFILTERED REPLICATE SAMPLE

NOO3 - UNFILTERED REPLICATE SAMPLE

NOO4 - UNFILTERED REPLICATE SAMPLE

DATA FILE NAME: IDARTIMON01 ISWQ10002.DAT 



\section{SOIL CHEMISTRY DATA BY LOCATION}

SOUP ReV. 0 dato

SITE: MONO1 MONUMENT VALLEY

LOCATION: 0620

NORTH COORDINATE: 70685.0 FT

EAST COORDINATE: $\quad 90918.8$ FT

06/28/93 TO 07/01/93

REPORT DATE: 06/22/195

\begin{tabular}{|c|c|c|c|c|c|c|c|c|c|c|}
\hline PARAMETER NAME & LOOG OATE| & SAMP & $\begin{array}{l}\text { DEPTH RANGE } \\
\text { (FT) }\end{array}$ & $\begin{array}{l}\text { DIG. } \\
\text { CODE }\end{array}$ & $\begin{array}{l}\text { SAMP } \\
\text { DESC }\end{array}$ & $\begin{array}{l}\text { UHITS OF } \\
\text { MEASURE }\end{array}$ & PVI & $\begin{array}{l}\text { ARAMETER } \\
\text { VALUE FLAGS }\end{array}$ & $\begin{array}{l}\text { DETECTION } \\
\text { LIMIT }\end{array}$ & $\begin{array}{l}\text { PARAMETER } \\
\text { UMCERT. }\end{array}$ \\
\hline$\%$ SOLIDS & $06 / 30 / 93$ & NO01 & - & & & $\%$ & & 66.9 & 0.1 & - \\
\hline ARSEHIC (TOTAL) & $06 / 30 / 93$ & NO01 & - & & & HG/KG & & 4. & 2. & - \\
\hline NITRATE (TOTAL) & $\mid 06 / 30 / 93$ & N001 & - & & & MG/KG & & 0.9 & 0.1 & - \\
\hline STRONTIUM (TOTAL) & $06 / 30 / 93$ & NO01 & - & & & MG/KG & & 28. & 1. & - \\
\hline SULFATE (TOTAL) & $06 / 30 / 93$ & N001 & - & & & MG/KG & & 48. & 10. & - \\
\hline URANIUM (TOTAL) & $06 / 30 / 93$ & N001 & - & & & $M G / K G$ & & 1.0 & 1.0 & - \\
\hline VANADIUM (TOTAL) & $|06 / 30 / 93|$ & NOO1 & - & & & MG/KG & & 9. & 1. & - \\
\hline ZINC (TOTAL) & $\mid 06 / 30 / 93$ & N001 & - & & & $M G / K G$ & & 18.7 & 0.5 & - \\
\hline
\end{tabular}

PARAMETER VALUE IHDICATOR (PVI): < - LESS THAN DETECTION LIMIT

SAMPLE DIGESTION TYPE CODES:

SAMPLE DESCRIPTIOH COOES: 
SOIL. CHEMISTRY DATA BY LOCATION

SOWP ReV. $O$ dato

S1TE: MONO1 MONUMENT VALLEY

LOCATION: 0623 RESERVED FOR RPAPUSCH

NORTH COORDINATE: UNKNOWN

EAST COORDINATE: UNKNOWN

06/28/93 TO 07/01/93

REPORT DATE: $06 / 22 / 95$

\begin{tabular}{|c|c|c|c|c|c|c|c|c|c|c|}
\hline PARAMETER NAME & LOG DATE & $\begin{array}{c}\text { SAMP } \\
\text { ID }\end{array}$ & $\begin{array}{l}\text { DEPTH RANGE } \\
\text { (FT) }\end{array}$ & $\left|\begin{array}{l}\text { DIG. } \\
\text { CODE }\end{array}\right|$ & $\begin{array}{l}\text { SAMP } \\
\text { DESC }\end{array}$ & $\begin{array}{l}\text { UNITS OF } \\
\text { MEASURE }\end{array}$ & PVI & $\begin{array}{l}\text { PARAMETER } \\
\text { VALUE FLAGS }\end{array}$ & $\begin{array}{l}\text { DETECTION } \\
\text { LIMIT }\end{array}$ & $\begin{array}{l}\text { PARAMETER } \\
\text { UNCERT. }\end{array}$ \\
\hline$\%$ SOLIDS & $06 / 28 / 93$ & NOO1 & - & & & $\%$ & & 64.5 & 0.1 & - \\
\hline ARSENIC (TOTAL) & $06 / 28 / 93$ & N001 & - & & & MG/KG & & 0.9 & 0.5 & - \\
\hline NITRATE (TOTAL) & $06 / 28 / 93$ & N001 & - & & & MG/KG & & 1.8 & 0.1 & - \\
\hline STRONTIUM (TOTAL) & $06 / 28 / 93$ & NO01 & - & & & MG/KG & & 116. & 1. & - \\
\hline SULFATE (TOTAL) & $06 / 28 / 93$ & N001 & - & & & MG/KG & & 771. & 250. & - \\
\hline URANIUM (TOTAL) & $06 / 28 / 93$ & N001 & - & & & MG/KG & $<$ & 1.0 & 1.0 & - \\
\hline VANADIUM (TOTAL) & $06 / 28 / 93$ & N001 & - & & & $M G / K G$ & & 6. & 1. & - \\
\hline ZINC (TOTAL) & $06 / 28 / 93$ & N001 & - & & & MG/KG & & 10.7 & 0.5 & - \\
\hline
\end{tabular}

PARAMETER VALUE INDICATOR (PVI): < - LESS THAN DETECTION LIMIT

SAMPLE DIGESTION TYPE CODES:

SAMPLE DESCRIPTION CODES:

$-$ 
SOIL CHEMISTRY DATA BY LOCATION

SOMP Rev. 0 dato

SITE: MONO1 MONUNEHT VALLEY

LOCATION: 0626 RESERVED FOR RPAPUSCH

NORTH COORDINATE: UNKHOWN

EAST COORDINATE: UNKNOWH

06/28/93 TO 07/01/93

REPORT DATE: 06/22/95

\begin{tabular}{|c|c|c|c|c|c|c|c|c|c|c|}
\hline PARAMETER NAME & LOG DATE & $\begin{array}{c}\text { SAMP } \\
\text { ID }\end{array}$ & $\begin{array}{l}\text { DEPTH RANGE } \\
\text { (FT) }\end{array}$ & $\begin{array}{l}\text { DIG. } \\
\text { CODE }\end{array}$ & $\begin{array}{l}\text { SAMP } \\
\text { DESC }\end{array}$ & $\begin{array}{l}\text { UNITS OF } \\
\text { MEASURE }\end{array}$ & PVI & $\begin{array}{l}\text { PARAMETER } \\
\text { VALUE }\end{array}$ & $\begin{array}{l}\text { DETECTION } \\
\text { LIMIT }\end{array}$ & $\begin{array}{l}\text { PARAMETER } \\
\text { UNCERT. }\end{array}$ \\
\hline \% SOLIDS & $06 / 28 / 93$ & NOO1 & - & & & $x$ & & 76.8 & 0.1 & $\cdot$ \\
\hline ARSENIC (TOTAL) & $06 / 28 / 93$ & N001 & - & & & MG/KG & & 0.8 & 0.5 & - \\
\hline NITRATE (TOTAL) & $06 / 28 / 93$ & N001 & - & & & $M G / K G$ & & 0.4 & 0.1 & - \\
\hline STRONTIUM (TOTAL) & $06 / 28 / 93$ & NOO1 & - & & & MG/KG & & 26. & 1. & - \\
\hline SULFATE (TOTAL) & $06 / 28 / 93$ & N001 & - & & & $M G / K G$ & & 42. & 10. & - \\
\hline URANIUM (TOTAL) & $06 / 28 / 93$ & N001 & . - & & & $M G / K G$ & $<$ & 1.0 & 1.0 & $\cdot$ \\
\hline VANADIUM (TOTAL) & $06 / 28 / 93$ & No01 & $\cdot$ & & & $M G / K G$ & & 5. & 1. & $\cdot$ \\
\hline Z̈INC (TOTAL) & $06 / 28 / 93$ & Np01 & - & & & $M G / K G$ & & $6.8^{\circ}$ & 0.5 & $\cdot$ \\
\hline
\end{tabular}

PARAMETER VALUE INDICATOR (PVI): < - LESS THAN DETECTION LIMIT

SAMPLE DIGESTION TYPE CODES:

SAMPLE DESCRIPTION COOES: 
SOIL CHEMISTRY DATA BY LOCATIOM

SOWP Rev. 0 data

SITE: MONO1 MONUMENT VALLEY

LOCATION: 0624 RESERVED FOR RPAPUSCH

NORTH COORDINATE: UNKNOWN

EAST COORDINATE: UNKNOWN

$06 / 28 / 93$ TO $07 / 01 / 93$

REPORT DATE : 06/22/95

\begin{tabular}{|c|c|c|c|c|c|c|c|c|c|c|}
\hline PARAMETER HAME & LOG DATE & $\begin{array}{c}\text { SAMP } \\
10\end{array}$ & $\begin{array}{l}\text { DEPTH RANGE } \\
\text { (FT) }\end{array}$ & DIG. & $\begin{array}{l}\text { SAMP } \\
\text { DESC }\end{array}$ & $\begin{array}{l}\text { UNITS OF } \\
\text { MEASURE }\end{array}$ & PVI & $\begin{array}{l}\text { PARAMETER } \\
\text { VALUE FLAGS }\end{array}$ & $\begin{array}{l}\text { DETECTION } \\
\text { LIMIT }\end{array}$ & $\begin{array}{l}\text { PARAMETER } \\
\text { UNCERT. }\end{array}$ \\
\hline$\%$ SOLIDS & $06 / 28 / 93$ & N001 & - & & & $\%$ & & 76.2 & 0.1 & - \\
\hline ARSENIC (TOTAL) & $06 / 28 / 93$ & N001 & - & & & MG/KG & & 0.6 & 0.5 & - \\
\hline NITRATE (TOTAL) & $06 / 28 / 93$ & Noo1 & - & & & $M G / K G$ & & 3.8 & 0.1 & - \\
\hline STRONTIUM (TOTAL) & $06 / 28 / 93$ & N001 & - & & & MG/KG & & 46. & 1. & - \\
\hline SULFATE (TOTAL) & $06 / 28 / 93$ & N001 & - & & & $M G / K G$ & & 935. & 250. & - \\
\hline URANIUM (TOTAL) & $06 / 28 / 93$ & NO01 & - & & & $\mathrm{MG} / \mathrm{KG}$ & $<$ & 1.0 & 1.0 & - \\
\hline VANADIUM (TOTAL) & $06 / 28 / 93$ & NOO1 & - & & & MG/KG & & 3. & 1. & $\cdot$ \\
\hline ZINC (TOTAL) & $06 / 28 / 93$ & N001 & - & & & MG/KG & & 10.8 & 0.5 & - \\
\hline
\end{tabular}

PARAMETER VALUE INDICATOR (PVI): < - LESS THAN OETECTION LIMIT

SAMPLE DIGESTION TYPE CODES:

SAMPLE DESCRIPTION COOES: 
SOIL CHEMISTRY DATA BY LOCATIOH

SOWP ReY. 0 dato

SITE: MOHO1 MONUMENT VALLEY

SITE: MONOT WONUMENT VALLEY PPAPUSCH

NORTH COORDINATE: UNKNOWN

EAST COORDINATE: UNKNOWN

$06 / 28 / 93$ TO $07 / 01 / 93$

REPORT DATE: $06 / 22 / 95$

\begin{tabular}{|c|c|c|c|c|c|c|c|c|c|c|}
\hline PARAMETER NAME & LOG DATE & $\begin{array}{l}\text { SAMP } \\
\text { ID }\end{array}$ & $\begin{array}{l}\text { DEPTH RANGE } \\
\text { (FT) }\end{array}$ & $\begin{array}{l}\text { DIG. } \\
\text { CODE }\end{array}$ & $\begin{array}{l}\text { SAMP } \\
\text { DESC }\end{array}$ & $\begin{array}{l}\text { UNITS OF } \\
\text { MEASURE }\end{array}$ & PVI & $\begin{array}{l}\text { ARAMETER } \\
\text { VALUE FLAGS }\end{array}$ & $\begin{array}{l}\text { DETECTION } \\
\text { LIMIT }\end{array}$ & $\begin{array}{l}\text { PARAMETER } \\
\text { UNCERT. }\end{array}$ \\
\hline$\%$ SOLIOS & $07 / 01 / 93$ & N001 & - & & & $\%$ & & 68.2 & 0.1 & - \\
\hline ARSENIC (TOTAL) & $07 / 01 / 93$ & N001 & - & & & MG/KG & & 0.9 & 0.5 & - \\
\hline NITRATE (TOTAL) & |07/01/93 & N001 & - & & & MG/KG & & 0.9 & 0.1 & - \\
\hline STRONTIUM (TOTAL) & 07/01/93| & N001 & - & & & |MG/KG & & 54. & 1. & - \\
\hline SULFATE (TOTAL) & 07/01/93 & N001 & - & & & MG/KG & & 35. & 10. & $=$ \\
\hline URANIUM (TOTAL) & $06 / 30 / 93$ & NOOI & - & & & MG/KG & $<$ & 1.0 & 1.0 & - \\
\hline VANADIUM (TOTAL) & 07/01/93 & N001 & - & & & MG/KG & & 9. & 1. & - \\
\hline ZINC (TOTAL) & $07 / 01 / 93$ & N001 & - & & & MG/KG & & 29.2 & 0.5 & - \\
\hline
\end{tabular}

PARAMETER VALUE INDICATOR (PVI): < - LESS THAN DETECTION LIMIT

SAMPLE DIGESTION TYPE CODES:

SAMPLE DESCRIPTION COOES:

DATA FILE NAME: IDARTIMONO1ISCI10000.DAT 
$\therefore$ 
GROUHDHATER OUALITY DATA BY LOCATION

SOMP Rev. O Data

SITE: MONOI MONUMENT VALLEY

LOCATION: 0601

NORTH COORDINATE: 54980.4 FT

EAST COORDINATE: 88017.7 FT

$04 / 21 / 88$ TO $12 / 13 / 94$

REPORT DATE: $06 / 20 / 95$

FORMATIOH OF COMPLETION: SHIHARUAP MEMBER OF THE CHINLE FORMATION (SR) HYORAULIC FLON RELATIOHSHIP: UPGRADIENT (U)

\begin{tabular}{|c|c|c|c|c|c|c|c|c|}
\hline PARAMETER MAME & LOS DATE & $\begin{array}{c}\text { SAMPLE } \\
10\end{array}$ & $\begin{array}{l}\text { UNITS OF } \\
\text { MEASURE }\end{array}$ & PVI & $\begin{array}{l}\text { PARAMETER } \\
\text { VALUE }\end{array}$ & FLAGS & $\begin{array}{l}\text { DETECTIOH } \\
\text { LIMIT }\end{array}$ & $\begin{array}{l}\text { PARAMETER } \\
\text { UNCERTAIHTY }\end{array}$ \\
\hline ALKALIKITY & $\begin{array}{l}05 / 31 / 91 \\
08 / 11 / 92 \\
11 / 22 / 92 \\
06 / 29 / 93\end{array}$ & $\begin{array}{l}0001 \\
0001 \\
0001 \\
\text { N001 }\end{array}$ & $\mathrm{MG} / \mathrm{L} \mathrm{CACO} 3$ & & $\begin{array}{l}280 . \\
302 \\
253 \\
281\end{array}$ & $\mathbf{L}$ & : & $\begin{array}{l}- \\
- \\
-\end{array}$ \\
\hline ALUMIRUA & $\begin{array}{l}05 / 31 / 91 \\
02 / 23 / 92 \\
08 / 11 / 92\end{array}$ & $\begin{array}{l}0001 \\
0001 \\
0001\end{array}$ & MG/L & $\begin{array}{l}< \\
<\end{array}$ & $\begin{array}{l}0.05 \\
0.07 \\
0.2\end{array}$ & $L$ & $\begin{array}{l}0.05 \\
0.05 \\
0.2\end{array}$ & : \\
\hline ANTONILM & $\begin{array}{l}05 / 31 / 91 \\
02 / 23 / 92\end{array}$ & $\begin{array}{l}0001 \\
0001\end{array}$ & HG/L & $<$ & $\begin{array}{l}0.2 \\
0.1\end{array}$ & L & $\begin{array}{l}0.1 \\
0.1\end{array}$ & - \\
\hline AMONIUM (TOTAL) & $06 / 29 / 93$ & NoOI & $M G / L$ & $<$ & 0.1 & & 0.1 & - \\
\hline AKT IMONY & $\begin{array}{l}05 / 31 / 91 \\
02 / 23 / 92 \\
08 / 11 / 92\end{array}$ & $\begin{array}{l}0001 \\
0001 \\
0001\end{array}$ & $M G / L$ & $<$ & $\begin{array}{l}0.003 \\
0.004 \\
0.06\end{array}$ & L & $\begin{array}{l}0.003 \\
0.003 \\
0.06\end{array}$ & : \\
\hline ARSENIC & $\begin{array}{l}05 / 31 / 91 \\
02 / 23 / 92 \\
08 / 91 / 92 \\
11 / 22 / 92 \\
06 / 29 / 93\end{array}$ & $\begin{array}{l}0001 \\
0001 \\
0001 \\
0001 \\
0001\end{array}$ & $M G / L$ & $\begin{array}{l}< \\
< \\
< \\
< \\
<\end{array}$ & $\begin{array}{l}0.01 \\
0.01 \\
0.01 \\
0.0015 \\
0.005\end{array}$ & L & $\begin{array}{l}0.01 \\
0.01 \\
0.01 \\
0.0015 \\
0.005\end{array}$ & $\begin{array}{l}- \\
- \\
- \\
-\end{array}$ \\
\hline ARSEHIC (TOTAL) & $\begin{array}{l}11 / 22 / 92 \\
06 / 29 / 93\end{array}$ & $\begin{array}{l}\text { N001 } \\
\text { NOO1 }\end{array}$ & $M G / L$ & $<$ & $\begin{array}{l}0.0015 \\
0.017\end{array}$ & + & $\begin{array}{l}0.0015 \\
0.005\end{array}$ & $\dot{-}$ \\
\hline BARIUM & $\begin{array}{l}05 / 31 / 91 \\
02 / 23 / 92 \\
08 / 11 / 92 \\
11 / 22 / 92 \\
06 / 29 / 93\end{array}$ & $\begin{array}{l}0001 \\
0001 \\
0001 \\
0001 \\
0001\end{array}$ & MG/L & $<$ & $\begin{array}{l}0.04 \\
0.04 \\
0.2 \\
0.052 \\
0.1\end{array}$ & $\mathbf{L}$ & $\begin{array}{l}0.01 \\
0.01 \\
0.2 \\
0.0015 \\
0.1\end{array}$ & $\begin{array}{l}- \\
- \\
-\end{array}$ \\
\hline BARIUM (TOTAL) & $\begin{array}{l}11 / 22 / 92 \\
06 / 29 / 93\end{array}$ & $\begin{array}{l}\text { HOO1 } \\
\text { HOOI }\end{array}$ & $M G / L$ & & $\begin{array}{l}0.19 \\
2.0\end{array}$ & & $\begin{array}{l}0.0015 \\
0.1\end{array}$ & $\dot{-}$ \\
\hline BERYLLIUM & $\begin{array}{l}05 / 31 / 91 \\
08 / 11 / 92\end{array}$ & $\begin{array}{l}0001 \\
0001\end{array}$ & MG/L & $<$ & $\begin{array}{l}0.005 \\
0.005\end{array}$ & $L$ & $\begin{array}{l}0.005 \\
0.005\end{array}$ & $\overline{-}$ \\
\hline BORON & $05 / 31 / 91$ & 0001 & MG/L & & 0.05 & $\mathbf{L}$ & 0.05 & - \\
\hline BROMIDE & $05 / 31 / 91$ & 0001 & $M G / L$ & $<$ & 0.1 & $L$ & 0.1 & - \\
\hline CADMIUN & $\begin{array}{l}05 / 31 / 91 \\
02 / 23 / 92 \\
08 / 11 / 92 \\
11 / 22 / 92\end{array}$ & $\begin{array}{l}0001 \\
0001 \\
0001 \\
0001\end{array}$ & MG/L & $\begin{array}{l}< \\
< \\
< \\
<\end{array}$ & $\begin{array}{l}0.001 \\
0.0005 \\
0.005 \\
0.00013\end{array}$ & L & $\begin{array}{l}0.001 \\
0.0005 \\
0.005 \\
0.00013\end{array}$ & $\begin{array}{l}- \\
-\end{array}$ \\
\hline CADMIUY (TOTAL) & $11 / 22 / 92$ & Noo1 & MG/L & & 0.00030 & $H$ & 0.00013 & - \\
\hline CALCIUA & $05 / 31 / 91$ & 0001 & $M E / L$ & & 29.9 & $\mathbf{L}$ & 0.5 & - \\
\hline
\end{tabular}

PARAYETER VALUE IMDICATOR (PVI): < - LESS THAN DETECTION LIMIT

OTHER PARAMETER VALUE FLAGS:

- DUPLICATE ANALYSIS NOT WITHIN CONTROL LIHITS

SAMPLE ID COOES:

OOO1 - FILTERED SAMPLE (.45 MICRONS)

+ - CORRELATIOH COEFFICIENT FOR MSA < 0.995

E - ESTIMATED VALUE BECAUSE OF INTERFERENCE, SEE CASE MARRATIVE

$L$ - LESS THAH THREE BORE VOLLHES REMOVED BEFORE SAMPLIHG

N - SPIKE SAMPLE RECOVERY NOT WITHIN CONTROL LIMITS

- $H$ - POST-DIGEST SPIKE OUT OF CHTR LIM HHILE SAMP ABS < 50\% SPIKE 
GROUMDWATER QUALITY DATA BY LOCATION

SOMP ReV. O Data

SITE: MONOY MONUMENT VALLEY

LOCATION: 0601

NORTH COORDINATE: $\quad 54980.4$ FT

EAST COORDINATE: 88017.7 FT

$04 / 21 / 88$ TO $12 / 13 / 94$

REPORT DATE: 06/20/95

FORMATION OF COMPLETION: SHIMARLAP MEMBER OF THE CHIHLE FORMATION (SR)

HYDRAULIC FLON RELATIONSHIP: UPGRADIENT (U)

\begin{tabular}{|c|c|c|c|c|c|c|c|c|}
\hline PARAMETER MAME & LOG DATE & $\underset{\text { SAMPLE }}{\text { SD }}$ & $\begin{array}{l}\text { UNITS OF } \\
\text { MEASURE }\end{array}$ & PVI & $\begin{array}{l}\text { PARAMETER } \\
\text { VALUE }\end{array}$ & FLAGS & $\begin{array}{l}\text { DETECTION } \\
\text { LIMIT }\end{array}$ & $\begin{array}{l}\text { PARAMETER } \\
\text { UNCERTAINTY }\end{array}$ \\
\hline CALCIUN & $\begin{array}{l}02 / 23 / 92 \\
08 / 11 / 92 \\
06 / 29 / 93\end{array}$ & $\begin{array}{l}0001 \\
0001 \\
0001\end{array}$ & MG/L & & $\begin{array}{l}27.4 \\
29 \\
27.8\end{array}$ & & $\begin{array}{l}0.5 \\
5 \\
0.5\end{array}$ & : \\
\hline CALCIUN (TOTAL) & $06 / 29 / 93$ & NoO1 & MG/L & & 318 & & 0.5 & - \\
\hline CHLORIDE & $\begin{array}{l}05 / 31 / 91 \\
02 / 23 / 92 \\
08 / 11 / 92\end{array}$ & $\begin{array}{l}0001 \\
0001 \\
0001\end{array}$ & $M G / L$ & & $\begin{array}{l}15.2 \\
15.1 \\
14\end{array}$ & $\mathbf{L}$ & $\begin{array}{l}0.5 \\
0.5 \\
0.016\end{array}$ & $\dot{-}$ \\
\hline CHLORIDE (TOTAL) & $06 / 29 / 93$ & H001 & MG/L & & 14.8 & & 0.5 & - \\
\hline CHROMIU:A & $\begin{array}{l}05 / 31 / 91 \\
02 / 23 / 92 \\
08 / 11 / 92 \\
11 / 22 / 92\end{array}$ & $\begin{array}{l}0001 \\
0001 \\
0001 \\
0001\end{array}$ & MG/L & $\begin{array}{l}< \\
< \\
<\end{array}$ & $\begin{array}{l}0.01 \\
0.01 \\
0.01 \\
0.0053\end{array}$ & $\mathbf{L}$ & $\begin{array}{l}0.01 \\
0.01 \\
0.01 \\
0.0053\end{array}$ & $\begin{array}{l}- \\
-\end{array}$ \\
\hline CHROHIUM (TOTAL) & $11 / 22 / 92$ & N001 & $M G / L$ & $<$ & 0.0053 & & 0.0053 & - \\
\hline $\operatorname{COBALT}$ & $05 / 31 / 91$ & 0001 & $M G / L$ & $<$ & 0.03 & $\mathbf{L}$ & 0.03 & - \\
\hline COPPER & $05 / 31 / 91$ & 0001 & MG/L & $<$ & 0.01 & $\mathbf{L}$ & 0.09 & - \\
\hline DISSOLVED OXYGEN & $11 / 22 / 92$ & 0001 & $M G / L$ & & 0.7 & & - & - \\
\hline FLUORIDE & $05 / 31 / 91$ & 0001 & $M G / L$ & & 0.2 & $\mathbf{L}$ & 0.1 & - \\
\hline GROSS ALPHA & $\begin{array}{l}05 / 31 / 91 \\
02 / 23 / 92 \\
08 / 11 / 92 \\
11 / 22 / 92 \\
06 / 29 / 93\end{array}$ & $\begin{array}{l}0001 \\
0001 \\
0001 \\
0001 \\
0001\end{array}$ & $\mathrm{PCI} / \mathrm{L}$ & & $\begin{array}{r}0.0 \\
0.6 \\
7.1 \\
3.7 \\
11\end{array}$ & L & $\begin{array}{l}1 . \\
1.0 \\
5.7 \\
3.2 \\
3\end{array}$ & $\begin{array}{l}5.3 \\
4.3 \\
4.7 \\
2.7 \\
6\end{array}$ \\
\hline GROSS ALPHA (TOTAL) & $\begin{array}{l}11 / 22 / 92 \\
06 / 29 / 93\end{array}$ & $\begin{array}{l}\text { No01 } \\
\text { N001 }\end{array}$ & $\mathrm{PCI} / \mathrm{L}$ & & 415 & H & $44^{9.2}$ & 118 \\
\hline GROSS BETA & $\begin{array}{l}05 / 31 / 91 \\
02 / 23 / 92 \\
08 / 11 / 92 \\
11 / 22 / 92 \\
06 / 29 / 93\end{array}$ & $\begin{array}{l}0001 \\
0001 \\
0001 \\
0001 \\
0001\end{array}$ & $\begin{array}{c}\mathrm{PCI} / \mathrm{L} \\
.\end{array}$ & • & $\begin{array}{c}2.2 \\
1.6 \\
10 \\
0.0 \\
2\end{array}$ & L & $\begin{array}{l}0.5 \\
0.5 \\
5 \\
5.7 \\
4\end{array}$ & $\begin{array}{l}3.2 \\
2.5 \\
4 \\
3.3 \\
4\end{array}$ \\
\hline GROSS BETA (TOTAL) & $\begin{array}{l}11 / 22 / 92 \\
06 / 29 / 93\end{array}$ & $\begin{array}{l}\text { N001 } \\
\text { N001 }\end{array}$ & $\mathrm{PCI} / \mathrm{L}$ & & $182^{0.7}$ & H & $\begin{array}{l}6.6 \\
46\end{array}$ & $\begin{array}{l}4.0 \\
50^{-0}\end{array}$ \\
\hline IRON & $\begin{array}{l}05 / 31 / 91 \\
02 / 23 / 92 \\
08 / 11 / 92 \\
05 / 29 / 93\end{array}$ & $\begin{array}{l}0001 \\
0001 \\
0001 \\
0001\end{array}$ & $M G / L$ & $\begin{array}{l}< \\
< \\
<\end{array}$ & $\begin{array}{l}0.03 \\
0.03 \\
0.1 \\
0.03\end{array}$ & L & $\begin{array}{l}0.03 \\
0.03 \\
0.1 \\
0.03\end{array}$ & : \\
\hline IRON (TOTAL) & $06 / 29 / 93$ & NOO1 & $M G / L$ & & 107 & & 0.03 & - \\
\hline LEAD & $\begin{array}{l}05 / 31 / 91 \\
11 / 22 / 92\end{array}$ & $\begin{array}{l}0001 \\
0001\end{array}$ & $M G / L$ & $<$ & $\begin{array}{l}0.005 \\
0.0015\end{array}$ & $L$ & $\begin{array}{l}0.005 \\
0.0015\end{array}$ & - \\
\hline
\end{tabular}

PARAMETER VALUE IMDICATOR (PVI): < - LESS THAN DETECTION LIMIT

SAMPLE ID COOES:

OTHER PARAMETER VALUE FLAGS:

O001 - FILTERED SNMPLE (.45 MICRONS)

$\downarrow$ - ESTIMATED VALUE

MOO1 - UMFILTERED SAMPLE

L - LESS THAN THREE BORE VOLUMES REHOVED BEFORE SAMPLING

H - SPIKE SAMPLE RECOVERY NOT UITHIN CONTROL LIHITS 
GROUMDHATER QUALITY DATA BY LOCATIOH

SOMP ReV. O Data

SITE: MONOI MOHLMENT VALLEY

LOCATION: 0601

NORTH COORDIHATE: 54980.4 FT

EAST COORDIHATE: 88017.7 FT

$04 / 21 / 88$ TO $12 / 13 / 94$

REPORT DATE: 06/20/95

FORMATION OF COMPLETION: SHIMARLMP MEMBER OF THE CHIHLE FORMATION (SR) HYDRAULIC FLOW RELATIOHSHIP: UPGRADIEHT (U)

\begin{tabular}{|c|c|c|c|c|c|c|c|c|}
\hline PARAMETER MNME & LOG DATE & $\underset{\text { ID }}{\text { SAMPLE }}$ & $\begin{array}{l}\text { UNITS OF } \\
\text { MEASURE }\end{array}$ & PVI & $\begin{array}{l}\text { PARAMETER } \\
\text { VALUE }\end{array}$ & FLAGS & $\begin{array}{l}\text { DETECTION } \\
\text { LIMIT }\end{array}$ & $\begin{array}{l}\text { PARAMETER } \\
\text { UNCERTAIHTY }\end{array}$ \\
\hline LEAD (TOTAL) & $11 / 22 / 92$ & HoO1 & $M G / L$ & \multicolumn{3}{|c|}{0.0049} & 0.0015 & - \\
\hline LEAD-290 (TOTAL) & $19 / 22 / 92$ & NoO1 & $\mathrm{PCI} / \mathrm{L}$ & \multicolumn{3}{|c|}{9.1} & 2.0 & 1.2 \\
\hline MAGKESIUM (TOTAL) & $06 / 29 / 93$ & N001 & MG/L & \multicolumn{3}{|c|}{47.1} & 0.1 & - \\
\hline MAKGAKESE & $\begin{array}{l}05 / 31 / 91 \\
02 / 23 / 92 \\
08 / 11 / 92 \\
19 / 22 / 92 \\
06 / 29 / 93\end{array}$ & $\begin{array}{l}0001 \\
0001 \\
0001 \\
0001 \\
0001\end{array}$ & MG/L & $\begin{array}{l}< \\
<\end{array}$ & $\begin{array}{l}0.01 \\
0.01 \\
0.01 \\
0.0037 \\
0.27\end{array}$ & $\mathbf{L}$ & $\begin{array}{l}0.01 \\
0.01 \\
0.01 \\
0.0015 \\
0.01\end{array}$ & : \\
\hline HOLYBDEKUM & $\begin{array}{l}05 / 31 / 91 \\
02 / 23 / 92 \\
08 / 11 / 92 \\
11 / 22 / 92 \\
06 / 29 / 93\end{array}$ & $\begin{array}{l}0001 \\
0001 \\
0001 \\
0001 \\
0001\end{array}$ & $M G / L$ & $\begin{array}{l}< \\
< \\
<\end{array}$ & $\begin{array}{l}0.01 \\
0.01 \\
0.007 \\
0.0049 \\
0.01\end{array}$ & $\begin{array}{l}\text { L } \\
\text { N }\end{array}$ & $\begin{array}{l}0.01 \\
0.01 \\
0.007 \\
0.0049 \\
0.01\end{array}$ & $\begin{array}{l}- \\
- \\
- \\
-\end{array}$ \\
\hline MOLYBOENUM (TOTAL) & $\begin{array}{l}11 / 22 / 92 \\
06 / 29 / 93\end{array}$ & $\begin{array}{l}\text { N001 } \\
\text { N001 }\end{array}$ & $M G / L$ & $<$ & \multicolumn{2}{|c|}{$\begin{array}{l}0.0049 N \\
0.01\end{array}$} & $\begin{array}{l}0.0049 \\
0.01\end{array}$ & - \\
\hline NET GROSS ALPHA $\bullet$ & $\begin{array}{l}05 / 31 / 91 \\
02 / 23 / 92 \\
11 / 22 / 92 \\
06 / 29 / 93\end{array}$ & $\begin{array}{l}0001 \\
0001 \\
0001 \\
0001\end{array}$ & $\mathrm{PCl} / \mathrm{L}$ & \multicolumn{3}{|c|}{$\begin{array}{r}-0.34 \\
-3.52 \\
-0.42 \\
8.26\end{array}$} & :- & : \\
\hline MITRATE (TOTAL) & $06 / 29 / 93$ & NoO1 & MG/L & & 3 & & 9 & - \\
\hline HITRITE NHD MITRATE & $05 / 31 / 91$ & 0001 & MG/L & & 1.22 & $\mathbf{L}$ & 0.05 & - \\
\hline PH & $05 / 31 / 91$ & 0001 & SU & & 7.85 & $\mathbf{L}$ & - & - \\
\hline
\end{tabular}

- MET GROSS ALPHA (GROSS ALPHA - URAHIUN) UITH 1 MG URAHILH = 686 PCI

* MET GROSS ALPHA (TOTAL) (TOTAL GROSS ALPHA - TOTAL URANIUH)

PARAMETER VALUE INDICATOR (PVI): < - LESS THAN DETECTION LIMIT

OTHER PARUMETER VALUE FLAGS:

L - LESS THAH THREE BORE VOLLUES REHOVED BEFORE SAMPLIMG

W - SPIKE SAMPLE RECOVERY HOT HITHIN CONTROL LIMITS
SAMPLE ID COOES:

0001 - FILTERED SAMPLE (.45 HICROHS)

NOOI - UWFILTERED SAMPLE 
GROUNDWATER QUALITY DATA BY LOCATION

SOWP ReV. O Data

SITE: MONO1 MONLMENT VALLEY

LOCATION: 0601

NORTH COORDIKATE: 54980.4 FT

EAST COORDINATE: 88017.7 FT

$04 / 21 / 88$ TO $12 / 13 / 94$

REPORT DATE: $06 / 20 / 95$

FORMATION OF COMPLETIOH: SHIHARUMP MEMBER OF THE CHIMLE FORMATION (SR) HYDRAULIC FLON RELATIOHSHIP: UPGRADIEHT (U)

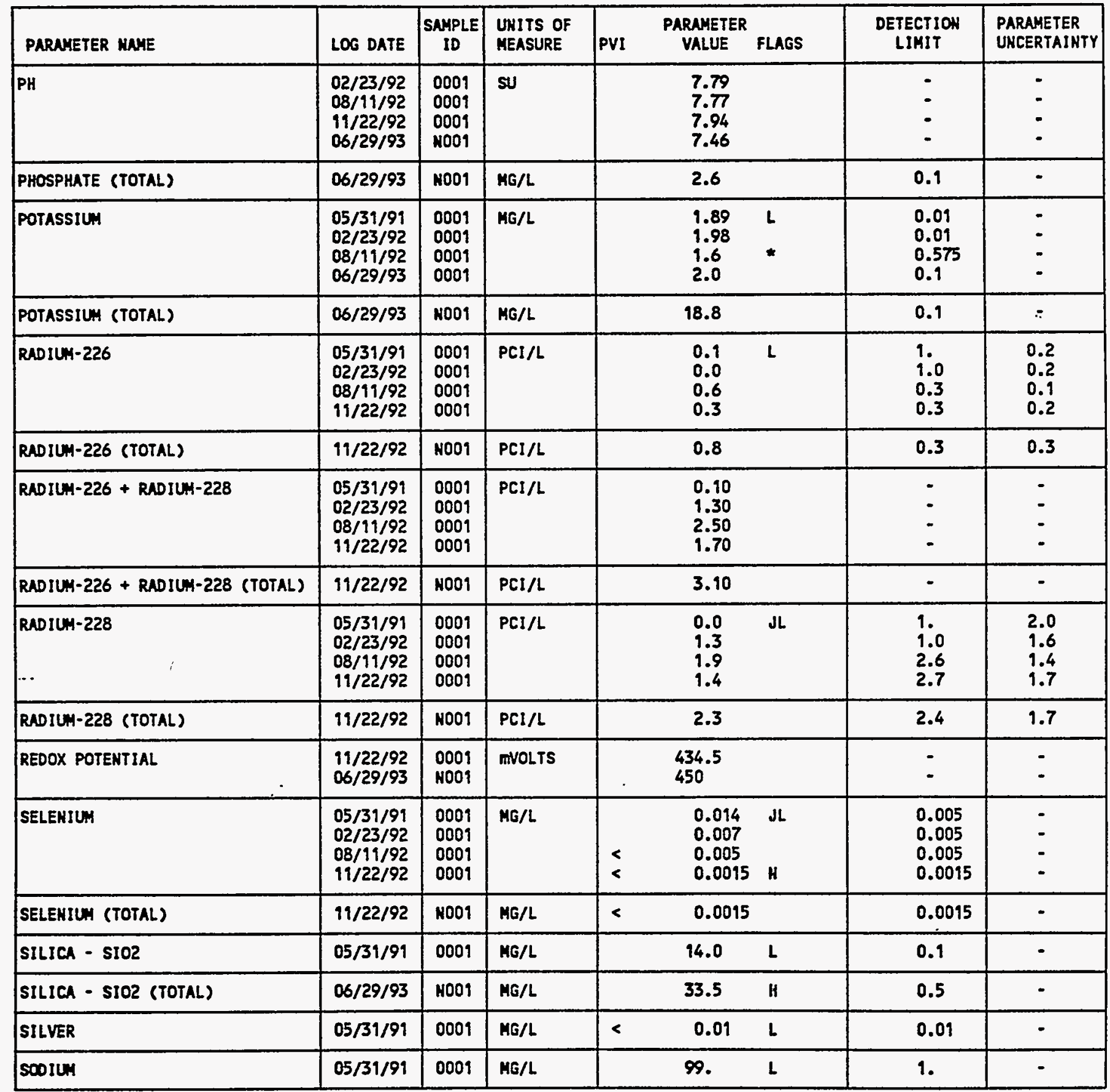

PARAMETER VALUE IMDICATOR (PVI): < - LESS THAN DETECTION LIMIT

- - DUPLICATE AMALYSIS nOT UITHIK CONTROL LIMITS

H - HOLD TIME EXPIRED, VALUE SUSPECT

d - ESTIMTED VALUE

L - LESS THAN THREE BORE VOLUMES REHOVED BEFORE SAMPLING

- SPIKE SNMPLE RECOVERY NOT MITHIN CONTROL LIMITS 
GROUNDHATER OUALITY DATA BY LOCATIOW

SOUP REV. O Dato

SITE: MOHOI HONUMENT VALLEY

LOCATION: 0601

NORTH COORDIHATE: $\quad 54980.4$ FT

EAST COORDIHATE: $\quad 88017.7$ FT

$04 / 21 / 88$ TO $12 / 13 / 94$

REPORT DATE: $06 / 20 / 95$

FORMATION OF COMPLETION: SHIMARLAP MEMBER OF THE CHINLE FORMATIOH (SR) HYDRAULIC FLON RELATIONSHIP: UPGRADIENT (U)

\begin{tabular}{|c|c|c|c|c|c|c|c|c|}
\hline PARAMETER MAME & LOG DATE & $\underset{\text { ID }}{\text { SAMPLE }}$ & $\begin{array}{l}\text { UNITS OF } \\
\text { MEASURE }\end{array}$ & PVI & $\begin{array}{l}\text { PARAMETER } \\
\text { VALUE }\end{array}$ & FLAGS & $\begin{array}{l}\text { DETECTION } \\
\text { LIMIT }\end{array}$ & $\begin{array}{l}\text { PARAMETER } \\
\text { UNCERTAINTY }\end{array}$ \\
\hline SOOIUA & $\begin{array}{l}02 / 23 / 92 \\
08 / 11 / 92 \\
06 / 29 / 93\end{array}$ & $\begin{array}{l}0001 \\
0001 \\
0001\end{array}$ & MG/L & & $\begin{array}{l}99 . \\
97 \\
98\end{array}$ & & $\begin{array}{l}1 . \\
5 \\
1\end{array}$ & $\begin{array}{l}- \\
-\end{array}$ \\
\hline SOOIUM (TOTAL) & $06 / 29 / 93$ & N001 & MG/L & & 102 & & 1 & - \\
\hline SPECIFIC CONDUCTAKCE & $\begin{array}{l}05 / 31 / 91 \\
02 / 23 / 92 \\
08 / 11 / 92 \\
11 / 22 / 92 \\
06 / 29 / 93\end{array}$ & $\begin{array}{l}0001 \\
0001 \\
0001 \\
0001 \\
1001\end{array}$ & UHHO/CH & & $\begin{array}{l}750 . \\
515 \\
553 \\
581 \\
532\end{array}$ & $\mathbf{L}$ & $\begin{array}{l}- \\
- \\
- \\
-\end{array}$ & $\begin{array}{l}- \\
- \\
-\end{array}$ \\
\hline STRONTIUM & $\begin{array}{l}05 / 31 / 91 \\
02 / 23 / 92 \\
08 / 11 / 92 \\
11 / 22 / 92 \\
06 / 29 / 93\end{array}$ & $\begin{array}{l}0001 \\
0001 \\
0001 \\
0001 \\
0001\end{array}$ & $M G / L$ & & $\begin{array}{l}0.28 \\
0.28 \\
0.30 \\
0.32 \\
0.29\end{array}$ & $L$ & $\begin{array}{l}0.01 \\
0.01 \\
0.2 \\
0.00035 \\
0.01\end{array}$ & $\begin{array}{l}- \\
- \\
-\end{array}$ \\
\hline STROHTILM (TOTAL) & $\begin{array}{l}11 / 22 / 92 \\
06 / 29 / 93\end{array}$ & $\begin{array}{l}\text { No01 } \\
\text { N001 }\end{array}$ & MG/L & & $\begin{array}{l}0.36 \\
0.84\end{array}$ & & $\begin{array}{l}0.00035 \\
0.01\end{array}$ & - \\
\hline SULFATE & $\begin{array}{l}05 / 31 / 91 \\
02 / 23 / 92 \\
08 / 11 / 92 \\
11 / 22 / 92\end{array}$ & $\begin{array}{l}0001 \\
0001 \\
0001 \\
0001\end{array}$ & $M G / L$ & & $\begin{array}{l}60 . \\
131 . \\
120 \\
130\end{array}$ & $\mathbf{L}$ & $\begin{array}{l}0.1 \\
10 . \\
0.059 \\
1.0\end{array}$ & $\dot{-}$ \\
\hline SULFATE (TOTAL) & $\begin{array}{l}11 / 22 / 92 \\
06 / 29 / 93\end{array}$ & $\begin{array}{l}\text { No01 } \\
\text { N001 }\end{array}$ & MG/L & & $\begin{array}{l}130 \\
124\end{array}$ & & 1.0 & : \\
\hline SULFIDE & $05 / 31 / 91$ & 0001 & MG/L & $<$ & 1. & $I J L$ & 1. & - \\
\hline TEMPERATURE & $\begin{array}{l}05 / 31 / 91 \\
02 / 23 / 92 \\
08 / 11 / 92 \\
11 / 22 / 92 \\
06 / 29 / 93\end{array}$ & $\begin{array}{l}0001 \\
0001 \\
0001 \\
0001 \\
N 001\end{array}$ & C - DEGREE & & $\begin{array}{l}15.0 \\
15.0 \\
17.7 \\
16.8 \\
16.4\end{array}$ & $L$ & $\begin{array}{l}- \\
- \\
- \\
-\end{array}$ & $\begin{array}{l}- \\
\dot{-} \\
\dot{-}\end{array}$ \\
\hline THALLIUM & $05 / 31 / 91$ & 0001 & $M G / L$ & $<$ & 0.09 & $L$ & 0.01 & - \\
\hline THORIU:-230 & $05 / 31 / 91$ & 0001 & $\mathrm{PCI} / \mathrm{L}$ & & 0.5 & $L$ & 1. & 0.4 \\
\hline TIH & $05 / 31 / 91$ & 0001 & MG/L & $<$ & 0.01 & Il & 0.01 & - \\
\hline TOTAL DISSOLVED SOLIDS & $\begin{array}{l}05 / 31 / 91 \\
02 / 23 / 92 \\
08 / 11 / 92\end{array}$ & $\begin{array}{l}0001 \\
0001 \\
0001\end{array}$ & MG/L & & $\begin{array}{l}436 . \\
429 . \\
380\end{array}$ & $\mathbf{L}$ & $\begin{array}{l}10 . \\
10 . \\
42\end{array}$ & - \\
\hline TOTAL DISSOLVED SOLIOS (TOTAL) & $06 / 29 / 93$ & noo1 & $M G / L$ & & 440 & H & 10 & - \\
\hline TOTAL ORGANIC CARBON & $\begin{array}{l}05 / 31 / 91 \\
02 / 23 / 92 \\
08 / 11 / 92\end{array}$ & $\begin{array}{l}0001 \\
0001 \\
0001\end{array}$ & MG/L & $<$ & $\begin{array}{l}1 . \\
1.0 \\
1.0\end{array}$ & $\mathbf{L}$ & $\begin{array}{l}1 . \\
1.0\end{array}$ & $\because$ \\
\hline URAKILY & $05 / 31 / 91$ & 0001 & MG/L & $<$ & 0.001 & $L$ & 0.001 & - \\
\hline
\end{tabular}

PARAMETER VALUE INDICATOR (PVI): < - LESS THAH DETECTIOH LIMIT

SAMPLE ID COOES:

OTHER PARAMETER VALUE FLAGS:

0001 - FILTERED SAMPLE (.45 HICRONS)

H - HOLD TIME EXPIRED, VALUE SUSPECT

NOOI - UNFILTERED SAMPLE

I - INCREASED DETECTION LIMIT DUE TO REOUIRED DILUTIOH

J - ESTIMATED VALUE

L - LESS THAN THREE BORE VOLUMES REMOVED BEFORE SAMPLIKG 
GROUMDHATER QUALITY DATA BY LOCATION

SOWP ReV. O Data

SITE: MONO1 MONUMENT VALLEY

LOCATION: 0601

NORTH COORDINATE: $\quad 54980.4$ FT

EAST COORDINATE: 88017.7 FT

04/21/88 TO $12 / 13 / 94$

REPORT DATE: 06/20/95

FORMATION OF COMPLETION: SHIMARUAP MEMBER OF THE CHINLE FORMATION (SR) HYDRAULIC FLOW RELATIOHSHIP: UPGRADIENT (U)

\begin{tabular}{|c|c|c|c|c|c|c|c|c|}
\hline PARAMETER MAME & LOS DATE & $\underset{\text { SAMPLE }}{\text { ID }}$ & $\begin{array}{l}\text { UNITS OF } \\
\text { MEASURE }\end{array}$ & PVI & $\begin{array}{l}\text { PARAMETER } \\
\text { VALUE }\end{array}$ & FLAGS & $\begin{array}{l}\text { DETECTION } \\
\text { LIMIT }\end{array}$ & $\begin{array}{l}\text { PARAMETER } \\
\text { UHCERTAINTY }\end{array}$ \\
\hline URAKIUYY & $\begin{array}{l}02 / 23 / 92 \\
11 / 22 / 92 \\
06 / 29 / 93\end{array}$ & $\begin{array}{l}0001 \\
0001 \\
0001\end{array}$ & $M G / L$ & & $\begin{array}{l}0.006 \\
0.006 \\
0.004\end{array}$ & & $\begin{array}{l}0.001 \\
0.001 \\
0.001\end{array}$ & : \\
\hline URAKIU: (TOTAL) & $\begin{array}{l}11 / 22 / 92 \\
06 / 29 / 93\end{array}$ & $\begin{array}{l}\text { NoO1 } \\
\text { N001 }\end{array}$ & MG/L & & $\begin{array}{l}0.004 \\
0.017\end{array}$ & & $\begin{array}{l}0.001 \\
0.001\end{array}$ & - \\
\hline VAKADILM & $\begin{array}{l}05 / 31 / 91 \\
02 / 23 / 92 \\
08 / 11 / 92 \\
11 / 22 / 92 \\
06 / 29 / 93\end{array}$ & $\begin{array}{l}0001 \\
0001 \\
0001 \\
0001 \\
0001\end{array}$ & $M G / L$ & $\begin{array}{l}< \\
< \\
< \\
< \\
<\end{array}$ & $\begin{array}{l}0.01 \\
0.01 \\
0.05 \\
0.0019 \\
0.01\end{array}$ & $L$ & $\begin{array}{l}0.09 \\
0.09 \\
0.05 \\
0.0019 \\
0.09\end{array}$ & $\begin{array}{l}: \\
: \\
-\end{array}$ \\
\hline VAKADIUH (TOTAL) & $\begin{array}{l}11 / 22 / 92 \\
06 / 29 / 93\end{array}$ & $\begin{array}{l}\text { NOOI } \\
\text { NOOI }\end{array}$ & $M G / L$ & & $\begin{array}{l}0.019 \\
0.14\end{array}$ & & $\begin{array}{l}0.0019 \\
0.09\end{array}$ & - \\
\hline ZINC & $\begin{array}{l}05 / 31 / 91 \\
02 / 23 / 92 \\
08 / 11 / 92 \\
11 / 22 / 92 \\
06 / 29 / 93\end{array}$ & $\begin{array}{l}0001 \\
0001 \\
0001 \\
0001 \\
0001\end{array}$ & $M G / L$ & $\begin{array}{l}< \\
< \\
< \\
<\end{array}$ & $\begin{array}{l}0.005 \\
0.005 \\
0.02 \\
0.0010 \\
0.011\end{array}$ & $\mathbf{L}$ & $\begin{array}{l}0.005 \\
0.005 \\
0.02 \\
0.0010 \\
0.005\end{array}$ & $\begin{array}{l}- \\
- \\
- \\
-\end{array}$ \\
\hline ZINC (TOTAL) & $\begin{array}{l}11 / 22 / 92 \\
06 / 29 / 93\end{array}$ & $\begin{array}{l}\text { NoO1 } \\
\text { NOOI }\end{array}$ & HG/L & & $\begin{array}{l}0.014 \\
0.271\end{array}$ & $J$ & $\begin{array}{l}0.0010 \\
0.005\end{array}$ & - \\
\hline
\end{tabular}

PARAMETER VALUE INDICATOR (PVI): < - LESS THAN DETECTION LIMIT

SAMPLE IO COOES:

OOOI - FILTERED SAMPLE (.45 MICRONS)

OTHER PARAMETER VALUE FLAGS:

NOO1 - UNFILTERED SAMPLE

J - ESTIMUTED VALUE

L - LESS THAN THREE BORE VOLUHES REMOVED BEFORE SAMPLING

DATA FILE MAME: IDARTYMOHOIYGLA10007.DAT 
GROUNDWATER QUALITY DATA BY LOCATION

SOWP ReV. O DETE

SITE: MONO1 MOWUMENT VALLEY

LOCATIOH: 0602

MORTH COORDIHATE: 56377.4 FT

EAST COORDIHATE: 88661.2 FT

$04 / 21 / 88$ TO $12 / 13 / 94$

REPORT DATE: 06/20/95

FORMATION OF COMPLETIOH: ALLUVIUM (AL)

HYDRAULIC FLON RELATIOHSHIP: UHKNOWN (H)

\begin{tabular}{|c|c|c|c|c|c|c|c|c|}
\hline PARAMETER MAHE & LOG DATE & $\begin{array}{c}\text { SAMPLE } \\
\text { ID }\end{array}$ & $\begin{array}{l}\text { UHITS OF } \\
\text { MEASURE }\end{array}$ & PVI & $\begin{array}{l}\text { PARAMETER } \\
\text { VALUE }\end{array}$ & FLAGS & $\begin{array}{l}\text { DETECTION } \\
\text { LIMIT }\end{array}$ & $\begin{array}{l}\text { PARAMETER } \\
\text { UNCERTAINTY }\end{array}$ \\
\hline ALKALIHITY & $\begin{array}{l}04 / 22 / 88 \\
06 / 05 / 89 \\
05 / 30 / 91 \\
02 / 25 / 92 \\
08 / 10 / 92 \\
11 / 22 / 92 \\
02 / 02 / 93 \\
06 / 30 / 93\end{array}$ & $\begin{array}{l}0001 \\
0001 \\
0001 \\
0001 \\
0001 \\
0001 \\
0001 \\
\text { N001 }\end{array}$ & MG/L CACO3 & & $\begin{array}{l}218 . \\
219 . \\
219 . \\
218 \\
213 \\
216 \\
231 \\
199\end{array}$ & $\begin{array}{l}L \\
L\end{array}$ & $\begin{array}{l}: \\
: \\
: \\
-\end{array}$ & $\begin{array}{l}: \\
: \\
: \\
: \\
- \\
-\end{array}$ \\
\hline ALGMINUM & $\begin{array}{l}06 / 05 / 89 \\
05 / 30 / 99 \\
02 / 25 / 92 \\
08 / 10 / 92\end{array}$ & $\begin{array}{l}0001 \\
0001 \\
0001 \\
0001\end{array}$ & MG/L & $\begin{array}{l}< \\
< \\
<\end{array}$ & $\begin{array}{l}0.1 \\
0.05 \\
0.05 \\
0.2\end{array}$ & $\begin{array}{l}\mathrm{L} \\
\star \mathrm{L}\end{array}$ & $\begin{array}{l}0.1 \\
0.05 \\
0.05 \\
0.2\end{array}$ & $\begin{array}{l}- \\
-\end{array}$ \\
\hline AMHONIU⿴囗十⺝ & $\begin{array}{l}04 / 22 / 88 \\
06 / 05 / 89 \\
05 / 30 / 91 \\
02 / 25 / 92 \\
02 / 02 / 93\end{array}$ & $\begin{array}{l}0001 \\
0001 \\
0001 \\
0001 \\
0001\end{array}$ & $M G / L$ & $\begin{array}{l}< \\
< \\
< \\
< \\
<\end{array}$ & $\begin{array}{l}0.1 \\
0.1 \\
0.1 \\
0.1 \\
0.1\end{array}$ & $\mathbf{L}$ & $\begin{array}{l}0.1 \\
0.1 \\
0.1 \\
0.1 \\
0.1\end{array}$ & $\begin{array}{l}- \\
- \\
- \\
-\end{array}$ \\
\hline AMYOHIU (TOTAL) & $06 / 30 / 93$ & HOOI & MG/L & $<$ & 0.1 & & 0.1 & - \\
\hline AHTIMOHY & $\begin{array}{l}06 / 05 / 89 \\
05 / 30 / 91 \\
02 / 25 / 92 \\
08 / 10 / 92\end{array}$ & $\begin{array}{l}0001 \\
0001 \\
0001 \\
0001\end{array}$ & MG/L & $\begin{array}{l}< \\
< \\
<\end{array}$ & $\begin{array}{l}0.003 \\
0.003 \\
0.003 \\
0.06\end{array}$ & $\begin{array}{l}L \\
\text { HL }\end{array}$ & $\begin{array}{l}0.003 \\
0.003 \\
0.003 \\
0.06\end{array}$ & $\begin{array}{l}- \\
-\end{array}$ \\
\hline $\begin{array}{c}\text { ARSEHIC } \\
\text {. }\end{array}$ & $\begin{array}{l}04 / 22 / 88 \\
06 / 05 / 89 \\
05 / 30 / 91 \\
02 / 25 / 92 \\
08 / 10 / 92 \\
11 / 22 / 92 \\
06 / 30 / 93\end{array}$ & $\begin{array}{l}0001 \\
0001 \\
0001 \\
0001 \\
0001 \\
0001 \\
0001\end{array}$ & MG/L & $\begin{array}{l}< \\
< \\
< \\
< \\
< \\
< \\
<\end{array}$ & $\begin{array}{l}0.001 \\
0.01 \\
0.01 \\
0.01 \\
0.01 \\
0.0015 \\
0.005\end{array}$ & $\begin{array}{l}J \\
L \\
H L\end{array}$ & $\begin{array}{l}0.01 \\
0.01 \\
0.01 \\
0.01 \\
0.01 \\
0.0015 \\
0.005\end{array}$ & $\begin{array}{l}- \\
- \\
- \\
- \\
-\end{array}$ \\
\hline ARSENIC (TOTAL) & $\begin{array}{l}11 / 22 / 92 \\
06 / 30 / 93\end{array}$ & $\begin{array}{l}\text { N001 } \\
\text { N001 }\end{array}$ & ME/L & $<$ & $\begin{array}{l}0.0026 \\
0.005\end{array}$ & & $\begin{array}{l}0.0015 \\
0.005\end{array}$ & - \\
\hline BARIUM & $\begin{array}{l}06 / 05 / 89 \\
05 / 30 / 91 \\
02 / 25 / 92 \\
08 / 10 / 92 \\
11 / 22 / 92 \\
06 / 30 / 93\end{array}$ & $\begin{array}{l}0001 \\
0001 \\
0001 \\
0001 \\
0001 \\
0001\end{array}$ & $M G / L$ & $\begin{array}{l}< \\
< \\
<\end{array}$ & $\begin{array}{l}0.1 \\
0.05 \\
0.05 \\
0.2 \\
0.049 \\
0.1\end{array}$ & $\begin{array}{l}L \\
L\end{array}$ & $\begin{array}{l}0.1 \\
0.01 \\
0.01 \\
0.2 \\
0.0015 \\
0.1\end{array}$ & $\begin{array}{l}- \\
- \\
- \\
-\end{array}$ \\
\hline BARIUH (TOTAL) & $\begin{array}{l}11 / 22 / 92 \\
06 / 30 / 93\end{array}$ & $\begin{array}{l}\text { No01 } \\
\text { N001 }\end{array}$ & HG/L & $<$ & $\begin{array}{l}0.055 \\
0.1\end{array}$ & & $\begin{array}{l}0.0015 \\
0.1\end{array}$ & - \\
\hline BERYLLIUM & $\begin{array}{l}06 / 05 / 89 \\
05 / 30 / 91 \\
08 / 10 / 92\end{array}$ & $\begin{array}{l}0001 \\
0001 \\
0001\end{array}$ & $M G / L$ & $\begin{array}{l}< \\
< \\
<\end{array}$ & $\begin{array}{l}0.09 \\
0.005 \\
0.005\end{array}$ & $L$ & $\begin{array}{l}0.01 \\
0.005 \\
0.005\end{array}$ & : \\
\hline BOROH & $06 / 05 / 89$ & 0009 & MG/L & $<$ & 0.1 & & 0.1 & - \\
\hline
\end{tabular}

PARAMETER VALUE IMDICATOR (PVI): < - LESS THAN DETECTION LIMIT

OTHER PARAMETER VALUE FLAGS:

SUMPLE ID COOES:

ODOI - FILTERED SAMPLE (.45 MICROHS)

- - DUPLICATE AMALYSIS NOT MITHIK CONTROL LIMITS

HOO1 - UAFILTERED SAMPLE

$J$ - ESTIHATED VALUE

L - LESS THAH THREE BORE VOLUMES REMOVED BEFORE SAMPLING

- $W$ - POST-DIGEST SPIKE OUT OF CNTR LIK MHILE SAMP ABS < 50\% SPIKE 
GROUNDWATER QUALITY DATA BY LOCATION

SOWP Rev. O Data

SITE: MONO1 MOHUMENT VALLEY

LOCATION: 0602

NORTH COORDINATE: 56377.4 FT

EAST COORDINATE: $88661.2 \mathrm{FT}$

$04 / 21 / 88$ TO $12 / 13 / 94$

REPORT DATE: 06/20/95

FORMATIOH OF COMPLETION: ALLUVILY (AL)

HYDRAULIC FLON RELATIONSHIP: UNKHOWN (N)

\begin{tabular}{|c|c|c|c|c|c|c|c|c|}
\hline PARAMETER NAME & LOE DATE & $\underset{\text { ID }}{\text { SAMPLE }}$ & $\begin{array}{l}\text { UNITS OF } \\
\text { MEASURE }\end{array}$ & PVI & $\begin{array}{l}\text { PARAMETER } \\
\text { VALUE }\end{array}$ & FLAGS & $\begin{array}{l}\text { DETECTION } \\
\text { LIMIT }\end{array}$ & $\begin{array}{l}\text { PARAMETER } \\
\text { UHCERTAIHTY }\end{array}$ \\
\hline Boron & $05 / 30 / 91$ & 0001 & MG/L & $<$ & 0.05 & $L$ & 0.05 & - \\
\hline BROAIDE & $05 / 30 / 91$ & 0001 & MG/L & & 0.2 & $L$ & 0.1 & - \\
\hline CADMIUNA & $\begin{array}{l}04 / 22 / 88 \\
06 / 05 / 89 \\
05 / 30 / 91 \\
02 / 25 / 92 \\
08 / 10 / 92 \\
11 / 22 / 92\end{array}$ & $\begin{array}{l}0001 \\
0001 \\
0001 \\
0001 \\
0001 \\
0001\end{array}$ & MG/L & $\begin{array}{l}< \\
< \\
< \\
<\end{array}$ & $\begin{array}{l}0.005 \\
0.001 \\
0.001 \\
0.0005 \\
0.005 \\
0.00013\end{array}$ & $\begin{array}{l}L \\
E L\end{array}$ & $\begin{array}{l}0.001 \\
0.001 \\
0.001 \\
0.0005 \\
0.005 \\
0.00013\end{array}$ & $\begin{array}{l}- \\
- \\
- \\
-\end{array}$ \\
\hline CADHILM (TOTAL) & $11 / 22 / 92$ & HOO1 & $M G / L$ & $<$ & 0.00013 & 3 NH & 0.00013 & - \\
\hline CALCIUM & $\begin{array}{l}04 / 22 / 88 \\
06 / 05 / 89 \\
05 / 30 / 91 \\
02 / 25 / 92 \\
08 / 10 / 92 \\
02 / 02 / 93 \\
06 / 30 / 93\end{array}$ & $\begin{array}{l}0001 \\
0001 \\
0001 \\
0001 \\
0001 \\
0001 \\
0001\end{array}$ & MG/L & & $\begin{array}{l}28.2 \\
28.7 \\
29.4 \\
27.4 \\
27 \\
26.5 \\
27.9\end{array}$ & $\begin{array}{l}L \\
L\end{array}$ & $\begin{array}{l}0.01 \\
0.01 \\
0.5 \\
0.5 \\
5 \\
0.5 \\
0.5\end{array}$ & $\begin{array}{l}- \\
- \\
- \\
- \\
-\end{array}$ \\
\hline CALCIUM (TOTAL) & $06 / 30 / 93$ & HOOI & $M G / L$ & & 27.5 & & 0.5 & - \\
\hline CHLORIDE & $\begin{array}{l}04 / 22 / 88 \\
06 / 05 / 89 \\
05 / 30 / 91 \\
02 / 25 / 92 \\
08 / 10 / 92 \\
02 / 02 / 93\end{array}$ & $\begin{array}{l}0001 \\
0001 \\
0001 \\
0001 \\
0001 \\
0001\end{array}$ & $M G / L$ & & $\begin{array}{l}17 . \\
15 . \\
15.5 \\
15.9 \\
14 \\
15.3\end{array}$ & $\begin{array}{l}L \\
L\end{array}$ & $\begin{array}{l}1 . \\
1 . \\
0.5 \\
0.5 \\
0.016 \\
0.5\end{array}$ & $\begin{array}{l}- \\
- \\
- \\
-\end{array}$ \\
\hline CHLORIDE (TOTAL) & $06 / 30 / 93$ & NOO1 & $M G / L$ & & 14.9 & & 0.5 & - \\
\hline CHROHIUM & $\begin{array}{l}06 / 05 / 89 \\
05 / 30 / 91 \\
02 / 25 / 92 \\
08 / 10 / 92 \\
11 / 22 / 92\end{array}$ & $\begin{array}{l}0001 \\
0001 \\
0001 \\
0001 \\
0001\end{array}$ & $M G / L$ & $\begin{array}{l}< \\
< \\
< \\
<\end{array}$ & $\begin{array}{l}0.01 \\
0.01 \\
0.01 \\
0.01 \\
0.0053\end{array}$ & $\begin{array}{l}L \\
L\end{array}$ & $\begin{array}{l}0.01 \\
0.01 \\
0.01 \\
0.01 \\
0.0053\end{array}$ & $\begin{array}{l}- \\
- \\
- \\
-\end{array}$ \\
\hline CHROMIUM (TOTAL) & $11 / 22 / 92$ & N001 & MG/L & $<$ & 0.0053 & & 0.0053 & $:-$ \\
\hline COBALT & $\begin{array}{l}06 / 05 / 89 \\
05 / 30 / 91\end{array}$ & $\begin{array}{l}0001 \\
0001\end{array}$ & $M G / L$ & $<$ & $\begin{array}{l}0.05 \\
0.03\end{array}$ & $\mathbf{L}$ & $\begin{array}{l}0.05 \\
0.03\end{array}$ & - \\
\hline COPPER & $\begin{array}{l}06 / 05 / 89 \\
05 / 30 / 91\end{array}$ & $\begin{array}{l}0001 \\
0001\end{array}$ & $M G / L$ & $<$ & $\begin{array}{l}0.02 \\
0.01\end{array}$ & $\mathbf{L}$ & $\begin{array}{l}0.02 \\
0.01\end{array}$ & - \\
\hline CYANIDE & $06 / 05 / 89$ & 0001 & $M G / L$ & $<$ & 0.01 & & 0.01 & - \\
\hline DISSOLVED OXYGEN & $\begin{array}{l}11 / 22 / 92 \\
06 / 30 / 93\end{array}$ & $\begin{array}{l}0001 \\
\text { N001 }\end{array}$ & MG/L & & $\begin{array}{l}2.2 \\
3.6\end{array}$ & & - & $\dot{-}$ \\
\hline FLLORIDE & $\begin{array}{l}06 / 05 / 89 \\
05 / 30 / 91\end{array}$ & $\begin{array}{l}0001 \\
0001\end{array}$ & MG/L & & $\begin{array}{l}0.3 \\
0.1\end{array}$ & $L$ & $\begin{array}{l}0.1 \\
0.1\end{array}$ & - \\
\hline GROSS ALPHA & $04 / 22 / 88$ & 0001 & $\mathrm{PCI} / \mathrm{L}$ & & 8.3 & & 0.2 & 4.1 \\
\hline
\end{tabular}

PARAMETER VALUE INDICATOR (PVI): < - LESS THAN DETECTIOH LIMIT

SAMPLE ID COOES:

OTHER PARAMETER VALUE FLAGS:

E - estimated VALUE because of IMTERFEREnCE, SEe case harRative

ODO - FILTERED SAMPLE (.45 MICRONS)

NOO1 - UMFILTERED SAMPLE

$L$ - LESS THAN THREE BORE VOLUMES REMOVED BEFORE SAMPLIMG

N - SPIKE SAMPLE RECOVERY NOT WITHIN CONTROL LIMITS

$W$ - POST-DIGEST SPIKE OUT OF CHTR LIM WHILE SNMP ABS < 50\% SPIKE 
GROUNDHATER QUALITY DATA BY LOCATION

SOWP ReV. $O$ Data

SITE: MONO1 MONUMENT VALLEY

LOCATION: 0.602

NORTH COORDINATE: 56377.4 FT

EAST COORDIHATE: 88661.2 FT

04/21/88 TO 12/13/94

REPORT DATE: 06/20/95

FORHATIOH OF COMPLETION: ALLUVIUM (AL)

HYORAULIC FLON RELATIONSHIP: UNKNOWN (N)

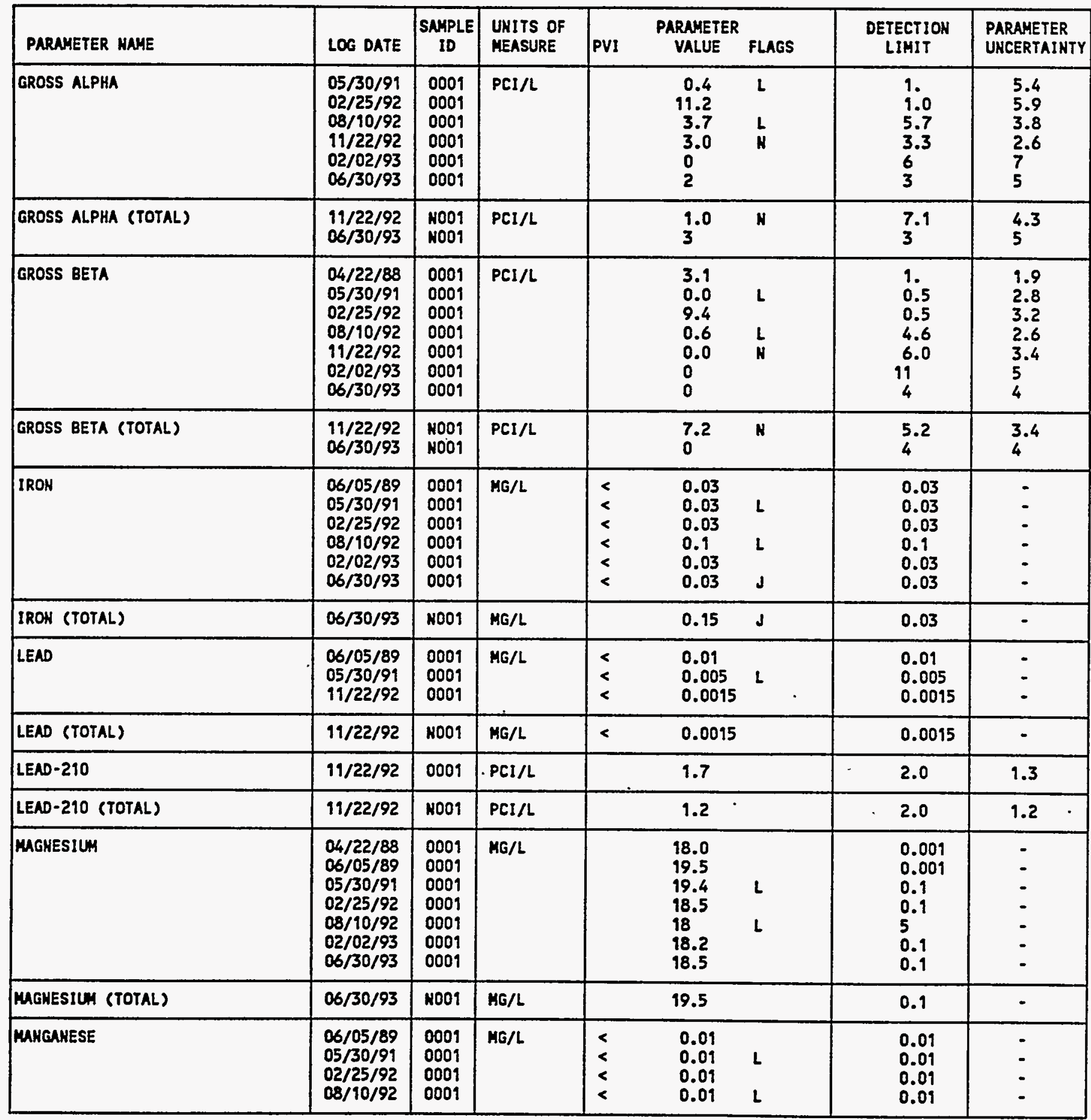

PARAMETER VALUE IHDICATOR (PVI): < - LESS THAN DETECTION LIMIT

SNMPLE ID CODES:

OTHER PARAMETER VALUE FLAGS:

0001 - FILTERED SAMPLE (.45 MICRONS)

J - ESTIMATED VALUE

HOO1 - UNFILTERED SAMPLE

$L$ - LESS THAH THREE BORE VOLUMES REMOVED BEFORE SAMPLIHG

* - SPIKE SAHPLE RECOVERY NOT MITHIN CONTROL LIMITS 
GROUNDHATER QUALITY DATA BY LOCATION

SOWP ReV. O Data

SITE: MONOI MONUMENT VALLEY

LOCATION: 0602

MORTH COORDINATE: $\quad 56377.4$ FT

EAST COORDIHATE: 88661.2 FT

$04 / 21 / 88$ TO $12 / 13 / 94$

REPORT DATE: $06 / 20 / 95$

FORMATION OF COHPLETION: ALLUVIUM (AL)

HYDRAULIC FLON RELATIONSHIP: UHKNOWN (N)

\begin{tabular}{|c|c|c|c|c|c|c|c|c|}
\hline PARANETER MAME & LOG DATE & $\underset{\text { SN }}{\text { SAMPLE }}$ & $\begin{array}{l}\text { UHITS OF } \\
\text { MEASURE }\end{array}$ & PVI & $\begin{array}{l}\text { PARAMETER } \\
\text { VALUE }\end{array}$ & FLAGS & $\begin{array}{l}\text { DETECTION } \\
\text { LIMIT }\end{array}$ & $\begin{array}{l}\text { PARAMETER } \\
\text { UNCERTAINTY }\end{array}$ \\
\hline MUNGAHESE & $\begin{array}{l}11 / 22 / 92 \\
02 / 02 / 93 \\
06 / 30 / 93\end{array}$ & $\begin{array}{l}0001 \\
0001 \\
0001\end{array}$ & MG/L & $\begin{array}{l}< \\
< \\
<\end{array}$ & $\begin{array}{l}0.0015 \\
0.01 \\
0.01\end{array}$ & & $\begin{array}{l}0.0015 \\
0.01 \\
0.01\end{array}$ & - \\
\hline WUNGANESE (TOTAL) & $\begin{array}{l}11 / 22 / 92 \\
06 / 30 / 93\end{array}$ & $\begin{array}{l}\text { No01 } \\
\text { N001 }\end{array}$ & MG/L & $\begin{array}{l}< \\
<\end{array}$ & $\begin{array}{l}0.0015 \\
0.01\end{array}$ & & $\begin{array}{l}0.0015 \\
0.01\end{array}$ & - \\
\hline MERCURY & $06 / 05 / 89$ & 0001 & MG/L & $<$ & 0.0002 & & 0.0002 & - \\
\hline MOLYBDENUM & $\begin{array}{l}04 / 22 / 88 \\
06 / 05 / 89 \\
05 / 30 / 91 \\
02 / 25 / 92 \\
08 / 90 / 92 \\
11 / 22 / 92 \\
06 / 30 / 93\end{array}$ & $\begin{array}{l}0001 \\
0001 \\
0001 \\
0001 \\
0001 \\
0001 \\
0001\end{array}$ & MG/L & $\begin{array}{l}< \\
< \\
< \\
< \\
< \\
<\end{array}$ & $\begin{array}{l}0.02 \\
0.01 \\
0.01 \\
0.01 \\
0.007 \\
0.0049 \\
0.01\end{array}$ & $\begin{array}{l}L \\
L \\
H\end{array}$ & $\begin{array}{l}0.01 \\
0.01 \\
0.01 \\
0.01 \\
0.007 \\
0.0049 \\
0.01\end{array}$ & $\begin{array}{l}- \\
- \\
- \\
-\end{array}$ \\
\hline MOLYBDEHUM (TOTAL) & $\begin{array}{l}11 / 22 / 92 \\
06 / 30 / 93\end{array}$ & $\begin{array}{l}\text { No01 } \\
\text { N001 }\end{array}$ & $M G / L$ & $<$ & $\begin{array}{l}0.0049 \\
0.01\end{array}$ & H & $\begin{array}{l}0.0049 \\
0.01\end{array}$ & : \\
\hline KET GROSS ALPHA * & $\begin{array}{l}04 / 22 / 88 \\
05 / 30 / 91 \\
02 / 25 / 92 \\
11 / 22 / 92 \\
02 / 02 / 93 \\
06 / 30 / 93\end{array}$ & $\begin{array}{l}0001 \\
0001 \\
0009 \\
0001 \\
0001 \\
0001\end{array}$ & $\mathrm{PCI} / \mathrm{L}$ & & $\begin{array}{r}5.28 \\
0.06 \\
7.77 \\
-0.43 \\
-2.74 \\
-0.74\end{array}$ & & $\begin{array}{l}- \\
: \\
- \\
-\end{array}$ & $\begin{array}{l}: \\
: \\
:\end{array}$ \\
\hline NET GROSS ALPHA (TOTAL) ** & $\begin{array}{l}11 / 22 / 92 \\
06 / 30 / 93\end{array}$ & $\begin{array}{l}\text { NoOI } \\
\text { N001 }\end{array}$ & $\mathrm{PCI} / \mathrm{L}$ & & $\begin{array}{r}-1.06 \\
0.26\end{array}$ & & - & $\dot{-}$ \\
\hline HICKEL & $\begin{array}{l}06 / 05 / 89 \\
05 / 30 / 91\end{array}$ & $\begin{array}{l}0001 \\
0001\end{array}$ & $M G / L$ & $<$ & $\begin{array}{l}0.04 \\
0.04\end{array}$ & $L$ & $\begin{array}{l}0.04 \\
0.04\end{array}$ & $\dot{-}$ \\
\hline HITRATE & $\begin{array}{l}04 / 22 / 88 \\
06 / 05 / 89 \\
05 / 30 / 91 \\
02 / 25 / 92 \\
08 / 10 / 92 \\
11 / 22 / 92 \\
02 / 02 / 93\end{array}$ & $\begin{array}{l}0001 \\
0001 \\
0001 \\
0001 \\
0001 \\
0001 \\
0001\end{array}$ & $M G / L$ & & $\begin{array}{l}4.1 \\
8.4 \\
7.4 \\
2.7 \\
5.8 \\
3.8 \\
4\end{array}$ & $\begin{array}{l}L \\
L\end{array}$ & $\begin{array}{l}1 . \\
1 . \\
1.0 \\
1.0 \\
0.075 \\
0.044 \\
1\end{array}$ & $\begin{array}{l}- \\
- \\
- \\
- \\
-\end{array}$ \\
\hline NITRATE (TOTAL) & $06 / 30 / 93$ & N001 & MG/L & & 2 & & 1 & - \\
\hline MITRITE AND NITRATE & $05 / 30 / 99$ & 0001 & $M G / L$ & & 1.68 & $\mathbf{L}$ & 0.05 & - \\
\hline PH & $\begin{array}{l}04 / 22 / 88 \\
06 / 05 / 89 \\
05 / 30 / 91 \\
02 / 25 / 92 \\
08 / 10 / 92 \\
11 / 22 / 92\end{array}$ & $\begin{array}{l}0001 \\
0001 \\
0001 \\
0001 \\
0001 \\
0001\end{array}$ & Su & & $\begin{array}{l}7.83 \\
7.83 \\
7.91 \\
7.78 \\
7.88 \\
8.01\end{array}$ & $L$ & $\begin{array}{l}- \\
- \\
- \\
-\end{array}$ & $\begin{array}{l}- \\
- \\
- \\
-\end{array}$ \\
\hline
\end{tabular}

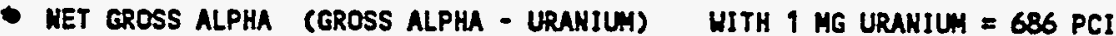

* MET GROSS ALPHA (TOTAL) (TOTAL GROSS ALPHA - TOTAL URANIUN)

PARAMETER VALUE INDICATOR (PVI): < - LESS THAN DETECTION LIMIT

SAMPLE ID COOES:

OTHER PARAMETER VALUE FLAGS:

L - LESS THAN THREE BORE VOLIMES REMOVED BEFORE SAMPLIMG

DOO1 - FILTERED SAMPLE (.45 MICRONS)

N - SPIKE SAMPLE RECOVERY NOT WITHIH CONTROL LIMITS

NOOS - UNFILTERED SAMPLE 
GROUMDHATER QUALITY DATA BY LOCATION

SOWP REV. O Data

SITE: MONO1 MONUMENT VALLEY

LOCATIOH: 0602

MORTH COORDIHATE: $\quad 56377.4$ FT

EAST COORDINATE: 88661.2 FT

$04 / 21 / 88$ TO $12 / 13 / 94$

REPORT DATE: $06 / 20 / 95$

FORHATION OF COAPLETION: ALLUVIUN (AL)

HYDRAULIC FLOM RELATIONSHIP: UNKNOWN (N)

\begin{tabular}{|c|c|c|c|c|c|c|c|c|}
\hline PARAMETER NAME & LOG DATE & $\begin{array}{c}\text { SAMPLE } \\
10\end{array}$ & $\begin{array}{l}\text { UHITS OF } \\
\text { MEASURE }\end{array}$ & PVI & $\begin{array}{l}\text { PARAMETER } \\
\text { VALUE }\end{array}$ & FLAGS & $\begin{array}{l}\text { DETECTION } \\
\text { LIMIT }\end{array}$ & $\begin{array}{l}\text { PARAMETER } \\
\text { UNCERTAINTY }\end{array}$ \\
\hline PH & $\begin{array}{l}02 / 02 / 93 \\
06 / 30 / 93\end{array}$ & $\begin{array}{l}0009 \\
\text { N001 }\end{array}$ & su & & $\begin{array}{l}7.80 \\
7.39\end{array}$ & & $\dot{-}$ & - \\
\hline PHOSPHATE & $06 / 05 / 89$ & 0001 & MG/L & & 0.6 & & 0.9 & - \\
\hline PHOSPHATE (TOTAL) & $06 / 30 / 93$ & NOO1 & MG/L & & 0.2 & & 0.1 & - \\
\hline POTASSIU:Y & $\begin{array}{l}04 / 22 / 88 \\
06 / 05 / 89 \\
05 / 30 / 91 \\
02 / 25 / 92 \\
08 / 10 / 92 \\
02 / 02 / 93 \\
06 / 30 / 93\end{array}$ & $\begin{array}{l}0001 \\
0001 \\
0001 \\
0009 \\
0001 \\
0009 \\
0001\end{array}$ & MG/L & & $\begin{array}{l}1.80 \\
2.1 \\
1.89 \\
1.93 \\
0.99 \\
1.9 \\
1.9\end{array}$ & $\begin{array}{l}L \\
\star L\end{array}$ & $\begin{array}{l}0.01 \\
0.01 \\
0.01 \\
0.01 \\
0.575 \\
0.1 \\
0.1\end{array}$ & $\begin{array}{l}- \\
- \\
- \\
-\end{array}$ \\
\hline POTASSIUM (TOTAL) & $06 / 30 / 93$ & N001 & MG/L & & 1.8 & & 0.1 & - \\
\hline RADIUM-226 & $\begin{array}{l}06 / 05 / 89 \\
05 / 30 / 91 \\
02 / 25 / 92 \\
08 / 10 / 92 \\
11 / 22 / 92\end{array}$ & $\begin{array}{l}0009 \\
0001 \\
0001 \\
0001 \\
0001\end{array}$ & PCI/L & & $\begin{array}{l}0.5 \\
0.7 \\
1.0 \\
0.6 \\
0.8\end{array}$ & $L$ & $\begin{array}{l}1 . \\
1.0 \\
1.0 \\
0.3 \\
0.3\end{array}$ & $\begin{array}{l}0.3 \\
0.2 \\
0.5 \\
0.1 \\
0.3\end{array}$ \\
\hline RADIUH-226 (TOTAL) & $11 / 22 / 92$ & N001 & $\mathrm{PCI} / \mathrm{L}$ & & 0.8 & & 0.3 & 0.3 \\
\hline RADIUM-226 + RADILN-228 & $\begin{array}{l}06 / 05 / 89 \\
05 / 30 / 91 \\
02 / 25 / 92 \\
08 / 10 / 92 \\
11 / 22 / 92\end{array}$ & $\begin{array}{l}0001 \\
0001 \\
0001 \\
0001 \\
0001\end{array}$ & PCI/L & & $\begin{array}{l}0.50 \\
0.70 \\
2.20 \\
3.00 \\
1.20\end{array}$ & & $\begin{array}{l}- \\
- \\
-\end{array}$ & $\begin{array}{l}- \\
- \\
-\end{array}$ \\
\hline RADIUA-226 + RADIUN-228 (TOTAL) & $11 / 22 / 92$ & NOOI & $\mathrm{PCI} / \mathrm{L}$ & & 4.00 & & - & - \\
\hline RADIUA-228 & $\begin{array}{l}06 / 05 / 89 \\
05 / 30 / 91 \\
02 / 25 / 92 \\
08 / 10 / 92 \\
11 / 22 / 92\end{array}$ & $\begin{array}{l}0001 \\
0001 \\
0001 \\
0001 \\
0001\end{array}$ & $\mathrm{PCI} / \mathrm{L}$ & . & $\begin{array}{l}0.0 \\
0.0 \\
1.2 \\
2.4 \\
0.4\end{array}$ & $\begin{array}{l}J L \\
L\end{array}$ & $\begin{array}{l}1 . \\
1.0 \\
1.0 \\
5.0 \\
2.7\end{array}$ & $\begin{array}{l}0.9 \\
1.9 \\
1.7 \\
2.7 \\
1.6\end{array}$ \\
\hline RADIUN-228 (TOTAL) & $11 / 22 / 92$ & N001 & $\mathrm{PCI} / \mathrm{L}$ & & 3.2 & & 2.4 & 1.7 \\
\hline REDOX POTENTIAL & $\begin{array}{l}11 / 22 / 92 \\
06 / 30 / 93\end{array}$ & $\begin{array}{l}0001 \\
\text { N001 }\end{array}$ & mVOLTS & & $\begin{array}{l}458.0 \\
424\end{array}$ & & - & - \\
\hline SELENIUY & $\begin{array}{l}04 / 22 / 88 \\
06 / 05 / 89 \\
05 / 30 / 91 \\
02 / 25 / 92 \\
08 / 10 / 92 \\
11 / 22 / 92\end{array}$ & $\begin{array}{l}0001 \\
0001 \\
0001 \\
0001 \\
0001 \\
0001\end{array}$ & MG/L & $\begin{array}{l}< \\
< \\
<\end{array}$ & $\begin{array}{l}0.006 \\
0.005 \\
0.009 \\
0.006 \\
0.005 \\
0.0015\end{array}$ & $\begin{array}{l}\mathbf{J} \mathbf{L} \\
\mathbf{L} \\
\mathbf{H}\end{array}$ & $\begin{array}{l}0.005 \\
0.005 \\
0.005 \\
0.005 \\
0.005 \\
0.0015\end{array}$ & $\begin{array}{l}- \\
\vdots \\
\vdots \\
-\end{array}$ \\
\hline SELENIUN (TOTAL) & $11 / 22 / 92$ & N001 & MG/L & & 0.0024 & & 0.0015 & - \\
\hline SILICA - SIOZ & $06 / 05 / 89$ & 0001 & MG/L & & 14. & & 2. & - \\
\hline
\end{tabular}

PARAMETER VALUE IMDICATOR (PVI): < - LESS THAN DETECTION LIMIT

SAMPLE ID COOES:

OTHER PARAMETER VALUE FLAGS:

0001 - FILTERED SAMPLE (.45 HICRONS)

* - DUPLICATE AMALYSIS MOT MITHIN COATROL LIHITS

HOO1 - UMFILTERED SAMPLE

J. EstIMATED VALUE

L - LESS THAH THREE BORE VOLLMES REMOVED BEFORE SAMPLIHG

H - SPIKE SAHPLE RECOVERY NOT WITHIN CONTROL LIMITS 
GROWNDWATER OUALITY DATA BY LOCATION

SOUP ReV. $O$ Data

SITE: MONO1 MONLMENT VALLEY

LOCATION: 0602

MORTH COORDIHATE: $\quad 56377.4$ FT

EAST COORDINATE: 88661.2 FT

$04 / 21 / 88$ TO $12 / 13 / 94$

REPORT DATE: 06/20/95

FORHATION OF COMPLETION: ALLUVIUM (AL)

HYDRAULIC FLOU RELATIONSHIP: UNKMOWN (N)

\begin{tabular}{|c|c|c|c|c|c|c|c|c|}
\hline PARAMETER MAME & LOG DATE & $\underset{I D}{\text { SAMPLE }}$ & $\begin{array}{l}\text { UHITS OF } \\
\text { MEASURE }\end{array}$ & PVI & $\begin{array}{l}\text { PARAMETER } \\
\text { VALUE }\end{array}$ & FLAGS & $\begin{array}{l}\text { DETECTION } \\
\text { LIHIT }\end{array}$ & $\begin{array}{l}\text { PARAMETER } \\
\text { UHCERTAINTY }\end{array}$ \\
\hline SILICA - SIO2 & $05 / 30 / 91$ & 0009 & MG/L & & 13.6 & $L$ & 0.1 & - \\
\hline SILICA - SIO2 (TOTAL) & $06 / 30 / 93$ & NoOI & $M G / L$ & & 13.7 & H & 0.5 & - \\
\hline SILVER & $\begin{array}{l}06 / 05 / 89 \\
05 / 30 / 91\end{array}$ & $\begin{array}{l}0001 \\
0001\end{array}$ & MG/L & $<$ & $\begin{array}{l}0.01 \\
0.01\end{array}$ & $\mathbf{L}$ & $\begin{array}{l}0.01 \\
0.01\end{array}$ & $\because$ \\
\hline SCOIUN & $\begin{array}{l}04 / 22 / 88 \\
06 / 05 / 89 \\
05 / 30 / 91 \\
02 / 25 / 92 \\
08 / 10 / 92 \\
02 / 02 / 93 \\
06 / 30 / 93\end{array}$ & $\begin{array}{l}0001 \\
0001 \\
0001 \\
0001 \\
0001 \\
0001 \\
0001\end{array}$ & MG/L & & $\begin{array}{c}97.4 \\
100 . \\
112 . \\
109 . \\
99 \\
101 \\
99\end{array}$ & $\begin{array}{l}\mathbf{L} \\
\mathbf{L}\end{array}$ & $\begin{array}{l}0.002 \\
0.002 \\
5 . \\
5 . \\
5 \\
0.1 \\
9\end{array}$ & $\begin{array}{l}- \\
- \\
- \\
- \\
-\end{array}$ \\
\hline SCOIUM (TOTAL) & $06 / 30 / 93$ & HOOI & MG/L & & 101 & & 1 & - \\
\hline SPECIFIC CONDUCTAKCE & $\begin{array}{l}04 / 22 / 88 \\
06 / 05 / 89 \\
05 / 30 / 91 \\
02 / 25 / 92 \\
08 / 10 / 92 \\
11 / 22 / 92 \\
02 / 02 / 93 \\
06 / 30 / 93\end{array}$ & $\begin{array}{l}0001 \\
0001 \\
0001 \\
0001 \\
0001 \\
0001 \\
0001 \\
1001\end{array}$ & UNHO/CM & & $\begin{array}{l}525 . \\
470 . \\
722 . \\
568 \\
603 \\
580 \\
575 \\
382\end{array}$ & $\begin{array}{l}L \\
L\end{array}$ & $\begin{array}{l}- \\
: \\
: \\
- \\
-\end{array}$ & $\begin{array}{l}- \\
= \\
= \\
- \\
-\end{array}$ \\
\hline STRONTILA & $\begin{array}{l}05 / 30 / 91 \\
02 / 25 / 92 \\
08 / 10 / 92 \\
11 / 22 / 92 \\
06 / 30 / 93\end{array}$ & $\begin{array}{l}0001 \\
0001 \\
0001 \\
0001 \\
0001\end{array}$ & $M G / L$ & & $\begin{array}{l}0.28 \\
0.27 \\
0.29 \\
0.36 \\
0.28\end{array}$ & $\begin{array}{l}L \\
L\end{array}$ & $\begin{array}{l}0.01 \\
0.01 \\
0.2 \\
0.00035 \\
0.01\end{array}$ & $\begin{array}{l}- \\
- \\
-\end{array}$ \\
\hline STRONTIUN (TOTAL) & $\begin{array}{l}11 / 22 / 92 \\
06 / 30 / 93\end{array}$ & $\begin{array}{l}\text { NOO1 } \\
\text { N001 }\end{array}$ & MG/L & & $\begin{array}{l}0.34 \\
0.29\end{array}$ & & $\begin{array}{l}0.00035 \\
0.01\end{array}$ & 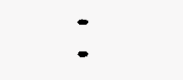 \\
\hline SULFATE & $\begin{array}{l}04 / 22 / 88 \\
06 / 05 / 89 \\
05 / 30 / 91 \\
02 / 25 / 92 \\
08 / 10 / 92 \\
11 / 22 / 92 \\
02 / 02 / 93\end{array}$ & $\begin{array}{l}0001 \\
0001 \\
0001 \\
0001 \\
0001 \\
0001 \\
0001\end{array}$ & MG/L & . & $\begin{array}{l}136 . \\
139 . \\
164 . \\
127 . \\
120 \\
130 \\
126\end{array}$ & $\begin{array}{l}\mathbf{L} \\
\mathbf{L}\end{array}$ & $\begin{array}{c}0.1 \\
0.1 \\
0.1 \\
20 . \\
0.059 \\
1.0 \\
1\end{array}$ & $\begin{array}{l}: \\
: \\
:\end{array}$ \\
\hline SULFATE (TOTAL) & $\begin{array}{l}11 / 22 / 92 \\
06 / 30 / 93\end{array}$ & $\begin{array}{l}\text { N001 } \\
\text { N001 }\end{array}$ & MG/L & & $\begin{array}{l}130 \\
125\end{array}$ & & 1.0 & - \\
\hline SULFIDE & $\begin{array}{l}06 / 05 / 89 \\
05 / 30 / 91\end{array}$ & $\begin{array}{l}0009 \\
0001\end{array}$ & MG/L & $<$ & $\begin{array}{l}0.1 \\
1 .\end{array}$ & IJL & $\begin{array}{l}0.1 \\
1 .\end{array}$ & $\dot{-}$ \\
\hline TEMPERATURE & $\begin{array}{l}04 / 22 / 88 \\
06 / 05 / 89 \\
05 / 30 / 91 \\
02 / 25 / 92 \\
08 / 10 / 92\end{array}$ & $\begin{array}{l}0001 \\
0001 \\
0001 \\
0001 \\
0001\end{array}$ & C - DEGREE & & $\begin{array}{l}13.5 \\
14.8 \\
16.3 \\
14.2 \\
16.8\end{array}$ & $\begin{array}{l}L \\
L\end{array}$ & $\begin{array}{l}- \\
- \\
- \\
-\end{array}$ & $\begin{array}{l}. \\
\dot{-} \\
. \\
-\end{array}$ \\
\hline
\end{tabular}

PARAMETER VALUE INDICATOR (PVI): < - LESS THAN DETECTION LIMIT

SAMPLE ID COOES:

OTHER PARAMETER VALUE FLAGS:

0001 - FILTERED SAMPLE (.45 MICROHS)

H - HOLD TIME EXPIRED, VALUE SUSPECT

NOO1 - UHFILTERED SAMPLE

1 - IMCREASED DETECTION LIMIT DUE TO REQUIRED DILUTION

J - ESTIMATED VALUE

L - LESS tHAN THREE BORE VOLUMES REMOVED BEFORE SNMLIHO 
GROUNDWATER OUALITY DATA BY LOCATION

SOMP ReV. O Data

SITE: MONO 1 HOHIMENT VALLEY

LOCATION: 0602

MORTH COORDINATE: 56377.4 FT

EAST COORDINATE: 88661.2 FT

$04 / 21 / 88$ TO $12 / 13 / 94$

REPORT DATE: 06/20/95

FORHATION OF COMPLETION: ALLUVIUM (AL)

HYDRAULIC FLOW RELATIONSHIP: UNKHOWN (H)

\begin{tabular}{|c|c|c|c|c|c|c|c|c|}
\hline PARAMETER MAME & LOG DATE & $\underset{\text { SAMPLE }}{\text { SO }}$ & $\begin{array}{l}\text { UNITS OF } \\
\text { MEASURE }\end{array}$ & PVI & $\begin{array}{l}\text { PARAMETER } \\
\text { VALUE }\end{array}$ & FLAGS & $\begin{array}{l}\text { DETECTION } \\
\text { LIMIT }\end{array}$ & $\begin{array}{l}\text { PARAMETER } \\
\text { UNCERTAINTY }\end{array}$ \\
\hline TEMPERATURE & $\begin{array}{l}11 / 22 / 92 \\
02 / 02 / 93 \\
06 / 30 / 93\end{array}$ & $\begin{array}{l}0001 \\
0001 \\
\text { N001 }\end{array}$ & C - DEGREE & & $\begin{array}{l}16.0 \\
14.8 \\
15.9\end{array}$ & & $\dot{-}$ & $\dot{-}$ \\
\hline THALLIUA & $\begin{array}{l}06 / 05 / 89 \\
05 / 30 / 99\end{array}$ & $\begin{array}{l}0001 \\
0001\end{array}$ & MG/L & $<$ & $\begin{array}{l}0.01 \\
0.01\end{array}$ & $\mathbf{L}$ & $\begin{array}{l}0.01 \\
0.01\end{array}$ & $\dot{-}$ \\
\hline THORIUN-230 & $05 / 30 / 91$ & 0001 & PCI/L & & 1.0 & $L$ & 1. & 0.5 \\
\hline TIH & $\begin{array}{l}06 / 05 / 89 \\
05 / 30 / 91\end{array}$ & $\begin{array}{l}0001 \\
0001\end{array}$ & MG/L & $<$ & $\begin{array}{l}0.005 \\
0.01\end{array}$ & IL & $\begin{array}{l}0.005 \\
0.01\end{array}$ & $\dot{-}$ \\
\hline TOTAL DISSOLVED SOLIDS & $\begin{array}{l}04 / 22 / 88 \\
06 / 05 / 89 \\
05 / 30 / 91 \\
02 / 25 / 92 \\
08 / 10 / 92 \\
02 / 02 / 93\end{array}$ & $\begin{array}{l}0001 \\
0001 \\
0001 \\
0001 \\
0009 \\
0001\end{array}$ & MG/L & & $\begin{array}{l}444 . \\
433 . \\
439 . \\
449 . \\
530 \\
400\end{array}$ & $L$ & $\begin{array}{l}10 . \\
10 . \\
10 . \\
10 . \\
42 \\
10\end{array}$ & $\begin{array}{l}- \\
- \\
- \\
-\end{array}$ \\
\hline TOTAL DISSOLVED SOLIDS (TOTAL) & $06 / 30 / 93$ & NOOI & MG/L & & 420 & H & 10 & - \\
\hline TOTAL ORGAHIC CARBON & $\begin{array}{l}04 / 22 / 88 \\
05 / 30 / 91 \\
02 / 25 / 92 \\
08 / 10 / 92\end{array}$ & $\begin{array}{l}0001 \\
0001 \\
0001 \\
0001\end{array}$ & MG/L & $\begin{array}{l}< \\
<\end{array}$ & $\begin{array}{l}61.2 \\
1 . \\
1.0\end{array}$ & $\begin{array}{l}L \\
L\end{array}$ & $\begin{array}{l}1 . \\
1 . \\
1 . \\
1.0\end{array}$ & $\begin{array}{l}: \\
-\end{array}$ \\
\hline URAKIUM & $\begin{array}{l}04 / 22 / 88 \\
06 / 05 / 89 \\
05 / 30 / 91 \\
02 / 25 / 92 \\
11 / 22 / 92 \\
02 / 02 / 93 \\
06 / 30 / 93\end{array}$ & $\begin{array}{l}0009 \\
0001 \\
0001 \\
0009 \\
0001 \\
0001 \\
0001\end{array}$ & $M G / L$ & $<$ & $\begin{array}{l}0.0044 \\
0.0035 \\
0.001 \\
0.005 \\
0.005 \\
0.004 \\
0.004\end{array}$ & $\mathbf{L}$ & $\begin{array}{l}0.003 \\
0.003 \\
0.001 \\
0.001 \\
0.001 \\
0.001 \\
0.001\end{array}$ & $\begin{array}{l}- \\
- \\
- \\
- \\
-\end{array}$ \\
\hline URAHILH (TOTAL) & $\begin{array}{l}11 / 22 / 92 \\
06 / 30 / 93\end{array}$ & $\begin{array}{l}\text { NO01 } \\
\text { HOO1 }\end{array}$ & $M G / L$ & & $\begin{array}{l}0.003 \\
0.004\end{array}$ & & $\begin{array}{l}0.001 \\
0.001\end{array}$ & - \\
\hline VAKADILM & $\begin{array}{l}06 / 05 / 89 \\
05 / 30 / 91 \\
02 / 25 / 92 \\
08 / 10 / 92 \\
11 / 22 / 92 \\
06 / 30 / 93\end{array}$ & $\begin{array}{l}0001 \\
0001 \\
0001 \\
0001 \\
0001 \\
0001\end{array}$ & $M G / L$ & $\begin{array}{l}< \\
< \\
< \\
< \\
<\end{array}$ & $\begin{array}{l}0.01 \\
0.01 \\
0.01 \\
0.05 \\
0.0019 \\
0.01\end{array}$ & $L$ & $\begin{array}{l}0.01 \\
0.01 \\
0.01 \\
0.05 \\
0.0019 \\
0.01\end{array}$ & $\begin{array}{l}- \\
- \\
- \\
-\end{array}$ \\
\hline VAHADIUA (TOTAL) & $\begin{array}{l}19 / 22 / 92 \\
06 / 30 / 93\end{array}$ & $\begin{array}{l}\text { N001 } \\
\text { N001 }\end{array}$ & MG/L & $<$ & $\begin{array}{l}0.0020 \\
0.01\end{array}$ & & $\begin{array}{l}0.0019 \\
0.01\end{array}$ & $\div$ \\
\hline ZINC & $\begin{array}{l}06 / 05 / 89 \\
05 / 30 / 91 \\
02 / 25 / 92 \\
08 / 10 / 92 \\
11 / 22 / 92 \\
06 / 30 / 93\end{array}$ & $\begin{array}{l}0001 \\
0001 \\
0001 \\
0001 \\
0001 \\
0001\end{array}$ & MG/L & $\begin{array}{l}< \\
< \\
<\end{array}$ & $\begin{array}{l}0.005 \\
0.005 \\
0.005 \\
0.02 \\
0.0012 \\
0.019\end{array}$ & $\begin{array}{l}L \\
L \\
J\end{array}$ & $\begin{array}{l}0.005 \\
0.005 \\
0.005 \\
0.02 \\
0.0010 \\
0.005\end{array}$ & $\begin{array}{l}- \\
- \\
-\end{array}$ \\
\hline
\end{tabular}

PARAMETER VALUE INDICATOR (PVI): < - LESS THAN DETECTION LIMIT

SAMPLE ID COOES:

OTHER PARAMETER VALUE FLAGS:

0001 - FILTERED SAMPLE (.45 MICRONS)

H - HOLD TIME EXPIRED, VALUE SUSPECT

NOO1 - UNFILTERED SAMPLE

I. INCREASED DETECTION LIMIT DUE TO REOUIRED DILUTION

$J$ - ESTIMATED VALUE

- L - LESS THAN THREE BORE VOLLMES REMOVEd BEFORE SAMPLIKG 
GROUHDWATER QUALITY DATA BY LOCATION

SOUP Rev. 0 Data

SITE: MONO1 MONUMENT VALLEY

LOCATIOH: 0602

MORTH COORDIHATE: 56377.4 FT

EAST COORDINATE: 88661.2 FT

04/21/88 TO $12 / 13 / 94$

REPORT DATE: 06/20/95

FORMATIOH OF COAPLETIOH: ALLUVIUM (AL)

HYDRAULIC FLON RELATIONSHIP: UHKHOWH (N)

\begin{tabular}{|c|c|c|c|c|c|c|c|}
\hline PARAMETER HAME & LOG DATE & $\begin{array}{c}\text { SAMPLE } \\
\text { ID }\end{array}$ & $\begin{array}{l}\text { UNITS OF } \\
\text { MEASURE }\end{array}$ & PVI & $\begin{array}{l}\text { PARAMETER } \\
\text { VALUE FLAGS }\end{array}$ & $\begin{array}{l}\text { DETECTION } \\
\text { LIMIT }\end{array}$ & $\begin{array}{l}\text { PARAMETER } \\
\text { UNCERTAINTY }\end{array}$ \\
\hline ZINC (TOTAL) & $\begin{array}{l}11 / 22 / 92 \\
06 / 30 / 93\end{array}$ & $\begin{array}{l}\text { NOO1 } \\
\text { NOO1 }\end{array}$ & $M G / L$ & & $\begin{array}{l}0.0050 \\
0.024 \quad \mathrm{~J}\end{array}$ & $\begin{array}{l}0.0010 \\
0.005\end{array}$ & $\dot{-}$ \\
\hline
\end{tabular}

PARAMETER VALUE INDICATOR (PVI):

< - LESS Than DETECTION LIMIT

SAMPLE ID COOES:

NOO1 - UHFILTERED SAMPLE

OTHER PARAMETER VALUe flags:

J - estimated VALUE 
GROUMDUATER OUALITY DATA BY LOCATION

SOUP ReV. O Data

SITE: MONO1 MONLWENT VALLEY

LOCATIOH: 0603

NORTH COORDINATE: 57812.7 FT

EAST COORDINATE: 89036.9 FT

O4/21/88 TO $12 / 13 / 94$

REPORT DATE: 06/20/95

FORMATION OF COMPLETIOH: ALLUVIUM (AL)

HYDRAULIC FLOW RELATIOHSHIP: CROSS GRADIENT (C)

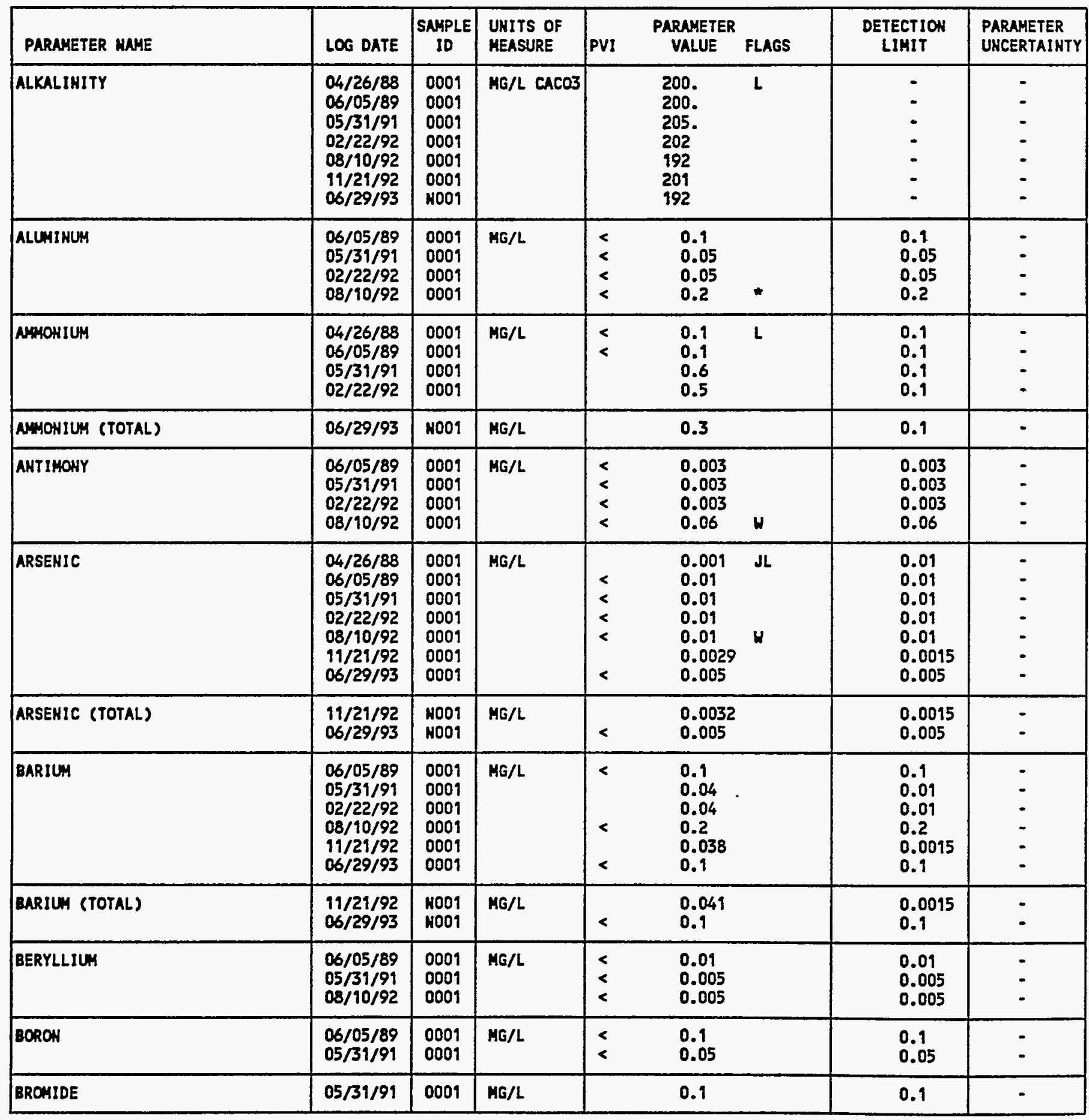

PARAMETER VALUE IMDICATOR (PVI): < - LESS THAN DETECTION LIMIT

SAMPLE ID COOES:

OTHER PARAMETER VALUE FLAGS:

0009 - FILTERED SAMPLE (.45 HICRONS)

- DUPLICATE AMALYSIS NOT HITHIN CONTROL LIMITS

NOO - UAFILTERED SNMPLE

J - ESTIMRTED VALUE

L - LESS THAN THREE BORE VOLUAES REMOVED BEFORE SAMPLIHG

W - POST-DIGEST SPIKE OUT OF CHTR LIM WHILE SAMP ABS < 50\% SPIKE 
GROUNDWATER OUALITY DATA BY LOCATION

SOUP Rev. O Data

SITE: MONO1 MONUWENT VALLEY

LOCATION: 0603

MORTH COORDIMATE: 57812.7 FT

EAST COORDIHATE: 89036.9 FT

$04 / 21 / 88$ TO $12 / 13 / 94$

REPORT DATE: 06/20/95

FORMATION OF COMPLETION: ALLUVIUM (AL)

HYDRAULIC FLON RELATIONSHIP: CROSS GRADIENT (C)

\begin{tabular}{|c|c|c|c|c|c|c|c|c|}
\hline PARAMETER MNHE & LOG DATE & $\underset{\text { ID }}{\text { SAMPLE }}$ & $\begin{array}{l}\text { UNITS OF } \\
\text { MEASURE }\end{array}$ & PVI & $\begin{array}{l}\text { PARAMETER } \\
\text { VALUE }\end{array}$ & FLAGS & $\begin{array}{l}\text { DETECTION } \\
\text { LIMIT }\end{array}$ & $\begin{array}{l}\text { PARAMETER } \\
\text { UHCERTAINTY }\end{array}$ \\
\hline CADHIU: & $\begin{array}{l}04 / 26 / 88 \\
06 / 05 / 89 \\
05 / 31 / 91 \\
02 / 22 / 92 \\
08 / 10 / 92 \\
11 / 21 / 92\end{array}$ & $\begin{array}{l}0001 \\
0001 \\
0001 \\
0001 \\
0001 \\
0001\end{array}$ & MG/L & $\begin{array}{l}< \\
< \\
< \\
<\end{array}$ & $\begin{array}{l}0.005 \\
0.001 \\
0.001 \\
0.0005 \\
0.005 \\
0.00013\end{array}$ & $\begin{array}{l}L \\
\end{array}$ & $\begin{array}{l}0.001 \\
0.001 \\
0.001 \\
0.0005 \\
0.005 \\
0.00013\end{array}$ & $\begin{array}{l}: \\
: \\
:\end{array}$ \\
\hline CADHIU: (TOTAL) & $11 / 21 / 92$ & NOOI & $M G / L$ & $<$ & \multicolumn{2}{|c|}{$0.00013 \mathrm{NH}$} & 0.00013 & - \\
\hline CALCIUH & $\begin{array}{l}04 / 26 / 88 \\
06 / 05 / 89 \\
05 / 31 / 91 \\
02 / 22 / 92 \\
08 / 10 / 92 \\
06 / 29 / 93\end{array}$ & $\begin{array}{l}0001 \\
0001 \\
0001 \\
0001 \\
0001 \\
0001\end{array}$ & $M G / L$ & & $\begin{array}{l}19.3 \\
19.5 \\
19.0 \\
17.9 \\
20 \\
19.4\end{array}$ & $L$ & $\begin{array}{l}0.01 \\
0.01 \\
0.1 \\
0.1 \\
5 \\
0.5\end{array}$ & $\begin{array}{l}. \\
: \\
: \\
.\end{array}$ \\
\hline CALCIUH (TOTAL) & $06 / 29 / 93$ & HOOT & $M G / L$ & \multicolumn{3}{|c|}{17.9} & 0.5 & - \\
\hline CHLORIDE & $\begin{array}{l}04 / 26 / 88 \\
06 / 05 / 89 \\
05 / 31 / 91 \\
02 / 22 / 92 \\
08 / 10 / 92\end{array}$ & $\begin{array}{l}0001 \\
0001 \\
0001 \\
0001 \\
0001\end{array}$ & MG/L & & $\begin{array}{l}13 . \\
11 . \\
12.6 \\
19.9 \\
11\end{array}$ & $L$ & $\begin{array}{l}1 . \\
1 . \\
0.5 \\
0.5 \\
0.016\end{array}$ & $\begin{array}{l}- \\
- \\
- \\
-\end{array}$ \\
\hline CHLORIDE (TOTAL) & $06 / 29 / 93$ & NOO1 & $M G / L$ & \multicolumn{3}{|c|}{12.9} & 0.5 & - \\
\hline CHROMIUN & $\begin{array}{l}06 / 05 / 89 \\
05 / 31 / 91 \\
02 / 22 / 92 \\
08 / 10 / 92 \\
11 / 21 / 92\end{array}$ & $\begin{array}{l}0001 \\
0001 \\
0001 \\
0001 \\
0001\end{array}$ & $M G / L$ & $\begin{array}{l}< \\
< \\
< \\
<\end{array}$ & \multicolumn{2}{|l|}{$\begin{array}{l}0.01 \\
0.01 \\
0.01 \\
0.01 \\
0.0053\end{array}$} & $\begin{array}{l}0.01 \\
0.01 \\
0.01 \\
0.01 \\
0.0053\end{array}$ & $\begin{array}{l}- \\
- \\
- \\
-\end{array}$ \\
\hline CHROHIUM (TOTAL) & $11 / 21 / 92$ & NoO1 & $M G / L$ & $<$ & \multicolumn{2}{|l|}{0.0053} & 0.0053 & - \\
\hline COBALT & $\begin{array}{l}06 / 05 / 89 \\
05 / 31 / 91\end{array}$ & $\begin{array}{l}0001 \\
0001\end{array}$ & $M G / L$ & $<$ & \multicolumn{2}{|l|}{$\begin{array}{l}0.05 \\
0.03\end{array}$} & $\begin{array}{l}0.05 \\
0.03\end{array}$ & - \\
\hline COPPER & $\begin{array}{l}06 / 05 / 89 \\
05 / 31 / 91\end{array}$ & $\begin{array}{l}0001 \\
0001\end{array}$ & $M G / L$ & $<$ & \multicolumn{2}{|l|}{$\begin{array}{l}0.02 \\
0.01\end{array}$} & $\begin{array}{l}0.02 \\
0.01\end{array}$ & $\dot{-}$ \\
\hline CYAKIDE & $06 / 05 / 89$ & 0001 & $M G / L$ & $<$ & \multicolumn{2}{|l|}{0.01} & 0.01 & - \\
\hline DISSOLVED OXYGEN & $\begin{array}{l}11 / 21 / 92 \\
06 / 29 / 93\end{array}$ & $\begin{array}{l}0001 \\
\mathrm{HOO1}\end{array}$ & MG/L & & \multicolumn{2}{|l|}{$\begin{array}{l}0.0 \\
0.6\end{array}$} & : & - \\
\hline FLUORIDE & $\begin{array}{l}06 / 05 / 89 \\
05 / 31 / 91\end{array}$ & $\begin{array}{l}0001 \\
0001\end{array}$ & MG/L & & \multicolumn{2}{|l|}{$\begin{array}{l}0.2 \\
0.1\end{array}$} & $\begin{array}{l}0.1 \\
0.1\end{array}$ & $\because$ \\
\hline GROSS ALPHA & $\begin{array}{l}04 / 26 / 88 \\
05 / 31 / 91 \\
02 / 22 / 92 \\
08 / 10 / 92 \\
11 / 21 / 92\end{array}$ & $\begin{array}{l}0001 \\
0001 \\
0001 \\
0001 \\
0001\end{array}$ & $\mathrm{PCI} / \mathrm{L}$ & & $\begin{array}{l}5.9 \\
0.0 \\
1.3 \\
1.1 \\
6.6\end{array}$ & $\begin{array}{l}\mathbf{L} \\
\mathbf{l} \\
\mathbf{N}\end{array}$ & $\begin{array}{l}0.2 \\
1.10 \\
1.0 \\
4.2 \\
3.1\end{array}$ & $\begin{array}{l}3.3 \\
4.8 \\
4.1 \\
2.2 \\
3.1\end{array}$ \\
\hline
\end{tabular}

PARAMETER VALUE IMDICATOR (PVI): < - LESS THAH DETECTION LIMIT

OTHER PARNGETER VALUE FLAGS:

E - ESTIMUTED VALUE BECAUSE OF INTERFERENCE, SEE CASE MARRATIVE

I - INCREASED DETECTION LIMIT DUE TO REOUIRED DILUTION

L - LESS THAN THREE BORE VOLUAES REMOVED BEFORE SAHPLIHG

M - SPIKE SNMPLE RECOVERY NOT WITHIN CONTROL LIMITS

$H$ - POST-DIGEST SPIKE OUT OF CHTR LIM WHILE SAMP ABS < 50\% SPIKE
SUMPLE ID COOES:

0001 - FILTERED SAMPLE (.45 MICROHS)

NOO1 - UKFILTERED SAMPLE 
GROUNDUATER QUALITY DATA BY LOCATION

SOMP ReV. O Data

SITE: MONO I MONUWENT VALLEY

LOCATIOH: 0603

HORTH COORDINATE: 57812.7 FT

EAST COORDINATE: 89036.9 FT

$04 / 21 / 88$ TO $12 / 13 / 94$

REPORT DATE: $06 / 20 / 95$

FORMATION OF COMPLETIOH: ALLUVIUM (AL)

HYDRAULIC FLON RELATIONSHIP: CROSS GRADIENT (C)

\begin{tabular}{|c|c|c|c|c|c|c|c|c|}
\hline PARAMETER NAME & LOG DATE & $\underset{\text { ID }}{\text { SAMPLE }}$ & $\begin{array}{l}\text { UHITS OF } \\
\text { MEASURE }\end{array}$ & PVI & $\begin{array}{l}\text { PARAMETER } \\
\text { VALUE }\end{array}$ & FLAGS & $\begin{array}{l}\text { DETECTIOH } \\
\text { LIMIT }\end{array}$ & $\begin{array}{l}\text { PARAHETER } \\
\text { UNCERTAINTY }\end{array}$ \\
\hline GROSS ALPHA & $06 / 29 / 93$ & 0001 & PCI/L & & 9 & & 3 & 6 \\
\hline CROSS ALPHA (TOTAL) & $\begin{array}{l}11 / 21 / 92 \\
06 / 29 / 93\end{array}$ & $\begin{array}{l}\text { NoO1 } \\
\text { N001 }\end{array}$ & $\mathrm{PCI} / \mathrm{L}$ & & $\begin{array}{l}0.7 \\
5\end{array}$ & N & $\begin{array}{l}6.7 \\
3\end{array}$ & $\begin{array}{l}4.1 \\
5\end{array}$ \\
\hline GROSS BETA & $\begin{array}{l}04 / 26 / 88 \\
05 / 31 / 91 \\
02 / 22 / 92 \\
08 / 10 / 92 \\
11 / 21 / 92 \\
06 / 29 / 93\end{array}$ & $\begin{array}{l}0001 \\
0001 \\
0001 \\
0001 \\
0001 \\
0001\end{array}$ & PCI/L & & $\begin{array}{l}4.5 \\
3.1 \\
2.2 \\
5.6 \\
0.0 \\
4\end{array}$ & $\begin{array}{l}\text { L } \\
\text { I }\end{array}$ & $\begin{array}{l}1 . \\
0.5 \\
0.5 \\
4.4 \\
5.6 \\
4\end{array}$ & $\begin{array}{l}1.7 \\
2.9 \\
2.3 \\
2.9 \\
3.2 \\
4\end{array}$ \\
\hline GROSS BETA (TOTAL) & $\begin{array}{l}11 / 21 / 92 \\
06 / 29 / 93\end{array}$ & $\begin{array}{l}\text { YOOI } \\
\text { NOOI }\end{array}$ & PCI/L & & $\begin{array}{l}4.0 \\
5\end{array}$ & $\mathbf{H}$ & $\begin{array}{l}4.9 \\
4\end{array}$ & 3.1 \\
\hline IRON & $\begin{array}{l}06 / 05 / 89 \\
05 / 31 / 91 \\
02 / 22 / 92 \\
08 / 10 / 92 \\
06 / 29 / 93\end{array}$ & $\begin{array}{l}0001 \\
0001 \\
0001 \\
0001 \\
0001\end{array}$ & MG/L & $\begin{array}{l}< \\
< \\
< \\
< \\
<\end{array}$ & $\begin{array}{l}0.03 \\
0.03 \\
0.03 \\
0.01 \\
0.03\end{array}$ & $\begin{array}{l}J \\
J\end{array}$ & $\begin{array}{l}0.03 \\
0.03 \\
0.03 \\
0.1 \\
0.03\end{array}$ & : \\
\hline IRON (TOTAL) & $06 / 29 / 93$ & NOOS & MG/L & & 0.12 & J & 0.03 & - \\
\hline LEAD & $\begin{array}{l}06 / 05 / 89 \\
05 / 31 / 91 \\
11 / 21 / 92\end{array}$ & $\begin{array}{l}0001 \\
0001 \\
0001\end{array}$ & HG/L & $\begin{array}{l}< \\
<\end{array}$ & $\begin{array}{l}0.01 \\
0.005 \\
0.0015\end{array}$ & & $\begin{array}{l}0.01 \\
0.005 \\
0.0015\end{array}$ & - \\
\hline LEAD (TOTAL) & $19 / 21 / 92$ & NOOI & $M G / L$ & $<$ & 0.0015 & & 0.0015 & - \\
\hline LEAD-210 & $11 / 21 / 92$ & 0001 & PCI/L & & 0.0 & & 2.0 & 1.2 \\
\hline LEAD-210 (TOTAL) & $11 / 21 / 92$ & K001 & PCI/L & & 1.2 & & 2.0 & 9.2 \\
\hline MAGHESIUM & $\begin{array}{l}04 / 26 / 88 \\
06 / 05 / 89 \\
05 / 31 / 91 \\
02 / 22 / 92 \\
08 / 10 / 92 \\
06 / 29 / 93\end{array}$ & $\begin{array}{l}0001 \\
0001 \\
0001 \\
0001 \\
0001 \\
0001\end{array}$ & $M G / L$ & & $\begin{array}{l}13.2 \\
14.1 \\
14.4 \\
13.8 \\
14 \\
14.1\end{array}$ & $\mathbf{L}$ & $\begin{array}{l}0.001 \\
0.001 \\
0.1 \\
0.1 \\
5 \\
0.1\end{array}$ & $\begin{array}{l}- \\
: \\
- \\
-\end{array}$ \\
\hline MAGNESIUN (TOTAL) & $06 / 29 / 93$ & N001 & MG/L & & 13.3 & & 0.1 & - \\
\hline MAKGAHESE & $\begin{array}{l}06 / 05 / 89 \\
05 / 31 / 91 \\
02 / 22 / 92 \\
08 / 10 / 92 \\
111 / 21 / 92 \\
06 / 29 / 93\end{array}$ & $\begin{array}{l}0001 \\
0001 \\
0001 \\
0001 \\
0001 \\
0001\end{array}$ & MG/L & $\begin{array}{l}< \\
< \\
< \\
<\end{array}$ & $\begin{array}{l}0.01 \\
0.01 \\
0.01 \\
0.01 \\
0.0038 \\
0.01\end{array}$ & & $\begin{array}{l}0.01 \\
0.01 \\
0.01 \\
0.01 \\
0.0015 \\
0.01\end{array}$ & $\begin{array}{l}- \\
\dot{-} \\
\dot{-} \\
\dot{-}\end{array}$ \\
\hline MANGANESE (TOTAL) & $\begin{array}{l}11 / 29 / 92 \\
06 / 29 / 93\end{array}$ & $\begin{array}{l}\text { N001 } \\
\text { N001 }\end{array}$ & $M G / L$ & $<$ & $\begin{array}{l}0.0040 \\
0.09\end{array}$ & & $\begin{array}{l}0.0015 \\
0.09\end{array}$ & - \\
\hline MERCURY & $06 / 05 / 89$ & 0001 & MG/L & & 0.0002 & & 0.0002 & - \\
\hline
\end{tabular}

PARAMETER VALUE INDICATOR (PVI): < - LESS THAN DETECTION LIMIT

SAHPLE ID COOES:

0001 - FILTERED SAMPLE (.45 MICRONS)

OTHER PARAMETER VALUE FLAGS:

NOOI - UMFILTERED SNMPLE

1 - IMCREASED DETECTION LIMIT DUE TO REQUIRED DILUTION

J - ESTIMATED VALUE

L - LESS THAH THREE BORE VOLUMES REMOVED BEFORE SMMPLIHG

H - SPIKE SAMPLE RECOVERY MOT MITHIN CONTROL LIMITS 
SITE: MOHOI MONUMENT VALLEY

LOCATIOH: 0603

NORTH COORDINATE: 57812.7 FT

EAST COORDINATE: 89036.9 FT

04/21/88 TO $12 / 13 / 94$

REPORT DATE: $06 / 20 / 95$

FORMATION OF COMPLETION: ALLUVIUN (AL)

HYORAULIC FLOW RELATIONSHIP: CROSS GRADIENT (C)

\begin{tabular}{|c|c|c|c|c|c|c|c|c|}
\hline PARANETER NAME & LOG DATE & $\mid \begin{array}{c}\text { SAMPLE } \\
\text { ID }\end{array}$ & $\begin{array}{l}\text { UHITS OF } \\
\text { MEASURE }\end{array}$ & PVI & $\begin{array}{l}\text { PARAMETER } \\
\text { VALUE }\end{array}$ & FLAGS & $\begin{array}{l}\text { DETECTION } \\
\text { LIMIT }\end{array}$ & $\begin{array}{l}\text { PARAMETER } \\
\text { UNCERTAINTY }\end{array}$ \\
\hline MOLYBDEKUU & $\begin{array}{l}04 / 26 / 88 \\
06 / 05 / 89 \\
05 / 31 / 91 \\
02 / 22 / 92 \\
08 / 10 / 92 \\
11 / 21 / 92 \\
06 / 29 / 93\end{array}$ & $\begin{array}{l}0001 \\
0001 \\
0001 \\
0001 \\
0001 \\
0001 \\
0001\end{array}$ & MG/L & $\begin{array}{l}< \\
< \\
< \\
<\end{array}$ & $\begin{array}{l}0.02 \\
0.01 \\
0.01 \\
0.01 \\
0.007 \\
0.0049 \\
0.01\end{array}$ & N & $\begin{array}{l}0.01 \\
0.01 \\
0.01 \\
0.01 \\
0.007 \\
0.0049 \\
0.01\end{array}$ & $\begin{array}{l}- \\
- \\
- \\
- \\
-\end{array}$ \\
\hline MOLYBDENUM (TOTAL) & $\begin{array}{l}11 / 21 / 92 \\
06 / 29 / 93\end{array}$ & $\begin{array}{l}\text { NOOI } \\
\text { N001 }\end{array}$ & $M G / L$ & $<$ & $\begin{array}{l}0.0049 \\
0.01\end{array}$ & N & $\begin{array}{l}0.0049 \\
0.01\end{array}$ & - \\
\hline NET GROSS ALPHA • & $\begin{array}{l}04 / 26 / 88 \\
05 / 31 / 91 \\
02 / 22 / 92 \\
11 / 21 / 92 \\
06 / 29 / 93\end{array}$ & $\begin{array}{l}0001 \\
0001 \\
0001 \\
0009 \\
0009\end{array}$ & PCI/L & & $\begin{array}{r}3.84 \\
-0.34 \\
-0.76 \\
3.17 \\
6.94\end{array}$ & & - & $\begin{array}{l}- \\
- \\
- \\
.\end{array}$ \\
\hline HET GROSS ALPHA (TOTAL) ** & $\begin{array}{l}11 / 21 / 92 \\
06 / 29 / 93\end{array}$ & $\begin{array}{l}\text { NOOI } \\
\text { NO01 }\end{array}$ & PCI/L & & $\begin{array}{r}-1.36 \\
3.63\end{array}$ & & $\dot{-}$ & - \\
\hline HICKEL & $\begin{array}{l}06 / 05 / 89 \\
05 / 31 / 91\end{array}$ & $\begin{array}{l}0009 \\
0001\end{array}$ & $M G / L$ & $<$ & $\begin{array}{l}0.04 \\
0.04\end{array}$ & & $\begin{array}{l}0.04 \\
0.04\end{array}$ & - \\
\hline NITRATE & $\begin{array}{l}04 / 26 / 88 \\
06 / 05 / 89 \\
05 / 31 / 91 \\
02 / 22 / 92 \\
08 / 10 / 92 \\
11 / 21 / 92\end{array}$ & $\begin{array}{l}0009 \\
0001 \\
0001 \\
0001 \\
0001 \\
0001\end{array}$ & $M G / L$ & & $\begin{array}{r}2.0 \\
10.6 \\
4.0 \\
1.0 \\
4.9 \\
44\end{array}$ & $L$ & $\begin{array}{l}1 . \\
1 . \\
1.0 \\
1.0 \\
0.075 \\
0.044\end{array}$ & $\begin{array}{l}- \\
- \\
- \\
-\end{array}$ \\
\hline HITRATE (TOTAL) & $06 / 29 / 93$ & NoO1 & $M G / L$ & & 1 & & 1 & - \\
\hline MITRITE AND NITRATE & $05 / 31 / 91$ & 0001 & $M G / L$ & & 0.90 & & 0.05 & - \\
\hline PH & $\begin{array}{l}04 / 26 / 88 \\
06 / 05 / 89 \\
05 / 31 / 91 \\
02 / 22 / 92 \\
08 / 10 / 92 \\
11 / 21 / 92 \\
06 / 29 / 93\end{array}$ & $\begin{array}{l}0001 \\
0001 \\
0001 \\
0001 \\
0001 \\
0001 \\
\text { N001 }\end{array}$ & su & . & $\begin{array}{l}7.95 \\
7.79 \\
7.96 \\
7.83 \\
7.73 \\
8.12 \\
7.60\end{array}$ & $\mathbf{L}$ & $\begin{array}{l}- \\
- \\
- \\
-\end{array}$ & $\begin{array}{l}- \\
- \\
- \\
- \\
-\end{array}$ \\
\hline PHOSPHATE & $06 / 05 / 89$ & 0001 & MG/L & & 0.6 & & 0.1 & - \\
\hline PHOSPHATE (TOTAL) & $06 / 29 / 93$ & N001 & HG/L & & 0.3 & & 0.1 & - \\
\hline POTASSIUM & $\begin{array}{l}04 / 26 / 88 \\
06 / 05 / 89 \\
05 / 31 / 91 \\
02 / 22 / 92 \\
08 / 10 / 92\end{array}$ & $\begin{array}{l}0001 \\
0001 \\
0001 \\
0001 \\
0001\end{array}$ & $M G / L$ & & $\begin{array}{l}2.40 \\
2.3 \\
2.6 \\
2.57 \\
1.9\end{array}$ & $\star$ & $\begin{array}{l}0.01 \\
0.01 \\
0.01 \\
0.01 \\
0.575\end{array}$ & : \\
\hline
\end{tabular}

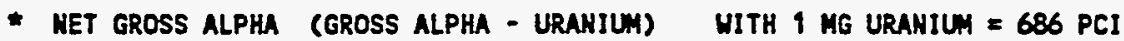

* MET GROSS ALPHA (TOTAL) (TOTAL GROSS ALPHA - TOTAL URANIUH)

PARAHETER VALUE IMDICATOR (PVI): < - LESS THAN DETECTION LIMIT

OTHER PARAMETER VALUE FLAGS:

- DUPLICATE AMALYSIS hOT HITHIM CONTROL LIMITS

L - LESS THAN THREE BORE VOLUMES REMOVED BEFORE SAMPLING

N - SPIKE SAMPLE RECOVERY NOT MITHIN CONTROL LIMITS
SAMPLE ID COOES:

ODOI - FILTERED SAMPLE (.45 MICRONS)

NOO1 - UNFILTERED SAMPLE 
GROUKDUATER QUALITY DATA BY LOCATION

SOUP ReV. O Data

SITE: HONOI MONUNENT VALLEY

LOCATION: 0603

NORTH COORDINATE: 57812.7 FT

EAST COORDINATE: 89036.9 FT

04/21/88 TO 12/13/94

REPORT DATE: 06/20/95

FORMATION OF COMPLETIOH: ALLUVIUM (AL)

HYDRAULIC FLON RELATIOHSHIP: CROSS GRADIENT (C)

\begin{tabular}{|c|c|c|c|c|c|c|c|c|}
\hline PARAMETER MAME & LOG DATE & $\underset{\text { SD }}{\text { SAMPLE }}$ & $\begin{array}{l}\text { UNITS OF } \\
\text { MEASURE }\end{array}$ & PVI & $\begin{array}{l}\text { PARAMETER } \\
\text { VALUE }\end{array}$ & FLAGS & $\begin{array}{l}\text { DETECTION } \\
\text { LIMIT }\end{array}$ & $\begin{array}{l}\text { PARAMETER } \\
\text { UNCERTAINTY }\end{array}$ \\
\hline POTASSIUM & $06 / 29 / 93$ & 0001 & MG/L & & 2.5 & & 0.1 & - \\
\hline POTASSIUH (TOTAL) & $06 / 29 / 93$ & K001 & $M G / L$ & & 2.3 & & 0.1 & - \\
\hline RADIUH-226 & $\begin{array}{l}06 / 05 / 89 \\
05 / 31 / 91 \\
02 / 22 / 92 \\
08 / 10 / 92 \\
11 / 21 / 92\end{array}$ & $\begin{array}{l}0001 \\
0001 \\
0001 \\
0001 \\
0001\end{array}$ & $\mathrm{PCI} / \mathrm{L}$ & & $\begin{array}{l}0.3 \\
0.3 \\
0.6 \\
0.43 \\
0.9\end{array}$ & & $\begin{array}{l}1 . \\
1 . \\
1.0 \\
0.48 \\
0.3\end{array}$ & $\begin{array}{l}0.2 \\
0.2 . \\
0.4 \\
0.07 \\
0.3\end{array}$ \\
\hline RADIUA-226 (TOTAL) & $19 / 21 / 92$ & NoO1 & $\mathrm{PCI} / \mathrm{L}$ & & 1.2 & & 0.3 & 0.4 \\
\hline RADIUN-226 + RADIUM-228 & $\begin{array}{l}06 / 05 / 89 \\
05 / 31 / 91 \\
02 / 22 / 92 \\
08 / 10 / 92 \\
11 / 21 / 92\end{array}$ & $\begin{array}{l}0001 \\
0001 \\
0001 \\
0001 \\
0001\end{array}$ & $\mathrm{PCI} / \mathrm{L}$ & & $\begin{array}{l}0.30 \\
0.30 \\
2.60 \\
0.73 \\
1.20\end{array}$ & & $\begin{array}{l}- \\
- \\
- \\
-\end{array}$ & $\begin{array}{l}- \\
- \\
- \\
-\end{array}$ \\
\hline RADIUH-226 + RADIUH-228 (TOTAL) & $19 / 21 / 92$ & NoOI & $\mathrm{PCI} / \mathrm{L}$ & & 6.00 & & - & - \\
\hline RADIUA-228 & $\begin{array}{l}06 / 05 / 89 \\
05 / 31 / 91 \\
02 / 22 / 92 \\
08 / 10 / 92 \\
11 / 21 / 92\end{array}$ & $\begin{array}{l}0001 \\
0001 \\
0001 \\
0001 \\
0001\end{array}$ & $\mathrm{PCI} / \mathrm{L}$ & & $\begin{array}{l}0.0 \\
0.0 \\
2.0 \\
0.3 \\
0.3\end{array}$ & $J$ & $\begin{array}{l}1 . \\
1.0 \\
1.0 \\
2.3 \\
2.7\end{array}$ & $\begin{array}{l}0.9 \\
1.9 \\
1.6 \\
1.2 \\
1.6\end{array}$ \\
\hline RADIUN-228 (TOTAL) & $11 / 21 / 92$ & N001 & $\mathrm{PCI} / \mathrm{L}$ & & 4.8 & & 2.4 & 1.9 \\
\hline REDOX POTENTIAL & $\begin{array}{l}11 / 21 / 92 \\
06 / 29 / 93\end{array}$ & $\begin{array}{l}0001 \\
\text { N001 }\end{array}$ & mVOLTS & & $\begin{array}{l}437.4 \\
462\end{array}$ & & - & - \\
\hline SELENIUM & $\begin{array}{l}04 / 26 / 88 \\
06 / 05 / 89 \\
05 / 31 / 91 \\
02 / 22 / 92 \\
08 / 10 / 92 \\
14 / 21 / 92\end{array}$ & $\begin{array}{l}0001 \\
0001 \\
0001 \\
0001 \\
0001 \\
0001\end{array}$ & MG/L & $\begin{array}{l}< \\
< \\
< \\
<\end{array}$ & $\begin{array}{l}0.005 \\
0.005 \\
0.007 \\
0.005 \\
0.005 \\
0.0015\end{array}$ & $\begin{array}{l}\text { L } \\
J \\
H \\
H\end{array}$ & $\begin{array}{l}0.005 \\
0.005 \\
0.005 \\
0.005 \\
0.005 \\
0.0015\end{array}$ & $\begin{array}{l}- \\
- \\
- \\
-\end{array}$ \\
\hline SELENIUM (TOTAL) & $11 / 21 / 92$ & NoO1 & MG/L & $<$ & 0.0015 & & 0.0015 & - \\
\hline SILICA - SIOZ & $\begin{array}{l}06 / 05 / 89 \\
05 / 31 / 99\end{array}$ & $\begin{array}{l}0009 \\
0001\end{array}$ & $M G / L$ & & $\begin{array}{l}12 . \\
12.5\end{array}$ & & $\begin{array}{l}2 . \\
0.1\end{array}$ & - \\
\hline SILICA - SIOZ (TOTAL) & $06 / 29 / 93$ & No09 & MG/L & & 13.1 & H & 0.5 & - \\
\hline SILVER & $\begin{array}{l}06 / 05 / 89 \\
05 / 31 / 91\end{array}$ & $\begin{array}{l}0001 \\
0001\end{array}$ & $M G / L$ & $<$ & $\begin{array}{l}0.01 \\
0.01\end{array}$ & & $\begin{array}{l}0.01 \\
0.01\end{array}$ & - \\
\hline scotury & $\begin{array}{l}04 / 26 / 88 \\
06 / 05 / 89 \\
05 / 31 / 91 \\
02 / 22 / 92\end{array}$ & $\begin{array}{l}0001 \\
0001 \\
0001 \\
0001\end{array}$ & $M G / L$ & & $\begin{array}{c}94.0 \\
99.5 \\
112 . \\
99 .\end{array}$ & $L$ & $\begin{array}{l}0.002 \\
0.002 \\
5 . \\
1 .\end{array}$ & : \\
\hline
\end{tabular}

PARAMETER VALUE IMDICATOR (PVI): < - LESS THAN DETECTION LIMIT

SAMPLE ID COOES:

OTHER PARAMETER VALUE FLAGS:

H - HOLD TIME EXPIRED, VALUE SUSPECT

OOO1 - FILTERED SAMPLE (.45 MICRONS)

J - ESTIMATED VALUE

L - LESS THAM THREE BORE VOLLMES REMOVED BEFORE SAMPLIHG

N - SPIKE SAMPLE RECOVERY MOT HITHIH CONTROL LIMITS

- $W$ - POST-DIGEST SPIKE OUT OF CHTR LIM WHILE SAMP ABS < 50\% SPIKE 
GROUNDWATER OUALITY DATA BY LOCATION

SOUP ReV. O Data

SITE: MONOI MONUMENT VALLEY

LOCATION: 0603

MORTH COORDINATE: 57812.7 FT

EAST COORDINATE: 89036.9 FT

04/21/88 TO $12 / 13 / 94$

REPORT DATE: $06 / 20 / 95$

FORHATION OF COMPLETION: ALLUVIUM (AL)

HYDRAULIC FLOU RELATIOHSHIP: CROSS GRADIENT (C)

\begin{tabular}{|c|c|c|c|c|c|c|c|c|}
\hline PARAMETER MAME & LOG DATE & $\begin{array}{c}\text { SAMPLE } \\
\text { ID }\end{array}$ & $\begin{array}{l}\text { UNITS OF } \\
\text { MEASURE }\end{array}$ & PVI & $\begin{array}{l}\text { PARAMETER } \\
\text { VALUE }\end{array}$ & FLAGS & $\begin{array}{l}\text { DETECTION } \\
\text { LIMIT }\end{array}$ & $\begin{array}{l}\text { PARAMETER } \\
\text { UHCERTAIHTY }\end{array}$ \\
\hline SOOIUH & $\begin{array}{l}08 / 10 / 92 \\
06 / 29 / 93\end{array}$ & $\begin{array}{l}0001 \\
0001\end{array}$ & $M G / L$ & & $\begin{array}{l}100.7 \\
103\end{array}$ & & $\begin{array}{l}5 \\
1\end{array}$ & : \\
\hline SOOIUM (TOTAL) & $06 / 29 / 93$ & N001 & MG/L & & 99 & & 1 & - \\
\hline SPECIFIC CONDUCTAKCE & $\begin{array}{l}04 / 26 / 88 \\
06 / 05 / 89 \\
05 / 31 / 91 \\
02 / 22 / 92 \\
08 / 10 / 92 \\
11 / 21 / 92 \\
06 / 29 / 93\end{array}$ & $\begin{array}{l}0001 \\
0001 \\
0001 \\
0001 \\
0001 \\
0001 \\
\text { N001 }\end{array}$ & UHHO/CM & & $\begin{array}{l}500 . \\
415 . \\
650 . \\
528 \\
534 \\
528 \\
528\end{array}$ & $L$ & $\begin{array}{l}- \\
: \\
: \\
-\end{array}$ & $\begin{array}{l}- \\
- \\
- \\
- \\
-\end{array}$ \\
\hline STRONTIUN & $\begin{array}{l}05 / 31 / 91 \\
02 / 22 / 92 \\
08 / 10 / 92 \\
11 / 21 / 92 \\
06 / 29 / 93\end{array}$ & $\begin{array}{l}0001 \\
0001 \\
0001 \\
0001 \\
0001\end{array}$ & MG/L & & $\begin{array}{l}0.23 \\
0.22 \\
0.24 \\
0.28 \\
0.24\end{array}$ & & $\begin{array}{l}0.01 \\
0.01 \\
0.2 \\
0.00035 \\
0.01\end{array}$ & $\begin{array}{l}- \\
- \\
-\end{array}$ \\
\hline STRONTIU: (TOTAL) & $\begin{array}{l}11 / 21 / 92 \\
0.6 / 29 / 93\end{array}$ & $\begin{array}{l}\text { NoO1 } \\
\text { N001 }\end{array}$ & HG/L & & $\begin{array}{l}0.26 \\
0.22\end{array}$ & & $\begin{array}{l}0.00035 \\
0.01\end{array}$ & : \\
\hline SULFATE & $\begin{array}{l}04 / 26 / 88 \\
06 / 05 / 89 \\
05 / 31 / 91 \\
02 / 22 / 92 \\
08 / 10 / 92 \\
11 / 21 / 92\end{array}$ & $\begin{array}{l}0001 \\
0001 \\
0001 \\
0001 \\
0001 \\
0001\end{array}$ & $M G / L$ & & $\begin{array}{l}112 . \\
117 . \\
151 . \\
101 . \\
100 \\
120\end{array}$ & \llcorner & $\begin{array}{c}0.1 \\
0.1 \\
0.1 \\
10 . \\
0.059 \\
1.0\end{array}$ & $\begin{array}{l}- \\
- \\
- \\
-\end{array}$ \\
\hline SULFATE (TOTAL) & $\begin{array}{l}11 / 21 / 92 \\
06 / 29 / 93\end{array}$ & $\begin{array}{l}\text { N001 } \\
\text { N001 }\end{array}$ & MG/L & & $\begin{array}{l}120 \\
111\end{array}$ & & 1.0 & - \\
\hline SULFIDE & $\begin{array}{l}06 / 05 / 89 \\
05 / 31 / 91\end{array}$ & $\begin{array}{l}0001 \\
0001\end{array}$ & MG/L & $<$ & $\begin{array}{l}0.1 \\
1 .\end{array}$ & IJ & $\begin{array}{l}0.1 \\
1 .\end{array}$ & - \\
\hline TEMPERATURE & $\begin{array}{l}04 / 26 / 88 \\
06 / 05 / 89 \\
05 / 31 / 91 \\
02 / 22 / 92 \\
08 / 10 / 92 \\
11 / 21 / 92 \\
06 / 29 / 93\end{array}$ & $\begin{array}{l}0001 \\
0001 \\
0001 \\
0001 \\
0001 \\
0001 \\
N 001\end{array}$ & C - DEGREE & & $\begin{array}{l}15.0 \\
15.2 \\
15.4 \\
15.4 \\
16.0 \\
15.4 \\
16.6\end{array}$ & \llcorner & $\begin{array}{l}- \\
- \\
- \\
- \\
-\end{array}$ & $\begin{array}{l}- \\
\because \\
\because \\
-\end{array}$ \\
\hline THALLIUY & $\begin{array}{l}06 / 05 / 89 \\
05 / 31 / 91\end{array}$ & $\begin{array}{l}0001 \\
0001\end{array}$ & ME/L & $<$ & $\begin{array}{l}0.09 \\
0.01\end{array}$ & & $\begin{array}{l}0.01 \\
0.01\end{array}$ & $\dot{-}$ \\
\hline THORIUH-230 & $05 / 31 / 91$ & 0001 & $\mathrm{PCI} / \mathrm{L}$ & & 0.4 & & 1. & 0.4 \\
\hline TIN & $\begin{array}{l}06 / 05 / 89 \\
05 / 31 / 91\end{array}$ & $\begin{array}{l}0001 \\
0001\end{array}$ & MG/L & $<$ & $\begin{array}{l}0.005 \\
0.01\end{array}$ & 1 & $\begin{array}{l}0.005 \\
0.01\end{array}$ & - \\
\hline TOTAL DISSOLVED SOLIDS & $\begin{array}{l}04 / 26 / 88 \\
06 / 05 / 89 \\
05 / 31 / 91 \\
02 / 22 / 92\end{array}$ & $\begin{array}{l}0001 \\
0001 \\
0001 \\
0001\end{array}$ & MG/L & & $\begin{array}{l}418 . \\
386 \\
396 \\
390\end{array}$ & $\mathbf{L}$ & $\begin{array}{l}10 . \\
10 . \\
10 . \\
10 .\end{array}$ & : \\
\hline
\end{tabular}

PARAMETER VALUE INDICATOR (PVI): < - LESS THAN DETECTIOH LIMIT

SAMPLE IO COOES

OTHER PARAMETER VALUE FLAGS:

0001 - FILTERED SAMPLE (.45 MICROHS)

I - IMCREASED DETECTION LIMIT DUE TO REQUIRED DILUTION

N001 - UAFILTERED SAMPLE

J ESTIMATEd VALUE

L - LESS THAH THREE BORE VOLUMES REMOVED BEFORE SAMPLIHG 
SOUP ReV. O Data

SITE: HONO 1 HOHUMENT VALLEY

LOCATION: 0603

NORTH COORDIHATE: 57812.7 FT

EAST COORDINATE: 89036.9 FT

$04 / 21 / 88$ TO $12 / 13 / 94$

REPORT DATE: $06 / 20 / 95$

FORHATION OF COMPLETIOH: ALLUVIUM (AL)

HYDRAULIC FLON RELATIOHSHIP: CROSS GRADIEHT (C)

\begin{tabular}{|c|c|c|c|c|c|c|c|c|}
\hline PARAMETER MAME & LOG DATE & $\begin{array}{c}\text { SAMPLE } \\
\text { ID }\end{array}$ & $\begin{array}{l}\text { UNITS OF } \\
\text { MEASURE }\end{array}$ & PVI & $\begin{array}{l}\text { PARAMETER } \\
\text { VALUE }\end{array}$ & FLAGS & $\begin{array}{l}\text { DETECTIOH } \\
\text { LIMIT }\end{array}$ & $\begin{array}{l}\text { PARAMETER } \\
\text { UNCERTAINTY }\end{array}$ \\
\hline TOTAL DISSOLVED SOLIDS & $08 / 10 / 92$ & 0001 & MG/L & \multicolumn{3}{|c|}{470} & 42 & - \\
\hline TOTAL DISSOLVED SOLIOS (TOTAL) & $06 / 29 / 93$ & N001 & MG/L & & 380 & H & 10 & - \\
\hline TOTAL ORGANIC CARBOH & $\begin{array}{l}04 / 26 / 88 \\
05 / 31 / 91 \\
02 / 22 / 92 \\
08 / 10 / 92\end{array}$ & $\begin{array}{l}0001 \\
0001 \\
0001 \\
0001\end{array}$ & MG/L & $\begin{array}{l}< \\
<\end{array}$ & $\begin{array}{l}56.1 \\
1 . \\
1 . \\
1.0\end{array}$ & 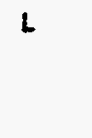 & $\begin{array}{l}1 . \\
1 . \\
1.0\end{array}$ & : \\
\hline URAHILH & $\begin{array}{l}04 / 26 / 88 \\
06 / 05 / 89 \\
05 / 31 / 91 \\
02 / 22 / 92 \\
11 / 21 / 92 \\
06 / 29 / 93\end{array}$ & $\begin{array}{l}0001 \\
0001 \\
0001 \\
0001 \\
0001 \\
0001\end{array}$ & $M G / L$ & $<$ & $\begin{array}{l}0.0030 \\
0.0023 \\
0.001 \\
0.003 \\
0.005 \\
0.003\end{array}$ & $\mathbf{L}$ & $\begin{array}{l}0.003 \\
0.003 \\
0.001 \\
0.001 \\
0.001 \\
0.001\end{array}$ & $\begin{array}{l}: \\
: \\
:\end{array}$ \\
\hline URANIUM (TOTAL) & $\begin{array}{l}11 / 21 / 92 \\
06 / 29 / 93\end{array}$ & $\begin{array}{l}\text { N001 } \\
\text { N001 }\end{array}$ & MG/L & & $\begin{array}{l}0.003 \\
0.002\end{array}$ & & $\begin{array}{l}0.001 \\
0.001\end{array}$ & $\dot{-}$ \\
\hline VAKADIUH & $\begin{array}{l}06 / 05 / 89 \\
05 / 31 / 91 \\
02 / 22 / 92 \\
08 / 10 / 92 \\
11 / 21 / 92 \\
06 / 29 / 93\end{array}$ & $\begin{array}{l}0001 \\
0001 \\
0001 \\
0001 \\
0001 \\
0001\end{array}$ & MG/L & $\begin{array}{l}< \\
< \\
< \\
< \\
< \\
<\end{array}$ & $\begin{array}{l}0.01 \\
0.01 \\
0.01 \\
0.05 \\
0.0019 \\
0.01\end{array}$ & & $\begin{array}{l}0.01 \\
0.01 \\
0.01 \\
0.05 \\
0.0019 \\
0.01\end{array}$ & $\begin{array}{l}- \\
- \\
-\end{array}$ \\
\hline VAKADIUM (TOTAL) & $\begin{array}{l}11 / 21 / 92 \\
06 / 29 / 93\end{array}$ & $\begin{array}{l}\text { NOO1 } \\
\text { HOOI }\end{array}$ & MG/L & $<$ & $\begin{array}{l}0.0030 \\
0.01\end{array}$ & & $\begin{array}{l}0.0019 \\
0.01\end{array}$ & - \\
\hline ZIKC & $\begin{array}{l}06 / 05 / 89 \\
05 / 31 / 91 \\
02 / 22 / 92 \\
08 / 10 / 92 \\
11 / 21 / 92 \\
06 / 29 / 93\end{array}$ & $\begin{array}{l}0001 \\
0001 \\
0001 \\
0009 \\
0001 \\
0001\end{array}$ & $M G / L$ & $\begin{array}{l}< \\
< \\
< \\
<\end{array}$ & $\begin{array}{l}0.005 \\
0.014 \\
0.005 \\
0.02 \\
0.0010 \\
0.011\end{array}$ & 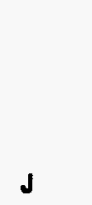 & $\begin{array}{l}0.005 \\
0.005 \\
0.005 \\
0.02 \\
0.0010 \\
0.005\end{array}$ & $\begin{array}{l}: \\
: \\
-\end{array}$ \\
\hline ZIMC (TOTAL) & $\begin{array}{l}11 / 21 / 92 \\
06 / 29 / 93\end{array}$ & $\begin{array}{l}\text { N001 } \\
\text { N001 }\end{array}$ & MG/L & & $\begin{array}{l}0.0040 \\
0.009 .\end{array}$ & 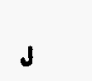 & $\begin{array}{l}0.0010 \\
0.005\end{array}$ & - \\
\hline
\end{tabular}

PARAMETER VALUE IKDICATOR (PVI): \& - LESS THAN DETECTION LIMIT

SAMPLE ID COOES:

OTHER PARAMETER VALUE FLAGS:

H - HOLD TIME EXPIRED, VALUE SUSPECT

O001 - FILTERED SAMPLE (.45 HICRONS)

J - ESTIMUTED VALUE

L - LESS THAN THREE BORE VOLUHES REHOVED BEFORE SAMPLING 
GROUNDWATER QUALITY DATA BY LOCATION

SONP REV. $O$ Data

SITE: MONO1 MONUMENT VALLEY

LOCATION: 0604

NORTH COORDINATE: 58397.0 FT

EAST COORDINATE: 89424.6 FT

04/21/88 TO $12 / 13 / 94$

REPORT DATE: 06/20/95

FORMATION OF COMPLETION: ALLUVIUH (AL)

HYDRAULIC FLON RELATIONSHIP: CROSS GRADIENT (C)

\begin{tabular}{|c|c|c|c|c|c|c|c|c|}
\hline PARAMETER NAME & LOG DATE & $\begin{array}{c}\text { SAHPLE } \\
\text { ID }\end{array}$ & $\begin{array}{l}\text { UNITS OF } \\
\text { MEASURE }\end{array}$ & PVI & $\begin{array}{l}\text { PARAMETER } \\
\text { VALUE }\end{array}$ & FLAGS & $\begin{array}{l}\text { DETECTIOH } \\
\text { LIMIT }\end{array}$ & $\begin{array}{l}\text { PARAMETER } \\
\text { UNCERTAIHTY }\end{array}$ \\
\hline ALKALINITY & $\begin{array}{l}06 / 09 / 89 \\
05 / 31 / 91 \\
02 / 22 / 92 \\
08 / 11 / 92 \\
11 / 19 / 92 \\
02 / 02 / 93 \\
06 / 26 / 93\end{array}$ & $\begin{array}{l}0001 \\
0001 \\
0001 \\
0001 \\
0001 \\
0001 \\
\text { N001 }\end{array}$ & $\mathrm{MG} / \mathrm{L} \mathrm{CACO}$ & & $\begin{array}{l}202 . \\
204 . \\
196 \\
189 \\
191 \\
207 \\
200\end{array}$ & $\mathbf{L}$ & $\begin{array}{l}- \\
- \\
- \\
-\end{array}$ & $\begin{array}{l}- \\
- \\
- \\
-\end{array}$ \\
\hline ALUMINUM & $\begin{array}{l}06 / 09 / 89 \\
05 / 31 / 91 \\
02 / 22 / 92 \\
08 / 11 / 92\end{array}$ & $\begin{array}{l}0001 \\
0001 \\
0001 \\
0001\end{array}$ & MG/L & $\begin{array}{l}< \\
< \\
< \\
<\end{array}$ & $\begin{array}{l}0.1 \\
0.05 \\
0.05 \\
0.2\end{array}$ & *L & $\begin{array}{l}0.1 \\
0.05 \\
0.05 \\
0.2\end{array}$ & : \\
\hline AMYONIUM & $\begin{array}{l}06 / 09 / 89 \\
05 / 31 / 91 \\
02 / 22 / 92\end{array}$ & $\begin{array}{l}0001 \\
0001 \\
0001\end{array}$ & $\mathrm{MG} / \mathrm{L}$ & $<$ & $\begin{array}{l}0.1 \\
0.3 \\
0.2\end{array}$ & & $\begin{array}{l}0.1 \\
0.1 \\
0.1\end{array}$ & 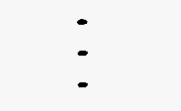 \\
\hline AMONIUM (TOTAL) & $06 / 26 / 93$ & N001 & MG/L & $<$ & 0.1 & & 0.1 & - \\
\hline AKTIMONY & $\begin{array}{l}06 / 09 / 89 \\
05 / 31 / 91 \\
02 / 22 / 92 \\
08 / 11 / 92\end{array}$ & $\begin{array}{l}0001 \\
0001 \\
0001 \\
0001\end{array}$ & MG/L & $\begin{array}{l}< \\
< \\
<\end{array}$ & $\begin{array}{l}0.003 \\
0.005 \\
0.003 \\
0.06\end{array}$ & UL & $\begin{array}{l}0.003 \\
0.003 \\
0.003 \\
0.06\end{array}$ & - \\
\hline ARSENIC & $\begin{array}{l}06 / 09 / 89 \\
05 / 31 / 91 \\
02 / 22 / 92 \\
08 / 11 / 92 \\
11 / 19 / 92 \\
06 / 26 / 93\end{array}$ & $\begin{array}{l}0001 \\
0001 \\
0001 \\
0001 \\
0001 \\
0001\end{array}$ & MG/L & $\begin{array}{l}< \\
< \\
< \\
<\end{array}$ & $\begin{array}{l}0.01 \\
0.01 \\
0.01 \\
0.01 \\
0.0027 \\
0.005\end{array}$ & $\begin{array}{l}\mathbf{W L} \\
\mathbf{W}\end{array}$ & $\begin{array}{l}0.01 \\
0.01 \\
0.01 \\
0.01 \\
0.0015 \\
0.005\end{array}$ & $\begin{array}{l}- \\
- \\
-\end{array}$ \\
\hline ARSENIC (TOTAL) & $\begin{array}{l}11 / 19 / 92 \\
06 / 26 / 93\end{array}$ & $\begin{array}{l}\text { No01 } \\
\text { N001 }\end{array}$ & MG/L & $<$ & $\begin{array}{l}0.0030 \\
0.005\end{array}$ & & $\begin{array}{l}0.0015 \\
0.005\end{array}$ & - \\
\hline BARIUM & $\begin{array}{l}06 / 09 / 89 \\
05 / 31 / 91 \\
02 / 22 / 92 \\
08 / 11 / 92 \\
11 / 19 / 92 \\
06 / 26 / 93\end{array}$ & $\begin{array}{l}0001 \\
0001 \\
0001 \\
0001 \\
0001 \\
0001\end{array}$ & $M G / L$ & $\begin{array}{l}< \\
< \\
<\end{array}$ & $\begin{array}{l}0.1 \\
0.04 \\
0.04 \\
0.2 \\
0.038 \\
0.1\end{array}$ & $L$ & $\begin{array}{l}0.1 \\
0.01 \\
0.01 \\
0.2 \\
0.0015 \\
0.1\end{array}$ & $\begin{array}{l}- \\
- \\
- \\
-\end{array}$ \\
\hline BARIUN (TOTAL) & $\begin{array}{l}11 / 19 / 92 \\
06 / 26 / 93\end{array}$ & $\begin{array}{l}\text { NoO1 } \\
\text { NO01 }\end{array}$ & MG/L & & $\begin{array}{l}0.075 \\
0.1\end{array}$ & & $\begin{array}{l}0.0015 \\
0.1\end{array}$ & - \\
\hline BERYLLIUY & $\begin{array}{l}06 / 09 / 89 \\
05 / 31 / 91 \\
08 / 11 / 92\end{array}$ & $\begin{array}{l}0001 \\
0001 \\
0001\end{array}$ & $M G / L$ & $\begin{array}{l}< \\
< \\
<\end{array}$ & $\begin{array}{l}0.01 \\
0.005 \\
0.005\end{array}$ & $\mathbf{L}$ & $\begin{array}{l}0.01 \\
0.005 \\
0.005\end{array}$ & 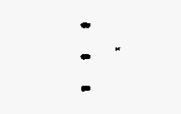 \\
\hline BORON & $\begin{array}{l}06 / 09 / 89 \\
05 / 31 / 91\end{array}$ & $\begin{array}{l}0001 \\
0001\end{array}$ & $M G / L$ & $\leqslant$ & $\begin{array}{l}0.1 \\
0.05\end{array}$ & & $\begin{array}{l}0.1 \\
0.05\end{array}$ & - \\
\hline BRONIDE & $05 / 31 / 91$ & 0001 & $M G / L$ & $<$ & 0.1 & & 0.1 & - \\
\hline CADMILA & $06 / 09 / 89$ & 0001 & MG/L & $<$ & 0.001 & & 0.001 & - \\
\hline
\end{tabular}

PARAMETER VALUE IMDICATOR (PVI): < - LESS THAN DETECTION LIMIT

SAMPLE ID COOES:

OTHER PARAMETER VALUE FLAGS:

OOOI - FILTERED SAMPLE (.45 MICRONS)

- - DUPLICATE ANALYSIS ROT WITHIN CONTROL LIMITS

NOO - UMFILTERED SAMPLE

L - LESS THAN THREE BORE VOLUNES REMOVED BEFORE SAMPLING

W - POST-DIGEST SPIKE OUT OF CHTR LIM HHILE SAMP ABS < 50\% SPIKE 
GROUNDHATER OUALITY DATA BY LOCATION

SOMP REV. O Dato

SITE: MOHOI MONUMENT VALLEY,

LOCATIOH: 0604

MORTH COORDINATE: $\quad 58397.0$ FT

EAST COORDIHATE: 89424.6 FT

04/21/88 TO $12 / 13 / 94$

REPORT DATE: $06 / 20 / 95$

FORMATIOH OF COMPLETIOH: ALLUVIUM (AL)

HYDRAULIC FLON RELATIONSHIP: CROSS GRADIENT (C)

\begin{tabular}{|c|c|c|c|c|c|c|c|c|}
\hline PARAMETER NAME & LOG DATE & $\begin{array}{c}\text { SAMPLE } \\
\text { ID }\end{array}$ & $\begin{array}{l}\text { UWITS OF } \\
\text { MEASURE }\end{array}$ & PVI & $\begin{array}{l}\text { PARAMETER } \\
\text { VALUE }\end{array}$ & FLAGS & $\begin{array}{l}\text { DETECTION } \\
\text { LIMIT }\end{array}$ & $\begin{array}{l}\text { PARAMETER } \\
\text { UNCERTAINTY }\end{array}$ \\
\hline CAOMIUH & $\begin{array}{l}05 / 31 / 91 \\
02 / 22 / 92 \\
08 / 11 / 92 \\
11 / 19 / 92\end{array}$ & $\begin{array}{l}0001 \\
0001 \\
0001 \\
0001\end{array}$ & MG/L & $\begin{array}{l}< \\
< \\
< \\
<\end{array}$ & $\begin{array}{l}0.001 \\
0.0005 \\
0.005 \\
0.00013\end{array}$ & EL & $\begin{array}{l}0.001 \\
0.0005 \\
0.005 \\
0.00013\end{array}$ & $\begin{array}{l}- \\
- \\
-\end{array}$ \\
\hline CADHIUH (TOTAL) & $11 / 19 / 92$ & no01 & $M G / L$ & & 0.00014 & $\mathrm{~N}$ & 0.00013 & - \\
\hline CALCIUM & $\begin{array}{l}06 / 09 / 89 \\
05 / 31 / 91 \\
02 / 22 / 92 \\
08 / 11 / 92 \\
02 / 02 / 93 \\
06 / 26 / 93\end{array}$ & $\begin{array}{l}0001 \\
0001 \\
0001 \\
0001 \\
0001 \\
0001\end{array}$ & $M G / L$ & & $\begin{array}{l}20.1 \\
18.8 \\
17.3 \\
20 \\
19.2 \\
19.1\end{array}$ & $L$ & $\begin{array}{l}0.01 \\
0.1 \\
0.1 \\
5 \\
0.5 \\
0.5\end{array}$ & $\begin{array}{l}- \\
- \\
- \\
-\end{array}$ \\
\hline CALCIUH (TOTAL) & $06 / 26 / 93$ & K001 & MG $/ L$ & & 22.8 & & 0.5 & - \\
\hline CHLORIDE & $\begin{array}{l}06 / 09 / 89 \\
05 / 31 / 91 \\
02 / 22 / 92 \\
08 / 11 / 92 \\
02 / 02 / 93\end{array}$ & $\begin{array}{l}0001 \\
0001 \\
0001 \\
0001 \\
0001\end{array}$ & $M G / L$ & & $\begin{array}{l}11 . \\
11.6 \\
12.1 \\
11 \\
11.5\end{array}$ & $\mathbf{L}$ & $\begin{array}{l}1 . \\
0.5 \\
0.5 \\
0.016 \\
0.5\end{array}$ & $\begin{array}{l}\dot{-} \\
\dot{-} \\
\dot{-}\end{array}$ \\
\hline CHLORIDE (TOTAL) & $06 / 26 / 93$ & No01 & HG/L & & 10.8 & & 0.5 & - \\
\hline CHROMIUM & $\begin{array}{l}06 / 09 / 89 \\
05 / 31 / 91 \\
02 / 22 / 92 \\
08 / 11 / 92 \\
11 / 19 / 92\end{array}$ & $\begin{array}{l}0001 \\
0001 \\
0001 \\
0001 \\
0001\end{array}$ & $M G / L$ & $\begin{array}{l}< \\
< \\
< \\
< \\
<\end{array}$ & $\begin{array}{l}0.01 \\
0.01 \\
0.01 \\
0.01 \\
0.0053\end{array}$ & $L$ & $\begin{array}{l}0.01 \\
0.01 \\
0.01 \\
0.01 \\
0.0053\end{array}$ & $\begin{array}{l}- \\
- \\
-\end{array}$ \\
\hline CHRONIUM (TOTAL) & $11 / 19 / 92$ & N001 & $M G / L$ & $<$ & 0.0053 & & 0.0053 & $\cdot$ \\
\hline $\operatorname{CosALT}$ & $\begin{array}{l}06 / 09 / 89 \\
05 / 31 / 91\end{array}$ & $\begin{array}{l}0001 \\
0001\end{array}$ & MG/L & $<$ & $\begin{array}{l}0.05 \\
0.03\end{array}$ & & $\begin{array}{l}0.05 \\
0.03\end{array}$ & : \\
\hline COPPER & $\begin{array}{l}06 / 09 / 89 \\
05 / 31 / 91\end{array}$ & $\begin{array}{l}0001 \\
0001\end{array}$ & MG/L & $<$ & $\begin{array}{l}0.02 \\
0.01\end{array}$ & & $\begin{array}{l}0.02 \\
0.01\end{array}$ & $\dot{-}$ \\
\hline CYAHIDE & $06 / 09 / 89$ & 0001 & ME/L & $<$ & 0.01 & & 0.01 & - \\
\hline DISSOLVED OXYGEN & $\begin{array}{l}11 / 19 / 92 \\
06 / 26 / 93\end{array}$ & $\begin{array}{l}0001 \\
\text { N001 }\end{array}$ & $M G / L$ & & $\begin{array}{l}0.4 \\
0.0\end{array}$ & & : & - \\
\hline FLLORIDE & $\begin{array}{l}06 / 09 / 89 \\
05 / 31 / 91\end{array}$ & $\begin{array}{l}0001 \\
0001\end{array}$ & $M G / L$ & & $\begin{array}{l}0.2 \\
0.1\end{array}$ & & $\begin{array}{l}0.1 \\
0.1\end{array}$ & 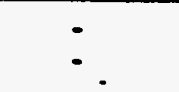 \\
\hline GROSS ALPHA & $\begin{array}{l}05 / 31 / 91 \\
02 / 22 / 92 \\
08 / 11 / 92 \\
11 / 19 / 92 \\
02 / 02 / 93 \\
06 / 26 / 93\end{array}$ & $\begin{array}{l}0001 \\
0001 \\
0001 \\
0001 \\
0001 \\
0001\end{array}$ & PCI/L & & $\begin{array}{l}5.0 \\
0.5 \\
1.2 \\
3.9 \\
0 \\
6\end{array}$ & $\stackrel{L}{H}$ & $\begin{array}{l}1 . \\
1.0 \\
4.1 \\
3.2 \\
6 \\
3\end{array}$ & $\begin{array}{l}5.4 \\
3.9 \\
2.2 \\
2.7 \\
6 \\
5\end{array}$ \\
\hline CROSS ALPHA (TOTAL) & $\begin{array}{l}11 / 19 / 92 \\
06 / 26 / 93\end{array}$ & $\begin{array}{l}\text { No01 } \\
\text { N001 }\end{array}$ & $\mathrm{PCI} / \mathrm{L}$ & & $\begin{array}{l}0.9 \\
4\end{array}$ & H & $\begin{array}{l}6.6 \\
3\end{array}$ & 4.0 \\
\hline
\end{tabular}

PARAMETER VALUE IMDICATOR (PVI): < - LeSS thaN DETECTION LIMIT

SAMPLE ID COOES:

0009 - FILTERED SAMPLE (.45 MICRONS)

OTHER PARAMETER VALUE FLAGS:

NOOI - UNFILTERED SAMPLE

E - ESTIMATED VALUE BECAUSE OF INTERFEREHCE, SEE CASE HARRATIVE

L - LESS THAH THREE BORE VOLUAES REMOVED BEFORE SAMPLIHG

$N$ - SPIKE SAMPLE RECOVERY NOT HITHIN CONTROL LIMITS 
GROUNDWATER QUALITY DATA BY LOCATION

SOWP ReV. 0 Dat8

SITE: MONO1 MONUMENT VALLEY

LOCATION: 0604

NORTH COORDINATE: 58397.0 FT

EAST COORDINATE: 89424.6 FT

04/21/88 TO 12/13/94

REPORT DATE: $06 / 20 / 95$

FORMATION OF COMPLETION: ALLUVILM (AL)

HYDRAULIC FLOW RELATIONSHIP: CROSS GRADIENT (C)

\begin{tabular}{|c|c|c|c|c|c|c|c|c|}
\hline PARAMETER NAME & LOG DATE & $\begin{array}{c}\text { SAMPLE } \\
\text { ID }\end{array}$ & $\begin{array}{l}\text { UNITS OF } \\
\text { MEASURE }\end{array}$ & PVI & $\begin{array}{l}\text { PARAMETER } \\
\text { VALUE }\end{array}$ & FLAGS & $\begin{array}{l}\text { DETECTION } \\
\text { LIMIT }\end{array}$ & $\begin{array}{l}\text { PARAMETER } \\
\text { UNCERTAINTY }\end{array}$ \\
\hline GROSS BETA & $\begin{array}{l}05 / 31 / 91 \\
02 / 22 / 92 \\
08 / 19 / 92 \\
11 / 19 / 92 \\
02 / 02 / 93 \\
06 / 26 / 93\end{array}$ & $\begin{array}{l}0001 \\
0001 \\
0001 \\
0001 \\
0001 \\
0001\end{array}$ & $\mathrm{PCI} / \mathrm{L}$ & & $\begin{array}{l}1.7 \\
1.8 \\
2.3 \\
0.0 \\
0 \\
3\end{array}$ & $\mathbf{L}$ & $\begin{array}{c}0.5 \\
0.5 \\
4.0 \\
5.6 \\
11 \\
4\end{array}$ & $\begin{array}{l}2.9 \\
2.2 \\
2.4 \\
3.1 \\
5 \\
4\end{array}$ \\
\hline GROSS BETA (TOTAL) & $\begin{array}{l}19 / 19 / 92 \\
06 / 26 / 93\end{array}$ & $\begin{array}{l}\text { No01 } \\
\text { No01 }\end{array}$ & $\mathrm{PCI} / \mathrm{L}$ & & $\begin{array}{l}8.9 \\
1\end{array}$ & N & $\begin{array}{l}5.1 \\
4\end{array}$ & $\begin{array}{l}3.4 \\
4\end{array}$ \\
\hline IRON & $\begin{array}{l}06 / 09 / 89 \\
05 / 31 / 91 \\
02 / 22 / 92 \\
08 / 11 / 92 \\
02 / 02 / 93 \\
06 / 26 / 93\end{array}$ & $\begin{array}{l}0009 \\
0001 \\
0001 \\
0001 \\
0009 \\
0001\end{array}$ & MG/L & $\begin{array}{l}< \\
< \\
< \\
< \\
< \\
<\end{array}$ & $\begin{array}{l}0.03 \\
0.03 \\
0.03 \\
0.1 \\
0.03 \\
0.03\end{array}$ & $\begin{array}{l}\text { J } \\
J\end{array}$ & $\begin{array}{l}0.03 \\
0.03 \\
0.03 \\
0.1 \\
0.03 \\
0.03\end{array}$ & $\begin{array}{l}: \\
: \\
: \\
:\end{array}$ \\
\hline IRON (TOTAL) & $06 / 26 / 93$ & HOO1 & $M G / L$ & & 1.88 & & 0.03 & - \\
\hline LEAD & $\begin{array}{l}05 / 09 / 89 \\
05 / 31 / 91 \\
11 / 19 / 92\end{array}$ & $\begin{array}{l}0001 \\
0001 \\
0001\end{array}$ & $M G / L$ & $\begin{array}{l}< \\
< \\
<\end{array}$ & $\begin{array}{l}0.01 \\
0.005 \\
0.0015\end{array}$ & & $\begin{array}{l}0.01 \\
0.005 \\
0.0015\end{array}$ & - \\
\hline LEND (TOTAL) & $11 / 19 / 92$ & N001 & MG/L & $<$ & 0.0015 & & 0.0015 & - \\
\hline LEAD-210 & $11 / 19 / 92$ & 0001 & $\mathrm{PCI} / \mathrm{L}$ & & 0.1 & & 2.0 & 1.2 \\
\hline LEAD-210 (TOTAL) & $11 / 19 / 92$ & NOO1 & $\mathrm{PCI} / \mathrm{L}$ & & 1.1 & & 2.0 & 1.2 \\
\hline MAGHESIUH & $\begin{array}{l}06 / 09 / 89 \\
05 / 31 / 91 \\
02 / 22 / 92 \\
08 / 11 / 92 \\
02 / 02 / 93 \\
06 / 26 / 93\end{array}$ & $\begin{array}{l}0001 \\
0001 \\
0001 \\
0001 \\
0001 \\
0001\end{array}$ & MG/L & & $\begin{array}{l}11.9 \\
11.8 \\
11.1 \\
12 \\
11.4 \\
11.4\end{array}$ & $L$ & $\begin{array}{l}0.001 \\
0.1 \\
0.1 \\
5 \\
0.1 \\
0.1\end{array}$ & : \\
\hline MAGHESIU: (TOTAL) & $06 / 26 / 93$ & N001 & $M G / L$ & & 12.4 & & 0.1 & - \\
\hline MAMGAHESE & $\begin{array}{l}06 / 09 / 89 \\
05 / 31 / 91 \\
02 / 22 / 92 \\
08 / 11 / 92 \\
11 / 19 / 92 \\
02 / 02 / 93 \\
06 / 26 / 93\end{array}$ & $\begin{array}{l}0001 \\
0001 \\
0001 \\
0001 \\
0001 \\
0001 \\
0001\end{array}$ & MG/L & $<$ & $\begin{array}{l}0.01 \\
0.01 \\
0.01 \\
0.011 \\
0.012 \\
0.01 \\
0.01\end{array}$ & $L$ & $\begin{array}{l}0.01 \\
0.01 \\
0.01 \\
0.01 \\
0.0015 \\
0.01 \\
0.01\end{array}$ & :- \\
\hline MANGANESE (TOTAL) & $\begin{array}{l}11 / 19 / 92 \\
06 / 26 / 93\end{array}$ & $\begin{array}{l}\text { No01 } \\
\text { N001 }\end{array}$ & MG/L & & $\begin{array}{l}0.025 \\
0.05\end{array}$ & & $\begin{array}{l}0.0015 \\
0.01\end{array}$ & - \\
\hline MERCURY & $06 / 09 / 89$ & 0001 & MG/L & $<$ & 0.0002 & & 0.0002 & - \\
\hline MOLYBDENUM & $\begin{array}{l}06 / 09 / 89 \\
05 / 31 / 91 \\
02 / 22 / 92\end{array}$ & $\begin{array}{l}0001 \\
0001 \\
0001\end{array}$ & MG/L & $\begin{array}{l}< \\
< \\
<\end{array}$ & $\begin{array}{l}0.01 \\
0.01 \\
0.01\end{array}$ & & $\begin{array}{l}0.01 \\
0.01 \\
0.01\end{array}$ & : \\
\hline
\end{tabular}

PARAMETER VALUE INDICATOR (PVI): < - LESS THAN DETECTION LIMIT

SNMPLE ID COOES:

0001 - FILTERED SAMPLE (.45 MICRONS)

OTHER PARAMETER VALUE FLAGS:

NOO1 - UHFILTERED SAMPLE

J - ESTIMATED VALUE

l - LESS THAH THREE BORE VOLUMES REMOVED BEFORE SAMPLING

H - SPIKE SAMPLE RECOVERY NOT HITHIM CONTROL LIMITS 
GROUNDWATER QUALITY DATA BY LOCATION

SOMP Rev. 0 Data

SITE: MOHO1 MONUHENT VALLEY

LOCATIOH: 0604

MORTH COORDINATE: 58397.0 FT

EAST COORDINATE: 89424.6 FT

$04 / 21 / 88$ T0 $12 / 13 / 94$

REPORT DATE: 06/20/95

FORMATION OF COMPLETION: ALLUVIUM (AL)

HYDRAULIC FLOW RELATIOHSHIP: CROSS GRADIENT (C)

\begin{tabular}{|c|c|c|c|c|c|c|c|c|}
\hline PARAMETER MAME & LOG DATE & $\begin{array}{c}\text { SAMPLE } \\
\text { ID }\end{array}$ & $\begin{array}{l}\text { UNITS OF } \\
\text { MEASURE }\end{array}$ & PVI & $\begin{array}{l}\text { PARAMETER } \\
\text { VALUE }\end{array}$ & FLAGS & $\begin{array}{l}\text { DETECTION } \\
\text { LIMIT }\end{array}$ & $\begin{array}{l}\text { PARAMETER } \\
\text { UNCERTAIUTY }\end{array}$ \\
\hline MOLYBDENUM & $\begin{array}{l}08 / 11 / 92 \\
11 / 19 / 92 \\
06 / 26 / 93\end{array}$ & $\begin{array}{l}0001 \\
0001 \\
0001\end{array}$ & KG/L & $\begin{array}{l}< \\
< \\
<\end{array}$ & $\begin{array}{l}0.007 \\
0.0049 \\
0.01\end{array}$ & $\mathbf{L}$ & $\begin{array}{l}0.007 \\
0.0049 \\
0.01\end{array}$ & : \\
\hline MOLYBOENLA (TOTAL) & $\begin{array}{l}11 / 19 / 92 \\
06 / 26 / 93\end{array}$ & $\begin{array}{l}\text { NoO1 } \\
\text { N001 }\end{array}$ & MG/L & $<$ & $\begin{array}{l}0.0049 \\
0.01\end{array}$ & N & $\begin{array}{l}0.0049 \\
0.01\end{array}$ & - \\
\hline HET GROSS ALPHA * & $\begin{array}{l}05 / 31 / 91 \\
02 / 22 / 92 \\
11 / 19 / 92 \\
02 / 02 / 93 \\
06 / 26 / 93\end{array}$ & $\begin{array}{l}0001 \\
0001 \\
0001 \\
0001 \\
0001\end{array}$ & $\mathrm{PCI} / \mathrm{L}$ & & $\begin{array}{r}4.66 \\
-0.87 \\
9.84 \\
-2.06 \\
4.63\end{array}$ & & $\begin{array}{l}- \\
- \\
-\end{array}$ & $\begin{array}{l}- \\
\dot{-} \\
\dot{-} \\
-\end{array}$ \\
\hline NET GROSS ALPHA (TOTAL) ** & $\begin{array}{l}19 / 19 / 92 \\
06 / 26 / 93\end{array}$ & $\begin{array}{l}\text { No01 } \\
\text { N001 }\end{array}$ & $\mathrm{PCI} / \mathrm{L}$ & & $\begin{array}{r}-1.16 \\
2.63\end{array}$ & & - & : \\
\hline HICKEL & $\begin{array}{l}06 / 09 / 89 \\
05 / 31 / 91\end{array}$ & $\begin{array}{l}0001 \\
0001\end{array}$ & MG/L & $<$ & $\begin{array}{l}0.04 \\
0.04\end{array}$ & & $\begin{array}{l}0.04 \\
0.04\end{array}$ & - \\
\hline HITRATE & $\begin{array}{l}06 / 09 / 89 \\
05 / 31 / 91 \\
02 / 22 / 92 \\
08 / 11 / 92 \\
11 / 19 / 92 \\
02 / 02 / 93\end{array}$ & $\begin{array}{l}0001 \\
0001 \\
0001 \\
0001 \\
0001 \\
0001\end{array}$ & MG/L & $\begin{array}{l}< \\
<\end{array}$ & $\begin{array}{l}5.3 \\
2.3 \\
1.0 \\
2.6 \\
1.06 \\
1\end{array}$ & $\mathbf{L}$ & $\begin{array}{l}1 . \\
1 . \\
1.0 \\
0.075 \\
0.044 \\
1\end{array}$ & $\begin{array}{l}\dot{-} \\
\dot{-} \\
\dot{-}\end{array}$ \\
\hline HITRATE (TOTAL) & $06 / 26 / 93$ & HoO1 & $M G / L$ & $<$ & 1 & & 1 & - \\
\hline HITRITE AHO NITRATE & $05 / 31 / 91$ & 0001 & $M G / L$ & & 0.53 & & 0.05 & - \\
\hline $\mathrm{PH}$ & $\begin{array}{l}06 / 09 / 89 \\
05 / 31 / 91 \\
02 / 22 / 92 \\
08 / 11 / 92 \\
11 / 19 / 92 \\
02 / 02 / 93 \\
06 / 26 / 93\end{array}$ & $\begin{array}{l}0001 \\
0001 \\
0001 \\
0001 \\
0001 \\
0001 \\
N 001\end{array}$ & SU & & $\begin{array}{l}8.12 \\
8.31 \\
8.18 \\
8.17 \\
8.26 \\
8.13 \\
8.03\end{array}$ & $\mathbf{L}$ & $\begin{array}{l}. \\
- \\
- \\
- \\
-\end{array}$ & $\begin{array}{l}- \\
- \\
- \\
- \\
-\end{array}$ \\
\hline PHOSPHATE & $06 / 09 / 89$ & 0001 & $M G / L$ & & 0.6 & & 0.1 & - \\
\hline PHOSPHATE (TOTAL) & $06 / 26 / 93$ & NOOI & MG/L & & 0.4 & & 0.1 & - \\
\hline POTASSIUM & $\begin{array}{l}06 / 09 / 89 \\
05 / 31 / 91 \\
02 / 22 / 92 \\
08 / 11 / 92 \\
02 / 02 / 93 \\
06 / 26 / 93\end{array}$ & $\begin{array}{l}0001 \\
0001 \\
0001 \\
0001 \\
0001 \\
0001\end{array}$ & MG/L & & $\begin{array}{l}1.4 \\
1.78 \\
1.90 \\
1.2 \\
1.7 \\
1.8\end{array}$ & $\star_{L}$ & $\begin{array}{l}0.01 \\
0.01 \\
0.01 \\
0.575 \\
0.1 \\
0.1\end{array}$ & $\begin{array}{l}- \\
- \\
- \\
-\end{array}$ \\
\hline POTASSIUM (TOTAL) & $06 / 26 / 93$ & noo1 & MG/L & & 2.3 & & 0.1 & - \\
\hline
\end{tabular}

- MET gROSS ALPHA (GROSS ALPHA - URAHIUM) HITH 1 MG URANIUH = 686 PCI

* HET GROSS ALPHA (TOTAL) (TOTAL GROSS ALPHA - TOTAL URAHIUM)

PARAMETER VALUE INDICATOR (PVI): < - LESS THAN DETECTION LIHIT

SAMPLE ID CODES:

other PARAMETER VALUE FLAGS:

0001 - FILTERED SAMPLE (.45 MICRONS)

- DUPLICATE aHALYSIS hot hithin CONTROL LIMITS

NOO1 - UNFILTERED SAMPLE

L - LESS THAK THREE BORE VOLUMES REMOVED BEFORE SAMPLIHG

H - SPIKE SAMPLE RECOVERY MOT WITHIN COHTROL LIMITS 
GROUNOWATER QUALITY DATA BY LOCATION

SOMP REV. O Data

SITE: MONO1 MONUMENT VALLEY

LOCATIOH: 0604

MORTH COORDIHATE: 58397.0 FT

EAST COORDINATE: 89424.6 FT

04/21/88 TO $12 / 13 / 94$

REPORT DATE: 06/20/95

FORMATION OF COMPLETIOH: ALLUVIUM (AL)

HYDRAULIC FLOW RELATIONSHIP: CROSS GRADIENT (C)

\begin{tabular}{|c|c|c|c|c|c|c|c|c|}
\hline PARAMETER NAME & LOG DATE & $\underset{\text { SAMPLE }}{\text { ID }}$ & $\begin{array}{l}\text { UNITS OF } \\
\text { MEASURE }\end{array}$ & PV1 & $\begin{array}{l}\text { PARAMETER } \\
\text { VALUE }\end{array}$ & FLAGS & $\begin{array}{l}\text { DETECTIOH } \\
\text { LIMIT }\end{array}$ & $\begin{array}{l}\text { PARAMETER } \\
\text { UNCERTAINTY }\end{array}$ \\
\hline RADIUN-226 & $\begin{array}{l}06 / 09 / 89 \\
05 / 31 / 91 \\
02 / 22 / 92 \\
08 / 11 / 92 \\
11 / 19 / 92\end{array}$ & $\begin{array}{l}0001 \\
0001 \\
0001 \\
0001 \\
0001\end{array}$ & $\mathrm{PCI} / \mathrm{L}$ & & $\begin{array}{l}0.1 \\
0.2 \\
0.0 \\
0.1 \\
0.1\end{array}$ & $L$ & $\begin{array}{l}1 . \\
1.0 \\
1.0 \\
0.3 \\
0.3\end{array}$ & $\begin{array}{l}0.2 \\
0.2 \\
0.3 \\
0.1 \\
0.2\end{array}$ \\
\hline RADIUA-226 (TOTAL) & $11 / 19 / 92$ & NoOI & $\mathrm{PCI} / \mathrm{L}$ & & 0.6 & & 0.3 & 0.3 \\
\hline RADIUH-226 + RADIUN-228 & $\begin{array}{l}06 / 09 / 89 \\
05 / 31 / 91 \\
02 / 22 / 92 \\
08 / 11 / 92 \\
11 / 19 / 92\end{array}$ & $\begin{array}{l}0001 \\
0001 \\
0001 \\
0001 \\
0001\end{array}$ & $\mathrm{PCI} / \mathrm{L}$ & & $\begin{array}{l}0.10 \\
0.20 \\
0.20 \\
1.90 \\
2.00\end{array}$ & & : & : \\
\hline RADIUN-226 + RADIUN-228 (TOTAL) & $11 / 19 / 92$ & NoOI & $\mathrm{PCI} / \mathrm{L}$ & & 1.30 & & - & * \\
\hline RADIUN-228 & $\begin{array}{l}06 / 09 / 89 \\
05 / 31 / 91 \\
02 / 22 / 92 \\
08 / 11 / 92 \\
11 / 19 / 92\end{array}$ & $\begin{array}{l}0001 \\
0001 \\
0001 \\
0001 \\
0001\end{array}$ & $\mathrm{PCl} / \mathrm{L}$ & & $\begin{array}{l}0.0 \\
0.0 \\
0.2 \\
1.8 \\
1.9\end{array}$ & $\begin{array}{l}J \\
L\end{array}$ & $\begin{array}{r}1 . \\
1.0 \\
1.0 \\
2.0 \\
2.7\end{array}$ & $\begin{array}{l}0.9 \\
1.9 \\
1.6 \\
1.1 \\
1.7\end{array}$ \\
\hline RADIUH-228 (TOTAL) & $11 / 19 / 92$ & NOOI & $\mathrm{PCI} / \mathrm{L}$ & & 0.7 & & 2.4 & 1.5 \\
\hline REDOX POTENTIAL & $\begin{array}{l}19 / 99 / 92 \\
06 / 26 / 93\end{array}$ & $\begin{array}{l}0001 \\
\text { N001 }\end{array}$ & mVOLTS & & $\begin{array}{l}453.1 \\
395\end{array}$ & & - & - \\
\hline SELENIUM & $\begin{array}{l}06 / 09 / 89 \\
05 / 31 / 91 \\
02 / 22 / 92 \\
08 / 11 / 92 \\
11 / 19 / 92\end{array}$ & $\begin{array}{l}0001 \\
0001 \\
0001 \\
0001 \\
0001\end{array}$ & $M G / L$ & $\begin{array}{l}< \\
< \\
<\end{array}$ & $\begin{array}{l}0.005 \\
0.006 \\
0.007 \\
0.005 \\
0.0015\end{array}$ & $\begin{array}{l}\text { J } \\
\text { WL } \\
\text { NW }\end{array}$ & $\begin{array}{l}0.005 \\
0.005 \\
0.005 \\
0.005 \\
0.0015\end{array}$ & $\begin{array}{l}\dot{-} \\
\dot{-} \\
\dot{-}\end{array}$ \\
\hline SELENIUM (TOTAL) & $11 / 19 / 92$ & NoOI & $M G / L$ & & 0.0015 & $y$ & 0.0015 & - \\
\hline SILICA - SIO2 & $\begin{array}{l}06 / 09 / 89 \\
05 / 31 / 91\end{array}$ & $\begin{array}{l}0001 \\
0001\end{array}$ & $M G / L$ & & $\begin{array}{l}13 . \\
13.2\end{array}$ & & $\begin{array}{l}2 . \\
0.1\end{array}$ & - \\
\hline SILICA - SIO2 (TOTAL) & $06 / 26 / 93$ & N001 & $M G / L$ & & 24.5 & H & 0.5 & $\because$ \\
\hline SILVER & $\begin{array}{l}06 / 09 / 89 \\
05 / 31 / 91\end{array}$ & $\begin{array}{l}0001 \\
0001\end{array}$ & MG/L & $<$ & $\begin{array}{l}0.01 \\
0.01\end{array}$ & & $\begin{array}{l}0.01 \\
0.01\end{array}$ & - \\
\hline sooluy & $\begin{array}{l}06 / 09 / 89 \\
05 / 31 / 91 \\
02 / 22 / 92 \\
08 / 11 / 92 \\
02 / 02 / 93 \\
06 / 26 / 93\end{array}$ & $\begin{array}{l}0001 \\
0001 \\
0001 \\
0001 \\
0001 \\
0001\end{array}$ & MG/L & & $\begin{array}{c}97.9 \\
112 . \\
110 . \\
98 \\
103 \\
101\end{array}$ & $L$ & $\begin{array}{l}0.002 \\
5 . \\
5 . \\
5 \\
0.1 \\
1\end{array}$ & $\begin{array}{l}: \\
: \\
: \\
-\end{array}$ \\
\hline SOOIUH (TOTAL) & $06 / 26 / 93$ & N001 & MG/L & & 97 & & 1 & - \\
\hline SPECIFIC CONDUCTANCE & $06 / 09 / 89$ & 0001 & ЧHНО/CN & & 385. & & - & - \\
\hline
\end{tabular}

PARAMETER VALUE IMDICATOR (PVI): < - LESS THAN DETECTION LIMIT

OTHER PARAMETER VALUE FLAGS:

H - HOLD TIME EXPIRED, VALUE SUSPECT

SAMPLE ID COOES:

0001 - FILTERED SAMPLE (.45 MICROHS)

J - ESTIMATED VALUE

L - LESS THAN THREE BORE VOLUMES REMOVED BEFORE SAMPLING

- H - SPIKE SNMPLE RECOVERY NOT WITHIN CONTROL LIMITS

- POST-DIGEST SPIKE WTT OF CHTR LIM WHILE SNIP ABS < 50\% SPIKE 
GROUNDHATER QUALITY DATA BY LOCATION

SOWP ReV. O Data

SITE: HONO1 MONUMENT VALLEY

LOCATION: 0604

NORTH COORDINATE: 58397.0 FT

EAST COORDINATE: 89424.6 FT

$04 / 21 / 88$ TO $12 / 13 / 94$

REPORT DATE: 06/20/95

FORMATIOH OF COMPLETION: ALLUVIUM (AL)

HYDRAULIC FLON RELATIOHSHIP: CROSS GRADIEKT (C)

\begin{tabular}{|c|c|c|c|c|c|c|c|c|}
\hline PARAMETER MAME & LOG DATE & $\begin{array}{c}\text { SAMPLE } \\
10\end{array}$ & $\begin{array}{l}\text { UNITS OF } \\
\text { MEASURE }\end{array}$ & PVI & $\begin{array}{l}\text { PARAMETER } \\
\text { VALUE }\end{array}$ & FLAGS & $\begin{array}{l}\text { DETECTION } \\
\text { LIMIT }\end{array}$ & $\begin{array}{l}\text { PARAMETER } \\
\text { UNCERTAINTY }\end{array}$ \\
\hline SPECIFIC CONDUCTANCE & $\begin{array}{l}05 / 31 / 91 \\
02 / 22 / 92 \\
08 / 11 / 92 \\
11 / 19 / 92 \\
02 / 02 / 93 \\
06 / 26 / 93\end{array}$ & $\begin{array}{l}0001 \\
0001 \\
0001 \\
0001 \\
0001 \\
1001\end{array}$ & $\mathrm{UHHO} / \mathrm{CH}$ & & $\begin{array}{l}632 . \\
507 \\
438 \\
517 \\
510 \\
520\end{array}$ & $L$ & $\begin{array}{l}- \\
: \\
-\end{array}$ & $\begin{array}{l}- \\
- \\
- \\
-\end{array}$ \\
\hline STRONTIU:A & $\begin{array}{l}05 / 31 / 91 \\
02 / 22 / 92 \\
08 / 11 / 92 \\
11 / 19 / 92 \\
06 / 26 / 93\end{array}$ & $\begin{array}{l}0001 \\
0001 \\
0001 \\
0001 \\
0001\end{array}$ & $M G / L$ & $<$ & $\begin{array}{l}0.18 \\
0.17 \\
0.2 \\
0.24 \\
0.19\end{array}$ & $\frac{L}{S}$ & $\begin{array}{l}0.01 \\
0.01 \\
0.2 \\
0.00035 \\
0.01\end{array}$ & $\begin{array}{l}- \\
- \\
- \\
-\end{array}$ \\
\hline STROHTIUH (TOTAL) & $\begin{array}{l}11 / 19 / 92 \\
06 / 26 / 93\end{array}$ & $\begin{array}{l}\text { N001 } \\
\text { N001 }\end{array}$ & $M G / L$ & & $\begin{array}{l}0.25 \\
0.20\end{array}$ & & $\begin{array}{l}0.00035 \\
0.01\end{array}$ & - \\
\hline SULFATE & $\begin{array}{l}06 / 09 / 89 \\
05 / 31 / 91 \\
02 / 22 / 92 \\
08 / 11 / 92 \\
11 / 19 / 92 \\
02 / 02 / 93\end{array}$ & $\begin{array}{l}0001 \\
0001 \\
0001 \\
0001 \\
0001 \\
0001\end{array}$ & MG/L & & $\begin{array}{c}119 . \\
158 . \\
95 . \\
100 \\
110 \\
112\end{array}$ & $L$ & $\begin{array}{l}0.1 \\
0.1 \\
10 . \\
0.059 \\
1.0 \\
1\end{array}$ & $\begin{array}{l}- \\
: \\
-\end{array}$ \\
\hline SULFATE (TOTAL) & $\begin{array}{l}11 / 19 / 92 \\
06 / 26 / 93\end{array}$ & $\begin{array}{l}\text { N001 } \\
\text { N001 }\end{array}$ & MG/L & & $\begin{array}{l}120 \\
108\end{array}$ & & 1.0 & - \\
\hline SULFIDE & $\begin{array}{l}06 / 09 / 89 \\
05 / 31 / 91\end{array}$ & $\begin{array}{l}0001 \\
0001\end{array}$ & $M G / L$ & $<$ & $\begin{array}{l}0.1 \\
5.14\end{array}$ & 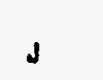 & $\begin{array}{l}0.1 \\
i .\end{array}$ & : \\
\hline TEMPERATURE & $\begin{array}{l}06 / 09 / 89 \\
05 / 31 / 91 \\
02 / 22 / 92 \\
08 / 11 / 92 \\
11 / 19 / 92 \\
02 / 02 / 93 \\
06 / 26 / 93\end{array}$ & $\begin{array}{l}0001 \\
0001 \\
0001 \\
0001 \\
0001 \\
0001 \\
N 001\end{array}$ & C - DEGREE & & $\begin{array}{l}15.3 \\
14.3 \\
14.8 \\
15.4 \\
15.8 \\
15.2 \\
17.4\end{array}$ & $\mathbf{L}$ & $\begin{array}{l}- \\
- \\
- \\
-\end{array}$ & $\begin{array}{l}- \\
- \\
- \\
-\end{array}$ \\
\hline THALLIUM & $\begin{array}{l}06 / 09 / 89 \\
05 / 31 / 91\end{array}$ & $\begin{array}{l}0001 \\
0001\end{array}$ & MG/L & $<$ & $\begin{array}{l}0.09 \\
0.09\end{array}$ & & $\begin{array}{l}0.01 \\
0.01\end{array}$ & $\therefore$ \\
\hline THORIUH-230 & $05 / 31 / 91$ & 0001 & $\mathrm{PCI} / \mathrm{L}$ & & 0.4 & & 1. & 0.3 \\
\hline TIH & $\begin{array}{l}06 / 09 / 89 \\
05 / 31 / 91\end{array}$ & $\begin{array}{l}0001 \\
0001\end{array}$ & $M G / L$ & $<$ & $\begin{array}{l}0.005 \\
0.01\end{array}$ & 1 & $\begin{array}{l}0.005 \\
0.01\end{array}$ & - \\
\hline TOTAL DISSOLVED SOLIDS & $\begin{array}{l}06 / 09 / 89 \\
05 / 31 / 91 \\
02 / 22 / 92 \\
08 / 19 / 92 \\
02 / 02 / 93\end{array}$ & $\begin{array}{l}0001 \\
0001 \\
0001 \\
0001 \\
0001\end{array}$ & HG/L & & $\begin{array}{l}389 . \\
383 . \\
385 . \\
390 \\
370\end{array}$ & $\mathbf{L}$ & $\begin{array}{l}10 . \\
10 . \\
10 . \\
42 \\
10\end{array}$ & - \\
\hline TOTAL DISSOLVED SOLIDS (TOTAL) & $06 / 26 / 93$ & No01 & MG/L & & 390 & & 10 & - \\
\hline TOTAL ORGANIC CARBON & $05 / 31 / 91$ & 0001 & MG/L & & 2. & & 1. & - \\
\hline
\end{tabular}

PARAMETER VALUE IMDICATOR (PVI): < - LESS THAN OETECTION LIMIT

SAMPLE ID COOES:

0001 - FILTERED SAMPLE (.45 MICROHS)

OTHER PARAMETER VALUE FLAGS:

HOO1 - UMFILTERED SAMPLE

1 - IMCREASED DETECTION LIMIT OUE TO REQUIRED DILUTION

J - ESTIMATED VALUE

L - LESS THAK THREE BORE VOLUNES REMOVED BEFORE SUMLIHG

S - REPORTED VALUE DETERMINED USING METHOO OF STD ADDITION (MSA) 
GROUNDHATER QUALITY DATA BY LOCATION

SOUP Rev. O Data

SITE: MOHOI MONUMENT VALLEY

LOCATION: 0604

NORTH COORDINATE: 58397.0 FT

EAST COORDINATE: 89424.6 FT

$04 / 21 / 88$ TO $12 / 13 / 94$

REPORT DATE: 06/20/95

FORMATION OF COMPLETION: ALLUVIUM (AL)

HYORAULIC FLON RELATIOHSHIP: CROSS GRADIENT (C)

\begin{tabular}{|c|c|c|c|c|c|c|c|c|}
\hline PARAMETER NAME & LOG DATE & $\underset{\text { ID }}{\text { SAMPLE }}$ & $\begin{array}{l}\text { UNITS OF } \\
\text { MEASURE }\end{array}$ & PVI & $\begin{array}{l}\text { PARAMETER } \\
\text { VALUE }\end{array}$ & FLAGS & $\begin{array}{l}\text { DETECTIOH } \\
\text { LIMIT }\end{array}$ & $\begin{array}{l}\text { PARAMETER } \\
\text { UHCERTAIHTY }\end{array}$ \\
\hline TOTAL ORGAKIC CARBON & $\begin{array}{l}02 / 22 / 92 \\
08 / 11 / 92\end{array}$ & $\begin{array}{l}0001 \\
0001\end{array}$ & $M G / L$ & $<$ & 1.0 & $\mathbf{L}$ & 1.0 & : \\
\hline URAKIUA & $\begin{array}{l}06 / 09 / 89 \\
05 / 31 / 91 \\
02 / 22 / 92 \\
11 / 19 / 92 \\
02 / 02 / 93 \\
06 / 26 / 93\end{array}$ & $\begin{array}{l}0001 \\
0001 \\
0001 \\
0001 \\
0001 \\
0001\end{array}$ & MG/L & $<$ & $\begin{array}{l}0.0051 \\
0.001 \\
0.002 \\
0.003 \\
0.003 \\
0.002\end{array}$ & & $\begin{array}{l}0.003 \\
0.001 \\
0.001 \\
0.001 \\
0.001 \\
0.001\end{array}$ & $\begin{array}{l}: \\
- \\
- \\
-\end{array}$ \\
\hline URAHIUN (TOTAL) & $\begin{array}{l}19 / 19 / 92 \\
06 / 26 / 93\end{array}$ & $\begin{array}{l}\text { Y001 } \\
\text { NO01 }\end{array}$ & MG/L & & $\begin{array}{l}0.003 \\
0.002\end{array}$ & & $\begin{array}{l}0.001 \\
0.001\end{array}$ & - \\
\hline VANADIUN & $\begin{array}{l}06 / 09 / 89 \\
05 / 31 / 91 \\
02 / 22 / 92 \\
08 / 11 / 92 \\
11 / 19 / 92 \\
06 / 26 / 93\end{array}$ & $\begin{array}{l}0001 \\
0001 \\
0001 \\
0001 \\
0001 \\
0001\end{array}$ & MG/L & $\begin{array}{l}< \\
< \\
< \\
< \\
<\end{array}$ & $\begin{array}{l}0.01 \\
0.01 \\
0.01 \\
0.05 \\
0.0019 \\
0.01\end{array}$ & $L$ & $\begin{array}{l}0.01 \\
0.01 \\
0.01 \\
0.05 \\
0.0019 \\
0.01\end{array}$ & $\begin{array}{l}: \\
: \\
: \\
-\end{array}$ \\
\hline VAHADIUH (TOTAL) & $\begin{array}{l}11 / 19 / 92 \\
06 / 26 / 93\end{array}$ & $\begin{array}{l}\text { NOO1 } \\
\text { N001 }\end{array}$ & $M G / L$ & $<$ & $\begin{array}{l}0.0020 \\
0.01\end{array}$ & & $\begin{array}{l}0.0019 \\
0.01\end{array}$ & - \\
\hline ZINC & $\begin{array}{l}05 / 09 / 89 \\
05 / 31 / 91 \\
02 / 22 / 92 \\
08 / 11 / 92 \\
11 / 19 / 92 \\
06 / 26 / 93\end{array}$ & $\begin{array}{l}0001 \\
0001 \\
0001 \\
0001 \\
0001 \\
0001\end{array}$ & MG/L & $\begin{array}{l}< \\
< \\
< \\
<\end{array}$ & $\begin{array}{l}0.005 \\
0.005 \\
0.005 \\
0.02 \\
0.012 \\
0.005\end{array}$ & $\begin{array}{l}\mathrm{L} \\
\mathrm{J}\end{array}$ & $\begin{array}{l}0.005 \\
0.005 \\
0.005 \\
0.02 \\
0.0010 \\
0.005\end{array}$ & $\begin{array}{l}: \\
: \\
:\end{array}$ \\
\hline ZIHC (TOTAL) & $\begin{array}{l}11 / 19 / 92 \\
06 / 26 / 93\end{array}$ & $\begin{array}{l}\text { No01 } \\
\text { N001 }\end{array}$ & $M G / L$ & & $\begin{array}{l}0.0060 \\
0.008\end{array}$ & \rfloor & $\begin{array}{l}0.0010 \\
0.005\end{array}$ & - \\
\hline
\end{tabular}

PARAMETER VALUE IHOICATOR (PVI): < - LESS THAN DETECTION LIMIT

SAMPLE ID COOES:

0001 - FILTERED SAMPLE (.45 HICRONS)

OTHER PARAMETER VALUE FLAGS:

NOO1 - UNFILTERED SAMPLE

J - ESTIMATED VALUE

L - LESS THAN THREE BORE VOLUHES REMOVED BEFORE SAMPLING 
GROUNOWATER QUALITY DATA BY LOCATION

SOMP ReV. O Data

SITE: HOHO1 MONUNENT VALLEY

LOCATIOH: 0605

MORTH COORDINATE: 58707.6 FT

EAST COORDIHATE: 90066.7 FT

$04 / 21 / 88$ TO $12 / 13 / 94$

REPORT DATE: 06/20/95

FORMATIOH OF COMPLETION: ALLUVIUM (AL)

HYDRAULIC FLON RELATIONSHIP: DOWN GRADIENT (D)

\begin{tabular}{|c|c|c|c|c|c|c|c|c|}
\hline PARAMETER MAME & LOG DATE & $\underset{\text { SN }}{\text { SAMPLE }}$ & $\begin{array}{l}\text { UWITS OF } \\
\text { MEASURE }\end{array}$ & PVI & $\begin{array}{l}\text { PARAMETER } \\
\text { VALUE }\end{array}$ & FLAGS & $\begin{array}{l}\text { DETECTION } \\
\text { LIMIT }\end{array}$ & $\begin{array}{l}\text { PARAMETER } \\
\text { UNCERTAINTY }\end{array}$ \\
\hline ALKALIHITY & $\begin{array}{l}04 / 22 / 88 \\
06 / 09 / 89 \\
12 / 04 / 89 \\
06 / 21 / 90 \\
01 / 29 / 91 \\
05 / 31 / 91 \\
11 / 22 / 92 \\
02 / 02 / 93 \\
06 / 26 / 93\end{array}$ & $\begin{array}{l}0001 \\
0001 \\
0001 \\
0001 \\
0001 \\
0001 \\
0001 \\
0001 \\
1001\end{array}$ & MG/L CACO3 & & $\begin{array}{l}204 . \\
219 . \\
331 . \\
240 . \\
312 . \\
301 . \\
349 \\
393 \\
372\end{array}$ & L & $\begin{array}{l}- \\
\dot{-} \\
\dot{-} \\
\dot{-} \\
\dot{-} \\
\dot{-}\end{array}$ & $\begin{array}{l}- \\
- \\
- \\
- \\
- \\
-\end{array}$ \\
\hline ALUHIHUM & $\begin{array}{l}06 / 09 / 89 \\
12 / 04 / 89 \\
06 / 21 / 90 \\
01 / 29 / 91 \\
05 / 31 / 91\end{array}$ & $\begin{array}{l}0001 \\
0001 \\
0001 \\
0001 \\
0001\end{array}$ & MG/L & $\begin{array}{l}< \\
< \\
< \\
< \\
<\end{array}$ & $\begin{array}{l}0.1 \\
0.1 \\
0.1 \\
0.1 \\
0.05\end{array}$ & & $\begin{array}{l}0.1 \\
0.1 \\
0.1 \\
0.1 \\
0.05\end{array}$ & $\begin{array}{l}\because \\
\because- \\
-\end{array}$ \\
\hline AMHONIUM & $\begin{array}{l}04 / 22 / 88 \\
06 / 09 / 89 \\
12 / 04 / 89 \\
06 / 21 / 90 \\
01 / 29 / 91 \\
05 / 31 / 91\end{array}$ & $\begin{array}{l}0001 \\
0001 \\
0001 \\
0001 \\
0001 \\
0001\end{array}$ & MG/L & $<$ & $\begin{array}{l}0.1 \\
0.5 \\
0.5 \\
0.6 \\
2.2 \\
0.5\end{array}$ & $H$ & $\begin{array}{l}0.1 \\
0.1 \\
0.1 \\
0.1 \\
0.1 \\
0.1\end{array}$ & $\begin{array}{l}: \\
: \\
:\end{array}$ \\
\hline AMOHIUA (TOTAL) & $06 / 26 / 93$ & NOOI & MG/L & & 0.1 & & 0.1 & - \\
\hline ANTIMONY & $\begin{array}{l}06 / 09 / 89 \\
12 / 04 / 89 \\
06 / 21 / 90 \\
01 / 29 / 91 \\
05 / 31 / 91\end{array}$ & $\begin{array}{l}0001 \\
0001 \\
0001 \\
0001 \\
0001\end{array}$ & $M G / L$ & $\begin{array}{l}< \\
< \\
< \\
<\end{array}$ & $\begin{array}{l}0.003 \\
0.003 \\
0.006 \\
0.003 \\
0.003\end{array}$ & a & $\begin{array}{l}0.003 \\
0.003 \\
0.003 \\
0.003 \\
0.003\end{array}$ & $\begin{array}{l}- \\
- \\
-\end{array}$ \\
\hline ARSENIC & $\begin{array}{l}04 / 22 / 88 \\
06 / 09 / 89 \\
12 / 04 / 89 \\
06 / 21 / 90 \\
01 / 29 / 91 \\
05 / 31 / 91 \\
11 / 22 / 92 \\
06 / 26 / 93\end{array}$ & $\begin{array}{l}0001 \\
0001 \\
0001 \\
0001 \\
0001 \\
0001 . \\
0001 \\
0001\end{array}$ & MG/L & $\begin{array}{l}< \\
< \\
< \\
< \\
< \\
<\end{array}$ & $\begin{array}{l}0.019 \\
0.01 \\
0.09 \\
0.01 \\
0.01 \\
0.01 \\
0.0015 \\
0.005\end{array}$ & $L$ & $\begin{array}{l}0.01 \\
0.01 \\
0.01 \\
0.01 \\
0.01 \\
0.01 \\
0.0015 \\
0.005\end{array}$ & $\begin{array}{l}- \\
- \\
- \\
- \\
-\end{array}$ \\
\hline ARSENIC (TOTAL) & $\begin{array}{l}11 / 22 / 92 \\
06 / 26 / 93\end{array}$ & $\begin{array}{l}\text { No01 } \\
\text { N001 }\end{array}$ & $M G / L$ & $<$ & $\begin{array}{l}0.0015 \\
0.005\end{array}$ & $\mathbf{L}$ & $\begin{array}{l}0.0015 \\
0.005\end{array}$ & - \\
\hline BARILA & $\begin{array}{l}06 / 09 / 89 \\
12 / 04 / 89 \\
06 / 21 / 90 \\
01 / 29 / 91 \\
05 / 31 / 91 \\
11 / 22 / 92 \\
06 / 26 / 93\end{array}$ & $\begin{array}{l}0001 \\
0001 \\
0001 \\
0001 \\
0001 \\
0001 \\
0001\end{array}$ & MG/L & $\begin{array}{l}< \\
< \\
< \\
<\end{array}$ & $\begin{array}{l}0.1 \\
0.1 \\
0.1 \\
0.1 \\
0.03 \\
0.023 \\
0.1\end{array}$ & $\mathbf{L}$ & $\begin{array}{l}0.1 \\
0.1 \\
0.1 \\
0.1 \\
0.01 \\
0.0015 \\
0.1\end{array}$ & $\begin{array}{l}- \\
:- \\
- \\
-\end{array}$ \\
\hline BARIUA (TOTAL) & $\begin{array}{l}11 / 22 / 92 \\
06 / 26 / 93\end{array}$ & $\begin{array}{l}\text { N009 } \\
\text { N001 }\end{array}$ & $M G / L$ & $<$ & $\begin{array}{l}0.022 \\
0.1\end{array}$ & $L$ & $\begin{array}{l}0.0015 \\
0.1\end{array}$ & $\dot{-}$ \\
\hline BERYLLIUYA & $06 / 09 / 89$ & 0009 & $M G / L$ & $<$ & 0.01 & & 0.01 & - \\
\hline
\end{tabular}

PARAMETER VALUE INDICATOR (PVI): < - LESS THAH DETECTION LIMIT

SAMPLE ID COOES

OTHER PARAMETER VALUE FLAGS:

O001 - FILTERED SAMPLE (.45 MICRONS)

H - HOLD TIME EXPIRED, VALUE SUSPECT

NO01 - UNFILTERED SAMPLE

J - ESTIMUTED VALLE

L - LESS THAK THREE BORE VOLLMES REMOVED BEFORE SAMPLIHG 
GROUNDWATER OUALITY DATA BY LOCATION

SOWP Rev. O Data

SITE: MONO1 MONLWENT VALLEY

LOCATION: 0605

MORTH COORDIMATE: 58707.6 FT

EAST COORDINATE: $\quad 90066.7$ FT

$04 / 21 / 88$ TO $12 / 13 / 94$

REPORT DATE: 06/20/95

FORMATION OF COAPLETION: ALLUVIUM (AL)

HYDRAULIC FLON RELATIONSHIP: DOUH GRADIENT (D)

\begin{tabular}{|c|c|c|c|c|c|c|c|}
\hline PARAMETER MAME & LOG DATE & $\mid \begin{array}{c}\text { SAMPLE } \\
\text { ID }\end{array}$ & $\begin{array}{l}\text { UNITS OF } \\
\text { MEASURE }\end{array}$ & PVI & $\begin{array}{l}\text { PARAMETER } \\
\text { VALUE FLAGS }\end{array}$ & $\begin{array}{l}\text { DETECTION } \\
\text { LIMIT }\end{array}$ & $\begin{array}{l}\text { PARAMETER } \\
\text { UNCERTAINTY }\end{array}$ \\
\hline BERYLLIUH & $\begin{array}{l}12 / 04 / 89 \\
06 / 21 / 90 \\
01 / 29 / 91 \\
05 / 31 / 91\end{array}$ & $\begin{array}{l}0001 \\
0001 \\
0001 \\
0001\end{array}$ & MG/L & $\begin{array}{l}< \\
< \\
< \\
<\end{array}$ & $\begin{array}{l}0.005 \\
0.01 \\
0.01 \\
0.005\end{array}$ & $\begin{array}{l}0.005 \\
0.01 \\
0.01 \\
0.005\end{array}$ & $\begin{array}{l}- \\
- \\
-\end{array}$ \\
\hline BORON & $\begin{array}{l}06 / 09 / 89 \\
12 / 04 / 89 \\
06 / 21 / 90 \\
01 / 29 / 91 \\
05 / 31 / 91\end{array}$ & $\begin{array}{l}0001 \\
0001 \\
0001 \\
0001 \\
0001\end{array}$ & MG/L & $\begin{array}{l}< \\
<\end{array}$ & $\begin{array}{l}0.1 \\
0.2 \\
0.1 \\
0.2 \\
0.21\end{array}$ & $\begin{array}{l}0.1 \\
0.1 \\
0.1 \\
0.1 \\
0.05\end{array}$ & $\begin{array}{l}- \\
\dot{-} \\
\dot{-} \\
-\end{array}$ \\
\hline BROMIDE & $\begin{array}{l}01 / 29 / 91 \\
05 / 31 / 91\end{array}$ & $\begin{array}{l}0001 \\
0001\end{array}$ & MG/L & $<$ & $\begin{array}{l}0.1 \\
1.1\end{array}$ & $\begin{array}{l}0.1 \\
0.1\end{array}$ & $\dot{-}$ \\
\hline CADMIUH & $\begin{array}{l}04 / 22 / 88 \\
06 / 09 / 89 \\
12 / 04 / 89 \\
06 / 21 / 90 \\
01 / 29 / 91 \\
05 / 31 / 91 \\
11 / 22 / 92\end{array}$ & $\begin{array}{l}0001 \\
0001 \\
0001 \\
0001 \\
0001 \\
0001 \\
0001\end{array}$ & MG/L & $\begin{array}{l}< \\
< \\
< \\
< \\
<\end{array}$ & $\begin{array}{l}0.017 \\
0.002 \\
0.001 \\
0.001 \\
0.001 \\
0.001 \\
0.00013 L\end{array}$ & $\begin{array}{l}0.001 \\
0.001 \\
0.001 \\
0.001 \\
0.001 \\
0.001 \\
0.00013\end{array}$ & $\begin{array}{l}- \\
- \\
- \\
- \\
-\end{array}$ \\
\hline CADMILM (TOTAL) & $11 / 22 / 92$ & Hoo1 & ME/L & & $0.00014 \mathrm{NL}$ & 0.00013 & - \\
\hline CALCIUA & $\begin{array}{l}04 / 22 / 88 \\
06 / 09 / 89 \\
12 / 04 / 89 \\
06 / 21 / 90 \\
01 / 29 / 91 \\
05 / 31 / 91 \\
02 / 02 / 93 \\
06 / 26 / 93\end{array}$ & $\begin{array}{l}0001 \\
0001 \\
0001 \\
0001 \\
0001 \\
0001 \\
0001 \\
0001\end{array}$ & MG/L & & $\begin{array}{l}106 . \\
116 . \\
162 . \\
130 . \\
146 . \\
130 . \\
112 \\
118\end{array}$ & $\begin{array}{l}0.01 \\
0.01 \\
0.01 \\
0.01 \\
0.01 \\
0.5 \\
0.5 \\
0.5\end{array}$ & $\begin{array}{l}- \\
- \\
- \\
- \\
- \\
-\end{array}$ \\
\hline CALCIUM (TOTAL) & $06 / 26 / 93$ & No01 & MG/L & & 121 & 0.5 & - \\
\hline CHLORIDE & $\begin{array}{l}04 / 22 / 88 \\
06 / 09 / 89 \\
12 / 04 / 89 \\
06 / 21 / 90 \\
01 / 29 / 91 \\
05 / 31 / 91 \\
02 / 02 / 93\end{array}$ & $\begin{array}{l}0001 \\
0001 \\
0001 \\
0001 \\
0001 \\
0001 \\
0001\end{array}$ & $M G / L$ & & $\begin{array}{l}110 . \\
120 . \\
212 . \\
124 . \\
259 . \\
195 . \\
232\end{array}$ & $\begin{array}{l}i . \\
i . \\
i . \\
i .5 \\
0.5 \\
0.5\end{array}$ & $\begin{array}{l}- \\
- \\
- \\
- \\
-\end{array}$ \\
\hline CHLORIDE (TOTAL) & $06 / 26 / 93$ & noo1 & MG/L & & 248 & 0.5 & - \\
\hline CHROAIUN & $\begin{array}{l}06 / 09 / 89 \\
12 / 04 / 89 \\
06 / 21 / 90 \\
01 / 29 / 91 \\
05 / 31 / 91 \\
11 / 22 / 92\end{array}$ & $\begin{array}{l}0001 \\
0001 \\
0001 \\
0001 \\
0001 \\
0001\end{array}$ & HG/L & $\begin{array}{l}< \\
< \\
< \\
< \\
< \\
<\end{array}$ & $\begin{array}{l}0.01 \\
0.01 \\
0.01 \\
0.01 \\
0.01 \\
0.0053 \quad L\end{array}$ & $\begin{array}{l}0.01 \\
0.01 \\
0.01 \\
0.01 \\
0.01 \\
0.0053\end{array}$ & $\begin{array}{l}- \\
- \\
- \\
-\end{array}$ \\
\hline CHROMIUH (TOTAL) & $11 / 22 / 92$ & NoO1 & MG/L & $<$ & $0.0053 \mathrm{~L}$ & 0.0053 & - \\
\hline COBALT & $06 / 09 / 89$ & 0001 & MG/L & $<$ & 0.05 & 0.05 & - \\
\hline
\end{tabular}

PARAMETER VALUE INDICATOR (PVI): < - LESS THAN DETECTION LIMIT

SAMPLE ID COOES:

0001 - FILTERED SAMPLE (.45 MICRONS)

OTHER PARAMETER VALUE FLAGS:

NOO1 - UMFILTERED SAMPLE

J - ESTIMATED VALUE

L - LESS THAH THREE BORE VOLUMES REMOVED BEFORE SAMPLIMG

W - SPIKE SNMPLE RECOVERY HOT UITHIN CONTROL LIMITS 
GROUHDHATER QUALITY DATA BY LOCATIOH

SOWP ReV. O Data

SITE: MONO MONUMENT VALLEY

LOCATION: 0605

MORTH COORDIHATE: 58707.6 FT

EAST COORDINATE: $\quad 90066.7$ FT

$04 / 21 / 88$ TO $12 / 13 / 94$

REPORT DATE: 06/20/95

FORMATION OF COMPLETION: ALLUVIUM (AL)

HYDRAULIC FLON RELATIONSHIP: DONM GRADIENT (D)

\begin{tabular}{|c|c|c|c|c|c|c|c|c|}
\hline PARNHETER MAME & LOS DATE & $\begin{array}{c}\text { SAMPLE } \\
10\end{array}$ & $\begin{array}{l}\text { UNITS OF } \\
\text { MEASURE }\end{array}$ & PVI & $\begin{array}{l}\text { PARAMETER } \\
\text { VALUE }\end{array}$ & FLAGS & $\begin{array}{l}\text { DETECTION } \\
\text { LIMIT }\end{array}$ & $\begin{array}{l}\text { PARAMETER } \\
\text { UNCERTAINTY }\end{array}$ \\
\hline $\cos A L T$ & $\begin{array}{l}12 / 04 / 89 \\
06 / 21 / 90 \\
01 / 29 / 91 \\
05 / 31 / 91\end{array}$ & $\begin{array}{l}0001 \\
0001 \\
0001 \\
0001\end{array}$ & MG $/ L$ & $\begin{array}{l}< \\
< \\
< \\
<\end{array}$ & $\begin{array}{l}0.05 \\
0.05 \\
0.05 \\
0.03\end{array}$ & & $\begin{array}{l}0.05 \\
0.05 \\
0.05 \\
0.03\end{array}$ & $\begin{array}{l}- \\
- \\
-\end{array}$ \\
\hline COPPER & $\begin{array}{l}06 / 09 / 89 \\
12 / 04 / 89 \\
06 / 21 / 90 \\
01 / 29 / 91 \\
05 / 31 / 91\end{array}$ & $\begin{array}{l}0001 \\
0001 \\
0001 \\
0001 \\
0001\end{array}$ & HG/L & $\begin{array}{l}< \\
< \\
< \\
< \\
<\end{array}$ & $\begin{array}{l}0.02 \\
0.02 \\
0.02 \\
0.02 \\
0.01\end{array}$ & & $\begin{array}{l}0.02 \\
0.02 \\
0.02 \\
0.02 \\
0.01\end{array}$ & $\begin{array}{l}- \\
\dot{-} \\
\dot{-}\end{array}$ \\
\hline CYAHIDE & $\begin{array}{l}06 / 09 / 89 \\
12 / 04 / 89\end{array}$ & $\begin{array}{l}0001 \\
0001\end{array}$ & $\mathrm{MG} / \mathrm{L}$ & $<$ & $\begin{array}{l}0.01 \\
0.01\end{array}$ & & $\begin{array}{l}0.09 \\
0.09\end{array}$ & : \\
\hline DISSOLVED OXYGEH & $\begin{array}{l}11 / 22 / 92 \\
06 / 26 / 93\end{array}$ & $\begin{array}{l}0001 \\
\text { K001 }\end{array}$ & $M G / L$ & & $\begin{array}{l}0.0 \\
0.0\end{array}$ & $\mathbf{L}$ & - & - \\
\hline FLUORIDE & $\begin{array}{l}06 / 09 / 89 \\
12 / 04 / 89 \\
06 / 21 / 90 \\
01 / 29 / 91 \\
05 / 31 / 91\end{array}$ & $\begin{array}{l}0001 \\
0001 \\
0001 \\
0001 \\
0001\end{array}$ & MG/L & & $\begin{array}{l}0.3 \\
0.3 \\
0.3 \\
0.3 \\
0.2\end{array}$ & & $\begin{array}{l}0.1 \\
0.1 \\
0.1 \\
0.1 \\
0.1\end{array}$ & $\begin{array}{l}- \\
- \\
- \\
-\end{array}$ \\
\hline GROSS ALPHA & $\begin{array}{l}04 / 22 / 88 \\
01 / 29 / 91 \\
05 / 31 / 91 \\
11 / 22 / 92 \\
02 / 02 / 93 \\
06 / 26 / 93\end{array}$ & $\begin{array}{l}0001 \\
0001 \\
0001 \\
0001 \\
0001 \\
0001\end{array}$ & $\mathrm{PCI} / \mathrm{L}$ & & $\begin{array}{l}38 . \\
14 . \\
0.0 \\
60.0 \\
12 \\
39\end{array}$ & NL & $\begin{array}{l}0.2 \\
1 . \\
1 . \\
24.0 \\
40 \\
41\end{array}$ & $\begin{array}{l}13 . \\
26 . \\
26.3 \\
24.9 \\
45 \\
70\end{array}$ \\
\hline GROSS ALPHA (TOTAL) & $\begin{array}{l}11 / 22 / 92 \\
06 / 26 / 93\end{array}$ & $\begin{array}{l}\text { No01 } \\
\text { N001 }\end{array}$ & PCI/L & & $\begin{array}{l}0.0 \\
0\end{array}$ & NL & $\begin{array}{l}52.8 \\
41\end{array}$ & $\begin{array}{l}27.8 \\
61\end{array}$ \\
\hline GROSS BETA & $\begin{array}{l}04 / 22 / 88 \\
01 / 29 / 91 \\
05 / 31 / 91 \\
11 / 22 / 92 \\
02 / 02 / 93 \\
06 / 26 / 93\end{array}$ & $\begin{array}{l}0001 \\
0001 \\
0001 \\
0001 \\
0001 \\
0001\end{array}$ & PCI/L & • & $\begin{array}{c}9.0 \\
22 . \\
0.0 \\
9.7 \\
0 \\
0\end{array}$ & NL & $\begin{array}{l}1 . \\
0.5 \\
0.5 \\
41.1 \\
58 \\
45\end{array}$ & $\begin{array}{l}5.5 \\
19 . \\
15.8 \\
24.8 \\
25 \\
40\end{array}$ \\
\hline GROSS BETA (TOTAL) & $\begin{array}{l}11 / 22 / 92 \\
06 / 26 / 93\end{array}$ & $\begin{array}{l}\text { NOO1 } \\
\text { N001 }\end{array}$ & PCI/L & & $\begin{array}{l}0.0 \\
0\end{array}$ & ML & $\begin{array}{l}39.0 \\
45\end{array}$ & $\begin{array}{l}23.1 \\
41\end{array}$ \\
\hline IRON & $\begin{array}{l}06 / 09 / 89 \\
12 / 04 / 89 \\
06 / 21 / 90 \\
01 / 29 / 91 \\
05 / 31 / 91 \\
02 / 02 / 93 \\
06 / 26 / 93\end{array}$ & $\begin{array}{l}0001 \\
0001 \\
0001 \\
0001 \\
0001 \\
0001 \\
0001\end{array}$ & MG/L & $<$ & $\begin{array}{l}0.14 \\
0.14 \\
0.11 \\
0.12 \\
0.06 \\
0.03 \\
0.03\end{array}$ & J & $\begin{array}{l}0.03 \\
0.03 \\
0.03 \\
0.03 \\
0.03 \\
0.03 \\
0.03\end{array}$ & $\begin{array}{l}: \\
\dot{-} \\
\dot{-} \\
-\end{array}$ \\
\hline IRON (TOTAL) & $06 / 26 / 93$ & N001 & MG/L & & 0.08 & J & 0.03 & - \\
\hline LEAD & $06 / 09 / 89$ & 0001 & $M G / L$ & $<$ & 0.01 & & 0.01 & - \\
\hline
\end{tabular}

PARAMETER VALUE IMDICATOR (PVI): < - LESS THAN DETECTION LIMIT

SAMPLE ID COOES:

OTHER PARAMETER VALUE FLAGS:

0001 - FILTERED SAMPLE (.45 MICRONS)

J - ESTIMATED VALUE

NOO - UNFILTERED SAMPLE

L - LESS THAH THREE BORE VOLLMES REMOVED BEFORE SAMPLING

\# - SPIKE SAMPLE RECOVERY HOT HITHIH CONTROL LIMITS 
GROUNDWATER QUALITY DATA BY LOCATION

SOWP ReV. $O$ Data

SITE: MONO1 MONUMENT VALLEY

LOCATION: 0605

HORTH COORDINATE: 58707.6 FT

EAST COORDINATE: 90066.7 FT

$04 / 21 / 88$ TO $12 / 13 / 94$

REPORT DATE: 06/20/95

FORMATIOH OF COMPLETIOH: ALLUVIUM (AL)

HYDRAULIC FLON RELATIONSHIP: DOWN GRADIENT (D)

\begin{tabular}{|c|c|c|c|c|c|c|c|c|}
\hline PARAMETER MUME & LOG DATE & $\begin{array}{c}\text { SAMPLE } \\
\text { ID }\end{array}$ & $\begin{array}{l}\text { UNITS OF } \\
\text { MEASURE }\end{array}$ & PVI & $\begin{array}{l}\text { PARAMETER } \\
\text { VALUE }\end{array}$ & FLAGS & $\begin{array}{l}\text { DETECTIOH } \\
\text { LIMIT }\end{array}$ & $\begin{array}{l}\text { PARAMETER } \\
\text { UNCERTAINTY }\end{array}$ \\
\hline LEAD & $\begin{array}{l}12 / 04 / 89 \\
06 / 21 / 90 \\
01 / 29 / 91 \\
05 / 31 / 91 \\
11 / 22 / 92\end{array}$ & $\begin{array}{l}0001 \\
0001 \\
0001 \\
0001 \\
0001\end{array}$ & $M G / L$ & $\begin{array}{l}< \\
< \\
< \\
<\end{array}$ & $\begin{array}{l}0.01 \\
0.01 \\
0.01 \\
0.005 \\
0.0015\end{array}$ & $\begin{array}{l}\text { J } \\
\text { L }\end{array}$ & $\begin{array}{l}0.01 \\
0.01 \\
0.01 \\
0.005 \\
0.0015\end{array}$ & $\begin{array}{l}- \\
- \\
-\end{array}$ \\
\hline LEAD (TOTAL) & $11 / 22 / 92$ & N001 & MG/L & $<$ & 0.0015 & HL & 0.0015 & - \\
\hline LEAD-210 & $11 / 22 / 92$ & 0001 & $\mathrm{PCI} / \mathrm{L}$ & & 0.6 & $\mathbf{L}$ & 2.0 & 1.2 \\
\hline LEAD-210 (TOTAL) & $11 / 22 / 92$ & N001 & PCI/L & & 5.4 & $\mathbf{L}$ & 0.3 & 0.9 \\
\hline MAGKESIUM & $\begin{array}{l}04 / 22 / 88 \\
06 / 09 / 89 \\
12 / 04 / 89 \\
06 / 21 / 90 \\
01 / 29 / 91 \\
05 / 31 / 91 \\
02 / 02 / 93 \\
06 / 26 / 93\end{array}$ & $\begin{array}{l}0001 \\
0001 \\
0001 \\
0001 \\
0001 \\
0001 \\
0001 \\
0001\end{array}$ & $M G / L$ & & $\begin{array}{l}87.5 \\
108 . \\
175 . \\
112 . \\
154 . \\
145 . \\
145 \\
148\end{array}$ & & $\begin{array}{l}0.001 \\
0.001 \\
0.001 \\
0.001 \\
0.001 \\
0.5 \\
0.1 \\
0.1\end{array}$ & $\begin{array}{l}- \\
- \\
- \\
- \\
-\end{array}$ \\
\hline MAGHESIUH (TOTAL) & $06 / 26 / 93$ & N001 & $M G / L$ & & 153 & & 0.1 & - \\
\hline MANGANESE & $\begin{array}{l}06 / 09 / 89 \\
12 / 04 / 89 \\
06 / 21 / 90 \\
01 / 29 / 91 \\
05 / 31 / 91 \\
11 / 22 / 92 \\
02 / 02 / 93 \\
06 / 26 / 93\end{array}$ & $\begin{array}{l}0001 \\
0001 \\
0001 \\
0001 \\
0001 \\
0001 \\
0001 \\
0001\end{array}$ & MG/L & & $\begin{array}{l}0.06 \\
0.03 \\
0.04 \\
0.05 \\
0.02 \\
0.022 \\
0.02 \\
0.02\end{array}$ & $\mathbf{L}$ & $\begin{array}{l}0.01 \\
0.01 \\
0.01 \\
0.01 \\
0.01 \\
0.0015 \\
0.01 \\
0.01\end{array}$ & $\begin{array}{l}: \\
: \\
: \\
: \\
-\end{array}$ \\
\hline MAHGANESE (TOTAL) & $\begin{array}{l}11 / 22 / 92 \\
06 / 26 / 93\end{array}$ & $\begin{array}{l}\text { No01 } \\
\text { N001 }\end{array}$ & $M G / L$ & & $\begin{array}{l}0.019 \\
0.02\end{array}$ & $\mathbf{L}$ & $\begin{array}{l}0.0015 \\
0.01\end{array}$ & - \\
\hline MERCURY & $\begin{array}{l}06 / 09 / 89 \\
12 / 04 / 89 \\
06 / 21 / 90\end{array}$ & $\begin{array}{l}0001 \\
0001 \\
0001\end{array}$ & MG/L & $\begin{array}{l}< \\
< \\
<\end{array}$ & $\begin{array}{l}0.0002 \\
0.0002 \\
0.0002\end{array}$ & & $\begin{array}{l}0.0002 \\
0.0002 \\
0.0002\end{array}$ & - \\
\hline MOLYBDENUM & $\begin{array}{l}04 / 22 / 88 \\
06 / 09 / 89 \\
12 / 04 / 89 \\
06 / 21 / 90 \\
01 / 29 / 99 \\
05 / 31 / 91 \\
11 / 22 / 92 \\
06 / 26 / 93\end{array}$ & $\begin{array}{l}0001 \\
0001 \\
0001 \\
0009 \\
0001 \\
0009 \\
0001 \\
0001\end{array}$ & $M G / L$ & $<$ & $\begin{array}{l}0.04 \\
0.01 \\
0.02 \\
0.01 \\
0.07 \\
0.03 \\
0.043 \\
0.06\end{array}$ & ML. & $\begin{array}{l}0.01 \\
0.01 \\
0.01 \\
0.01 \\
0.01 \\
0.01 \\
0.0049 \\
0.01\end{array}$ & $\begin{array}{l}- \\
- \\
- \\
- \\
-\end{array}$ \\
\hline MOLYBDENUNA (TOTAL) & $\begin{array}{l}11 / 22 / 92 \\
06 / 26 / 93\end{array}$ & $\begin{array}{l}\text { No01 } \\
\text { N009 }\end{array}$ & HG/L & & $\begin{array}{l}0.044 \\
0.06\end{array}$ & HL & $\begin{array}{l}0.0049 \\
0.01\end{array}$ & : \\
\hline MET GROSS ALPHA * & $04 / 22 / 88$ & 0001 & $\mathrm{PCI} / \mathrm{L}$ & & 35.53 & & - & - \\
\hline
\end{tabular}

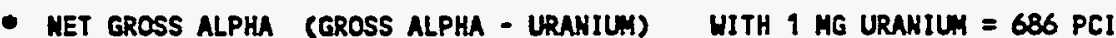

PARAMETER VALUE INDICATOR (PVI): < - LESS than DETECTION LIMIT

OTHER PARAMETER VALUE FLAGS:

J - ESTIMUTED VALUE

L - LESS THAH THREE BORE VOLUMES REMOVED BEFORE SAMPLIHG

H - SPIKE SAYPLE RECOVERY NOT WITHIN CONTROL LIMITS

$W$ - POST-DIGEST SPIKE OUT OF CNTR LIM WHILE SAMP ABS < 50\% SPIKE
SAMPLE ID COOES:

OOO1 - FILTERED SAMPLE (.45 MICROHS)

NOO1 - UNFILTERED SAMPLE 
GROUWDHATER QUALITY DATA BY LOCATION

SOWP ReV. O Data

SITE: MONO1 MONLMENT VALLEY

LOCATIOH: 0605

HORTH COORDIHATE: 58707.6 FT

EAST COORDINATE: $\quad 90066.7$ FT

$04 / 21 / 88$ TO $12 / 13 / 94$

REPORT DATE: $06 / 20 / 95$

FORMATIOH OF COMPLETION: ALLUVIUM (AL)

HYDRAULIC FLOM RELATIONSHIP: DOWN GRADIENT (D)

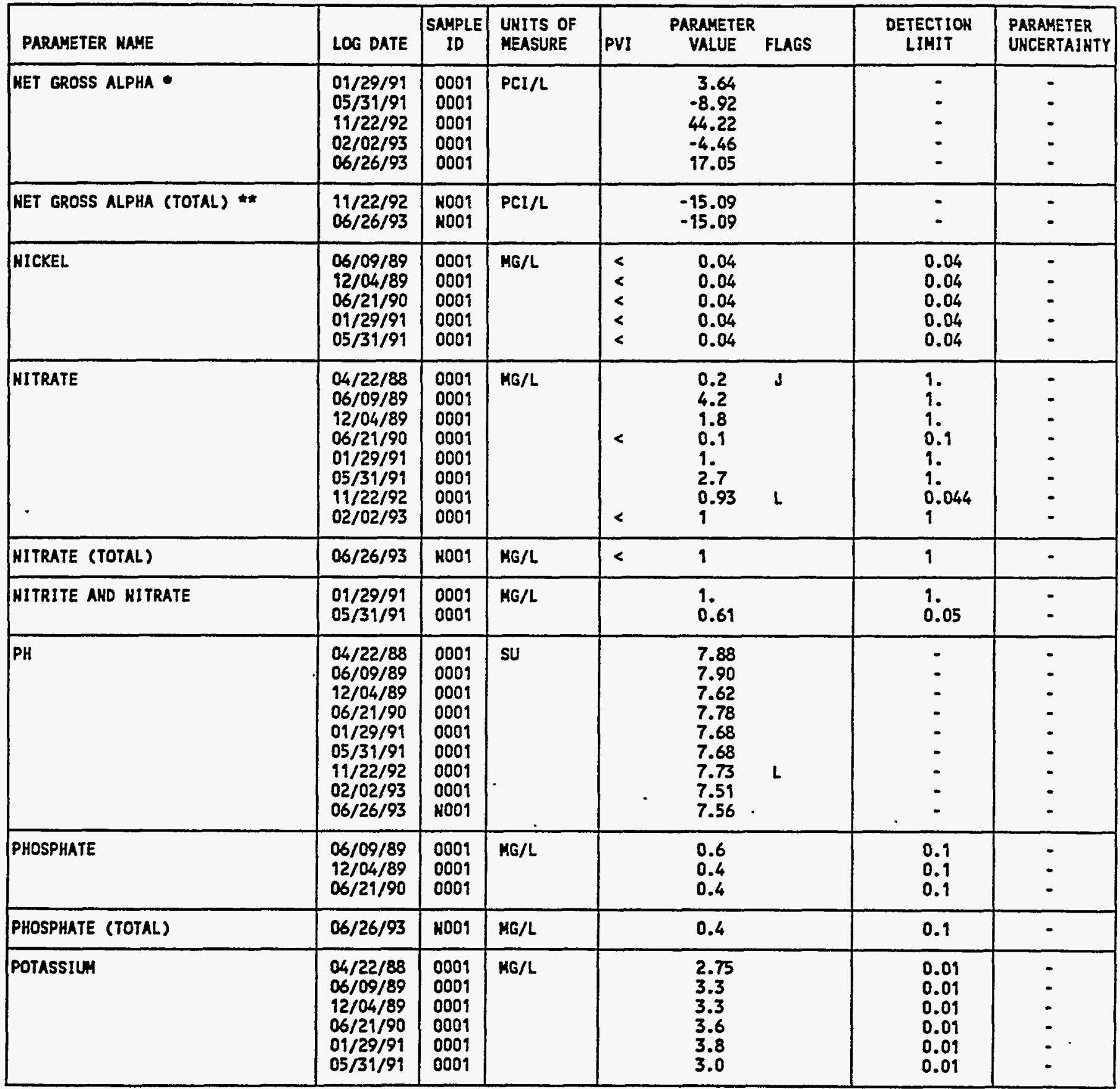

- MET GROSS ALPHA (GROSS ALPHA - URANIUM) WITH 1 MG URANILH = 686 PCI

* MET GROSS ALPHA (TOTAL) (TOTAL GROSS ALPHA - TOTAL URAKIUM)

PARAMETER VALUE INDICATOR (PVI): < - LESS THAN DETECTION LIMIT

SAMPLE ID COOES:

0001 - FILTERED SAMPLE (.45 MICRONS)

OTHER PARAMETER VALUE FLAGS:

MOOI - UMFILTERED SAMPLE

J - ESTIMATED VALUE

L - LESS THAH THREE BORE VOLLAES REMOVED BEFORE SAMPLIMG 
GROUNDHATER OUALITY DATA BY LOCATION

SOUP Rev. 0 Data

SITE: MOHOY MOHUHENT VALLEY

LOCATION: 0605

HORTH COORDIHATE: $\quad 58707.6$ FT

EAST COORDINATE: 90066.7 FT

04/21/88 TO $12 / 13 / 94$

REPORT DATE: $06 / 20 / 95$

FORHATION OF COAPLETION: ALLUVIUH (AL)

HYORAULIC FLON RELATIONSHIP: DOWN GRADIEHT (D)

\begin{tabular}{|c|c|c|c|c|c|c|c|c|}
\hline PARAMETER MAME & LOG DATE & $\underset{\text { ID }}{\text { SAMPLE }}$ & $\begin{array}{l}\text { UNITS OF } \\
\text { MEASURE }\end{array}$ & PVI & $\begin{array}{l}\text { PARAMETER } \\
\text { VALUE }\end{array}$ & FLAGS & $\begin{array}{l}\text { DETECTIOH } \\
\text { LIMIT }\end{array}$ & $\begin{array}{l}\text { PARAMETER } \\
\text { UHCERTAINTY }\end{array}$ \\
\hline POTASSILA & $\begin{array}{l}02 / 02 / 93 \\
06 / 26 / 93\end{array}$ & $\begin{array}{l}0001 \\
0001\end{array}$ & MG/L & & $\begin{array}{l}2.8 \\
3.2\end{array}$ & & $\begin{array}{l}0.1 \\
0.1\end{array}$ & : \\
\hline POTASSILH (TOTAL) & $06 / 26 / 93$ & N001 & $M G / L$ & & 3.1 & & 0.1 & - \\
\hline RADIUA-226 & $\begin{array}{l}06 / 09 / 89 \\
12 / 04 / 89 \\
06 / 21 / 90 \\
01 / 29 / 91 \\
05 / 31 / 91 \\
11 / 22 / 92\end{array}$ & $\begin{array}{l}0001 \\
0001 \\
0001 \\
0001 \\
0001 \\
0001\end{array}$ & PCI/L & & $\begin{array}{l}0.1 \\
0.1 \\
0.1 \\
0.1 \\
0.0 \\
0.0\end{array}$ & $\mathbf{L}$ & $\begin{array}{l}1 . \\
1 . \\
1 . \\
1 . \\
1 . \\
0.3\end{array}$ & $\begin{array}{l}0.2 \\
0.2 \\
0.2 \\
0.5 \\
0.1 \\
0.2\end{array}$ \\
\hline RADIUH-226 (TOTAL) & $19 / 22 / 92$ & N001 & $\mathrm{PCI} / \mathrm{L}$ & & 0.2 & $L$ & 0.3 & 0.2 \\
\hline RADIUH-226 + RADIUM-228 & $\begin{array}{l}06 / 09 / 89 \\
12 / 04 / 89 \\
06 / 21 / 90 \\
01 / 29 / 91 \\
05 / 31 / 91 \\
11 / 22 / 92\end{array}$ & $\begin{array}{l}0001 \\
0001 \\
0001 \\
0001 \\
0001 \\
0001\end{array}$ & PCI/L & & $\begin{array}{l}0.10 \\
0.10 \\
0.50 \\
1.50 \\
0.00 \\
1.90\end{array}$ & & $\begin{array}{l}\dot{-} \\
\dot{-} \\
\dot{-} \\
.\end{array}$ & $\begin{array}{l}- \\
- \\
- \\
-\end{array}$ \\
\hline RADIUH-226 + RADIUH-228 (TOTAL) & $11 / 22 / 92$ & HoO1 & PCI/L & & 3.70 & & - & - \\
\hline RADIUN-228 & $\begin{array}{l}06 / 09 / 89 \\
12 / 04 / 89 \\
06 / 21 / 90 \\
01 / 29 / 91 \\
05 / 31 / 91 \\
11 / 22 / 92\end{array}$ & $\begin{array}{l}0001 \\
0001 \\
0001 \\
0001 \\
0001 \\
0001\end{array}$ & $\mathrm{PCI} / \mathrm{L}$ & & $\begin{array}{l}0.0 \\
0.0 \\
0.4 \\
1.4 \\
0.0 \\
1.9\end{array}$ & $\mathfrak{d}$ & $\begin{array}{l}1 . \\
1 . \\
1 . \\
1 . \\
1 . \\
2.7\end{array}$ & $\begin{array}{l}0.8 \\
1.7 \\
1.2 \\
1.1 \\
2.0 \\
1.7\end{array}$ \\
\hline RNDIUH-228 (TOTAL) & $11 / 22 / 92$ & No01 & $\mathrm{PCI} / \mathrm{L}$ & & 3.5 & $L$ & 1.9 & 1.4 \\
\hline REDOX POTENTIAL & $\begin{array}{l}19 / 22 / 92 \\
06 / 26 / 93\end{array}$ & $\begin{array}{l}\text { C001 } \\
\text { N001 }\end{array}$ & mVOLTS & & $\begin{array}{l}441.5 \\
213\end{array}$ & \llcorner & - & - \\
\hline SELENIU: (TOTAL) & $11 / 22 / 92$ & HOOI & MG/L & $<$ & 0.0015 & HL & 0.0015 & - \\
\hline sILICA - sioz & $\begin{array}{l}06 / 09 / 89 \\
12 / 04 / 89 \\
06 / 21 / 90 \\
01 / 29 / 91 \\
05 / 31 / 91\end{array}$ & $\begin{array}{l}0001 \\
0001 \\
0001 \\
0001 \\
0001\end{array}$ & MG/L & & $\begin{array}{l}26 . \\
33 . \\
0.28 \\
30 . \\
29.5\end{array}$ & 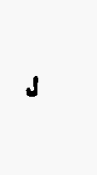 & $\begin{array}{l}2 . \\
2 . \\
2 . \\
2 . \\
0.1\end{array}$ & $:$ \\
\hline SILICA - SIO2 (TOTAL) & $06 / 26 / 93$ & N001 & $M G / L$ & & 33.0 & H & 0.5 & - \\
\hline SILVER & $06 / 09 / 89$ & 0001 & MG/L & $<$ & 0.01 & $L$ & 0.01 & - \\
\hline
\end{tabular}

PARAMETER VALUE INDICATOR (PVI): < - LESS THAN DETECTION LIMIT

SAMPLE ID COOES:

ODO1 - FILTERED SAMPLE (.45 MICROHS)

OTHER PARAHETER VALUE FLAGS:

NOO1 - UHFILTERED SAMPLE

H - HOLD TIME EXPIRED, VALUE SUSPECT

1 - INCREASED DETECTION LIMIT DUE TO REQUIRED DILUTION

J - estIMATED VALUE

L - LESS THAH THREE BORE VOLUMES REMOVED BEFORE SAMPLIHG

N - SPIKE SAMPLE RECOVERY NOT WITHIN CONTROL LIMITS

U - POST-DIGEST SPIKE OUT OF CHTR LIM HHILE SAMP ABS < 50\% SPIKE 
GROUNDWATER QUALITY DATA BY LOCATION

SOWP ReV. O Data

SITE: MONO1 MOHLMENT VALLEY

LOCATIOH: 0605

NORTH COORDIHATE: 58707.6 FT

EAST COORDIHATE: 90066.7 FT

$04 / 21 / 88$ TO $12 / 13 / 94$

REPORT DATE: 06/20/95

FORMATION OF COMPLETIOH: ALLUVIUN (AL)

HYDRAULIC FLON RELATIONSHIP: DOUN GRADIENT (D)

\begin{tabular}{|c|c|c|c|c|c|c|c|c|}
\hline PARAMETER HAHE & LOG DATE & $\mid \begin{array}{c}\text { SAMPLE } \\
\text { ID }\end{array}$ & $\begin{array}{l}\text { UHITS OF } \\
\text { MEASURE }\end{array}$ & PVI & $\begin{array}{l}\text { PARAMETER } \\
\text { VALUE }\end{array}$ & FLAGS & $\begin{array}{l}\text { DETECTION } \\
\text { LIHIT }\end{array}$ & $\begin{array}{l}\text { PARAMETER } \\
\text { UNCERTAINTY }\end{array}$ \\
\hline SILVER & $\begin{array}{l}12 / 04 / 89 \\
06 / 21 / 90 \\
01 / 29 / 91 \\
05 / 31 / 91\end{array}$ & $\begin{array}{l}0001 \\
0001 \\
0001 \\
0001\end{array}$ & MG/L & $\begin{array}{l}< \\
< \\
< \\
<\end{array}$ & $\begin{array}{l}0.01 \\
0.01 \\
0.01 \\
0.01\end{array}$ & & $\begin{array}{l}0.01 \\
0.01 \\
0.01 \\
0.01\end{array}$ & : \\
\hline SOOIUM & $\begin{array}{l}04 / 22 / 88 \\
06 / 09 / 89 \\
12 / 04 / 89 \\
06 / 21 / 90 \\
01 / 29 / 91 \\
05 / 31 / 91 \\
02 / 02 / 93 \\
06 / 26 / 93\end{array}$ & $\begin{array}{l}0001 \\
0001 \\
0001 \\
0001 \\
0001 \\
0001 \\
0001 \\
0001\end{array}$ & MG/L & & $\begin{array}{l}257 . \\
279 . \\
617 . \\
302 . \\
592 . \\
625 . \\
701 \\
828\end{array}$ & & $\begin{array}{l}0.002 \\
0.002 \\
0.002 \\
0.002 \\
0.002 \\
5 . \\
0.1 \\
1\end{array}$ & $\begin{array}{l}- \\
: \\
: \\
- \\
-\end{array}$ \\
\hline SCOIU: (TOTAL) & $06 / 26 / 93$ & NOO1 & $M G / L$ & & 860 & & 1 & - \\
\hline SPECIFIC CONDUCTAKCE & $\begin{array}{l}04 / 22 / 88 \\
06 / 09 / 89 \\
12 / 04 / 89 \\
06 / 21 / 90 \\
01 / 29 / 91 \\
05 / 31 / 91 \\
11 / 22 / 92 \\
02 / 02 / 93 \\
06 / 26 / 93\end{array}$ & $\begin{array}{l}0001 \\
0001 \\
0001 \\
0001 \\
0001 \\
0001 \\
0001 \\
0009 \\
1001\end{array}$ & UHKO/CH & & $\begin{array}{l}1550 . \\
1490 . \\
3050 . \\
1950 . \\
3890 . \\
3950 . \\
3630 \\
3560 \\
2890\end{array}$ & $\mathbf{L}$ & $\begin{array}{l}- \\
- \\
\dot{-} \\
\dot{-} \\
\dot{-} \\
\dot{-}\end{array}$ & $\begin{array}{l}- \\
- \\
- \\
- \\
- \\
-\end{array}$ \\
\hline STRONTIUM & $\begin{array}{l}06 / 21 / 90 \\
01 / 29 / 91 \\
05 / 31 / 91 \\
11 / 22 / 92 \\
06 / 26 / 93\end{array}$ & $\begin{array}{l}0001 \\
0001 \\
0001 \\
0001 \\
0001\end{array}$ & MG/L & & $\begin{array}{l}1.54 \\
2.3 \\
1.86 \\
2.9 \\
2.23\end{array}$ & • & $\begin{array}{l}0.1 \\
0.1 \\
0.01 \\
0.00035 \\
0.01\end{array}$ & $\begin{array}{l}- \\
- \\
-\end{array}$ \\
\hline STROHTIUM (TOTAL) & $\begin{array}{l}11 / 22 / 92 \\
06 / 26 / 93\end{array}$ & $\begin{array}{l}\text { No01 } \\
\text { N001 }\end{array}$ & MG/L & & $\begin{array}{l}2.7 \\
2.27\end{array}$ & $L$ & $\begin{array}{l}0.00035 \\
0.01\end{array}$ & - \\
\hline SULFATE & $\begin{array}{l}04 / 22 / 88 \\
06 / 09 / 89 \\
12 / 04 / 89 \\
06 / 21 / 90 \\
01 / 29 / 91 \\
05 / 31 / 91 \\
11 / 22 / 92 \\
02 / 02 / 93\end{array}$ & $\begin{array}{l}0001 \\
0001 \\
0001 \\
0001 \\
0001 \\
0001 \\
0001 \\
0001\end{array}$ & MG/L & & $\begin{array}{l}906 . \\
1030 . \\
1720 . \\
1080 . \\
1770 . \\
1690 . \\
1900 \\
1950\end{array}$ & 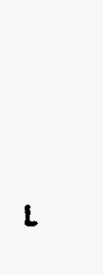 & $\begin{array}{c}0.1 \\
0.1 \\
0.1 \\
0.1 \\
0.1 \\
10 . \\
1.0 \\
1\end{array}$ & $\begin{array}{l}- \\
- \\
- \\
- \\
- \\
-\end{array}$ \\
\hline SULFATE (TOTAL) & $\begin{array}{l}11 / 22 / 92 \\
06 / 26 / 93\end{array}$ & $\begin{array}{l}\text { No01 } \\
\text { n001 }\end{array}$ & $M G / L$ & & $\begin{array}{l}1900 \\
2130\end{array}$ & L & 9.0 & $\because$ \\
\hline SULFIDE & $\begin{array}{l}06 / 09 / 89 \\
12 / 04 / 89 \\
01 / 29 / 91 \\
05 / 31 / 91\end{array}$ & $\begin{array}{l}0001 \\
0001 \\
0001 \\
0001\end{array}$ & $M G / L$ & $\begin{array}{l}< \\
<\end{array}$ & $\begin{array}{r}0.1 \\
0.4 \\
0.1 \\
13.2\end{array}$ & J & $\begin{array}{l}0.1 \\
0.1 \\
0.1 \\
1 .\end{array}$ & : \\
\hline TEMPERATURE & $\begin{array}{l}04 / 22 / 88 \\
06 / 09 / 89\end{array}$ & $\begin{array}{l}0001 \\
0001\end{array}$ & C - DEGREE & & $\begin{array}{l}12.5 \\
16.0\end{array}$ & & $\because$ & : \\
\hline
\end{tabular}

PARAMETER VALUE INDICATOR (PVI): < - LESS THAH DETECTION LIMIT

SAMPLE ID COOES

OTHER PARAMETER VALUE FLAGS:

DOOI - FILTERED SAMPLE (.45 MICRONS)

- DUPLICATE aHALYSIS nOt HITHIN CONTROL LIMITS

NOO1 - UMFILTERED SAMPLE

J. estimated value

$L$ - LESS THAH THREE GORE VOLUMES REMOVED BEFORE SAMPLIKG 
GROUNDHATER QUALITY DATA BY LOCATION

SOUP Rev. O Data

SITE: MONOI MONUMENT VALLEY

LOCATION: 0605

NORTH COORDINATE: 58707.6 FT

EAST COORDINATE: 90066.7 FT

$04 / 21 / 88$ TO $12 / 13 / 94$

REPORT DATE: $06 / 20 / 95$

FORMATION OF COHPLETION: ALLUVIUH (AL)

HYDRAULIC FLON RELATIOHSHIP: DOWN GRADIENT (D)

\begin{tabular}{|c|c|c|c|c|c|c|c|c|}
\hline PARAMETER MAME & LOG DATE & $\underset{\text { SAMPLE }}{\text { ID }}$ & $\begin{array}{l}\text { UNITS OF } \\
\text { MEASURE }\end{array}$ & PVI & $\begin{array}{l}\text { PARAMETER } \\
\text { VALUE }\end{array}$ & FLAGS & $\begin{array}{l}\text { DETECTION } \\
\text { LIMIT }\end{array}$ & $\begin{array}{l}\text { PARAMETER } \\
\text { UNCERTAINTY }\end{array}$ \\
\hline TEMPERATURE & $\begin{array}{l}12 / 04 / 89 \\
06 / 21 / 90 \\
01 / 29 / 91 \\
05 / 31 / 91 \\
11 / 22 / 92 \\
02 / 02 / 93 \\
06 / 26 / 93\end{array}$ & $\begin{array}{l}0001 \\
0001 \\
0001 \\
0001 \\
0001 \\
0001 \\
N 001\end{array}$ & C - DEGREE & & $\begin{array}{l}16.5 \\
16.0 \\
13.4 \\
13.5 \\
16.9 \\
14.4 \\
16.1\end{array}$ & $L$ & $\begin{array}{l}- \\
- \\
- \\
-\end{array}$ & $\begin{array}{l}- \\
- \\
- \\
-\end{array}$ \\
\hline THALLIUH & $\begin{array}{l}06 / 09 / 89 \\
12 / 04 / 89 \\
06 / 21 / 90 \\
01 / 29 / 91 \\
05 / 31 / 91\end{array}$ & $\begin{array}{l}0001 \\
0001 \\
0001 \\
0001 \\
0001\end{array}$ & $M G / L$ & $\begin{array}{l}< \\
< \\
< \\
< \\
<\end{array}$ & $\begin{array}{l}0.01 \\
0.1 \\
0.01 \\
0.01 \\
0.01\end{array}$ & & $\begin{array}{l}0.01 \\
0.1 \\
0.01 \\
0.01 \\
0.01\end{array}$ & $\begin{array}{l}- \\
- \\
-\end{array}$ \\
\hline THORILA-230 & $\begin{array}{l}01 / 29 / 91 \\
05 / 31 / 91\end{array}$ & $\begin{array}{l}0001 \\
0001\end{array}$ & PCI/L & & $\begin{array}{l}0.0 \\
0.8\end{array}$ & & 1. & $\begin{array}{l}0.3 \\
0.3\end{array}$ \\
\hline TIN & $\begin{array}{l}06 / 09 / 89 \\
12 / 04 / 89 \\
06 / 21 / 90 \\
01 / 29 / 91 \\
05 / 31 / 91\end{array}$ & $\begin{array}{l}0001 \\
0001 \\
0001 \\
0001 \\
0001\end{array}$ & MG/L & $\begin{array}{l}< \\
< \\
< \\
<\end{array}$ & $\begin{array}{l}0.005 \\
0.01 \\
0.006 \\
0.005 \\
0.01\end{array}$ & 1 & $\begin{array}{l}0.005 \\
0.01 \\
0.005 \\
0.005 \\
0.01\end{array}$ & : \\
\hline TOTAL DISSOLVED SOLIOS & $\begin{array}{l}04 / 22 / 88 \\
06 / 09 / 89 \\
12 / 04 / 89 \\
06 / 21 / 90 \\
01 / 29 / 91 \\
05 / 31 / 91 \\
02 / 02 / 93\end{array}$ & $\begin{array}{l}0001 \\
0001 \\
0001 \\
0001 \\
0001 \\
0001 \\
0001\end{array}$ & $M G / L$ & & $\begin{array}{l}1660 . \\
1860 . \\
3280 . \\
1780 . \\
2250 . \\
3080 . \\
3390\end{array}$ & & $\begin{array}{l}10 . \\
10 . \\
10 . \\
10 . \\
10 \\
10 \\
10\end{array}$ & $\begin{array}{l}- \\
- \\
- \\
- \\
-\end{array}$ \\
\hline TOTAL DISSOLVED SOLIDS (TOTAL) & $06 / 26 / 93$ & NOO1 & MG/L & & 3870 & & 10 & - \\
\hline TOTAL ORGANIC CARBON & $\begin{array}{l}04 / 22 / 88 \\
01 / 29 / 91 \\
05 / 31 / 91\end{array}$ & $\begin{array}{l}0001 \\
0001 \\
0001\end{array}$ & MG/L & & $\begin{array}{c}62.6 \\
1.8 \\
2 .\end{array}$ & & $\begin{array}{l}1 . \\
1 . \\
1 .\end{array}$ & : \\
\hline URANIUN & $\begin{array}{l}04 / 22 / 88 \\
06 / 09 / 89 \\
12 / 04 / 89 \\
06 / 21 / 90 \\
01 / 29 / 91 \\
05 / 31 / 91 \\
11 / 22 / 92 \\
02 / 02 / 93 \\
06 / 26 / 93\end{array}$ & $\begin{array}{l}0001 \\
0001 \\
0001 \\
0001 \\
0001 \\
0001 \\
0001 \\
0001 \\
0001\end{array}$ & $M G / L$ & & $\begin{array}{l}0.0036 \\
0.0044 \\
0.017 \\
0.0042 \\
0.0151 \\
0.013 \\
0.023 \\
0.024 \\
0.032\end{array}$ & $\mathbf{L}$ & $\begin{array}{l}0.003 \\
0.003 \\
0.003 \\
0.003 \\
0.0003 \\
0.001 \\
0.001 \\
0.001 \\
0.001\end{array}$ & $\begin{array}{l}- \\
- \\
- \\
- \\
- \\
-\end{array}$ \\
\hline URANIUA (TOTAL) & $\begin{array}{l}11 / 22 / 92 \\
06 / 26 / 93\end{array}$ & $\begin{array}{l}\text { N001 } \\
\text { N001 }\end{array}$ & MG/L & & $\begin{array}{l}0.022 \\
0.022\end{array}$ & $\mathbf{L}$ & $\begin{array}{l}0.001 \\
0.001\end{array}$ & $\dot{-}$ \\
\hline VANADIU: & $\begin{array}{l}06 / 09 / 89 \\
12 / 04 / 89 \\
06 / 21 / 90 \\
01 / 29 / 91 \\
05 / 31 / 99\end{array}$ & $\begin{array}{l}0001 \\
0001 \\
0001 \\
0001 \\
0001\end{array}$ & MG/L & $\begin{array}{l}< \\
< \\
<\end{array}$ & $\begin{array}{l}0.03 \\
0.01 \\
0.02 \\
0.01 \\
0.01\end{array}$ & & $\begin{array}{l}0.01 \\
0.01 \\
0.01 \\
0.01 \\
0.01\end{array}$ & $\begin{array}{l}- \\
- \\
-\end{array}$ \\
\hline
\end{tabular}

PARAMETER VALUE IMDICATOR (PVI): < - LESS THAN DETECTION LIMIT

SMMPLE ID COOES:

OOO1 - FILTERED SAMPLE (.45 HICRONS)

OTHER PARAHETER VALUE FLAGS:

NOO1 - UNFILTERED SAMPLE

I - IMCREASED DETECTION LIMIT DUE TO REQUIRED DILUTION

L - LESS THAH THREE BORE VOLUMES REMOVED BEFORE SAMPLING 
GROUNDWATER QUALITY DATA BY LOCATION

SOUP ReV. O Data

SITE: HONOI HONUMENT VALLEY,

LOCATION: 0605

MORTH COORDIHATE: 58707.6 FT

EAST COORDINATE: $\quad 90066.7$ FT

04/21/88 TO $12 / 13 / 94$

REPORT DATE: 06/20/95

FORMATION OF COMPLETIOH: ALLUVIUM (AL)

HYDRAULIC FLON RELATIOHSHIP:! DOWN GRADIEHT (D)

\begin{tabular}{|c|c|c|c|c|c|c|c|}
\hline PARAMETER MAME & LOG DATE & $\begin{array}{c}\text { SAMPLE } \\
\text { ID }\end{array}$ & $\begin{array}{l}\text { UHITS OF } \\
\text { MEASURE }\end{array}$ & PVI & $\begin{array}{l}\text { PARAMETER } \\
\text { VALUE FLAGS }\end{array}$ & $\begin{array}{l}\text { DETECTION } \\
\text { LIMIT }\end{array}$ & $\begin{array}{l}\text { PARAMETER } \\
\text { UNCERTAINTY }\end{array}$ \\
\hline VARADIUN & $\begin{array}{l}11 / 22 / 92 \\
06 / 26 / 93\end{array}$ & $\begin{array}{l}0001 \\
0001\end{array}$ & MG/L & $<$ & $\begin{array}{l}0.0019 \quad L \\
0.01\end{array}$ & $\begin{array}{l}0.0019 \\
0.01\end{array}$ & - \\
\hline VAHADILA (TOTAL) & $\begin{array}{l}11 / 22 / 92 \\
06 / 26 / 93\end{array}$ & $\begin{array}{l}\text { No01 } \\
\text { Y001 }\end{array}$ & $M G / L$ & $<$ & $\begin{array}{l}0.0040 \mathrm{~L} \\
0.01\end{array}$ & $\begin{array}{l}0.0019 \\
0.01\end{array}$ & - \\
\hline ZIHC (TOTAL) & $\begin{array}{l}91 / 22 / 92 \\
06 / 26 / 93\end{array}$ & $\begin{array}{l}\text { NO01 } \\
\text { N001 }\end{array}$ & $M G / L$ & $<$ & $\begin{array}{ll}0.0030 & \mathrm{~L} \\
0.005 & \mathrm{~J}\end{array}$ & $\begin{array}{l}0.0010 \\
0.005\end{array}$ & 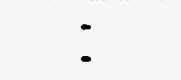 \\
\hline
\end{tabular}

PARAMETER VALUE INDICATOR (PVI): < - LESS THAK DETECTION LIHIT

SAMPLE ID COOES:

OTHER PARAMETER VALUE FLAGS:

0001 - FILTERED SAMPLE (.45 MICRONS)

J - ESTIMATED VALUE

NOO1 - UNFILTERED SAMPLE

L. - LESS THAN THREE BORE VOLUMES REMOVED BEFORE SAMPLING 
GROUNDWATER OUALITY DATA BY LOCATIOH

SOWP ReV. O Data

SITE: MONO1 MONUMENT VALLEY

LOCATION: 0606

MORTH COORDINATE: 59034.2 FT

EAST COORDINATE: 88634.0 FT

04/21/88 TO $12 / 13 / 94$

REPORT DATE: 06/20/95

FORMATION OF COMPLETION: ALLUVIUH (AL)

MYDRAULIC FLOW RELATIONSHIP: DOWN GRADIENT (D)

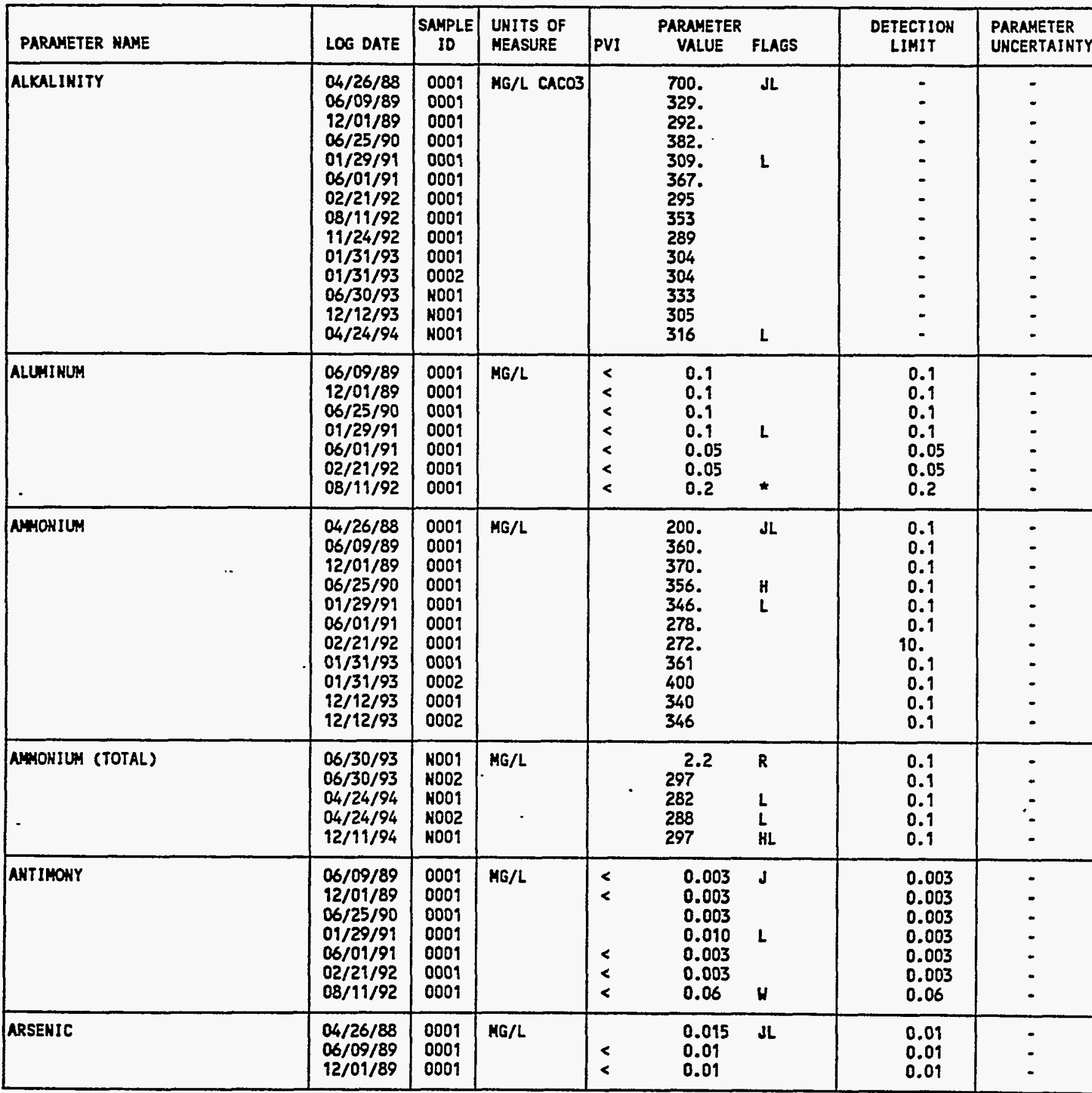

PARNETER VALUE IHDICATOR (PVI): < - LESS THAN DETECTIOH LIMIT

SAMPLE ID COOES:

OTHER PARAMETER VALUE FLAGS:

- - DUPLICATE akALYSIS hot MITHIN CONTROL limits

0001 - FILTERED SAMPLE (.45 MICROHS)

H - HOLD TIME EXPIRED, VALUE SUSPECT

0002 - FILTERED REPLICATE SAMPLE (.45 MICRONS)

NOO1 - UNFILTERED SAMPLE

MOO2 - UMFILTERED REPLICATE SAMPLE

J - ESTIMATED VALUE

L - LESS THAH THREE BORE VOLUMES REMOVED BEFORE SAMPLIHG

$R$ - UHUSABLE DATA POINT

$U$ - POST-DIGEST SPIKE OUT OF CNTR LIM WHILE SAMP ABS < 50\% SPIKE 
GROUHDWATER QUALITY DATA BY LOCATIOH

SOWP ReV. 0 Data

SITE: MOHO1 MONUNENT VALLEY

LOCATIOH: 0606

HORTH COORDINATE: 59034.2 FT

EAST COORDIHATE: 88634.0 FT

$04 / 21 / 88$ TO $12 / 13 / 94$

REPORT DATE: 06/20/95

FORMATION OF COMPLETIOH: ALLUVIUM (AL)

HYDRAULIC FLOW RELATIOHSHIP: DOWH GRADIEHT (D)

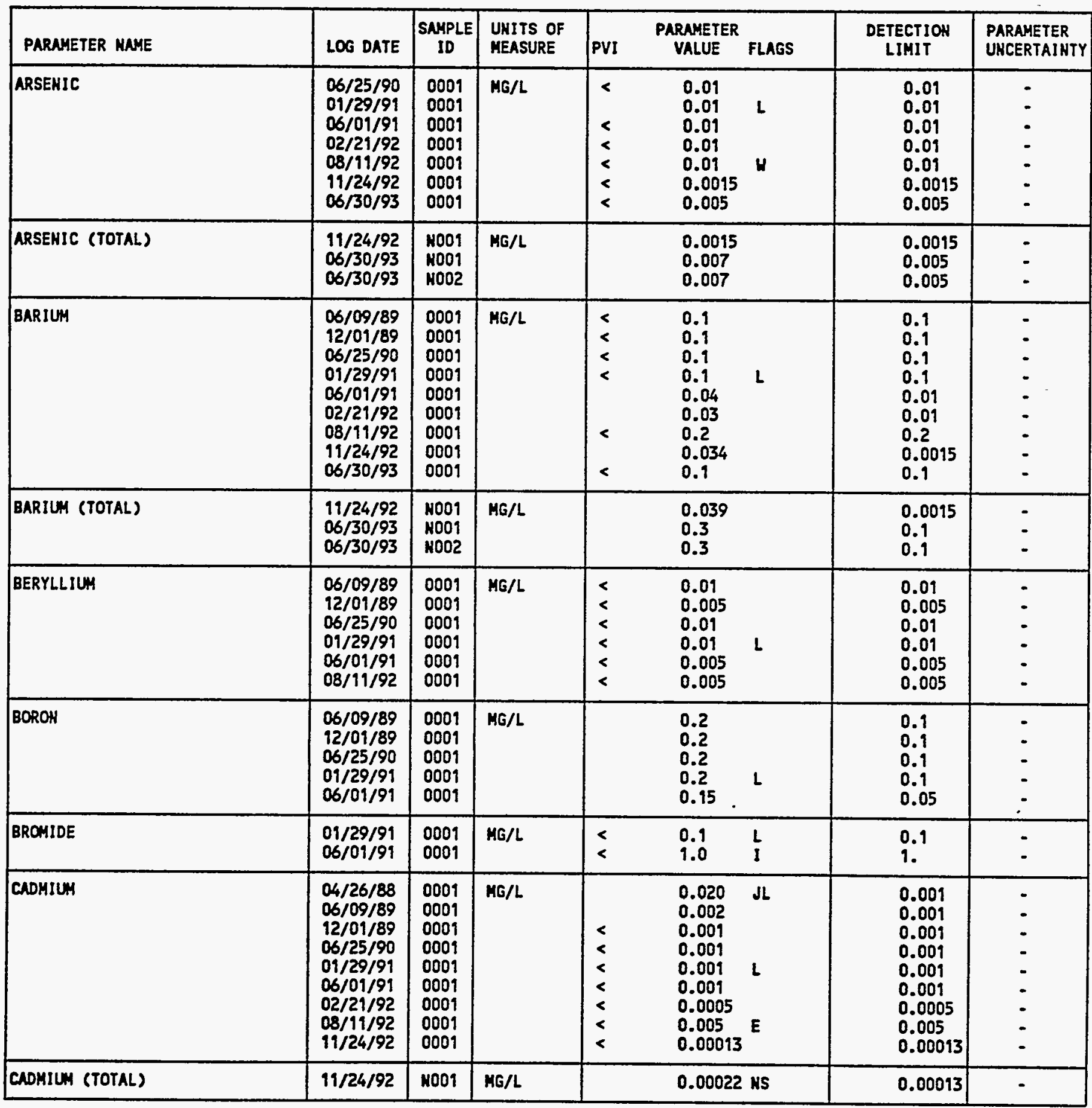

PARAMETER VALUE IMDICATOR (PVI): < - LESS thAH DETECTION LIMIT

OTHER PARAMETER VALUE FLAGS:

E - ESTIMUTED VALUE BECAUSE OF INTERFERENCE, SEE CASE MARRATIVE

I - INCREASED DETECTION LIMIT DUE TO REOUIRED DILUTION

J - estimated VALUE

L - LESS THAN THREE BORE VOLUMES REMOVED BEFORE SAMPLIHG

H - SPIKE SAMPLE RECOVERY NOT WITHIN CONTROL LIMITS

S - REPORTED VALUE DETERMIHED USING METHOD OF STD ADDITION (MSA)

$U$ - POST-DIGEST SPIKE OUT OF CHTR LIM UHILE SAMP ABS < 50\% SPIKE
SAMPLE ID COOES:

0001 - FILTERED SAMPLE (.45 MICROWS)

NOO1 - UMFILTERED SAMPLE

HOO2 - UNFILTERED REPLICATE SAMPLE 
GROUNDHATER QUALITY DATA BY LOCATION

SOMP ReV, 0 Data

SITE: MONOI MONUNENT VALLEY

LOCATION: 0606

MORTH COORDINATE: 59034.2 FT

EAST COORDIMATE: 88634.0 FT

$04 / 21 / 88$ TO $12 / 13 / 94$

REPORT DATE: 06/20/95

FORMATION OF COMPLETIOH: ALLUVIUM (AL)

HYDRAULIC FLOW RELATIONSHIP: DONN GRADIENT (D)

\begin{tabular}{|c|c|c|c|c|c|c|c|c|}
\hline PARAMETER MAME & LOG DATE & $\mid \begin{array}{c}\text { SAMPLE } \\
\text { ID }\end{array}$ & $\begin{array}{l}\text { UNITS OF } \\
\text { MEASURE }\end{array}$ & PVI & $\begin{array}{l}\text { PARAMETER } \\
\text { VALUE }\end{array}$ & FLAGS & $\begin{array}{l}\text { DETECTIOH } \\
\text { LIMIT }\end{array}$ & $\begin{array}{l}\text { PARAMETER } \\
\text { UHCERTAINTY }\end{array}$ \\
\hline Calciun & $\begin{array}{l}04 / 26 / 88 \\
06 / 09 / 89 \\
12 / 01 / 89 \\
06 / 25 / 90 \\
01 / 29 / 91 \\
06 / 01 / 91 \\
02 / 21 / 92 \\
08 / 91 / 92 \\
01 / 31 / 93 \\
01 / 31 / 93 \\
06 / 30 / 93 \\
12 / 12 / 93 \\
12 / 12 / 93 \\
04 / 24 / 94 \\
04 / 24 / 94 \\
12 / 91 / 94\end{array}$ & $\begin{array}{l}0001 \\
0001 \\
0001 \\
0001 \\
0001 \\
0001 \\
0001 \\
0001 \\
0001 \\
0002 \\
0001 \\
0001 \\
0002 \\
0001 \\
0002 \\
0001\end{array}$ & $M G / L$ & & $\begin{array}{l}660 . \\
226 . \\
266 . \\
254 . \\
220 . \\
283 . \\
211 . \\
200 \\
199 \\
202 \\
237 \\
203 \\
207 \\
221 \\
222 \\
262\end{array}$ & $\begin{array}{l}d L \\
L\end{array}$ & $\begin{array}{l}0.01 \\
0.01 \\
0.01 \\
0.01 \\
0.01 \\
0.5 \\
0.5 \\
5 \\
0.5 \\
0.5 \\
0.5 \\
0.5 \\
0.5 \\
0.5 \\
0.5 \\
0.5\end{array}$ & $\begin{array}{l}: \\
: \\
: \\
: \\
: \\
: \\
: \\
\vdots \\
-\end{array}$ \\
\hline CALCIUH (TOTAL) & $\begin{array}{l}06 / 30 / 93 \\
06 / 30 / 93 \\
12 / 12 / 93 \\
04 / 24 / 94 \\
04 / 24 / 94\end{array}$ & $\begin{array}{l}\text { NoO1 } \\
\text { N002 } \\
\text { NO01 } \\
\text { N001 } \\
\text { NOO2 }\end{array}$ & MG/L & & $\begin{array}{l}271 \\
255 \\
230 \\
263 \\
268\end{array}$ & $L$ & $\begin{array}{l}0.5 \\
0.5 \\
0.5 \\
0.5 \\
0.5\end{array}$ & $\begin{array}{l}- \\
\dot{-} \\
\dot{-}\end{array}$ \\
\hline CHLORIDE & $\begin{array}{l}04 / 26 / 88 \\
06 / 09 / 89 \\
12 / 01 / 89 \\
06 / 25 / 90 \\
01 / 29 / 91 \\
06 / 01 / 91 \\
02 / 21 / 92 \\
08 / 11 / 92 \\
01 / 31 / 93 \\
01 / 31 / 93 \\
12 / 12 / 93 \\
12 / 12 / 93 \\
04 / 24 / 94 \\
04 / 24 / 94 \\
12 / 11 / 94\end{array}$ & $\begin{array}{l}0001 \\
0001 \\
0001 \\
0001 \\
0001 \\
0001 \\
0001 \\
0001 \\
0001 \\
0002 \\
0001 \\
0002 \\
0001 \\
0002 \\
0001\end{array}$ & MG/L & & $\begin{array}{l}21 . \\
20 . \\
17.5 \\
18 . \\
16 . \\
17.0 \\
15.9 \\
18 \\
16.2 \\
16.2 \\
14.2 \\
14.5 \\
15.7 \\
15.8 \\
14\end{array}$ & $\begin{array}{l}\mathrm{JL} \\
\mathrm{JL}\end{array}$ & $\begin{array}{l}1 . \\
1 . \\
1 . \\
1 . \\
1 . \\
0.5 \\
0.5 \\
0.016 \\
0.5 \\
0.5 \\
0.5 \\
0.5 \\
0.5 \\
0.5 \\
0.5\end{array}$ & $\begin{array}{l}- \\
- \\
- \\
- \\
- \\
- \\
- \\
- \\
-\end{array}$ \\
\hline CHLORIDE (TOTAL) & $\begin{array}{l}06 / 30 / 93 \\
06 / 30 / 93\end{array}$ & $\begin{array}{l}\text { N001 } \\
\text { N002 }\end{array}$ & MG/L & & $\begin{array}{l}16.4 \\
17.5\end{array}$ & & $\begin{array}{l}0.5 \\
0.5\end{array}$ & - \\
\hline CHROHIUN & $\begin{array}{l}06 / 09 / 89 \\
12 / 01 / 89 \\
06 / 25 / 90 \\
01 / 29 / 91 \\
06 / 01 / 91 \\
02121 / 92 \\
08 / 11 / 92 \\
11 / 24 / 92\end{array}$ & $\begin{array}{l}0001 \\
0001 \\
0001 \\
0001 \\
0001 \\
0001 \\
0001 \\
0001\end{array}$ & $M G / L$ & $\begin{array}{l}< \\
5 \\
5 \\
5 \\
5 \\
5 \\
5\end{array}$ & $\begin{array}{l}0.01 \\
0.01 \\
0.01 \\
0.01 \\
0.01 \\
0.01 \\
0.01 \\
0.0053\end{array}$ & $L$ & $\begin{array}{l}0.01 \\
0.01 \\
0.09 \\
0.01 \\
0.09 \\
0.09 \\
0.01 \\
0.0053\end{array}$ & $\begin{array}{l}: \\
: \\
: \\
: \\
-\end{array}$ \\
\hline CHROMIUH (TOTAL) & $11 / 24 / 92$ & $N 001$ & $M G / L$ & $<$ & 0.0053 & & 0.0053 & - \\
\hline COBALT & $\begin{array}{l}06 / 09 / 89 \\
12 / 01 / 89 \\
06 / 25 / 90\end{array}$ & $\begin{array}{l}0001 \\
0001 \\
0001\end{array}$ & MG/L & $\begin{array}{l}< \\
<\end{array}$ & $\begin{array}{l}0.05 \\
0.05 \\
0.05\end{array}$ & & $\begin{array}{l}0.05 \\
0.05 \\
0.05\end{array}$ & $:$ \\
\hline
\end{tabular}

paraMeter VAlue Indicator (PVI): < - LeSS than Detection LIMIT

SAMPLE IO COOES:

OTHER PARMYTER VALUE fLAGS:

DOO1 - FILTERED SAMPLE (.45 MICRONS)

OOO2 - FILTERED REPLICATE SMPLE (.45 MICRONS)

J - ESTIMATED VALUE

L - LESS THAN THREE BORE VOLUAES REMOVED BEFORE SAMPLING

NOO1 - LMFILTERED SAMPLE

NOO2 - UNFILTERED REPLICATE SAMPLE 
GROUNDUATER QUALITY DATA BY LOCATIOH

SOUP ReV. O Data

SITE: MONO 1 MONUMENT VALLEY

LOCATION: 0606

MORTH COORDIHATE: 59034.2 FT

EAST COORDINATE: 88634.0 FT

04/21/88 TO $12 / 13 / 94$

REPORT DATE: $06 / 20 / 95$

FORMATION OF COAPLETIOH: ALLUVIUM (AL)

HYDRAULIC FLOM RELATIOHSHIP: DOWN GRADIEHT (D)

\begin{tabular}{|c|c|c|c|c|c|c|c|c|}
\hline PARAMETER MAME & LOG DATE & $\underset{\text { SAMPLE }}{\text { ID }}$ & $\begin{array}{l}\text { UHITS OF } \\
\text { MEASURE }\end{array}$ & PVI & $\begin{array}{l}\text { PARAMETER } \\
\text { VALUE }\end{array}$ & FLAGS & $\begin{array}{l}\text { DETECTIOH } \\
\text { LIHIT }\end{array}$ & $\begin{array}{l}\text { PARAMETER } \\
\text { UNCERTAINTY }\end{array}$ \\
\hline COBALT & $\begin{array}{l}01 / 29 / 99 \\
06 / 01 / 99\end{array}$ & $\begin{array}{l}0001 \\
0001\end{array}$ & $M G / L$ & $<$ & $\begin{array}{l}0.05 \\
0.03\end{array}$ & $\ell$ & $\begin{array}{l}0.05 \\
0.03\end{array}$ & - \\
\hline COPPER & $\begin{array}{l}06 / 09 / 89 \\
12 / 01 / 89 \\
06 / 25 / 90 \\
01 / 29 / 91 \\
06 / 01 / 91\end{array}$ & $\begin{array}{l}0001 \\
0001 \\
0001 \\
0001 \\
0001\end{array}$ & MG/L & $\begin{array}{l}< \\
< \\
<\end{array}$ & $\begin{array}{l}0.02 \\
0.02 \\
0.02 \\
0.02 \\
0.01\end{array}$ & $L$ & $\begin{array}{l}0.02 \\
0.02 \\
0.02 \\
0.02 \\
0.01\end{array}$ & $\begin{array}{l}- \\
- \\
-\end{array}$ \\
\hline CYAHIDE & $\begin{array}{l}06 / 09 / 89 \\
12 / 01 / 89\end{array}$ & $\begin{array}{l}0009 \\
0001\end{array}$ & MG/L & $<$ & $\begin{array}{l}0.01 \\
0.01\end{array}$ & & $\begin{array}{l}0.01 \\
0.01\end{array}$ & - \\
\hline DISSOLVED OXYGEN & $11 / 24 / 92$ & 0001 & MG/L & & 0.7 & & - & - \\
\hline FLUORIDE & $\begin{array}{l}06 / 09 / 89 \\
12 / 01 / 89 \\
06 / 25 / 90 \\
01 / 29 / 91 \\
06 / 01 / 91\end{array}$ & $\begin{array}{l}0001 \\
0001 \\
0001 \\
0001 \\
0001\end{array}$ & MG/L & & $\begin{array}{l}0.5 \\
0.6 \\
0.6 \\
0.5 \\
0.4\end{array}$ & $L$ & $\begin{array}{l}0.1 \\
0.1 \\
0.1 \\
0.1 \\
0.1\end{array}$ & : \\
\hline GROSS ALPHA & $\begin{array}{l}04 / 26 / 88 \\
01 / 29 / 91 \\
06 / 01 / 91 \\
02 / 21 / 92 \\
08 / 11 / 92 \\
11 / 24 / 92 \\
011 / 31 / 93 \\
01 / 31 / 93 \\
06 / 30 / 93\end{array}$ & $\begin{array}{l}0001 \\
0001 \\
0001 \\
0001 \\
0001 \\
0001 \\
0001 \\
0002 \\
0001\end{array}$ & $\mathrm{PCI} / \mathrm{L}$ & & $\begin{array}{l}38 . \\
7 . \\
0.0 \\
14.7 \\
16 \\
11.5 \\
18 \\
29 \\
51\end{array}$ & $\begin{array}{l}\mathrm{JL} \\
\mathrm{L}\end{array}$ & $\begin{array}{l}0.2 \\
1 . \\
1 . \\
1.0 \\
26 \\
10.8 \\
33 \\
35 \\
16\end{array}$ & $\begin{array}{l}24 . \\
11 . \\
22.4 \\
25.5 \\
17 \\
8.8 \\
38 \\
41 \\
32\end{array}$ \\
\hline GROSS ALPHA (TOTAL) & $\begin{array}{l}11 / 24 / 92 \\
06 / 30 / 93 \\
06 / 30 / 93\end{array}$ & $\begin{array}{l}\text { NOO1 } \\
\text { NOO1 } \\
\text { NOO2 }\end{array}$ & PCI/L & & $\begin{array}{l}0.0 \\
0 \\
7\end{array}$ & $\mathbf{H}$ & $\begin{array}{l}25.4 \\
20 \\
22\end{array}$ & $\begin{array}{l}12.8 \\
28 \\
35\end{array}$ \\
\hline GROSS BETA & $\begin{array}{l}04 / 26 / 88 \\
01 / 29 / 91 \\
06 / 01 / 91 \\
02 / 21 / 92 \\
08 / 11 / 92 \\
11 / 24 / 92 \\
01 / 31 / 93 \\
01 / 31 / 93 \\
06 / 30 / 93\end{array}$ & $\begin{array}{l}0001 \\
0001 \\
0001 \\
0001 \\
0001 \\
0001 \\
0001 \\
0002 \\
0001\end{array}$ & PCI/L & & $\begin{array}{l}23 . \\
17 . \\
16.1 \\
26.0 \\
12 \\
0.0 \\
10 \\
18 \\
5\end{array}$ & $\begin{array}{l}\mathrm{JL} \\
\mathrm{L}\end{array}$ & $\begin{array}{l}1 . \\
0.5 \\
0.5 \\
0.5 \\
22 \\
19.6 \\
40 \\
40 \\
22\end{array}$ & $\begin{array}{l}11 . \\
7 . \\
14.2 \\
17.3 \\
13 \\
11.6 \\
18 \\
18 \\
21\end{array}$ \\
\hline GROSS EETA (TOTAL) & $\begin{array}{l}11 / 24 / 92 \\
06 / 30 / 93 \\
06 / 30 / 93\end{array}$ & $\begin{array}{l}\text { No01 } \\
\text { No01 } \\
\text { no2 }\end{array}$ & PCI/L & & $\begin{array}{l}4.0 \\
0 \\
19\end{array}$ & H & $\begin{array}{l}18.6 \\
23 \\
23\end{array}$ & $\begin{array}{l}11.2 . \\
20 \\
22 .\end{array}$ \\
\hline IRON & $\begin{array}{l}06 / 09 / 89 \\
12 / 01 / 89 \\
06 / 25 / 90 \\
01 / 29 / 91 \\
06 / 01 / 91 \\
02 / 21 / 92\end{array}$ & $\begin{array}{l}0001 \\
0001 \\
0001 \\
0001 \\
0001 \\
0001\end{array}$ & $M G / L$ & $\begin{array}{l}< \\
< \\
<\end{array}$ & $\begin{array}{l}0.06 \\
0.03 \\
0.07 \\
0.06 \\
0.03 \\
0.03\end{array}$ & $\mathbf{L}$ & $\begin{array}{l}0.03 \\
0.03 \\
0.03 \\
0.03 \\
0.03 \\
0.03\end{array}$ & $\begin{array}{l}- \\
- \\
- \\
-\end{array}$ \\
\hline
\end{tabular}

PARAHETER VALUE INDICATOR (PVI): < - LESS THAN DETECTION LIMIT

SAMPLE TD COOES:

OTHER PARAMETER VALUE FLAGS:

J - ESTIMATED VALUE

L - LESS THAN THREE BORE VOLLMES REMOVED BEFORE SAMPLIMG

0001 - FILTERED SAMPLE (.45 MICROHS)

0002 - FILTERED REPLICATE SAMPLE (.45 MICRONS)

MOOI - UHFILTERED SAMPLE

MOO2 - UAFILTERED REPLICATE SAMPLE

K - SPIKE SAMPLE RECOVERY NOT WITHIN CONTROL LIMITS 
GROUNDWATER QUALITY DATA BY LOCATION

SOMP Rev. O Data

SITE: MONO1 MONUMENT VALLEY

LOCATION: 0606

MORTH COORDINATE: 59034.2 FT

EAST COORDINATE: 88634.0 FT

04/21/88 TO $12 / 13 / 94$

REPORT DATE: 06/20/95

FORMATION OF COHPLETION: ALLUVIUM (AL)

HYDRAULIC FLOW RELATIONSHIP: DOWN GRADIENT (D)

\begin{tabular}{|c|c|c|c|c|c|c|c|c|c|}
\hline PARAMETER KAME & & LOG DATE & $\underset{\text { SAMPLE }}{\text { SA }}$ & $\begin{array}{l}\text { UNITS OF } \\
\text { MEASURE }\end{array}$ & PVI & $\begin{array}{l}\text { PARAMETER } \\
\text { VALUE }\end{array}$ & FLAGS & $\begin{array}{l}\text { DETECTION } \\
\text { LIMIT }\end{array}$ & $\begin{array}{l}\text { PARAMETER } \\
\text { UNCERTAINTY }\end{array}$ \\
\hline IROH & & $\begin{array}{l}08 / 11 / 92 \\
01 / 31 / 93 \\
01 / 31 / 93 \\
06 / 30 / 93\end{array}$ & $\begin{array}{l}0001 \\
0001 \\
0002 \\
0001\end{array}$ & MG/L & $\begin{array}{l}< \\
< \\
<\end{array}$ & $\begin{array}{l}0.1 \\
0.03 \\
0.03 \\
0.03\end{array}$ & $J$ & $\begin{array}{l}0.1 \\
0.03 \\
0.03 \\
0.03\end{array}$ & : \\
\hline IRON (TOTAL) & & $\begin{array}{l}06 / 30 / 93 \\
06 / 30 / 93\end{array}$ & $\begin{array}{l}\text { N001 } \\
\text { N002 }\end{array}$ & $M G / L$ & & $\begin{array}{l}10.2 \\
10.3\end{array}$ & & $\begin{array}{l}0.03 \\
0.03\end{array}$ & - \\
\hline LEAD & & $\begin{array}{l}06 / 09 / 89 \\
12 / 01 / 89 \\
06 / 25 / 90 \\
01 / 29 / 91 \\
06 / 01 / 91 \\
11 / 24 / 92\end{array}$ & $\begin{array}{l}0001 \\
0001 \\
0001 \\
0001 \\
0001 \\
0001\end{array}$ & $M G / L$ & $\begin{array}{l}< \\
< \\
< \\
< \\
<\end{array}$ & $\begin{array}{l}0.01 \\
0.01 \\
0.01 \\
0.01 \\
0.005 \\
0.0015\end{array}$ & J & $\begin{array}{l}0.01 \\
0.01 \\
0.01 \\
0.01 \\
0.005 \\
0.0015\end{array}$ & $\begin{array}{l}- \\
- \\
- \\
-\end{array}$ \\
\hline LEAD (TOTAL) & & $11 / 24 / 92$ & HOOS & $M G / L$ & $<$ & 0.0015 & $\mathbf{H}$ & 0.0015 & - \\
\hline LEAD-210 & & $11 / 24 / 92$ & 0009 & $\mathrm{PCI} / \mathrm{L}$ & & 1.9 & & 3.6 & 2.2 \\
\hline LEAD-210 (TOTAL) & & $11 / 24 / 92$ & NoOT & PCI/L & & 5.6 & & 0.3 & 0.9 \\
\hline MAGNESIUH & & $\begin{array}{l}04 / 26 / 88 \\
06 / 09 / 89 \\
12 / 01 / 89 \\
06 / 25 / 90 \\
01 / 29 / 91 \\
06 / 01 / 91 \\
02 / 21 / 92 \\
08 / 11 / 92 \\
01 / 31 / 93 \\
01 / 31 / 93 \\
06 / 30 / 93 \\
12 / 12 / 93 \\
12 / 12 / 93 \\
04 / 24 / 94 \\
04 / 24 / 94 \\
12 / 19 / 94\end{array}$ & $\begin{array}{l}0001 \\
0001 \\
0001 \\
0001 \\
0001 \\
0001 \\
0001 \\
0001 \\
0001 \\
0002 \\
0001 \\
0001 \\
0002 \\
0001 \\
0002 \\
0001\end{array}$ & $M G / L$ & & $\begin{array}{l}144 . \\
137 . \\
155 . \\
132 . \\
114 . \\
121 . \\
122 . \\
120 \\
119 \\
120 \\
130 \\
118 \\
117 \\
120 \\
121 \\
135\end{array}$ & $\begin{array}{l}\text { JL } \\
L\end{array}$ & $\begin{array}{l}0.001 \\
0.001 \\
0.001 \\
0.001 \\
0.001 \\
0.5 \\
0.5 \\
5 \\
0.1 \\
0.1 \\
0.1 \\
0.1 \\
0.1 \\
0.1 \\
0.1 \\
0.1\end{array}$ & $\begin{array}{l}- \\
- \\
- \\
- \\
- \\
- \\
- \\
- \\
- \\
- \\
-\end{array}$ \\
\hline MAGHESIUM (TOTAL) & & $\begin{array}{l}06 / 30 / 93 \\
06 / 30 / 93 \\
12 / 12 / 93 \\
12 / 12 / 93 \\
04 / 24 / 94 \\
04 / 24 / 94\end{array}$ & $\begin{array}{l}\text { NOO1 } \\
\text { NOO2 } \\
\text { NOO1 } \\
\text { NOO2 } \\
\text { NOO1 } \\
\text { NOO2 }\end{array}$ & MG/L & & $\begin{array}{l}144 \\
139 \\
121 \\
120 \\
119 \\
120\end{array}$ & $\begin{array}{l}L \\
L\end{array}$ & $\begin{array}{l}0.1 \\
0.1 \\
0.1 \\
0.1 \\
0.1 \\
0.1\end{array}$ & $\begin{array}{l}- \\
- \\
- \\
-\end{array}$ \\
\hline mAMGAHESE & & $\begin{array}{l}06 / 09 / 89 \\
12 / 01 / 89 \\
06 / 25 / 90 \\
01 / 29 / 91 \\
06 / 01 / 91 \\
02 / 29 / 92 \\
08 / 11 / 92 \\
11 / 24 / 92 \\
01 / 31 / 93\end{array}$ & $\begin{array}{l}0001 \\
0001 \\
0001 \\
0001 \\
0001 \\
0001 \\
0001 \\
0001 \\
0001\end{array}$ & MG/L & & $\begin{array}{l}0.17 \\
0.14 \\
0.15 \\
0.13 \\
0.14 \\
0.09 \\
0.11 \\
0.12 \\
0.13\end{array}$ & $\mathbf{L}$ & $\begin{array}{l}0.01 \\
0.01 \\
0.01 \\
0.01 \\
0.01 \\
0.01 \\
0.01 \\
0.0015 \\
0.01\end{array}$ & $\begin{array}{l}- \\
- \\
- \\
- \\
- \\
-\end{array}$ \\
\hline
\end{tabular}

PARAMETER VALUE INDICATOR (PVI): < - LESS THAN DETECTION LIMIT

OTHER PARAMETER VALUE FLAGS:

J - ESTIMUTED VALUE

L - LESS THAN THREE BORE VOLUMES REWOVED BEFORE SAMPLIHG

$W$ - POST-DIGEST SPIKE OUT OF CNTR LIM WHILE SAMP ABS < 50\% SPIKE
SNMPLE ID COOES:

0001 - FILTERED SAMPLE (.45 MICROHS)

0002 - FILTERED REPLICATE SAMPLE (.45 MICROHS)

NOO1 - UHFILTERED SAMPLE

MOO2 - UHFILTERED REPLICATE SAMPLE 
GROUHDWATER QUALITY DATA BY LOCATION

SOMP ReV. O Data

SITE: MOHO1 MOHLMEHT VALLEY

LOCATIOH: 0606

MORTH COORDINATE: 59034.2 FT

EAST COOROINATE: 88634.0 FT

$04 / 21 / 88$ TO $12 / 13 / 94$

REPORT DATE: $06 / 20 / 95$

FORMATION OF COMPLETION: ALLUVIUM (AL)

HYDRAULIC FLON RELATIOHSHIP: DOWN GRADIENT (D)

\begin{tabular}{|c|c|c|c|c|c|c|c|c|}
\hline PARAMETER NAME & LOG DATE & $\underset{\text { SAMPL }}{\text { SA }}$ & $\begin{array}{l}\text { UNITS DF } \\
\text { MEASURE }\end{array}$ & PVI & $\begin{array}{l}\text { PARAMETER } \\
\text { VALUE }\end{array}$ & FLAGS & $\begin{array}{l}\text { DETECTION } \\
\text { LIMIT }\end{array}$ & $\begin{array}{l}\text { PARAMETER } \\
\text { UNCERTAINTY }\end{array}$ \\
\hline MANGAMESE & $\begin{array}{l}01 / 31 / 93 \\
06 / 30 / 93 \\
12 / 12 / 93 \\
12 / 12 / 93 \\
04 / 24 / 94 \\
04 / 24 / 94 \\
12 / 11 / 94\end{array}$ & $\begin{array}{l}0002 \\
0001 \\
0001 \\
0002 \\
0001 \\
0002 \\
0001\end{array}$ & MG/L & & $\begin{array}{l}0.13 \\
0.16 \\
0.12 \\
0.12 \\
0.14 \\
0.14 \\
0.26\end{array}$ & $\begin{array}{l}L \\
L \\
L\end{array}$ & $\begin{array}{l}0.01 \\
0.01 \\
0.01 \\
0.01 \\
0.01 \\
0.01 \\
0.01\end{array}$ & $\begin{array}{l}- \\
- \\
- \\
- \\
-\end{array}$ \\
\hline MANGAHESE (TOTAL) & $\begin{array}{l}11 / 24 / 92 \\
06 / 30 / 93 \\
06 / 30 / 93 \\
12 / 12 / 93 \\
12 / 12 / 93 \\
04 / 24 / 94 \\
04 / 24 / 94\end{array}$ & $\begin{array}{l}\text { No01 } \\
\text { No01 } \\
\text { N002 } \\
\text { NO01 } \\
\text { NO02 } \\
\text { NO01 } \\
\text { NO02 }\end{array}$ & MG/L & & $\begin{array}{l}0.11 \\
0.31 \\
0.30 \\
0.19 \\
0.19 \\
0.21 \\
0.22\end{array}$ & $\begin{array}{l}L \\
L\end{array}$ & $\begin{array}{l}0.0015 \\
0.01 \\
0.01 \\
0.01 \\
0.01 \\
0.01 \\
0.01\end{array}$ & $\begin{array}{l}- \\
- \\
- \\
- \\
-\end{array}$ \\
\hline MOLYBDENUM & $\begin{array}{l}04 / 26 / 88 \\
06 / 09 / 89 \\
12 / 01 / 89 \\
06 / 25 / 90 \\
01 / 29 / 91 \\
06 / 01 / 99 \\
02 / 21 / 92 \\
08 / 11 / 92 \\
11 / 24 / 92 \\
06 / 30 / 93 \\
12 / 11 / 94\end{array}$ & $\begin{array}{l}0001 \\
0001 \\
0001 \\
0001 \\
0001 \\
0001 \\
0001 \\
0001 \\
0001 \\
0001 \\
0001\end{array}$ & MG/L & $\begin{array}{l}< \\
< \\
< \\
< \\
< \\
< \\
< \\
<\end{array}$ & $\begin{array}{l}0.06 \\
0.01 \\
0.01 \\
0.01 \\
0.04 \\
0.01 \\
0.01 \\
0.013 \\
0.0049 \\
0.01 \\
0.01\end{array}$ & $\begin{array}{l}\text { JL } \\
\text { L } \\
\text { N } \\
\text { L }\end{array}$ & $\begin{array}{l}0.01 \\
0.01 \\
0.01 \\
0.01 \\
0.01 \\
0.01 \\
0.01 \\
0.007 \\
0.0049 \\
0.01 \\
0.01\end{array}$ & $\begin{array}{l}- \\
- \\
- \\
- \\
- \\
- \\
-\end{array}$ \\
\hline $\begin{array}{l}\text { MOLYBDENUM (TOTAL) } \\
\text {. }\end{array}$ & $\begin{array}{l}11 / 24 / 92 \\
06 / 30 / 93 \\
06 / 30 / 93\end{array}$ & $\begin{array}{l}\text { No01 } \\
\text { N001 } \\
\text { N002 }\end{array}$ & MG/L & $<$ & $\begin{array}{l}0.0080 \\
0.01 \\
0.01\end{array}$ & N & $\begin{array}{l}0.0049 \\
0.01 \\
0.01\end{array}$ & $\dot{-}$ \\
\hline MET GROSS ALPHA (TOTAL) ** & $\begin{array}{l}11 / 24 / 92 \\
06 / 30 / 93 \\
06 / 30 / 93\end{array}$ & $\begin{array}{l}N 001 \\
N 001 \\
\text { NO02 }\end{array}$ & PCI/L & & $\begin{array}{l}-8.23 \\
-8.92 \\
-1.23\end{array}$ & & - & $\begin{array}{l}- \\
-\end{array}$ \\
\hline HICKEL & $06 / 09 / 89$ & 0001 & MG/L & $<$ & 0.04 & & 0.04 & - \\
\hline
\end{tabular}

- WET GROSS ALPHA (GROSS ALPHA - URANIUH) WITH 1 MG URANIUM = 686 PCI

* .NET GROSS ALPHA (TOTAL) (TOTAL EROSS ALPHA - TOTAL URAKILA)

PARAMETER VALUE INDICATOR (PVI): < - LESS THAH DETECTION LIHIT

SAMPLE ID COOES:

OTHER PARAMETER VALUE FLAGS:

$J$ - ESTIMATED VALUE

L - LESS THAN THREE BORE VOLUMES REMOVED BEFORE SAMPLIHG

N - SPIKE SAMPLE RECOVERY NOT HITHIN CONTROL LIHITS

OOD1 - FILTERED SAMPLE (.45 MICRONS)

0002 - FILTERED REPLICATE SNPLE (.45 MICRONS)

NOO9 - UHFILTERED SAMPLE

NOO2 - UHFILTERED REPLICATE SMMPLE 
FORMATIOH OF COHPLETION: ALLUVIUN (AL) HYDRAULIC FLON RELATIONSHIP: DONN GRADIENT (D)

\begin{tabular}{|c|c|c|c|c|c|c|c|c|}
\hline PARAMETER NAME & LOG DATE & $\underset{\text { ID }}{\text { SAMPLE }}$ & $\begin{array}{l}\text { UHITS OF } \\
\text { MEASURE }\end{array}$ & PVI & $\begin{array}{l}\text { PARAMETER } \\
\text { VALUE }\end{array}$ & FLAGS & $\begin{array}{l}\text { DETECTIOH } \\
\text { LIMIT }\end{array}$ & $\begin{array}{l}\text { PARAMETER } \\
\text { UNCERTAIHTY }\end{array}$ \\
\hline HICKEL & $\begin{array}{l}12 / 01 / 89 \\
06 / 25 / 90 \\
01 / 29 / 91 \\
06 / 01 / 91\end{array}$ & $\begin{array}{l}0001 \\
0001 \\
0001 \\
0001\end{array}$ & $M G / L$ & $\begin{array}{l}< \\
< \\
< \\
<\end{array}$ & $\begin{array}{l}0.04 \\
0.04 \\
0.04 \\
0.04\end{array}$ & $\mathbf{L}$ & $\begin{array}{l}0.04 \\
0.04 \\
0.04 \\
0.04\end{array}$ & : \\
\hline NITRATE & $\begin{array}{l}04 / 26 / 88 \\
06 / 09 / 89 \\
12 / 01 / 89 \\
06 / 25 / 90 \\
01 / 29 / 91 \\
06 / 01 / 91 \\
02 / 21 / 92 \\
08 / 11 / 92 \\
11 / 24 / 92 \\
01 / 31 / 93 \\
01 / 31 / 93 \\
12 / 12 / 93 \\
12 / 12 / 93\end{array}$ & $\begin{array}{l}0001 \\
0001 \\
0009 \\
0009 \\
0009 \\
0001 \\
0009 \\
0001 \\
0001 \\
0001 \\
0002 \\
0001 \\
0002\end{array}$ & $M G / L$ & & $\begin{array}{l}1600 . \\
1200 . \\
1200 . \\
1120 . \\
1080 . \\
974 . \\
1060 . \\
1100 \\
1600 \\
1160 \\
1960 \\
1120 \\
1260\end{array}$ & $\begin{array}{l}\text { JL } \\
\text { L } \\
\text { I }\end{array}$ & $\begin{array}{c}1 . \\
1 . \\
1 . \\
1 . \\
1 . \\
1 . \\
150 . \\
0.75 \\
0.044 \\
1 \\
1 \\
1 \\
1\end{array}$ & $\begin{array}{l}: \\
: \\
: \\
: \\
: \\
: \\
: \\
-\end{array}$ \\
\hline NITRATE (TOTAL) & $\begin{array}{l}06 / 30 / 93 \\
06 / 30 / 93 \\
04 / 24 / 94 \\
04 / 24 / 94 \\
12 / 11 / 94\end{array}$ & $\begin{array}{l}\text { NOOI } \\
\text { HOO2 } \\
\text { HOOI } \\
\text { NOO2 } \\
\text { NOOI }\end{array}$ & MG/L & & $\begin{array}{l}1170 \\
1160 \\
1180 \\
1130 \\
1360\end{array}$ & $\begin{array}{l}L \\
L \\
\text { HJLA }\end{array}$ & $\begin{array}{l}1 \\
1 \\
1 \\
1 \\
1\end{array}$ & $\begin{array}{l}- \\
- \\
-\end{array}$ \\
\hline MITRITE AND MITRATE & $\begin{array}{l}01 / 29 / 91 \\
06 / 01 / 91\end{array}$ & $\begin{array}{l}0001 \\
0001\end{array}$ & MG/L & & $\begin{array}{l}1080 . \\
215 .\end{array}$ & $L$ & $\begin{array}{l}1.05 \\
0.05\end{array}$ & $\dot{-}$ \\
\hline $\mathrm{PH}$ & $\begin{array}{l}04 / 26 / 88 \\
06 / 09 / 89 \\
12 / 01 / 89 \\
06 / 25 / 90 \\
01 / 29 / 91 \\
06 / 01 / 91 \\
02 / 21 / 92 \\
08 / 11 / 92 \\
11 / 24 / 92 \\
01 / 31 / 93 \\
01 / 31 / 93 \\
06 / 30 / 93 \\
12 / 12 / 93 \\
04 / 24 / 94 \\
12 / 11 / 94\end{array}$ & $\begin{array}{l}0001 \\
0001 \\
0001 \\
0001 \\
0001 \\
0001 \\
0001 \\
0001 \\
0001 \\
0001 \\
0002 \\
\text { NO01 } \\
\text { NO01 } \\
\text { NO01 } \\
\text { NO01 }\end{array}$ & su & & $\begin{array}{l}7.20 \\
7.33 \\
7.15 \\
7.26 \\
7.02 \\
7.20 \\
7.06 \\
7.20 \\
7.32 \\
6.98 \\
6.98 \\
6.98 \\
7.26 \\
7.16 \\
7.24\end{array}$ & $\begin{array}{l}L \\
L\end{array}$ & $\begin{array}{l}- \\
- \\
- \\
- \\
- \\
- \\
- \\
- \\
- \\
- \\
- \\
0.10\end{array}$ & $\begin{array}{l}- \\
- \\
- \\
- \\
- \\
- \\
- \\
- \\
- \\
- \\
-\end{array}$ \\
\hline PHOSPHATE & $\begin{array}{l}06 / 09 / 89 \\
12 / 01 / 89 \\
06 / 25 / 90\end{array}$ & $\begin{array}{l}0001 \\
0001 \\
0001\end{array}$ & $M G / L$ & & $\begin{array}{l}0.6 \\
0.3 \\
0.4\end{array}$ & & $\begin{array}{l}0.1 \\
0.1 \\
0.1\end{array}$ & - \\
\hline PHOSPHATE (TOTAL) & $\begin{array}{l}06 / 30 / 93 \\
06 / 30 / 93\end{array}$ & $\begin{array}{l}\mathrm{HOO1} \\
\mathrm{NOO2}\end{array}$ & $M G / L$ & & $\begin{array}{l}1.0 \\
1.0\end{array}$ & & $\begin{array}{l}0.1 \\
0.1\end{array}$ & 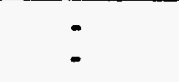 \\
\hline POTASSIU: & $\begin{array}{l}04 / 26 / 88 \\
06 / 09 / 89\end{array}$ & $\begin{array}{l}0001 \\
0001\end{array}$ & MG/L & & $\begin{array}{l}10.5 \\
13.8\end{array}$ & لL & $\begin{array}{l}0.01 \\
0.01\end{array}$ & - \\
\hline
\end{tabular}

PARAMETER VALUE INDICATOR (PVI): < - LESS THAK DETECTION LIMIT

OTHER PARAMETER VALUE FLAGS:

H - HOLD TIME EXPIRED, VALUE SUSPECT

1 - INCREASED DETECTION LIMIT DUE TO REQUIRED DILUTION

J - ESTIMATED VALUE

L - LESS THAN THREE BORE VOLLHES REMOVED BEFORE SAMPLING

N - SPIKE SAMPLE RECOVERY NOT WITHIN CONTROL LIMITS
SAMPLE ID COOES:

0001 - FILTERED SAMPLE (.45 MICRONS)

OOD2 - FILTERED REPLICATE SAMPLE (.45 MICRONS) HOOI - UHFILTERED SAMPLE

NOD2 - UNFILTERED REPLICATE SAMPLE 
GROUNDHATER QUALITY DATA BY LOCATION

SOUP Rev. O Data

SITE: MONO 1 MONUMENT VALLEY

LOCATION: 0606

MORTH COORDINATE: 59034.2 FT

EAST COORDINATE: 88634.0 FT

$04 / 21 / 88$ TO $12 / 13 / 94$

REPORT DATE: 06/20/95

FORMATIOH OF CONPLETION: ALLUVIUM (AL)

HYDRAULIC FLON RELATIONSHIP: DOWH GRADIENT (D)

\begin{tabular}{|c|c|c|c|c|c|c|c|c|}
\hline PARAMETER MAME & LOG DATE & $\mid \begin{array}{c}\text { SAMPLE } \\
10\end{array}$ & $\begin{array}{l}\text { UNITS OF } \\
\text { MEASURE }\end{array}$ & PVI & $\begin{array}{l}\text { PARAMETER } \\
\text { VALUE }\end{array}$ & FLAGS & $\begin{array}{l}\text { DETECTION } \\
\text { LIMIT }\end{array}$ & $\begin{array}{l}\text { PARAMETER } \\
\text { UNCERTAINTY }\end{array}$ \\
\hline POTASSIUH & $\begin{array}{l}12 / 01 / 89 \\
06 / 25 / 90 \\
01 / 29 / 91 \\
06 / 01 / 91 \\
02 / 21 / 92 \\
08 / 11 / 92 \\
01 / 31 / 93 \\
01 / 31 / 93 \\
06 / 30 / 93 \\
12 / 12 / 93 \\
12 / 12 / 93 \\
04 / 24 / 94 \\
04 / 24 / 94 \\
12 / 11 / 94\end{array}$ & $\begin{array}{l}0001 \\
0001 \\
0001 \\
0001 \\
0001 \\
0001 \\
0001 \\
0002 \\
0001 \\
0001 \\
0002 \\
0001 \\
0002 \\
0001\end{array}$ & MG/L & & $\begin{array}{l}12.2 \\
12.7 \\
12.3 \\
13 . \\
11 . \\
12 \\
12.1 \\
12.4 \\
14.1 \\
9.4 \\
9.2 \\
11.6 \\
11.6 \\
14.4\end{array}$ & L & $\begin{array}{l}0.01 \\
0.01 \\
0.01 \\
5 . \\
5 . \\
0.575 \\
0.1 \\
0.1 \\
0.1 \\
0.1 \\
0.1 \\
0.1 \\
0.1 \\
0.1\end{array}$ & $\begin{array}{l}- \\
- \\
- \\
- \\
- \\
- \\
- \\
- \\
-\end{array}$ \\
\hline POTASSILA (TOTAL) & $\begin{array}{l}06 / 30 / 93 \\
06 / 30 / 93 \\
12 / 12 / 93 \\
12 / 12 / 93 \\
04 / 24 / 94 \\
04 / 24 / 94\end{array}$ & $\begin{array}{l}\text { NOO1 } \\
\text { NOO2 } \\
\text { NOO1 } \\
\text { NOO2 } \\
\text { NO01 } \\
\text { N002 }\end{array}$ & $M G / L$ & & $\begin{array}{l}16.7 \\
16.9 \\
10.3 \\
10.3 \\
11.8 \\
12.7\end{array}$ & $L$ & $\begin{array}{l}0.1 \\
0.1 \\
0.1 \\
0.1 \\
0.1 \\
0.1\end{array}$ & $\begin{array}{l}- \\
: \\
: \\
-\end{array}$ \\
\hline RADIUA-226 & $\begin{array}{l}06 / 09 / 89 \\
12 / 01 / 89 \\
06 / 25 / 90 \\
01 / 29 / 91 \\
06 / 01 / 91 \\
02 / 21 / 92 \\
08 / 11 / 92 \\
11 / 24 / 92\end{array}$ & $\begin{array}{l}0001 \\
0001 \\
0001 \\
0001 \\
0001 \\
0009 \\
0001 \\
0001\end{array}$ & PCI/L & & $\begin{array}{l}0.4 \\
0.2 \\
0.1 \\
1.0 \\
0.3 \\
0.2 \\
0.4 \\
0.7\end{array}$ & $\mathbf{L}$ & $\begin{array}{l}1 . \\
1 . \\
1 . \\
1 . \\
1 . \\
1.0 \\
0.5 \\
0.3\end{array}$ & $\begin{array}{l}0.2 \\
0.2 \\
0.2 \\
0.9 \\
0.3 \\
0.3 \\
0.1 \\
0.3\end{array}$ \\
\hline RADIUA-226 (TOTAL) & $11 / 24 / 92$ & N001 & $\mathrm{PCI} / \mathrm{L}$ & & 1.0 & & 0.3 & 0.4 \\
\hline RADIUH-226 + RADIUH-228 & $\begin{array}{l}06 / 09 / 89 \\
12 / 01 / 89 \\
06 / 25 / 90 \\
01 / 29 / 91 \\
06 / 01 / 91 \\
02 / 21 / 92 \\
08 / 11 / 92 \\
11 / 24 / 92\end{array}$ & $\begin{array}{l}0001 \\
0001 \\
0001 \\
0001 \\
0001 \\
0001 \\
0001 \\
0001\end{array}$ & $\mathrm{PCI} / \mathrm{L}$ & & $\begin{array}{r}0.40 \\
1.00 \\
1.50 \\
2.50 \\
15.50 \\
2.10 \\
0.40 \\
3.00\end{array}$ & & $\begin{array}{l}- \\
- \\
- \\
- \\
-\end{array}$ & $\begin{array}{l}- \\
- \\
- \\
- \\
-\end{array}$ \\
\hline RADIUH-226 + RADIUH-228 (TOTAL) & $11 / 24 / 92$ & HOOI & PCI/L & & 3.20 & & - & - \\
\hline RADIUH-228 & $\begin{array}{l}06 / 09 / 89 \\
12 / 01 / 89 \\
06 / 25 / 90 \\
01 / 29 / 91 \\
06 / 01 / 91 \\
02 / 21 / 92 \\
08 / 11 / 92 \\
11 / 24 / 92\end{array}$ & $\begin{array}{l}0001 \\
0001 \\
0001 \\
0001 \\
0001 \\
0001 \\
0001 \\
0001\end{array}$ & $\mathrm{PCI} / \mathrm{L}$ & & $\begin{array}{r}0.0 \\
0.8 \\
1.4 \\
1.5 \\
15.2 \\
1.9 \\
0.0 \\
2.3\end{array}$ & L & $\begin{array}{l}1 . \\
1 . \\
1 . \\
1 . \\
1.0 \\
1.0 \\
1.9 \\
2.0\end{array}$ & $\begin{array}{l}0.9 \\
1.8 \\
0.9 \\
0.8 \\
2.2- \\
1.6 \\
1.0 \\
1.4\end{array}$ \\
\hline RADIUH-228 (TOTAL) & $11 / 24 / 92$ & n001 & $\mathrm{PCI} / \mathrm{L}$ & & 2.2 & & 2.0 & 1.4 \\
\hline
\end{tabular}

PARAMETER VALUE INOICATOR (PVI): < - LESS thaH DETECTION LIMIT

SAMPLE IO COOES:

0001 - FILTERED SAMPLE (.45 MICRONS)

OTHER PARAMETER VALUE FLAGS:

- DUPLICATE akALYSIS nOt uITHIN COHTROL LIHITS

OOO2 - FILTERED REPLICATE SAMPLE (.45 MICRONS)

\& - estimated VALUE

L - LÉSS THAN THREE BORE VOLUNES REMOVED BEFORE SAMPLING

MOOI - UNFILTERED SAMPLE

MOOZ - UAFILTERED REPLICATE SAMPLE 
GROUNDHATER QUALITY DATA BY LOCATION

SOUP ReV. O Data

SITE: MONO I MONUMENT VALLEY

LOCATION: 0606

HORTH COORDINATE: 59034.2 FT

EAST COORDIHATE: 88634.0 FT

04/21/88 TO $12 / 13 / 94$

REPORT DATE: $06 / 20 / 95$

FORMATION OF COMPLETION: ALLUVIUM (AL)

HYORAULIC FLON RELATIOHSHIP: DONN GRADIENT (D)

\begin{tabular}{|c|c|c|c|c|c|c|c|c|}
\hline PARAMETER HAME & LOG DATE & $\left|\begin{array}{c}\text { SNMPLE } \\
\text { ID }\end{array}\right|$ & $\begin{array}{l}\text { UNITS OF } \\
\text { MEASURE }\end{array}$ & PVI & $\begin{array}{l}\text { PARAMETER } \\
\text { VALUE }\end{array}$ & FLAGS & $\begin{array}{l}\text { DETECTION } \\
\text { LIMIT }\end{array}$ & $\begin{array}{l}\text { PARAMETER } \\
\text { UHCERTAIHTY }\end{array}$ \\
\hline REDOX POTENTIAL & $\begin{array}{l}11 / 24 / 92 \\
12 / 11 / 94\end{array}$ & $\begin{array}{l}0001 \\
\text { NOO1 }\end{array}$ & ㄸNOLTS & & $\begin{array}{l}481.7 \\
426\end{array}$ & $L$ & - & - \\
\hline SELENIUH & $\begin{array}{l}04 / 26 / 88 \\
06 / 09 / 89 \\
12 / 01 / 89 \\
06 / 25 / 90 \\
01 / 29 / 91 \\
06 / 01 / 91 \\
02 / 21 / 92 \\
08 / 11 / 92 \\
11 / 24 / 92\end{array}$ & $\begin{array}{l}0001 \\
0001 \\
0001 \\
0001 \\
0001 \\
0001 \\
0001 \\
0001 \\
0001\end{array}$ & MG/L & $\begin{array}{l}< \\
< \\
< \\
< \\
<\end{array}$ & $\begin{array}{l}0.009 \\
0.015 \\
0.005 \\
0.005 \\
0.005 \\
0.007 \\
0.03 \\
0.005 \\
0.0015\end{array}$ & $\begin{array}{l}\text { JL } \\
\text { L } \\
\text { I } \\
\text { HW }\end{array}$ & $\begin{array}{l}0.005 \\
0.005 \\
0.005 \\
0.005 \\
0.005 \\
0.005 \\
0.03 \\
0.005 \\
0.0015\end{array}$ & $\begin{array}{l}- \\
- \\
- \\
- \\
- \\
-\end{array}$ \\
\hline SELEHIUM (TOTAL) & $11 / 24 / 92$ & NOOI & MG/L & $<$ & \multicolumn{2}{|l|}{0.0015} & 0.0015 & - \\
\hline SILICA - SIO2 & $\begin{array}{l}06 / 09 / 89 \\
12 / 01 / 89 \\
06 / 25 / 90 \\
01 / 29 / 91 \\
06 / 01 / 91 \\
12 / 12 / 93 \\
12 / 12 / 93 \\
12 / 11 / 94\end{array}$ & $\begin{array}{l}0001 \\
0001 \\
0001 \\
0001 \\
0001 \\
0001 \\
0002 \\
0001\end{array}$ & MG/L & \multicolumn{2}{|r|}{$\begin{array}{l}21 . \\
21 . \\
23 . \\
21 . \\
22.5 \\
21.8 \\
22.1 \\
21.6\end{array}$} & $\mathbf{L}$ & $\begin{array}{l}2 . \\
2 . \\
2 . \\
2 . \\
0.1 \\
0.1 \\
0.1 \\
0.1\end{array}$ & $\begin{array}{l}: \\
: \\
: \\
: \\
-\end{array}$ \\
\hline SILICA - SIO2 (TOTAL) & $\begin{array}{l}06 / 30 / 93 \\
06 / 30 / 93 \\
12 / 12 / 93 \\
12 / 12 / 93\end{array}$ & $\begin{array}{l}\text { No01 } \\
\text { N002 } \\
\text { N001 } \\
\text { N002 }\end{array}$ & ME/L & \multicolumn{3}{|c|}{$\begin{array}{l}26.7 \\
46.8 \\
41.7 \\
43.8\end{array}$} & $\begin{array}{l}0.5 \\
0.5 \\
0.1 \\
0.1\end{array}$ & $\begin{array}{l}- \\
-\end{array}$ \\
\hline SILVER & $\begin{array}{l}06 / 09 / 89 \\
12 / 01 / 89 \\
06 / 25 / 90 \\
01 / 29 / 91 \\
06 / 01 / 91\end{array}$ & $\begin{array}{l}0001 \\
0001 \\
0001 \\
0001 \\
0001\end{array}$ & MG/L & $\begin{array}{l}< \\
< \\
< \\
< \\
<\end{array}$ & \multicolumn{2}{|l|}{$\begin{array}{l}0.01 \\
0.01 \\
0.01 \\
0.01 \\
0.01\end{array}$} & $\begin{array}{l}0.01 \\
0.01 \\
0.01 \\
0.01 \\
0.01\end{array}$ & $\begin{array}{l}- \\
- \\
-\end{array}$ \\
\hline SCOIUA & $\begin{array}{l}04 / 26 / 88 \\
06 / 09 / 89 \\
12 / 01 / 89 \\
06 / 25 / 90 \\
01 / 29 / 91 \\
06 / 01 / 91 \\
02 / 21 / 92 \\
08 / 11 / 92 \\
01 / 31 / 93 \\
01 / 31 / 93 \\
06 / 30 / 93 \\
12 / 12 / 93 \\
12 / 12 / 93 \\
04 / 24 / 94 \\
04 / 24 / 94 \\
12 / 11 / 94\end{array}$ & $\begin{array}{l}0001 \\
0001 \\
0001 \\
0001 \\
0001 \\
0001 \\
0001 \\
0001 \\
0001 \\
0002 \\
0001 \\
0001 \\
0002 \\
0001 \\
0002 \\
0001\end{array}$ & MG/L & & $\begin{array}{c}98.7 \\
98.8 \\
106 . \\
102 . \\
97.3 \\
96 . \\
95 . \\
95 \\
95 \\
96.2 \\
94 \\
92 \\
90 \\
94 \\
95 \\
100\end{array}$ & $\begin{array}{l}L \\
L \\
L\end{array}$ & $\begin{array}{l}0.002 \\
0.002 \\
0.002 \\
0.002 \\
0.002 \\
1 . \\
1 . \\
5 \\
0.1 \\
0.1 \\
1 \\
1 \\
1 \\
1 \\
1 \\
1\end{array}$ & $\begin{array}{l}- \\
: \\
: \\
: \\
: \\
- \\
- \\
- \\
- \\
-\end{array}$ \\
\hline SOOIUH (TOTAL) & $06 / 30 / 93$ & No01 & $M G / L$ & \multicolumn{3}{|c|}{96} & 1 & - \\
\hline
\end{tabular}

PARAMETER VALUE INDICATOR (PVI): < - LESS THAN DETECTION LIMIT

SNMPLE ID COOES:

DO01 - FILTERED SAMPLE (.45 MICROHS)

OTHER PARAMETER VALUE FLAGS:

DOO2 - FILTERED REPLICATE SAMPLE (.45 MICRONS)

NOO1 - UHFILTERED SAMPLE

H - HOLD TIME EXPIRED, VALUE SUSPECT

I - INCREASED DETECTION LIMIT DUE TO REQUIRED DILUTION

NOO2 - UNFILTERED REPLICATE SAMPLE

J. ESTIMUTED VALUE

L - LESS THAH THREE BORE VOLUMES REMOVED BEFORE SAMPLING

H - SPIKE SAMPLE RECOVERY MOT HITHIH CONTROL LIMITS

$W$ - POST-DIGEST SPIKE OUT OF CNTR LIH HHILE SAMP ABS < 50\% SPIKE 
GROUKDHATER OUALITY DATA BY LOCATION

SOWP Rev. O Data

SITE: MONO1 MONUMENT VALLEY

LOCATION: 0606

NORTH COORDIHATE: 59034.2 FT

EAST COORDINATE: 88634.0 FT

04/21/88 TO $12 / 13 / 94$

REPORT DATE: 06/20/95

FORMATION OF COMPLETION: ALLUVIUM (AL)

HYDRAULIC FLOW RELATIOHSHIP: DOWN GRADIENT (D)

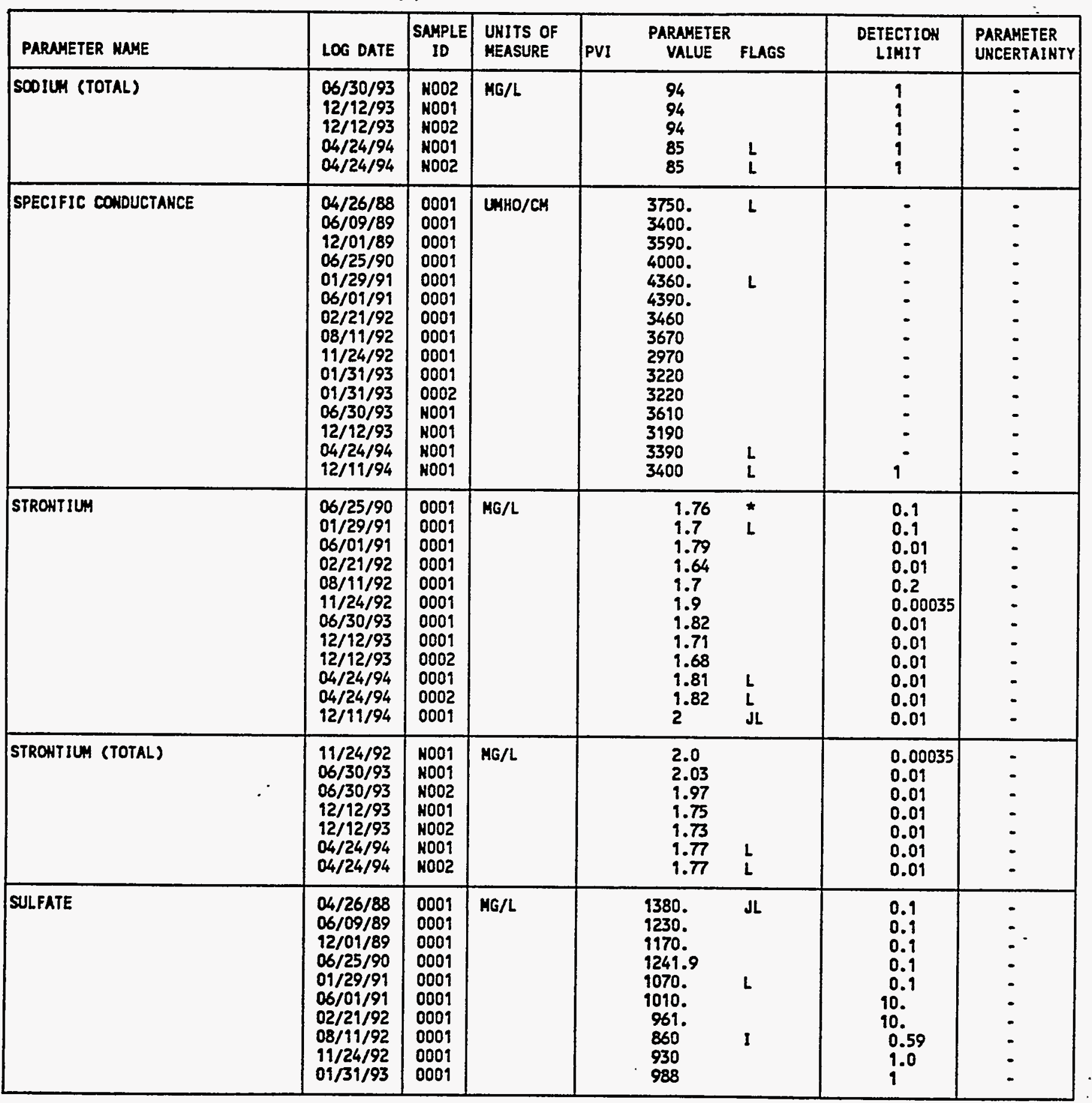

PARAMETER VALUE INDICATOR (PVI): < - LESS THAN DETECTION LIMIT

SAMPLE ID COOES:

OTHER PARAMETER VALUE FLAGS:

- DUPLICATE aMALYSIS NOT MITHIN CONTROL LIHITS

0001 - FILTERED SAHPLE (.45 HICRONS)

1 - IMCREASED DETECTION LIMIT DUE TO REQUIRED OILUTIOH

J - ESTIMLTED VALUE

NOOI - UNFILTERED SAMPLE

NOO2 - UNFILTERED REPLICATE SAHPLE

L - LESS THAN THREE BORE VOLUMES REMOVED BEFORE SAMPLIHG 
GROUNDHATER QUALITY DATA BY LOCATION

SOMP ReV. O Data

SITE: MONO1 MONUMENT VALLEY

LOCATIOH: 0606

NORTH COORDINATE: 59034.2 FT

EAST COORDINATE: 88634.0 FT

04/21/88 To $12 / 13 / 94$

REPORT DATE: $06 / 20 / 95$

FORHATION OF COHPLETION: ALLUVIUM (AL)

HYORAULIC FLON RELATIOHSHIP: DOWN GRADIEHT (D)

\begin{tabular}{|c|c|c|c|c|c|c|c|c|}
\hline PARALETER NAME & LOG DATE & $\underset{\text { SD }}{\text { SUMPLE }}$ & $\begin{array}{l}\text { UNITS OF } \\
\text { MEASURE }\end{array}$ & PVI & $\begin{array}{l}\text { PARAMETER } \\
\text { VALUE }\end{array}$ & FLAGS & $\begin{array}{l}\text { DETECTION } \\
-\quad \text { LIMIT }\end{array}$ & $\begin{array}{l}\text { PARAMETER } \\
\text { UHCERTAINTY }\end{array}$ \\
\hline SULFATE & $\begin{array}{l}01 / 31 / 93 \\
12 / 12 / 93 \\
12 / 12 / 93 \\
04 / 24 / 94 \\
04 / 24 / 94 \\
12 / 11 / 94\end{array}$ & $\begin{array}{l}0002 \\
0001 \\
0002 \\
0001 \\
0002 \\
0001\end{array}$ & $M G / L$ & & $\begin{array}{l}990 \\
805 \\
805 \\
861 \\
822 \\
857\end{array}$ & $\begin{array}{l}L \\
L\end{array}$ & $\begin{array}{l}1 \\
1 \\
1 \\
1 \\
1 \\
1\end{array}$ & $\begin{array}{l}- \\
- \\
- \\
- \\
-\end{array}$ \\
\hline SULFATE (TOTAL) & $\begin{array}{l}11 / 24 / 92 \\
06 / 30 / 93 \\
06 / 30 / 93\end{array}$ & $\begin{array}{l}\text { No01 } \\
\text { NO01 } \\
\text { NO02 }\end{array}$ & MG/L & & $\begin{array}{l}920 \\
884 \\
932\end{array}$ & & $\begin{array}{l}9.0 \\
1 \\
1\end{array}$ & - \\
\hline SULFIDE & $\begin{array}{l}06 / 09 / 89 \\
12 / 01 / 89 \\
01 / 29 / 91 \\
06 / 01 / 91\end{array}$ & $\begin{array}{l}0001 \\
0001 \\
0001 \\
0001\end{array}$ & $M G / L$ & $\begin{array}{l}< \\
<\end{array}$ & $\begin{array}{r}0.1 \\
0.1 \\
0.1 \\
11.5\end{array}$ & J & $\begin{array}{l}0.1 \\
0.1 \\
0.1 \\
1 .\end{array}$ & $\begin{array}{l}- \\
- \\
-\end{array}$ \\
\hline TEMPERATURE & $\begin{array}{l}04 / 26 / 88 \\
06 / 09 / 89 \\
12 / 01 / 89 \\
06 / 25 / 90 \\
01 / 29 / 91 \\
06 / 01 / 91 \\
02 / 21 / 92 \\
08 / 11 / 92 \\
11 / 24 / 92 \\
01 / 31 / 93 \\
01 / 31 / 93 \\
06 / 30 / 93 \\
12 / 12 / 93 \\
04 / 24 / 94 \\
12 / 11 / 94\end{array}$ & $\begin{array}{l}0001 \\
0001 \\
0001 \\
0001 \\
0001 \\
0001 \\
0001 \\
0001 \\
0001 \\
0001 \\
0002 \\
N 001 \\
N 001 \\
\text { No01 } \\
\text { N001 }\end{array}$ & $C$ - DEGREE & & $\begin{array}{l}15.0 \\
17.0 \\
16.0 \\
22 . \\
11.1 \\
15.4 \\
14.2 \\
17.7 \\
8.3 \\
12.2 \\
12.2 \\
16.4 \\
15.0 \\
15.4 \\
14.2\end{array}$ & $\begin{array}{l}\mathbf{L} \\
\mathbf{L}\end{array}$ & $\begin{array}{l}- \\
- \\
- \\
- \\
- \\
- \\
- \\
- \\
- \\
- \\
0.1\end{array}$ & $\begin{array}{l}- \\
- \\
- \\
- \\
- \\
- \\
- \\
- \\
- \\
- \\
- \\
-\end{array}$ \\
\hline THALLIUM & $\begin{array}{l}06 / 09 / 89 \\
12 / 01 / 89 \\
06 / 25 / 90 \\
01 / 29 / 91 \\
06 / 01 / 91 \\
02 / 21 / 92\end{array}$ & $\begin{array}{l}0001 \\
0001 \\
0001 \\
0001 \\
0001 \\
0001\end{array}$ & MG/L & $\begin{array}{l}< \\
< \\
< \\
<\end{array}$ & $\begin{array}{l}0.01 \\
0.1 \\
0.01 \\
0.01 \\
0.01 \\
0.01\end{array}$ & $L$ & $\begin{array}{l}0.01 \\
0.1 \\
0.01 \\
0.01 \\
0.01 \\
0.01\end{array}$ & $\begin{array}{l}- \\
- \\
- \\
- \\
-\end{array}$ \\
\hline THORIUN-230 & $\begin{array}{l}01 / 29 / 91 \\
06 / 01 / 91\end{array}$ & $\begin{array}{l}0001 \\
0001\end{array}$ & PCI $/ L$ & & $\begin{array}{l}0.0 \\
0.5\end{array}$ & 1 & 1. & $\begin{array}{l}0.3 \\
0.3\end{array}$ \\
\hline TIN & $\begin{array}{l}06 / 09 / 89 \\
12 / 01 / 89 \\
06 / 25 / 90 \\
01 / 29 / 91 \\
06 / 01 / 91 \\
02 / 21 / 92\end{array}$ & $\begin{array}{l}0001 \\
0001 \\
0001 \\
0001 \\
0001 \\
0001\end{array}$ & MG/L & $\begin{array}{l}< \\
< \\
< \\
< \\
<\end{array}$ & $\begin{array}{l}0.005 \\
0.005 \\
0.013 \\
0.005 \\
0.01 \\
0.005\end{array}$ & $\frac{L}{I}$ & $\begin{array}{l}0.005 \\
0.005 \\
0.005 \\
0.005 \\
0.01 \\
0.005\end{array}$ & $\begin{array}{l}- \\
- \\
- \\
- \\
-\end{array}$ \\
\hline TOTAL DISSOLVED SOLIDS & $\begin{array}{l}04 / 26 / 88 \\
06 / 09 / 89 \\
12 / 01 / 89 \\
06 / 25 / 90 \\
01 / 29 / 91\end{array}$ & $\begin{array}{l}0001 \\
0001 \\
0001 \\
0001 \\
0001\end{array}$ & MG/L & & $\begin{array}{l}3410 . \\
2410 \\
2520 \\
2068 . \\
1980\end{array}$ & $\mathbf{L}$ & $\begin{array}{l}10 . \\
10 . \\
10 . \\
10 . \\
10 .\end{array}$ & $\begin{array}{l}\ddot{-} \\
\ddot{-} \\
-\end{array}$ \\
\hline
\end{tabular}

PARAMETER VALUE IMDICATOR (PVI): < - LESS THAH DETECTION LIHIT

SNMPLE ID COOES:

0001 - FILTERED SAMPLE (.45 MICRONS)

OTHER PARAMETER VALUE FLAGS:

0002 - FILTERED REPLICATE SAMPLE (.45 MICRONS)

I - IMCREASED DETECTION LIMIT DUE TO REQUIRED DILUTIOH

J - ESTIMATED VALUE

NDO1 - UNFILTERED SAMPLE

NO02 - UMFILTERED REPLICATE SAMPLE

L - LESS THAH THREE BORE VOLUHES REMOVED BEFORE SAMLING 
GROUNDHATER QUALITY DATA BY LOCATION

SOMP ReV. $O$ Data

SITE: MONOI MONLMENT VALLEY

LOCATION: 0606

MORTH COORDIKATE: 59034.2 FT

EAST COORDINATE: 88634.0 FT

$04 / 21 / 88$ TO $12 / 13 / 94$

REPORT DATE: $06 / 20 / 95$

FORMATION OF COMPLETIOH: ALLUVIUM (AL)

HYDRAULIC FLOH RELATIOHSHIP: DOWN GRADIEHT (D)

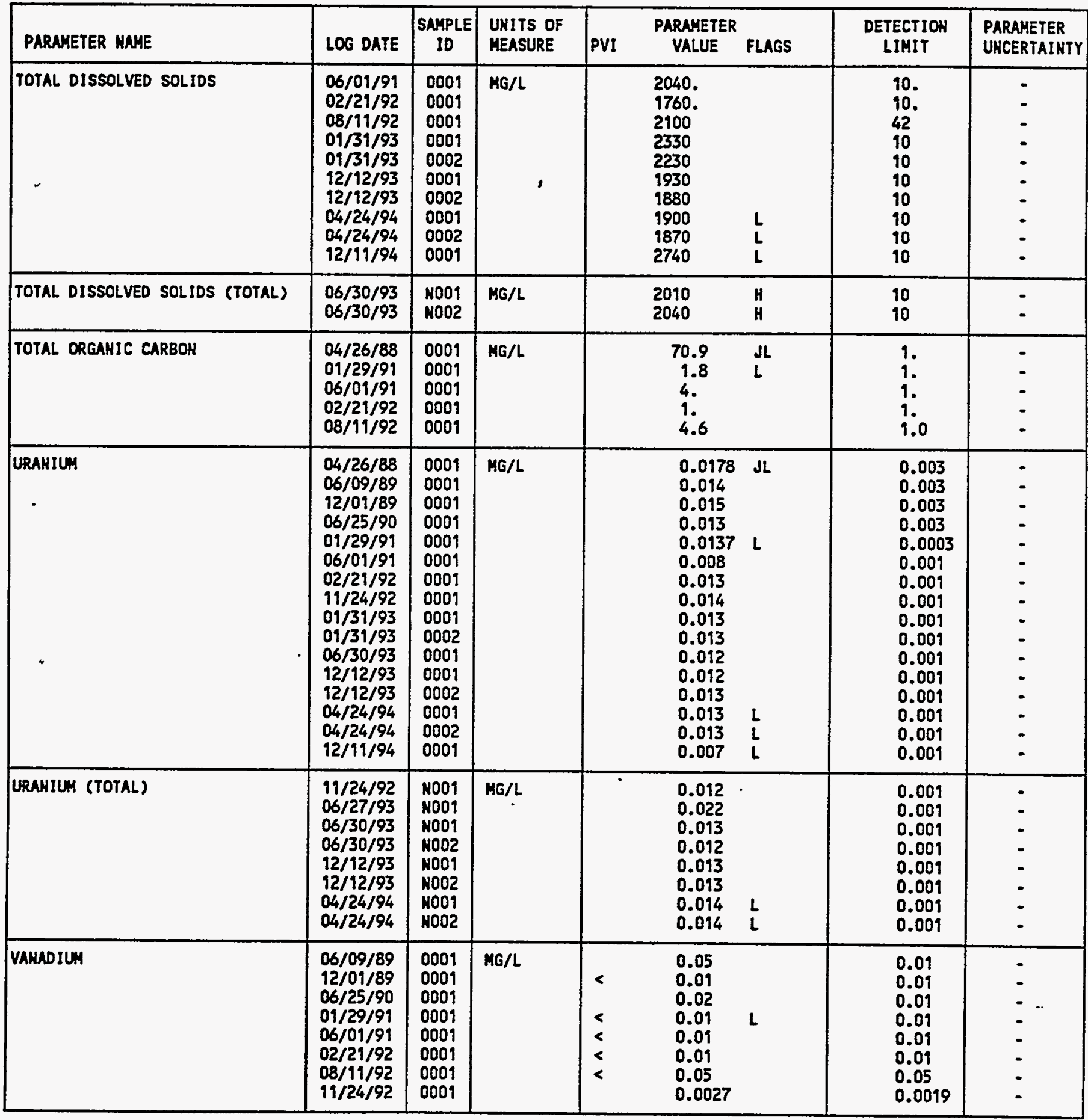

PARAMETER VALUE IMDICATOR (PVI): \& - LESS THAN DETECTION LIMIT

SAMPLE ID COOES:

OTHER PARAMETER VALUE FLAGS:

H - HOLD TIME EXPIRED, VALUE SUSPECT

0001 - FILTERED SAMPLE (.45 MICRONS)

J - estimated VALUE

0002 - FILTERED REPLICATE SAMPLE (.45 MICRONS)

NOO - UHFILTERED SAMPLE

MOO2 - UAFILTERED REPLICATE SAMPLE

- L - LESS THAH THREE bORE VOLLMES REMOVED BEFORE SAMPLIHG 
GROUNDHATER QUALITY DATA BY LOCATION

SOUP ReV. O Data

SITE: HONO1 MONUMENT VALLEY

LOCATION: 0606

NORTH COORDINATE: 59034.2 FT

EAST COORDIHATE: 88634.0 FT

04/21/88 TO $12 / 13 / 94$

REPORT DATE: 06/20/95

FORMATION OF COMPLETION: ALLUVIUH (AL)

HYDRAULIC FLOW RELATIONSHIP: DOWN GRADIENT (D)

\begin{tabular}{|c|c|c|c|c|c|c|c|c|}
\hline PARAMETER MAME & LOG DATE & $\mid \begin{array}{c}\text { SAMPLE } \\
\text { ID }\end{array}$ & $\begin{array}{l}\text { UNITS OF } \\
\text { MEASURE }\end{array}$ & PVI & $\begin{array}{l}\text { PARANETER } \\
\text { VALUE }\end{array}$ & FLAGS & $\begin{array}{l}\text { DETECTION } \\
\text { LIMIT }\end{array}$ & $\begin{array}{l}\text { PARAMETER } \\
\text { UHCERTAINTY }\end{array}$ \\
\hline VAHADIUY & $\begin{array}{l}06 / 30 / 93 \\
12 / 12 / 93 \\
12 / 12 / 93 \\
04 / 24 / 94 \\
04 / 24 / 94 \\
12 / 11 / 94\end{array}$ & $\begin{array}{l}0001 \\
0001 \\
0002 \\
0001 \\
0002 \\
0001\end{array}$ & $M G / L$ & $\begin{array}{l}< \\
< \\
< \\
< \\
<\end{array}$ & $\begin{array}{l}0.01 \\
0.01 \\
0.01 \\
0.01 \\
0.01 \\
0.01\end{array}$ & $\begin{array}{l}\mathbf{L} \\
\mathbf{L} \\
\mathbf{L}\end{array}$ & $\begin{array}{l}0.01 \\
0.01 \\
0.01 \\
0.01 \\
0.01 \\
0.01\end{array}$ & $\begin{array}{l}- \\
- \\
- \\
-\end{array}$ \\
\hline VAHADIUM (TOTAL) & $\begin{array}{l}11 / 24 / 92 \\
06 / 30 / 93 \\
06 / 30 / 93 \\
12 / 12 / 93 \\
12 / 12 / 93 \\
04 / 24 / 94 \\
04 / 24 / 94\end{array}$ & $\begin{array}{l}\text { No01 } \\
\text { NOO1 } \\
\text { NO02 } \\
\text { NO01 } \\
\text { NO02 } \\
\text { NO01 } \\
\text { NO02 }\end{array}$ & MG/L & $\begin{array}{l}< \\
< \\
< \\
<\end{array}$ & $\begin{array}{l}0.0030 \\
0.01 \\
0.01 \\
0.01 \\
0.01 \\
0.01 \\
0.01\end{array}$ & $L$ & $\begin{array}{l}0.0019 \\
0.01 \\
0.01 \\
0.01 \\
0.01 \\
0.01 \\
0.01\end{array}$ & $\begin{array}{l}- \\
- \\
- \\
-\end{array}$ \\
\hline ZIHC & $\begin{array}{l}06 / 09 / 89 \\
12 / 01 / 89 \\
06 / 25 / 90 \\
01 / 29 / 91 \\
06 / 01 / 91 \\
02 / 21 / 92 \\
08 / 11 / 92 \\
11 / 24 / 92 \\
06 / 30 / 93\end{array}$ & $\begin{array}{l}0001 \\
0001 \\
0001 \\
0001 \\
0001 \\
0001 \\
0001 \\
0001 \\
0001\end{array}$ & MG/L & $\begin{array}{l}< \\
< \\
<\end{array}$ & $\begin{array}{l}0.017 \\
0.005 \\
0.005 \\
0.005 \\
0.024 \\
0.031 \\
0.02 \\
0.0021 \\
0.012\end{array}$ & J & $\begin{array}{l}0.005 \\
0.005 \\
0.005 \\
0.005 \\
0.005 \\
0.005 \\
0.02 \\
0.0010 \\
0.005\end{array}$ & $\begin{array}{l}- \\
- \\
- \\
\dot{-} \\
- \\
-\end{array}$ \\
\hline ZINC (TOTAL) & $\begin{array}{l}11 / 24 / 92 \\
06 / 30 / 93 \\
06 / 30 / 93\end{array}$ & $\begin{array}{l}\text { NOO1 } \\
\text { NOO1 } \\
\text { N002 }\end{array}$ & $M G / L$ & & $\begin{array}{l}0.0060 \\
0.051 \\
0.046\end{array}$ & $\begin{array}{l}\mathrm{J} \\
\mathrm{J}\end{array}$ & $\begin{array}{l}0.0010 \\
0.005 \\
0.005\end{array}$ & : \\
\hline
\end{tabular}

PARAMETER VALUE INDICATOR (PVI): < - LESS THAN DETECTIOH LIMIT

SAMPLE ID COOES:

OtHer PARAMETER VALUe flags:

J - ESTIMUTED VALUE

$L$ - LESS THAN THREE BORE VOLUMES REMOVED BEFORE SAMPLING

0001 - FILTERED SAMPLE (.45 MICRONS)

OOO2 - FILTERED REPLICATE SAMPLE (.45 MICROHS) NOO1 - UHFILTERED SAMPLE

MOO2 - UNFILTERED REPLICATE SAMPLE 
GROUMDHATER OUALITY DATA BY LOCATION

SOUP Rev. O Data

SITE: MONO 1 MONUMENT VALLEY

LOCATION: O619

MORTH COORDINATE: 57811.0 FT

EAST COORDINATE: 89017.5 FT

$04 / 21 / 88$ TO $12 / 13 / 94$

REPORT DATE: 06/20/95

FORMATION OF COMPLETIOH: OECHELLEY MEMBER OF THE CUTLER FORHATION (DC) HYDRAULIC FLON RELATIOHSHIP: UNKHOWN (N)

\begin{tabular}{|c|c|c|c|c|c|c|c|}
\hline PARAHETER MAME & LOG DATE & $\underset{\text { SD }}{\text { SAMPLE }}$ & $\begin{array}{l}\text { UNITS OF } \\
\text { MEASURE }\end{array}$ & PVI & $\begin{array}{l}\text { PARAMETER } \\
\text { VALUE FLAGS }\end{array}$ & $\begin{array}{l}\text { DETECTION } \\
\text { LIHIT }\end{array}$ & $\begin{array}{l}\text { PARAMETER } \\
\text { UNCERTAINTY }\end{array}$ \\
\hline ALKALIHITY & $\begin{array}{l}06 / 09 / 89 \\
06 / 29 / 93\end{array}$ & $\begin{array}{l}0009 \\
N 001\end{array}$ & MG/L CACO3 & & $\begin{array}{l}207 . \\
195\end{array}$ & : & - \\
\hline ALUHINUM & $06 / 09 / 89$ & 0001 & MG/L & $<$ & 0.1 & 0.1 & - \\
\hline Amonsur & $06 / 09 / 89$ & 0001 & MG/L & $<$ & 0.1 & 0.1 & - \\
\hline ANTIIMONY & $06 / 09 / 89$ & 0001 & $M G / L$ & $<$ & 0.003 & 0.003 & - \\
\hline ARSEHIC & $\begin{array}{l}06 / 09 / 89 \\
06 / 29 / 93\end{array}$ & $\begin{array}{l}0001 \\
0001\end{array}$ & MG/L & $<$ & $\begin{array}{l}0.01 \\
0.005\end{array}$ & $\begin{array}{l}0.01 \\
0.005\end{array}$ & - \\
\hline ARSENIC (TOTAL) & $06 / 29 / 93$ & NoO1 & $M G / L$ & $<$ & $0.005 \quad U$ & 0.005 & - \\
\hline BARIU: (TOTAL) & $06 / 29 / 93$ & NOOI & $M G / L$ & $<$ & 0.1 & 0.1 & - \\
\hline BERYLLIUM & $06 / 09 / 89$ & 0001 & $M G / L$ & $<$ & 0.01 & 0.01 & - \\
\hline BOROH & $06 / 09 / 89$ & 0001 & $M G / L$ & $<$ & 0.9 & 0.1 & - \\
\hline CADMIU: & $06 / 09 / 89$ & 0001 & MG/L & $<$ & 0.001 & 0.001 & - \\
\hline CALCIUM & $\begin{array}{l}06 / 09 / 89 \\
06 / 29 / 93\end{array}$ & $\begin{array}{l}0001 \\
0001\end{array}$ & MG/L & & $\begin{array}{l}16.8 \\
15.9\end{array}$ & $\begin{array}{l}0.01 \\
0.5\end{array}$ & - \\
\hline CALCIUH (TOTAL) & $06 / 29 / 93$ & HODI & MG/L & & 14.5 & 0.5 & - \\
\hline CHLORIDE & $06 / 09 / 89$ & 0001 & $M G / L$ & & 7.4 & 1. & - \\
\hline CYAKIDE & $06 / 09 / 89$ & 0001 & $M G / L$ & $<$ & 0.01 & 0.01 & - \\
\hline DISSOLVED OXYGEH & $06 / 29 / 93$ & M001 & $M G / L$ & & 0.3 & - & - \\
\hline FLUORIDE & $06 / 09 / 89$ & 0001 & $M G / L$ & & 0.3 & 0.1 & - \\
\hline GROSS ALPHA & $06 / 29 / 93$ & 0001 & $\mathrm{PCI} / \mathrm{L}$ & & 6 & 3 & 5 \\
\hline GROSS ALPHA (TOTAL) & $06 / 29 / 93$ & NOO1 & $\mathrm{PCI} / \mathrm{L}$ & & 13 & 3 & 6 \\
\hline GROSS BETA & $06 / 29 / 93$ & 0001 & $\mathrm{PCI} / \mathrm{L}$ & & 7 & 4 & 4 \\
\hline GROSS BETA (TOTAL) & $06 / 29 / 93$ & N001 & $\mathrm{PCI} / \mathrm{L}$ & & 4 & 4 & 4 \\
\hline IRON & $06 / 09 / 89$ & 0001 & MG/L & & 0.04 & 0.03 & - \\
\hline
\end{tabular}

PARAMETER VALUE IHDICATOR (PVI): \& - LESS THAN DETECTION LIMIT

SAMPLE ID COOES:

OOO1 - FILTERED SAMPLE (.45 MICRONS)

OTHER PARAMETER VALUE FLAGS:

$W$ - POST-DIGEST SPIKE OUT OF CHTR LIH WHILE SAMP ABS < 50\% SPIKE

NOOI - UNFILTERED SAKPLE 
GROUHDHATER QUALITY DATA BY LOCATION

SOMP ReV. 0 Data

SITE: MONO1 MONUMENT VALLEY

LOCATION: 0611

HORTH COORDINATE: $\quad 57811.0$ FT

EAST COORDINATE: 89017.5 FT

$04 / 21 / 88$ TO $12 / 13 / 94$

REPORT DATE: 06/20/95

FORMATION OF CONPLETION: DECHELLEY MEMBER OF THE CUTLER FORMATION (DC) HYDRAULIC FLON RELATIONSHIP: UNKMONN (N)

\begin{tabular}{|c|c|c|c|c|c|c|c|}
\hline PARAMETER MAME & LOG DATE & $\mid \begin{array}{c}\text { SAMPLE } \\
\text { ID }\end{array}$ & $\begin{array}{l}\text { UHITS OF } \\
\text { MEASURE }\end{array}$ & PVI & $\begin{array}{l}\text { PARAMETER } \\
\text { VALUE FLAGS }\end{array}$ & $\begin{array}{l}\text { DETECTIOH } \\
\text { LIMIT }\end{array}$ & $\begin{array}{l}\text { PARAMETER } \\
\text { UHCERTAINTY }\end{array}$ \\
\hline IRON & $06 / 29 / 93$ & 0001 & MG/L & $<$ & 0.03 & 0.03 & - \\
\hline IRON (TOTAL) & $06 / 29 / 93$ & No01 & MG/L & & $0.08 \mathrm{~J}$ & 0.03 & - \\
\hline LEAD & $06 / 09 / 89$ & 0001 & MG/L & $<$ & 0.01 & 0.01 & - \\
\hline MAGMESIUM & $\begin{array}{l}06 / 09 / 89 \\
06 / 29 / 93\end{array}$ & $\begin{array}{l}0001 \\
0001\end{array}$ & MG/L & & $\begin{array}{l}22.8 \\
21.5\end{array}$ & $\begin{array}{l}0.001 \\
0.1\end{array}$ & $\dot{-}$ \\
\hline MAGMESIU: (TOTAL) & $06 / 29 / 93$ & HoO1 & MG/L & & 20.1 & 0.1 & - \\
\hline MANGANESE & $\begin{array}{l}06 / 09 / 89 \\
06 / 29 / 93\end{array}$ & $\begin{array}{l}0001 \\
0001\end{array}$ & MG/L & $<$ & $\begin{array}{l}0.01 \\
0.01\end{array}$ & $\begin{array}{l}0.01 \\
0.01\end{array}$ & : \\
\hline MANGAKESE (TOTAL) & $06 / 29 / 93$ & HOOI & MG/L & $<$ & 0.01 & 0.01 & - \\
\hline MERCURY & $06 / 09 / 89$ & 0001 & MG/L & $<$ & 0.0002 & 0.0002 & - \\
\hline MOLYBDEKUY & $\begin{array}{l}06 / 09 / 89 \\
06 / 29 / 93\end{array}$ & $\begin{array}{l}0001 \\
0001\end{array}$ & $M G / L$ & $\begin{array}{l}< \\
<\end{array}$ & $\begin{array}{l}0.01 \\
0.01\end{array}$ & $\begin{array}{l}0.01 \\
0.01\end{array}$ & : \\
\hline MOLYBDENUM (TOTAL) & $06 / 29 / 93$ & N001 & $M G / L$ & $<$ & 0.01 & 0.01 & - \\
\hline MET GROSS ALPHA * & $06 / 29 / 93$ & 0001 & $P C I / L$ & & 0.51 & - & $\cdot$ \\
\hline NET GROSS ALPHA (TOTAL) ** & $06 / 29 / 93$ & NOOI & $\mathrm{PCI} / \mathrm{L}$ & & 8.20 & - & - \\
\hline HICKEL & $06 / 09 / 89$ & 0001 & $M G / L$ & $<$ & 0.04 & 0.04 & - \\
\hline NITRATE & $06 / 09 / 89$ & 0001 & MG/L & & 5.0 & 1. & - \\
\hline MITRATE (TOTAL) & $06 / 29 / 93$ & N001 & $M G / L$ & & 1 & 1 & - \\
\hline PH & $\begin{array}{l}06 / 09 / 89 \\
06 / 29 / 93\end{array}$ & $\begin{array}{l}0001 \\
\text { N001 }\end{array}$ & SU & & $\begin{array}{l}7.76 \\
7.93\end{array}$ & - & - \\
\hline PHOSPHATE & $06 / 09 / 89$ & 0001 & MG/L & & 1.0 & 0.1 & - \\
\hline PHOSPHATE (TOTAL) & $06 / 29 / 93$ & No01 & MG/L & & 0.6 & 0.1 & - \\
\hline Potassivi & $\begin{array}{l}06 / 09 / 89 \\
06 / 29 / 93\end{array}$ & $\begin{array}{l}0001 \\
0001\end{array}$ & MG/L & & $\begin{array}{l}2.8 \\
2.8\end{array}$ & $\begin{array}{l}0.01 \\
0.1\end{array}$ & 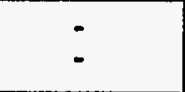 \\
\hline POTASSIL: (TOTAL) & $06 / 29 / 93$ & N001 & $M G / L$ & & 2.1 & 0.1 & - \\
\hline RADIUH-226 & $06 / 09 / 89$ & 0001 & $\mathrm{PCI} / \mathrm{L}$ & & 2.0 & 1. & 0.5 \\
\hline RADIUH-226 + RADIUN-228 & $06 / 09 / 89$ & 0001 & PCI/L & & 2.30 & - & - \\
\hline RADIUN-228 & $06 / 09 / 89$ & 0001 & $\mathrm{PCl} / \mathrm{L}$ & & 0.3 & 1. & 1.0 \\
\hline REDOX POTENTIAL & $06 / 29 / 93$ & No01 & nIVOLTS & & 446 & - & - \\
\hline
\end{tabular}

- NET gROSS ALPHA (GROSS ALPHA - URANILN) WITH I MG URANIUM = 686 PCI

* NET gROSS ALPHA (TOTAL) (TOTAL gROSS ALPHA - TOTAL URANIUM)

PARAMETER VALUE IRDICATOR (PVI): < - LESS THAN DETECTION LIMIT

SAMPLE ID COOES:

OTHER PARAMETER VALUE FLAGS:

0001 - FILTERED SMMPLE (.45 MICRONS)

$J$ - ESTIMTIED VALUE

MOO1 - LNFILTERED SAMPLE 
GROUNDWATER QUALITY DATA BY LOCATION

SOMP ReV. O Data

SITE: MONO1 MONUMENT VALLEY

LOCATIOH: 0611

NORTH COORDIMATE: 57811.0 FT

EAST COORDIKATE: 89017.5 FT

$04 / 21 / 88$ TO $12 / 13 / 94$

REPORT DATE: 06/20/95

FORMATION OF COAPLETION: DECHELLEY MEMBER OF THE CUTLER FORMATION (DC) HYDRAULIC FLON RELATIOHSHIP: UNKHOWN (N)

\begin{tabular}{|c|c|c|c|c|c|c|c|}
\hline PARAMETER NAME & LOG DATE & $\underset{\text { ID }}{\text { SAMPLE }}$ & $\begin{array}{l}\text { UNITS OF } \\
\text { MEASURE }\end{array}$ & PVI & $\begin{array}{l}\text { PARAMETER } \\
\text { VALUE FLAGS }\end{array}$ & $\begin{array}{l}\text { DETECTION } \\
\text { LIMIT }\end{array}$ & $\begin{array}{l}\text { PARANETER } \\
\text { UNCERTAINTY }\end{array}$ \\
\hline SELENIUH & $06 / 09 / 89$ & 0001 & MG/L & $<$ & 0.005 & 0.005 & - \\
\hline SILICA - SIO2 & $06 / 09 / 89$ & 0001 & MG/L & & 12. & 2. & - \\
\hline SILICA - SIO2 (TOTAL) & $06 / 29 / 93$ & HOO1 & MG/L & & 11.2 & 0.5 & - \\
\hline SILVER & $06 / 09 / 89$ & 0001 & $M G / L$ & $<$ & 0.01 & 0.01 & - \\
\hline Soorlum & $\begin{array}{l}06 / 09 / 89 \\
06 / 29 / 93\end{array}$ & $\begin{array}{l}0009 \\
0001\end{array}$ & MG/L & & $\begin{array}{l}76.3 \\
74\end{array}$ & 0.002 & - \\
\hline SODIUM (TOTAL) & $06 / 29 / 93$ & N001 & $\mathrm{MG} / \mathrm{L}$ & & 72 & 1 & - \\
\hline SPECIFIC CONDUCTAKCE & $\begin{array}{l}06 / 09 / 89 \\
06 / 29 / 93\end{array}$ & $\begin{array}{l}0001 \\
\text { N001 }\end{array}$ & UHHO/CM & & $\begin{array}{l}379 . \\
470\end{array}$ & - & $\because$ \\
\hline STRONTILA & $06 / 29 / 93$ & 0001 & MG/L & & 0.35 & 0.01 & - \\
\hline STRONTIUM (TOTAL) & $06 / 29 / 93$ & No01 & $M G / L$ & & 0.32 & 0.01 & - \\
\hline SULFATE & $06 / 09 / 89$ & 0001 & MG/L & & 77. & 0.1 & - \\
\hline SULFATE (TOTAL) & $06 / 29 / 93$ & N001 & MG/L & & 73 & 1 & - \\
\hline SULFIDE & $06 / 09 / 89$ & 0001 & MG/L & $<$ & 0.1 & 0.1 & - \\
\hline TEHPERATURE & $\begin{array}{l}06 / 09 / 89 \\
06 / 29 / 93\end{array}$ & $\begin{array}{l}0001 \\
\text { N001 }\end{array}$ & C - DEGREE & & $\begin{array}{l}17.0 \\
18.0\end{array}$ & - & - \\
\hline THALLIUN & $06 / 09 / 89$ & 0009 & MG/L & $<$ & 0.01 & 0.01 & - \\
\hline TIH & $06 / 09 / 89$ & 0001 & $M G / L$ & $<$ & 0.005 & 0.005 & - \\
\hline TOTAL DISSOLVED SOLIDS & $06 / 09 / 89$ & 0001 & $M G / L$ & & 313. & 90. & - \\
\hline TOTAL DISSOLVED SOLIDS (TOTAL) & $06 / 29 / 93$ & No01. & MG/L & & 310 & 10 & - \\
\hline URAKIUN & $\begin{array}{l}06 / 09 / 89 \\
06 / 29 / 93\end{array}$ & $\begin{array}{l}0001 \\
0001\end{array}$ & $M G / L$ & & $\begin{array}{l}0.0099 \\
0.008\end{array}$ & $\begin{array}{l}0.003 \\
0.001\end{array}$ & - \\
\hline URAKIUA (TOTAL) & $06 / 29 / 93$ & N001 & MG/L & & 0.007 & 0.001 & - \\
\hline VAKADIUA & $\begin{array}{l}06 / 09 / 89 \\
06 / 29 / 93\end{array}$ & $\begin{array}{l}0001 \\
0001\end{array}$ & MG/L & $<$ & $\begin{array}{l}0.01 \\
0.01\end{array}$ & $\begin{array}{l}0.01 \\
0.01\end{array}$ & - \\
\hline VAKADILM (TOTAL) & $06 / 29 / 93$ & N001 & $M G / L$ & $<$ & 0.01 & 0.01 & - \\
\hline ZINC & $\begin{array}{l}06 / 09 / 89 \\
06 / 29 / 93\end{array}$ & $\begin{array}{l}0001 \\
0001\end{array}$ & MG/L & $<$ & $\begin{array}{l}0.005 \\
0.085\end{array}$ & $\begin{array}{l}0.005 \\
0.005\end{array}$ & - \\
\hline ZIHC (TOTAL) & $06 / 29 / 93$ & NOO1 & MG/L & & 0.101 & 0.005 & - \\
\hline
\end{tabular}

PARAMETER VALUE IHDICATOR (PVI): < - LESS THAH DETECTION LIMIT

SAMPLE ID COOES:

OTHER PARAMETER VALUE FLAGS:

H - HOLD TIME EXPIRED, VALUE SUSPECT

OOOI - FILTERED SAMPLE (.45 MICROHS)

$J$ - Estimated VALUE

NOO1 - UHFILTERED SAMPLE 
GROUNDHATER QUALITY DATA BY LOCATIOH

SOUP ReV. O Data

SITE: MONO MONLHENT VALLEY

LOCATION: 0612

MORTH COORDINATE: $\quad 58437.2$ FT

EAST COORDINATE: 85614.8 FT

$04 / 21 / 88$ TO $12 / 13 / 94$

REPORT DATE: $06 / 20 / 95$

FORMATION OF COMPLETION: DECHELLEY MEMBER OF THE CUTLER FORMATION (DC)

HYDRAULIC FLOW RELATIONSHIP: UNKMONN (H)

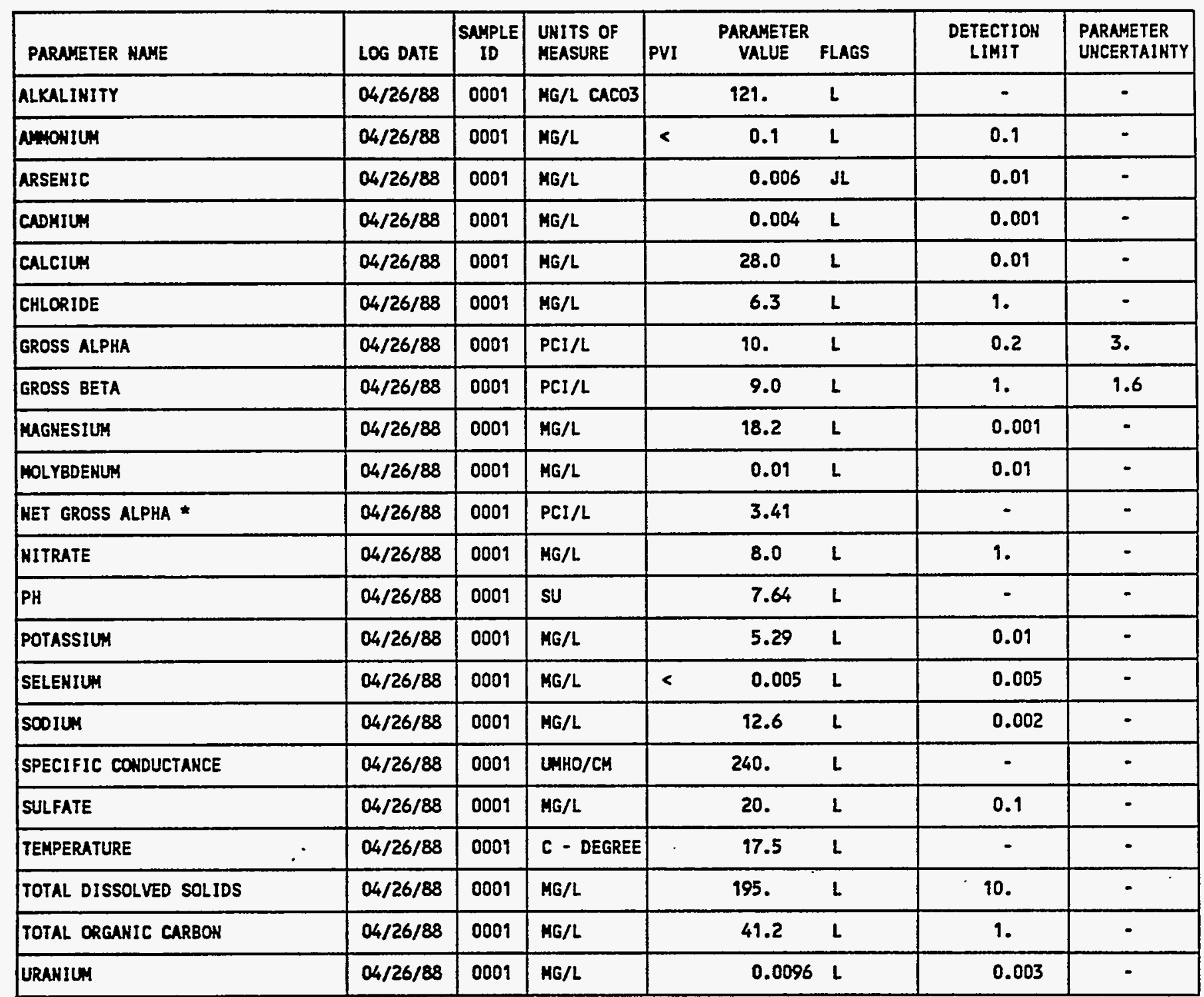

- MET GROSS ALPHA (GROSS ALPHA - URAMIUM) HITH I MG URAMIUM = 686 PCI

PARAYETER VALUE IMDICATOR (PVI): < - LESS THAN DETECTION LIMIT

OTHER PARAMETER VALUE FLAGS:

J - estimated value

L - LESS THAH THREE BORE VOLLHES REMOVED BEFORE SAMPLIHG 
GROUHDWATER QUALITY DATA BY LOCATION

SONP REV. O Data

SITE: MONO1 MOHUHENT VALLEY

LOCATION: 0613

NORTH COORDIKATE: 56377.8 FT

EAST COORDIHATE: 88643.7 FT

$04 / 21 / 88$ TO $12 / 13 / 94$

REPORT DATE: $06 / 20 / 95$

FORMATION OF COMPLETION: DECHELLEY MEMBER OF THE CUTLER FORMATION (DC) HYDRAULIC FLOW RELATIONSHIP: UNKHOWN (N)

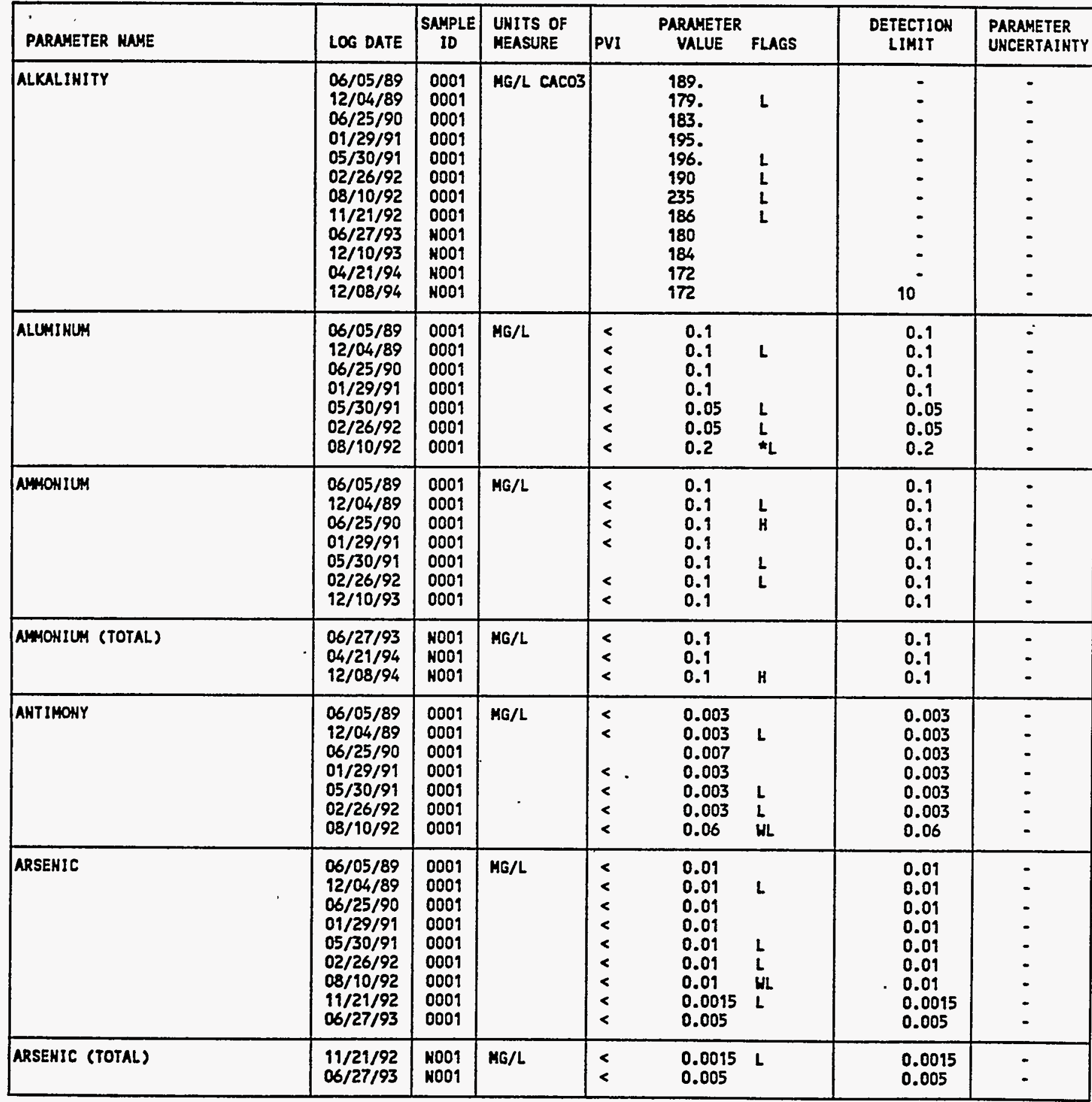

PARAMETER VALUE IMOICATOR (PVI): < - LESS THAH DETECTION LIMIT

\section{OTHER PARAMETER VALUE FLAGS:}

- - DUPLICATE AHALYSIS MOT HITHIN CONTROL LIHITS

H - HOLD TIME EXPIRED, VALUE SUSPECT

L - LESS THAM THREE BORE VOLLMES REMOVED BEFORE SMMPLING

- $W$ - POST-DIGEST SPIKE OUT OF CHTR LIM MHILE SAMP ABS $<50 \%$ SPIKE
SAMPLE ID COOES:

0001 - FILTERED SAMPLE (.45 MICRONS)

NDO - UHFILTERED SAMPLE 
GROUNDWATER QUALITY DATA BY LOCATION

SOWP ReV. O Data

SITE: MONO1 MONUMENT VALLEY

LOCATIOH: 0613

MORTH COORDINATE: 56377.8 FT

EAST COORDINATE: 88643.7 FT

$04 / 21 / 88$ TO $12 / 13 / 94$

REPORT DATE: 06/20/95

FORMATION OF COMPLETION: DECHELLEY MEMBER OF THE CUTLER FORMATION (DC) HYDRAULIC FLON RELATIONSHIP: UNKHOWN (N)

\begin{tabular}{|c|c|c|c|c|c|c|c|c|}
\hline PARNYETER MAME & LOG DATE & $\begin{array}{c}\text { SNMPLE } \\
10\end{array}$ & $\begin{array}{l}\text { UHITS OF } \\
\text { MEASURE }\end{array}$ & PVI & $\begin{array}{l}\text { PARANAETER } \\
\text { VALUE }\end{array}$ & FLAGS & $\begin{array}{l}\text { DETECTIOH } \\
\text { LIMIT }\end{array}$ & $\begin{array}{l}\text { PARAMETER } \\
\text { UNCERTAIHTY }\end{array}$ \\
\hline BARIUA & $\begin{array}{l}06 / 05 / 89 \\
12 / 04 / 89 \\
06 / 25 / 90 \\
01 / 29 / 91 \\
05 / 30 / 91 \\
02 / 26 / 92 \\
08 / 10 / 92 \\
11 / 21 / 92 \\
06 / 27 / 93\end{array}$ & $\begin{array}{l}0001 \\
0001 \\
0001 \\
0001 \\
0001 \\
0001 \\
0001 \\
0001 \\
0001\end{array}$ & $M G / L$ & $\begin{array}{l}< \\
< \\
< \\
< \\
< \\
<\end{array}$ & $\begin{array}{l}0.1 \\
0.1 \\
0.1 \\
0.1 \\
0.04 \\
0.04 \\
0.2 \\
0.036 \\
0.1\end{array}$ & $\begin{array}{l}L \\
L \\
L \\
L \\
L\end{array}$ & $\begin{array}{l}0.1 \\
0.1 \\
0.1 \\
0.1 \\
0.09 \\
0.01 \\
0.2 \\
0.0015 \\
0.1\end{array}$ & $\begin{array}{l}- \\
- \\
- \\
- \\
- \\
-\end{array}$ \\
\hline BARIUA (TOTAL) & $\begin{array}{l}11 / 21 / 92 \\
06 / 27 / 93\end{array}$ & $\begin{array}{l}\text { NOO1 } \\
\text { NOO1 }\end{array}$ & $M G / L$ & $<$ & $\begin{array}{l}0.038 \\
0.1\end{array}$ & 2 & $\begin{array}{l}0.0015 \\
0.1\end{array}$ & $\dot{-}$ \\
\hline BERYLLIUH & $\begin{array}{l}06 / 05 / 89 \\
12 / 04 / 89 \\
06 / 25 / 90 \\
01 / 29 / 91 \\
05 / 30 / 91 \\
08 / 10 / 92\end{array}$ & $\begin{array}{l}0001 \\
0001 \\
0001 \\
0001 \\
0001 \\
0001\end{array}$ & $M G / L$ & $\begin{array}{l}< \\
< \\
< \\
< \\
<\end{array}$ & $\begin{array}{l}0.01 \\
0.005 \\
0.01 \\
0.01 \\
0.005 \\
0.005\end{array}$ & $\begin{array}{l}L \\
L \\
L\end{array}$ & $\begin{array}{l}0.01 \\
0.005 \\
0.01 \\
0.09 \\
0.005 \\
0.005\end{array}$ & $\begin{array}{l}- \\
- \\
- \\
-\end{array}$ \\
\hline BORON & $\begin{array}{l}06 / 05 / 89 \\
12 / 04 / 89 \\
06 / 25 / 90 \\
01 / 29 / 91 \\
05 / 30 / 91\end{array}$ & $\begin{array}{l}0001 \\
0001 \\
0001 \\
0001 \\
0001\end{array}$ & $M G / L$ & $\begin{array}{l}< \\
< \\
< \\
<\end{array}$ & $\begin{array}{l}0.1 \\
0.1 \\
0.1 \\
0.1 \\
0.05\end{array}$ & $\begin{array}{l}\mathbf{L} \\
\mathbf{L}\end{array}$ & $\begin{array}{l}0.1 \\
0.1 \\
0.1 \\
0.1 \\
0.05\end{array}$ & $\begin{array}{l}- \\
- \\
- \\
-\end{array}$ \\
\hline BROMIOE & $\begin{array}{l}01 / 29 / 91 \\
05 / 30 / 91\end{array}$ & $\begin{array}{l}0001 \\
0001\end{array}$ & MG/L & $<$ & $\begin{array}{l}0.1 \\
0.1\end{array}$ & $L$ & $\begin{array}{l}0.1 \\
0.1\end{array}$ & - \\
\hline CADHIUM & $\begin{array}{l}06 / 05 / 89 \\
12 / 04 / 89 \\
06 / 25 / 90 \\
01 / 29 / 91 \\
05 / 30 / 91 \\
02 / 26 / 92 \\
08 / 10 / 92 \\
11 / 21 / 92\end{array}$ & $\begin{array}{l}0001 \\
0001 \\
0001 \\
0001 \\
0001 \\
0001 \\
0001 \\
0001\end{array}$ & MG/L & $\begin{array}{l}< \\
< \\
< \\
< \\
< \\
< \\
<\end{array}$ & $\begin{array}{l}0.001 \\
0.001 \\
0.001 \\
0.001 \\
0.001 \\
0.001 \\
0.005 \\
0.00013\end{array}$ & $\begin{array}{l}L \\
L \\
L L \\
L\end{array}$ & $\begin{array}{l}0.001 \\
0.001 \\
0.001 \\
0.001 \\
0.001 \\
0.001 \\
0.005 \\
0.00013\end{array}$ & $\begin{array}{l}: \\
: \\
: \\
-\end{array}$ \\
\hline CADMIUM (TOTAL) & $11 / 21 / 92$ & NOOI & $M G / L$ & $<$ & 0.00013 & $\mathrm{NL}$ & 0.00013 & $\therefore$ \\
\hline CALCIUn & $\begin{array}{l}06 / 05 / 89 \\
12 / 04 / 89 \\
06 / 25 / 90 \\
01 / 29 / 91 \\
05 / 30 / 91 \\
02 / 26 / 92 \\
08 / 10 / 92 \\
06 / 27 / 93 \\
12 / 10 / 93 \\
04 / 21 / 94 \\
12 / 08 / 94\end{array}$ & $\begin{array}{l}0001 \\
0001 \\
0001 \\
0001 \\
0001 \\
0001 \\
0001 \\
0001 \\
0001 \\
0001 \\
0001\end{array}$ & MG/L & & $\begin{array}{l}17.3 \\
16.9 \\
16.3 \\
16.1 \\
15.9 \\
15.7 \\
16 \\
15.7 \\
14.6 \\
15.2 \\
16.4\end{array}$ & $\begin{array}{l}L \\
L \\
L \\
L\end{array}$ & $\begin{array}{l}0.01 \\
0.01 \\
0.01 \\
0.01 \\
0.1 \\
0.1 \\
5 \\
0.5 \\
0.5 \\
0.5 \\
0.5\end{array}$ & $\begin{array}{l}- \\
: \\
: \\
: \\
: \\
- \\
-\end{array}$ \\
\hline CALCIUH (TOTAL) & $\begin{array}{l}06 / 27 / 93 \\
12 / 10 / 93\end{array}$ & $\begin{array}{l}\text { No01 } \\
\text { H001 }\end{array}$ & MG/L & & $\begin{array}{l}15.2 \\
15.3\end{array}$ & & $\begin{array}{l}0.5 \\
0.5\end{array}$ & 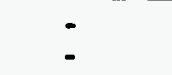 \\
\hline
\end{tabular}

PARAMETER VALUE INDICATOR (PVI): < - LESS THAN DETECTION LIMIT

SAMPLE ID COOES:

OTHER PARAMETER VALUE FLAGS:

0001 - FILTERED SAMPLE (.45 MICRONS)

NOOI - UNFILTERED SAMPLE

E - estIMATED VALUE BECAUSE OF INTERFERENCE, SEe CASE haRRATIVE

L - LESS THAN THREE BORE VOLLMES REMOVED BEFORE SAMPLING

N - SPIKE SAMPLE RECOVERY NOT HITHIN CONTROL LIMITS 
GROUHDWATER QUALITY DATA BY LOCATIOH

SOUP Rev. O Data

SITE: MOHOI MONUNENT VALLEY

LOCATION: 0613

NORTH COORDIKATE: 56377.8 FT

EAST COORDINATE: 88643.7 FT

04/21/88 TO 12/13/94

REPORT DATE: 06/20/95

FORMATIOH OF COMPLETION: DECHELLEY MEMBER OF THE CUTLER FORHATION (DC) HYDRAULIC FLOW RELATIOHSHIP: UHKHOWN (N)

\begin{tabular}{|c|c|c|c|c|c|c|c|c|}
\hline PARNMETER NAME & LOG DATE & $\mid \begin{array}{c}\text { SAMPLE } \\
\text { ID }\end{array}$ & $\begin{array}{l}\text { UNITS OF } \\
\text { MEASURE }\end{array}$ & PVI & $\begin{array}{l}\text { PARAMETER } \\
\text { VALUE }\end{array}$ & FLAGS & $\begin{array}{l}\text { DETECTION } \\
\text { LIMIT }\end{array}$ & $\begin{array}{l}\text { PARAMETER } \\
\text { UNCERTAINTY }\end{array}$ \\
\hline CALCIUA (TOTAL) & $\begin{array}{l}04 / 21 / 94 \\
12 / 08 / 94\end{array}$ & $\begin{array}{l}\text { NOOI } \\
\text { NOOI }\end{array}$ & $M G / L$ & & $\begin{array}{l}15.2 \\
95.8\end{array}$ & & $\begin{array}{l}0.5 \\
0.5\end{array}$ & $\dot{-}$ \\
\hline CHLORIDE & $\begin{array}{l}06 / 05 / 89 \\
12 / 04 / 89 \\
06 / 25 / 90 \\
01 / 29 / 91 \\
05 / 30 / 91 \\
02 / 26 / 92 \\
08 / 10 / 92 \\
12 / 10 / 93 \\
04 / 21 / 94\end{array}$ & $\begin{array}{l}0001 \\
0001 \\
0001 \\
0001 \\
0001 \\
0001 \\
0001 \\
0001 \\
0001\end{array}$ & $M G / L$ & & $\begin{array}{l}7.4 \\
8.7 \\
8 . \\
4 . \\
8.2 \\
9.3 \\
8.0 \\
34.7 \\
7.2\end{array}$ & $\begin{array}{l}L \\
J \\
L \\
L \\
L\end{array}$ & $\begin{array}{l}1 . \\
1 . \\
1 . \\
1 . \\
0.5 \\
0.5 \\
0.016 \\
0.5 \\
0.5\end{array}$ & $\begin{array}{l}- \\
- \\
- \\
- \\
- \\
-\end{array}$ \\
\hline CHLORIDE (TOTAL) & $\begin{array}{l}06 / 27 / 93 \\
12 / 08 / 94\end{array}$ & $\begin{array}{l}\text { NOO1 } \\
\text { H001 }\end{array}$ & $M G / L$ & & $\begin{array}{l}8.0 \\
5\end{array}$ & & $\begin{array}{l}0.5 \\
0.5\end{array}$ & - \\
\hline CHROMIUM & $\begin{array}{l}06 / 05 / 89 \\
12 / 04 / 89 \\
06 / 25 / 90 \\
01 / 29 / 91 \\
05 / 30 / 91 \\
02 / 26 / 92 \\
08 / 10 / 92 \\
11 / 21 / 92\end{array}$ & $\begin{array}{l}0001 \\
0001 \\
0001 \\
0001 \\
0001 \\
0001 \\
0001 \\
0001\end{array}$ & $M G / L$ & $\begin{array}{l}< \\
< \\
< \\
< \\
< \\
< \\
<\end{array}$ & $\begin{array}{l}0.01 \\
0.01 \\
0.01 \\
0.01 \\
0.01 \\
0.09 \\
0.1 \\
0.0053\end{array}$ & $\begin{array}{l}L \\
L \\
L \\
L \\
L\end{array}$ & $\begin{array}{l}0.01 \\
0.01 \\
0.01 \\
0.01 \\
0.01 \\
0.01 \\
0.01 \\
0.0053\end{array}$ & $\begin{array}{l}- \\
- \\
- \\
- \\
-\end{array}$ \\
\hline CHROMIUM (TOTAL) & $11 / 21 / 92$ & K001 & $M G / L$ & $<$ & 0.0053 & $L$ & 0.0053 & - \\
\hline COBALT & $\begin{array}{l}06 / 05 / 89 \\
12 / 04 / 89 \\
06 / 25 / 90 \\
01 / 29 / 91 \\
05 / 30 / 91\end{array}$ & $\begin{array}{l}0001 \\
0001 \\
0001 \\
0001 \\
0001\end{array}$ & $M G / L$ & $\begin{array}{l}< \\
< \\
< \\
< \\
<\end{array}$ & $\begin{array}{l}0.05 \\
0.05 \\
0.05 \\
0.05 \\
0.03\end{array}$ & $\begin{array}{l}\mathbf{L} \\
\mathbf{L}\end{array}$ & $\begin{array}{l}0.05 \\
0.05 \\
0.05 \\
0.05 \\
0.03\end{array}$ & $\begin{array}{l}- \\
- \\
- \\
-\end{array}$ \\
\hline COPPER & $\begin{array}{l}06 / 05 / 89 \\
12 / 04 / 89 \\
06 / 25 / 90 \\
01 / 29 / 91 \\
05 / 30 / 99\end{array}$ & $\begin{array}{l}0001 \\
0001 \\
0001 \\
0001 \\
0001\end{array}$ & $M G / L$ & $\begin{array}{l}< \\
< \\
< \\
< \\
<\end{array}$ & $\begin{array}{l}0.02 \\
0.02 \\
0.02 \\
0.02 \\
0.01\end{array}$ & $\begin{array}{l}L \\
L\end{array}$ & $\begin{array}{l}0.02 \\
0.02 \\
0.02 \\
0.02 \\
0.01\end{array}$ & $\begin{array}{l}- \\
- \\
- \\
-\end{array}$ \\
\hline CYANIDE & $\begin{array}{l}06 / 05 / 89 \\
12 / 04 / 89\end{array}$ & $\begin{array}{l}0001 \\
0001\end{array}$ & MG/L & $<$ & $\begin{array}{l}0.09 \\
0.09\end{array}$ & $\mathbf{L}$ & $\begin{array}{l}0.09 \\
0.09\end{array}$ & - \\
\hline DISSOLVED OXYGEK & $\begin{array}{l}06 / 27 / 93 \\
12 / 10 / 93 \\
04 / 21 / 94\end{array}$ & $\begin{array}{l}\text { No01 } \\
\text { N001 } \\
\text { N001 }\end{array}$ & HG/L & & $\begin{array}{l}3.2 \\
3.2 \\
3.4\end{array}$ & & $\dot{-}$ & - \\
\hline FLUORIDE & $\begin{array}{l}06 / 05 / 89 \\
12 / 04 / 89 \\
06 / 25 / 90 \\
01 / 29 / 91 \\
05 / 30 / 91\end{array}$ & $\begin{array}{l}0001 \\
0001 \\
0001 \\
0001 \\
0001\end{array}$ & $M G / L$ & & $\begin{array}{l}0.3 \\
0.4 \\
0.4 \\
0.3 \\
0.2\end{array}$ & $\begin{array}{l}L \\
L\end{array}$ & $\begin{array}{l}0.1 \\
0.1 \\
0.1 \\
0.1 \\
0.1\end{array}$ & $\begin{array}{l}- \\
\vdots \\
- \\
-\end{array}$ \\
\hline GROSS ALPHA & $\begin{array}{l}01 / 29 / 91 \\
05 / 30 / 91 \\
02 / 26 / 92\end{array}$ & $\begin{array}{l}0001 \\
0001 \\
0001\end{array}$ & PCI/L & & $\begin{array}{l}1.9 \\
0.0 \\
5.2\end{array}$ & $l$ & $\begin{array}{l}1 . \\
1.0\end{array}$ & $\begin{array}{l}2.3= \\
4.1 \\
4.3\end{array}$ \\
\hline
\end{tabular}

PARAMETER VALUE IHDICATOR (PVI): < - LESS THAN DETECTION LIMIT

l - LESS THAH THREE BORE VOLUMES REMOVED BEFORE SAMPLIHG 
GROUHDWATER QUALITY DATA BY LOCATIOH

SOWP REV. O Dato

SITE: HONO1 MOHUMENT VALLEY

LOCATION: 0613

NORTH COORDINATE: 56377.8 FT

EAST COORDINATE: 88643.7 FT

04/21/88 TO 12/13/94

REPORT DATE: 06/20/95

FORMATION OF COMPLETION: DECHELLEY MEMBER OF THE CUTLER FORMATION (DC) HYDRAULIC FLOH RELATIOHSHIP: UNKHONN (N)

\begin{tabular}{|c|c|c|c|c|c|c|c|c|}
\hline PARNMETER NAME & LOG DATE & $\begin{array}{c}\text { SAMPLE } \\
\text { I0 }\end{array}$ & $\begin{array}{l}\text { UNITS OF } \\
\text { MEASURE }\end{array}$ & PVI & $\begin{array}{l}\text { PARAMETER } \\
\text { VALUE }\end{array}$ & FLAGS & $\begin{array}{l}\text { DETECTION } \\
\text { LIMIT }\end{array}$ & $\begin{array}{l}\text { PARAMETER } \\
\text { UNCERTAINTY }\end{array}$ \\
\hline GROSS ALPHA & $\begin{array}{l}08 / 10 / 92 \\
11 / 21 / 92 \\
06 / 27 / 93\end{array}$ & $\begin{array}{l}0001 \\
0001 \\
0001\end{array}$ & PCI/L & & $\begin{array}{l}2.6 \\
7.4 \\
7\end{array}$ & IL & $\begin{array}{l}3.9 \\
3.0 \\
3\end{array}$ & $\begin{array}{l}2.7 \\
3.1 \\
5\end{array}$ \\
\hline GROSS ALPHA (TOTAL) & $\begin{array}{l}11 / 21 / 92 \\
06 / 27 / 93\end{array}$ & $\begin{array}{l}\text { N001 } \\
\text { N001 }\end{array}$ & PCI/L & & $\begin{array}{l}0.0 \\
4\end{array}$ & ML & $\begin{array}{l}6.6 \\
3\end{array}$ & $\begin{array}{l}3.6 \\
5\end{array}$ \\
\hline GROSS BETA & $\begin{array}{l}01 / 29 / 91 \\
05 / 30 / 91 \\
02 / 26 / 92 \\
08 / 10 / 92 \\
11 / 21 / 92 \\
06 / 27 / 93\end{array}$ & $\begin{array}{l}0001 \\
0001 \\
0001 \\
0001 \\
0001 \\
0001\end{array}$ & $\mathrm{PCI} / \mathrm{L}$ & & $\begin{array}{l}2.9 \\
1.5 \\
6.9 \\
5.7 \\
5.6 \\
5\end{array}$ & $\begin{array}{l}L \\
L \\
I L\end{array}$ & $\begin{array}{l}0.5 \\
0.5 \\
0.5 \\
4.2 \\
5.4 \\
4\end{array}$ & $\begin{array}{l}1.8 \\
2.7 \\
2.4 \\
2.8 \\
3.4 \\
4 .\end{array}$ \\
\hline GROSS BETA (TOTAL) & $\begin{array}{l}11 / 21 / 92 \\
06 / 27 / 93\end{array}$ & $\begin{array}{l}\text { HOO1 } \\
\text { HOO1 }\end{array}$ & $\mathrm{PCI} / \mathrm{L}$ & & $\begin{array}{l}0.0 \\
0\end{array}$ & HL & $\begin{array}{l}4.8 \\
4\end{array}$ & $\begin{array}{l}2.8 \\
4\end{array}$ \\
\hline IRON & $\begin{array}{l}06 / 05 / 89 \\
12 / 04 / 89 \\
06 / 25 / 90 \\
01 / 29 / 91 \\
05 / 30 / 91 \\
02 / 26 / 92 \\
08 / 10 / 92 \\
06 / 27 / 93\end{array}$ & $\begin{array}{l}0001 \\
0001 \\
0001 \\
0001 \\
0001 \\
0001 \\
0001 \\
0001\end{array}$ & MG/L & $\begin{array}{l}< \\
< \\
< \\
< \\
< \\
< \\
< \\
<\end{array}$ & $\begin{array}{l}0.03 \\
0.03 \\
0.03 \\
0.03 \\
0.03 \\
0.03 \\
0.1 \\
0.03\end{array}$ & $\begin{array}{l}L \\
L \\
L \\
L \\
J\end{array}$ & $\begin{array}{l}0.03 \\
0.03 \\
0.03 \\
0.03 \\
0.03 \\
0.03 \\
0.9 \\
0.03\end{array}$ & $\begin{array}{l}: \\
: \\
: \\
- \\
-\end{array}$ \\
\hline IRON (TOTAL) & $06 / 27 / 93$ & N001 & ME/L & & 0.04 & J & 0.03 & - \\
\hline LEAD & $\begin{array}{l}06 / 05 / 89 \\
12 / 04 / 89 \\
06 / 25 / 90 \\
01 / 29 / 99 \\
05 / 30 / 99 \\
11 / 21 / 92\end{array}$ & $\begin{array}{l}0001 \\
0001 \\
0001 \\
0001 \\
0001 \\
0001\end{array}$ & $M G / L$ & $\begin{array}{l}< \\
< \\
< \\
< \\
< \\
<\end{array}$ & $\begin{array}{l}0.01 \\
0.01 \\
0.01 \\
0.01 \\
0.005 \\
0.0015\end{array}$ & $\begin{array}{l}L \\
L \\
L\end{array}$ & $\begin{array}{l}0.01 \\
0.01 \\
0.01 \\
0.01 \\
0.005 \\
0.0015\end{array}$ & $\begin{array}{l}- \\
\dot{-} \\
\dot{-}\end{array}$ \\
\hline LEAD (TOTAL) & $11 / 21 / 92$ & NOOI & MG/L & $<$ & 0.0015 & $\mathbf{L}$ & 0.0015 & - \\
\hline LEAD-210 & $11 / 21 / 92$ & 0001 & $\mathrm{PCI} / \mathrm{L}$ & & 0.3 & $\mathbf{L}$ & 2.0 & 1.2 \\
\hline LEAD-210 (TOTAL) & $11 / 21 / 92$ & N001 & $\mathrm{PCI} / \mathrm{L}$ & & 5.8 & $\mathbf{L}$ & 0.3 & 0.9 \\
\hline MGNESIUM & $\begin{array}{l}06 / 05 / 89 \\
12 / 04 / 89 \\
06 / 25 / 90 \\
01 / 29 / 91 \\
05 / 30 / 91 \\
02 / 26 / 92 \\
08 / 10 / 92 \\
06 / 27 / 93 \\
12 / 10 / 93 \\
04 / 21 / 94 \\
12 / 08 / 94\end{array}$ & $\begin{array}{l}0001 \\
0001 \\
0001 \\
0001 \\
0001 \\
0001 \\
0001 \\
0001 \\
0001 \\
0001 \\
0001\end{array}$ & MG/L & & $\begin{array}{l}29.9 \\
30 . \\
29.5 \\
31.9 \\
28.8 \\
28.7 \\
28 \\
27.3 \\
26.5 \\
26.5 \\
29.2\end{array}$ & $\begin{array}{l}L \\
L \\
L \\
L\end{array}$ & $\begin{array}{l}0.001 \\
0.001 \\
0.001 \\
0.001 \\
0.1 \\
0.1 \\
5 \\
0.1 \\
0.1 \\
0.1 \\
0.1\end{array}$ & $\begin{array}{l}- \\
- \\
- \\
- \\
- \\
- \\
-\end{array}$ \\
\hline MUGKESIUM (TOTAL) & $06 / 27 / 93$ & NoO1 & MG/L & & 26.9 & & 0.1 & - \\
\hline
\end{tabular}

PARAMETER VALUE IMDICATOR (PVI): < - LESS THAN DETECTIOH LIMIT

OTHER PARAMETER VALUE FLAGS:

1 - IHCREASED DETECTION LIMIT OUE TO REOUIRED DILUTION

J - ESTIMATED VALUE

L - LESS THAN THREE BORE VOLUMES REMOVED BEFORE SAMPLING

N - SPIKE SNMPLE RECOVERY HOT HITHIN CONTROL LIMITS
SAMPLE ID COOES:

OOO1 - FILTERED SAMPLE (.45 MICRONS)

NOO1 - UAFILTERED SAMPLE 
LOCATION: 0613

MORTH COORDINATE: 56377.8 FT

EAST COORDINATE: 88643.7 FT

$04 / 21 / 88$ TO $12 / 13 / 94$

REPORT DATE: OS/20/95

FORHATION OF COMPLETION: DECHELLEY MEHBER OF THE CUTLER FORMATION (DC) HYDRAULIC FLOH RELATIONSHIP: LHKNOWH (H)

\begin{tabular}{|c|c|c|c|c|c|c|c|c|}
\hline PARAMETER NAME & LOG DATE & $\mid \begin{array}{c}\text { SAMPLE } \\
\text { ID }\end{array}$ & $\begin{array}{l}\text { UHITS OF } \\
\text { MEASURE }\end{array}$ & PVI & $\begin{array}{l}\text { PARAMETER } \\
\text { VALUE }\end{array}$ & FLAGS & $\begin{array}{l}\text { DETECTION } \\
\text { LIHIT }\end{array}$ & $\begin{array}{l}\text { PARAMETER } \\
\text { UNCERTAINTY }\end{array}$ \\
\hline MAGNESIUN (TOTAL) & $\begin{array}{l}12 / 10 / 93 \\
04 / 21 / 94 \\
12 / 08 / 94\end{array}$ & $\begin{array}{l}\text { No01 } \\
\text { Ho01 } \\
\text { No01 }\end{array}$ & MG/L & & $\begin{array}{l}26.4 \\
26.3 \\
27.1\end{array}$ & & $\begin{array}{l}0.1 \\
0.1 \\
0.1\end{array}$ & - \\
\hline MUKGAKESE & $\begin{array}{l}06 / 05 / 89 \\
12 / 04 / 89 \\
06 / 25 / 90 \\
01 / 29 / 91 \\
05 / 30 / 91 \\
02 / 26 / 92 \\
08 / 10 / 92 \\
11 / 21 / 92 \\
06 / 27 / 93 \\
12 / 10 / 93 \\
04 / 21 / 94 \\
12 / 08 / 94\end{array}$ & $\begin{array}{l}0001 \\
0001 \\
0001 \\
0001 \\
0001 \\
0001 \\
0001 \\
0001 \\
0001 \\
0001 \\
0001 \\
0001\end{array}$ & $M G / L$ & $\begin{array}{l}< \\
< \\
< \\
< \\
< \\
< \\
< \\
< \\
< \\
< \\
< \\
<\end{array}$ & $\begin{array}{l}0.01 \\
0.01 \\
0.01 \\
0.01 \\
0.01 \\
0.01 \\
0.01 \\
0.0015 \\
0.01 \\
0.01 \\
0.01 \\
0.01\end{array}$ & $\begin{array}{l}\mathbf{L} \\
\mathbf{L} \\
\mathbf{L} \\
\mathrm{L} \\
\mathrm{HL}\end{array}$ & $\begin{array}{l}0.01 \\
0.01 \\
0.01 \\
0.01 \\
0.01 \\
0.01 \\
0.01 \\
0.0015 \\
0.01 \\
0.01 \\
0.01 \\
0.01\end{array}$ & $\begin{array}{l}- \\
- \\
- \\
- \\
- \\
- \\
- \\
-\end{array}$ \\
\hline MANGAHESE (TOTAL) & $\begin{array}{l}11 / 21 / 92 \\
06 / 27 / 93 \\
12 / 90 / 93 \\
04 / 21 / 94 \\
12 / 08 / 94\end{array}$ & $\begin{array}{l}\text { NOO1 } \\
\text { NO01 } \\
\text { NO01 } \\
\text { NOO1 } \\
\text { NO01 }\end{array}$ & $M G / L$ & $\begin{array}{l}< \\
< \\
< \\
< \\
<\end{array}$ & $\begin{array}{l}0.0015 \\
0.01 \\
0.01 \\
0.01 \\
0.01\end{array}$ & $\mathbf{L}$ & $\begin{array}{l}0.0015 \\
0.01 \\
0.01 \\
0.01 \\
0.01\end{array}$ & $\begin{array}{l}- \\
- \\
-\end{array}$ \\
\hline HERCURY & $\begin{array}{l}06 / 05 / 89 \\
12 / 04 / 89 \\
06 / 25 / 90\end{array}$ & $\begin{array}{l}0001 \\
0001 \\
0001\end{array}$ & $M G / L$ & $<$ & $\begin{array}{l}0.0002 \\
0.0002 \\
0.0002\end{array}$ & $\mathbf{L}$ & $\begin{array}{l}0.0002 \\
0.0002 \\
0.0002\end{array}$ & - \\
\hline MOLYBDEHUM & $\begin{array}{l}06 / 05 / 89 \\
12 / 04 / 89 \\
06 / 25 / 90 \\
01 / 29 / 91 \\
05 / 30 / 91 \\
02 / 26 / 92 \\
08 / 10 / 92 \\
11 / 21 / 92 \\
06 / 27 / 93 \\
12 / 08 / 94\end{array}$ & $\begin{array}{l}0001 \\
0001 \\
0001 \\
0001 \\
0001 \\
0001 \\
0001 \\
0001 \\
0001 \\
0001 .\end{array}$ & MG/L & $\begin{array}{l}< \\
< \\
< \\
< \\
< \\
< \\
<\end{array}$ & $\begin{array}{l}0.01 \\
0.01 \\
0.01 \\
0.01 \\
0.01 \\
0.01 \\
0.007 \\
0.0086 \\
0.01 \\
0.01\end{array}$ & $\begin{array}{l}\mathbf{L} \\
\mathbf{L} \\
\mathbf{L} \\
\mathbf{L} \\
\mathrm{HL}\end{array}$ & $\begin{array}{l}0.01 \\
0.01 \\
0.01 \\
0.01 \\
0.01 \\
0.01 \\
0.007 \\
0.0049 \\
0.01 \\
0.01\end{array}$ & $\begin{array}{l}- \\
: \\
- \\
- \\
- \\
-\end{array}$ \\
\hline MOLYBDEKUM (TOTAL) & $\begin{array}{l}11 / 21 / 92 \\
06 / 27 / 93 \\
12 / 08 / 94\end{array}$ & $\begin{array}{l}\text { No01 } \\
\text { N001 } \\
\text { N001 }\end{array}$ & MG/L & $\begin{array}{l}< \\
<\end{array}$ & $\begin{array}{l}0.0070 \\
0.01 \\
0.01\end{array}$ & HL & $\begin{array}{l}0.0049 \\
0.01 \\
0.01\end{array}$ & $:$ \\
\hline HET GROSS ALPHA & $\begin{array}{l}01 / 29 / 91 \\
05 / 30 / 91 \\
02 / 26 / 92 \\
11 / 21 / 92 \\
06 / 27 / 93\end{array}$ & $\begin{array}{l}0001 \\
0001 \\
0001 \\
0001 \\
0001\end{array}$ & $\mathrm{PCI} / \mathrm{L}$ & & $\begin{array}{l}-0.64 \\
-2.74 \\
3.14 \\
4.66 \\
4.26\end{array}$ & & $\begin{array}{l}- \\
- \\
-\end{array}$ & $\begin{array}{l}- \\
- \\
-\end{array}$ \\
\hline HET GROSS ALPHA (TOTAL) ** & $\begin{array}{l}11 / 21 / 92 \\
06 / 27 / 93\end{array}$ & $\begin{array}{l}\text { No01 } \\
\text { N001 }\end{array}$ & PCI/L & & $\begin{array}{r}-2.06 \\
1.94\end{array}$ & & - & - \\
\hline MICKEL & $06 / 05 / 89$ & 0001 & MG/L & $<$ & 0.04 & & 0.04 & - \\
\hline
\end{tabular}

- met cRoss alPHa (GROSS ALPHA - URAHIUH) UITH 1 MG URAMIUH = 686 PCI

* MET GROSS ALPHA (TOTAL) (TOTAL GROSS ALPHA - TOTAL URAHIUA)

PARAMETER VALUE IMDICATOR (PVI): < - LESS THAN DETECTION LIMIT

SAMPLE ID COOES:

OTHER PARAMETER VALUE FLAGS:

DOO1 - FILTERED SNMLE (.45 MICRONS)

L - LESS THAN THREE BORE VOLUHES REMOVED BEFORE SAMPLING

NOOI - UAFILTERED SAMPLE

H - SPIKE SAMLE RECOVERY NOT HITHIN CONTROL LIMITS 
GROUNDWATER QUALITY DATA BY LOCATIOH

SOMP ReV. 0 Data

SITE: MONOI MONUNENT VALLEY

LOCATION: 0613

MORTH COORDIHATE: 56377.8 FT

EAST COORDIHATE: 88643.7 FT

$04 / 21 / 88$ TO $12 / 13 / 94$

REPORT DATE: O6/20/95

FORMATION OF COAPLETION: OECHELLEY MEMBER OF THE CUTLER FORMATION (DC) HYDRAULIC FLOW RELATIONSHIP: UHKNOWN (N)

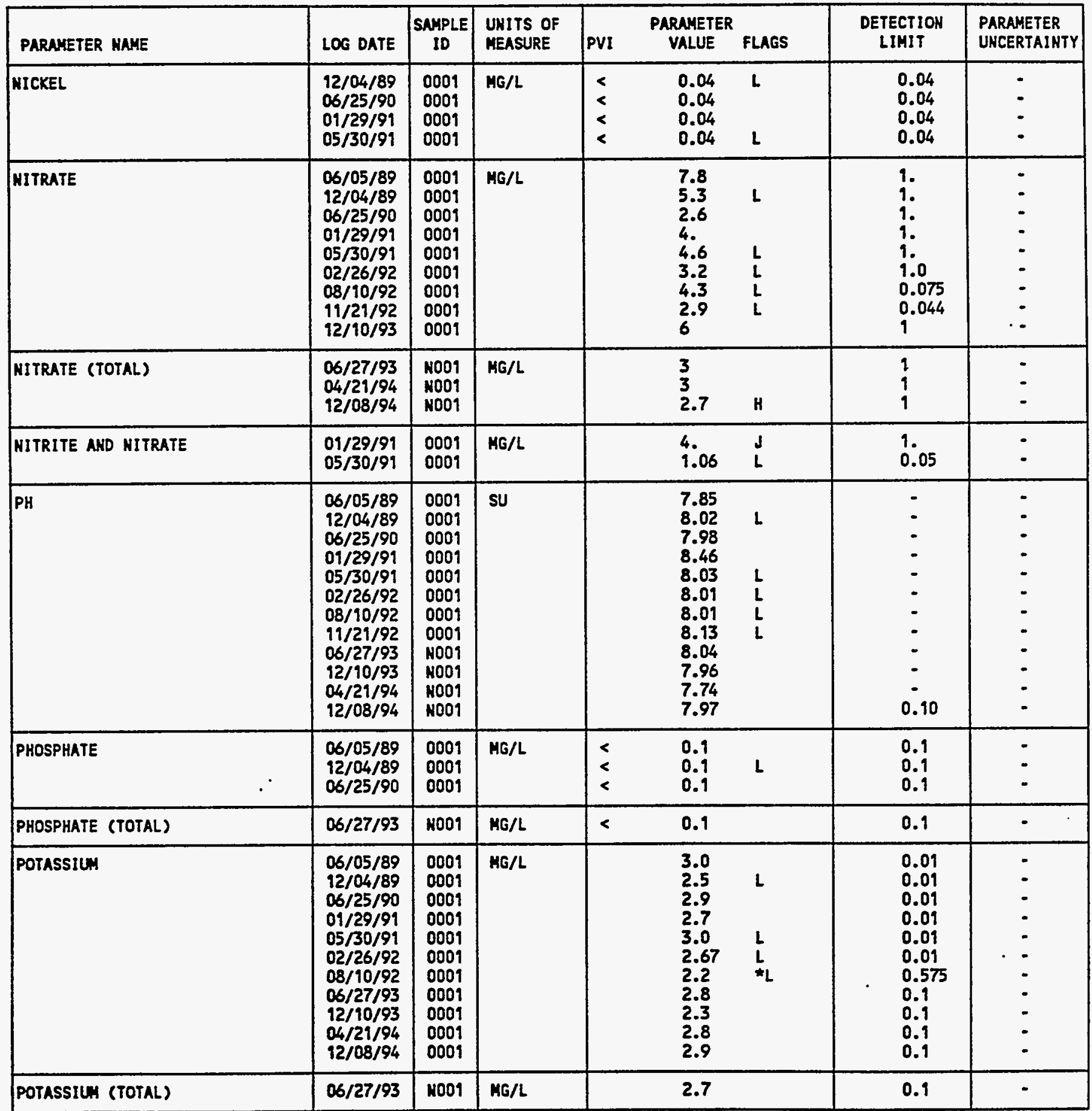

PARAMETER VALUE INDICATOR (PVI): < - LESS THAN DETECTION LIMIT

SMPLE ID COOES:

OTHER PARAMETER VALUE FLAGS:

DOO1 - FILTERED SAMPLE (.45 MICROHS)

MO01 - UNFILTERED SNMPLE

- DUPLICATE AHALYSIS MOT MITHIM CONTROL LIMITS

h - HOLD TIME EXPIRED, VALUE SUSPECT

J - ESTIMATEO VALUE

L - LESS THAN THREE BORE VOLUMES REMOVED BEFORE SAMPLING 
GROUMDHATER OUALITY DATA BY LOCATION

SOWP ReV. $O$ Data

SITE: MOHO1 MONUMEHT VALLEY

LOCATION: 0613

MORTH COORDIHATE: $\quad 56377.8$ FT

EAST COOROINATE: 88643.7 FT

O4/21/88 TO $12 / 13 / 94$

REPORT OATE: 06/20/95

FORMATION OF COMPLETION: DECHELLEY MEMBER OF THE CUTLER FORMATION (DC) HYORAULIC FLOW RELATIOHSHIP: UNKNOWN (N)

\begin{tabular}{|c|c|c|c|c|c|c|c|c|}
\hline PARAMETER MNHE & LOG DATE & $\begin{array}{c}\text { SAMPLE } \\
\text { ID }\end{array}$ & $\begin{array}{l}\text { UNITS OF } \\
\text { MEASURE }\end{array}$ & PVI & $\begin{array}{l}\text { PARAMETER } \\
\text { VALUE }\end{array}$ & FLAGS & $\begin{array}{l}\text { DETECTION } \\
\text { LIHIT }\end{array}$ & $\begin{array}{l}\text { PARAMETER } \\
\text { UNCERTAINTY }\end{array}$ \\
\hline POTASSIUN (TOTAL) & $\begin{array}{l}12 / 10 / 93 \\
04 / 21 / 94 \\
12 / 08 / 94\end{array}$ & $\begin{array}{l}\text { No01 } \\
\text { NO01 } \\
\text { No01 }\end{array}$ & MG/L & & $\begin{array}{l}2.3 \\
2.7 \\
2.7\end{array}$ & & $\begin{array}{l}0.1 \\
0.1 \\
0.1\end{array}$ & - \\
\hline RADIUH-226 & $\begin{array}{l}06 / 05 / 89 \\
12 / 04 / 89 \\
06 / 25 / 90 \\
01 / 29 / 91 \\
05 / 30 / 91 \\
02 / 26 / 92 \\
08 / 10 / 92 \\
11 / 21 / 92\end{array}$ & $\begin{array}{l}0001 \\
0001 \\
0001 \\
0001 \\
0001 \\
0001 \\
0001 \\
0001\end{array}$ & $\mathrm{PCI} / \mathrm{L}$ & & $\begin{array}{l}0.0 \\
0.1 \\
0.0 \\
0.0 \\
0.0 \\
0.0 \\
0.1 \\
0.2\end{array}$ & $\begin{array}{l}L \\
L \\
L \\
L\end{array}$ & $\begin{array}{l}1 . \\
1 . \\
1 . \\
1 . \\
1.0 \\
0.3 \\
0.3\end{array}$ & $\begin{array}{l}0.1 \\
0.2 \\
0.1 \\
0.1 \\
0.1 \\
0.2 \\
0.1 \\
0.2\end{array}$ \\
\hline RADIUA-226 (TOTAL) & $11 / 21 / 92$ & NOO1 & $\mathrm{PCI} / \mathrm{L}$ & & 0.3 & \llcorner & 0.3 & 0.2 \\
\hline RADIUH-226 + RADIUA-228 & $\begin{array}{l}06 / 05 / 89 \\
12 / 04 / 89 \\
06 / 25 / 90 \\
01 / 29 / 91 \\
05 / 30 / 91 \\
02 / 26 / 92 \\
08 / 10 / 92 \\
11 / 21 / 92\end{array}$ & $\begin{array}{l}0001 \\
0001 \\
0001 \\
0001 \\
0009 \\
0001 \\
0001 \\
0001\end{array}$ & PCI/L & & $\begin{array}{l}0.00 \\
0.90 \\
0.20 \\
0.70 \\
0.00 \\
1.70 \\
0.40 \\
3.00\end{array}$ & & $\begin{array}{l}- \\
: \\
: \\
: \\
-\end{array}$ & $\begin{array}{l}- \\
- \\
- \\
- \\
- \\
-\end{array}$ \\
\hline RADIUH-226 + RADIUN-22B (TOTAL) & $11 / 21 / 92$ & NOOI & PCI/L & & 3.00 & & - & - \\
\hline RADILH-228 & $\begin{array}{l}06 / 05 / 89 \\
12 / 04 / 89 \\
06 / 25 / 90 \\
01 / 29 / 91 \\
05 / 30 / 91 \\
02 / 26 / 92 \\
08 / 10 / 92 \\
11 / 21 / 92\end{array}$ & $\begin{array}{l}0001 \\
0001 \\
0001 \\
0001 \\
0001 \\
0001 \\
0001 \\
0001\end{array}$ & PCI/L & & $\begin{array}{l}0.0 \\
0.8 \\
0.2 \\
0.7 \\
0.0 \\
1.7 \\
0.3 \\
2.8\end{array}$ & $\begin{array}{l}L \\
\mathrm{JL} \\
L \\
L \\
L\end{array}$ & $\begin{array}{l}1 . \\
1 . \\
1 . \\
1 . \\
1.0 \\
2.2 \\
2.5\end{array}$ & $\begin{array}{l}0.9 \\
1.8 \\
0.7 \\
0.8 \\
1.2 \\
1.7 \\
1.1 \\
1.7\end{array}$ \\
\hline RNDIUA-228 (TOTAL) & $11 / 21 / 92$ & NOO1 & PCI/L & & 2.7 & $L$ & 1.9 & 1.4 \\
\hline REDOX POTEKTIAL & $\begin{array}{l}06 / 27 / 93 \\
12 / 10 / 93 \\
04 / 21 / 94 \\
12 / 08 / 94\end{array}$ & $\begin{array}{l}\text { NOO1 } \\
\text { NOO1 } \\
\text { NOO1 } \\
\text { NOO1 }\end{array}$ & mVOLTS & & $\begin{array}{l}466 \\
407 \\
445 \\
448\end{array}$ & - & : & $\begin{array}{l}- \\
- \\
-\end{array}$ \\
\hline SELENIUM & $\begin{array}{l}06 / 05 / 89 \\
12 / 04 / 89 \\
06 / 25 / 90 \\
01 / 29 / 91 \\
05 / 30 / 91 \\
02 / 26 / 92 \\
08 / 10 / 92 \\
11 / 21 / 92\end{array}$ & $\begin{array}{l}0001 \\
0001 \\
0001 \\
0001 \\
0001 \\
0001 \\
0001 \\
0001\end{array}$ & $M G / L$ & $\begin{array}{l}< \\
< \\
< \\
< \\
<\end{array}$ & $\begin{array}{l}0.005 \\
0.005 \\
0.005 \\
0.005 \\
0.005 \\
0.005 \\
0.005 \\
0.0015\end{array}$ & $\begin{array}{l}L \\
J L \\
L \\
U L \\
N L\end{array}$ & $\begin{array}{l}0.005 \\
0.005 \\
0.005 \\
0.005 \\
0.005 \\
0.005 \\
0.005 \\
0.0015\end{array}$ & $\begin{array}{l}- \\
: \\
: \\
: \\
-\end{array}$ \\
\hline SELENIUA (TOTAL) & $11 / 21 / 92$ & N001 & ME/L & & 0.0016 & $\mathbf{L}$ & 0.0015 & $\cdot$ \\
\hline SILICA - SIO2 & $06 / 05 / 89$ & 0001 & MG/L & & 11. & & 2. & - \\
\hline
\end{tabular}

PARAMETER VALUE IMDICATOR (PVI): < - LESS THAN DETECTION LIMIT

SMPLE ID COOES:

OTHER PARAMETER VALUE FLAGS:

0001 - FILTERED SAMPLE (.45 HICRONS)

J. ESTIKMTED VALUE

L - LESS THAN THREE BORE VOLUMES REHOVED BEFORE SNMPLING

- $N$ - SPIKE SAYPLE RECOVERY HOT WITHIH CONTROL LIMITS

$H$ - POST-DIGEST SPIKE OUT OF CNTR LIM UHILE SNMP ABS < 50\% SPIKE 
GROUNDHATER QUALITY DATA BY LOCATION

SOUP ReV. O Data

SITE: MOHOI MONLMENT VALLEY

LOCATION: 0613

NORTH COORDINATE: $\quad 56377.8$ FT

EAST COORDIHATE: 88643.7 FT

$04 / 21 / 88$ TO $12 / 13 / 94$

REPORT DATE: 06/20/95

FORMATION OF COMPLETION: DECHELLEY MEMBER OF THE CUTLER FORMATION (DC) HYDRAULIC FLOU RELATIONSHIP: UHKNOWN (N)

\begin{tabular}{|c|c|c|c|c|c|c|c|c|}
\hline PARAMETER MAME & LOG DATE & $\underset{\text { ID }}{\text { SAMPLE }}$ & $\begin{array}{l}\text { UNITS OF } \\
\text { MEASURE }\end{array}$ & PVI & $\begin{array}{l}\text { PARAMETER } \\
\text { VALUE }\end{array}$ & FLAGS & $\begin{array}{l}\text { DETECTIOH } \\
\text { LIMIT }\end{array}$ & $\begin{array}{l}\text { PARAMETER } \\
\text { UNCERTAINTY }\end{array}$ \\
\hline SILICA - SIOZ & $\begin{array}{l}12 / 04 / 89 \\
06 / 25 / 90 \\
01 / 29 / 91 \\
05 / 30 / 91 \\
12 / 10093 \\
12 / 08 / 94\end{array}$ & $\begin{array}{l}0009 \\
0001 \\
0001 \\
0001 \\
0001 \\
0001\end{array}$ & MG/L & & $\begin{array}{l}10.6 \\
11 . \\
10 . \\
10.8 \\
10.7 \\
11.5\end{array}$ & $\begin{array}{l}L \\
L\end{array}$ & $\begin{array}{l}2 . \\
2 . \\
2 . \\
0.1 \\
0.1 \\
0.1\end{array}$ & $:$ \\
\hline SILICA - SIO2 (TOTAL) & $\begin{array}{l}06 / 27 / 93 \\
12 / 10 / 93 \\
12 / 08 / 94\end{array}$ & $\begin{array}{l}\text { N001 } \\
\text { N001 } \\
\text { N001 }\end{array}$ & $M G / L$ & & $\begin{array}{r}10.4 \\
10.3 \\
9.4\end{array}$ & H & $\begin{array}{l}0.5 \\
0.1 \\
0.1\end{array}$ & : \\
\hline SILVER & $\begin{array}{l}06 / 05 / 89 \\
12 / 04 / 89 \\
06 / 25 / 90 \\
01 / 29 / 91 \\
05 / 30 / 91\end{array}$ & $\begin{array}{l}0001 \\
0001 \\
0001 \\
0001 \\
0001\end{array}$ & MG/L & $\begin{array}{l}< \\
< \\
< \\
<\end{array}$ & $\begin{array}{l}0.01 \\
0.01 \\
0.01 \\
0.01 \\
0.01\end{array}$ & $L$ & $\begin{array}{l}0.01 \\
0.01 \\
0.01 \\
0.01 \\
0.01\end{array}$ & : \\
\hline SOOIUM & $\begin{array}{l}06 / 05 / 89 \\
12 / 04 / 89 \\
06 / 25 / 90 \\
01 / 29 / 91 \\
05 / 30 / 91 \\
02 / 26 / 92 \\
08 / 10 / 92 \\
06 / 27 / 93 \\
12 / 10 / 93 \\
04 / 21 / 94 \\
12 / 08 / 94\end{array}$ & $\begin{array}{l}0009 \\
0001 \\
0001 \\
0001 \\
0001 \\
0001 \\
0001 \\
0009 \\
0001 \\
0001 \\
0001\end{array}$ & $M G / L$ & & $\begin{array}{l}51.7 \\
49 . \\
51.2 \\
51.6 \\
50 . \\
51 . \\
49 \\
49 \\
48 \\
49 \\
53.7\end{array}$ & $\begin{array}{l}L \\
L \\
L\end{array}$ & $\begin{array}{l}0.002 \\
0.002 \\
0.002 \\
0.002 \\
1 . \\
1 . \\
5 \\
1 \\
1 \\
1 \\
1\end{array}$ & $\begin{array}{l}: \\
: \\
: \\
: \\
:\end{array}$ \\
\hline SOOIUM (TOTAL) & $\begin{array}{l}06 / 27 / 93 \\
12 / 10 / 93 \\
04 / 21 / 94 \\
12 / 08 / 94\end{array}$ & $\begin{array}{l}\text { NOOI } \\
\text { NOO1 } \\
\text { N001 } \\
\text { NOO1 }\end{array}$ & MG/L & & $\begin{array}{l}50 \\
49 \\
48 \\
48.5\end{array}$ & & $\begin{array}{l}1 \\
1 \\
1 \\
1\end{array}$ & $:$ \\
\hline SPECIFIC CONDUCTANCE & $\begin{array}{l}06 / 05 / 89 \\
12 / 04 / 89 \\
06 / 25 / 90 \\
01 / 29 / 91 \\
05 / 30 / 91 \\
02 / 26 / 92 \\
08 / 10 / 92 \\
11 / 2192 \\
06 / 27 / 93 \\
12 / 90 / 93 \\
04 / 21 / 94 \\
12 / 08 / 94\end{array}$ & $\begin{array}{l}0001 \\
0001 \\
0001 \\
0001 \\
0001 \\
0001 \\
0001 \\
0001 \\
\text { No01 } \\
\text { NO01 } \\
\text { NO01 } \\
\text { No01 }\end{array}$ & UMHO/CM & & $\begin{array}{l}332 . \\
390 . \\
400 . \\
490 . \\
508 . \\
412 \\
421 \\
411 \\
422 \\
292 \\
370 \\
381\end{array}$ & $\begin{array}{l}L \\
L \\
L \\
L \\
L\end{array}$ & $\begin{array}{l}: \\
: \\
: \\
: \\
: \\
: \\
:\end{array}$ & $\begin{array}{l}: \\
\vdots \\
\vdots \\
: \\
:\end{array}$ \\
\hline STROATIUA & $\begin{array}{l}06 / 25 / 90 \\
01 / 29 / 91 \\
05 / 30 / 91 \\
02 / 26 / 92 \\
08 / 10 / 92 \\
11 / 21 / 92\end{array}$ & $\begin{array}{l}0001 \\
0001 \\
0001 \\
0001 \\
0001 \\
0001\end{array}$ & $M G / L$ & & $\begin{array}{l}0.36 \\
0.3 \\
0.35 \\
0.36 \\
0.3 \\
0.40\end{array}$ & $\begin{array}{l}- \\
L \\
L \\
L\end{array}$ & $\begin{array}{l}0.1 \\
0.1 \\
0.01 \\
0.01 \\
0.2 \\
0.00035\end{array}$ & $:$ \\
\hline
\end{tabular}

PARAMETER VALUE INDICATOR (PVI): < - LESS THAN DETECTION LIMIT

SAMPLE ID COOES:

OTHER PARAMETER VALUE FLAGS:

DOO1 - FILTERED SAMPLE (.45 MICRONS)

- DUPLICATE AMALYSIS nOT UITHIN CONTROL LIMITS

NOO1 - UHFILTERED SAMPLE

H - HOLD TIME EXPIRED, VALUE SUSPECT

J - estimated value

- $L$ - LESS THAN THREE BORE VOLLAES REMOVED BEFORE SAMPLING 
GROUNDWATER QUALITY DATA BY LOCATION

SOWP Rev. 0 Data

SITE: MONOI MONUNENT VALLEY

LOCATIOH: 0613

HORTH COORDINATE: 56377.8 FT

EAST COORDIHATE: 88643.7 FT

04/21/88 TO $12 / 13 / 94$

REPORT DATE: 06/20/95

FORMATION OF CONPLETIOH: DECHELLEY MEMBER OF THE CUTLER FORHATION (OC) HYDRAULIC FLON RELATIOHSHIP: UHKNOWN (N)

\begin{tabular}{|c|c|c|c|c|c|c|c|c|}
\hline PARANETER RAME & LOG DATE & $\underset{\text { ID }}{\text { SAMPLE }}$ & $\begin{array}{l}\text { UMITS OF } \\
\text { MEASURE }\end{array}$ & PVI & $\begin{array}{l}\text { PARAMETER } \\
\text { VALUE }\end{array}$ & FLAGS & $\begin{array}{l}\text { DETECTIOW } \\
\text { LIMIT }\end{array}$ & $\begin{array}{l}\text { PARAMETER } \\
\text { UHCERTAINTY }\end{array}$ \\
\hline STRONTIUM & $\begin{array}{l}06 / 27 / 93 \\
12 / 10 / 93 \\
04 / 21 / 94 \\
12 / 08 / 94\end{array}$ & $\begin{array}{l}0001 \\
0001 \\
0001 \\
0001\end{array}$ & MG/L & & $\begin{array}{l}0.35 \\
0.34 \\
0.35 \\
0.37\end{array}$ & $d$ & $\begin{array}{l}0.01 \\
0.01 \\
0.01 \\
0.01\end{array}$ & $\begin{array}{l}- \\
- \\
-\end{array}$ \\
\hline STRONTIUM (TOTAL) & $\begin{array}{l}11 / 21 / 92 \\
06 / 27 / 93 \\
12 / 10 / 93 \\
04 / 21 / 94 \\
12 / 08 / 94\end{array}$ & $\begin{array}{l}\text { noo1 } \\
\text { no01 } \\
\text { No01 } \\
\text { no01 } \\
\text { no01 }\end{array}$ & $M G / L$ & & $\begin{array}{l}0.39 \\
0.34 \\
0.34 \\
0.34 \\
0.36\end{array}$ & J & $\begin{array}{l}0.00035 \\
0.01 \\
0.01 \\
0.01 \\
0.01\end{array}$ & $\begin{array}{l}- \\
- \\
- \\
-\end{array}$ \\
\hline SULFATE & $\begin{array}{l}06 / 05 / 89 \\
12 / 04 / 89 \\
06 / 25 / 90 \\
01 / 29 / 91 \\
05 / 30 / 91 \\
02 / 26 / 92 \\
08 / 10 / 92 \\
11 / 21 / 92 \\
12 / 10 / 93 \\
04 / 21 / 94\end{array}$ & $\begin{array}{l}0001 \\
0001 \\
0001 \\
0001 \\
0001 \\
0001 \\
0001 \\
0001 \\
0001 \\
0001\end{array}$ & MG/L & & $\begin{array}{l}64 . \\
64 . \\
52.3 \\
61.7 \\
56 . \\
60 . \\
60 \\
65 \\
59 \\
61\end{array}$ & $\begin{array}{l}\mathrm{L} \\
\mathrm{L} \\
\mathrm{L} \\
\mathrm{L} \\
\mathrm{L}\end{array}$ & $\begin{array}{l}0.1 \\
0.1 \\
0.1 \\
0.1 \\
0.1 \\
10 . \\
0.059 \\
1.0 \\
1 \\
1\end{array}$ & $\begin{array}{l}- \\
: \\
- \\
: \\
- \\
-\end{array}$ \\
\hline SULFATE (TOTAL) & $\begin{array}{l}11 / 21 / 92 \\
06 / 27 / 93 \\
12 / 08 / 94\end{array}$ & $\begin{array}{l}\text { No01 } \\
\text { No01 } \\
\text { no01 }\end{array}$ & MG/L & & $\begin{array}{l}64 \\
61 \\
45\end{array}$ & $L$ & $\begin{array}{l}1.0 \\
1 \\
1\end{array}$ & - \\
\hline SULFIDE & $\begin{array}{l}06 / 05 / 89 \\
12 / 04 / 89 \\
01 / 29 / 91 \\
05 / 30 / 91\end{array}$ & $\begin{array}{l}0001 \\
0001 \\
0001 \\
0001\end{array}$ & $M G / L$ & $\begin{array}{l}< \\
<\end{array}$ & $\begin{array}{l}0.1 \\
0.1 \\
0.1 \\
4.9\end{array}$ & $\begin{array}{l}L \\
J \\
J L\end{array}$ & $\begin{array}{l}0.1 \\
0.1 \\
0.1 \\
1 .\end{array}$ & : \\
\hline TEMPERATURE & $\begin{array}{l}06 / 05 / 89 \\
12 / 04 / 89 \\
06 / 25 / 90 \\
01 / 29 / 91 \\
05 / 30 / 91 \\
02 / 26 / 92 \\
08 / 10 / 92 \\
11 / 21 / 92 \\
06 / 27 / 93 \\
12 / 10 / 93 \\
04 / 21 / 94 \\
12 / 08 / 94\end{array}$ & $\begin{array}{l}0001 \\
0001 \\
0001 \\
0001 \\
0001 \\
0001 \\
0001 \\
0001 \\
\text { N001 } \\
\text { N001 } \\
\text { H001 } \\
\text { H001 }\end{array}$ & C - DEGREE & & $\begin{array}{l}17.0 \\
15.0 \\
17.0 \\
13.7 \\
16.4 \\
15.4 \\
16.5 \\
15.4 \\
17.8 \\
15.2 \\
15.3 \\
13.8\end{array}$ & $\begin{array}{l}\text { L } \\
\text { L } \\
L \\
L \\
L\end{array}$ & $\begin{array}{c}- \\
- \\
- \\
- \\
- \\
= \\
- \\
- \\
0.1\end{array}$ & $\begin{array}{l}: \\
: \\
- \\
- \\
- \\
- \\
-\end{array}$ \\
\hline THALLIUA & $\begin{array}{l}06 / 05 / 89 \\
12 / 04 / 89 \\
06 / 25 / 90 \\
01 / 29 / 91 \\
05 / 30 / 91\end{array}$ & $\begin{array}{l}0001 \\
0001 \\
0001 \\
0001 \\
0001\end{array}$ & MG/L & $\begin{array}{l}< \\
< \\
< \\
< \\
<\end{array}$ & $\begin{array}{l}0.01 \\
0.1 \\
0.01 \\
0.01 \\
0.01\end{array}$ & L & $\begin{array}{l}0.01 \\
0.1 \\
0.01 \\
0.01 \\
0.01\end{array}$ & $\begin{array}{l}- \\
- \\
- \\
-\end{array}$ \\
\hline THORIUN-230 & $\begin{array}{l}01 / 29 / 91 \\
05 / 30 / 91\end{array}$ & $\begin{array}{l}0001 \\
0001\end{array}$ & $\mathrm{PCI} / \mathrm{L}$ & & $\begin{array}{l}0.0 \\
9.1\end{array}$ & $L$ & i. & $\begin{array}{l}0.3 \\
0.5\end{array}$ \\
\hline TIH & $\begin{array}{l}06 / 05 / 89 \\
12 / 04 / 89\end{array}$ & $\begin{array}{l}0001 \\
0001\end{array}$ & MG/L & $<$ & $\begin{array}{l}0.005 \\
0.005\end{array}$ & $L$ & $\begin{array}{l}0.005 \\
0.005\end{array}$ & - \\
\hline
\end{tabular}

PARAMETER VALUE IHOICATOR (PVI): < - LESS THAN DETECTION LIMIT

SAMPLE IO COOES:

OTHER PARAMETER VALUE FLAGS:

0001 - FILTERED SAMPLE (.45 MICROHS)

$J$ - ESTIMATED VALUE

NOO1 - LAFILTERED SAMPLE

$L$ - LESS THAH THREE BORE VOLGMES REMOVED BEFORE SAMPLIHG 
GROUNDWATER OUALITY DATA BY LOCATION

SOUP ReV. O Data

SITE: MONOS MONUHENT VALLEY

LOCATION: 0613

NORTH COORDINATE: 56377.8 FT

EAST COORDINATE: 88643.7 FT

$04 / 21 / 88$ TO $12 / 13 / 94$

REPORT DATE: $06 / 20 / 95$

FORMATION OF CONPLETION: DECHELLEY MEMBER OF THE CUTLER FORMATION (DC)

HYDRAULIC FLON RELATIONSHIP: UNKHOWN (N)

\begin{tabular}{|c|c|c|c|c|c|c|c|c|}
\hline PARAMETER MAME & LOG DATE & $\underset{10}{\operatorname{SNMPLE}}$ & $\begin{array}{l}\text { UNITS OF } \\
\text { MEASURE }\end{array}$ & PVI & $\begin{array}{l}\text { PARAMETER } \\
\text { VALUE }\end{array}$ & FLAGS & $\begin{array}{l}\text { DETECTION } \\
\text { LIMIT }\end{array}$ & $\begin{array}{l}\text { PARAMETER } \\
\text { UKCERTAINTY }\end{array}$ \\
\hline TIN & $\begin{array}{l}06 / 25 / 90 \\
01 / 29 / 91 \\
05 / 30 / 91\end{array}$ & $\begin{array}{l}0001 \\
0001 \\
0001\end{array}$ & MG/L & $\begin{array}{l}< \\
<\end{array}$ & $\begin{array}{l}0.005 \\
0.005 \\
0.01\end{array}$ & IL & $\begin{array}{l}0.005 \\
0.005 \\
0.01\end{array}$ & : \\
\hline TOTAL DISSOLVED SOLIDS & $\begin{array}{l}06 / 05 / 89 \\
12 / 04 / 89 \\
06 / 25 / 90 \\
01 / 29 / 91 \\
05 / 30 / 91 \\
02 / 26 / 92 \\
08 / 10 / 92 \\
12 / 10 / 93 \\
04 / 21 / 94 \\
12 / 08 / 94\end{array}$ & $\begin{array}{l}0001 \\
0001 \\
0001 \\
0001 \\
0001 \\
0001 \\
0001 \\
0001 \\
0001 \\
0001\end{array}$ & MG/L & & $\begin{array}{l}281 . \\
298 . \\
284 . \\
228 . \\
288 . \\
277 . \\
360 \\
300 \\
290 \\
294\end{array}$ & $\begin{array}{l}L \\
L \\
L \\
L \\
H\end{array}$ & $\begin{array}{l}10 . \\
10 . \\
10 . \\
10 . \\
10 . \\
10 . \\
42 \\
10 \\
10 \\
10\end{array}$ & $\begin{array}{l}: \\
: \\
- \\
: \\
: \\
\therefore\end{array}$ \\
\hline TOTAL DISSOLVED SOLIDS (TOTAL) & $06 / 27 / 93$ & N001 & $M G / L$ & & 270 & & 10 & $\cdot$ \\
\hline TOTAL ORGANIC CARBON & $\begin{array}{l}01 / 29 / 91 \\
05 / 30 / 91 \\
02 / 26 / 92 \\
08 / 90 / 92\end{array}$ & $\begin{array}{l}0001 \\
0001 \\
0001 \\
0001\end{array}$ & MG/L & $\begin{array}{l}< \\
<\end{array}$ & $\begin{array}{l}1 . \\
2 . \\
1.0\end{array}$ & $\begin{array}{l}J \\
L \\
L\end{array}$ & $\begin{array}{l}1 . \\
1 . \\
1.0\end{array}$ & : \\
\hline URAKILN & $\begin{array}{l}06 / 05 / 89 \\
12 / 04 / 89 \\
06 / 25 / 90 \\
01 / 29 / 91 \\
05 / 30 / 91 \\
02 / 26 / 92 \\
11 / 21 / 92 \\
06 / 27 / 93 \\
12 / 10 / 93 \\
04 / 21 / 94 \\
12 / 08 / 94\end{array}$ & $\begin{array}{l}0001 \\
0001 \\
0001 \\
0001 \\
0001 \\
0001 \\
0001 \\
0001 \\
0001 \\
0001 \\
0001\end{array}$ & MG/L & & $\begin{array}{l}0.0044 \\
0.005 \\
0.0042 \\
0.0037 \\
0.004 \\
0.003 \\
0.004 \\
0.004 \\
0.005 \\
0.004 \\
0.004\end{array}$ & $\begin{array}{l}\mathbf{L} \\
\mathbf{L} \\
\mathbf{L} \\
\mathbf{L}\end{array}$ & $\begin{array}{l}0.003 \\
0.003 \\
0.003 \\
0.0003 \\
0.001 \\
0.001 \\
0.001 \\
0.001 \\
0.001 \\
0.001 \\
0.001\end{array}$ & $\begin{array}{l}- \\
: \\
: \\
: \\
: \\
-\end{array}$ \\
\hline URAKIUW (TOTAL) & $\begin{array}{l}11 / 21 / 92 \\
06 / 27 / 93 \\
12 / 10 / 93 \\
04 / 21 / 94 \\
12 / 08 / 94\end{array}$ & $\begin{array}{l}\text { NOO1 } \\
\text { NOO1 } \\
\text { NOO1 } \\
\text { NOO1 } \\
\text { HOO1 }\end{array}$ & $M G / L$ & & $\begin{array}{l}0.003 \\
0.003 \\
0.004 \\
0.004 \\
0.003\end{array}$ & $L$ & $\begin{array}{l}0.001 \\
0.001 \\
0.001 \\
0.001 \\
0.001\end{array}$ & - \\
\hline VAKADIU: & $\begin{array}{l}06 / 05 / 89 \\
12 / 04 / 89 \\
06 / 25 / 90 \\
01 / 29 / 91 \\
05 / 30 / 91 \\
02 / 26 / 92 \\
08 / 10 / 92 \\
11 / 21 / 92 \\
06 / 27 / 93 \\
12 / 10 / 93 \\
04 / 21 / 94 \\
12 / 08 / 94\end{array}$ & $\begin{array}{l}0001 \\
0001 \\
0001 \\
0001 \\
0001 \\
0001 \\
0001 \\
0001 \\
0001 \\
0001 \\
0001 \\
0001\end{array}$ & $M G / L$ & $\begin{array}{l}< \\
< \\
< \\
< \\
< \\
< \\
< \\
< \\
< \\
< \\
<\end{array}$ & $\begin{array}{l}0.01 \\
0.01 \\
0.01 \\
0.01 \\
0.01 \\
0.01 \\
0.005 \\
0.0019 \\
0.01 \\
0.01 \\
0.01 \\
0.01\end{array}$ & $\begin{array}{l}\mathrm{L} \\
\mathrm{L} \\
\mathrm{L} \\
\mathrm{J} \\
\mathrm{L}\end{array}$ & $\begin{array}{l}0.09 \\
0.01 \\
0.09 \\
0.09 \\
0.01 \\
0.01 \\
0.05 \\
0.0019 \\
0.01 \\
0.09 \\
0.01 \\
0.01\end{array}$ & $\begin{array}{l}: \\
: \\
\vdots \\
: \\
: \\
:\end{array}$ \\
\hline VANADIUA (TOTAL) & $11 / 21 / 92$ & NoO1 & MG/L & & 0.0030 & $L$ & 0.0019 & - \\
\hline
\end{tabular}

PARAMETER VALUE INDICATOR (PVI): < - LESS THAN DETECTION LIMIT

SAPLE ID CODES:

OTHER PARAMETER VALUE FLAGS:

OOO1 - FILTERED SAMPLE (.45 MICROHS)

NOO1 - UMFILTERED SAMPLE

H - HOLD TIME EXPIRED, VALUE SUSPECT

I - INCREASED DETECTION LIMIT DUE TO REQUIRED DILUTION

d - ESTIMATED VALUE

L - LESS THAN THREE BORE VOLUHES REMOVED BEFORE SAMPLIMG 
GRONDWATER QUALITY DATA BY LOCATION

SOUP ReV. O Data

SITE: MOHO1 MONUMENT VALLEY

LOCATION: 0613

MORTH COORDINATE: 56377.8 FT

EAST COOROINATE: 88643.7 FT

O4/21/88 TO 12/13/94

REPORT DATE: 06/20/95

FORMATION OF COMPLETION: DECHELLEY MEMBER OF THE CUTLER FORMATION (DC) HYDRAULIC FLON RELATIONSHIP: UNKHOWN (N)

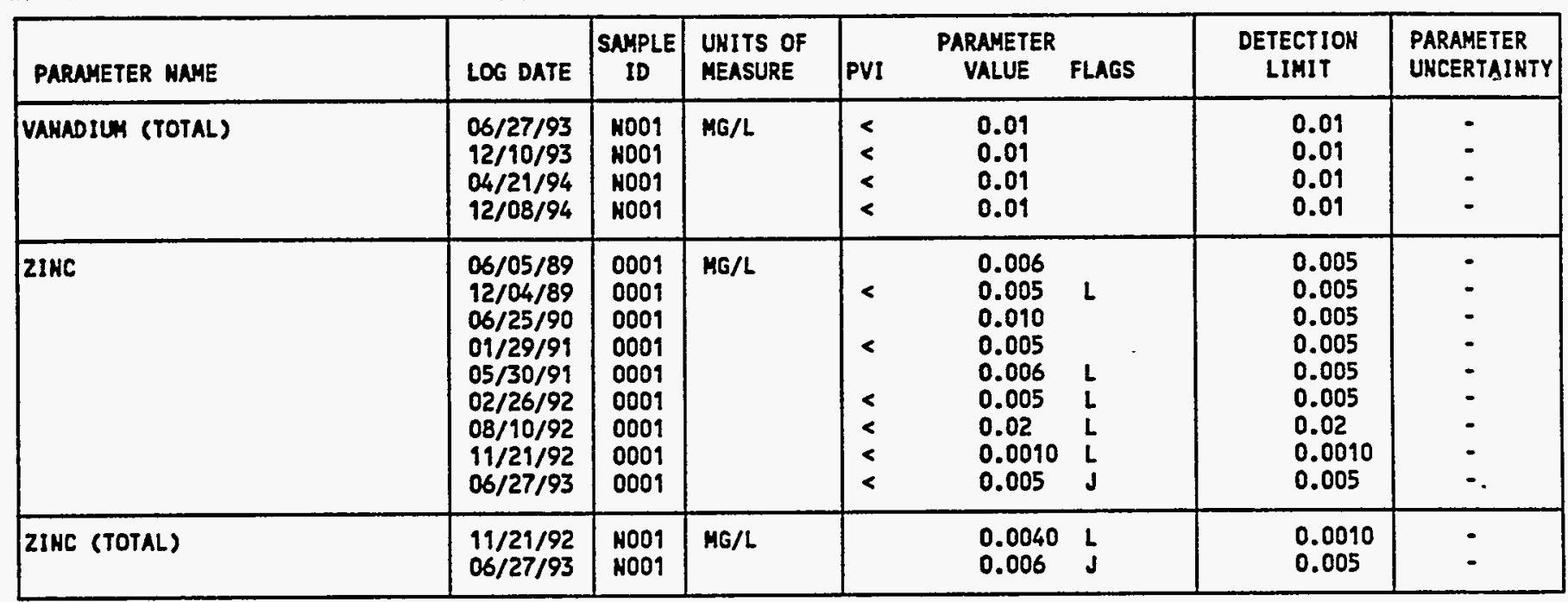

PARAMETER VALUE IHDICATOR (PVI): < - LESS THAN DETECTION LIMIT

SAMPLE ID CODES:

OTHER PARAMETER VALUE FLAGS:

OOO - FILTERED SAMPLE (.45 MICRONS)

J ESTIMATED VALUE

NOOI - UHFILTERED SAHPLE

L - LESS THAH THREE BORE VOLLMES REMOVED BEFORE SAMPLING 
GROUHDWATER QUALITY DATA BY LOCATION

SOUP ReV. 0 Data

SITE: MONO1 MONUMENT VALLEY

LOCATIOH: 0614

NORTH COORDIHATE: $\quad 60940.5$ FT

EAST COORDINATE: 87832.6 FT

04/21/88 TO 12/13/94

REPORT DATE: $06 / 20 / 95$

FORMATION OF COMPLETION: SHIMARUMIP MEMBER OF THE CHINLE FORMATION (SR)

HYDRAULIC FLON RELATIOHSHIP: DOUN GRADIENT (D)

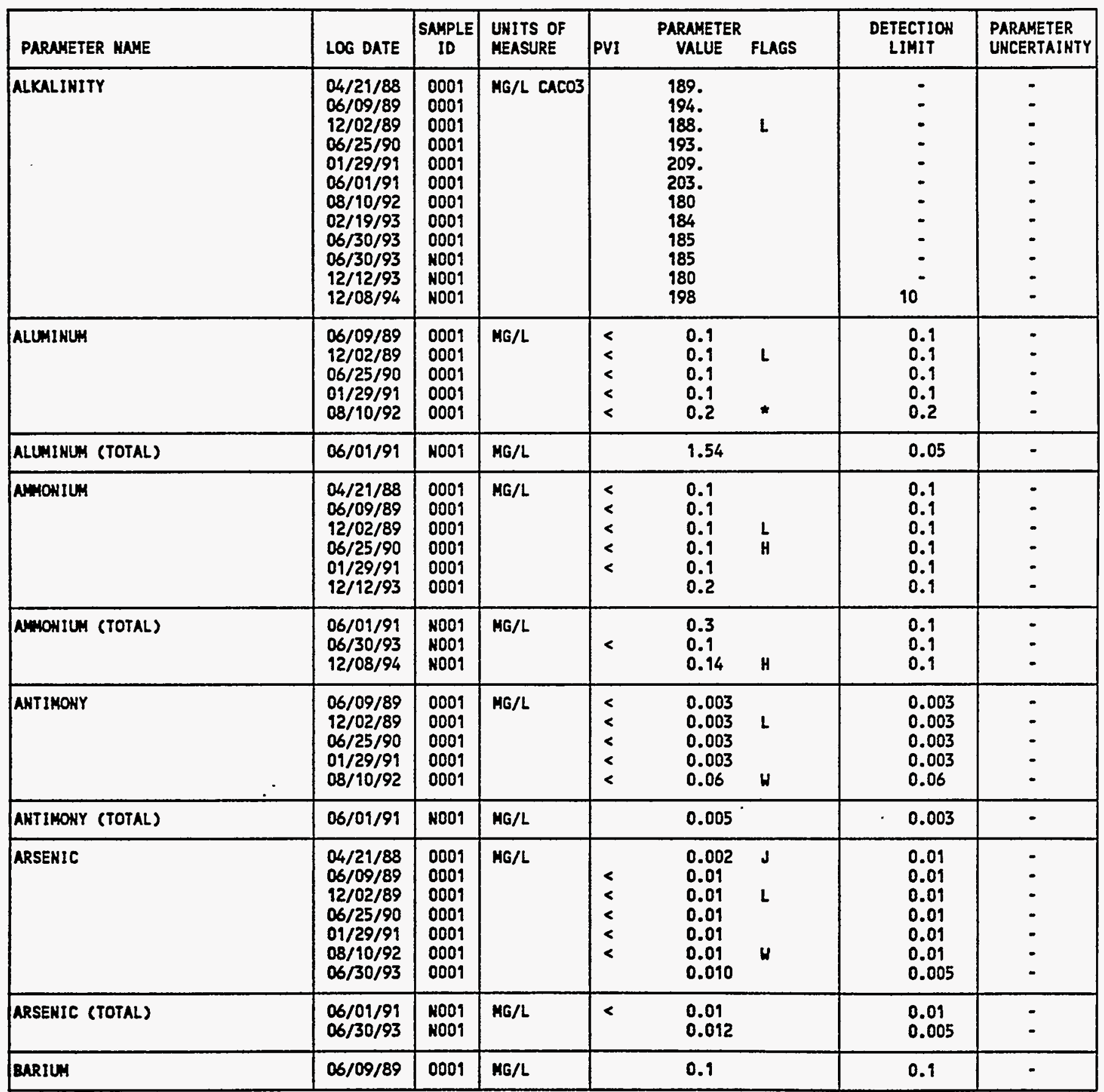

PARAMETER VALUE IMDICATOR (PVI): < - LESS THAN DETECTION LIMIT

SAMPLE ID COOES:

OOO1 - FILTERED SAMPLE (.45 MICROHS)

OTHER PARAMETER VALUE FLAGS:

MOO9 - UKFILTERED SAMPLE

- - DUPLICATE AMALYSIS NOT HITHIN CONTROL LIMITS

H - hOLd TIME EXPIRED, VALUE SUSPECT

J - ESTIMLTED VALUE

L - LESS THAN THREE BORE VOLLMES REMOVED BEFORE SAMPLING

W - POST-DIGEST SPIKE OUT OF CHTR LIM HHILE SAMP ABS < 50\% SPIKE 
GROUNDWATER OUALITY DATA BY LOCATION

SOWP ReV. 0 Date

SITE: MONO1 MONUMENT VALLEY

LOCATION: 0614

HORTH COORDIHATE: 60940.5 FT

EAST COORDIHATE: 87832.6 FT

$04 / 21 / 88$ TO $12 / 13 / 94$

REPORT DATE: $06 / 20 / 95$

FORMATION OF COHPLETION: SHIMARLAP MEMBER OF THE CHIHLE FOAMTION (SR) HYDRAULIC FLON RELATIONSHIP: DOWH GRADIENT (D)

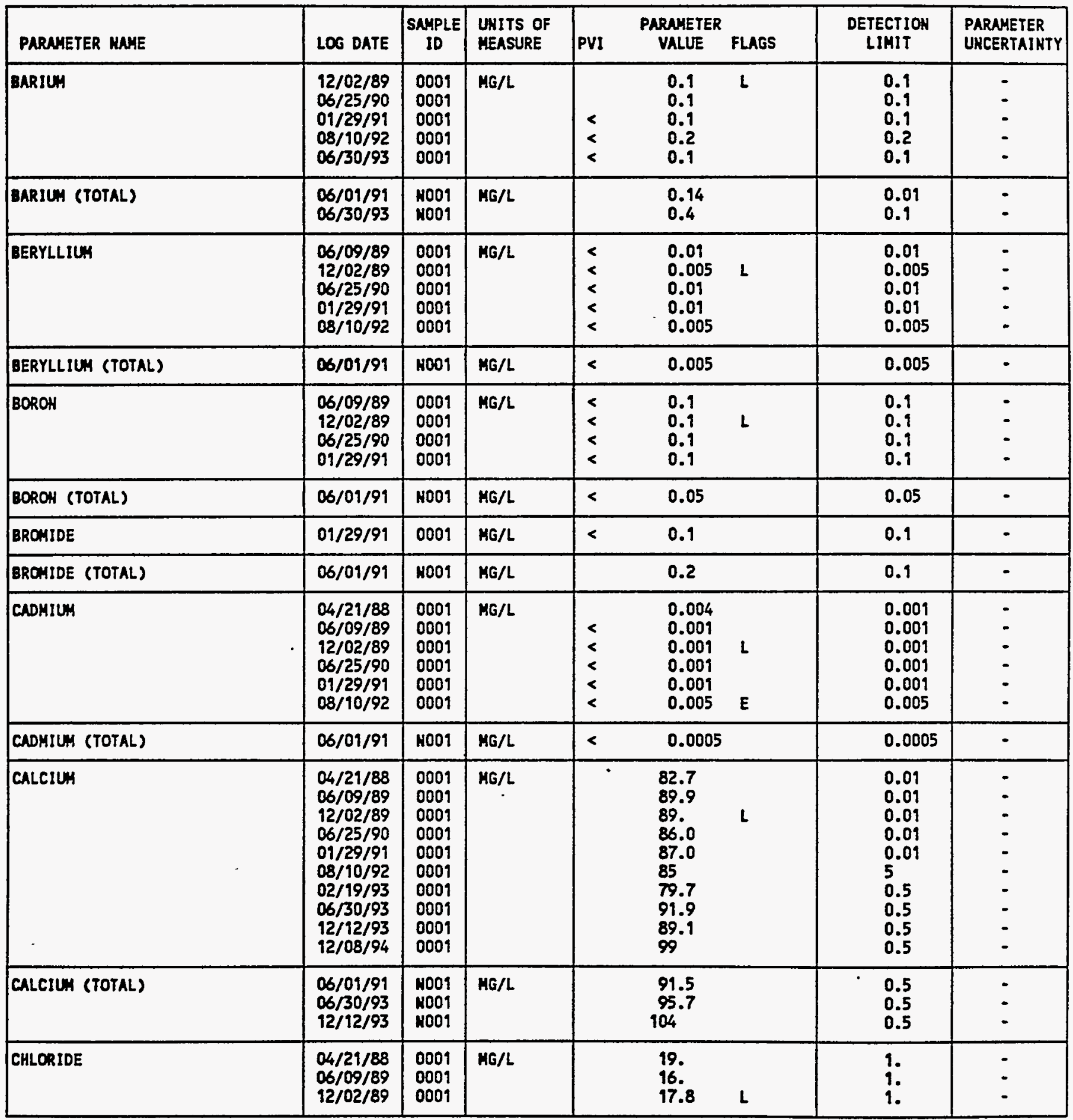

PARAYETER VALUE IMDICATOR (PVI): < - LESS THAN DETECTION LIMIT

SNMPLE ID COOES:

DTHER PARAMETER VALUE FLAGS:

0001 - FILTERED SNMLE (.45 MICRONS)

E - estIMLTED VALUE because of InTERfEREHCE, SEe CASE MARRATIVE

MDOI - LNFILTERED SNMPLE

- $L$ - LESS THAH THREE BORE VOLLMES REMOVED BEFORE SAMPLIHG 
GROUNDHATER QUALITY DATA BY LOCATION

SOUP ReV. 0 Data

SITE: HONOI MONUMENT VALLEY

LOCATION: 0614

NORTH COORDINATE: 60940.5 FT

EAST COORDINATE: 87832.6 FT

$04 / 21 / 88$ TO $12 / 13 / 94$

REPORT DATE: 06/20/95

FORHATION OF COHPLETION: SHINARUYP MEMBER OF THE CHIMLE FORMATION (SR) HYDRAULIC FLOW RELATIONSHIP: DONN GRADIENT (D)

\begin{tabular}{|c|c|c|c|c|c|c|c|c|}
\hline PARANETER NAME & LOS DATE & $\underset{\text { SOMPLE }}{\text { SD }}$ & $\begin{array}{l}\text { UNITS OF } \\
\text { MEASURE }\end{array}$ & PVI & $\begin{array}{l}\text { PARAMETER } \\
\text { VALUE }\end{array}$ & FLAGS & $\begin{array}{l}\text { DETECTIOH } \\
\text { LIMIT }\end{array}$ & $\begin{array}{l}\text { PARAMETEER } \\
\text { UACERTAIHTY }\end{array}$ \\
\hline CHLORIDE & $\begin{array}{l}06 / 25 / 90 \\
01 / 29 / 91 \\
08 / 10 / 92 \\
02 / 19 / 93 \\
12 / 12 / 93 \\
12 / 08 / 94\end{array}$ & $\begin{array}{l}0001 \\
0001 \\
0001 \\
0001 \\
0001 \\
0001\end{array}$ & MG/L & & $\begin{array}{l}21 . \\
20 . \\
17 \\
21.8 \\
56.7 \\
48\end{array}$ & $\mathbf{J}$ & $\begin{array}{l}9 . \\
1 . \\
0.016 \\
0.5 \\
0.5 \\
0.5\end{array}$ & $\begin{array}{l}- \\
: \\
: \\
-\end{array}$ \\
\hline CHLORIDE (TOTAL) & $\begin{array}{l}06 / 01 / 91 \\
06 / 30 / 93\end{array}$ & $\begin{array}{l}\text { HOO1 } \\
\text { N001 }\end{array}$ & MG/L & & $\begin{array}{l}27 . \\
39.7\end{array}$ & & $\begin{array}{l}1 . \\
0.5\end{array}$ & - \\
\hline CHROMIUM & $\begin{array}{l}06 / 09 / 89 \\
12 / 02 / 89 \\
06 / 25 / 90 \\
01 / 29 / 91 \\
08 / 10 / 92\end{array}$ & $\begin{array}{l}0001 \\
0001 \\
0001 \\
0001 \\
0001\end{array}$ & $M G / L$ & $\begin{array}{l}< \\
< \\
< \\
< \\
<\end{array}$ & $\begin{array}{l}0.01 \\
0.01 \\
0.01 \\
0.01 \\
0.01\end{array}$ & \llcorner & $\begin{array}{l}0.01 \\
0.01 \\
0.01 \\
0.01 \\
0.01\end{array}$ & $\begin{array}{l}- \\
- \\
- \\
-\end{array}$ \\
\hline CHROAIUN (TOTAL) & $06 / 01 / 91$ & N001 & HG/L & $<$ & 0.01 & & 0.01 & - \\
\hline $\cos A L T$ & $\begin{array}{l}06 / 09 / 89 \\
12 / 02 / 89 \\
06 / 25 / 90 \\
01 / 29 / 91\end{array}$ & $\begin{array}{l}0001 \\
0001 \\
0001 \\
0001\end{array}$ & $M G / L$ & $\begin{array}{l}< \\
< \\
< \\
<\end{array}$ & $\begin{array}{l}0.05 \\
0.05 \\
0.05 \\
0.05\end{array}$ & $L$ & $\begin{array}{l}0.05 \\
0.05 \\
0.05 \\
0.05\end{array}$ & - \\
\hline COBALT (TOTAL) & $06 / 01 / 91$ & N001 & $M G / L$ & $<$ & 0.03 & & 0.03 & - \\
\hline COPPER & $\begin{array}{l}06 / 09 / 89 \\
12 / 02 / 89 \\
06 / 25 / 90 \\
01 / 29 / 91\end{array}$ & $\begin{array}{l}0001 \\
0001 \\
0001 \\
0001\end{array}$ & $\mathbf{H G} / \mathbf{L}$ & $\begin{array}{l}< \\
< \\
< \\
<\end{array}$ & $\begin{array}{l}0.02 \\
0.02 \\
0.02 \\
0.02\end{array}$ & $L$ & $\begin{array}{l}0.02 \\
0.02 \\
0.02 \\
0.02\end{array}$ & $\begin{array}{l}- \\
-\end{array}$ \\
\hline COPPER (TOTAL) & $06 / 01 / 91$ & N001 & MG/L & & 0.01 & & 0.01 & - \\
\hline CYANIDE & $\begin{array}{l}06 / 09 / 89 \\
12 / 02 / 89\end{array}$ & $\begin{array}{l}0001 \\
0001\end{array}$ & $M G / L$ & $<$ & $\begin{array}{l}0.01 \\
0.01\end{array}$ & L & $\begin{array}{l}0.01 \\
0.01\end{array}$ & - \\
\hline FLLORIDE & $\begin{array}{l}06 / 09 / 89 \\
12 / 02 / 89 \\
06 / 25 / 90 \\
01 / 29 / 91\end{array}$ & $\begin{array}{l}0001 \\
0001 \\
0001 \\
0001\end{array}$ & MG/L & & $\begin{array}{l}0.2 \\
0.2 \\
0.2 \\
0.2\end{array}$ & $L$ & $\begin{array}{l}0.1 \\
0.1 \\
0.1 \\
0.1\end{array}$ & $\begin{array}{l}- \\
\therefore\end{array}$ \\
\hline FLUORIDE (TOTAL) & $06 / 01 / 99$ & No01 & MG/L & & 0.1 & & 0.1 & - \\
\hline GROSS ALPHA & $\begin{array}{l}04 / 21 / 88 \\
01 / 29 / 91 \\
06 / 01 / 91 \\
08 / 10 / 92 \\
02 / 19 / 93 \\
06 / 30 / 93\end{array}$ & $\begin{array}{l}0001 \\
0001 \\
0001 \\
0001 \\
0001 \\
0001\end{array}$ & $\mathrm{PCI} / \mathrm{L}$ & & $\begin{array}{l}24 . \\
28 . \\
35.9 \\
18 \\
22 \\
29\end{array}$ & $\frac{1}{1}$ & $\begin{array}{l}0.2 \\
1 . \\
1 . \\
8 \\
9 \\
5\end{array}$ & $\begin{array}{c}8 . \\
6 . \\
12.4 \\
8 \\
12 \\
11\end{array}$ \\
\hline GROSS ALPHA (TOTAL) & $07 / 01 / 93$ & N001 & PCI/L & & 54 & & 7 & 18 \\
\hline GROSS BETA & $\begin{array}{l}04 / 21 / 88 \\
01 / 29 / 99 \\
06 / 01 / 91\end{array}$ & $\begin{array}{l}0001 \\
0001 \\
0001\end{array}$ & PCI/L & & $\begin{array}{r}16 . \\
7.0 \\
13.3\end{array}$ & & $\begin{array}{l}1 . \\
0.5 \\
0.5\end{array}$ & $\begin{array}{l}2 . \\
2.8 \\
5.9\end{array}$ \\
\hline
\end{tabular}

PARAMETER VALUE INDICATOR (PVI): < - LESS THAN DETECTION LIMIT

SAMPLE ID COOES:

OTHER PARAMETER VALUE FLAGS:

SOOPL - FILTERED SAMPLE (.45 MICRONS)

I - IMCREASED DETECTIOH LIMIT DUE TO REQUIRED DILUTION

MOO1 - UNFILTERED SAMPLE

d - ESTIMLTED VALUE

l - LESS THAN THREE BORE VOLLMES REMOVED BEFORE SAMPLIHG 
GROUNDUATER QUALITY DATA BY LOCATION

SOUP Rev. O Data

SITE: MOHOI MOHUMENT VALLEY

LOCATIOH: 0614

MORTH COORDINATE: 60940.5 FT

EAST COORDINATE: 87832.6 FT

04/21/88 TO $12 / 13 / 94$

REPORT DATE: $06 / 20 / 95$

FORMATION OF COMPLETIOH: SHIHARUMP MEMBER OF THE CHINLE FORMATION (SR) HYDRAULIC FLON RELATIOHSHIP: DOWN GRADIENT (D)

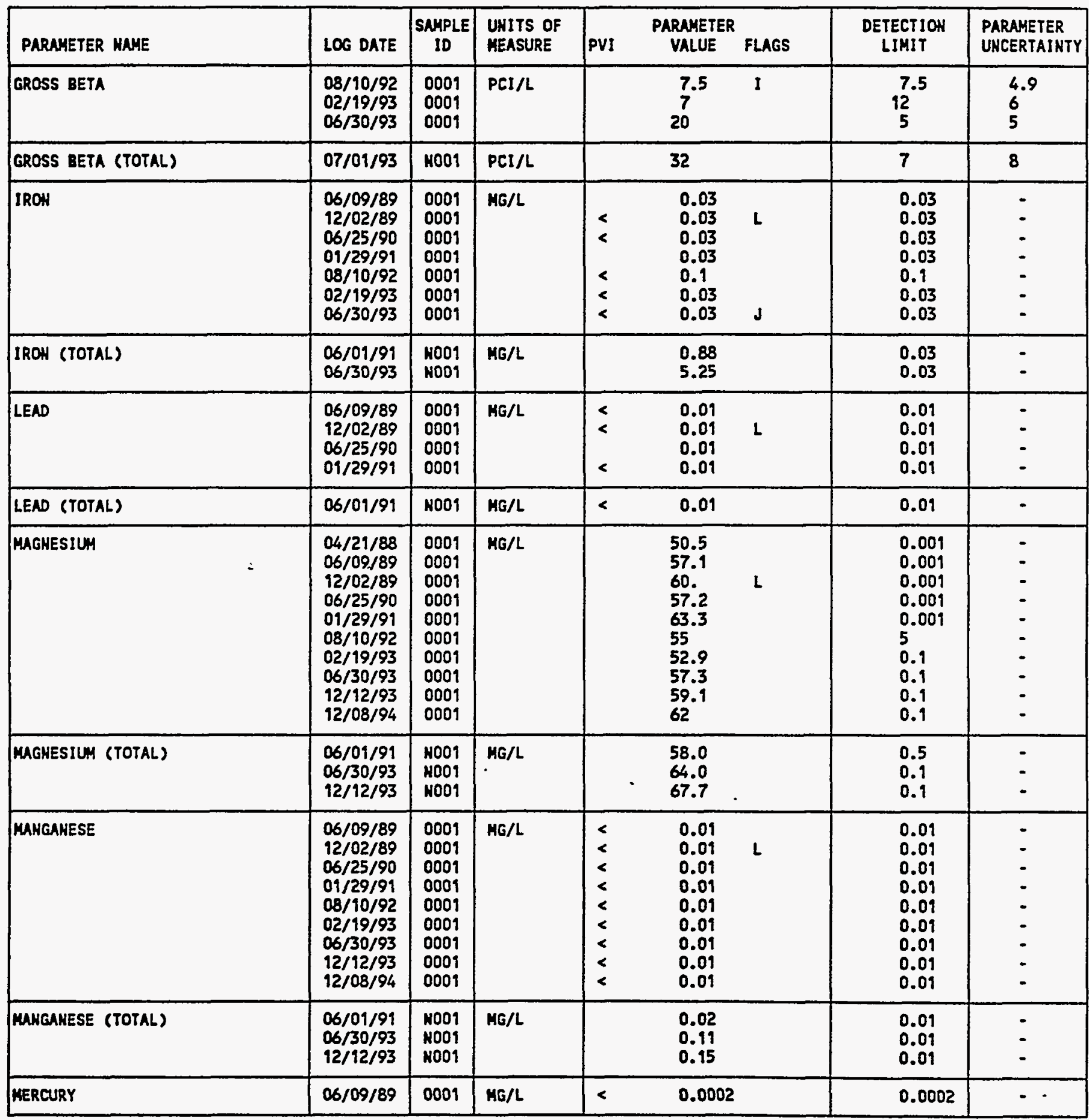

PARAMETER VALUE INOICATOR (PVI): < - LESS THAN DETECTION LIMIT

OTHER PARAMETER VALUE FLAGS:

SAMPLE IO COOES:

ODO1 - FILTERED SAMPLE (.45 MICRONS)

1 - IMCREASED DETECTION LIMIT DUE TO REQUIRED DILUTIOH

NOO1 - UNFILTERED SAMPLE

J - estimated VALUE

L. LESS THAM THREE BORE VOLUMES REMOVED BEFORE SAMPLIHG 
GROUNDWATER QUALITY DATA BY LOCATIOH

SOMP ReV. O Data

SITE: MONO1 MONUHENT VALLEY

LOCATIOH: 0614

MORTH COORDINATE: 60940.5 FT

EAST COORDINATE: 87832.6 FT

$04 / 21 / 88$ TO $12 / 13 / 94$

REPORT DATE: $06 / 20 / 95$

FORMATION OF COMPLETION: SHIMARLAP MEMBER OF THE CHIHLE FORMATION (SR) HYORAULIC FLOW RELATIOHSHIP: DOWH GRADIENT (D)

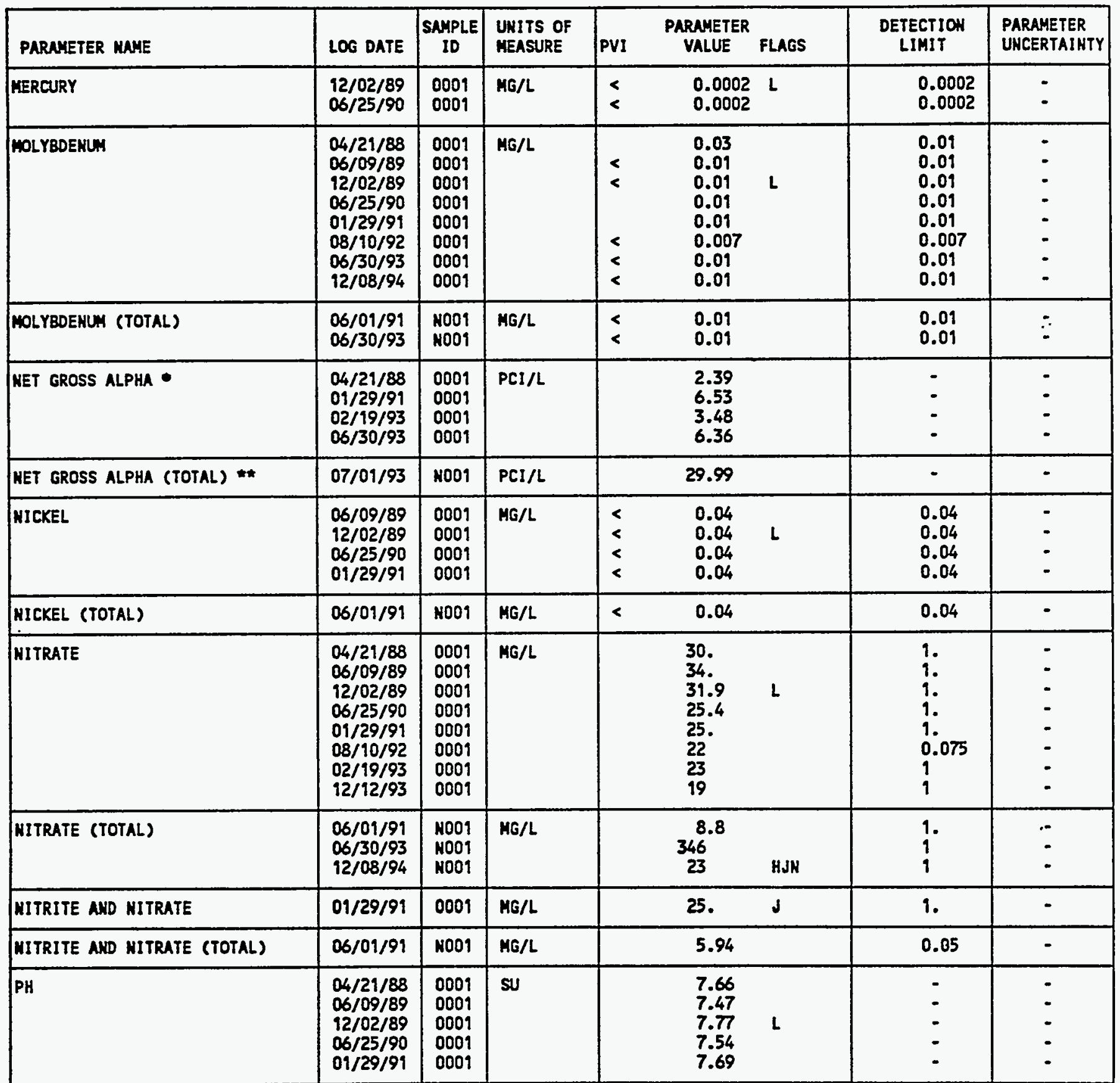

- NET gRoss alPHa (GROSS ALPHA - LRANIUM) MITH I MG URANIUM = 686 PCI

* MET GROSS ALPHA (TOTAL) (TOTAL GROSS ALPHA - TOTAL URANIUA)

PARAHETER VALUE INDICATOR (PVI): < - LESS THAN DETECTION LIMIT

SUMPLE ID COOES:

OTHER PARAMETER VALUE FLAGS:

0001 - FILTERED SAMPLE (.45 MICRONS)

H - HOLD TIME EXPIRED, VALUE SUSPECT

NOO1 - UMFILTERED SAMPLE

J - estimated VALUE

l - LESS THAN THREE BORE VOLLMES RENOVED BEFORE SAMPLING

- SPIKE SAHPLE RECOVERY NOT WITHIN CONTROL LIMITS 
GROMHDWATER QUALITY DATA BY LOCATION

SOMP Rev. $O$ Data

SITE: MONO1 MONUNENT VALLEY

LOCATION: 0614

MORTH COORDINATE: 60940.5 FT

EAST COORDIHATE: 87832.6 FT

$04 / 21 / 88$ TO $12 / 13 / 94$

REPORT DATE: $06 / 20 / 95$

FORMATIOH OF COMPLETION: SHIMARLMP MEMBER OF THE CHIMLE FORMATION (SR) HYDRAULIC FLON RELATIOHSHIP: DONN GRADIENT (D)

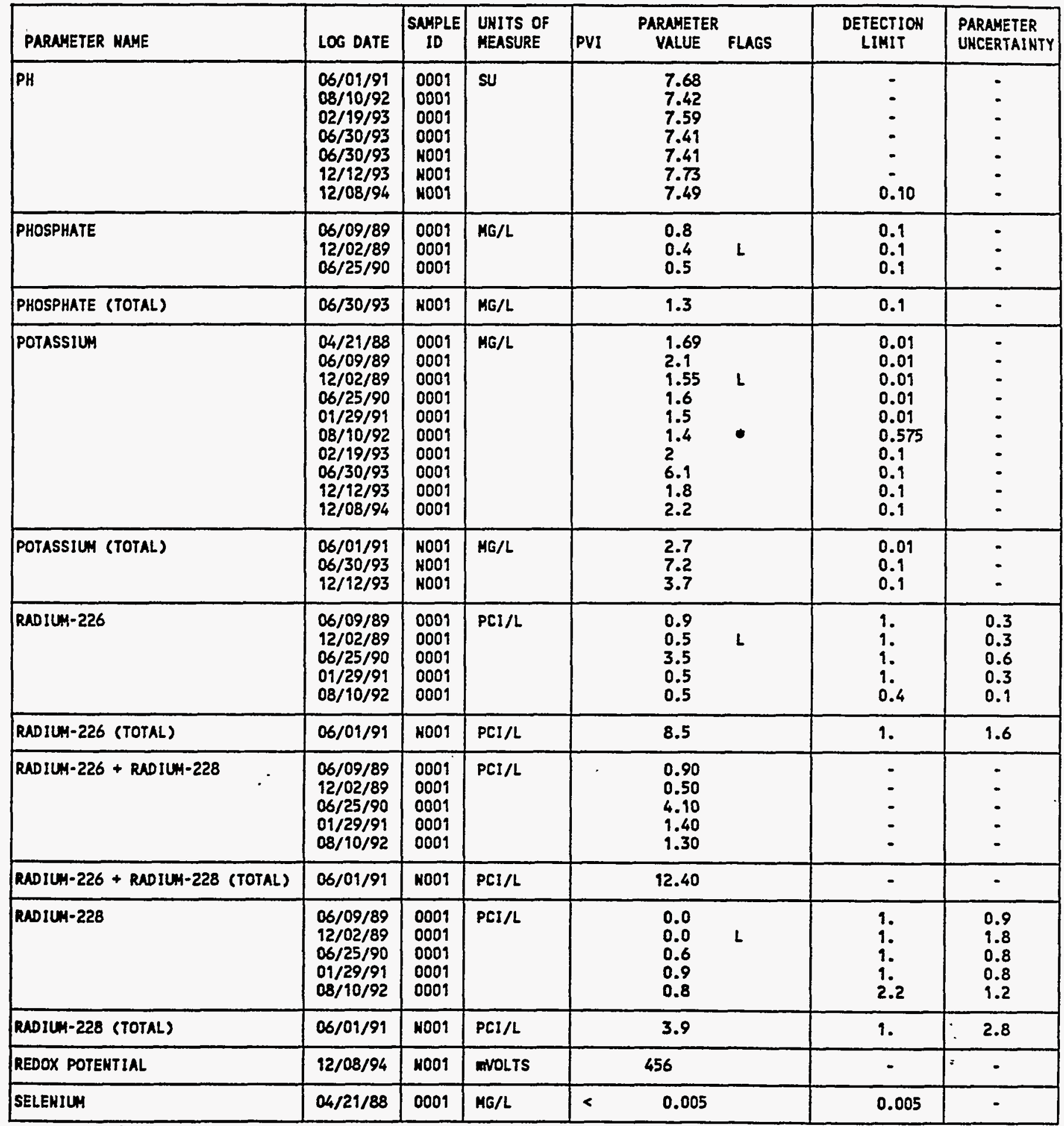

PARAMETER VALUE INDICATOR (PVI): < - LESS THAN DETECTIOH LIMIT

SMPLE ID COOES:

OTHER PARAMETER VALUE FLAGS:

OOD1 - FILTERED SAMPLE (.45 MICRONS)

- DUPLICATE AHALYSIS hOT HITHIH CONTROL LIMITS

NOO1 - UNFILTERED SAMPLE

L - LESS THAN THREE BORE VOLUHES REMOVED BEFORE SAMPLING 
GRONDHATER QUALITY DATA BY LOCATION

SOMP REV. 0 Data

SITE: MONOI MONUMEKT VALLEY

LOCATION: 0614

NORTH COORDINATE: $\quad 60940.5$ FT

EAST COORDINATE: 87832.6 FT

$04 / 21 / 88$ To $12 / 13 / 94$

REPORT DATE: 06/20/95

FORMATION OF COMPLETIOH: SHIHARUMP MEMBER OF THE CHIHLE FORMATION (SR) HYORAULIC FLON RELATIONSHIP: DOWN GRADIENT (D)

\begin{tabular}{|c|c|c|c|c|c|c|c|c|}
\hline PARAMETER MAME & LOG DATE & $\begin{array}{c}\text { SAMPLE } \\
\text { ID }\end{array}$ & $\begin{array}{l}\text { UNITS OF } \\
\text { MEASURE }\end{array}$ & PVI & $\begin{array}{l}\text { PARAMETER } \\
\text { VALUE }\end{array}$ & FLAGS & $\begin{array}{l}\text { DETECTION } \\
\text { LIMIT }\end{array}$ & $\begin{array}{l}\text { PARAMETER } \\
\text { UNCERTAINTY }\end{array}$ \\
\hline SELENIUY & $\begin{array}{l}06 / 09 / 89 \\
12 / 02 / 89 \\
06 / 25 / 90 \\
01 / 29 / 91 \\
08 / 10 / 92\end{array}$ & $\begin{array}{l}0001 \\
0001 \\
0001 \\
0001 \\
0001\end{array}$ & $M G / L$ & $\begin{array}{l}< \\
< \\
<\end{array}$ & $\begin{array}{l}0.008 \\
0.005 \\
0.007 \\
0.005 \\
0.005\end{array}$ & $\begin{array}{l}1 \\
4\end{array}$ & $\begin{array}{l}0.005 \\
0.005 \\
0.005 \\
0.005 \\
0.005\end{array}$ & $\begin{array}{l}- \\
- \\
-\end{array}$ \\
\hline SELEMIUN (TOTAL) & $06 / 01 / 91$ & No01 & MG/L & & 0.010 & & 0.005 & - \\
\hline SILICA - SIOZ & $\begin{array}{l}06 / 09 / 89 \\
12 / 02 / 89 \\
06 / 25 / 90 \\
01 / 29 / 91 \\
12 / 12 / 93 \\
12 / 08 / 94\end{array}$ & $\begin{array}{l}0001 \\
0001 \\
0001 \\
0001 \\
0001 \\
0001\end{array}$ & $M G / L$ & & $\begin{array}{l}14 . \\
13.9 \\
14 . \\
13 . \\
14.3 \\
14.9\end{array}$ & $\ell$ & $\begin{array}{l}2 . \\
2 . \\
2 . \\
2 . \\
0.1 \\
0.1\end{array}$ & $\begin{array}{l}- \\
- \\
- \\
. \\
-\end{array}$ \\
\hline SILICA - SIOZ (TOTAL) & $\begin{array}{l}06 / 01 / 91 \\
06 / 30 / 93 \\
12 / 12 / 93\end{array}$ & $\begin{array}{l}\text { No01 } \\
\text { NOOI } \\
\text { NOOI }\end{array}$ & $M G / L$ & & $\begin{array}{l}19.9 \\
26.0 \\
51.0\end{array}$ & $\mathbf{H}$ & $\begin{array}{l}0.1 \\
0.5 \\
0.1\end{array}$ & - \\
\hline SILVER & $\begin{array}{l}06 / 09 / 89 \\
12 / 02 / 89 \\
06 / 25 / 90 \\
01 / 29 / 91\end{array}$ & $\begin{array}{l}0001 \\
0001 \\
0001 \\
0001\end{array}$ & $M G / L$ & $\begin{array}{l}< \\
< \\
< \\
<\end{array}$ & $\begin{array}{l}0.01 \\
0.01 \\
0.01 \\
0.01\end{array}$ & $\mathbf{L}$ & $\begin{array}{l}0.01 \\
0.01 \\
0.01 \\
0.01\end{array}$ & $\begin{array}{l}- \\
- \\
-\end{array}$ \\
\hline SILVER (TOTAL) & $08 / 01 / 91$ & No01 & MG/L & $<$ & 0.01 & & 0.01 & - \\
\hline SooIum & $\begin{array}{l}04 / 21 / 88 \\
06 / 09 / 89 \\
12 / 02 / 89 \\
06 / 25 / 90 \\
01 / 29 / 91 \\
08 / 10 / 92 \\
02 / 19 / 93 \\
06 / 30 / 93 \\
12 / 12 / 93 \\
12 / 08 / 94\end{array}$ & $\begin{array}{l}0001 \\
0001 \\
0001 \\
0001 \\
0001 \\
0001 \\
0001 \\
0001 \\
0001 \\
0001\end{array}$ & $M G / L$ & & $\begin{array}{l}27.2 \\
29.8 \\
29 . \\
31.5 \\
31.4 \\
30 \\
29.3 \\
34 \\
40 \\
43.7\end{array}$ & $\mathbf{L}$ & $\begin{array}{l}0.002 \\
0.002 \\
0.002 \\
0.002 \\
0.002 \\
5 \\
0.1 \\
1 \\
1 \\
1\end{array}$ & $\begin{array}{l}- \\
- \\
- \\
- \\
- \\
- \\
-\end{array}$ \\
\hline SODTUN (TOTAL) & $\begin{array}{l}06 / 01 / 91 \\
06 / 30 / 93 \\
12 / 12 / 93\end{array}$ & $\begin{array}{l}\text { No01 } \\
\text { No01 } \\
\text { N001 }\end{array}$ & MG/L & & $\begin{array}{l}33 \\
34 \\
43\end{array}$ & & $\begin{array}{l}1 . \\
1 \\
1\end{array}$ & $\begin{array}{l}- \\
-\end{array}$ \\
\hline SPECIFIC CONDUCTAHCE & $\begin{array}{l}04 / 21 / 88 \\
06 / 09 / 89 \\
12 / 02 / 89 \\
06 / 25 / 90 \\
01 / 29 / 91 \\
06 / 01 / 91 \\
08 / 10 / 92 \\
02 / 19 / 93 \\
06 / 30 / 93 \\
06 / 30 / 93 \\
12 / 12 / 93 \\
12 / 08 / 94\end{array}$ & $\begin{array}{l}0001 \\
0001 \\
0001 \\
0001 \\
0001 \\
0001 \\
0001 \\
0001 \\
0001 \\
N 001 \\
\text { HoO1 } \\
\text { No01 }\end{array}$ & UNHO/CH & & $\begin{array}{l}700 . \\
510 . \\
700 . \\
780 \\
925 . \\
950 . \\
853 \\
719 \\
850 \\
850 \\
878 \\
856\end{array}$ & J & $\begin{array}{l}- \\
- \\
- \\
- \\
- \\
- \\
- \\
- \\
-\end{array}$ & $\begin{array}{l}- \\
- \\
- \\
- \\
- \\
- \\
- \\
- \\
- \\
-\end{array}$ \\
\hline
\end{tabular}

PARAMETER VALUE INDICATOR (PVI): < - LESS THAN DETECTIOH LIHIT

SAMPLE ID CODES:

OOO1 - FILTERED SAMPLE (.45 MICROAS)

OTHER PARNAETER VALUE FLAGS:

NOO1 - UAFILTERED SAMPLE

H - HOLD TIME EXPIRED, VALUE SUSPECT

d - ESTIMATED VALUE

L - LESS THAH THREE BORE VOLUAES REMOVED BEFORE SAMPLIHG

4 - POST-DIGEST SPIKE OUT OF CHTR LIM HHILE SHP ABS < 50\% SPIKE 
GROUMDHATER QUALITY DATA BY LOCATION

SOUP Rev. 0 Data

SITE: MONO1 MONUAENT VALLEY

LOCATIOH: 0614

MORTH COORDINATE: 60940.5 FT

EAST COORDIHATE: 87832.6 FT

Q4/21/88 TO $12 / 13 / 94$

REPORT DATE: 06/20/95

FORHATION OF COMPLETIOH: SHINARUMP MEMBER OF THE CHINLE FORMATION (SR) HYDRAULIC FLOW RELATIONSHIP: DOWN GRADIENT (D)

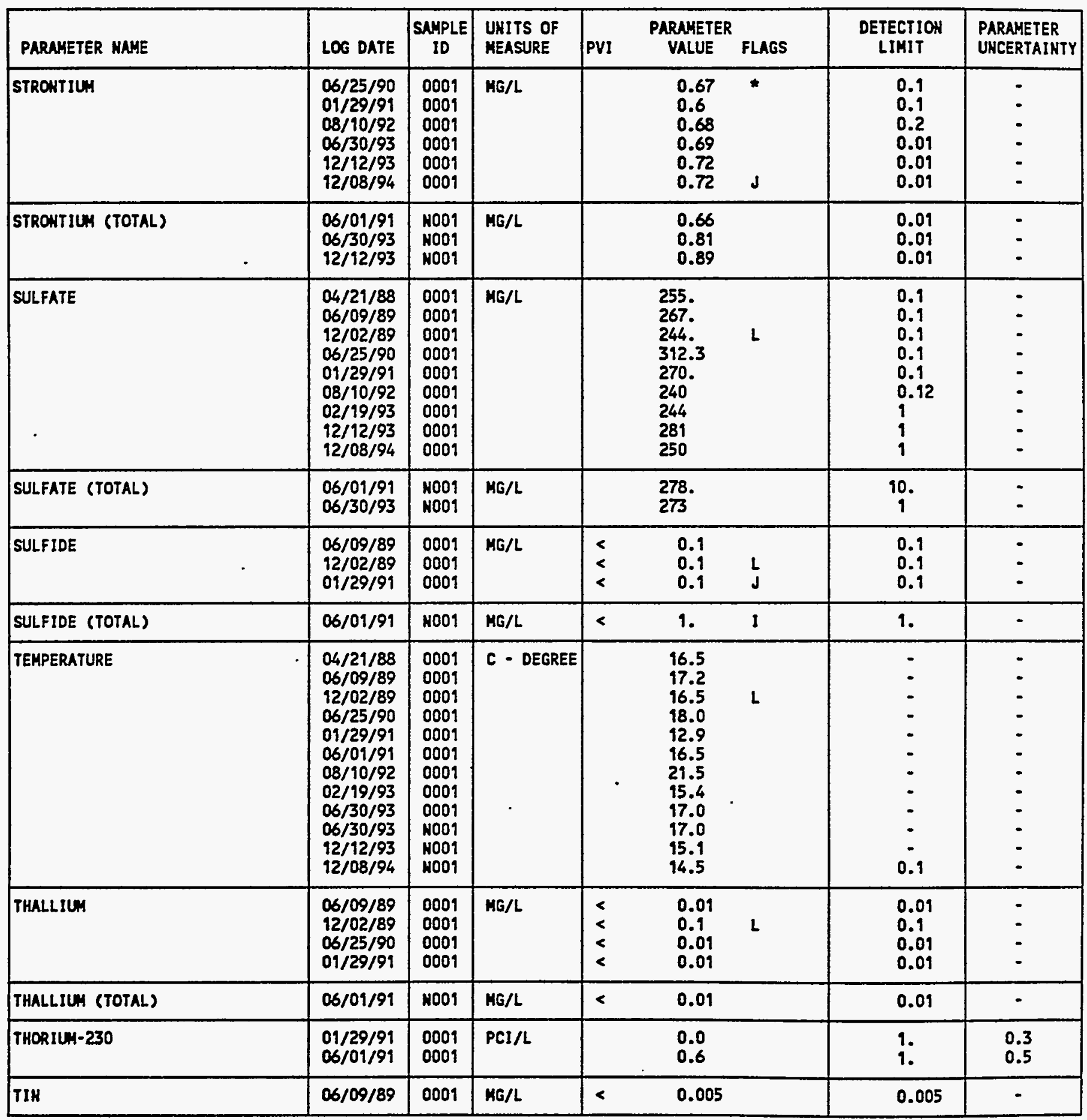

PARAMETER VALUE IMDICATOR (PVI): < - LESS than DETECTION LIMIT

SAMPLE ID COOES:

OTHER PARUMETER VALUE FLAGS:

O001 - FILTERED SAMPLE (.45 MICROHS)

- - OUPLICATE aHALYSIS NOT HITHIN CONTROL LIMITS

NOOI - UAFILTERED SAMPLE

1 - IHCREASED DETECTION LIMIT DUE TO REQUIRED DILUTION

J - ESTIMATED VALUE

L - LESS THAN THREE BORE VOLLHES REMOVED BEFORE SAMPLING 
GROUNDWATER QUALITY DATA BY LOCATION

SOWP ReV. O Data

SITE: MONOI MONLWENT VALLEY

LOCATIOH: 0614

MORTH COORDINATE: 60940.5 FT

EAST COORDIHATE: $\quad 87832.6$ FT

04/21/88 TO 12/13/94

REPORT DATE: $06 / 20 / 95$

FORMATION OF COMPLETION: SHIMARLMP MEMBER OF THE CHIMLE FORMATION (SR) HYDRAULIC FLON RELATIONSHIP: DOWN GRADIENT (D)

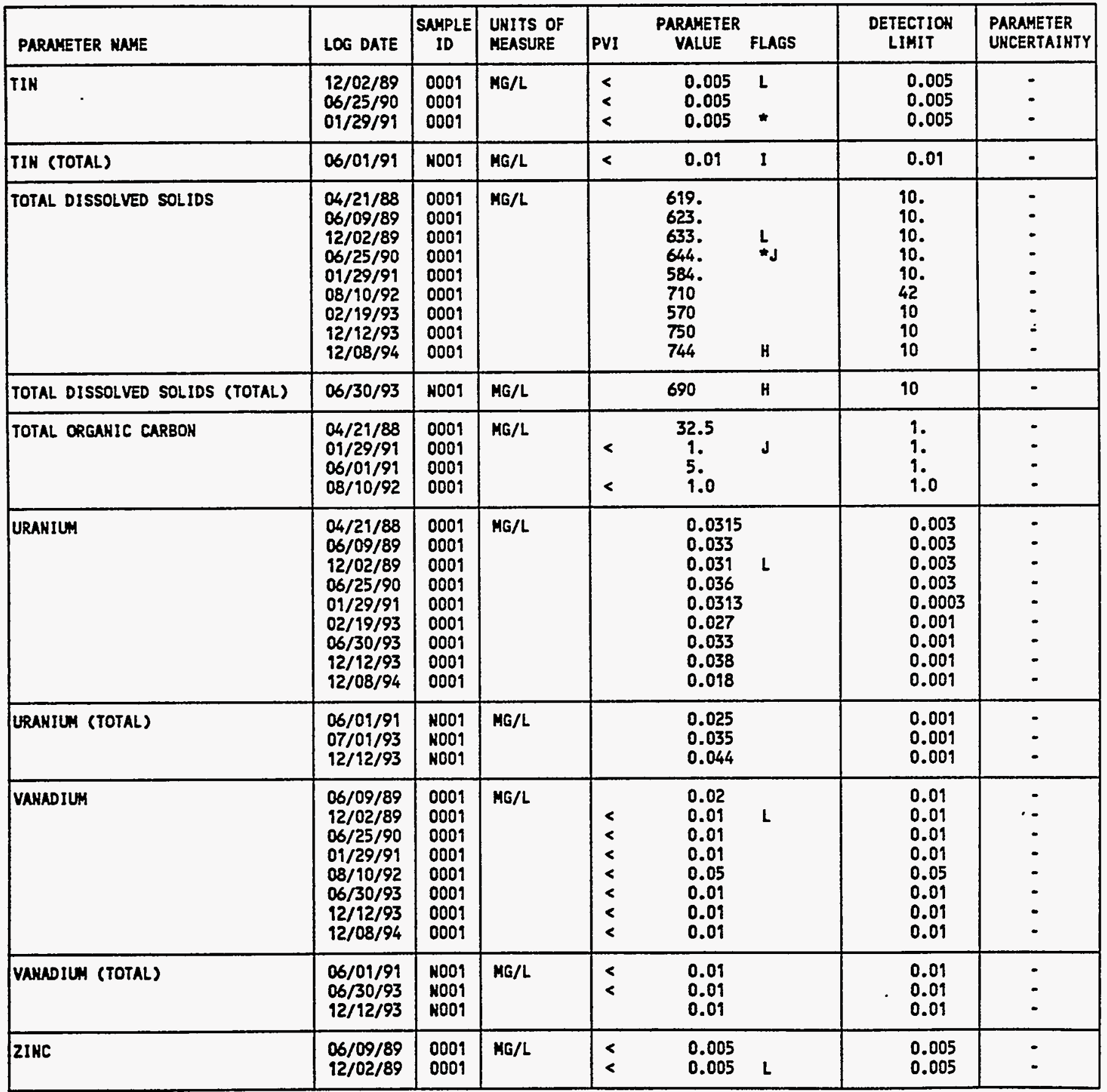

PARAMETER VALUE IMDICATOR (PVI): < - LESS THAN DETECTION LIMIT

SAMPLE IO COOES:

DTHER PARAMETER VALUE FLAGS:

0001 - FILTERED SAMPLE (.45 MICRONS)

* - DUPLICATE AMALYSIS NOT MITHIN CONTROL LIMITS

NOO1 - UHFILTERED SAMPLE

H - HOLD TIME EXPIRED, VALUE SUSPECT

I - IMCREASED DETECTION LIMIT DUE TO REQUIRED DILUTION

J - ESTIMATED VALUE

L - LESS THAN THREE BORE VOLLHES REMOVED BEFORE SAMPLIHG 
GROUMDHATER QUALITY DATA BY LOCATION

SOWP Rev. 0 Data

SITE: MONO1 MONUNENT VALLEY

LOCATION: 0614

MORTH COORDIMATE: $\quad 60940.5$ FT

EAST COORDINATE: 87832.6 FT

O4/21/88 TO 12/13/94

REPORT DATE: 06/20/95

FORMATION OF COMPLETION: SHIHARUMP MEMBER OF THE CHIMLE FORMATION (SR) HYORAULIC FLON RELATIOHSHIP: DOWN GRADIENT (D)

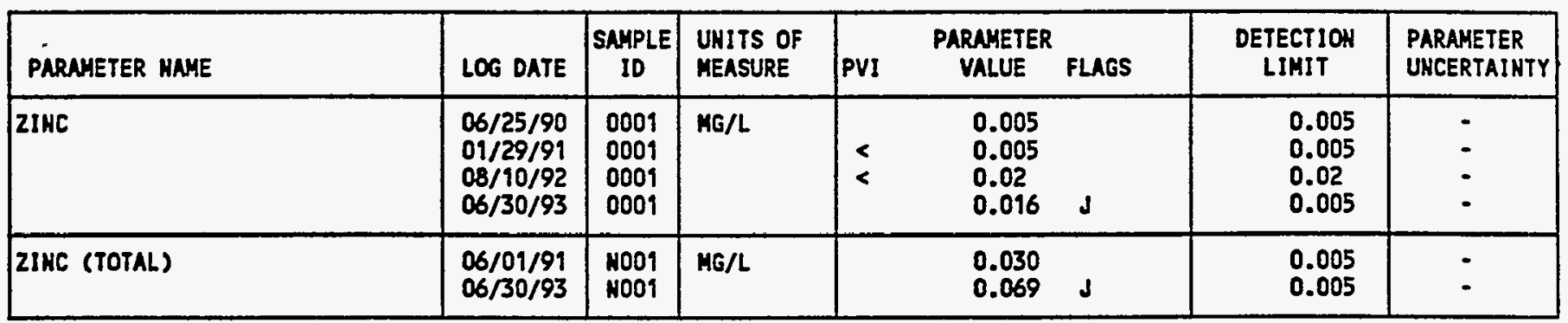

PARAMETER VALUE IMDICATOR (PVI):

< - LESS than detection limit

SNMPLE ID COOES:

OTHER PARAMETER VALUE fLAGS:

OOO1 - FILTERED SAMPLE (.45 MICRONS)

d- ESTIMATED VALUE

NOD1 - UNFILTERED SAMPLE 
GROUNOHATER QUALITY DATA BY LOCATION

SOWP ReV. O Data

SITE: MONO1 HONLNENT VALLEY

LOCATIOH: 0615

MORTH COOROINATE: 57794.4 FT

EAST COORDIHATE: 88980.7 FT

$04 / 21 / 88$ TO $12 / 13 / 94$

REPORT DATE: 06/20/95

FORMATIOH OF COMPLETION: SHIMARUMP MEMBER OF THE CHINLE FORHATION (SR) HYDRAULIC FLOU RELATIONSHIP: CROSS GRADIENT (C)

\begin{tabular}{|c|c|c|c|c|c|c|c|c|}
\hline PARANETER NAME & LOG DATE & $\mid \begin{array}{c}\text { SAMPLE } \\
\text { ID }\end{array}$ & $\begin{array}{l}\text { UNITS OF } \\
\text { MEASURE }\end{array}$ & PVI & $\begin{array}{c}\text { PARAMETER } \\
\text { VALUE }\end{array}$ & FLAGS & $\begin{array}{l}\text { DETECTION } \\
\text { LIMIT }\end{array}$ & $\begin{array}{l}\text { PARAMETER } \\
\text { UHCERTAIHTY }\end{array}$ \\
\hline ALKALINITY & $\begin{array}{l}06 / 09 / 89 \\
11 / 23 / 92 \\
06 / 29 / 93\end{array}$ & $\begin{array}{l}0001 \\
0001 \\
1001\end{array}$ & MG/L CACO3 & & $\begin{array}{l}230 . \\
226 \\
212\end{array}$ & $\begin{array}{l}1 \\
l\end{array}$ & - & - \\
\hline ALUHIRUM & $06 / 09 / 89$ & 0001 & $M G / L$ & $<$ & 0.1 & & 0.1 & - \\
\hline AMHONIU⿴囗十⺝ & $06 / 09 / 89$ & 0009 & $M G / L$ & $<$ & 0.9 & & 0.1 & - \\
\hline AMSONIUH (TOTAL) & $06 / 29 / 93$ & NoOI & $M G / L$ & $<$ & 0.1 & $\mathbf{L}$ & 0.1 & - \\
\hline ANTIMONY & $06 / 09 / 89$ & 0001 & HG/L & $<$ & 0.003 & & 0.003 & - \\
\hline ARSENIC & $\begin{array}{l}06 / 09 / 89 \\
11 / 23 / 92 \\
06 / 29 / 93\end{array}$ & $\begin{array}{l}0001 \\
0001 \\
0001\end{array}$ & MG/L & $\begin{array}{l}< \\
<\end{array}$ & $\begin{array}{l}0.01 \\
0.0015 \\
0.005\end{array}$ & $L$ & $\begin{array}{l}0.01 \\
0.0015 \\
0.005\end{array}$ & : \\
\hline ARSENIC (TOTAL) & $\begin{array}{l}19 / 23 / 92 \\
06 / 29 / 93\end{array}$ & $\begin{array}{l}\text { NOO1 } \\
\text { HOO1 }\end{array}$ & MG/L & & $\begin{array}{l}0.022 \\
0.019\end{array}$ & $L$ & $\begin{array}{l}0.0015 \\
0.005\end{array}$ & $\dot{-}$ \\
\hline BARIUA & $\begin{array}{l}06 / 09 / 89 \\
11 / 23 / 92 \\
06 / 29 / 93\end{array}$ & $\begin{array}{l}0001 \\
0001 \\
0001\end{array}$ & $M G / L$ & $<$ & $\begin{array}{l}0.1 \\
0.038 \\
0.9\end{array}$ & $L$ & $\begin{array}{l}0.1 \\
0.0015 \\
0.1\end{array}$ & $\begin{array}{l}- \\
-\end{array}$ \\
\hline BARIUH (TOTAL) & $\begin{array}{l}19 / 23 / 92 \\
06 / 29 / 93\end{array}$ & $\begin{array}{l}\text { NOOI } \\
\text { HOOI }\end{array}$ & $M G / L$ & $<$ & $\begin{array}{l}0.070 \\
0.9\end{array}$ & $L$ & $\begin{array}{l}0.0015 \\
0.1\end{array}$ & $\dot{-}$ \\
\hline BERYLLIUN & $06 / 09 / 89$ & 0001 & $M G / L$ & $<$ & 0.01 & & 0.01 & - \\
\hline BORON & $06 / 09 / 89$ & 0001 & $M G / L$ & $<$ & 0.1 & & 0.1 & - \\
\hline CADHILA & $\begin{array}{l}06 / 09 / 89 \\
11 / 23 / 92\end{array}$ & $\begin{array}{l}0001 \\
0001\end{array}$ & MG/L & $<$ & $\begin{array}{l}0.009 \\
0.00013\end{array}$ & 31 & $\begin{array}{l}0.001 \\
0.00013\end{array}$ & : \\
\hline CADMIUN (TOTAL) & $91 / 23 / 92$ & NoOr & MG/L & & 0.00032 & 2 NSL & 0.00013 & - \\
\hline calcius & $\begin{array}{l}06 / 09 / 89 \\
06 / 29 / 93\end{array}$ & $\begin{array}{l}0001 \\
0001\end{array}$ & MG/L & - & $\begin{array}{l}18.9 \\
16.9\end{array}$ & $L$ & $\begin{array}{l}0.01 \\
0.5\end{array}$ & - \\
\hline CALCIUN (TOTAL) & $06 / 29 / 93$ & ห०O & MG/L & & 16.1 & $L$ & 0.5 & - \\
\hline CHLORIDE & $06 / 09 / 89$ & 0001 & $M G / L$ & & 9.9 & & 1. & - \\
\hline CHLORIDE (TOTAL) & $06 / 29 / 93$ & NoOI & MG/L & & 9.6 & $\mathbf{L}$ & 0.5 & - \\
\hline ChROHIUH & $\begin{array}{l}06 / 09 / 89 \\
11 / 23 / 92\end{array}$ & $\begin{array}{l}0001 \\
0001\end{array}$ & $M G / L$ & $<$ & $\begin{array}{l}0.01 \\
0.0053\end{array}$ & $\mathbf{L}$ & $\begin{array}{l}0.09 \\
0.0053\end{array}$ & - \\
\hline CHROHIUH (TOTAL) & $11 / 23 / 92$ & NoO1 & $M G / L$ & $<$ & 0.0053 & $L$ & 0.0053 & - \\
\hline COBALT & $06 / 09 / 89$ & 0001 & $M G / L$ & $<$ & 0.05 & & 0.05 & - \\
\hline COPPER & $06 / 09 / 89$ & 0001 & MG/L & $<$ & 0.02 & & 0.02 & - \\
\hline CranioE & $06 / 09 / 89$ & 0001 & $M G / L$ & $<$ & 0.01 & & 0.01 & - \\
\hline DISSOLVED OXYGEN & $06 / 29 / 93$ & N001 & MG/L & & 1.1 & $L$ & - & - \\
\hline
\end{tabular}

PARAMETER VALUE IHDICATOR (PVI): < - LESS THAH DETECTION LIHIT

OTHER PARAYETER VALUE FLAGS:

- L - LESS THAN THREE BORE VOLUMES REMOVED BEFORE SAMPLING

SNMPLE ID CODES:

0001 - FILTERED SAMPLE (.45 HICROMS)

NOO1 - LHFILTERED SAMPLE

H - SPIKE SAMPLE RECONERY NOT HITHIK CONTROL LIHITS

S - REPORTED VALUE DETERMIHED USIMG RETHOO OF STO ADDITION (MSA) 
GROUNDHATER QUALITY DATA BY LOCATION

SOWP Rev. O Data

SITE: MONO MONUMENT VALLEY

LOCATIOH: 0615

NORTH COORDINATE: 57794.4 FT

EAST COORDINATE: 88980.7 FT

$04 / 21 / 88$ TO $12 / 13 / 94$

REPORT DATE: $06 / 20 / 95$

FORMATION OF COMPLETIOH: SHIMARUMP MEMBER OF THE CHIMLE FORMATIOH (SR)

HYORAULIC FLOW RELATIONSHIP: CROSS GRADIENT (C)

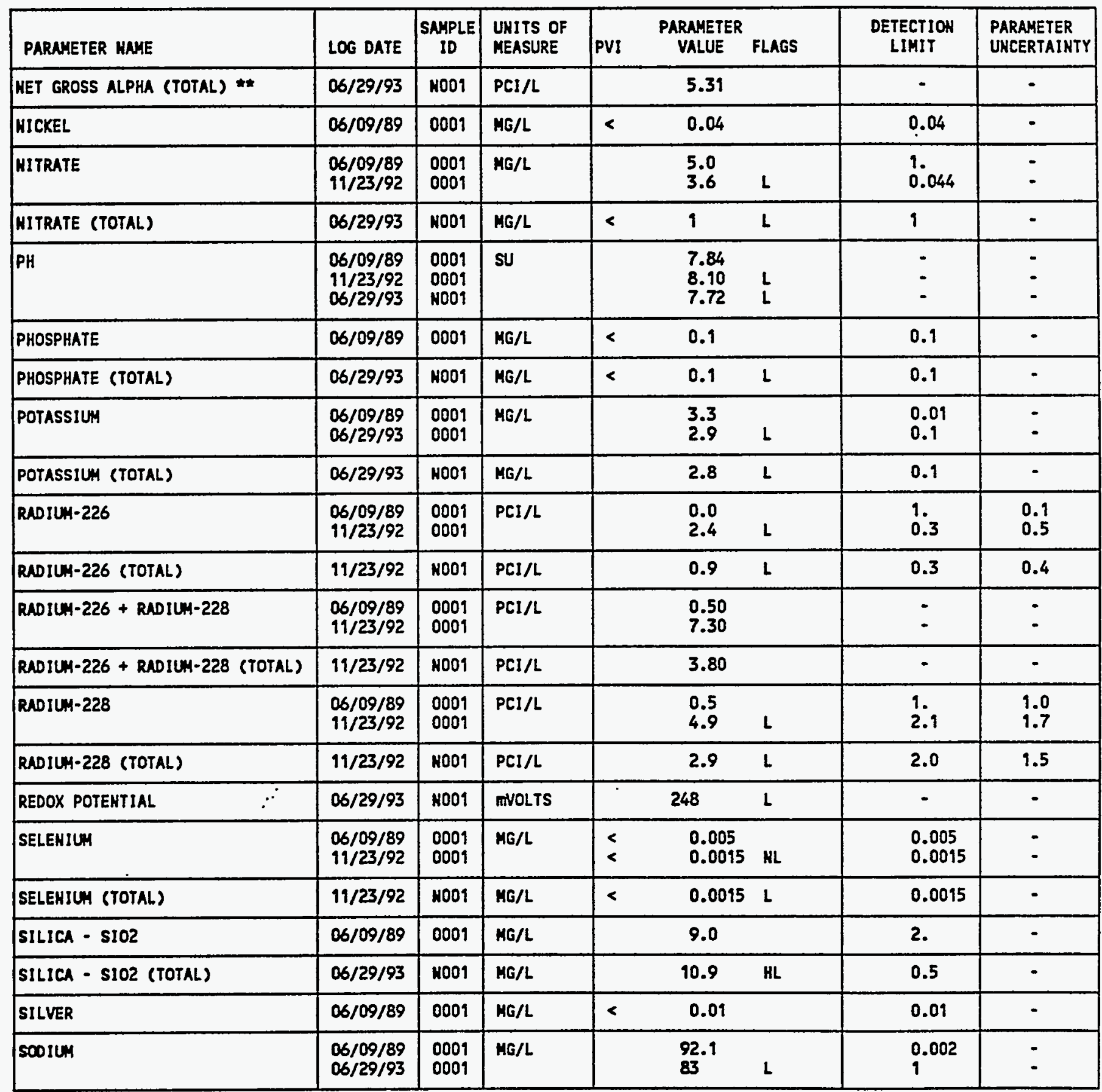

* NET GROSS ALPHA (TOTAL) (TOTAL GROSS ALPHA - TOTAL URAMIUM)

PARAMETER VALUE IMDICATOR (PVI): < - LESS THAN DETECTION LIMIT

H - HOLD TIME EXPIRED, VALUE SUSPECT

0001 - FILTERED SAMPLE (.45 MICRONS)

NOO1 - UAFILTERED SAMPLE

L - LESS THAN THREE BORE VOLUMES REMOVED BEFORE SAMPLIHG

H - SPIKE SAMPLE RECOVERY NOT UITHIN CONTROL LIMITS 
GROUNDHATER QUALITY DATA BY LOCATIOH

SOWP ReV. O Data

SITE: HONO1 MONUMENT VALLEY

LOCATION: 0615

MORTH COORDIHATE: 57794.4 FT

EAST COORDINATE: $\quad 88980.7$ FT

$04 / 21 / 88$ TO $12 / 13 / 94$

REPORT DATE: $06 / 20 / 95$

FORMATION OF CONPLETION: SHIHARUAP MEMBER OF THE CHINLE FORMATIOH (SR) HYDRAULIC FLON RELATIONSHIP: CROSS GRADIENT (C)

\begin{tabular}{|c|c|c|c|c|c|c|c|c|}
\hline PARAMETER NAME & LOG DATE & $\underset{10}{\text { SAMPLE }}$ & $\begin{array}{l}\text { UNITS OF } \\
\text { MEASURE }\end{array}$ & PVI & $\begin{array}{l}\text { PARAMETER } \\
\text { VALUE }\end{array}$ & FLAGS & $\begin{array}{l}\text { DETECTION } \\
\text { LIMIT }\end{array}$ & $\begin{array}{l}\text { PARAMETER } \\
\text { UHCERTAINTY }\end{array}$ \\
\hline FLUORIDE & $06 / 09 / 89$ & 0001 & MG/L & & 0.4 & & 0.1 & - \\
\hline GROSS ALPHA & $\begin{array}{l}11 / 23 / 92 \\
06 / 29 / 93\end{array}$ & $\begin{array}{l}0001 \\
0001\end{array}$ & $\mathrm{PCl} / \mathrm{L}$ & & $\begin{array}{l}3.5 \\
0\end{array}$ & $\begin{array}{l}\text { KL } \\
L\end{array}$ & $\begin{array}{l}3.0 \\
3\end{array}$ & $\begin{array}{l}2.5 \\
4\end{array}$ \\
\hline GROSS ALPHA (TOTAL) & $\begin{array}{l}11 / 23 / 92 \\
06 / 29 / 93\end{array}$ & $\begin{array}{l}\text { N001 } \\
\text { N001 }\end{array}$ & $\mathrm{PCI} / \mathrm{L}$ & & $\begin{array}{l}0.0 \\
6\end{array}$ & $\stackrel{M L}{L}$ & $\begin{array}{l}6.7 \\
3\end{array}$ & $\begin{array}{l}3.6 \\
5\end{array}$ \\
\hline GROSS BETA & $\begin{array}{l}11 / 23 / 92 \\
06 / 29 / 93\end{array}$ & $\begin{array}{l}0001 \\
0001\end{array}$ & $\mathrm{PCI} / \mathrm{L}$ & & $\begin{array}{l}1.0 \\
0\end{array}$ & $L$ & $\begin{array}{l}3.6 \\
4\end{array}$ & $\begin{array}{l}2.2 \\
4\end{array}$ \\
\hline GROSS BETA (TOTAL) & $\begin{array}{l}11 / 23 / 92 \\
06 / 29 / 93\end{array}$ & $\begin{array}{l}\text { No01 } \\
\text { N001 }\end{array}$ & $\mathrm{PCI} / \mathrm{L}$ & & 1.5 & $\begin{array}{l}\text { HL } \\
L\end{array}$ & $\begin{array}{l}5.0 \\
4\end{array}$ & $\begin{array}{l}3.1 \\
4\end{array}$ \\
\hline IROH & $\begin{array}{l}06 / 09 / 89 \\
06 / 29 / 93\end{array}$ & $\begin{array}{l}0001 \\
0001\end{array}$ & MG/L & $<$ & $\begin{array}{l}0.25 \\
0.03\end{array}$ & JL & $\begin{array}{l}0.03 \\
0.03\end{array}$ & $\dot{-}$ \\
\hline IRON (TOTAL) & $06 / 29 / 93$ & N001 & $M G / L$ & & 2.95 & $\mathbf{L}$ & 0.03 & - \\
\hline LEAD & $\begin{array}{l}06 / 09 / 89 \\
11 / 23 / 92\end{array}$ & $\begin{array}{l}0001 \\
0001\end{array}$ & $M G / L$ & $<$ & $\begin{array}{l}0.01 \\
0.0015\end{array}$ & $\mathbf{L}$ & $\begin{array}{l}0.01 \\
0.0015\end{array}$ & - \\
\hline LEAD (TOTAL) & $11 / 23 / 92$ & NOOI & $M G / L$ & & 0.0022 & $L$ & 0.0015 & - \\
\hline LEAD-210 & $11 / 23 / 92$ & 0001 & $\mathrm{PCI} / \mathrm{L}$ & & 1.0 & $\mathbf{L}$ & 7.3 & 4.4 \\
\hline LEAD-290 (TOTAL) & $11 / 23 / 92$ & N001 & PCI/L & & 7.2 & $\mathbf{L}$ & 0.3 & 1.0 \\
\hline MGGESIUN & $\begin{array}{l}06 / 09 / 89 \\
06 / 29 / 93\end{array}$ & $\begin{array}{l}0001 \\
0001\end{array}$ & $M G / L$ & & $\begin{array}{l}16.2 \\
16.0\end{array}$ & $\mathbf{L}$ & $\begin{array}{l}0.001 \\
0.1\end{array}$ & - \\
\hline MAGHESIUM (TOTAL) & $06 / 29 / 93$ & N001 & $M G / L$ & & 15.4 & $L$ & 0.1 & - \\
\hline $\begin{array}{c}\text { MUNGANESE } \\
.\end{array}$ & $\begin{array}{l}06 / 09 / 89 \\
11 / 23 / 92 \\
06 / 29 / 93\end{array}$ & $\begin{array}{l}0001 \\
0001 \\
0001\end{array}$ & $M G / L$ & $<$ & $\begin{array}{l}0.01 \\
0.0017 \\
0.01\end{array}$ & $L$ & $\begin{array}{l}0.01 \\
0.0015 \\
0.01\end{array}$ & $\dot{-}$ \\
\hline MANGAKESE (TOTAL) & $\begin{array}{l}11 / 23 / 92 \\
06 / 29 / 93\end{array}$ & $\begin{array}{l}\text { No01 } \\
\text { N001 }\end{array}$ & $M G / L$ & & $\begin{array}{l}0.27 \\
0.42\end{array}$ & $\begin{array}{l}L \\
L\end{array}$ & $\begin{array}{l}0.0015 \\
0.01\end{array}$ & $\ddot{-}$ \\
\hline MERCURY & $06 / 09 / 89$ & 0001 & $M G / L$ & $<$ & 0.0002 & & 0.0002 & - \\
\hline MOLYBDENUN & $\begin{array}{l}06 / 09 / 89 \\
11 / 23 / 92 \\
06 / 29 / 93\end{array}$ & $\begin{array}{l}0001 \\
0001 \\
0001\end{array}$ & MG/L & $\begin{array}{l}< \\
< \\
<\end{array}$ & $\begin{array}{l}0.01 \\
0.0049 \\
0.01\end{array}$ & $\begin{array}{l}N L \\
L\end{array}$ & $\begin{array}{l}0.01 \\
0.0049 \\
0.01\end{array}$ & . \\
\hline MOLYBDENUN (TOTAL) & $\begin{array}{l}11 / 23 / 92 \\
06 / 29 / 93\end{array}$ & $\begin{array}{l}\text { N001 } \\
\text { N001 }\end{array}$ & $\mathrm{MG} / \mathrm{L}$ & $<$ & $\begin{array}{l}0.0060 \\
0.01\end{array}$ & $\begin{array}{l}M L \\
L\end{array}$ & $\begin{array}{l}0.0049 \\
0.01\end{array}$ & - \\
\hline HET GROSS ALPHA * & $\begin{array}{l}11 / 23 / 92 \\
06 / 29 / 93\end{array}$ & $\begin{array}{l}0001 \\
0001\end{array}$ & PCI/L & & $\begin{array}{r}2.81 \\
-1.37\end{array}$ & & - & - \\
\hline HET. GROSS ALPHA (TOTAL) \# & $11 / 23 / 92$ & no01 & PCI/L & & -0.69 & & - & - \\
\hline
\end{tabular}

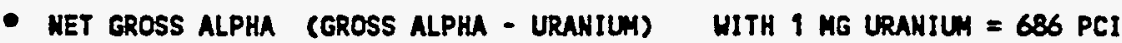

- hET gROSS ALPHA (TOTAL) (TOTAL GROSS ALPHA - TOTAL URANIUM)

PARAMETER VALUE INDICATOR (PVI): < - LESS THAN DETECTION LIMIT

SAMPLE ID COOES:

other parameter VALUE fLAGS:

O001 - FILTERED SNMPLE (.45 MICRONS)

J - ESTIMUTED VALUE

NOOI - UAFILTERED SAMPLE

L - LESS THAN THREE BORE VOLUMES REMOVED BEFORE SAMPLING

N - SPIKE SNMPLE RECOVERY NOT HITHIN CONTROL LIMITS 
GROUHDWATER QUALITY DATA BY LOCATIOH

SOWP ReV. O Dats

SITE: HOWO

LOCATIOH: 0615

HORTH COORDIHATE: 57794.4 FT

EAST COORDINATE: 88980.7 FT

$04 / 21 / 88$ TO $12 / 13 / 94$

REPORT DATE: 06/20/95

FORMATION OF COAPLETION: SHIMARUMP MEMBER OF THE CHINLE FORMATION (SR) HYDRAULIC FLON RELATIONSHIP: CROSS GRADIENT (C)

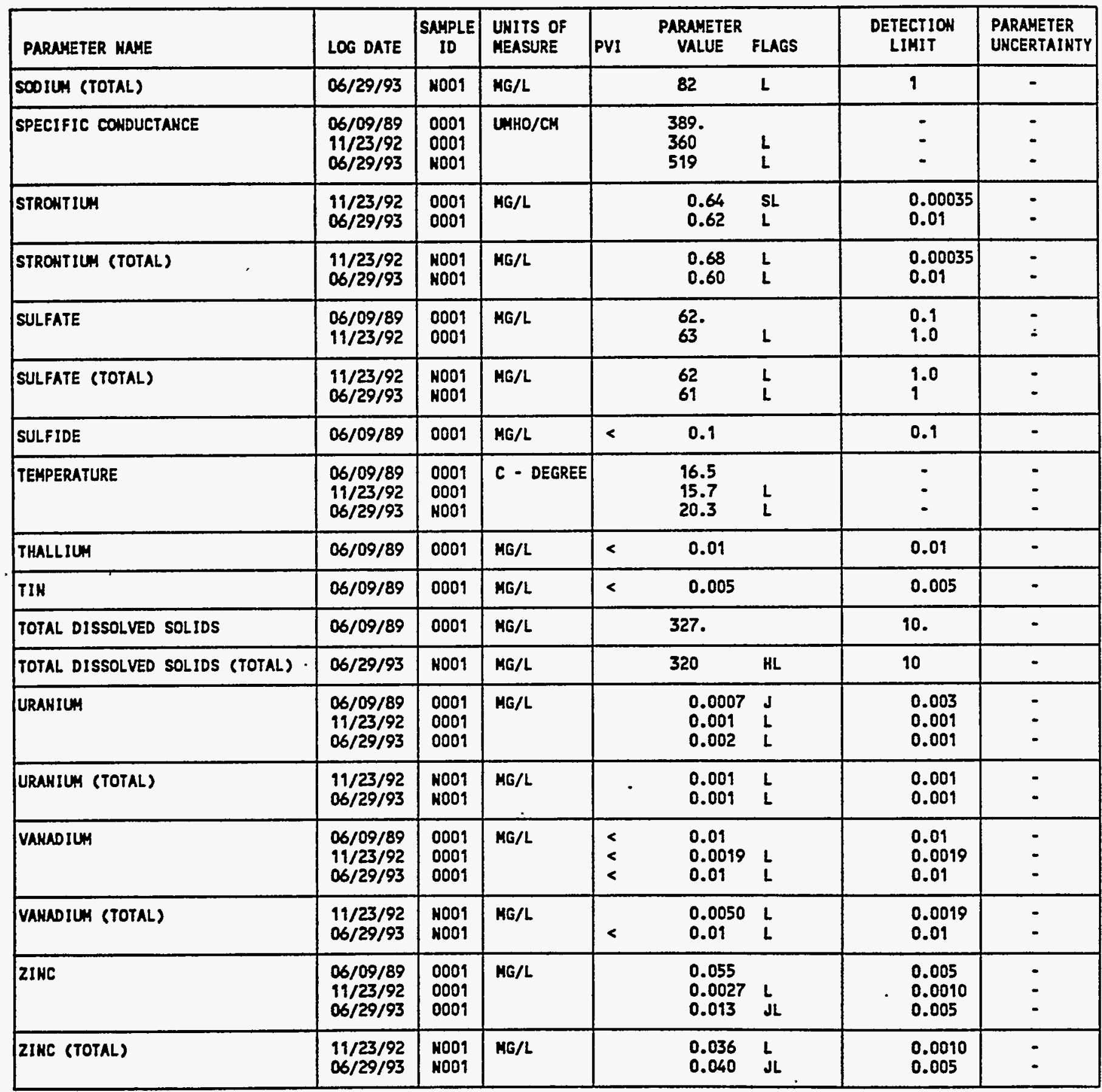

PARAMETER VALUe INOICATOR (PVI): < - LeSS than DETECTION LIMIT

OTHER PARAMETER VALUE FLAGS:

H - HOLD TIKE EXPIRED, VALUE SUSPECT

J - ESTIMLTED VALUE

L - LESS THAN THREE gORE VOLLMES REMOVED BEFORE SAHPLING

S - REPORTED VALUE DETERHIMED USIHG METHDO OF STD ADDITION (MSA)
SAMPLE ID COOES:

OODI - FILTERED SAMPLE (.45 MICRONS)

NOO1 - LNFILTERED SAMPLE 
GROUNDWATER QUALITY DATA BY LOCATION

Soup Rev. O Data

SITE: MOHOI MONLMENT VALLEY

LOCNTIOH: 0616

NORTH COORDIHATE: 56747.3 FT

EAST COORDINATE: 87987.6 FT

O4/21/88 TO $12 / 13 / 94$

REPORT DATE: 06/20/95

FORHATION OF COAPLETION: ALLUVIUM (AL)

HYDRAULIC FLOH RELATIONSHIP: UNKHON (N)

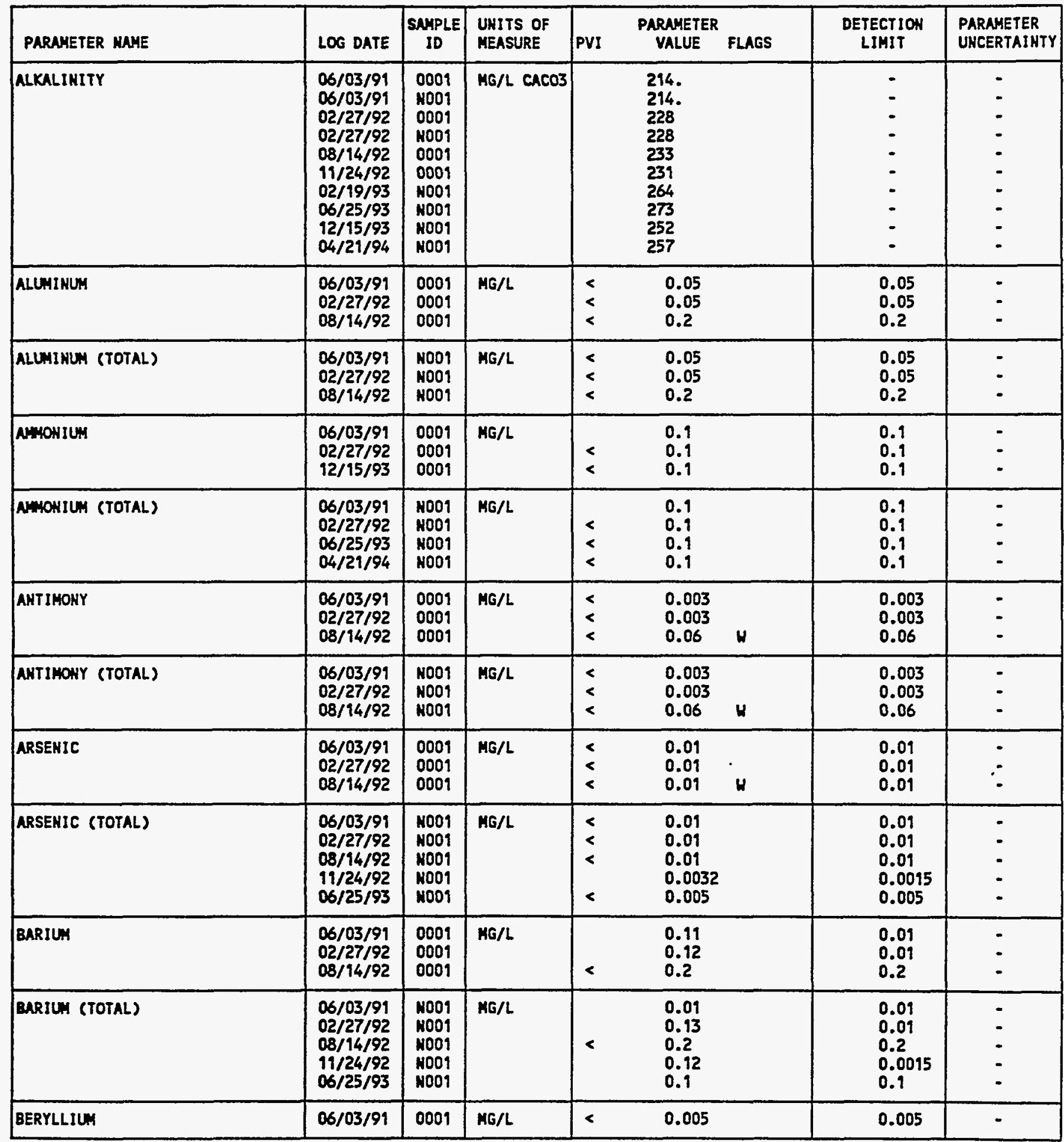

PARAMETER VALUE INDICATOR (PVI): < - LESS THAN DETECTION LIMIT

SNYPLE ID COOES:

0001 - FILTERED SNMPLE (.45 MICRONS)

OTHER PARAMETER VALUE FLAGS:

NOO1 - UNFILTERED SAMPLE

W - POST-DIGEST SPIKE OUT OF CNTR LIM UHILE SAMP ABS < 50\% SPIKE 
GROUNDHATER QUALITY DATA BY LOCATION

SOMP Rev. 0 Data

SITE: HONO1 MONLNENT VALLEY

LOCATION: 0616

NORTH COORDINATE: 56747.3 FT

EAST COORDINATE: 87987.6 FT

$04 / 21 / 88$ TO $12 / 13 / 94$

REPORT DATE: 06/20/95

FORMATION OF COMPLETION: ALLUVIUM (AL)

HYORAULIC FLON RELATIOHSHIP: UNKNONH (H)

\begin{tabular}{|c|c|c|c|c|c|c|c|}
\hline PARAMETER MAME & LOG DATE & $\underset{10}{\text { SNAPLE }}$ & $\begin{array}{l}\text { UNITS OF } \\
\text { MEASURE }\end{array}$ & PVI & $\begin{array}{l}\text { PARAMETER } \\
\text { VALUE FLAGS }\end{array}$ & $\begin{array}{l}\text { DETECTION } \\
\text { LIHIT }\end{array}$ & $\begin{array}{l}\text { PARAMETER } \\
\text { UHCERTAINTY }\end{array}$ \\
\hline BERYLLIUM & $08 / 14 / 92$ & 0001 & $M G / L$ & $<$ & 0.005 & 0.005 & - \\
\hline BERYLLIUM (TOTAL) & $\begin{array}{l}06 / 03 / 91 \\
08 / 14 / 92\end{array}$ & $\begin{array}{l}\text { No01 } \\
\text { N001 }\end{array}$ & MG/L & $<$ & $\begin{array}{l}0.005 \\
0.005\end{array}$ & $\begin{array}{l}0.005 \\
0.005\end{array}$ & - \\
\hline BOROH & $06 / 03 / 99$ & 0001 & MG/L & $<$ & 0.05 & 0.05 & - \\
\hline BORON (TOTAL) & $06 / 03 / 91$ & No01 & MG/L & & 0.19 & 0.05 & - \\
\hline BRONIDE & $06 / 03 / 91$ & 0001 & MG/L & $<$ & 0.1 & 0.1 & - \\
\hline BROHIDE (TOTAL) & $06 / 03 / 91$ & N001 & MG/L & $<$ & 0.1 & 0.1 & - \\
\hline CADHIUM & $\begin{array}{l}06 / 03 / 91 \\
02 / 27 / 92 \\
08 / 14 / 92\end{array}$ & $\begin{array}{l}0001 \\
0001 \\
0001\end{array}$ & MG/L & $\begin{array}{l}< \\
< \\
<\end{array}$ & $\begin{array}{l}0.001 \\
0.001 \\
0.005\end{array}$ & $\begin{array}{l}0.001 \\
0.001 \\
0.005\end{array}$ & : \\
\hline CADHILA (TOTAL) & $\begin{array}{l}06 / 03 / 91 \\
02 / 27 / 92 \\
08 / 14 / 92 \\
11 / 24 / 92\end{array}$ & $\begin{array}{l}\text { NOO1 } \\
\text { NOO1 } \\
\text { NO01 } \\
\text { NO01 }\end{array}$ & $M G / L$ & $\begin{array}{l}< \\
< \\
<\end{array}$ & $\begin{array}{l}0.001 \\
0.001 \\
0.005 \\
0.00013 \mathrm{H}\end{array}$ & $\begin{array}{l}0.001 \\
0.001 \\
0.005 \\
0.00013\end{array}$ & : \\
\hline CALCIUM & $\begin{array}{l}06 / 03 / 91 \\
02 / 27 / 92 \\
08 / 14 / 92 \\
12 / 15 / 93 \\
04 / 21 / 94\end{array}$ & $\begin{array}{l}0001 \\
0001 \\
0001 \\
0001 \\
0001\end{array}$ & $M G / L$ & & $\begin{array}{l}35.6 \\
30.3 \\
37 \\
33.4 \\
39.7\end{array}$ & $\begin{array}{l}0.5 \\
0.5 \\
5 \\
0.5 \\
0.5\end{array}$ & $\begin{array}{l}- \\
- \\
- \\
-\end{array}$ \\
\hline CALCIUA (TOTAL) & $\begin{array}{l}06 / 03 / 91 \\
02 / 27 / 92 \\
08 / 14 / 92 \\
02 / 19 / 93 \\
06 / 25 / 93 \\
12 / 15 / 93 \\
04 / 21 / 94\end{array}$ & $\begin{array}{l}\text { No01 } \\
\text { N001 } \\
\text { HO01 } \\
\text { Ho01 } \\
\text { N001 } \\
\text { NO01 } \\
\text { N001 }\end{array}$ & $M G / L$ & & $\begin{array}{l}4.5 \\
31.5 \\
35 \\
16 \\
66.9 \\
35.2 \\
39.6\end{array}$ & $\begin{array}{l}0.1 \\
0.5 \\
5 \\
0.5 \\
0.5 \\
0.5 \\
0.5\end{array}$ & $\begin{array}{l}- \\
- \\
- \\
- \\
-\end{array}$ \\
\hline CHLORIDE & $\begin{array}{l}06 / 03 / 91 \\
02 / 27 / 92 \\
08 / 14 / 92 \\
12 / 15 / 93 \\
04 / 21 / 94\end{array}$ & $\begin{array}{l}0001 \\
0001 \\
0001 \\
0001 \\
0001\end{array}$ & ME/L & - & $\begin{array}{c}11.4 \\
9.7 \\
12 \\
22.2 \\
31.9\end{array}$ & $\begin{array}{l}0.5 \\
0.5 \\
0.016 \\
0.5 \\
0.5\end{array}$ & $\begin{array}{l}- \\
- \\
-\end{array}$ \\
\hline CHLORIDE (TOTAL) & $\begin{array}{l}06 / 03 / 91 \\
02 / 27 / 92 \\
08 / 14 / 92 \\
02 / 19 / 93 \\
06 / 25 / 93\end{array}$ & $\begin{array}{l}\text { nO01 } \\
\text { HOO1 } \\
\text { N001 } \\
\text { N001 } \\
\text { N001 }\end{array}$ & $M G / L$ & & $\begin{array}{c}11.3 \\
9.8 \\
11 \\
14.4 \\
68.3\end{array}$ & $\begin{array}{l}0.5 \\
0.5 \\
0.016 \\
0.5 \\
0.5\end{array}$ & $\begin{array}{l}- \\
- \\
-\end{array}$ \\
\hline CHROMIU:H & $\begin{array}{l}06 / 03 / 91 \\
02 / 27 / 92 \\
08 / 14 / 92\end{array}$ & $\begin{array}{l}0001 \\
0001 \\
0001\end{array}$ & $M G / L$ & & $\begin{array}{l}0.01 \\
0.01 \\
0.01\end{array}$ & $\begin{array}{l}0.01 \\
0.01 \\
0.01\end{array}$ & - \\
\hline CHROMIUH (TOTAL) & $\begin{array}{l}06 / 03 / 91 \\
02 / 27 / 92 \\
08 / 14 / 92\end{array}$ & $\begin{array}{l}\text { No01 } \\
\text { N001 } \\
\text { N001 }\end{array}$ & MG/L & & $\begin{array}{l}0.01 \\
0.01 \\
0.01\end{array}$ & $\begin{array}{l}0.01 \\
0.01 \\
0.01\end{array}$ & : \\
\hline
\end{tabular}

PARAMETER VALUE IHDICATOR (PVI): < - LESS THAN DETECTION LIHIT

SAMPLE ID COOES:

OTHER PARAMETER VALUE FLAGS:

E - ESTIMATEd VALUE BECAUSE OF INTERFEREnCE, SEE CASE MaRRATIVE

DOO1 - FILTERED SAMPLE (.45 MICRONS)

W - SPIIKE SAMPLE RECOVERY NOT WITHIN CONTROL LIMITS 
GRONDWATER QUALITY DATA BY LOCATIOH

SOWP ReV. O Data

SITE: MONO1 MONUMENT VALLEY

LOCATIOH: 0616

HORTH COORDINATE: 56747.3 FT

EAST COORDINATE: 87987.6 FT

$04 / 21 / 88$ TO $12 / 13 / 94$

REPORT DATE: OS/20/95

FORMATION OF COAPLETION: ALLUVIUM (AL)

HYDRAULIC FLON RELATIONSHIP: UNKHOWN (N)

\begin{tabular}{|c|c|c|c|c|c|c|c|c|}
\hline PARAMETER MAME & LOG DATE & $\underset{\text { SNMPLE }}{\text { SN }}$ & $\begin{array}{l}\text { UNITS OF } \\
\text { MEASURE }\end{array}$ & PVI & $\begin{array}{l}\text { PARAMETER } \\
\text { VALUEE }\end{array}$ & FLAGS & $\begin{array}{l}\text { DETECTION } \\
\text { LIMIT }\end{array}$ & $\begin{array}{l}\text { PARAMETER } \\
\text { UNCERTAINTY }\end{array}$ \\
\hline CHROMIUH (TOTAL) & $11 / 24 / 92$ & N001 & $M G / L$ & $<$ & 0.0053 & & 0.0053 & - \\
\hline COBALT & $06 / 03 / 91$ & 0001 & MG/L & $<$ & 0.03 & & 0.03 & - \\
\hline COBALT (TOTAL) & $06 / 03 / 91$ & N001 & MG/L & $<$ & 0.03 & & 0.03 & - \\
\hline COPPER & $06 / 03 / 91$ & 0001 & MG/L & $<$ & 0.01 & & 0.01 & - \\
\hline COPPER (TOTAL) & $06 / 03 / 91$ & NO01 & MG/L & $<$ & 0.01 & & 0.01 & - \\
\hline FLUORIDE & $06 / 03 / 91$ & 0001 & MG/L & & 0.3 & & 0.1 & - \\
\hline FLUORIDE (TOTAL) & $06 / 03 / 91$ & No01 & HG/L & & 0.3 & & 0.9 & - \\
\hline GROSS ALPHA & $\begin{array}{l}06 / 03 / 91 \\
02 / 27 / 92 \\
08 / 14 / 92\end{array}$ & $\begin{array}{l}0001 \\
0001 \\
0001\end{array}$ & PCI/L & & $\begin{array}{r}10.7 \\
1.2 \\
3.1\end{array}$ & & $\begin{array}{l}1 . \\
1.0 \\
6.1\end{array}$ & $\begin{array}{l}6.2 \\
3.9 \\
3.8\end{array}$ \\
\hline GROSS ALPHA (TOTAL) & $\begin{array}{l}06 / 03 / 91 \\
02 / 27 / 92 \\
08 / 14 / 92 \\
11 / 24 / 92 \\
02 / 19 / 93 \\
06 / 25 / 93\end{array}$ & $\begin{array}{l}\text { NOO1 } \\
\text { HOO1 } \\
\text { HOO1 } \\
\text { NO01 } \\
\text { NO01 } \\
\text { NO01 }\end{array}$ & PCI/L & & $\begin{array}{l}6.5 \\
3.7 \\
5.3 \\
0.0 \\
0 \\
8\end{array}$ & N & $\begin{array}{l}1 . \\
1.0 \\
5.8 \\
6.8 \\
7 \\
5\end{array}$ & $\begin{array}{l}7.1 \\
4.2 \\
4.2 \\
3.8 \\
7 \\
9\end{array}$ \\
\hline GROSS BETA & $\begin{array}{l}06 / 03 / 91 \\
02 / 27 / 92 \\
08 / 14 / 92\end{array}$ & $\begin{array}{l}0001 \\
0001 \\
0001\end{array}$ & $\mathrm{PCI} / \mathrm{L}$ & & $\begin{array}{l}3.5 \\
1.8 \\
4.6\end{array}$ & & $\begin{array}{l}0.5 \\
0.5 \\
6.2\end{array}$ & $\begin{array}{l}3.2 \\
2.6 \\
3.8\end{array}$ \\
\hline GROSS BETA (TOTAL) & $\begin{array}{l}06 / 03 / 91 \\
02 / 27 / 92 \\
08 / 14 / 92 \\
11 / 24 / 92 \\
02 / 19 / 93 \\
06 / 25 / 93\end{array}$ & $\begin{array}{l}\text { NO01 } \\
\text { NO01 } \\
\text { NO01 } \\
\text { NO01 } \\
\text { N001 } \\
\text { N001 }\end{array}$ & $\mathrm{PCI} / \mathrm{L}$ & & $\begin{array}{l}4.4 \\
2.2 \\
1.9 \\
7.0 \\
0 \\
2\end{array}$ & H & $\begin{array}{c}0.5 \\
0.5 \\
5.0 \\
5.3 \\
12 \\
5\end{array}$ & $\begin{array}{l}3.5 \\
2.6 \\
3.0 \\
3.5 \\
5 \\
5\end{array}$ \\
\hline IROH & $\begin{array}{l}06 / 03 / 91 \\
02 / 27 / 92 \\
08 / 14 / 92\end{array}$ & $\begin{array}{l}0001 \\
0001 \\
0001\end{array}$ & $M G / L$ & $\begin{array}{l}< \\
<\end{array}$ & $\begin{array}{l}0.03 \\
0.03 \\
0.1\end{array}$ & & $\begin{array}{l}0.03 \\
0.03 \\
0.1\end{array}$ & : \\
\hline IRON (TOTAL) & $\begin{array}{l}06 / 03 / 91 \\
02 / 27 / 92 \\
08 / 14 / 92 \\
02 / 99 / 93 \\
06 / 25 / 93\end{array}$ & $\begin{array}{l}\text { HOO1 } \\
\text { NOO1 } \\
\text { NO01 } \\
\text { NOO1 } \\
\text { NO01 }\end{array}$ & $M G / L$ & $<$ & $\begin{array}{l}0.16 \\
0.14 \\
0.01 \\
0.17 \\
0.08\end{array}$ & J & $\begin{array}{l}0.03 \\
0.03 \\
0.1 \\
0.03 \\
0.03\end{array}$ & $\begin{array}{l}- \\
- \\
-\end{array}$ \\
\hline LEAD & $06 / 03 / 91$ & 0001 & MG/L & $<$ & 0.005 & & 0.005 & - \\
\hline LEAD (TOTAL) & $\begin{array}{l}06 / 03 / 91 \\
11 / 24 / 92\end{array}$ & $\begin{array}{l}\text { N001 } \\
\text { N001 }\end{array}$ & MG/L & $<$ & $\begin{array}{l}0.01 \\
0.0015\end{array}$ & & $\begin{array}{l}0.09 \\
0.0015\end{array}$ & - \\
\hline LEAD-210 (TOTAL) & $11 / 24 / 92$ & No01 & PCI/L & & 5.3 & & 0.3 & 0.8 \\
\hline MAGKESIUY & $\begin{array}{l}06 / 03 / 91 \\
02 / 27 / 92\end{array}$ & $\begin{array}{l}0001 \\
0001\end{array}$ & MG/L & & $\begin{array}{l}28.4 \\
25.6\end{array}$ & & $\begin{array}{l}0.1 \\
0.1\end{array}$ & - \\
\hline
\end{tabular}

PARAMETER VALUE INDICATOR (PVI): < - LeSS THAN DETECTION LIMIT

SAMPLE ID COOES:

OTHER PARAMETER VALUE FLAGS:

0001 - FILTERED SAMPLE (.45 MICRONS)

J - ESTIMUTED VALUE

NOD1 - UMFJLTERED SAMPLE

N - SPIKE SAMPLE RECOVERY NOT WITHIN CONTROL LIMITS 
GROUNDHATER QUALITY DATA BY LOCATION

SOWP ReV. O Data

SITE: MONO1 MOHUWENT VALLEY

LOCATION: 0616

MORTH COORDINATE: 56747.3 FT

EAST COORDIHATE: 87987.6 FT

$04 / 21 / 88$ TO $12 / 13 / 94$

REPORT DATE: OS/20/95

FORMATION OF COMPLETION: ALLUVIUM (AL)

HYDRAULIC FLON RELATIOHSHIP: UHKHOWN (H)

\begin{tabular}{|c|c|c|c|c|c|c|c|}
\hline PARAMETER MAME & LOG DATE & $\mid \begin{array}{c}\text { SAMPLE } \\
\text { ID }\end{array}$ & $\begin{array}{l}\text { UHITS OF } \\
\text { MEASURE }\end{array}$ & PVI & $\begin{array}{l}\text { ARAMETER } \\
\text { VALUE FLAGS }\end{array}$ & $\begin{array}{l}\text { DETECTION } \\
\text { LIMIT }\end{array}$ & $\begin{array}{l}\text { PARAMETER } \\
\text { UNCERTAINTY }\end{array}$ \\
\hline MAGNESIUY & $\begin{array}{l}08 / 14 / 92 \\
12 / 15 / 93 \\
04 / 21 / 94\end{array}$ & $\begin{array}{l}0001 \\
0001 \\
0001\end{array}$ & $M G / L$ & & $\begin{array}{l}30 \\
33.4 \\
36.3\end{array}$ & $\begin{array}{l}5 \\
0.1 \\
0.1\end{array}$ & : \\
\hline MGGESIUH (TOTAL) & $\begin{array}{l}06 / 03 / 91 \\
02 / 27 / 92 \\
08 / 14 / 92 \\
02 / 19 / 93 \\
06 / 25 / 93 \\
12 / 15 / 93 \\
04 / 21 / 94\end{array}$ & $\begin{array}{l}\text { No01 } \\
\text { N001 } \\
\text { N001 } \\
\text { N001 } \\
\text { N001 } \\
\text { N001 } \\
\text { N001 }\end{array}$ & $M G / L$ & & $\begin{array}{l}26.1 \\
28.2 \\
29 \\
33.1 \\
60.1 \\
34.1 \\
36.2\end{array}$ & $\begin{array}{l}0.1 \\
0.1 \\
5 \\
0.1 \\
0.1 \\
0.1 \\
0.1\end{array}$ & $\begin{array}{l}- \\
: \\
: \\
:\end{array}$ \\
\hline MAHGAHESE & $\begin{array}{l}06 / 03 / 91 \\
02 / 27 / 92 \\
08 / 14 / 92 \\
12 / 15 / 93 \\
04 / 21 / 94\end{array}$ & $\begin{array}{l}0001 \\
0001 \\
0001 \\
0001 \\
0001\end{array}$ & MG/L & $\begin{array}{l}< \\
< \\
< \\
< \\
<\end{array}$ & $\begin{array}{l}0.01 \\
0.01 \\
0.01 \\
0.01 \\
0.01\end{array}$ & $\begin{array}{l}0.01 \\
0.01 \\
0.01 \\
0.01 \\
0.01\end{array}$ & $\begin{array}{l}- \\
- \\
-\end{array}$ \\
\hline MOLYBDENUH & $\begin{array}{l}06 / 03 / 91 \\
02 / 27 / 92 \\
08 / 14 / 92\end{array}$ & $\begin{array}{l}0001 \\
0001 \\
0001\end{array}$ & $M G / L$ & $<$ & $\begin{array}{l}0.01 \\
0.01 \\
0.007\end{array}$ & $\begin{array}{l}0.01 \\
0.01 \\
0.007\end{array}$ & $\stackrel{-}{-}$ \\
\hline MOLYBDENUM (TOTAL) & $\begin{array}{l}06 / 03 / 91 \\
02 / 27 / 92 \\
08 / 14 / 92 \\
11 / 24 / 92 \\
06 / 25 / 93\end{array}$ & $\begin{array}{l}\text { No01 } \\
\text { NO01 } \\
\text { HOO1 } \\
\text { No01 } \\
\text { N001 }\end{array}$ & $M G / L$ & $\begin{array}{l}< \\
< \\
< \\
< \\
<\end{array}$ & $\begin{array}{l}0.01 \\
0.01 \\
0.007 \\
0.0049 \quad \mathrm{~N} \\
0.01\end{array}$ & $\begin{array}{l}0.01 \\
0.01 \\
0.007 \\
0.0049 \\
0.01\end{array}$ & $\begin{array}{l}- \\
- \\
-\end{array}$ \\
\hline MICKELL & $06 / 03 / 91$ & 0001 & MG/L & $<$ & 0.04 & 0.04 & - \\
\hline HICKEL (TOTAL) & $06 / 03 / 91$ & No01 & MG/L & $<$ & 0.04 & 0.04 & - \\
\hline MITRATE & $06 / 03 / 91$ & 0001 & $M G / L$ & & 6.2 & 1. & - \\
\hline
\end{tabular}

- HET GROSS ALPHA (GROSS ALPHA - URANIUM) HITH I MG URANIUM = 686 PCI

HET GROSS ALPHA (TOTAL) (TOTAL GROSS ALPHA - TOTAL URANIUH)

PARAMETER VALUE IMDICATOR (PVI): < - LESS THAN DETECTION LIMIT

OTHER PARAMETER VALUE FLAGS:

H - SPIKE SAMPLE RECOVERY NOT UITHIN CONTROL LIHITS
SAMPLE ID COOES:

0001 - FILTERED SAHPLE (.45 MICROHS)

NOD1 - UNFILTERED SAMPLE 
GROUNDWATER QUALITY DATA BY LOCATION

SOMP REV. 0 Dato

SITE: MONOI MONUMENT VALLEY

LOCATION: 0616

NORTH COORDINATE: 56747.3 FT

EAST COORDINATE: 87987.6 FT

$04 / 21 / 88$ TO $12 / 13 / 94$

REPORT DATE: $06 / 20 / 95$

FORMATION OF COMPLETION: ALLUVIUM (AL)

HYDRAULIC FLON RELATIONSHIP: UNKNOWN (N)

\begin{tabular}{|c|c|c|c|c|c|c|c|}
\hline PARAMETER MANE & LOG DATE & $\underset{10}{\text { SAMPLE }}$ & $\begin{array}{l}\text { UNITS OF } \\
\text { MEASURE }\end{array}$ & PVI & $\begin{array}{l}\text { PARAMETER } \\
\text { VALUE FLAGS }\end{array}$ & $\begin{array}{l}\text { DETECTION } \\
\text { LIMIT }\end{array}$ & $\begin{array}{l}\text { PARAMETER } \\
\text { UNCERTAIHTY }\end{array}$ \\
\hline NITRATE & $\begin{array}{l}02 / 27 / 92 \\
08 / 94 / 92 \\
11 / 24 / 92 \\
12 / 15 / 93\end{array}$ & $\begin{array}{l}0001 \\
0001 \\
N 001 \\
0001\end{array}$ & MG/L & & $\begin{array}{l}2.4 \\
5.8 \\
3.9 \\
6\end{array}$ & $\begin{array}{l}1.0 \\
0.075 \\
0.044 \\
1\end{array}$ & $\begin{array}{l}- \\
-\end{array}$ \\
\hline NITRATE (TOTAL) & $\begin{array}{l}06 / 03 / 91 \\
02 / 27 / 92 \\
02 / 19 / 93 \\
06 / 25 / 93 \\
04 / 21 / 94\end{array}$ & $\begin{array}{l}\text { NOO1 } \\
\text { NOO1 } \\
\text { NOO1 } \\
\text { NOO1 } \\
\text { HOO1 }\end{array}$ & MG/L & & $\begin{array}{l}5.7 \\
4.9 \\
4 \\
6 \\
7\end{array}$ & $\begin{array}{l}1.0 \\
1 \\
1 \\
1\end{array}$ & $\begin{array}{l}- \\
- \\
-\end{array}$ \\
\hline NITRITE AND HITRATE & $06 / 03 / 91$ & 0001 & MG/L & & 1.40 & 0.05 & - \\
\hline MITRITE AND NITRATE (TOTAL) & $06 / 03 / 91$ & NOOT & $M G / L$ & & 1.29 & 0.05 & - \\
\hline $\mathrm{PH}$ & $\begin{array}{l}06 / 03 / 91 \\
06 / 03 / 91 \\
02 / 27 / 92 \\
02 / 27 / 92 \\
08 / 14 / 92 \\
11 / 24 / 92 \\
02 / 99 / 93 \\
06 / 25 / 93 \\
12 / 15 / 93 \\
04 / 21 / 94\end{array}$ & $\begin{array}{l}0001 \\
\text { HOO1 } \\
0001 \\
\text { HOO1 } \\
0001 \\
0001 \\
\text { NO01 } \\
\text { NOO1 } \\
\text { NO01 } \\
\text { NO01 }\end{array}$ & SU & & $\begin{array}{l}7.89 \\
7.89 \\
8.25 \\
8.25 \\
7.95 \\
8.90 \\
8.23 \\
8.38 \\
8.12 \\
7.85\end{array}$ & $\begin{array}{l}- \\
: \\
: \\
: \\
: \\
-\end{array}$ & $\begin{array}{l}- \\
: \\
: \\
: \\
- \\
-\end{array}$ \\
\hline PHOSPHATE (TOTAL) & $06 / 25 / 93$ & HOOI & $M G / L$ & $<$ & 0.1 & 0.9 & - \\
\hline $\begin{array}{l}\text { POTASSIUM } \\
-\end{array}$ & $\begin{array}{l}06 / 03 / 91 \\
02 / 27 / 92 \\
08 / 14 / 92 \\
12 / 15 / 93 \\
04 / 21 / 94\end{array}$ & $\begin{array}{l}0001 \\
0001 \\
0001 \\
0001 \\
0001\end{array}$ & MG/L & & $\begin{array}{l}1.06 \\
0.87 \\
2.2 \\
0.9 \\
1.4\end{array}$ & $\begin{array}{l}0.01 \\
0.01 \\
0.575 \\
0.1 \\
0.1\end{array}$ & $\begin{array}{l}- \\
- \\
-\end{array}$ \\
\hline POTASSIUN (TOTAL) & $\begin{array}{l}06 / 03 / 91 \\
02 / 27 / 92 \\
08 / 14 / 92 \\
02 / 19 / 93 \\
06 / 25 / 93 \\
12 / 15 / 93 \\
04 / 21 / 94\end{array}$ & $\begin{array}{l}\text { NOO1 } \\
\text { NOO1 } \\
\text { NO01 } \\
\text { NO01 } \\
\text { NOO1 } \\
\text { NOO1 } \\
\text { NOO1 }\end{array}$ & MG/L & & $\begin{array}{l}1.28 \\
0.84 \\
2.2 \\
1.3 \\
1.4 \\
0.9 \\
1.4\end{array}$ & $\begin{array}{l}0.01 \\
0.01 \\
0.575 \\
0.1 \\
0.1 \\
0.1 \\
0.1\end{array}$ & $\begin{array}{l}- \\
- \\
- \\
-\end{array}$ \\
\hline RADIUH-226 & $\begin{array}{l}06 / 03 / 91 \\
02 / 27 / 92 \\
08 / 14 / 92\end{array}$ & $\begin{array}{l}0001 \\
0001 \\
0001\end{array}$ & PCI/L & & $\begin{array}{l}0.0 \\
0.0 \\
0.3\end{array}$ & $\begin{array}{l}1 . \\
1.0 \\
0.5\end{array}$ & $\begin{array}{l}0.2 \\
0.2 \\
0.1\end{array}$ \\
\hline RADILH-226 (TOTAL) & $\begin{array}{l}06 / 03 / 91 \\
02 / 27 / 92 \\
08 / 14 / 92 \\
11 / 24 / 92\end{array}$ & $\begin{array}{l}\text { HOOI } \\
\text { HOOI } \\
\text { NOOI } \\
\text { NOOI }\end{array}$ & PCI/L & & $\begin{array}{l}0.1 \\
0.0 \\
0.3 \\
1.2\end{array}$ & $\begin{array}{l}1 . \\
1.0 \\
0.4 \\
0.3\end{array}$ & $\begin{array}{l}0.3 \\
0.1 \\
0.1 \\
0.4\end{array}$ \\
\hline RADIUH-226 + RADIUH-228 & $\begin{array}{l}06 / 03 / 91 \\
02 / 27 / 92 \\
08 / 14 / 92\end{array}$ & $\begin{array}{l}0001 \\
0001 \\
0001\end{array}$ & PCI/L & & $\begin{array}{l}0.40 \\
2.30 \\
0.30\end{array}$ & $\ddot{-}$ & - \\
\hline RADIUH-226 + RADIUM-228 (TOTAL) & $\begin{array}{l}06 / 03 / 91 \\
02 / 27 / 92\end{array}$ & $\begin{array}{l}\text { No01 } \\
\text { N001 }\end{array}$ & PCI/L & & $\begin{array}{l}7.80 \\
0.00\end{array}$ & $\ddot{-}$ & $\dot{-}$ \\
\hline
\end{tabular}

PARAMETER VALUE INDICATOR (PVI): < - LESS THAN DETECTION LIMIT

SAMPLE ID COOES:

ODO - FILTERED SAMPLE (.45 MICRONS)

MOO1 - UNFILTERED SAMPLE 
GROUHDWATER QUALITY DATA BY LOCATIOH

SOUP Rev. O Data

SITE: MONO1 MONUMENT VALLEY

LOCATION: 0616

NORTH COORDIMATE: 56747.3 FT

EAST COORDINATE: B7987.6 FT

$04 / 21 / 88$ TO $12 / 13 / 94$

REPORT DATE: $0.6 / 20 / 95$

FORMATION OF COMPLETION: ALLUVIUM (AL)

HYDRAULIC FLON RELATIOHSHIP: UHKNOWN (N)

\begin{tabular}{|c|c|c|c|c|c|c|c|}
\hline PARAMETER MAME & LOG DATE & $\underset{\text { ID }}{\text { SAMPLE }}$ & $\begin{array}{l}\text { UHITS OF } \\
\text { MEASURE }\end{array}$ & PVI & $\begin{array}{l}\text { PARAMETER } \\
\text { VALUE FLAGS }\end{array}$ & $\begin{array}{l}\text { DETECTION } \\
\text { LIMIT }\end{array}$ & $\begin{array}{l}\text { PARAMETER } \\
\text { UHCERTAINTY }\end{array}$ \\
\hline RADIUH-226 + RADILH-228 (TOTAL) & $\begin{array}{l}08 / 94 / 92 \\
11 / 24 / 92\end{array}$ & $\begin{array}{l}\text { NOO1 } \\
\text { N001 }\end{array}$ & $\mathrm{PCI} / \mathrm{L}$ & & $\begin{array}{l}0.30 \\
4.40\end{array}$ & $\dot{-}$ & $\dot{-}$ \\
\hline RADIUH-228 & $\begin{array}{l}06 / 03 / 91 \\
02 / 27 / 92 \\
08 / 14 / 92\end{array}$ & $\begin{array}{l}0001 \\
0001 \\
0001\end{array}$ & $\mathrm{PCI} / \mathrm{L}$ & & $\begin{array}{l}0.4 \\
2.3 \\
0.0\end{array}$ & $\begin{array}{l}1.0 \\
1.0 \\
2.0\end{array}$ & $\begin{array}{l}1.6 \\
1.1 \\
1.1\end{array}$ \\
\hline RADIUH-228 (TOTAL) & $\begin{array}{l}06 / 03 / 91 \\
02 / 27 / 92 \\
08 / 14 / 92 \\
11 / 24 / 92\end{array}$ & $\begin{array}{l}\text { HOO1 } \\
\text { NOO1 } \\
\text { HOO } \\
\text { NO01 }\end{array}$ & PCI/L & & $\begin{array}{l}7.7 \\
0.0 \\
0.0 \\
3.2\end{array}$ & $\begin{array}{l}1.0 \\
1.0 \\
1.8 \\
2.0\end{array}$ & $\begin{array}{l}2.3 \\
1.8 \\
0.9 \\
1.5\end{array}$ \\
\hline SELENIUA & $\begin{array}{l}06 / 03 / 91 \\
02 / 27 / 92 \\
08 / 14 / 92\end{array}$ & $\begin{array}{l}0001 \\
0001 \\
0001\end{array}$ & $M G / L$ & $<$ & $\begin{array}{l}0.005 \\
0.005 \\
0.005\end{array}$ & $\begin{array}{l}0.005 \\
0.005 \\
0.005\end{array}$ & - \\
\hline SELENIUH (TOTAL) & $\begin{array}{l}06 / 03 / 91 \\
02 / 27 / 92 \\
08 / 14 / 92 \\
11 / 24 / 92\end{array}$ & $\begin{array}{l}\text { NOO1 } \\
\text { HOO1 } \\
\text { NO01 } \\
\text { NO01 }\end{array}$ & MG/L & $\begin{array}{l}< \\
<\end{array}$ & $\begin{array}{l}0.006 \\
0.005 \\
0.005 \\
0.0015\end{array}$ & $\begin{array}{l}0.005 \\
0.005 \\
0.005 \\
0.0015\end{array}$ & - \\
\hline SILICA - SIO2 & $\begin{array}{l}06 / 03 / 91 \\
12 / 15 / 93\end{array}$ & $\begin{array}{l}0001 \\
0001\end{array}$ & MG/L & & $\begin{array}{l}26.9 \\
26.4\end{array}$ & $\begin{array}{l}0.1 \\
0.1\end{array}$ & $\dot{-}$ \\
\hline SILICA - SIOZ (TOTAL) & $\begin{array}{l}06 / 03 / 99 \\
06 / 25 / 93 \\
12 / 95 / 93\end{array}$ & $\begin{array}{l}\text { NOO1 } \\
\text { NOO1 } \\
\text { HOO1 }\end{array}$ & $M G / L$ & & $\begin{array}{r}8.7 \\
24.2 \\
26.6\end{array}$ & $\begin{array}{l}0.1 \\
0.5 \\
0.1\end{array}$ & : \\
\hline SILVER & $06 / 03 / 91$ & 0001 & MG/L & $<$ & 0.01 & 0.01 & - \\
\hline SILVER (TOTAL) & $06 / 03 / 91$ & HOO1 & MG/L & $<$ & 0.01 & 0.01 & - \\
\hline SOOIUN & $\begin{array}{l}06 / 03 / 91 \\
02 / 27 / 92 \\
08 / 14 / 92 \\
12 / 15 / 93 \\
04 / 21 / 94\end{array}$ & $\begin{array}{l}0001 \\
0001 \\
0001 \\
0001 \\
0001\end{array}$ & $M G / L$ & & $\begin{array}{c}66 . \\
57 . \\
72 \\
95 \\
108\end{array}$ & $\begin{array}{l}1 . \\
1 . \\
5 \\
1 \\
1\end{array}$ & $\begin{array}{l}- \\
- \\
-\end{array}$ \\
\hline SOOIUH (TOTAL) & $\begin{array}{l}06 / 03 / 91 \\
02 / 27 / 92 \\
08 / 14 / 92 \\
02 / 19 / 93 \\
06 / 25 / 93 \\
12 / 15 / 93 \\
04 / 21 / 94\end{array}$ & $\begin{array}{l}\text { NOO1 } \\
\text { NOO1 } \\
\text { NOO1 } \\
\text { NOO1 } \\
\text { HOO1 } \\
\text { NOO1 } \\
\text { NOOI }\end{array}$ & MG/L & & $\begin{array}{c}63 . \\
61 . \\
73 \\
108 \\
113 \\
100 \\
105\end{array}$ & $\begin{array}{l}1 . \\
1 . \\
5 \\
0.1 \\
1 \\
1 \\
1\end{array}$ & $\begin{array}{l}- \\
- \\
- \\
- \\
-\end{array}$ \\
\hline SPECIFIC COHDUCTANCE & $\begin{array}{l}06 / 03 / 91 \\
06 / 03 / 91 \\
02 / 27 / 92 \\
02 / 27 / 92 \\
08 / 14 / 92 \\
11 / 24 / 92 \\
02 / 19 / 93 \\
06 / 25 / 93 \\
12 / 15 / 93\end{array}$ & $\begin{array}{l}0001 \\
\text { NoO1 } \\
0001 \\
\text { No01 } \\
0001 \\
0001 \\
\text { No01 } \\
\text { NO01 } \\
\text { No01 }\end{array}$ & UMHO/CH & & $\begin{array}{l}602 . \\
602 . \\
397 \\
397 \\
605 \\
400 \\
516 \\
821 \\
582\end{array}$ & $\begin{array}{l}- \\
- \\
- \\
- \\
-\end{array}$ & $\begin{array}{l}- \\
- \\
- \\
- \\
- \\
-\end{array}$ \\
\hline
\end{tabular}

PARAMETER VALUE INDICATOR (PVI): < - LESS THAN DETECTION LIHIT

SUMPLE ID COOES:

OTHER PARAMETER VALUE FLAGS:

H - HOLD TIME EXPIRED, VALUE SUSPECT

ODO1 - FILTERED SAMPLE (.45 HICROHS)

NOOI - UNFILTERED SAMPLE

-J - estimated VALUE 
GROUKDUATER QUALITY DATA BY LOCATION

SOWP ReV. 0 Data

SITE: MONO1 MONLWENT VALLEY

LOCATION: 0616

MORTH COORDINATE: 56747.3 FT

EAST COORDINATE: 87987.6 FT

$04 / 21 / 88$ TO $12 / 13 / 94$

REPORT DATE: $06 / 20 / 95$

FORMATION OF COMPLETION: ALLUVIUM (AL)

HYDRAULIC FLON RELATIONSHIP: UHKHOWH (N)

\begin{tabular}{|c|c|c|c|c|c|c|c|c|}
\hline PARAMETER MAME & LOG DATE & $\begin{array}{c}\text { SAMPLE } \\
\text { ID }\end{array}$ & $\begin{array}{l}\text { UNITS OF } \\
\text { MEASURE }\end{array}$ & PVI & $\begin{array}{l}\text { PARAMETER } \\
\text { VALUE }\end{array}$ & FLAGS & $\begin{array}{l}\text { DETECTION } \\
\text { LIMIT }\end{array}$ & $\begin{array}{l}\text { PARAMETER } \\
\text { UHCERTAINTY }\end{array}$ \\
\hline SPECIFIC CONDUCTANCE & $04 / 21 / 94$ & NoO1 & UHН/CN & \multicolumn{3}{|c|}{742} & - & - \\
\hline $\begin{array}{l}\text { STRONTIUH } \\
.\end{array}$ & $\begin{array}{l}06 / 03 / 91 \\
02 / 27 / 92 \\
08 / 14 / 92 \\
12 / 15 / 93 \\
04 / 21 / 94\end{array}$ & $\begin{array}{l}0001 \\
0001 \\
0001 \\
0001 \\
0001\end{array}$ & $M G / L$ & \multicolumn{3}{|c|}{$\begin{array}{l}0.43 \\
0.40 \\
0.5 \\
0.47 \\
0.52\end{array}$} & $\begin{array}{l}0.01 \\
0.01 \\
0.2 \\
0.01 \\
0.01\end{array}$ & $\begin{array}{l}- \\
- \\
- \\
-\end{array}$ \\
\hline STRONTIUH (TOTAL) & $\begin{array}{l}06 / 03 / 91 \\
02 / 27 / 92 \\
08 / 14 / 92 \\
11 / 24 / 92 \\
06 / 25 / 93 \\
12 / 15 / 93 \\
04 / 21 / 94\end{array}$ & $\begin{array}{l}\text { NOO1 } \\
\text { HOO1 } \\
\text { NOO1 } \\
\text { NO01 } \\
\text { NO01 } \\
\text { NOO1 } \\
\text { NO01 }\end{array}$ & $M G / L$ & \multicolumn{3}{|c|}{$\begin{array}{l}0.05 \\
0.44 \\
0.48 \\
0.51 \\
0.82 \\
0.48 \\
0.50\end{array}$} & $\begin{array}{l}0.01 \\
0.01 \\
0.2 \\
0.00035 \\
0.01 \\
0.01 \\
0.01\end{array}$ & $\begin{array}{l}- \\
- \\
\vdots \\
\vdots \\
-\end{array}$ \\
\hline SULFATE & $\begin{array}{l}06 / 03 / 91 \\
02 / 27 / 92 \\
08 / 14 / 92 \\
12 / 15 / 93 \\
04 / 21 / 94\end{array}$ & $\begin{array}{l}0001 \\
0001 \\
0001 \\
0001 \\
0001\end{array}$ & MG/L & \multicolumn{3}{|c|}{$\begin{array}{c}134 . \\
69 \\
86 \\
136 \\
183\end{array}$} & $\begin{array}{l}10 . \\
10 . \\
0.059 \\
1 \\
1\end{array}$ & $\begin{array}{l}- \\
- \\
- \\
-\end{array}$ \\
\hline SULFATE (TOTAL) & $\begin{array}{l}06 / 03 / 91 \\
02 / 27 / 92 \\
08 / 94 / 92 \\
11 / 24 / 92 \\
02 / 19 / 93 \\
06 / 25 / 93\end{array}$ & $\begin{array}{l}\text { NOO1 } \\
\text { NOO1 } \\
\text { NOO1 } \\
\text { NOO1 } \\
\text { NOO1 } \\
\text { NOOI }\end{array}$ & $M G / L$ & \multicolumn{3}{|c|}{$\begin{array}{c}82 . \\
77 \\
85 \\
79 \\
102 \\
346\end{array}$} & $\begin{array}{l}0.1 \\
10 . \\
0.059 \\
1.0 \\
1 \\
1\end{array}$ & $\begin{array}{l}- \\
: \\
-\end{array}$ \\
\hline SULFIDE & $06 / 03 / 91$ & 0001 & $M G / L$ & $<$ & 1. & IJ & 1. & - \\
\hline SULLIDE (TOTAL) & $06 / 03 / 91$ & N001 & MG/L & $<$ & 1. & 1 & 1. & - \\
\hline TEMPERATURE & $\begin{array}{l}06 / 03 / 91 \\
06 / 03 / 91 \\
02 / 27 / 92 \\
02 / 27 / 92 \\
08 / 14 / 92 \\
11 / 24 / 92 \\
02 / 19 / 93 \\
06 / 25 / 93 \\
12 / 15 / 93 \\
04 / 21 / 94\end{array}$ & 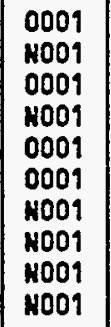 & C - DEGREE & \multicolumn{3}{|c|}{$\begin{array}{r}15.7 \\
15.7 \\
8.6 \\
8.6 \\
19.3 \\
9.0 \\
11.6 \\
15.7 \\
9.1 \\
14.4\end{array}$} & $\begin{array}{l}- \\
: \\
- \\
: \\
- \\
-\end{array}$ & $\begin{array}{l}- \\
: \\
- \\
- \\
- \\
-\end{array}$ \\
\hline THALLIUA & $06 / 03 / 91$ & 0001 & MG/L & $<$ & \multicolumn{2}{|l|}{0.01} & 0.01 & - \\
\hline THALLIUA (TOTAL) & $06 / 03 / 91$ & N001 & MG/L & $<$ & \multicolumn{2}{|l|}{0.01} & 0.01 & - \\
\hline THORIUA-230 & $06 / 03 / 91$ & 0001 & PCI/L & \multicolumn{3}{|c|}{0.8} & 1. & 0.3 \\
\hline THORIUH-230 (TOTAL) & $06 / 03 / 91$ & No01 & PCI/L & \multicolumn{3}{|c|}{1.4} & 1. & 0.5 \\
\hline TIN & $06 / 03 / 91$ & 0001 & MG/L & $<$ & 0.01 & 1 & 0.01 & - \\
\hline TIN (TOTAL) & $06 / 03 / 91$ & NoO1 & MG/L & $<$ & 0.01 & 1 & 0.09 & - \\
\hline
\end{tabular}

PARAMETER VALUE INDICATOR (PVI): < - LESS THAH DETECTION LIMIT

SAMPLE ID COOES

OTHER PARAMETER VALUE FLAGS:

DOO1 - FILTERED SAMPLE (.45 MICRONS)

- - DUPLICATE ANALYSIS NOT HITHIN CONTROL LIHITS

NOO1 - UNFILTERED SAMPLE

I - INCREASED DETECTION LIKIT DUE TO REOUIRED DILUTION

J - estimated value 
GROUNDUATER OUALITY DATA BY LOCATION

SOWP ReV. O Data

SITE: HOHOI MOHUMENT VALLEY

LOCATION: 0616

HORTH COOROIHATE: 56747.3 FT

EAST COORDINATE: 87987.6 FT

$04 / 21 / 88$ TO $12 / 13 / 94$

REPORT DATE: 06/20/95

FORMATION OF COMPLETION: ALLUVIUH (AL)

HYDRAULIC FLOW RELATIONSHIP': UNKHOWN (N)

\begin{tabular}{|c|c|c|c|c|c|c|c|}
\hline PARAMETER NAHE & LOG DATE & $\underbrace{\text { SAMPLE }}_{\text {ID }}$ & $\begin{array}{l}\text { UNITS OF } \\
\text { MEASURE }\end{array}$ & PVI & $\begin{array}{l}\text { PARAMETER } \\
\text { VALUE FLAGS }\end{array}$ & $\begin{array}{l}\text { DETECTION } \\
\text { LIHIT }\end{array}$ & $\begin{array}{l}\text { PARAMETER } \\
\text { UNCERTAINTY }\end{array}$ \\
\hline TOTAL DISSOLVED SOLIOS & $\begin{array}{l}06 / 03 / 91 \\
02 / 27 / 92 \\
08 / 14 / 92 \\
12 / 15 / 93 \\
04 / 21 / 94\end{array}$ & $\begin{array}{l}0001 \\
0001 \\
0001 \\
0001 \\
0001\end{array}$ & MG/L & & $\begin{array}{l}378 . \\
349 . \\
420 \\
530 \\
590\end{array}$ & $\begin{array}{l}10 . \\
10 . \\
42 \\
10 \\
10\end{array}$ & $\begin{array}{l}- \\
- \\
-\end{array}$ \\
\hline TOTAL DISSOLVED SOLIDS (TOTAL) & $\begin{array}{l}08 / 14 / 92 \\
02 / 19 / 93 \\
06 / 25 / 93\end{array}$ & $\begin{array}{l}\text { No01 } \\
\text { No01 } \\
\text { No01 }\end{array}$ & $M G / L$ & & $\begin{array}{l}400 \\
420 \\
880\end{array}$ & $\begin{array}{l}42 \\
10 \\
10\end{array}$ & $\begin{array}{l}- \\
-\end{array}$ \\
\hline TOTAL ORGAHIC CARBOH & $\begin{array}{l}06 / 03 / 91 \\
06 / 03 / 91 \\
02 / 27 / 92 \\
02 / 27 / 92 \\
08 / 14 / 92 \\
08 / 14 / 92\end{array}$ & $\begin{array}{l}0001 \\
N 001 \\
0001 \\
\text { No01 } \\
0001 \\
\text { N001 }\end{array}$ & MG/L & $\begin{array}{l}< \\
< \\
< \\
< \\
<\end{array}$ & $\begin{array}{l}1 . \\
1 . \\
1 . \\
1.0 \\
1.0 \\
1.0\end{array}$ & $\begin{array}{l}1 . \\
1 . \\
1 . \\
1.0 \\
1.0 \\
1.0\end{array}$ & $\begin{array}{l}- \\
- \\
- \\
- \\
-\end{array}$ \\
\hline URAKIUN & $\begin{array}{l}06 / 03 / 91 \\
02 / 27 / 92 \\
08 / 14 / 92 \\
12 / 15 / 93 \\
04 / 21 / 94\end{array}$ & $\begin{array}{l}0001 \\
0001 \\
0001 \\
0001 \\
0001\end{array}$ & MG/L & & $\begin{array}{l}0.001 \\
0.004 \\
0.004 \\
0.006 \\
0.007\end{array}$ & $\begin{array}{l}0.001 \\
0.001 \\
0.001 \\
0.001 \\
0.001\end{array}$ & $\begin{array}{l}- \\
- \\
-\end{array}$ \\
\hline URANIUM (TOTAL) & $\begin{array}{l}06 / 03 / 91 \\
02 / 27 / 92 \\
08 / 14 / 92 \\
11 / 24 / 92 \\
02 / 19 / 93 \\
06 / 25 / 93 \\
12 / 15 / 93 \\
04 / 21 / 94\end{array}$ & $\begin{array}{l}\text { HO01 } \\
\text { No01 } \\
\text { N001 } \\
\text { HO01 } \\
\text { No01 } \\
\text { HO01 } \\
\text { HO01 } \\
\text { NO01 }\end{array}$ & MG/L & $<$ & $\begin{array}{l}0.001 \\
0.004 \\
0.004 \\
0.006 \\
0.005 \\
0.006 \\
0.006 \\
0.007\end{array}$ & $\begin{array}{l}0.001 \\
0.001 \\
0.001 \\
0.001 \\
0.001 \\
0.001 \\
0.001 \\
0.001\end{array}$ & $\begin{array}{l}- \\
- \\
- \\
- \\
- \\
-\end{array}$ \\
\hline VANADIUM & $\begin{array}{l}06 / 03 / 91 \\
02 / 27 / 92 \\
08 / 14 / 92 \\
12 / 15 / 93 \\
04 / 21 / 94\end{array}$ & $\begin{array}{l}0001 \\
0001 \\
0001 \\
0001 \\
0001\end{array}$ & MG/L & $\begin{array}{l}< \\
< \\
< \\
< \\
<\end{array}$ & $\begin{array}{l}0.01 \\
0.01 \\
0.05 \\
0.01 \\
0.01\end{array}$ & $\begin{array}{l}0.01 \\
0.01 \\
0.05 \\
0.01 \\
0.01\end{array}$ & $\begin{array}{l}- \\
- \\
- \\
-\end{array}$ \\
\hline VAKADIUM (TOTAL) & $\begin{array}{l}06 / 03 / 91 \\
02 / 27 / 92 \\
08 / 14 / 92 \\
11 / 24 / 92 \\
06 / 25 / 93 \\
12 / 15 / 93 \\
04 / 21 / 94\end{array}$ & $\begin{array}{l}\text { No01 } \\
\text { No01 } \\
\text { No01 } \\
\text { No01 } \\
\text { No01 } \\
\text { No01 } \\
\text { No01 }\end{array}$ & MG/L & $\begin{array}{l}< \\
< \\
< \\
< \\
< \\
<\end{array}$ & $\begin{array}{l}0.01 \\
0.01 \\
0.05 \\
0.010 \\
0.01 \\
0.01 \\
0.01\end{array}$ & $\begin{array}{l}0.01 \\
0.01 \\
0.05 \\
0.0019 \\
0.01 \\
0.01 \\
0.01\end{array}$ & $\begin{array}{l}- \\
- \\
- \\
- \\
-\end{array}$ \\
\hline ZIHC & $\begin{array}{l}06 / 03 / 91 \\
02 / 27 / 92 \\
08 / 14 / 92\end{array}$ & $\begin{array}{l}0001 \\
0001 \\
0001\end{array}$ & $M G / L$ & $\begin{array}{l}< \\
< \\
<\end{array}$ & $\begin{array}{l}0.005 \\
0.005 \\
0.02\end{array}$ & $\begin{array}{l}0.005 \\
0.005 \\
0.02\end{array}$ & - \\
\hline ZINC (TOTAL) & $\begin{array}{l}06 / 03 / 91 \\
02 / 27 / 92 \\
08 / 14 / 92 \\
11 / 24 / 92 \\
06 / 25 / 93\end{array}$ & $\begin{array}{l}\text { No01 } \\
\text { No01 } \\
\text { N001 } \\
\text { N001 } \\
\text { N001 }\end{array}$ & HG/L & $<$ & $\begin{array}{l}0.005 \\
0.039 \\
0.021 \\
0.069 \\
0.099\end{array}$ & $\begin{array}{l}0.005 \\
0.005 \\
0.02 \\
0.0010 \\
0.005\end{array}$ & $\begin{array}{l}- \\
- \\
-\end{array}$ \\
\hline
\end{tabular}

PARAMETER VALUE INDICATOR (PVI): < - LESS THAN DETECTION LIMIT

SAMPLE ID CODES:

OTHER PARANETER VALUE FLAGS:

0001 - FILTERED SAMPLE (.45 MICRONS)

- DUPLICATE AMALYSIS NOT HITHIN COHTROL LIHITS

N001 - UNFILTERED SAMPLE

$d$ - ESTIMATED VALUE 
GROUNDWATER QUALITY DATA BY LOCATION

SOUP ReV. O Data

SITE: MONO1 MONUMENT VALLEY

LOCATION: 0616

NORTH COORDINATE: 56747.3 FT

EAST COORDINATE: 87987.6 FT

04/21/88 TO $12 / 13 / 94$

REPORT DATE: 06/20/95

FORMATION OF CONPLETION: ALLUVIUM (AL)

HYDRAULIC FLOW RELATIOHSHIP: UHKHOWN (H)

\begin{tabular}{|c|c|c|c|c|c|c|c|c|}
\hline PARNMETER MAME & LOG DATE & $\left|\begin{array}{c}\text { SAMPLE } \\
\text { ID }\end{array}\right|$ & $\begin{array}{l}\text { UNITS OF } \\
\text { MEASURE }\end{array}$ & PVI & $\begin{array}{l}\text { PARAMETES } \\
\text { VALUE }\end{array}$ & FLAGS & $\begin{array}{l}\text { DETECTION } \\
\text { LIMIT }\end{array}$ & $\begin{array}{l}\text { PARAMETER } \\
\text { UNCERTAINTY }\end{array}$ \\
\hline
\end{tabular}

PARAMETER VALUE INDICATOR (PVI): < - LESS THAN DETECTION LIMIT SAMPLE ID COOES: 
FORMATION OF CONPLETION: ALLUVIUM (AL)

HYORAULIC FLON RELATIOHSHIP: UHKHOWN (K)

\begin{tabular}{|c|c|c|c|c|c|c|c|c|}
\hline PARAMETER HAME & LOG DATE & $\underset{\text { ID }}{\text { SAMPLE }}$ & $\begin{array}{l}\text { UMITS OF } \\
\text { MEASURE }\end{array}$ & PVI & $\begin{array}{l}\text { PARAMETER } \\
\text { VALUE }\end{array}$ & FLAGS & $\begin{array}{l}\text { DETECTION } \\
\text { LIMIT }\end{array}$ & $\begin{array}{l}\text { PARAMETER } \\
\text { UNCERTAINTY }\end{array}$ \\
\hline ALKALIKITY & $\begin{array}{l}02 / 27 / 92 \\
02 / 27 / 92 \\
08 / 14 / 92 \\
11 / 24 / 92 \\
02 / 19 / 93 \\
06 / 25 / 93 \\
12 / 12 / 93 \\
04 / 21 / 94 \\
12 / 09 / 94\end{array}$ & $\begin{array}{l}0001 \\
\text { NO01 } \\
0001 \\
0001 \\
\text { NO01 } \\
\text { NO01 } \\
\text { NO01 } \\
\text { NO01 } \\
\text { HOO1 }\end{array}$ & $M G / L C A C O 3$ & & $\begin{array}{l}268 \\
268 \\
239 \\
271 \\
289 \\
277 \\
252 \\
248 \\
271\end{array}$ & & $\begin{array}{l}= \\
= \\
= \\
10\end{array}$ & $\begin{array}{l}- \\
- \\
- \\
- \\
-\end{array}$ \\
\hline ALUNIHUM & $\begin{array}{l}02 / 27 / 92 \\
08 / 14 / 92\end{array}$ & $\begin{array}{l}0001 \\
0001\end{array}$ & $M G / L$ & $<$ & $\begin{array}{l}0.05 \\
0.2\end{array}$ & $J$ & $\begin{array}{l}0.05 \\
0.2\end{array}$ & - \\
\hline ALUMINUM (TOTAL) & $\begin{array}{l}02 / 27 / 92 \\
08 / 14 / 92\end{array}$ & $\begin{array}{l}\text { N001 } \\
\text { N001 }\end{array}$ & $M G / L$ & $<$ & $\begin{array}{l}0.05 \\
0.2\end{array}$ & & $\begin{array}{l}0.05 \\
0.2\end{array}$ & $\therefore$ \\
\hline AmонILY & $\begin{array}{l}02 / 27 / 92 \\
12 / 12 / 93\end{array}$ & $\begin{array}{l}0001 \\
0001\end{array}$ & $M G / L$ & $<$ & $\begin{array}{l}0.1 \\
0.1\end{array}$ & & $\begin{array}{l}0.1 \\
0.1\end{array}$ & : \\
\hline AMOHIUM (TOTAL) & $\begin{array}{l}02 / 27 / 92 \\
06 / 25 / 93 \\
04 / 21 / 94 \\
12 / 09 / 94\end{array}$ & $\begin{array}{l}\text { NOO1 } \\
\text { NOO1 } \\
\text { HOOI } \\
\text { NOO1 }\end{array}$ & $M G / L$ & $\begin{array}{l}< \\
< \\
< \\
<\end{array}$ & $\begin{array}{l}0.1 \\
0.1 \\
0.1 \\
0.1\end{array}$ & H & $\begin{array}{l}0.1 \\
0.1 \\
0.1 \\
0.1\end{array}$ & $:$ \\
\hline ANTIMONY & $\begin{array}{l}02 / 27 / 92 \\
08 / 14 / 92\end{array}$ & $\begin{array}{l}0001 \\
0001\end{array}$ & MG/L & $<$ & $\begin{array}{l}0.003 \\
0.06\end{array}$ & & $\begin{array}{l}0.003 \\
0.06\end{array}$ & $\dot{-}$ \\
\hline ANTIMONY (TOTAL) & $\begin{array}{l}02 / 27 / 92 \\
08 / 14 / 92\end{array}$ & $\begin{array}{l}\text { N001 } \\
\text { H001 }\end{array}$ & MG/L & $<$ & $\begin{array}{l}0.003 \\
0.06\end{array}$ & $\mathbf{W}$ & $\begin{array}{l}0.003 \\
0.06\end{array}$ & : \\
\hline ARSEHIC & $\begin{array}{l}02 / 27 / 92 \\
08 / 14 / 92\end{array}$ & $\begin{array}{l}0001 \\
0001\end{array}$ & $M G / L$ & $<$ & $\begin{array}{l}0.01 \\
0.01\end{array}$ & $\boldsymbol{H}$ & $\begin{array}{l}0.09 \\
0.01\end{array}$ & : \\
\hline ARSEHIC (TOTAL) & $\begin{array}{l}02 / 27 / 92 \\
08 / 14 / 92 \\
11 / 24 / 92 \\
06 / 25 / 93\end{array}$ & $\begin{array}{l}\text { HOO1 } \\
\text { KOO1 } \\
\text { HOO1 } \\
\text { NOO1 }\end{array}$ & $M G / L$ & $<$ & $\begin{array}{l}0.01 \\
0.01 \\
0.0054 \\
0.006\end{array}$ & $\mathbf{H}$ & $\begin{array}{l}0.01 \\
0.01 \\
0.0015 \\
0.005\end{array}$ & $\ddot{-}$ \\
\hline BARIUN & $\begin{array}{l}02 / 27 / 92 \\
08 / 14 / 92\end{array}$ & $\begin{array}{l}0001 \\
0001\end{array}$ & $M G / L$ & $<$ & $\begin{array}{l}0.05 \\
0.2\end{array}$ & & $\begin{array}{l}0.01 \\
0.2\end{array}$ & - \\
\hline BARIUN (TOTAL) & $\begin{array}{l}02 / 27 / 92 \\
08 / 14 / 92 \\
11 / 24 / 92 \\
06 / 25 / 93\end{array}$ & $\begin{array}{l}\text { NoO1 } \\
\text { NO01 } \\
\text { No01 } \\
\text { NOO1 }\end{array}$ & $M G / L$ & $<$ & $\begin{array}{l}0.05 \\
0.2 \\
0.056 \\
0.1\end{array}$ & & $\begin{array}{l}0.01 \\
0.2 \\
0.0015 \\
0.1\end{array}$ & $\begin{array}{l}- \\
- \\
-\end{array}$ \\
\hline BERYLLIUM & $08 / 14 / 92$ & 0001 & MG/L & $<$ & 0.005 & & 0.005 & - \\
\hline BERYLLIUA (TOTAL) & $08 / 14 / 92$ & NOOI & MG/L & $<$ & 0.005 & & 0.005 & - \\
\hline CADHIU: & $\begin{array}{l}02 / 27 / 92 \\
08 / 14 / 92\end{array}$ & $\begin{array}{l}0001 \\
0001\end{array}$ & MG/L & $<$ & $\begin{array}{l}0.001 \\
0.005\end{array}$ & $\mathbf{E}$ & $\begin{array}{l}0.001 \\
0.005\end{array}$ & - \\
\hline CADHIU: (TOTAL) & $02 / 27 / 92$ & MOOI & MG/L & $<$ & 0.009 & & 0.001 & - \\
\hline
\end{tabular}

PARAMETER VALUE INDICATOR (PVI): < - LESS THAH DETECTION LIMIT

OTHER PARAMETER VALUE FLAGS:

E - estiMATEd VALUE becAuse of INTERfERENCE, SEe case harRative

H - HOLD TIME EXPIRED, VALUE SUSPECT

J- estimated value

- POST-DIGEST SPIKE OUT OF CNTR LIM UHILE SAMP ABS < 50\% SPIKE
SAMPLE ID COOES:

0001 - FILTERED SAMPLE (.45 MICRONS)

NO01 - UHFILTERED SAMPLE 
GROUNDHATER QUALITY DATA BY LOCATION

SOWP REV. O Data

SITE: MONO9 MONUWENT VALLEY

LOCATION: 0617

MORTH COORDINATE: 52090.6 FT

EAST COORDINATE: 87094.4 FT

04/21/88 TO 12/13/94

REPORT DATE: 06/20/95

FORMATION OF COAPLETION: ALLUVIUN (AL)

HYDRAULIC FLON RELATIONSHIP: UNKHONN (N)

\begin{tabular}{|c|c|c|c|c|c|c|c|}
\hline PARAMETER NAME & LOG DATE & $\underset{\text { ID }}{\text { SAMPLE }}$ & $\begin{array}{l}\text { UNITS OF } \\
\text { MEASURE }\end{array}$ & PVI & $\begin{array}{l}\text { PARAMETER } \\
\text { VALUE FLAGS }\end{array}$ & $\begin{array}{l}\text { DETECTION } \\
\text { LIMIT }\end{array}$ & $\begin{array}{l}\text { PARAMETER } \\
\text { UHCERTAINTY }\end{array}$ \\
\hline CNOKIUN (TOTAL) & $\begin{array}{l}08 / 14 / 92 \\
11 / 24 / 92\end{array}$ & $\begin{array}{l}\text { No01 } \\
\text { No01 }\end{array}$ & MG/L & $<$ & $\begin{array}{ll}0.005 & E \\
0.00019 & \text { KS }\end{array}$ & $\begin{array}{l}0.005 \\
0.00013\end{array}$ & $\dot{-}$ \\
\hline calcilor & $\begin{array}{l}02 / 27 / 92 \\
08 / 14 / 92 \\
12 / 12 / 93 \\
04 / 21 / 94 \\
12 / 09 / 94\end{array}$ & $\begin{array}{l}0001 \\
0001 \\
0001 \\
0001 \\
0001\end{array}$ & MG/L & & $\begin{array}{l}18.1 \\
23 \\
22.8 \\
22.0 \\
25.2\end{array}$ & $\begin{array}{l}0.1 \\
5 \\
0.5 \\
0.5 \\
0.5\end{array}$ & : \\
\hline CALCIUN (TOTAL) & $\begin{array}{l}02 / 27 / 92 \\
08 / 14 / 92 \\
02 / 19 / 93 \\
06 / 25 / 93 \\
12 / 12 / 93 \\
04 / 21 / 94 \\
12 / 09 / 94\end{array}$ & $\begin{array}{l}\text { No01 } \\
\text { N001 } \\
\text { N001 } \\
\text { N001 } \\
\text { N001 } \\
\text { N001 } \\
\text { N001 }\end{array}$ & MG/L & & $\begin{array}{l}18.3 \\
22 \\
16.2 \\
19.4 \\
23.4 \\
22.5 \\
24.2\end{array}$ & $\begin{array}{l}0.1 \\
5 \\
0.5 \\
0.5 \\
0.5 \\
0.5 \\
0.5\end{array}$ & $\begin{array}{l}- \\
: \\
- \\
- \\
-\end{array}$ \\
\hline CHLORIDE & $\begin{array}{l}02 / 27 / 92 \\
08 / 14 / 92 \\
12 / 12 / 93 \\
04 / 21 / 94\end{array}$ & $\begin{array}{l}0001 \\
0001 \\
0001 \\
0001\end{array}$ & MG/L & & $\begin{array}{l}15.5 \\
14 \\
32.3 \\
29.5\end{array}$ & $\begin{array}{l}0.5 \\
0.016 \\
0.5 \\
0.5\end{array}$ & $\dot{-}$ \\
\hline CHLORIDE (TOTAL) & $\begin{array}{l}02 / 27 / 92 \\
08 / 14 / 92 \\
02 / 19 / 93 \\
06 / 25 / 93 \\
12 / 09 / 94\end{array}$ & $\begin{array}{l}\text { No01 } \\
\text { No01 } \\
\text { NO01 } \\
\text { N001 } \\
\text { No01 }\end{array}$ & ME/L & & $\begin{array}{l}15.8 \\
14 \\
23.5 \\
35.1 \\
24\end{array}$ & $\begin{array}{l}0.5 \\
0.016 \\
0.5 \\
0.5 \\
0.5\end{array}$ & $\begin{array}{l}- \\
- \\
-\end{array}$ \\
\hline CHROHIUH & $\begin{array}{l}02 / 27 / 92 \\
08 / 14 / 92\end{array}$ & $\begin{array}{l}0001 \\
0001\end{array}$ & MG/L & $<$ & $\begin{array}{l}0.01 \\
0.01\end{array}$ & $\begin{array}{l}0.01 \\
0.01\end{array}$ & $\because$ \\
\hline CHROHIUH (TOTAL) & $\begin{array}{l}02 / 27 / 92 \\
08 / 14 / 92 \\
11 / 24 / 92\end{array}$ & $\begin{array}{l}\text { N001 } \\
\text { N001 } \\
\text { N001 }\end{array}$ & $M G / L$ & $\begin{array}{l}< \\
<\end{array}$ & $\begin{array}{l}0.01 \\
0.01 \\
0.0053\end{array}$ & $\begin{array}{l}0.01 \\
0.01 \\
0.0053\end{array}$ & : \\
\hline $\begin{array}{l}\text { GROSS ALPHA } \\
\ldots\end{array}$ & $\begin{array}{l}02 / 27 / 92 \\
08 / 14 / 92\end{array}$ & $\begin{array}{l}0001 \\
0001\end{array}$ & $\mathrm{PCI} / \mathrm{L}$ & & $\begin{array}{l}3.3 \\
3.6\end{array}$ & $\begin{array}{l}1.0 \\
6.6\end{array}$ & $\begin{array}{l}5.4 \\
4.2\end{array}$ \\
\hline GROSS ALPHA (TOTAL) & $\begin{array}{l}02 / 27 / 92 \\
08 / 14 / 92 \\
11 / 24 / 92 \\
02 / 19 / 93 \\
06 / 25 / 93\end{array}$ & $\begin{array}{l}\text { No01 } \\
\text { N001 } \\
\text { N001 } \\
\text { N001 } \\
\text { N001 }\end{array}$ & $\mathrm{PCI} / \mathrm{L}$ & & $\begin{array}{l}0.0 \\
4.2 \\
0.0 \\
0 \\
14\end{array}$ & $\begin{array}{l}1.0 \\
6.0 \\
7.2 \\
8 \\
5\end{array}$ & $\begin{array}{l}5.2 \\
4.1 \\
3.9 \\
9 \\
10\end{array}$ \\
\hline GROSS BETA & $\begin{array}{l}02 / 27 / 92 \\
08 / 14 / 92\end{array}$ & $\begin{array}{l}0001 \\
0001\end{array}$ & $\mathrm{PCI} / \mathrm{L}$ & & $\begin{array}{l}0.0 \\
3.8\end{array}$ & $\begin{array}{l}0.5 \\
6.0\end{array}$ & $\begin{array}{l}2.8 \\
3.7\end{array}$ \\
\hline GROSS BETA (TOTAL) & $\begin{array}{l}02 / 27 / 92 \\
08 / 14 / 92 \\
11 / 24 / 92 \\
02 / 19 / 93 \\
06 / 25 / 93\end{array}$ & $\begin{array}{l}\text { No01 } \\
\text { No01 } \\
\text { N001 } \\
\text { No01 } \\
\text { No01 }\end{array}$ & $\mathrm{PCI} / \mathrm{L}$ & & $\begin{array}{l}2.5 \\
3.8 \\
0.0 \\
0 \\
1\end{array}$ & $\begin{array}{c}0.5 \\
6.5 \\
5.4 \\
12 \\
5\end{array}$ & $\begin{array}{l}3.1 \\
3.9 \\
3.1 \\
5 \\
4\end{array}$ \\
\hline IROH & $\begin{array}{l}02 / 27 / 92 \\
08 / 14 / 92\end{array}$ & $\begin{array}{l}0001 \\
0001\end{array}$ & MG/L & $<$ & $\begin{array}{l}0.03 \\
0.1\end{array}$ & $\begin{array}{l}0.03 \\
0.1\end{array}$ & $\dot{-}$ \\
\hline
\end{tabular}

PARAMETER VALUE INDICATOR (PVI): < - LESS THAN DETECTION LIMIT

SAMPLE IO COOES:

OTHER PARAMETER VALUE FLAGS:

E - estimated VALUE becAuse of INTERFERENCE, SEe CASE marRatIVE

O001 - FILTERED SAMPLE (.45 MICRONS)

MOO1 - UNFILTERED SAMPLE

H - SPIKE SAMPLE RECOVERY NOT HITHIN CONTROL LIMITS

S - REPORTED VALUE DETERHINED USING METHOO OF STD ADDITION (MSA) 
GROUHDWATER QUALITY DATA BY LOCATION

SOMP ReY. O Data

SITE: MONOY MOHUMEHT VALLEY

LOCATION: 0617

NORTH COORDIHATE: $\quad 52090.6$ FT

EAST COORDINATE: 87094.4 FT

$04 / 21 / 88$ TO $12 / 13 / 94$

REPORT DATE: 06/20/95

FORMATION OF COAPLETION: ALLUVIUM (AL)

HYORAULIC FLON RELATIONSHIP: UNKHOWH (N)

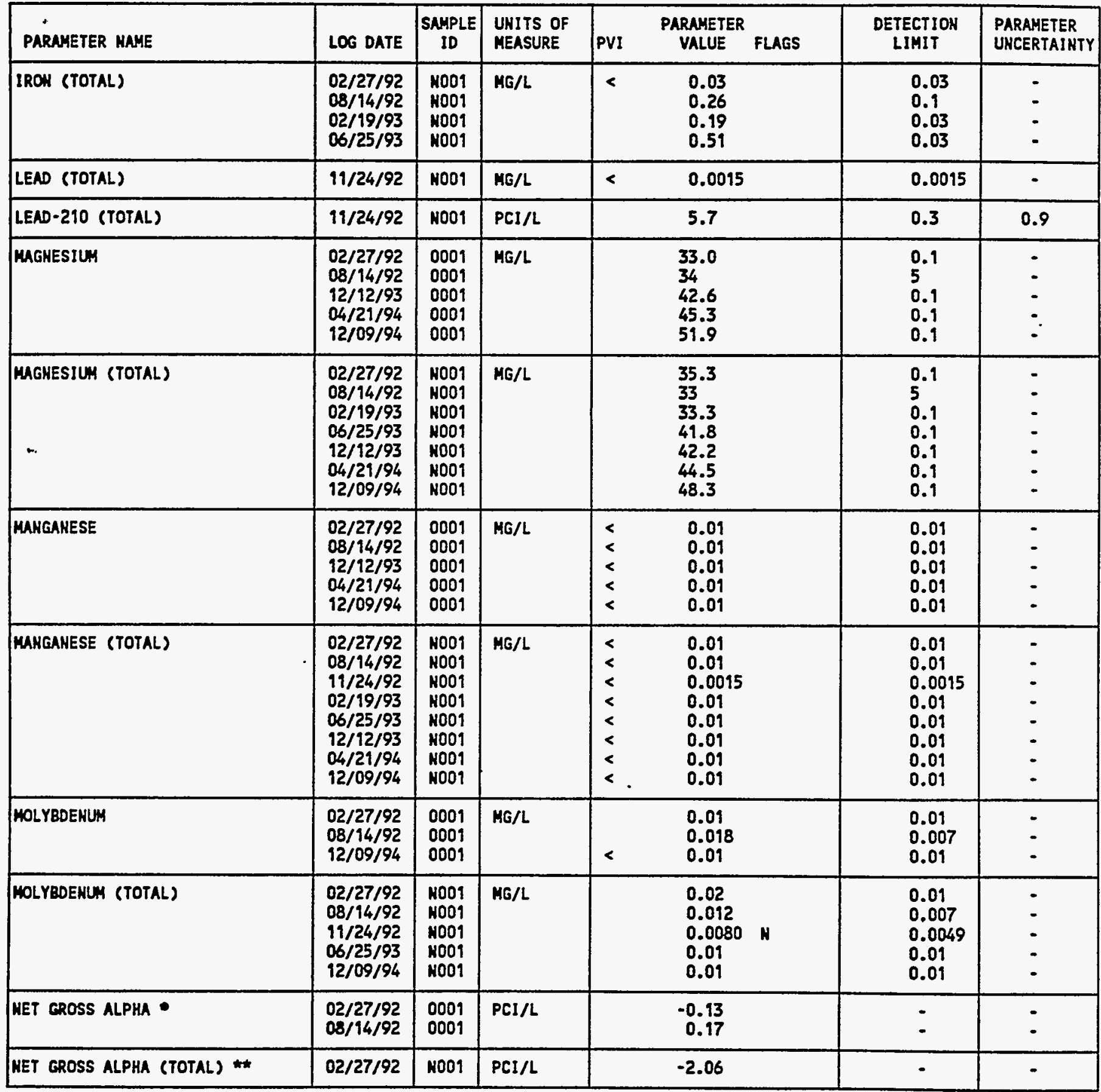

- NET GROSS ALPHA (GROSS ALPHA - URANIUM) MITH 1 MG URANILM = 686 PCI

* HET GROSS ALPHA (TOTAL) (TOTAL GROSS ALPHA - TOTAL URAHIUM)

PARAMETER VALUE IHDICATOR (PVI): < - LESS THAN DETECTION LIMIT

SUPLE ID COOES:

OTHER PARAMETER VALUE fLAGS:

H - SPIKE SAMPLE RECOVERY HOT HITHIM CONTROL LIMITS

O001 - FILTERED SAMPLE (.45 MICROWS)

HOO1 - UWFILTERED SAMPLE 
GROUMDHATER QUALITY DATA BY LOCATIOH

SOMP Rev. O Data

SITE: MOHOI MONUWENT VALLEY

LOCATIOH: 0617

NORTH COORDIHATE: $\quad 52090.6$ FT

EAST COORDINATE: 87094.4 FT

$04 / 21 / 88$ TO $12 / 13 / 94$

REPORT DATE: $06 / 20 / 95$

FORMATION OF COMPLETION: ALLUVIUM (AL)

HYDRAULIC FLON RELATIONSHIP: UNKNOWN (N)

\begin{tabular}{|c|c|c|c|c|c|c|c|}
\hline PARAMETER NAME & LOG DATE & $\begin{array}{c}\text { SAMPLE } \\
\text { ID }\end{array}$ & $\begin{array}{l}\text { UNITS OF } \\
\text { MEASURE }\end{array}$ & PVI & $\begin{array}{l}\text { PARAMETER } \\
\text { VALUE FLAGS }\end{array}$ & $\begin{array}{l}\text { DETECTION } \\
\text { LIMIT }\end{array}$ & $\begin{array}{l}\text { PARAMETER } \\
\text { UHCERTAINTY }\end{array}$ \\
\hline NET GROSS ALPHA (TOTAL) ** & $\begin{array}{l}08 / 14 / 92 \\
11 / 24 / 92 \\
02 / 19 / 93 \\
06 / 25 / 93\end{array}$ & $\begin{array}{l}\text { No01 } \\
\text { Ho01 } \\
\text { N001 } \\
\text { N001 }\end{array}$ & PCI/L & & $\begin{array}{r}0.77 \\
-4.12 \\
-4.80 \\
9.88\end{array}$ & $\begin{array}{l}- \\
- \\
-\end{array}$ & : \\
\hline MITRATE & $\begin{array}{l}02 / 27 / 92 \\
08 / 14 / 92 \\
11 / 24 / 92 \\
12 / 12 / 93\end{array}$ & $\begin{array}{l}0001 \\
0001 \\
N 001 \\
0001\end{array}$ & MG/L & $<$ & $\begin{array}{l}12.6 \\
15 \\
0.044 \\
35\end{array}$ & $\begin{array}{l}1.0 \\
0.075 \\
0.044 \\
1\end{array}$ & - \\
\hline WITRATE (TOTAL) & $\begin{array}{l}02 / 27 / 92 \\
02 / 19 / 93 \\
06 / 25 / 93 \\
04 / 21 / 94 \\
12 / 09 / 94\end{array}$ & $\begin{array}{l}\text { NOO1 } \\
\text { NO01 } \\
\text { NO01 } \\
\text { NO01 } \\
\text { NO01 }\end{array}$ & $M G / L$ & & $\begin{array}{l}13.0 \\
25 \\
47 \\
29 \\
36.3\end{array}$ & $\begin{array}{l}1.0 \\
1 \\
1 \\
1 \\
1\end{array}$ & $\begin{array}{l}- \\
- \\
-\end{array}$ \\
\hline PH & $\begin{array}{l}02 / 27 / 92 \\
02 / 27 / 92 \\
08 / 14 / 92 \\
11 / 24 / 92 \\
02 / 19 / 93 \\
06 / 25 / 93 \\
12 / 12 / 93 \\
04 / 21 / 94 \\
12 / 09 / 94\end{array}$ & $\begin{array}{l}0001 \\
\text { NOO1 } \\
0001 \\
0001 \\
\text { NOO1 } \\
\text { NOO1 } \\
\text { NOO1 } \\
\text { NOO1 } \\
\text { HOOI }\end{array}$ & su & & $\begin{array}{l}8.39 \\
8.39 \\
8.17 \\
8.57 \\
8.41 \\
7.91 \\
8.17 \\
8.02 \\
8.23\end{array}$ & $\begin{array}{l}- \\
- \\
= \\
= \\
= \\
0.10\end{array}$ & $\begin{array}{l}- \\
- \\
- \\
- \\
- \\
-\end{array}$ \\
\hline PHOSPHATE (TOTAL) & $06 / 25 / 93$ & NOO1 & MG/L & & 0.1 & 0.1 & - \\
\hline POTASSIUH & $\begin{array}{l}02 / 27 / 92 \\
08 / 14 / 92 \\
12 / 12 / 93 \\
04 / 21 / 94 \\
12 / 09 / 94\end{array}$ & $\begin{array}{l}0001 \\
0001 \\
0001 \\
0001 \\
0001\end{array}$ & MG/L & & $\begin{array}{l}0.55 \\
0.78 \\
0.5 \\
0.6 \\
0.9\end{array}$ & $\begin{array}{l}0.01 \\
0.575 \\
0.1 \\
0.1 \\
0.1\end{array}$ & : \\
\hline POTASSIUM (TOTAL) & $\begin{array}{l}02 / 27 / 92 \\
08 / 14 / 92 \\
02 / 19 / 93 \\
06 / 25 / 93 \\
12 / 12 / 93 \\
04 / 21 / 94 \\
12 / 09 / 94\end{array}$ & $\begin{array}{l}\text { NO01 } \\
\text { NO01 } \\
\text { No01 } \\
\text { NO01 } \\
\text { NO01 } \\
\text { NO01 } \\
\text { NO01 }\end{array}$ & MG/L & & $\begin{array}{l}0.63 \\
1.1 \\
1.4 \\
0.6 \\
0.5 \\
0.6 \\
0.6\end{array}$ & $\begin{array}{l}0.01 \\
0.575 \\
0.1 \\
0.1 \\
0.1 \\
0.1 \\
0.1\end{array}$ & $\begin{array}{l}- \\
- \\
\dot{-} \\
-\end{array}$ \\
\hline RADIUA-226 & $\begin{array}{l}02 / 27 / 92 \\
08 / 14 / 92\end{array}$ & $\begin{array}{l}0009 \\
0001\end{array}$ & PCI/L & & $\begin{array}{l}0.1 \\
5.7\end{array}$ & $\begin{array}{l}1.0 \\
0.7\end{array}$ & $\begin{array}{l}0.2 \\
0.3\end{array}$ \\
\hline RADIUH-226 (TOTAL) & $\begin{array}{l}02 / 27 / 92 \\
08 / 14 / 92 \\
11 / 24 / 92\end{array}$ & $\begin{array}{l}\text { No01 } \\
\text { No01 } \\
\text { No01 }\end{array}$ & PCI/L & & $\begin{array}{l}0.0 \\
0.1 \\
0.3\end{array}$ & $\begin{array}{l}1.0 \\
0.7 \\
0.3\end{array}$ & $\begin{array}{l}0.2 \\
0.1 \\
0.2\end{array}$ \\
\hline RADIUA-226 + RADILA-228 & $\begin{array}{l}02 / 27 / 92 \\
08 / 14 / 92\end{array}$ & $\begin{array}{l}0001 \\
0001\end{array}$ & PCI/L & & $\begin{array}{l}1.70 \\
5.90\end{array}$ & $\dot{-}$ & $\dot{-}$ \\
\hline RADIUN-226 + RADIUH-228 (TOTAL) & $02 / 27 / 92$ & No01 & $\mathrm{PCI} / \mathrm{L}$ & & 0.00 & - & - \\
\hline
\end{tabular}

* NET gROSS ALPHA (TOTAL) (TOTAL GROSS ALPHA - TOTAL URANIUH)
PARAMETER VALUE INDICATOR (PVI): < - LESS THAN DETECTION LIMIT
SNMPLE ID COOES:
OTHER PARAMETER VALUE FLAGS
OOO1 - FILTERED SAMPLE (.45 MICROHS)
NO01 - UHFILTERED SAMPLE

H - HOLD TIME EXPIRED, VALUE SUSPECT

J - estimated VALUE 
GROUKDWATER QUALITY DATA BY LOCATION

SOMP ReV. 0 Data

SITE: MONO1 MOHUNENT VALLEY

LOCATIOH: 0617

MORTH COORDINATE: $\quad 52090.6$ FT

EAST COORDIHATE: 87094.4 FT

04/21/88 TO $12 / 13 / 94$

REPORT DATE: 06/20/95

FORMATION OF COHPLETION: ALLUVIUH (AL)

HYDRAULIC FLOM RELATIONSHIP: UHKHOWN (K)

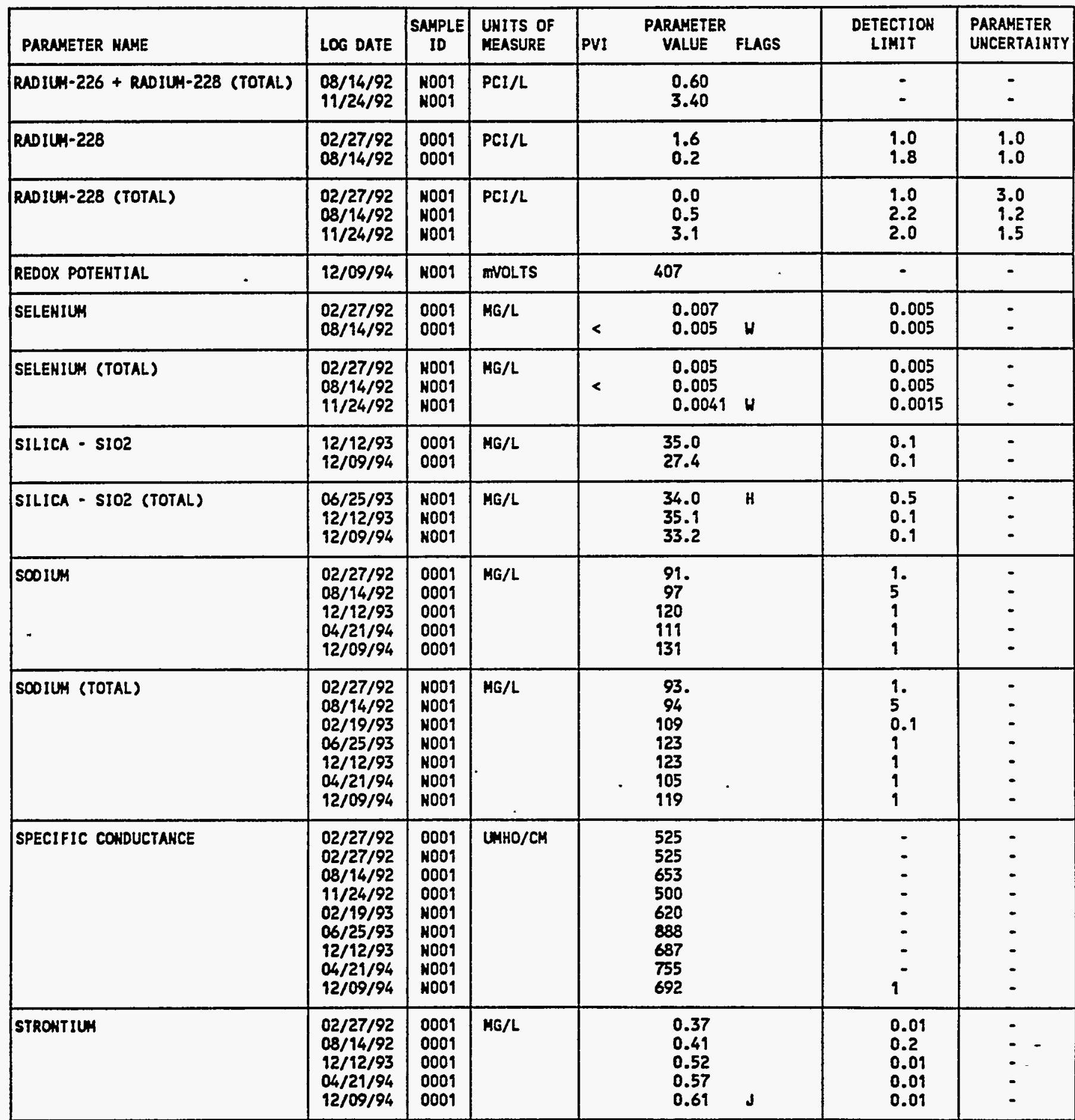

PARAMETER VALUE IMDICATOR (PVI): < - LESS THAN DETECTION LIMIT

SAMPLE ID COOES:

OTHER PARAHETER VALUE FLAGS:

0009 - FILTERED SAMPLE (.45 MICRONS)

H - HOLD TIME EXPIRED, VALUE SUSPECT

NOOI - UNFILTERED SNMPLE

J - estimated VALUE

$W$ - POST-DIGEST SPIKE OUT OF CNTR LIM UHILE SAMP ABS < 50\% SPIKE 
GROUHDWATER QUALITY DATA BY LOCATION

SOWP ReV. O Data

SITE: MONO1 MONUMENT VALLEY

LOCATION: 0617

HORTH COORDINATE: 52090.6 FT

EAST COORDINATE: 87094.4 FT

$04 / 21 / 88$ TO $12 / 13 / 94$

REPORT DATE: 06/20/95

FORMATION OF COMPLETIOH: ALLUVIUM (AL)

HYDRAULIC FLON RELATIONSHIP: UHKNOWH (K)

\begin{tabular}{|c|c|c|c|c|c|c|c|}
\hline PARUNETER NRHE & LOG DATE & $\begin{array}{c}\text { SAMPLE } \\
\text { ID }\end{array}$ & $\begin{array}{l}\text { UNITS OF } \\
\text { MEASURE }\end{array}$ & PVI & $\begin{array}{l}\text { PARAMETER } \\
\text { VALUE FLAGS }\end{array}$ & $\begin{array}{l}\text { DETECTION } \\
\text { LIMIT }\end{array}$ & $\begin{array}{l}\text { PARAMETER } \\
\text { UNCERTAINTY }\end{array}$ \\
\hline STRONTIU: (TOTAL) & $\begin{array}{l}02 / 27 / 92 \\
08 / 14 / 92 \\
11 / 24 / 92 \\
06 / 25 / 93 \\
12 / 12 / 93 \\
04 / 21 / 94 \\
12 / 09 / 94\end{array}$ & $\begin{array}{l}\text { NOO1 } \\
\text { NOO1 } \\
\text { N001 } \\
\text { NOO1 } \\
\text { No01 } \\
\text { NOO1 } \\
\text { NOO1 }\end{array}$ & MG/L & & $\begin{array}{l}0.39 \\
0.40 \\
0.48 \\
0.52 \\
0.51 \\
0.55 \\
0.59\end{array}$ & $\begin{array}{l}0.01 \\
0.2 \\
0.00035 \\
0.01 \\
0.01 \\
0.01 \\
0.01\end{array}$ & $\begin{array}{l}. \\
- \\
. \\
- \\
- \\
-\end{array}$ \\
\hline SULFATE & $\begin{array}{l}02 / 27 / 92 \\
08 / 14 / 92 \\
12 / 12 / 93 \\
04 / 21 / 94\end{array}$ & $\begin{array}{l}0001 \\
0001 \\
0001 \\
0001\end{array}$ & MG/L & & $\begin{array}{c}85 . \\
80 \\
162 \\
169\end{array}$ & $\begin{array}{l}10 . \\
0.059 \\
1 \\
1\end{array}$ & : \\
\hline SULFATE (TOTAL) & $\begin{array}{l}02 / 27 / 92 \\
08 / 14 / 92 \\
11 / 24 / 92 \\
02 / 19 / 93 \\
06 / 25 / 93 \\
12 / 09 / 94\end{array}$ & $\begin{array}{l}\text { NOO1 } \\
\text { NOO1 } \\
\text { NOO1 } \\
\text { HOO1 } \\
\text { HOO1 } \\
\text { HOOI }\end{array}$ & MG/L & & $\begin{array}{c}91 . \\
84 \\
91 \\
116 \\
159 \\
149\end{array}$ & $\begin{array}{l}10 . \\
0.059 \\
1.0 \\
1 \\
1 \\
1\end{array}$ & $\begin{array}{l}: \\
: \\
:\end{array}$ \\
\hline TEKPERATURE & $\begin{array}{l}02 / 27 / 92 \\
02 / 27 / 92 \\
08 / 14 / 92 \\
11 / 24 / 92 \\
02 / 19 / 93 \\
06 / 25 / 93 \\
12 / 12 / 93 \\
04 / 21 / 94 \\
12 / 09 / 94\end{array}$ & $\begin{array}{l}0001 \\
\text { NO01 } \\
0001 \\
0001 \\
\text { NO01 } \\
\text { NO01 } \\
\text { NO01 } \\
\text { NO01 } \\
\text { NO01 }\end{array}$ & C - DEGREE & & $\begin{array}{r}9.7 \\
9.7 \\
19.3 \\
10.1 \\
11.6 \\
7.8 \\
10.3 \\
13.9 \\
10.0\end{array}$ & $\begin{array}{l}- \\
- \\
- \\
- \\
- \\
- \\
- \\
0.1\end{array}$ & $\begin{array}{l}- \\
- \\
- \\
- \\
- \\
-\end{array}$ \\
\hline TOTAL DISSOLVED SOLIDS & $\begin{array}{l}02 / 27 / 92 \\
08 / 14 / 92 \\
12 / 12 / 93 \\
04 / 21 / 94 \\
12 / 09 / 94\end{array}$ & $\begin{array}{l}0001 \\
0001 \\
0001 \\
0001 \\
0001\end{array}$ & $M G / L$ & & $\begin{array}{l}442 . \\
450 \\
640 \\
580 \\
438\end{array}$ & $\begin{array}{l}10 . \\
42 \\
10 \\
10 \\
10\end{array}$ & $\begin{array}{l}: \\
: \\
-\end{array}$ \\
\hline TOTAL DISSOLVED SOLIDS (TOTAL) & $\begin{array}{l}08 / 14 / 92 \\
02 / 19 / 93 \\
06 / 25 / 93\end{array}$ & $\begin{array}{l}\text { NOO1 } \\
\text { NOOI } \\
\text { HOOI }\end{array}$ & MG/L & & $\begin{array}{l}470 \\
490 \\
630\end{array}$ & $\begin{array}{l}42 \\
10 \\
10\end{array}$ & - \\
\hline TOTAL ORGANIC CARBON & $\begin{array}{l}02 / 27 / 92 \\
02 / 27 / 92 \\
08 / 14 / 92 \\
08 / 14 / 92\end{array}$ & $\begin{array}{l}0001 \\
\text { NOO1 } \\
0001 \\
\text { N001 }\end{array}$ & MG/L & $\begin{array}{l}< \\
< \\
<\end{array}$ & $\begin{array}{l}1 . \\
1.0 \\
1.0 \\
1.0\end{array}$ & $\begin{array}{l}1 . \\
1.0 \\
1.0 \\
1.0\end{array}$ & : \\
\hline URAKIUH & $\begin{array}{l}02 / 27 / 92 \\
08 / 14 / 92 \\
12 / 12 / 93 \\
04 / 21 / 94 \\
12 / 09 / 94\end{array}$ & $\begin{array}{l}0001 \\
0001 \\
0001 \\
0001 \\
0001\end{array}$ & MG/L & & $\begin{array}{l}0.005 \\
0.005 \\
0.008 \\
0.007 \\
0.007\end{array}$ & $\begin{array}{l}0.001 \\
0.001 \\
0.001 \\
0.001 \\
0.001\end{array}$ & $\begin{array}{l}- \\
- \\
- \\
-\end{array}$ \\
\hline URAKIUH (TOTAL) & $\begin{array}{l}02 / 27 / 92 \\
08 / 14 / 92 \\
11 / 24 / 92 \\
02 / 19 / 93 \\
06 / 25 / 93\end{array}$ & $\begin{array}{l}\text { No01 } \\
\text { No01 } \\
\text { No01 } \\
\text { No01 } \\
\text { No01 }\end{array}$ & MG/L & & $\begin{array}{l}0.003 \\
0.005 \\
0.006 \\
0.007 \\
0.006\end{array}$ & $\begin{array}{l}0.001 \\
0.001 \\
0.001 \\
0.001 \\
0.001\end{array}$ & $\begin{array}{l}: \\
: \\
:\end{array}$ \\
\hline
\end{tabular}

PARAMETER VALUE IMDICATOR (PVI): < - LESS THAN DETECTION LIMIT SAMPLE TD COOES:

0001 - FILTERED SAMPLE (.45 MICRONS)

OTHER PARAMETER VALUE FLAGS:

NOO1 - UNFILTERED SAMPLE

J - estimated VALUE 
GROUHDHATER QUALITY DATA BY LOCATIOM

SOUP Rev. 0 Data

SITE: MONO1 MONUMENT VALLEY

LOCATION: 0697

HORTH COORDIHATE: 52090.6 FT

EAST COORDINATE: 87094.4 FT

$04 / 21 / 88$ TO $12 / 13 / 94$

REPORT DATE: OS/20/95

FORMATION OF COMPLETION: ALLUVIUM (AL)

HYDRAULIC FLON RELATIONSHIP: UNKHOWN (H)

\begin{tabular}{|c|c|c|c|c|c|c|c|c|}
\hline PARAMETER MAME & LOG DATE & $\begin{array}{c}\text { SAMPLE } \\
\text { ID }\end{array}$ & $\begin{array}{l}\text { UWITS OF } \\
\text { MEASURE }\end{array}$ & PVI & $\begin{array}{l}\text { PARAMETER } \\
\text { VALUE }\end{array}$ & FLAGS & $\begin{array}{l}\text { DETECTION } \\
\text { LIMIT }\end{array}$ & $\begin{array}{l}\text { PARAMETER } \\
\text { UNCERTAINTY }\end{array}$ \\
\hline URAKIUN (TOTAL) & $\begin{array}{l}12 / 12 / 93 \\
04 / 21 / 94 \\
12 / 09 / 94\end{array}$ & $\begin{array}{l}\text { No01 } \\
\text { No01 } \\
\text { No01 }\end{array}$ & MG/L & & $\begin{array}{l}0.007 \\
0.007 \\
0.006\end{array}$ & & $\begin{array}{l}0.001 \\
0.001 \\
0.001\end{array}$ & : \\
\hline VAMADILM & $\begin{array}{l}02 / 27 / 92 \\
08 / 14 / 92 \\
12 / 12 / 93 \\
04 / 21 / 94 \\
12 / 09 / 94\end{array}$ & $\begin{array}{l}0001 \\
0001 \\
0001 \\
0001 \\
0001\end{array}$ & MG/L & $<$ & $\begin{array}{l}0.02 \\
0.05 \\
0.01 \\
0.01 \\
0.01\end{array}$ & - & $\begin{array}{l}0.01 \\
0.05 \\
0.01 \\
0.01 \\
0.01\end{array}$ & $\begin{array}{l}\dot{-} \\
\dot{-} \\
\dot{-}\end{array}$ \\
\hline VAHADIUA (TOTAL) & $\begin{array}{l}02 / 27 / 92 \\
08 / 14 / 92 \\
11 / 24 / 92 \\
06 / 25 / 93 \\
12 / 12 / 93 \\
04 / 21 / 94 \\
12 / 09 / 94\end{array}$ & $\begin{array}{l}\text { No01 } \\
\text { NO01 } \\
\text { N001 } \\
\text { H001 } \\
\text { N001 } \\
\text { N001 } \\
\text { N001 }\end{array}$ & MG/L & $<$ & $\begin{array}{l}0.01 \\
0.05 \\
0.018 \\
0.02 \\
0.02 \\
0.01 \\
0.01\end{array}$ & * & $\begin{array}{l}0.01 \\
0.05 \\
0.0019 \\
0.01 \\
0.01 \\
0.01 \\
0.01\end{array}$ & $\begin{array}{l}: \\
: \\
:\end{array}$ \\
\hline ZIHC & $\begin{array}{l}02 / 27 / 92 \\
08 / 14 / 92\end{array}$ & $\begin{array}{l}0001 \\
0001\end{array}$ & ME/L & $<$ & $\begin{array}{l}0.005 \\
0.02\end{array}$ & & $\begin{array}{l}0.005 \\
0.02\end{array}$ & $\dot{-}$ \\
\hline ZIMC (TOTAL) & $\begin{array}{l}02 / 27 / 92 \\
08 / 14 / 92 \\
11 / 24 / 92 \\
06 / 25 / 93\end{array}$ & $\begin{array}{l}\text { No01 } \\
\text { N001 } \\
\text { N001 } \\
\text { H001 }\end{array}$ & MG/L & $<$ & $\begin{array}{l}0.005 \\
0.057 \\
0.029 \\
0.079\end{array}$ & J & $\begin{array}{l}0.005 \\
0.02 \\
0.0010 \\
0.005\end{array}$ & $\begin{array}{l}- \\
-\end{array}$ \\
\hline
\end{tabular}

PARAMETER VALUE IMDICATOR (PVI): < - LESS THAM DETECTION LIMIT

SAMPLE ID COOES:

OTHER PARAHETER VALUE FLAGS:

OOO1 - FILTERED SAMPLE (.45 HICRONS)

- DUPLICATE aMALYSIS hOt UITHIN COHTROL limits

NOO1 - UNFILTERED SAMPLE

$J$ - estimated value 
GROUNDWATER QUALITY DATA BY LOCATIOH

SOMP Rev. O Date

SITE: MONOI MONUMENT VALLEY

LOCATION: 0619

NORTH COORDIHATE: 58876.5 FT

EAST COORDINATE: 87587.9 FT

04/21/88 TO 12/13/94

REPORT DATE: 06/20/95

FORMATION OF COMPLETION: DECHELLEY MEMBER OF THE CUTLER FORMATION (DC) HYDRAULIC FLON RELATIOHSHIP: ON-SITE (O)

\begin{tabular}{|c|c|c|c|c|c|c|c|}
\hline PARAMETER MAME & LOG DATE & $\underset{\text { ID }}{\text { SAMPLE }}$ & $\begin{array}{l}\text { UNITS OF } \\
\text { MEASURE }\end{array}$ & PVI & $\begin{array}{l}\text { PARAMETER } \\
\text { VALUE FLAGS }\end{array}$ & $\begin{array}{l}\text { DETECTION } \\
\text { LIHIT }\end{array}$ & $\begin{array}{l}\text { PARAMETER } \\
\text { UNCERTAINTY }\end{array}$ \\
\hline ALKALINITY & $06 / 29 / 93$ & No01 & MG/L CACO3 & \multicolumn{2}{|r|}{189} & - & - \\
\hline Amontun & $12 / 13 / 93$ & 0001 & MG/L & $<$ & 0.1 & 0.1 & - \\
\hline AmEONILH (TOTAL) & $06 / 29 / 93$ & N001 & $M G / L$ & $<$ & 0.1 & 0.1 & - \\
\hline ARSEKIC & $06 / 29 / 93$ & 0001 & $M G / L$ & $<$ & 0.005 & 0.005 & - \\
\hline ARSENIC (TOTAL) & $06 / 29 / 93$ & NoO1 & $M G / L$ & $<$ & 0.005 & 0.005 & - \\
\hline BARIUH & $06 / 29 / 93$ & 0001 & $M G / L$ & $<$ & 0.1 & 0.1 & - \\
\hline BARIUI (TOTAL) & $06 / 29 / 93$ & NOO1 & $M G / L$ & $<$ & 0.1 & 0.1 & - \\
\hline CALCIU: & $\begin{array}{l}06 / 29 / 93 \\
12 / 13 / 93\end{array}$ & $\begin{array}{l}0001 \\
0001\end{array}$ & MG/L & & $\begin{array}{l}66.0 \\
46.3\end{array}$ & $\begin{array}{l}0.5 \\
0.5\end{array}$ & - \\
\hline CALCIUH (TOTAL) & $\begin{array}{l}06 / 29 / 93 \\
12 / 13 / 93\end{array}$ & $\begin{array}{l}\text { NOO1 } \\
\text { NOO1 }\end{array}$ & $M G / L$ & & $\begin{array}{l}62.8 \\
49.4\end{array}$ & $\begin{array}{l}0.5 \\
0.5\end{array}$ & $\because$ \\
\hline CHLORIDE & $12 / 13 / 93$ & 0001 & MG/L & & 7.1 & 0.5 & - \\
\hline CHLORIDE (TOTAL) & $06 / 29 / 93$ & N001 & $M G / L$ & & 7.5 & 0.5 & - \\
\hline DISSOLVED OXYGEN & $06 / 29 / 93$ & NOO1 & MG/L & & 6.6 & - & - \\
\hline GROSS ALPHA & $06 / 29 / 93$ & 0001 & $\mathrm{PCl} / \mathrm{L}$ & & 90 & 4 & 14 \\
\hline GROSS ALPHA (TOTAL) & $06 / 29 / 93$ & N001 & $\mathrm{PCI} / \mathrm{L}$ & & 91 & 4 & 14 \\
\hline GROSS BETA & $06 / 29 / 93$ & 0009 & $\mathrm{PCI} / \mathrm{L}$ & & 45 & 5 & 6 \\
\hline GROSS BETA (TOTAL) & $06 / 29 / 93$ & N001 & $\mathrm{PCI} / \mathrm{L}$ & & 43 & 5 & 6 \\
\hline IRON & $06 / 29 / 93$ & 0001 & MG/L & $<$ & 0.03 & 0.03 & - \\
\hline IRON (TOTAL) & $06 / 29 / 93$ & NOO1. & $M G / L$ & $<$ & 0.03 & 0.03 & - \\
\hline MAGHESIU: & $\begin{array}{l}06 / 29 / 93 \\
12 / 13 / 93\end{array}$ & $\begin{array}{l}0001 \\
0001\end{array}$ & MG/L & & $\begin{array}{l}36.4 \\
27.0\end{array}$ & $\begin{array}{l}0.1 \\
0.1\end{array}$ & : \\
\hline MAGHESIUH (TOTAL) & $\begin{array}{l}06 / 29 / 93 \\
12 / 13 / 93\end{array}$ & $\begin{array}{l}\text { N001 } \\
\text { N001 }\end{array}$ & MG/L & & $\begin{array}{l}35.9 \\
28.8\end{array}$ & $\begin{array}{l}0.1 \\
0.1\end{array}$ & - \\
\hline MANGANESE & $\begin{array}{l}06 / 29 / 93 \\
12 / 13 / 93\end{array}$ & $\begin{array}{l}0001 \\
0001\end{array}$ & $M G / L$ & $<$ & $\begin{array}{l}0.01 \\
0.01\end{array}$ & $\begin{array}{l}0.01 \\
0.01\end{array}$ & - \\
\hline MAKGAKESE (TOTAL) & $\begin{array}{l}06 / 29 / 93 \\
12 / 13 / 93\end{array}$ & $\begin{array}{l}\text { NOOI } \\
\text { N001 }\end{array}$ & $M G / L$ & $<$ & $\begin{array}{l}0.01 \\
0.01\end{array}$ & $\begin{array}{l}0.01 \\
0.01\end{array}$ & : \\
\hline MOLYBOEKUM & $06 / 29 / 93$ & 0009 & MG/L & & 0.03 & 0.01 & - \\
\hline MOLYBDEKUN (TOTAL) & $06 / 29 / 93$ & NOOI & MG/L & $<$ & 0.01 & 0.01 & $\cdot$ \\
\hline NET GROSS ALPHA * & $06 / 29 / 93$ & 0001 & $\mathrm{PCI} / \mathrm{L}$ & & -1.24 & - & - \\
\hline
\end{tabular}

* Net gross ALPHA (GROSS ALPHA - URANIUH) HITH I MG URANIUH = 686 PCI

PARAMETER VALUE INDICATOR (PVI): < - LESS THAN DETECTION LIMIT

OTHER PARAMETER VALUE FLAGS:

J - ESTIMATED VALUE
SAMPLE ID COOES:

0001 - FILTERED SAMPLE (.45 MICRONS)

NOO1 - UAFILTERED SAMPLE 
GROUNDHATER QUALITY DATA BY LOCATION

SOMP ReV. O Data

SITE: MOHOI HONUMENT VALLEY

LOCATION: 0619

NORTH COORDIHATE: $\quad 58876.5$ FT

EAST COORDIHATE: 87587.9 FT

$04 / 21 / 88$ TO $12 / 13 / 94$

REPORT DATE: OS/20/95

FORHATION OF COMPLETION: DECHELLEY MEMBER OF THE CUTLER FORMATION (DC) HYDRAULIC FLOW RELATIONSHIP: OH-SITE (O)

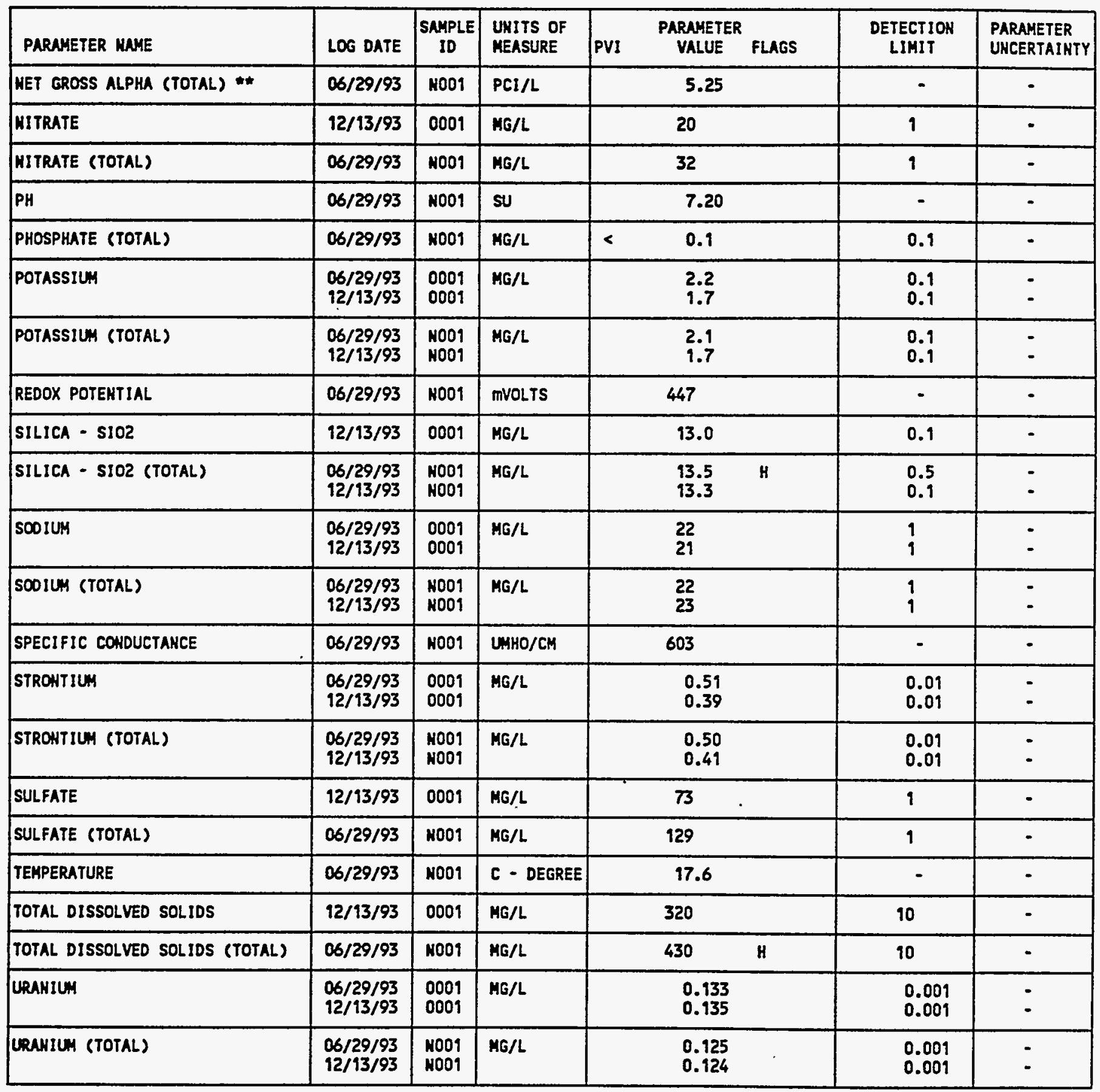

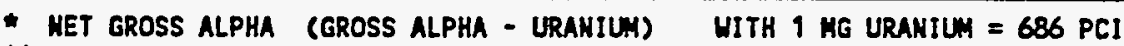

* NET GROSS ALPHA (TOTAL) (TOTAL GROSS ALPHA - TOTAL URANIUA)

PARAMETER VALUE IHDICATOR (PVI): < - LESS THAN DETECTION LIMIT

SUMPLE ID COOES:

OTHER PARAMETER VALUE FLAGS:

. H - hold TIME EXPIRED, VALUE SUSPECT

0001 - FILTERED SNMPLE (.45 MICRONS)

HOO1 - UHFILTERED SAMPLE 
GROUNDUATER OUALITY DATA BY LOCATION

SOWP Rev. O Data

SITE: MONO1 MOHUMENT VALLEY

LOCATION: 0619

NORTH COORDINATE: 58876.5 FT

EAST COORDINATE: 87587.9 FT

04/21/88 TO $12 / 93 / 94$

REPORT DATE: 06/20/95

FORMATION OF COMPLETION: DECHELLEY MEMBER OF THE CUTLER FORMATION (DC) HYDRAULIC FLON RELATIONSHIP: ON-SITE (O)

\begin{tabular}{|c|c|c|c|c|c|c|c|}
\hline PARAMETER MAME & LOG DATE & $\underset{\text { SAMPLE }}{\text { ID }}$ & $\begin{array}{l}\text { UNITS OF } \\
\text { MEASURE }\end{array}$ & PVI & $\begin{array}{l}\text { PARAMETER } \\
\text { VALUE FLAGS }\end{array}$ & $\begin{array}{l}\text { DETECTION } \\
\text { LIMIT }\end{array}$ & $\begin{array}{l}\text { PARAMETER } \\
\text { UHCERTAINTY }\end{array}$ \\
\hline VAHADIUA & $\begin{array}{l}06 / 29 / 93 \\
12 / 93 / 93\end{array}$ & $\begin{array}{l}0001 \\
0001\end{array}$ & MG/L & & $\begin{array}{l}0.02 \\
0.02\end{array}$ & $\begin{array}{l}0.01 \\
0.01\end{array}$ & : \\
\hline VANADIUH (TOTAL) & $\begin{array}{l}06 / 29 / 93 \\
12 / 13 / 93\end{array}$ & $\begin{array}{l}\text { N001 } \\
\text { N001 }\end{array}$ & MG/L & & $\begin{array}{l}0.02 \\
0.02\end{array}$ & $\begin{array}{l}0.01 \\
0.01\end{array}$ & : \\
\hline ZINC & $06 / 29 / 93$ & 0009 & MG/L & & 0.105 & 0.005 & - \\
\hline ZINC (TOTAL) & $06 / 29 / 93$ & N001 & MG/L & & 0.035 & 0.005 & - \\
\hline
\end{tabular}

PARAMETER VALUE INDICATOR (PVI): < - LESS THAN DETECTION LIMIT

SAMPLE 10 COOES:

OTHER PARAMETER VALUE FLAGS:

0001 - FILTERED SAMPLE (.45 MICROHS)

J - estimated VAlUe

NOO1 - UNFILTERED SAMPLE 
GROUHDHATER OUALITY DATA BY LOCATIOH

SOUP ReV. 0 Data

SITE: MONO MONUMENT VALLEY

LOCATION: 0625

NORTH COORDINATE: 58272.5 FT

EAST COORDINATE: 89803.9 FT

04/21/88 TO $12 / 13 / 94$

REPORT DATE: 06/20/95

FORHATION OF COMPLETION: DECHELLEY MEMBER OF THE CUTLER FORMATION (DC) HYDRAULIC FLOW RELATIONSHIP: CROSS GRADIENT (C)

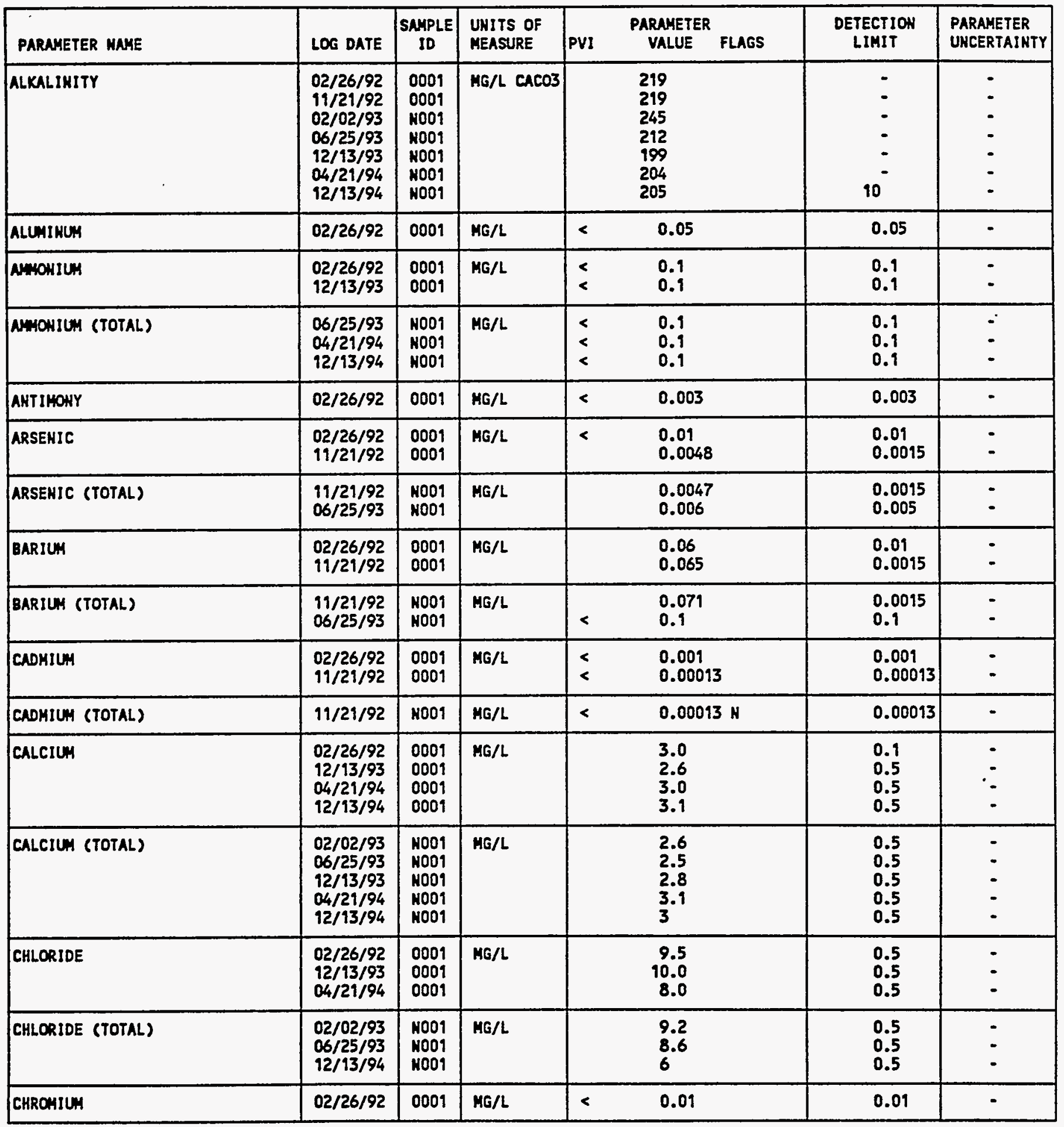

PARAMETER VALUE IMDICATOR (PVI): < - LESS THAN DETECTION LIMIT

SAMPLE ID COOES:

OTHER PARAMETER VALUE FLAGS:

0001 - FILTERED SNMPLE (.45 MICRONS)

W - SPIKE SAMPLE RECOVERY NOT WITHIN CONTROL LIMITS

MO01 - UMFILTERED SAMPLE 
GROUNDWATER QUALITY DATA BY LOCATION

SOUP ReV. 0 Data

SITE: HOHO1 MONUMENT VALLEY

LOCATION: 0625

NORTH COORDINATE: $\quad 58272.5$ FT

EAST COORDINATE: 89803.1 FT

$04 / 21 / 88$ TO $12 / 13 / 94$

REPORT DATE: 06/20/95

FORMATION OF COMPLETION: DECHELLEY MEMBER OF THE CUTLER FORMATION (DC) HYDRAULIC FLON RELATIONSHIP: CROSS GRADIENT (C)

\begin{tabular}{|c|c|c|c|c|c|c|c|}
\hline PARAMETER NAME & LOG DATE & $\underset{\text { SN }}{\text { SNMPLE }}$ & $\begin{array}{l}\text { UNITS OF } \\
\text { MEASURE }\end{array}$ & PVI & $\begin{array}{l}\text { PARAMETER } \\
\text { VALUE FLAGS }\end{array}$ & $\begin{array}{l}\text { DETECTION } \\
\text { LIMIT }\end{array}$ & $\begin{array}{l}\text { PARAMETER } \\
\text { UNCERTAIHTY }\end{array}$ \\
\hline CHROHIUN & $11 / 21 / 92$ & 0001 & MG/L & $<$ & 0.0053 & 0.0053 & - \\
\hline CHROMIUN (TOTAL) & $11 / 21 / 92$ & NoOI & ME/L & $<$ & 0.0053 & 0.0053 & - \\
\hline GROSS ALPHA & $\begin{array}{l}02 / 26 / 92 \\
11 / 21 / 92\end{array}$ & $\begin{array}{l}0001 \\
0001\end{array}$ & $\mathrm{PCI} / \mathrm{L}$ & & $\begin{array}{l}9.5 \\
6.7\end{array}$ & $\begin{array}{l}1.0 \\
3.0\end{array}$ & $\begin{array}{l}4.7 \\
3.0\end{array}$ \\
\hline GROSS ALPHA (TOTAL) & $\begin{array}{l}11 / 21 / 92 \\
02 / 02 / 93 \\
06 / 25 / 93\end{array}$ & $\begin{array}{l}\text { NoO1 } \\
\text { NOOI } \\
\text { NO01 }\end{array}$ & PCI/L & & $\begin{array}{l}0.2 \\
0 \\
5\end{array}$ & $\begin{array}{l}6.8 \\
5 \\
2\end{array}$ & $\begin{array}{l}4.1 \\
6 \\
5\end{array}$ \\
\hline GROSS BETA & $\begin{array}{l}02 / 26 / 92 \\
11 / 21 / 92\end{array}$ & $\begin{array}{l}0001 \\
0001\end{array}$ & PCI/L & & $\begin{array}{l}5.6 \\
1.5\end{array}$ & $\begin{array}{l}0.5 \\
5.3\end{array}$ & $\begin{array}{l}2.7 \\
3.2\end{array}$ \\
\hline GROSS BETA (TOTAL) & $\begin{array}{l}11 / 21 / 92 \\
02 / 02 / 93 \\
06 / 25 / 93\end{array}$ & $\begin{array}{l}\text { NOO1 } \\
\text { N001 } \\
\text { N001 }\end{array}$ & $\mathrm{PCI} / \mathrm{L}$ & & $\begin{array}{l}3.1 \quad N \\
0 \\
5\end{array}$ & $\begin{array}{c}5.0 \\
11 \\
4\end{array}$ & $\begin{array}{l}3.1 \\
5 \\
4\end{array}$ \\
\hline IRON & $02 / 26 / 92$ & 0001 & MG/L & $<$ & 0.03 & 0.03 & - \\
\hline IRON (TOTAL) & $\begin{array}{l}02 / 02 / 93 \\
06 / 25 / 93\end{array}$ & $\begin{array}{l}\text { N001 } \\
\text { N001 }\end{array}$ & MG/L & $<$ & $\begin{array}{l}0.03 \\
0.03\end{array}$ & $\begin{array}{l}0.03 \\
0.03\end{array}$ & : \\
\hline LEAD & $11 / 21 / 92$ & 0001 & MG/L & $<$ & 0.0015 & 0.0015 & - \\
\hline LEAD (TOTAL) & $19 / 21 / 92$ & NOO1 & MG/L & $<$ & 0.0015 & 0.0015 & - \\
\hline LEAD-210 & $11 / 21 / 92$ & 0001 & PCI/L & & 1.3 & 2.0 & 1.2 \\
\hline LEAD-210 (TOTAL) & $19 / 21 / 92$ & N001 & PCI/L & & 6.3 & 0.3 & 0.9 \\
\hline MAGNESIUH & $\begin{array}{l}02 / 26 / 92 \\
12 / 93 / 93 \\
04 / 21 / 94 \\
12 / 13 / 94\end{array}$ & $\begin{array}{l}0001 \\
0001 \\
0001 \\
0001\end{array}$ & $M G / L$ & & $\begin{array}{l}3.2 \\
2.5 \\
2.9 \\
3.2\end{array}$ & $\begin{array}{l}0.1 \\
0.1 \\
0.1 \\
0.1\end{array}$ & $\begin{array}{l}: \\
:\end{array}$ \\
\hline MAGNESIUM (TOTAL) & $\begin{array}{l}02 / 02 / 93 \\
06 / 25 / 93 \\
12 / 13 / 93 \\
04 / 21 / 94 \\
12 / 13 / 94\end{array}$ & $\begin{array}{l}\text { No01 } \\
\text { No01 } \\
\text { No01 } \\
\text { No01 } \\
\text { No01 }\end{array}$ & MG/L & - & $\begin{array}{l}2.8 \\
2.4 \\
2.5 \\
2.9 \\
3\end{array}$ & $\begin{array}{l}0.1 \\
0.1 \\
0.1 \\
0.1 \\
0.1\end{array}$ & $\begin{array}{l}- \\
- \\
-\end{array}$ \\
\hline MUNGAKESE & $\begin{array}{l}02 / 26 / 92 \\
11 / 21 / 92 \\
12 / 13 / 93 \\
04 / 21 / 94 \\
12 / 13 / 94\end{array}$ & $\begin{array}{l}0001 \\
0001 \\
0001 \\
0001 \\
0001\end{array}$ & MG/L & $\begin{array}{l}< \\
< \\
< \\
<\end{array}$ & $\begin{array}{l}0.01 \\
0.0044 \\
0.01 \\
0.01 \\
0.01\end{array}$ & $\begin{array}{l}0.01 \\
0.0015 \\
0.01 \\
0.01 \\
0.01\end{array}$ & $\begin{array}{l}- \\
\therefore \\
-\end{array}$ \\
\hline MANGAMESE (TOTAL) & $\begin{array}{l}11 / 21 / 92 \\
02 / 02 / 93 \\
06 / 25 / 93 \\
12 / 13 / 93 \\
04 / 21 / 94 \\
12 / 13 / 94\end{array}$ & $\begin{array}{l}\text { NO01 } \\
\text { NO01 } \\
\text { NO01 } \\
\text { N009 } \\
\text { N001 } \\
\text { NO01 }\end{array}$ & MG/L & $\begin{array}{l}< \\
< \\
< \\
<\end{array}$ & $\begin{array}{l}0.0015 \\
0.01 \\
0.01 \\
0.09 \\
0.01 \\
0.01\end{array}$ & $\begin{array}{l}0.0015 \\
0.01 \\
0.01 \\
0.01 \\
0.01 \\
0.01\end{array}$ & $\begin{array}{l}: \\
: \\
:\end{array}$ \\
\hline MOLYBDENUM & $02 / 26 / 92$ & 0009 & MG/L & $<$ & 0.01 & 0.01 & - \\
\hline
\end{tabular}

PARAMETER VALUE IMDICATOR (PVI): < - LESS THAN DETECTION LIMIT

SAMPLE ID COOES:

OOO1 - FILTERED SAMPLE (.45 MICRONS)

OTHER PARAMETER VALUE FLAGS:

NOOI - UNFILTERED SAMPLE

$\mathrm{J}$ - ESTIMATED VALUE

N - SPIKE SNMPLE RECOVERY NOT HITHIN CONTROL LIMITS 
GROUNDHATER QUALITY DATA BY LOCATIOH

SOUP REV. O Data

SITE: HOHO9 MOHUMENT VALLEY

LOCATION: 0625

MORTH COORDIHATE: 58272.5 FT

EAST COORDIHATE: B9803.1 FT

04/21/88 TO $12 / 13 / 94$

REPORT DATE: $06 / 20 / 95$

FORHATION OF COMPLETION: DECHELLEY MEMBER OF THE CUTLER FORMATION (DC) HYORAULIC FLOW RELATIOHSHIP: CROSS GRADIENT (C)

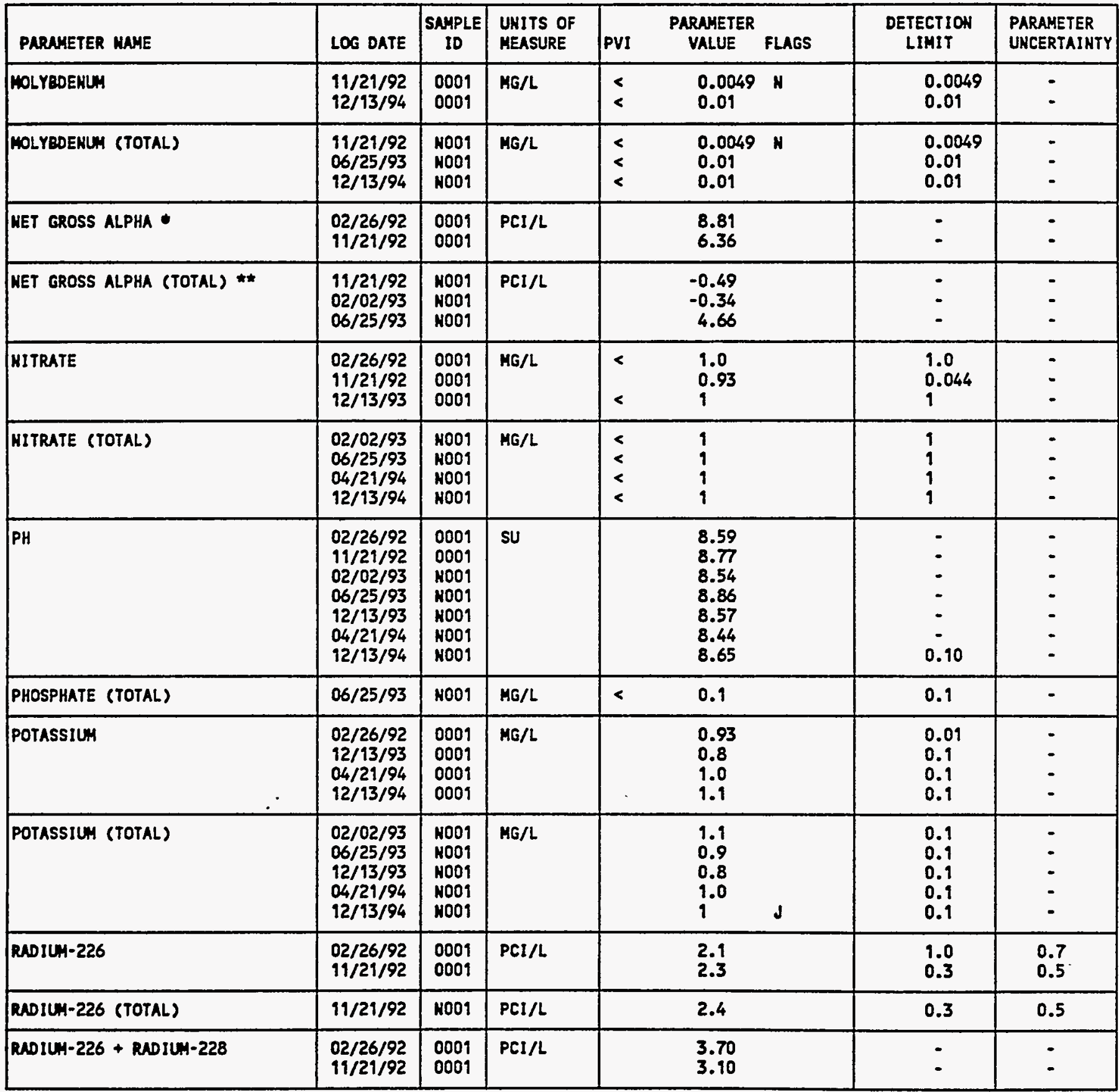

- MET gross alpha (GROSS ALPHA - URANIUM) MITH 1 Mg URAKIUM = 686 PCI

* MET GROSS ALPHA (TOTAL) (TOTAL GROSS ALPHA - TOTAL URANIUH)

PARAMETER VALUE IMDICATOR (PVI): < - LESS THAN DETECTION LIMIT

OTHER PARAMETER VALUE FLAGS:

SAMPLE ID COOES:

OOO1 - FILTERED SAMPLE (.45 MICROHS)

J - ESTIMATED VALUE

MO01 - UAFILTERED SNMPLE

H - SPIKE SAMPLE RECOVERY NOT MITHIM CONTROL LIMITS 
GROUHDWATER QUALITY DATA BY LOCATION

SOUP REV. O Dots

SITE: MONOI MONLMENT VALLEY

LOCATION: 0625

NORTH COORDINATE: $\quad 58272.5 \mathrm{FT}$

EAST COORDINATE: 89803.1 FT

$04 / 21 / 88$ TO $12 / 13 / 94$

REPORT DATE: $06 / 20 / 95$

FORMATION OF CONPLETION: DECHELLEY MEMBER OF THE CUTLER FORMATION (DC)

HYDRAULIC FLOU RELATIONSHIP: CROSS GRADIENT (C)

\begin{tabular}{|c|c|c|c|c|c|c|c|c|}
\hline PARAMETER MAME & LOG DATE & ID & $\begin{array}{l}\text { UNITS OF } \\
\text { MEASURE }\end{array}$ & PVI & $\begin{array}{l}\text { PARAMETER } \\
\text { VALUE }\end{array}$ & FLAGS & $\begin{array}{l}\text { DETECTIOH } \\
\text { LIMIT }\end{array}$ & $\begin{array}{l}\text { PARAMETER } \\
\text { UNCERTAINTY }\end{array}$ \\
\hline RADIUH-226 + RADIUH-228 (TOTAL) & $11 / 21 / 92$ & NOOI & $\mathrm{PCI} / \mathrm{L}$ & & 5.90 & & - & - \\
\hline RADIUN-228 & $\begin{array}{l}02 / 26 / 92 \\
11 / 21 / 92\end{array}$ & $\begin{array}{l}0001 \\
0001\end{array}$ & $\mathrm{PCI} / \mathrm{L}$ & & $\begin{array}{l}1.6 \\
0.8\end{array}$ & & $\begin{array}{l}1.0 \\
2.5\end{array}$ & $\begin{array}{l}1.7 \\
1.6\end{array}$ \\
\hline RADIUA-228 (TOTAL) & $11 / 21 / 92$ & NoOI & $\mathrm{PCI} / \mathrm{L}$ & & 3.5 & & 1.9 & 1.4 \\
\hline REDOX POTENTIAL & $12 / 13 / 94$ & N001 & mVOLTS & & 167 & & - & - \\
\hline SELENIUH & $\begin{array}{l}02 / 26 / 92 \\
11 / 21 / 92\end{array}$ & $\begin{array}{l}0001 \\
0001\end{array}$ & $M G / L$ & $<$ & $\begin{array}{l}0.005 \\
0.0015\end{array}$ & N & $\begin{array}{l}0.005 \\
0.0015\end{array}$ & - \\
\hline SELENIUM (TOTAL) & $11 / 21 / 92$ & N001 & MG/L & $<$ & 0.0015 & & 0.0015 & - \\
\hline SILICA - SIO2 & $\begin{array}{l}12 / 13 / 93 \\
12 / 13 / 94\end{array}$ & $\begin{array}{l}0001 \\
0001\end{array}$ & MG/L & & $\begin{array}{l}13.3 \\
13.2\end{array}$ & & $\begin{array}{l}0.1 \\
0.1\end{array}$ & - \\
\hline SILICA - SIOZ (TOTAL) & $\begin{array}{l}06 / 25 / 93 \\
12 / 13 / 93 \\
12 / 13 / 94\end{array}$ & $\begin{array}{l}\text { No01 } \\
\text { N001 } \\
\text { N001 }\end{array}$ & MG/L & & $\begin{array}{l}12.7 \\
13.2 \\
11.2\end{array}$ & H & $\begin{array}{l}0.5 \\
0.1 \\
0.1\end{array}$ & $\div$ \\
\hline SOOIUM & $\begin{array}{l}02 / 26 / 92 \\
12 / 13 / 93 \\
04 / 21 / 94 \\
12 / 13 / 94\end{array}$ & $\begin{array}{l}0001 \\
0001 \\
0001 \\
0001\end{array}$ & $M G / L$ & & $\begin{array}{l}124 . \\
118 \\
121 \\
127\end{array}$ & & $\begin{array}{l}5 . \\
1 \\
1 \\
1\end{array}$ & $\begin{array}{l}- \\
- \\
-\end{array}$ \\
\hline SCOIU: (TOTAL) & $\begin{array}{l}02 / 02 / 93 \\
06 / 25 / 93 \\
12 / 13 / 93 \\
04 / 21 / 94 \\
12 / 13 / 94\end{array}$ & $\begin{array}{l}\text { N001 } \\
\text { No01 } \\
\text { N001 } \\
\text { N001 } \\
\text { N001 }\end{array}$ & MG/L & & $\begin{array}{l}110 \\
117 \\
120 \\
107 \\
115\end{array}$ & & $\begin{array}{l}0.1 \\
1 \\
1 \\
1 \\
1\end{array}$ & $\begin{array}{l}: \\
: \\
:\end{array}$ \\
\hline SPECIFIC COHDUCTANCE & $\begin{array}{l}02 / 26 / 92 \\
11 / 21 / 92 \\
02 / 02 / 93 \\
06 / 25 / 93 \\
12 / 13 / 93 \\
04 / 21 / 94 \\
12 / 13 / 94\end{array}$ & $\begin{array}{l}0001 \\
0001 \\
\text { NOO1 } \\
\text { N001 } \\
\text { N001 } \\
\text { NOO1 } \\
\text { NO01 }\end{array}$ & UNHO/CM & & $\begin{array}{l}456 \\
455 \\
454 \\
460 \\
420 \\
462 \\
429\end{array}$ & & $\begin{array}{l}- \\
- \\
i \\
i\end{array}$ & : \\
\hline STROATIUN & $\begin{array}{l}02 / 26 / 92 \\
11 / 21 / 92 \\
12 / 13 / 93 \\
04 / 21 / 94 \\
12 / 13 / 94\end{array}$ & $\begin{array}{l}0001 \\
0001 \\
0001 \\
0001 \\
0001\end{array}$ & MG/L & & $\begin{array}{l}0.08 \\
0.10 \\
0.07 \\
0.08 \\
0.08\end{array}$ & $d$ & $\begin{array}{l}0.01 \\
0.00035 \\
0.01 \\
0.01 \\
0.01\end{array}$ & $\begin{array}{l}- \\
- \\
-\end{array}$ \\
\hline STRONTILA (TOTAL) & $\begin{array}{l}11 / 21 / 92 \\
06 / 25 / 93 \\
12 / 13 / 93 \\
04 / 21 / 94 \\
12 / 13 / 94\end{array}$ & $\begin{array}{l}\text { No01 } \\
\text { N001 } \\
\text { N001 } \\
\text { N001 } \\
\text { NO01 }\end{array}$ & $M G / L$ & & $\begin{array}{l}0.096 \\
0.06 \\
0.07 \\
0.08 \\
0.08\end{array}$ & J & $\begin{array}{l}0.00035 \\
0.01 \\
0.01 \\
0.01 \\
0.01\end{array}$ & $\begin{array}{l}- \\
- \\
-\end{array}$ \\
\hline SULFATE & $\begin{array}{l}02 / 26 / 92 \\
11 / 21 / 92\end{array}$ & $\begin{array}{l}0001 \\
0001\end{array}$ & MG/L & & $\begin{array}{l}54 . \\
58\end{array}$ & & 10. & $\ddot{-}$ \\
\hline
\end{tabular}

PARAMETER VALUE IMDICATOR (PVI): < - LESS THAN DETECTION LIMIT SAMPLE ID COOES:

OTHER PARAMETER VALUE FLAGS:

0001 - FILTERED SAMPLE (.45 MICRONS)

H - HOLD TIME EXPIRED, VALUE SUSPECT

NOO - UMFILTERED SAMPLE

J - estimated VALUE

N - SṔIKE SAMPLE RECOVERY NOT UITHIN CONTROL LIMITS 
GROUNDHATER QUALITY DATA BY LOCATIOH

SOUP REV. $O$ Data

SITE: MONO1 MONUNENT VALLEY

LOCATION: 0625

HORTH COORDIMATE: 58272.5 FT

EAST COORDIHATE: 89803.1 FT

04/21/88 TO $12 / 13 / 94$

REPORT DATE: 06/20/95

FORMATION OF COMPLETION: DECHELLEY MEMBER OF THE CUTLER FORMATION (DC) HYDRAULIC FLON RELATIOHSHIP: CROSS GRADIEKT (C)

\begin{tabular}{|c|c|c|c|c|c|c|c|}
\hline PARAMETER NAME & LOE DATE & $\begin{array}{c}\text { SAMPLE } \\
\text { ID }\end{array}$ & $\begin{array}{l}\text { UHITS OF } \\
\text { MEASURE }\end{array}$ & PVI & $\begin{array}{l}\text { PARAMETER } \\
\text { VALUE FLAGS }\end{array}$ & $\begin{array}{l}\text { DETECTION } \\
\text { LIMIT }\end{array}$ & $\begin{array}{l}\text { PARAMETER } \\
\text { UNCERTAIHTY }\end{array}$ \\
\hline SULFATE & $\begin{array}{l}12 / 13 / 93 \\
04 / 21 / 94\end{array}$ & $\begin{array}{l}0001 \\
0001\end{array}$ & MG/L & & $\begin{array}{l}55 \\
54\end{array}$ & $\begin{array}{l}1 \\
1\end{array}$ & $\dot{-}$ \\
\hline SULFATE (TOTAL) & $\begin{array}{l}11 / 21 / 92 \\
02 / 02 / 93 \\
06 / 25 / 93 \\
12 / 13 / 94\end{array}$ & $\begin{array}{l}\text { No01 } \\
\text { No01 } \\
\text { N001 } \\
\text { N001 }\end{array}$ & MG/L & & $\begin{array}{l}57 \\
46 \\
51 \\
42\end{array}$ & $\begin{array}{l}1.0 \\
1 \\
1 \\
1\end{array}$ & : \\
\hline TEMPERATURE & $\begin{array}{l}02 / 26 / 92 \\
11 / 21 / 92 \\
02 / 02 / 93 \\
06 / 25 / 93 \\
12 / 13 / 93 \\
04 / 21 / 94 \\
12 / 13 / 94\end{array}$ & $\begin{array}{l}\text { Do01 } \\
0001 \\
\text { No01 } \\
\text { N001 } \\
\text { N001 } \\
\text { N001 } \\
\text { N001 }\end{array}$ & C - DEGREE & & $\begin{array}{l}15.6 \\
15.7 \\
15.7 \\
16.9 \\
13.0 \\
16.0 \\
14.4\end{array}$ & $\begin{array}{c}- \\
\therefore \\
- \\
- \\
0.1\end{array}$ & $\begin{array}{l}- \\
- \\
- \\
\vdots \\
-\end{array}$ \\
\hline TOTAL DISSOLVED SOLIDS & $\begin{array}{l}02 / 26 / 92 \\
12 / 13 / 93 \\
04 / 21 / 94 \\
12 / 13 / 94\end{array}$ & $\begin{array}{l}0001 \\
0001 \\
0001 \\
0001\end{array}$ & $M G / L$ & & $\begin{array}{l}339 . \\
330 \\
340 \\
330\end{array}$ & $\begin{array}{l}10 . \\
10 \\
10 \\
10\end{array}$ & $\begin{array}{l}- \\
\dot{-} \\
\dot{-}\end{array}$ \\
\hline TOTAL DISSOLVED SOLIDS (TOTAL) & $\begin{array}{l}02 / 02 / 93 \\
06 / 25 / 93\end{array}$ & $\begin{array}{l}\text { N001 } \\
\text { H001 }\end{array}$ & $M G / L$ & & $\begin{array}{l}340 \\
310\end{array}$ & $\begin{array}{l}10 \\
10\end{array}$ & - \\
\hline TOTAL DRGAHIC CARBON & $02 / 26 / 92$ & 0001 & $M G / L$ & $<$ & 9. & 1. & - \\
\hline URAKILM & $\begin{array}{l}02 / 26 / 92 \\
11 / 21 / 92 \\
12 / 13 / 93 \\
04 / 21 / 94 \\
12 / 13 / 94\end{array}$ & $\begin{array}{l}0001 \\
0001 \\
0001 \\
0001 \\
0001\end{array}$ & $M G / L$ & $\begin{array}{l}< \\
< \\
< \\
<\end{array}$ & $\begin{array}{l}0.001 \\
0.001 \\
0.001 \\
0.001 \\
0.001\end{array}$ & $\begin{array}{l}0.001 \\
0.001 \\
0.001 \\
0.001 \\
0.001\end{array}$ & $\begin{array}{l}- \\
- \\
. \\
.\end{array}$ \\
\hline URAKIUH (TOTAL) & $\begin{array}{l}11 / 21 / 92 \\
02 / 02 / 93 \\
06 / 25 / 93 \\
12 / 13 / 93 \\
04 / 21 / 94 \\
12 / 13 / 94\end{array}$ & $\begin{array}{l}\text { HOO1 } \\
\text { NO01 } \\
\text { NO01 } \\
\text { NO01 } \\
\text { N001 } \\
\text { N001 }\end{array}$ & MG/L & $\begin{array}{l}< \\
< \\
< \\
< \\
<\end{array}$ & $\begin{array}{l}0.001 \\
0.001 \\
0.001 \\
0.001 \\
0.001 \\
0.001\end{array}$ & $\begin{array}{l}0.001 \\
0.001 \\
0.001 \\
0.001 \\
0.001 \\
0.001\end{array}$ & $\begin{array}{l}- \\
- \\
- \\
-\end{array}$ \\
\hline VAKADIUM & $\begin{array}{l}02 / 26 / 92 \\
11 / 21 / 92 \\
12 / 13 / 93 \\
04 / 21 / 94 \\
12 / 13 / 94\end{array}$ & $\begin{array}{l}0001 \\
0001 \\
0001 \\
0001 \\
0001\end{array}$ & ME/L & $\begin{array}{l}< \\
< \\
< \\
< \\
<\end{array}$ & $\begin{array}{l}0.01 \\
0.0019 \\
0.01 \\
0.01 \\
0.01\end{array}$ & $\begin{array}{l}0.01 \\
0.0019 \\
0.01 \\
0.01 \\
0.01\end{array}$ & $\begin{array}{l}- \\
- \\
- \\
-\end{array}$ \\
\hline VAHADIUM (TOTAL) & $\begin{array}{l}11 / 21 / 92 \\
06 / 25 / 93 \\
12 / 13 / 93 \\
04 / 21 / 94 \\
12 / 13 / 94\end{array}$ & $\begin{array}{l}\text { No01 } \\
\text { No01 } \\
\text { No01 } \\
\text { No01 } \\
\text { No01 }\end{array}$ & MG/L & $\begin{array}{l}< \\
< \\
< \\
< \\
<\end{array}$ & $\begin{array}{l}0.0019 \\
0.01 \\
0.01 \\
0.01 \\
0.01\end{array}$ & $\begin{array}{l}0.0019 \\
0.01 \\
0.01 \\
0.01 \\
0.01\end{array}$ & $\begin{array}{l}- \\
: \\
-\end{array}$ \\
\hline ZINC & $\begin{array}{l}02 / 26 / 92 \\
11 / 21 / 92\end{array}$ & $\begin{array}{l}0001 \\
0001\end{array}$ & $M G / L$ & $<$ & $\begin{array}{l}0.005 \\
0.0010\end{array}$ & $\begin{array}{l}0.005 \\
0.0010\end{array}$ & $\dot{-}$ \\
\hline 2IMC (TOTAL) & $\begin{array}{l}11 / 21 / 92 \\
06 / 25 / 93\end{array}$ & $\begin{array}{l}\text { N001 } \\
\text { N001 }\end{array}$ & MG/L & $<$ & $\begin{array}{l}0.0030 \\
0.005 \mathrm{~J}\end{array}$ & $\begin{array}{l}0.0010 \\
0.005\end{array}$ & 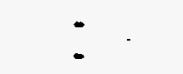 \\
\hline
\end{tabular}

PARAMETER VALUE IHDICATOR (PVI): < - LESS THAN DETECTION LIMIT

SAMPLE ID COOES:

DOO1 - FILTERED SAMLE (.45 MICRONS)

OTHER PARAMETER VALUE FLAGS:

NOO1 - UNFILTERED SAMPLE

H - hOLD TIME EXPIRED, VALUE SUSPECT

J - estimated VALUe 
GROUNDWATER QUALITY DATA BY LOCATION

SOWP ReV. O Data

SITE: MOHO1 MONUMENT VALLEY

LOCATION: 0625

NORTH COORDINATE: 58272.5 FT

EAST COORDINATE: 89803.1 FT

$04 / 21 / 88$ TO $12 / 13 / 94$

REPORT DATE: $06 / 20 / 95$

FORMATION OF COAPLETION: DECHELLEY MEMBER OF THE CUTLER FORMATION (DC) HYDRAULIC FLON RELATIONSHIP: CROSS GRADIENT (C)

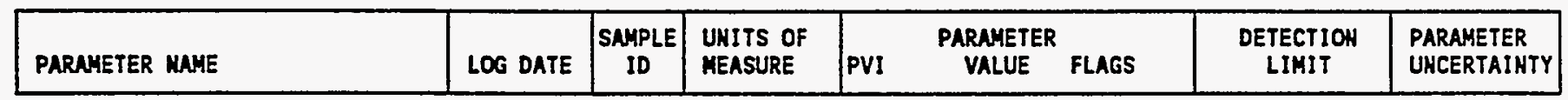

PARAMETER VALUE INDICATOR (PVI):

< - LESS THAN DETECTION LIMIT

SNMPLE ID COOES: 
GROUMDUATER QUALITY DATA BY LOCATION

SOLP REV. 0 Data

SITE: MONOI MONUMENT VALLEY

LOCATIOH: OS40 RESERVED FOR AMILLER

MORTH COORDIHATE: UNKHOWN

EAST COORDIHATE: UNKNOWN

04/21/88 TO $12 / 13 / 94$

REPORT DATE: $06 / 20 / 95$

FORHATIOH OF COHPLETIOH: NO RECOVERY OF OATA FOR CLASSIFYIHG (HR) HYDRAULIC FLOW RELATIONSHIP: UNKHOWN (N)

\begin{tabular}{|c|c|c|c|c|c|c|c|c|}
\hline ' PARAHETER NAME & LOG DATE & $\begin{array}{c}\text { SAMPLE } \\
10\end{array}$ & $\begin{array}{l}\text { UNITS OF } \\
\text { MEASURE }\end{array}$ & PVI & $\begin{array}{l}\text { PARAMETER } \\
\text { VALUE }\end{array}$ & FLAGS & $\begin{array}{l}\text { DETECTION } \\
\text { LIMIT }\end{array}$ & $\begin{array}{l}\text { PARAMETER } \\
\text { UHCERTAIUTY }\end{array}$ \\
\hline ALKALINITY & $12 / 12 / 93$ & NOOI & $\mathrm{MG} / \mathrm{L} \mathrm{CACO} 3$ & & 372 & RX & - & - \\
\hline Ammoniun & $12 / 12 / 93$ & 0001 & $M G / L$ & $<$ & 0.1 & RX & 0.1 & - \\
\hline Calcilu & $12 / 12 / 93$ & 0001 & MG/L & & 56.2 & RX & 0.5 & - \\
\hline CALCIUA (TOTAL) & $12 / 92 / 93$ & N001 & MG/L & & 57.9 & RX & 0.5 & - \\
\hline CHLORIDE & $12 / 12 / 93$ & 0001 & MG/L & & 113 & $\mathbf{R X}$ & 0.5 & - \\
\hline MAGHESIUH & $12 / 12 / 93$ & 0001 & $M G / L$ & & 107 & $R X$ & 0.1 & - \\
\hline MUGNESIUM (TOTAL) & $12 / 12 / 93$ & NOO1 & $M G / L$ & & 105 & RX & 0.1 & $\therefore$ \\
\hline MAHGAHESE & $12 / 12 / 93$ & 0001 & $M G / L$ & $<$ & 0.01 & $\mathbf{R X}$ & 0.01 & - \\
\hline MAKGAKESE (TOTAL) & $12 / 12 / 93$ & N001 & $M G / L$ & $<$ & 0.09 & RX & 0.01 & - \\
\hline HITRATE & $12 / 12 / 93$ & 0001 & MG/L & & 19 & $R X$ & 1 & - \\
\hline PH & $12 / 12 / 93$ & N001 & SU & & 7.87 & RX & - & - \\
\hline POTASSIUA & $12 / 12 / 93$ & 0009 & MG/L & & 0.8 & $\mathbf{R X}$ & 0.1 & - \\
\hline POTASSILM (TOTAL) & $12 / 12 / 93$ & No01 & MG/L & & 0.9 & RX & 0.1 & - \\
\hline SILICA - SIOZ & $12 / 12 / 93$ & 0001 & MG/L & & 54.0 & RX & 0.1 & - \\
\hline SILICA - SIOL (TOTAL) & $12 / 12 / 93$ & NOOI & $M G / L$ & & 52.9 & RX & 0.1 & - \\
\hline SCOIUH & $12 / 12 / 93$ & 0001 & $M G / L$ & & 299 & RX & 1 & $\cdot$ \\
\hline SOOIUM (TOTAL) & $12 / 12 / 93$ & N001 & $M G / L$ & & 298 & RX & 1 & - \\
\hline SPECIFIC CONDUCTAHCE & $12 / 12 / 93$ & N001 & ЧNHO/CM & & 1667 & RX & - & $\cdot$ \\
\hline STROHTIUM & $12 / 12 / 93$ & 0001 & MG/L & & 1.65 & RX & 0.01 & - \\
\hline STRONTIUM (TOTAL) & $12 / 12 / 93$ & M001 & $M G / L$ & & 1.59 & RX & 0.01 & - \\
\hline SULFATE & $12 / 12 / 93$ & 0001 & $M G / L$ & & 668 & $R X$ & 1 & - \\
\hline TEHPERATURE & $12 / 12 / 93$ & N001 & C - DEGREE & & 11.6 & RX & - & - \\
\hline TOTAL DISSOLVED SOLIDS & $12 / 12 / 93$ & 0001 & MG/L & & 1590 & $\mathbf{R X}$ & 10 & - \\
\hline LRakitu & $12 / 12 / 93$ & 0001 & MG/L & & 0.021 & $\mathbf{R X}$ & 0.001 & - \\
\hline URAKILH (TOTAL) & $12 / 12 / 93$ & Y009 & MG/L & & 0.019 & RX & 0.001 & - \\
\hline VAMADIUA & $12 / 12 / 93$ & 0001 & $M G / L$ & & 0.01 & RX & 0.01 & - \\
\hline VANADIUN (TOTAL) & $12 / 12 / 93$ & N001 & MG/L & & 0.02 & RX & 0.09 & $=$ \\
\hline
\end{tabular}

PARAMETER VALUE INDICATOR (PVI): < - LESS THAN DETECTION LIMIT

SUMPLE ID COOES:

OTHER PARAMETER VALUE FLAGS:

0001 - FILTERED SAMPLE (.45 HICRONS)

$R$ - UNUSABLE DATA POIHT

HOO1 - UHFILTERED SAMPLE

$X$ - LOCATION UMDEFIMED 
GROUNDWATER QUALITY DATA BY LOCATIOH

SOUP ReV. 0 Data

SITE: MONO1 MONLWENT VALLEY

LOCATION: 0.550

MORTH COORDINATE: 64970.2 FT

EAST COORDINATE: 89923.8 FT

$04 / 21 / 88$ TO $12 / 13 / 94$

REPORT DATE: OS/20/95

FORMATIOH OF COMPLETIOH: ALLUVIUM (AL)

HYDRAULIC FLON RELATIONSHIP: DOWN GRNDIENT (D)

\begin{tabular}{|c|c|c|c|c|c|c|c|c|}
\hline PARAMETER NAME & LOS DATE & $\underset{\text { SNMPLE }}{\text { ID }}$ & $\begin{array}{l}\text { UNITS OF } \\
\text { MEASURE }\end{array}$ & PVI & $\begin{array}{l}\text { PARAMETER } \\
\text { VALUE }\end{array}$ & FLAGS & $\begin{array}{l}\text { DETECTION } \\
\text { LIMIT }\end{array}$ & $\begin{array}{l}\text { PARAMETER } \\
\text { UHCERTAIHTY }\end{array}$ \\
\hline ALKALIKITY & $\begin{array}{l}04 / 26 / 88 \\
06 / 09 / 89 \\
12 / 14 / 93\end{array}$ & $\begin{array}{l}0001 \\
0001 \\
\text { N001 }\end{array}$ & $\mathrm{MG} / \mathrm{L} \quad \mathrm{CACO} 3$ & & $\begin{array}{l}205 . \\
219 . \\
204\end{array}$ & $\mathbf{L}$ & : & $:$ \\
\hline ALUAINUM & $06 / 09 / 89$ & 0001 & MG/L & $<$ & 0.1 & $\mathbf{L}$ & 0.1 & - \\
\hline AmeNIUA & $\begin{array}{l}04 / 26 / 88 \\
06 / 09 / 89 \\
12 / 14 / 93\end{array}$ & $\begin{array}{l}0001 \\
0001 \\
0001\end{array}$ & MG/L & $\begin{array}{l}< \\
<\end{array}$ & $\begin{array}{l}0.1 \\
0.1 \\
0.1\end{array}$ & $L$ & $\begin{array}{l}0.1 \\
0.1 \\
0.1\end{array}$ & : \\
\hline AKTIMONY & $06 / 09 / 89$ & 0001 & MG/L & $<$ & 0.003 & $L$ & 0.003 & - \\
\hline ARSENIC & $\begin{array}{l}04 / 26 / 88 \\
06 / 09 / 89\end{array}$ & $\begin{array}{l}0001 \\
0001\end{array}$ & $M G / L$ & $<$ & $\begin{array}{l}0.003 \\
0.01\end{array}$ & $\begin{array}{l}3 \\
L\end{array}$ & $\begin{array}{l}0.01 \\
0.01\end{array}$ & $\because$ \\
\hline BARIUM & $06 / 09 / 89$ & 0001 & MG/L & $<$ & 0.1 & $\mathbf{L}$ & 0.9 & - \\
\hline BERYLLIUM & $06 / 09 / 89$ & 0001 & MG/L & $<$ & 0.01 & $L$ & 0.01 & - \\
\hline BORON & $06 / 09 / 89$ & 0001 & $M G / L$ & $<$ & 0.1 & $L$ & 0.1 & - \\
\hline $\begin{array}{l}\text { CADMIUH } \\
. .\end{array}$ & $\begin{array}{l}04 / 26 / 88 \\
06 / 09 / 89\end{array}$ & $\begin{array}{l}0001 \\
0001\end{array}$ & MG/L & $<$ & $\begin{array}{l}0.004 \\
0.001\end{array}$ & $L$ & $\begin{array}{l}0.001 \\
0.001\end{array}$ & - \\
\hline $\begin{array}{l}\text { CALCIUN } \\
\ldots\end{array}$ & $\begin{array}{l}04 / 26 / 88 \\
06 / 09 / 89 \\
12 / 14 / 93\end{array}$ & $\begin{array}{l}0001 \\
0001 \\
0001\end{array}$ & MG/L & & $\begin{array}{l}9.00 \\
9.48 \\
8.3\end{array}$ & $L$ & $\begin{array}{l}0.01 \\
0.01 \\
0.5\end{array}$ & : \\
\hline CALCIUM (TOTAL) & $12 / 14 / 93$ & NoO1 & MG/L & & 9.2 & & 0.5 & - \\
\hline CHLORIDE & $\begin{array}{l}04 / 26 / 88 \\
06 / 09 / 89 \\
12 / 14 / 93\end{array}$ & $\begin{array}{l}0001 \\
0001 \\
0001\end{array}$ & MG/L & & $\begin{array}{r}8.2 \\
6.1 \\
11.6\end{array}$ & $L$ & $\begin{array}{l}1 . \\
0.5\end{array}$ & : \\
\hline CHROMIUM & $06 / 09 / 89$ & 0001 & MG/L & $<$ & 0.01 & $L$ & 0.01 & - \\
\hline $\operatorname{COBALT}$ & $06 / 09 / 89$ & 0001 . & $M G / L$ & $<$ & 0.05 & $\mathbf{L}$ & 0.05 & - \\
\hline COPPER & $06 / 09 / 89$ & 0001 & MG/L & $<$ & 0.02 & $L$ & 0.02 & $\cdots$ \\
\hline CYANIDE & $06 / 09 / 89$ & 0001 & MG/L & $<$ & 0.01 & $L$ & 0.01 & - \\
\hline DISSOLVED OXYGEN & $12 / 14 / 93$ & No01 & MG/L & $<$ & 0.6 & & - & - \\
\hline FLUORIDE & $06 / 09 / 89$ & 0001 & MG/L & & 0.4 & $\mathbf{L}$ & 0.1 & - \\
\hline GROSS ALPHA & $04 / 26 / 88$ & 0001 & $\mathrm{PCI} / \mathrm{L}$ & & 3.0 & & 0.2 & 2.6 \\
\hline GROSS BETA & $04 / 26 / 88$ & 0001 & PCI/L & & 2.9 & & 1. & 1.3 \\
\hline IRON & $06 / 09 / 89$ & 0001 & MG/L & $<$ & 0.03 & $\mathbf{L}$ & 0.03 & - \\
\hline LEAD & $06 / 09 / 89$ & 0001 & MG/L & $<$ & 0.01 & $L$ & 0.01 & - \\
\hline MGEESIU: & $04 / 26 / 88$ & 0001 & MG/L & & 6.23 & & 0.001 & - \\
\hline
\end{tabular}

PARAMETER VALUE IHDICATOR (PVI):

$<$ - LESS THAN DETECTION LIMIT

SNMPLE ID COOES:

OTHER PARAYETER VALUE FLAGS:

OOO1 - FILTERED SAMPLE (.45 MICRONS)

NO01 - UNFILTERED SAMPLE

J - ESTIMUTED VALUE

L - LESS THAH THREE BORE VOLLMES REMOVED BEFORE SNMPLING 
GROUHDHATER QUALITY DATA BY LOCATION

SOWP REV. O Data

SITE: MONO1 MONUMENT VALLEY

LOCATION: 0650

NORTH COORDINATE: 64970.2 FT

EAST COORDIHATE: 89923.8 FT

04/21/88 TO $12 / 13 / 94$

REPORT DATE: 06/20/95

FORMATION OF COMPLETION: ALLUVIUM (AL)

HYDRAULIC FLON RELATIONSHIP: DOWN GRADIEHT (D)

\begin{tabular}{|c|c|c|c|c|c|c|c|c|}
\hline PARAMETER MAME & LOG DATE & $\underset{\text { SAMPLE }}{\text { ID }}$ & $\begin{array}{l}\text { UHITS OF } \\
\text { MEASURE }\end{array}$ & PVI & $\begin{array}{l}\text { PARAMETER } \\
\text { VALUE }\end{array}$ & FLAGS & $\begin{array}{l}\text { DETECTIOH } \\
\text { LIMIT }\end{array}$ & $\begin{array}{l}\text { PARAMETER } \\
\text { UHCERTAINTY }\end{array}$ \\
\hline MAGHESIUM & $\begin{array}{l}06 / 09 / 89 \\
12 / 14 / 93\end{array}$ & $\begin{array}{l}0001 \\
0001\end{array}$ & $M G / L$ & & $\begin{array}{l}6.51 \\
6.1\end{array}$ & $\mathbf{L}$ & $\begin{array}{l}0.001 \\
0.1\end{array}$ & - \\
\hline MGGESIUM (TOTAL) & $12 / 14 / 93$ & N001 & MG/L & & 7.0 & & 0.1 & - \\
\hline MAKGAKESE & $\begin{array}{l}06 / 09 / 89 \\
12 / 14 / 93\end{array}$ & $\begin{array}{l}0001 \\
0001\end{array}$ & MG/L & $<$ & $\begin{array}{l}0.01 \\
0.01\end{array}$ & $\mathbf{L}$ & $\begin{array}{l}0.09 \\
0.01\end{array}$ & - \\
\hline MAKGAKESE (TOTAL) & $12 / 14 / 93$ & noo1 & Mo/L & $<$ & 0.01 & & 0.01 & - \\
\hline MERCURY & $06 / 09 / 89$ & 0001 & $M G / L$ & $<$ & 0.0002 & $\mathbf{L}$ & 0.0002 & - \\
\hline MOLYBDEKUM & $\begin{array}{l}04 / 26 / 88 \\
06 / 09 / 89\end{array}$ & $\begin{array}{l}0001 \\
0001\end{array}$ & MG/L & $<$ & $\begin{array}{l}0.01 \\
0.01\end{array}$ & $L$ & $\begin{array}{l}0.01 \\
0.01\end{array}$ & - \\
\hline HET GROSS ALPHA * & $04 / 26 / 88$ & 0001 & $\mathrm{PCI} / \mathrm{L}$ & & 1.42 & & - & - \\
\hline MICKEL & $06 / 09 / 89$ & 0001 & MG/L & $<$ & 0.04 & $\mathbf{L}$ & 0.04 & - \\
\hline MITRATE & $\begin{array}{l}04 / 26 / 88 \\
06 / 09 / 89 \\
12 / 14 / 93\end{array}$ & $\begin{array}{l}0001 \\
0001 \\
0001\end{array}$ & $M G / L$ & & $\begin{array}{l}0.5 \\
5.3 \\
1\end{array}$ & $j$ & 1. & : \\
\hline PH & $\begin{array}{l}04 / 26 / 88 \\
06 / 09 / 89 \\
12 / 14 / 93\end{array}$ & $\begin{array}{l}0001 \\
0001 \\
\text { N001 }\end{array}$ & su & & $\begin{array}{l}8.52 \\
8.16 \\
8.32\end{array}$ & $\mathbf{L}$ & $:$ & : \\
\hline PHOSPHATE & $06 / 09 / 89$ & 0001 & $M G / L$ & & 0.6 & $\mathbf{L}$ & 0.1 & - \\
\hline POTASSIUA & $\begin{array}{l}04 / 26 / 88 \\
06 / 09 / 89 \\
12 / 14 / 93\end{array}$ & $\begin{array}{l}0001 \\
0001 \\
0001\end{array}$ & $M G / L$ & & $\begin{array}{l}1.02 \\
1.0 \\
0.9\end{array}$ & $\mathbf{L}$ & $\begin{array}{l}0.01 \\
0.01 \\
0.1\end{array}$ & : \\
\hline POTASSIUA (TOTAL) & $12 / 14 / 93$ & N001 & $M G / L$ & & 14.6 & & 0.1 & - \\
\hline RADIUH-226 & $06 / 09 / 89$ & 0001 & $\mathrm{PCI} / \mathrm{L}$ & & 0.1 & $\mathbf{L}$ & 1. & 0.2 \\
\hline RADILM-226 + RADIUM-228 & $06 / 09 / 89$ & 0001 & $\mathrm{PCI} / \mathrm{L}$ & & 0.10 & & - & - \\
\hline RADIUM-228 & $06 / 09 / 89$ & 0001 & $\mathrm{PCI} / \mathrm{L}$ & & 0.0 & $\mathbf{L}$ & 9. & 0.7 \\
\hline REDOX POTENTIAL & $12 / 14 / 93$ & No01 & NVOLTS & & 376 & & - & - \\
\hline SELENIUM & $\begin{array}{l}04 / 26 / 88 \\
06 / 09 / 89\end{array}$ & $\begin{array}{l}0001 \\
0001\end{array}$ & MG/L & $<$ & $\begin{array}{l}0.005 \\
0.005\end{array}$ & $\mathbf{L}$ & $\begin{array}{l}0.005 \\
0.005\end{array}$ & - \\
\hline SILICA - SIO2 & $\begin{array}{l}06 / 09 / 89 \\
12 / 14 / 93\end{array}$ & $\begin{array}{l}0001 \\
0001\end{array}$ & MG/L & & $\begin{array}{l}12 . \\
12.6\end{array}$ & $L$ & $\begin{array}{l}2 . \\
0.1\end{array}$ & - \\
\hline SILICA - SIO2 (TOTAL) & $12 / 14 / 93$ & N001 & MG/L & & 12.3 & & 0.1 & - \\
\hline SILVER & $06 / 09 / 89$ & 0009 & $M G / L$ & $<$ & 0.01 & $\mathbf{L}$ & 0.01 & - \\
\hline SOOIU, & $04 / 26 / 88$ & 0001 & MG/L & & 93.6 & & 0.002 & - \\
\hline
\end{tabular}

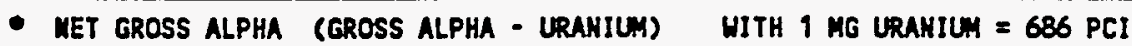

PARAMETER VALUE IHDICATOR (PVI): < - LESS THAN DETECTIOH LIMIT

L - LESS THAH THREE BORE VOLUMES REMOVED BEFORE SAMPLIMG 
GROUNDHATER QUALITY DATA BY LOCATIOH

SONP ReV. O Data

SITE: MONOI MONUMENT VALLEY

LOCATION: 0650

NORTH COORDINATE: 64970.2 FT

EAST COORDINATE: 89923.8 FT

$04 / 21 / 88$ TO $12 / 13 / 94$

REPORT DATE: $06 / 20 / 95$

FORMATION OF COMPLETION: ALLUVIUM (AL)

HYDRAULIC FLOW RELATIONSHIP: DOWN GRADIENT (D)

\begin{tabular}{|c|c|c|c|c|c|c|c|c|}
\hline PARAMETER MAME & LOG DATE & $\begin{array}{c}\text { SAMPLE } \\
\text { ID }\end{array}$ & $\begin{array}{l}\text { UNITS OF } \\
\text { MEASURE }\end{array}$ & PVI & $\begin{array}{l}\text { PARAMETER } \\
\text { VALUE }\end{array}$ & FLAGS & $\begin{array}{l}\text { DETECTION } \\
\text { LIMIT }\end{array}$ & $\begin{array}{l}\text { PARAMETER } \\
\text { UNCERTAINTY }\end{array}$ \\
\hline SOOIUH & $\begin{array}{l}06 / 09 / 89 \\
12 / 14 / 93\end{array}$ & $\begin{array}{l}0001 \\
0001\end{array}$ & MG/L & & $\begin{array}{l}91.2 \\
90\end{array}$ & $L$ & 0.002 & - \\
\hline SOOILH (TOTAL) & $12 / 14 / 93$ & N001 & HG/L & & 157 & & 1 & - \\
\hline SPECIFIC CONDUCTANCE & $\begin{array}{l}04 / 26 / 88 \\
06 / 09 / 89 \\
12 / 14 / 93\end{array}$ & $\begin{array}{l}0001 \\
0001 \\
\text { N001 }\end{array}$ & Іино/сн & & $\begin{array}{l}360 . \\
340 . \\
393\end{array}$ & $\mathbf{L}$ & : & $\ddot{-}$ \\
\hline STRONTIU:M & $12 / 14 / 93$ & 0001 & $M G / L$ & \multicolumn{3}{|c|}{0.10} & 0.01 & - \\
\hline STRONTIUM (TOTAL) & $12 / 14 / 93$ & HoOI & $M G / L$ & \multicolumn{3}{|c|}{0.91} & 0.01 & - \\
\hline SULFATE & $\begin{array}{l}04 / 26 / 88 \\
06 / 09 / 89 \\
12 / 14 / 93\end{array}$ & $\begin{array}{l}0001 \\
0001 \\
0001\end{array}$ & $\mathrm{MG} / \mathrm{L}$ & \multicolumn{3}{|c|}{$\begin{array}{l}28 . \\
31 . \\
31\end{array}$} & $\begin{array}{l}0.1 \\
0.1 \\
1\end{array}$ & : \\
\hline SULFIDE & $06 / 09 / 89$ & 0001 & $M G / L$ & $<$ & 0.1 & $L$ & 0.1 & - \\
\hline TEMPERATURE & $\begin{array}{l}04 / 26 / 88 \\
06 / 09 / 89 \\
12 / 14 / 93\end{array}$ & $\begin{array}{l}0001 \\
0001 \\
\text { N001 }\end{array}$ & C - DEGREE & \multicolumn{3}{|c|}{$\begin{array}{l}16.5 \\
17.0 \\
15.2\end{array}$} & : & : \\
\hline THALLIUM & $06 / 09 / 89$ & 0001 & $M G / L$ & $<$ & 0.01 & $L$ & 0.01 & - \\
\hline TIN & $06 / 09 / 89$ & 0001 & MG/L & $<$ & 0.005 & \llcorner & 0.005 & - \\
\hline TOTAL OISSOLVEO SOLIDS & $\begin{array}{l}04 / 26 / 88 \\
06 / 09 / 89 \\
12 / 14 / 93\end{array}$ & $\begin{array}{l}0001 \\
0001 \\
0001\end{array}$ & $M G / L$ & \multicolumn{3}{|c|}{$\begin{array}{l}319 . \\
262 . \\
300\end{array}$} & $\begin{array}{l}10 . \\
10 . \\
10\end{array}$ & - \\
\hline TOTAL ORGANIC CARBON & $04 / 26 / 88$ & 0001 & MG/L & \multicolumn{3}{|c|}{58.5} & 1. & - \\
\hline URAKILA & $\begin{array}{l}04 / 26 / 88 \\
06 / 09 / 89 \\
12 / 14 / 93\end{array}$ & $\begin{array}{l}0001 \\
0001 \\
0001\end{array}$ & $M G / L$ & \multicolumn{3}{|c|}{$\begin{array}{l}0.0023 \\
0.0041 \\
0.002\end{array}$} & $\begin{array}{l}0.003 \\
0.003 \\
0.001\end{array}$ & $\ddot{-}$ \\
\hline URANIUM (TOTAL) & $12 / 14 / 93$ & NOO1 & MG/L & \multicolumn{3}{|c|}{0.003} & 0.001 & - \\
\hline VARADIUAM & $\begin{array}{l}06 / 09 / 89 \\
12 / 14 / 93\end{array}$ & $\begin{array}{l}0001 \\
0001\end{array}$ & MG/L & $<$ & $\begin{array}{l}0.01 \\
0.01\end{array}$ & $L$ & $\begin{array}{l}0.01 \\
0.01\end{array}$ & - \\
\hline VAKADIUA (TOTAL) & $12 / 14 / 93$ & NOOI & MG/L & $<$ & \multicolumn{2}{|l|}{0.01} & 0.01 & - \\
\hline ZINC & $06 / 09 / 89$ & 0009 & $M G / L$ & \multicolumn{3}{|c|}{$0.617 \quad L$} & 0.005 & - \\
\hline
\end{tabular}

PARAMETER VALUE INDICATOR (PVI): < - LESS THAN DETECTIOH LIMIT

SAMPLE ID COOES

OTHER PARAMETER VALUE FLAGS:

0009 - FILTERED SAMPLE (.45 MICROHS)

J - ESTIMATED VALUE

NOOI - UNFILTERED SAMPLE

L - LESS THAH THREE BORE VOLUMES REMOVED BEFORE SAMPLIHG 
GROUNDLATER QUALITY DATA BY LOCATION

SOUP ReV. O Data

SITE: MONOI MONUMENT VALLEY

LOCATIOH: 0651

MORTH COORDINATE: 63788.9 FT

EAST COORDINATE: 92734.3 FT

04/21/88 TO $12 / 13 / 94$

REPORT DATE: 06/20/95

FORMATION OF COAPLETION: ALLUVILM (AL)

HYDRAULIC FLON RELATIONSHIP: DOWH GRADIENT (D)

\begin{tabular}{|c|c|c|c|c|c|c|c|c|}
\hline PARAMETER MAME & LOG DATE & $\begin{array}{c}\text { SAMPLE } \\
\text { ID }\end{array}$ & $\begin{array}{l}\text { UNITS OF } \\
\text { MEASURE }\end{array}$ & PVI & $\begin{array}{l}\text { PARAMETER } \\
\text { VALUE }\end{array}$ & FLAGS & $\begin{array}{l}\text { DETECTIOH } \\
\text { LIMIT }\end{array}$ & $\begin{array}{l}\text { PARAMETER } \\
\text { UNCERTAINTY }\end{array}$ \\
\hline ALKALINITY & $\begin{array}{l}04 / 21 / 88 \\
06 / 05 / 89 \\
06 / 05 / 89 \\
06 / 05 / 89 \\
06 / 05 / 89 \\
06 / 05 / 89 \\
12 / 03 / 89 \\
06 / 25 / 90 \\
01 / 29 / 91 \\
06 / 02 / 91 \\
02 / 25 / 92 \\
08 / 13 / 92 \\
11 / 21 / 92 \\
07 / 01 / 93 \\
04 / 20 / 94 \\
12 / 11 / 94\end{array}$ & $\begin{array}{l}0001 \\
0001 \\
0002 \\
0003 \\
0004 \\
0005 \\
0001 \\
0001 \\
0001 \\
0001 \\
0001 \\
0001 \\
0001 \\
N 001 \\
N 001 \\
N 001\end{array}$ & MG/L CaCO3 & & $\begin{array}{l}192 . \\
198 . \\
198 . \\
198 . \\
198 . \\
198 . \\
194 . \\
175 . \\
206 . \\
199 . \\
199 \\
172 \\
195 \\
188 \\
180 \\
191\end{array}$ & $\begin{array}{l}L \\
L \\
L \\
L \\
L \\
L \\
L \\
L \\
L \\
L\end{array}$ & $\begin{array}{c}- \\
- \\
\vdots \\
\vdots \\
\vdots \\
\vdots \\
= \\
= \\
\end{array}$ & $\begin{array}{l}- \\
- \\
- \\
- \\
- \\
- \\
- \\
- \\
- \\
- \\
-\end{array}$ \\
\hline$\left\{\begin{array}{l}\text { ALLMIHUM } \\
-\end{array}\right.$ & $\begin{array}{l}06 / 05 / 89 \\
06 / 05 / 89 \\
06 / 05 / 89 \\
06 / 05 / 89 \\
06 / 05 / 89 \\
12 / 03 / 89 \\
06 / 25 / 90 \\
01 / 29 / 91 \\
06 / 02 / 91 \\
02 / 25 / 92 \\
08 / 13 / 92\end{array}$ & $\begin{array}{l}0001 \\
0002 \\
0003 \\
0004 \\
0005 \\
0001 \\
0001 \\
0001 \\
0001 \\
0001 \\
0001\end{array}$ & $M G / L$ & $\begin{array}{l}< \\
< \\
< \\
< \\
< \\
< \\
< \\
< \\
< \\
< \\
<\end{array}$ & $\begin{array}{l}0.9 \\
0.1 \\
0.9 \\
0.1 \\
0.1 \\
0.1 \\
0.1 \\
0.1 \\
0.05 \\
0.05 \\
0.2\end{array}$ & $\begin{array}{l}L \\
L \\
L \\
L \\
L \\
L \\
L \\
L \\
L\end{array}$ & $\begin{array}{l}0.1 \\
0.1 \\
0.1 \\
0.1 \\
0.1 \\
0.1 \\
0.1 \\
0.1 \\
0.05 \\
0.05 \\
0.2\end{array}$ & $\begin{array}{l}- \\
- \\
- \\
- \\
- \\
- \\
- \\
-\end{array}$ \\
\hline AพAOHIUH & $\begin{array}{l}04 / 21 / 88 \\
06 / 05 / 89 \\
06 / 05 / 89 \\
06 / 05 / 89 \\
06 / 05 / 89 \\
06 / 05 / 89 \\
12 / 03 / 89 \\
06 / 25 / 90 \\
01 / 29 / 91 \\
06 / 02 / 91 \\
02 / 25 / 92\end{array}$ & $\begin{array}{l}0001 \\
0001 \\
0002 \\
0003 \\
0004 \\
0005 \\
0001 \\
0001 \\
0001 \\
0001 \\
0001\end{array}$ & MG/L & $\begin{array}{l}< \\
< \\
< \\
< \\
< \\
< \\
<\end{array}$ & $\begin{array}{l}0.1 \\
0.1 \\
0.1 \\
0.1 \\
0.2 \\
0.2 \\
0.1 \\
0.1 \\
0.1 \\
0.1 \\
0.1\end{array}$ & $\begin{array}{l}L \\
L \\
L \\
L \\
L \\
L \\
H L \\
L \\
L \\
L\end{array}$ & $\begin{array}{l}0.1 \\
0.1 \\
0.1 \\
0.1 \\
0.1 \\
0.1 \\
0.1 \\
0.1 \\
0.1 \\
0.1 \\
0.1\end{array}$ & $\begin{array}{l}- \\
- \\
- \\
- \\
- \\
- \\
- \\
-\end{array}$ \\
\hline AmoHIUH (TOTAL) & $\begin{array}{l}07 / 01 / 93 \\
04 / 20 / 94 \\
12 / 11 / 94\end{array}$ & $\begin{array}{l}\text { No01 } \\
\text { Ho01 } \\
\text { Ho01 }\end{array}$ & MG/L & $<$ & $\begin{array}{l}0.1 \\
0.1 \\
0.19\end{array}$ & FH & $\begin{array}{l}0.1 \\
0.1 \\
0.1\end{array}$ & : \\
\hline ANTIMOHY & $\begin{array}{l}06 / 05 / 89 \\
06 / 05 / 89 \\
06 / 05 / 89 \\
06 / 05 / 89 \\
06 / 05 / 89 \\
12 / 03 / 89 \\
06 / 25 / 90 \\
01 / 29 / 91\end{array}$ & $\begin{array}{l}0001 \\
0002 \\
0003 \\
0004 \\
0005 \\
0001 \\
0001 \\
0001\end{array}$ & $M G / L$ & $\begin{array}{l}< \\
< \\
< \\
< \\
< \\
< \\
< \\
<\end{array}$ & $\begin{array}{l}0.003 \\
0.003 \\
0.003 \\
0.003 \\
0.003 \\
0.003 \\
0.003 \\
0.003\end{array}$ & $\begin{array}{l}2 \\
2 \\
1 \\
1 \\
1 \\
2 \\
L\end{array}$ & $\begin{array}{l}0.003 \\
0.003 \\
0.003 \\
0.003 \\
0.003 \\
0.003 \\
0.003 \\
0.003\end{array}$ & $\begin{array}{l}- \\
: \\
: \\
: \\
-\end{array}$ \\
\hline
\end{tabular}

PARAMETER VALUE INDICATOR (PVI): < - LESS THAK DETECTION LIMIT

\section{OTHER PARAMETER VALUE FLAGS:}

- DUPLICATE akalysis nOt hithin COATROL limits

F - LOW-FLON SAMPLIMG/DEDICATED PUAP

H - HOLD TIME EXPIRED, VALUE SUSPECT

$L$ - LESS THAK THREE BORE VOLLMES REMOVED BEFORE SAMPLIHG
SNMPLE ID COOES:

0001 - FILTERED SNMPLE (.45 MICRONS)

0002 - FILTERED REPLICATE SAMPLE (.45 MICROHS)

0003 - FILTERED REPLICATE SAMPLE (.45 MICROHS)

0004 - FILTERED REPLICATE SAMPLE (.45 MICRONS)

MOO1 - UMFILTERED SMMPLE 
GROUHDHATER OUALITY DATA BY LOCATION

SOMP ReV. O Data

SITE: HONO1 MONUMENT VALLEY

LOCATIOH: 0651

HORTH COORDIHATE: 63788.9 FT

EAST COORDINATE: 92734.3 FT

04/21/88 TO $12 / 13 / 94$

REPORT DATE: $06 / 20 / 95$

FORMATION OF COMPLETION: ALLUVIUM (AL)

HYORAULIC FLOU RELATIONSHIP: DONK GRADIENT (D)

\begin{tabular}{|c|c|c|c|c|c|c|c|c|}
\hline PARAMETER MAME & LOG DATE & $\underset{\text { SD }}{\text { SAMPLE }}$ & $\begin{array}{l}\text { UNITS OF } \\
\text { MEASURE }\end{array}$ & PVI & $\begin{array}{l}\text { PARAMETER } \\
\text { VALUE }\end{array}$ & FLAGS & $\begin{array}{l}\text { DETECTION } \\
\text { LIMIT }\end{array}$ & $\begin{array}{l}\text { PARAMETER } \\
\text { UNCERTAIHTY }\end{array}$ \\
\hline ANTIMONY & $\begin{array}{l}06 / 02 / 91 \\
02 / 25 / 92 \\
08 / 13 / 92\end{array}$ & $\begin{array}{l}0001 \\
0001 \\
0001\end{array}$ & $M G / L$ & $<$ & $\begin{array}{l}0.003 \\
0.003 \\
0.06\end{array}$ & $\stackrel{L}{H L}$ & $\begin{array}{l}0.003 \\
0.003 \\
0.06\end{array}$ & : \\
\hline ARSENIC & $\begin{array}{l}04 / 21 / 88 \\
06 / 05 / 89 \\
06 / 05 / 89 \\
06 / 05 / 89 \\
06 / 05 / 89 \\
06 / 05 / 89 \\
12 / 03 / 89 \\
06 / 25 / 90 \\
01 / 29 / 91 \\
06 / 02 / 91 \\
02 / 25 / 92 \\
08 / 13 / 92 \\
07 / 01 / 93\end{array}$ & $\begin{array}{l}0001 \\
0001 \\
0002 \\
0003 \\
0004 \\
0005 \\
0001 \\
0001 \\
0001 \\
0001 \\
0001 \\
0001 \\
0001\end{array}$ & MG/L & $\begin{array}{l}< \\
< \\
< \\
< \\
< \\
< \\
< \\
< \\
< \\
< \\
< \\
<\end{array}$ & $\begin{array}{l}0.002 \\
0.01 \\
0.01 \\
0.01 \\
0.01 \\
0.01 \\
0.01 \\
0.01 \\
0.01 \\
0.01 \\
0.09 \\
0.01 \\
0.005\end{array}$ & $\begin{array}{l}J L \\
L \\
L \\
L \\
L \\
L \\
L \\
L \\
L \\
H L \\
H\end{array}$ & $\begin{array}{l}0.01 \\
0.01 \\
0.01 \\
0.01 \\
0.01 \\
0.01 \\
0.01 \\
0.01 \\
0.01 \\
0.01 \\
0.01 \\
0.01 \\
0.005\end{array}$ & $\begin{array}{l}- \\
- \\
- \\
- \\
- \\
- \\
- \\
-\end{array}$ \\
\hline ARSEKIC (TOTAL) & $\begin{array}{l}11 / 21 / 92 \\
07 / 01 / 93\end{array}$ & $\begin{array}{l}\text { NO01 } \\
\text { N001 }\end{array}$ & $M G / L$ & $<$ & \multicolumn{2}{|l|}{$\begin{array}{l}0.0045 \\
0.005\end{array}$} & $\begin{array}{l}0.0015 \\
0.005\end{array}$ & - \\
\hline BARIUM & $\begin{array}{l}06 / 05 / 89 \\
06 / 05 / 89 \\
06 / 05 / 89 \\
06 / 05 / 89 \\
06 / 05 / 89 \\
12 / 03 / 89 \\
06 / 25 / 90 \\
01 / 29 / 91 \\
06 / 02 / 91 \\
02 / 25 / 92 \\
08 / 13 / 92 \\
07 / 01 / 93\end{array}$ & $\begin{array}{l}0001 \\
0002 \\
0003 \\
0004 \\
0005 \\
0001 \\
0001 \\
0001 \\
0001 \\
0001 \\
0001 \\
0001\end{array}$ & $M G / L$ & $\begin{array}{l}< \\
< \\
< \\
< \\
< \\
< \\
< \\
< \\
< \\
<\end{array}$ & $\begin{array}{l}0.1 \\
0.1 \\
0.1 \\
0.1 \\
0.1 \\
0.1 \\
0.1 \\
0.1 \\
0.04 \\
0.03 \\
0.2 \\
0.1\end{array}$ & $\begin{array}{l}L \\
L \\
L \\
L \\
L \\
L \\
L \\
L \\
L\end{array}$ & $\begin{array}{l}0.1 \\
0.1 \\
0.1 \\
0.1 \\
0.1 \\
0.1 \\
0.1 \\
0.1 \\
0.01 \\
0.01 \\
0.2 \\
0.1\end{array}$ & $\begin{array}{l}- \\
- \\
- \\
- \\
- \\
- \\
- \\
-\end{array}$ \\
\hline BARIUM (TOTAL) & $\begin{array}{l}11 / 21 / 92 \\
07 / 01 / 93\end{array}$ & $\begin{array}{l}\text { NOOI } \\
\text { N001 }\end{array}$ & $M G / L$ & $<$ & \multicolumn{2}{|l|}{$\begin{array}{l}0.037 \\
0.1\end{array}$} & $\begin{array}{l}0.0015 \\
0.1\end{array}$ & - \\
\hline BERYLLIUM & $\begin{array}{l}06 / 05 / 89 \\
06 / 05 / 89 \\
06 / 05 / 89 \\
06 / 05 / 89 \\
06 / 05 / 89 \\
12 / 03 / 89 \\
06 / 25 / 90 \\
01 / 29 / 91 \\
06 / 02 / 91 \\
08 / 13 / 92\end{array}$ & $\begin{array}{l}0001 \\
0002 \\
0003 \\
0004 \\
0005 \\
0001 \\
0001 \\
0001 \\
0001 \\
0001\end{array}$ & $M G / L$ & $\begin{array}{l}< \\
< \\
< \\
< \\
< \\
< \\
< \\
< \\
<\end{array}$ & $\begin{array}{l}0.01 \\
0.01 \\
0.01 \\
0.01 \\
0.01 \\
0.005 \\
0.01 \\
0.01 \\
0.005 \\
0.005\end{array}$ & $\begin{array}{l}L \\
L \\
L \\
L \\
L \\
L \\
L \\
L\end{array}$ & $\begin{array}{l}0.01 \\
0.01 \\
0.01 \\
0.09 \\
0.09 \\
0.005 \\
0.09 \\
0.01 \\
0.005 \\
0.005\end{array}$ & $\begin{array}{l}- \\
: \\
: \\
: \\
- \\
-\end{array}$ \\
\hline BORON & $\begin{array}{l}06 / 05 / 89 \\
06 / 05 / 89 \\
06 / 05 / 89 \\
06 / 05 / 89 \\
06 / 05 / 89 \\
12 / 03 / 89\end{array}$ & $\begin{array}{l}0001 \\
0002 \\
0003 \\
0004 \\
0005 \\
0001\end{array}$ & $M G / L$ & $\begin{array}{l}< \\
< \\
< \\
< \\
<\end{array}$ & $\begin{array}{l}0.1 \\
0.1 \\
0.1 \\
0.1 \\
0.1 \\
0.1\end{array}$ & $\begin{array}{l}L \\
L \\
L \\
L \\
L\end{array}$ & $\begin{array}{l}0.1 \\
0.1 \\
0.1 \\
0.1 \\
0.1 \\
0.1\end{array}$ & $\begin{array}{l}- \\
: \\
-\end{array}$ \\
\hline
\end{tabular}

PARAMETER VALUE IHDICATOR (PVI): < - LESS THAN DETECTION LIMIT

SAMPLE ID COOES:

OTHER PARAMETER VALUE FLAGS:

J - ESTIMATED VALUE

L - LESS THAK THREE BORE VOLLMES REMOVED BEFORE SAMPLING

W - POST-DIGEST SPIKE OUT OF CKTR LIM MHILE SAMP ABS < 50\% SPIKE

OOO1 - FILTERED SAMPLE (.45 MICRONS)

O002 - FILTERED REPLICATE SAMPLE (.45 MICRONS)

0003 - FILTERED REPLICATE SAMPLE (.45 MICRONS)

0004 - FILTERED REPLICATE SAMPLE (.45 MICRONS)

MOOI - UMFILTERED SAMPLE 
SOWP ReV. O Data

SITE: MONOI HONUMENT VALLEY

LOCATION: 0651

HORTH COORDIHATE: 63788.9 FT

EAST COOROINATE: 92734.3 FT

$04 / 21 / 88$ TO $12 / 13 / 94$

REPORT DATE: OS/20/95

FORMATIOH OF COMPLETION: ALLUVIUM (AL)

HYDRAULIC FLOU RELATIONSHIP: DOWN GRADIENT (D)

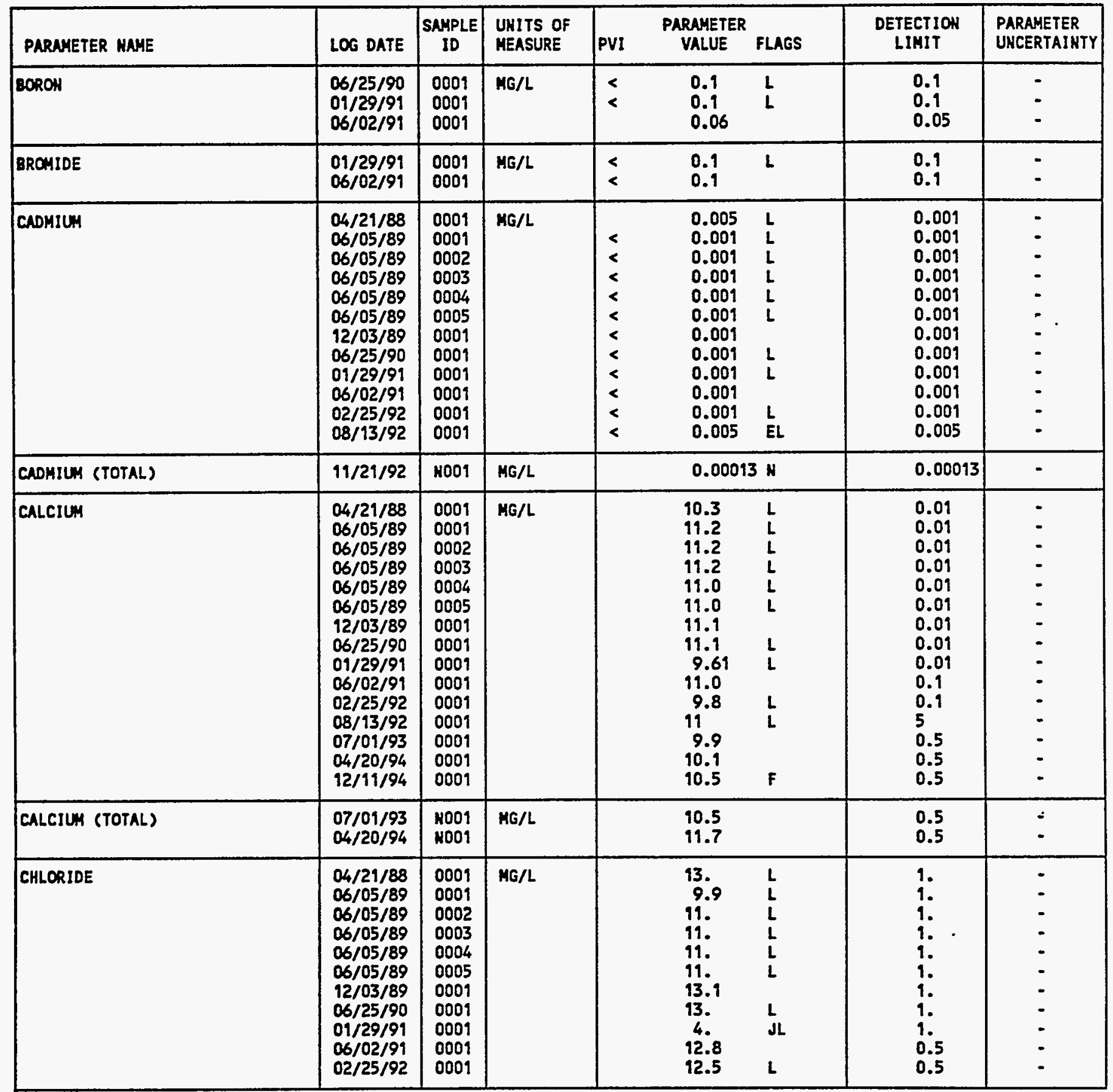

PARAMETER VAlUE INDICATOR (PVI): < - LESS THAH DETECTION LIMIT

OTHER PARAMETER VALUE FLAGS:

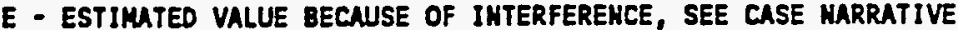

F - LOW-FLON SAMPLING/DEDICATED PLMP

- ESTIMUTED VALUE

L - LESS tHAH THREE BORE VOLUMES REMOVEd BEFORE SAMPLING

W - SPIKE SAMPLE RECONERY NOT WITHIN CONTROL LIMITS
SAMPLE ID COOES:

0001 - FILTERED SAMPLE (.45 MICROWS)

O0O2 - FILTERED REPLICATE SAMPLE (.45 MICRONS)

0003 - FILTERED REPLICATE SAMPLE (.45 MICRONS)

OOO4 - FILTERED REPLICATE SAMPLE (.45 MICRONS)

MOO1 - LNFILTERED SNMPLE 
GROUWDWATER QUALITY DATA BY LOCATION

SOUP REV. 0 Data

SITE: MONO1 MONLMENT VALLEY

LOCATION: 0651

MORTH COORDINATE: 63788.9 FT

EAST COORDINATE: 92734.3 FT

04/21/88 TO 12/13/94

REPORT DATE: 06/20/95

FORMATIOH OF COHPLETIOH: ALLUVIUH (AL)

HYDRAULIC FLON RELATIONSHIP: DOWN GRADIENT (D)

\begin{tabular}{|c|c|c|c|c|c|c|c|c|}
\hline PARAMETER NAME & LOG DATE & $\underset{\text { ID }}{\text { SNMPLE }}$ & $\begin{array}{l}\text { UNITS OF } \\
\text { MEASURE }\end{array}$ & PVI & $\begin{array}{l}\text { PARAMETER } \\
\text { VALUE }\end{array}$ & FLAGS & $\begin{array}{l}\text { DETECTIOH } \\
\text { LIMIT }\end{array}$ & $\begin{array}{l}\text { PARAMETER } \\
\text { UHCERTAINTY }\end{array}$ \\
\hline CHLORIDE & $\begin{array}{l}08 / 13 / 92 \\
04 / 20 / 94 \\
12 / 11 / 94\end{array}$ & $\begin{array}{l}0001 \\
0001 \\
0001\end{array}$ & HG/L & & $\begin{array}{l}12 \\
11.6 \\
8\end{array}$ & $\begin{array}{l}L \\
F\end{array}$ & $\begin{array}{l}0.016 \\
0.5 \\
0.5\end{array}$ & : \\
\hline CHLORIDE (TOTAL) & $07 / 01 / 93$ & N001 & $M G / L$ & & 13.9 & & 0.5 & - \\
\hline CHROHIUM & $\begin{array}{l}06 / 05 / 89 \\
06 / 05 / 89 \\
06 / 05 / 89 \\
06 / 05 / 89 \\
06 / 05 / 89 \\
12 / 03 / 89 \\
06 / 25 / 90 \\
01 / 29 / 91 \\
06 / 02 / 99 \\
02 / 25 / 92 \\
08 / 13 / 92\end{array}$ & $\begin{array}{l}0001 \\
0002 \\
0003 \\
0004 \\
0005 \\
0001 \\
0001 \\
0001 \\
0001 \\
0001 \\
0001\end{array}$ & $M G / L$ & $\begin{array}{l}< \\
< \\
< \\
< \\
< \\
< \\
< \\
< \\
< \\
< \\
<\end{array}$ & $\begin{array}{l}0.01 \\
0.01 \\
0.01 \\
0.01 \\
0.01 \\
0.01 \\
0.01 \\
0.01 \\
0.01 \\
0.01 \\
0.01\end{array}$ & $\begin{array}{l}L \\
L \\
L \\
L \\
L \\
L \\
L \\
L \\
L\end{array}$ & $\begin{array}{l}0.01 \\
0.01 \\
0.01 \\
0.01 \\
0.01 \\
0.01 \\
0.01 \\
0.01 \\
0.01 \\
0.01 \\
0.01\end{array}$ & $\begin{array}{l}- \\
- \\
- \\
- \\
- \\
- \\
-\end{array}$ \\
\hline CHROHIUH (TOTAL) & $11 / 21 / 92$ & N001 & MG/L & $<$ & \multicolumn{2}{|l|}{0.0053} & 0.0053 & - \\
\hline COBALT & $\begin{array}{l}06 / 05 / 89 \\
06 / 05 / 89 \\
06 / 05 / 89 \\
06 / 05 / 89 \\
06 / 05 / 89 \\
12 / 03 / 89 \\
06 / 25 / 90 \\
01 / 29 / 91 \\
06 / 02 / 91\end{array}$ & $\begin{array}{l}0001 \\
0002 \\
0003 \\
0004 \\
0005 \\
0001 \\
0001 \\
0001 \\
0001\end{array}$ & MG/L & $\begin{array}{l}< \\
< \\
< \\
< \\
< \\
< \\
< \\
< \\
<\end{array}$ & $\begin{array}{l}0.05 \\
0.05 \\
0.05 \\
0.05 \\
0.05 \\
0.05 \\
0.05 \\
0.05 \\
0.03\end{array}$ & $\begin{array}{l}\mathbf{L} \\
\mathbf{L} \\
\mathbf{L} \\
\mathbf{L} \\
\mathbf{L} \\
\mathbf{L} \\
\mathbf{L}\end{array}$ & $\begin{array}{l}0.05 \\
0.05 \\
0.05 \\
0.05 \\
0.05 \\
0.05 \\
0.05 \\
0.05 \\
0.03\end{array}$ & $\begin{array}{l}- \\
- \\
- \\
- \\
- \\
-\end{array}$ \\
\hline COPPER & $\begin{array}{l}06 / 05 / 89 \\
06 / 05 / 89 \\
06 / 05 / 89 \\
06 / 05 / 89 \\
06 / 05 / 89 \\
12 / 03 / 89 \\
06 / 25 / 90 \\
01 / 29 / 91 \\
06 / 02 / 91\end{array}$ & $\begin{array}{l}0001 \\
0002 \\
0003 \\
0004 \\
0005 \\
0001 \\
0001 \\
0001 \\
0001\end{array}$ & $M G / L$ & $\begin{array}{l}< \\
< \\
< \\
< \\
< \\
< \\
< \\
< \\
<\end{array}$ & $\begin{array}{l}0.02 \\
0.02 \\
0.02 \\
0.02 \\
0.02 \\
0.02 \\
0.02 \\
0.02 \\
0.01\end{array}$ & $\begin{array}{l}L \\
L \\
L \\
L \\
L \\
L \\
L\end{array}$ & $\begin{array}{l}0.02 \\
0.02 \\
0.02 \\
0.02 \\
0.02 \\
0.02 \\
0.02 \\
0.02 \\
0.01\end{array}$ & $\begin{array}{l}- \\
- \\
- \\
- \\
- \\
-\end{array}$ \\
\hline CYAKIDE & $\begin{array}{l}06 / 05 / 89 \\
06 / 05 / 89 \\
06 / 05 / 89 \\
06 / 05 / 89 \\
06 / 05 / 89 \\
12 / 03 / 89\end{array}$ & $\begin{array}{l}0001 \\
0002 \\
0003 \\
0004 \\
0005 \\
0001\end{array}$ & $M G / L$ & $\begin{array}{l}< \\
< \\
< \\
< \\
<\end{array}$ & $\begin{array}{l}0.01 \\
0.01 \\
0.01 \\
0.01 \\
0.01 \\
0.01\end{array}$ & $\begin{array}{l}L \\
L \\
L \\
L \\
L\end{array}$ & $\begin{array}{l}0.01 \\
0.01 \\
0.01 \\
0.01 \\
0.01 \\
0.01\end{array}$ & $\begin{array}{l}: \\
: \\
- \\
-\end{array}$ \\
\hline OISSOLVED OXYGEN & $\begin{array}{l}07 / 01 / 93 \\
04 / 20 / 94 \\
12 / 191 / 94\end{array}$ & $\begin{array}{l}\text { No01 } \\
\text { No01 } \\
\text { N001 }\end{array}$ & MG/L & $<$ & $\begin{array}{l}0.0 \\
0.054 \\
0.02\end{array}$ & $\mathbf{F}$ & $\dot{-}$ & : \\
\hline FLLORIDE & $\begin{array}{l}06 / 05 / 89 \\
06 / 05 / 89 \\
06 / 05 / 89\end{array}$ & $\begin{array}{l}0001 \\
0002 \\
0003\end{array}$ & MG/L & & $\begin{array}{l}0.2 \\
0.2 \\
0.2\end{array}$ & $\begin{array}{l}L \\
L\end{array}$ & $\begin{array}{l}0.1 \\
0.1 \\
0.1\end{array}$ & $\div$ \\
\hline
\end{tabular}

PARAHETER VALUE IMDICATOR (PVI): < - LESS THAN DETECTION LIMIT

SAMPLE ID COOES:

OTHER PARAMETER VALUE FLAGS:

F - LOW-FLON SAMPLING/DEDICATED PLMP

OOO1 - FILTERED SAMPLE (.45 MICROWS)

L - LESS THAN THREE BORE VOLLUES REMOVED BEFORE SAMPLING

OOO2 - FILTERED REPLICATE SAMPLE (.45 MICRONS)

0003 - FILTERED REPLICATE SAMPLE (.45 MICRONS)

0004 - FILTERED REPLICATE SAMPLE (.45 MICRONS)

NODI - LMFILTERED SAMPLE 
37dwys 098317IJNn - LOOH

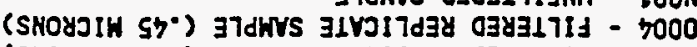

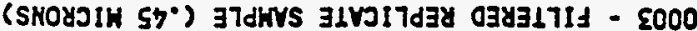

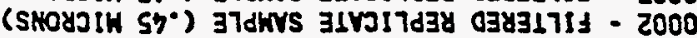

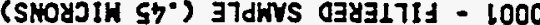

:S300J al 37dins

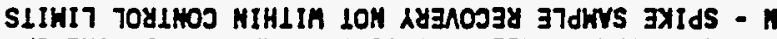

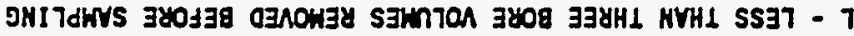

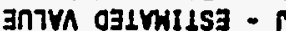

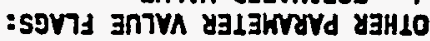

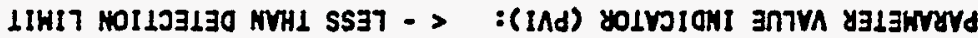

\begin{tabular}{|c|c|c|c|c|c|c|c|c|}
\hline- & $5100^{\circ} 0$ & & $\$ 100 \% 0$ & $>$ & T/פH & bOON & $26 / 12 / 16$ & (7V101) 0V37 \\
\hline $\begin{array}{l}- \\
- \\
- \\
\div \\
: \\
-\end{array}$ & $\begin{array}{r}500.0 \\
10.0 \\
10^{\circ} 0 \\
10^{\circ} 0 \\
10.0 \\
10.0 \\
10 \% 0 \\
10.0 \\
10.0\end{array}$ & $\begin{array}{l}7 \\
7 \\
7 \\
7 \\
7 \\
7 \\
7\end{array}$ & $\begin{array}{l}500^{\circ} 0 \\
10^{\circ} 0 \\
60^{\circ} 0 \\
10^{\circ} 0 \\
10^{\circ} 0 \\
10^{\circ} 0 \\
10 \% 0 \\
60^{\circ} 0 \\
10^{\circ} 0\end{array}$ & $\begin{array}{l}> \\
> \\
> \\
> \\
> \\
> \\
> \\
> \\
>\end{array}$ & 1/פH & $\begin{array}{l}1000 \\
1000 \\
1000 \\
1000 \\
5000 \\
+000 \\
5000 \\
2000 \\
1000\end{array}$ & $\begin{array}{l}16 / 20 / 90 \\
16 / 62 / 10 \\
06 / 52 / 90 \\
68 / 50 / 21 \\
68 / 50 / 90 \\
68 / 50 / 90 \\
68 / 50 / 90 \\
68 / 50 / 90 \\
68 / 50 / 90\end{array}$ & ava7 \\
\hline - & $\varepsilon 0^{\circ} \mathrm{O}$ & $r$ & $90^{\circ} 0$ & & $7 / 9 H$ & bOON & $26 / 10 / 10$ & (7V101) HOY1 \\
\hline $\begin{array}{l}- \\
- \\
- \\
- \\
- \\
- \\
- \\
- \\
-\end{array}$ & 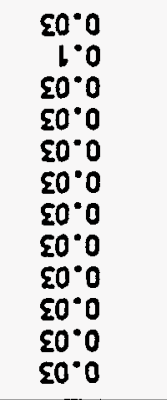 & $\begin{array}{l}p \\
7 \\
7 \\
7 \\
7 \\
7 \\
7 \\
7 \\
7 \\
7\end{array}$ & 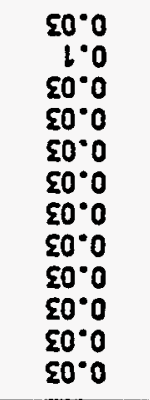 & $\begin{array}{l}> \\
> \\
> \\
> \\
> \\
> \\
> \\
> \\
> \\
> \\
> \\
>\end{array}$ & $7 / 9 N$ & $\begin{array}{l}1000 \\
1000 \\
1000 \\
1000 \\
6000 \\
1000 \\
1000 \\
5000 \\
7000 \\
5000 \\
2000 \\
1000\end{array}$ & $\begin{array}{l}\varepsilon 6 / 10 / 10 \\
26 / 51 / 80 \\
26 / 52 / 20 \\
16 / 20 / 90 \\
16 / 62 / 10 \\
06 / 52 / 90 \\
68 / 50 / 21 \\
68 / 50 / 90 \\
68 / 50 / 90 \\
68 / 50 / 90 \\
68 / 50 / 90 \\
68 / 50 / 90\end{array}$ & HOYI \\
\hline$\rightarrow \cdot \frac{7}{\varepsilon}$ & $\varepsilon \cdot \stackrel{7}{s}$ & $n$ & $\begin{aligned} 2 \\
\varepsilon^{\prime}\end{aligned}$ & & $7 / 10 d$ & $\begin{array}{l}\text { LOON } \\
\text { LOOH }\end{array}$ & $\begin{array}{l}86 / 60 / 20 \\
26 / 62 / 66\end{array}$ & (7Y101) V138 SSO49 \\
\hline $\begin{array}{r}7 \\
9 \cdot 2 \\
9 \cdot 2 \\
2 \cdot 2 \\
2 \cdot 2 \\
0 \cdot 2 \\
8 \cdot 1\end{array}$ & $\begin{array}{r}67 \\
6 \cdot 5 \\
\varepsilon \cdot 4 \\
5 \cdot 0 \\
5 \cdot 0 \\
5 \cdot 0 \\
\cdot 1\end{array}$ & $\begin{array}{l}7 \\
7 \\
7 \\
7\end{array}$ & $\begin{array}{l}1 \\
b \cdot 7 \\
2 \cdot 2 \\
9 \cdot 9 \\
0.4 \\
0.5 \\
0.8\end{array}$ & & $7 / 13 d$ & $\begin{array}{l}1000 \\
1000 \\
6000 \\
1000 \\
1000 \\
1000 \\
1000\end{array}$ & $\begin{array}{l}\varepsilon 6 / 10 / 10 \\
26 / 12 / 16 \\
26 / \varepsilon 1 / 80 \\
26 / 52 / 20 \\
16 / 20 / 90 \\
16 / 62 / 10 \\
88 / 12 / 40\end{array}$ & Y138 S5049 \\
\hline$\eta \cdot \frac{s}{\varepsilon}$ & $\downarrow \cdot \frac{\varepsilon}{L}$ & N & 0.6 & & $7 / 13 d$ & $\begin{array}{l}\text { LOON } \\
\text { LOON }\end{array}$ & $\begin{array}{l}56 / 60 / 20 \\
26 / 12 / 66\end{array}$ & (TY101) YHdרY SSOYง \\
\hline $\begin{array}{r}5 \\
7 \cdot \frac{5}{8} \\
0 \cdot 5 \\
6 \cdot 7 \\
2 \cdot 5 \\
8 \cdot \frac{5}{5}\end{array}$ & $\begin{array}{r}\varepsilon \\
7 \cdot \frac{\varepsilon}{8} \\
b \cdot \frac{7}{0} \\
0.6 \\
\cdot 1 \\
2 \cdot 0\end{array}$ & $\begin{array}{l}\mathbf{H} \\
7 \\
7 \\
7 \\
7\end{array}$ & 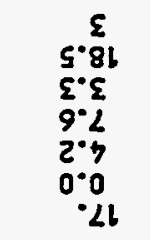 & & $7 / 10 d$ & $\begin{array}{l}1000 \\
1000 \\
1000 \\
1000 \\
1000 \\
1000 \\
1000\end{array}$ & $\begin{array}{l}56 / 10 / 20 \\
26 / 12 / 16 \\
26 / \varepsilon b / 80 \\
26 / 52 / 20 \\
16 / 20 / 90 \\
16 / 62 / 60 \\
88 / 12 / 40\end{array}$ & VHdTY Ssoyg \\
\hline $\begin{array}{l}- \\
: \\
:\end{array}$ & $\begin{array}{l}b \cdot 0 \\
b \cdot 0 \\
b \cdot 0 \\
b \cdot 0 \\
b \cdot 0 \\
b \cdot 0\end{array}$ & $\begin{array}{l}7 \\
1 \\
7 \\
7\end{array}$ & $\begin{array}{l}1 \cdot 0 \\
2 \cdot 0 \\
2 \cdot 0 \\
2 \cdot 0 \\
2 \cdot 0 \\
2 \cdot 0\end{array}$ & $>$ & רופ & $\begin{array}{l}1000 \\
1000 \\
1000 \\
1000 \\
5000 \\
5000\end{array}$ & $\begin{array}{l}16 / 20 / 90 \\
16 / 62 / 60 \\
06 / 52 / 90 \\
68 / 50 / 26 \\
68 / 50 / 90 \\
68 / 50 / 90\end{array}$ & 30ixonา \\
\hline $\begin{array}{r}\text { AINIYIYZJNก } \\
\text { YヨIJWYYYd }\end{array}$ & $\begin{array}{c}\text { LIHIT } \\
\text { NOI1ว3130 }\end{array}$ & sפYาt & 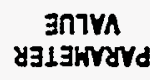 & Ind & $\begin{array}{l}\text { Jynsyan } \\
\text { jo SIIKn }\end{array}$ & or & J1v0 507 & 3WYM y \\
\hline
\end{tabular}

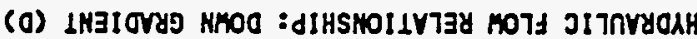
(TV) WกIAกาר :

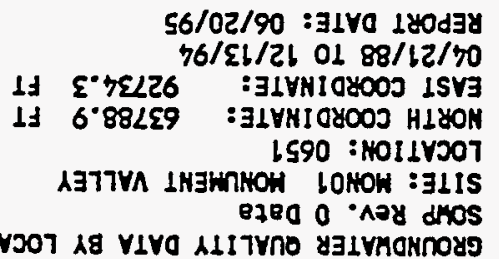


GROUNDHATER QUALITY DATA BY LOCATION

SOWP REV. $O$ Data

SITE: MONOI MONUMENT VALLEY

LOCATION: 0651

MORTH COORDIHATE: 63788.9 FT

EAST COORDINATE: 92734.3 FT

$04 / 21 / 88$ TO $12 / 13 / 94$

REPORT DATE : 06/20/95

FORHATION OF COAPLETION: ALLUVIUM (AL)

HYDRAULIC FLOW RELATIONSHIP: DOWH GRADIENT (D)

\begin{tabular}{|c|c|c|c|c|c|c|c|c|}
\hline PARAHETER MAME & LOG DATE & $\begin{array}{c}\text { SAMPLE } \\
\text { ID }\end{array}$ & $\begin{array}{l}\text { UHITS OF } \\
\text { MEASURE }\end{array}$ & PVI & $\begin{array}{l}\text { PARAMETER } \\
\text { VALUE }\end{array}$ & FLAGS & $\begin{array}{l}\text { DETECTION } \\
\text { LIMIT }\end{array}$ & $\begin{array}{l}\text { PARAMETER } \\
\text { UNCERTAINTY }\end{array}$ \\
\hline LEAD-210 & $11 / 21 / 92$ & 0001 & PCI/L & \multicolumn{3}{|c|}{0.0} & 2.0 & 1.2 \\
\hline LEAD-210 (TOTAL) & $11 / 21 / 92$ & NoOI & $\mathrm{PCI} / \mathrm{L}$ & \multicolumn{3}{|c|}{5.8} & 0.3 & 0.9 \\
\hline MAGNESIUM & $\begin{array}{l}04 / 21 / 88 \\
06 / 05 / 89 \\
06 / 05 / 89 \\
06 / 05 / 89 \\
06 / 05 / 89 \\
06 / 05 / 89 \\
12 / 03 / 89 \\
06 / 25 / 90 \\
01 / 29 / 91 \\
06 / 02 / 91 \\
02 / 25 / 92 \\
08 / 13 / 92 \\
07 / 01 / 93 \\
04 / 20 / 94 \\
12 / 11 / 94\end{array}$ & $\begin{array}{l}0001 \\
0001 \\
0002 \\
0003 \\
0004 \\
0005 \\
0001 \\
0001 \\
0001 \\
0001 \\
0001 \\
0001 \\
0001 \\
0001 \\
0001\end{array}$ & MG/L & & $\begin{array}{l}5.32 \\
5.21 \\
5.23 \\
5.21 \\
5.15 \\
5.11 \\
5.61 \\
5.49 \\
5.01 \\
5.4 \\
4.9 \\
5.2 \\
4.7 \\
4.8 \\
4.9\end{array}$ & $\begin{array}{l}L \\
L \\
L \\
L \\
L \\
L \\
L \\
L \\
L \\
L\end{array}$ & $\begin{array}{l}0.001 \\
0.001 \\
0.001 \\
0.001 \\
0.001 \\
0.001 \\
0.001 \\
0.001 \\
0.001 \\
0.1 \\
0.1 \\
5 \\
0.1 \\
0.1 \\
0.1\end{array}$ & $\begin{array}{l}- \\
- \\
- \\
: \\
- \\
- \\
- \\
- \\
-\end{array}$ \\
\hline MAGHESIUH (TOTAL) & $\begin{array}{l}07 / 09 / 93 \\
04 / 20 / 94\end{array}$ & $\begin{array}{l}\text { NOO1 } \\
\text { HOO1 }\end{array}$ & $M G / L$ & \multicolumn{3}{|c|}{$\begin{array}{l}4.9 \\
5.1\end{array}$} & $\begin{array}{l}0.1 \\
0.1\end{array}$ & $\dot{-}$ \\
\hline MANGANESE & $\begin{array}{l}06 / 05 / 89 \\
06 / 05 / 89 \\
06 / 05 / 89 \\
06 / 05 / 89 \\
06 / 05 / 89 \\
12 / 03 / 89 \\
06 / 25 / 90 \\
01 / 29 / 99 \\
06 / 02 / 91 \\
02 / 25 / 92 \\
08 / 13 / 92 \\
07 / 01 / 93 \\
04 / 20 / 94 \\
12 / 19 / 94\end{array}$ & $\begin{array}{l}0001 \\
0002 \\
0003 \\
0004 \\
0005 \\
0001 \\
0001 \\
0001 \\
0009 \\
0001 \\
0001 \\
0001 \\
0001 \\
0001\end{array}$ & $M G / L$ & $<$ & $\begin{array}{l}0.03 \\
0.03 \\
0.03 \\
0.03 \\
0.03 \\
0.03 \\
0.03 \\
0.02 \\
0.03 \\
0.02 \\
0.026 \\
0.02 \\
0.03 \\
0.01\end{array}$ & $\begin{array}{l}L \\
L \\
L \\
L \\
L \\
L \\
L \\
L \\
L\end{array}$ & $\begin{array}{l}0.01 \\
0.01 \\
0.01 \\
0.01 \\
0.01 \\
0.01 \\
0.01 \\
0.01 \\
0.01 \\
0.01 \\
0.01 \\
0.01 \\
0.01 \\
0.01\end{array}$ & $\begin{array}{l}- \\
: \\
- \\
- \\
- \\
- \\
- \\
- \\
-\end{array}$ \\
\hline MUMGAHESE (TOTAL) & $\begin{array}{l}11 / 21 / 92 \\
07 / 01 / 93 \\
04 / 20 / 94\end{array}$ & $\begin{array}{l}\text { No01 } \\
\text { No01 } \\
\text { No01 }\end{array}$ & $M G / L$ & \multicolumn{3}{|c|}{$\begin{array}{l}0.024 \\
0.03 \\
0.04\end{array}$} & $\begin{array}{l}0.0015 \\
0.01 \\
0.01\end{array}$ & - \\
\hline MERCURY & $\begin{array}{l}06 / 05 / 89 \\
06 / 05 / 89 \\
06 / 05 / 89 \\
06 / 05 / 89 \\
06 / 05 / 89 \\
12 / 03 / 89 \\
06 / 25 / 90\end{array}$ & $\begin{array}{l}0001 \\
0002 \\
0003 \\
0004 \\
0005 \\
0001 \\
0001\end{array}$ & MG/L & $\begin{array}{l}< \\
< \\
< \\
< \\
< \\
< \\
<\end{array}$ & $\begin{array}{l}0.0002 \\
0.0002 \\
0.0002 \\
0.0002 \\
0.0002 \\
0.0002 \\
0.0002\end{array}$ & $\begin{array}{l}L \\
L \\
L \\
L \\
L \\
L\end{array}$ & $\begin{array}{l}0.0002 \\
0.0002 \\
0.0002 \\
0.0002 \\
0.0002 \\
0.0002 \\
0.0002\end{array}$ & $\begin{array}{l}- \\
- \\
- \\
- \\
-\end{array}$ \\
\hline MOLYBOENUN & $\begin{array}{l}04 / 21 / 88 \\
06 / 05 / 89 \\
06 / 05 / 89 \\
06 / 05 / 89\end{array}$ & $\begin{array}{l}0001 \\
0001 \\
0002 \\
0003\end{array}$ & MG/L & $\begin{array}{l}< \\
<\end{array}$ & $\begin{array}{l}0.01 \\
0.01 \\
0.01 \\
0.01\end{array}$ & $\begin{array}{l}L \\
L \\
L \\
L\end{array}$ & $\begin{array}{l}0.01 \\
0.01 \\
0.01 \\
0.01\end{array}$ & : \\
\hline
\end{tabular}

PARAMETER VALUE INDICATOR (PVI): < - LESS THAN DETECTION LIMIT

SAMPLE TO COOES:

0001 - FILTERED SAMPLE (.45 MICRONS)

OTHER PARAMETER VALUE FLAGS:

F - LOU-FLOU SAMPLING/DEDICATED PLMP

0002 - FILTERED REPLICATE SAMPLE (.45 MICRONS)

0003 - FILTERED REPLICATE SAMLE (.45 MICROHS)

L - LESS tHAN THREE BORE VOLUMES REMOVED BEFORE SNMPLING

0004 - FILTERED REPLICATE SAMPLE (.45 MICROHS)

NOO1 - UMFILTERED SNMPLE 
GROUNDWATER QUALITY DATA BY LOCATION

SOMP ReV. $O$ Data

SITE: MONO1 MONUMENT VALLEY

LOCATION: 0651

HORTH COORDIHATE: 63788.9 FT

EAST COORDINATE: 92734.3 FT

04/21/88 TO $12 / 13 / 94$

REPORT DATE: 06/20/95

FORMATION OF COMPLETION: ALLUVIUN (AL)

HYDRAULIC FLON RELATIONSHIP: DOWH GRADIEHT (D)

\begin{tabular}{|c|c|c|c|c|c|c|c|c|}
\hline PARNMETER NAME & LOG DATE & $\underset{\text { ID }}{\text { SAMPLE }}$ & $\begin{array}{l}\text { UNITS OF } \\
\text { MEASURE }\end{array}$ & PV! & $\begin{array}{l}\text { PARAMETER } \\
\text { VALUE }\end{array}$ & FLAGS & $\begin{array}{l}\text { DETECTION } \\
\text { LIMIT }\end{array}$ & $\begin{array}{l}\text { PARAMETER } \\
\text { UKCERTAINTY }\end{array}$ \\
\hline MOL YBDEELUM & $\begin{array}{l}06 / 05 / 89 \\
06 / 05 / 89 \\
12 / 03 / 89 \\
06 / 25 / 90 \\
01 / 29 / 91 \\
06 / 02 / 91 \\
02 / 25 / 92 \\
08 / 13 / 92 \\
07 / 01 / 93 \\
12 / 11 / 94\end{array}$ & $\begin{array}{l}0004 \\
0005 \\
0001 \\
0001 \\
0001 \\
0001 \\
0001 \\
0001 \\
0001 \\
0001\end{array}$ & MG/L & $\begin{array}{l}< \\
< \\
< \\
< \\
< \\
< \\
< \\
< \\
<\end{array}$ & $\begin{array}{l}0.01 \\
0.01 \\
0.01 \\
0.01 \\
0.01 \\
0.01 \\
0.01 \\
0.007 \\
0.01 \\
0.01\end{array}$ & $\begin{array}{l}L \\
L \\
L \\
L \\
L \\
L \\
F\end{array}$ & $\begin{array}{l}0.01 \\
0.01 \\
0.01 \\
0.01 \\
0.01 \\
0.01 \\
0.01 \\
0.007 \\
0.01 \\
0.01\end{array}$ & $\begin{array}{l}- \\
- \\
\vdots \\
- \\
- \\
-\end{array}$ \\
\hline MOLYBDEKUH (TOTAL) & $\begin{array}{l}11 / 21 / 92 \\
07 / 01 / 93\end{array}$ & $\begin{array}{l}\text { NOO1 } \\
\text { N001 }\end{array}$ & MG/L & $<$ & $\begin{array}{l}0.0049 \\
0.01\end{array}$ & $\mathbf{H}$ & $\begin{array}{l}0.0049 \\
0.01\end{array}$ & : \\
\hline HET GROSS ALPHA.* & $\begin{array}{l}04 / 21 / 88 \\
01 / 29 / 91 \\
06 / 02 / 91 \\
02 / 25 / 92 \\
11 / 21 / 92 \\
07 / 01 / 93\end{array}$ & $\begin{array}{l}0001 \\
0001 \\
0001 \\
0001 \\
0001 \\
0001\end{array}$ & $\mathrm{PCI} / \mathrm{L}$ & & $\begin{array}{r}15.70 \\
-0.96 \\
3.86 \\
6.91 \\
15.07 \\
1.63\end{array}$ & & $\begin{array}{l}- \\
- \\
- \\
-\end{array}$ & $\begin{array}{l}- \\
- \\
-\end{array}$ \\
\hline HET GROSS ALPHA (TOTAL) \# & $\begin{array}{l}11 / 21 / 92 \\
07 / 01 / 93\end{array}$ & $\begin{array}{l}\text { N001 } \\
\text { N001 }\end{array}$ & $\mathrm{PCI} / \mathrm{L}$ & & $\begin{array}{l}-1.37 \\
-0.37\end{array}$ & & - & - \\
\hline HICKEL & $\begin{array}{l}06 / 05 / 89 \\
06 / 05 / 89 \\
06 / 05 / 89 \\
06 / 05 / 89 \\
06 / 05 / 89 \\
12 / 03 / 89 \\
06 / 25 / 90 \\
01 / 29 / 91 \\
06 / 02 / 91\end{array}$ & $\begin{array}{l}0001 \\
0002 \\
0003 \\
0004 \\
0005 \\
0001 \\
0001 \\
0001 \\
0001\end{array}$ & MG/L & $\begin{array}{l}< \\
< \\
< \\
< \\
< \\
< \\
< \\
< \\
<\end{array}$ & $\begin{array}{l}0.04 \\
0.04 \\
0.04 \\
0.04 \\
0.04 \\
0.04 \\
0.04 \\
0.04 \\
0.04\end{array}$ & $\begin{array}{l}L \\
L \\
L \\
L \\
L \\
L \\
L L\end{array}$ & $\begin{array}{l}0.04 \\
0.04 \\
0.04 \\
0.04 \\
0.04 \\
0.04 \\
0.04 \\
0.04 \\
0.04\end{array}$ & $\begin{array}{l}- \\
- \\
- \\
- \\
- \\
-\end{array}$ \\
\hline MITRATE (TOTAL) & $07 / 01 / 93$ & N001 & HG/L & \multicolumn{3}{|c|}{2} & 9 & - \\
\hline
\end{tabular}

- MET GROSS ALPHA (GROSS ALPHA - URANIUN) WITH 1 MG URANIUM = 686 PCI

* NET GROSS ALPHA (TOTAL) (TOTAL GROSS ALPHA - TOTAL URAHIUH)

PARAMETER VALUE INDICATOR (PVI): < - LESS THAN DETECTION LIMIT

\section{OTHER PARAMETER VALUE FLAGS:}

F - LOU-FLON SAMPLIHG/DEDICATED PLMP

J - ESTIMATED VALUE

L - LESS THAH THREE BORE VOLUMES REMOVED BEFORE SAMPLIHG

$\checkmark$ - SPIKE SAMPLE RECOVERY NOT HITHIN CONTROL LIMITS
SNMPLE ID COOES:

0001 - FILTERED SAMPLE (.45 MICRONS)

0002 - FILTERED REPLICATE SMMPLE (.45 MICRONS)

0003 - FILTERED REPLICATE SAMPLE (.45 MICRONS)

0004 - FILTERED REPLICATE SAMPLE (.45 MICRONS)

HOO - UHFILTERED SAMPLE 
GROUHDWATER QUALITY DATA BY LOCATION

SOLP Rev. O Data

SITE: MONO9 MONUMENT VALLEY

LOCATION: 0.551

MORTH COORDIHATE: 63788.9 FT

EAST COORDIHATE: 92734.3 FT

04/21/88 TO $12 / 13 / 94$

REPORT DATE: $06 / 20 / 95$

FORMATION OF COAPLETION: ALLUVIUM (AL)

HYDRAULIC FLON RELATIONSHIP: DOWN GRADIEHT (D)

\begin{tabular}{|c|c|c|c|c|c|c|c|c|c|}
\hline PARAMETER MAME & . & LOG DATE & $\begin{array}{c}\text { SAMPLE } \\
\text { ID }\end{array}$ & $\begin{array}{l}\text { UNITS OF } \\
\text { MEASURE }\end{array}$ & PVI & $\begin{array}{l}\text { PARAMETER } \\
\text { VALUE }\end{array}$ & FLAGS & $\begin{array}{l}\text { DETECTION } \\
\text { LIMIT }\end{array}$ & $\begin{array}{l}\text { PARAMETER } \\
\text { UHCERTAINTY }\end{array}$ \\
\hline NITRATE (TOTAL) & & $\begin{array}{l}04 / 20 / 94 \\
12 / 11 / 94\end{array}$ & $\begin{array}{l}\text { NoO1 } \\
\text { N001 }\end{array}$ & MG/L & & $\begin{array}{l}9 \\
1\end{array}$ & FHJH & $\begin{array}{l}1 \\
1\end{array}$ & - \\
\hline MITRITE AND NITRATE & & $\begin{array}{l}01 / 29 / 91 \\
06 / 02 / 91\end{array}$ & $\begin{array}{l}0009 \\
0001\end{array}$ & MG/L & $<$ & 1.97 & JL & $\begin{array}{l}1.05 \\
0.05\end{array}$ & $\dot{-}$ \\
\hline $\mathrm{PH}$ & & $\begin{array}{l}04 / 21 / 88 \\
06 / 05 / 89 \\
06 / 05 / 89 \\
06 / 05 / 89 \\
06 / 05 / 89 \\
06 / 05 / 89 \\
12 / 03 / 89 \\
06 / 25 / 90 \\
01 / 29 / 91 \\
06 / 02 / 91 \\
02 / 25 / 92 \\
08 / 13 / 92 \\
11 / 21 / 92 \\
07 / 01 / 93 \\
04 / 20 / 94 \\
12 / 11 / 94\end{array}$ & $\begin{array}{l}0001 \\
0001 \\
0002 \\
0003 \\
0004 \\
0005 \\
0001 \\
0001 \\
0001 \\
0001 \\
0001 \\
0001 \\
0001 \\
\text { N001 } \\
\text { N001 } \\
\text { N001 }\end{array}$ & SU & & $\begin{array}{l}8.24 \\
8.07 \\
8.07 \\
8.07 \\
8.07 \\
8.07 \\
8.48 \\
8.19 \\
8.23 \\
8.33 \\
8.34 \\
8.13 \\
8.59 \\
8.52 \\
8.17 \\
8.19\end{array}$ & $\begin{array}{l}L \\
L \\
L \\
L \\
L \\
L \\
L \\
L \\
L \\
L\end{array}$ & $\begin{array}{l}- \\
- \\
- \\
- \\
- \\
- \\
- \\
- \\
- \\
- \\
- \\
0.10\end{array}$ & $\begin{array}{l}- \\
- \\
- \\
- \\
- \\
- \\
- \\
- \\
- \\
- \\
- \\
-\end{array}$ \\
\hline PHOSPHATE & $=$ & $\begin{array}{l}06 / 05 / 89 \\
06 / 05 / 89 \\
06 / 05 / 89 \\
06 / 05 / 89 \\
06 / 05 / 89 \\
12 / 03 / 89 \\
06 / 25 / 90\end{array}$ & $\begin{array}{l}0001 \\
0002 \\
0003 \\
0004 \\
0005 \\
0001 \\
0001\end{array}$ & MG/L & & $\begin{array}{l}0.6 \\
0.6 \\
0.6 \\
0.6 \\
0.6 \\
0.2 \\
0.3\end{array}$ & $\begin{array}{l}L \\
L \\
L \\
L \\
L \\
L\end{array}$ & $\begin{array}{l}0.1 \\
0.1 \\
0.1 \\
0.1 \\
0.1 \\
0.1 \\
0.1\end{array}$ & $\begin{array}{l}- \\
- \\
- \\
- \\
-\end{array}$ \\
\hline PHOSPHATE (TOTAL) & & $07 / 01 / 93$ & H0O1 & $M G / L$ & & 0.2 & & 0.1 & - \\
\hline POTASSIUA & & $\begin{array}{l}04 / 21 / 88 \\
06 / 05 / 89 \\
06 / 05 / 89 \\
06 / 05 / 89 \\
06 / 05 / 89 \\
06 / 05 / 89 \\
12 / 03 / 89 \\
06 / 25 / 90 \\
01 / 29 / 91 \\
06 / 02 / 91 \\
02 / 25 / 92 \\
08 / 13 / 92 \\
07 / 01 / 93 \\
04 / 20 / 94 \\
12 / 11 / 94\end{array}$ & $\begin{array}{l}0001 \\
0001 \\
0002 \\
0003 \\
0004 \\
0005 \\
0001 \\
0001 \\
0001 \\
0001 \\
0001 \\
0001 \\
0001 \\
0001 \\
0001\end{array}$ & $M G / L$ & & $\begin{array}{l}1.85 \\
2.9 \\
1.8 \\
2.0 \\
2.1 \\
1.8 \\
1.76 \\
2.1 \\
1.8 \\
2.1 \\
1.96 \\
1.7 \\
2.1 \\
2.1 \\
1.9\end{array}$ & $\begin{array}{l}L \\
L \\
L \\
L \\
L \\
L \\
L \\
L \\
L \\
* L \\
F\end{array}$ & $\begin{array}{l}0.01 \\
0.01 \\
0.01 \\
0.01 \\
0.01 \\
0.01 \\
0.01 \\
0.01 \\
0.01 \\
0.01 \\
0.01 \\
0.575 \\
0.1 \\
0.1 \\
0.1\end{array}$ & $\begin{array}{l}: \\
: \\
: \\
: \\
: \\
: \\
- \\
- \\
- \\
-\end{array}$ \\
\hline POTASSIU: (TOTAL) & & $\begin{array}{l}07 / 01 / 93 \\
04 / 20 / 94\end{array}$ & $\begin{array}{l}\text { N001 } \\
\text { H001 }\end{array}$ & $M G / L$ & & $\begin{array}{l}2.0 \\
2.2\end{array}$ & & $\begin{array}{l}0.1 \\
0.1\end{array}$ & - \\
\hline RNDIUH-226 & & $\begin{array}{l}06 / 05 / 89 \\
06 / 05 / 89\end{array}$ & $\begin{array}{l}0001 \\
0002\end{array}$ & $\mathrm{PCI} / \mathrm{L}$ & & $\begin{array}{l}0.0 \\
0.0\end{array}$ & $L$ & i. & $\begin{array}{l}0.1 \\
0.1\end{array}$ \\
\hline
\end{tabular}

PARAMETER VALUE INDICATOR (PVI): < - LESS THAH DETECTION LIMIT

OTHER PARAMETER VALUE FLAGS:

- - DUPLICATE AHALYSIS NOT UITHIN CONTROL LIMITS

F - LON-FLON SAMPLIHG/DEDICATED PUMP

H - HOLD TIME EXPIRED, VALUE SUSPECT

J - ESTIMATED VALUE

L - LESS THAH THREE BORE VOLUMES REMOVED BEFORE SAMPLIHG

N - SPIKE SNMPLE RECOVERY NOT WITHIN CONTROL LIMITS
SNMPLE ID COOES:

O001 - FILTERED SAMPLE (.45 MICROHS)

O002 - FILTERED REPLICATE SAMPLE (.45 MICRONS)

0003 - FILTERED REPLICATE SAMPLE (.45 MICRONS)

0004 - FILTERED REPLICATE SNMLL (.45 MICROHS)

NOO1 - UWFILTERED SAMPLE 
GROUHDWATER QUALITY DATA BY LOCATIOH

SOMP ReV. O Data

SITE: MOHOI MONUMENT VALLEY

LOCATION: 065!

HORTH COORDIHATE: 63788.9 FT

EAST COORDIHATE: 92734.3 FT

$04 / 21 / 88$ TO $12 / 93 / 94$

REPORT DATE: 06/20/95

FORMATIOH OF COHPLETIOH: ALLUVIUH (AL)

HYDRAULIC FLOM RELATIOHSHIP: DOW GRADIENT (D)

\begin{tabular}{|c|c|c|c|c|c|c|c|c|}
\hline PARAHETER MAME & LOG DATE & $\begin{array}{c}\text { SANPLE } \\
\text { ID }\end{array}$ & $\begin{array}{l}\text { UNITS OF } \\
\text { MEASURE }\end{array}$ & PVI & $\begin{array}{l}\text { PARAMETER } \\
\text { VALUE }\end{array}$ & FLAGS & $\begin{array}{l}\text { DETECTION } \\
\text { LIMIT }\end{array}$ & $\begin{array}{l}\text { PARAMETER } \\
\text { UNCERTAINTY }\end{array}$ \\
\hline RADIUA-226 & $\begin{array}{l}06 / 05 / 89 \\
06 / 05 / 89 \\
06 / 05 / 89 \\
12 / 03 / 89 \\
06 / 25 / 90 \\
01 / 29 / 91 \\
06 / 02 / 91 \\
02 / 25 / 92 \\
08 / 13 / 92 \\
11 / 21 / 92\end{array}$ & $\begin{array}{l}0003 \\
0004 \\
0005 \\
0001 \\
0001 \\
0001 \\
0001 \\
0001 \\
0009 \\
0001\end{array}$ & PCI/L & & $\begin{array}{l}0.0 \\
0.1 \\
0.0 \\
0.0 \\
0.0 \\
0.0 \\
0.0 \\
0.1 \\
0.1 \\
0.4\end{array}$ & $\begin{array}{l}L \\
L \\
L \\
L \\
L \\
L \\
L\end{array}$ & $\begin{array}{l}1 . \\
9 . \\
9 . \\
1 . \\
1 . \\
1.0 \\
1.0 \\
0.6 \\
0.3\end{array}$ & $\begin{array}{l}0.1 \\
0.2 \\
0.1 \\
0.1 \\
0.1 \\
0.1 \\
0.2 \\
0.3 \\
0.1 \\
0.3\end{array}$ \\
\hline RADIUA-226 (TOTAL) & $11 / 21 / 92$ & No01 & PCI/L & \multicolumn{3}{|c|}{0.0} & 0.3 & 0.1 \\
\hline RADILH-226 + RADIUH -228 & $\begin{array}{l}06 / 05 / 89 \\
06 / 05 / 89 \\
06 / 05 / 89 \\
06 / 05 / 89 \\
06 / 05 / 89 \\
12 / 03 / 89 \\
06 / 25 / 90 \\
01 / 29 / 91 \\
06 / 02 / 91 \\
02 / 25 / 92 \\
08 / 13 / 92 \\
11 / 21 / 92\end{array}$ & $\begin{array}{l}0001 \\
0002 \\
0003 \\
0004 \\
0005 \\
0001 \\
0001 \\
0001 \\
0001 \\
0001 \\
0001 \\
0001\end{array}$ & PCI/L & \multicolumn{3}{|c|}{$\begin{array}{l}0.00 \\
0.00 \\
0.00 \\
0.10 \\
0.00 \\
0.00 \\
0.30 \\
0.40 \\
0.90 \\
2.10 \\
0.30 \\
3.40\end{array}$} & $\begin{array}{l}- \\
- \\
- \\
- \\
- \\
- \\
- \\
- \\
-\end{array}$ & $\begin{array}{l}- \\
- \\
- \\
- \\
- \\
- \\
-\end{array}$ \\
\hline RADIUHA-226 + RADIUH-228 (TOTAL) & $11 / 21 / 92$ & N001 & $\mathrm{PCI} / \mathrm{L}$ & \multicolumn{3}{|c|}{3.90} & - & - \\
\hline RADIUH-228 & $\begin{array}{l}06 / 05 / 89 \\
06 / 05 / 89 \\
06 / 05 / 89 \\
06 / 05 / 89 \\
06 / 05 / 89 \\
12 / 03 / 89 \\
06 / 25 / 90 \\
01 / 29 / 91 \\
06 / 02 / 91 \\
02 / 25 / 92 \\
08 / 13 / 92 \\
11 / 21 / 92\end{array}$ & $\begin{array}{l}0001 \\
0002 \\
0003 \\
0004 \\
0005 \\
0001 \\
0001 \\
0001 \\
0001 \\
0001 \\
0001 \\
0001\end{array}$ & PCI/L & & $\begin{array}{l}0.0 \\
0.0 \\
0.0 \\
0.0 \\
0.0 \\
0.0 \\
0.3 \\
0.4 \\
0.9 \\
2.0 \\
0.2 \\
3.0\end{array}$ & $\begin{array}{l}L \\
L \\
L \\
L \\
L \\
L \\
L \\
L \\
J \\
L \\
L\end{array}$ & $\begin{array}{l}1 . \\
1 . \\
1 . \\
1 . \\
1 . \\
1 . \\
1 . \\
1 . \\
1.0 \\
1.9 \\
2.9\end{array}$ & $\begin{array}{l}0.8 \\
0.7 \\
0.7 \\
0.8 \\
0.7 \\
1.9 \\
0.9 \\
0.7 \\
1.6 \\
1.8 \\
1.0 \\
1.5\end{array}$ \\
\hline RADIUK-228 (TOTAL) & $11 / 21 / 92$ & NoOI & $\mathrm{PCI} / \mathrm{L}$ & & 3.9 & & 1.9 & 1.5 \\
\hline REDOX POTENTIAL & $\begin{array}{l}07 / 01 / 93 \\
04 / 20 / 94 \\
12 / 11 / 94\end{array}$ & $\begin{array}{l}\text { No01 } \\
\text { No01 } \\
\text { No01 }\end{array}$ & anOLTS & & $\begin{array}{l}413 \\
377 \\
338\end{array}$ & $\mathbf{F}$ & - & $\begin{array}{l}- \\
-\end{array}$ \\
\hline SELEKIU: & $\begin{array}{l}04 / 29 / 88 \\
06 / 05 / 89 \\
06 / 05 / 89 \\
06 / 05 / 89 \\
06 / 05 / 89 \\
06 / 05 / 89 \\
12 / 03 / 89\end{array}$ & $\begin{array}{l}0001 \\
0001 \\
0002 \\
0003 \\
0004 \\
0005 \\
0001\end{array}$ & MG/L & $\begin{array}{l}< \\
< \\
< \\
< \\
< \\
<\end{array}$ & $\begin{array}{l}0.005 \\
0.005 \\
0.005 \\
0.005 \\
0.005 \\
0.005 \\
0.005\end{array}$ & $\begin{array}{l}L \\
L \\
L \\
L \\
L \\
L\end{array}$ & $\begin{array}{l}0.005 \\
0.005 \\
0.005 \\
0.005 \\
0.005 \\
0.005 \\
0.005\end{array}$ & $\begin{array}{l}- \\
- \\
- \\
- \\
-\end{array}$ \\
\hline
\end{tabular}

PARAMETER VALUE IMDICATOR (PVI): c - LESS THAN DETECTION LIMIT

SUYPLE 10 COOES:

OTHER PARAMETER VALUE FLAGS:

OO01 - FILTERED SAMPLE (.45 MICRONS)

0002 - FILTERED REPLICATE SAMPLE (.45 MICRONS)

0003 - FILTERED REPLICATE SAMPLE (.45 MICRONS)

0004 - FILTERED REPLICATE SNMPLE (.45 MICRONS)

$J$ - ESTIMUTED VALUE

L - LESS THAN THREE BORE VOLUMES REMOVED BEFORE SAMPLING

MDOI - UAFILTERED SAMPLE 
GROUMDHATER QUALITY DATA BY LOCATION

SOWP Rev. O Data

SITE: MONOT MONUMENT VALLEY

LOCATION: 0651

NORTH COORDINATE: 63788.9 FT

EAST COORDINATE: 92734.3 FT

04/21/88 TO $12 / 13 / 94$

REPORT DATE: $06 / 20 / 95$

FORMATION OF COAPLETION: ALLUVIUM (AL)

HYDRAULIC FLON RELATIOHSHIP: DOWH GRADIENT (D)

\begin{tabular}{|c|c|c|c|c|c|c|c|c|}
\hline PARAMETER MAME & LOG DATE & $\underset{\text { SAMPLE }}{\text { ID }}$ & $\begin{array}{l}\text { UNITS OF } \\
\text { MEASURE }\end{array}$ & PVI & $\begin{array}{l}\text { PARAMETER } \\
\text { VALUE }\end{array}$ & FLAGS & $\begin{array}{l}\text { DETECTION } \\
\text { LIMIT }\end{array}$ & $\begin{array}{l}\text { PARAMETER } \\
\text { UHCERTAIHTY }\end{array}$ \\
\hline SELEHIUA & $\begin{array}{l}06 / 25 / 90 \\
01 / 29 / 91 \\
06 / 02 / 91 \\
02 / 25 / 92 \\
08 / 13 / 92\end{array}$ & $\begin{array}{l}0001 \\
0001 \\
0001 \\
0001 \\
0001\end{array}$ & MG/L & $\begin{array}{l}< \\
< \\
< \\
< \\
<\end{array}$ & $\begin{array}{l}0.005 \\
0.005 \\
0.005 \\
0.005 \\
0.005\end{array}$ & $\begin{array}{l}L \\
L \\
d \\
L \\
L\end{array}$ & $\begin{array}{l}0.005 \\
0.005 \\
0.005 \\
0.005 \\
0.005\end{array}$ & $\begin{array}{l}- \\
- \\
- \\
-\end{array}$ \\
\hline SELENIUM (TOTAL) & $11 / 21 / 92$ & NoO1 & MG/L & \multicolumn{3}{|c|}{0.0015} & 0.0015 & - \\
\hline SILICA - SIO2 & $\begin{array}{l}06 / 05 / 89 \\
06 / 05 / 89 \\
06 / 05 / 89 \\
06 / 05 / 89 \\
06 / 05 / 89 \\
12 / 03 / 89 \\
06 / 25 / 90 \\
01 / 29 / 91 \\
06 / 02 / 91 \\
12 / 11 / 94\end{array}$ & $\begin{array}{l}0001 \\
0002 \\
0003 \\
0004 \\
0005 \\
0001 \\
0001 \\
0001 \\
0001 \\
0001\end{array}$ & MG/L & & $\begin{array}{l}11 . \\
11 . \\
11 . \\
11 . \\
11 . \\
11.2 \\
11 . \\
11 . \\
11.3 \\
19.1\end{array}$ & $\begin{array}{l}L \\
L \\
L \\
L \\
L \\
L \\
L \\
F\end{array}$ & $\begin{array}{l}2 . \\
2 . \\
2 . \\
2 . \\
2 . \\
2 . \\
2 . \\
2 . \\
0.1 \\
0.1\end{array}$ & $\begin{array}{l}: \\
: \\
: \\
: \\
- \\
-\end{array}$ \\
\hline SILICA - SIO2 (TOTAL) & $07 / 01 / 93$ & NOOI & MG/L & \multicolumn{3}{|c|}{99.0} & 0.5 & - \\
\hline SILVER & $\begin{array}{l}06 / 05 / 89 \\
06 / 05 / 89 \\
06 / 05 / 89 \\
06 / 05 / 89 \\
06 / 05 / 89 \\
12 / 03 / 89 \\
06 / 25 / 90 \\
01 / 29 / 91 \\
06 / 02 / 99\end{array}$ & $\begin{array}{l}0001 \\
0002 \\
0003 \\
0004 \\
0005 \\
0001 \\
0001 \\
0001 \\
0001\end{array}$ & MG/L & $\begin{array}{l}< \\
< \\
< \\
< \\
< \\
< \\
< \\
< \\
<\end{array}$ & $\begin{array}{l}0.01 \\
0.01 \\
0.01 \\
0.01 \\
0.01 \\
0.01 \\
0.01 \\
0.01 \\
0.01\end{array}$ & $\begin{array}{l}L \\
L \\
L \\
L \\
L \\
L \\
L\end{array}$ & $\begin{array}{l}0.01 \\
0.01 \\
0.01 \\
0.01 \\
0.01 \\
0.01 \\
0.01 \\
0.01 \\
0.01\end{array}$ & $\begin{array}{l}- \\
\dot{-} \\
\dot{-} \\
\dot{-} \\
\dot{-}\end{array}$ \\
\hline SOOILM & $\begin{array}{l}04 / 21 / 88 \\
06 / 05 / 89 \\
06 / 05 / 89 \\
06 / 05 / 89 \\
06 / 05 / 89 \\
06 / 05 / 89 \\
12 / 03 / 89 \\
06 / 25 / 90 \\
01 / 29 / 99 \\
06 / 02 / 99 \\
02 / 25 / 92 \\
08 / 13 / 92 \\
07 / 01 / 93 \\
04 / 20 / 94 \\
12 / 11 / 94\end{array}$ & $\begin{array}{l}0001 \\
0001 \\
0002 \\
0003 \\
0004 \\
0005 \\
0001 \\
0001 \\
0001 \\
0001 \\
0001 \\
0001 \\
0001 \\
0001 \\
0001\end{array}$ & $M G / L$ & & $\begin{array}{l}128 . \\
121 . \\
122 . \\
120 . \\
119 . \\
118 . \\
128 . \\
124 . \\
132 . \\
143 . \\
130 . \\
130 \\
131 \\
129 \\
129\end{array}$ & $\begin{array}{l}L \\
L \\
L \\
L \\
L \\
L \\
L \\
L \\
L \\
L\end{array}$ & $\begin{array}{l}0.002 \\
0.002 \\
0.002 \\
0.002 \\
0.002 \\
0.002 \\
0.002 \\
0.002 \\
0.002 \\
5 . \\
5 . \\
5 \\
1 . \\
1 . \\
1\end{array}$ & $\begin{array}{l}- \\
- \\
- \\
: \\
- \\
- \\
- \\
- \\
-\end{array}$ \\
\hline SOOIUM (TOTAL) & $\begin{array}{l}07 / 01 / 93 \\
04 / 20 / 94\end{array}$ & $\begin{array}{l}\text { NOOS } \\
\text { NOOI }\end{array}$ & MG/L & & $\begin{array}{l}123 \\
126\end{array}$ & & $\begin{array}{l}1 \\
1\end{array}$ & $\dot{-}$ \\
\hline SPECIFIC CONDUCTANCE & $\begin{array}{l}04 / 21 / 88 \\
06 / 05 / 89 \\
06 / 05 / 89 \\
06 / 05 / 89\end{array}$ & $\begin{array}{l}0001 \\
0001 \\
0002 \\
0003\end{array}$ & UHHO/CM & & $\begin{array}{l}460 \\
450 \\
450 \\
450\end{array}$ & $\begin{array}{l}L \\
L \\
L \\
L\end{array}$ & $\begin{array}{l}\ddot{-} \\
\dot{.}\end{array}$ & $\dot{-}$ \\
\hline
\end{tabular}

PARAMETER VALUE INDICATOR (PVI): < - LESS THAN DETECTION LIMIT SAMPLE ID COOES:

OTHER PARAMETER VALUE FLAGS:

0009 - FILTERED SAMLE (.45 MICRONS)

F - LOU-FLON SAYPLING/DEDICATED PUMP

0002 - FILTERED REPLICATE SAMPLE (.45 MICROHS)

J - estiMTEd VALUE

L - LESS THAN THREE BORE VDLUMES REMOVED BEFORE SAMPLIHG

OOO3 - FILTERED REPLICATE SAMPLE (.45 MICROHS)

0004 - FILTERED REPLICATE SAMPLE (.45 MICROHS)

NOOI - UNFILTERED SAMPLE 
GROUNDWATER QUALITY DATA BY LOCATION

SOWP REV. 0 Data

SITE: HOHOI MOHUMENT VALLEY

LOCATION: 0651

NORTH COORDINATE: 63788.9 FT

EAST COORDIHATE: 92734.3 FT

$04 / 21 / 88$ TO $12 / 13 / 94$

REPORT DATE: $06 / 20 / 95$

FORMATIOH OF COMPLETION: ALLUVIUM (AL)

HYDRAULIC FLOW RELATIONSHIP: DOWN GRADIENT (D)

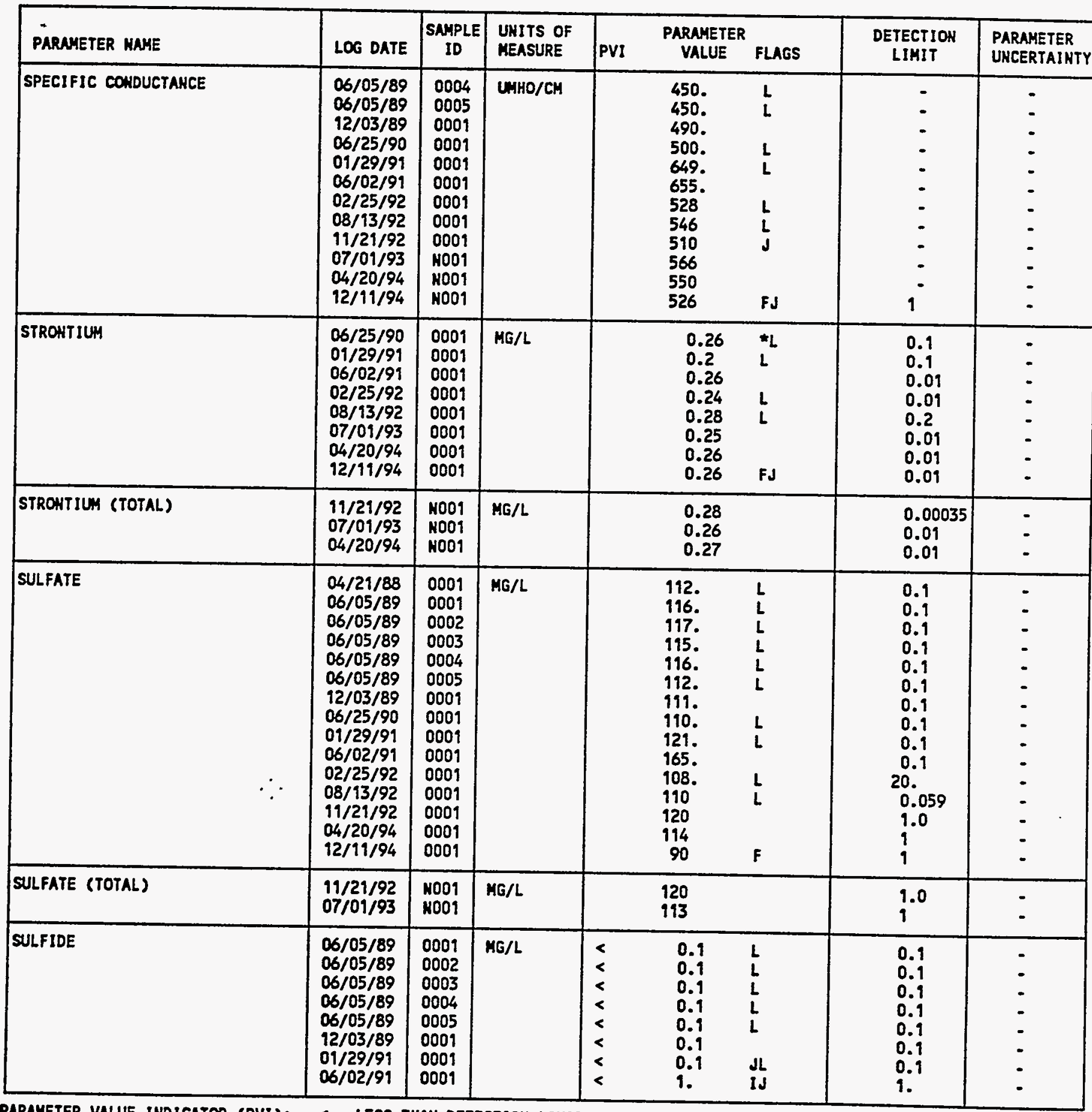

PARAMETER VALUE IMDICATOR (PVI): < - LESS THAN DETECTION LIMIT

SAMPLE ID COOES:

OTHER PARAMETER YALUE FLAGS:

- DUPLICATE aHALYSIS hOT MITHIH CONTROL LIMITS

F - LON-FLON SAMPLIMG/DEDICATEO PUMP

I - INCREASED OETECTION LIMIT DUE TO REQUIRED DILUTION

J - estiMated VALUE

O001 - FILTERED SAMPLE (.45 MICRONS)

0002 - FILTERED REPLICATE SAMPLE (.45 MICRONS)

0003 - FILTERED REPLICATE SAMPLE (.45 MICRONS)

0004 - FILTERED REPLICATE SAMPLE (.45 MICRONS)

NOOI - UHFILTERED SAMPLE

$l$ - LESS THAH THREE BORE YOLLMES REMOVED BEFORE SAMPLING 
GROUNDHATER QUALITY DATA BY LOCATION

SOMP REV. 0 Data

SITE: MONO1 MONUNENT VALLEY

LOCATION: 0651

NORTH COORDINATE: 63788.9 FT

EAST COORDINATE: 92734.3 FT

04/21/88 TO $12 / 13 / 94$

REPORT DATE: $06 / 20 / 95$

FORMATION OF COHPLETION: ALLUVIUM (AL)

HYDRAULIC FLON RELATIONSHIP: DONN GRADIENT (D)

\begin{tabular}{|c|c|c|c|c|c|c|c|c|}
\hline PARANETER RAME & LOG DATE & $\underset{\text { SNMPLE }}{\text { SO }}$ & $\begin{array}{l}\text { UNITS OF } \\
\text { MEASURE }\end{array}$ & PVI & $\begin{array}{l}\text { PARAMETER } \\
\text { VALUE }\end{array}$ & FLAGS & $\begin{array}{l}\text { DETECTIOH } \\
\text { LIMIT }\end{array}$ & $\begin{array}{l}\text { PARAMETER } \\
\text { UNCERTAINTY }\end{array}$ \\
\hline TEMPERATURE & $\begin{array}{l}04 / 21 / 88 \\
06 / 05 / 89 \\
06 / 05 / 89 \\
06 / 05 / 89 \\
06 / 05 / 89 \\
06 / 05 / 89 \\
12 / 03 / 89 \\
06 / 25 / 90 \\
01 / 29 / 91 \\
06 / 02 / 91 \\
02 / 25 / 92 \\
08 / 13 / 92 \\
11 / 21 / 92 \\
07 / 01 / 93 \\
04 / 20 / 94 \\
12 / 11 / 94\end{array}$ & $\begin{array}{l}0001 \\
0001 \\
0002 \\
0003 \\
0004 \\
0005 \\
0001 \\
0001 \\
0001 \\
0001 \\
0001 \\
0001 \\
0001 \\
N 001 \\
H 001 \\
N 001\end{array}$ & C - DEGREE & & $\begin{array}{l}15.0 \\
17.0 \\
17.0 \\
17.0 \\
17.0 \\
17.0 \\
15.0 \\
16.0 \\
14.1 \\
15.5 \\
15.1 \\
16.6 \\
14.1 \\
18.4 \\
17.2 \\
15.1\end{array}$ & $\begin{array}{l}L \\
L \\
L \\
L \\
L \\
L \\
L \\
L \\
L \\
L \\
L\end{array}$ & $\begin{array}{c}- \\
- \\
- \\
\vdots \\
- \\
- \\
- \\
- \\
- \\
- \\
0.1\end{array}$ & $\begin{array}{l}- \\
: \\
- \\
- \\
- \\
- \\
- \\
- \\
- \\
- \\
-\end{array}$ \\
\hline THALLIUN & $\begin{array}{l}06 / 05 / 89 \\
06 / 05 / 89 \\
06 / 05 / 89 \\
06 / 05 / 89 \\
06 / 05 / 89 \\
12 / 03 / 89 \\
06 / 25 / 90 \\
01 / 29 / 91 \\
06 / 02 / 91\end{array}$ & $\begin{array}{l}0001 \\
0002 \\
0003 \\
0004 \\
0005 \\
0001 \\
0001 \\
0001 \\
0001\end{array}$ & MG/L & $\begin{array}{l}< \\
< \\
< \\
< \\
< \\
< \\
< \\
< \\
<\end{array}$ & $\begin{array}{l}0.01 \\
0.01 \\
0.01 \\
0.01 \\
0.01 \\
0.1 \\
0.01 \\
0.01 \\
0.01\end{array}$ & $\begin{array}{l}L \\
L \\
L \\
L \\
L \\
L \\
L\end{array}$ & $\begin{array}{l}0.01 \\
0.01 \\
0.01 \\
0.01 \\
0.01 \\
0.1 \\
0.01 \\
0.01 \\
0.01\end{array}$ & $\begin{array}{l}- \\
- \\
- \\
- \\
- \\
-\end{array}$ \\
\hline THORIUM-230 & $\begin{array}{l}01 / 29 / 91 \\
06 / 02 / 91\end{array}$ & $\begin{array}{l}0001 \\
0001\end{array}$ & PCI/L & & $\begin{array}{l}0.0 \\
0.5\end{array}$ & $\mathbf{L}$ & 1. & $\begin{array}{l}0.3 \\
0.3\end{array}$ \\
\hline TIK & $\begin{array}{l}06 / 05 / 89 \\
06 / 05 / 89 \\
06 / 05 / 89 \\
06 / 05 / 89 \\
06 / 05 / 89 \\
12 / 03 / 89 \\
06 / 25 / 90 \\
01 / 29 / 99 \\
06 / 02 / 91\end{array}$ & $\begin{array}{l}0001 \\
0002 \\
0003 \\
0004 \\
0005 \\
0001 \\
0001 \\
0001 \\
0001\end{array}$ & MG/L & $\begin{array}{l}< \\
< \\
< \\
< \\
< \\
< \\
< \\
< \\
< \\
<\end{array}$ & $\begin{array}{l}0.005 \\
0.005 \\
0.005 \\
0.005 \\
0.005 \\
0.005 \\
0.005 \\
0.005 \\
0.01\end{array}$ & $\begin{array}{l}L \\
L \\
L \\
L \\
L \\
L \\
L \\
I\end{array}$ & $\begin{array}{l}0.005 \\
0.005 \\
0.005 \\
0.005 \\
0.005 \\
0.005 \\
0.005 \\
0.005 \\
0.01\end{array}$ & $\begin{array}{l}- \\
- \\
- \\
- \\
- \\
-\end{array}$ \\
\hline TOTAL DISSOLVED SOLIDS & $\begin{array}{l}04 / 21 / 88 \\
06 / 05 / 89 \\
06 / 05 / 89 \\
06 / 05 / 89 \\
06 / 05 / 89 \\
06 / 05 / 89 \\
12 / 03 / 89 \\
06 / 25 / 90 \\
01 / 29 / 91 \\
06 / 02 / 91 \\
02 / 25 / 92 \\
08 / 13 / 92 \\
04 / 20 / 94 \\
12 / 11 / 94\end{array}$ & $\begin{array}{l}0001 \\
0001 \\
0002 \\
0003 \\
0004 \\
0005 \\
0001 \\
0001 \\
0001 \\
0001 \\
0001 \\
0001 \\
0001 \\
0001\end{array}$ & $M G / L$ & & $\begin{array}{l}430 . \\
389 . \\
382 . \\
376 . \\
368 . \\
387 . \\
415 . \\
440 . \\
348 . \\
395 . \\
401 . \\
420 \\
410 \\
402\end{array}$ & $\begin{array}{l}L \\
L \\
L \\
L \\
L \\
L \\
L \\
L \\
L \\
L \\
F\end{array}$ & $\begin{array}{l}10 . \\
10 . \\
10 . \\
10 . \\
10 . \\
10 . \\
10 . \\
10 . \\
10 . \\
10 . \\
10 . \\
42 \\
10 \\
10\end{array}$ & $\begin{array}{l}- \\
: \\
: \\
: \\
: \\
- \\
- \\
- \\
- \\
-\end{array}$ \\
\hline
\end{tabular}

PARAMETER VALUE IMDICATOR (PVI): < - LESS THAN DETECTION LIMIT

SAHPLE ID COOES:

O001 - FILTERED SAMPLE (.45 MICROHS)

OTHER PARAMETER VALUE FLAGS:

F - LOW-FLON SAMPLING/DEDICATED PUIP

I - INCREASED DETECTION LIMIT DUE TO REOUIRED DILUTION

- $L$ - LESS THAN THREE BORE VOLUMES REMOVED BEFORE SAMPLIHG

0002 - FILTERED REPLICATE SAMPLE (.45 MICRONS) 0003 - FILTERED REPLICATE SAMPLE (.45 MICRONS) 0004 - FILTERED REPLICATE SAMPLE (.45 MICRONS) NOOI - UHFILTERED SAMPLE 
GROUKDWATER QUALITY DATA BY LOCATION

SOWP ReV. O Data

SITE: HOHO 9 MONLMENT VALLEY

LOCATION: 0651

NORTH COORDINATE: 63788.9 FT

EAST COORDINATE: 92734.3 FT

Q4/21/88 TO $12 / 13 / 94$

REPORT DATE: 06/20/95

FORMATIOH OF COHPLETIOH: ALLUVIUM (AL)

HYDRAULIC FLON RELATIONSHIP: DOWN GRADIEHT (D)

\begin{tabular}{|c|c|c|c|c|c|c|c|c|}
\hline PARAMETER NAME & LOG DATE & $\mid \begin{array}{c}\text { SAMPLE } \\
\text { ID }\end{array}$ & $\begin{array}{l}\text { UNITS OF } \\
\text { MEASURE }\end{array}$ & PVI & $\begin{array}{l}\text { PARAMETER } \\
\text { VALUE }\end{array}$ & FLAGS & $\begin{array}{l}\text { DETECTION } \\
\text { LIMIT }\end{array}$ & $\begin{array}{l}\text { PARAMETER } \\
\text { UHCERTAINTY }\end{array}$ \\
\hline TOTAL DISSOLVED SOLIDS (TOTAL) & $07 / 01 / 93$ & No01 & $M G / L$ & & 390 & & 10 & - \\
\hline TOTAL ORGANIC CARBOH & $\begin{array}{l}04 / 21 / 88 \\
01 / 29 / 91 \\
06 / 02 / 91 \\
02 / 25 / 92 \\
08 / 13 / 92\end{array}$ & $\begin{array}{l}0001 \\
0001 \\
0001 \\
0001 \\
0001\end{array}$ & $M G / L$ & $\begin{array}{l}< \\
< \\
<\end{array}$ & $\begin{array}{r}103 . \\
1 . \\
1 . \\
1.0\end{array}$ & $\begin{array}{l}L \\
J L \\
L \\
G L\end{array}$ & $\begin{array}{l}1 . \\
1 . \\
1 . \\
1.0\end{array}$ & $\begin{array}{l}\dot{-} \\
\dot{-} \\
\dot{-}\end{array}$ \\
\hline TURBIDITY & $12 / 11 / 94$ & nOO1 & NTU & & 8 & $\mathbf{F}$ & 1 & - \\
\hline URANILY & $\begin{array}{l}04 / 21 / 88 \\
06 / 05 / 89 \\
06 / 05 / 89 \\
06 / 05 / 89 \\
06 / 05 / 89 \\
06 / 05 / 89 \\
12 / 03 / 89 \\
06 / 25 / 90 \\
01 / 29 / 91 \\
06 / 02 / 91 \\
02 / 25 / 92 \\
11 / 21 / 92 \\
07 / 01 / 93 \\
04 / 20 / 94 \\
12 / 11 / 94\end{array}$ & $\begin{array}{l}0001 \\
0001 \\
0002 \\
0003 \\
0004 \\
0005 \\
0009 \\
0001 \\
0001 \\
0001 \\
0009 \\
0001 \\
0009 \\
0001 \\
0009\end{array}$ & MG/L & $<$ & $\begin{array}{l}0.0019 \\
0.0090 \\
0.059 \\
0.019 \\
0.018 \\
0.011 \\
0.003 \\
0.0023 \\
0.0014 \\
0.001 \\
0.001 \\
0.005 \\
0.002 \\
0.002 \\
0.001\end{array}$ & $\begin{array}{l}J L \\
L \\
L \\
L \\
L \\
L \\
J L \\
L \\
L \\
J L \\
F\end{array}$ & $\begin{array}{l}0.003 \\
0.003 \\
0.003 \\
0.003 \\
0.003 \\
0.003 \\
0.003 \\
0.003 \\
0.0003 \\
0.001 \\
0.001 \\
0.001 \\
0.001 \\
0.001 \\
0.001\end{array}$ & $\begin{array}{l}- \\
: \\
- \\
: \\
- \\
- \\
- \\
- \\
- \\
-\end{array}$ \\
\hline URAHIUM (TOTAL) & $\begin{array}{l}11 / 21 / 92 \\
07 / 01 / 93 \\
04 / 20 / 94\end{array}$ & $\begin{array}{l}\text { No01 } \\
\text { No0? } \\
\text { No01 }\end{array}$ & MG/L & & $\begin{array}{l}0.002 \\
0.002 \\
0.002\end{array}$ & & $\begin{array}{l}0.001 \\
0.001 \\
0.001\end{array}$ & $:$ \\
\hline VAHADIUN & $\begin{array}{l}06 / 05 / 89 \\
06 / 05 / 89 \\
06 / 05 / 89 \\
06 / 05 / 89 \\
06 / 05 / 89 \\
12 / 03 / 89 \\
06 / 25 / 90 \\
01 / 29 / 91 \\
06 / 02 / 91 \\
02 / 25 / 92 \\
08 / 13 / 92 \\
07 / 01 / 93 \\
04 / 20 / 94 \\
12 / 11 / 94\end{array}$ & $\begin{array}{l}0001 \\
0002 \\
0003 \\
0004 \\
0005 \\
0001 \\
0001 \\
0009 \\
0001 \\
0001 \\
0001 \\
0009 \\
0001 \\
0001\end{array}$ & MG/L & $\begin{array}{l}< \\
< \\
< \\
< \\
< \\
<\end{array}$ & $\begin{array}{l}0.01 \\
0.01 \\
0.01 \\
0.01 \\
0.01 \\
0.01 \\
0.01 \\
0.01 \\
0.01 \\
0.01 \\
0.05 \\
0.01 \\
0.01 \\
0.01\end{array}$ & $\begin{array}{l}L \\
L \\
L \\
L \\
L \\
L \\
L \\
L \\
L\end{array}$ & $\begin{array}{l}0.01 \\
0.01 \\
0.01 \\
0.01 \\
0.01 \\
0.01 \\
0.01 \\
0.01 \\
0.01 \\
0.01 \\
0.05 \\
0.01 \\
0.01 \\
0.01\end{array}$ & $\begin{array}{l}- \\
- \\
- \\
- \\
- \\
\therefore \\
- \\
- \\
-\end{array}$ \\
\hline VAKADILA (TOTAL) & $\begin{array}{l}11 / 21 / 92 \\
07 / 01 / 93 \\
04 / 20 / 94\end{array}$ & $\begin{array}{l}\text { No01 } \\
\text { No01 } \\
\text { No01 }\end{array}$ & $M G / L$ & & $\begin{array}{l}0.011 \\
0.01 \\
0.01\end{array}$ & & $\begin{array}{l}0.0019 \\
0.01 \\
0.01\end{array}$ & $\ddot{-}$ \\
\hline ZIMC & $\begin{array}{l}06 / 05 / 89 \\
06 / 05 / 89 \\
06 / 05 / 89 \\
06 / 05 / 89\end{array}$ & $\begin{array}{l}0001 \\
0002 \\
0003 \\
0004\end{array}$ & MG/L & $\begin{array}{l}< \\
<\end{array}$ & $\begin{array}{l}0.005 \\
0.005 \\
0.059 \\
0.005\end{array}$ & $\begin{array}{l}L \\
L \\
L \\
L\end{array}$ & $\begin{array}{l}0.005 \\
0.005 \\
0.005 \\
0.005\end{array}$ & $\dot{-}$ \\
\hline
\end{tabular}

PARAMETER VALUE INDICATOR (PVI): < - LESS THAN DETECTION LIMIT

SAMPLE ID COOES:

O0O1 - FILTERED SAMPLE (.45 MICRONS)

OTHER PARAMETER VALUE FLAGS:

F - LOW-FLON SAMPLING/DEDICATED PUMP

0002 - FILTERED REPLICATE SAMPLE (.45 MICRONS)

0003 - FILTERED REPLICATE SAMPLE (.45 MICRONS)

G - PH > 9, POSSIBLE GROUT CONTNMIHATION

J - ESTIMATED VALUE

L - LESS THAK THREE BORE VOLUMES REMOVED BEFORE SNMPLIHG

OOO4 - FILTERED REPLICATE SAMPLE (.45 MICRONS)

NOOI - UNFILTERED SAMPLE 
GROUNDWATER QUALITY DATA BY LOCATION

SOWP REV. O Data

SITE: HOHOI MONUMENT VALLEY

LOCATION: 0659

MORTH COORDINATE: 63788.9 FT

EAST COORDINATE: 92734.3 FT

$04 / 29 / 88$ TO $12 / 13 / 94$

REPORT DATE: $06 / 20 / 95$

FORHATIOH OF COMPLETION: ALLUVIUH (AL)

HYDRAULIC FLON RELATIONSHIP: DOWN GRADIENT (D)

\begin{tabular}{|c|c|c|c|c|c|c|c|c|}
\hline PARAMETER MAHE & LOG DATE & $\begin{array}{c}\text { SAMPLE } \\
\text { TO }\end{array}$ & $\begin{array}{l}\text { UNITS OF } \\
\text { MEASURE }\end{array}$ & PVI & $\begin{array}{l}\text { PARAMETER } \\
\text { VALUE }\end{array}$ & FLAGS & $\begin{array}{l}\text { DETECTION } \\
\text { LIMIT }\end{array}$ & $\begin{array}{l}\text { PARAMETER } \\
\text { UNCERTAINTY }\end{array}$ \\
\hline ZIMC & $\begin{array}{l}06 / 05 / 89 \\
12 / 03 / 89 \\
06 / 25 / 90 \\
01 / 29 / 91 \\
06 / 02 / 91 \\
02 / 25 / 92 \\
08 / 13 / 92 \\
07 / 01 / 93\end{array}$ & $\begin{array}{l}0005 \\
0001 \\
0001 \\
0001 \\
0001 \\
0001 \\
0001 \\
0001\end{array}$ & $M G / L$ & $\begin{array}{l}< \\
< \\
< \\
< \\
<\end{array}$ & $\begin{array}{l}0.005 \\
0.005 \\
0.009 \\
0.005 \\
0.005 \\
0.005 \\
0.02 \\
0.022\end{array}$ & $\begin{array}{l}L \\
L \\
L \\
L \\
L \\
J\end{array}$ & $\begin{array}{l}0.005 \\
0.005 \\
0.005 \\
0.005 \\
0.005 \\
0.005 \\
0.02 \\
0.005\end{array}$ & $\begin{array}{l}- \\
- \\
- \\
- \\
-\end{array}$ \\
\hline ZINC (TOTAL) & $\begin{array}{l}11 / 21 / 92 \\
07 / 01 / 93\end{array}$ & $\begin{array}{l}\text { NO01 } \\
\text { NO01 }\end{array}$ & $M G / L$ & & $\begin{array}{l}0.0040 \\
0.023\end{array}$ & $J$ & $\begin{array}{l}0.0010 \\
0.005\end{array}$ & $\dot{-}$ \\
\hline
\end{tabular}

PARAMETER VALUE INDICATOR (PVI): < - LESS THAN DETECTION LIMIT

SAMPLE ID COOES

OTHER PARAMETER VALUE FLAGS:

OOO1 - FILTERED SAMPLE (.45 MICRONS)

J - ESTIMATED VALUE

NOO - UNFILTERED SAMPLE

L - LESS THAN THREE BORE VOLLMES REMOVED BEFORE SAMPLING 
GROUNDHATER QUALITY DATA BY LOCATION

SOMP REV. O Data

SITE: MOHO1 MOHLMENT VALLEY

LOCATIOH: 0652

MORTH COORDIHATE: 62581.5 FT

EAST COORDIHATE: $\quad 93759.3$ FT

$04 / 21 / 88$ TO $12 / 13 / 94$

REPORT DATE: 06/20/95

FORMATION OF COMPLETION: ALLUVIUN (AL)

HYDRAULIC FLOW RELATIONSHIP: DONN GRADIENT (D)

\begin{tabular}{|c|c|c|c|c|c|c|c|c|}
\hline PARAMETER KAME & LOG DATE & $\begin{array}{c}\text { SAMPLE } \\
10\end{array}$ & $\begin{array}{l}\text { UNITS OF } \\
\text { MEASURE }\end{array}$ & PVI & $\begin{array}{l}\text { PARAMETER } \\
\text { VALUE }\end{array}$ & FLAGS & $\begin{array}{l}\text { DETECTION } \\
\text { LIMIT }\end{array}$ & $\begin{array}{l}\text { PARAMETER } \\
\text { UNCERTAIHTY }\end{array}$ \\
\hline ALKaLIMITY & $\begin{array}{l}06 / 01 / 91 \\
02 / 23 / 92 \\
08 / 12 / 92 \\
11 / 21 / 92 \\
02 / 19 / 93 \\
07 / 01 / 93 \\
12 / 08 / 94\end{array}$ & $\begin{array}{l}0001 \\
0001 \\
0001 \\
0001 \\
0001 \\
\text { No01 } \\
\text { NO01 }\end{array}$ & MG/L CACO3 & & $\begin{array}{l}205 . \\
195 \\
177 \\
190 \\
196 \\
187 \\
173\end{array}$ & $\mathbf{L}$ & $\begin{array}{r}- \\
\div \\
10\end{array}$ & $\begin{array}{l}: \\
: \\
: \\
:\end{array}$ \\
\hline ALUMIKLM & $\begin{array}{l}06 / 01 / 91 \\
02 / 23 / 92 \\
08 / 12 / 92\end{array}$ & $\begin{array}{l}0001 \\
0001 \\
0001\end{array}$ & MG/L & $\begin{array}{l}< \\
< \\
<\end{array}$ & $\begin{array}{l}0.05 \\
0.05 \\
0.2\end{array}$ & $\begin{array}{l}L \\
*\end{array}$ & $\begin{array}{l}0.05 \\
0.05 \\
0.2\end{array}$ & : \\
\hline AMONIUM & $\begin{array}{l}06 / 01 / 91 \\
02 / 23 / 92\end{array}$ & $\begin{array}{l}0001 \\
0001\end{array}$ & $M G / L$ & $<$ & $\begin{array}{l}0.1 \\
0.1\end{array}$ & $\mathbf{L}$ & $\begin{array}{l}0.1 \\
0.1\end{array}$ & $\because$ \\
\hline AMYOHIUM (TOTAL) & $\begin{array}{l}07 / 01 / 93 \\
12 / 08 / 94\end{array}$ & $\begin{array}{l}\text { NOO1 } \\
\text { N001 }\end{array}$ & MG/L & $<$ & $\begin{array}{l}0.1 \\
0.12\end{array}$ & H & $\begin{array}{l}0.1 \\
0.1\end{array}$ & - \\
\hline АКT IMONY & $\begin{array}{l}06 / 01 / 91 \\
02 / 23 / 92 \\
08 / 12 / 92\end{array}$ & $\begin{array}{l}0001 \\
0001 \\
0001\end{array}$ & $M G / L$ & $\begin{array}{l}< \\
<\end{array}$ & $\begin{array}{l}0.003 \\
0.003 \\
0.06\end{array}$ & $\begin{array}{l}L \\
H\end{array}$ & $\begin{array}{l}0.003 \\
0.003 \\
0.06\end{array}$ & : \\
\hline ARSENIC & $\begin{array}{l}06 / 01 / 91 \\
02 / 23 / 92 \\
08 / 12 / 92 \\
11 / 21 / 92 \\
07 / 01 / 93\end{array}$ & $\begin{array}{l}0001 \\
0001 \\
0001 \\
0001 \\
0001\end{array}$ & $M G / L$ & $\begin{array}{l}< \\
< \\
< \\
<\end{array}$ & $\begin{array}{l}0.01 \\
0.01 \\
0.01 \\
0.0015 \\
0.005\end{array}$ & L & $\begin{array}{l}0.01 \\
0.01 \\
0.01 \\
0.0015 \\
0.005\end{array}$ & : \\
\hline ARSENIC (TOTAL) & $\begin{array}{l}11 / 21 / 92 \\
07 / 01 / 93\end{array}$ & $\begin{array}{l}\text { No01 } \\
\text { NOO1 }\end{array}$ & $M G / L$ & $<$ & $\begin{array}{l}0.0015 \\
0.005\end{array}$ & & $\begin{array}{l}0.0015 \\
0.005\end{array}$ & - \\
\hline BARIUM & $\begin{array}{l}06 / 01 / 91 \\
02 / 23 / 92 \\
08 / 12 / 92 \\
11 / 21 / 92 \\
07 / 01 / 93\end{array}$ & $\begin{array}{l}0001 \\
0001 \\
0001 \\
0001 \\
0001\end{array}$ & MG/L & $<$ & $\begin{array}{l}0.15 \\
0.13 \\
0.2 \\
0.14 \\
0.1\end{array}$ & $\mathbf{L}$ & $\begin{array}{l}0.01 \\
0.01 \\
0.2 \\
0.0015 \\
0.1\end{array}$ & $\begin{array}{l}- \\
- \\
- \\
-\end{array}$ \\
\hline BARIUH (TOTAL) & $\begin{array}{l}11 / 21 / 92 \\
07 / 01 / 93\end{array}$ & $\begin{array}{l}\text { No01 } \\
\text { No01 }\end{array}$ & $M G / L$ & & $\begin{array}{l}0.13 \\
0.1\end{array}$ & & $\begin{array}{l}0.0015 \\
0.1\end{array}$ & $\therefore$ \\
\hline BERYLLIUY & $\begin{array}{l}06 / 01 / 91 \\
08 / 12 / 92\end{array}$ & $\begin{array}{l}0001 \\
0001\end{array}$ & MG/L & $<$ & $\begin{array}{l}0.005 \\
0.005\end{array}$ & $L$ & $\begin{array}{l}0.005 \\
0.005\end{array}$ & 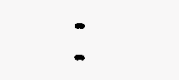 \\
\hline BORON & $06 / 01 / 91$ & 0001 & $M G / L$ & & 0.08 & $\mathbf{L}$ & 0.05 & - \\
\hline BROMIDE & $06 / 01 / 91$ & 0001 & MG/L & & 0.1 & $\mathbf{L}$ & 0.1 & - \\
\hline CADHIUH & $\begin{array}{l}06 / 01 / 91 \\
02 / 23 / 92 \\
08 / 12 / 92 \\
11 / 21 / 92\end{array}$ & $\begin{array}{l}0001 \\
0001 \\
0001 \\
0001\end{array}$ & $M G / L$ & $\begin{array}{l}< \\
< \\
<\end{array}$ & $\begin{array}{l}0.001 \\
0.0005 \\
0.005 \\
0.00013\end{array}$ & L & $\begin{array}{l}0.001 \\
0.0005 \\
0.005 \\
0.00013\end{array}$ & $\begin{array}{l}- \\
- \\
-\end{array}$ \\
\hline CADHIUH (TOTAL) & $11 / 21 / 92$ & No01 & MG/L & $<$ & 0.00013 & $\mathrm{H}$ & 0.00013 & - \\
\hline
\end{tabular}

PARAMETER VALUE INDICATOR (PVI): < - LESS thaM DETECTION LIMIT

SAMPLE ID COOES:

OTHER PARAMETER VALUE FLAGS:

OOO1 - FILTERED SAMPLE (.45 MICRONS)

- - duplicate analysis hot uithin control limits

NOO1 - UHFILTERED SAMPLE

E - ESTIMATED VALUE BECAUSE OF IHTERFEREHCE, SEE CASE MARRATIVE

H - HOLD TIME EXPIRED, VALUE SUSPECT

L - LESS THAK THREE BORE VOLLMES REMOVEO BEFORE SAMPLIMG

H - SPIKE SAMPLE RECOVERY NOT WITHIN COHTROL LIMITS

$W$ - POST-DIGEST SPIKE OUT OF CHTR LIM WHILE SAMP ABS < 50\% SPIKE 
GROUHDWATER QUALITY DATA BY LOCATION

SOWP REV. D Data

SITE: MONOT MONLMENT VALLEY

LOCATION: 0652

MORTH COORDINATE: 62581.5 FT

EAST COOROINATE: $\quad 93759.3$ FT

O4/21/88 TO $12 / 13 / 94$

REPORT DATE: $06 / 20 / 95$

FORMATION OF COMPLETION: ALLUVIUM (AL)

HYDRAULIC FLON RELATIONSHIP: DOWN GRADIENT (D)

\begin{tabular}{|c|c|c|c|c|c|c|c|c|}
\hline PARAMETER NAME & LOG DATE & $\begin{array}{c}\text { SNMPLE } \\
10\end{array}$ & $\begin{array}{l}\text { UNITS OF } \\
\text { MEASURE }\end{array}$ & PVI & $\begin{array}{l}\text { PARAMETER } \\
\text { VALUE }\end{array}$ & FLAGS & $\begin{array}{l}\text { DETECTION } \\
\text { LIMIT }\end{array}$ & $\begin{array}{l}\text { PARAMETER } \\
\text { UNCERTAINTY }\end{array}$ \\
\hline CALCIUM & $\begin{array}{l}06 / 01 / 91 \\
02 / 23 / 92 \\
08 / 12 / 92 \\
02 / 19 / 93 \\
07 / 01 / 93 \\
12 / 08 / 94\end{array}$ & $\begin{array}{l}0001 \\
0001 \\
0001 \\
0001 \\
0001 \\
0001\end{array}$ & $M G / L$ & & $\begin{array}{l}29.6 \\
26.7 \\
28 \\
26.3 \\
26.6 \\
27.3\end{array}$ & $\mathbf{L}$ & $\begin{array}{l}0.5 \\
0.5 \\
5 \\
0.5 \\
0.5 \\
0.5\end{array}$ & $\begin{array}{l}: \\
: \\
:\end{array}$ \\
\hline CALCIUA (TOTAL) & $07 / 01 / 93$ & HoOI & MG/L & & 26.1 & & 0.5 & - \\
\hline CHLORIDE & $\begin{array}{l}06 / 01 / 91 \\
02 / 23 / 92 \\
08 / 12 / 92 \\
02 / 19 / 93 \\
12 / 08 / 94\end{array}$ & $\begin{array}{l}0001 \\
0001 \\
0001 \\
0001 \\
0001\end{array}$ & $M G / L$ & & $\begin{array}{l}15.3 \\
15.3 \\
14 \\
15 \\
9\end{array}$ & \llcorner & $\begin{array}{l}0.5 \\
0.5 \\
0.016 \\
0.5 \\
0.5\end{array}$ & $\begin{array}{l}- \\
- \\
-\end{array}$ \\
\hline CHLORIDE (TOTAL) & $07 / 01 / 93$ & N001 & MG/L & & 15.1 & & 0.5 & - \\
\hline CHROMILM & $\begin{array}{l}06 / 01 / 91 \\
02 / 23 / 92 \\
08 / 12 / 92 \\
11 / 21 / 92\end{array}$ & $\begin{array}{l}0001 \\
0001 \\
0001 \\
0001\end{array}$ & $M G / L$ & $\begin{array}{l}< \\
< \\
< \\
<\end{array}$ & $\begin{array}{l}0.01 \\
0.01 \\
0.01 \\
0.0053\end{array}$ & $L$ & $\begin{array}{l}0.01 \\
0.01 \\
0.01 \\
0.0053\end{array}$ & $\begin{array}{l}- \\
- \\
-\end{array}$ \\
\hline CHROMIUA (TOTAL) & $11 / 21 / 92$ & No01 & $M G / L$ & $<$ & 0.0053 & & 0.0053 & - \\
\hline $\operatorname{COBALT}$ & $06 / 01 / 91$ & 0001 & MG/L & $<$ & 0.03 & $L$ & 0.03 & - \\
\hline COPPER & $06 / 01 / 91$ & 0001 & MG/L & $<$ & 0.01 & $L$ & 0.01 & - \\
\hline DISSOLVED OXYGEN & $\begin{array}{l}07 / 01 / 93 \\
12 / 08 / 94\end{array}$ & $\begin{array}{l}\text { NOO1 } \\
\text { N001 }\end{array}$ & MG/L & $<$ & $\begin{array}{l}0.1 \\
0.02\end{array}$ & & 0.02 & - \\
\hline FLUORIDE & $06 / 01 / 91$ & 0001 & $\mathrm{MG} / \mathrm{L}$ & & 0.1 & $L$ & 0.1 & - \\
\hline GROSS ALPHA & $\begin{array}{l}06 / 01 / 91 \\
02 / 23 / 92 \\
08 / 12 / 92 \\
11 / 21 / 92 \\
02 / 19 / 93 \\
07 / 01 / 93\end{array}$ & $\begin{array}{l}0001 \\
0001 \\
0001 \\
0001 \\
0001 \\
0001\end{array}$ & PCI/L & . & $\begin{array}{l}6.8 \\
1.6 \\
2.9 \\
9.4 \\
0 \\
2\end{array}$ & L & $\begin{array}{l}1.0 \\
1.0 \\
4.3 \\
3.0 \\
5 \\
3\end{array}$ & $\begin{array}{l}5.1 \\
3.8 \\
2.9 \\
3.4 \\
5 \\
4\end{array}$ \\
\hline GROSS ALPHA (TOTAL) & $\begin{array}{l}11 / 21 / 92 \\
06 / 30 / 93\end{array}$ & $\begin{array}{l}\text { No01 } \\
\text { N001 }\end{array}$ & $\mathrm{PCI} / \mathrm{L}$ & & $\begin{array}{l}0.0 \\
9\end{array}$ & H & $\begin{array}{l}6.8 \\
2\end{array}$ & $\begin{array}{l}3.8 \\
5\end{array}$ \\
\hline GROSS BETA & $\begin{array}{l}06 / 01 / 91 \\
02 / 23 / 92 \\
08 / 12 / 92 \\
11 / 21 / 92 \\
02 / 19 / 93 \\
07 / 01 / 93\end{array}$ & $\begin{array}{l}0001 \\
0001 \\
0001 \\
0001 \\
0001 \\
0001\end{array}$ & PCI/L & & $\begin{array}{l}7.4 \\
3.3 \\
3.6 \\
5.9 \\
0 \\
0\end{array}$ & L & $\begin{array}{c}0.5 \\
0.5 \\
4.1 \\
5.5 \\
11 \\
4\end{array}$ & $\begin{array}{l}2.9 \\
2.2 \\
2.6 \\
3.5 \\
5 \\
4\end{array}$ \\
\hline GROSS BETA (TOTAL) & $\begin{array}{l}19 / 21 / 92 \\
06 / 30 / 93\end{array}$ & $\begin{array}{l}\text { NoO9 } \\
\text { nOOI }\end{array}$ & PCI/L & & $\begin{array}{l}6.4 \\
1\end{array}$ & "I & $\begin{array}{l}5.0 \\
4\end{array}$ & $\begin{array}{l}3.3 \\
4\end{array}$ \\
\hline IRON & $06 / 01 / 91$ & 0001 & MG/L & $<$ & 0.03 & L & 0.03 & - \\
\hline
\end{tabular}

PARAMETER VALUE IMDICATOR (PVI):

< - LESS THAN DETECTION LIMIT

SAMPLE ID COOES:

OTHER PARAMETER VALUE FLAGS:

OOO1 - FILTERED SAMPLE (.45 MICRONS)

- DUPLICATE anALYSIS nOT HITHIN CONTROL LIMITS

MOO1 - UNFILTERED SAMPLE

$L$ - LESS THAN THREE BORE VOLLMES REMOVED BEFORE SAMPLIHG

- N - SPIKE SAMPLE RECOVERY NOT WITHIN CONTROL LIMITS 
GROUKDWATER QUALITY DATA BY LOCATIOH

SOUP Rev. O Data

SITE: MONO MONUHENT VALLEY

LOCATION: 0652

HORTH COORDIHATE: 62581.5 FT

EAST COORDINATE: 93759.3 FT

04/21/88 TO 12/13/94

REPORT DATE: 06/20/95

FORMATION OF COMPLETION: ALLUVIUM (AL)

HYDRAULIC FLON RELATIONSHIP: DOWN GRADIENT (D)

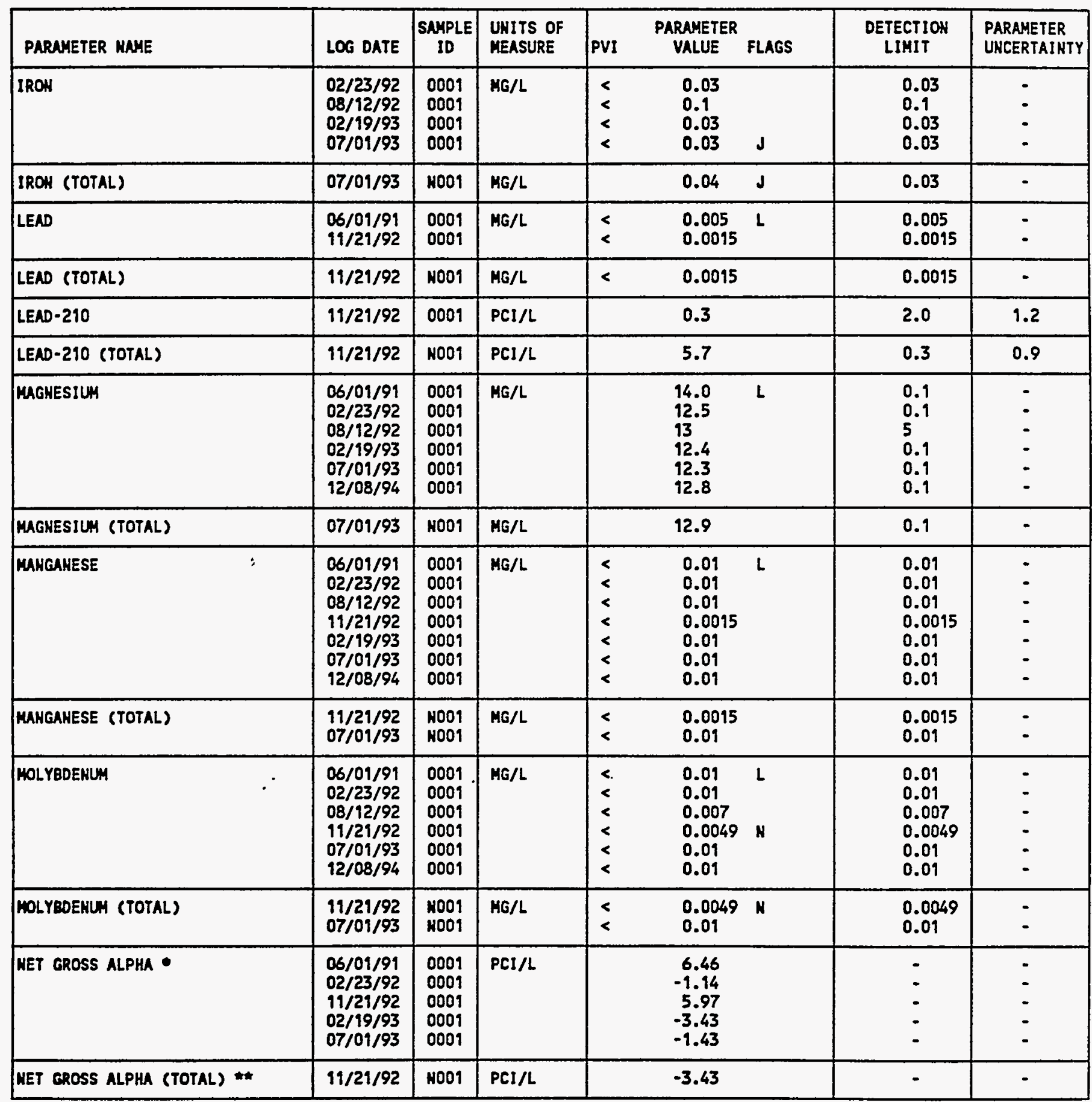

- het gross aLPHA (GROSS ALPHA - URAKIUH) HITH I MG URANIUH = 686 PCI

* MET GROSS ALPHA (TOTAL) (TOTAL GROSS ALPHA - TOTAL URANIUH)

PARAMETER VALUE INDICATOR (PVI): < - LESS THAN DETECTIOH LIMIT

$J$ - ESTIMATED VALUE

L - LESS THAN THREE BORE VOLUMES REMOVED BEFORE SAMPLING

H - SPIKE SAMPLE RECOVERY NOT WITHIN CONTROL LIMITS 


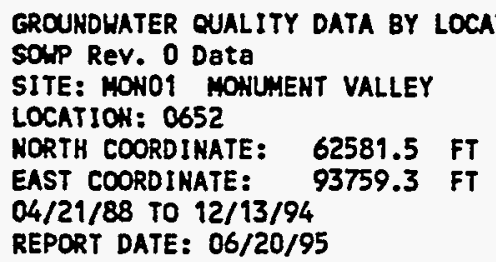

FORMATIOH OF COMPLETIOH: ALLUVIUH (AL)

HYDRAULIC FLON RELATIONSHIP: DOWN GRADIENT (D)

\begin{tabular}{|c|c|c|c|c|c|c|c|c|}
\hline PARAMETER RNME & LOG DATE & $\underset{\text { ID }}{\text { SAMPLE }}$ & $\begin{array}{l}\text { UNITS OF } \\
\text { MEASURE }\end{array}$ & PVI & $\begin{array}{l}\text { PARAMETER } \\
\text { VALUE }\end{array}$ & FLAGS & $\begin{array}{l}\text { DETECTIOH } \\
\text { LIMIT }\end{array}$ & $\begin{array}{l}\text { PARAMETER } \\
\text { UNCERTAINTY }\end{array}$ \\
\hline NET GROSS ALPHA (TOTAL) * & $06 / 30 / 93$ & NoOs & $\mathrm{PCI} / \mathrm{L}$ & \multicolumn{3}{|c|}{6.26} & - & - \\
\hline MICKEL & $06 / 01 / 91$ & 0001 & MG/L & $<$ & 0.04 & $\mathbf{L}$ & 0.04 & - \\
\hline NITRATE & $\begin{array}{l}06 / 01 / 91 \\
02 / 23 / 92 \\
08 / 12 / 92 \\
11 / 21 / 92 \\
02 / 19 / 93\end{array}$ & $\begin{array}{l}0001 \\
0001 \\
0001 \\
0001 \\
0001\end{array}$ & MG/L & & $\begin{array}{l}21.6 \\
20.1 \\
19 \\
7.1 \\
20\end{array}$ & $L$ & $\begin{array}{l}1 . \\
1.0 \\
0.075 \\
0.044 \\
1\end{array}$ & $\begin{array}{l}- \\
- \\
-\end{array}$ \\
\hline MITRATE (TOTAL) & $\begin{array}{l}07 / 01 / 93 \\
12 / 08 / 94\end{array}$ & $\begin{array}{l}\text { N001 } \\
\text { NOOI }\end{array}$ & $M G / L$ & & $\begin{array}{l}19 \\
21\end{array}$ & HJN & $\begin{array}{l}1 \\
1\end{array}$ & - \\
\hline HITRITE AND HITRATE & $06 / 01 / 91$ & 0001 & MG/L & & 4.87 & \llcorner & 0.05 & - \\
\hline PH & $\begin{array}{l}06 / 01 / 91 \\
02 / 23 / 92 \\
08 / 12 / 92 \\
11 / 21 / 92 \\
02 / 19 / 93 \\
07 / 01 / 93 \\
12 / 08 / 94\end{array}$ & $\begin{array}{l}0001 \\
0001 \\
0001 \\
0001 \\
0001 \\
\text { N001 } \\
\text { N001 }\end{array}$ & SU & & $\begin{array}{l}7.99 \\
7.93 \\
8.01 \\
8.06 \\
7.93 \\
8.11 \\
7.97\end{array}$ & $L$ & $\begin{array}{l}- \\
- \\
- \\
- \\
0.10\end{array}$ & $\begin{array}{l}- \\
: \\
: \\
-\end{array}$ \\
\hline PHOSPHATE (TOTAL) & $07 / 01 / 93$ & NoO1 & MG/L & & 0.2 & & 0.1 & - \\
\hline POTASSIUM & $\begin{array}{l}06 / 01 / 91 \\
02 / 23 / 92 \\
08 / 12 / 92 \\
02 / 19 / 93 \\
07 / 01 / 93 \\
12 / 08 / 94\end{array}$ & $\begin{array}{l}0001 \\
0001 \\
0009 \\
0009 \\
0001 \\
0001\end{array}$ & MG/L & & $\begin{array}{l}2.8 \\
3.09 \\
2.0 \\
2.7 \\
2.8 \\
2.7\end{array}$ & $\begin{array}{l}\mathbf{L} \\
\star\end{array}$ & $\begin{array}{l}0.01 \\
0.01 \\
0.575 \\
0.1 \\
0.1 \\
0.1\end{array}$ & $\begin{array}{l}- \\
- \\
- \\
-\end{array}$ \\
\hline POTASSILN (TOTAL) & $07 / 01 / 93$ & Hoo & MG/L & & 2.6 & & 0.1 & - \\
\hline RADIUA-226 & $\begin{array}{l}06 / 01 / 91 \\
02 / 23 / 92 \\
08 / 12 / 92 \\
11 / 21 / 92\end{array}$ & $\begin{array}{l}0001 \\
0001 \\
0001 \\
0001\end{array}$ & $\mathrm{PCI} / \mathrm{L}$ & . & $\begin{array}{l}0.2 \\
0.0 \\
0.12 \\
0.3\end{array}$ & $L$ & $\begin{array}{l}1 . \\
1.0 \\
0.41 \\
0.3\end{array}$ & $\begin{array}{l}0.3 \\
0.2 \\
0.05 \\
0.2\end{array}$ \\
\hline RADIUN-226 (TOTAL) & $11 / 21 / 92$ & NoO1 & $\mathrm{PCI} / \mathrm{L}$ & & 9.2 & & 0.3 & 0.4 \\
\hline RADIUH-226 + RADIUH-228 & $\begin{array}{l}06 / 01 / 91 \\
02 / 23 / 92 \\
08 / 12 / 92 \\
11 / 21 / 92\end{array}$ & $\begin{array}{l}0001 \\
0001 \\
0001 \\
0001\end{array}$ & $\mathrm{PCI} / \mathrm{L}$ & & $\begin{array}{l}0.60 \\
1.30 \\
1.12 \\
2.30\end{array}$ & & : & : \\
\hline RADIUA-226 + RADIUN-228 (TOTAL) & $11 / 21 / 92$ & N001 & PCI/L & & 4.10 & & - & - \\
\hline RADIUH-228 & $\begin{array}{l}06 / 01 / 91 \\
02 / 23 / 92 \\
08 / 12 / 92 \\
11 / 21 / 92\end{array}$ & $\begin{array}{l}0001 \\
0001 \\
0001 \\
0009\end{array}$ & $\mathrm{PCI} / \mathrm{L}$ & & $\begin{array}{l}0.4 \\
1.3 \\
1.0 \\
2.0\end{array}$ & JL & $\begin{array}{l}1 . \\
1.0 \\
1.8 \\
2.5\end{array}$ & $\begin{array}{l}1.6 \\
1.0 \\
1.0 \\
1.7\end{array}$ \\
\hline
\end{tabular}

* HET GROSS ALPHA (TOTAL) (TOTAL GROSS ALPHA - TOTAL URAHIUN)

PARAMETER VALUE IMDICATOR (PVI): < - LESS THAN DETECTION LIHIT

SAMPLE ID COOES:

OTHER PARAMETER VALUE FLAGS:

OOO1 - FILTERED SAMPLE (.45 MICRONS)

- DUPLICATE AMALYSIS nOT HITHIN COHTROL LIHITS

NOO1 - UNFILTERED SAMPLE

H - HOLD TIME EXPIRED, VALUE SUSPECT

- J - EsTIMKTED VALUE

L - LESS THAH THREE BORE VOLUHES REHOVED BEFORE SAMPLING

N - SPIKE SAMPLE RECOVERY NOT UITHIN CONTROL LIMITS 
GROHNDWATER OUALITY DATA BY LOCATION

SOMP ReV. $O$ Data

SITE: MONO1 MOHUMENT VALLEY

LOCATION: 0652

NORTH COORDIHATE: 62581.5 FT

EAST COORDINATE: $\quad 93759.3$ FT

$04 / 21 / 88$ TO $12 / 13 / 94$

REPORT OATE: $06 / 20 / 95$

FORMATION OF COMPLETION: ALLUVILM (AL)

HYDRAULIC FLON RELATIONSHIP: DONH GRADIENT (D)

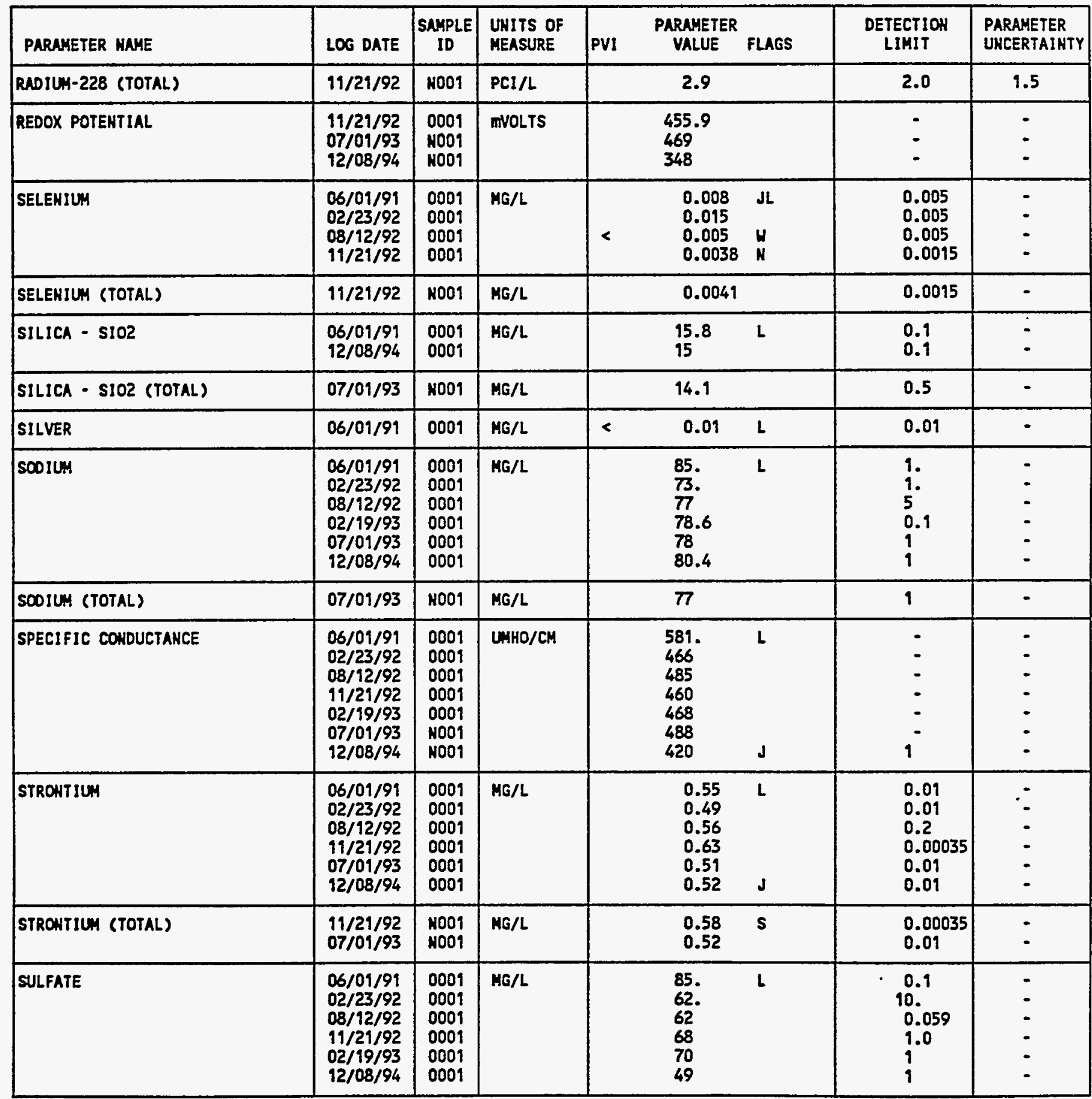

PARAMETER VALUE INDICATOR (PVI): < - LESS THAN DETECTION LIMIT

SAMPLE ID COOES

OTHER PARAMETER VALUE FLAGS:

O001 - FILTERED SAMPLE (.45 MICRONS)

$J$ - ESTIMATED VALUE

NOO1 - UMFILTERED SAMPLE

L - LESS THAN THREE BORE VOLUHES REMOVED BEFORE SMMPLING

N - SPIKE SAYPLE RECOVERY NOT WITHIN CONTROL LIMITS

S - REPORTED VALUE DETERMIMED USING METHOO OF STD NDDITION (MSA)

W - POST-DIGEST SPIKE OUT OF CHTR LIM WHILE SAMP ABS < 50\% SPIKE 
GROUHDHATER QUALITY DATA BY LOCATION

SOWP ReV. 0 Data

SITE: MOHOI HONUNENT VALLEY

LOCATION: 0652

MORTK COORDINATE: 62581.5 FT

EAST COORDINATE: 93759.3 FT

04/21/88 TO $12 / 13 / 94$

REPORT DATE: $06 / 20 / 95$

FORMATION OF COMPLETION: ALLUVIUH (AL)

HYDRAULIC FLOW RELATIONSHIP: DOWN GRADIENT (D)

\begin{tabular}{|c|c|c|c|c|c|c|c|c|}
\hline PARAMETER MAME & LOG DATE & $\begin{array}{c}\text { SAMPLE } \\
\text { ID }\end{array}$ & $\begin{array}{l}\text { UNITS OF } \\
\text { MEASURE }\end{array}$ & PVI & $\begin{array}{l}\text { PARAMETER } \\
\text { VALUE }\end{array}$ & FLAGS & $\begin{array}{l}\text { DETECTION } \\
\text { LIMIT }\end{array}$ & $\begin{array}{l}\text { PARAMETER } \\
\text { UHCERTAINTY }\end{array}$ \\
\hline SULFATE (TOTAL) & $\begin{array}{l}11 / 21 / 92 \\
07 / 01 / 93\end{array}$ & $\begin{array}{l}\text { NOO1 } \\
\text { nOO1 }\end{array}$ & $M G / L$ & & $\begin{array}{l}67 \\
64\end{array}$ & & 9.0 & $\dot{-}$ \\
\hline SULFIDE & $06 / 01 / 91$ & 0001 & MG/L & $<$ & 1. & IJL & 1. & - \\
\hline TEMPERATURE & $\begin{array}{l}06 / 01 / 91 \\
02 / 23 / 92 \\
08 / 12 / 92 \\
11 / 21 / 92 \\
02 / 19 / 93 \\
07 / 01 / 93 \\
12 / 08 / 94\end{array}$ & $\begin{array}{l}0001 \\
0001 \\
0001 \\
0001 \\
0001 \\
\text { No01 } \\
\text { No01 }\end{array}$ & C - DEGREE & & $\begin{array}{l}14.6 \\
15.2 \\
16.7 \\
14.5 \\
15.7 \\
17.7 \\
11.4\end{array}$ & $L$ & $\begin{array}{l}- \\
\therefore \\
\therefore \\
\therefore \\
0.1\end{array}$ & $\begin{array}{l}: \\
\dot{-} \\
\dot{-} \\
\dot{-}\end{array}$ \\
\hline THALLIUM & $06 / 01 / 91$ & 0001 & MG/L & $<$ & 0.01 & $L$ & 0.01 & - \\
\hline THORIUH-230 & $06 / 01 / 91$ & 0001 & $\mathrm{PCI} / \mathrm{L}$ & & 0.7 & $L$ & १. & 0.4 \\
\hline TIK & $06 / 01 / 91$ & 0001 & MG/L & $<$ & 0.01 & IL & 0.01 & - \\
\hline TOTAL DISSOLVED SOLIDS & $\begin{array}{l}06 / 01 / 91 \\
02 / 23 / 92 \\
08 / 12 / 92 \\
02 / 19 / 93 \\
12 / 08 / 94\end{array}$ & $\begin{array}{l}0001 \\
0001 \\
0001 \\
0001 \\
0001\end{array}$ & $M G / L$ & & $\begin{array}{l}344 . \\
346 . \\
380 \\
330 \\
346\end{array}$ & $\begin{array}{l}\mathbf{L} \\
\mathrm{H}\end{array}$ & $\begin{array}{l}10 . \\
10 . \\
42 \\
10 \\
10\end{array}$ & $\begin{array}{l}- \\
- \\
- \\
-\end{array}$ \\
\hline TOTAL DISSOLVED SOLIDS (TOTAL) & $07 / 01 / 93$ & N001 & MG/L & & 330 & & 10 & - \\
\hline TOTAL ORGAMIC CARBOH & $\begin{array}{l}06 / 01 / 91 \\
02 / 23 / 92 \\
08 / 12 / 92\end{array}$ & $\begin{array}{l}0001 \\
0001 \\
0001\end{array}$ & MG/L & $\begin{array}{l}< \\
< \\
<\end{array}$ & $\begin{array}{l}1 . \\
1.0\end{array}$ & $L$ & $\begin{array}{l}1 . \\
1.0\end{array}$ & : \\
\hline URAKIU: & $\begin{array}{l}06 / 01 / 91 \\
02 / 23 / 92 \\
11 / 21 / 92 \\
02 / 19 / 93 \\
07 / 01 / 93 \\
12 / 08 / 94\end{array}$ & $\begin{array}{l}0001 \\
0001 \\
0001 \\
0001 \\
0001 \\
0001\end{array}$ & $M G / L$ & $<$ & $\begin{array}{l}0.001 \\
0.004 \\
0.005 \\
0.005 \\
0.005 \\
0.002\end{array}$ & $L$ & $\begin{array}{l}0.001 \\
0.001 \\
0.001 \\
0.001 \\
0.001 \\
0.001\end{array}$ & $\begin{array}{l}- \\
- \\
-\end{array}$ \\
\hline URAKIUM (TOTAL) & $\begin{array}{l}11 / 21 / 92 \\
06 / 30 / 93\end{array}$ & $\begin{array}{l}\text { No01 } \\
\text { N001 }\end{array}$ & MG/L & & $\begin{array}{l}0.005^{\circ} \\
0.004\end{array}$ & & $\begin{array}{l}0.001 \\
0.001\end{array}$ & $\dot{-}$ \\
\hline VARADIUAS & $\begin{array}{l}06 / 01 / 91 \\
02 / 23 / 92 \\
08 / 12 / 92 \\
11 / 21 / 92 \\
07 / 01 / 93 \\
12 / 08 / 94\end{array}$ & $\begin{array}{l}0001 \\
0001 \\
0001 \\
0001 \\
0001 \\
0001\end{array}$ & $M G / L$ & $<$ & $\begin{array}{l}0.01 \\
0.01 \\
0.05 \\
0.012 \\
0.01 \\
0.01\end{array}$ & $L$ & $\begin{array}{l}0.01 \\
0.01 \\
0.05 \\
0.0019 \\
0.01 \\
0.01\end{array}$ & $\begin{array}{l}: \\
: \\
: \\
-\end{array}$ \\
\hline VRNADIUA (TOTAL) & $\begin{array}{l}11 / 21 / 92 \\
07 / 01 / 93\end{array}$ & $\begin{array}{l}\text { No01 } \\
\text { No01 }\end{array}$ & MG/L & $<$ & $\begin{array}{l}0.013 \\
0.01\end{array}$ & & $\begin{array}{l}0.0019 \\
0.01\end{array}$ & - \\
\hline ZINC & $\begin{array}{l}06 / 01 / 91 \\
02 / 23 / 92 \\
08 / 12 / 92 \\
11 / 21 / 92\end{array}$ & $\begin{array}{l}0001 \\
0001 \\
0001 \\
0001\end{array}$ & $M G / L$ & $\begin{array}{l}< \\
< \\
< \\
<\end{array}$ & $\begin{array}{l}0.005 \\
0.005 \\
0.02 \\
0.0010\end{array}$ & $\mathbf{L}$ & $\begin{array}{l}0.005 \\
0.005 \\
0.02 \\
0.0010\end{array}$ & : \\
\hline
\end{tabular}

PARAMETER VALUE INDICATOR (PVI): < - LESS THAN DETECTION LIMIT

SAMPLE ID COOES:

OTHER PARAMETER VALUE FLAGS:

DOO1 - FILTERED SAMPLE (.45 MICROHS)

H - KOLD TIME EXPIRED, VALUE SUSPECT

NOO1 - UNFILTERED SAMPLE

I - INCREASED DETECTIOA LIMIT DUE TO REQUIRED DILUTION

J - ESTIMATED VALUE

L - LESS THAN THREE BORE VOLUMES REMOVED BEFORE SAMPLING 
GROUNDWATER QUALITY DATA BY LOCATION

SOUP ReV. O Data

SITE: MOHO MONLMENT VALLEY

LOCATION: 0652

HORTH COORDINATE: 62581.5 FT

EAST COORDINATE: 93759.3 FT

04/21/88 TO $12 / 13 / 94$

REPORT DATE: 06/20/95

FORMATION OF COAPLETION: ALLUVIUAN (AL)

HYDRAULIC FLON RELATIONSHIP: DOWN GRADIENT (D)

\begin{tabular}{|c|c|c|c|c|c|c|c|}
\hline PARANETER NAME & LOG DATE & $\begin{array}{c}\text { SNMPLE } \\
\text { ID }\end{array}$ & $\begin{array}{l}\text { UHITS OF } \\
\text { MEASURE }\end{array}$ & PVI & $\begin{array}{l}\text { PARAMETER } \\
\text { VALUE FLAGS }\end{array}$ & $\begin{array}{l}\text { DETECTION } \\
\text { LIMIT }\end{array}$ & $\begin{array}{l}\text { PARAMETER } \\
\text { UHCERTAINTY }\end{array}$ \\
\hline ZINC & $07 / 01 / 93$ & 0009 & $M G / L$ & & 0.023 & 0.005 & - \\
\hline ZINC (TOTAL) & $\begin{array}{l}11 / 21 / 92 \\
07 / 01 / 93\end{array}$ & $\begin{array}{l}\text { N001 } \\
\text { N001 }\end{array}$ & MG/L & & $\begin{array}{l}0.0030 \\
0.024 \mathrm{~J}\end{array}$ & $\begin{array}{l}0.0010 \\
0.005\end{array}$ & $\dot{-}$ \\
\hline
\end{tabular}

PARAMETER VALUE IHDICATOR (PVI): < - LESS THAN DETECTION LIMIT SAMPLE ID COOES:

OTHER PARAMETER VALUE FLAGS:

OOO1 - FILTERED SAMPLE (.45 MICROHS)

OTHER PARAMETER VALUE
$\mathrm{J}$ - ESTIMATED VALUE

HOO1 - UNFILTERED SNMPLE 
GROUNDHATER QUALITY DATA BY LOCATION

SOWP ReV. 0 Data

SITE: MONO 1 MOHUMENT VALLEY

LOCATION: 0653

NORTH COORDINATE: 61249.3 FT

EAST COORDINATE: 89596.3 FT

$04 / 21 / 88$ TO $12 / 13 / 94$

REPORT DATE: 06/20/95

FORHATION OF COMPLETION: ALLUVIUH (AL)

HYDRAULIC FLON RELATIONSHIP: DOWN GRADIENT (D)

\begin{tabular}{|c|c|c|c|c|c|c|c|c|}
\hline PARAMETER MAME & LOG DATE & $\begin{array}{c}\text { SAMPLE } \\
\text { ID }\end{array}$ & $\begin{array}{l}\text { UNITS OF } \\
\text { MEASURE }\end{array}$ & PVI & $\begin{array}{l}\text { PARAMETER } \\
\text { VALUE }\end{array}$ & FLAGS & $\begin{array}{l}\text { DETECTION } \\
\text { LIMIT }\end{array}$ & $\begin{array}{l}\text { PARAMETER } \\
\text { UNCERTAINTY }\end{array}$ \\
\hline ALKALINITY & $\begin{array}{l}04 / 22 / 88 \\
06 / 25 / 89 \\
12 / 01 / 89 \\
06 / 21 / 90 \\
06 / 21 / 90 \\
06 / 21 / 90 \\
06 / 21 / 90 \\
06 / 21 / 90 \\
01 / 29 / 91 \\
06 / 04 / 91 \\
02 / 25 / 92 \\
11 / 99 / 92 \\
02 / 19 / 93 \\
06 / 30 / 93 \\
04 / 24 / 94 \\
12 / 13 / 94\end{array}$ & $\begin{array}{l}0009 \\
0001 \\
0009 \\
0001 \\
0002 \\
0003 \\
0004 \\
0005 \\
0009 \\
0009 \\
0001 \\
0009 \\
0009 \\
H 009 \\
\text { NO09 } \\
\text { NOO9 }\end{array}$ & MG/L CACO3 & & $\begin{array}{l}173 . \\
201 . \\
197 . \\
175 . \\
175 . \\
175 . \\
175 . \\
175 . \\
206 . \\
215 . \\
209 \\
196 \\
209 \\
202 \\
206 \\
214\end{array}$ & $\begin{array}{l}\mathrm{L} \\
\mathrm{JL} \\
\mathrm{JL} \\
\mathrm{JL} \\
\mathrm{JL} \\
\mathrm{J} \\
\mathrm{L}\end{array}$ & $\begin{array}{r}= \\
= \\
= \\
= \\
= \\
= \\
= \\
= \\
10\end{array}$ & $\begin{array}{l}: \\
: \\
: \\
: \\
: \\
: \\
- \\
- \\
-\end{array}$ \\
\hline ALUHINUM & $\begin{array}{l}06 / 25 / 89 \\
12 / 01 / 89 \\
06 / 21 / 90 \\
06 / 21 / 90 \\
06 / 21 / 90 \\
06 / 21 / 90 \\
06 / 21 / 90 \\
09 / 29 / 91 \\
06 / 04 / 91 \\
02 / 25 / 92\end{array}$ & $\begin{array}{l}0001 \\
0001 \\
0009 \\
0002 \\
0003 \\
0004 \\
0005 \\
0001 \\
0001 \\
0001\end{array}$ & $M G / L$ & $\begin{array}{l}< \\
< \\
< \\
< \\
< \\
< \\
< \\
<\end{array}$ & $\begin{array}{l}0.1 \\
0.1 \\
0.1 \\
0.1 \\
0.1 \\
0.1 \\
0.1 \\
0.1 \\
0.05 \\
0.05\end{array}$ & $\begin{array}{l}\text { LR } \\
\text { JL } \\
\text { JL } \\
\mathrm{JL} \\
\mathrm{JL} \\
\mathrm{JL} \\
\mathrm{L}\end{array}$ & $\begin{array}{l}0.1 \\
0.1 \\
0.1 \\
0.1 \\
0.1 \\
0.1 \\
0.1 \\
0.1 \\
0.05 \\
0.05\end{array}$ & $\begin{array}{l}\text { - } \\
- \\
- \\
- \\
- \\
-\end{array}$ \\
\hline Amsonium & $\begin{array}{l}04 / 22 / 88 \\
06 / 25 / 89 \\
12 / 01 / 89 \\
06 / 21 / 90 \\
06 / 21 / 90 \\
06 / 21 / 90 \\
06 / 29 / 90 \\
06 / 21 / 90 \\
01 / 29 / 91 \\
06 / 04 / 91 \\
02 / 25 / 92\end{array}$ & $\begin{array}{l}0001 \\
0001 \\
0001 \\
0001 \\
0002 \\
0003 \\
0004 \\
0005 \\
0001 . \\
0001 \\
0001\end{array}$ & MG/L & $\begin{array}{l}< \\
< \\
< \\
< \\
< \\
< \\
< \\
< \\
< \\
< \\
<\end{array}$ & $\begin{array}{l}0.1 \\
0.1 \\
0.1 \\
0.1 \\
0.1 \\
0.1 \\
0.1 \\
0.1 \\
0.1 \\
0.1 \\
0.1\end{array}$ & $\begin{array}{l}\text { L } \\
\text { HJL } \\
\text { HJL } \\
\text { HJL } \\
\text { HJL } \\
\text { HJL } \\
\text { L }\end{array}$ & $\begin{array}{l}0.1 \\
0.1 \\
0.1 \\
0.1 \\
0.1 \\
0.1 \\
0.1 \\
0.1 \\
0.1 \\
0.1 \\
0.1\end{array}$ & $\begin{array}{l}- \\
- \\
- \\
- \\
- \\
- \\
-\end{array}$ \\
\hline AMONIUN (TOTAL) & $\begin{array}{l}06 / 30 / 93 \\
06 / 30 / 93 \\
04 / 24 / 94 \\
04 / 24 / 94 \\
12 / 13 / 94 \\
12 / 13 / 94\end{array}$ & $\begin{array}{l}\text { NOO1 } \\
\text { NO02 } \\
\text { NO01 } \\
\text { NO02 } \\
\text { NO01 } \\
\text { NO02 }\end{array}$ & MG/L & $\begin{array}{l}< \\
< \\
< \\
<\end{array}$ & $\begin{array}{l}0.1 \\
0.1 \\
0.1 \\
0.1 \\
0.15 \\
0.12\end{array}$ & & $\begin{array}{l}0.1 \\
0.1 \\
0.1 \\
0.1 \\
0.1 \\
0.1\end{array}$ & $\begin{array}{l}- \\
- \\
- \\
-\end{array}$ \\
\hline ANTIMONY & $\begin{array}{l}06 / 25 / 89 \\
12 / 01 / 89 \\
06 / 21 / 90 \\
06 / 21 / 90 \\
06 / 21 / 90 \\
06 / 21 / 90\end{array}$ & $\begin{array}{l}0001 \\
0001 \\
0009 \\
0002 \\
0003 \\
0004\end{array}$ & MG/L & $\begin{array}{l}< \\
< \\
< \\
< \\
<\end{array}$ & $\begin{array}{l}0.003 \\
0.003 \\
0.003 \\
0.003 \\
0.003 \\
0.003\end{array}$ & $\begin{array}{l}\mathrm{JL} \\
\mathrm{JL} \\
\mathrm{JL} \\
\mathrm{JL} \\
\mathrm{JL}\end{array}$ & $\begin{array}{l}0.003 \\
0.003 \\
0.003 \\
0.003 \\
0.003 \\
0.003\end{array}$ & $\begin{array}{l}- \\
- \\
- \\
-\end{array}$ \\
\hline
\end{tabular}

PARAMETER VALUE INDICATOR (PVI): < - LESS THAN DETECTION LIMIT

SAMPE ID COOES:

OOO1 - FILTERED SAMPLE (.45 MICRONS)

OTHER PARAMETER VALUE FLAGS:

H - HOLD TIME EXPIRED, VALUE SUSPECT

0002 - FILTERED REPLICATE SAMPLE (.45 MICRONS)

0003 - FILTERED REPLICATE SAMPLE (.45 MICRONS)

0004 - FILTERED REPLICATE SMMPLE (.45 MICROHS)

J - ESTIMATED YALUE

L - LESS THAH THREE BORE VOLLMES REMOVED BEFORE SAMPLING

NOO - UNFILTERED SAMPLE

NOO2 - UHFILTERED REPLICATE SAMPLE 
GROUMDWATER QUALITY DATA BY LOCATION

SOWP ReV. 0 Data

SITE: MONO 1 MONUMENT VALLEY

LOCATION: 0653

NORTH COORDIHATE: 61249.3 FT

EAST COORDIMATE: 89596.3 FT

$04 / 21 / 88$ TO $12 / 13 / 94$

REPORT DATE: $06 / 20 / 95$

FORMATION OF COMPLETIOH: ALLUVIUM (AL)

HYDRAULIC FLON RELATIOHSHIP: DOWN GRADIENT (D)

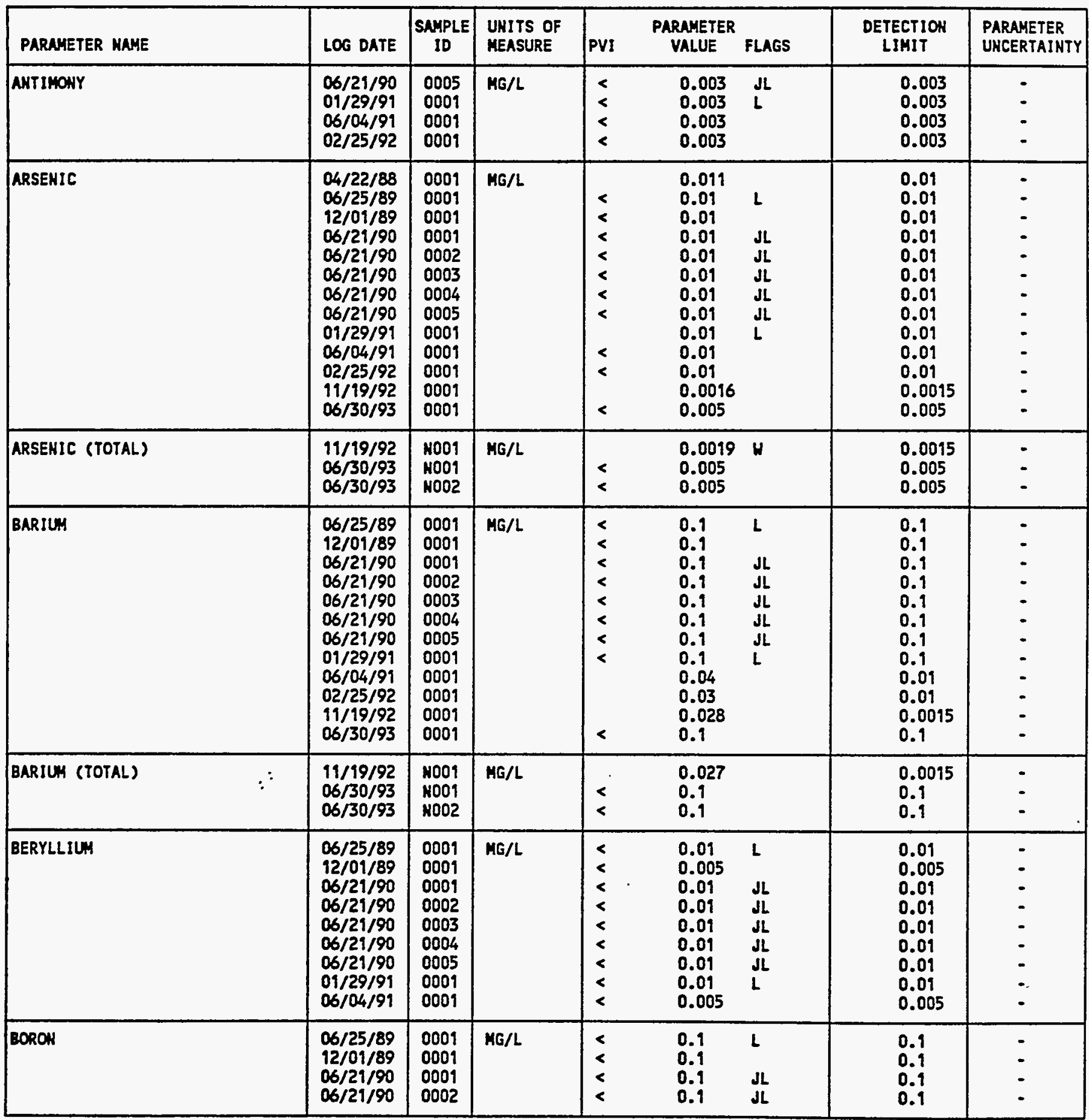

PARAMETER VALUE ILOICATOR (PVI): < - LESS THAN DETECTION LIMIT

OTHER PARAMETER VALUE FLAGS:

J - ESTIMATED VALUE

L - LESS THAH THREE BORE VOLUMES REMOVED BEFORE SAMPLIHG

$W$ - POST-DIGEST SPIKE OUT OF CHTR LIM WHILE SAMP ABS < 50\% SPIKE
SAMPLE ID COOES:

OOO1 - FILTERED SAMPLE (.45 MICRONS)

0002 - FILTERED REPLICATE SAMPLE (.45 MICRONS)

0003 - FILTERED REPLICATE SNMPLE (.45 MICRONS)

0004 - FILTERED REPLICATE SNIPLE (.45 MICRONS)

NOO1 - UNFILTERED SNMLE

NOO2 - UNFILTERED REPLICATE SAMPLE 
GROUNDHATER QUALITY DATA BY LOCATION

SOWP ReV. O Data

SITE: MONOI HONUMENT VALLEY

LOCATION: 0653

NORTH COORDIMATE: 61249.3 FT

EAST COORDIHATE: 89596.3 FT

$04 / 21 / 88$ TO $12 / 13 / 94$

REPORT DATE: 06/20/95

FORHATION OF COHPLETION: ALLUVIUM (AL)

HYDRAULIC FLON RELATIONSHIP: DOWN GRADIENT (D)

\begin{tabular}{|c|c|c|c|c|c|c|c|c|c|}
\hline PARAMETER NAME & & LOG DATE & $\begin{array}{c}\text { SAMPLE } \\
\text { ID }\end{array}$ & $\begin{array}{l}\text { UNITS OF } \\
\text { MEASURE }\end{array}$ & PVI & $\begin{array}{l}\text { PARAMETER } \\
\text { VALUE }\end{array}$ & FLAGS & $\begin{array}{l}\text { DETECTION } \\
\text { LIMIT }\end{array}$ & $\begin{array}{l}\text { PARAMETER } \\
\text { UHCERTAINTY }\end{array}$ \\
\hline BORON & & $\begin{array}{l}06 / 21 / 90 \\
06 / 21 / 90 \\
06 / 21 / 90 \\
01 / 29 / 91 \\
06 / 04 / 91\end{array}$ & $\begin{array}{l}0003 \\
0004 \\
0005 \\
0001 \\
0001\end{array}$ & $M G / L$ & $\begin{array}{l}< \\
< \\
< \\
<\end{array}$ & $\begin{array}{l}0.1 \\
0.1 \\
0.1 \\
0.1 \\
0.05\end{array}$ & $\begin{array}{l}J L \\
J L \\
J L \\
L\end{array}$ & $\begin{array}{l}0.1 \\
0.1 \\
0.1 \\
0.1 \\
0.05\end{array}$ & $\begin{array}{l}- \\
- \\
- \\
-\end{array}$ \\
\hline BROAIDE & & $\begin{array}{l}01 / 29 / 91 \\
06 / 04 / 91\end{array}$ & $\begin{array}{l}0001 \\
0001\end{array}$ & MG/L & $<$ & $\begin{array}{l}0.1 \\
0.2\end{array}$ & $L$ & $\begin{array}{l}0.1 \\
0.9\end{array}$ & - \\
\hline CADMIUM & $\ldots-$ & $\begin{array}{l}04 / 22 / 88 \\
06 / 25 / 89 \\
12 / 01 / 89 \\
06 / 21 / 90 \\
06 / 21 / 90 \\
06 / 21 / 90 \\
06 / 21 / 90 \\
06 / 21 / 90 \\
01 / 29 / 91 \\
06 / 04 / 91 \\
02 / 25 / 92 \\
11 / 19 / 92\end{array}$ & $\begin{array}{l}0001 \\
0001 \\
0001 \\
0001 \\
0002 \\
0003 \\
0004 \\
0005 \\
0001 \\
0001 \\
0001 \\
0001\end{array}$ & $M G / L$ & $\begin{array}{l}< \\
< \\
< \\
< \\
< \\
< \\
< \\
< \\
< \\
<\end{array}$ & $\begin{array}{l}0.003 \\
0.001 \\
0.001 \\
0.001 \\
0.001 \\
0.001 \\
0.001 \\
0.001 \\
0.001 \\
0.001 \\
0.001 \\
0.00013\end{array}$ & $\begin{array}{l}L \\
J L \\
J L \\
J L \\
J L \\
J L \\
L \\
H\end{array}$ & $\begin{array}{l}0.001 \\
0.001 \\
0.001 \\
0.001 \\
0.001 \\
0.001 \\
0.001 \\
0.001 \\
0.001 \\
0.001 \\
0.001 \\
0.00013\end{array}$ & $\begin{array}{l}- \\
- \\
- \\
- \\
- \\
- \\
- \\
-\end{array}$ \\
\hline CADMIUM (TOTAL) & & $11 / 19 / 92$ & NoOI & $M G / L$ & $<$ & 0.00013 & $\mathrm{H}$ & 0.00013 & - \\
\hline CALCIUM & & $\begin{array}{l}04 / 22 / 88 \\
06 / 25 / 89 \\
12 / 01 / 89 \\
06 / 21 / 90 \\
06 / 21 / 90 \\
06 / 21 / 90 \\
06 / 21 / 90 \\
06 / 21 / 90 \\
01 / 29 / 91 \\
06 / 04 / 91 \\
02 / 25 / 92 \\
02 / 19 / 93 \\
06 / 30 / 93 \\
04 / 24 / 94 \\
04 / 24 / 94 \\
12 / 13 / 94 \\
12 / 13 / 94\end{array}$ & $\begin{array}{l}0001 \\
0001 \\
0001 \\
0001 \\
0002 \\
0003 \\
0004 \\
0005 \\
0001 \\
0001 \\
0001 \\
0001 \\
0001 \\
0001 \\
0002 \\
0001 \\
0002\end{array}$ & $M G / L$ & & $\begin{array}{l}151 . \\
156 . \\
202 . \\
194 . \\
190 . \\
188 . \\
193 . \\
200 . \\
200 . \\
246 . \\
251 . \\
247 \\
252 \\
261 \\
256 \\
276 \\
271\end{array}$ & $\begin{array}{l}L \\
J L \\
J L \\
J L \\
J L \\
J L \\
L\end{array}$ & $\begin{array}{l}0.01 \\
0.01 \\
0.01 \\
0.01 \\
0.01 \\
0.01 \\
0.01 \\
0.01 \\
0.01 \\
0.5 \\
0.5 \\
0.5 \\
0.5 \\
0.5 \\
0.5 \\
0.5 \\
0.5\end{array}$ & $\begin{array}{l}- \\
: \\
: \\
: \\
: \\
: \\
- \\
- \\
: \\
- \\
-\end{array}$ \\
\hline CALCIUA (TOTAL) & & $\begin{array}{l}06 / 30 / 93 \\
06 / 30 / 93 \\
04 / 24 / 94 \\
04 / 24 / 94\end{array}$ & $\begin{array}{l}\text { NOO1 } \\
\text { HOO2 } \\
\text { NOO1 } \\
\text { NOO2 }\end{array}$ & $M G / L$ & & $\begin{array}{l}253 \\
248 \\
284 \\
291\end{array}$ & & $\begin{array}{l}0.5 \\
0.5 \\
0.5 \\
0.5\end{array}$ & $\ddot{-}$ \\
\hline CHLORIDE & & $\begin{array}{l}04 / 22 / 88 \\
06 / 25 / 89 \\
12 / 01 / 89 \\
06 / 21 / 90 \\
06 / 21 / 90 \\
06 / 21 / 90 \\
06 / 21 / 90\end{array}$ & $\begin{array}{l}0001 \\
0001 \\
0001 \\
0001 \\
0002 \\
0003 \\
0004\end{array}$ & MG/L & & $\begin{array}{l}32 . \\
36 . \\
33 . \\
38 . \\
37 . \\
37 . \\
37 .\end{array}$ & $\begin{array}{l}L \\
J L \\
J L \\
J L \\
J L\end{array}$ & $\begin{array}{l}1 . \\
1 . \\
1 . \\
1 .\end{array}$ & $\begin{array}{l}- \\
- \\
- \\
- \\
-\end{array}$ \\
\hline
\end{tabular}

PARAMETER VALUE INDICATOR (PVI): < - LESS THAH DETECTION LIMIT

OTHER PARAMETER VALUE FLAGS:

J - ESTIMATED VALUE

- L - LESS THAH THREE BORE VOLUMES REMOVED BEFORE SAMPLIMG

K - SPIKE SAMPLE RECOVERY HOT HITHIN CONTROL LIMITS

$H$ - POST-DIGEST SPIKE WIT OF CNTR LIM WHILE SAMP ABS < $50 \%$ SPIKE
SAMPLE ID COOES:

O001 - FILTERED SAMPLE (.45 MICRONS)

0002 - FILTERED REPLICATE SAMPLE (.45 MICRONS)

0003 - FILTERED REPLICATE SAMPLE (.45 MICRONS)

0004 - FILTERED REPLICATE SAMPLE (.45 MICRONS)

NOO1 - UNFILTERED SAMPLE

NOO2 - UNFILTERED REPLICATE SAMPLE 
GROUHDHATER QUALITY DATA BY LOCATION

SOUP ReV. O Data

SITE: MONO 1 MOHUHENT VALLEY

LOCATIOH: 0653

HORTH COORDIHATE: 61249.3 FT

EAST COORDINATE: 89596.3 FT

04/21/88 TO $12 / 13 / 94$

REPORT DATE: 06/20/95

FORHATIOH OF COHPLETIOH: ALLUVIUM (AL)

HYDRAULIC FLON RELATIOHSHIP: DOWN GRADIEHT (D)

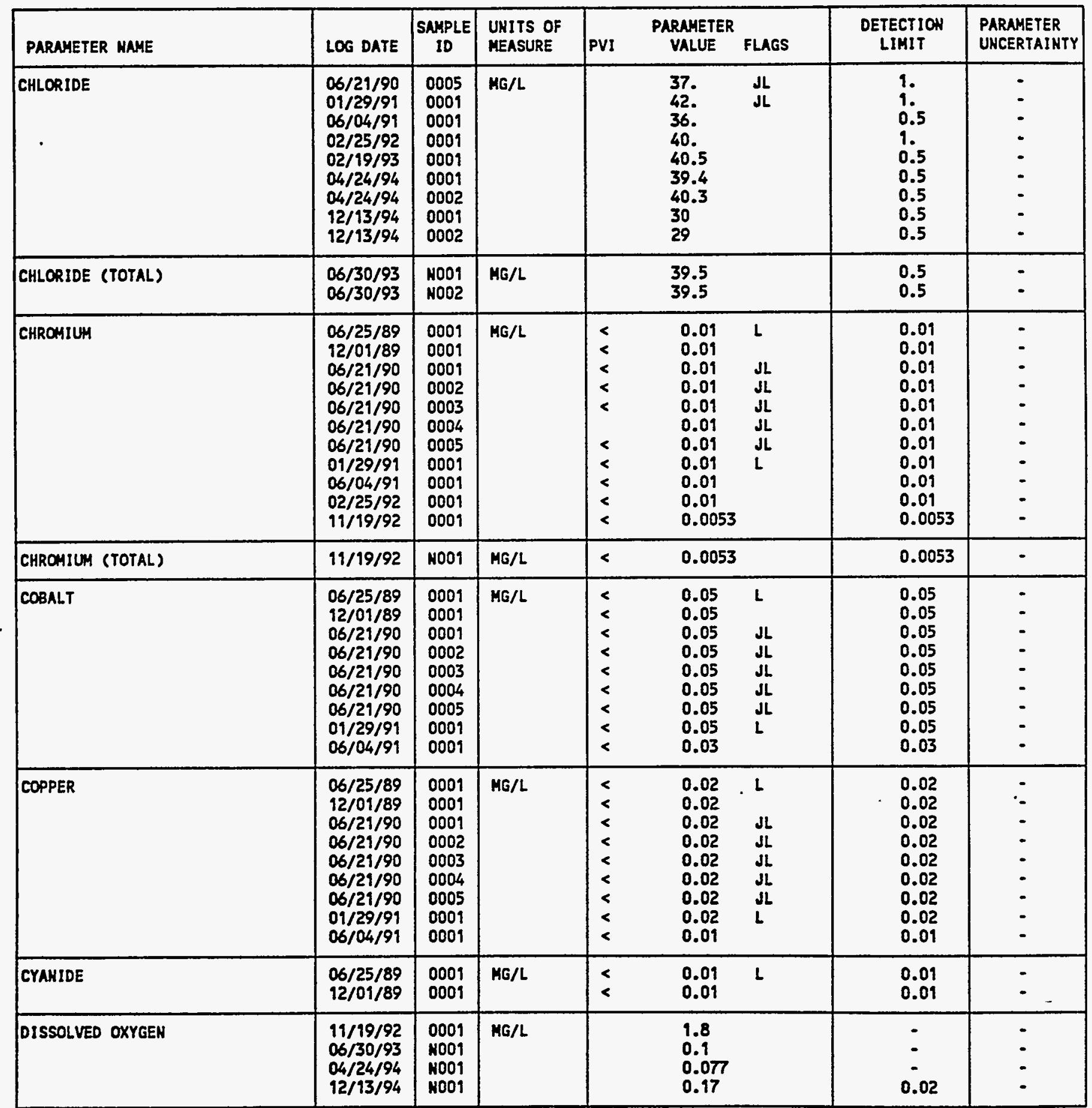

PARAMETER VALUE INDICATOR (PVI): < - LESS THAN DETECTION LIMIT

OTHER PARAMETER VALUE FLAGS:

J. ESTIMATED VALUE

L - LESS THAN THREE BORE VOLUMES REMOVED BEFORE SNMPLIMG
SAMPLE ID COOES:

0001 - FILTEREO SNMPLE (.45 MICRONS)

0002 - FILTERED REPLICATE SAMPLE (.45 MICRONS)

OOO3 - FILTERED REPLICATE SAMPLE (.45 MICRONS)

0004 - FILTERED REPLICATE SAMPLE (.45 MICRONS)

MOOI - UMFILTERED SAMPLE

HOO2 - UNFILTERED REPLICATE SAMPLE 
GROUNDWATER OUALITY DATA BY LOCATION

SOUP ReV. $O$ Data

SITE: MONO1 MONUMENT VALLEY

LOCATION: 0653

MORTH COORDINATE: 61249.3 FT

EAST COORDIHATE: 89596.3 FT

$04 / 21 / 88$ TO $12 / 13 / 94$

REPORT DATE: $06 / 20 / 95$

FORMATION OF COMPLETION: ALLUVIUN (AL)

HYDRAULIC FLON RELATIONSHIP: DOWN GRADIENT (O)

\begin{tabular}{|c|c|c|c|c|c|c|c|c|}
\hline PARANETER NAME & LOG DATE & $\mid \begin{array}{c}\text { SAMPLE } \\
\text { ID }\end{array}$ & $\begin{array}{l}\text { UHITS OF } \\
\text { MEASURE }\end{array}$ & PVI & $\begin{array}{l}\text { PARAMETER } \\
\text { VALUE }\end{array}$ & FLAGS & $\begin{array}{l}\text { DETECTION } \\
\text { LIMIT }\end{array}$ & $\begin{array}{l}\text { PARAMETER } \\
\text { UHCERTAINTY }\end{array}$ \\
\hline FLUORIDE & $\begin{array}{l}06 / 25 / 89 \\
12101 / 89 \\
06 / 21 / 90 \\
06 / 21 / 90 \\
06 / 21 / 90 \\
06 / 21 / 90 \\
06 / 21 / 90 \\
01 / 29 / 91 \\
06 / 04 / 91\end{array}$ & $\begin{array}{l}0001 \\
0001 \\
0001 \\
0002 \\
0003 \\
0004 \\
0005 \\
0001 \\
0001\end{array}$ & MG/L & $<$ & $\begin{array}{l}0.2 \\
0.2 \\
0.2 \\
0.2 \\
0.2 \\
0.2 \\
0.2 \\
0.2 \\
0.1\end{array}$ & $\begin{array}{l}L \\
J L \\
d L \\
J L \\
J L \\
J L \\
L\end{array}$ & $\begin{array}{l}0.1 \\
0.1 \\
0.1 \\
0.1 \\
0.1 \\
0.1 \\
0.1 \\
0.1 \\
0.1\end{array}$ & $\begin{array}{l}- \\
- \\
- \\
- \\
- \\
-\end{array}$ \\
\hline GROSS ALPHA & $\begin{array}{l}04 / 22 / 88 \\
01 / 29 / 91 \\
06 / 04 / 91 \\
02 / 26 / 92 \\
11 / 19 / 92 \\
02 / 19 / 93 \\
06 / 30 / 93\end{array}$ & $\begin{array}{l}0001 \\
0001 \\
0001 \\
0001 \\
0001 \\
0001 \\
0001\end{array}$ & PCI/L & & $\begin{array}{c}26 . \\
4 . \\
7.2 \\
112 . \\
20.9 \\
0 \\
0\end{array}$ & L & $\begin{array}{l}0.2 \\
1 . \\
1 . \\
1.0 \\
16.2 \\
31 \\
14\end{array}$ & $\begin{array}{l}12 . \\
19 . \\
21.5 \\
58.0 \\
13.8 \\
32 \\
22\end{array}$ \\
\hline GROSS ALPHA (TOTAL) & $\begin{array}{l}11 / 19 / 92 \\
06 / 30 / 93 \\
06 / 30 / 93\end{array}$ & $\begin{array}{l}\text { NOO1 } \\
\text { N001 } \\
\text { HOO2 }\end{array}$ & PCI/L & & $\begin{array}{l}0.0 \\
14 \\
18\end{array}$ & N & $\begin{array}{l}38.1 \\
13 \\
14\end{array}$ & $\begin{array}{l}19.7 \\
22 \\
25\end{array}$ \\
\hline GROSS BETA & $\begin{array}{l}04 / 22 / 88 \\
01 / 29 / 91 \\
06 / 04 / 91 \\
02 / 26 / 92 \\
11 / 19 / 92 \\
02 / 19 / 93 \\
06 / 30 / 93\end{array}$ & $\begin{array}{l}0001 \\
0001 \\
0001 \\
0001 \\
0009 \\
0001 \\
0001\end{array}$ & PCI/L & & $\begin{array}{l}9.8 \\
3 . \\
4.0 \\
60.8 \\
0.0 \\
0 \\
0\end{array}$ & $\begin{array}{l}L \\
N\end{array}$ & $\begin{array}{l}1 . \\
0.5 \\
0.5 \\
0.5 \\
28.3 \\
39 \\
15\end{array}$ & $\begin{array}{l}5.8 \\
15 . \\
11.7 \\
31.1 \\
15.2 \\
17 \\
14\end{array}$ \\
\hline GROSS BETA (TOTAL) & $\begin{array}{l}11 / 19 / 92 \\
06 / 30 / 93 \\
06 / 30 / 93\end{array}$ & $\begin{array}{l}\text { NoO1 } \\
\text { No01 } \\
\text { NO02 }\end{array}$ & PCI/L & & $\begin{array}{l}0.4 \\
0 \\
0\end{array}$ & N & $\begin{array}{l}28.2 \\
15 \\
15\end{array}$ & $\begin{array}{l}16.8 \\
13 \\
13\end{array}$ \\
\hline IROH & $\begin{array}{l}06 / 25 / 89 \\
12 / 01 / 89 \\
06 / 21 / 90 \\
06 / 21 / 90 \\
06 / 21 / 90 \\
06 / 21 / 90 \\
06 / 21 / 90 \\
01 / 29 / 91 \\
06 / 04 / 91 \\
02 / 25 / 92 \\
02 / 19 / 93 \\
06 / 30 / 93\end{array}$ & $\begin{array}{l}0001 \\
0001 \\
0001 \\
0002 \\
0003 \\
0004 \\
0005 \\
0001 \\
0001 \\
0001 \\
0001 \\
0001\end{array}$ & $M G / L$ & $\begin{array}{l}< \\
< \\
< \\
<\end{array}$ & $\begin{array}{l}3.50 \\
0.03 \\
0.06 \\
0.04 \\
0.04 \\
0.06 \\
0.07 \\
0.06 \\
0.03 \\
0.03 \\
0.03 \\
0.03\end{array}$ & $\begin{array}{l}L \\
J L \\
J L \\
J L \\
J L \\
J L \\
L\end{array}$ & $\begin{array}{l}0.03 \\
0.03 \\
0.03 \\
0.03 \\
0.03 \\
0.03 \\
0.03 \\
0.03 \\
0.03 \\
0.03 \\
0.03 \\
0.03\end{array}$ & $\begin{array}{l}- \\
: \\
: \\
- \\
- \\
- \\
- \\
-\end{array}$ \\
\hline IRON (TOTAL) & $\begin{array}{l}06 / 30 / 93 \\
06 / 30 / 93\end{array}$ & $\begin{array}{l}\text { NOO1 } \\
\text { N002 }\end{array}$ & MG/L & $<$ & $\begin{array}{l}0.03 \\
0.03\end{array}$ & J & $\begin{array}{l}0.03 \\
0.03\end{array}$ & $\dot{-}$ \\
\hline LEAD & $\begin{array}{l}06 / 25 / 89 \\
12 / 01 / 89 \\
06 / 21 / 90\end{array}$ & $\begin{array}{l}0001 \\
0001 \\
0001\end{array}$ & $M G / L$ & $\begin{array}{l}< \\
<\end{array}$ & $\begin{array}{l}0.09 \\
0.09 \\
0.01\end{array}$ & $\begin{array}{l}L \\
J\end{array}$ & $\begin{array}{l}0.01 \\
0.01 \\
0.01\end{array}$ & : \\
\hline
\end{tabular}

PARAMETER VALUE INDICATOR (PVI): < - LESS THAH DETECTION LIMIT

SAMPLE ID CODES:

0001 - FILTERED SAMPLE (.45 MICROHS)

OTHER PARAKETER VALUE FLAGS:

$J$ - ESTIMLTED VALUE

0002 - FILTERED REPLICATE SAMPLE (.45 MICROHS)

0003 - FILTERED REPLICATE SAMPLE (.45 MICROHS)

L - LESS THAH THREE BCRE VOLUHES REMOVED BEFORE SAMPLING

DOO4 - FILTERED REPLICATE SAMPLE (.45 MICRONS)

H - SPIKE SNMPLE RECOVERY NOT WITHIH CONTROL LIHITS

MOO1 - UMFILTERED SAMPLE

NOO2 - UHFILTERED REPLICATE SNMPLE 
GROUNDHATER QUALITY DATA BY LOCATIOH

SOWP REV. O DBta

SITE: MONO1 HOHUMENT VALLEY

LOCATION: 0653

NORTH COORDIKATE: 61249.3 FT

EAST COORDIKATE: 89596.3 FT

$04 / 21 / 88$ TO $12 / 13 / 94$

REPORT DATE: $06 / 20 / 95$

FORMATION OF COMPLETION: ALLUVIUM (AL)

HYDRAULIC FLON RELATIONSHIP: DONN GRADIENT (D)

\begin{tabular}{|c|c|c|c|c|c|c|c|c|}
\hline PARAMETER MAME & LOG DATE & $\begin{array}{c}\text { SNMPLE } \\
\text { ID }\end{array}$ & $\begin{array}{l}\text { UNITS OF } \\
\text { MEASURE }\end{array}$ & PVI & $\begin{array}{l}\text { PARAMETER } \\
\text { VALUE }\end{array}$ & FLAGS & $\begin{array}{l}\text { DETECTION } \\
\text { LIMIT }\end{array}$ & $\begin{array}{l}\text { PARAMETER } \\
\text { UNCERTAIHTY }\end{array}$ \\
\hline LEAD & $\begin{array}{l}06 / 21 / 90 \\
06 / 21 / 90 \\
06 / 21 / 90 \\
06 / 21 / 90 \\
01 / 29 / 91 \\
06 / 04 / 91 \\
11 / 19 / 92\end{array}$ & $\begin{array}{l}0002 \\
0003 \\
0004 \\
0005 \\
0001 \\
0001 \\
0001\end{array}$ & MG/L & $\begin{array}{l}< \\
< \\
< \\
< \\
< \\
<\end{array}$ & $\begin{array}{l}0.01 \\
0.01 \\
0.01 \\
0.01 \\
0.01 \\
0.005 \\
0.0015\end{array}$ & $\begin{array}{l}\mathbf{J} L \\
\mathbf{J L} \\
\mathbf{J} \mathbf{L} \\
\mathbf{J} \\
\mathbf{L}\end{array}$ & $\begin{array}{l}0.01 \\
0.01 \\
0.01 \\
0.01 \\
0.01 \\
0.005 \\
0.0015\end{array}$ & $\begin{array}{l}- \\
- \\
- \\
- \\
-\end{array}$ \\
\hline LEAD (TOTAL) & $11 / 19 / 92$ & N001 & MG/L & $<$ & 0.0015 & $\boldsymbol{H}$ & 0.0015 & - \\
\hline LEAD-210 & $11 / 99 / 92$ & 0001 & $\mathrm{PCl} / \mathrm{L}$ & & 0.1 & & 2.0 & 1.2 \\
\hline LEAD-210 (TOTAL) & $11 / 19 / 92$ & No01 & $\mathrm{PCI} / \mathrm{L}$ & & 5.9 & & 0.3 & 0.9 \\
\hline MAGKESIUH & $\begin{array}{l}04 / 22 / 88 \\
06 / 25 / 89 \\
12 / 01 / 89 \\
06 / 21 / 90 \\
06 / 21 / 90 \\
06 / 21 / 90 \\
06 / 21 / 90 \\
06 / 21 / 90 \\
01 / 29 / 91 \\
06 / 04 / 91 \\
02 / 25 / 92 \\
02 / 19 / 93 \\
06 / 30 / 93 \\
04 / 24 / 94 \\
04 / 24 / 94 \\
12 / 13 / 94 \\
12 / 13 / 94\end{array}$ & $\begin{array}{l}0001 \\
0001 \\
0001 \\
0001 \\
0002 \\
0003 \\
0004 \\
0005 \\
0001 \\
0001 \\
0001 \\
0001 \\
0001 \\
0001 \\
0002 \\
0001 \\
0002\end{array}$ & $M G / L$ & & $\begin{array}{l}113 . \\
133 . \\
160 . \\
150 . \\
150 . \\
148 . \\
152 . \\
160 . \\
160 . \\
194 . \\
200 . \\
198 \\
195 \\
209 \\
208 \\
218 \\
216\end{array}$ & $\begin{array}{l}L \\
J L \\
J L \\
J L \\
J L \\
J L \\
L\end{array}$ & $\begin{array}{l}0.001 \\
0.001 \\
0.001 \\
0.001 \\
0.001 \\
0.001 \\
0.001 \\
0.001 \\
0.001 \\
0.5 \\
0.5 \\
0.1 \\
0.1 \\
0.1 \\
0.1 \\
0.1 \\
0.1\end{array}$ & $\begin{array}{l}- \\
: \\
: \\
: \\
: \\
: \\
- \\
- \\
- \\
- \\
-\end{array}$ \\
\hline MAGMESIUM (TOTAL) & $\begin{array}{l}06 / 30 / 93 \\
06 / 30 / 93 \\
04 / 24 / 94 \\
04 / 24 / 94\end{array}$ & $\begin{array}{l}\text { NOO1 } \\
\text { NOO2 } \\
\text { NOO1 } \\
\text { NOO2 }\end{array}$ & MG/L & & $\begin{array}{l}205 \\
204 \\
196 \\
204\end{array}$ & & $\begin{array}{l}0.1 \\
0.1 \\
0.1 \\
0.1\end{array}$ & $\begin{array}{l}- \\
- \\
-\end{array}$ \\
\hline
\end{tabular}

PARAMETER VALUE IMDICATOR (PVI): < - LESS THAN DETECTION LIMIT

SAMPLE ID COOES:

OTHER PARAMETER VALUE FLAGS:

OOO1 - FILTERED SAMPLE (.45 MICROHS)

0002 - FILTERED REPLICATE SAMPLE (.45 MICRONS)

J - ESTIMATED VALUE

L - LESS THAK THREE GORE VOLLAES REMOVED BEFORE SMMPLIHG

$H$ - POST-DIGEST SPIKE OUT OF CNTR LIM MHILE SAMP ABS < 50\% SPIKE

0003 - FILTERED REPLICATE SAMPLE (.45 MICRONS)

0004 - FILTERED REPLICATE SAMPLE (.45 MICRONS)

NOO1 - UNFILTERED SAMPLE

NOO2 - UNFILTERED REPLICATE SAMPLE 
GROUHDHATER QUALITY DATA BY LOCATIOH

SOMP ReV. O Data

SITE: HONOY MONLAMEHT VALLEY

LOCATION: 0653

NORTH COORDIHATE: 61249.3 FT

EAST COORDINATE: 89596.3 FT

04/21/88 TO $12 / 13 / 94$

REPORT DATE: $06 / 20 / 95$

FORHATION OF COMPLETION: ALLUVIUN (AL)

HYDRAULIC FLON RELATIOHSHIP: DOWN GRADIENT (D)

\begin{tabular}{|c|c|c|c|c|c|c|c|c|}
\hline PARAMETER NAME & LOG DATE & $\begin{array}{c}\text { SAMPLE } \\
\text { ID }\end{array}$ & $\begin{array}{l}\text { UNITS OF } \\
\text { MEASURE }\end{array}$ & PVI & $\begin{array}{l}\text { PARAMETER } \\
\text { VALUE }\end{array}$ & FLAGS & $\begin{array}{l}\text { DETECTION } \\
\text { LIMIT }\end{array}$ & $\begin{array}{l}\text { PARAMETER } \\
\text { UHCERTAINTY }\end{array}$ \\
\hline MAKGAMESE (TOTAL) & $\begin{array}{l}11 / 99 / 92 \\
06 / 30 / 93 \\
06 / 30 / 93 \\
04 / 24 / 94 \\
04 / 24 / 94\end{array}$ & $\begin{array}{l}\text { No01 } \\
\text { N001 } \\
\text { No02 } \\
\text { No01 } \\
\text { No02 }\end{array}$ & MG/L & $\begin{array}{l}< \\
<\end{array}$ & $\begin{array}{l}0.0015 \\
0.01 \\
0.01 \\
0.03 \\
0.03\end{array}$ & & $\begin{array}{l}0.0015 \\
0.01 \\
0.01 \\
0.01 \\
0.01\end{array}$ & $\begin{array}{l}- \\
- \\
-\end{array}$ \\
\hline MERCURY & $\begin{array}{l}06 / 25 / 89 \\
12 / 01 / 89 \\
06 / 21 / 90 \\
06 / 21 / 90 \\
06 / 21 / 90 \\
06 / 21 / 90 \\
06 / 21 / 90\end{array}$ & $\begin{array}{l}0001 \\
0001 \\
0001 \\
0002 \\
0003 \\
0004 \\
0005\end{array}$ & $M G / L$ & $\begin{array}{l}< \\
< \\
< \\
< \\
< \\
<\end{array}$ & $\begin{array}{l}0.0002 \\
0.0002 \\
0.0002 \\
0.0002 \\
0.0002 \\
0.0002 \\
0.0002\end{array}$ & $\begin{array}{l}L \\
J L \\
J L \\
J L \\
J L \\
J L\end{array}$ & $\begin{array}{l}0.0002 \\
0.0002 \\
0.0002 \\
0.0002 \\
0.0002 \\
0.0002 \\
0.0002\end{array}$ & $\begin{array}{l}- \\
- \\
- \\
- \\
-\end{array}$ \\
\hline MOLYBDENUM & $\begin{array}{l}04 / 22 / 88 \\
06 / 25 / 89 \\
12 / 01 / 89 \\
06 / 21 / 90 \\
06 / 21 / 90 \\
06 / 21 / 90 \\
06 / 21 / 90 \\
06 / 21 / 90 \\
01 / 29 / 91 \\
06 / 04 / 91 \\
02 / 25 / 92 \\
11 / 19 / 92 \\
06 / 30 / 93 \\
12 / 13 / 94 \\
12 / 13 / 94\end{array}$ & $\begin{array}{l}0001 \\
0001 \\
0001 \\
0001 \\
0002 \\
0003 \\
0004 \\
0005 \\
0001 \\
0001 \\
0001 \\
0001 \\
0001 \\
0001 \\
0002\end{array}$ & $M G / L$ & $\begin{array}{l}< \\
< \\
< \\
< \\
< \\
< \\
< \\
< \\
< \\
< \\
<\end{array}$ & $\begin{array}{l}0.04 \\
0.01 \\
0.01 \\
0.01 \\
0.01 \\
0.01 \\
0.01 \\
\quad 0.01 \\
0.01 \\
0.01 \\
0.03 \\
0.01 \\
0.01 \\
0.07 \\
0.049 \\
0.01\end{array}$ & $\begin{array}{l}\mathbf{L} \\
\mathbf{J} L \\
\mathrm{JL} \\
\mathrm{JL} \\
\mathrm{JL} \\
\mathrm{JL} \\
\mathbf{L} \\
\mathbf{N}\end{array}$ & $\begin{array}{l}0.01 \\
0.01 \\
0.01 \\
0.01 \\
0.01 \\
0.01 \\
0.01 \\
0.01 \\
0.01 \\
0.01 \\
0.01 \\
0.0049 \\
0.01 \\
0.01 \\
0.01\end{array}$ & $\begin{array}{l}- \\
: \\
: \\
: \\
: \\
: \\
- \\
- \\
- \\
-\end{array}$ \\
\hline MOLYBDENUM (TOTAL) & $\begin{array}{l}11 / 19 / 92 \\
06 / 30 / 93 \\
06 / 30 / 93\end{array}$ & $\begin{array}{l}\text { No01 } \\
\text { NOO1 } \\
\text { N002 }\end{array}$ & MG/L & $\begin{array}{l}< \\
<\end{array}$ & $\begin{array}{l}0.0049 \\
0.01 \\
0.01\end{array}$ & H & $\begin{array}{l}0.0049 \\
0.01 \\
0.01\end{array}$ & $\div$ \\
\hline NET GROSS ALPHA $\bullet$ & $\begin{array}{l}04 / 22 / 88 \\
01 / 29 / 91 \\
06 / 04 / 91 \\
02 / 26 / 92 \\
11 / 19 / 92 \\
02 / 19 / 93 \\
06 / 30 / 93\end{array}$ & $\begin{array}{l}0001 \\
0001 \\
0001 . \\
0001 \\
0001 \\
0001 \\
0001\end{array}$ & $P C I / L$ & & $\begin{array}{r}21.06 \\
-2.45 \\
-2.40 \\
105.14 \\
12.67 \\
-8.92 \\
-7.55\end{array}$ & & $\begin{array}{l}- \\
- \\
- \\
- \\
-\end{array}$ & $\begin{array}{l}- \\
- \\
- \\
- \\
-\end{array}$ \\
\hline MET GROSS ALPHA (TOTAL) & $\begin{array}{l}11 / 99 / 92 \\
06 / 30 / 93 \\
06 / 30 / 93\end{array}$ & $\begin{array}{l}\text { NoO1 } \\
\text { NOO1 } \\
\text { NOO2 }\end{array}$ & PCI/L & & $\begin{array}{r}-7.55 \\
7.14 \\
11.14\end{array}$ & & $\begin{array}{l}- \\
-\end{array}$ & $\begin{array}{l}- \\
-\end{array}$ \\
\hline MICKEL & $\begin{array}{l}06 / 25 / 89 \\
12 / 01 / 89 \\
06 / 21 / 90\end{array}$ & $\begin{array}{l}0001 \\
0001 \\
0001\end{array}$ & MG/L & $\begin{array}{l}< \\
<\end{array}$ & $\begin{array}{l}0.04 \\
0.04 \\
0.04\end{array}$ & $\begin{array}{l}L \\
J L\end{array}$ & $\begin{array}{l}0.04 \\
0.04 \\
0.04\end{array}$ & : \\
\hline
\end{tabular}

* MET GROSS ALPHA (GROSS ALPHA - URANIUM) WITH 1 MG URAMIUM = 686 PCI

* NET GROSS ALPHA (TOTAL) (TOTAL GROSS ALPHA - TOTAL URANIUM)

PARAMETER VALUE IMDICATOR (PVI): < - LESS THAN DETECTION LIMIT

OTHER PARAMETER VALUE FLAGS:

J - ESTIMATED VALUE

L - LESS THAN THREE BORE VOLUMES REMOVED BEFORE SNMPLING

N - SPIKE SAMPLE RECOVERY MOT HITHIN CONTROL LIMITS
SAMPLE ID COOES:

0001 - FILTERED SAMPLE (.45 HICROHS)

OOO2 - FILTERED REPLICATE SAMPLE (.45 MICROHS)

ODO3 - FILTERED REPLICATE SNMPLE (.45 MICRONS)

0004 - FILTERED REPLICATE SAMPLE (.45 MICRONS)

Y001 - UNFILTERED SAMPLE

NOO2 - LMFILTERED REPLICATE SAMPLE 
GROUHDWATER QUALITY DATA BY LOCATIOH

SOMP ReV. O Data

SITE: MONO MOHUMENT VALLEY

LOCATION: 0653

HORTH COORDINATE: 61249.3 FT

EAST COORDIHATE: 89596.3 FT

$04 / 21 / 88$ TO $12 / 13 / 94$

REPORT DATE: $06 / 20 / 95$

FORMATION OF COMPLETION: ALLUVIUM (AL)

HYDRAULIC FLON RELATIOHSHIP: DOWN GRADIENT (D)

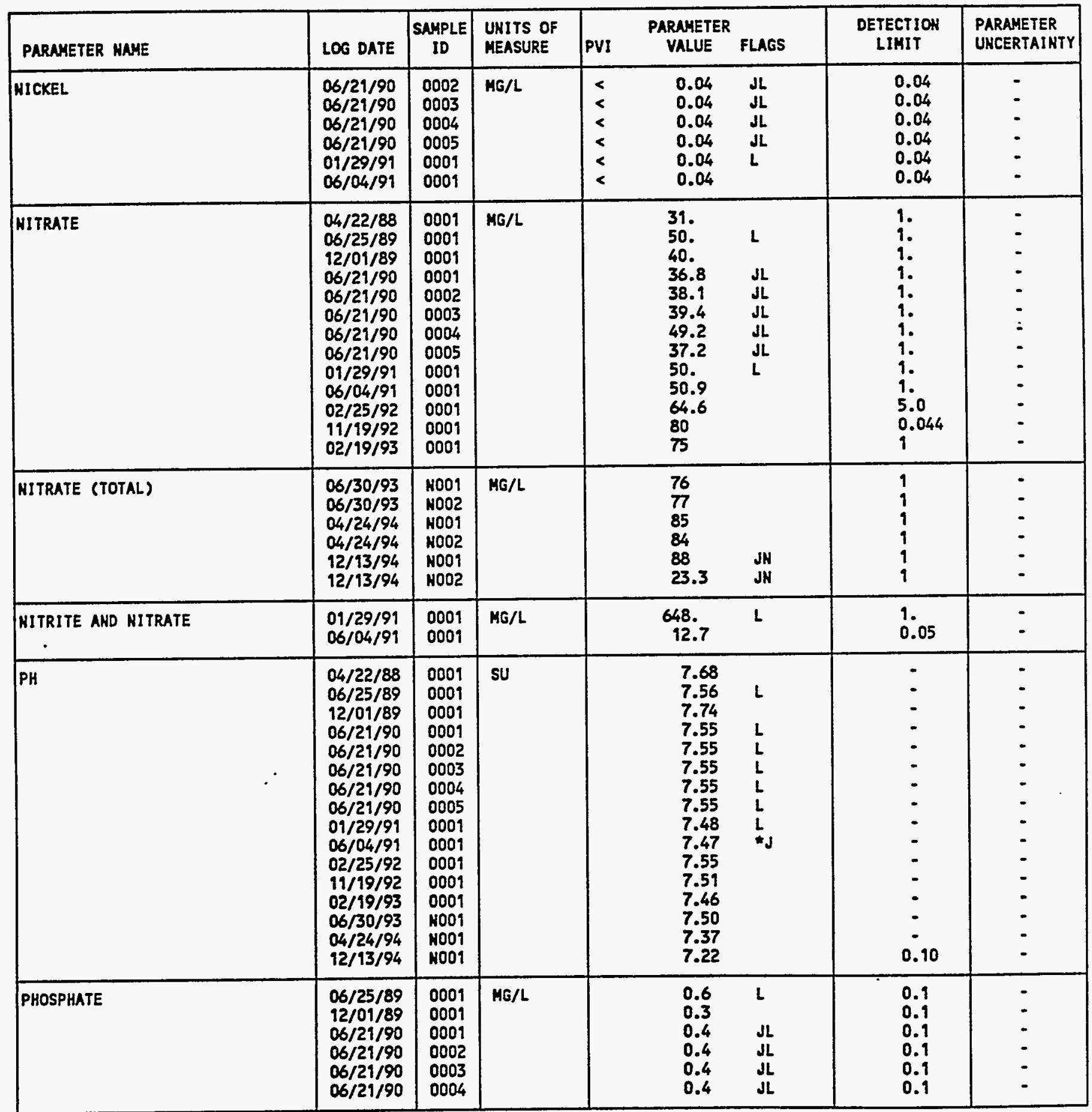

PARAMETER VALUE IMDICATOR (PVI): < - LESS THAN DETECTION LIMIT

SAMPLE ID COOES:

OOO1 - FILTERED SAMPLE (.45 MICRONS)

OTHER PARAMETER VALUE FLAGS:

0002 - FILTERED REPLICATE SNMPLE (.45 MICRONS)

0003 - FILTERED REPLICATE SAMPLE (.45 MICRONS)

0004 - FILTERED REPLICATE SAMPLE (.45 MICRONS)

J- DUPLICATE AMALYSIMTED VALUE

L - LESS THAH THREE BORE VOLUHES REMOVED BEFORE SAMPLING

N - SPIKE SAMPLE RECOVERY MOT HITHIN CONTROL LIMITS 
GROUNDWATER QUALITY DATA BY LOCATION

SOUP ReV. 0 Data

SITE: MONO1 MONUMENT VALLEY

LOCATION: 0653

MORTH COORDINATE: 61249.3 FT

EAST COORDINATE: 89596.3 FT

$04 / 21 / 88$ TO $12 / 13 / 94$

REPORT DATE: 06/20/95

FORMATIOH OF COMPLETION: ALLUVIUM (AL)

HYDRAULIC FLON RELATIOMSHIP: DOWN GRADIENT (D)

\begin{tabular}{|c|c|c|c|c|c|c|c|c|}
\hline PARAMETER MAME & LOG DATE & $\begin{array}{c}\text { SAMPLE } \\
\text { ID }\end{array}$ & $\begin{array}{l}\text { UNITS OF } \\
\text { MEASURE }\end{array}$ & PVI & $\begin{array}{l}\text { PARAMETE } \\
\text { VALUE }\end{array}$ & FLAGS & $\begin{array}{l}\text { DETECTIOH } \\
\text { LIMIT }\end{array}$ & $\begin{array}{l}\text { PARAMETER } \\
\text { UHCERTAINTY }\end{array}$ \\
\hline PHOSPHATE & $06 / 21 / 90$ & 0005 & MG/L & & 0.4 & JL & 0.1 & - \\
\hline PHOSPHATE (TOTAL) & $\begin{array}{l}06 / 30 / 93 \\
06 / 30 / 93\end{array}$ & $\begin{array}{l}\text { NOO1 } \\
\text { N002 }\end{array}$ & $M G / L$ & & $\begin{array}{l}0.3 \\
0.3\end{array}$ & & $\begin{array}{l}0.1 \\
0.1\end{array}$ & : \\
\hline POTASSIUY & $\begin{array}{l}04 / 22 / 88 \\
06 / 25 / 89 \\
12 / 01 / 89 \\
06 / 21 / 90 \\
06 / 21 / 90 \\
06 / 21 / 90 \\
06 / 21 / 90 \\
06 / 21 / 90 \\
01 / 29 / 91 \\
06 / 04 / 91 \\
02 / 25 / 92 \\
02 / 99 / 93 \\
06 / 30 / 93 \\
04 / 24 / 94 \\
04 / 24 / 94 \\
12 / 13 / 94 \\
12 / 13 / 94\end{array}$ & $\begin{array}{l}0001 \\
0001 \\
0001 \\
0001 \\
0002 \\
0003 \\
0004 \\
0005 \\
0001 \\
0001 \\
0009 \\
0001 \\
0001 \\
0001 \\
0002 \\
0001 \\
0002\end{array}$ & MG/L & & $\begin{array}{l}3.24 \\
3.6 \\
3.3 \\
4.0 \\
4.0 \\
3.8 \\
4.1 \\
4.4 \\
3.7 \\
4.0 \\
4.19 \\
4.2 \\
4.5 \\
4.6 \\
4.5 \\
4 \\
4.4\end{array}$ & $\begin{array}{l}\mathrm{L} \\
\mathbf{L} \\
\mathrm{JL} \\
\mathrm{JL} \\
\mathrm{JL} \\
\mathrm{JL} \\
\mathrm{JL} \\
\mathrm{L}\end{array}$ & $\begin{array}{l}0.01 \\
0.01 \\
0.01 \\
0.01 \\
0.01 \\
0.01 \\
0.01 \\
0.01 \\
0.01 \\
0.01 \\
0.01 \\
0.1 \\
0.1 \\
0.1 \\
0.1 \\
0.1 \\
0.1\end{array}$ & $\begin{array}{l}- \\
- \\
- \\
- \\
- \\
\vdots \\
- \\
- \\
- \\
- \\
- \\
-\end{array}$ \\
\hline POTASSIUH (TOTAL) & $\begin{array}{l}06 / 30 / 93 \\
06 / 30 / 93 \\
04 / 24 / 94 \\
04 / 24 / 94\end{array}$ & $\begin{array}{l}\text { NoO1 } \\
\text { NOO2 } \\
\text { NOOI } \\
\text { No02 }\end{array}$ & $M G / L$ & & $\begin{array}{l}4.2 \\
4.2 \\
4.5 \\
4.5\end{array}$ & & $\begin{array}{l}0.1 \\
0.1 \\
0.1 \\
0.1\end{array}$ & $\begin{array}{l}- \\
\dot{-} \\
\dot{-}\end{array}$ \\
\hline $\begin{array}{l}\text { RADIUH-226 } \\
t \\
\end{array}$ & $\begin{array}{l}06 / 25 / 89 \\
12 / 01 / 89 \\
06 / 21 / 90 \\
06 / 21 / 90 \\
06 / 21 / 90 \\
06 / 21 / 90 \\
06 / 21 / 90 \\
01 / 29 / 91 \\
06 / 04 / 91 \\
02 / 26 / 92 \\
11 / 19 / 92\end{array}$ & $\begin{array}{l}0001 \\
0001 \\
0001 \\
0002 \\
0003 \\
0004 \\
0005 \\
0001 \\
0001 \\
0001 \\
0001\end{array}$ & PCI/L & & $\begin{array}{l}0.1 \\
0.2 \\
0.2 \\
0.1 \\
0.2 \\
0.3 \\
0.1 \\
0.4 \\
0.5 \\
0.0 \\
0.4\end{array}$ & $\begin{array}{l}L \\
\mathrm{JL} \\
\mathrm{JL} \\
\mathrm{JL} \\
\mathrm{JL} \\
\mathrm{JL} \\
\mathrm{L}\end{array}$ & $\begin{array}{l}1 . \\
1 . \\
1 . \\
1 . \\
1 . \\
1 . \\
1 . \\
1.0 \\
0.3\end{array}$ & $\begin{array}{l}0.2 \\
0.2 \\
0.2 \\
0.1 \\
0.2 \\
0.2 \\
0.2 \\
0.7 \\
0.4 \\
0.3 \\
0.3\end{array}$ \\
\hline RADIUN-226 (TOTAL) & $11 / 19 / 92$ & NOOI & $\mathrm{PCI} / \mathrm{L}$ & & 0.1 & & 0.3 & 0.2 \\
\hline RADIUA-226 + RADIUH-228 & $\begin{array}{l}06 / 25 / 89 \\
12 / 01 / 89 \\
06 / 21 / 90 \\
06 / 21 / 90 \\
06 / 21 / 90 \\
06 / 21 / 90 \\
06 / 21 / 90 \\
01 / 29 / 91 \\
06 / 04 / 91 \\
02 / 26 / 92 \\
11 / 19 / 92\end{array}$ & $\begin{array}{l}0001 \\
0001 \\
0001 \\
0002 \\
0003 \\
0004 \\
0005 \\
0001 \\
0001 \\
0001 \\
0001\end{array}$ & PCI/L & & $\begin{array}{l}0.10 \\
0.60 \\
1.00 \\
1.20 \\
1.10 \\
1.00 \\
1.40 \\
0.70 \\
6.10 \\
2.40 \\
3.00\end{array}$ & & $\begin{array}{l}- \\
- \\
- \\
- \\
- \\
- \\
-\end{array}$ & $\begin{array}{l}- \\
- \\
- \\
- \\
- \\
- \\
-\end{array}$ \\
\hline RADIUH-226 + RADIUH-228 (TOTAL) & $11 / 19 / 92$ & м001 & PCI/L & & 4.60 & & - & - \\
\hline
\end{tabular}

PARAMETER VALUE IHDICATOR (PVI): < - LESS THAN DETECTION LIMIT

SAMPLE ID COOES:

OTHER PARUYETER VALUE FLAGS:

J. ESTIMUTED VALUE

- L - LESS THAM THREE BORE VOLUMES REMOVED BEFORE SAMPLING

0001 - FILTERED SNHPLE (.45 MICRONS)

0002 - FILTERED REPLICATE SAMPLE (.45 MICRONS)

0003 - FILTERED REPLICATE SAMPLE (.45 MICRONS)

0004 - FILTERED REPLICATE SAMPLE (.45 MICRONS)

NOO1 - UNFILTERED SAMPLE

HOO2 - UNFILTERED REPLICATE SAMPLE 
GROUHDWATER QUALITY OATA BY LOCATION

SOUP ReV. O Data

SITE: MONO MONUMENT VALLEY

LOCATION: 0653

MORTH COORDINATE: 61249.3 FT

EAST COORDIHATE: 89596.3 FT

$04 / 21 / 88$ TO $12 / 13 / 94$

REPORT DATE: O6/20/95

FORMATION OF COMPLETIOH: ALLUVIUM (AL)

HYDRAULIC FLOH RELATIONSHIP: DOWN GRADIENT (D)

\begin{tabular}{|c|c|c|c|c|c|c|c|c|}
\hline PARAMETER HAHE & LDG DATE & $\begin{array}{c}\text { SAMPLE } \\
\text { ID }\end{array}$ & $\begin{array}{l}\text { UNITS OF } \\
\text { MEASUIRE }\end{array}$ & PVI & $\begin{array}{l}\text { PARAMETER } \\
\text { VALUE }\end{array}$ & FLAGS & $\begin{array}{l}\text { DETECTION } \\
\text { LIHIT }\end{array}$ & $\begin{array}{l}\text { PARAMETER } \\
\text { UHCERTAINTY }\end{array}$ \\
\hline RADIUH-228 & $\begin{array}{l}06 / 25 / 89 \\
12 / 01 / 89 \\
06 / 21 / 90 \\
06 / 21 / 90 \\
06 / 21 / 90 \\
06 / 21 / 90 \\
06 / 21 / 90 \\
01 / 29 / 91 \\
06 / 04 / 91 \\
02 / 26 / 92 \\
11 / 19 / 92\end{array}$ & $\begin{array}{l}0001 \\
0001 \\
0001 \\
0002 \\
0003 \\
0004 \\
0005 \\
0001 \\
0001 \\
0001 \\
0001\end{array}$ & PCI/L & & $\begin{array}{l}0.0 \\
0.4 \\
0.8 \\
1.1 \\
0.9 \\
0.7 \\
1.3 \\
0.3 \\
5.6 \\
2.4 \\
2.6\end{array}$ & $\begin{array}{l}L \\
J L \\
J L \\
J L \\
J L \\
J L \\
L \\
J\end{array}$ & $\begin{array}{l}1 . \\
1 . \\
1 . \\
1 . \\
1 . \\
1 . \\
1 . \\
1 . \\
1.0 \\
2.5\end{array}$ & $\begin{array}{l}0.9 \\
1.9 \\
0.9 \\
0.9 \\
0.9 \\
0.9 \\
0.7 \\
0.8 \\
2.2 \\
1.8 \\
1.7\end{array}$ \\
\hline RADIUH-228 (TOTAL) & $11 / 19 / 92$ & NOOI & $\mathrm{PCI} / \mathrm{L}$ & & 4.5 & & 2.0 & 1.6 \\
\hline REDOX POTENTIAL & $\begin{array}{l}11 / 19 / 92 \\
06 / 30 / 93 \\
04 / 24 / 94 \\
12 / 13 / 94\end{array}$ & $\begin{array}{l}\text { No01 } \\
\text { No01 } \\
\text { NO01 } \\
\text { HO01 }\end{array}$ & mVOLTS & & $\begin{array}{l}443.1 \\
227 \\
367 \\
293\end{array}$ & & $:$ & : \\
\hline SELENIUA & $\begin{array}{l}04 / 22 / 88 \\
06 / 25 / 89 \\
12 / 01 / 89 \\
06 / 21 / 90 \\
06 / 21 / 90 \\
06 / 21 / 90 \\
06 / 21 / 90 \\
06 / 21 / 90 \\
01 / 29 / 91 \\
06 / 04 / 91 \\
02 / 25 / 92 \\
11 / 19 / 92\end{array}$ & $\begin{array}{l}0001 \\
0001 \\
0001 \\
0001 \\
0002 \\
0003 \\
0004 \\
0005 \\
0001 \\
0001 \\
0001 \\
0001\end{array}$ & $M G / L$ & $\begin{array}{l}< \\
< \\
< \\
< \\
< \\
< \\
< \\
< \\
<\end{array}$ & $\begin{array}{l}0.007 \\
0.016 \\
0.005 \\
0.005 \\
0.005 \\
0.005 \\
0.005 \\
0.005 \\
0.005 \\
0.005 \\
0.005 \\
0.015\end{array}$ & $\begin{array}{l}L \\
J L \\
J L \\
J L \\
J L \\
J L \\
L \\
J \\
I N\end{array}$ & $\begin{array}{l}0.005 \\
0.005 \\
0.005 \\
0.005 \\
0.005 \\
0.005 \\
0.005 \\
0.005 \\
0.005 \\
0.005 \\
0.005 \\
0.015\end{array}$ & $\begin{array}{l}- \\
- \\
- \\
- \\
- \\
- \\
- \\
- \\
-\end{array}$ \\
\hline SELENIUM (TOTAL) & $11 / 19 / 92$ & NOOI & MG/L & $<$ & \multicolumn{2}{|l|}{0.0015} & 0.0015 & - \\
\hline SILICA - SIOZ & $\begin{array}{l}06 / 25 / 89 \\
12 / 01 / 89 \\
06 / 21 / 90 \\
06 / 21 / 90 \\
06 / 21 / 90 \\
06 / 21 / 90 \\
06 / 21 / 90 \\
01 / 29 / 91 \\
06 / 04 / 91 \\
12 / 13 / 94 \\
12 / 13 / 94\end{array}$ & $\begin{array}{l}0001 \\
0001 \\
0001 \\
0002 \\
0003 \\
0004 \\
0005 \\
0001 \\
0001 \\
0001 \\
0002\end{array}$ & MG/L & • & $\begin{array}{l}15 . \\
15.9 \\
15 . \\
15 . \\
15 . \\
16 . \\
16 . \\
14 . \\
15.8 \\
16.1 \\
16.3\end{array}$ & $\begin{array}{l}\mathrm{L} \\
\mathrm{JL} \\
\mathrm{JL} \\
\mathrm{JL} \\
\mathrm{JL} \\
\mathrm{JL} \\
\mathbf{L}\end{array}$ & $\begin{array}{l}2 . \\
2 . \\
2 . \\
2 . \\
2 . \\
2 . \\
2 . \\
2 . \\
0.1 \\
0.1 \\
0.1\end{array}$ & $\begin{array}{l}- \\
- \\
\therefore \\
- \\
- \\
- \\
-\end{array}$ \\
\hline SILICA - SIO2 (TOTAL) & $\begin{array}{l}06 / 30 / 93 \\
06 / 30 / 93\end{array}$ & $\begin{array}{l}\text { NOOI } \\
\text { N002 }\end{array}$ & $M G / L$ & & $\begin{array}{l}15.6 \\
15.4\end{array}$ & $\begin{array}{l}H \\
H\end{array}$ & $\begin{array}{l}0.5 \\
0.5\end{array}$ & 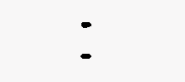 \\
\hline SILVER & $\begin{array}{l}06 / 25 / 89 \\
12 / 01 / 89 \\
06 / 21 / 90\end{array}$ & $\begin{array}{l}0001 \\
0001 \\
0001\end{array}$ & $M G / L$ & $\begin{array}{l}< \\
< \\
<\end{array}$ & $\begin{array}{l}0.01 \\
0.01 \\
0.01\end{array}$ & $\begin{array}{l}\mathrm{L} \\
\mathrm{JL}\end{array}$ & $\begin{array}{l}0.01 \\
0.01 \\
0.01\end{array}$ & 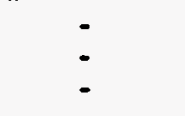 \\
\hline
\end{tabular}

PARAMETER VALUE IHDICATOR (PVI): < - LESS tHAN DETECTION LIMIT

\section{OTHER PARAMETER VALUE FLAGS:}

H - HOLD TIME EXPIRED, VALUE SUSPECT

I - IMCREASED DETECTIÓn LIMIT DUE TO REQUIRED DILUTION

J - ESTIMATED VALUE

L - LESS THAN THREE BORE VOLUAES REHOVED BEFORE SAMPLING

- $N$ - SPIKE SAMPLE RECOVERY NOT WITHIN CONTROL LIMITS
SAMPLE ID COOES:

0001 - FILTERED SAMPLE (.45 MICRONS)

ODO2 - FILTERED REPLICATE SAMPLE (.45 MICRONS)

0003 - FILTERED REPLICATE SAMPLE (.45 MICRONS)

0004 - FILTERED REPLICATE SMMPLE (.45 MICRONS)

MOO1 - UNFILTERED SAMPLE

MOO2 - UMFILTERED REPLICATE SAMPLE 
GROUNDHATER QUALITY DATA BY LOCATION

SONP ReV. O Data

SITE: MOHO1 HOHUMENT VALLEY

LOCATION: 0653

NORTH COORDINATE: 61249.3 FT

EAST COORDINATE: 89596.3 FT

$04 / 21 / 88$ TO $12 / 13 / 94$

REPORT DATE: $06 / 20 / 95$

FORMATION OF COAPLETION: ALLUVIUM (AL)

HYDRAULIC FLON RELATIONSHIP: DONN GRADIENT (D)

\begin{tabular}{|c|c|c|c|c|c|c|c|c|}
\hline PARAMETER NAME & LOS DATE & $\underset{\text { SOMPLE }}{\text { SO }}$ & $\begin{array}{l}\text { UNITS OF } \\
\text { MEASURE }\end{array}$ & PVI & $\begin{array}{l}\text { PARAMETE } \\
\text { VALUE }\end{array}$ & FLAGS & $\begin{array}{l}\text { DETECTION } \\
\text { LIMIT }\end{array}$ & $\begin{array}{l}\text { PARAMETER } \\
\text { UNCERTAINTY }\end{array}$ \\
\hline SILVER & $\begin{array}{l}06 / 29 / 90 \\
06 / 29 / 90 \\
06 / 29 / 90 \\
06 / 21 / 90 \\
01 / 29 / 91 \\
06 / 04 / 91\end{array}$ & $\begin{array}{l}0002 \\
0003 \\
0004 \\
0005 \\
0001 \\
0009\end{array}$ & $M G / L$ & $\begin{array}{l}< \\
< \\
< \\
< \\
<\end{array}$ & $\begin{array}{l}0.01 \\
0.01 \\
0.01 \\
0.01 \\
0.01 \\
0.01\end{array}$ & $\begin{array}{l}d L \\
d L \\
J L \\
d L \\
L\end{array}$ & $\begin{array}{l}0.01 \\
0.01 \\
0.01 \\
0.01 \\
0.01 \\
0.01\end{array}$ & $\begin{array}{l}- \\
- \\
- \\
-\end{array}$ \\
\hline SOOIUM & $\begin{array}{l}04 / 22 / 88 \\
06 / 25 / 89 \\
12 / 01 / 89 \\
06 / 21 / 90 \\
06 / 21 / 90 \\
06 / 21 / 90 \\
06 / 21 / 90 \\
06 / 21 / 90 \\
01 / 29 / 91 \\
06 / 04 / 91 \\
02 / 25 / 92 \\
02 / 19 / 93 \\
06 / 30 / 93 \\
04 / 24 / 94 \\
04 / 24 / 94 \\
12 / 13 / 94 \\
12 / 13 / 94\end{array}$ & $\begin{array}{l}0001 \\
0001 \\
0001 \\
0001 \\
0002 \\
0003 \\
0004 \\
0005 \\
0001 \\
0001 \\
0001 \\
0001 \\
0001 \\
0001 \\
0002 \\
0001 \\
0002\end{array}$ & MG/L & & $\begin{array}{l}210 . \\
211 . \\
242 . \\
231 . \\
234 . \\
231 . \\
238 . \\
249 . \\
231 . \\
267 . \\
256 . \\
248 \\
249 \\
258 \\
261 \\
261 \\
259\end{array}$ & $\begin{array}{l}L \\
J L \\
J L \\
J L \\
J L \\
J L \\
L\end{array}$ & $\begin{array}{l}0.002 \\
0.002 \\
0.002 \\
0.002 \\
0.002 \\
0.002 \\
0.002 \\
0.002 \\
0.002 \\
5 . \\
5 . \\
0.1 \\
1 \\
1 \\
1 \\
1 \\
1\end{array}$ & $\begin{array}{l}- \\
: \\
: \\
: \\
: \\
: \\
: \\
- \\
- \\
-\end{array}$ \\
\hline SOOIUA (TOTAL) & $\begin{array}{l}06 / 30 / 93 \\
06 / 30 / 93 \\
04 / 24 / 94 \\
04 / 24 / 94\end{array}$ & $\begin{array}{l}N 001 \\
N 002 \\
N 001 \\
\text { NOO2 }\end{array}$ & MG/L & & $\begin{array}{l}244 \\
243 \\
225 \\
233\end{array}$ & & $\begin{array}{l}1 \\
1 \\
1 \\
1\end{array}$ & $\begin{array}{l}- \\
- \\
-\end{array}$ \\
\hline $\begin{array}{l}\text { SPECIFIC CONDUCTAKCE } \\
-.\end{array}$ & $\begin{array}{l}04 / 22 / 88 \\
06 / 25 / 89 \\
12 / 01 / 89 \\
06 / 21 / 90 \\
06 / 21 / 90 \\
06 / 21 / 90 \\
06 / 21 / 90 \\
06 / 21 / 90 \\
01 / 29 / 91 \\
06 / 04 / 91 \\
02 / 25 / 92 \\
11 / 19 / 92 \\
02 / 19 / 93 \\
06 / 30 / 93 \\
04 / 24 / 94 \\
12 / 13 / 94\end{array}$ & $\begin{array}{l}0001 \\
0001 \\
0001 \\
0001 \\
0002 \\
0003 \\
0004 \\
0005 \\
0001 \\
0001 \\
0001 \\
0001 \\
0001 \\
\text { No01 } \\
\text { No01 } \\
\text { N001 }\end{array}$ & UMHO/CH & . & $\begin{array}{l}1650 . \\
1660 . \\
2000 . \\
2000 . \\
2000 . \\
2000 . \\
2000 . \\
2000 . \\
2790 . \\
2890 . \\
2510 \\
2510 \\
2510 \\
2610 \\
2670 \\
2650\end{array}$ & $\begin{array}{l}L \\
L \\
L \\
L \\
L \\
L \\
L\end{array}$ & 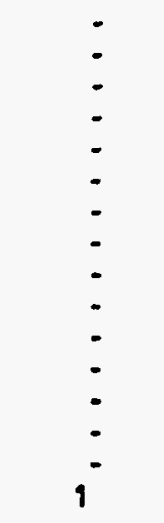 & $\begin{array}{l}- \\
- \\
- \\
- \\
- \\
- \\
- \\
- \\
- \\
- \\
-\end{array}$ \\
\hline STROHTIUN & $\begin{array}{l}06 / 21 / 90 \\
06 / 21 / 90 \\
06 / 21 / 90 \\
06 / 21 / 90 \\
06 / 21 / 90 \\
01 / 29 / 91 \\
06 / 04 / 91\end{array}$ & $\begin{array}{l}0001 \\
0002 \\
0003 \\
0004 \\
0005 \\
0001 \\
0001\end{array}$ & $\mathrm{HG} / \mathrm{L}$ & & $\begin{array}{l}7.55 \\
1.59 \\
1.57 \\
1.62 \\
1.69 \\
1.8 \\
1.95\end{array}$ & $\begin{array}{l}* J L \\
\star J L \\
* J L \\
\star J L \\
* J L \\
L\end{array}$ & $\begin{array}{l}0.1 \\
0.1 \\
0.1 \\
0.1 \\
0.1 \\
0.1 \\
0.01\end{array}$ & $\begin{array}{l}- \\
: \\
- \\
- \\
-\end{array}$ \\
\hline
\end{tabular}

PARAMETER VALUE INDICATOR (PVI): < - LESS THAN DETECTION LIMIT

SAMPLE IO COOES:

OTHER PARAMETER VALUE FLAGS:

* DUPLICATE AHALYSIS nOT HITHIN CONTROL LIMITS

O001 - FILTERED SAMPLE (.45 MICRONS)

0002 - FILTERED REPLICATE SAMPLE (.45 MICRONS)

0003 - FILTERED REPLICATE SNMPLE (.45 MICRONS)

3 - estimated VALUE

- L - LESS THAN THREE BORE VOLLMES REMOVED BEFORE SAMPLING

0004 - FILTERED REPLICATE SAMPLE (.45 MICRONS)

MOO1 - UAFILTERED SMMPLE

HOD2 - INFILTERED REPLICATE SAMPLE 
GROUHDWATER QUALITY DATA BY LOCATION

SOWP REV. 0 Deto

SITE: HOHOI MOHLWENT VALLEY

LOCATION: 0.653

MORTH COOROIHATE: 61249.3 FT

EAST COORDINATE: 89596.3 FT

$04 / 21 / 88$ TO $12 / 13 / 94$

REPORT DATE: 06/20/95

FORMATIOH OF COMPLETION: ALLUVIUM (AL)

HYDRAULIC FLON RELATIOHSHIP: DOWH GRADIENT (D)

\begin{tabular}{|c|c|c|c|c|c|c|c|c|}
\hline PARAMETER MAME & LOG DATE & $\underset{\text { SN }}{\text { SNMPLE }}$ & $\begin{array}{l}\text { UNITS OF } \\
\text { MEASURE }\end{array}$ & PVI & $\begin{array}{l}\text { PARANETER } \\
\text { VALUE }\end{array}$ & FLAGS & $\begin{array}{l}\text { DETECTION } \\
\text { LIMIT }\end{array}$ & $\begin{array}{l}\text { PARANETER } \\
\text { UNCERTAINTY }\end{array}$ \\
\hline STROHTILA & $\begin{array}{l}02 / 25 / 92 \\
11 / 19 / 92 \\
06 / 30 / 93 \\
04 / 24 / 94 \\
04 / 24 / 94 \\
12 / 13 / 94 \\
12 / 13 / 94\end{array}$ & $\begin{array}{l}0001 \\
0001 \\
0001 \\
0001 \\
0002 \\
0001 \\
0002\end{array}$ & $M G / L$ & & $\begin{array}{l}1.97 \\
2.9 \\
2.00 \\
2.19 \\
2.18 \\
2.19 \\
2.13\end{array}$ & & $\begin{array}{l}0.01 \\
0.00035 \\
0.01 \\
0.01 \\
0.01 \\
0.01 \\
0.01\end{array}$ & $\begin{array}{l}- \\
: \\
: \\
-\end{array}$ \\
\hline STROHTIUY (TOTAL) & $\begin{array}{l}11 / 19 / 92 \\
06 / 30 / 93 \\
06 / 30 / 93 \\
04 / 24 / 94 \\
04 / 24 / 94\end{array}$ & $\begin{array}{l}\text { NOO1 } \\
\text { NOO1 } \\
\text { N002 } \\
\text { NO01 } \\
\text { NOO2 }\end{array}$ & MG/L & & $\begin{array}{l}2.4 \\
2.10 \\
2.08 \\
1.99 \\
2.08\end{array}$ & & $\begin{array}{l}0.00035 \\
0.01 \\
0.01 \\
0.01 \\
0.01\end{array}$ & : \\
\hline SULFATE & $\begin{array}{l}04 / 22 / 88 \\
06 / 25 / 89 \\
12 / 01 / 89 \\
06 / 21 / 90 \\
06 / 21 / 90 \\
06 / 21 / 90 \\
06 / 21 / 90 \\
06 / 21 / 90 \\
01 / 29 / 91 \\
06 / 04 / 91 \\
02 / 25 / 92 \\
11 / 19 / 92 \\
02 / 19 / 93 \\
04 / 24 / 94 \\
04 / 24 / 94 \\
12 / 13 / 94 \\
12 / 13 / 94\end{array}$ & $\begin{array}{l}0001 \\
0001 \\
0001 \\
0001 \\
0002 \\
0003 \\
0004 \\
0005 \\
0001 \\
0001 \\
0001 \\
0001 \\
0001 \\
0001 \\
0002 \\
0001 \\
0002\end{array}$ & $M G / L$ & & $\begin{array}{l}1060 . \\
1240 . \\
1330 . \\
1120 . \\
1457.5 \\
1460.8 \\
1360 . \\
1471.5 \\
1530 . \\
1520 . \\
1690 . \\
1700 \\
1790 \\
1660 \\
1700 \\
1800 \\
1720\end{array}$ & $\begin{array}{l}L \\
J L \\
J L \\
J L \\
J L \\
J L \\
L\end{array}$ & $\begin{array}{c}0.1 \\
0.1 \\
0.1 \\
0.1 \\
0.1 \\
0.1 \\
0.1 \\
0.1 \\
0.1 \\
10.1 \\
10 . \\
1.0 \\
1 \\
1 \\
1 \\
1 \\
1\end{array}$ & $\begin{array}{l}- \\
: \\
: \\
: \\
- \\
: \\
: \\
- \\
- \\
- \\
-\end{array}$ \\
\hline SULFATE (TOTAL) & $\begin{array}{l}11 / 19 / 92 \\
06 / 30 / 93 \\
06 / 30 / 93\end{array}$ & $\begin{array}{l}\text { NOO1 } \\
\text { NOO1 } \\
\text { NO02 }\end{array}$ & HG/L & & $\begin{array}{l}1600 \\
1650 \\
1660\end{array}$ & & $\begin{array}{l}1.0 \\
1 \\
1\end{array}$ & $\dot{-}$ \\
\hline
\end{tabular}

PARAMETER VALUE IMDICATOR (PVI): < - LESS THAH DETECTION LIMIT

OTHER PARAMETER VALUE FLAGS:

J - ESTIMATED VALUE

L - LESS tHAN THREE BORE VOLUMES REMOVED BEFORE SAMPLING
SAMPLE ID COOES:

OOO1 - FILTERED SAMPLE (.45 MICRONS)

0002 - FILTERED REPLICATE SAMPLE (.45 MICRONS)

0003 - FILTERED REPLICATE SAMPLE (.45 MICROHS)

0004 - FILTERED REPLICATE SAMPLE (.45 MICRONS)

NOO1 - UMFILTERED SAMPLE

HOO2 - UNFILTERED REPLICATE SAMPLE 
GROUHDWATER QUALITY DATA BY LOCATION

SOWP Rev. O Data

SITE: MOHO1 MOHUMENT VALLEY

LOCATION: 0653

NORTH COORDINATE: 61249.3 FT

EAST COORDINATE: 89596.3 FT

Q4/21/88 TO $12 / 13 / 94$

REPORT DATE: 06/20/95

FORMATION OF COMPLETION: ALLUVIUM (AL)

HYDRAULIC FLON RELATIONSHIP: DOWN GRADIENT (D)

\begin{tabular}{|c|c|c|c|c|c|c|c|c|}
\hline PARAMETER MAME & LOG DATE & $\begin{array}{c}\text { SNYPLE } \\
\text { ID }\end{array}$ & $\begin{array}{l}\text { UNITS OF } \\
\text { MEASURE }\end{array}$ & PVI & $\begin{array}{l}\text { PARAMETER } \\
\text { VALUE }\end{array}$ & FLAGS & $\begin{array}{l}\text { DETECTION } \\
\text { LIMIT }\end{array}$ & $\begin{array}{l}\text { PARAMETER } \\
\text { UNCERTAINTY }\end{array}$ \\
\hline TEMPERATURE & $\begin{array}{l}02 / 19 / 93 \\
06 / 30 / 93 \\
04 / 24 / 94 \\
12 / 13 / 94\end{array}$ & $\begin{array}{l}0001 \\
\text { N001 } \\
\text { N001 } \\
\text { N001 }\end{array}$ & C - DEGREE & & $\begin{array}{l}15.4 \\
18.7 \\
16.4 \\
15.3\end{array}$ & & $\begin{array}{c}- \\
0.1\end{array}$ & : \\
\hline THALLIUH & $\begin{array}{l}06 / 25 / 89 \\
12 / 01 / 89 \\
06 / 21 / 90 \\
06 / 21 / 90 \\
06 / 21 / 90 \\
06 / 21 / 90 \\
06 / 21 / 90 \\
01 / 29 / 91 \\
06 / 04 / 91\end{array}$ & $\begin{array}{l}0001 \\
0001 \\
0001 \\
0002 \\
0003 \\
0004 \\
0005 \\
0001 \\
0001\end{array}$ & MG/L & $\begin{array}{l}< \\
< \\
5 \\
< \\
< \\
< \\
< \\
< \\
<\end{array}$ & $\begin{array}{l}0.01 \\
0.1 \\
0.01 \\
0.01 \\
0.01 \\
0.01 \\
0.01 \\
0.01 \\
0.01\end{array}$ & $\begin{array}{l}\mathbf{L} \\
J L \\
J L \\
J L \\
J L \\
J L \\
L\end{array}$ & $\begin{array}{l}0.01 \\
0.1 \\
0.01 \\
0.01 \\
0.01 \\
0.01 \\
0.01 \\
0.01 \\
0.01\end{array}$ & $\begin{array}{l}- \\
- \\
- \\
- \\
\dot{-} \\
-\end{array}$ \\
\hline THORIUM-230 & $\begin{array}{l}01 / 29 / 91 \\
06 / 04 / 91\end{array}$ & $\begin{array}{l}0001 \\
0001\end{array}$ & $\mathrm{PCI} / \mathrm{L}$ & & $\begin{array}{l}0.0 \\
0.3\end{array}$ & $\mathbf{L}$ & 1. & $\begin{array}{l}0.3 \\
0.3\end{array}$ \\
\hline TIN & $\begin{array}{l}06 / 25 / 89 \\
12 / 01 / 89 \\
06 / 21 / 90 \\
06 / 21 / 90 \\
06 / 21 / 90 \\
06 / 21 / 90 \\
06 / 21 / 90 \\
01 / 29 / 91 \\
06 / 04 / 91 \\
02 / 25 / 92\end{array}$ & $\begin{array}{l}0001 \\
0001 \\
0001 \\
0002 \\
0003 \\
0004 \\
0005 \\
0001 \\
0001 \\
0001\end{array}$ & MG/L & $\begin{array}{l}< \\
<\end{array}$ & $\begin{array}{l}0.024 \\
0.005 \\
0.020 \\
0.019 \\
0.020 \\
0.018 \\
0.019 \\
0.005 \\
0.01 \\
0.03\end{array}$ & $\begin{array}{l}L \\
J L \\
J L \\
J L \\
J L \\
J L \\
L \\
I \\
I\end{array}$ & $\begin{array}{l}0.005 \\
0.005 \\
0.005 \\
0.005 \\
0.005 \\
0.005 \\
0.005 \\
0.005 \\
0.01 \\
0.03\end{array}$ & $\begin{array}{l}: \\
: \\
: \\
: \\
: \\
- \\
-\end{array}$ \\
\hline TOTAL DISSOLVED SOLIDS & $\begin{array}{l}04 / 22 / 88 \\
06 / 25 / 89 \\
12 / 01 / 89 \\
06 / 21 / 90 \\
06 / 21 / 90 \\
06 / 21 / 90 \\
06 / 21 / 90 \\
06 / 21 / 90 \\
01 / 29 / 91 \\
06 / 04 / 91 \\
02 / 25 / 92 \\
02 / 19 / 93 \\
04 / 24 / 94 \\
04 / 24 / 94 \\
12 / 13 / 94 \\
12 / 13 / 94\end{array}$ & $\begin{array}{l}0001 \\
0001 \\
0001 \\
0001 \\
0002 \\
0003 \\
0004 \\
0005 \\
0001 \\
0001 \\
0001 \\
0001 \\
0001 \\
0002 \\
0001 \\
0002\end{array}$ & MG/L & & $\begin{array}{l}1730 . \\
2030 . \\
2290 . \\
2104 . \\
2068 . \\
2080 . \\
2028 . \\
1956 . \\
2320 . \\
2590 . \\
2640 . \\
2820 \\
2760 \\
2780 \\
2970 \\
2990\end{array}$ & $\begin{array}{l}\mathrm{L} \\
\mathrm{JL} \\
\mathrm{JL} \\
\mathrm{JL} \\
\mathrm{JL} \\
\mathrm{JL} \\
\mathrm{L}\end{array}$ & $\begin{array}{l}10 . \\
10 . \\
10 . \\
10 . \\
10 . \\
10 . \\
10 . \\
10 . \\
10 . \\
10 . \\
10 . \\
10 \\
10 \\
10 \\
10 \\
10\end{array}$ & $\begin{array}{l}- \\
- \\
- \\
- \\
- \\
- \\
- \\
- \\
- \\
- \\
-\end{array}$ \\
\hline TOTAL DISSOLVED SOLIDS (TOTAL) & $\begin{array}{l}06 / 30 / 93 \\
06 / 30 / 93\end{array}$ & $\begin{array}{l}\text { NoO1 } \\
\text { N002 }\end{array}$ & $M G / L$ & & $\begin{array}{l}2670 \\
2640\end{array}$ & $\begin{array}{l}H \\
H\end{array}$ & $\begin{array}{l}10 \\
10\end{array}$ & - \\
\hline TOTAL CRGAKIC CARBON & $\begin{array}{l}04 / 22 / 88 \\
01 / 29 / 91 \\
06 / 04 / 91 \\
02 / 25 / 92\end{array}$ & $\begin{array}{l}0001 \\
0001 \\
0001 \\
0001\end{array}$ & MG/L & $<$ & $\begin{array}{c}53.5 \\
9 . \\
1 . \\
1 .\end{array}$ & $\mathbf{L}$ & $\begin{array}{l}1 . \\
1 .\end{array}$ & : \\
\hline
\end{tabular}

PARAMETER VALUE INDICATOR (PVI): < - LESS THAN DETECTION LIHIT

SNYPLE ID COOES:

0001 - FILTERED SAMPLE (.45 MICRONS)

OTHER PARAMETER VALUE FLAGS:

0002 - FILTERED REPLICATE SAMPLE (.45 MICRONS)

0003 - FILTERED REPLICATE SNIPLE (.45 MICRONS)

I - INCREASED DETECTION LIMIT DUE TO REQUIRED DILUTION

J - estiMATED VALUE

0004 - FILTERED REPLICATE SAMPLE (.45 MICRONS)

MOO1 - UNFILTERED SAMPLE

L - LESS THAN THREE BORE VOLLMES REMOVED BEFORE SAMPLIHG

NOO2 - UAFILTERED REPLICATE SNMPLE 
GROUNDWATER QUALITY DATA BY LOCATION

SOMP ReV. O Data

SITE: HOHO1 MONUMENT VALLEY

LOCATIOH: 0653

MORTH COORDIHATE: 61249.3 FT

EAST COORDIHATE: 89596.3 FT

04/21/88 TO 12/13/94

REPORT DATE: $06 / 20 / 95$

FORMATIOH OF COAPLETIOH: ALLUVIUH (AL)

HYDRAULIC FLOW RELATIOHSHIP: DOWH GRADIENT (O)

\begin{tabular}{|c|c|c|c|c|c|c|c|c|}
\hline PARAMETER NAME & LOG DATE & $\begin{array}{c}\text { SAMPLE } \\
\text { ID }\end{array}$ & $\begin{array}{l}\text { UNITS OF } \\
\text { MEASURE }\end{array}$ & PVI & $\begin{array}{l}\text { PARAMETER } \\
\text { VALUE }\end{array}$ & FLAGS & $\begin{array}{l}\text { DETECTION } \\
\text { LIMIT }\end{array}$ & $\begin{array}{l}\text { PARAMETER } \\
\text { UNCERTAINTY }\end{array}$ \\
\hline TURBIDITY & $\begin{array}{l}04 / 24 / 94 \\
12 / 93 / 94\end{array}$ & $\begin{array}{l}\text { No01 } \\
\text { N001 }\end{array}$ & NTU & & $\begin{array}{r}9 \\
14\end{array}$ & & 1 & $\dot{-}$ \\
\hline URAKIUY & $\begin{array}{l}04 / 22 / 88 \\
06 / 25 / 89 \\
12 / 01 / 89 \\
06 / 21 / 90 \\
06 / 21 / 90 \\
06 / 21 / 90 \\
06 / 21 / 90 \\
06 / 21 / 90 \\
01 / 29 / 91 \\
06 / 04 / 91 \\
02 / 26 / 92 \\
11 / 19 / 92 \\
02 / 19 / 93 \\
06 / 30 / 93 \\
04 / 24 / 94 \\
04 / 24 / 94 \\
12 / 13 / 94 \\
12 / 13 / 94\end{array}$ & $\begin{array}{l}0001 \\
0001 \\
0001 \\
0001 \\
0002 \\
0003 \\
0004 \\
0005 \\
0001 \\
0001 \\
0001 \\
0001 \\
0001 \\
0001 \\
0001 \\
0002 \\
0001 \\
0002\end{array}$ & $M G / L$ & $<$ & $\begin{array}{l}0.0072 \\
0.0078 \\
0.009 \\
0.0068 \\
0.063 \\
0.0016 \\
0.0061 \\
0.0072 \\
0.0094 \\
0.014 \\
0.010 \\
0.012 \\
0.013 \\
0.011 \\
0.012 \\
0.012 \\
0.006 \\
0.001\end{array}$ & $\begin{array}{l}L \\
\text { JL } \\
J L \\
J L \\
J L \\
J L \\
L\end{array}$ & $\begin{array}{l}0.003 \\
0.003 \\
0.003 \\
0.003 \\
0.003 \\
0.003 \\
0.003 \\
0.003 \\
0.0003 \\
0.001 \\
0.001 \\
0.001 \\
0.005 \\
0.001 \\
0.001 \\
0.001 \\
0.001 \\
0.001\end{array}$ & $\begin{array}{l}- \\
i \\
- \\
- \\
- \\
- \\
- \\
- \\
- \\
- \\
- \\
- \\
-\end{array}$ \\
\hline URANIUM (TOTAL) & $\begin{array}{l}11 / 19 / 92 \\
06 / 30 / 93 \\
06 / 30 / 93 \\
04 / 24 / 94 \\
04 / 24 / 94\end{array}$ & $\begin{array}{l}\text { NOO1 } \\
\text { NO01 } \\
\text { NOO2 } \\
\text { NOO1 } \\
\text { NOO2 }\end{array}$ & $M G / L$ & & $\begin{array}{l}0.011 \\
0.09 \\
0.01 \\
0.012 \\
0.012\end{array}$ & & $\begin{array}{l}0.001 \\
0.001 \\
0.001 \\
0.001 \\
0.001\end{array}$ & $\begin{array}{l}- \\
- \\
- \\
-\end{array}$ \\
\hline $\begin{array}{l}\text { VAHADIUN } \\
*\end{array}$ & $\begin{array}{l}06 / 25 / 89 \\
12 / 01 / 89 \\
06 / 21 / 90 \\
06 / 21 / 90 \\
06 / 21 / 90 \\
06 / 21 / 90 \\
06 / 21 / 90 \\
01 / 29 / 91 \\
06 / 04 / 91 \\
02 / 25 / 92 \\
11 / 19 / 92 \\
06 / 30 / 93 \\
04 / 24 / 94 \\
04 / 24 / 94 \\
12 / 13 / 94 \\
12 / 13 / 94\end{array}$ & $\begin{array}{l}0001 \\
0001 \\
0001 \\
0002 \\
0003 \\
0004 \\
0005 \\
0001 \\
0001 \\
0001 \\
0001 \\
0001 \\
0001 \\
0002 \\
0001 \\
0002\end{array}$ & $M G / L$ & $\begin{array}{l}< \\
< \\
< \\
< \\
< \\
<\end{array}$ & $\begin{array}{l}0.05 \\
0.01 \\
0.03 \\
0.02 \\
0.02 \\
0.02 \\
0.02 \\
0.01 \\
0.01 \\
0.01 \\
0.0093 \\
0.01 \\
0.01 \\
0.01 \\
0.01 \\
0.01\end{array}$ & $\begin{array}{l}L \\
J L \\
J L \\
J L \\
J L \\
J L \\
L \\
L\end{array}$ & 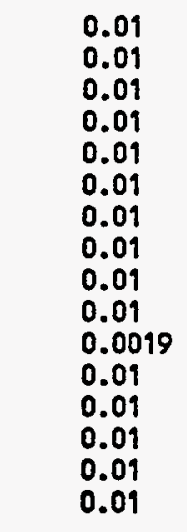 & $\begin{array}{l}- \\
- \\
: \\
: \\
- \\
- \\
- \\
- \\
- \\
- \\
-\end{array}$ \\
\hline VAHADIUA (TOTAL) & $\begin{array}{l}11 / 19 / 92 \\
06 / 30 / 93 \\
06 / 30 / 93 \\
04 / 24 / 94 \\
04 / 24 / 94\end{array}$ & $\begin{array}{l}\text { No01 } \\
\text { No01 } \\
\text { No02 } \\
\text { No01 } \\
\text { No02 }\end{array}$ & MG/L & $\begin{array}{l}< \\
< \\
<\end{array}$ & $\begin{array}{l}0.0080 \\
0.01 \\
0.01 \\
0.01 \\
0.01\end{array}$ & & $\begin{array}{l}0.0019 \\
0.01 \\
0.01 \\
0.01 \\
0.01\end{array}$ & : \\
\hline ZINC & $\begin{array}{l}06 / 25 / 89 \\
12 / 01 / 89\end{array}$ & $\begin{array}{l}0001 \\
0001\end{array}$ & MG/L & $<$ & $\begin{array}{l}0.005 \\
0.005\end{array}$ & $\mathbf{L}$ & $\begin{array}{l}0.005 \\
0.005\end{array}$ & $\dot{-}$ \\
\hline
\end{tabular}

PARAMETER VALUE IMDICATOR (PVI): < - LESS THAN DETECTION LIMIT

SAMPLE ID COOES:

0001 - FILTERED SAMPLE (.45 MICRONS)

OTHER PARAMETER VALUE FLAGS:

J- estimuted VALUE

L - LESS THAH THREE BORE VOLLWES REHOVED BEFORE SAMPLIMG

0002 - FILTERED REPLICATE SAMPLE (.45 MICRONS)

0003 - FILTERED REPLICATE SAMPLE (.45 MICRONS)

0004 - FILTERED REPLICATE SAMPLE (.45 MICRONS)

NOOI - UNFILTERED SAMPLE

NOO2 - UHFILTERED REPLICATE SNMPLE 
GRQUNDWATER QUALITY DATA BY LOCATION

SOUP REV. O Data

SITE: MONOI MOHLMENT VALLEY

LOCATIOH: 0653

NORTH COORDINATE: 61249.3 FT

EAST COORDINATE: 89596.3 FT

$04 / 21 / 88$ TO $12 / 13 / 94$

REPORT DATE: $06 / 20 / 95$

FORMATION OF CONPLETION: ALLUNIUH (AL)

HYDRAULIC FLOM RELATIOHSHIP: DON GRAOIENT (D)

\begin{tabular}{|c|c|c|c|c|c|c|c|c|}
\hline PARAMETER NAME & LOG DATE & $\underset{\text { SAMPLE }}{\text { SD }}$ & $\begin{array}{l}\text { UNITS OF } \\
\text { MEASURE }\end{array}$ & PVI & $\begin{array}{l}\text { PARAMETER } \\
\text { VALUE }\end{array}$ & FLAGS & $\begin{array}{l}\text { DETECTIOH } \\
\text { LIMIT }\end{array}$ & $\begin{array}{l}\text { PARAMETER } \\
\text { UHCERTAIHTY }\end{array}$ \\
\hline ZIHC & $\begin{array}{l}06 / 21 / 90 \\
06 / 21 / 90 \\
06 / 21 / 90 \\
06 / 21 / 90 \\
06 / 21 / 90 \\
01 / 29 / 91 \\
06 / 04 / 91 \\
02 / 25 / 92 \\
11 / 19 / 92 \\
06 / 30 / 93\end{array}$ & $\begin{array}{l}0001 \\
0002 \\
0003 \\
0004 \\
0005 \\
0001 \\
0001 \\
0001 \\
0001 \\
0001\end{array}$ & $M G / L$ & $\begin{array}{l}< \\
< \\
< \\
< \\
< \\
<\end{array}$ & $\begin{array}{l}0.005 \\
0.005 \\
0.005 \\
0.082 \\
0.005 \\
0.005 \\
0.005 \\
0.019 \\
0.0048 \\
0.016\end{array}$ & $\begin{array}{l}J L \\
J L \\
J L \\
J L \\
J L \\
L\end{array}$ & $\begin{array}{l}0.005 \\
0.005 \\
0.005 \\
0.005 \\
0.005 \\
0.005 \\
0.005 \\
0.005 \\
0.0010 \\
0.005\end{array}$ & $\begin{array}{l}: \\
: \\
: \\
: \\
: \\
-\end{array}$ \\
\hline 2INC (TOTAL) & $\begin{array}{l}11 / 19 / 92 \\
06 / 30 / 93 \\
06 / 30 / 93\end{array}$ & $\begin{array}{l}\text { NOO1 } \\
\text { N001 } \\
\text { HOO2 }\end{array}$ & $M G / L$ & & $\begin{array}{l}0.0070 \\
0.020 \\
0.020\end{array}$ & J & $\begin{array}{l}0.0010 \\
0.005 \\
0.005\end{array}$ & : \\
\hline
\end{tabular}

PARAMETER VALUE IHDICATOR (PVI): < - LESS THAN DETECTION LIMIT

SAMPLE ID COOES:

OTHER PARAMETER VALUE FLAGS:

J. ESTIMATED VALUE

l - LESS THAN THREE BORE VOLLMES REMOVED BEFORE SAMPLING

0001 - FILTERED SAMPLE (.45 MICRONS)

DOO2 - FILTERED REPLICATE SAMPLE (.45 MICROHS)

0003 - FILTERED REPLICATE SAMPLE (.45 MICRONS)

0004 - FILTERED REPLICATE SAMPLE (.45 MICRONS)

NOO1 - UMFILTERED SAMPLE

HOOZ - UHFILTERED REPLICATE SAMPLE 
SITE: MONO1 HONUMENT VALLEY

LOCATION: 0654

MORTH COORDIHATE: 59351.4 FT

EAST COORDIMATE: $\quad 91063.4$ FT

$04 / 21 / 88$ TO $12 / 13 / 94$

REPORT DATE: O6/20/95

FORHATION OF COMPLETION: ALLUVIUN (AL)

HYDRAULIC FLON RELATIONSHIP: CROSS GRADIENT (C)

\begin{tabular}{|c|c|c|c|c|c|c|c|c|}
\hline PARAMETER MAME & LOG DATE & $\underset{\text { ID }}{\text { SAMPLE }}$ & $\begin{array}{l}\text { UHITS OF } \\
\text { MEASURE }\end{array}$ & PVI & $\begin{array}{l}\text { ARANETER } \\
\text { VALUE }\end{array}$ & FLAGS & $\begin{array}{l}\text { DETECTION } \\
\text { LIMIT }\end{array}$ & $\begin{array}{l}\text { PARAMETER } \\
\text { UNCERTAINTY }\end{array}$ \\
\hline ALKALINITY & $\begin{array}{l}04 / 26 / 88 \\
06 / 09 / 89 \\
12 / 03 / 89 \\
06 / 21 / 90 \\
01 / 29 / 91 \\
06 / 02 / 91 \\
08 / 12 / 92 \\
11 / 22 / 92 \\
02 / 18 / 93 \\
06 / 30 / 93\end{array}$ & $\begin{array}{l}0001 \\
0001 \\
0001 \\
0001 \\
0001 \\
0001 \\
0001 \\
0001 \\
0001 \\
N 001\end{array}$ & $\mathrm{MG} / \mathrm{L} \mathrm{CACO} 3$ & & $\begin{array}{l}179 . \\
193 . \\
159 . \\
170 . \\
168 . \\
176 . \\
174 \\
184 \\
184 \\
175\end{array}$ & $\begin{array}{l}L \\
L \\
L \\
L \\
L \\
L\end{array}$ & $\begin{array}{l}- \\
- \\
- \\
- \\
- \\
- \\
-\end{array}$ & $\begin{array}{l}- \\
- \\
- \\
- \\
- \\
- \\
-\end{array}$ \\
\hline ALCUIMU: & $\begin{array}{l}06 / 09 / 89 \\
12 / 03 / 89 \\
06 / 21 / 90 \\
01 / 29 / 91 \\
06 / 02 / 91 \\
08 / 12 / 92\end{array}$ & $\begin{array}{l}0001 \\
0001 \\
0001 \\
0001 \\
0001 \\
0001\end{array}$ & MG/L & $\begin{array}{l}< \\
< \\
< \\
< \\
< \\
<\end{array}$ & $\begin{array}{l}0.1 \\
0.1 \\
0.1 \\
0.1 \\
0.05 \\
0.2\end{array}$ & $\begin{array}{l}L \\
L \\
L \\
L \\
L\end{array}$ & $\begin{array}{l}0.1 \\
0.9 \\
0.1 \\
0.1 \\
0.05 \\
0.2\end{array}$ & $\begin{array}{l}- \\
- \\
- \\
-\end{array}$ \\
\hline AmonILA & $\begin{array}{l}04 / 26 / 88 \\
06 / 09 / 89 \\
12 / 03 / 89 \\
06 / 21 / 90 \\
01 / 29 / 91 \\
06 / 02 / 91\end{array}$ & $\begin{array}{l}0001 \\
0001 \\
0001 \\
0001 \\
0001 \\
0001\end{array}$ & MG/L & $\begin{array}{l}< \\
< \\
< \\
<\end{array}$ & $\begin{array}{l}0.1 \\
0.1 \\
0.1 \\
0.1 \\
0.1 \\
0.2\end{array}$ & $\begin{array}{l}L \\
L \\
H L \\
L \\
L\end{array}$ & $\begin{array}{l}0.1 \\
0.1 \\
0.1 \\
0.1 \\
0.1 \\
0.1\end{array}$ & $\begin{array}{l}- \\
- \\
- \\
- \\
-\end{array}$ \\
\hline AพyOHIUH (TOTAL) & $06 / 30 / 93$ & No01 & $M G / L$ & $<$ & 0.1 & & 0.1 & - \\
\hline ANTIMONY & $\begin{array}{l}06 / 09 / 89 \\
12 / 03 / 89 \\
06 / 21 / 90 \\
01 / 29 / 91 \\
06 / 02 / 91 \\
08 / 12 / 92\end{array}$ & $\begin{array}{l}0001 \\
0001 \\
0001 \\
0001 \\
0001 \\
0001\end{array}$ & MG/L & $\begin{array}{l}< \\
< \\
< \\
< \\
<\end{array}$ & $\begin{array}{l}0.003 \\
0.003 \\
0.003 \\
0.003 \\
0.003 \\
0.06\end{array}$ & $\begin{array}{l}L \\
L \\
L \\
L \\
L\end{array}$ & $\begin{array}{l}0.003 \\
0.003 \\
0.003 \\
0.003 \\
0.003 \\
0.06\end{array}$ & $\begin{array}{l}- \\
- \\
- \\
-\end{array}$ \\
\hline ARSENIC & $\begin{array}{l}04 / 26 / 88 \\
06 / 09 / 89 \\
12 / 03 / 89 \\
06 / 21 / 90 \\
01 / 29 / 91 \\
06 / 02 / 91 \\
08 / 12 / 92 \\
11 / 22 / 92 \\
06 / 30 / 93\end{array}$ & $\begin{array}{l}0001 \\
0001 \\
0001 \\
0001 \\
0001 \\
0001 \\
0001 \\
0001 \\
0001\end{array}$ & MG/L & $\begin{array}{l}< \\
< \\
< \\
< \\
< \\
< \\
< \\
< \\
<\end{array}$ & $\begin{array}{l}0.001 \\
0.01 \\
0.01 \\
0.01 \\
0.01 \\
0.01 \\
0.01 \\
0.0015 \\
0.005\end{array}$ & $\begin{array}{l}J L \\
L \\
L \\
L \\
L \\
W L\end{array}$ & $\begin{array}{l}0.01 \\
0.01 \\
0.01 \\
0.01 \\
0.01 \\
0.01 \\
0.01 \\
0.0015 \\
0.005\end{array}$ & $\begin{array}{l}- \\
- \\
\therefore \\
- \\
- \\
-\end{array}$ \\
\hline ARSENIC (TOTAL) & $\begin{array}{l}11 / 22 / 92 \\
06 / 30 / 93\end{array}$ & $\begin{array}{l}\text { NOO9 } \\
\text { N009 }\end{array}$ & MG/L & $<$ & $\begin{array}{l}0.0015 \\
0.005\end{array}$ & *HJL & $\begin{array}{l}0.0015 \\
0.005\end{array}$ & $\dot{-}$ \\
\hline BARILH & $\begin{array}{l}06 / 09 / 89 \\
12 / 03 / 89 \\
06 / 21 / 90 \\
01 / 29 / 91 \\
06 / 02 / 91\end{array}$ & $\begin{array}{l}0001 \\
0001 \\
0001 \\
0001 \\
0001\end{array}$ & MG/L & & $\begin{array}{l}0.1 \\
0.1 \\
0.2 \\
0.2 \\
0.15\end{array}$ & $\begin{array}{l}L \\
L \\
L \\
L\end{array}$ & $\begin{array}{l}0.1 \\
0.1 \\
0.1 \\
0.1 \\
0.01\end{array}$ & $\begin{array}{l}- \\
- \\
-\end{array}$ \\
\hline
\end{tabular}

PARAMETER VALUE IMDICATOR (PVI): < - LESS THAN DETECTION LIMIT

\section{OTHER PARAMETER VALUE FLAGS:}

- - DUPLICATE AMALYSIS hOT UITHIM CONTROL LIMITS

H - HOLD TIME EXPIRED, VALUE SUSPECT

J - ESTIMATED VALUE

L - LESS THAK THREE BORE VOLLMES REMOVED BEFORE SAMPLING

- POST-DIGEST SPIKE OUT OF CHTR LIM WHILE SAMP ABS < 50\% SPIKE
SAYPLE ID CODES:

0001 - FILTERED SAMPLE (.45 MICRONS)

NOO1 - UHFILTERED SMMPLE 
GROUNDWATER QUALITY DATA BY LOCATION

SOWP ReV. 0 Data

SITE: MONOY MONUMENT VALLEY

LOCATIOH: 0654

MORTH COORDINATE: 59359.4 FT

EAST COORDIHATE: $\quad 91063.4$ FT

$04 / 21 / 88$ TO $12 / 13 / 94$

REPORT DATE: 06/20/95

FORMATIOH OF COMPLETION: ALLUVIUM (AL)

HYDRAULIC FLOW RELATIONSHIP: CROSS GRADIEHT (C)

\begin{tabular}{|c|c|c|c|c|c|c|c|c|}
\hline PARAMETER MAME & LOG DATE & $\underset{10}{\text { SAMPLE }}$ & $\begin{array}{l}\text { UNITS OF } \\
\text { MEASURE }\end{array}$ & PVI & $\begin{array}{l}\text { PARAMETER } \\
\text { VALUE }\end{array}$ & FLAGS & $\begin{array}{l}\text { DETECTION } \\
\text { LIMIT }\end{array}$ & $\begin{array}{l}\text { PARAMETER } \\
\text { UHCERTAINTY }\end{array}$ \\
\hline BARIUM & $\begin{array}{l}08 / 12 / 92 \\
11 / 22 / 92 \\
06 / 30 / 93\end{array}$ & $\begin{array}{l}0001 \\
0001 \\
0001\end{array}$ & MG/L & $<$ & $\begin{array}{l}0.2 \\
0.14 \\
0.1\end{array}$ & L & $\begin{array}{l}0.2 \\
0.0015 \\
0.1\end{array}$ & : \\
\hline BARIUN (TOTAL) & $\begin{array}{l}11 / 22 / 92 \\
06 / 30 / 93\end{array}$ & $\begin{array}{l}\text { NOO1 } \\
\text { NOO1 }\end{array}$ & ME/L & & $\begin{array}{l}0.94 \\
0.9\end{array}$ & & $\begin{array}{l}0.0015 \\
0.1\end{array}$ & $\dot{-}$ \\
\hline BERYLLIUH & $\begin{array}{l}06 / 09 / 89 \\
12 / 03 / 89 \\
06 / 21 / 90 \\
01 / 29 / 91 \\
06 / 02 / 91 \\
08 / 12 / 92\end{array}$ & $\begin{array}{l}0001 \\
0001 \\
0001 \\
0001 \\
0001 \\
0001\end{array}$ & $M G / L$ & $\begin{array}{l}< \\
< \\
< \\
< \\
<\end{array}$ & $\begin{array}{l}0.01 \\
0.005 \\
0.01 \\
0.01 \\
0.005 \\
0.005\end{array}$ & $\begin{array}{l}L \\
L \\
L \\
L \\
L\end{array}$ & $\begin{array}{l}0.01 \\
0.005 \\
0.01 \\
0.01 \\
0.005 \\
0.005\end{array}$ & $\begin{array}{l}- \\
: \\
- \\
-\end{array}$ \\
\hline BORON & $\begin{array}{l}06 / 09 / 89 \\
12 / 03 / 89 \\
06 / 21 / 90 \\
01 / 29 / 91 \\
06 / 02 / 91\end{array}$ & $\begin{array}{l}0001 \\
0001 \\
0001 \\
0001 \\
0001\end{array}$ & $M G / L$ & $\begin{array}{l}< \\
< \\
< \\
<\end{array}$ & $\begin{array}{l}0.1 \\
0.1 \\
0.1 \\
0.1 \\
0.05\end{array}$ & $\begin{array}{l}L \\
L \\
L \\
L\end{array}$ & $\begin{array}{l}0.1 \\
0.1 \\
0.1 \\
0.1 \\
0.05\end{array}$ & : \\
\hline BROHIDE & $\begin{array}{l}01 / 29 / 91 \\
06 / 02 / 91\end{array}$ & $\begin{array}{l}0001 \\
0001\end{array}$ & $M G / L$ & $<$ & $\begin{array}{l}0.1 \\
0.1\end{array}$ & 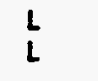 & $\begin{array}{l}0.1 \\
0.1\end{array}$ & $\dot{-}$ \\
\hline CADMILM & $\begin{array}{l}04 / 26 / 88 \\
06 / 09 / 89 \\
12 / 03 / 89 \\
06 / 21 / 90 \\
01 / 29 / 91 \\
06 / 02 / 91 \\
08 / 12 / 92 \\
11 / 22 / 92\end{array}$ & $\begin{array}{l}0001 \\
0001 \\
0001 \\
0001 \\
0001 \\
0001 \\
0001 \\
0001\end{array}$ & $M G / L$ & $\begin{array}{l}< \\
< \\
< \\
< \\
< \\
<\end{array}$ & $\begin{array}{l}0.002 \\
0.001 \\
0.001 \\
0.001 \\
0.001 \\
0.001 \\
0.005 \\
0.00013\end{array}$ & $\begin{array}{l}L \\
L \\
L \\
L \\
L \\
L\end{array}$ & $\begin{array}{l}0.001 \\
0.001 \\
0.001 \\
0.001 \\
0.001 \\
0.001 \\
0.005 \\
0.00013\end{array}$ & $\begin{array}{l}- \\
- \\
- \\
- \\
-\end{array}$ \\
\hline CADHIUM (TOTAL) & $11 / 22 / 92$ & NOOI & MG/L & \multicolumn{3}{|c|}{$0.00021 \mathrm{HS}$} & 0.00013 & - \\
\hline CALCIUH & $\begin{array}{l}04 / 26 / 88 \\
06 / 09 / 89 \\
12 / 03 / 89 \\
06 / 21 / 90 \\
01 / 29 / 91 \\
06 / 02 / 91 \\
08 / 12 / 92 \\
02 / 18 / 93 \\
06 / 30 / 93\end{array}$ & $\begin{array}{l}0001 \\
0001 \\
0001 \\
0001 \\
0001 \\
0001 \\
0001 \\
0001 \\
0001\end{array}$ & $M G / L$ & & $\begin{array}{l}40.9 \\
42.1 \\
42 . \\
41.9 \\
42.6 \\
45.5 \\
42 \\
38.1 \\
41.3\end{array}$ & $\begin{array}{l}L \\
L \\
L \\
L \\
L \\
L\end{array}$ & $\begin{array}{l}0.01 \\
0.01 \\
0.01 \\
0.01 \\
0.01 \\
0.5 \\
5 \\
0.5 \\
0.5\end{array}$ & $\begin{array}{l}- \\
- \\
- \\
- \\
- \\
-\end{array}$ \\
\hline CALCILH (TOTAL) & $06 / 30 / 93$ & NOOI & $M G / L$ & & 38.7 & & 0.5 & - \\
\hline CHLORIOE & $\begin{array}{l}04 / 26 / 88 \\
06 / 09 / 89 \\
12 / 03 / 89 \\
06 / 21 / 90 \\
01 / 29 / 91 \\
06 / 02 / 91 \\
08 / 12 / 92 \\
02 / 18 / 93\end{array}$ & $\begin{array}{l}0001 \\
0001 \\
0001 \\
0001 \\
0001 \\
0001 \\
0001 \\
0001\end{array}$ & MG/L & $<$ & $\begin{array}{l}5.7 \\
3.6 \\
5.4 \\
6 . \\
1 . \\
5.3 \\
5.6 \\
5.8\end{array}$ & $\begin{array}{l}L \\
L \\
L \\
J L \\
L \\
L\end{array}$ & $\begin{array}{l}1 . \\
1 . \\
1 . \\
1 . \\
1 . \\
0.5 \\
0.016 \\
0.5\end{array}$ & $\begin{array}{l}- \\
: \\
: \\
-\end{array}$ \\
\hline
\end{tabular}

PARAMETER VALUE IMDICATOR (PVI): < - LESS THAN DETECTION LIHIT

SAMPLE ID COOES:

OTHER PARAMETER VALUE FLAGS:

0001 - FILTERED SAMPLE (.45 MICRONS)

$J$ - ESTIMATED VALUE

NOD1 - UAFILTERED SAMPLE'

$\iota$ - LESS THAH THREE BORE VOLUMES REMOVED BEFORE SAMPLING

H - SPIKE SAHPLE RECOVERY NOT MITHIH COHTROL LIMITS

S - REPORTED VALUE DETERHIHED USING METHOO OF STD ADDITION (MSA) 
GROUNDUATER OUALITY DATA BY LOCATION

SOWP ReV. O Data

SITE: MONOI HOHUMENT VALLEY

LOCATIOH: 0654

MORTH COOROINATE: 59351.4 FT

EAST COOROINATE: $\quad 91063.4$ FT

$04 / 21 / 88$ TO $12 / 13 / 94$

REPORT DATE: 06/20/95

FORMATION OF COMPLETIOH: ALLUVIUN (AL)

HYDRAULIC FLON RELATIONSHIP: CROSS GRADIEHT (C)

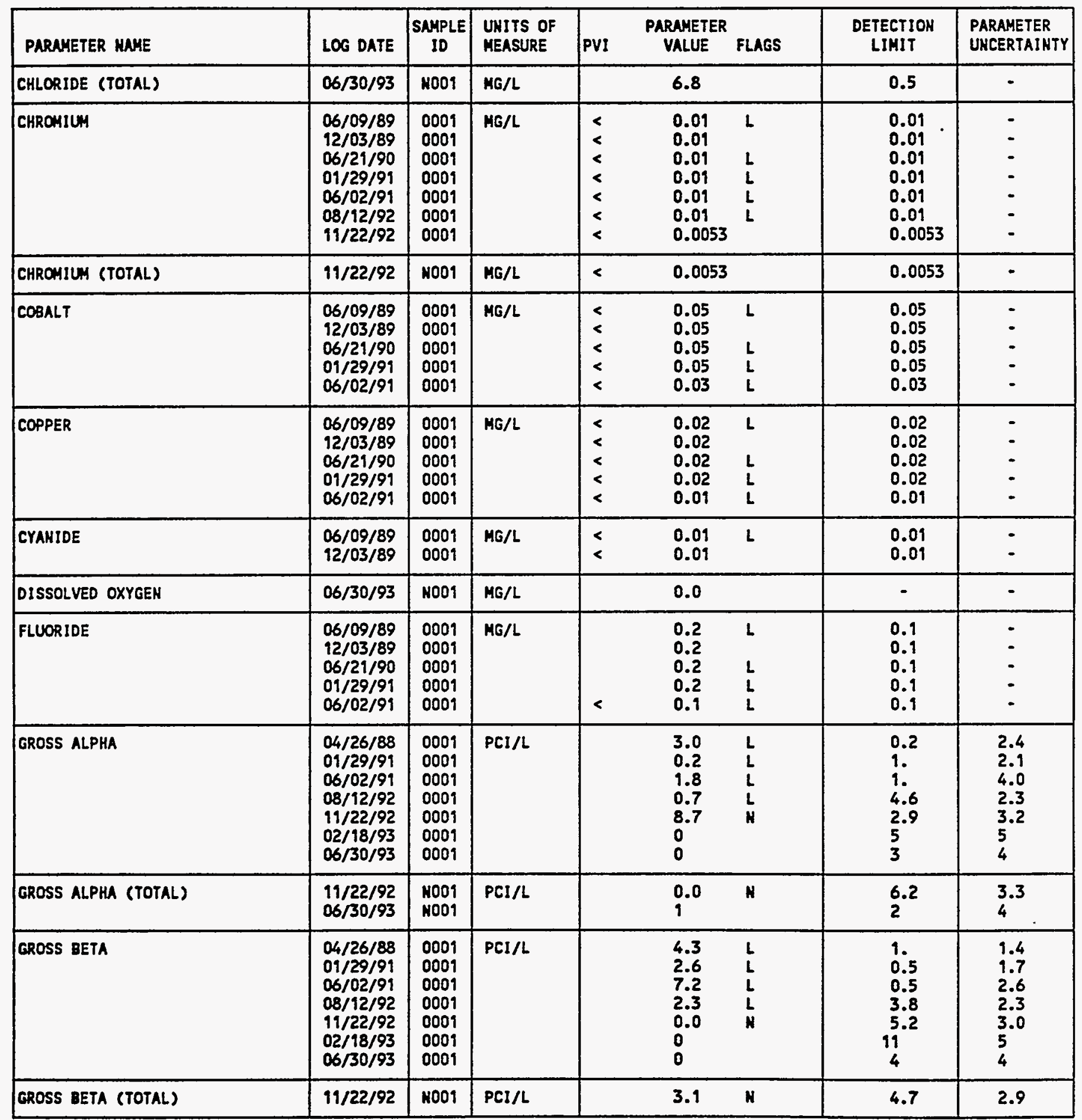

PARAMETER VALUE INDICATOR (PVI): < - LESS THAM DETECTION LIMIT

SNMPLE ID COOES:

OTHER PARAMETER VALUE FLAGS:

O001 - FILTERED SAMPLE (.45 MICRONS)

$L$ - LESS THAH THREE BORE VOLUMES REHOVED BEFORE SAMPLIHG

MOO1 - UHFILTERED SAMPLE

H - SPIKE SAIPLE RECOVERY HOT HITHIN CONTROL LIMITS 
GROUNDWATER QUALITY DATA BY LOCATION

SOWP Rev. O Data

SITE: MONOI MONUMENT VALLEY

LOCATION: 0654

MORTH COORDINATE: 59351.4 FT

EAST COORDIHATE: $\quad 91063.4$ FT

$04 / 21 / 88$ TO $12 / 13 / 94$

REPORT DATE: 06/20/95

FORMATION OF COMPLETION: ALLUVIUM (AL)

HYDRAULIC FLOW RELATIONSHIP: CROSS GRADIENT (C)

\begin{tabular}{|c|c|c|c|c|c|c|c|c|}
\hline PARNAETER NAME & LOG DATE & $\mid \begin{array}{c}\text { SAMPLE } \\
\text { ID }\end{array}$ & $\begin{array}{l}\text { UHITS OF } \\
\text { MEASURE }\end{array}$ & PVI & $\begin{array}{l}\text { PARAMETER } \\
\text { VALUE }\end{array}$ & FLAGS & $\begin{array}{l}\text { OETECTION } \\
\text { LIMIT }\end{array}$ & $\begin{array}{l}\text { PARAMETER } \\
\text { UHCERTAINTY }\end{array}$ \\
\hline GROSS BETA (TOTAL) & $06 / 30 / 93$ & No01 & PCI/L & & 0 & & 4 & 4 \\
\hline IRON & $\begin{array}{l}06 / 09 / 89 \\
12 / 03 / 89 \\
06 / 21 / 90 \\
01 / 29 / 91 \\
06 / 02 / 91 \\
08 / 12 / 92 \\
02 / 18 / 93 \\
06 / 30 / 93\end{array}$ & $\begin{array}{l}0001 \\
0001 \\
0001 \\
0001 \\
0001 \\
0001 \\
0001 \\
0001\end{array}$ & $M G / L$ & $\begin{array}{l}< \\
< \\
< \\
< \\
<\end{array}$ & $\begin{array}{l}0.04 \\
0.03 \\
0.03 \\
0.06 \\
0.03 \\
0.1 \\
0.03 \\
0.03\end{array}$ & $\begin{array}{l}L \\
L \\
L \\
L \\
L \\
J\end{array}$ & $\begin{array}{l}0.03 \\
0.03 \\
0.03 \\
0.03 \\
0.03 \\
0.1 \\
0.03 \\
0.03\end{array}$ & $\begin{array}{l}- \\
: \\
- \\
- \\
- \\
-\end{array}$ \\
\hline IRON (TOTAL) & $06 / 30 / 93$ & NoO1 & MG/L & & 0.06 & J & 0.03 & - \\
\hline LEAD & $\begin{array}{l}06 / 09 / 89 \\
12 / 03 / 89 \\
06 / 21 / 90 \\
01 / 29 / 91 \\
06 / 02 / 99 \\
11 / 22 / 92\end{array}$ & $\begin{array}{l}0001 \\
0001 \\
0009 \\
0009 \\
0001 \\
0001\end{array}$ & MG/L & $\begin{array}{l}< \\
< \\
< \\
< \\
<\end{array}$ & $\begin{array}{l}0.01 \\
0.01 \\
0.01 \\
0.01 \\
0.005 \\
0.0015\end{array}$ & $\begin{array}{l}L \\
L \\
L \\
L \\
H\end{array}$ & $\begin{array}{l}0.01 \\
0.01 \\
0.01 \\
0.01 \\
0.005 \\
0.0015\end{array}$ & $\begin{array}{l}: \\
: \\
:\end{array}$ \\
\hline LEAD (TOTAL) & $11 / 22 / 92$ & NoOI & MG/L & $<$ & 0.0015 & & 0.0015 & - \\
\hline LEAD-210 & $91 / 22 / 92$ & 0001 & PCI/L & & 0.6 & & 2.0 & 1.2 \\
\hline LEAD-210 (TOTAL) & $11 / 22 / 92$ & NoOI & PCI/L & & 4.7 & & 0.3 & 0.8 \\
\hline MAGNESIUH & $\begin{array}{l}04 / 26 / 88 \\
06 / 09 / 89 \\
12 / 03 / 89 \\
06 / 21 / 90 \\
01 / 29 / 91 \\
06 / 02 / 91 \\
08 / 12 / 92 \\
02 / 18 / 93 \\
06 / 30 / 93\end{array}$ & $\begin{array}{l}0001 \\
0001 \\
0001 \\
0001 \\
0001 \\
0001 \\
0001 \\
0001 \\
0001\end{array}$ & MG/L & & $\begin{array}{l}17.6 \\
18.1 \\
18.5 \\
18.5 \\
20.5 \\
19.2 \\
18 \\
16.5 \\
17.4\end{array}$ & $\begin{array}{l}L \\
L \\
L \\
L \\
L \\
L\end{array}$ & $\begin{array}{l}0.001 \\
0.001 \\
0.001 \\
0.001 \\
0.001 \\
0.1 \\
5 \\
0.1 \\
0.1\end{array}$ & $\begin{array}{l}- \\
: \\
: \\
: \\
:\end{array}$ \\
\hline MAGNESIUM (TOTAL) & $06 / 30 / 93$ & NoO1: & MG/L & & 17.4 & & 0.1 & - \\
\hline MANGANESE & $\begin{array}{l}06 / 09 / 89 \\
12 / 03 / 89 \\
06 / 21 / 90 \\
01 / 29 / 91 \\
06 / 02 / 91 \\
08 / 12 / 92 \\
11 / 22 / 92 \\
02 / 18 / 93 \\
06 / 30 / 93\end{array}$ & $\begin{array}{l}0001 \\
0001 \\
0001 \\
0001 \\
0001 \\
0009 \\
0001 \\
0001 \\
0001\end{array}$ & $M G / L$ & & $\begin{array}{l}0.05 \\
0.04 \\
0.04 \\
0.04 \\
0.04 \\
0.038 \\
0.033 \\
0.03 \\
0.04\end{array}$ & $\begin{array}{l}L \\
L \\
L \\
L \\
L\end{array}$ & $\begin{array}{l}0.01 \\
0.01 \\
0.01 \\
0.01 \\
0.01 \\
0.01 \\
0.0015 \\
0.01 \\
0.01\end{array}$ & $\begin{array}{l}- \\
- \\
- \\
- \\
- \\
-\end{array}$ \\
\hline MANGAKESE (TOTAL) & $\begin{array}{l}11 / 22 / 92 \\
06 / 30 / 93\end{array}$ & $\begin{array}{l}\text { NoO9 } \\
\text { N001 }\end{array}$ & MG/L & & $\begin{array}{l}0.029 \\
0.03\end{array}$ & & $\begin{array}{l}0.0015 \\
0.01\end{array}$ & - \\
\hline MERCURY & $\begin{array}{l}06 / 09 / 89 \\
12 / 03 / 89 \\
06 / 21 / 90\end{array}$ & $\begin{array}{l}0001 \\
0009 \\
0001\end{array}$ & $M G / L$ & $\begin{array}{l}< \\
<\end{array}$ & $\begin{array}{l}0.0002 \\
0.0002 \\
0.0002\end{array}$ & $\begin{array}{l}L \\
L\end{array}$ & $\begin{array}{l}0.0002 \\
0.0002 \\
0.0002\end{array}$ & : \\
\hline
\end{tabular}

PARAMETER VALUE INDICATOR (PVI): < - LESS THAN DETECTION LIHIT

SNMPLE ID COOES:

OTHER PARAMETER VALUE FLAGS:

0001 - FILTERED SAMPLE (.45 MICRONS)

NOO - UHFILTERED SNMPLE

$J$ - ESTIHATED VALUE

l - LESS THAH THREE BORE VOLUMES REMOVED BEFORE SAMPLING

$H$ - POST-DIGEST SPIKE OUT OF CNTR LIM WHILE SAMP ABS < 50\% SPIKE 
GROUMDHATER QUALITY DATA BY LOCATIOH

SOWP ReV. O Data

SITE: MONO1 MONLMENT VALLEY

LOCATION: 0654

NORTH COORDINATE: 59351.4 FT

EAST COORDIHATE: 91063.4 FT

$04 / 21 / 88$ TO $12 / 13 / 94$

REPORT DATE: 06/20/95

FORMATION OF COMPLETIOH: ALLUVIUM (AL)

HYDRAULIC FLON RELATIONSHIP: CROSS GRNDIENT (C)

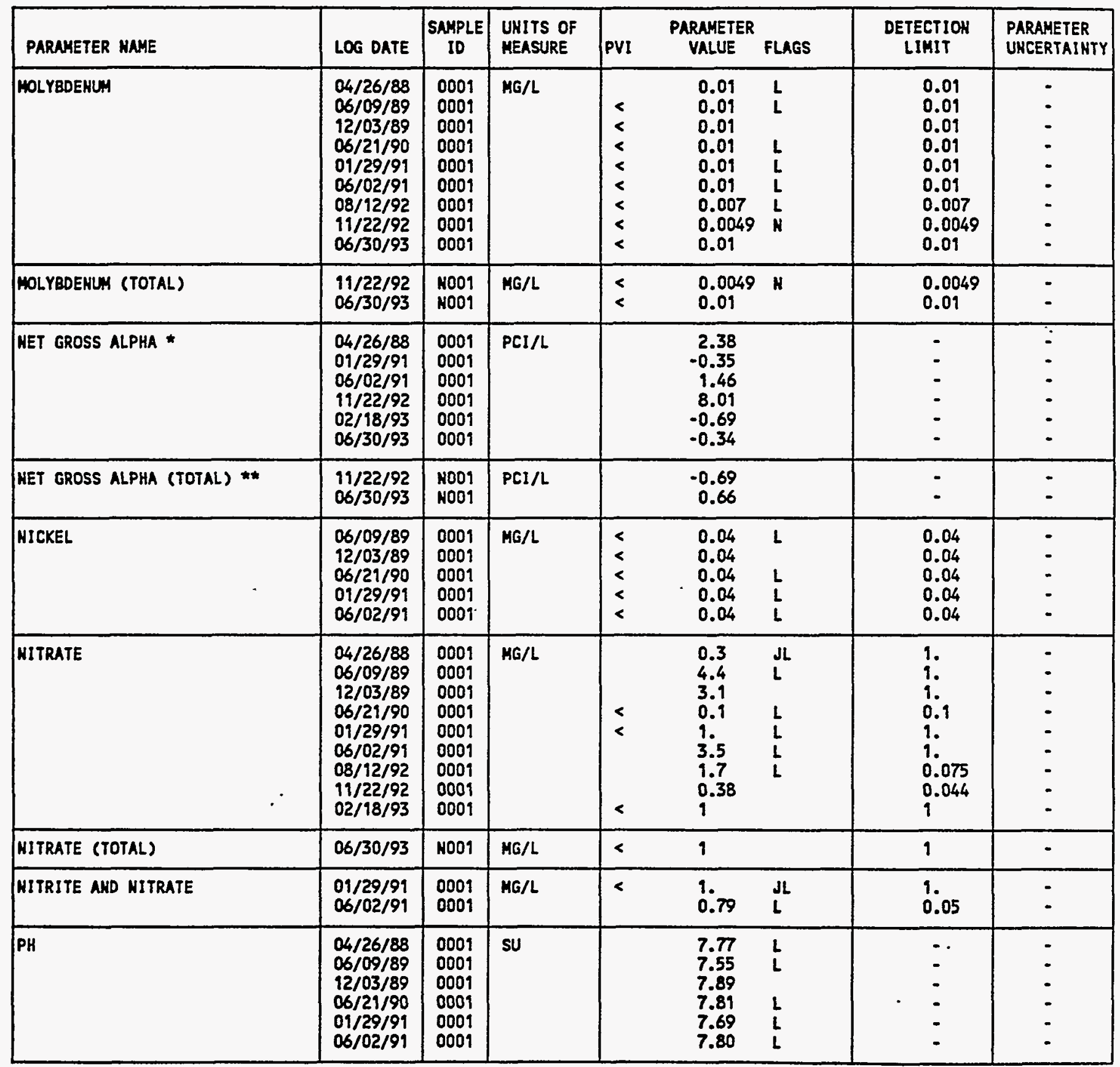

- HET GROSS ALPHA (GROSS ALPHA - LRANIUH) UITH 1 MG URANIUH = 686 PCI

* MET GROSS ALPHA (TOTAL) (TOTAL gROSS ALPHA - TOTAL URAHIUA)

PARNETER VALUE IMDICATOR (PVI): < - LESS THAN DETECTION LIMIT

OTHER PARAMETER VALUE FLAGS:

0001 - FILTERED SNAPLE (.45 MICRONS)

J - estIMATED VALUE

NOO1 - UNFILTERED SAMPLE

l. - LESS THAH THREE BORE VOLUHES REMOVED BEFORE SAMPLING

H - SPIKE SAYPLE RECOVERY NOT MITHIN CONTROL LIMITS 
GROWDWATER QUALITY DATA BY LOCATION

SOMP Rev. D Data

SITE: MONOI MONLMENT VALLEY

LOCATION: 0654

NORTH COORDINATE: $\quad 59351.4$ FT

EAST COORDINATE: 91063.4 FT

$04 / 21 / 88$ TO $12 / 13 / 94$

REPORT DATE: 06/20/95

FORMATION OF COMPLETION: ALLUVIUM (AL)

HYDRAULIC FLON RELATIOHSHIP: CROSS GRADIENT (C)

\begin{tabular}{|c|c|c|c|c|c|c|c|c|}
\hline PARAMETER MAME & LOG DATE & $\begin{array}{c}\text { SAMPLE } \\
\text { ID }\end{array}$ & $\begin{array}{l}\text { UNITS OF } \\
\text { MEASURE }\end{array}$ & PVI & $\begin{array}{l}\text { PARAMETER } \\
\text { VALUE }\end{array}$ & FLAGS & $\begin{array}{l}\text { DETECTION } \\
\text { LIMIT }\end{array}$ & $\begin{array}{l}\text { PARAMETER } \\
\text { UNCERTAINTY }\end{array}$ \\
\hline PH & $\begin{array}{l}08 / 12 / 92 \\
11 / 22 / 92 \\
02 / 18 / 93 \\
06 / 30 / 93\end{array}$ & $\begin{array}{l}0001 \\
0001 \\
0001 \\
\text { No01 }\end{array}$ & SU & & $\begin{array}{l}7.61 \\
7.74 \\
7.69 \\
7.86\end{array}$ & $\mathbf{L}$ & $\begin{array}{l}- \\
- \\
-\end{array}$ & . \\
\hline PHOSPHATE & $\begin{array}{l}06 / 09 / 89 \\
12 / 03 / 89 \\
06 / 21 / 90\end{array}$ & $\begin{array}{l}0001 \\
0001 \\
0001\end{array}$ & $M G / L$ & $<$ & $\begin{array}{l}0.6 \\
0.9 \\
0.1\end{array}$ & $\begin{array}{l}L \\
L\end{array}$ & $\begin{array}{l}0.1 \\
0.1 \\
0.1\end{array}$ & - \\
\hline PHOSPHATE (TOTAL) & $06 / 30 / 93$ & NoOI & MG/L & $<$ & 0.1 & & 0.1 & - \\
\hline POTASSIUM & $\begin{array}{l}04 / 26 / 88 \\
06 / 09 / 89 \\
12 / 03 / 89 \\
06 / 21 / 90 \\
01 / 29 / 91 \\
06 / 02 / 91 \\
08 / 12 / 92 \\
02 / 18 / 93 \\
06 / 30 / 93\end{array}$ & $\begin{array}{l}0001 \\
0001 \\
0001 \\
0001 \\
0001 \\
0001 \\
0001 \\
0001 \\
0001\end{array}$ & MG/L & & $\begin{array}{l}2.47 \\
2.5 \\
2.2 \\
2.5 \\
2.4 \\
2.6 \\
2.2 \\
2.3 \\
2.6\end{array}$ & $\begin{array}{l}L \\
L \\
L \\
L \\
L\end{array}$ & $\begin{array}{l}0.01 \\
0.01 \\
0.01 \\
0.01 \\
0.01 \\
0.01 \\
0.575 \\
0.1 \\
0.1\end{array}$ & $\begin{array}{l}- \\
\dot{-} \\
\dot{-} \\
\dot{-} \\
-\end{array}$ \\
\hline POTASSIUN (TOTAL) & $06 / 30 / 93$ & No01 & MG/L & & 2.4 & & 0.1 & - \\
\hline RADIUN-226 & $\begin{array}{l}06 / 09 / 89 \\
12 / 03 / 89 \\
06 / 21 / 90 \\
01 / 29 / 91 \\
06 / 02 / 91 \\
08 / 12 / 92 \\
11 / 22 / 92\end{array}$ & $\begin{array}{l}0001 \\
0001 \\
0001 \\
0001 \\
0001 \\
0001 \\
0001\end{array}$ & $\mathrm{PCl} / \mathrm{L}$ & & $\begin{array}{l}0.3 \\
0.3 \\
0.1 \\
0.2 \\
0.4 \\
0.07 \\
0.6\end{array}$ & $\begin{array}{l}L \\
L \\
L \\
L \\
L\end{array}$ & $\begin{array}{l}1 . \\
1 . \\
1 . \\
1 . \\
1 . \\
0.42 \\
0.3\end{array}$ & $\begin{array}{l}0.2 \\
0.2 \\
0.2 \\
0.2 \\
0.4 \\
0.04 \\
0.3\end{array}$ \\
\hline RADILA-226 (TOTAL) & $11 / 22 / 92$ & NoD1 & PCI/L & & 0.9 & & 0.3 & 0.4 \\
\hline RADILH-226 + RADILAS-228 & $\begin{array}{l}06 / 09 / 89 \\
12 / 03 / 89 \\
06 / 21 / 90 \\
01 / 29 / 91 \\
06 / 02 / 91 \\
08 / 12 / 92 \\
11 / 22 / 92\end{array}$ & $\begin{array}{l}0001 \\
0001 \\
0001 \\
0001 \\
0001 \\
0001 \\
0001\end{array}$ & $\mathrm{PCI} / \mathrm{L}$ & - & $\begin{array}{l}0.30 \\
0.30 \\
0.80 \\
1.30 \\
0.50 \\
0.47 \\
3.40\end{array}$ & & $\begin{array}{l}- \\
- \\
- \\
- \\
- \\
-\end{array}$ & $\begin{array}{l}- \\
- \\
- \\
- \\
-\end{array}$ \\
\hline RADIUA-226 + RADIUH-228 (TOTAL) & $11 / 22 / 92$ & No01 & $\mathrm{PCI} / \mathrm{L}$ & & 4.40 & & - & - \\
\hline RADIUS-228 & $\begin{array}{l}06 / 09 / 89 \\
12 / 03 / 89 \\
06 / 21 / 90 \\
01 / 29 / 91 \\
06 / 02 / 91 \\
08 / 12 / 92 \\
11 / 22 / 92\end{array}$ & $\begin{array}{l}0001 \\
0001 \\
0001 \\
0001 \\
0001 \\
0001 \\
0001\end{array}$ & PCI/L & & $\begin{array}{l}0.0 \\
0.0 \\
0.7 \\
1.1 \\
0.1 \\
0.4 \\
2.8\end{array}$ & $\begin{array}{l}L \\
L \\
L \\
J L \\
L\end{array}$ & $\begin{array}{l}1 . \\
1 . \\
1 . \\
1 . \\
1.0 \\
2.0 \\
2.5\end{array}$ & $\begin{array}{l}0.7 \\
1.9 \\
0.9 \\
0.8 \\
1.5 \\
1.0 \\
1.7\end{array}$ \\
\hline RADIUH-228 (TOTAL) & $11 / 22 / 92$ & KOOI & $\mathrm{PCI} / \mathrm{L}$ & & 3.5 & & 2.0 & 1.5 \\
\hline REDOX POTENTIAL & $11 / 22 / 92$ & 0001 & mNOLTS & & 111.7 & & - & $\cdot$ \\
\hline
\end{tabular}

PARAMETER VALUE INDICATOR (PVI): < - LESS THAN DETECTION LIMIT

SAMPLE ID COOES:

OTHER PARAMETER VALUE FLAGS:

- DUPLICATE aMALYSIS hOT MITHIN CONTROL LIMITS

0001 - FILTERED SAMPLE (.45 MICRONS)

$J$ - eSTIMATED VALUE

L - LESS THAN THREE BORE VOLLMES REMOVED BEFORE SAMPLIHG 
GROUNDHATER QUALITY DATA BY LOCATION

SOWP ReV. O Data

SITE: MONOI MONUNENT VALLEY

LOCATION: 0654

HORTH COORDINATE: 59351.4 FT

EAST COORDINATE: 91063.4 FT

$04 / 21 / 88$ TO $12 / 13 / 94$

REPORT DATE: 06/20/95

FORMATION OF COHPLETION: ALLUVIUM (AL)

HYDRAULIC FLOH RELATIONSHIP: CROSS GRADIENT (C)

\begin{tabular}{|c|c|c|c|c|c|c|c|c|}
\hline PARAMETER NAME & LOG DATE & $\underset{\text { ID }}{\text { SAMPLE }}$ & $\begin{array}{l}\text { UNITS OF } \\
\text { MEASURE }\end{array}$ & PVI & $\begin{array}{l}\text { PARAMETER } \\
\text { VALUE }\end{array}$ & FLAGS & $\begin{array}{l}\text { DETECTION } \\
\text { LIMIT }\end{array}$ & $\begin{array}{l}\text { PARAMETER } \\
\text { UNCERTAINTY }\end{array}$ \\
\hline REDOX POTENTIAL & $06 / 30 / 93$ & N001 & mVOLTS & \multicolumn{3}{|c|}{70} & - & - \\
\hline SELEHIUH & $\begin{array}{l}04 / 26 / 88 \\
06 / 09 / 89 \\
12 / 03 / 89 \\
06 / 21 / 90 \\
01 / 29 / 91 \\
06 / 02 / 91 \\
08 / 12 / 92 \\
11 / 22 / 92\end{array}$ & $\begin{array}{l}0001 \\
0001 \\
0001 \\
0001 \\
0001 \\
0001 \\
0001 \\
0001\end{array}$ & $M G / L$ & $\begin{array}{l}< \\
< \\
< \\
< \\
< \\
< \\
<\end{array}$ & $\begin{array}{l}0.005 \\
0.005 \\
0.005 \\
0.005 \\
0.005 \\
0.005 \\
0.005 \\
0.0015\end{array}$ & $\begin{array}{l}L \\
L \\
L \\
L \\
J L \\
H L \\
N H\end{array}$ & $\begin{array}{l}0.005 \\
0.005 \\
0.005 \\
0.005 \\
0.005 \\
0.005 \\
0.005 \\
0.0015\end{array}$ & $\begin{array}{l}- \\
- \\
- \\
- \\
-\end{array}$ \\
\hline SELEHIUM (TOTAL) & $11 / 22 / 92$ & N001 & $M G / L$ & $<$ & \multicolumn{2}{|l|}{0.0015} & 0.0015 & - \\
\hline SILICA - SIOZ & $\begin{array}{l}06 / 09 / 89 \\
12 / 03 / 89 \\
06 / 21 / 90 \\
01 / 29 / 91 \\
06 / 02 / 91\end{array}$ & $\begin{array}{l}0001 \\
0001 \\
0001 \\
0001 \\
0001\end{array}$ & MG/L & & $\begin{array}{l}13 . \\
13.0 \\
13 . \\
13 . \\
13.6\end{array}$ & $\begin{array}{l}L \\
L \\
L \\
L\end{array}$ & $\begin{array}{l}2 . \\
2 . \\
2 . \\
2 . \\
0.1\end{array}$ & $\begin{array}{l}- \\
- \\
-\end{array}$ \\
\hline SILICA - SIOL (TOTAL) & $06 / 30 / 93$ & NOO1 & $M G / L$ & \multicolumn{3}{|c|}{12.4} & 0.5 & - \\
\hline SILVER & $\begin{array}{l}06 / 09 / 89 \\
12 / 03 / 89 \\
06 / 21 / 90 \\
01 / 29 / 91 \\
06 / 02 / 91\end{array}$ & $\begin{array}{l}0001 \\
0001 \\
0001 \\
0001 \\
0001\end{array}$ & $M G / L$ & $\begin{array}{l}< \\
< \\
< \\
< \\
<\end{array}$ & $\begin{array}{l}0.01 \\
0.01 \\
0.01 \\
0.01 \\
0.01\end{array}$ & $\begin{array}{l}L \\
L \\
L \\
L\end{array}$ & $\begin{array}{l}0.01 \\
0.01 \\
0.01 \\
0.01 \\
0.01\end{array}$ & $\begin{array}{l}- \\
- \\
- \\
-\end{array}$ \\
\hline SODIUM & $\begin{array}{l}04 / 26 / 88 \\
06 / 09 / 89 \\
12 / 03 / 89 \\
06 / 21 / 90 \\
01 / 29 / 91 \\
06 / 02 / 91 \\
08 / 12 / 92 \\
02 / 18 / 93 \\
06 / 30 / 93\end{array}$ & $\begin{array}{l}0001 \\
0001 \\
0001 \\
0001 \\
0001 \\
0001 \\
0001 \\
0001 \\
0001\end{array}$ & $M G / L$ & & $\begin{array}{l}23.4 \\
22.2 \\
21 . \\
22.9 \\
23.5 \\
24 . \\
24 \\
24.8 \\
24\end{array}$ & $\begin{array}{l}L \\
L \\
L \\
L \\
L \\
L\end{array}$ & $\begin{array}{l}0.002 \\
0.002 \\
0.002 \\
0.002 \\
0.002 \\
1 . \\
5 \\
0.1 \\
1\end{array}$ & $\begin{array}{l}- \\
- \\
- \\
- \\
- \\
-\end{array}$ \\
\hline SODIUM (TOTAL) & $06 / 30 / 93$ & H0O1 & $M G / L$ & & 24 & & 1 & - \\
\hline SPECIFIC CONDUCTANCE & $\begin{array}{l}04 / 26 / 88 \\
06 / 09 / 89 \\
12 / 03 / 89 \\
06 / 21 / 90 \\
01 / 29 / 91 \\
06 / 02 / 91 \\
08 / 12 / 92 \\
11 / 22 / 92 \\
02 / 18 / 93 \\
06 / 30 / 93\end{array}$ & $\begin{array}{l}0001 \\
0001 \\
0001 \\
0001 \\
0001 \\
0001 \\
0001 \\
0001 \\
0001 \\
\text { N001 }\end{array}$ & UMHO/CH & & $\begin{array}{l}300 . \\
298 . \\
325 . \\
335 . \\
423 . \\
430 \\
365 \\
340 \\
347 \\
370\end{array}$ & $\begin{array}{l}L \\
L \\
L \\
L \\
L \\
L\end{array}$ & $\begin{array}{l}- \\
- \\
- \\
- \\
- \\
- \\
-\end{array}$ & $\begin{array}{l}- \\
- \\
- \\
- \\
- \\
- \\
-\end{array}$ \\
\hline STRONTIUM & $\begin{array}{l}06 / 21 / 90 \\
01 / 29 / 91\end{array}$ & $\begin{array}{l}0001 \\
0001\end{array}$ & MG/L & & $\begin{array}{l}0.39 \\
0.4\end{array}$ & ${ }^{*} L$ & $\begin{array}{l}0.1 \\
0.1\end{array}$ & - \\
\hline
\end{tabular}

PARAMETER VALUE IMDICATOR (PVI): < - LESS THAN DETECTION LIMIT

SAMPLE ID CODES:

OTHER PARAMETER VALUE FLAGS:

0001 - FILTERED SAMPLE (.45 MICRONS)

* - DUPLICATE ANALYSIS NOT WITHIN CONTROL LIMITS

N001 - UNFILTERED SAMPLE

J - ESTIMATED VALUE

L - LESS THAN THREE BORE VOLUMES REMOVED BEFORE SAMPLING

$N$ - SPIKE SAMPLE RECOVERY NOT HITHIN CONTROL LIMITS

$W$ - POST-DIGEST SPIKE OUT OF CHTR LIM WHILE SAMP ABS < 50\% SPIKE 
GROUNDHATER QUALITY DATA BY LOCATION

SOWP ReV. O Data

SITE: MONOI MONUMENT VALLEY

LOCATION: 0654

HORTH COORDINATE: 59351.4 FT

EAST COOROINATE: $\quad 91063.4$ FT

$04 / 21 / 88$ TO $12 / 13 / 94$

REPORT DATE: 06/20/95

FORHATION OF COMPLETION: ALLUVIUH (AL)

HYDRAULIC FLON RELATIONSHIP: CROSS GRADIENT (C)

\begin{tabular}{|c|c|c|c|c|c|c|c|c|}
\hline PARAMETER NAME & LOG DATE & $\begin{array}{c}\text { SAMPLE } \\
\text { ID }\end{array}$ & $\begin{array}{l}\text { UNITS OF } \\
\text { MEASURE }\end{array}$ & PVI & $\begin{array}{l}\text { PARAMETER } \\
\text { VALUE }\end{array}$ & FLAGS & $\begin{array}{l}\text { DETECTION } \\
\text { LIHIT }\end{array}$ & $\begin{array}{l}\text { PARAMETER } \\
\text { UNCERTAINTY }\end{array}$ \\
\hline STRONTIUM & $\begin{array}{l}06 / 02 / 91 \\
08 / 12 / 92 \\
11 / 22 / 92 \\
06 / 30 / 93\end{array}$ & $\begin{array}{l}0001 \\
0001 \\
0001 \\
0001\end{array}$ & $M G / L$ & & $\begin{array}{l}0.40 \\
0.41 \\
0.43 \\
0.38\end{array}$ & $\begin{array}{l}\mathrm{L} \\
\mathrm{L} \\
+\end{array}$ & $\begin{array}{l}0.01 \\
0.2 \\
0.00035 \\
0.01\end{array}$ & $\begin{array}{l}- \\
- \\
-\end{array}$ \\
\hline STRONTIUN (TOTAL) & $\begin{array}{l}11 / 22 / 92 \\
06 / 30 / 93\end{array}$ & $\begin{array}{l}\text { No01 } \\
\text { N001 }\end{array}$ & $M G / L$ & & $\begin{array}{l}0.38 \\
0.38\end{array}$ & + & $\begin{array}{l}0.00035 \\
0.01\end{array}$ & - \\
\hline SULFATE & $\begin{array}{l}04 / 26 / 88 \\
06 / 09 / 89 \\
12 / 03 / 89 \\
06 / 21 / 90 \\
01 / 29 / 91 \\
06 / 02 / 91 \\
08 / 12 / 92 \\
11 / 22 / 92 \\
02 / 18 / 93\end{array}$ & $\begin{array}{l}0001 \\
0001 \\
0001 \\
0001 \\
0001 \\
0001 \\
0001 \\
0001 \\
0001\end{array}$ & MG/L & & $\begin{array}{l}27 . \\
40 . \\
41 . \\
38.3 \\
40.3 \\
40 . \\
42 \\
43 \\
48\end{array}$ & $\begin{array}{l}\mathbf{L} \\
\mathbf{L} \\
\mathbf{L} \\
\mathbf{L} \\
\mathbf{L} \\
\mathbf{L}\end{array}$ & $\begin{array}{l}0.1 \\
0.1 \\
0.1 \\
0.1 \\
0.1 \\
0.1 \\
0.059 \\
1.0 \\
1\end{array}$ & $\begin{array}{l}- \\
- \\
- \\
- \\
- \\
- \\
-\end{array}$ \\
\hline SULFATE (TOTAL) & $\begin{array}{l}11 / 22 / 92 \\
06 / 30 / 93\end{array}$ & $\begin{array}{l}\text { No01 } \\
\text { NO01 }\end{array}$ & MG/L & & $\begin{array}{l}43 \\
42\end{array}$ & & 1.0 & - \\
\hline SULFIDE & $\begin{array}{l}06 / 09 / 89 \\
12 / 03 / 89 \\
01 / 29 / 91 \\
06 / 02 / 91\end{array}$ & $\begin{array}{l}0001 \\
0001 \\
0001 \\
0001\end{array}$ & MG/L & $\begin{array}{l}< \\
< \\
<\end{array}$ & $\begin{array}{l}0.1 \\
0.4 \\
0.1 \\
1 .\end{array}$ & $\begin{array}{l}L \\
\text { JL } \\
\text { IJL }\end{array}$ & $\begin{array}{l}0.1 \\
0.1 \\
0.1 \\
1 .\end{array}$ & $\begin{array}{l}- \\
- \\
-\end{array}$ \\
\hline TEMPERATURE & $\begin{array}{l}04 / 26 / 88 \\
06 / 09 / 89 \\
12 / 03 / 89 \\
06 / 21 / 90 \\
01 / 29 / 91 \\
06 / 02 / 91 \\
08 / 12 / 92 \\
11 / 22 / 92 \\
02 / 18 / 93 \\
06 / 30 / 93\end{array}$ & $\begin{array}{l}0001 \\
0001 \\
0001 \\
0001 \\
0001 \\
0001 \\
0001 \\
0001 \\
0001 \\
\text { No01 }\end{array}$ & C - DEGREE & & $\begin{array}{l}15.5 \\
17.0 \\
15.5 \\
16.0 \\
14.0 \\
15.7 \\
17.4 \\
14.8 \\
15.3 \\
18.9\end{array}$ & $\begin{array}{l}L \\
L \\
L \\
L \\
L \\
L\end{array}$ & $\begin{array}{l}- \\
- \\
- \\
- \\
- \\
- \\
-\end{array}$ & $\begin{array}{l}- \\
- \\
- \\
- \\
- \\
- \\
-\end{array}$ \\
\hline THALLIUM & $\begin{array}{l}06 / 09 / 89 \\
12 / 03 / 89 \\
06 / 21 / 90 \\
01 / 29 / 91 \\
06 / 02 / 91\end{array}$ & $\begin{array}{l}0001 \\
0001 \\
0001 \\
0001 \\
0001\end{array}$ & MG/L & $\begin{array}{l}< \\
< \\
< \\
< \\
<\end{array}$ & $\begin{array}{l}0.01 \\
0.1 \\
0.01 \\
0.01 \\
0.01\end{array}$ & $\begin{array}{l}L \\
L \\
L \\
L\end{array}$ & $\begin{array}{l}0.01 \\
0.1 \\
0.01 \\
0.01 \\
0.01\end{array}$ & $\begin{array}{l}- \\
- \\
- \\
-\end{array}$ \\
\hline THORIUM-230 & $\begin{array}{l}01 / 29 / 91 \\
06 / 02 / 91\end{array}$ & $\begin{array}{l}0001 \\
0001\end{array}$ & $\mathrm{PCI} / \mathrm{L}$ & & $\begin{array}{l}0.2 \\
0.5\end{array}$ & $\begin{array}{l}L \\
L\end{array}$ & 1. & $\begin{array}{l}0.4 \\
0.3\end{array}$ \\
\hline TIN & $\begin{array}{l}06 / 09 / 89 \\
12 / 03 / 89 \\
06 / 21 / 90 \\
01 / 29 / 91 \\
06 / 02 / 91\end{array}$ & $\begin{array}{l}0001 \\
0001 \\
0001 \\
0001 \\
0001\end{array}$ & $M G / L$ & $\begin{array}{l}< \\
< \\
< \\
< \\
<\end{array}$ & $\begin{array}{l}0.005 \\
0.005 \\
0.005 \\
0.005 \\
0.01\end{array}$ & $\begin{array}{l}L \\
L \\
L \\
I L\end{array}$ & $\begin{array}{l}0.005 \\
0.005 \\
0.005 \\
0.005 \\
0.01\end{array}$ & $\begin{array}{l}- \\
- \\
-\end{array}$ \\
\hline TOTAL DISSOLVED SOLIDS & $04 / 26 / 88$ & 0001 & MG/L & & 241. & $\mathbf{L}$ & 10. & - \\
\hline
\end{tabular}

PARAMETER VALUE INDICATOR (PVI): < - LESS THAN DETECTION LIMIT

SAMPLE ID CODES:

OTHER PARAMETER VALUE FLAGS:

0001 - FILTERED SAMPLE (.45 MICRONS)

+ - CORRELATION COEFFICIENT FOR MSA $<0.995$

NOD1 - UMFILTERED SAMPLE

1 - INCREASED DETECTION LIMIT DUE TO REQUIRED DILUTION

J - ESTIMATED VALUE

L - LESS THAN THREE BORE VOLUMES REMOVED BEFORE SAMPLING 
GROUNDWATER QUALITY DATA BY LOCATION

SOWP ReV. 0 Data

SITE: NONO1 HONUMENT VALLEY

LOCATION: 0654

NORTH COOROINATE: 59351.4 FT

EAST COOROINATE: $\quad 91063.4$ FT

$04 / 21 / 88$ TO $12 / 13 / 94$

REPORT DATE: 06/20/95

FORMATION OF COMPLETION: ALLUVIUM (AL)

HYDRAULIC FLOH RELATIONSHIP: CROSS GRADIENT (C)

\begin{tabular}{|c|c|c|c|c|c|c|c|c|}
\hline PARAMETER NAME & LOG DATE & $\begin{array}{c}\text { SAMPLE } \\
\text { ID }\end{array}$ & $\begin{array}{l}\text { UNITS OF } \\
\text { MEASURE }\end{array}$ & PVI & $\begin{array}{l}\text { PARAHETER } \\
\text { VALUE }\end{array}$ & FLAGS & $\begin{array}{l}\text { DETECTION } \\
\text { LIHIT }\end{array}$ & $\begin{array}{l}\text { PARAMETER } \\
\text { UNCERTAINTY }\end{array}$ \\
\hline TOTAL DISSOLVED SOLIDS & $\begin{array}{l}06 / 09 / 89 \\
12 / 03 / 89 \\
06 / 21 / 90 \\
01 / 29 / 91 \\
06 / 02 / 91 \\
08 / 12 / 92 \\
02 / 18 / 93\end{array}$ & $\begin{array}{l}0001 \\
0001 \\
0001 \\
0001 \\
0001 \\
0001 \\
0001\end{array}$ & MG/L & & $\begin{array}{l}227 . \\
244 . \\
254 . \\
204 . \\
252 . \\
280 \\
230\end{array}$ & $\begin{array}{l}L \\
L \\
L \\
L \\
L\end{array}$ & $\begin{array}{l}10 . \\
10 . \\
10 . \\
10 . \\
10 . \\
42 \\
10\end{array}$ & $\begin{array}{l}- \\
- \\
- \\
- \\
- \\
-\end{array}$ \\
\hline TOTAL DISSOLVED SOLIDS (TOTAL) & $06 / 30 / 93$ & NoO1 & $M G / L$ & & 230 & H & 10 & - \\
\hline TOTAL ORGANIC CARBOH & $\begin{array}{l}04 / 26 / 88 \\
01 / 29 / 91 \\
06 / 02 / 91 \\
08 / 12 / 92\end{array}$ & $\begin{array}{l}0001 \\
0001 \\
0001 \\
0001\end{array}$ & MG/L & $\begin{array}{l}< \\
< \\
<\end{array}$ & $\begin{array}{c}46.1 \\
1 . \\
1 . \\
1.0\end{array}$ & $\begin{array}{l}L \\
J L \\
L \\
L\end{array}$ & $\begin{array}{l}1 . \\
1 . \\
1 . \\
1.0\end{array}$ & $\begin{array}{l}- \\
- \\
-\end{array}$ \\
\hline URANIUM & $\begin{array}{l}04 / 26 / 88 \\
06 / 09 / 89 \\
12 / 03 / 89 \\
06 / 21 / 90 \\
01 / 29 / 91 \\
06 / 02 / 91 \\
11 / 22 / 92 \\
02 / 18 / 93 \\
06 / 30 / 93\end{array}$ & $\begin{array}{l}0001 \\
0001 \\
0001 \\
0001 \\
0001 \\
0001 \\
0001 \\
0001 \\
0001\end{array}$ & MG/L & $\begin{array}{l}< \\
<\end{array}$ & $\begin{array}{l}0.0009 \\
0.0037 \\
0.001 \\
0.0018 \\
0.0008 \\
0.001 \\
0.001 \\
0.001 \\
0.001\end{array}$ & $\begin{array}{l}\text { JL } \\
\mathbf{L} \\
\mathbf{J} \\
\mathrm{J} L \\
\mathbf{L} \\
\mathbf{L}\end{array}$ & $\begin{array}{l}0.003 \\
0.003 \\
0.003 \\
0.003 \\
0.0003 \\
0.001 \\
0.001 \\
0.001 \\
0.001\end{array}$ & $\begin{array}{l}- \\
- \\
- \\
- \\
- \\
- \\
- \\
-\end{array}$ \\
\hline URANIUH (TOTAL) & $\begin{array}{l}11 / 22 / 92 \\
06 / 30 / 93\end{array}$ & $\begin{array}{l}\text { No01 } \\
\text { N001 }\end{array}$ & $M G / L$ & $<$ & $\begin{array}{l}0.001 \\
0.001\end{array}$ & & $\begin{array}{l}0.001 \\
0.001\end{array}$ & - \\
\hline VANADIUH & $\begin{array}{l}06 / 09 / 89 \\
12 / 03 / 89 \\
06 / 21 / 90 \\
01 / 29 / 91 \\
06 / 02 / 91 \\
08 / 12 / 92 \\
11 / 22 / 92 \\
06 / 30 / 93\end{array}$ & $\begin{array}{l}0001 \\
0001 \\
0001 \\
0001 \\
0001 \\
0001 \\
0001 \\
0001\end{array}$ & MG/L & $\begin{array}{l}< \\
< \\
< \\
< \\
< \\
< \\
< \\
<\end{array}$ & $\begin{array}{l}0.01 \\
0.01 \\
0.01 \\
0.01 \\
0.01 \\
0.05 \\
0.0019 \\
0.01\end{array}$ & $\begin{array}{l}\mathbf{L} \\
\mathbf{L} \\
\mathbf{L} \\
\mathbf{L} \\
\mathbf{L}\end{array}$ & $\begin{array}{l}0.01 \\
0.01 \\
0.01 \\
0.01 \\
0.01 \\
0.05 \\
0.0019 \\
0.01\end{array}$ & $\begin{array}{l}- \\
- \\
- \\
- \\
- \\
- \\
-\end{array}$ \\
\hline VANADIUM (TOTAL) & $\begin{array}{l}11 / 22 / 92 \\
06 / 30 / 93\end{array}$ & $\begin{array}{l}\text { NO01 } \\
\text { N001 }\end{array}$ & $M G / L$ & $\begin{array}{l}< \\
<\end{array}$ & $\begin{array}{l}0.0019 \\
0.01\end{array}$ & & $\begin{array}{l}0.0019 \\
0.01\end{array}$ & - \\
\hline ZINC & $\begin{array}{l}06 / 09 / 89 \\
12 / 03 / 89 \\
06 / 21 / 90 \\
01 / 29 / 91 \\
06 / 02 / 91 \\
08 / 12 / 92 \\
11 / 22 / 92 \\
06 / 30 / 93\end{array}$ & $\begin{array}{l}0001 \\
0001 \\
0001 \\
0001 \\
0001 \\
0001 \\
0001 \\
0001\end{array}$ & MG/L & $\begin{array}{l}< \\
< \\
< \\
< \\
< \\
< \\
<\end{array}$ & $\begin{array}{l}0.005 \\
0.005 \\
0.005 \\
0.005 \\
0.005 \\
0.02 \\
0.0010 \\
0.026\end{array}$ & $\begin{array}{l}L \\
L \\
L \\
L \\
L \\
J\end{array}$ & $\begin{array}{l}0.005 \\
0.005 \\
0.005 \\
0.005 \\
0.005 \\
0.02 \\
0.0010 \\
0.005\end{array}$ & $\begin{array}{l}- \\
- \\
- \\
- \\
- \\
- \\
-\end{array}$ \\
\hline ZINC (TOTAL) & $\begin{array}{l}11 / 22 / 92 \\
06 / 30 / 93\end{array}$ & $\begin{array}{l}\text { No01 } \\
\text { N001 }\end{array}$ & $M G / L$ & & $\begin{array}{l}0.0030 \\
0.033\end{array}$ & $\mathbf{J}$ & $\begin{array}{l}0.0010 \\
0.005\end{array}$ & - \\
\hline
\end{tabular}

PARAMETER VALUE INDICATOR (PVI): < - LESS THAN DETECTION LIMIT

OTHER PARAMETER VALUE FLAGS:

SAMPLE ID CODES:

0001 - FILTERED SAMPLE (.45 MICRONS)

H - HOLD TIME EXPIRED, VALUE SUSPECT

NO01 - UNFILTERED SAMPLE

- ESTIMATED VALUE

L - LESS THAN THREE BORE VOLUMES REMOVED BEFORE SAMPLING 
GROUNDHATER OUALITY DATA BY LOCATION

SOWP REV. 0 Data

SITE: MONOY MONUNENT VALLEY

LOCATION: 0655

MORTH COORDINATE: 59734.6 FT

EAST COORDINATE: 88624.1 FT

$04 / 21 / 88$ TO $12 / 13 / 94$

REPORT DATE: 06/20/95

FORMATION OF COAPLETIOH: ALLUVIUM (AL)

HYDRAULIC FLOW RELATIONSHIP: DONH GRADIENT (D)

\begin{tabular}{|c|c|c|c|c|c|c|c|c|}
\hline PARAHETER NAME & LOG DATE & $\mid \begin{array}{c}\text { SAMPLE } \\
\text { ID }\end{array}$ & $\begin{array}{l}\text { UNITS OF } \\
\text { MEASURE }\end{array}$ & PVI & $\begin{array}{l}\text { PARAMETER } \\
\text { VALUE }\end{array}$ & FLAGS & $\begin{array}{l}\text { DETECTION } \\
\text { LIMIT }\end{array}$ & $\begin{array}{l}\text { PARAMETER } \\
\text { UNCERTAIHTY }\end{array}$ \\
\hline ALKALIHITY & $\begin{array}{l}04 / 21 / 88 \\
04 / 21 / 88 \\
04 / 21 / 88 \\
04 / 21 / 88 \\
04 / 21 / 88 \\
06 / 09 / 89 \\
06 / 09 / 89 \\
06 / 09 / 89 \\
06 / 09 / 89 \\
06 / 09 / 89 \\
12 / 03 / 89 \\
12 / 03 / 89 \\
12 / 03 / 89 \\
12 / 03 / 89 \\
12 / 03 / 89 \\
06 / 25 / 90 \\
01 / 29 / 91 \\
06 / 02 / 91 \\
02 / 22 / 92 \\
08 / 13 / 92 \\
11 / 19 / 92 \\
02 / 19 / 93 \\
06 / 26 / 93 \\
04 / 24 / 94\end{array}$ & $\begin{array}{l}0001 \\
0002 \\
0003 \\
0004 \\
0005 \\
0001 \\
0002 \\
0003 \\
0004 \\
0005 \\
0001 \\
0002 \\
0003 \\
0004 \\
0005 \\
0001 \\
0001 \\
0001 \\
0001 \\
0001 \\
0001 \\
0001 \\
N 001 \\
N 001\end{array}$ & MG/L CACO3 & & $\begin{array}{l}358 . \\
358 . \\
358 . \\
358 . \\
358 . \\
325 . \\
325 . \\
325 . \\
325 . \\
325 . \\
325 . \\
325 . \\
325 . \\
325 . \\
325 . \\
347 . \\
299 . \\
356 . \\
313 \\
282 \\
300 \\
293 \\
296 \\
287\end{array}$ & $\begin{array}{l}L \\
L \\
L \\
L \\
L \\
L \\
L \\
L \\
L \\
L\end{array}$ & $\begin{array}{l}- \\
- \\
i \\
- \\
i \\
- \\
- \\
- \\
- \\
- \\
- \\
- \\
- \\
- \\
- \\
- \\
- \\
-\end{array}$ & $\begin{array}{l}- \\
- \\
- \\
- \\
\vdots \\
- \\
\vdots \\
\vdots \\
\vdots \\
- \\
- \\
- \\
- \\
- \\
- \\
-\end{array}$ \\
\hline ALUALHUM & $\begin{array}{l}06 / 09 / 89 \\
06 / 09 / 89 \\
06 / 09 / 89 \\
06 / 09 / 89 \\
06 / 09 / 89 \\
12 / 03 / 89 \\
12 / 03 / 89 \\
12 / 03 / 89 \\
12 / 03 / 89 \\
12 / 03 / 89 \\
06 / 25 / 90 \\
01 / 29 / 91 \\
06 / 02 / 91 \\
02 / 22 / 92 \\
08 / 13 / 92\end{array}$ & $\begin{array}{l}0001 \\
0002 \\
0003 \\
0004 \\
0005 \\
0001 \\
0002 \\
0003 \\
0004 \\
0005 \\
0001 \\
0001 \\
0001 \\
0001 \\
0009\end{array}$ & $M G / L$ & $\begin{array}{l}< \\
< \\
< \\
< \\
< \\
< \\
< \\
< \\
< \\
< \\
< \\
< \\
< \\
< \\
<\end{array}$ & $\begin{array}{l}0.1 \\
0.1 \\
0.1 \\
0.1 \\
0.1 \\
0.1 \\
0.1 \\
0.1 \\
0.1 \\
0.1 \\
0.1 \\
0.1 \\
0.05 \\
0.05 \\
0.2\end{array}$ & $\begin{array}{l} \\
\star L\end{array}$ & $\begin{array}{l}0.1 \\
0.1 \\
0.1 \\
0.1 \\
0.1 \\
0.1 \\
0.1 \\
0.1 \\
0.1 \\
0.1 \\
0.1 \\
0.1 \\
0.05 \\
0.05 \\
0.2\end{array}$ & $\begin{array}{l}- \\
- \\
- \\
- \\
- \\
- \\
- \\
- \\
- \\
-\end{array}$ \\
\hline
\end{tabular}

PARAMETER VALUE IMDICATOR (PVI): < - LESS THAN DETECTION LIMIT

SAMPLE ID COOES:

OTHER PARAMETER VALUE FLAgS:

- - DUPLICATE aMalysis hot hithIn CONTROL limits

L - LESS THAN THREE BORE VOLUMES REHOVED BEFORE SAMPLIMG 
GROUNDWATER QUALITY DATA BY LOCATION

SOMP ReV. O Data

SITE: MONO MONLMENT VALLEY

LOCATION: 0655

NORTH COORDINATE: 59754.6 FT

EAST COORDINATE: 88624.1 FT

$04 / 21 / 88$ TO $12 / 13 / 94$

REPORT DATE: 06/20/95

FORMATION OF COHPLETION: ALLUVIUN (AL)

HYDRAULIC FLON RELATIONSHIP: DOWN GRADIENT (D)

\begin{tabular}{|c|c|c|c|c|c|c|c|c|}
\hline PARAMETER MAME & LOG DATE & $\underset{\text { ID }}{\text { SAMPLE }}$ & $\begin{array}{l}\text { UNITS OF } \\
\text { MEASURE }\end{array}$ & PVI & $\begin{array}{l}\text { PARAMETER } \\
\text { VALUE }\end{array}$ & FLAGS & $\begin{array}{l}\text { DETECTION } \\
\text { LIMIT }\end{array}$ & $\begin{array}{l}\text { PARAMETER } \\
\text { UKCERTAIHTY }\end{array}$ \\
\hline AmENIUN & $\begin{array}{l}12 / 03 / 89 \\
12 / 03 / 89 \\
06 / 25 / 90 \\
01 / 29 / 91 \\
06 / 02 / 91 \\
02 / 22 / 92 \\
02 / 19 / 93\end{array}$ & $\begin{array}{l}0004 \\
0005 \\
0001 \\
0001 \\
0001 \\
0001 \\
0001\end{array}$ & MG/L & \multicolumn{3}{|c|}{$\begin{array}{l}340 . \\
330 . \\
297 . \\
239 . \\
43.4 \\
184 . \\
245\end{array}$} & $\begin{array}{c}0.1 \\
0.1 \\
0.1 \\
0.1 \\
0.1 \\
10 . \\
0.1\end{array}$ & $\begin{array}{l}: \\
: \\
: \\
:\end{array}$ \\
\hline (AмонIUH (TOTAL) & $\begin{array}{l}06 / 26 / 93 \\
04 / 24 / 94\end{array}$ & $\begin{array}{l}\text { N001 } \\
\text { N001 }\end{array}$ & $M G / L$ & \multicolumn{3}{|c|}{$\begin{array}{l}189 \\
154\end{array}$} & $\begin{array}{l}0.1 \\
0.1\end{array}$ & : \\
\hline АКТ IHOAY & $\begin{array}{l}06 / 09 / 89 \\
06 / 09 / 89 \\
06 / 09 / 89 \\
06 / 09 / 89 \\
06 / 09 / 89 \\
12 / 03 / 89 \\
12 / 03 / 89 \\
12 / 03 / 89 \\
12 / 03 / 89 \\
12 / 03 / 89 \\
06 / 25 / 90 \\
01 / 29 / 91 \\
06 / 02 / 91 \\
02 / 22 / 92 \\
08 / 13 / 92\end{array}$ & $\begin{array}{l}0001 \\
0002 \\
0003 \\
0004 \\
0005 \\
0001 \\
0002 \\
0003 \\
0004 \\
0005 \\
0001 \\
0001 \\
0001 \\
0001 \\
0001\end{array}$ & MG/L & $\begin{array}{l}< \\
< \\
< \\
< \\
< \\
< \\
< \\
< \\
< \\
< \\
< \\
< \\
< \\
< \\
<\end{array}$ & $\begin{array}{l}0.0003 \\
0.003 \\
0.0003 \\
0.003 \\
0.003 \\
0.003 \\
0.003 \\
0.003 \\
0.003 \\
0.003 \\
0.003 \\
0.003 \\
0.003 \\
0.003 \\
0.06\end{array}$ & $\begin{array}{l}J L \\
J L \\
J L \\
J L \\
d L\end{array}$ & $\begin{array}{l}0.003 \\
0.003 \\
0.003 \\
0.003 \\
0.003 \\
0.003 \\
0.003 \\
0.003 \\
0.003 \\
0.003 \\
0.003 \\
0.003 \\
0.003 \\
0.003 \\
0.06\end{array}$ & $\begin{array}{l}- \\
: \\
: \\
: \\
: \\
- \\
- \\
- \\
- \\
-\end{array}$ \\
\hline ARSENIC & $\begin{array}{l}04 / 21 / 88 \\
04 / 21 / 88 \\
04 / 21 / 88 \\
04 / 21 / 88 \\
04 / 21 / 88 \\
06 / 09 / 89 \\
06 / 09 / 89 \\
06 / 09 / 89 \\
06 / 09 / 89 \\
06 / 09 / 89 \\
12 / 03 / 89 \\
12 / 03 / 89 \\
12 / 03 / 89 \\
12 / 03 / 89 \\
12 / 03 / 89 \\
06 / 25 / 90 \\
01 / 29 / 91 \\
06 / 02 / 91 \\
02 / 22 / 92 \\
08 / 13 / 92 \\
11 / 19 / 92 \\
06 / 26 / 93\end{array}$ & $\begin{array}{l}0001 \\
0002 \\
0003 \\
0004 \\
0005 \\
0001 \\
0002 \\
0003 \\
0004 \\
0005 \\
0001 \\
0002 \\
0003 \\
0004 \\
0005 \\
0001 \\
0001 \\
0001 \\
0001 \\
0001 \\
0001 \\
0001\end{array}$ & MG/L & $\begin{array}{l}< \\
< \\
< \\
< \\
< \\
< \\
< \\
< \\
< \\
< \\
< \\
< \\
< \\
< \\
< \\
< \\
<\end{array}$ & $\begin{array}{l}0.012 \\
0.013 \\
0.016 \\
0.015 \\
0.014 \\
0.01 \\
0.01 \\
0.01 \\
0.01 \\
0.01 \\
0.01 \\
0.01 \\
0.01 \\
0.01 \\
0.01 \\
0.01 \\
0.01 \\
0.05 \\
0.01 \\
0.01 \\
0.0015 \\
0.005\end{array}$ & $\begin{array}{l}J \\
1 \\
M L\end{array}$ & $\begin{array}{l}0.01 \\
0.01 \\
0.01 \\
0.01 \\
0.01 \\
0.01 \\
0.01 \\
0.01 \\
0.01 \\
0.01 \\
0.01 \\
0.01 \\
0.01 \\
0.01 \\
0.01 \\
0.01 \\
0.01 \\
0.05 \\
0.01 \\
0.01 \\
0.0015 \\
0.005\end{array}$ & $\begin{array}{l}: \\
: \\
: \\
: \\
: \\
- \\
: \\
- \\
- \\
- \\
- \\
- \\
- \\
- \\
-\end{array}$ \\
\hline ARSENIC (TOTAL) & $\begin{array}{l}11 / 19 / 92 \\
06 / 26 / 93\end{array}$ & $\begin{array}{l}\text { N001 } \\
\text { N001 }\end{array}$ & MG/L & $<$ & $\begin{array}{l}0.0015 \\
0.005\end{array}$ & & $\begin{array}{l}0.0015 \\
0.005\end{array}$ & $\dot{-}$ \\
\hline BARIUA & $06 / 09 / 89$ & 0001 & MG/L & $<$ & 0.1 & $\mathbf{L}$ & 0.1 & - \\
\hline
\end{tabular}

PARAMETER VALUE IMDICATOR (PVI): < - LESS THAN DETECTION LIMIT

SNMPLE IO COOES:

OTHER PARMAETER VALUE FLAGS:

H - HOLD TIME EXPIRED, VALUE SUSPECT

I - IMCREASED DETECTION LIMIT DUE TO REQUIRED DILUTION

: $J$ - ESTIHUTED VALUE

L - LESS THAN THREE BORE VOLUMES REMOVED BEFORE SAMPLIKG

0001 - FILTERED SAMPLE (.45 MICRONS)

0002 - FILTERED REPLICATE SAMPLE (.45 MICROHS)

0003 - FILTERED REPLICATE SAYPLE (.45 MICRONS)

0004 - FILTERED REPLICATE SAMPLE (.45 MICRONS)

NOOI - UHFILTERED SAMPLE

W - POST-DIGEST SPIKE OUT DF CNTR LIM WHILE SAMP ABS < 50\% SPIKE 
GROUNDWATER OUALITY DATA BY LOCATION

SONP REV. D Data

SITE: MOHO1 MOHUHENT VALLEY

LOCATION: 0655

MORTH COORDINATE: 59754.6 FT

EAST COORDIHATE: 88624.1 FT

04/21/88 TO 12/13/94

REPORT DATE: O6/20/95

FORMATIOH OF COMPLETION: ALLUVIUM (AL)

HYORAULIC FLON RELATIONSHIP: DOWN GRADIENT (D)

\begin{tabular}{|c|c|c|c|c|c|c|c|c|c|}
\hline PARAMETER MAME & & LOG DATE & $\mid \begin{array}{c}\text { SAMPLE } \\
10\end{array}$ & $\begin{array}{l}\text { UHITS OF } \\
\text { MEASURE }\end{array}$ & PVI & $\begin{array}{l}\text { PARAMETER } \\
\text { VALUE }\end{array}$ & FLAGS & $\begin{array}{l}\text { DETECTIOH } \\
\text { LIMIT }\end{array}$ & $\begin{array}{l}\text { PARAMETER } \\
\text { UNCERTAINTY }\end{array}$ \\
\hline BARILA & & $\begin{array}{l}06 / 09 / 89 \\
06 / 09 / 89 \\
06 / 09 / 89 \\
06 / 09 / 89 \\
12 / 03 / 89 \\
12 / 03 / 89 \\
12 / 03 / 89 \\
12 / 03 / 89 \\
12 / 03 / 89 \\
06 / 25 / 90 \\
01 / 29 / 91 \\
06 / 02 / 91 \\
02 / 22 / 92 \\
08 / 13 / 92 \\
11 / 19 / 92 \\
06 / 26 / 93\end{array}$ & $\begin{array}{l}0002 \\
0003 \\
0004 \\
0005 \\
0001 \\
0002 \\
0003 \\
0004 \\
0005 \\
0001 \\
0001 \\
0001 \\
0001 \\
0001 \\
0001 \\
0001\end{array}$ & MG/L & $\begin{array}{l}< \\
< \\
< \\
< \\
< \\
< \\
< \\
< \\
< \\
< \\
< \\
<\end{array}$ & $\begin{array}{l}0.001 \\
0.1 \\
0.1 \\
0.1 \\
0.1 \\
0.1 \\
0.1 \\
0.1 \\
0.1 \\
0.1 \\
0.1 \\
0.02 \\
0.02 \\
0.2 \\
0.018 \\
0.1\end{array}$ & $\begin{array}{l}J L \\
L \\
L \\
L\end{array}$ & $\begin{array}{l}0.1 \\
0.1 \\
0.1 \\
0.1 \\
0.1 \\
0.1 \\
0.1 \\
0.1 \\
0.1 \\
0.1 \\
0.1 \\
0.01 \\
0.01 \\
0.2 \\
0.0015 \\
0.1\end{array}$ & $\begin{array}{l}- \\
- \\
- \\
- \\
- \\
- \\
- \\
- \\
- \\
- \\
-\end{array}$ \\
\hline BARIUM (TOTAL) & & $\begin{array}{l}11 / 19 / 92 \\
06 / 26 / 93\end{array}$ & $\begin{array}{l}\text { N001 } \\
\text { N001 }\end{array}$ & $M G / L$ & $<$ & $\begin{array}{l}0.020 \\
0.1\end{array}$ & & $\begin{array}{l}0.0015 \\
0.1\end{array}$ & - \\
\hline BERYLLIUM & $=$ & $\begin{array}{l}06 / 09 / 89 \\
06 / 09 / 89 \\
06 / 09 / 89 \\
06 / 09 / 89 \\
06 / 09 / 89 \\
12 / 03 / 89 \\
12 / 03 / 89 \\
12 / 03 / 89 \\
12 / 03 / 89 \\
12 / 03 / 89 \\
06 / 25 / 90 \\
01 / 29 / 91 \\
06 / 02 / 91 \\
08 / 13 / 92\end{array}$ & $\begin{array}{l}0001 \\
0002 \\
0003 \\
0004 \\
0005 \\
0001 \\
0002 \\
0003 \\
0004 \\
0005 \\
0001 \\
0001 \\
0001 \\
0001\end{array}$ & $M G / L$ & $\begin{array}{l}< \\
< \\
< \\
< \\
< \\
< \\
< \\
< \\
< \\
< \\
< \\
< \\
< \\
<\end{array}$ & $\begin{array}{l}0.01 \\
0.01 \\
0.01 \\
0.01 \\
0.01 \\
0.005 \\
0.005 \\
0.005 \\
0.005 \\
0.005 \\
0.01 \\
0.01 \\
0.005 \\
0.005\end{array}$ & $\begin{array}{l}L \\
L \\
L \\
L \\
L\end{array}$ & $\begin{array}{l}0.01 \\
0.01 \\
0.01 \\
0.01 \\
0.01 \\
0.005 \\
0.005 \\
0.005 \\
0.005 \\
0.005 \\
0.01 \\
0.01 \\
0.005 \\
0.005\end{array}$ & $\begin{array}{l}- \\
- \\
- \\
- \\
- \\
- \\
- \\
- \\
-\end{array}$ \\
\hline BORON & $\therefore$ & $\begin{array}{l}06 / 09 / 89 \\
06 / 09 / 89 \\
06 / 09 / 89 \\
06 / 09 / 89 \\
06 / 09 / 89 \\
12 / 03 / 89 \\
12 / 03 / 89 \\
12 / 03 / 89 \\
12 / 03 / 89 \\
12 / 03 / 89 \\
06 / 25 / 90 \\
01 / 29 / 91 \\
06 / 02 / 91\end{array}$ & $\begin{array}{l}0009 \\
0002 \\
0003 \\
0004 \\
0005 \\
0001 \\
0002 \\
0003 \\
0004 \\
0005 \\
0001 \\
0001 \\
0001\end{array}$ & $M G / L$ & $\begin{array}{l}< \\
< \\
< \\
< \\
< \\
< \\
< \\
< \\
< \\
< \\
< \\
< \\
<\end{array}$ & $\begin{array}{l}0.1 \\
0.1 \\
0.1 \\
0.1 \\
0.1 \\
0.1 \\
0.1 \\
0.1 \\
0.1 \\
0.1 \\
0.1 \\
0.1 \\
0.05\end{array}$ & $\begin{array}{l}L \\
L \\
L \\
L \\
L\end{array}$ & $\begin{array}{l}0.1 \\
0.1 \\
0.1 \\
0.1 \\
0.1 \\
0.1 \\
0.1 \\
0.1 \\
0.1 \\
0.1 \\
0.1 \\
0.1 \\
0.05\end{array}$ & $\begin{array}{l}- \\
- \\
- \\
- \\
- \\
- \\
- \\
- \\
-\end{array}$ \\
\hline BROMIDE & & $\begin{array}{l}01 / 29 / 91 \\
06 / 02 / 91\end{array}$ & $\begin{array}{l}0001 \\
0001\end{array}$ & $M G / L$ & $<$ & $\begin{array}{l}0.1 \\
0.5\end{array}$ & & $\begin{array}{l}0.1 \\
0.1\end{array}$ & - \\
\hline CADMIUA & & $\begin{array}{l}04 / 21 / 88 \\
04 / 21 / 88 \\
04 / 21 / 88\end{array}$ & $\begin{array}{l}0001 \\
0002 \\
0003\end{array}$ & MG/L & & $\begin{array}{l}0.030 \\
0.030 \\
0.029\end{array}$ & $\begin{array}{l}L \\
L \\
L\end{array}$ & $\begin{array}{l}0.001 \\
0.001 \\
0.001\end{array}$ & 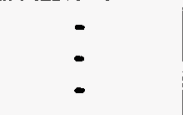 \\
\hline
\end{tabular}

PARAMETER VALUE IMOICATOR (PVI): < - LESS THAN DETECTION LIMIT

SAMPLE TO COOES:

OTHER PARAMETER VALUE FLAGS:

0001 - FILTEREO SAMPLE (.45 MICRONS)

OOO2 - FILTERED REPLICATE SAMPLE (.45 MICRONS)

O003 - FILTERED REPLICATE SAMPLE (.45 MICROHS)

0004 - FILTERED REPLICATE SAMPLE (.45 MICRONS)

NOO1 - UNFILTERED SNMPLE 
GROUNDHATER QUALITY DATA BY LOCATION

SOUP Rev. O Data

SITE: MONO MOHLNENT VALLEY

LOCATION: 0655

HORTH COORDIHATE: 59754.6 FT

EAST COORDIHATE: 88624.1 FT

$04 / 21 / 88$ TO $12 / 13 / 94$

REPORT DATE: 06/20/95

FORHATION OF COHPLETION: ALLUVIUM (AL)

HYDRAULIC FLON RELATIONSHIP: DOWN GRADIENT (D)

\begin{tabular}{|c|c|c|c|c|c|c|c|c|}
\hline PARAMETER MAHE & LOG DATE & $\begin{array}{c}\text { SAMPLE } \\
\text { ID }\end{array}$ & $\begin{array}{l}\text { UNITS OF } \\
\text { MEASURE }\end{array}$ & PVI & $\begin{array}{l}\text { PARAMETER } \\
\text { VALUE }\end{array}$ & FLAGS & $\begin{array}{l}\text { DETECTION } \\
\text { LIMIT }\end{array}$ & $\begin{array}{l}\text { PARAMETER } \\
\text { UNCERTAIHTY }\end{array}$ \\
\hline CADHIUA & $\begin{array}{l}04 / 21 / 88 \\
04 / 21 / 88 \\
06 / 09 / 89 \\
06 / 09 / 89 \\
06 / 09 / 89 \\
06 / 09 / 89 \\
06 / 09 / 89 \\
12 / 03 / 89 \\
12 / 03 / 89 \\
12 / 03 / 89 \\
12 / 03 / 89 \\
12 / 03 / 89 \\
06 / 25 / 90 \\
01 / 29 / 91 \\
06 / 02 / 91 \\
02 / 22 / 92 \\
08 / 13 / 92 \\
11 / 19 / 92\end{array}$ & $\begin{array}{l}0004 \\
0005 \\
0001 \\
0002 \\
0003 \\
0004 \\
0005 \\
0001 \\
0002 \\
0003 \\
0004 \\
0005 \\
0001 \\
0001 \\
0001 \\
0001 \\
0001 \\
0001\end{array}$ & MG/L & $\begin{array}{l}< \\
< \\
< \\
< \\
< \\
< \\
< \\
< \\
< \\
< \\
< \\
< \\
< \\
< \\
< \\
<\end{array}$ & $\begin{array}{l}0.030 \\
0.031 \\
0.120 \\
0.12 \\
0.001 \\
0.001 \\
0.001 \\
0.001 \\
0.001 \\
0.001 \\
0.001 \\
0.001 \\
0.001 \\
0.001 \\
0.001 \\
0.0005 \\
0.005 \\
0.00013\end{array}$ & $\begin{array}{l}L \\
L \\
L \\
I L \\
L \\
L \\
L \\
\\
W \\
W\end{array}$ & $\begin{array}{l}0.001 \\
0.001 \\
0.001 \\
0.12 \\
0.001 \\
0.001 \\
0.001 \\
0.001 \\
0.001 \\
0.001 \\
0.001 \\
0.001 \\
0.001 \\
0.001 \\
0.001 \\
0.0005 \\
0.005 \\
0.00013\end{array}$ & $\begin{array}{l}- \\
: \\
- \\
: \\
- \\
- \\
- \\
- \\
- \\
- \\
- \\
-\end{array}$ \\
\hline CADHIUN (TOTAL) & $11 / 19 / 92$ & HOOI & MG/L & $<$ & 0.00013 & 3 NH & 0.00013 & - \\
\hline Calcium & $\begin{array}{l}04 / 21 / 88 \\
04 / 29 / 88 \\
04 / 29 / 88 \\
04 / 21 / 88 \\
04 / 29 / 88 \\
06 / 09 / 89 \\
06 / 09 / 89 \\
06 / 09 / 89 \\
06 / 09 / 89 \\
06 / 09 / 89 \\
12 / 03 / 89 \\
12 / 03 / 89 \\
12 / 03 / 89 \\
12 / 03 / 89 \\
12 / 03 / 89 \\
06 / 25 / 90 \\
01 / 29 / 91 \\
06 / 02 / 99 \\
02 / 22 / 92 \\
08 / 13 / 92 \\
02 / 19 / 93 \\
06 / 26 / 93 \\
04 / 24 / 94\end{array}$ & $\begin{array}{l}0001 \\
0002 \\
0003 \\
0004 \\
0005 \\
0001 \\
0002 \\
0003 \\
0004 \\
0005 \\
0001 \\
0002 \\
0003 \\
0004 \\
0005 \\
0001 \\
0001 \\
0001 \\
0001 \\
0001 \\
0001 \\
0001 \\
0001\end{array}$ & MG/L & & $\begin{array}{l}518 . \\
521 . \\
530 . \\
539 . \\
506 . \\
429 . \\
405 . \\
407 . \\
411 . \\
416 . \\
433 . \\
417 . \\
426 . \\
406 . \\
404 . \\
395 . \\
420 . \\
463 . \\
390 . \\
410 \\
372 \\
370 \\
375\end{array}$ & $\begin{array}{l}L \\
L \\
L \\
L \\
L \\
L \\
L \\
L \\
L \\
L\end{array}$ & $\begin{array}{l}0.01 \\
0.01 \\
0.01 \\
0.01 \\
0.01 \\
0.01 \\
0.01 \\
0.01 \\
0.01 \\
0.01 \\
0.01 \\
0.01 \\
0.01 \\
0.01 \\
0.01 \\
0.01 \\
0.01 \\
0.5 \\
0.5 \\
5 \\
0.5 \\
0.5 \\
0.5\end{array}$ & $\begin{array}{l}- \\
- \\
- \\
- \\
- \\
- \\
- \\
- \\
- \\
- \\
- \\
- \\
- \\
- \\
- \\
-\end{array}$ \\
\hline CALCIUN (TOTAL) & $\begin{array}{l}06 / 26 / 93 \\
04 / 24 / 94\end{array}$ & $\begin{array}{l}\text { NoO9 } \\
\text { NO01 }\end{array}$ & MG/L & & $\begin{array}{l}350 \\
427\end{array}$ & - & $\begin{array}{l}0.5 \\
0.5\end{array}$ & - \\
\hline CHLORIDE & $\begin{array}{l}04 / 21 / 88 \\
04 / 21 / 88 \\
04 / 21 / 88 \\
04 / 21 / 88 \\
04 / 21 / 88\end{array}$ & $\begin{array}{l}0001 \\
0002 \\
0003 \\
0004 \\
0005\end{array}$ & MG/L & & $\begin{array}{l}34 . \\
34 . \\
35 \\
35 \\
35\end{array}$ & $\begin{array}{l}L \\
L \\
L \\
L \\
L\end{array}$ & $\begin{array}{l}1 . \\
1 . \\
1 . \\
1 .\end{array}$ & : \\
\hline
\end{tabular}

PARAMETER VALUE INDICATOR (PVI): < - LESS THAN DETECTION LIMIT

SAMPLE ID COOES:

OTHER PARAMETER VALUE FLAGS:

1 - IMCREASED DETECTION LIMIT DUE TO REQUIRED DILUTION

L - LESS THAN THREE BORE VOLUWES RENOVED BEFORE SAMPLIHG

N - SPIKE SAMPLE RECOVERY NOT WITHIN CONTROL LIMITS

$W$ - POST-DIGEST SPIKE OUT OF CWTR LIM WHILE SAMP ABS < 50\% SPIKE

0001 - FILTERED SAMPLE (.45 MICROHS)

0002 - FILTERED REPLICATE SAMPLE (.45 MICRONS)

0003 - FILTERED REPLICATE SAMPLE (.45 MICROHS)

0004 - FILTERED REPLICATE SAMPLE (.45 MICRONS)

NOO1 - LAFILTERED SNMPLE 
GROUNDHATER QUALITY DATA BY LOCATION

SOUP ReV. 0 Data

SITE: MONO1 MOHUMEKT VALLEY

LOCATIOH: 0655

MORTH COORDIHATE: 59754.6 FT

EAST COORDIHATE: 88624.1 FT

$04 / 21 / 88$ TO $12 / 13 / 94$

REPORT DATE: $06 / 20 / 95$

FORMATIOW OF COMPLETIOH: ALLUVIUH (AL)

HYDRAULIC FLON RELATIONSHIP: DOWH GRADIENT (D)

\begin{tabular}{|c|c|c|c|c|c|c|c|c|}
\hline PARAMETER HAME & LOG DATE & $\begin{array}{c}\text { SAMPLE } \\
\text { ID }\end{array}$ & $\begin{array}{l}\text { UNITS OF } \\
\text { MEASURE }\end{array}$ & PVI & $\begin{array}{l}\text { PARAMETER } \\
\text { VALUE }\end{array}$ & FLAGS & $\begin{array}{l}\text { DETECTION } \\
\text { LIMIT }\end{array}$ & $\begin{array}{l}\text { PARAMETER } \\
\text { UNCERTAIKTY }\end{array}$ \\
\hline CHLORIDE & $\begin{array}{l}06 / 09 / 89 \\
06 / 09 / 89 \\
06 / 09 / 89 \\
06 / 09 / 89 \\
06 / 09 / 89 \\
12 / 03 / 89 \\
12 / 03 / 89 \\
12 / 03 / 89 \\
12 / 03 / 89 \\
12 / 03 / 89 \\
06 / 25 / 90 \\
01 / 29 / 99 \\
06 / 02 / 99 \\
02 / 22 / 92 \\
08 / 13 / 92 \\
02 / 19 / 93 \\
04 / 24 / 94\end{array}$ & $\begin{array}{l}0001 \\
0002 \\
0003 \\
0004 \\
0005 \\
0001 \\
0002 \\
0003 \\
0004 \\
0005 \\
0001 \\
0001 \\
0001 \\
0001 \\
0001 \\
0001 \\
0001\end{array}$ & MG/L & & $\begin{array}{l}36 . \\
36 . \\
36 . \\
36 . \\
36 . \\
26.6 \\
31 . \\
32 . \\
31 . \\
31 . \\
35 . \\
38 . \\
33 . \\
30 . \\
29 \\
29.8 \\
25.8\end{array}$ & $\begin{array}{l}L \\
L \\
L \\
L \\
L\end{array}$ & $\begin{array}{l}1 . \\
i . \\
i . \\
1 . \\
i . \\
i . \\
1 . \\
i . \\
i . \\
i . \\
0.5 \\
1 . \\
0.016 \\
0.5 \\
0.5\end{array}$ & $\begin{array}{l}- \\
- \\
- \\
- \\
- \\
- \\
- \\
- \\
- \\
- \\
- \\
-\end{array}$ \\
\hline CHLORIDE (TOTAL) & $06 / 26 / 93$ & N001 & $M G / L$ & & 27.7 & & 0.5 & - \\
\hline CHROMIUH & $\begin{array}{l}06 / 09 / 89 \\
06 / 09 / 89 \\
06 / 09 / 89 \\
06 / 09 / 89 \\
12 / 03 / 89 \\
12 / 03 / 89 \\
12 / 03 / 89 \\
12 / 03 / 89 \\
12 / 03 / 89 \\
06 / 25 / 90 \\
01 / 29 / 91 \\
06 / 02 / 91 \\
02 / 22 / 92 \\
08 / 13 / 92 \\
11 / 19 / 92\end{array}$ & $\begin{array}{l}0001 \\
0003 \\
0004 \\
0005 \\
0001 \\
0002 \\
0003 \\
0004 \\
0005 \\
0001 \\
0001 \\
0001 \\
0001 \\
0001 \\
0001\end{array}$ & $M G / L$ & $\begin{array}{l}< \\
< \\
< \\
< \\
< \\
< \\
< \\
< \\
< \\
< \\
< \\
< \\
< \\
<\end{array}$ & $\begin{array}{l}0.01 \\
0.01 \\
0.09 \\
0.01 \\
0.01 \\
0.09 \\
0.01 \\
0.01 \\
0.01 \\
0.01 \\
0.01 \\
0.01 \\
0.09 \\
0.01\end{array}$ & $\begin{array}{l}L \\
L \\
L \\
L\end{array}$ & $\begin{array}{l}0.01 \\
0.01 \\
0.01 \\
0.01 \\
0.01 \\
0.09 \\
0.01 \\
0.01 \\
0.01 \\
0.01 \\
0.01 \\
0.09 \\
0.09 \\
0.01 \\
0.0053\end{array}$ & $\begin{array}{l}- \\
- \\
- \\
- \\
- \\
- \\
- \\
- \\
- \\
- \\
-\end{array}$ \\
\hline CHROMIUN (TOTAL) & $11 / 19 / 92$ & N001 & MG/L & $<$ & 0.0053 & & 0.0053 & - \\
\hline COBALT & $\begin{array}{l}06 / 09 / 89 \\
06 / 09 / 89 \\
06 / 09 / 89 \\
06 / 09 / 89 \\
06 / 09 / 89 \\
12 / 03 / 89 \\
12 / 03 / 89 \\
12 / 03 / 89 \\
12 / 03 / 89 \\
12 / 03 / 89 \\
06 / 25 / 90 \\
01 / 29 / 91 \\
06 / 02 / 91\end{array}$ & $\begin{array}{l}0001 \\
0002 \\
0003 \\
0004 \\
0005 \\
0001 \\
0002 \\
0003 \\
0004 \\
0005 \\
0001 \\
0001 \\
0001\end{array}$ & MG/L & $\begin{array}{l}< \\
< \\
< \\
< \\
< \\
< \\
< \\
< \\
< \\
< \\
< \\
< \\
<\end{array}$ & $\begin{array}{l}0.05 \\
0.05 \\
0.05 \\
0.05 \\
0.05 \\
0.05 \\
0.05 \\
0.05 \\
0.05 \\
0.05 \\
0.05 \\
0.05 \\
0.03\end{array}$ & $\begin{array}{l}L \\
L \\
L \\
L \\
L\end{array}$ & $\begin{array}{l}0.05 \\
0.05 \\
0.05 \\
0.05 \\
0.05 \\
0.05 \\
0.05 \\
0.05 \\
0.05 \\
0.05 \\
0.05 \\
0.05 \\
0.03\end{array}$ & $\begin{array}{l}- \\
- \\
- \\
- \\
- \\
- \\
- \\
-\end{array}$ \\
\hline COPPER & $\begin{array}{l}06 / 09 / 89 \\
06 / 09 / 89\end{array}$ & $\begin{array}{l}0001 \\
0002\end{array}$ & $M G / L$ & & $\begin{array}{l}0.02 \\
0.02\end{array}$ & $\frac{1}{L}$ & $\begin{array}{l}0.02 \\
0.02\end{array}$ & - \\
\hline
\end{tabular}

PARAKETER VALUE INDICATOR (PVI): < - LESS thaN DETECTION LIMIT

OTHER PARAMETER VALUE FLAGS:

J- ESTIMATED VALUE

L - LESS THAN THREE BORE VOLUAES REMOVED BEFORE SAMPLIHG
SANPLE ID COOES:

O001 - FILTERED SAMPLE (.45 MICRONS)

O002 - FILTERED REPLICATE SAMPLE (.45 MICRONS)

0003 - FILTERED REPLICATE SAMPLE (.45 MICRONS)

0004 - FILTERED REPLICATE SAMPLE (.45 MICRONS) NOO1 - UAFILTERED SAMPLE 
GROUHDWATER QUALITY DATA BY LOCATION

SOMP ReV. O Data

SITE: MONO1 MONUMENT VALLEY

LOCATION: 0655

MORTH COORDINATE: 59754.6 FT

EAST COORDIMATE: 88624.9 FT

04/21/88 TO $12 / 13 / 94$

REPORT DATE: $06 / 20 / 95$

FORMATION OF COMPLETION: ALLUVIUM (AL)

HYDRAULIC FLON RELATIONSHIP: DOW GRADIENT (D)

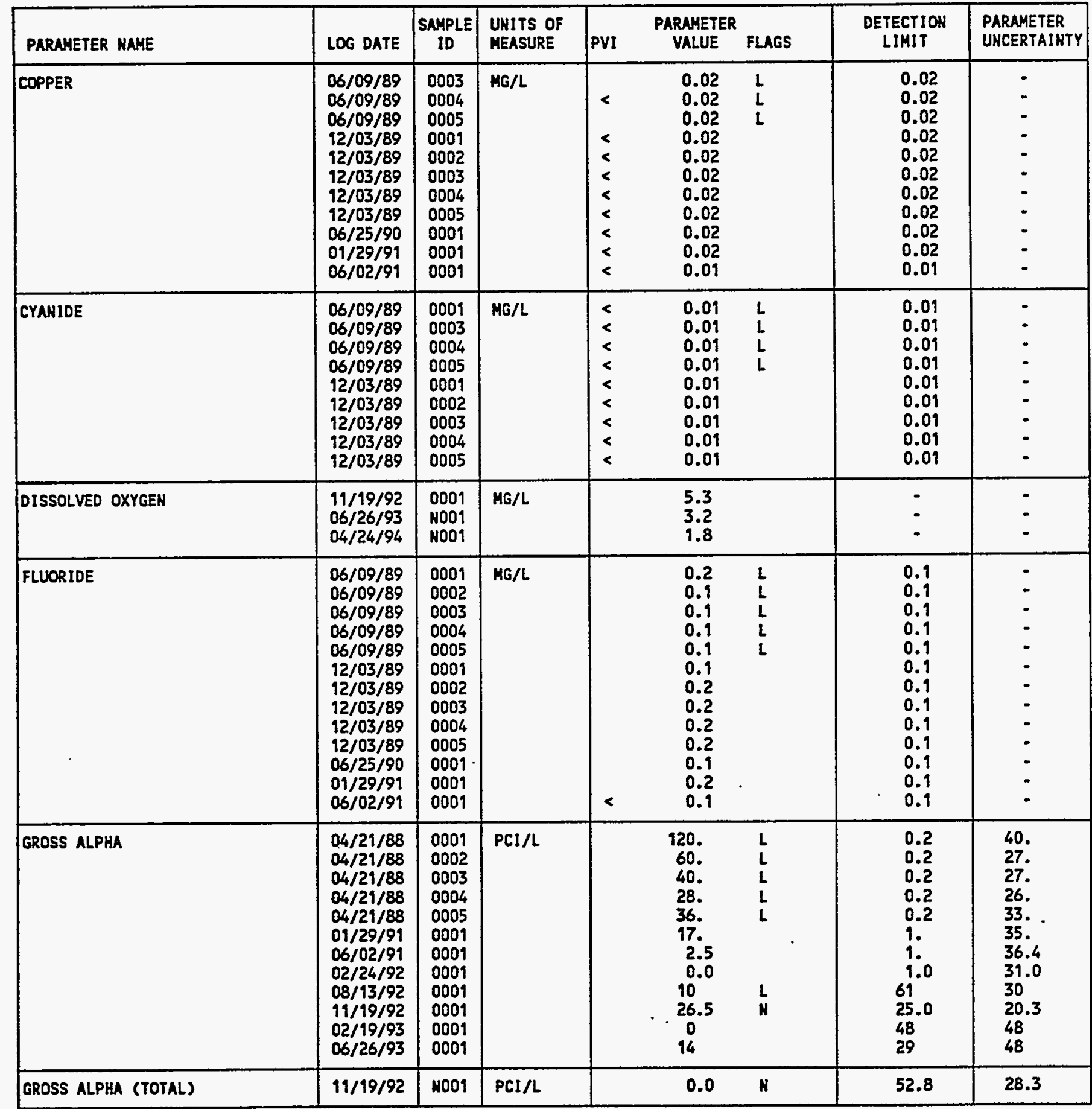

PARAMETER VALUE IMDICATOR (PVI): < - LESS THAN DETECTION LIMIT

SAMPLE ID COOES:

OTHER PARAMETER VALUE FLAGS:

L - LESS THAN THREE BORE VOLUMES REMOVED BEFORE SAMPLIHG

- SPIKE SAMPLE RECOVERY NOT WITHIN CONTROL LIMITS

0001 - FILTERED SAMPLE (.45 MICRONS)

0002 - FILTERED REPLICATE SAMPLE (.45 HICRONS)

0003 - FILTERED REPLICATE SAMPLE (.45 MICROHS)

0004 - FILTERED REPLICATE SAMPLE (.45 MICRONS)

NOO1 - UHFILTERED SAYPLE 
Sorp Rev. 0 Data

SITE: HONO1 MOHUHENT YALLEY

LOCATION: 0655

MORTH COORDINATE: 59754.6 FT

EAST COORDINATE: 88624.1 FT

$04 / 21 / 88$ To $12 / 13 / 94$

REPORT DATE: $06 / 20 / 95$

FORMATION OF COMPLETION: ALLUVIUM (AL)

HYDRAULIC FLON RELATIOHSHIP: DOMN GRADIENT (D)

\begin{tabular}{|c|c|c|c|c|c|c|c|c|}
\hline PARAMETER MANE & LOS DATE & $\begin{array}{c}\text { SAMPLE } \\
\text { ID }\end{array}$ & $\begin{array}{l}\text { UHITS OF } \\
\text { MEASURE }\end{array}$ & PVI & $\begin{array}{l}\text { PARAMETER } \\
\text { VALUE }\end{array}$ & FLAGS & $\begin{array}{l}\text { DETECTION } \\
\text { LIMIT }\end{array}$ & $\begin{array}{l}\text { PARAMETER } \\
\text { UNCERTAINTY }\end{array}$ \\
\hline GROSS ALPHA (TOTAL) & $06 / 26 / 93$ & No01 & PCI/L & \multicolumn{3}{|c|}{25} & 26 & 45 \\
\hline CROSS BETA & $\begin{array}{l}04 / 21 / 88 \\
04 / 21 / 88 \\
04 / 21 / 88 \\
04 / 21 / 88 \\
04 / 21 / 88 \\
01 / 29 / 91 \\
06 / 02 / 91 \\
02 / 24 / 92 \\
08 / 13 / 92 \\
11 / 19 / 92 \\
02 / 19 / 93 \\
06 / 26 / 93\end{array}$ & $\begin{array}{l}0001 \\
0002 \\
0003 \\
0004 \\
0005 \\
0001 \\
0001 \\
0001 \\
0001 \\
0001 \\
0001 \\
0001\end{array}$ & $\mathrm{PCI} / \mathrm{L}$ & \multicolumn{3}{|c|}{$\begin{array}{l}62 . \\
67 . \\
60 . \\
67 . \\
60 . \\
37 . \\
24.1 \\
24.8 \\
15 \\
0.0 \\
1 \\
35\end{array}$} & $\begin{array}{l}1 . \\
1 . \\
1 . \\
1 . \\
1 . \\
0.5 \\
0.5 \\
0.5 \\
71 \\
42.7 \\
59 \\
44\end{array}$ & $\begin{array}{l}16 . \\
13 . \\
13 . \\
15 . \\
17 . \\
24 . \\
21.3 \\
22.0 \\
41 \\
24.9 \\
26 . \\
42\end{array}$ \\
\hline GROSS BETA (TOTAL) & $\begin{array}{l}11 / 19 / 92 \\
06 / 26 / 93\end{array}$ & $\begin{array}{l}\text { N001 } \\
\text { N001 }\end{array}$ & $\mathrm{PCI} / \mathrm{L}$ & \multicolumn{2}{|r|}{$\underset{0}{25.5}$} & N & $\begin{array}{l}39.0 \\
44\end{array}$ & $\begin{array}{l}24.4 \\
40\end{array}$ \\
\hline IROH & $\begin{array}{l}06 / 09 / 89 \\
06 / 09 / 89 \\
06 / 09 / 89 \\
06 / 09 / 89 \\
06 / 09 / 89 \\
12 / 03 / 89 \\
12 / 03 / 89 \\
12 / 03 / 89 \\
12 / 03 / 89 \\
12 / 03 / 89 \\
06 / 25 / 90 \\
01 / 29 / 91 \\
06 / 02 / 91 \\
02 / 22 / 92 \\
08 / 13 / 92 \\
02 / 19 / 93 \\
06 / 26 / 93\end{array}$ & $\begin{array}{l}0001 \\
0002 \\
0003 \\
0004 \\
0005 \\
0001 \\
0002 \\
0003 \\
0004 \\
0005 \\
0001 \\
0001 \\
0001 \\
0001 \\
0001 \\
0001 \\
0001\end{array}$ & MG/L & \multicolumn{2}{|r|}{$\begin{array}{l}\quad 0.09 \\
0.08 \\
0.09 \\
0.08 \\
0.52 \\
0.03 \\
0.03 \\
0.03 \\
0.03 \\
0.03 \\
0.09 \\
0.09 \\
0.03 \\
0.03 \\
0.1 \\
0.03\end{array}$} & $\begin{array}{l}L \\
L \\
L \\
L \\
L\end{array}$ & $\begin{array}{l}0.03 \\
0.03 \\
0.03 \\
0.03 \\
0.03 \\
0.03 \\
0.03 \\
0.03 \\
0.03 \\
0.03 \\
0.03 \\
0.03 \\
0.03 \\
0.03 \\
0.1 \\
0.03 \\
0.03\end{array}$ & $\begin{array}{l}- \\
- \\
- \\
- \\
- \\
- \\
- \\
- \\
- \\
- \\
- \\
- \\
-\end{array}$ \\
\hline IROW (TOTAL) & $06 / 26 / 93$ & HOOI & $M G / L$ & \multicolumn{2}{|r|}{0.06} & J & 0.03 & - \\
\hline LEAD & $\begin{array}{l}06 / 09 / 89 \\
06 / 09 / 89 \\
06 / 09 / 89 \\
06 / 09 / 89 \\
06 / 09 / 89 \\
12 / 03 / 89 \\
12 / 03 / 89 \\
12 / 03 / 89 \\
12 / 03 / 89 \\
12 / 03 / 89 \\
06 / 25 / 90 \\
01 / 29 / 91 \\
06 / 02 / 91 \\
11 / 19 / 92\end{array}$ & $\begin{array}{l}0001 \\
0002 \\
0003 \\
0004 \\
0005 \\
0001 \\
0002 \\
0003 \\
0004 \\
0005 \\
0001 \\
0001 \\
0001 \\
0001\end{array}$ & MG/L & \multicolumn{2}{|r|}{$\begin{array}{l}0.01 \\
0.01 \\
0.01 \\
0.01 \\
0.01 \\
0.01 \\
0.01 \\
0.01 \\
0.01 \\
0.01 \\
0.01 \\
0.01 \\
0.005 \\
0.0015\end{array}$} & $\begin{array}{l}L \\
L \\
L \\
L \\
L\end{array}$ & $\begin{array}{l}0.01 \\
0.01 \\
0.01 \\
0.01 \\
.0 .01 \\
0.01 \\
0.01 \\
0.01 \\
0.01 \\
0.01 \\
0.01 \\
0.01 \\
0.005 \\
0.0015\end{array}$ & $\begin{array}{l}- \\
- \\
- \\
- \\
- \\
- \\
- \\
- \\
-\end{array}$ \\
\hline LEAD (TOTAL) & $11 / 19 / 92$ & noot & MG/L & \multicolumn{3}{|c|}{$0.0015 \mathrm{~W}$} & 0.0015 & - \\
\hline $\begin{array}{l}\text { PARAMETER VALUE IHDIC } \\
\text { OTHER PARANETER VALUE } \\
\text { J - ESTIMATED VALUE } \\
\text { - LESS THAN THREE B } \\
\text { - SPIKE SAMPLE RECO } \\
\text { - POST-DIGEST SPIKE }\end{array}$ & $<$ - LESS & THAN DET & ECTION LII & & $\begin{array}{l}\text { SAMPLE ID C } \\
0001 \text { - FILT } \\
0002 \text { - FILT } \\
0003 \text { - FILT } \\
0004 \text { - FILT } \\
\text { NOO1 - UNFI }\end{array}$ & $\begin{array}{l}\text { COOES: } \\
\text { TERED S } \\
\text { TERED } \\
\text { TERED } \\
\text { TERED } \\
\text { TER } \\
\text { ILTERED }\end{array}$ & $\begin{array}{l}\text { C.45 MICRON } \\
\text { TE SAMPLE } \\
\text { TE SAMPLE } \\
\text { TE SAMPLE }\end{array}$ & $\begin{array}{l}5 \text { MICRONS) } \\
5 \text { MICRONS) } \\
5 \text { MICRONS) }\end{array}$ \\
\hline
\end{tabular}


GROUNDHATER QUALITY DATA BY LOCATION

SOUP Rev. O Data

SITE: MONOI MONUMENT VALLEY

LOCATION: 0655

HORTH COORDINATE: 59754.6 FT

EAST COORDIHATE: 88624.1 FT

$04 / 21 / 88$ TO $12 / 13 / 94$

REPORT DATE: $06 / 20 / 95$

FORMATION OF COHPLETION: ALLUUILM (AL)

HYDRAULIC FLON RELATIONSHIP: DOWN GRADIENT (D)

\begin{tabular}{|c|c|c|c|c|c|c|c|c|}
\hline PARAMETER MAME & LOG DATE & $\underset{10}{\text { SAMPLE }}$ & $\begin{array}{l}\text { UHITS OF } \\
\text { MEASURE }\end{array}$ & PVI & $\begin{array}{l}\text { PARAMETER } \\
\text { VALUE }\end{array}$ & FLAGS & $\begin{array}{l}\text { DETECTIOW } \\
\text { LIMIT }\end{array}$ & $\begin{array}{l}\text { PARAMETER } \\
\text { UNCERTAINTY }\end{array}$ \\
\hline LEAD-210 & $11 / 19 / 92$ & 0001 & PCI/L & & 0.3 & & 2.0 & 1.2 \\
\hline LEAD-210 (TOTAL) & $11 / 99 / 92$ & N001 & PCI/L & & 5.2 & & 0.3 & 0.8 \\
\hline MAGNESIUH & $\begin{array}{l}04 / 21 / 88 \\
04 / 21 / 88 \\
04 / 21 / 88 \\
04 / 21 / 88 \\
04 / 21 / 88 \\
06 / 09 / 89 \\
06 / 09 / 89 \\
06 / 09 / 89 \\
06 / 09 / 89 \\
06 / 09 / 89 \\
12 / 03 / 89 \\
12 / 03 / 89 \\
12 / 03 / 89 \\
12 / 03 / 89 \\
12 / 03 / 89 \\
06 / 25 / 90 \\
01 / 29 / 91 \\
06 / 02 / 91 \\
02 / 22 / 92 \\
08 / 13 / 92 \\
02 / 19 / 93 \\
06 / 26 / 93 \\
04 / 24 / 94\end{array}$ & $\begin{array}{l}0001 \\
0002 \\
0003 \\
0004 \\
0005 \\
0001 \\
0002 \\
0003 \\
0004 \\
0005 \\
0001 \\
0002 \\
0003 \\
0004 \\
0005 \\
0001 \\
0009 \\
0001 \\
0001 \\
0001 \\
0001 \\
0001 \\
0001\end{array}$ & $\mathrm{MG} / \mathrm{L}$ & & $\begin{array}{l}295 . \\
290 . \\
294 . \\
288 . \\
300 . \\
311 . \\
313 . \\
306 . \\
321 . \\
317 . \\
321 . \\
319 . \\
326 . \\
319 . \\
310 . \\
306 . \\
319 . \\
350 . \\
301 . \\
320 \\
288 \\
271 \\
254\end{array}$ & $\begin{array}{l}L \\
L \\
L \\
L \\
L \\
L \\
L \\
L \\
L \\
L\end{array}$ & $\begin{array}{l}0.001 \\
0.001 \\
0.001 \\
0.001 \\
0.001 \\
0.001 \\
0.001 \\
0.001 \\
0.001 \\
0.001 \\
0.001 \\
0.001 \\
0.001 \\
0.001 \\
0.001 \\
0.001 \\
0.001 \\
0.5 \\
0.5 \\
5 \\
0.1 \\
0.1 \\
0.1\end{array}$ & $\begin{array}{l}: \\
: \\
: \\
: \\
\vdots \\
: \\
: \\
: \\
: \\
: \\
:\end{array}$ \\
\hline MAGNESIUM (TOTAL) & $\begin{array}{l}06 / 26 / 93 \\
04 / 24 / 94\end{array}$ & $\begin{array}{l}\text { NoO1 } \\
\text { N001 }\end{array}$ & $M G / L$ & & $\begin{array}{l}266 \\
249\end{array}$ & & $\begin{array}{l}0.9 \\
0.1\end{array}$ & : \\
\hline MANGAMESE & $\begin{array}{l}06 / 09 / 89 \\
06 / 09 / 89 \\
06 / 09 / 89 \\
06 / 09 / 89 \\
06 / 09 / 89 \\
12 / 03 / 89 \\
12 / 03 / 89 \\
12 / 03 / 89 \\
12 / 03 / 89 \\
12 / 03 / 89 \\
06 / 25 / 90 \\
01 / 29 / 91 \\
06 / 02 / 91 \\
02 / 22 / 92 \\
08 / 13 / 92 \\
11 / 19 / 92 \\
02 / 19 / 93 \\
06 / 26 / 93 \\
04 / 24 / 94\end{array}$ & $\begin{array}{l}0001 \\
0002 \\
0003 \\
0004 \\
0005 \\
0001 \\
0002 \\
0003 \\
0004 \\
0005 \\
0001 \\
0001 \\
0001 \\
0001 \\
0001 \\
0001 \\
0001 \\
0001 \\
0001\end{array}$ & $M G / L$ & & $\begin{array}{l}0.12 \\
0.12 \\
0.12 \\
0.12 \\
0.12 \\
0.07 \\
0.07 \\
0.07 \\
0.07 \\
0.07 \\
0.08 \\
0.08 \\
0.08 \\
0.04 \\
0.068 \\
0.055 \\
0.04 \\
0.05 \\
0.04\end{array}$ & $\begin{array}{l}L \\
L \\
L \\
L \\
L\end{array}$ & $\begin{array}{l}0.09 \\
0.01 \\
0.01 \\
0.01 \\
0.01 \\
0.01 \\
0.01 \\
0.01 \\
0.01 \\
0.01 \\
0.09 \\
0.01 \\
.0 .09 \\
0.01 \\
0.01 \\
0.0095 \\
0.01 \\
0.01 \\
0.01\end{array}$ & $\begin{array}{l}: \\
: \\
: \\
: \\
: \\
: \\
: \\
: \\
: \\
-\end{array}$ \\
\hline MUHGAHESE (TOTAL) & $\begin{array}{l}11 / 99 / 92 \\
06 / 26 / 93 \\
04 / 24 / 94\end{array}$ & $\begin{array}{l}\text { N001 } \\
\text { N001 } \\
\text { N001 }\end{array}$ & MG/L & & $\begin{array}{l}0.051 \\
0.05 \\
0.05\end{array}$ & & $\begin{array}{l}0.0015 \\
0.01 \\
0.01\end{array}$ & : \\
\hline
\end{tabular}

PARAMETER VALUE IMDICATOR (PVI): < - LESS THAN DETECTION LIHIT

SAMPLE ID CODES:

OTHER PARAMETER VALUE FLAGS:

0001 - FILTERED SAMPLE (.45 MICRONS)

OOO2 - FILTERED REPLICATE SAMPLE (.45 MICRONS)

0003 - FILTERED REPLICATE SAMPLE (.45 MICRONS)

0004 - FILTERED REPLICATE SAMPLE (.45 HICRONS)

NOO1 - UAFILTERED SAMPLE 
GROUMDHATER QUALITY DATA BY LOCATIOH

SOMP ReV. O Data

SITE: HONO1 MONLAENT VALLEY

LOCATION: 0655

MORTH COORDIHATE: 59754.6 FT

EAST COORDINATE: 88624.1 FT

04/21/88 TO $12 / 13 / 94$

REPORT DATE: $06 / 20 / 95$

FORMATION OF COAPLETION: ALLUVIUM (AL)

HYDRAULIC FLON RELATIONSHIP: DOWN GRADIENT (D)

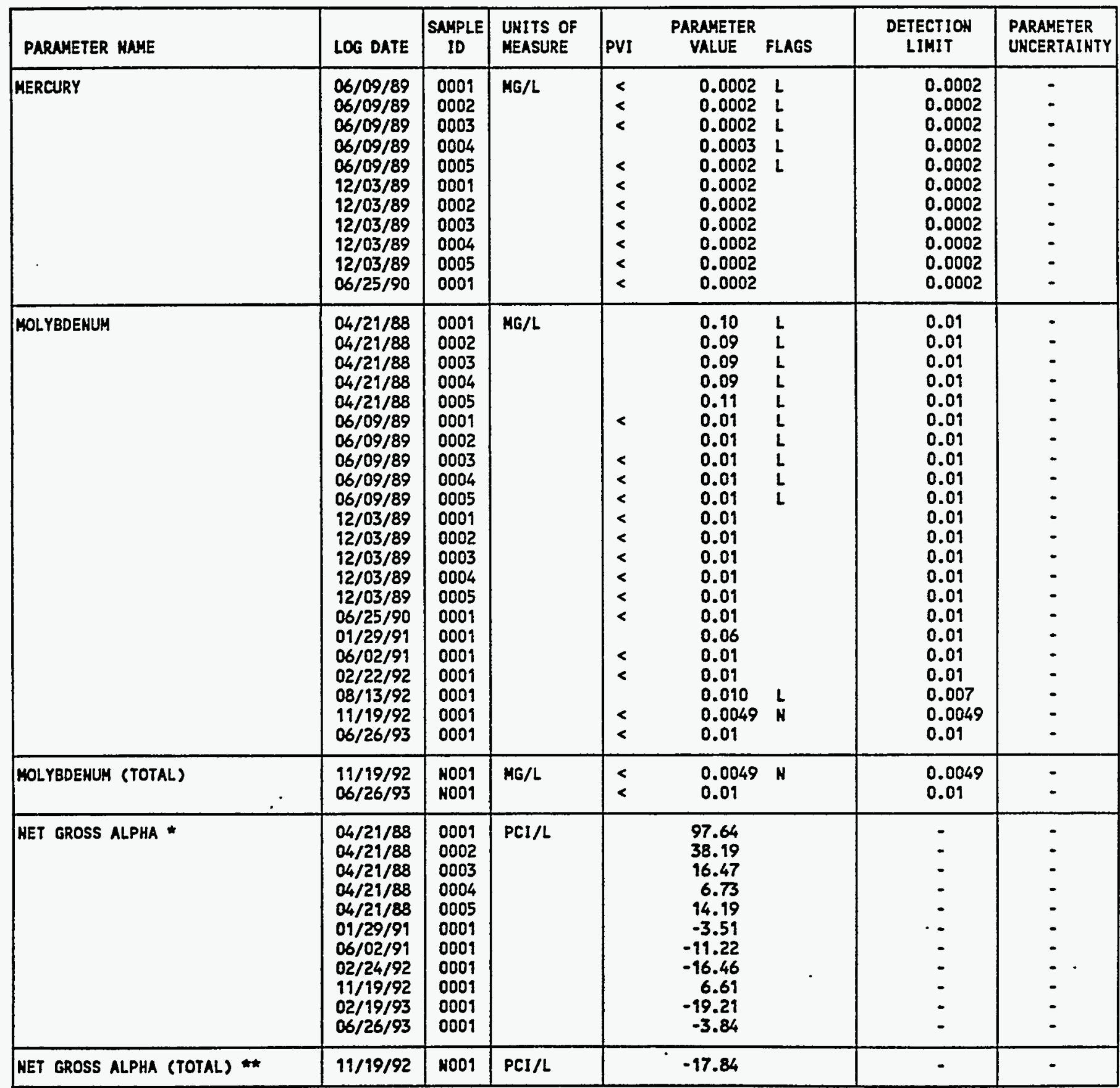

- MET GROSS ALPHA (GROSS ALPHA - URAHIUM) UITH 1 MG URANIUM = 686 PCI

* HET GROSS ALPHA (TOTAL) (TOTAL GROSS ALPHA - TOTAL URAMIUM)

PARAMETER VALUE IMDICATOR (PVI): < - LESS THAH DETECTION LIMIT

SAMPLE ID COOES:

ODO1 - FILTERED SAMPLE (.45 MICRONS)

OTHER PARAMETER VALUE FLAGS:

L - LESS THAH THREE BORE VOLUMES REMOVED BEFORE SAMPLING

0002 - FILTERED REPLICATE SAMPLE (.45 MICRONS)

0003 - FILTERED REPLICATE SAMPLE (.45 MICRONS)

0004 - FILTERED REPLICATE SNMPLE (.45 MICRONS)

M - SPIKE SAMPLE RECOVERY NOT MITHIH CONTROL LIMITS

NOO1 - UNFILTERED SNMPLE 
GROUNDHATER QUALITY DATA BY LOCATION

SOWP Rev. O Data

SITE: MONOI MONUMENT VALLEY

LOCATION: 0655

MORTH COORDINATE: 59754.6 FT

EAST COORDINATE: 88624.1 FT

$04 / 21 / 88$ TO $12 / 13 / 94$

REPORT DATE: 06/20/95

FORMATION OF COMPLETION: ALLUVIUM (AL)

HYDRAULIC FLON RELATIONSHIP: DOWN GRADIENT (D)

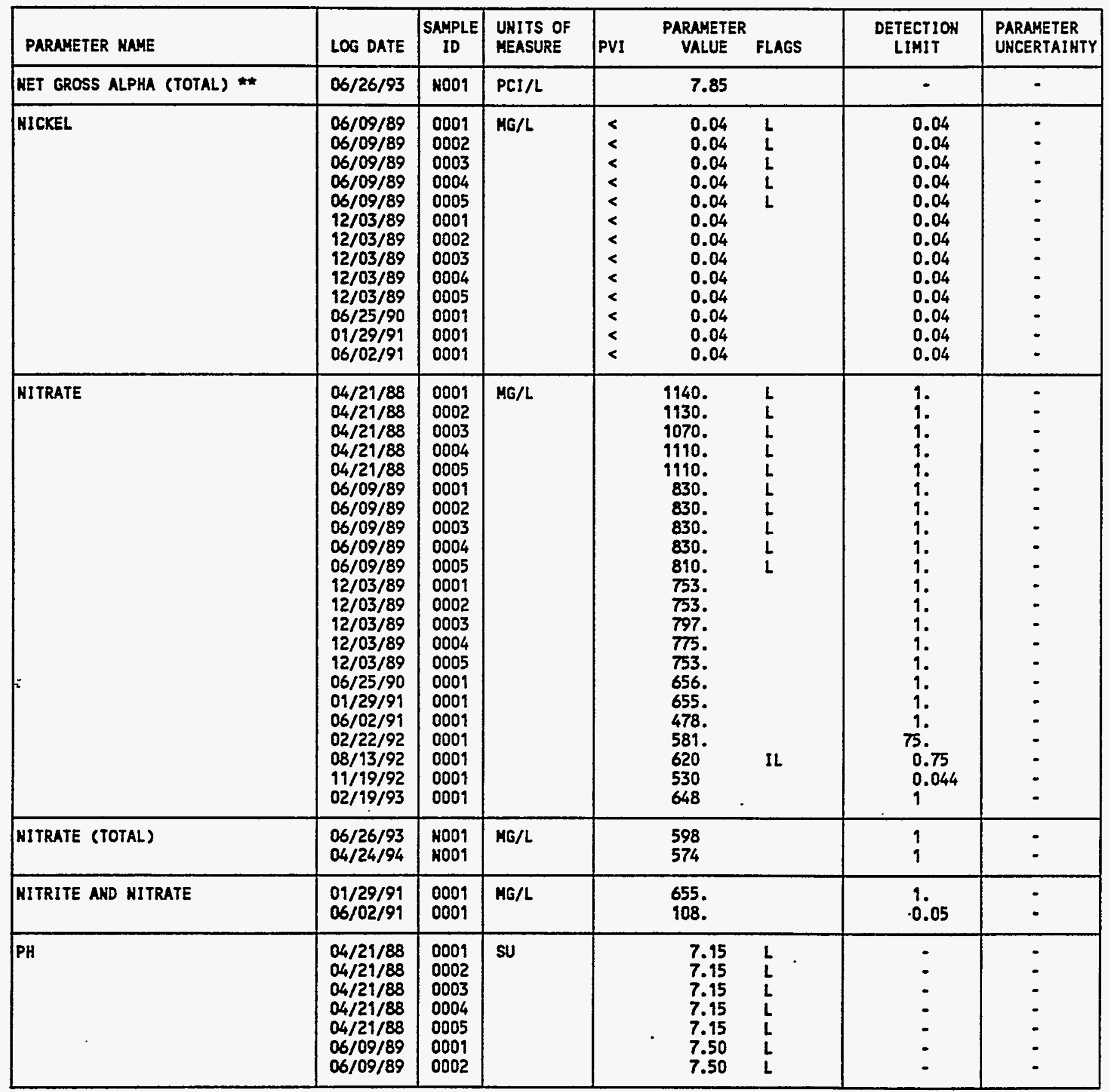

* het gROSS ALPHA (TOTAL) (TOTAL GROSS ALPHA - TOTAL URAMIUM)

PARAMETER VALUE INDICATOR (PVI): < - LESS THAK DETECTION LIMIT

OTHER PARAMETER VALUE FLAGS:

1 - INCREASED DETECTION LIMIT DUE TO REQUIRED DILUTION

L - LESS THAN THREE BORE VOLUMES RENOVEO BEFORE SAMPLIHG
SAMPLE ID COOES:

DOOI - FILTERED SAMPLE (.45 MICRONS)

0002 - FILTERED REPLICATE SAMPLE (.45 MICRONS)

0003 - FILTERED REPLICATE SAMPLE (.45 MICRONS)

0004 - FILTERED REPLICATE SAMPLE (.45 MICRONS)

N001 - LAFILTERED SAMPLE 
GROUMDHATER QUALITY DATA BY LOCATION

SOMP REV. O Data

SITE: MONO1 MONUMENT VALLEY

LOCATION: 0.655

MORTH COORDINATE: 59754.6 FT

EAST COORDINATE: 88624.1 FT

04/21/88 TO 12/13/94

REPORT DATE: 06/20/95

FORMATION OF COHPLETION: ALLUVIUN (AL)

HYORAULIC FLON RELATIONSHIP: DON GRADIENT (D)

\begin{tabular}{|c|c|c|c|c|c|c|c|c|}
\hline PARAMETER MANE & LOG DATE & $\begin{array}{c}\text { SAMPLE } \\
\text { ID }\end{array}$ & $\begin{array}{l}\text { UNITS OF } \\
\text { MEASURE }\end{array}$ & PVI & $\begin{array}{l}\text { PARAMETER } \\
\text { VALUE }\end{array}$ & FLAGS & $\begin{array}{l}\text { DETECTION } \\
\text { LIMIT }\end{array}$ & $\begin{array}{l}\text { PARAMETER } \\
\text { UHCERTAINTY }\end{array}$ \\
\hline PH & $\begin{array}{l}06 / 09 / 89 \\
06 / 09 / 89 \\
06 / 09 / 89 \\
12 / 03 / 89 \\
12 / 03 / 89 \\
12 / 03 / 89 \\
12 / 03 / 89 \\
12 / 03 / 89 \\
06 / 25 / 90 \\
01 / 29 / 91 \\
06 / 02 / 91 \\
02 / 22 / 92 \\
08 / 13 / 92 \\
11 / 19 / 92 \\
02 / 19 / 93 \\
06 / 26 / 93 \\
04 / 24 / 94\end{array}$ & $\begin{array}{l}0003 \\
0004 \\
0005 \\
0001 \\
0002 \\
0003 \\
0004 \\
0005 \\
0001 \\
0001 \\
0001 \\
0001 \\
0001 \\
0001 \\
0001 \\
N 001 \\
1001\end{array}$ & SU & & $\begin{array}{l}7.50 \\
7.50 \\
7.50 \\
7.21 \\
7.21 \\
7.21 \\
7.21 \\
7.21 \\
7.04 \\
7.08 \\
7.26 \\
7.05 \\
7.31 \\
7.33 \\
7.14 \\
7.23 \\
7.06\end{array}$ & $\begin{array}{l}L \\
L\end{array}$ & 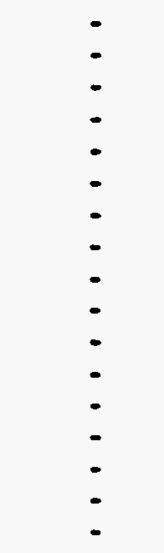 & $\begin{array}{l}- \\
- \\
- \\
- \\
- \\
: \\
- \\
: \\
- \\
- \\
-\end{array}$ \\
\hline PHOSPHATE (TOTAL) & $06 / 26 / 93$ & NOO1 & MG/L & & 0.3 & & 0.1 & - \\
\hline POTASSIUM & $\begin{array}{l}04 / 21 / 88 \\
04 / 21 / 88 \\
04 / 21 / 88 \\
04 / 21 / 88 \\
04 / 21 / 88 \\
06 / 09 / 89 \\
06 / 09 / 89 \\
06 / 09 / 89 \\
06 / 09 / 89 \\
06 / 09 / 89 \\
12 / 03 / 89 \\
12 / 03 / 89 \\
12 / 03 / 89 \\
12 / 03 / 89 \\
12 / 03 / 89 \\
06 / 25 / 90 \\
01 / 29 / 91 \\
06 / 02 / 91 \\
02 / 22 / 92 \\
08 / 13 / 92 \\
02 / 19 / 93 \\
06 / 26 / 93\end{array}$ & $\begin{array}{l}0001 \\
0002 \\
0003 \\
0004 \\
0005 \\
0001 \\
0002 \\
0003 \\
0004 \\
0005 \\
0001 \\
0002 \\
0003 \\
0004 \\
0005 \\
0001 \\
0001 \\
0001 \\
0001 \\
0001 \\
0001 \\
0001\end{array}$ & MG/L & & $\begin{array}{l}43.4 \\
41.8 \\
42.5 \\
45.9 \\
42.2 \\
36.6 \\
352 . \\
34.3 \\
36.9 \\
35.7 \\
30 . \\
31 . \\
31 . \\
30 . \\
30 . \\
32.8 \\
25.8 \\
27 . \\
25 . \\
30 \\
26.7 \\
26.6\end{array}$ & $\begin{array}{l}L \\
L \\
L \\
L \\
L \\
L \\
L \\
L \\
L \\
L\end{array}$ & $\begin{array}{l}0.01 \\
0.01 \\
0.01 \\
0.01 \\
0.01 \\
0.01 \\
0.01 \\
0.01 \\
0.01 \\
0.01 \\
0.01 \\
0.01 \\
0.01 \\
0.01 \\
0.01 \\
0.01 \\
0.01 \\
5 . \\
5 . \\
0.575 \\
0.9 \\
0.1\end{array}$ & $\begin{array}{l}- \\
- \\
- \\
: \\
\vdots \\
- \\
- \\
- \\
- \\
- \\
- \\
- \\
- \\
- \\
-\end{array}$ \\
\hline
\end{tabular}

PARAMETER VALUE INDICATOR (PVI): < - LESS THAN DETECTION LIMIT

SAMPLE ID COOES:

OTHER PARAMETER VALUE FLAGS:

- DUPLICATE ANALYSIS nOT UITHIK CONTROL LIMITS

L - LESS THAN THREE BORE VOLUMES REMOVED BEFORE SAMPLIHG

DOO1 - FILTERED SAMPLE (.45 MICRONS)

0002 - FILTERED REPLICATE SAMPLE (. 45 MICRONS)

0003 - FILTERED REPLICATE SAMPLE (.45 MICROHS)

0004 - FILTERED REPLICATE SAMPLE (.45 MICRONS) HOOI - UNFILTERED SMMPLE 
GROUNDWATER QUALITY DATA BY LOCATION

SOMP ReV. O Data

SITE: MONOI HOWUAENT VALLEY

LOCATION: 0655

MORTH COORDINATE: 59754.6 FT

EAST COORDINATE: 88624.1 FT

$04 / 21 / 88$ TO $12 / 13 / 94$

REPORT DATE: 06/20/95

FORMATION OF COMPLETION: ALLUVIUH (AL)

HYDRAULIC FLOW RELATIONSHIP: DOWN GRADIENT (D)

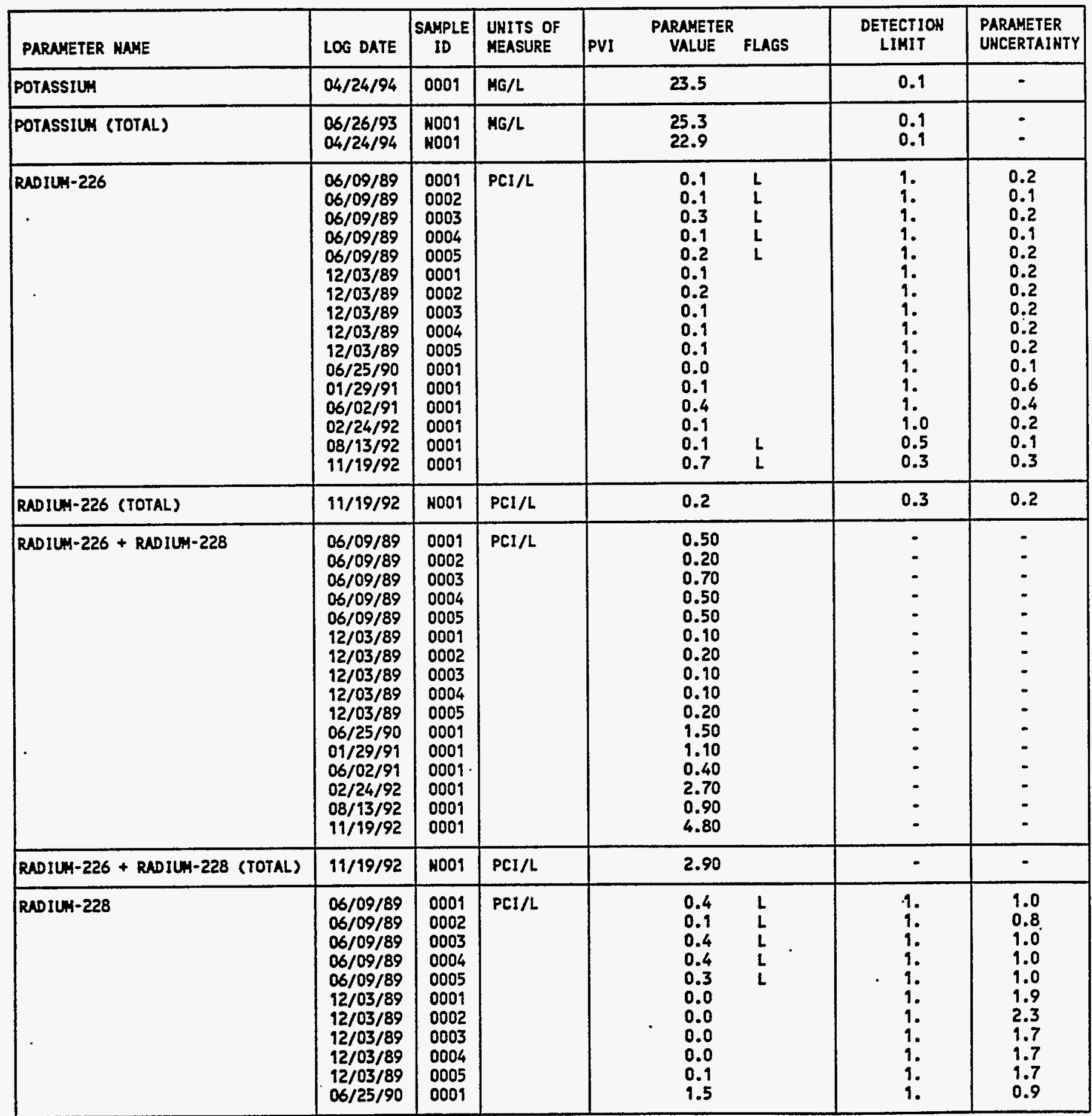

PARAMETER VALUE INDICATOR (PVI): \& - LESS THAN DETECTION LIMIT

SAMPLE ID COOES:

OOO1 - FILTERED SAMPLE (.45 MICRONS)

OTHER PARAYETER VALUE FLAGS:

$L$ - LESS THAN THREE BORE VOLUAES REMOVED BEFORE SAMPLIMG

0002 - FILTERED REPLICATE SAMPLE (.45 MICROHS)

0003 - FILTERED REPLICATE SAMPLE (.45 MICRONS)

0004 - FILTERED REPLICATE SAMPLE (.45 MICRONS)

NOD1 - UNFILTERED SAMPLE 
GROUNDWATER QUALITY DATA BY LOCATION

SOMP ReV. O Data

SITE: MONO 1 MONUMENT VALLEY

LOCATION: 0655

MORTH COORDINATE: 59754.6 FT

EAST COORDINATE: 88624.1 FT

$04 / 21 / 88$ TO $12 / 13 / 94$

REPORT DATE: $06 / 20 / 95$

FORHATION OF COMPLETIOH: ALLUVIUM (AL)

HYDRAULIC FLON RELATIOHSHIP: DOWN GRADIENT (D)

\begin{tabular}{|c|c|c|c|c|c|c|c|c|}
\hline PARAMETER MAME & LOG DATE & $\underset{\text { ID }}{\text { SAMPLE }}$ & $\begin{array}{l}\text { UHITS OF } \\
\text { MEASURE }\end{array}$ & PVI & $\begin{array}{l}\text { PARANETER } \\
\text { VALUE }\end{array}$ & FLAGS & $\begin{array}{l}\text { DETECTION } \\
\text { LIHIT }\end{array}$ & $\begin{array}{l}\text { PARAMETER } \\
\text { UNCERTAIHTY }\end{array}$ \\
\hline RADILW-228 & $\begin{array}{l}01 / 29 / 91 \\
06 / 02 / 91 \\
02 / 24 / 92 \\
08 / 13 / 92 \\
11 / 19 / 92\end{array}$ & $\begin{array}{l}0001 \\
0001 \\
0001 \\
0009 \\
0001\end{array}$ & PCI/L & & $\begin{array}{l}1.0 \\
0.0 \\
2.6 \\
0.8 \\
4.1\end{array}$ & $\begin{array}{l}J \\
L\end{array}$ & $\begin{array}{l}1 . \\
1.0 \\
1.0 \\
1.8 \\
2.1\end{array}$ & $\begin{array}{l}0.9 \\
1.7 \\
1.1 \\
1.0 \\
1.7\end{array}$ \\
\hline RADIUY-228 (TOTAL) & $11 / 19 / 92$ & NOO1 & PCI/L & & 2.7 & & 2.0 & 1.4 \\
\hline REDOX POTENTIAL & $\begin{array}{l}11 / 19 / 92 \\
06 / 26 / 93 \\
04 / 24 / 94\end{array}$ & $\begin{array}{l}0001 \\
\text { N001 } \\
\text { N001 }\end{array}$ & mVOLTS & & $\begin{array}{l}457.9 \\
460 \\
415\end{array}$ & & $\dot{-}$ & : \\
\hline SELENIUH & $\begin{array}{l}04 / 21 / 88 \\
04 / 21 / 88 \\
04 / 21 / 88 \\
04 / 21 / 88 \\
04 / 21 / 88 \\
06 / 09 / 89 \\
06 / 09 / 89 \\
06 / 09 / 89 \\
06 / 09 / 89 \\
06 / 09 / 89 \\
12 / 03 / 89 \\
12 / 03 / 89 \\
12 / 03 / 89 \\
12 / 03 / 89 \\
12 / 03 / 89 \\
06 / 25 / 90 \\
01 / 29 / 91 \\
06 / 02 / 91 \\
02 / 22 / 92 \\
08 / 13 / 92 \\
11 / 19 / 92\end{array}$ & $\begin{array}{l}0001 \\
0002 \\
0003 \\
0004 \\
0005 \\
0001 \\
0002 \\
0003 \\
0004 \\
0005 \\
0001 \\
0002 \\
0003 \\
0004 \\
0005 \\
0001 \\
0009 \\
0001 \\
0001 \\
0001 \\
0001\end{array}$ & MG/L & $\begin{array}{l}< \\
< \\
< \\
< \\
< \\
< \\
< \\
< \\
< \\
< \\
<\end{array}$ & $\begin{array}{l}0.010 \\
0.009 \\
0.010 \\
0.010 \\
0.010 \\
0.006 \\
0.019 \\
0.020 \\
0.020 \\
0.020 \\
0.005 \\
0.005 \\
0.005 \\
0.005 \\
0.005 \\
0.005 \\
0.005 \\
0.005 \\
0.005 \\
0.005 \\
0.015\end{array}$ & $\begin{array}{l}L \\
L \\
L \\
L \\
L \\
L \\
L \\
L \\
L \\
L\end{array}$ & $\begin{array}{l}0.005 \\
0.005 \\
0.005 \\
0.005 \\
0.005 \\
0.005 \\
0.005 \\
0.005 \\
0.005 \\
0.005 \\
0.005 \\
0.005 \\
0.005 \\
0.005 \\
0.005 \\
0.005 \\
0.005 \\
0.005 \\
0.005 \\
0.005 \\
0.015\end{array}$ & $\begin{array}{l}: \\
- \\
- \\
- \\
: \\
- \\
- \\
- \\
- \\
- \\
- \\
- \\
- \\
-\end{array}$ \\
\hline SELEHIUN (TOTAL) & $11 / 19 / 92$ & NOOI & $M G / L$ & $<$ & \multicolumn{2}{|l|}{0.0015} & 0.0015 & - \\
\hline SILICA - SIOZ & $\begin{array}{l}06 / 09 / 89 \\
06 / 09 / 89 \\
06 / 09 / 89 \\
06 / 09 / 89 \\
06 / 09 / 89 \\
12 / 03 / 89 \\
12 / 03 / 89 \\
12 / 03 / 89 \\
12 / 03 / 89 \\
12 / 03 / 89 \\
06 / 25 / 90 \\
01 / 29 / 91 \\
06 / 02 / 91\end{array}$ & $\begin{array}{l}0001 \\
0002 \\
0003 \\
0004 \\
0005 \\
0009 \\
0002 \\
0003 \\
0004 \\
0005 \\
0001 \\
0001 \\
0001\end{array}$ & $\begin{array}{c}\text { MG/L } \\
.\end{array}$ & & $\begin{array}{l}16 . \\
16 . \\
16 . \\
16 . \\
16 . \\
15.6 \\
16.3 \\
15.0 \\
15.4 \\
14.1 \\
16 . \\
14 . \\
15.7\end{array}$ & $\begin{array}{l}L \\
L \\
L \\
L \\
L\end{array}$ & $\begin{array}{l}2 . \\
2 . \\
2 . \\
2 . \\
2 . \\
2 . \\
2 . \\
2 . \\
2 . \\
2 . \\
2 . \\
2 . \\
0.1\end{array}$ & $\begin{array}{l}: \\
: \\
: \\
: \\
: \\
- \\
- \\
-\end{array}$ \\
\hline SILICA - SIOZ (TOTAL) & $06 / 26 / 93$ & NOO1 & MG/L & & 14.9 & H & 0.5 & - \\
\hline SILVER & $06 / 09 / 89$ & 0001 & $M G / L$ & $<$ & 0.01 & $\mathbf{L}$ & 0.01 & - \\
\hline
\end{tabular}

PARAMETER VALUE IMDICATOR (PVI): < - LESS THAN DETECTION LIMIT

OTHER PARAMETER VALUE FLAGS:

H - HOLD TIME EXPIRED, VALUE SUSPECT

1 - IMCREASED DETECTION LIMIT DUE TO REQUIRED DILUTION

J - estiMTTED VALUE

L - LESS THAK THREE BORE VOLLMES REMOVED BEFORE SAMPLIHG

U - SPIKE SNMPLE RECOVERY NOT WITHIN CONTROL LIMITS

SAMPLE ID COOES:

0001 - FILTERED SAMPLE (.45 MICRONS)

0002 - FILTERED REPLICATE SAMPLE (.45 MICRONS)

0003 - FILTERED REPLICATE SAMPLE (.45 MICRONS)

OOO4 - FILTERED REPLICATE SNMPLE (.45 MICRONS)

MOOI - UNFILTERED SNMPLE 
GROUNDHATER OUALITY DATA BY LOCATION

SOUP ReV. O Data

SITE: MONO1 MONUMENT VALLEY

LOCATION: 0655

NORTH COORDIHATE: 59754.6 FT

EAST COORDIHATE: 88624.1 FT

$04 / 21 / 88$ TO $12 / 13 / 94$

REPORT DATE: 06/20/95

FORMATION OF COMPLETION: ALLUVIUM (AL)

HYDRAULIC FLOW RELATIONSHIP: DOWH GRADIENT (D)

\begin{tabular}{|c|c|c|c|c|c|c|c|c|}
\hline PARAMETER MAME & LOG DATE & $\begin{array}{c}\text { SAMPLE } \\
\text { ID }\end{array}$ & $\begin{array}{l}\text { UNITS OF } \\
\text { MEASURE }\end{array}$ & PVI & $\begin{array}{l}\text { PARANETER } \\
\text { VALUE }\end{array}$ & FLAGS & $\begin{array}{l}\text { DETECTION } \\
\text { LIMIT }\end{array}$ & $\begin{array}{l}\text { PARAMETER } \\
\text { UNCERTAINTY }\end{array}$ \\
\hline SILVER & $\begin{array}{l}06 / 09 / 89 \\
06 / 09 / 89 \\
06 / 09 / 89 \\
06 / 09 / 89 \\
12 / 03 / 89 \\
12 / 03 / 89 \\
12 / 03 / 89 \\
12 / 03 / 89 \\
12 / 03 / 89 \\
06 / 25 / 90 \\
01 / 29 / 99 \\
06 / 02 / 91\end{array}$ & $\begin{array}{l}0002 \\
0003 \\
0004 \\
0005 \\
0001 \\
0002 \\
0003 \\
0004 \\
0005 \\
0001 \\
0001 \\
0001\end{array}$ & MG/L & $\begin{array}{l}< \\
< \\
< \\
< \\
< \\
< \\
< \\
< \\
< \\
< \\
<\end{array}$ & $\begin{array}{l}0.01 \\
0.01 \\
0.01 \\
0.01 \\
0.01 \\
0.01 \\
0.01 \\
0.01 \\
0.01 \\
0.01 \\
0.01 \\
0.01\end{array}$ & $\begin{array}{l}\mathbf{L} \\
\mathbf{L} \\
\mathbf{L} \\
\mathbf{L}\end{array}$ & $\begin{array}{l}0.01 \\
0.01 \\
0.01 \\
0.01 \\
0.01 \\
0.01 \\
0.01 \\
0.01 \\
0.01 \\
0.01 \\
0.01 \\
0.01\end{array}$ & $\begin{array}{l}- \\
- \\
- \\
- \\
: \\
- \\
- \\
-\end{array}$ \\
\hline SCOIUA & $\begin{array}{l}04 / 21 / 88 \\
04 / 21 / 88 \\
04 / 21 / 88 \\
04 / 21 / 88 \\
04 / 21 / 88 \\
06 / 09 / 89 \\
06 / 09 / 89 \\
06 / 09 / 89 \\
06 / 09 / 89 \\
06 / 09 / 89 \\
12 / 03 / 89 \\
12 / 03 / 89 \\
12 / 03 / 89 \\
12 / 03 / 89 \\
12 / 03 / 89 \\
06 / 25 / 90 \\
01 / 29 / 91 \\
06 / 02 / 91 \\
02 / 22 / 92 \\
08 / 13 / 92 \\
02 / 19 / 93 \\
06 / 26 / 93 \\
04 / 24 / 94\end{array}$ & $\begin{array}{l}0001 \\
0002 \\
0003 \\
0004 \\
0005 \\
0001 \\
0002 \\
0003 \\
0004 \\
0005 \\
0001 \\
0002 \\
0003 \\
0004 \\
0005 \\
0001 \\
0001 \\
0001 \\
0001 \\
0001 \\
0001 \\
0001 \\
0001\end{array}$ & MG/L & & $\begin{array}{l}149 . \\
144 . \\
145 . \\
140 . \\
137 . \\
177 . \\
163 . \\
158 . \\
169 . \\
165 . \\
166 . \\
170 . \\
172 . \\
167 . \\
166 . \\
165 . \\
185 . \\
190 . \\
163 . \\
170 \\
157 \\
150 \\
143\end{array}$ & $\begin{array}{l}L \\
L \\
L \\
L \\
L \\
L \\
L \\
L \\
L \\
L\end{array}$ & $\begin{array}{l}0.002 \\
0.002 \\
0.002 \\
0.002 \\
0.002 \\
0.002 \\
0.002 \\
0.002 \\
0.002 \\
0.002 \\
0.002 \\
0.002 \\
0.002 \\
0.002 \\
0.002 \\
0.002 \\
0.002 \\
5 . \\
5 . \\
5 \\
0.1 \\
1 \\
1\end{array}$ & $\begin{array}{l}- \\
: \\
: \\
\vdots \\
- \\
- \\
- \\
- \\
- \\
- \\
- \\
- \\
- \\
- \\
-\end{array}$ \\
\hline SOOIUA (TOTAL) & $\begin{array}{l}06 / 26 / 93 \\
04 / 24 / 94\end{array}$ & $\begin{array}{l}\text { N001 } \\
\text { N001 }\end{array}$ & $M G / L$ & & $\begin{array}{l}154 \\
129\end{array}$ & & $\begin{array}{l}1 \\
1\end{array}$ & : \\
\hline SPECIFIC CONDUCTANCE & $\begin{array}{l}04 / 21 / 88 \\
04 / 21 / 88 \\
04 / 21 / 88 \\
04 / 21 / 88 \\
04 / 21 / 88 \\
06 / 09 / 89 \\
06 / 09 / 89 \\
06 / 09 / 89 \\
06 / 09 / 89 \\
06 / 09 / 89 \\
12 / 03 / 89 \\
12 / 03 / 89 \\
12 / 03 / 89 \\
12 / 03 / 89\end{array}$ & $\begin{array}{l}0001 \\
0002 \\
0003 \\
0004 \\
0005 \\
0001 \\
0002 \\
0003 \\
0004 \\
0005 \\
0001 \\
0002 \\
0003 \\
0004\end{array}$ & ЧнНО/СК & & $\begin{array}{l}4950 . \\
4950 . \\
4950 . \\
4950 . \\
4950 . \\
4950 . \\
4150 . \\
4150 . \\
4150 . \\
4150 . \\
4650 . \\
4650 . \\
4650 . \\
4650 .\end{array}$ & $\begin{array}{l}L \\
L \\
J L \\
L \\
L \\
L \\
L \\
L \\
L \\
L\end{array}$ & $\begin{array}{l}- \\
- \\
- \\
- \\
- \\
- \\
- \\
- \\
-\end{array}$ & $\begin{array}{l}: \\
: \\
: \\
: \\
- \\
- \\
- \\
-\end{array}$ \\
\hline
\end{tabular}

PARAMETER VALUE INDICATOR (PVI): < - LESS THAN DETECTION LIMIT

SUMPLE ID COOES:

OTHER PARAMETER VALUE FLAGS:

O001 - FILTERED SAMPLE (.45 MICRONS)

O002 - FILTERED REPLICATE SAMPLE (. 45 MICROHS)

J - ESTIMATED VALUE

L - LESS THAH THREE BORE VOLUNES REMOVED BEFORE SNMPLIHG

0003 - FILTERED REPLICATE SAMPLE (.45 MICRONS)

0004 - FILTERED REPLICATE SAMPLE (.45 MICROHS)

NOO1 - UNFILTERED SAMPLE 
GROUHDHATER QUALITY DATA BY LOCATION

SOUP ReV. O Data

SITE: MOHOI MOHUMENT VALLEY

LOCATION: 0655

MORTH COORDIHATE: 59754.6 FT

EAST COORDINATE: 88624.9 FT

04/21/88 TO $12 / 13 / 94$

REPORT DATE: $06 / 20 / 95$

FORHATION OF COMPLETION: ALLUVIUM (AL)

HYDRAULIC FLON RELATIOHSHIP: DOWN GRADIENT (D)

\begin{tabular}{|c|c|c|c|c|c|c|c|c|}
\hline PARAMETER HAME & LOG DATE & $\underset{\text { SAMPLE }}{\text { ID }}$ & $\begin{array}{l}\text { UNITS OF } \\
\text { MEASURE }\end{array}$ & PVI & $\begin{array}{l}\text { PARAMETE } \\
\text { VALUE }\end{array}$ & FLAGS & $\begin{array}{l}\text { DETECTION } \\
\text { LIMIT }\end{array}$ & $\begin{array}{l}\text { PARAMETER } \\
\text { UHCERTAINTY }\end{array}$ \\
\hline SPECIFIC CONDUCTAHCE & $\begin{array}{l}12 / 03 / 89 \\
06 / 25 / 90 \\
01 / 29 / 91 \\
06 / 02 / 91 \\
02 / 22 / 92 \\
08 / 13 / 92 \\
11 / 19 / 92 \\
02 / 19 / 93 \\
06 / 26 / 93 \\
04 / 24 / 94\end{array}$ & $\begin{array}{l}0005 \\
0001 \\
0001 \\
0001 \\
0001 \\
0001 \\
0001 \\
0001 \\
\text { NO01 } \\
\text { N001 }\end{array}$ & UNHO/CM & \multicolumn{3}{|c|}{$\begin{array}{r}4650 . \\
4550 . \\
5300 . \\
5190 . \\
4260 \\
449 \\
3990 \\
3910 \\
424 \\
3770\end{array}$} & $\begin{array}{l}- \\
- \\
- \\
- \\
- \\
-\end{array}$ & $\begin{array}{l}- \\
: \\
: \\
- \\
-\end{array}$ \\
\hline STRONTIUM & $\begin{array}{l}06 / 25 / 90 \\
01 / 29 / 91 \\
06 / 02 / 91 \\
02 / 22 / 92 \\
08 / 13 / 92 \\
11 / 19 / 92 \\
06 / 26 / 93 \\
04 / 24 / 94\end{array}$ & $\begin{array}{l}0001 \\
0001 \\
0001 \\
0001 \\
0001 \\
0001 \\
0001 \\
0001\end{array}$ & $M G / L$ & \multicolumn{3}{|c|}{$\begin{array}{l}2.68 \\
3.0 \\
2.98 \\
2.54 \\
3.0 \\
3.2 \\
2.55 \\
2.88\end{array}$} & $\begin{array}{l}0.1 \\
0.1 \\
0.01 \\
0.01 \\
0.2 \\
0.00035 \\
0.01 \\
0.01\end{array}$ & $\begin{array}{l}- \\
: \\
: \\
: \\
-\end{array}$ \\
\hline STRONTIUM (TOTAL) & $\begin{array}{l}11 / 19 / 92 \\
06 / 26 / 93 \\
04 / 24 / 94\end{array}$ & $\begin{array}{l}\text { No01 } \\
\text { Ho01 } \\
\text { No01 }\end{array}$ & MG/L & \multicolumn{3}{|c|}{$\begin{array}{l}3.4 \\
2.50 \\
2.73\end{array}$} & $\begin{array}{l}0.00035 \\
0.01 \\
0.01\end{array}$ & : \\
\hline SULFATE & $\begin{array}{l}04 / 21 / 88 \\
04 / 21 / 88 \\
04 / 21 / 88 \\
04 / 21 / 88 \\
04 / 21 / 88 \\
06 / 09 / 89 \\
06 / 09 / 89 \\
06 / 09 / 89 \\
06 / 09 / 89 \\
06 / 09 / 89 \\
12 / 03 / 89 \\
12 / 03 / 89 \\
12 / 03 / 89 \\
12 / 03 / 89 \\
12 / 03 / 89 \\
06 / 25 / 90 \\
01 / 29 / 91 \\
06 / 02 / 91 \\
02 / 22 / 92 \\
08 / 13 / 92 \\
11 / 19 / 92 \\
02 / 19 / 93 \\
04 / 24 / 94\end{array}$ & $\begin{array}{l}0001 \\
0002 \\
0003 \\
0004 \\
0005 \\
0001 \\
0002 \\
0003 \\
0004 \\
0005 \\
0001 \\
0002 \\
0003 \\
0004 \\
0005 \\
0001 \\
0001 \\
0001 \\
0001 \\
0001 \\
0001 \\
0001 \\
0001\end{array}$ & MG/L & \multicolumn{3}{|c|}{$\begin{array}{l}2950 . \\
2910 . \\
2950 . \\
2960 . \\
2930 . \\
2770 . \\
2790 . \\
2780 . \\
2790 . \\
2800 . \\
2730 . \\
2670 . \\
2660 . \\
2590 . \\
2410 . \\
2660 . \\
2760 . \\
3540 . \\
2370 . \\
2000 \\
2400 \\
2470 \\
1950\end{array}$} & $\begin{array}{l}0.1 \\
0.1 \\
0.1 \\
0.1 \\
0.1 \\
0.1 \\
0.1 \\
0.1 \\
0.1 \\
0.1 \\
0.1 \\
0.1 \\
0.1 \\
0.1 \\
0.1 \\
0.1 \\
0.1 \\
10 . \\
10 . \\
0.59 \\
1.0 \\
1 \\
1\end{array}$ & $\begin{array}{l}- \\
- \\
- \\
- \\
- \\
- \\
- \\
- \\
- \\
- \\
- \\
- \\
- \\
- \\
- \\
-\end{array}$ \\
\hline SULFATE (TOTAL) & $\begin{array}{l}11 / 19 / 92 \\
06 / 26 / 93\end{array}$ & $\begin{array}{l}\text { No01 } \\
\text { N001 }\end{array}$ & $M G / L$ & \multicolumn{3}{|c|}{$\begin{array}{l}2400 \\
2140\end{array}$} & 1.0 & - \\
\hline SULFIDE & $06 / 09 / 89$ & 0001 & $M G / L$ & $<$ & 0.1 & $L$ & 0.1 & - \\
\hline
\end{tabular}

PARAMETER VALUE IHDICATOR (PVI): < - LESS THAN DETECTIOH LIMIT

SAMPLE IO COOES:

0001 - FILTERED SAMPLE (.45 MICRONS)

OTHER PARAMETER VALUE FLAGS:

- - DUPLICATE AMALYSIS NOT HITHIN CONTROL LIMITS

OOO2 - FILTERED REPLICATE SAMPLE (.45 MICRONS)

0003 - FILTERED REPLICATE SAMPLE (.45 MICROHS)

0004 - FILTERED REPLICATE SAMPLE (.45 HICROHS)

I - INCREASED OETECTION LIMIT DUE TO REQUIRED DILUTION

R - IMUSABLE DATA POIHT

NOO1 - UNFILTERED SAMPLE

S - REPORTED VALUE DETERHIHED USING METHOO OF STD ADDITION (HSA) 
GROUNDHATER QUALITY DATA BY LOCATION

SOMP ReV. 0 Data

SITE: MONO1 HONUMENT VALLEY

LOCATION: 0655

NORTH COORDINATE: 59754.6 FT

EAST COORDINATE: B8624.1 FT

04/21/88 TO 12/13/94

REPORT DATE: 06/20/95

FORMATION OF COMPLETIOH: ALLUVIUM (AL)

HYDRAULIC FLON RELATIONSHIP: DOWN GRADIENT (D)

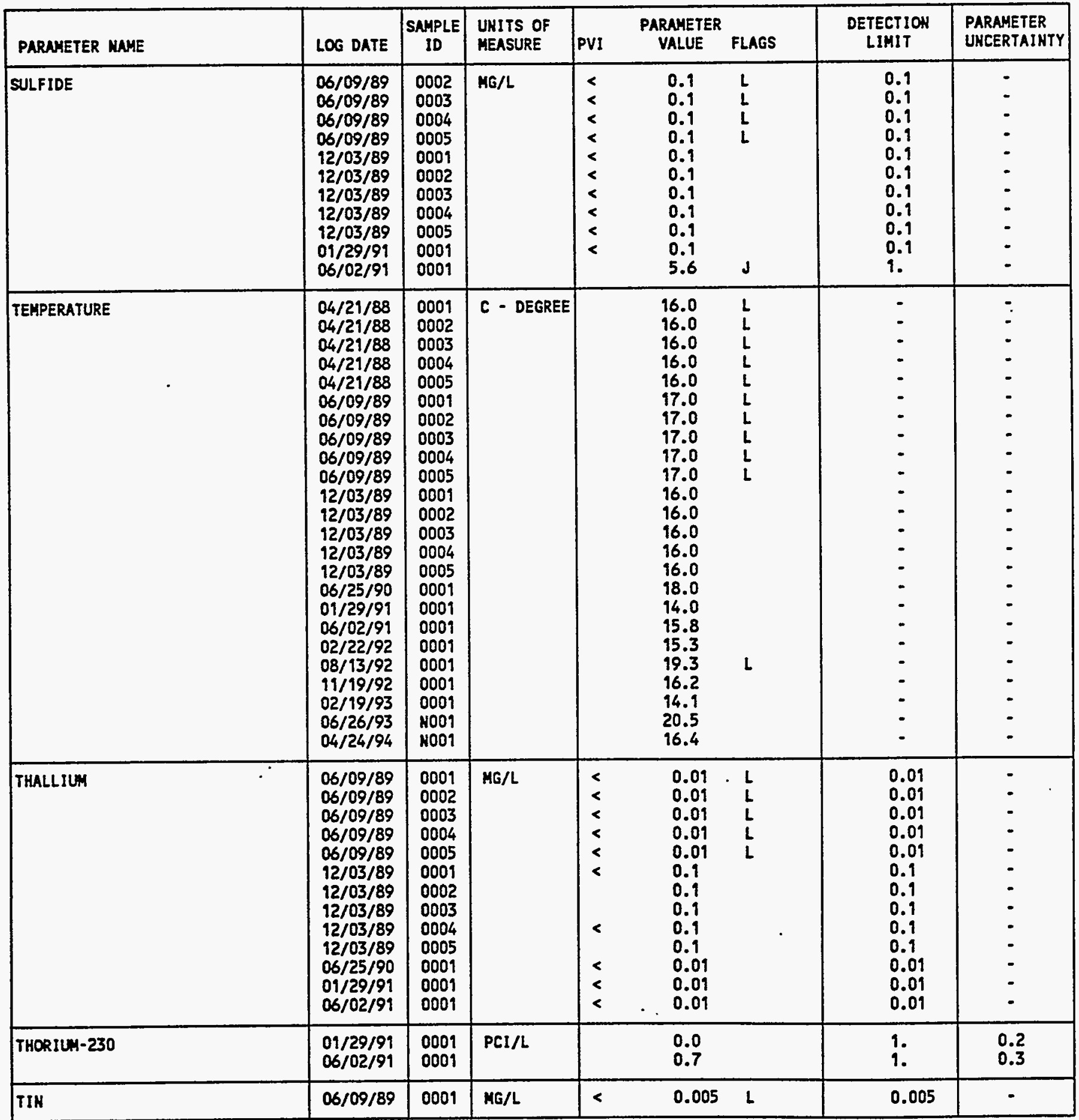

PARANETER VALUE INDICATOR (PVI): < - LESS THAN DETECTIOH LIMIT

SAMPLE ID COOES:

OTHER PARAMETER VALUE fLAGS:

0009 - FILTERED SNMPLE (.45 MICRONS)

0002 - FILTERED REPLICATE SAMPLE (.45 MICRONS)

$\mathrm{J}$ - ESTIMLTED VALUE

0003 - FILTERED REPLICATE SAMPLE (.45 MICRONS)

L - LESS THAN THREE BORE VOLUHES REMOVED BEFORE SAMLIHG 
GROUHDHATER QUALITY DATA BY LOCATION

SOWP ReV. O Data

SITE: MONO1 MONLMENT VALLEY

LOCATION: 0655

NORTH COORDIHATE: 59754.6 FT

EAST COORDIHATE: 88624.1 FT

$04 / 21 / 88$ TO $12 / 13 / 94$

REPORT DATE: 06/20/95

FORMATION OF COMPLETION: ALLUVIUM (AL)

HYDRAULIC FLOW RELATIONSHIP: DOWN GRADIENT (D)

\begin{tabular}{|c|c|c|c|c|c|c|c|c|}
\hline PARAMETER MAME & LOG DATE & $\underset{\text { SD }}{\text { SAMPLE }}$ & $\begin{array}{l}\text { UNITS OF } \\
\text { MEASURE }\end{array}$ & PVI & $\begin{array}{l}\text { PARAMETER } \\
\text { VALUE }\end{array}$ & FLAGS & $\begin{array}{l}\text { DETECTION } \\
\text { LIMIT }\end{array}$ & $\begin{array}{l}\text { PARAMETER } \\
\text { UNCERTAINTY }\end{array}$ \\
\hline TIK & $\begin{array}{l}06 / 09 / 89 \\
06 / 09 / 89 \\
06 / 09 / 89 \\
06 / 09 / 89 \\
12 / 03 / 89 \\
12 / 03 / 89 \\
12 / 03 / 89 \\
12 / 03 / 89 \\
12 / 03 / 89 \\
06 / 25 / 90 \\
01 / 29 / 91 \\
06 / 02 / 91 \\
02 / 22 / 92\end{array}$ & $\begin{array}{l}0002 \\
0003 \\
0004 \\
0005 \\
0001 \\
0002 \\
0003 \\
0004 \\
0005 \\
0001 \\
0001 \\
0001 \\
0001\end{array}$ & $M G / L$ & $\begin{array}{l}< \\
< \\
< \\
< \\
< \\
< \\
< \\
< \\
< \\
< \\
< \\
<\end{array}$ & $\begin{array}{l}0.005 \\
0.005 \\
0.005 \\
0.005 \\
0.005 \\
0.005 \\
0.005 \\
0.005 \\
0.005 \\
0.063 \\
0.005 \\
0.01 \\
0.03\end{array}$ & $\begin{array}{l}L \\
L \\
L \\
L\end{array}$ & $\begin{array}{l}0.005 \\
0.005 \\
0.005 \\
0.005 \\
0.005 \\
0.005 \\
0.005 \\
0.005 \\
0.005 \\
0.005 \\
0.005 \\
0.01 \\
0.03\end{array}$ & $\begin{array}{l}- \\
i \\
- \\
- \\
- \\
- \\
- \\
- \\
-\end{array}$ \\
\hline TOTAL DISSOLVED SOLIDS & $\begin{array}{l}04 / 21 / 88 \\
04 / 21 / 88 \\
04 / 21 / 88 \\
04 / 21 / 88 \\
04 / 21 / 88 \\
06 / 09 / 89 \\
06 / 09 / 89 \\
06 / 09 / 89 \\
06 / 09 / 89 \\
06 / 09 / 89 \\
12 / 03 / 89 \\
12 / 03 / 89 \\
12 / 03 / 89 \\
12 / 03 / 89 \\
12 / 03 / 89 \\
06 / 25 / 90 \\
01 / 29 / 91 \\
06 / 02 / 91 \\
02 / 22 / 92 \\
08 / 13 / 92 \\
02 / 19 / 93 \\
04 / 24 / 94\end{array}$ & $\begin{array}{l}0001 \\
0002 \\
0003 \\
0004 \\
0005 \\
0001 \\
0002 \\
0003 \\
0004 \\
0005 \\
0001 \\
0002 \\
0003 \\
0004 \\
0005 \\
0001 \\
0001 \\
0001 \\
0001 \\
0001 \\
0001 \\
0001\end{array}$ & MG/L & & $\begin{array}{l}4400 . \\
4350 . \\
4390 . \\
4420 . \\
4430 . \\
4490 . \\
4680 . \\
4500 . \\
4550 . \\
4640 . \\
4410 . \\
4440 . \\
4460 . \\
4440 . \\
4400 . \\
3940 . \\
4170 . \\
4160 . \\
3790 . \\
4200 \\
3870 \\
3410\end{array}$ & $\begin{array}{l}\mathbf{L} \\
\mathbf{L} \\
\mathbf{L} \\
\mathbf{L} \\
\mathbf{L} \\
\mathbf{L} \\
\mathbf{L} \\
\mathbf{L} \\
\mathbf{L} \\
\mathbf{L}\end{array}$ & $\begin{array}{l}10 . \\
10 . \\
10 . \\
10 . \\
10 . \\
10 . \\
10 . \\
10 . \\
10 . \\
10 . \\
10 . \\
10 . \\
10 . \\
10 . \\
10 . \\
10 . \\
10 . \\
10 . \\
10 . \\
42 \\
10 \\
10\end{array}$ & $\begin{array}{l}- \\
- \\
: \\
- \\
- \\
= \\
- \\
- \\
- \\
- \\
- \\
- \\
- \\
- \\
- \\
-\end{array}$ \\
\hline TOTAL DISSOLVED SOLIDS (TOTAL) & $06 / 26 / 93$ & No01 & $M G / L$ & & 3680 & & 10 & - \\
\hline TOTAL ORGAHIC CARBOA & $\begin{array}{l}04 / 21 / 88 \\
04 / 21 / 88 \\
04 / 21 / 88 \\
04 / 21 / 88 \\
04 / 21 / 88 \\
01 / 29 / 91 \\
06 / 02 / 91 \\
02 / 22 / 92 \\
08 / 13 / 92\end{array}$ & $\begin{array}{l}0001 \\
0002 \\
0003 \\
0004 \\
0005 \\
0001 \\
0001 \\
0001 \\
0001\end{array}$ & MG/L & & $\begin{array}{c}101 . \\
96.0 \\
91.5 \\
91.5 \\
98.8 \\
1.2 \\
3 . \\
1 . \\
1.5\end{array}$ & $\begin{array}{l}L \\
L \\
L \\
L \\
L\end{array}$ & $\begin{array}{l}1 . \\
1 . \\
1 . \\
1 . \\
1 . \\
1 . \\
1 . \\
1.0\end{array}$ & $\begin{array}{l}- \\
- \\
- \\
- \\
- \\
-\end{array}$ \\
\hline TURBIDITY & $04 / 24 / 94$ & N001 & NTU & & $\mathbf{0}$ & & - & - \\
\hline URANIUA & $\begin{array}{l}04 / 21 / 88 \\
04 / 21 / 88 \\
04 / 21 / 88\end{array}$ & $\begin{array}{l}0001 \\
0002 \\
0003\end{array}$ & MG/L & & $\begin{array}{l}0.0326 \\
0.0318 \\
0.0343\end{array}$ & $\begin{array}{l}L \\
L\end{array}$ & $\begin{array}{l}0.003 \\
0.003 \\
0.003\end{array}$ & : \\
\hline
\end{tabular}

PARAMETER VALUE IMDICATOR (PVI): < - LESS THAN DETECTION LIMIT

SNYPLE ID COOES:

OTHER PARAMETER VALUE FLAGS:

1 - IMCREASED DETECTION LIMIT DUE TO REQUIRED DILUTION

L - LESS THAH THREE BORE VOLUNES REMOVED BEFORE SAMPLIMG 
GROUNDHATER QUALITY DATA BY LOCATION

SOMP ReV. O Data

SITE: HONO MONUNENT VALLEY

LOCATION: 0655

NORTH COORDIHATE: 59754.6 FT

EAST COORDINATE: 88624.1 FT

04/21/88 TO $12 / 13 / 94$

REPORT DATE: $06 / 20 / 95$

FORMATION OF COAPLETION: ALLUVIUN (AL)

HYDRAULIC FLON RELATIOHSHIP: DOWN GRADIENT (D)

\begin{tabular}{|c|c|c|c|c|c|c|c|c|}
\hline PARAMETER MAME & LOG DATE & $\begin{array}{c}\text { SAMPLE } \\
\text { ID }\end{array}$ & $\begin{array}{l}\text { UNITS OF } \\
\text { MEASURE }\end{array}$ & PVI & $\begin{array}{l}\text { PARAMETER } \\
\text { VALUE }\end{array}$ & FLAGS & $\begin{array}{l}\text { DETECTION } \\
\text { LIMIT }\end{array}$ & $\begin{array}{l}\text { PARAMETER } \\
\text { UNCERTAINTY }\end{array}$ \\
\hline URANIUN & $\begin{array}{l}04 / 21 / 88 \\
04 / 21 / 88 \\
06 / 09 / 89 \\
06 / 09 / 89 \\
06 / 09 / 89 \\
06 / 09 / 89 \\
06 / 09 / 89 \\
12 / 03 / 89 \\
12 / 03 / 89 \\
12 / 03 / 89 \\
12 / 03 / 89 \\
12 / 03 / 89 \\
06 / 25 / 90 \\
01 / 29 / 91 \\
06 / 02 / 91 \\
02 / 24 / 92 \\
11 / 19 / 92 \\
02 / 19 / 93 \\
06 / 26 / 93 \\
04 / 24 / 94\end{array}$ & $\begin{array}{l}0004 \\
0005 \\
0001 \\
0002 \\
0003 \\
0004 \\
0005 \\
0001 \\
0002 \\
0003 \\
0004 \\
0005 \\
0001 \\
0001 \\
0001 \\
0001 \\
0001 \\
0001 \\
0001 \\
0001\end{array}$ & ME/L & & $\begin{array}{l}0.0310 \\
0.0318 \\
0.028 \\
0.028 \\
0.027 \\
0.028 \\
0.031 \\
0.030 \\
0.028 \\
0.029 \\
0.028 \\
0.028 \\
0.019 \\
0.0299 \\
0.020 \\
0.024 \\
0.029 \\
0.028 \\
0.026 \\
0.027\end{array}$ & $\begin{array}{l}L \\
L \\
L \\
L \\
L \\
L \\
L\end{array}$ & $\begin{array}{l}0.003 \\
0.003 \\
0.003 \\
0.003 \\
0.003 \\
0.003 \\
0.003 \\
0.003 \\
0.003 \\
0.003 \\
0.003 \\
0.003 \\
0.003 \\
0.0003 \\
0.001 \\
0.001 \\
0.001 \\
0.005 \\
0.001 \\
0.001\end{array}$ & $\begin{array}{l}- \\
i \\
i \\
i \\
i \\
i \\
- \\
- \\
- \\
- \\
- \\
-\end{array}$ \\
\hline URAKIUM (TOTAL) & $\begin{array}{l}11 / 19 / 92 \\
06 / 26 / 93 \\
04 / 24 / 94\end{array}$ & $\begin{array}{l}\text { NO01 } \\
\text { NO01 } \\
\text { NO01 }\end{array}$ & MG/L & & $\begin{array}{l}0.026 \\
0.025 \\
0.027\end{array}$ & & $\begin{array}{l}0.001 \\
0.001 \\
0.001\end{array}$ & : \\
\hline VANADIUM & $\begin{array}{l}06 / 09 / 89 \\
06 / 09 / 89 \\
06 / 09 / 89 \\
06 / 09 / 89 \\
06 / 09 / 89 \\
12 / 03 / 89 \\
12 / 03 / 89 \\
12 / 03 / 89 \\
12 / 03 / 89 \\
12 / 03 / 89 \\
06 / 25 / 90 \\
01 / 29 / 91 \\
06 / 02 / 91 \\
02 / 22 / 92 \\
08 / 13 / 92 \\
11 / 19 / 92 \\
06 / 26 / 93 \\
04 / 24 / 94\end{array}$ & $\begin{array}{l}0001 \\
0002 \\
0003 \\
0004 \\
0005 \\
0001 \\
0002 \\
0003 \\
0004 \\
0005 \\
0001 \\
0001 \\
0001 \\
0001 \\
0001 \\
0001 \\
0001 \\
0001\end{array}$ & ME/L & $\begin{array}{l}< \\
< \\
< \\
< \\
< \\
< \\
< \\
< \\
< \\
<\end{array}$ & $\begin{array}{l}0.08 \\
0.08 \\
0.08 \\
0.07 \\
0.08 \\
0.01 \\
0.01 \\
0.01 \\
0.01 \\
0.01 \\
0.02 \\
0.01 \\
0.01 \\
0.02 \\
0.05 \\
0.0083 \\
0.01 \\
0.01\end{array}$ & $\begin{array}{l}L \\
L \\
L \\
L \\
L\end{array}$ & $\begin{array}{l}0.01 \\
0.01 \\
0.01 \\
0.01 \\
0.01 \\
0.01 \\
0.01 \\
0.01 \\
0.01 \\
0.01 \\
0.01 \\
0.01 \\
0.01 \\
0.01 \\
0.05 \\
0.0019 \\
0.01\end{array}$ & $\begin{array}{l}- \\
- \\
- \\
- \\
- \\
- \\
- \\
- \\
- \\
- \\
- \\
-\end{array}$ \\
\hline VAHADIUH (TOTAL) & $\begin{array}{l}11 / 19 / 92 \\
06 / 26 / 93 \\
04 / 24 / 94\end{array}$ & $\begin{array}{l}\text { NOO1 } \\
\text { NOOI } \\
\text { HOOI }\end{array}$ & ME/L & $<$ & $\begin{array}{l}0.0070 \\
0.01 \\
0.01\end{array}$ & & $\begin{array}{l}0.0019 \\
0.01 \\
0.01\end{array}$ & 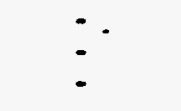 \\
\hline ZIMC & $\begin{array}{l}06 / 09 / 89 \\
06 / 09 / 89 \\
06 / 09 / 89 \\
06 / 09 / 89 \\
06 / 09 / 89 \\
12 / 03 / 89\end{array}$ & $\begin{array}{l}0001 \\
0002 \\
0003 \\
0004 \\
0005 \\
0001\end{array}$ & $M G / L$ & $\begin{array}{l}< \\
< \\
< \\
< \\
< \\
<\end{array}$ & $\begin{array}{l}0.005 \\
0.005 \\
0.005 \\
0.005 \\
0.005 \\
0.005\end{array}$ & $\begin{array}{l}L \\
L \\
L \\
L \\
L\end{array}$ & $\begin{array}{l}0.005 \\
0.005 \\
0.005 \\
0.005 \\
0.005 \\
0.005\end{array}$ & $\begin{array}{l}: \\
: \\
:\end{array}$ \\
\hline
\end{tabular}

PARAMETER VALUE INDICATOR (PVI): < - LESS THAM DETECTION LIMIT

SAMPLE ID COOES:

OTHER PARAMETER VALUE FLAGS:

0002 - FILTERED REPLICATE SAMPLE (.45 MICRONS)

0003 - FILTERED REPLICATE SANPLE (.45 MICRONS)

0004 - FILTERED REPLICATE SAMPLE (.45 MICROHS) NOO1 - UNFILTERED SAMPLE 
GROUNDGATER QUALITY DATA BY LOCATION

SOWP REV. 0 Data

SITE: HONO1 MOHUNENT VALLEY

LOCATION: 0655

HORTH COORDINATE: 59754.6 FT

EAST COORDIHATE: 88624.1 FT

$04 / 21 / 88$ TO $12 / 13 / 94$

REPORT DATE: 06/20/95

FORMATION OF COMPLETIOH: ALLUVIUN (AL)

HYDRAULIC FLOW RELATIOHSHIP: DONN GRADIENT (D)

\begin{tabular}{|c|c|c|c|c|c|c|c|c|}
\hline PARAMETER MAME & LOG DATE & $\underset{\text { SN }}{\text { SNMLE }}$ & $\begin{array}{l}\text { UNITS OF } \\
\text { MEASURE }\end{array}$ & PVI & $\begin{array}{l}\text { PARAMETER } \\
\text { VALUE }\end{array}$ & FLAGS & $\begin{array}{l}\text { DETECTION } \\
\text { LIMIT }\end{array}$ & $\begin{array}{l}\text { PARAMETER } \\
\text { UNCERTAIHTY }\end{array}$ \\
\hline ZINC & $\begin{array}{l}12 / 03 / 89 \\
12 / 03 / 89 \\
12 / 03 / 89 \\
12 / 03 / 89 \\
06 / 25 / 90 \\
01 / 29 / 91 \\
06 / 02 / 91 \\
02 / 22 / 92 \\
08 / 13 / 92 \\
11 / 19 / 92 \\
06 / 26 / 93\end{array}$ & $\begin{array}{l}0002 \\
0003 \\
0004 \\
0005 \\
0001 \\
0001 \\
0001 \\
0001 \\
0001 \\
0001 \\
0001\end{array}$ & $M G / L$ & $\begin{array}{l}< \\
< \\
< \\
< \\
< \\
< \\
<\end{array}$ & $\begin{array}{l}0.005 \\
0.005 \\
0.005 \\
0.005 \\
0.005 \\
0.005 \\
0.005 \\
0.059 \\
0.030 \\
0.0071 \\
0.019\end{array}$ & $\begin{array}{l}\text { L } \\
\text { J }\end{array}$ & $\begin{array}{l}0.005 \\
0.005 \\
0.005 \\
0.005 \\
0.005 \\
0.005 \\
0.005 \\
0.005 \\
0.02 \\
0.0010 \\
0.005\end{array}$ & $\begin{array}{l}- \\
- \\
- \\
- \\
- \\
- \\
-\end{array}$ \\
\hline ZINC (TOTAL) & $\begin{array}{l}11 / 19 / 92 \\
06 / 26 / 93\end{array}$ & $\begin{array}{l}\text { NoO1 } \\
\text { HOO1 }\end{array}$ & $M G / L$ & & $\begin{array}{l}0.0050 \\
0.033\end{array}$ & ง & $\begin{array}{l}0.0010 \\
0.005\end{array}$ & $\because$ \\
\hline
\end{tabular}

PARAMETER VALUE IHDICATOR (PVI): < - LESS THAN DETECTIOH LIMIT

SAMPLE ID COOES:

OTHER PARAMETER VALUE fLAGS:

$J$ - ESTIMATED VALUE

L - LESS THAN THREE BORE VOLLMES REMOVED BEFORE SAMPLIHG

DOO1 - FILTERED SAMPLE (.45 MICRONS)

0002 - FILTERED REPLICATE SAMPLE (.45 MICRONS)

0003 - FILTERED REPLICATE SAMPLE (.45 MICRONS)

0004 - FILTERED REPLICATE SAMPLE (.45 MICRONS)

NOO1 - UAFILTERED SAMPLE 
GROUHDHATER QUALITY DATA BY LOCATION

SOWP ReV. O Data

SITE: HONOI HOHLNENT VALLEY

LOCATION: 0656

NORTH COORDIHATE: 59545.2 FT

EAST COORDINATE: 89175.1 FT

$04 / 21 / 88$ TO $12 / 13 / 94$

REPORT DATE: 06/20/95

FORMATION OF COMPLETION: ALLUVIUH (AL)

HYDRAULIC FLON RELATIONSHIP: DOWN GRADIENT (D)

\begin{tabular}{|c|c|c|c|c|c|c|c|c|}
\hline PARNMETER MAME & LOG DATE & $\underset{\text { SAMPLE }}{\text { SAPL }}$ & $\begin{array}{l}\text { UNITS OF } \\
\text { MEASURE }\end{array}$ & PVI & $\begin{array}{l}\text { PARAMETER } \\
\text { VALUE }\end{array}$ & FLAGS & $\begin{array}{l}\text { DETECTION } \\
\text { LIHIT }\end{array}$ & $\begin{array}{l}\text { PARAMETER } \\
\text { UHCERTAINTY }\end{array}$ \\
\hline ALKALINITY & $\begin{array}{l}06 / 25 / 89 \\
11 / 22 / 92 \\
02 / 19 / 93 \\
06 / 29 / 93\end{array}$ & $\begin{array}{l}0001 \\
0001 \\
0001 \\
1001\end{array}$ & MG/L Caco3 & & $\begin{array}{l}262 . \\
260 \\
260 \\
241\end{array}$ & $\mathbf{L}$ & $\begin{array}{l}- \\
-\end{array}$ & $\begin{array}{l}- \\
-\end{array}$ \\
\hline ALUAINUM & $06 / 25 / 89$ & 0001 & MG/L & $<$ & 0.1 & $\mathbf{L}$ & 0.1 & - \\
\hline AmHONIUA & $\begin{array}{l}06 / 25 / 89 \\
02 / 19 / 93\end{array}$ & $\begin{array}{l}0001 \\
0001\end{array}$ & ME/L & & $\begin{array}{l}120 . \\
117\end{array}$ & $L$ & $\begin{array}{l}0.1 \\
0.1\end{array}$ & $\ddot{-}$ \\
\hline AMHOWIUM (TOTAL) & $0.6 / 29 / 93$ & No01 & MG/L & & 118 & & 0.1 & - \\
\hline ANTIMONY & $06 / 25 / 89$ & 0001 & $M G / L$ & & 0.008 & JL & 0.003 & - \\
\hline ARSENIC & $\begin{array}{l}06 / 25 / 89 \\
11 / 22 / 92 \\
06 / 29 / 93\end{array}$ & $\begin{array}{l}0001 \\
0001 \\
0001\end{array}$ & $M G / L$ & $<$ & $\begin{array}{l}0.01 \\
0.0015 \\
0.005\end{array}$ & $L$ & $\begin{array}{l}0.01 \\
0.0015 \\
0.005\end{array}$ & : \\
\hline ARSENIC (TOTAL) & $\begin{array}{l}11 / 22 / 92 \\
06 / 29 / 93\end{array}$ & $\begin{array}{l}\text { NOO1 } \\
\text { N001 }\end{array}$ & MG/L & $<$ & $\begin{array}{l}0.0015 \\
0.005\end{array}$ & & $\begin{array}{l}0.0015 \\
0.005\end{array}$ & - \\
\hline BARIUM & $\begin{array}{l}06 / 25 / 89 \\
11 / 22 / 92 \\
06 / 29 / 93\end{array}$ & $\begin{array}{l}0009 \\
0001 \\
0001\end{array}$ & MG/L & $<$ & $\begin{array}{l}0.1 \\
0.028 \\
0.1\end{array}$ & $\mathbf{L}$ & $\begin{array}{l}0.1 \\
0.0015 \\
0.1\end{array}$ & - \\
\hline BARIUH (TOTAL) & $\begin{array}{l}11 / 22 / 92 \\
06 / 29 / 93\end{array}$ & $\begin{array}{l}\text { NO01 } \\
\text { N001 }\end{array}$ & MG/L & $<$ & $\begin{array}{l}0.040 \\
0.1\end{array}$ & & $\begin{array}{l}0.0015 \\
0.1\end{array}$ & - \\
\hline BERYLLIUN & $06 / 25 / 89$ & 0001 & MG/L & $<$ & 0.01 & $L$ & 0.01 & - \\
\hline BORON & $06 / 25 / 89$ & 0001 & $M G / L$ & $<$ & 0.1 & $L$ & 0.1 & - \\
\hline CADMIUM & $\begin{array}{l}06 / 25 / 89 \\
11 / 22 / 92\end{array}$ & $\begin{array}{l}0001 \\
0001\end{array}$ & $M G / L$ & $<$ & $\begin{array}{l}0.002 \\
0.00013\end{array}$ & $3^{L}$ & $\begin{array}{l}0.001 \\
0.00013\end{array}$ & - \\
\hline CADMIUH (TOTAL) & $11 / 22 / 92$ & N001 & MG/L & $<$ & 0.00013 & $3 \mathrm{~N}$ & 0.00013 & - \\
\hline CALCIUM & $\begin{array}{l}06 / 25 / 89 \\
02 / 19 / 93 \\
06 / 29 / 93\end{array}$ & $\begin{array}{l}0001 \\
0001 \\
0001\end{array}$ & $M G / L$ & & $\begin{array}{l}74.7 \\
61.1 \\
65.0\end{array}$ & $\mathbf{L}$ & $\begin{array}{l}0.01 \\
0.5 \\
0.5\end{array}$ & - \\
\hline CALCIUN (TOTAL) & $06 / 29 / 93$ & NOO1 & $M G / L$ & & 61.3 & & 0.5 & - \\
\hline CHLORIDE & $\begin{array}{l}06 / 25 / 89 \\
02 / 19 / 93\end{array}$ & $\begin{array}{l}0001 \\
0001\end{array}$ & $M G / L$ & & $\begin{array}{l}21 . \\
18.1\end{array}$ & $\mathbf{L}$ & 1.5 & - \\
\hline CHLORIOE (TOTAL) & $06 / 29 / 93$ & N001 & MG/L & & 17.1 & & 0.5 & - \\
\hline Chrouriur & $\begin{array}{l}06 / 25 / 89 \\
11 / 22 / 92\end{array}$ & $\begin{array}{l}0001 \\
0001\end{array}$ & HG/L & $<$ & $\begin{array}{l}0.01 \\
0.0053\end{array}$ & 1 & $\begin{array}{l}0.01 \\
0.0053\end{array}$ & - \\
\hline CHRONIUN (TOTAL) & $11 / 22 / 92$ & No01 & MG/L & $<$ & 0.0053 & & 0.0053 & - \\
\hline COBALT & $06 / 25 / 89$ & 0001 & MG/L & $<$ & 0.05 & $L$ & 0.05 & - \\
\hline
\end{tabular}

PARAMETER VALUE INDICATOR (PVI): < - LESS THAN DETECTION LIMIT

SAMPLE ID COOES:

OTHER PARAMETER VALUE FLAGS:

0001 - FILTERED SNMPLE (.45 MICRONS)

J - ESTIMATED VALUE

NOD1 - UWFILTERED SAMPLE

L - LESS THAN THREE BORE VOLUMES REHOVED BEFORE SAMPLING

- $N$ - SPIKE SAMPLE RECOVERY NOT WITHIH CONTROL LIMITS 
GROUNDWATER QUALITY DATA BY LOCATIOH

SOMP Rev. O Data

SITE: MONOI MONUMENT VALLEY

LOCATIOH: 0656

MORTH COORDINATE: 59545.2 FT

EAST COORDINATE: 89175.1 FT

$04 / 21 / 88$ To $12 / 13 / 94$

REPORT DATE: $06 / 20 / 95$

FORMATIOH OF COMPLETION: ALLUVIUM (AL)

HYDRAULIC FLON RELATIONSHIP: DOWN GRADIENT (D)

\begin{tabular}{|c|c|c|c|c|c|c|c|c|}
\hline PARAMETER HAME & LOG DATE & $\begin{array}{c}\text { SAMPLE } \\
\text { ID }\end{array}$ & $\begin{array}{l}\text { UNITS OF } \\
\text { MEASURE }\end{array}$ & PVI & $\begin{array}{l}\text { PARAMETER } \\
\text { VALUE }\end{array}$ & FLAGS & $\begin{array}{l}\text { DETECTION } \\
\text { LIMIT }\end{array}$ & $\begin{array}{l}\text { PARAMETER } \\
\text { UNCERTAINTY }\end{array}$ \\
\hline COPPER & $06 / 25 / 89$ & 0001 & MG/L & & 0.03 & $\mathbf{L}$ & 0.02 & - \\
\hline CYANIDE & $06 / 25 / 89$ & 0001 & MG/L & $<$ & 0.01 & $L$ & 0.01 & - \\
\hline DISSOLVED OXYGEN & $06 / 29 / 93$ & Noo1 & MG/L & & 0.0 & & - & - \\
\hline FLUORIDE & $06 / 25 / 89$ & 0001 & MG/L & & 0.2 & $\mathbf{L}$ & 0.1 & - \\
\hline GROSS ALPHA & $\begin{array}{l}11 / 22 / 92 \\
02 / 19 / 93 \\
06 / 29 / 93\end{array}$ & $\begin{array}{l}0001 \\
0001 \\
0001\end{array}$ & PCI/L & & $\begin{array}{l}8.5 \\
0 \\
0\end{array}$ & H & $\begin{array}{c}4.8 \\
4\end{array}$ & $\begin{array}{l}4.5 \\
14 \\
7\end{array}$ \\
\hline GROSS ALPHA (TOTAL) & $\begin{array}{l}11 / 22 / 92 \\
06 / 29 / 93\end{array}$ & $\begin{array}{l}\text { No01 } \\
\text { NOO1 }\end{array}$ & PCI/L & & $\begin{array}{l}0.0 \\
4\end{array}$ & N & $\frac{12.3}{3}$ & $\frac{6.6}{6}$ \\
\hline GROSS BETA & $\begin{array}{l}11 / 22 / 92 \\
02 / 19 / 93 \\
06 / 29 / 93\end{array}$ & $\begin{array}{l}0001 \\
0001 \\
0001\end{array}$ & PCI/L & & $\begin{array}{l}8.7 \\
4 \\
14\end{array}$ & N & $\frac{8.9}{5}$ & $\begin{array}{c}5.7 \\
10 \\
5\end{array}$ \\
\hline GROSS BETA (TOTAL) & $\begin{array}{l}11 / 22 / 92 \\
06 / 29 / 93\end{array}$ & $\begin{array}{l}\text { No01 } \\
\text { N001 }\end{array}$ & PCI/L & & $\begin{array}{l}13.2 \\
15\end{array}$ & $\mathbf{N}$ & $\begin{array}{l}9.1 \\
5\end{array}$ & $\begin{array}{l}6.0 \\
5\end{array}$ \\
\hline IROH & $\begin{array}{l}06 / 25 / 89 \\
02 / 19 / 93 \\
06 / 29 / 93\end{array}$ & $\begin{array}{l}0001 \\
0001 \\
0001\end{array}$ & MG/L & $\begin{array}{l}< \\
< \\
<\end{array}$ & $\begin{array}{l}0.03 \\
0.03 \\
0.03\end{array}$ & $\begin{array}{l}L \\
J\end{array}$ & $\begin{array}{l}0.03 \\
0.03 \\
0.03\end{array}$ & - \\
\hline IROH (TOTAL) & $06 / 29 / 93$ & M001 & $M G / L$ & & 0.52 & & 0.03 & - \\
\hline LEAD & $\begin{array}{l}06 / 25 / 89 \\
11 / 22 / 92\end{array}$ & $\begin{array}{l}0001 \\
0001\end{array}$ & $M G / L$ & $<$ & $\begin{array}{l}0.01 \\
0.0015\end{array}$ & $L$ & $\begin{array}{l}0.01 \\
0.0015\end{array}$ & 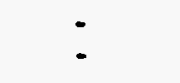 \\
\hline LEAD (TOTAL) & $11 / 22 / 92$ & N001 & MG/L & $<$ & 0.0015 & & 0.0015 & - \\
\hline LEAD-210 & $11 / 22 / 92$ & 0001 & PCI/L & & 1.0 & & 2.0 & 9.2 \\
\hline LEAD-210 (TOTAL) & $11 / 22 / 92$ & No01 & $\mathrm{PCI} / \mathrm{L}$ & & 5.9 & & 0.3 & 0.9 \\
\hline MAGNESIUM & $\begin{array}{l}06 / 25 / 89 \\
02 / 19 / 93 \\
06 / 29 / 93\end{array}$ & $\begin{array}{l}0001 \\
0001 \\
0001\end{array}$ & MG/L & & $\begin{array}{l}65.7 \\
50.6 \\
52.0\end{array}$ & 2 & $\begin{array}{l}0.001 \\
0.1 \\
0.1\end{array}$ & - \\
\hline MAGHESIUA (TOTAL) & $06 / 29 / 93$ & N001 & MG/L & & 50.3 & & 0.1 & - \\
\hline MANGAMESE & $\begin{array}{l}06 / 25 / 89 \\
11 / 22 / 92 \\
02 / 19 / 93 \\
06 / 29 / 93\end{array}$ & $\begin{array}{l}0001 \\
0001 \\
0001 \\
0001\end{array}$ & MG/L & $\begin{array}{l}< \\
<\end{array}$ & $\begin{array}{l}0.01 \\
0.0044 \\
0.01 \\
0.01\end{array}$ & $\begin{array}{l}\mathbf{L} \\
\mathbf{J}\end{array}$ & $\begin{array}{l}0.01 \\
0.0015 \\
0.01 \\
0.01\end{array}$ & $\begin{array}{l}- \\
- \\
- \\
-\end{array}$ \\
\hline MAMGAHESE (TOTAL) & $\begin{array}{l}11 / 22 / 92 \\
06 / 29 / 93\end{array}$ & $\begin{array}{l}\text { No01 } \\
\text { No01 }\end{array}$ & $M G / L$ & & $\begin{array}{l}0.0070 \\
0.01\end{array}$ & & $\begin{array}{l}0.0015 \\
0.01\end{array}$ & $\dot{-}$ \\
\hline MERCURY & $06 / 25 / 89$ & 0001 & MG/L & $<$ & 0.0002 & $\mathbf{L}$ & 0.0002 & - \\
\hline MOLYAOENUM & $06 / 25 / 89$ & 0001 & $M G / L$ & $<$ & 0.01 & $\mathbf{L}$ & 0.01 & - \\
\hline
\end{tabular}

PARAMETER VALUE IHDICATOR (PVI): < - LESS THAH DETECTIOH LIHIT

SAMPLE ID COOES:

0001 - FILTERED SAMPLE (.45 MICRONS)

OTHER PARAMETER VALUE FLAGS:

NODI - UNFILTERED SAMPLE

J- estimateo value

L - LESS THAN THREE BORE VOLUMES REMOVED BEFORE SHMPLIMG

N - SPIKE SAHPLE RECOVERY NOT HITHIN CONTROL LIHITS 
GROUNDWATER QUALITY DATA BY LOCATION

SOWP Rev. O Data

SITE: MONO1 MONLWENT VALLEY

LOCATION: 0656

MORTH COORDINATE: 59545.2 FT

EAST COORDINATE: 89173.1 FT

$04 / 21 / 88$ TO $12 / 13 / 94$

REPORT DATE: $06 / 20 / 95$

FORMATION OF COMPLETIOH: ALLUVIUM (AL)

HYDRAULIC FLON RELATIOHSHIP: DOWN GRADIENT (D)

\begin{tabular}{|c|c|c|c|c|c|c|c|}
\hline PARAMETER MAME & LOG DATE & $\mid \begin{array}{c}\text { SAMPLE } \\
\text { ID }\end{array}$ & $\begin{array}{l}\text { UNITS OF } \\
\text { MEASURE }\end{array}$ & PVI & $\begin{array}{l}\text { PARAMETER } \\
\text { VALUE FLAGS }\end{array}$ & $\begin{array}{l}\text { DETECTIOH } \\
\text { LIMIT }\end{array}$ & $\begin{array}{l}\text { PARAMETER } \\
\text { UHCERTAINTY }\end{array}$ \\
\hline HOLYBOENUM & $\begin{array}{l}11 / 22 / 92 \\
06 / 29 / 93\end{array}$ & $\begin{array}{l}0001 \\
0001\end{array}$ & MG/L & $<$ & $\begin{array}{l}0.0049 \quad \mathrm{~N} \\
0.01\end{array}$ & $\begin{array}{l}0.0049 \\
0.01\end{array}$ & $\dot{-}$ \\
\hline MOLYBOENUM (TOTAL) & $\begin{array}{l}11 / 22 / 92 \\
06 / 29 / 93\end{array}$ & $\begin{array}{l}\text { NOO1 } \\
\text { N001 }\end{array}$ & MG/L & $<$ & $\begin{array}{l}0.0049 \quad \mathrm{H} \\
0.01\end{array}$ & $\begin{array}{l}0.0049 \\
0.01\end{array}$ & 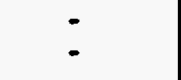 \\
\hline WET GROSS ALPHA * & $\begin{array}{l}11 / 22 / 92 \\
02 / 19 / 93 \\
06 / 29 / 93\end{array}$ & $\begin{array}{l}0001 \\
0001 \\
0001\end{array}$ & PCI/L & & $\begin{array}{r}3.01 \\
-5.49 \\
-5.49\end{array}$ & : & 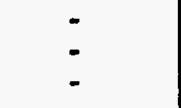 \\
\hline NET GROSS ALPHA (TOTAL) *t & $\begin{array}{l}11 / 22 / 92 \\
06 / 29 / 93\end{array}$ & $\begin{array}{l}\text { N001 } \\
\text { N001 }\end{array}$ & PCI/L & & $\begin{array}{l}-5.49 \\
-0.80\end{array}$ & - & - \\
\hline HICKEL & $06 / 25 / 89$ & 0001 & $M G / L$ & $<$ & 0.04 & 0.04 & - \\
\hline HITRATE & $\begin{array}{l}06 / 25 / 89 \\
11 / 22 / 92 \\
02 / 19 / 93\end{array}$ & $\begin{array}{l}0001 \\
0001 \\
0001\end{array}$ & MG/L & & $\begin{array}{l}460 . \\
415 \\
339\end{array}$ & $\begin{array}{l}9 . \\
0.044 \\
9\end{array}$ & : \\
\hline NITRATE (TOTAL) & $06 / 29 / 93$ & N001 & MG/L & & 254 & 1 & - \\
\hline PH & $\begin{array}{l}06 / 25 / 89 \\
11 / 22 / 92 \\
02 / 19 / 93 \\
06 / 29 / 93\end{array}$ & $\begin{array}{l}0001 \\
0001 \\
0001 \\
\text { N001 }\end{array}$ & su & & $\begin{array}{l}7.44 \\
7.68 \\
7.55 \\
7.29\end{array}$ & : & - \\
\hline PHOSPHATE & $06 / 25 / 89$ & 0001 & MG/L & $<$ & 0.1 & 0.1 & - \\
\hline PHOSPHATE (TOTAL) & $06 / 29 / 93$ & NOO1 & $M G / L$ & & 0.2 & 0.1 & - \\
\hline POTASSIU: & $\begin{array}{l}06 / 25 / 89 \\
02 / 19 / 93 \\
06 / 29 / 93\end{array}$ & $\begin{array}{l}0001 \\
0001 \\
0001\end{array}$ & MG/L & & $\begin{array}{l}18.3 \\
14 \\
15.4\end{array}$ & $\begin{array}{l}0.01 \\
0.1 \\
0.1\end{array}$ & - \\
\hline POTASSIU: (TOTAL) & $0.6 / 29 / 93$ & NO01 & MG/L & & 14.3 & 0.1 & - \\
\hline RADIUA-226 & $\begin{array}{l}06 / 25 / 89 \\
11 / 22 / 92\end{array}$ & $\begin{array}{l}0001 \\
0001\end{array}$ & PCI/L & & $\begin{array}{ll}0.1 & L \\
0.3 .\end{array}$ & 1.3 & $\begin{array}{l}0.2 \\
0.2\end{array}$ \\
\hline RADIUH-226 (TOTAL) & $11 / 22 / 92$ & NOO1 & PCI/L & & 0.8 & 0.3 & 0.3 \\
\hline RADIUA -226 + RADIUA -228 & $\begin{array}{l}06 / 25 / 89 \\
11 / 22 / 92\end{array}$ & $\begin{array}{l}0001 \\
0001\end{array}$ & PCI/L & & $\begin{array}{l}0.10 \\
1.80\end{array}$ & $\dot{-}$ & : \\
\hline RADIUH-226 + RADIUN-228 (TOTAL) & $11 / 22 / 92$ & N001 & PCI/L & & 3.40 & - & - \\
\hline RADIUH-228 & $\begin{array}{l}06 / 25 / 89 \\
11 / 22 / 92\end{array}$ & $\begin{array}{l}0001 \\
0001\end{array}$ & PCI/L & & $\begin{array}{l}0.0 \\
1.5\end{array}$ & $\begin{array}{l}1.9 \\
2.9\end{array}$ & $\begin{array}{l}0.8 \\
1.8\end{array}$ \\
\hline RADIUM-228 (TOTAL) & $11 / 22 / 92$ & N001 & $\mathrm{PCI} / \mathrm{L}$ & & 2.6 & 2.0 & 1.4 \\
\hline REDOX POTENTIAL & $11 / 22 / 92$ & 0001 & INOLTS & & 423.7 & - & - \\
\hline
\end{tabular}

- NET gROSS ALPHA (GROSS ALPHA - URANILM) WITH I MG URAHIUM = 685 PCI

** MET GROSS ALPHA (TOTAL) (TOTAL GROSS ALPHA - TOTAL URANIUH)

PARAMETER VALUE INDICATOR (PVI): < - LESS THAN DETECTION LIMIT

SAMPLE ID COOES:

OTHER PARAYETER VALUE FLAGS:

0001 - FILTERED SAMPLE (.45 MICROHS)

L - LESS THAN THREE BORE VOLUMES REMOVED BEFORE SAMPLIHG

MOO1 - UHFILTERED SAMPLE

И - SPIKE SAMPLE RECOVERY NOT MITHIN CONTROL LIMITS 
GROUNDWATER QUALITY DATA BY LOCATION

SOWP ReV. O Data

SITE: MONO1 MONUWENT VALLEY

LOCATION: 0656

MORTH COORDINATE: 59545.2 FT

EAST COORDINATE: 89173.1 FT

$04 / 21 / 88$ TO $12 / 13 / 94$

REPORT DATE: 06/20/95

FORMATION OF COAPLETION: ALLUVIUM (AL)

HYDRAULIC FLOW RELATIONSHIP: DONN GRADIENT (D)

\begin{tabular}{|c|c|c|c|c|c|c|c|c|}
\hline PARAMETER HAME & LOG DATE & $\begin{array}{c}\text { SNMPLE } \\
10\end{array}$ & $\begin{array}{l}\text { UNITS OF } \\
\text { MEASURE }\end{array}$ & PVI & $\begin{array}{c}\text { PARAHETER } \\
\text { VALUE }\end{array}$ & FLAGS & $\begin{array}{l}\text { DETECTIOH } \\
\text { LIMIT }\end{array}$ & $\begin{array}{l}\text { PARAMETER } \\
\text { UNCERTAIHTY }\end{array}$ \\
\hline REDOX POTENTIAL & $06 / 29 / 93$ & NoO1 & mVOLTS & & 490 & & - & - \\
\hline SELENIU:H & $\begin{array}{l}06 / 25 / 89 \\
11 / 22 / 92\end{array}$ & $\begin{array}{l}0001 \\
0001\end{array}$ & MG/L & $<$ & $\begin{array}{l}0.011 \\
0.0015\end{array}$ & $\underset{\text { NW }}{\mathbf{L}}$ & $\begin{array}{l}0.005 \\
0.0015\end{array}$ & - \\
\hline SELENIUH (TOTAL) & $11 / 22 / 92$ & N001 & $M G / L$ & $<$ & 0.0015 & & 0.0015 & - \\
\hline SILICA - SIO2 & $06 / 25 / 89$ & 0001 & $M G / L$ & & 13. & $\mathbf{L}$ & 2. & - \\
\hline SILICA - SIOZ (TOTAL) & $06 / 29 / 93$ & NOO1 & MG/L & & 13.7 & H & 0.5 & - \\
\hline SILVER & $06 / 25 / 89$ & 0001 & $M G / L$ & $<$ & 0.01 & $\mathbf{L}$ & 0.01 & - \\
\hline soolum & $\begin{array}{l}06 / 25 / 89 \\
02 / 19 / 93 \\
06 / 29 / 93\end{array}$ & $\begin{array}{l}0001 \\
0001 \\
0001\end{array}$ & $M G / L$ & & $\begin{array}{l}148 . \\
118 \\
119\end{array}$ & $\mathbf{L}$ & $\begin{array}{l}0.002 \\
0.1 \\
1\end{array}$ & $:$ \\
\hline SOOIUM (TOTAL) & $06 / 29 / 93$ & H001 & MG/L & & 119 & & 9 & - \\
\hline SPECIFIC CONDUCTANCE & $\begin{array}{l}06 / 25 / 89 \\
11 / 22 / 92 \\
02 / 19 / 93 \\
06 / 29 / 93\end{array}$ & $\begin{array}{l}0001 \\
0001 \\
0001 \\
\text { N001 }\end{array}$ & UNHO/CM & & $\begin{array}{l}1700 . \\
1650 \\
1645 \\
1868\end{array}$ & $L$ & $\begin{array}{l}- \\
-\end{array}$ & : \\
\hline STRONTIUA & $\begin{array}{l}11 / 22 / 92 \\
06 / 29 / 93\end{array}$ & $\begin{array}{l}0001 \\
0001\end{array}$ & MG/L & & $\begin{array}{l}0.71 \\
0.63\end{array}$ & $\mathbf{s}$ & $\begin{array}{l}0.00035 \\
0.01\end{array}$ & - \\
\hline STROHTILM (TOTAL) & $\begin{array}{l}11 / 22 / 92 \\
06 / 29 / 93\end{array}$ & $\begin{array}{l}\text { N001 } \\
\text { N001 }\end{array}$ & $M G / L$ & & $\begin{array}{l}0.71 \\
0.61\end{array}$ & $\mathbf{s}$ & $\begin{array}{l}0.00035 \\
0.01\end{array}$ & - \\
\hline SULFATE & $\begin{array}{l}06 / 25 / 89 \\
11 / 22 / 92 \\
02 / 19 / 93\end{array}$ & $\begin{array}{l}0001 \\
0001 \\
0001\end{array}$ & MG/L & & $\begin{array}{l}571 . \\
440 \\
466\end{array}$ & \llcorner & $\begin{array}{l}0.1 \\
1.0 \\
1\end{array}$ & : \\
\hline SULFATE (TOTAL) & $\begin{array}{l}91 / 22 / 92 \\
06 / 29 / 93\end{array}$ & $\begin{array}{l}\text { N001 } \\
\text { n001 }\end{array}$ & MG/L & & $\begin{array}{l}440 \\
414\end{array}$ & & 1.0 & - \\
\hline SULFIDE & $06 / 25 / 89$ & 0001 & $\mathrm{MG} / \mathrm{L}$ & $<$ & 0.1 & $\mathbf{L}$ & 0.1 & $\therefore$ \\
\hline TEMPERATURE & $\begin{array}{l}06 / 25 / 89 \\
11 / 22 / 92 \\
02 / 19 / 93 \\
06 / 29 / 93\end{array}$ & $\begin{array}{l}0001 \\
0001 \\
0001 \\
\text { N001 }\end{array}$ & C - DEGREE & & $\begin{array}{l}18.0 \\
14.8 \\
15.3 \\
20.9\end{array}$ & $\mathbf{L}$ & $\begin{array}{l}- \\
- \\
-\end{array}$ & $\begin{array}{l}- \\
- \\
-\end{array}$ \\
\hline THALLIU:A & $06 / 25 / 89$ & 0001 & $M G / L$ & $<$ & 0.01 & $L$ & 0.01 & - \\
\hline TIK & $06 / 25 / 89$ & 0001 & MG/L & $<$ & 0.005 & $L$ & 0.005 & - \\
\hline TOTAL DISSOLVED SOLIDS & $\begin{array}{l}06 / 25 / 89 \\
02 / 19 / 93\end{array}$ & $\begin{array}{l}0001 \\
0001\end{array}$ & MG/L & & $\begin{array}{c}1340 \\
.990\end{array}$ & $\mathbf{L}$ & $\begin{array}{l}10 . \\
10\end{array}$ & - \\
\hline TOTAL DISSOLVED SOLIDS (TOTAL) & $06 / 29 / 93$ & No01 & MG/L & & 990 & H & 10 & - \\
\hline URANIUA & $06 / 25 / 89$ & 0001 & MG/L & & 0.0087 & $L$ & 0.003 & - \\
\hline
\end{tabular}

PARAMETER VALUE IMDICATOR (PVI): " < - LESS THAN DETECTION LIMIT

SAMPLE ID COOES:

OTHER PARAHETER VALUE FLAGS:

0001 - FILTERED SAMPLE (.45 MICROHS)

H - HOLD TIME EXPIRED, VALUE SUSPECT

MOO1 - UMFILTERED SAMPLE

L - LESS THAH THREE BORE VOLUMES REMOVED BEFORE SAMPLIHG

H - SPIKE SAMPLE RECOVERY NOT HITHIN CONTROL LIMITS

S - REPORTED VALUE DETERMINED USING METHOO OF STD ADDITION (MSA)

H - POST-DIGEST SPIKE OUT OF CNTR LIM UHILE SNMP ABS < 50\% SPIKE 
GROUNDWATER OUALITY DATA BY LOCATION

SOWP ReV. O DatB

SITE: MONOI MOHLMENT VALLEY

LOCATION: 0656

NORTH COOROINATE: 59545.2 FT

EAST COORDINATE: 89175.1 FT

04/21/88 TO 12/13/94

REPORT DATE: $06 / 20 / 95$

FORHATION OF COHPLETIOH: ALLUVIUH (AL)

HYDRAULIC FLON RELATIONSHIP: DOWN GRADIENT (D)

\begin{tabular}{|c|c|c|c|c|c|c|c|c|}
\hline PARAMETER HAME & LOG DATE & $\begin{array}{l}\text { SAMPLE } \\
\text { ID }\end{array}$ & $\begin{array}{l}\text { UHITS OF } \\
\text { MEASURE }\end{array}$ & PVI & $\begin{array}{l}\text { PARAMETER } \\
\text { VALUE }\end{array}$ & FLAGS & $\begin{array}{l}\text { DETECTIOA } \\
\text { LIMIT }\end{array}$ & $\begin{array}{l}\text { PARAMETER } \\
\text { UHCERTAINTY }\end{array}$ \\
\hline URANIUM & $\begin{array}{l}11 / 22 / 92 \\
02 / 19 / 93 \\
06 / 29 / 93\end{array}$ & $\begin{array}{l}0001 \\
0001 \\
0001\end{array}$ & $M G / L$ & & $\begin{array}{l}0.008 \\
0.008 \\
0.008\end{array}$ & & $\begin{array}{l}0.001 \\
0.001 \\
0.001\end{array}$ & - \\
\hline URANIUM (TOTAL) & $\begin{array}{l}11 / 22 / 92 \\
06 / 29 / 93\end{array}$ & $\begin{array}{l}\text { NoO1 } \\
\text { N001 }\end{array}$ & MG/L & & $\begin{array}{l}0.008 \\
0.007\end{array}$ & & $\begin{array}{l}0.001 \\
0.001\end{array}$ & - \\
\hline VAHRDIUA & $\begin{array}{l}06 / 25 / 89 \\
11 / 22 / 92 \\
06 / 29 / 93\end{array}$ & $\begin{array}{l}0001 \\
0001 \\
0001\end{array}$ & MG/L & $<$ & $\begin{array}{l}0.03 \\
0.0019 \\
0.01\end{array}$ & L & $\begin{array}{l}0.01 \\
0.0019 \\
0.01\end{array}$ & : \\
\hline VAHADIUA (TOTAL) & $\begin{array}{l}11 / 22 / 92 \\
06 / 29 / 93\end{array}$ & $\begin{array}{l}\text { HOOI } \\
\text { HOO1 }\end{array}$ & $M G / L$ & $<$ & $\begin{array}{l}0.0020 \\
0.01\end{array}$ & & $\begin{array}{l}0.0019 \\
0.09\end{array}$ & : \\
\hline ZINC & $\begin{array}{l}06 / 25 / 89 \\
11 / 22 / 92 \\
06 / 29 / 93\end{array}$ & $\begin{array}{l}0001 \\
0001 \\
0001\end{array}$ & $M G / L$ & $<$ & $\begin{array}{l}0.005 \\
0.0010 \\
0.006\end{array}$ & L & $\begin{array}{l}0.005 \\
0.0010 \\
0.005\end{array}$ & $\dot{-}$ \\
\hline ZIHC (TOTAL) & $\begin{array}{l}11 / 22 / 92 \\
06 / 29 / 93\end{array}$ & $\begin{array}{l}\text { NOO1 } \\
\text { HOO1 }\end{array}$ & $M G / L$ & & $\begin{array}{l}0.0050 \\
0.019\end{array}$ & $J$ & $\begin{array}{l}0.0010 \\
0.005\end{array}$ & $\dot{-}$ \\
\hline
\end{tabular}

PARAMETER VALUE INDICATOR (PVI): < - LESS THAN DETECTION LIMIT

SAMPLE ID COOES

OTHER PARAMETER VALUE FLAGS:

0001 - FILTERED SAMPLE (.45 MICRONS)

$J$ - ESTIMATED VALUE

NOO1 - UNFILTERED SAMPLE

L - LESS THAM THREE BORE VOLUMES REMOVED BEFORE SAMPLING 
GROUHDHATER QUALITY DATA BY LOCATIOW

SOWP ReV. O Data

SITE: MOHO1 MONUMEHT VALLEY

LOCATION: 0657

MORTH COORDINATE: 59266.0 FT

EAST COORDIHATE: $\quad 87596.5$ FT

$04 / 21 / 88$ TO $12 / 13 / 94$

REPORT DATE: O6/20/95

FORMATION OF COMPLETIOH: DECHELLEY MEMBER OF THE CUTLER FORMATION (DC) HYDRAULIC FLOH RELATIOHSHIP: OH-SITE (O)

\begin{tabular}{|c|c|c|c|c|c|c|c|c|}
\hline PARAMETER MAME & LOG DATE & $\begin{array}{c}\text { SAMPLEE } \\
\text { ID }\end{array}$ & $\begin{array}{l}\text { UNITS OF } \\
\text { MEASURE }\end{array}$ & PVI & $\begin{array}{l}\text { PARAMETER } \\
\text { VALUE }\end{array}$ & FLAGS & $\begin{array}{l}\text { DETECTION } \\
\text { LIMIT }\end{array}$ & $\begin{array}{l}\text { PARAMETER } \\
\text { UNCERTAINTY }\end{array}$ \\
\hline ALKALINITY & $\begin{array}{l}04 / 26 / 88 \\
06 / 25 / 89 \\
12 / 04 / 89 \\
06 / 25 / 90 \\
01 / 29 / 91 \\
02 / 18 / 93 \\
06 / 29 / 93 \\
12 / 13 / 93 \\
04 / 23 / 94 \\
12 / 09 / 94\end{array}$ & $\begin{array}{l}0009 \\
0009 \\
0009 \\
0009 \\
0009 \\
0009 \\
\text { N009 } \\
\text { N001 } \\
\text { N001 } \\
\text { N001 }\end{array}$ & MG/L CACO3 & & $\begin{array}{l}194 . \\
200 . \\
193 . \\
189 . \\
207 . \\
190 \\
186 \\
193 \\
189 \\
195\end{array}$ & $\begin{array}{l}L \\
L\end{array}$ & $\begin{array}{c}: \\
\vdots \\
\vdots \\
\vdots \\
\vdots \\
10\end{array}$ & $:$ \\
\hline ALLMINGLA & $\begin{array}{l}06 / 25 / 89 \\
12 / 04 / 89 \\
06 / 25 / 90 \\
01 / 29 / 91\end{array}$ & $\begin{array}{l}0009 \\
0001 \\
0001 \\
0001\end{array}$ & MG/L & $\begin{array}{l}< \\
< \\
<\end{array}$ & $\begin{array}{l}0.1 \\
0.1 \\
0.1 \\
0.1\end{array}$ & $\begin{array}{l}L \\
L\end{array}$ & $\begin{array}{l}0.1 \\
0.1 \\
0.1 \\
0.1\end{array}$ & : \\
\hline ANOWIUA & $\begin{array}{l}04 / 26 / 88 \\
06 / 25 / 89 \\
12 / 04 / 89 \\
06 / 25 / 90 \\
01 / 29 / 91 \\
12 / 13 / 93\end{array}$ & $\begin{array}{l}0001 \\
0001 \\
0001 \\
0001 \\
0001 \\
0001\end{array}$ & MG/L & $\begin{array}{l}< \\
< \\
<\end{array}$ & $\begin{array}{l}0.1 \\
0.1 \\
0.1 \\
1.4 \\
0.1 \\
0.1\end{array}$ & $\begin{array}{l}L \\
L \\
H L\end{array}$ & $\begin{array}{l}0.1 \\
0.1 \\
0.1 \\
0.1 \\
0.1 \\
0.1\end{array}$ & $:$ \\
\hline AMOHIUM (TOTAL) & $\begin{array}{l}06 / 29 / 93 \\
04 / 23 / 94 \\
12 / 09 / 94\end{array}$ & $\begin{array}{l}\text { NoO1 } \\
\text { N001 } \\
\text { N001 }\end{array}$ & MG/L & $\begin{array}{l}< \\
<\end{array}$ & $\begin{array}{l}0.1 \\
0.1 \\
0.1\end{array}$ & $\begin{array}{l}\text { L } \\
H\end{array}$ & $\begin{array}{l}0.1 \\
0.1 \\
0.1\end{array}$ & : \\
\hline AHTIMONY & $\begin{array}{l}06 / 25 / 89 \\
12 / 04 / 89 \\
06 / 25 / 90 \\
01 / 29 / 91\end{array}$ & $\begin{array}{l}0001 \\
0001 \\
0001 \\
0001\end{array}$ & MG/L & $\begin{array}{l}< \\
< \\
<\end{array}$ & $\begin{array}{l}0.003 \\
0.003 \\
0.003 \\
0.003\end{array}$ & $\begin{array}{l}L \\
L\end{array}$ & $\begin{array}{l}0.003 \\
0.003 \\
0.003 \\
0.003\end{array}$ & : \\
\hline ARSEHIC & $\begin{array}{l}04 / 26 / 88 \\
06 / 25 / 89 \\
12 / 04 / 89 \\
06 / 25 / 90 \\
01 / 29 / 91 \\
06 / 29 / 93\end{array}$ & $\begin{array}{l}0001 \\
0001 \\
0001 \\
0001 \\
0001 \\
0001\end{array}$ & $\mathrm{MG} / \mathrm{L}$ & $\begin{array}{l}< \\
< \\
< \\
<\end{array}$ & $\begin{array}{l}0.001 \\
0.01 \\
0.01 \\
0.01 \\
0.01 \\
0.005\end{array}$ & $\begin{array}{l}J \\
L \\
L \\
L \\
L\end{array}$ & $\begin{array}{l}0.01 \\
0.01 \\
0.01 \\
0.01 \\
0.01 \\
0.005\end{array}$ & $:$ \\
\hline ARSENIC (TOTAL) & $06 / 29 / 93$ & NOO1 & MG/L & $<$ & 0.005 & $\mathbf{L}$ & 0.005 & - \\
\hline BARIUM & $\begin{array}{l}06 / 25 / 89 \\
12 / 04 / 89 \\
06 / 25 / 90 \\
01 / 29 / 91 \\
06 / 29 / 93\end{array}$ & $\begin{array}{l}0001 \\
0001 \\
0001 \\
0001 \\
0001\end{array}$ & MG/L & & $\begin{array}{l}0.2 \\
0.2 \\
0.2 \\
0.2 \\
0.2\end{array}$ & $\begin{array}{l}L \\
L \\
L\end{array}$ & $\begin{array}{l}0.1 \\
0.1 \\
0.1 \\
0.1 \\
0.1\end{array}$ & : \\
\hline BARIUM (TOTAL) & $06 / 29 / 93$ & No01 & $M G / L$ & & 0.2 & $L$ & 0.9 & - \\
\hline DERYLLIUM & $\begin{array}{l}06 / 25 / 89 \\
12 / 04 / 89 \\
06 / 25 / 90 \\
01 / 29 / 91\end{array}$ & $\begin{array}{l}0001 \\
0001 \\
0009 \\
0001\end{array}$ & $M G / L$ & $\begin{array}{l}< \\
< \\
<\end{array}$ & $\begin{array}{l}0.01 \\
0.005 \\
0.01 \\
0.01\end{array}$ & $\begin{array}{l}L \\
L\end{array}$ & $\begin{array}{l}0.01 \\
0.005 \\
0.01 \\
0.01\end{array}$ & 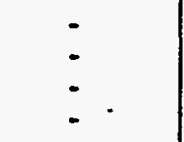 \\
\hline COROH & $06 / 25 / 89$ & 0001 & MG/L & $<$ & 0.9 & $L$ & 0.1 & - \\
\hline
\end{tabular}

PARAHETER VALUE IMDICATOR (PVI): \& - LESS THAN DETECTION LIMIT

SAHPLE ID COOES:

OTHER PARAMETER VALUE FLAGS:

0001 - FILTERED SAMPLE (.45 MICRONS)

1 H - HOLD TIME EXPIRED, VALUE SUSPECT

NOO1 - UNFILTERED SNMPLE

$J$ - ESTIMATED VALUE

L - LESS THAN THREE BORE VOLUMES REMOVED BEFORE SAMPLING 
GROUNDHATER QUALITY DATA BY LOCATION

SOWP ReV. O Data

SITE: MONO MONUNENT VALLEY

LOCATION: 0657

MORTH COORDINATE: 59266.0 FT

EAST COORDINATE: 87596.5 FT

$04 / 21 / 88$ TO $12 / 93 / 94$

REPORT DATE: 06/20/95

FORMATION OF COMPLETION: DECHELLEY MEMBER OF THE CUTLER FORMATION (DC) HYDRAULIC FLON RELATIONSHIP: OH-SITE (O)

\begin{tabular}{|c|c|c|c|c|c|c|c|c|}
\hline PARAYETER NAME & LOG DATE & $\underset{\text { ID }}{\text { SAMPLE }}$ & $\begin{array}{l}\text { UNITS OF } \\
\text { MEASURE }\end{array}$ & PVI & $\begin{array}{l}\text { PARAMETER } \\
\text { VALUE }\end{array}$ & FLAGS & $\begin{array}{l}\text { DETECTIOH } \\
\text { LIMIT }\end{array}$ & $\begin{array}{l}\text { PARAMETER } \\
\text { UNCERTAINTY }\end{array}$ \\
\hline BOROH & $\begin{array}{l}12 / 04 / 89 \\
06 / 25 / 90 \\
01 / 29 / 91\end{array}$ & $\begin{array}{l}0001 \\
0001 \\
0001\end{array}$ & $M G / L$ & $\begin{array}{l}< \\
< \\
<\end{array}$ & $\begin{array}{l}0.1 \\
0.1 \\
0.1\end{array}$ & $L$ & $\begin{array}{l}0.1 \\
0.1 \\
0.1\end{array}$ & : \\
\hline BROHIDE & $01 / 29 / 91$ & 0001 & MG/L & $<$ & 0.1 & & 0.1 & - \\
\hline CADHIUH & $\begin{array}{l}04 / 26 / 88 \\
06 / 25 / 89 \\
12 / 04 / 89 \\
06 / 25 / 90 \\
01 / 29 / 91\end{array}$ & $\begin{array}{l}0009 \\
0009 \\
0001 \\
0001 \\
0001\end{array}$ & $M G / L$ & $\begin{array}{l}< \\
< \\
< \\
<\end{array}$ & $\begin{array}{l}0.005 \\
0.001 \\
0.001 \\
0.001 \\
0.001\end{array}$ & $\begin{array}{l}L \\
L \\
L\end{array}$ & $\begin{array}{l}0.001 \\
0.001 \\
0.001 \\
0.001 \\
0.001\end{array}$ & $\begin{array}{l}- \\
- \\
-\end{array}$ \\
\hline $\begin{array}{l}\text { CALCIUH } \\
-\end{array}$ & $\begin{array}{l}04 / 26 / 88 \\
06 / 25 / 89 \\
12 / 04 / 89 \\
06 / 25 / 90 \\
01 / 29 / 91 \\
02 / 18 / 93 \\
06 / 29 / 93 \\
12 / 13 / 93 \\
04 / 23 / 94 \\
12 / 09 / 94\end{array}$ & $\begin{array}{l}0001 \\
0001 \\
0001 \\
0001 \\
0001 \\
0001 \\
0001 \\
0001 \\
0001 \\
0001\end{array}$ & ME/L & & $\begin{array}{l}62.2 \\
65.5 \\
69.5 \\
64.8 \\
69.6 \\
59.1 \\
63.9 \\
57.2 \\
60.7 \\
64.5\end{array}$ & $\begin{array}{l}L \\
L \\
L \\
L\end{array}$ & $\begin{array}{l}0.01 \\
0.01 \\
0.01 \\
0.01 \\
0.01 \\
0.5 \\
0.5 \\
0.5 \\
0.5 \\
0.5\end{array}$ & $\begin{array}{l}- \\
: \\
: \\
: \\
: \\
- \\
-\end{array}$ \\
\hline CALCIUN (TOTAL) & $\begin{array}{l}06 / 29 / 93 \\
12 / 13 / 93 \\
04 / 23 / 94\end{array}$ & $\begin{array}{l}\text { NOO1 } \\
\text { NOO1 } \\
\text { NOO1 }\end{array}$ & MG/L & & $\begin{array}{l}60.7 \\
62.6 \\
63.7\end{array}$ & $\mathbf{L}$ & $\begin{array}{l}0.5 \\
0.5 \\
0.5\end{array}$ & : \\
\hline - & $\begin{array}{l}04 / 26 / 88 \\
06 / 25 / 89 \\
12 / 04 / 89 \\
06 / 25 / 90 \\
01 / 29 / 91 \\
02 / 18 / 93 \\
12 / 13 / 93 \\
04 / 23 / 94 \\
12 / 09 / 94\end{array}$ & $\begin{array}{l}0001 \\
0001 \\
0001 \\
0001 \\
0001 \\
0001 \\
0001 \\
0001 \\
0001\end{array}$ & MG/L & & $\begin{array}{l}8.2 \\
7.4 \\
8.5 \\
8 . \\
5 . \\
7.6 \\
7.6 \\
7.2 \\
4\end{array}$ & $\begin{array}{l}L \\
L \\
L \\
J\end{array}$ & $\begin{array}{l}1 . \\
1 . \\
1 . \\
1 . \\
1 . \\
0.5 \\
0.5 \\
0.5 \\
0.5\end{array}$ & $\begin{array}{l}- \\
: \\
: \\
- \\
- \\
-\end{array}$ \\
\hline CHLORIDE (TOTAL) & $06 / 29 / 93$ & NoOI & HG/L & : & 9.7 & $L$ & 0.5 & - \\
\hline CHROMIUY & $\begin{array}{l}06 / 25 / 89 \\
12 / 04 / 89 \\
06 / 25 / 90 \\
01 / 29 / 91\end{array}$ & $\begin{array}{l}0001 \\
0001 \\
0001 \\
0001\end{array}$ & MG/L & $\begin{array}{l}< \\
< \\
<\end{array}$ & $\begin{array}{l}0.01 \\
0.01 \\
0.01 \\
0.01\end{array}$ & $\begin{array}{l}L \\
L\end{array}$ & $\begin{array}{l}0.01 \\
0.01 \\
0.01 \\
0.01\end{array}$ & - \\
\hline COBALT & $\begin{array}{l}06 / 25 / 89 \\
12 / 04 / 89 \\
06 / 25 / 90 \\
01 / 29 / 91\end{array}$ & $\begin{array}{l}0001 \\
0001 \\
0001 \\
0001\end{array}$ & MG/L & $\begin{array}{l}< \\
< \\
< \\
<\end{array}$ & $\begin{array}{l}0.05 \\
0.05 \\
0.05 \\
0.05\end{array}$ & $\begin{array}{l}L \\
L\end{array}$. & $\begin{array}{l}0.05 \\
0.05 \\
0.05 \\
0.05\end{array}$ & $\begin{array}{l}- \\
- \\
-\end{array}$ \\
\hline COPPER & $\begin{array}{l}06 / 25 / 89 \\
12 / 04 / 89 \\
06 / 25 / 90 \\
01 / 29 / 91\end{array}$ & $\begin{array}{l}0001 \\
0001 \\
0001 \\
0001\end{array}$ & MG/L & $\begin{array}{l}< \\
< \\
< \\
<\end{array}$ & $\begin{array}{l}0.02 \\
0.02 \\
0.02 \\
0.02\end{array}$ & $\begin{array}{l}L \\
L \\
L\end{array}$ & $\begin{array}{l}0.02 \\
0.02 \\
0.02 \\
0.02\end{array}$ & $\dot{-}$ \\
\hline CYANIDE & $06 / 25 / 89$ & 0001 & MG/L & $<$ & 0.01 & $\mathbf{L}$ & 0.01 & - \\
\hline
\end{tabular}

PARAMETER VALUE INDICATOR (PVI): < - LESS THAN DETECTIOH LIMIT

OTHER PARAHETER VALUE FLAGS:

SAMPLE IO COOES:

OOO1 - FILTERED SAMPLE (.45 MICROHS)

J - ESTIMATED VALUE

MOO1 - UMFILTERED SAMPLE

- L - LESS THAN THREE BORE VOLUMES RENOVED BEFORE SAMPLIMG 
GROUHDUATER QUALITY DATA BY LOCATIOH

SOUP ReV. O Data

SITE: MONOI MONUMENT VALLEY

LOCATION: 0657

HORTH COORDINATE: 59266.0 FT

EAST COORDIHATE: 87596.5 FT

04/21/88 TO $12 / 13 / 94$

REPORT DATE: 06/20/95

FORHATIOH OF COAPLETION: DECHELLEY MEMBER OF THE CUTLER FORMATION (DC) HYDRAULIC FLON RELATIOHSHIP: ON-SITE (O)

\begin{tabular}{|c|c|c|c|c|c|c|c|c|}
\hline PARAMETER NAME & LOG DATE & $\underset{\text { SD }}{\text { SAMPLE }}$ & $\begin{array}{l}\text { UNITS OF } \\
\text { MEASURE }\end{array}$ & PVI & $\begin{array}{l}\text { PARAMETER } \\
\text { VALUE }\end{array}$ & FLAGS & $\begin{array}{l}\text { DETECTIOH } \\
\text { LIMIT }\end{array}$ & $\begin{array}{l}\text { PARAMETER } \\
\text { UNCERTAIHTY }\end{array}$ \\
\hline CYAHIDE & $12 / 04 / 89$ & 0001 & MG/L & $<$ & 0.01 & $L$ & 0.01 & - \\
\hline DISSOLVED OXYGEN & $\begin{array}{l}06 / 29 / 93 \\
12 / 13 / 93 \\
04 / 23 / 94 \\
12 / 09 / 94\end{array}$ & $\begin{array}{l}\text { NOOI } \\
\text { NOOI } \\
\text { N009 } \\
\text { N001 }\end{array}$ & MG/L & $<$ & $\begin{array}{l}7.0 \\
6.8 \\
6.6 \\
0.6\end{array}$ & $\mathbf{L}$ & $\begin{array}{c}- \\
0.6\end{array}$ & : \\
\hline FLUORIDE & $\begin{array}{l}06 / 25 / 89 \\
12 / 04 / 89 \\
06 / 25 / 90 \\
01 / 29 / 91\end{array}$ & $\begin{array}{l}0009 \\
0001 \\
0001 \\
0001\end{array}$ & MG/L & & $\begin{array}{l}0.2 \\
0.2 \\
0.1 \\
0.2\end{array}$ & $\begin{array}{l}L \\
L \\
L\end{array}$ & $\begin{array}{l}0.1 \\
0.1 \\
0.1 \\
0.1\end{array}$ & : \\
\hline GROSS ALPHA & $\begin{array}{l}04 / 26 / 88 \\
01 / 29 / 91 \\
02 / 18 / 93 \\
06 / 29 / 93\end{array}$ & $\begin{array}{l}0009 \\
0001 \\
0001 \\
0001\end{array}$ & PCI/L & & $\begin{array}{l}39 . \\
49 . \\
50 \\
45\end{array}$ & $L$ & $\begin{array}{l}0.2 \\
9 . \\
7 \\
4\end{array}$ & $\begin{array}{c}7 . \\
7 . \\
12 \\
11\end{array}$ \\
\hline GROSS ALPHA (TOTAL) & $06 / 29 / 93$ & N001 & PCI/L & & 54 & $L$ & 4 & 11 \\
\hline GROSS BETA & $\begin{array}{l}04 / 26 / 88 \\
01 / 29 / 91 \\
02 / 18 / 93 \\
06 / 29 / 93\end{array}$ & $\begin{array}{l}0001 \\
0001 \\
0001 \\
0001\end{array}$ & PCI/L & & $\begin{array}{l}19 . \\
14 . \\
15 \\
22\end{array}$ & $L$ & $\begin{array}{c}1 . \\
0.5 \\
12 \\
5\end{array}$ & $\begin{array}{l}2 . \\
2 . \\
6 \\
5\end{array}$ \\
\hline GROSS BETA (TOTAL) & $06 / 29 / 93$ & No01 & $\mathrm{PCl} / \mathrm{L}$ & & 29 & $L$ & 5 & 5 \\
\hline IROH & $\begin{array}{l}06 / 25 / 89 \\
12 / 04 / 89 \\
06 / 25 / 90 \\
01 / 29 / 91 \\
02 / 18 / 93 \\
06 / 29 / 93\end{array}$ & $\begin{array}{l}0001 \\
0001 \\
0001 \\
0001 \\
0001 \\
0001\end{array}$ & MG/L & $\begin{array}{l}< \\
< \\
< \\
< \\
<\end{array}$ & $\begin{array}{l}0.03 \\
0.03 \\
0.03 \\
0.03 \\
0.03 \\
0.03\end{array}$ & $\begin{array}{l}L \\
L \\
L \\
J L\end{array}$ & $\begin{array}{l}0.03 \\
0.03 \\
0.03 \\
0.03 \\
0.03 \\
0.03\end{array}$ & : \\
\hline IRON (TOTAL) & $06 / 29 / 93$ & NOOI & MG/L & & 0.06 & JL & 0.03 & - \\
\hline LEAD & $\begin{array}{l}06 / 25 / 89 \\
12 / 04 / 89 \\
06 / 25 / 90 \\
01 / 29 / 91\end{array}$ & $\begin{array}{l}0001 \\
0001 \\
0001 \\
0001\end{array}$ & MG/L & $\begin{array}{l}5 \\
5 \\
5\end{array}$ & $\begin{array}{l}0.01 \\
0.01 \\
0.01 \\
0.01\end{array}$ & $\begin{array}{l}L \\
L \\
L\end{array}$ & $\begin{array}{l}0.01 \\
0.01 \\
0.01 \\
0.01\end{array}$ & : \\
\hline MAGKESIUH & $\begin{array}{l}04 / 26 / 88 \\
06 / 25 / 89 \\
12 / 04 / 89 \\
06 / 25 / 90 \\
01 / 29 / 91 \\
02 / 18 / 93 \\
06 / 29 / 93 \\
12 / 13 / 93 \\
04 / 23 / 94 \\
12 / 09 / 94\end{array}$ & $\begin{array}{l}0001 \\
0001 \\
0001 \\
0001 \\
0001 \\
0001 \\
0001 \\
0001 \\
0001 \\
0001\end{array}$ & HG/L & & $\begin{array}{l}38.1 \\
42.3 \\
48 . \\
42.9 \\
50.4 \\
38.4 \\
41.2 \\
38.6 \\
39.6 \\
41.2\end{array}$ & $\begin{array}{l}L \\
L \\
L\end{array}$ & $\begin{array}{l}0.001 \\
0.001 \\
0.001 \\
0.001 \\
0.001 \\
0.9 \\
0.1 \\
0.1 \\
0.9 \\
0.9\end{array}$ & $\begin{array}{l}: \\
: \\
: \\
:\end{array}$ \\
\hline MGKESIUN (TOTAL) & $\begin{array}{l}06 / 29 / 93 \\
12 / 13 / 93 \\
04 / 23 / 94\end{array}$ & $\begin{array}{l}\text { No01 } \\
\text { nO01 } \\
\text { nO01 }\end{array}$ & $M G / L$ & & $\begin{array}{l}40.9 \\
39.1 \\
38.4\end{array}$ & $L$ & $\begin{array}{l}0.1 \\
0.1 \\
0.1\end{array}$ & $:$ \\
\hline MANGAHESE & $06 / 25 / 89$ & 0009 & MG/L & & 0.02 & $L$ & 0.01 & - \\
\hline
\end{tabular}

PARMAETER VALUE IHDICATOR (PVI): < - LESS THAN DETECTION LIMIT

SAMPLE ID COOES:

OTHER PARAMETER VALUE FLAGS:

OOO1 - FILTERED SUMPLE (.45 MICRONS)

J - ESTIMATED VALUE

NDO - UMFILTERED SAMPLE

L - LESS THAN THREE BORE VOLUMES REMOVED BEFORE SMMPLIMG 
GROUNDHATER OUALITY DATA BY LOCATION

SOMP Rev. O Data

SITE: MONO1 MONLWEHT VALLEY

LOCATION: 0657

MORTH COORDIHATE: 59266.0 FT

EAST COORDINATE: 87596.5 FT

$04 / 21 / 88$ TO $12 / 93 / 94$

REPORT DATE: 06/20/95

FORMATION OF COMPLETION: DECHELLEY MEMBER OF THE CUTLER FORMATION (DC) HYDRAULIC FLOW RELATIONSHIP: ON-SITE (O)

\begin{tabular}{|c|c|c|c|c|c|c|c|c|}
\hline PARAMETER MAME & LOS DATE & $\underset{\text { ID }}{\text { SAMPLE }}$ & $\begin{array}{l}\text { UNITS OF } \\
\text { MEASURE }\end{array}$ & PVI & $\begin{array}{l}\text { PARAMETER } \\
\text { VALUE }\end{array}$ & FLAGS & $\begin{array}{l}\text { DETECTION } \\
\text { LIMIT }\end{array}$ & $\begin{array}{l}\text { PARAMETER } \\
\text { UNCERTAINTY }\end{array}$ \\
\hline MANGANESE & $\begin{array}{l}12 / 04 / 89 \\
06 / 25 / 90 \\
01 / 29 / 91 \\
02 / 18 / 93 \\
06 / 29 / 93 \\
12 / 13 / 93 \\
04 / 23 / 94 \\
12 / 09 / 94\end{array}$ & $\begin{array}{l}0001 \\
0001 \\
0001 \\
0001 \\
0001 \\
0001 \\
0001 \\
0001\end{array}$ & MG/L & $\begin{array}{l}< \\
< \\
< \\
< \\
< \\
<\end{array}$ & $\begin{array}{l}0.01 \\
0.01 \\
0.01 \\
0.01 \\
0.01 \\
0.01 \\
0.01 \\
0.01\end{array}$ & $\begin{array}{l}L \\
L\end{array}$ & $\begin{array}{l}0.09 \\
0.01 \\
0.01 \\
0.09 \\
0.01 \\
0.09 \\
0.09 \\
0.01\end{array}$ & $\begin{array}{l}: \\
: \\
:\end{array}$ \\
\hline |MANGANESE (TOTAL) & $\begin{array}{l}06 / 29 / 93 \\
12 / 13 / 93 \\
04 / 23 / 94\end{array}$ & $\begin{array}{l}\text { NOO1 } \\
\text { N001 } \\
\text { N001 }\end{array}$ & MG/L & $<$ & $\begin{array}{l}0.01 \\
0.01 \\
0.01\end{array}$ & 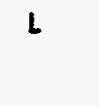 & $\begin{array}{l}0.09 \\
0.09 \\
0.01\end{array}$ & !- \\
\hline MERCURY & $\begin{array}{l}06 / 25 / 89 \\
12 / 04 / 89 \\
06 / 25 / 90\end{array}$ & $\begin{array}{l}0001 \\
0001 \\
0001\end{array}$ & MG/L & $<$ & $\begin{array}{l}0.0002 \\
0.0002 \\
0.0002\end{array}$ & $\begin{array}{l}L \\
L \\
L\end{array}$ & $\begin{array}{l}0.0002 \\
0.0002 \\
0.0002\end{array}$ & : \\
\hline HOLYBDENUM & $\begin{array}{l}04 / 26 / 88 \\
06 / 25 / 89 \\
12 / 04 / 89 \\
06 / 25 / 90 \\
01 / 29 / 91 \\
06 / 29 / 93 \\
12 / 09 / 94\end{array}$ & $\begin{array}{l}0001 \\
0001 \\
0001 \\
0001 \\
0001 \\
0001 \\
0001\end{array}$ & $\mathrm{MG} / \mathrm{L}$ & $\begin{array}{l}< \\
<\end{array}$ & $\begin{array}{l}0.02 \\
0.09 \\
0.09 \\
0.01 \\
0.09 \\
0.01 \\
0.09\end{array}$ & $\begin{array}{l}L \\
L \\
L \\
L\end{array}$ & $\begin{array}{l}0.01 \\
0.01 \\
0.01 \\
0.01 \\
0.01 \\
0.01 \\
0.01\end{array}$ & : \\
\hline HOLYBDENUM (TOTAL) & $06 / 29 / 93$ & N001 & $M G / L$ & $<$ & 0.09 & 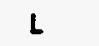 & 0.01 & - \\
\hline NET GROSS ALPHA • & $\begin{array}{l}04 / 26 / 88 \\
01 / 29 / 91 \\
02 / 18 / 93 \\
06 / 29 / 93\end{array}$ & $\begin{array}{l}0001 \\
0001 \\
0001 \\
0001\end{array}$ & PCI/L & & $\begin{array}{r}11.90 \\
3.52 \\
3.35 \\
-7.14\end{array}$ & & $:$ & 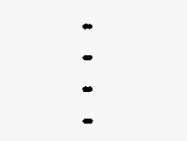 \\
\hline NET GROSS ALPHA (TOTAL) ** & $06 / 29 / 93$ & n001 & $\mathrm{PCI} / \mathrm{L}$ & & 49.20 & & - & - \\
\hline MICKEL & $\begin{array}{l}06 / 25 / 89 \\
12 / 04 / 89 \\
06 / 25 / 90 \\
01 / 29 / 91\end{array}$ & $\begin{array}{l}0009 \\
0009 \\
0001 \\
0009\end{array}$ & MG/L & $\begin{array}{l}< \\
< \\
<\end{array}$ & $\begin{array}{l}0.04 \\
0.04 \\
0.04 \\
0.04\end{array}$ & $\begin{array}{l}L \\
L \\
L\end{array}$ & $\begin{array}{l}0.04 \\
0.04 \\
0.04 \\
0.04\end{array}$ & $:$ \\
\hline HITRATE & $\begin{array}{l}04 / 26 / 88 \\
06 / 25 / 89 \\
12 / 04 / 89 \\
06 / 25 / 90 \\
01 / 29 / 91 \\
02 / 18 / 93 \\
12 / 13 / 93\end{array}$ & $\begin{array}{l}0009 \\
0009 \\
0001 \\
0009 \\
0001 \\
0009 \\
0009\end{array}$ & MG/L & & $\begin{array}{l}22 . \\
29 . \\
23.9 \\
19.9 \\
19 . \\
19 \\
19\end{array}$ & $\begin{array}{l}L \\
L \\
L\end{array}$ & $\begin{array}{l}1 . \\
1 . \\
1 . \\
9 \\
1 \\
1\end{array}$ & $\begin{array}{l}: \\
: \\
: \\
-\end{array}$ \\
\hline MITRATE (TOTAL) & $\begin{array}{l}06 / 29 / 93 \\
04 / 23 / 94 \\
12 / 09 / 94\end{array}$ & $\begin{array}{l}\text { No09 } \\
\text { N001 } \\
\text { N001 }\end{array}$ & MG/L & & $\begin{array}{l}19 \\
19 \\
20\end{array}$ & $\begin{array}{l}\text { L } \\
\text { HJN }\end{array}$ & $\begin{array}{l}1 \\
1 \\
1\end{array}$ & : \\
\hline WITRITE AND MITRATE & $01 / 29 / 91$ & 0009 & MG/L & & 19. & J & 1. & - \\
\hline
\end{tabular}

- MET GROSS ALPHA (GROSS ALPHA - LRANIUNI) UITH I MG URANIUM = 686 PCI

- MET GROSS ALPHA (TOTAL) (TOTAL GROSS ALPHA - TOTAL URANIUM)

PARAMETER VALUE IhDICATOR (PVI): < - LeSS than DETECTION LIMIT

SAMPLE IO COOES:

OTHER PARAMETER VALUE FLAGS:

OOO1 - FILTERED SAMPLE (.45 MICRONS)

H - MOLD TIME EXPIRED, VALUE SUSPECT

NOO1 - UNFILTERED SAMPLE

J - est IMATED VALUE

L - LESS THAN THREE BORE VOLUHES REMOVED BEFORE SAMPLING

W - SPIKE SNIPLE RECOVERY NOT WITHIH CONTROL LIMITS 
GROUKDHATER QUALITY DATA BY LOCATIOH

SOUP ReV. 0 Data

SITE: MONO1 MONUMENT VALLEY

LOCATIOH: 0657

NORTH COORDINATE: 59266.0 FT

EAST COORDINATE: 87596.5 FT

$04 / 21 / 88$ TO $12 / 13 / 94$

REPORT DATE: 06/20/95

FORMATION OF COAPLETION: DECHELLEY MEMBER OF THE CUTLER FORMATION (DC) HYORAULIC FLON RELATIOHSHIP: OH-SITE (O)

\begin{tabular}{|c|c|c|c|c|c|c|c|c|}
\hline PARAMETER MAME & LOG DATE & $\begin{array}{c}\text { SAMPLE } \\
10\end{array}$ & $\begin{array}{l}\text { UNITS OF } \\
\text { MEASURE }\end{array}$ & PVI & $\begin{array}{l}\text { PARAMETER } \\
\text { VALUE }\end{array}$ & FLAGS & $\begin{array}{l}\text { DETECTION } \\
\text { LIMIT }\end{array}$ & $\begin{array}{l}\text { PARAMETER } \\
\text { UNCERTAINTY }\end{array}$ \\
\hline PH & $\begin{array}{l}04 / 26 / 88 \\
06 / 25 / 89 \\
12 / 04 / 89 \\
06 / 25 / 90 \\
01 / 29 / 91 \\
02 / 18 / 93 \\
06 / 29193 \\
12 / 13 / 93 \\
04 / 23 / 94 \\
12 / 09 / 94\end{array}$ & $\begin{array}{l}0001 \\
0001 \\
0001 \\
0001 \\
0001 \\
0001 \\
0001 \\
\text { HoO1 } \\
\text { NOO1 } \\
\text { NO01 }\end{array}$ & sU & & $\begin{array}{l}7.33 \\
7.49 \\
7.37 \\
7.48 \\
7.29 \\
7.53 \\
7.45 \\
7.33 \\
7.36 \\
7.50\end{array}$ & $\begin{array}{l}L \\
L \\
L\end{array}$ & $\begin{array}{l}\vdots \\
\vdots \\
\vdots \\
\vdots \\
\vdots \\
0.10\end{array}$ & $\begin{array}{l}: \\
\vdots \\
\vdots \\
:\end{array}$ \\
\hline PHOSPHATE & $\begin{array}{l}06 / 25 / 89 \\
12 / 04 / 89 \\
06 / 25 / 90\end{array}$ & $\begin{array}{l}0001 \\
0001 \\
0001\end{array}$ & MG/L & < & $\begin{array}{l}0.1 \\
0.1 \\
0.1\end{array}$ & $\begin{array}{l}L \\
L \\
L\end{array}$ & $\begin{array}{l}0.1 \\
0.1 \\
0.1\end{array}$ & : \\
\hline PHOSPHATE (TOTAL) & $06 / 29 / 93$ & noo1 & MG/L & $<$ & 0.1 & $L$ & 0.1 & - \\
\hline POTASSIUNA & $\begin{array}{l}04 / 26 / 88 \\
06 / 25 / 89 \\
12 / 04 / 89 \\
06 / 25 / 90 \\
01 / 29 / 91 \\
02 / 18993 \\
06 / 29 / 93 \\
12 / 13 / 93 \\
04 / 23 / 94 \\
12 / 09 / 94\end{array}$ & $\begin{array}{l}0009 \\
0001 \\
0001 \\
0001 \\
0009 \\
0001 \\
0001 \\
0001 \\
0001 \\
0001\end{array}$ & MG/L & & $\begin{array}{l}1.69 \\
2.9 \\
1.74 \\
1.8 \\
1.8 \\
1.8 \\
2.1 \\
1.6 \\
2.0 \\
2\end{array}$ & $\begin{array}{l}L \\
L \\
L\end{array}$ & $\begin{array}{l}0.01 \\
0.01 \\
0.01 \\
0.01 \\
0.01 \\
0.1 \\
0.1 \\
0.1 \\
0.1 \\
0.1\end{array}$ & $\begin{array}{l}: \\
\vdots \\
\vdots \\
\vdots\end{array}$ \\
\hline POTASSIUM (TOTAL) & $\begin{array}{l}06 / 29 / 93 \\
12 / 13 / 93 \\
04 / 23 / 94\end{array}$ & $\begin{array}{l}\text { NOOI } \\
\text { NOOI } \\
\text { HOOI }\end{array}$ & MG/L & & $\begin{array}{l}1.9 \\
1.4 \\
1.9\end{array}$ & $L$ & $\begin{array}{l}0.1 \\
0.1 \\
0.1\end{array}$ & - \\
\hline RADIUH-226 & $\begin{array}{l}06 / 25 / 89 \\
12 / 04 / 89 \\
06 / 25 / 90 \\
01 / 29 / 91\end{array}$ & $\begin{array}{l}0009 \\
0009 \\
0001 \\
0001\end{array}$ & PCI/L & & $\begin{array}{l}0.0 \\
0.3 \\
0.4 \\
0.1\end{array}$ & $\begin{array}{l}L \\
L\end{array}$ & $\begin{array}{l}1 . \\
1 . \\
1 .\end{array}$ & $\begin{array}{l}0.1 \\
0.2 \\
0.2 \\
0.2\end{array}$ \\
\hline RADILH-226 + RADIUH-228 & $\begin{array}{l}06 / 25 / 89 \\
12 / 04 / 89 \\
06 / 25 / 90 \\
01 / 29 / 91\end{array}$ & $\begin{array}{l}0001 \\
0001 \\
0001 \\
0001\end{array}$ & $\mathrm{PCI} / \mathrm{L}$ & & $\begin{array}{l}0.00 \\
0.30 \\
1.40 \\
1.10\end{array}$ & & $:$ & $\begin{array}{l}- \\
. \\
-\end{array}$ \\
\hline RADIUA-228 & $\begin{array}{l}06 / 25 / 89 \\
12 / 04 / 89 \\
06 / 25 / 90 \\
01 / 29 / 91\end{array}$ & $\begin{array}{l}0001 \\
0001 \\
0001 \\
0001\end{array}$ & PCI/L & & $\begin{array}{l}0.0 \\
0.0 \\
1.0 \\
1.0\end{array}$ & $\begin{array}{l}L \\
L \\
L\end{array}$ & $\begin{array}{l}1 . \\
1 . \\
1 .\end{array}$ & $\begin{array}{l}0.7 \\
1.9 \\
0.9 \\
0.9\end{array}$ \\
\hline REDOX POTENTIAL & $\begin{array}{l}06 / 29 / 93 \\
12 / 13 / 93 \\
04 / 23 / 94 \\
12 / 09 / 94\end{array}$ & $\begin{array}{l}\text { NOO1 } \\
\text { HOO1 } \\
\text { NO01 } \\
\text { NO01 }\end{array}$ & NOLLTS & & $\begin{array}{l}430 \\
447 \\
340 \\
.431\end{array}$ & $\mathbf{L}$ & : & $\begin{array}{l}- \\
-\end{array}$ \\
\hline SELENIUM & $\begin{array}{l}04 / 26 / 88 \\
06 / 25 / 89 \\
12 / 04 / 89 \\
06 / 25 / 90\end{array}$ & $\begin{array}{l}0001 \\
0001 \\
0001 \\
0001\end{array}$ & MG/L & $\begin{array}{l}< \\
< \\
<\end{array}$ & $\begin{array}{l}0.005 \\
0.005 \\
0.005 \\
0.005\end{array}$ & $\begin{array}{l}L \\
L \\
L\end{array}$ & $\begin{array}{l}0.005 \\
0.005 \\
0.005 \\
0.005\end{array}$ & : \\
\hline
\end{tabular}

PARAMETER VALUE INDICATOR (PVI): < - LESS THAN DETECTION LIMIT

SAMPLE ID COOES:

OTHER PARAMETER VALUE FLAGS:

O001 - FILTERED SAMPLE (.45 MICRONS)

L - LESS THAH THREE BORE VOLLAES REMOVED BEFORE SAMPLING

NOO1 - UMFILTERED SAMPLE 
GROUNDHATER QUALITY DATA BY LOCATION

SOWP ReV. O Data

SITE: MONOI MONUMENT VALLEY

LOCATIOH: 0657

NORTH COOROINATE: 59266.0 FT

EAST COORDIHATE: 87596.5 FT

Q4/21/88 TO $12 / 13 / 94$

REPORT DATE: $06 / 20 / 95$

FORMATION OF COMPLETION: DECHELLEY MEMBER OF THE CUTLER FORMATION (DC) HYORAULIC FLON RELATIONSHIP: OH-SITE (O)

\begin{tabular}{|c|c|c|c|c|c|c|c|c|}
\hline PARAMETER MAME & LOG DATE & $\begin{array}{c}\text { SAMPLE } \\
\text { ID }\end{array}$ & $\begin{array}{l}\text { UNITS OF } \\
\text { MEASURE }\end{array}$ & PVI & $\begin{array}{l}\text { PARAMETER } \\
\text { VALUE }\end{array}$ & FLAGS & $\begin{array}{l}\text { DETECTION } \\
\text { LIMIT }\end{array}$ & $\begin{array}{l}\text { PARAMETER } \\
\text { UNCERTAINTY }\end{array}$ \\
\hline SELENIUN & $01 / 29 / 91$ & 0001 & $M G / L$ & $<$ & \multicolumn{2}{|l|}{0.005} & 0.005 & - \\
\hline silica - sioz & $\begin{array}{l}06 / 25 / 89 \\
12 / 04 / 89 \\
06 / 25 / 90 \\
01 / 29 / 91 \\
12 / 13 / 93 \\
12 / 09 / 94\end{array}$ & $\begin{array}{l}0001 \\
0001 \\
0001 \\
0001 \\
0001 \\
0001\end{array}$ & MG/L & & $\begin{array}{l}14 . \\
13.9 \\
14 . \\
13 . \\
13.4 \\
14.9\end{array}$ & $\begin{array}{l}L \\
L \\
L\end{array}$ & $\begin{array}{l}2 . \\
2 . \\
2 . \\
2 . \\
0.1 \\
0.1\end{array}$ & $\begin{array}{l}- \\
- \\
-\end{array}$ \\
\hline SILICA - SIOZ (TOTAL) & $\begin{array}{l}06 / 29 / 93 \\
12 / 13 / 93\end{array}$ & $\begin{array}{l}\text { NOO1 } \\
\text { N001 }\end{array}$ & $M G / L$ & & $\begin{array}{l}13.2 \\
13.5\end{array}$ & HL & $\begin{array}{l}0.5 \\
0.1\end{array}$ & $\dot{-}$ \\
\hline SILVER & $\begin{array}{l}06 / 25 / 89 \\
12 / 04 / 89 \\
06 / 25 / 90 \\
01 / 29 / 91\end{array}$ & $\begin{array}{l}0001 \\
0001 \\
0001 \\
0001\end{array}$ & MG/L & $\begin{array}{l}< \\
< \\
<\end{array}$ & $\begin{array}{l}0.01 \\
0.01 \\
0.01 \\
0.01\end{array}$ & $\begin{array}{l}L \\
L \\
L\end{array}$ & $\begin{array}{l}0.01 \\
0.01 \\
0.01 \\
0.01\end{array}$ & $\begin{array}{l}- \\
-\end{array}$ \\
\hline SCOIUN & $\begin{array}{l}04 / 26 / 88 \\
06 / 25 / 89 \\
12 / 04 / 89 \\
06 / 25 / 90 \\
01 / 29 / 91 \\
02 / 18 / 93 \\
06 / 29 / 93 \\
12 / 13 / 93 \\
04 / 23 / 94 \\
12 / 09 / 94\end{array}$ & $\begin{array}{l}0001 \\
0001 \\
0009 \\
0001 \\
0001 \\
0001 \\
0001 \\
0001 \\
0001 \\
0001\end{array}$ & MG/L & & $\begin{array}{l}14.7 \\
15.4 \\
17 . \\
16.0 \\
16.4 \\
13.9 \\
14 \\
14 \\
15 \\
14.9\end{array}$ & $\begin{array}{l}L \\
L \\
L \\
L\end{array}$ & $\begin{array}{l}0.002 \\
0.002 \\
0.002 \\
0.002 \\
0.002 \\
0.1 \\
1 \\
1 \\
1 \\
1\end{array}$ & $\begin{array}{l}- \\
- \\
- \\
- \\
- \\
- \\
-\end{array}$ \\
\hline SCOIUN (TOTAL) & $\begin{array}{l}06 / 29 / 93 \\
12 / 13 / 93 \\
04 / 23 / 94\end{array}$ & $\begin{array}{l}\text { NOO1 } \\
\text { NO01 } \\
\text { NO01 }\end{array}$ & $M G / L$ & & $\begin{array}{l}15 \\
14 \\
14\end{array}$ & $\mathbf{L}$ & $\begin{array}{l}1 \\
1 \\
1\end{array}$ & $\dot{-}$ \\
\hline SPECIFIC CONDUCTANCE & $\begin{array}{l}04 / 26 / 88 \\
06 / 25 / 89 \\
12 / 04 / 89 \\
06 / 25 / 90 \\
01 / 29 / 91 \\
02 / 18 / 93 \\
06 / 29 / 93 \\
12 / 13 / 93 \\
04 / 23 / 94 \\
12 / 09 / 94\end{array}$ & $\begin{array}{l}0001 \\
0001 \\
0001 \\
0001 \\
0001 \\
0001 \\
\text { N001 } \\
\text { N001 } \\
\text { N001 } \\
\text { N001 }\end{array}$ & UMHO/CM & & $\begin{array}{l}470 . \\
410 . \\
450 . \\
510 . \\
698 . \\
546 \\
595 \\
536 \\
573 \\
521\end{array}$ & $\begin{array}{l}L \\
L \\
L \\
L\end{array}$ & $\begin{array}{l}- \\
- \\
- \\
- \\
- \\
\\
\end{array}$ & $\begin{array}{l}: \\
: \\
: \\
: \\
: \\
-\end{array}$ \\
\hline STRONTIU: & $\begin{array}{l}06 / 25 / 90 \\
01 / 29 / 91 \\
06 / 29 / 93 \\
12 / 13 / 93 \\
04 / 23 / 94 \\
12 / 09 / 94\end{array}$ & $\begin{array}{l}0001 \\
0001 \\
0001 \\
0001 \\
0001 \\
0001\end{array}$ & MG/L & & $\begin{array}{l}0.51 \\
0.5 \\
0.51 \\
0.48 \\
0.52 \\
0.51\end{array}$ & $\begin{array}{l}* L \\
L \\
J\end{array}$ & $\begin{array}{l}0.1 \\
0.1 \\
0.01 \\
0.01 \\
0.01 \\
0.01\end{array}$ & $\begin{array}{l}- \\
: \\
- \\
-\end{array}$ \\
\hline STRONTIUN (TOTAL) & $\begin{array}{l}06 / 29 / 93 \\
12 / 13 / 93 \\
04 / 23 / 94\end{array}$ & $\begin{array}{l}\text { NOO1 } \\
\text { NOO1 } \\
\text { NO01 }\end{array}$ & MG/L & & $\begin{array}{l}0.49 \\
0.48 \\
0.48\end{array}$ & $L$ & $\begin{array}{l}0.01 \\
0.01 \\
0.01\end{array}$ & - \\
\hline
\end{tabular}

PARAMETER VALUE INDICATOR (PVI): < - LESS THAN DETECTION LIMIT

SNMPLE ID COOES:

0001 - FILTERED SAMPLE (.45 HICRONS)

OTHER PARAMETER VALUE FLAGS:

MOO1 - LNFILTERED SAMPLE

- DUPLICATE AKALYSIS HOT UITHIN CONTROL LIMITS

H - HOLD TIME EXPIRED, VALUE SUSPECT

J- ESTIMATED VALUE

L - LESS thaN THREE BORE VOLUHES REMOVED BEFORE SAMPLIMG 
GROUKDHATER QUALITY DATA BY LOCATION

SOWP Rev. 0 Data

SITE: MONOY MONUMENT VALLEY

LOCATION: 0657

NORTH COORDIHATE: 59266.0 FT

EAST COORDINATE: 87596.5 FT

O4/21/88 TO 12/13/94

REPORT DATE: 06/20/95

FORMATION OF COMPLETION: DECHELLEY MEMBER OF THE CUTLER FORMATION (DC) HYDRAULIC FLON RELATIOHSHIP: OH-SITE (O)

\begin{tabular}{|c|c|c|c|c|c|c|c|c|}
\hline PARAMETER NAME & LOG DATE & $\underset{\text { SAMPE }}{\text { SAMP }}$ & $\begin{array}{l}\text { UNITS OF } \\
\text { MEASURE }\end{array}$ & PVI & $\begin{array}{l}\text { PARANETER } \\
\text { VALUE }\end{array}$ & FLAGS & $\begin{array}{l}\text { DETECTION } \\
\text { LIMIT }\end{array}$ & $\begin{array}{l}\text { PARAMETER } \\
\text { UNCERTAINTY }\end{array}$ \\
\hline SULFATE & $\begin{array}{l}04 / 26 / 88 \\
06 / 25 / 89 \\
12 / 04 / 89 \\
06 / 25 / 90 \\
01 / 29 / 91 \\
02 / 18 / 93 \\
12 / 13 / 93 \\
04 / 23 / 94 \\
12 / 09 / 94\end{array}$ & $\begin{array}{l}0001 \\
0001 \\
0001 \\
0001 \\
0001 \\
0001 \\
0001 \\
0001 \\
0001\end{array}$ & HG/L & & $\begin{array}{l}127 . \\
122 . \\
153 . \\
187.6 \\
149 . \\
138 \\
122 \\
125 \\
97\end{array}$ & $\begin{array}{l}L \\
L \\
L\end{array}$ & $\begin{array}{l}0.1 \\
0.1 \\
0.1 \\
0.1 \\
0.1 \\
1 \\
1 \\
1 \\
1\end{array}$ & $\begin{array}{l}- \\
: \\
- \\
- \\
- \\
-\end{array}$ \\
\hline SULFATE (TOTAL) & $06 / 29 / 93$ & nool & MG/L & & 130 & $L$ & 1 & - \\
\hline SULFIDE & $\begin{array}{l}06 / 25 / 89 \\
12 / 04 / 89 \\
01 / 29 / 91\end{array}$ & $\begin{array}{l}0001 \\
0001 \\
0001\end{array}$ & $M G / L$ & $\begin{array}{l}< \\
< \\
<\end{array}$ & $\begin{array}{l}0.1 \\
0.1 \\
0.1\end{array}$ & $\begin{array}{l}L \\
J\end{array}$ & $\begin{array}{l}0.1 \\
0.1 \\
0.1\end{array}$ & $\begin{array}{l}\vdots \\
-\end{array}$ \\
\hline THALLIUM & $\begin{array}{l}06 / 25 / 89 \\
12 / 04 / 89 \\
06 / 25 / 90 \\
01 / 29 / 91\end{array}$ & $\begin{array}{l}0001 \\
0001 \\
0001 \\
0001\end{array}$ & MG/L & $\begin{array}{l}< \\
< \\
< \\
<\end{array}$ & $\begin{array}{l}0.01 \\
0.1 \\
0.01 \\
0.01\end{array}$ & $\begin{array}{l}L \\
L\end{array}$ & $\begin{array}{l}0.01 \\
0.1 \\
0.01 \\
0.01\end{array}$ & $\begin{array}{l}- \\
-\end{array}$ \\
\hline THORIU:-230 & $01 / 29 / 91$ & 0001 & $\mathrm{PCI} / \mathrm{L}$ & & 0.0 & & 1. & 0.3 \\
\hline TOTAL DISSOLVED SOLIDS (TOTAL) & $06 / 29 / 93$ & N001 & MG/L & & 410 & HL & 10 & - \\
\hline TOTAL ORGANIC CARBON & $\begin{array}{l}04 / 26 / 88 \\
01 / 29 / 91\end{array}$ & $\begin{array}{l}0001 \\
0001\end{array}$ & MG/L & $<$ & $\begin{array}{l}45.0 \\
1 .\end{array}$ & J & i. & 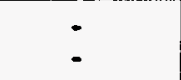 \\
\hline TURBIDITY & $04 / 23 / 94$ & n001 & NTU & & 0.0 & & - & - \\
\hline
\end{tabular}

PARAYETER VALUE INDICATOR (PVI): < - LESS THAN DETECTION LIMIT

OTHER PARAMETER VALUE FLAGS:

H - HOLD TIME EXPIRED, VALUE SUSPECT

J. estiMATED VALUE

L - LESS THAN THREE BORE VOLGMES REMOVED BEFORE SAMPLING
SNMPLE ID COOES:

OOO1 - FILTERED SNHPLE (.45 MICROHS)

NOO1 - UNFILTERED SAMPLE 
GROUNDWATER OUALITY DATA BY LOCATION

SOWP ReV. O Data

SITE: HONO1 HONUMENT VALLEY

LOCATION: 0657

NORTH COORDINATE: 59266.0 FT

EAST COORDINATE: 87596.5 FT

04/21/88 TO $12 / 13 / 94$

REPORT DATE: $06 / 20 / 95$

FORMATIOH OF CONPLETION: DECHELLEY MEMBER OF THE CUTLER FORMATION (DC) HYDRAULIC FLON RELATIONSHIP: OW-SITE (O)

\begin{tabular}{|c|c|c|c|c|c|c|c|c|}
\hline PARAMETER NAME & LOG DATE & $\underset{\text { SAMPLE }}{\operatorname{SAM}}$ & $\begin{array}{l}\text { UNITS OF } \\
\text { MEASURE }\end{array}$ & PVI & $\begin{array}{l}\text { PARAMETER } \\
\text { VALUE }\end{array}$ & FLAGS & $\begin{array}{l}\text { DETECTION } \\
\text { LIMIT }\end{array}$ & $\begin{array}{l}\text { PARAMETER } \\
\text { UHCERTAINTY }\end{array}$ \\
\hline TURBIDITY & $12 / 09 / 94$ & N001 & NTU & $<$ & 1 & & 1 & - \\
\hline URAKILM & $\begin{array}{l}04 / 26 / 88 \\
06 / 25 / 89 \\
12 / 04 / 89 \\
06 / 25 / 90 \\
01 / 29 / 91 \\
02 / 18 / 93 \\
06 / 29 / 93 \\
12 / 13 / 93 \\
04 / 23 / 94 \\
12 / 09 / 94\end{array}$ & $\begin{array}{l}0001 \\
0001 \\
0001 \\
0001 \\
0001 \\
0001 \\
0001 \\
0001 \\
0001 \\
0001\end{array}$ & MG/L & & $\begin{array}{l}0.0395 \\
0.040 \\
0.056 \\
0.061 \\
0.0663 \\
0.068 \\
0.076 \\
0.080 \\
0.078 \\
0.039\end{array}$ & $\begin{array}{l}L \\
L \\
L\end{array}$ & $\begin{array}{l}0.003 \\
0.003 \\
0.003 \\
0.003 \\
0.0003 \\
0.001 \\
0.001 \\
0.001 \\
0.001 \\
0.001\end{array}$ & $\begin{array}{l}\dot{-} \\
\vdots \\
\vdots \\
\vdots \\
\vdots\end{array}$ \\
\hline URANIUN (TOTAL) & $\begin{array}{l}06 / 29 / 93 \\
12 / 13 / 93 \\
04 / 23 / 94\end{array}$ & $\begin{array}{l}\text { N001 } \\
\text { N001 } \\
\text { N001 }\end{array}$ & $M G / L$ & & $\begin{array}{l}0.007 \\
0.083 \\
0.084\end{array}$ & $\mathbf{L}$ & $\begin{array}{l}0.001 \\
0.001 \\
0.001\end{array}$ & : \\
\hline VANADIUA & $\begin{array}{l}06 / 25 / 89 \\
12 / 04 / 89 \\
06 / 25 / 90 \\
01 / 29 / 91 \\
06 / 29 / 93 \\
12 / 13 / 93 \\
04 / 23 / 94 \\
12 / 09 / 94\end{array}$ & $\begin{array}{l}0001 \\
0001 \\
0001 \\
0001 \\
0001 \\
0001 \\
0001 \\
0001\end{array}$ & MG/L & $<$ & $\begin{array}{l}0.07 \\
0.01 \\
0.07 \\
0.06 \\
0.06 \\
0.05 \\
0.06 \\
0.06\end{array}$ & $\begin{array}{l}L \\
L \\
L \\
L\end{array}$ & $\begin{array}{l}0.01 \\
0.01 \\
0.01 \\
0.01 \\
0.01 \\
0.01 \\
0.01 \\
0.01\end{array}$ & $\begin{array}{l}- \\
- \\
- \\
- \\
-\end{array}$ \\
\hline VAHADIUH (TOTAL) & $\begin{array}{l}06 / 29 / 93 \\
12 / 13 / 93 \\
04 / 23 / 94\end{array}$ & $\begin{array}{l}\text { NOO1 } \\
\text { NOO1 } \\
\text { NOO1 }\end{array}$ & HG/L & $<$ & $\begin{array}{l}0.05 \\
0.06 \\
0.05\end{array}$ & $L$ & $\begin{array}{l}0.01 \\
0.01 \\
0.01\end{array}$ & $\ddot{-}$ \\
\hline ZINC & $\begin{array}{l}06 / 25 / 89 \\
12 / 04 / 89 \\
06 / 25 / 90 \\
01 / 29 / 91 \\
06 / 29 / 93\end{array}$ & $\begin{array}{l}0001 \\
0001 \\
0001 \\
0001 \\
0001\end{array}$ & MG/L & $<$ & $\begin{array}{l}0.012 \\
0.005 \\
0.005 \\
0.005 \\
0.048\end{array}$ & $\begin{array}{l}L \\
L \\
L \\
J L\end{array}$ & $\begin{array}{l}0.005 \\
0.005 \\
0.005 \\
0.005 \\
0.005\end{array}$ & $\begin{array}{l}- \\
- \\
-\end{array}$ \\
\hline ZINC (TOTAL) & $06 / 29 / 93$ & NoOI & $M G / L$ & & 0.197 & JL & 0.005 & - \\
\hline
\end{tabular}

PARAMETER VALUE IMDICATOR (PVI): < - LESS THAN DETECTION LIMIT

SAMPLE ID COOES:

0001 - FILTERED SAMPLE (.45 MICRONS)

OTHER PARAMETER VALUE FLAGS:

NOOI - UMFILTERED SAMPLE

J - ESTIMATED VALUE

L - LESS THAN THREE BORE YOLLHES REMOVED BEFORE SAMPLING 
GROUHDWATER QUALITY DATA BY LOCATION

SOWP ReV. O Data

SITE: MONO1 MONUAENT VALLEY

LOCATION: 0659

NORTH COORDINATE: 59069.8 FT

EAST COORDINATE: 88670.3 FT

$04 / 21 / 88$ TO $12 / 13 / 94$

REPORT DATE: $06 / 20 / 95$

FORMATIOH OF COMPLETION: SHIMARUMP MEMBER OF THE CHINLE FORMATION (SR) HYDRAULIC FLOW RELATIONSHIP: DOWN GRADIENT (D)

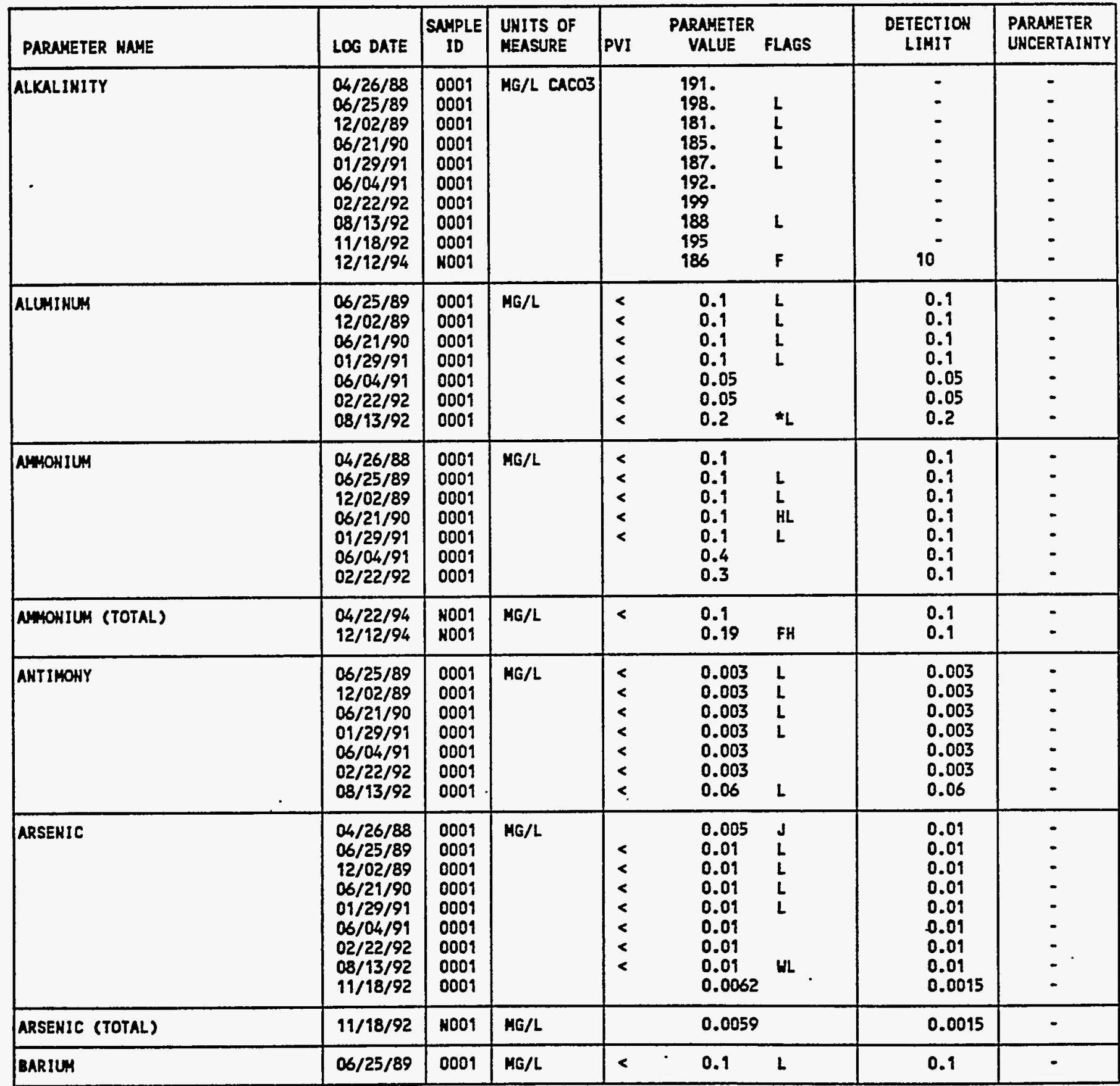

PARAMETER VALUE IMOICATOR (PVI): < - LESS THAN DETECTION LIMIT

SAMPLE ID COOES:

OTHER PARAMETER VALUE FLAGS:

O001 - FILTERED SAMPLE (.45 MICRONS)

- oUPLICATE AMALYSIS nOt hithIN CONTROL LIMITS

NOOI - UNFILTERED SAMPLE

F - LOH-FLOA SNMPLING/DEDICATED PUMP

H - HOLD TIME EXPIRED, VALUE SUSPECT

J - estimated VALUE

L - LESS THAH THREE BORE VOLUMES REMOVED BEFORE SAMPLING

$W$ - POST-DIGEST SPIKE OUT OF CHTR LIM WHILE SAMP ABS < 50\% SPIKE 
GROUNDWATER QUALITY OATA BY LOCATION

SOUP ReV. O Data

SITE: MOHOI MOHUWEHT VALLEY

LOCATION: 0659

NORTH COORDINATE: 59069.8 FT

EAST COORDINATE: 88670.3 FT

$04 / 21 / 88$ TO $12 / 13 / 94$

REPORT DATE: $06 / 20 / 95$

FORMATION OF COMPLETION: SHIMARUMP MEMBER OF THE CHIMLE FORMATION (SR) HYDRAULIC FLON RELATIONSHIP: DOWN GRADIENT (D)

\begin{tabular}{|c|c|c|c|c|c|c|c|c|}
\hline PARAMETER MAME & LOG DATE & $\mid \begin{array}{c}\text { SAMPLE } \\
\text { ID }\end{array}$ & $\begin{array}{l}\text { UNITS OF } \\
\text { MEASURE }\end{array}$ & PVI & $\begin{array}{l}\text { ARAMETER } \\
\text { VALUE }\end{array}$ & FLAGS & $\begin{array}{l}\text { DETECTION } \\
\text { LIMIT }\end{array}$ & $\begin{array}{l}\text { PARAMETER } \\
\text { UNCERTAINTY }\end{array}$ \\
\hline BARIUM & $\begin{array}{l}12 / 02 / 89 \\
06 / 21 / 90 \\
01 / 29 / 91 \\
06 / 04 / 91 \\
02 / 22 / 92 \\
08 / 13 / 92 \\
11 / 18 / 92\end{array}$ & $\begin{array}{l}0001 \\
0001 \\
0001 \\
0001 \\
0001 \\
0001 \\
0001\end{array}$ & ME/L & $\begin{array}{l}< \\
< \\
<\end{array}$ & $\begin{array}{l}0.1 \\
0.1 \\
0.1 \\
0.04 \\
0.04 \\
0.2 \\
0.038\end{array}$ & $\begin{array}{l}L \\
L \\
L \\
L\end{array}$ & $\begin{array}{l}0.1 \\
0.1 \\
0.1 \\
0.01 \\
0.01 \\
0.2 \\
0.0015\end{array}$ & $\begin{array}{l}- \\
- \\
- \\
- \\
-\end{array}$ \\
\hline BARIUN (TOTAL) & $11 / 18 / 92$ & HOO1 & MG/L & \multicolumn{3}{|c|}{0.046} & 0.0015 & - \\
\hline BERYLLIUH & $\begin{array}{l}06 / 25 / 89 \\
12 / 02 / 89 \\
06 / 21 / 90 \\
01 / 29 / 91 \\
06 / 04 / 91 \\
08 / 13 / 92\end{array}$ & $\begin{array}{l}0001 \\
0001 \\
0001 \\
0001 \\
0001 \\
0001\end{array}$ & $\mathrm{MG} / \mathrm{L}$ & $\begin{array}{l}< \\
< \\
< \\
< \\
<\end{array}$ & $\begin{array}{l}0.01 \\
0.005 \\
0.01 \\
0.01 \\
0.005 \\
0.005\end{array}$ & $\begin{array}{l}L \\
L \\
L \\
L \\
L\end{array}$ & $\begin{array}{l}0.01 \\
0.005 \\
0.01 \\
0.01 \\
0.005 \\
0.005\end{array}$ & $\begin{array}{l}- \\
- \\
-\end{array}$ \\
\hline BORON & $\begin{array}{l}06 / 25 / 89 \\
12 / 02 / 89 \\
06 / 21 / 90 \\
01 / 29 / 91 \\
06 / 04 / 91\end{array}$ & $\begin{array}{l}0001 \\
0001 \\
0001 \\
0001 \\
0001\end{array}$ & MG/L & $\begin{array}{l}< \\
< \\
< \\
< \\
<\end{array}$ & $\begin{array}{l}0.1 \\
0.1 \\
0.1 \\
0.1 \\
0.05\end{array}$ & $\begin{array}{l}L \\
L \\
L \\
L\end{array}$ & $\begin{array}{l}0.1 \\
0.1 \\
0.1 \\
0.1 \\
0.05\end{array}$ & $\begin{array}{l}- \\
- \\
-\end{array}$ \\
\hline BROMIDE & $\begin{array}{l}01 / 29 / 91 \\
06 / 04 / 91\end{array}$ & $\begin{array}{l}0001 \\
0001\end{array}$ & $M G / L$ & $<$ & $\begin{array}{l}0.1 \\
0.1\end{array}$ & \llcorner & $\begin{array}{l}0.1 \\
0.1\end{array}$ & : \\
\hline CADMIUA & $\begin{array}{l}04 / 26 / 88 \\
06 / 25 / 89 \\
12 / 02 / 89 \\
06 / 21 / 90 \\
01 / 29 / 91 \\
06 / 04 / 91 \\
02 / 22 / 92 \\
08 / 13 / 92 \\
11 / 18 / 92\end{array}$ & $\begin{array}{l}0001 \\
0001 \\
0001 \\
0001 \\
0001 \\
0001 \\
0001 \\
0001 \\
0001\end{array}$ & MG/L & $\begin{array}{l}< \\
< \\
< \\
< \\
< \\
< \\
< \\
<\end{array}$ & $\begin{array}{l}0.004 \\
0.001 \\
0.001 \\
0.001 \\
0.001 \\
0.001 \\
0.0005 \\
0.005 \\
0.00013\end{array}$ & $\begin{array}{l}L \\
L \\
L \\
L \\
L\end{array}$ & $\begin{array}{l}0.001 \\
0.001 \\
0.001 \\
0.001 \\
0.001 \\
0.001 \\
0.0005 \\
0.005 \\
0.00013\end{array}$ & $\begin{array}{l}- \\
- \\
- \\
- \\
- \\
-\end{array}$ \\
\hline CADMIUA (TOTAL) & $11 / 18 / 92$ & No01 & $M G / L$ & & 0.00020 & $N$ & 0.00013 & - \\
\hline CALCIUN & $\begin{array}{l}04 / 26 / 88 \\
06 / 25 / 89 \\
12 / 02 / 89 \\
06 / 21 / 90 \\
01 / 29 / 91 \\
06 / 04 / 91 \\
02 / 22 / 92 \\
08 / 13 / 92 \\
04 / 22 / 94 \\
12 / 12 / 94\end{array}$ & $\begin{array}{l}0009 \\
0001 \\
0001 \\
0001 \\
0001 \\
0001 \\
0009 \\
0001 \\
0001 \\
0001\end{array}$ & MG/L & & $\begin{array}{l}19.3 \\
23.7 \\
18.4 \\
20.1 \\
22.2 \\
25.0 \\
22.7 \\
23 \\
22.5 \\
22.9\end{array}$ & $\begin{array}{l}L \\
L \\
L \\
L \\
L \\
F\end{array}$ & $\begin{array}{l}0.01 \\
0.01 \\
0.01 \\
0.01 \\
0.01 \\
0.5 \\
0.5 \\
5 \\
0.5 \\
0.5\end{array}$ & $\begin{array}{l}: \\
: \\
: \\
: \\
: \\
-\end{array}$ \\
\hline CALCIUH (TOTAL) & $04 / 22 / 94$ & NOOI & MG/L & & 23.7 & & 0.5 & - \\
\hline CHLORIDE & $\begin{array}{l}04 / 26 / 88 \\
06 / 25 / 89 \\
12 / 02 / 89\end{array}$ & $\begin{array}{l}0009 \\
0009 \\
0009\end{array}$ & MG/L & & $\begin{array}{r}11 . \\
8.6 \\
9.5\end{array}$ & $\begin{array}{l}L \\
L\end{array}$ & $\begin{array}{l}1 . \\
1 .\end{array}$ & : \\
\hline
\end{tabular}

PARAMETER VALUE IMDICATOR (PVI): < - LESS THAN DETECTION LIMIT

SAMPLE IO COOES:

OTHER PARAMETER VALUE FLAGS:

0001 - FILTERED SNMPLE (.45 MICROWS)

F - LOU-FLOU SAMLIMG/DEDICATED PUMP

NOOI - UMFILTERED SNMPLE

L - LESS THAN THREE BORE VOLUWES RENOVED BEFORE SAMPLING

N - SPIKE SNMPLE RECOVERY NOT HITHIN CONTROL LIMITS 
GROUNDWATER QUALITY DATA BY LOCATION

SOMP ReV. O Data

SITE: HONO 9 MONUMENT VALLEY

LOCATION: 0659

HORTH COORDIHATE: 59069.8 FT

EAST COORDIHATE: 88670.3 FT

$04 / 21 / 88$ TO $12 / 13 / 94$

REPORT DATE: $06 / 20 / 95$

FORMATION OF COHPLETION: SHIMARLMP MEMBER OF THE CHIHLE FORHATION (SR) HYDRAULIC FLON RELATIONSHIP: DOWH GRADIENT (D)

\begin{tabular}{|c|c|c|c|c|c|c|c|c|c|}
\hline PARAMETER NAME & & LOG DATE & $\begin{array}{c}\text { SAMPLE } \\
\text { I0 }\end{array}$ & $\begin{array}{l}\text { UHITS OF } \\
\text { MEASURE }\end{array}$ & PVI & $\begin{array}{l}\text { PARANETERR } \\
\text { VALUE }\end{array}$ & FLAGS & $\begin{array}{l}\text { DETECTION } \\
\text { LIMIT }\end{array}$ & $\begin{array}{l}\text { PARAMETER } \\
\text { UNCERTAINTY }\end{array}$ \\
\hline CHLORIDE & & $\begin{array}{l}06 / 21 / 90 \\
01 / 29 / 91 \\
06 / 04 / 91 \\
02 / 22 / 92 \\
08 / 13 / 92 \\
04 / 22 / 94 \\
12 / 12 / 94\end{array}$ & $\begin{array}{l}0001 \\
0001 \\
0001 \\
0001 \\
0009 \\
0001 \\
0001\end{array}$ & MG/L & & $\begin{array}{l}9 . \\
6 . \\
9.6 \\
9.9 \\
8.8 \\
9.1 \\
6\end{array}$ & $\begin{array}{l}L \\
J L \\
L \\
F\end{array}$ & $\begin{array}{l}1 . \\
1 . \\
0.5 \\
0.5 \\
0.016 \\
0.5 \\
0.5\end{array}$ & $\begin{array}{l}- \\
- \\
- \\
-\end{array}$ \\
\hline CHROMIUY & & $\begin{array}{l}06 / 25 / 89 \\
12 / 02 / 89 \\
06 / 21 / 90 \\
01 / 29 / 91 \\
06 / 04 / 91 \\
02 / 22 / 92 \\
08 / 13 / 92 \\
11 / 18 / 92\end{array}$ & $\begin{array}{l}0001 \\
0001 \\
0001 \\
0001 \\
0001 \\
0001 \\
0001 \\
0001\end{array}$ & $M G / L$ & $\begin{array}{l}< \\
< \\
< \\
< \\
< \\
< \\
< \\
<\end{array}$ & $\begin{array}{l}0.01 \\
0.01 \\
0.01 \\
0.01 \\
0.01 \\
0.01 \\
0.01 \\
0.0053\end{array}$ & $\begin{array}{l}L \\
L \\
L \\
L\end{array}$ & $\begin{array}{l}0.01 \\
0.01 \\
0.01 \\
0.01 \\
0.01 \\
0.01 \\
0.01 \\
0.0053\end{array}$ & $\begin{array}{l}- \\
\vdots \\
\vdots \\
\vdots \\
-\end{array}$ \\
\hline COPPER & $\because$ & $\begin{array}{l}06 / 25 / 89 \\
12 / 02 / 89 \\
06 / 21 / 90 \\
01 / 29 / 91 \\
06 / 04 / 91\end{array}$ & $\begin{array}{l}0001 \\
0001 \\
0001 \\
0001 \\
0001\end{array}$ & $M G / L$ & $\begin{array}{l}< \\
< \\
< \\
< \\
<\end{array}$ & $\begin{array}{l}0.02 \\
0.02 \\
0.02 \\
0.02 \\
0.01\end{array}$ & $\begin{array}{l}1 \\
L \\
L \\
L\end{array}$ & $\begin{array}{l}0.02 \\
0.02 \\
0.02 \\
0.02 \\
0.01\end{array}$ & 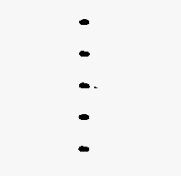 \\
\hline CYAHIDE & & $\begin{array}{l}06 / 25 / 89 \\
12 / 02 / 89\end{array}$ & $\begin{array}{l}0009 \\
0001\end{array}$ & MG/L & $<$ & $\begin{array}{l}0.01 \\
0.01\end{array}$ & $\begin{array}{l}L \\
L\end{array}$ & $\begin{array}{l}0.01 \\
0.01\end{array}$ & 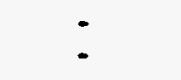 \\
\hline GROSS ALPHA & & $\begin{array}{l}04 / 26 / 88 \\
01 / 29 / 91 \\
06 / 04 / 91 \\
02 / 22 / 92 \\
08 / 13 / 92 \\
11 / 18 / 92\end{array}$ & $\begin{array}{l}0001 \\
0001 \\
0001 \\
0001 \\
0001 \\
0001\end{array}$ & PCI/L & & $\begin{array}{r}19 . \\
10 . \\
5.3 \\
8.6 \\
8.5 \\
13.0\end{array}$ & $\mathbf{L}$ & $\begin{array}{l}0.2 \\
1 . \\
1 . \\
1.0 \\
3.9 \\
2.7\end{array}$ & $\begin{array}{l}5 . \\
3 . \\
5.2 \\
4.8 \\
4.3 \\
3.7\end{array}$ \\
\hline GROSS ALPHA (TOTAL) & & $11 / 18 / 92$ & NOO1 & PCI/L & & 3.8 & H & 6.9 & 4.5 \\
\hline GROSS BETA & & $04 / 26 / 88$ & 0001 & $\mathrm{PCI} / \mathrm{L}$ & & 8.3 & & 1. & 1.6 \\
\hline
\end{tabular}

PARAMETER VALUE IMDICATOR (PVI): < - LESS THAH DETECTION LIHIT

MPLE ID COOES:

OTHER PARAMETER VALUE FLAGS:

0001 - FILTERED SAMPLE (.45 HICROHS)

F - LOW-FLOW SAMPLING/DEDICATED PUMP

NOO1 - UHFILTERED SAMPLE

J estimated VALUE

L - LESS THAH THREE BORE VOLLMES REMOVED BEFORE SAMPLING

H - SPIKE SAMLL RECOVERY NOT HITHIH CONTROL LIMITS 
GROUHDWATER QUALITY DATA BY LOCATION

SOWP ReV. O Data

SITE: MOHO1 MONUMENT VALLEY

LOCATIOH: 0659

NORTH COORDINATE: 59069.8 FT

EAST COORDINATE: 88670.3 FT

04/21/88 TO $12 / 13 / 94$

REPORT DATE: 06/20/95

FORMATION OF COHPLETION: SHIMARUAP MEMBER OF THE CHIHLE FORMATION (SR) HYORAULIC FLON RELATIONSHIP: DOWN GRADIENT (D)

\begin{tabular}{|c|c|c|c|c|c|c|c|c|}
\hline PARAMETER MAME & LOG DATE & $\underset{\text { SD }}{\text { SNMPLE }}$ & $\begin{array}{l}\text { UNITS OF } \\
\text { MEASURE }\end{array}$ & PVI & $\begin{array}{l}\text { PARAMETER } \\
\text { VALUE }\end{array}$ & FLAGS & $\begin{array}{l}\text { DETECTION } \\
\text { LIMIT }\end{array}$ & $\begin{array}{l}\text { PARAMETER } \\
\text { UNCERTAINTY }\end{array}$ \\
\hline GROSS BETA & $\begin{array}{l}01 / 29 / 91 \\
06 / 04 / 91 \\
02 / 22 / 92 \\
08 / 13 / 92 \\
11 / 18 / 92\end{array}$ & $\begin{array}{l}0001 \\
0001 \\
0001 \\
0001 \\
0001\end{array}$ & $\mathrm{PCl} / \mathrm{L}$ & & $\begin{array}{r}5.0 \\
7.8 \\
11.2 \\
7.8 \\
13.6\end{array}$ & $\begin{array}{l}\mathbf{L} \\
\mathbf{L}\end{array}$ & $\begin{array}{l}0.5 \\
0.5 \\
0.5 \\
4.7 \\
3.8\end{array}$ & $\begin{array}{l}1.9 \\
3.0 \\
3.1 \\
3.3 \\
2.9\end{array}$ \\
\hline GROSS BETA (TOTAL) & $11 / 18 / 92$ & N001 & $\mathrm{PCI} / \mathrm{L}$ & & 7.7 & N & 5.1 & 3.4 \\
\hline IRON & $\begin{array}{l}06 / 25 / 89 \\
12 / 02 / 89 \\
06 / 21 / 90 \\
01 / 29 / 91 \\
06 / 04 / 91 \\
02 / 22 / 92 \\
08 / 13 / 92\end{array}$ & $\begin{array}{l}0001 \\
0001 \\
0001 \\
0001 \\
0001 \\
0001 \\
0001\end{array}$ & $M G / L$ & & $\begin{array}{l}0.15 \\
0.12 \\
0.19 \\
0.21 \\
0.18 \\
0.10 \\
0.17\end{array}$ & $\begin{array}{l}L \\
L \\
L \\
L \\
L\end{array}$ & $\begin{array}{l}0.03 \\
0.03 \\
0.03 \\
0.03 \\
0.03 \\
0.03 \\
0.1\end{array}$ & $\begin{array}{l}: \\
\vdots \\
\vdots \\
\vdots\end{array}$ \\
\hline$\left[\begin{array}{c}\text { LEAD } \\
--\end{array}\right.$ & $\begin{array}{l}06 / 25 / 89 \\
12 / 02 / 89 \\
06 / 21 / 90 \\
01 / 29 / 91 \\
06 / 04 / 91 \\
11 / 18 / 92\end{array}$ & $\begin{array}{l}0001 \\
0001 \\
0001 \\
0001 \\
0001 \\
0001\end{array}$ & MG/L & $\begin{array}{l}< \\
< \\
< \\
< \\
<\end{array}$ & $\begin{array}{l}0.01 \\
0.01 \\
0.01 \\
0.01 \\
0.005 \\
0.0015\end{array}$ & $\begin{array}{l}\mathbf{L} \\
\mathbf{L} \\
\mathbf{L} \\
\mathbf{L}\end{array}$ & $\begin{array}{l}0.01 \\
0.01 \\
0.01 \\
0.01 \\
0.005 \\
0.0015\end{array}$ & $\begin{array}{l}- \\
: \\
- \\
-\end{array}$ \\
\hline LEAD (TOTAL) & $11 / 18 / 92$ & NOOT & MG/L & $<$ & \multicolumn{2}{|l|}{0.0015} & 0.0015 & - \\
\hline LEND-210 & $19 / 18 / 92$ & 0001 & $\mathrm{PCI} / \mathrm{L}$ & \multicolumn{3}{|c|}{1.6} & 2.0 & 1.2 \\
\hline LEAD-210 (TOTAL) & $11 / 18 / 92$ & HOOI & PCI/L & \multicolumn{3}{|c|}{2.0} & 2.0 & 9.3 \\
\hline MGGKESIUY & $\begin{array}{l}04 / 26 / 88 \\
06 / 25 / 89 \\
12 / 02 / 89 \\
06 / 21 / 90 \\
01 / 29 / 99 \\
06 / 04 / 99 \\
02 / 22 / 92 \\
08 / 13 / 92 \\
04 / 22 / 94 \\
12 / 12 / 94\end{array}$ & $\begin{array}{l}0009 \\
0001 \\
0001 \\
0001 \\
0001 \\
0009 \\
0001 \\
0009 . \\
0001 \\
0001\end{array}$ & MG/L & \multicolumn{3}{|c|}{$\begin{array}{l}20.9 \\
27.1 \\
24.9 \\
24.1 \\
29.1 \\
27.8 \\
26.3 \\
27 \\
26.6 \\
26.4\end{array}$} & $\begin{array}{l}0.001 \\
0.001 \\
0.001 \\
0.001 \\
0.001 \\
0.1 \\
0.1 \\
5 \\
0.1 \\
0.1\end{array}$ & $\begin{array}{l}- \\
: \\
: \\
: \\
: \\
-\end{array}$ \\
\hline MGKESIUM (TOTAL) & $04 / 22 / 94$ & No01 & MG/L & \multicolumn{3}{|c|}{25.2} & 0.1 & - \\
\hline MURGAHESE & $\begin{array}{l}06 / 25 / 89 \\
12 / 02 / 89 \\
06 / 21 / 90 \\
01 / 29 / 91 \\
06 / 04 / 91 \\
02 / 22 / 92 \\
08 / 13 / 92 \\
11 / 18 / 92 \\
04 / 22 / 94 \\
12 / 12 / 94\end{array}$ & $\begin{array}{l}0001 \\
0001 \\
0009 \\
0001 \\
0001 \\
0001 \\
0001 \\
0009 \\
0001 \\
0001\end{array}$ & MG/L & & $\begin{array}{l}0.12 \\
0.10 \\
0.10 \\
0.12 \\
0.11 \\
0.11 \\
0.12 \\
0.10 \\
0.13 \\
0.13\end{array}$ & $\begin{array}{l}L \\
L \\
L \\
L \\
L \\
F\end{array}$ & $\begin{array}{l}0.01 \\
0.01 \\
0.01 \\
0.01 \\
0.01 \\
0.01 \\
0.01 \\
0.0015 \\
0.01 \\
0.01\end{array}$ & $\begin{array}{l}- \\
: \\
: \\
: \\
- \\
-\end{array}$ \\
\hline MANGANESE (TOTAL) & $19 / 18 / 92$ & NoO1 & MG/L & & 0.10 & & 0.0015 & - \\
\hline
\end{tabular}

PARUMETER VALUE INDICATOR (PVI): < - LESS THAH DETECTION LIMIT

SAMPLE IO COOES

OOO1 - FILTERED SAMPLE (.45 MICRONS)

OTHER PARAMETER VALUE FLAGS:

MOO1 - UNFILTEREO SAMPLE

F - LON-FLON SNMPLING/DEDICATED PUMP

L - LESS THAH THREE BORE VOLUHES REMOVED BEFORE SAMPLING

N - SPIKE SAMPLE RECOVERY NOT HITHIN CONTROL LIMITS 
GROUMDWATER QUALITY DATA BY LOCATION

SOUP Rev. O Data

SITE: MOHO1 MOHUMENT VALLEY

LOCATION: 0659

MORTH COORDIMATE: 59069.8 FT

EAST COORDIHATE: $\quad 88670.3$ FT

$04 / 21 / 88$ TO $12 / 13 / 94$

REPORT DATE: $06 / 20 / 95$

FORMATION OF COMPLETION: SHIMARUMP MEMBER OF THE CHINLE FORMATION (SR) HYDRAULIC FLON RELATIONSHIP: DOWN GRADIENT (D)

\begin{tabular}{|c|c|c|c|c|c|c|c|c|}
\hline PARAMETER MAHE & LOG DATE & $\begin{array}{c}\text { SNHPLE } \\
\text { IO }\end{array}$ & $\begin{array}{l}\text { UNITS OF } \\
\text { MEASURE }\end{array}$ & PVI & $\begin{array}{l}\text { PARAMETER } \\
\text { VALUE }\end{array}$ & FLAGS & $\begin{array}{l}\text { DETECTION } \\
\text { LIHIT }\end{array}$ & $\begin{array}{l}\text { PARAMETER } \\
\text { UHCERTAINTY }\end{array}$ \\
\hline MANGAHESE (TOTAL) & $04 / 22 / 94$ & NOOI & MG/L & \multicolumn{3}{|c|}{0.13} & 0.01 & - \\
\hline MERCURY & $\begin{array}{l}06 / 25 / 89 \\
12 / 02 / 89 \\
06 / 21 / 90\end{array}$ & $\begin{array}{l}0001 \\
0001 \\
0001\end{array}$ & MG/L & $\begin{array}{l}< \\
< \\
<\end{array}$ & $\begin{array}{l}0.0002 \\
0.0002 \\
0.0002\end{array}$ & $\begin{array}{l}L \\
L \\
L\end{array}$ & $\begin{array}{l}0.0002 \\
0.0002 \\
0.0002\end{array}$ & $:$ \\
\hline NET GROSS ALPHA $\bullet$ & $\begin{array}{l}04 / 26 / 88 \\
01 / 29 / 91 \\
06 / 04 / 91 \\
02 / 22 / 92 \\
11 / 18 / 92\end{array}$ & $\begin{array}{l}0001 \\
0001 \\
0001 \\
0001 \\
0001\end{array}$ & $\mathrm{PCl} / \mathrm{L}$ & & $\begin{array}{r}17.90 \\
8.83 \\
4.96 \\
7.23 \\
10.94\end{array}$ & & $\begin{array}{l}- \\
- \\
-\end{array}$ & $\begin{array}{l}- \\
: \\
-\end{array}$ \\
\hline HET GROSS ALPHA (TOTAL) ** & $11 / 18 / 92$ & No01 & $\mathrm{PCI} / \mathrm{L}$ & & 3.11 & & - & - \\
\hline HICKEL & $\begin{array}{l}06 / 25 / 89 \\
12 / 02 / 89 \\
06 / 21 / 90 \\
01 / 29 / 91 \\
06 / 04 / 91\end{array}$ & $\begin{array}{l}0001 \\
0001 \\
0001 \\
0001 \\
0001\end{array}$ & MG/L & $\begin{array}{l}< \\
< \\
< \\
< \\
<\end{array}$ & $\begin{array}{l}0.04 \\
0.04 \\
0.04 \\
0.04 \\
0.04\end{array}$ & $\begin{array}{l}L \\
L \\
L \\
L\end{array}$ & $\begin{array}{l}0.04 \\
0.04 \\
0.04 \\
0.04 \\
0.04\end{array}$ & $\begin{array}{l}- \\
- \\
-\end{array}$ \\
\hline MITRATE (TOTAL) & $\begin{array}{l}04 / 22 / 94 \\
12 / 12 / 94\end{array}$ & $\begin{array}{l}\text { No01 } \\
\text { no01 }\end{array}$ & MG/L & $<$ & $\begin{array}{l}2 \\
1\end{array}$ & FHJK & $\begin{array}{l}1 \\
1\end{array}$ & . \\
\hline MITRITE AND MITRATE & $\begin{array}{l}01 / 29 / 91 \\
06 / 04 / 91\end{array}$ & $\begin{array}{l}0001 \\
0001\end{array}$ & MG/L & $<$ & 1.05 & JL & $\begin{array}{l}1 . \\
0.05\end{array}$ & - \\
\hline PH & $04 / 26 / 88$ & 0001 & SU & & 7.46 & & - & - \\
\hline
\end{tabular}

- HET GROSS ALPHA (GROSS ALPHA - URANIUM) HITH 9 MG URANILH = 686 PCI

* MET GROSS ALPHA (TOTAL) (TOTAL gROSS ALPHA - TOTAL URAHIUH)

PARAMETER VALUE INDICATOR (PVI): < - LESS THAN DETECTION LIMIT

OTHER PARAMETER VALUE FLAGS:

F - LON-FLON SAMPLING/DEDICATED PUMP

H - HOLD TIME EXPIRED, VALUE SUSPECT

J - estimated VALUE

L - LESS THAN THREE BORE VOLUHES REMOVED BEFORE SAMPLIHG

N - SPIKE SAMPLE RECOVERY HOT UITHIN CONTROL LIMITS
SAMPLE ID COOES:

0001 - FILTERED SAMPLE (.45 MICROHS)

HOD1 - UHFILTERED SAMPLE 
GROUNDHATER QUALITY DATA BY LOCATION

SOUP ReV. O Data

SITE: MONO1 MONUHENT VALLEY

LOCATION: 0659

NORTH COORDINATE: 59069.8 FT

EAST COORDINATE: 88670.3 FT

$04 / 21 / 88$ TO $12 / 13 / 94$

REPORT DATE: 06/20/95

FORHATION OF COMPLETION: SHIMARLNP MEHBER OF THE CHINLE FORMATION (SR) HYDRAULIC FLON RELATIONSHIP: DOWN GRADIENT (D)

\begin{tabular}{|c|c|c|c|c|c|c|c|c|}
\hline PARAMETER MNAE & LOG DATE & $\begin{array}{c}\text { SAMPLE } \\
\text { ID }\end{array}$ & $\begin{array}{l}\text { UHITS OF } \\
\text { MEASURE }\end{array}$ & PVI & $\begin{array}{l}\text { PARAMETER } \\
\text { VALUE }\end{array}$ & FLAGS & $\begin{array}{l}\text { DETECTION } \\
\text { LIMIT }\end{array}$ & $\begin{array}{l}\text { PARAMETER } \\
\text { UNCERTAINTY }\end{array}$ \\
\hline PH & $\begin{array}{l}06 / 25 / 89 \\
12 / 02 / 89 \\
06 / 21 / 90 \\
01 / 29 / 91 \\
06 / 04 / 91 \\
02 / 22 / 92 \\
08 / 13 / 92 \\
11 / 18 / 92 \\
04 / 22 / 94 \\
12 / 12 / 94\end{array}$ & $\begin{array}{l}0001 \\
0001 \\
0001 \\
0001 \\
0001 \\
0001 \\
0001 \\
0001 \\
\text { N001 } \\
\text { H001 }\end{array}$ & su & & $\begin{array}{l}7.43 \\
7.63 \\
7.33 \\
7.44 \\
7.41 \\
7.45 \\
7.25 \\
7.36 \\
7.30 \\
7.60\end{array}$ & $\begin{array}{l}L \\
L \\
L \\
L \\
L \\
F\end{array}$ & $\begin{array}{l}: \\
\vdots \\
\vdots \\
\vdots \\
\vdots \\
- \\
0.10\end{array}$ & $\begin{array}{l}- \\
: \\
: \\
: \\
: \\
- \\
-\end{array}$ \\
\hline PHOSPHATE & $\begin{array}{l}06 / 25 / 89 \\
12 / 02 / 89 \\
06 / 21 / 90\end{array}$ & $\begin{array}{l}0001 \\
0001 \\
0001\end{array}$ & MG/L & & $\begin{array}{l}1.2 \\
1.1 \\
1.4\end{array}$ & $\begin{array}{l}L \\
L \\
L\end{array}$ & $\begin{array}{l}0.1 \\
0.1 \\
0.1\end{array}$ & $\dot{-}$ \\
\hline POTASSIU: & $\begin{array}{l}04 / 26 / 88 \\
06 / 25 / 89 \\
12 / 02 / 89 \\
06 / 21 / 90 \\
01 / 29 / 91 \\
06 / 04 / 91 \\
02 / 22 / 92 \\
08 / 13 / 92 \\
04 / 22 / 94 \\
12 / 12 / 94\end{array}$ & $\begin{array}{l}0001 \\
0001 \\
0001 \\
0001 \\
0001 \\
0001 \\
0001 \\
0001 \\
0001 \\
0001\end{array}$ & MG/L & & $\begin{array}{l}2.56 \\
3.7 \\
2.6 \\
3.0 \\
3.1 \\
3.5 \\
3.87 \\
2.8 \\
3.4 \\
3.2\end{array}$ & $\begin{array}{l}L \\
L \\
L \\
L \\
* L \\
F\end{array}$ & $\begin{array}{l}0.01 \\
0.01 \\
0.01 \\
0.01 \\
0.01 \\
0.01 \\
0.01 \\
0.575 \\
0.1 \\
0.1\end{array}$ & $\begin{array}{l}: \\
: \\
: \\
- \\
- \\
-\end{array}$ \\
\hline POTASSIU: (TOTAL) & $04 / 22 / 94$ & NOOI & $M G / L$ & & 3.3 & & 0.1 & - \\
\hline RADIUAH-226 & $\begin{array}{l}06 / 25 / 89 \\
12 / 02 / 89 \\
06 / 21 / 90 \\
01 / 29 / 91 \\
06 / 04 / 91 \\
02 / 22 / 92 \\
08 / 13 / 92 \\
11 / 18 / 92 \\
06 / 26 / 93\end{array}$ & $\begin{array}{l}0001 \\
0001 \\
0001 \\
0001 \\
0001 \\
0001 \\
0001 \\
0001 \\
0001\end{array}$ & PCI/L & .. & $\begin{array}{l}3.9 \\
2.9 \\
5.5 \\
4.9 \\
6.9 \\
4.0 \\
5.1 \\
8.1 \\
7\end{array}$ & $\begin{array}{l}L \\
L \\
L \\
L \\
L\end{array}$ & $\begin{array}{l}1 . \\
1 . \\
1 . \\
1 . \\
1.0 \\
0.5 \\
0.3 \\
1\end{array}$ & $\begin{array}{l}0.7 \\
0.7 \\
0.8 \\
0.8 \\
1.3 \\
0.7 \\
0.2 \\
0.9 \\
1\end{array}$ \\
\hline RADILH-226 (TOTAL) & $\begin{array}{l}11 / 18 / 92 \\
06 / 26 / 93\end{array}$ & $\begin{array}{l}\text { No01 } \\
\text { HOOI }\end{array}$ & PCI/L & & $\begin{array}{l}6.4 \\
6\end{array}$ & & $i_{1}^{0.3}$ & $\mathbb{1}^{0.8}$ \\
\hline RADIUA-226 + RADIUH-228 & $\begin{array}{l}06 / 25 / 89 \\
12 / 02 / 89 \\
06 / 21 / 90 \\
01 / 29 / 91 \\
06 / 04 / 91 \\
02 / 22 / 92 \\
08 / 13 / 92 \\
11 / 18 / 92 \\
06 / 26 / 93\end{array}$ & $\begin{array}{l}0001 \\
0001 \\
0001 \\
0001 \\
0001 \\
0001 \\
0001 \\
0001 \\
0001\end{array}$ & PCI/L & & $\begin{array}{r}4.70 \\
4.90 \\
7.10 \\
6.10 \\
11.10 \\
5.50 \\
7.00 \\
12.50 \\
9.00\end{array}$ & - & $\begin{array}{l}- \\
- \\
- \\
: \\
-\end{array}$ & $\begin{array}{l}: \\
: \\
: \\
- \\
- \\
-\end{array}$ \\
\hline RADIUH-226 + RADIUH-228 (TOTAL) & $\begin{array}{l}11 / 18 / 92 \\
06 / 26 / 93\end{array}$ & $\begin{array}{l}\text { NoO? } \\
\text { N001 }\end{array}$ & PCI/L & & $\begin{array}{r}11.30 \\
9.00\end{array}$ & & $\because$ & : \\
\hline RADIUH-228 & $06 / 25 / 89$ & 0001 & PCI/L & & 0.8 & $\mathbf{L}$ & 1. & 0.8 \\
\hline
\end{tabular}

PARAMETER VALUE INDICATOR (PVI): < - LESS THAN DETECTION LIMIT

SNMPLE ID COOES:

OTHER PARAMETER VALUE FLAGS:

OOOI - FILTERED SNMPLE (.45 MICRONS)

NOOT - UNFILTERED SAMPLE

- DUPLICATE AHALYSIS hOT HITHIN CONTROL LIMITS

"F - LON-FLOU SAMPLIMG/DEDICATED PUMP

L - LESS THAH THREE BORE VOLUHES REMOVED BEFORE SAMPLING 
GROUNDWATER QUALITY DATA BY LOCATION

SOMP REV. O Dato

SITE: MONO1 MOHLMENT VALLEY

LOCATION: 0659

HORTH COORDIHATE: 59069.8 FT

EAST COORDIMATE: 88670.3 FT

$04 / 21 / 88$ TO $12 / 13 / 94$

REPORT DATE: $06 / 20 / 95$

FORMATION OF COAPLETION: SHIMARLMP MEMBER OF THE CHINLE FORMATION (SR) HYDRAULIC FLON RELATIOHSHIP: DOWN GRADIENT (D)

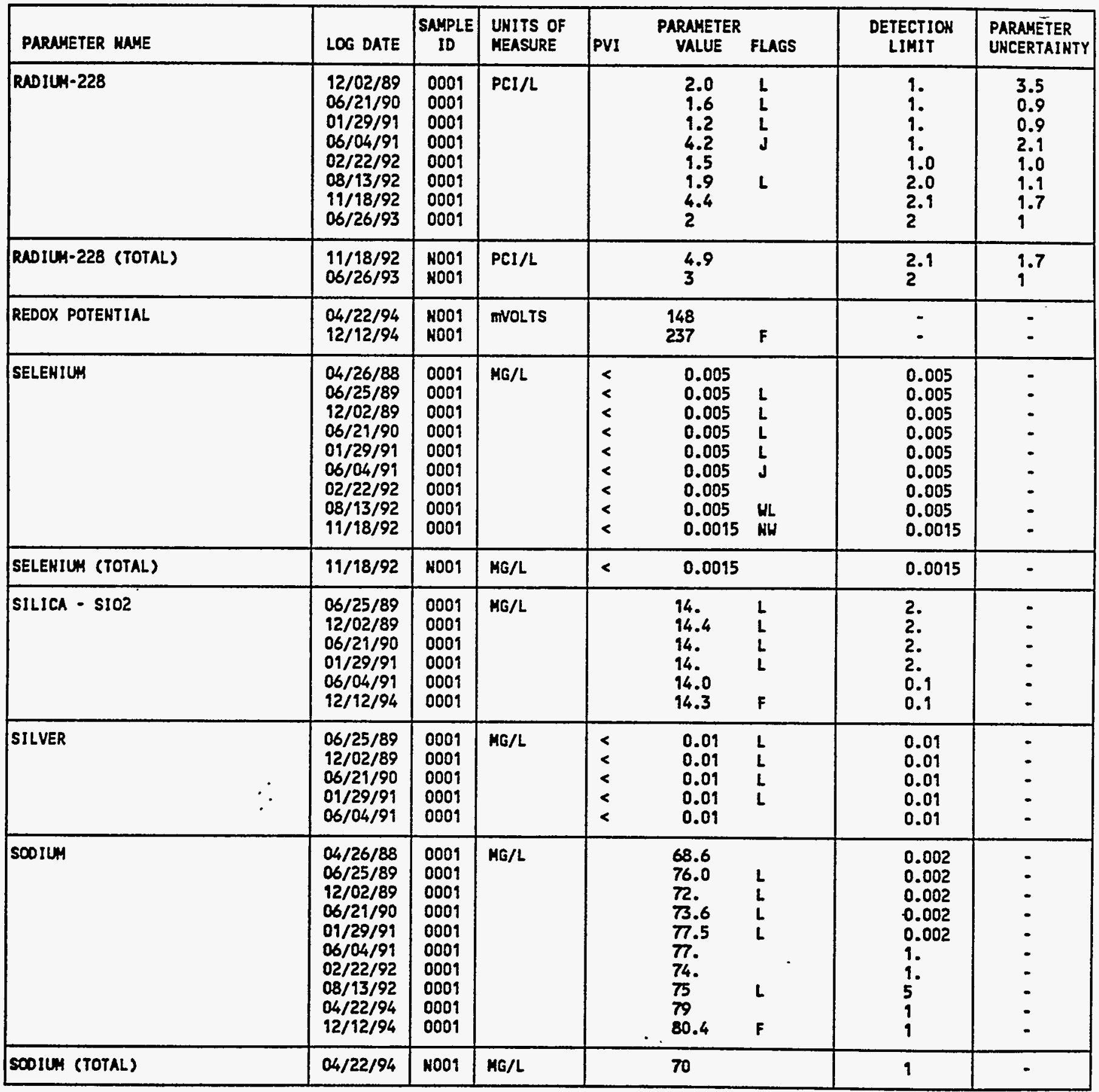

PARAMETER VALUE IHDICATOR (PVI): < - LESS THAH DETECTION LIMIT

OTHER PARAMETER VALUE FLAGS:

SAMPLE ID COOES:

0001 - FILTERED SAMPLE (.45 MICROHS)

F - LON-FLON SAMPLING/OEDICATED PUAP

MOO1 - UAFILTERED SAMPLE

- ESTIMATED VALUE

L - LESS THAH THREE BORE VOLUMES REHOVED BEFORE SAMPLIMG

H - SPIKE SAMPLE RECOVERY NOT WITHIN CONTROL LIMITS

W - POST-DIGEST SPIKE OUT OF CNTR LIM UHILE SAMP ABS < 50\% SPIKE 
GROUNDHATER QUALITY DATA BY LOCATIOH

SOMP REV. O Data

SITE: MONO1 MONLHEMT VALLEY

LOCATION: 0659

NORTH COORDINATE: 59069.8 FT

EAST COORDINATE: 88670.3 FT

$04 / 21 / 88$ TO $12 / 13 / 94$

REPORT DATE: $06 / 20 / 95$

FORMATION OF COHPLETION: SHIMARLMP MEMBER OF THE CHIMLE FORMATION (SR) HYDRAULIC FLON RELATIONSHIP: DOWN GRADIENT (D)

\begin{tabular}{|c|c|c|c|c|c|c|c|c|}
\hline PARAMETER MAME & LOG DATE & $\underset{\text { ID }}{\text { SAMPLE }}$ & $\begin{array}{l}\text { UNITS OF } \\
\text { MEASURE }\end{array}$ & PVI & $\begin{array}{l}\text { PARAMETER } \\
\text { VALUE }\end{array}$ & FLAGS & $\begin{array}{l}\text { DETECTION } \\
\text { LIMIT }\end{array}$ & $\begin{array}{l}\text { PARAMETER } \\
\text { UHCERTAINTY }\end{array}$ \\
\hline SPECIFIC CONDUCTANCE & $\begin{array}{l}04 / 26 / 88 \\
06 / 25 / 89 \\
12 / 02 / 89 \\
06 / 21 / 90 \\
01 / 29 / 91 \\
06 / 04 / 91 \\
02 / 22 / 92 \\
08 / 13 / 92 \\
11 / 18 / 92 \\
04 / 22 / 94 \\
12 / 12 / 94\end{array}$ & $\begin{array}{l}0001 \\
0001 \\
0001 \\
0001 \\
0001 \\
0001 \\
0001 \\
0001 \\
0001 \\
N 001 \\
N 001\end{array}$ & UHHO/CH & & $\begin{array}{l}410 . \\
455 . \\
455 . \\
470 . \\
614 . \\
628 . \\
524 \\
539 \\
510 \\
548 \\
280\end{array}$ & $\begin{array}{l}L \\
L \\
L \\
L \\
L \\
F\end{array}$ & $\begin{array}{l}- \\
: \\
\therefore \\
: \\
: \\
i\end{array}$ & $\begin{array}{l}- \\
: \\
: \\
: \\
: \\
- \\
-\end{array}$ \\
\hline STRONTIUM & $\begin{array}{l}06 / 21 / 90 \\
01 / 29 / 91 \\
06 / 04 / 91 \\
02 / 22 / 92 \\
08 / 13 / 92 \\
11 / 18 / 92 \\
04 / 22 / 94 \\
12 / 12 / 94\end{array}$ & $\begin{array}{l}0001 \\
0001 \\
0001 \\
0001 \\
0001 \\
0001 \\
0001 \\
0001\end{array}$ & $M G / L$ & & $\begin{array}{l}0.24 \\
0.2 \\
0.27 \\
0.26 \\
0.28 \\
0.25 \\
0.27 \\
0.27\end{array}$ & $\begin{array}{l}L_{L}^{L} \\
L \\
\text { FJ }\end{array}$ & $\begin{array}{l}0.1 \\
0.1 \\
0.01 \\
0.01 \\
0.2 \\
0.00035 \\
0.01 \\
0.01\end{array}$ & $\begin{array}{l}- \\
- \\
- \\
- \\
-\end{array}$ \\
\hline STRONTILH (TOTAL) & $\begin{array}{l}11 / 18 / 92 \\
04 / 22 / 94\end{array}$ & $\begin{array}{l}\text { NOO1 } \\
\text { NOOI }\end{array}$ & MG/L & & $\begin{array}{l}0.30 \\
0.25\end{array}$ & & $\begin{array}{l}0.00035 \\
0.01\end{array}$ & $\dot{-}$ \\
\hline SULFATE & $\begin{array}{l}04 / 26 / 88 \\
06 / 25 / 89 \\
12 / 02 / 89 \\
06 / 21 / 90 \\
01 / 29 / 91 \\
06 / 04 / 91 \\
02 / 22 / 92 \\
08 / 13 / 92 \\
11 / 18 / 92 \\
04 / 22 / 94 \\
12 / 12 / 94\end{array}$ & $\begin{array}{l}0001 \\
0001 \\
0001 \\
0001 \\
0001 \\
0001 \\
0001 \\
0001 \\
0001 \\
0001 \\
0001\end{array}$ & MG/L & & $\begin{array}{c}84 . \\
109 . \\
99 . \\
145.7 \\
113 . \\
104 . \\
112 . \\
110 \\
120 \\
128 \\
99\end{array}$ & $\begin{array}{l}L \\
L \\
L \\
L \\
L \\
F\end{array}$ & $\begin{array}{l}0.1 \\
0.1 \\
0.1 \\
0.1 \\
0.1 \\
0.1 \\
10 . \\
0.059 \\
1.0 \\
1 \\
1\end{array}$ & $\begin{array}{l}- \\
- \\
- \\
- \\
- \\
- \\
-\end{array}$ \\
\hline SULFATE (TOTAL) & $11 / 18 / 92$ & NoOi & $M G / L$ & & 120 & & 1.0 & - \\
\hline SULFIDE & $\begin{array}{l}06 / 25 / 89 \\
12 / 02 / 89 \\
01 / 29 / 91 \\
06 / 04 / 91\end{array}$ & $\begin{array}{l}0001 \\
0001 \\
0001 \\
0001\end{array}$ & MG/L & $\begin{array}{l}< \\
< \\
<\end{array}$ & $\begin{array}{l}0.1 \\
0.1 \\
0.1 \\
1 .\end{array}$ & $\begin{array}{l}L \\
L \\
J L \\
I J\end{array}$ & $\begin{array}{l}0.1 \\
0.1 \\
0.1 \\
1 .\end{array}$ & : \\
\hline TEMPERATURE & $\begin{array}{l}04 / 26 / 88 \\
06 / 25 / 89 \\
12 / 02 / 89 \\
06 / 21 / 90 \\
01 / 29 / 99 \\
06 / 04 / 91 \\
02 / 22 / 92 \\
08 / 13 / 92 \\
11 / 18 / 92\end{array}$ & $\begin{array}{l}0001 \\
0009 \\
0009 \\
0001 \\
0001 \\
0001 \\
0001 \\
0001 \\
0001\end{array}$ & C - DEGREE & & $\begin{array}{r}16.0 \\
18.0 \\
16.5 \\
17.0 \\
15.4 \\
16.8 \\
15.8 \\
17.2 \\
15.4\end{array}$ & $\begin{array}{l}L \\
L \\
L \\
L \\
L\end{array}$ & $\begin{array}{l}- \\
- \\
- \\
- \\
- \\
-\end{array}$ & $\begin{array}{l}- \\
- \\
- \\
- \\
- \\
-\end{array}$ \\
\hline
\end{tabular}

PARAKETER VALUE IMDICATOR (PVI): \& - LESS THAN DETECTION LIMIT

SAMPLE ID COOES:

OOO - FILTEREO SNMPLE (.45 MICROHS)

OTHER PARAMETER VALUE FLAGS:

NOOI - UNFILTERED SAMPLE

- DUPLICATE AMALYSIS nOt hITHIM CONTROL LIMITS

F - LON-FLON SAMPLING/DEDICATED PUMP

1 - INCREASED DETECTION LIMIT DUE TO REQUIRED DILUTION

J - ESTIMATED VALUE

$l$ - LESS THAN THREE BORE VOLLMES REMOVED BEFORE SAMPLING 
GROUNDWATER QUALITY DATA BY LOCATION

SOMP Rev. 0 Data

SITE: MONO1 MONLAENT VALLEY

LOCATION: 0659

MORTH COORDIHATE: 59069.8 FT

EAST COORDINATE: 88670.3 FT

$04 / 21 / 88$ TO $12 / 13 / 94$

REPORT DATE: OS/20/95

FORMATION OF COMPLETIOH: SHINARUMP MEMBER OF THE CHIMLE FORMATION (SR) HYDRAULIC FLOW RELATIONSHIP: DOWN GRADIENT (D)

\begin{tabular}{|c|c|c|c|c|c|c|c|c|}
\hline PARAMETER MAME & LOG DATE & $\begin{array}{c}\text { SAMPLE } \\
\text { ID }\end{array}$ & $\begin{array}{l}\text { UNITS OF } \\
\text { MEASURE }\end{array}$ & PVI & $\begin{array}{c}\text { PARAMETER } \\
\text { VALUE }\end{array}$ & FLAGS & $\begin{array}{l}\text { DETECTION } \\
\text { LIMIT }\end{array}$ & $\begin{array}{l}\text { PARAMETER } \\
\text { UNCERTAIHTY }\end{array}$ \\
\hline TEMPERATURE & $\begin{array}{l}04 / 22 / 94 \\
12 / 12 / 94\end{array}$ & $\begin{array}{l}\text { N001 } \\
\text { N001 }\end{array}$ & C - DEGREE & & $\begin{array}{l}17.0 \\
14.8\end{array}$ & $\mathbf{F}$ & 0.1 & - \\
\hline THALLIUA & $\begin{array}{l}06 / 25 / 89 \\
12 / 02 / 89 \\
06 / 21 / 90 \\
01 / 29 / 91 \\
06 / 04 / 91\end{array}$ & $\begin{array}{l}0001 \\
0001 \\
0001 \\
0001 \\
0001\end{array}$ & $M G / L$ & $\begin{array}{l}< \\
< \\
< \\
< \\
<\end{array}$ & $\begin{array}{l}0.01 \\
0.9 \\
0.01 \\
0.01 \\
0.01\end{array}$ & $\begin{array}{l}L \\
L \\
L \\
L\end{array}$ & $\begin{array}{l}0.01 \\
0.1 \\
0.01 \\
0.01 \\
0.01\end{array}$ & $\begin{array}{l}- \\
- \\
-\end{array}$ \\
\hline THORIUH-230 & $\begin{array}{l}01 / 29 / 91 \\
06 / 04 / 91\end{array}$ & $\begin{array}{l}0001 \\
0001\end{array}$ & $\mathrm{PCI} / \mathrm{L}$ & & $\begin{array}{l}0.0 \\
0.3\end{array}$ & $\mathbf{L}$ & 1. & $\begin{array}{l}0.3 \\
0.3\end{array}$ \\
\hline TIN & $\begin{array}{l}06 / 25 / 89 \\
12 / 02 / 89 \\
06 / 21 / 90 \\
01 / 29 / 91 \\
06 / 04 / 91\end{array}$ & $\begin{array}{l}0001 \\
0001 \\
0001 \\
0001 \\
0001\end{array}$ & $M G / L$ & $\begin{array}{l}< \\
< \\
< \\
< \\
<\end{array}$ & $\begin{array}{l}0.005 \\
0.005 \\
0.005 \\
0.005 \\
0.01\end{array}$ & $\begin{array}{l}L \\
L \\
L \\
L \\
l\end{array}$ & $\begin{array}{l}0.005 \\
0.005 \\
0.005 \\
0.005 \\
0.01\end{array}$ & $\begin{array}{l}- \\
- \\
- \\
-\end{array}$ \\
\hline TOTAL DISSOLVED SOLIDS & $\begin{array}{l}04 / 26 / 88 \\
06 / 25 / 89 \\
12 / 02 / 89 \\
06 / 21 / 90 \\
01 / 29 / 91 \\
06 / 04 / 91 \\
02 / 22 / 92 \\
08 / 13 / 92 \\
04 / 22 / 94 \\
12 / 12 / 94\end{array}$ & $\begin{array}{l}0001 \\
0001 \\
0001 \\
0001 \\
0001 \\
0001 \\
0001 \\
0001 \\
0001 \\
0001\end{array}$ & $M G / L$ & & $\begin{array}{l}340 . \\
348 . \\
355 \\
340 \\
316 \\
388 \\
383 \\
410 \\
410 \\
358\end{array}$ & $\begin{array}{l}L \\
L \\
L \\
L \\
L \\
F\end{array}$ & $\begin{array}{l}10 . \\
10 . \\
10 . \\
10 . \\
10 . \\
10 . \\
10 . \\
42 \\
10 \\
10\end{array}$ & $\begin{array}{l}- \\
: \\
: \\
: \\
- \\
-\end{array}$ \\
\hline TOTAL ORGAKIC CARBON & $\begin{array}{l}04 / 26 / 88 \\
01 / 29 / 91 \\
06 / 04 / 91 \\
02 / 22 / 92 \\
08 / 13 / 92\end{array}$ & $\begin{array}{l}0001 \\
0001 \\
0001 \\
0001 \\
0001\end{array}$ & $M G / L$ & $\begin{array}{l}< \\
< \\
< \\
<\end{array}$ & $\begin{array}{l}44.0 \\
1 . \\
1 . \\
1.0 \\
1.0\end{array}$ & $\begin{array}{l}\text { JL } \\
L\end{array}$ & $\begin{array}{l}1 . \\
1 . \\
1 . \\
1.0\end{array}$ & $\begin{array}{l}- \\
- \\
\dot{-} \\
-\end{array}$ \\
\hline TURBIDITY & $04 / 22 / 94$ & No01 & NTU & & 0 & & - & - \\
\hline URAHIUM & $\begin{array}{l}04 / 26 / 88 \\
06 / 25 / 89 \\
12 / 02 / 89 \\
06 / 21 / 90 \\
01 / 29 / 91 \\
06 / 04 / 91 \\
02 / 22 / 92 \\
11 / 18 / 92 \\
04 / 22 / 94 \\
12 / 12 / 94\end{array}$ & $\begin{array}{l}0001 \\
0001 \\
0001 \\
0001 \\
0001 \\
0001 \\
0001 \\
0001 \\
0001 \\
0001\end{array}$ & $\mathrm{HG} / \mathrm{L}$ & $<$ & $\begin{array}{l}0.0016 \\
0.0015 \\
0.001 \\
0.0022 \\
0.0017 \\
0.001 \\
0.002 \\
0.003 \\
0.002 \\
0.001\end{array}$ & $\begin{array}{l}J \\
J L \\
J L \\
J L \\
L\end{array}$ & $\begin{array}{l}0.003 \\
0.003 \\
0.003 \\
0.003 \\
0.0003 \\
0.001 \\
0.001 \\
0.001 \\
0.001 \\
0.001\end{array}$ & $\begin{array}{l}\because \\
: \\
\dot{-} \\
\dot{-} \\
- \\
-\end{array}$ \\
\hline URAHIUA (TOTAL) & $\begin{array}{l}11 / 18 / 92 \\
04 / 22 / 94\end{array}$ & $\begin{array}{l}\text { NOOI } \\
\text { NOOI }\end{array}$ & $M G / L$ & & $\begin{array}{l}0.001 \\
0.002\end{array}$ & & $\begin{array}{l}0.001 \\
0.001\end{array}$ & $\dot{-}$ \\
\hline VARNDIUH & $\begin{array}{l}06 / 25 / 89 \\
12 / 02 / 89\end{array}$ & $\begin{array}{l}0001 \\
0001\end{array}$ & $M G / L$ & $<$ & $\begin{array}{l}0.01 \\
0.01\end{array}$ & $\begin{array}{l}L \\
L\end{array}$ & $\begin{array}{l}0.01 \\
0.01\end{array}$ & $\dot{-}$ \\
\hline
\end{tabular}

PARAMETER VALUE INDICATOR (PVI): < - LESS THAN DETECTION LIMIT

SAMPLE ID COOES:

OTHER PARAMETER VALUE FLAGS:

0001 - FILTERED SAMPLE (.45 MICRONS)

F - LOH-FLON SAMPLING/DEDICATED PLMP

NOO1 - UMFILTERED SAMPLE

I - INCREASED OETECTION LIMIT DUE TO REQUIRED DILUTION

j - ESTIMATED VALUE

L - LESS THAN THREE BORE VOLUMES REMOVEd BEFORE SAMPLING 
GROUHDWATER QUALITY DATA BY LOCATION

SOUP ReV. O Data

SITE: MONO1 MONUWENT VALLEY

LOCATION: 0659

MORTH COORDINATE: 59069.8 FT

EAST COORDINATE: 88670.3 FT

$04 / 21 / 88$ TO $12 / 13 / 94$

REPORT DATE: 06/20/95

FORMATION OF COMPLETION: SHIMARUAP MEMBER OF THE CHIMLE FORMATION (SR) HYDRAULIC FLON RELATIONSHIP: DOWN GRADIENT (D)

\begin{tabular}{|c|c|c|c|c|c|c|c|c|}
\hline PARAMETER MAME & LOG DATE & $\underset{\text { SD }}{\text { SAMPLE }}$ & $\begin{array}{l}\text { UNITS OF } \\
\text { MEASURE }\end{array}$ & PVI & $\begin{array}{l}\text { PARAMETER } \\
\text { VALUE }\end{array}$ & FLAGS & $\begin{array}{l}\text { DETECTION } \\
\text { LIMIT }\end{array}$ & $\begin{array}{l}\text { PARANETER } \\
\text { UNCERTAIHTY }\end{array}$ \\
\hline VANADILA & $\begin{array}{l}06 / 21 / 90 \\
01 / 29 / 91 \\
06 / 04 / 91 \\
02 / 22 / 92 \\
08 / 13 / 92 \\
11 / 18 / 92 \\
04 / 22 / 94 \\
12 / 12 / 94\end{array}$ & $\begin{array}{l}0001 \\
0009 \\
0001 \\
0009 \\
0001 \\
0009 \\
0009 \\
0001\end{array}$ & MG/L & $\begin{array}{l}< \\
< \\
< \\
< \\
< \\
< \\
<\end{array}$ & $\begin{array}{l}0.01 \\
0.01 \\
0.01 \\
0.01 \\
0.05 \\
0.0019 \\
0.01 \\
0.01\end{array}$ & $\begin{array}{l}L \\
L \\
L \\
F\end{array}$ & $\begin{array}{l}0.01 \\
0.01 \\
0.09 \\
0.01 \\
0.05 \\
0.0019 \\
0.01 \\
0.01\end{array}$ & $\begin{array}{l}- \\
- \\
- \\
- \\
- \\
-\end{array}$ \\
\hline VANADIUH (TOTAL) & $\begin{array}{l}11 / 18 / 92 \\
04 / 22 / 94\end{array}$ & $\begin{array}{l}\text { No01 } \\
\text { N001 }\end{array}$ & MG/L & $<$ & \multicolumn{2}{|l|}{$\begin{array}{l}0.0020 \\
0.09\end{array}$} & $\begin{array}{l}0.0019 \\
0.01\end{array}$ & - \\
\hline ZINC & $\begin{array}{l}06 / 25 / 89 \\
12 / 02 / 89 \\
06 / 21 / 90 \\
01 / 29 / 91 \\
06 / 04 / 91 \\
02 / 22 / 92 \\
08 / 13 / 92 \\
11 / 18 / 92\end{array}$ & $\begin{array}{l}0001 \\
0001 \\
0001 \\
0001 \\
0001 \\
0001 \\
0001 \\
0001\end{array}$ & ME/L & $\begin{array}{l}< \\
< \\
< \\
< \\
<\end{array}$ & $\begin{array}{l}0.015 \\
0.005 \\
0.005 \\
0.005 \\
0.005 \\
0.005 \\
0.02 \\
0.17\end{array}$ & $\begin{array}{l}L \\
L \\
L \\
L \\
L\end{array}$ & $\begin{array}{l}0.005 \\
0.005 \\
0.005 \\
0.005 \\
0.005 \\
0.005 \\
0.02 \\
0.0010\end{array}$ & $\begin{array}{l}- \\
- \\
- \\
: \\
-\end{array}$ \\
\hline ZIMC (TOTAL) & $11 / 18 / 92$ & No01 & $M G / L$ & \multicolumn{3}{|c|}{0.0090} & 0.0010 & - \\
\hline
\end{tabular}

PARAMETER VALUE INDICATOR (PVI): < - LESS THAN DETECTION LIMIT

SAMPLE ID COOES:

OTHER PARAHETER VALUE FLAGS:

OOO1 - FILTERED SAMPLE (.45 MICROHS)

F - LOH-FLOW SAMPLIMG/DEDICATED PUMP

NOO1 - UNFILTERED SAMPLE

L - LESS THAN THREE BORE VOLLMES REMOVED BEFORE SAMPLING 
GROUNDWATER QUALITY DATA BY LOCATIOH

SOWP REV. O Dota

SITE: MOHOY MOHUMENT VALLEY

LOCATION: O660

NORTH COORDIHATE: $\quad 61303.4$ FT

EAST COORDIKATE: 89584.3 FT

$04 / 21 / 88$ TO $12 / 13 / 94$

REPORT DATE: 06/20/95

FORMATION OF COMPLETION: SHIMARUAP MEMBER OF THE CHIMLE FORMATION (SR) HYDRAULIC FLOH RELATIOHSHIP: DOWN GRADIENT (O)

\begin{tabular}{|c|c|c|c|c|c|c|c|c|}
\hline PARAMETER MAHE & LOS DATE & $\underset{\text { ID }}{\text { SNMPLE }}$ & $\begin{array}{l}\text { UKITS OF } \\
\text { MEASURE }\end{array}$ & PVI & $\begin{array}{l}\text { PARAMETER } \\
\text { YALUE }\end{array}$ & FLAGS & $\begin{array}{l}\text { DETECTION } \\
\text { LIMIT }\end{array}$ & $\begin{array}{l}\text { PARAMETER } \\
\text { UNCERTAINTY }\end{array}$ \\
\hline ALKALIKITY & $\begin{array}{l}04 / 21 / 88 \\
06 / 25 / 89 \\
12 / 01 / 89 \\
06 / 25 / 90 \\
01 / 29 / 91 \\
02 / 01 / 93 \\
06 / 27 / 93 \\
04 / 25 / 94 \\
12 / 13 / 94\end{array}$ & $\begin{array}{l}0001 \\
0001 \\
0001 \\
0001 \\
0001 \\
0001 \\
\text { No01 } \\
\text { N001 } \\
\text { N001 }\end{array}$ & MG/L CACo3 & & $\begin{array}{l}211 . \\
219 . \\
226 . \\
213 . \\
201 . \\
234 \\
209 \\
217 \\
207\end{array}$ & $\begin{array}{l}L \\
L \\
L \\
L \\
L \\
G \\
L \\
F\end{array}$ & $\begin{array}{c}- \\
- \\
- \\
\div \\
10\end{array}$ & $\begin{array}{l}- \\
: \\
: \\
- \\
- \\
-\end{array}$ \\
\hline ALUMIHUM & $\begin{array}{l}06 / 25 / 89 \\
12 / 01 / 89 \\
06 / 25 / 90 \\
01 / 29 / 91\end{array}$ & $\begin{array}{l}0001 \\
0001 \\
0001 \\
0001\end{array}$ & $M G / L$ & $\begin{array}{l}< \\
< \\
< \\
<\end{array}$ & $\begin{array}{l}0.1 \\
0.1 \\
0.1 \\
0.1\end{array}$ & $\begin{array}{l}L \\
L \\
L \\
L\end{array}$ & $\begin{array}{l}0.1 \\
0.1 \\
0.1 \\
0.1\end{array}$ & $\begin{array}{l}- \\
\vdots\end{array}$ \\
\hline AMMONIUN & $\begin{array}{l}04 / 21 / 88 \\
06 / 25 / 89 \\
12 / 01 / 89 \\
06 / 25 / 90 \\
01 / 29 / 91\end{array}$ & $\begin{array}{l}0001 \\
0001 \\
0001 \\
0001 \\
0001\end{array}$ & MG/L & $\begin{array}{l}< \\
< \\
< \\
< \\
<\end{array}$ & $\begin{array}{l}0.1 \\
0.1 \\
0.1 \\
0.1 \\
0.1\end{array}$ & $\begin{array}{l}L \\
L \\
L \\
H L \\
L\end{array}$ & $\begin{array}{l}0.1 \\
0.1 \\
0.1 \\
0.1 \\
0.1\end{array}$ & $\begin{array}{l}- \\
- \\
-\end{array}$ \\
\hline AMLNIUM (TOTAL) & $\begin{array}{l}06 / 27 / 93 \\
04 / 25 / 94 \\
12 / 13 / 94\end{array}$ & $\begin{array}{l}\text { NOO1 } \\
\text { N001 } \\
\text { NO09 }\end{array}$ & MG/L & $<$ & $\begin{array}{l}0.1 \\
0.1 \\
0.1\end{array}$ & $\begin{array}{l}G \\
L\end{array}$ & $\begin{array}{l}0.1 \\
0.1 \\
0.1\end{array}$ & - \\
\hline AнTIMOHY & $\begin{array}{l}06 / 25 / 89 \\
12 / 01 / 89 \\
06 / 25 / 90 \\
01 / 29 / 91\end{array}$ & $\begin{array}{l}0001 \\
0001 \\
0001 \\
0001\end{array}$ & ME/L & $\begin{array}{l}< \\
<\end{array}$ & $\begin{array}{l}0.003 \\
0.003 \\
0.003 \\
0.004\end{array}$ & $\begin{array}{l}l \\
L \\
l\end{array}$ & $\begin{array}{l}0.003 \\
0.003 \\
0.003 \\
0.003\end{array}$ & $\begin{array}{l}- \\
-\end{array}$ \\
\hline $\begin{array}{l}\text { ARSEHIC } \\
\therefore\end{array}$ & $\begin{array}{l}04 / 21 / 88 \\
06 / 25 / 89 \\
12 / 01 / 89 \\
06 / 25 / 90 \\
01 / 29 / 91 \\
06 / 27 / 93\end{array}$ & $\begin{array}{l}0001 \\
0001 \\
0001 \\
0001 \\
0001 \\
0001\end{array}$ & MG/L & $\begin{array}{l}< \\
< \\
< \\
< \\
<\end{array}$ & $\begin{array}{l}0.004 \\
0.01 \\
0.01 \\
0.01 \\
0.01 \\
0.005\end{array}$ & $\begin{array}{l}J L \\
L \\
L \\
L \\
L \\
G H\end{array}$ & $\begin{array}{l}0.01 \\
0.01 \\
0.01 \\
0.01 \\
0.01 \\
0.005\end{array}$ & $\begin{array}{l}- \\
- \\
- \\
-\end{array}$ \\
\hline ARSENIC (TOTAL) & $06 / 27 / 93$ & NoOI & MG/L & & 0.005 & G & 0.005 & - \\
\hline BARIUN & $\begin{array}{l}06 / 25 / 89 \\
12 / 01 / 89 \\
06 / 25 / 90 \\
01 / 29 / 91 \\
06 / 27 / 93\end{array}$ & $\begin{array}{l}0009 \\
0001 \\
0001 \\
0001 \\
0001\end{array}$ & $M G / L$ & $\begin{array}{l}< \\
< \\
< \\
< \\
<\end{array}$ & $\begin{array}{l}0.1 \\
0.1 \\
0.1 \\
0.1 \\
0.1\end{array}$ & $\begin{array}{l}L \\
L \\
L \\
L \\
G\end{array}$ & $\begin{array}{l}0.1 \\
0.1 \\
0.1 \\
0.1 \\
0.1\end{array}$ & $\begin{array}{l}- \\
- \\
-\end{array}$ \\
\hline BARIUM (TOTAL) & $06 / 27 / 93$ & No01 & $M G / L$ & $<$ & 0.1 & $\mathbf{G}$. & 0.1 & - \\
\hline BERYLLIUYM & $\begin{array}{l}06 / 25 / 89 \\
12 / 01 / 89 \\
06 / 25 / 90 \\
01 / 29 / 91\end{array}$ & $\begin{array}{l}0001 \\
0001 \\
0001 \\
0001\end{array}$ & MG/L & $\begin{array}{l}< \\
< \\
< \\
<\end{array}$ & $\begin{array}{l}0.01 \\
0.005 \\
0.01 \\
0.01\end{array}$ & $\begin{array}{l}L \\
L \\
L \\
L\end{array}$ & $\begin{array}{l}0.01 \\
0.005 \\
0.01 \\
0.01\end{array}$ & : \\
\hline
\end{tabular}

PARAMETER VALUE IMDICATOR (PVI): < - LESS THAN DETECTION LIMIT

OTHER PARAMETER VALUE FLAGS:

F - LOW-FLOW SUMPLING/DEDICATED PUMP

G - PH > 9, POSSIBLE GROUT CONTAMIHATION

H - HOLD TIME EXPIRED, VALUE SUSPECT

$J$ - ESTIMHTED VALUE

L - LESS THAH THREE BORE VOLUMES REMOVEO BEFORE SAMPLIHG

$W$ - POST-DIGEST SPIKE OUT OF CHTR LIH HHILE SAMP ABS < 50\% SPIKE
SAMPLE ID COOES:

0001 - FILTERED SAMPLE (.45 MICROMS)

NODI - UHFILTERED SAHPLE 
GROUMDUATER QUALITY DATA BY LOCATION

SOWP ReV. O Dats

SITE: HONO1 MONUMENT VALLEY

LOCATION: 0660

MORTH COORDIHATE: $\quad 61303.4$ FT

EAST COORDINATE: 89584.3 FT

$04 / 21 / 88$ TO $12 / 13 / 94$

REPORT DATE: $06 / 20 / 95$

FORMATION OF COMPLETION: SHIMARUMP MEMBER OF THE CHINLE FORMATION (SR)

HYDRAULIC FLON RELATIONSHIP: DON GRADIENT (D)

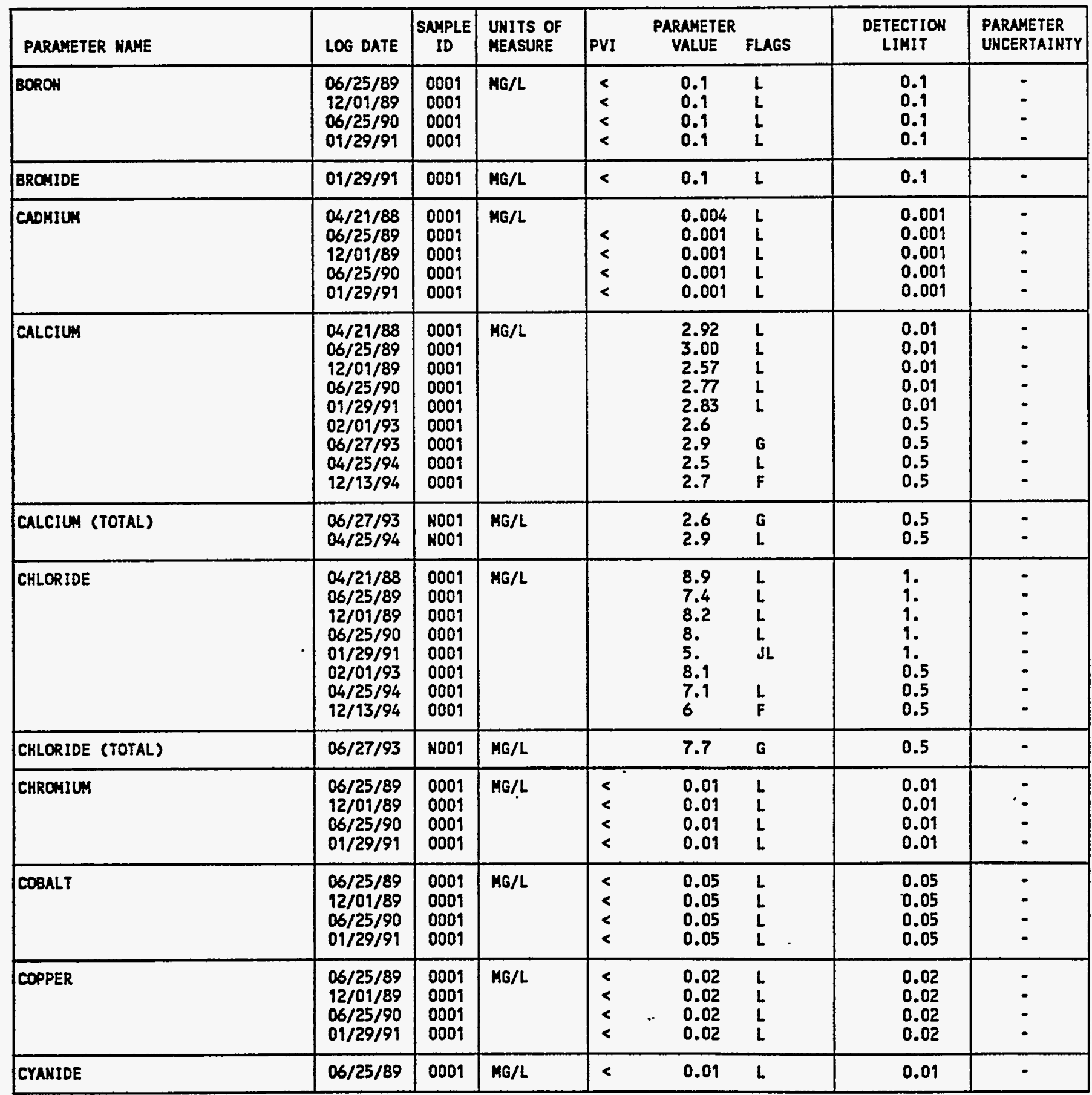

PARAMETER VALUE INDICATOR (PVI): < - LESS THAN DETECTION LIMIT

SAMPLE ID COOES:

OTHER PARAMETER VALUE FLAGS:

O001 - FILTERED SAMPLE (.45 MICRONS)

F - LOU-FLOU SAMPLIHG/DEDICATED PUHP

NOO1 - UAFILTERED SAMPLE

G - PH > 9, POSSIBLE GROUT CONTANIMATION

J - ESTIMATED VALUE

- $L$ - LESS THAN THREE BORE VOLLAES REMOVED BEFORE SAMPLING 
GROUHDWATER OUALITY DATA BY LOCATION

SOMP ReV. 0 Data

SITE: MONOI MONUNENT VALLEY

LOCATIOH: 0660

NORTH COORDINATE: 69303.4 FT

EAST COORDIHATE: 89584.3 FT

$04 / 21 / 88$ TO $12 / 13 / 94$

REPORT DATE: $06 / 20 / 95$

FORHATION OF CONPLETION: SHIHARLMP MEMBER OF THE CHIHLE FORHATION (SR) HYORAULIC FLOH RELATIOHSHIP: DONH GRADIENT $(D)$

\begin{tabular}{|c|c|c|c|c|c|c|c|c|c|}
\hline PARAHETER MAME & & LOG DATE & $\begin{array}{c}\text { SAMPLE } \\
\text { ID }\end{array}$ & $\begin{array}{l}\text { UWITS OF } \\
\text { MEASURE }\end{array}$ & PVI & $\begin{array}{l}\text { PARAMETER } \\
\text { VALUE }\end{array}$ & FLAGS & $\begin{array}{l}\text { DETECTION } \\
\text { LIMIT }\end{array}$ & $\begin{array}{l}\text { PARAMETER } \\
\text { UNCERTAINTY }\end{array}$ \\
\hline CYAKIDE & & $12 / 01 / 89$ & 0001 & MG/L & $<$ & 0.01 & $L$ & 0.01 & - \\
\hline DISSOLVED OXYGEN & & $\begin{array}{l}06 / 27 / 93 \\
04 / 25 / 94 \\
12 / 13 / 94\end{array}$ & $\begin{array}{l}\text { N001 } \\
\text { N001 } \\
\text { N001 }\end{array}$ & MG/L & $<$ & $\begin{array}{l}0.4 \\
0.029 \\
0.6\end{array}$ & $\begin{array}{l}G \\
L \\
F\end{array}$ & 0. & . \\
\hline GROSS ALPHA & & $\begin{array}{l}04 / 21 / 88 \\
01 / 29 / 91 \\
02 / 01 / 93 \\
06 / 27 / 93\end{array}$ & $\begin{array}{l}0001 \\
0001 \\
0001 \\
0001\end{array}$ & $\mathrm{PCI} / \mathrm{L}$ & & $\begin{array}{l}2.0 \\
0.0 \\
0 \\
0\end{array}$ & $\begin{array}{l}L \\
L \\
G\end{array}$ & $\begin{array}{l}0.2 \\
1 . \\
5 \\
2\end{array}$ & $\begin{array}{l}2.7 \\
2.2 \\
5 \\
4\end{array}$ \\
\hline GROSS BETA (TOTAL) & & $06 / 27 / 93$ & HoOI & $\mathrm{PCI} / \mathrm{L}$ & & 4 & G & 4 & 4 \\
\hline IROH & -. & $\begin{array}{l}06 / 25 / 89 \\
12 / 01 / 89 \\
06 / 25 / 90 \\
01 / 29 / 91 \\
02 / 01 / 93 \\
06 / 27 / 93\end{array}$ & $\begin{array}{l}0001 \\
0001 \\
0001 \\
0001 \\
0001 \\
0001\end{array}$ & MG/L & $\begin{array}{l}< \\
< \\
< \\
< \\
< \\
<\end{array}$ & $\begin{array}{l}0.03 \\
0.03 \\
0.03 \\
0.03 \\
0.03 \\
0.03\end{array}$ & $\begin{array}{l}L \\
L \\
L \\
L \\
\text { GJ }\end{array}$ & $\begin{array}{l}0.03 \\
0.03 \\
0.03 \\
0.03 \\
0.03 \\
0.03\end{array}$ & $\begin{array}{l}- \\
: \\
:\end{array}$ \\
\hline IRON (TOTAL) & & $06 / 27 / 93$ & NOOI & MG/L & $<$ & 0.03 & GJ & 0.03 & - \\
\hline MGGESIUH (TOTAL) & & $\begin{array}{l}06 / 27 / 93 \\
04 / 25 / 94\end{array}$ & $\begin{array}{l}\text { No01 } \\
\text { N001 }\end{array}$ & MG/L & & $\begin{array}{l}1.6 \\
1.5\end{array}$ & $\begin{array}{l}G \\
L\end{array}$ & $\begin{array}{l}0.1 \\
0.1\end{array}$ & - \\
\hline MAMGAKESE & & $06 / 25 / 89$ & 0001 & MG/L & $<$ & 0.01 & $L$ & 0.01 & - \\
\hline
\end{tabular}

PARAMETER VALUE IMDICATOR (PVI): < - LESS THAN DETECTION LIMIT

OTHER PARAMETER VALUE FLAGS:

SAMPLE ID COOES:

OOO1 - FILTERED SAMPLE (.45 MICRONS)

F - LOW-FLOW SAMPLIHG/OEDICATED PLMP

NOO1 - UNFILTERED SNMPLE

G - PH > 9, POSSIBLE GROUT CONTAMIHATIOH

J. estimated VALUE

L - LESS THAN THREE BORE VOLUMES REMOVED BEFORE SAMPLIHG 
GROUNDHATER OUALITY DATA BY LOCATION

SOMP ReV. O Data

SITE: MONO1 MONUMENT VALLEY

LOCATION: 0660

NORTH COORDINATE: 69303.4 FT

EAST COORDINATE: 89584.3 FT

04/21/88 TO $12 / 13 / 94$

REPORT DATE: 06/20/95

FORMATION OF COMPLETION: SHIMARUAP MEMBER OF THE CHINLE FORMATION (SR) HYDRAULIC FLON RELATIONSHIP: DOWN GRADIENT (D)

\begin{tabular}{|c|c|c|c|c|c|c|c|c|}
\hline PARAMETER MAME & LOG DATE & $\underset{\text { SAMPLE }}{\text { SD }}$ & $\begin{array}{l}\text { UNITS OF } \\
\text { MEASURE }\end{array}$ & PVI & $\begin{array}{l}\text { PARAMETER } \\
\text { VALUE }\end{array}$ & FLAGS & $\begin{array}{l}\text { DETECTION } \\
\text { LIMIT }\end{array}$ & $\begin{array}{l}\text { PARAMETER } \\
\text { UNCERTAINTY }\end{array}$ \\
\hline MUNGAKESE & $\begin{array}{l}12 / 01 / 89 \\
06 / 25 / 90 \\
01 / 29 / 91 \\
02 / 01 / 93 \\
06 / 27 / 93 \\
04 / 25 / 94 \\
12 / 13 / 94\end{array}$ & $\begin{array}{l}0001 \\
0001 \\
0001 \\
0001 \\
0001 \\
0001 \\
0001\end{array}$ & MG/L & $\begin{array}{l}< \\
< \\
< \\
< \\
< \\
< \\
<\end{array}$ & $\begin{array}{l}0.01 \\
0.01 \\
0.01 \\
0.01 \\
0.01 \\
0.01 \\
0.01\end{array}$ & $\begin{array}{l}L \\
L \\
L \\
G \\
L \\
F\end{array}$ & $\begin{array}{l}0.01 \\
0.01 \\
0.01 \\
0.01 \\
0.01 \\
0.01 \\
0.01\end{array}$ & $\begin{array}{l}- \\
: \\
: \\
-\end{array}$ \\
\hline MANGAKESE (TOTAL) & $\begin{array}{l}06 / 27 / 93 \\
04 / 25 / 94\end{array}$ & $\begin{array}{l}\text { NOO1 } \\
\text { NODI }\end{array}$ & MG/L & $<$ & $\begin{array}{l}0.01 \\
0.01\end{array}$ & $\begin{array}{l}\mathbf{G} \\
\mathbf{L}\end{array}$ & $\begin{array}{l}0.01 \\
0.01\end{array}$ & - \\
\hline MERCURY & $\begin{array}{l}06 / 25 / 89 \\
12 / 01 / 89 \\
06 / 25 / 90\end{array}$ & $\begin{array}{l}0001 \\
0001 \\
0001\end{array}$ & MG/L & $<$ & $\begin{array}{l}0.0002 \\
0.0002 \\
0.0002\end{array}$ & $\begin{array}{l}L \\
L \\
L\end{array}$ & $\begin{array}{l}0.0002 \\
0.0002 \\
0.0002\end{array}$ & : \\
\hline MOLYBDENUN & $\begin{array}{l}04 / 21 / 88 \\
06 / 25 / 89 \\
12 / 01 / 89 \\
06 / 25 / 90 \\
01 / 29 / 91 \\
06 / 27 / 93 \\
12 / 13 / 94\end{array}$ & $\begin{array}{l}0001 \\
0001 \\
0001 \\
0001 \\
0001 \\
0001 \\
0001\end{array}$ & $M G / L$ & $\begin{array}{l}< \\
< \\
< \\
< \\
<\end{array}$ & $\begin{array}{l}0.01 \\
0.01 \\
0.01 \\
0.01 \\
0.01 \\
0.01 \\
0.01\end{array}$ & $\begin{array}{l}L \\
L \\
L \\
L \\
L \\
G \\
F\end{array}$ & $\begin{array}{l}0.01 \\
0.01 \\
0.01 \\
0.01 \\
0.01 \\
0.01 \\
0.01\end{array}$ & $\begin{array}{l}- \\
- \\
- \\
-\end{array}$ \\
\hline MOLYBDENUM (TOTAL) & $06 / 27 / 93$ & NOO1 & MG/L & $<$ & 0.01 & G & 0.01 & - \\
\hline NET GROSS ALPHA • & $\begin{array}{l}04 / 21 / 88 \\
01 / 29 / 91 \\
02 / 01 / 93 \\
06 / 27 / 93\end{array}$ & $\begin{array}{l}0001 \\
0001 \\
0001 \\
0001\end{array}$ & $\mathrm{PCI} / \mathrm{L}$ & & $\begin{array}{r}1.66 \\
-0.10 \\
-0.34 \\
-0.34\end{array}$ & & $\dot{-}$ & $\begin{array}{l}- \\
-\end{array}$ \\
\hline HET GROSS ALPHA (TOTAL) ** & $06 / 27 / 93$ & N001 & $\mathrm{PCI} / \mathrm{L}$ & & 4.66 & & - & - \\
\hline NICKEL & $\begin{array}{l}06 / 25 / 89 \\
12 / 01 / 89 \\
06 / 25 / 90 \\
01 / 29 / 91\end{array}$ & $\begin{array}{l}0001 \\
0001 \\
0001 \\
0001\end{array}$ & MG/L & $\begin{array}{l}< \\
< \\
< \\
<\end{array}$ & $\begin{array}{l}0.04 \\
0.04 \\
0.04 \\
0.04\end{array}$ & $\begin{array}{l}L \\
L \\
L \\
L\end{array}$ & $\begin{array}{l}0.04 \\
0.04 \\
0.04 \\
0.04\end{array}$ & : \\
\hline NITRATE & $\begin{array}{l}04 / 21 / 88 \\
06 / 25 / 89 \\
12 / 01 / 89 \\
06 / 25 / 90 \\
01 / 29 / 91 \\
02 / 01 / 93\end{array}$ & $\begin{array}{l}0001 \\
0001 \\
0001 \\
0001 \\
0001 \\
0001\end{array}$ & HG/L & $<$ & $\begin{array}{l}0.7 \\
2.9 \\
5.8 \\
0.1 \\
4 . \\
1\end{array}$ & $\begin{array}{l}J L \\
L \\
L \\
L \\
L\end{array}$ & $\begin{array}{l}1 . \\
1 . \\
1.1 \\
1.1 \\
1\end{array}$ & $\begin{array}{l}- \\
- \\
-\end{array}$ \\
\hline HITRATE (TOTAL) & $\begin{array}{l}06 / 27 / 93 \\
04 / 25 / 94 \\
12 / 13 / 94\end{array}$ & $\begin{array}{l}\text { No01 } \\
\text { N001 } \\
\text { N001 }\end{array}$ & MG/L & $\begin{array}{l}< \\
< \\
<\end{array}$ & $\begin{array}{l}1 \\
1 \\
1\end{array}$ & $\begin{array}{l}\text { G } \\
\text { FJN }\end{array}$ & $\begin{array}{l}1 \\
1 \\
1\end{array}$ & $\ddot{-}$ \\
\hline MITRITE AND NITRATE & $01 / 29 / 91$ & 0001 & MG/L & $<$ & 1. & JL & 1. & - \\
\hline
\end{tabular}

- MET gross ALPHA (GROSS ALPHA - URANIUM) UITH 1 MG URANIUM = $686^{\circ}$ PCI

* MET GROSS ALPHA (TOTAL) (TOTAL GROSS ALPHA - TOTAL URANILH)

PARAMETER VALUE IMDICATOR (PVI): < - LESS THAN DETECTION LIMIT

OTHER PARAMETER VALUE FLAGS:

F - LOW-FLOW SAMPLING/DEDICATED PLMP

G - PH > 9, POSSIBLE GROUT CONTAMINATIOH

J - ESTIMATED VALUE

$l$ - LESS THAN THREE BORE VOLUMES REMOVED BEFORE SAMPLIHG

M - SPIKE SAMPLE RECOVERY NOT WITHIH CONTROL LIMITS
SAMPLE 10 COOES:

0001 - FILTERED SAMPLE (.45 MICRONS)

NOO1 - UNFILTERED SAMPLE 
GROUHDWATER QUALITY DATA BY LOCATION

SOWP ReV. 0 Data

SITE: MONOI MONUHENT VALLEY

LOCATION: 0660

MORTH COORDINATE: 61303.4 FT

EAST COORDINATE: 89584.3 FT

$04 / 21 / 88$ TO $12 / 13 / 94$

REPORT DATE: 06/20/95

FORMATION OF COMPLETIOH: SHIMARUMP MEMBER OF THE CHIMLE FORHATION (SR) HYORAULIC FLOW RELATIOHSHIP: DOWN GRADIEHT (D)

\begin{tabular}{|c|c|c|c|c|c|c|c|c|}
\hline PARAMETER HAME & LOG DATE & $\underset{\text { ID }}{\text { SAMPLE }}$ & $\begin{array}{l}\text { UWITS OF } \\
\text { MEASURE }\end{array}$ & PVI & $\begin{array}{l}\text { PARAMETER } \\
\text { VALUEE }\end{array}$ & FLAGS & $\begin{array}{l}\text { DETECTION } \\
\text { LIMIT }\end{array}$ & $\begin{array}{l}\text { PARAMETER } \\
\text { UHCERTAINTY }\end{array}$ \\
\hline PH & $\begin{array}{l}04 / 21 / 88 \\
06 / 25 / 89 \\
12 / 01 / 89 \\
06 / 25 / 90 \\
01 / 29 / 91 \\
02 / 01 / 93 \\
06 / 27 / 93 \\
04 / 25 / 94 \\
12 / 13 / 94\end{array}$ & $\begin{array}{l}0001 \\
0001 \\
0001 \\
0001 \\
0001 \\
0001 \\
\text { NoO1 } \\
\text { NoO1 } \\
\text { No01 }\end{array}$ & su & & $\begin{array}{l}8.69 \\
8.25 \\
8.88 \\
8.73 \\
8.62 \\
8.63 \\
9.01 \\
8.62 \\
8.88\end{array}$ & $\begin{array}{l}L \\
L \\
L \\
L \\
L \\
G \\
L \\
F\end{array}$ & $\begin{array}{c}: \\
\vdots \\
\vdots \\
\vdots \\
\vdots \\
0.10\end{array}$ & $\begin{array}{l}: \\
: \\
: \\
: \\
-\end{array}$ \\
\hline PHOSPHATE & $\begin{array}{l}06 / 25 / 89 \\
12 / 01 / 89 \\
06 / 25 / 90\end{array}$ & $\begin{array}{l}0001 \\
0001 \\
0001\end{array}$ & MG/L & $<$ & $\begin{array}{l}0.1 \\
0.3 \\
0.1\end{array}$ & $\begin{array}{l}L \\
L \\
L\end{array}$ & $\begin{array}{l}0.1 \\
0.1 \\
0.1\end{array}$ & $\therefore$ \\
\hline PHOSPHATE (TOTAL) & $06 / 27 / 93$ & N001 & MG/L & $<$ & 0.1 & G & 0.1 & - \\
\hline POTASSIUH & $\begin{array}{l}04 / 21 / 88 \\
06 / 25 / 89 \\
12 / 01 / 89 \\
06 / 25 / 90 \\
01 / 29 / 91 \\
02 / 01 / 93 \\
06 / 27 / 93 \\
04 / 25 / 94 \\
12 / 13 / 94\end{array}$ & $\begin{array}{l}0001 \\
0001 \\
0001 \\
0001 \\
0001 \\
0001 \\
0001 \\
0001 \\
0001\end{array}$ & $\mathrm{MG} / \mathrm{L}$ & & $\begin{array}{l}0.58 \\
0.7 \\
0.63 \\
0.51 \\
0.7 \\
0.6 \\
0.8 \\
0.8 \\
0.7\end{array}$ & $\begin{array}{l}L \\
L \\
L \\
L \\
L \\
G \\
L \\
\text { FJ }\end{array}$ & $\begin{array}{l}0.01 \\
0.01 \\
0.01 \\
0.01 \\
0.01 \\
0.1 \\
0.1 \\
0.1 \\
0.1\end{array}$ & $\begin{array}{l}: \\
: \\
: \\
:\end{array}$ \\
\hline POTASSIUH (TOTAL) & $\begin{array}{l}06 / 27 / 93 \\
04 / 25 / 94\end{array}$ & $\begin{array}{l}\text { NO01 } \\
\text { N001 }\end{array}$ & MG/L & & $\begin{array}{l}0.7 \\
0.7\end{array}$ & G & $\begin{array}{l}0.1 \\
0.1\end{array}$ & - \\
\hline RADIUN-226 & $\begin{array}{l}06 / 25 / 89 \\
12 / 01 / 89 \\
06 / 25 / 90 \\
01 / 29 / 99\end{array}$ & $\begin{array}{l}0001 \\
0001 \\
0001 \\
0001\end{array}$ & PCI/L & & $\begin{array}{l}0.5 \\
0.0 \\
0.8 \\
0.6\end{array}$ & $\begin{array}{l}L \\
L \\
L \\
L\end{array}$ & $\begin{array}{l}1 . \\
1 . \\
1 .\end{array}$ & $\begin{array}{l}0.3 \\
0.1 \\
0.3 \\
0.3\end{array}$ \\
\hline RADILH-226 + RADIUM-228 & $\begin{array}{l}06 / 25 / 89 \\
12 / 01 / 89 \\
06 / 25 / 90 \\
01 / 29 / 91\end{array}$ & $\begin{array}{l}0001 \\
0001 \\
0001 \\
0001\end{array}$ & PCI/L & & $\begin{array}{l}0.70 \\
2.10 \\
1.80 \\
1.50\end{array}$ & & : & $:$ \\
\hline RADIUM-228 & $\begin{array}{l}06 / 25 / 89 \\
12 / 01 / 89 \\
06 / 25 / 90 \\
01 / 29 / 91\end{array}$ & $\begin{array}{l}0009 \\
0001 \\
0001 \\
0001\end{array}$ & PCI/L & & $\begin{array}{l}0.2 \\
2.1 \\
1.0 \\
0.9\end{array}$ & $\begin{array}{l}L \\
L \\
L \\
L\end{array}$ & $\begin{array}{l}9 . \\
1 . \\
1 .\end{array}$ & $\begin{array}{l}0.7 \\
3.5 \\
0.8 \\
0.7\end{array}$ \\
\hline REDOX POTEHTIAL & $\begin{array}{l}06 / 27 / 93 \\
04 / 25 / 94 \\
12 / 13 / 94\end{array}$ & $\begin{array}{l}\text { HOOI } \\
\text { NOO1 } \\
\text { HOO1 }\end{array}$ & mOLTS & & $\begin{array}{r}64 \\
-25 \\
-57\end{array}$ & $\begin{array}{l}G \\
L \\
F\end{array}$ & - & : \\
\hline SELENILA & $\begin{array}{l}04 / 21 / 88 \\
06 / 25 / 89 \\
12 / 01 / 89 \\
06 / 25 / 90 \\
01 / 29 / 99\end{array}$ & $\begin{array}{l}0001 \\
0001 \\
0001 \\
0001 \\
0009\end{array}$ & $M G / L$ & $\begin{array}{l}< \\
< \\
<\end{array}$ & $\begin{array}{l}0.005 \\
0.005 \\
0.005 \\
0.005 \\
0.005\end{array}$ & $\begin{array}{l}L \\
L \\
L \\
L\end{array}$ & $\begin{array}{l}0.005 \\
0.005 \\
0.005 \\
0.005 \\
0.005\end{array}$ & $:$ \\
\hline
\end{tabular}

PARAMETER VALUE INDICATOR (PVI): < - LESS THAN DETECTION LIMIT

OTHER PARAMETER VALUE FLAGS:

SAMPLE ID COOES:

O001 - FILTERED SAMPLE (.45 MICRONS)

F - LON-FLON SAMPLIHG/DEDICATED PUMP

NODI - UNFILTERED SAMPLE

G - PH > 9, POSSIBLE GROUT CONTAHIHATION

J - estimated VALUe

L - LESS THAK THREE BORE VOLUMES REMOVED BEFORE SAMPLIMG 
GROUNDHATER QUALITY DATA BY LOCATION

SOWP ReV. O Data

SITE: MONO1 MONUHENT VALLEY

LOCATION: 0660

NORTH COORDIHATE: 61303.4 FT

EAST COORDINATE: 89584.3 FT

$04 / 21 / 88$ TO $12 / 13 / 94$

REPORT DATE: $06 / 20 / 95$

FORMATION OF CONPLETION: SHINARUMP MEMBER OF THE CHIHLE FORMATIOH (SR) HYDAULIC FLOW RELATIONSHIP: DONN GRAOIENT (D)

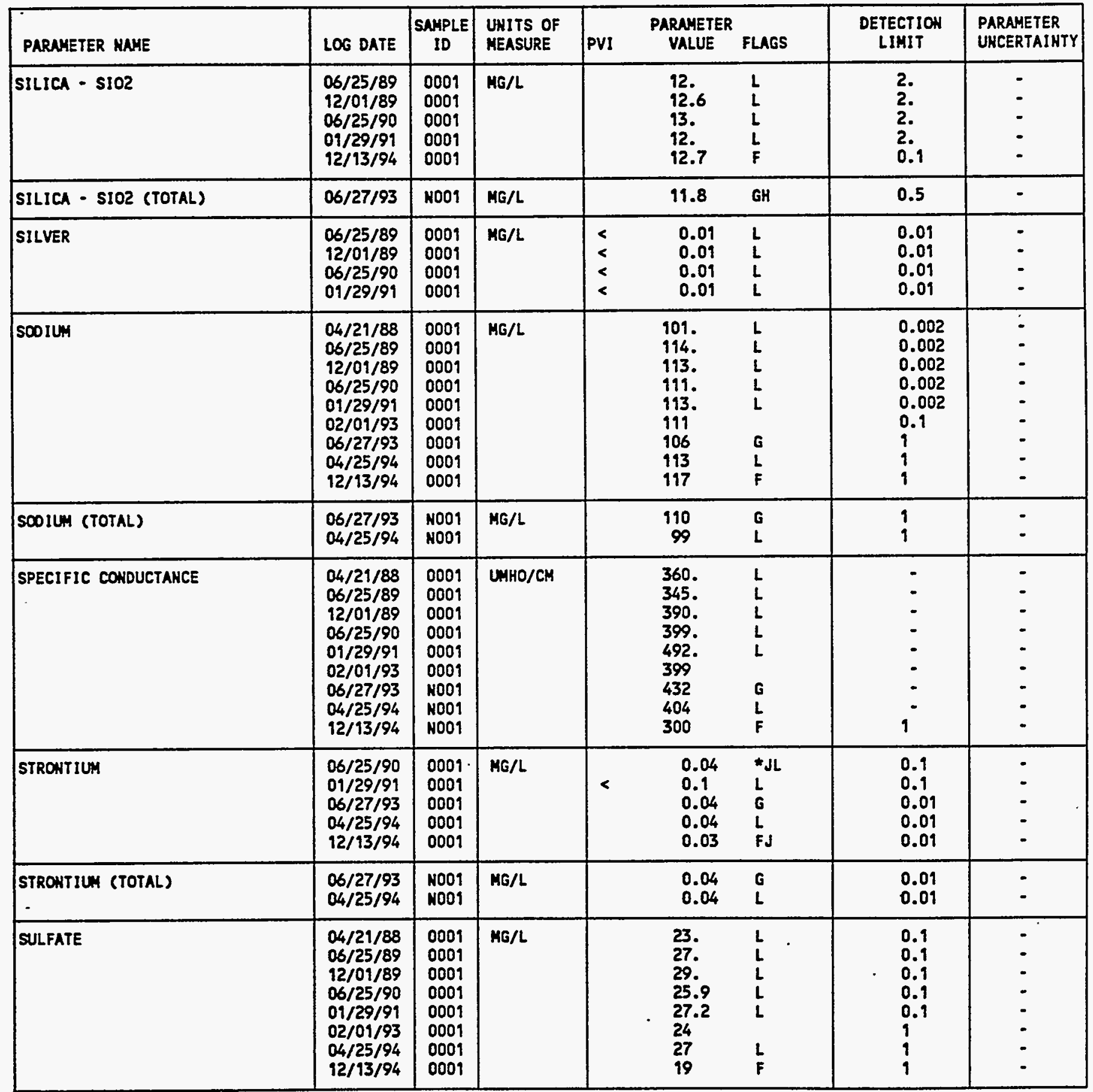

PARAMETER VALUE IMDICATOR (PVI): < - LESS THAN DETECTION LIMIT

SAMPLE ID COOES:

OTHER PARNAETER VALUE FLAGS:

0001 - FILTERED SAMPLE (.45 MICRONS)

* - DUPLICATE ANALYSIS MOT HITHIN CONTROL LIHITS

NOO1 - UHFILTERED SAMPLE

F - LON-FLON SAMPLING/DEDICATED PUMP

G - PH > 9, POSSIBLE GROUT CONTAMINATIOH

H - HOLD TIME EXPIRED, VALUE SUSPECT

J - ESTIMATED VALUE

L - LESS THAN THREE BORE VOLUHES REMOVED BEFORE SNMPLIKG 
GROUHDWATER QUALITY DATA BY LOCATION

SOUP REV. O Dat8

SITE: MONO1 MONUMENT VALLEY

LOCATION: 0660

MORTH COORDIHATE: 61303.4 FT

EAST COORDIHATE: 89584.3 FT

$04 / 21 / 88$ TO $12 / 13 / 94$

REPORT DATE: 06/20/95

FORHATION OF COMPLETION: SHIMARUMP MEMBER OF THE CHIHLE FORMATIOH (SR)

HYDRAULIC FLOU RELATIONSHIP: DOUN GRADIENT (D)

\begin{tabular}{|c|c|c|c|c|c|c|c|c|}
\hline PARAMETER MAME & LOG DATE & $\underset{\text { SOMLE }}{\text { SAMPLE }}$ & $\begin{array}{l}\text { UNITS OF } \\
\text { MEASURE }\end{array}$ & PVI & $\begin{array}{l}\text { PARAMETER } \\
\text { VALUE }\end{array}$ & FLAGS & $\begin{array}{l}\text { DETECTIOH } \\
\text { LIMIT }\end{array}$ & $\begin{array}{l}\text { PARAHETER } \\
\text { UNCERTAIIITY }\end{array}$ \\
\hline SULFATE (TOTAL) & $06 / 27 / 93$ & NoO1 & $\mathbf{H G} / \mathbf{L}$ & & 28 & G & 1 & - \\
\hline SULFIDE & $\begin{array}{l}06 / 25 / 89 \\
12 / 01 / 89 \\
01 / 29 / 91\end{array}$ & $\begin{array}{l}0001 \\
0001 \\
0001\end{array}$ & MG/L & $\begin{array}{l}< \\
<\end{array}$ & $\begin{array}{l}0.1 \\
0.1 \\
0.1\end{array}$ & $\begin{array}{l}L \\
L \\
J L\end{array}$ & $\begin{array}{l}0.1 \\
0.9 \\
0.1\end{array}$ & - \\
\hline TEMPERATURE & $\begin{array}{l}04 / 21 / 88 \\
06 / 25 / 89 \\
12 / 01 / 89 \\
06 / 25 / 90 \\
01 / 29 / 91 \\
02 / 01 / 93 \\
06 / 27 / 93 \\
04 / 25 / 94 \\
12 / 13 / 94\end{array}$ & $\begin{array}{l}0001 \\
0001 \\
0001 \\
0001 \\
0001 \\
0001 \\
N 001 \\
N 001 \\
N 001\end{array}$ & C - DEGREE & & $\begin{array}{l}16.0 \\
17.5 \\
16.0 \\
17.5 \\
15.6 \\
15.2 \\
18.8 \\
15.9 \\
15.7\end{array}$ & $\begin{array}{l}L \\
L \\
L \\
L \\
L \\
G \\
L \\
F\end{array}$ & $\begin{array}{l}- \\
= \\
= \\
= \\
0.1\end{array}$ & $\begin{array}{l}- \\
\dot{-} \\
\dot{-} \\
- \\
- \\
-\end{array}$ \\
\hline THALLILY & $\begin{array}{l}06 / 25 / 89 \\
12 / 01 / 89 \\
06 / 25 / 90 \\
01 / 29 / 91\end{array}$ & $\begin{array}{l}0001 \\
0001 \\
0001 \\
0001\end{array}$ & $M G / L$ & $\begin{array}{l}< \\
< \\
<\end{array}$ & $\begin{array}{l}0.01 \\
0.1 \\
0.01 \\
0.01\end{array}$ & $\begin{array}{l}L \\
L \\
L \\
L\end{array}$ & $\begin{array}{l}0.01 \\
0.1 \\
0.01 \\
0.01\end{array}$ & $\begin{array}{l}- \\
- \\
-\end{array}$ \\
\hline THORIUN-230 & $01 / 29 / 91$ & 0001 & $\mathrm{PCI} / \mathrm{L}$ & & 0.0 & $\mathbf{L}$ & 1. & 0.2 \\
\hline TIN & $\begin{array}{l}06 / 25 / 89 \\
12 / 01 / 89 \\
06 / 25 / 90 \\
01 / 29 / 91\end{array}$ & $\begin{array}{l}0001 \\
0001 \\
0001 \\
0001\end{array}$ & MG/L & $\begin{array}{l}< \\
< \\
<\end{array}$ & $\begin{array}{l}0.005 \\
0.005 \\
0.005 \\
0.005\end{array}$ & $\begin{array}{l}L \\
L \\
L \\
L\end{array}$ & $\begin{array}{l}0.005 \\
0.005 \\
0.005 \\
0.005\end{array}$ & $\begin{array}{l}- \\
- \\
-\end{array}$ \\
\hline TOTAL DISSOLVED SOLIDS & $\begin{array}{l}04 / 21 / 88 \\
06 / 25 / 89 \\
12 / 01 / 89 \\
06 / 25 / 90 \\
01 / 29 / 91 \\
02 / 01 / 93 \\
04 / 25 / 94 \\
12 / 13 / 94\end{array}$ & $\begin{array}{l}0001 \\
0001 \\
0001 \\
0001 \\
0001 \\
0001 \\
0001 \\
0001\end{array}$ & MG/L & & $\begin{array}{l}281 . \\
285 . \\
296 . \\
268 . \\
260 . \\
280 \\
280 \\
266\end{array}$ & $\begin{array}{l}L \\
L \\
L \\
L \\
L \\
L \\
F\end{array}$ & $\begin{array}{l}10 . \\
10 . \\
10 . \\
10 . \\
10 . \\
10 \\
10 \\
10\end{array}$ & $\begin{array}{l}- \\
- \\
- \\
- \\
-\end{array}$ \\
\hline TOTAL DISSOLVED SOLIDS (TOTAL) & $06 / 27 / 93$ & NoO1 & $M G / L$ & & 280 & G & 10 & - \\
\hline TOTAL ORGAMIC CARBON & $\begin{array}{l}04 / 21 / 88 \\
01 / 29 / 91\end{array}$ & $\begin{array}{l}0001 \\
0001\end{array}$ & MG/L & $<$ & $\begin{array}{c}55.7 \\
1 .\end{array}$ & $\stackrel{L}{J L}$ & 1. & - \\
\hline TURBIDITY & $12 / 13 / 94$ & 4001 & NTU & $<$ & 1 & $\mathbf{f}$ & 1 & - \\
\hline URAKIUN & $\begin{array}{l}04 / 21 / 88 \\
06 / 25 / 89 \\
12 / 01 / 89 \\
06 / 25 / 90 \\
01 / 29 / 91 \\
02 / 01 / 93 \\
06 / 27 / 93 \\
04 / 25 / 94 \\
12 / 13 / 94\end{array}$ & $\begin{array}{l}0001 \\
0001 \\
0001 \\
0001 \\
0001 \\
0001 \\
0001 \\
0001 \\
0001\end{array}$ & MG/L & $\begin{array}{l}< \\
< \\
< \\
< \\
< \\
<\end{array}$ & $\begin{array}{l}0.0005 \\
0.0003 \\
0.021 \\
0.0004 \\
0.0003 \\
0.001 \\
0.001 \\
0.001 \\
0.001\end{array}$ & $\begin{array}{l}J L \\
J L \\
L \\
J L \\
L \\
G \\
L \\
F\end{array}$ & $\begin{array}{l}0.003 \\
0.003 \\
0.003 \\
0.003 \\
0.0003 \\
0.001 \\
0.001 \\
0.001 \\
0.001\end{array}$ & $\begin{array}{l}- \\
- \\
- \\
- \\
-\end{array}$ \\
\hline
\end{tabular}

PARAMETER VALUE INDICATOR (PVI): < - LESS THAN DETECTION LIMIT

SAMPLE ID COOES:

0001 - FILTERED SAMPLE (.45 MICRONS)

OTHER PARAMETER VALUE FLAGS:

HOO1 - UHFILTERED SAMPLE

F - LON-FLON SAMPLIHG/DEDICATED PUMP

$G$ - PH > 9, POSSIBLE GROUT CONTAMIHATIOH

J - estimated VALUE

- $L$ - LESS THAH THREE BORE VOLLMES REMOVED BEFORE SAMPLING 
GROUNDHTER QUALITY DATA BY LOCATION

SOUP ReV. 0 Data

SITE: MONOI MOHLHENT VALLEY

LOCATIOH: 0660

MORTH COORDINATE: $\quad 61303.4$ FT

EAST COORDINATE: 89584.3 FT

$04 / 21 / 88$ TO $12 / 13 / 94$

REPORT DATE: 06/20/95

FORHATION OF COMPLETION: SHIMARLMP MEMBER OF THE CHINLE FORMATION (SR) HYDRAULIC FLOW RELATIOHSHIP: DOWN GRADIENT (D)

\begin{tabular}{|c|c|c|c|c|c|c|c|c|}
\hline PARNAETER MAME & LOG DATE & $\underset{10}{\text { SAMPLE }}$ & $\begin{array}{l}\text { UNITS OF } \\
\text { MEASURE }\end{array}$ & PVI & $\begin{array}{l}\text { PARAMETER } \\
\text { VALUE }\end{array}$ & FLAGS & $\begin{array}{l}\text { DETECTION } \\
\text { LIMIT }\end{array}$ & $\begin{array}{l}\text { PARAMETER } \\
\text { UHCERTAIHTY }\end{array}$ \\
\hline URAHIUA (TOTAL) & $\begin{array}{l}06 / 27 / 93 \\
04 / 25 / 94\end{array}$ & $\begin{array}{l}\text { No01 } \\
\text { N001 }\end{array}$ & MG/L & $<$ & $\begin{array}{l}0.001 \\
0.001\end{array}$ & $\begin{array}{l}G \\
1\end{array}$ & $\begin{array}{l}0.001 \\
0.001\end{array}$ & - \\
\hline VANADIUY & $\begin{array}{l}06 / 25 / 89 \\
12 / 01 / 89 \\
06 / 25 / 90 \\
01 / 29 / 91 \\
06 / 27 / 93 \\
04 / 25 / 94 \\
12 / 43 / 94\end{array}$ & $\begin{array}{l}0001 \\
0001 \\
0001 \\
0001 \\
0001 \\
0001 \\
0001\end{array}$ & MG/L & $\begin{array}{l}< \\
< \\
< \\
< \\
< \\
<\end{array}$ & $\begin{array}{l}0.01 \\
0.01 \\
0.01 \\
0.01 \\
0.01 \\
0.01 \\
0.01\end{array}$ & $\begin{array}{l}L \\
L \\
L \\
L \\
G \\
L \\
F\end{array}$ & $\begin{array}{l}0.01 \\
0.01 \\
0.01 \\
0.01 \\
0.01 \\
0.01 \\
0.01\end{array}$ & : \\
\hline VANADIU: (TOTAL) & $\begin{array}{l}06 / 27 / 93 \\
04 / 25 / 94\end{array}$ & $\begin{array}{l}\text { No01 } \\
\text { N001 }\end{array}$ & MG/L & $<$ & $\begin{array}{l}0.01 \\
0.01\end{array}$ & $\begin{array}{l}G \\
\text { L. }\end{array}$ & $\begin{array}{l}0.01 \\
0.01\end{array}$ & - \\
\hline ZINC & $\begin{array}{l}06 / 25 / 89 \\
12 / 01 / 89 \\
06 / 25 / 90 \\
01 / 29 / 91 \\
06 / 27 / 93\end{array}$ & $\begin{array}{l}0001 \\
0001 \\
0001 \\
0001 \\
0001\end{array}$ & MG/L & $\begin{array}{l}< \\
< \\
<\end{array}$ & $\begin{array}{l}0.005 \\
0.005 \\
0.006 \\
0.005 \\
0.005\end{array}$ & $\begin{array}{l}L \\
L \\
L \\
L \\
G J\end{array}$ & $\begin{array}{l}0.005 \\
0.005 \\
0.005 \\
0.005 \\
0.005\end{array}$ & $:$ \\
\hline ZINC (TOTAL) & $06 / 27 / 93$ & N001 & $M G / L$ & & 0.005 & GJ & 0.005 & - \\
\hline
\end{tabular}

PARAMETER VALUE IHDICATOR (PVI): < - LESS THAN DETECTION LIMIT

SAMPLE ID COOES:

OTHER PARAMETER VALUE FLAGS:

0001 - FILTERED SAMPLE (.45 MICRONS)

F - LON-FLON SAMPLING/DEDICATED PUMP

NOO1 - UNFILTERED SAMPLE

G - PH > 9, POSSIBLE GROUT CONTAMIHATION

J. ESTIMATED VALUE

L - LESS THAN THREE BORE VOLUMES REMOVED BEFORE SAMPLIHG 
GROUNDWATER QUALITY DATA BY LOCATION

SOWP Rev. O Data

SITE: MONOI MONUWENT VALLEY

LOCATION: 0662

NORTH COORDINATE: 59236.5 FT

EAST COORDIMATE: 87577.5 FT

$04 / 21 / 88$ TO $12 / 13 / 94$

REPORT DATE: 06/20/95

FORMATION OF COMPLETION: ALLUVIUH (AL)

HYORAULIC FLON RELATIOHSHIP: DOWN GRADIENT (D)

\begin{tabular}{|c|c|c|c|c|c|c|c|}
\hline PARAMETER HAHE & LOG DATE & $\underset{10}{\text { SAMPLE }}$ & $\begin{array}{l}\text { UNITS OF } \\
\text { MEASURE }\end{array}$ & PVI & $\begin{array}{l}\text { PARAMETER } \\
\text { VALUE FLAGS }\end{array}$ & $\begin{array}{l}\text { DETECTION } \\
\text { LIMIT }\end{array}$ & $\begin{array}{l}\text { PARAMETER } \\
\text { UHCERTAINTY }\end{array}$ \\
\hline ALKALIHITY & $\begin{array}{l}06 / 25 / 89 \\
06 / 05 / 91 \\
02 / 26 / 92 \\
11 / 23 / 92 \\
02 / 18 / 93 \\
06 / 29 / 93 \\
12 / 15 / 93 \\
04 / 23 / 94\end{array}$ & $\begin{array}{l}0001 \\
0001 \\
0001 \\
0001 \\
\text { O001 } \\
\text { N001 } \\
\text { N001 } \\
\text { N001 }\end{array}$ & MG/L CACO3 & & $\begin{array}{l}396 . \\
353 . \\
343 \\
325 \\
322 \\
292 \\
592 \\
267\end{array}$ & $\begin{array}{l}- \\
- \\
- \\
- \\
-\end{array}$ & : \\
\hline ALUMIHUM & $\begin{array}{l}06 / 25 / 89 \\
06 / 05 / 91 \\
02 / 26 / 92\end{array}$ & $\begin{array}{l}0001 \\
0001 \\
0001\end{array}$ & MG/L & $\begin{array}{l}< \\
< \\
<\end{array}$ & $\begin{array}{l}0.1 \\
0.05 \\
0.05\end{array}$ & $\begin{array}{l}0.1 \\
0.05 \\
0.05\end{array}$ & : \\
\hline AнтонIU⿴囗十⺝ & $\begin{array}{l}06 / 25 / 89 \\
06 / 05 / 91 \\
02 / 26 / 92 \\
12 / 15 / 93\end{array}$ & $\begin{array}{l}0001 \\
0001 \\
0001 \\
0001\end{array}$ & MG/L & $\begin{array}{l}< \\
<\end{array}$ & $\begin{array}{l}0.1 \\
0.1 \\
0.1 \\
0.1\end{array}$ & $\begin{array}{l}0.1 \\
0.1 \\
0.1 \\
0.1\end{array}$ & $i$ \\
\hline AMONIUM (TOTAL) & $\begin{array}{l}06 / 29 / 93 \\
04 / 23 / 94\end{array}$ & $\begin{array}{l}\text { NOOI } \\
\text { HOOI }\end{array}$ & MG/L & $<$ & $\begin{array}{l}0.1 \\
0.1\end{array}$ & $\begin{array}{l}0.1 \\
0.1\end{array}$ & : \\
\hline AKT IMONY & $\begin{array}{l}06 / 25 / 89 \\
06 / 05 / 91 \\
02 / 26 / 92\end{array}$ & $\begin{array}{l}0001 \\
0001 \\
0001\end{array}$ & MG/L & $\begin{array}{l}< \\
< \\
<\end{array}$ & $\begin{array}{l}0.003 \\
0.003 \\
0.003\end{array}$ & $\begin{array}{l}0.003 \\
0.003 \\
0.003\end{array}$ & : \\
\hline ARSENIC & $\begin{array}{l}06 / 25 / 89 \\
06 / 05 / 91 \\
02 / 26 / 92 \\
11 / 23 / 92 \\
06 / 29 / 93\end{array}$ & $\begin{array}{l}0001 \\
0001 \\
0001 \\
0001 \\
0001\end{array}$ & $M G / L$ & $\begin{array}{l}< \\
< \\
< \\
< \\
<\end{array}$ & $\begin{array}{l}0.01 \\
0.01 \\
0.01 \\
0.0015 \\
0.005\end{array}$ & $\begin{array}{l}0.01 \\
0.01 \\
0.01 \\
0.0015 \\
0.005\end{array}$ & $\begin{array}{l}- \\
- \\
-\end{array}$ \\
\hline ARSEKIC (TOTAL) & $\begin{array}{l}11 / 23 / 92 \\
06 / 29 / 93\end{array}$ & $\begin{array}{l}\text { NOOI } \\
\text { HOOI }\end{array}$ & MG/L & $<$ & $\begin{array}{l}0.0015 \\
0.005\end{array}$ & $\begin{array}{l}0.0015 \\
0.005\end{array}$ & - \\
\hline BARIUY & $\begin{array}{l}06 / 25 / 89 \\
06 / 05 / 91 \\
02 / 26 / 92 \\
11 / 23 / 92 \\
06 / 29 / 93\end{array}$ & $\begin{array}{l}0001 \\
0001 \\
0001 \\
0001 \\
0001\end{array}$ & $M G / L$ & $\begin{array}{l}< \\
<\end{array}$ & $\begin{array}{l}0.1 \\
0.03 \\
0.02 \\
0.023 \\
0.1\end{array}$ & $\begin{array}{l}0.1 \\
0.01 \\
0.01 \\
0.0015 \\
0.1\end{array}$ & $\begin{array}{l}- \\
- \\
- \\
-\end{array}$ \\
\hline BARIUM (TOTAL) & $\begin{array}{l}11 / 23 / 92 \\
06 / 29 / 93\end{array}$ & $\begin{array}{l}\text { NOOI } \\
\text { NOOI }\end{array}$ & $M G / L$ & $<$ & $\begin{array}{l}0.051 \\
0.1\end{array}$ & $\begin{array}{l}0.0015 \\
0.1\end{array}$ & - \\
\hline BERYLLIUA & $\begin{array}{l}06 / 25 / 89 \\
06 / 05 / 91\end{array}$ & $\begin{array}{l}0001 \\
0001\end{array}$ & $M G / L$ & $<$ & $\begin{array}{l}0.01 \\
0.005\end{array}$ & $\begin{array}{l}-0.01 \\
0.005\end{array}$ & - \\
\hline BORON & $\begin{array}{l}06 / 25 / 89 \\
06 / 05 / 91\end{array}$ & $\begin{array}{l}0001 \\
0001\end{array}$ & $M G / L$ & $<$ & $\begin{array}{l}0.1 \\
0.05\end{array}$ & $\begin{array}{l}0.1 \\
0.05\end{array}$ & - \\
\hline BROAIDE & $06 / 05 / 91$ & 0001 & MG/L & & 0.2 & 0.1 & - \\
\hline CADHIUM & $\begin{array}{l}06 / 25 / 89 \\
06 / 05 / 91 \\
02 / 26 / 92 \\
11 / 23 / 92\end{array}$ & $\begin{array}{l}0001 \\
0001 \\
0001 \\
0001\end{array}$ & $M G / L$ & $\begin{array}{l}< \\
< \\
< \\
<\end{array}$ & $\begin{array}{l}0.001 \\
0.001 \\
0.001 \\
0.00013\end{array}$ & $\begin{array}{l}0.001 \\
0.001 \\
0.001 \\
0.00013\end{array}$ & :- \\
\hline
\end{tabular}

PARAMETER VALUE INDICATOR (PVI): < - LESS THAN DETECTION LIHIT

SAMPLE ID COOES:

OTHER PARAMETER VALUE FLAGS:

0009 - FILTERED SAHPLE (.45 MICRONS)

J - ESTIMATED VALUE

NOOI - UMFILTERED SAMPLE

L - LESS THAH THREE BORE VOLUMES REHOVED BEFORE SAMPLING 
GROUNDWATER QUALITY DATA BY LOCATION

SOWP Rev. O Data

SITE: HONO1 MOHUMENT VALLEY

LOCATION: 0662

NORTH COORDINATE: 59236.5 FT

EAST COORDINATE: 87577.5 FT

04/21/88 TO 12/13/94

REPORT DATE: 06/20/95

FORHATION OF COMPLETIOH: ALLUVIUM (AL)

HYDRAULIC FLOW RELATIOASHIP: DOWN GRADIEKT (D)

\begin{tabular}{|c|c|c|c|c|c|c|c|}
\hline PARAMETER NAME & LOS DATE & $\begin{array}{c}\text { SAMPLE } \\
10\end{array}$ & $\begin{array}{l}\text { UNITS OF } \\
\text { MEASURE }\end{array}$ & PVI & $\begin{array}{l}\text { PARAMETER } \\
\text { VALUE FLAGS }\end{array}$ & $\begin{array}{l}\text { DETECTION } \\
\text { LIMIT }\end{array}$ & $\begin{array}{l}\text { PARAMETER } \\
\text { UHCERTAIHTY }\end{array}$ \\
\hline CADMIUA (TOTAL) & $11 / 23 / 92$ & No01 & $M G / L$ & $<$ & $0.00013 L$ & 0.00013 & - \\
\hline CALCILA & $\begin{array}{l}06 / 25 / 89 \\
06 / 05 / 91 \\
02 / 26 / 92 \\
02 / 18 / 93 \\
06 / 29 / 93 \\
12 / 15 / 93 \\
04 / 23 / 94\end{array}$ & $\begin{array}{l}0001 \\
0001 \\
0001 \\
0001 \\
0001 \\
0001 \\
0001\end{array}$ & $M G / L$ & \multicolumn{2}{|r|}{$\begin{array}{l}143 . \\
167 . \\
153 . \\
143 \\
173 \\
171 \\
186\end{array}$} & $\begin{array}{l}0.01 \\
0.5 \\
0.5 \\
0.5 \\
0.5 \\
0.5 \\
0.5\end{array}$ & $\begin{array}{l}- \\
- \\
- \\
- \\
-\end{array}$ \\
\hline CALCIUA (TOTAL) & $\begin{array}{l}06 / 29 / 93 \\
12 / 15 / 93 \\
04 / 23 / 94\end{array}$ & $\begin{array}{l}\text { NoO1 } \\
\text { NOO1 } \\
\text { NO01 }\end{array}$ & $M G / L$ & \multicolumn{2}{|r|}{$\begin{array}{l}166 \\
395 \\
200\end{array}$} & $\begin{array}{l}0.5 \\
0.5 \\
0.5\end{array}$ & - \\
\hline CHLORIDE & $\begin{array}{l}06 / 25 / 89 \\
06 / 05 / 91 \\
02 / 26 / 92 \\
02 / 18 / 93 \\
12 / 15 / 93 \\
04 / 23 / 94\end{array}$ & $\begin{array}{l}0001 \\
0001 \\
0001 \\
0001 \\
0001 \\
0001\end{array}$ & $M G / L$ & \multicolumn{2}{|r|}{$\begin{array}{l}20 . \\
17.4 \\
16.8 \\
18.3 \\
14.8 \\
14.6\end{array}$} & $\begin{array}{l}1 . \\
0.5 \\
0.5 \\
0.5 \\
0.5 \\
0.5\end{array}$ & $\begin{array}{l}- \\
- \\
- \\
- \\
-\end{array}$ \\
\hline CHLORIDE (TOTAL) & $06 / 29 / 93$ & NOOI & $M G / L$ & \multicolumn{2}{|r|}{18.2} & 0.5 & - \\
\hline CHROMIUM & $\begin{array}{l}06 / 25 / 89 \\
06 / 05 / 91 \\
02 / 26 / 92 \\
19 / 23 / 92\end{array}$ & $\begin{array}{l}0001 \\
0001 \\
0001 \\
0001\end{array}$ & MG/L & $\begin{array}{l}< \\
< \\
< \\
<\end{array}$ & $\begin{array}{l}0.01 \\
0.01 \\
0.01 \\
0.0053\end{array}$ & $\begin{array}{l}0.01 \\
0.01 \\
0.01 \\
0.0053\end{array}$ & $\begin{array}{l}- \\
- \\
- \\
-\end{array}$ \\
\hline CHROHIUM (TOTAL) & $19 / 23 / 92$ & No01 & $M G / L$ & $<$ & 0.0053 & 0.0053 & - \\
\hline COBALT & $\begin{array}{l}06 / 25 / 89 \\
06 / 05 / 91\end{array}$ & $\begin{array}{l}0001 \\
0001\end{array}$ & $M G / L$ & $<$ & $\begin{array}{l}0.05 \\
0.03\end{array}$ & $\begin{array}{l}0.05 \\
0.03\end{array}$ & $\dot{-}$ \\
\hline COPPER & $\begin{array}{l}06 / 25 / 89 \\
06 / 05 / 91\end{array}$ & $\begin{array}{l}0001 \\
0001\end{array}$ & MG/L & $<$ & $\begin{array}{l}0.02 \\
0.01\end{array}$ & $\begin{array}{l}0.02 \\
0.01\end{array}$ & - \\
\hline CYANIDE & $06 / 25 / 89$ & 0001 & MG/L & $<$ & 0.01 & 0.01 & - \\
\hline DISSOLVED OXYGEN & $\begin{array}{l}11 / 23 / 92 \\
06 / 29 / 93 \\
04 / 23 / 94\end{array}$ & $\begin{array}{l}0001 \\
\text { N001 } \\
\text { N001 }\end{array}$ & MG/L & & $\begin{array}{l}9.2 \\
8.0 \\
8.6\end{array}$ & - & $\begin{array}{l}- \\
- \\
-\end{array}$ \\
\hline FLUORIDE & $\begin{array}{l}06 / 25 / 89 \\
06 / 05 / 91\end{array}$ & $\begin{array}{l}0001 \\
0001\end{array}$ & MG/L & $\begin{array}{l}< \\
<\end{array}$ & $\begin{array}{l}0.1 \\
0.1\end{array}$ & $\begin{array}{l}0.1 \\
0.1\end{array}$ & - \\
\hline GROSS ALPHA & $\begin{array}{l}06 / 05 / 91 \\
02 / 26 / 92 \\
11 / 23 / 92 \\
02 / 18 / 93 \\
06 / 29 / 93\end{array}$ & $\begin{array}{l}0001 \\
0001 \\
0001 \\
0001 \\
0001\end{array}$ & PCI/L & & $\begin{array}{c}8.5 \\
27.3 \\
8.8 \\
0 \\
4\end{array}$ & $\begin{array}{c}1 . \\
1.0 \\
6.4 \\
16 \\
3\end{array}$ & $\begin{array}{c}12.2 \\
15.2 \\
5.6 \\
17 \\
5\end{array}$ \\
\hline GROSS ALPHA (TOTAL) & $\begin{array}{l}11 / 23 / 92 \\
06 / 29 / 93\end{array}$ & $\begin{array}{l}\text { No01 } \\
\text { No01 }\end{array}$ & $\mathrm{PCl} / \mathrm{L}$ & & $0.0 \quad N$ & $\begin{array}{c}14.0 \\
4\end{array}$ & $\begin{array}{l}7.5 \\
7\end{array}$ \\
\hline GROSS BETA & $06 / 05 / 91$ & 0001 & $\mathrm{PCI} / \mathrm{L}$ & & 9.7 & 0.5 & 6.7 \\
\hline
\end{tabular}

PARAMETER VALUE INDICATOR (PVI): < - LESS THAN DETECTION LIMIT

SAMPLE ID COOES:

0001 - FILTERED SAMPLE (.45 MICRONS)

OTHER PARAMETER VALUE FLAGS:

NOO1 - UNFILTERED SAMPLE

L - LESS THAH THREE BORE VOLUMES REMOVED BEFORE SAMPLIMG

N - SPIKE SHMLE RECOVERY NOT HITHIN CONTROL LIHITS 
GROUKDWATER QUALITY DATA BY LOCATION

SOUP ReV. O Data

SITE: MONOI MONLMENT VALLEY

LOCATIOH: 0662

NORTH COORDINATE: 59236.5 FT

EAST COORDINATE: 87577.5 FT

$04 / 21 / 88$ TO $12 / 13 / 94$

REPORT DATE: O6/20/95

FORMATIOH OF COAPLETIOH: ALLUVIUM (AL)

HYDRAULIC FLOH RELATIOHSHIP: DOWN GRADIENT (D)

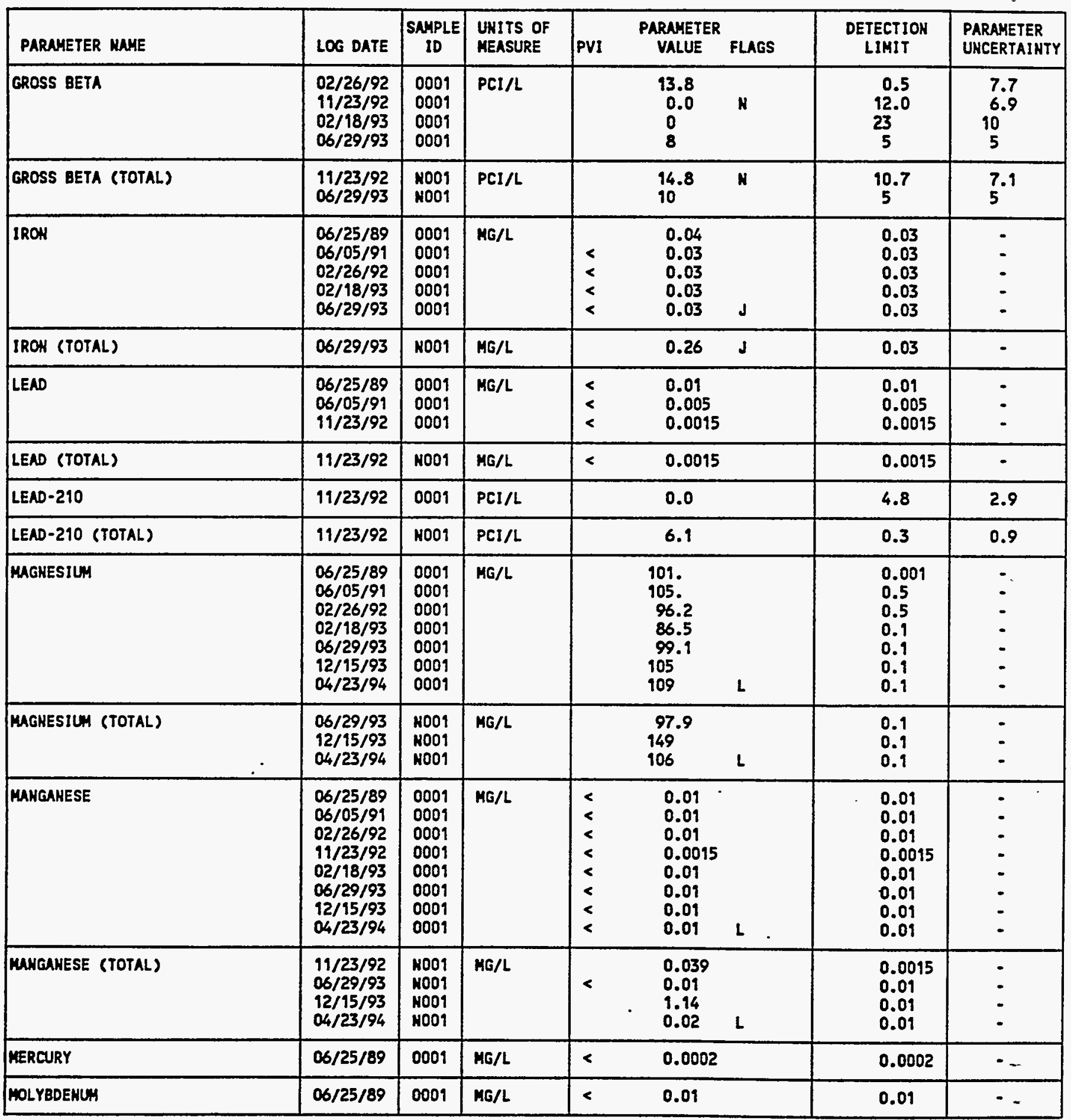

PARAMETER VALUE IMDICATOR (PVI): < - LESS THAN DETECTIOA LIMIT

OTHER PARAMETER VALUE FLAGS:

SAMPLE ID COOES:

0001 - FILTERED SNMPLE (.45 MICROHS)

J- ESTIMUTED VALUE

NDO1 - UMFILTERED SAMPLE

L - LESS THAH THREE BORE VOLUHES REMOVED BEFORE SAMPLING

H - SPIKE SAMPLE RECOVERY NOT MITHIN CONTROL LIMITS 
GROWNDATER QUALITY DATA BY LOCATION

SOMP Rev. O Data

SITE: MONOI MONUMENT VALLEY

LOCATION: 0662

MORTH COORDIHATE: $\quad 59236.5$ FT

EAST COOROINATE: 87577.5 FT

$04 / 21 / 88$ TO $12 / 13 / 94$

REPORT DATE: $06 / 20 / 95$

FORMATION OF COMPLETION: ALLUVIUM (AL)

HYDRAULIC FLON RELATIONSHIP: DONN GRADIEHT (D)

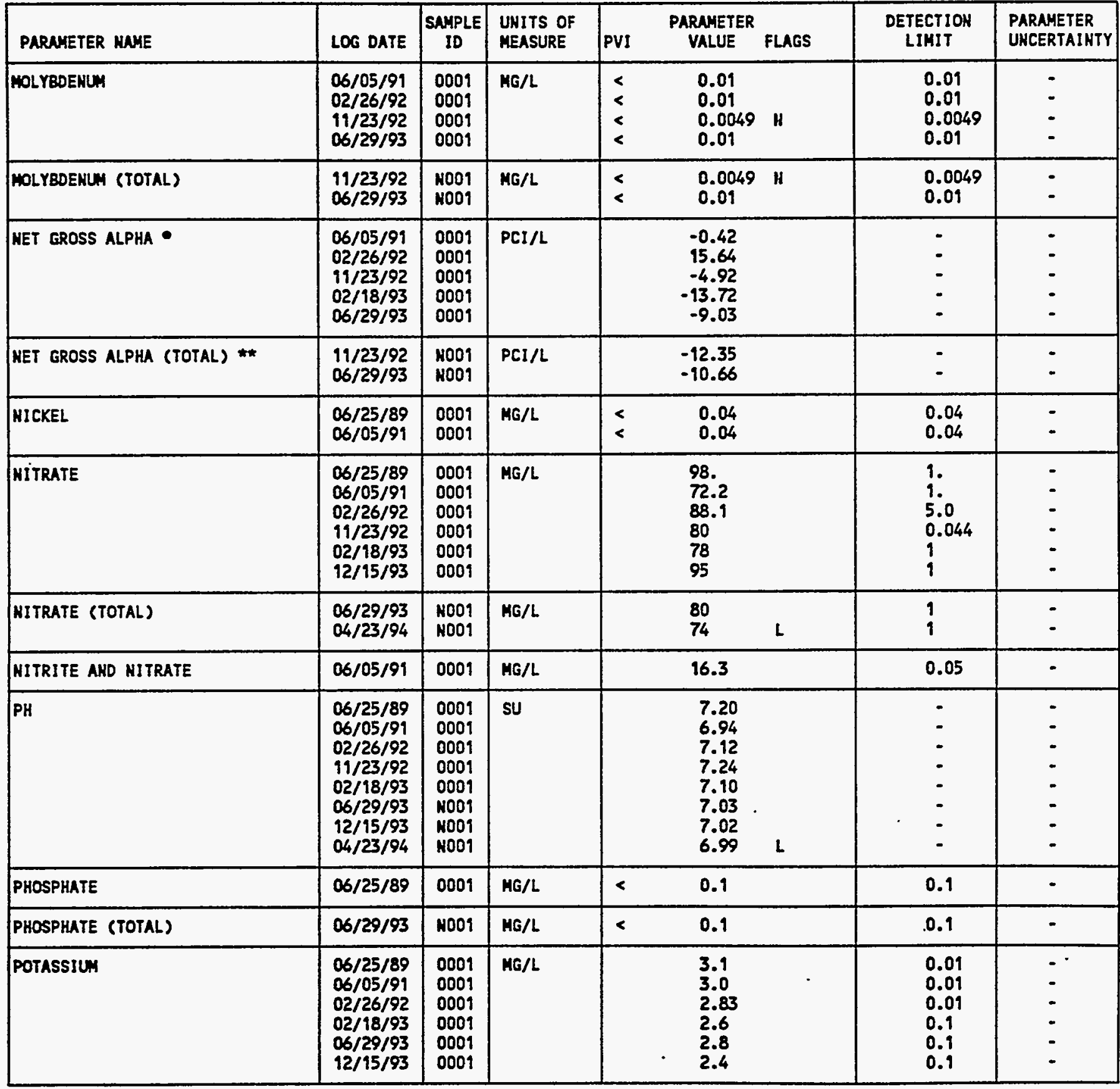

- het gROSS ALPHA (GROSS ALPHA - URANIUH) WITH 1 MG URAHIUM = 686 PCI

* MET GROSS ALPHA (TOTAL) (TOTAL GROSS ALPHA - TOTAL URANIUM)

PARAMETER VALUE IMDICATOR (PVI): < - LESS THAN DETECTION LIMIT

OTHER PARAMETER VALUE FLAGS:

$L$ - LESS THAN THREE BORE VDLLUES REMOVED BEFORE SAMPLING

N - SPIKE SNMPLE RECOVERY NOT WITHIN CONTROL LIMITS
SNMPLE ID CODES:

OOO1 - FILTERED SNMPLE (.45 MICRONS)

NOOI - UNFILTERED SNMPLE 
GROUHDWATER QUALITY DATA BY LOCATIOH

SOUP Rev. 0 Data

SITE: MONO1 MONUMENT VALLEY

LOCATION: 0662

NORTH COORDIHATE: $\quad 59236.5$ FT

EAST COORDINATE: 87577.5 FT

04/21/88 TO $12 / 13 / 94$

REPORT DATE: 06/20/95

FORHATION OF COMPLETIOH: ALLUVIUM (AL)

HYORAULIC FLON RELATIOHSHIP: DOWN GRADIEHT (D)

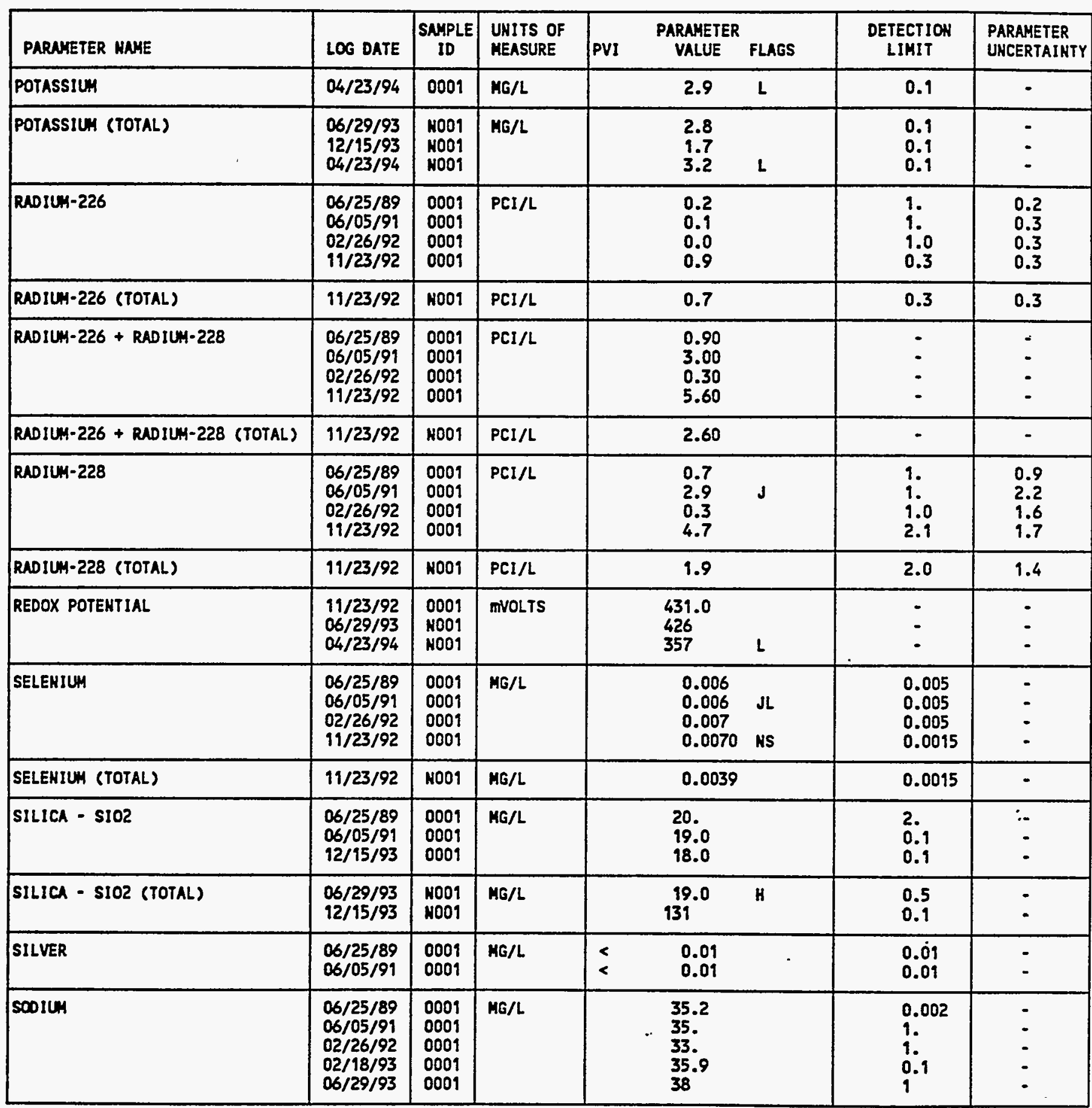

PARAMTER VALUE IMDICATOR (PVI): < - LESS THAN DETECTION LIMIT

SNMPLE ID COOES:

OTHER PARAMETER VALUE FLAGS:

0001 - FILTERED SAMPLE (.45 MICROHS)

H - HOLD TIHE EXPIRED, VALUE SUSPECT

NOO1 - UHFILTERED SAMPLE

- ESTIMATED VALUE

L - LESS THAN THREE BORE VOLUMES REMOVED BEFORE SAMPLIHG

H - SPIKE SAMPLE RECOVERY NOT WITHIN CONTROL LIMITS

S - REPORTED VALUE DETERMINED USING METHOO OF STD ADDITION (MSA) 
GROUNDWATER QUALITY DATA BY LOCATIOH

SOMP Rev. O Data

SITE: HOHO 9 HONUMENT VALLEY

LOCATION: 0662

NORTH COORDINATE: $\quad 59236.5$ FT

EAST COORDINATE: 87577.5 FT

04/21/88 TO $12 / 13 / 94$

REPORT DATE: 06/20/95

FORMATIOH OF COMPLETION: ALLUVIUH (AL)

HYDRAULIC FLON RELATIONSHIP: DOWN GRADIENT (D)

\begin{tabular}{|c|c|c|c|c|c|c|c|c|}
\hline PARAMETER MAME & LOG DATE & $\underset{\text { SN }}{\text { SAMPLE }}$ & $\begin{array}{l}\text { UMITS OF } \\
\text { MEASURE }\end{array}$ & PVI & $\begin{array}{l}\text { PARAMETEF } \\
\text { VALUE }\end{array}$ & FLAGS & $\begin{array}{l}\text { DETECTIOH } \\
\text { LIMIT }\end{array}$ & $\begin{array}{l}\text { PARAMETER } \\
\text { UHCERTAINTY }\end{array}$ \\
\hline SOOIU: & $\begin{array}{l}12 / 15 / 93 \\
04 / 23 / 94\end{array}$ & $\begin{array}{l}0001 \\
0001\end{array}$ & MG/L & & $\begin{array}{l}36 \\
38\end{array}$ & $\mathbf{L}$ & $\begin{array}{l}1 \\
1\end{array}$ & $\because$ \\
\hline SODILH (TOTAL) & $\begin{array}{l}06 / 29 / 93 \\
12 / 15 / 93 \\
04 / 23 / 94\end{array}$ & $\begin{array}{l}\text { NOO1 } \\
\text { N001 } \\
\text { NO01 }\end{array}$ & $M G / L$ & & $\begin{array}{l}39 \\
39 \\
35\end{array}$ & $\mathbf{L}$ & $\begin{array}{l}1 \\
1 \\
1\end{array}$ & $:$ \\
\hline SPECIFIC CONDUCTANCE & $\begin{array}{l}06 / 25 / 89 \\
06 / 05 / 91 \\
02 / 26 / 92 \\
11 / 23 / 92 \\
02 / 18 / 93 \\
06 / 29 / 93 \\
12 / 15 / 93 \\
04 / 23 / 94\end{array}$ & $\begin{array}{l}0001 \\
0001 \\
0001 \\
0001 \\
0009 \\
\text { NOO1 } \\
\text { N001 } \\
\text { NOOI }\end{array}$ & LMHO/CH & & $\begin{array}{l}920 . \\
1450 . \\
1182 \\
1095 \\
1161 \\
1420 \\
1346 \\
1465\end{array}$ & $\mathbf{L}$ & $\begin{array}{l}- \\
- \\
- \\
- \\
- \\
-\end{array}$ & $\begin{array}{l}: \\
: \\
: \\
: \\
-\end{array}$ \\
\hline STRONTIUH & $\begin{array}{l}06 / 05 / 91 \\
02 / 26 / 92 \\
11 / 23 / 92 \\
06 / 29 / 93 \\
12 / 15 / 93 \\
04 / 23 / 94\end{array}$ & $\begin{array}{l}0001 \\
0001 \\
0001 \\
0001 \\
0001 \\
0001\end{array}$ & MG/L & & $\begin{array}{l}1.00 \\
0.92 \\
1.1 \\
1.10 \\
1.16 \\
1.21\end{array}$ & $\mathbf{L}$ & $\begin{array}{l}0.01 \\
0.01 \\
0.00035 \\
0.01 \\
0.01 \\
0.01\end{array}$ & : \\
\hline STRONTIUN (TOTAL) & $\begin{array}{l}19 / 23 / 92 \\
06 / 29 / 93 \\
12 / 15 / 93 \\
04 / 23 / 94\end{array}$ & $\begin{array}{l}\text { No01 } \\
\text { N001 } \\
\text { N001 } \\
\text { N001 }\end{array}$ & MG/L & & $\begin{array}{l}1.1 \\
1.07 \\
2.01 \\
1.11\end{array}$ & $\mathbf{L}$ & $\begin{array}{l}0.00035 \\
0.01 \\
0.01 \\
0.01\end{array}$ & : \\
\hline SULFATE & $\begin{array}{l}06 / 25 / 89 \\
06 / 05 / 99 \\
02 / 26 / 92 \\
11 / 23 / 92 \\
02 / 18 / 93 \\
12 / 15 / 93 \\
04 / 23 / 94\end{array}$ & $\begin{array}{l}0001 \\
0001 \\
0001 \\
0001 \\
0009 \\
0001 \\
0001\end{array}$ & MG/L & & $\begin{array}{l}418 . \\
356 . \\
404 . \\
420 \\
412 \\
584 \\
635\end{array}$ & $\mathbf{L}$ & $\begin{array}{c}0.1 \\
0.1 \\
40 . \\
1.0 \\
1 \\
1 \\
1\end{array}$ & $\begin{array}{l}- \\
- \\
- \\
- \\
-\end{array}$ \\
\hline SULFATE (TOTAL) & $\begin{array}{l}11 / 23 / 92 \\
06 / 29 / 93\end{array}$ & $\begin{array}{l}\text { N001 } \\
\text { H0O1 }\end{array}$ & $M G / L$ & & $\begin{array}{l}380 \\
524\end{array}$ & & 1.0 & $\dot{-}$ \\
\hline SULFIDE & $\begin{array}{l}06 / 25 / 89 \\
06 / 05 / 91\end{array}$ & $\begin{array}{l}0001 \\
0001\end{array}$ & MG/L & $<$ & $\begin{array}{r}0.1 \\
11.7\end{array}$ & $J$ & $\begin{array}{l}0.1 \\
1 .\end{array}$ & $\dot{-}$ \\
\hline TEHPERATURE & $\begin{array}{l}06 / 25 / 89 \\
06 / 05 / 99 \\
02 / 26 / 92 \\
19 / 23 / 92 \\
02 / 18 / 93 \\
06 / 29 / 93 \\
12 / 15 / 93 \\
04 / 23 / 94\end{array}$ & $\begin{array}{l}0001 \\
0001 \\
0009 \\
0001 \\
0009 \\
\text { N001 } \\
\text { N009 } \\
\text { N001 }\end{array}$ & C - DEGREE & & $\begin{array}{l}17.8 \\
12.3 \\
15.1 \\
14.3 \\
15.5 \\
22.2 \\
15.5 \\
18.2\end{array}$ & $\mathbf{L}$ & $\begin{array}{l}- \\
: \\
: \\
-\end{array}$ & $\begin{array}{l}: \\
: \\
: \\
: \\
-\end{array}$ \\
\hline THALLIUH & $\begin{array}{l}06 / 25 / 89 \\
06 / 05 / 91\end{array}$ & $\begin{array}{l}0001 \\
0001\end{array}$ & $M G / L$ & $<$ & $\begin{array}{l}0.01 \\
0.01\end{array}$ & & $\begin{array}{l}0.01 \\
0.01\end{array}$ & $\dot{-}$ \\
\hline THORILH-230 & $06 / 05 / 99$ & 0001 & PCI/L & & 0.2 & & 1. & 0.2 \\
\hline
\end{tabular}

PARAMETER VALUE IMDICATOR (PVI): < - LESS THAN DETECTIOH LIMIT

SAMPLE ID COOES:

OTHER PARAMETER VALUE FLAGS:

OOO1 - FILTERED SAMPLE (.45 MICRONS)

NOO1 - UHFILTERED SAMPLE

J - ESTIMATED VALUE

- $l$ - LESS THAN THREE BORE VOLLMES REHOVED BEFORE SAMPLING 
GROUHDHATER OUALITY DATA BY LOCATION

SOWP ReV. 0 Data

SITE: MONO1 MONUNEHT VALLEY

LOCATIOH: 0662

NORTH COORDIHATE: 59236.5 FT

EAST COORDINATE: 87577.5 FT

$04 / 21 / 88$ TO $12 / 13 / 94$

REPORT DATE: 06/20/95

FORMATION OF COMPLETION: ALLUVIUM (AL)

HYORAULIC FLON RELATIOHSHIP: DOWN GRADIENT (D)

\begin{tabular}{|c|c|c|c|c|c|c|c|c|}
\hline PARAMETER MAME & LOS DATE & ${ }_{10}^{\text {SAMPLE }}$ & $\begin{array}{l}\text { UHITS OF } \\
\text { MEASUIRE }\end{array}$ & PVI & $\begin{array}{l}\text { PARAMETER } \\
\text { VALUE }\end{array}$ & FLAGS & $\begin{array}{l}\text { DETECTION } \\
\text { LIMIT }\end{array}$ & $\begin{array}{l}\text { PARAMETER } \\
\text { UNCERTAINTY }\end{array}$ \\
\hline TIN & $\begin{array}{l}06 / 25 / 89 \\
06 / 05 / 91\end{array}$ & $\begin{array}{l}0001 \\
0001\end{array}$ & $M G / L$ & $<$ & $\begin{array}{l}0.005 \\
0.01\end{array}$ & I & $\begin{array}{l}0.005 \\
0.01\end{array}$ & 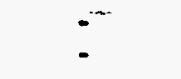 \\
\hline TOTAL DISSOLVED SOLIDS & $\begin{array}{l}06 / 25 / 89 \\
06 / 05 / 91 \\
02 / 26 / 92 \\
02 / 18 / 93 \\
12 / 15 / 93 \\
04 / 23 / 94\end{array}$ & $\begin{array}{l}0001 \\
0001 \\
0001 \\
0001 \\
0001 \\
0001\end{array}$ & MG/L & & $\begin{array}{c}1070 . \\
1070 . \\
1060 . \\
990 \\
1320 \\
1350\end{array}$ & $\mathbf{L}$ & $\begin{array}{l}10 . \\
10 . \\
10 . \\
10 \\
10 \\
10\end{array}$ & $\begin{array}{l}- \\
- \\
- \\
-\end{array}$ \\
\hline TOTAL DISSOLVED SOLIDS (TOTAL) & $06 / 29 / 93$ & N001 & $M G / L$ & & 1170 & H & 10 & - \\
\hline TOTAL ORGAHIC CARBOH & $02 / 26 / 92$ & 0001 & MG/L & $<$ & 1. & & 1. & $=$ \\
\hline TURBIDITY & $04 / 23 / 94$ & NOOI & NTU & & 36 & $\mathbf{L}$ & - & - \\
\hline URAHIUH (TOTAL) & $\begin{array}{l}11 / 23 / 92 \\
06 / 29 / 93 \\
12 / 15 / 93 \\
04 / 23 / 94\end{array}$ & $\begin{array}{l}\text { No01 } \\
\text { N001 } \\
\text { N001 } \\
\text { N001 }\end{array}$ & $M G / L$ & & $\begin{array}{l}0.018 \\
0.017 \\
0.028 \\
0.020\end{array}$ & $\mathbf{L}$ & $\begin{array}{l}0.001 \\
0.001 \\
0.001 \\
0.001\end{array}$ & $\vdots$ \\
\hline VAMADIUM & $\begin{array}{l}06 / 25 / 89 \\
06 / 05 / 91 \\
02 / 26 / 92 \\
11 / 23 / 92 \\
06 / 29 / 93 \\
12 / 15 / 93 \\
04 / 23 / 94\end{array}$ & $\begin{array}{l}0001 \\
0001 \\
0001 \\
0001 \\
0001 \\
0001 \\
0001 .\end{array}$ & MG/L & $<$ & $\begin{array}{l}0.05 \\
0.02 \\
0.01 \\
0.025 \\
0.02 \\
0.02 \\
0.02\end{array}$ & $\mathbf{L}$ & $\begin{array}{l}0.01 \\
0.01 \\
0.01 \\
0.0019 \\
0.01 \\
0.01 \\
0.01\end{array}$ & $\begin{array}{l}- \\
- \\
- \\
-\end{array}$ \\
\hline ZIMC & $\begin{array}{l}06 / 25 / 89 \\
06 / 05 / 91 \\
02 / 26 / 92 \\
11 / 23 / 92 \\
06 / 29 / 93\end{array}$ & $\begin{array}{l}0001 \\
0001 \\
0001 \\
0001 \\
0001\end{array}$ & $M G / L$ & $<$ & $\begin{array}{l}0.005 \\
0.005 \\
0.049 \\
0.0056 \\
0.053\end{array}$ & J & $\begin{array}{l}0.005 \\
0.005 \\
0.005 \\
0.0010 \\
0.005\end{array}$ & $\begin{array}{l}- \\
: \\
-\end{array}$ \\
\hline ZINC (TOTAL) & $\begin{array}{l}11 / 23 / 92 \\
06 / 29 / 93\end{array}$ & $\begin{array}{l}\text { No01 } \\
\text { N001 }\end{array}$ & MG/L & & $\begin{array}{l}0.010 \\
0.057\end{array}$ & $\mathbf{J}$ & $\begin{array}{l}0.0010 \\
0.005\end{array}$ & - \\
\hline
\end{tabular}

PARAMETER VALUE INDICATOR (PVI): < - LESS THAN DETECTION LIMIT

SUMPLE ID COOES:

OTHER PARAMETER VALUE FLAGS:

OOO1 - FILTERED SAMPLE (.45 MICRONS)

H - HOLD TIME EXPIRED, VALUE SUSPECT

NO01 - UHFILTERED SAMPLE

1 - INCREASED DETECTIOH LIMIT DUE TO REQUIRED DILUTION

d - estimated VALUE

L - LESS THAH THREE BORE VOLUAES REMOVED BEFORE SAMPLING 
GROUNDWATER OUALITY DATA BY LOCATION

SOWP Rev. O Data

SITE: MONO 9 MONLWENT VALLEY

LOCATION: 0663

MORTH COORDINATE: 59069.6 FT

EAST COORDIHATE: 88593.5 FT

$04 / 21 / 88$ TO $12 / 13 / 94$

REPORT DATE: $06 / 20 / 95$

FORMATION OF COHPLETION: DECHELLEY MEMBER OF THE CUTLER FORMATION (DC) HYDRAULIC FLOU RELATIONSHIP: DOWN GRADIENT (D)

\begin{tabular}{|c|c|c|c|c|c|c|c|c|}
\hline PARAMETER NAME & LOG DATE & $\underset{\text { SAMLE }}{\text { SAMPL }}$ & $\begin{array}{l}\text { UNITS OF } \\
\text { MEASURE }\end{array}$ & PVI & $\begin{array}{c}\text { PARAMETER } \\
\text { VALUE }\end{array}$ & FLAGS & $\begin{array}{l}\text { DETECTION } \\
\text { LIMIT }\end{array}$ & $\begin{array}{l}\text { PARAMETER } \\
\text { UNCERTAINTY }\end{array}$ \\
\hline ALKALINITY & $\begin{array}{l}04 / 26 / 88 \\
06 / 25 / 89 \\
12 / 02 / 89 \\
06 / 25 / 90 \\
01 / 29 / 91 \\
06 / 04 / 91 \\
02 / 20 / 92 \\
08 / 15 / 92 \\
11 / 20 / 92 \\
06 / 24 / 93 \\
12 / 11 / 93 \\
12 / 12 / 94\end{array}$ & $\begin{array}{l}0001 \\
0001 \\
0001 \\
0001 \\
0001 \\
0001 \\
0001 \\
0001 \\
0001 \\
N 001 \\
\text { NO01 } \\
\text { HOO1 }\end{array}$ & MG/L CACO3 & & $\begin{array}{l}141 . \\
187 . \\
187 . \\
167.0 \\
207 . \\
190 . \\
206 \\
172 \\
192 \\
189 \\
172 \\
187\end{array}$ & $\begin{array}{l}G L \\
L \\
L \\
J L \\
L \\
L \\
L \\
L \\
L \\
L \\
L \\
F\end{array}$ & $\begin{array}{c}- \\
= \\
= \\
= \\
= \\
= \\
10\end{array}$ & $\begin{array}{l}- \\
- \\
- \\
- \\
\dot{-} \\
\dot{-} \\
- \\
-\end{array}$ \\
\hline ALLMINUM & $\begin{array}{l}06 / 25 / 89 \\
12 / 02 / 89 \\
06 / 25 / 90 \\
01 / 29 / 91 \\
06 / 04 / 91 \\
02 / 20 / 92 \\
08 / 15 / 92\end{array}$ & $\begin{array}{l}0001 \\
0001 \\
0001 \\
0001 \\
0001 \\
0001 \\
0001\end{array}$ & MG/L & $\begin{array}{l}< \\
< \\
< \\
< \\
< \\
< \\
<\end{array}$ & $\begin{array}{l}0.1 \\
0.1 \\
0.1 \\
0.1 \\
0.05 \\
0.05 \\
0.2\end{array}$ & $\begin{array}{l}L \\
L \\
J L \\
L \\
L \\
L \\
L\end{array}$ & $\begin{array}{l}0.1 \\
0.1 \\
0.1 \\
0.1 \\
0.05 \\
0.05 \\
0.2\end{array}$ & $\begin{array}{l}. \\
- \\
- \\
- \\
-\end{array}$ \\
\hline AMHONIUH & $\begin{array}{l}04 / 26 / 88 \\
06 / 25 / 89 \\
12 / 02 / 89 \\
06 / 25 / 90 \\
01 / 29 / 91 \\
06 / 04 / 91 \\
02 / 20 / 92 \\
12 / 11 / 93\end{array}$ & $\begin{array}{l}0001 \\
0001 \\
0001 \\
0001 \\
0001 \\
0001 \\
0001 \\
0001\end{array}$ & MG/L & $\begin{array}{l}< \\
< \\
< \\
<\end{array}$ & $\begin{array}{l}0.1 \\
0.1 \\
0.1 \\
0.8 \\
0.1 \\
0.1 \\
0.1 \\
0.1\end{array}$ & $\begin{array}{l}G L \\
L \\
L \\
H J L \\
L \\
L \\
L \\
L\end{array}$ & $\begin{array}{l}0.1 \\
0.1 \\
0.1 \\
0.1 \\
0.1 \\
0.1 \\
0.1 \\
0.1\end{array}$ & $\begin{array}{l}- \\
\dot{-} \\
- \\
- \\
-\end{array}$ \\
\hline AะHONIUM (TOTAL) & $\begin{array}{l}06 / 24 / 93 \\
05 / 18 / 94 \\
12 / 12 / 94 \\
12 / 12 / 94\end{array}$ & $\begin{array}{l}\text { NO01 } \\
\text { N001 } \\
\text { N001 } \\
\text { N002 }\end{array}$ & MG/L & $<$ & $\begin{array}{l}0.1 \\
0.1 \\
0.13 \\
0.44\end{array}$ & $\begin{array}{l}\text { L } \\
\text { FH } \\
\text { FH }\end{array}$ & $\begin{array}{l}0.1 \\
0.1 \\
0.1 \\
0.1\end{array}$ & $\begin{array}{l}\text { - } \\
\dot{-}\end{array}$ \\
\hline ANTIMONY & $\begin{array}{l}06 / 25 / 89 \\
12 / 02 / 89 \\
06 / 25 / 90 \\
01 / 29 / 91 \\
06 / 04 / 91 \\
02 / 20 / 92 \\
08 / 15 / 92\end{array}$ & $\begin{array}{l}0001 \\
0001 \\
0001 \\
0001 \\
0001 \\
0001 \\
0001\end{array}$ & MG/L & $\begin{array}{l}< \\
< \\
< \\
< \\
< \\
<\end{array}$ & $\begin{array}{l}0.003 \\
0.003 \\
0.003 \\
0.003 \\
0.003 \\
0.003 \\
0.06\end{array}$ & $\begin{array}{l}L \\
L \\
J L \\
L \\
L \\
L \\
H L\end{array}$ & $\begin{array}{l}0.003 \\
0.003 \\
0.003 \\
0.003 \\
0.003 \\
0.003 \\
0.06\end{array}$ & $\begin{array}{l}- \\
- \\
- \\
- \\
-\end{array}$ \\
\hline ARSENIC & $\begin{array}{l}04 / 26 / 88 \\
06 / 25 / 89 \\
12 / 02 / 89 \\
06 / 25 / 90 \\
01 / 29 / 91 \\
06 / 04 / 91 \\
02 / 20 / 92 \\
08 / 15 / 92 \\
11 / 20 / 92\end{array}$ & $\begin{array}{l}0001 \\
0001 \\
0001 \\
0001 \\
0001 \\
0001 \\
0001 \\
0001 \\
0001\end{array}$ & $M G / L$ & $\begin{array}{l}< \\
< \\
< \\
< \\
< \\
< \\
< \\
<\end{array}$ & $\begin{array}{l}0.002 \\
0.01 \\
0.01 \\
0.01 \\
0.01 \\
0.01 \\
0.01 \\
0.01 \\
0.0015\end{array}$ & $\begin{array}{l}\text { GJL } \\
L \\
L \\
d L \\
L \\
L \\
L \\
H L \\
L\end{array}$ & $\begin{array}{l}0.01 \\
0.01 \\
0.01 \\
0.01 \\
0.01 \\
0.01 \\
0.01 \\
0.01 \\
0.0015\end{array}$ & $\begin{array}{l}- \\
\therefore \\
: \\
- \\
-\end{array}$ \\
\hline
\end{tabular}

PARAMETER VALUE INDICATOR (PVI): < - LESS THAN DETECTION LIMIT

OTHER PARAMETER VALUE FLAGS:

$F$ - LOH-FLOW SAMPLING/DEDICATED PUMP

G - PH > 9, POSSIBLE GROUT CONTAMINATION

H - HOLD TIME EXPIRED, VALUE SUSPECT

J - estIMATED VALUE

L - LESS THAN THREE BORE VOLUMES REMOVED BEFORE SAMPLING

4 - POST-DIGEST SPIKE OUT OF CHTR LIH MHILE SNMP ABS < 50\% SPIKE
SAMPLE ID COOES:

0001 - FILTERED SNMPLE (.45 HICRONS)

KOO1 - UMFILTERED SAMPLE

NOO2 - UNFILTERED REPLICATE SAMPLE 
GROUHDWATER QUALITY DATA BY LOCATION

SOWP REV. O Data

SITE: MONO1 MOHUMENT VALLEY

LOCATION: 0663

NORTH COORDIHATE: 59069.6 FT

EAST COORDINATE: 88593.5 FT

04/21/88 TO 12/13/94

REPORT DATE: 06/20/95

FORMATION OF COMPLETION: DECHELLEY MEMBER OF THE CUTLER FORMATION (DC) HYDRAULIC FLON RELATIONSHIP: DONH GRADIENT (D)

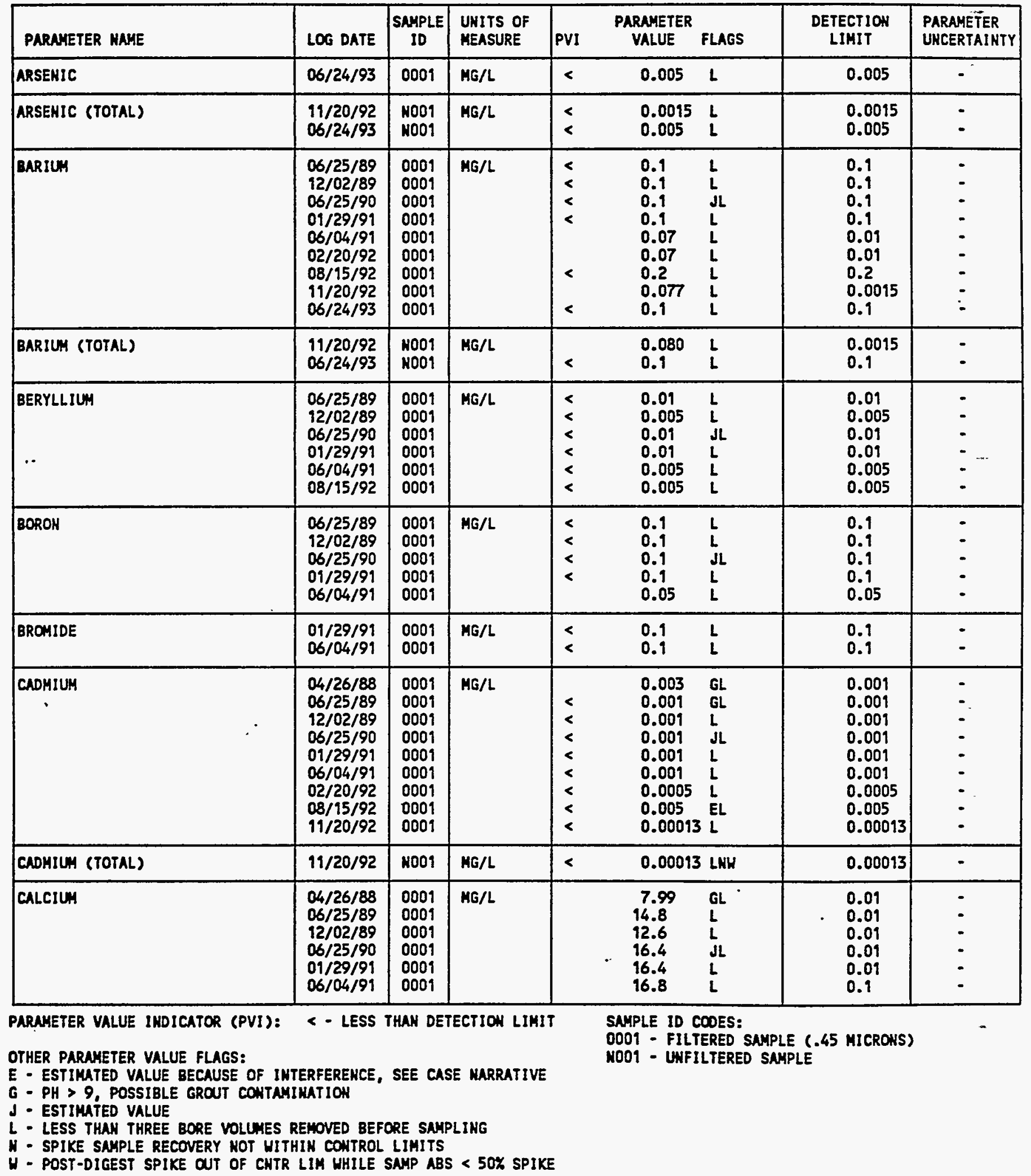


GROUNDWATER QUALITY DATA BY LOCATION

SOWP ReV. O Data

SITE: MONO1 MONLMENT VALLEY

LOCATION: 0663

MORTH COORDIHATE: 59069.6 FT

EAST COORDIHATE: $\quad 88593.5$ FT

$04 / 21 / 88$ TO $12 / 13 / 94$

REPORT DATE: $06 / 20 / 95$

FORMATION OF COMPLETION: DECHELLEY MEMBER OF THE CUTLER FORMATION (DC) HYDRAULIC FLON RELATIOKSHIP: DOWN GRADIENT (D)

\begin{tabular}{|c|c|c|c|c|c|c|c|c|}
\hline PARAMETER NAME & LOG DATE & $\begin{array}{c}\text { SAMPLE } \\
\text { ID }\end{array}$ & $\begin{array}{l}\text { UNITS OF } \\
\text { MEASURE }\end{array}$ & PVI & $\begin{array}{l}\text { PARAMETER } \\
\text { VALUE }\end{array}$ & FLAGS & $\begin{array}{l}\text { DETECTION } \\
\text { LIMIT }\end{array}$ & $\begin{array}{l}\text { PARAMETER } \\
\text { UNCERTAINTY }\end{array}$ \\
\hline calciun & $\begin{array}{l}02 / 20 / 92 \\
08 / 15 / 92 \\
06 / 24 / 93 \\
12 / 11 / 93 \\
05 / 14 / 94 \\
12 / 12 / 94 \\
12 / 12 / 94\end{array}$ & $\begin{array}{l}0001 \\
0001 \\
0001 \\
0001 \\
0001 \\
0001 \\
0002\end{array}$ & MG/L & & $\begin{array}{l}15.1 \\
17 \\
14.3 \\
15.2 \\
13.0 \\
17.9 \\
22.5\end{array}$ & $\begin{array}{l}L \\
L \\
L \\
L \\
F \\
F\end{array}$ & $\begin{array}{l}0.1 \\
5 \\
0.5 \\
0.5 \\
0.5 \\
0.5 \\
0.5\end{array}$ & $\begin{array}{l}- \\
: \\
: \\
-\end{array}$ \\
\hline CALCIUM (TOTAL) & $\begin{array}{l}06 / 24 / 93 \\
12 / 11 / 93 \\
05 / 14 / 94\end{array}$ & $\begin{array}{l}\text { No01 } \\
\text { NOO1 } \\
\text { N001 }\end{array}$ & MG/L & & $\begin{array}{l}14.2 \\
15.4 \\
14.2\end{array}$ & $\begin{array}{l}\mathbf{L} \\
\mathbf{L}\end{array}$ & $\begin{array}{l}0.5 \\
0.5 \\
0.5\end{array}$ & $:$ \\
\hline CHLORIDE & $\begin{array}{l}04 / 26 / 88 \\
06 / 25 / 89 \\
12 / 02 / 89 \\
06 / 25 / 90 \\
01 / 29 / 91 \\
06 / 04 / 91 \\
02 / 20 / 92 \\
08 / 15 / 92 \\
12 / 11 / 93 \\
05 / 14 / 94 \\
12 / 12 / 94 \\
12 / 12 / 94\end{array}$ & $\begin{array}{l}0001 \\
0001 \\
0001 \\
0001 \\
0001 \\
0001 \\
0001 \\
0001 \\
0001 \\
0001 \\
0001 \\
0002\end{array}$ & $M G / L$ & & $\begin{array}{c}11 . \\
8.6 \\
9.7 \\
10 . \\
7 . \\
9.8 \\
9.5 \\
8.8 \\
9.2 \\
10.3 \\
6 \\
6\end{array}$ & $\begin{array}{l}\text { GL } \\
L \\
L \\
J L \\
J L \\
L \\
L \\
L \\
L \\
F \\
F\end{array}$ & $\begin{array}{l}1 . \\
1 . \\
1 . \\
1 . \\
1 . \\
0.5 \\
0.5 \\
0.016 \\
0.5 \\
0.5 \\
0.5 \\
0.5\end{array}$ & $\begin{array}{l}- \\
- \\
- \\
- \\
- \\
- \\
- \\
- \\
-\end{array}$ \\
\hline CHLORIDE (TOTAL) & $06 / 24 / 93$ & NOO1 & $M G / L$ & & 9.4 & $L$ & 0.5 & - \\
\hline CHROMIUM & $\begin{array}{l}06 / 25 / 89 \\
12 / 02 / 89 \\
06 / 25 / 90 \\
01 / 29 / 91 \\
06 / 04 / 91 \\
02 / 20 / 92 \\
08 / 15 / 92 \\
11 / 20 / 92\end{array}$ & $\begin{array}{l}0001 \\
0001 \\
0001 \\
0001 \\
0001 \\
0001 \\
0001 \\
0001\end{array}$ & $M G / L$ & $\begin{array}{l}< \\
< \\
< \\
< \\
< \\
<\end{array}$ & $\begin{array}{l}0.01 \\
0.01 \\
0.01 \\
0.01 \\
0.01 \\
0.01 \\
0.01 \\
0.0053\end{array}$ & $\begin{array}{l}L \\
L \\
J L \\
L \\
L \\
L \\
L \\
L\end{array}$ & $\begin{array}{l}0.01 \\
0.01 \\
0.01 \\
0.01 \\
0.01 \\
0.01 \\
0.01 \\
0.0053\end{array}$ & $\begin{array}{l}- \\
- \\
- \\
: \\
-\end{array}$ \\
\hline CHROMIUN (TOTAL) & $11 / 20 / 92$ & N001 & $M G / L$ & $<$ & 0.0053 & $\mathbf{L}$ & 0.0053 & - \\
\hline COBALT & $\begin{array}{l}06 / 25 / 89 \\
12 / 02 / 89 \\
06 / 25 / 90 \\
01 / 29 / 91 \\
06 / 04 / 91\end{array}$ & $\begin{array}{l}0001 \\
0001 \\
0001 \\
0001 \\
0001\end{array}$ & $M G / L$ & $\begin{array}{l}< \\
< \\
< \\
< \\
<\end{array}$ & $\begin{array}{l}0.05 \\
0.05 \\
0.05 \\
0.05 \\
0.03\end{array}$ & $\begin{array}{l}L \\
L \\
J L \\
L \\
L\end{array}$ & $\begin{array}{l}0.05 \\
0.05 \\
0.05 \\
0.05 \\
0.03\end{array}$ & $\begin{array}{l}- \\
- \\
- \\
-\end{array}$ \\
\hline COPPER & $\begin{array}{l}06 / 25 / 89 \\
12 / 02 / 89 \\
06 / 25 / 90 \\
01 / 29 / 91 \\
06 / 04 / 91\end{array}$ & $\begin{array}{l}0001 \\
0001 \\
0001 \\
0001 \\
0001\end{array}$ & MG/L & $\begin{array}{l}< \\
< \\
< \\
<\end{array}$ & $\begin{array}{l}0.02 \\
0.02 \\
0.02 \\
0.02 \\
0.01\end{array}$ & $\begin{array}{l}L \\
L \\
J L \\
L \\
L\end{array}$ & $\begin{array}{l}0.02 \\
0.02 \\
0.02 \\
0.02 \\
0.01\end{array}$ & $\begin{array}{l}- \\
- \\
-\end{array}$ \\
\hline CYANIDE & $\begin{array}{l}06 / 25 / 89 \\
12 / 02 / 89\end{array}$ & $\begin{array}{l}0001 \\
0001\end{array}$ & MG/L & $<$ & $\begin{array}{l}0.01 \\
0.01\end{array}$ & $L$ & $\begin{array}{l}0.01 \\
0.01\end{array}$ & - \\
\hline DISSOLVED OXYGEN & $11 / 20 / 92$ & 0009 & $M G / L$ & & 1.6 & $\mathbf{L}$ & - & - \\
\hline
\end{tabular}

PARAMETER VALUE INDICATOR (PVI): < - LESS THAN DETECTION LIMIT

SUMPLE ID COOES:

OTHER PARAMETER VALUE FLAGS:

0001 - FILTERED SAMPLE (.45 MICRONS)

F - LON-FLON SAMPLING/DEDICATED PLAP

0002 - FILTERED REPLICATE SAMPLE (.45 MICRONS)

G - PH > 9, POSSIBLE GROUT CONTAMIMATION

MOO1 - UNFILTERED SNMPLE

$J$ - ESTIMATED VALUE

L - LESS THAN THREE BORE VOLUHES REMOVED BEFORE SAMPLING 
GROUNDWATER QUALITY DATA BY LOCATIOH

SOMP ReV. 0 Data

SITE: HOHO1 MOHLMEHT VALLEY

LOCATION: 0663

NORTH COORDINATE: $\quad 59069.6$ FT

EAST COORDINATE: 88593.5 FT

$04 / 21 / 88$ TO $12 / 93 / 94$

REPORT DATE: $06 / 20 / 95$

FORMATION OF COMPLETION: DECHELLEY MEMBER OF THE CUTLER FORMATION (DC) HYDRAULIC FLON RELATIOHSHIP: DOWN GRADIENT (D)

\begin{tabular}{|c|c|c|c|c|c|c|c|c|}
\hline PARAMETER MAME & LOG DATE & $\mid \begin{array}{c}\text { SNMPLE } \\
\text { ID }\end{array}$ & $\begin{array}{l}\text { UHITS OF } \\
\text { MEASURE }\end{array}$ & PVI & $\begin{array}{l}\text { PARAMETER } \\
\text { VALUE }\end{array}$ & FLAGS & $\begin{array}{l}\text { DETECTION } \\
\text { LIMIT }\end{array}$ & $\begin{array}{l}\text { PARAMETER } \\
\text { UHCERTAINTY }\end{array}$ \\
\hline DISSOLVED OXYGEN & $\begin{array}{l}06 / 24 / 93 \\
12 / 11 / 93 \\
05 / 14 / 94 \\
12 / 12 / 94\end{array}$ & $\begin{array}{l}\text { NoO1 } \\
\text { NOO1 } \\
\text { HOOI } \\
\text { HOOI }\end{array}$ & MG/L & $<$ & $\begin{array}{l}0.3 \\
0.6 \\
3.8 \\
1.3\end{array}$ & $\begin{array}{l}L \\
L\end{array}$ & $\dot{-}$ & $\begin{array}{l}- \\
- \\
-\end{array}$ \\
\hline FLUORIDE & $\begin{array}{l}06 / 25 / 89 \\
12 / 02 / 89 \\
06 / 25 / 90 \\
01 / 29 / 91 \\
06 / 04 / 91\end{array}$ & $\begin{array}{l}0001 \\
0001 \\
0001 \\
0001 \\
0001\end{array}$ & MG/L & & $\begin{array}{l}0.2 \\
0.3 \\
6.6 \\
0.2 \\
0.2\end{array}$ & $\begin{array}{l}L \\
L \\
d L \\
L \\
L\end{array}$ & $\begin{array}{l}0.1 \\
0.1 \\
0.1 \\
0.1 \\
0.1\end{array}$ & $\begin{array}{l}- \\
- \\
- \\
-\end{array}$ \\
\hline GROSS ALPHA & $\begin{array}{l}04 / 26 / 88 \\
01 / 29 / 91 \\
06 / 04 / 91 \\
02 / 20 / 92 \\
08 / 15 / 92 \\
11 / 20 / 92 \\
06 / 24 / 93\end{array}$ & $\begin{array}{l}0001 \\
0001 \\
0001 \\
0001 \\
0001 \\
0001 \\
0001\end{array}$ & $\mathrm{PCI} / \mathrm{L}$ & & $\begin{array}{l}9.0 \\
0.0 \\
4.0 \\
1.4 \\
0.0 \\
2.0 \\
1\end{array}$ & $\begin{array}{l}\text { GL } \\
L \\
L \\
L \\
L \\
L N \\
L\end{array}$ & $\begin{array}{l}0.2 \\
1 . \\
1.0 \\
1.0 \\
3.2 \\
2.7 \\
2\end{array}$ & $\begin{array}{l}2.1 \\
2.2 \\
4.3 \\
3.8 \\
1.2 \\
2.0 \\
4\end{array}$ \\
\hline GROSS BETA & $\begin{array}{l}04 / 26 / 88 \\
01 / 29 / 91 \\
06 / 04 / 91 \\
02 / 20 / 92 \\
08 / 15 / 92 \\
11 / 20 / 92 \\
06 / 24 / 93\end{array}$ & $\begin{array}{l}0001 \\
0001 \\
0001 \\
0001 \\
0001 \\
0001 \\
0001\end{array}$ & $\mathrm{PCI} / \mathrm{L}$ & & $\begin{array}{l}3.0 \\
2.8 \\
4.6 \\
4.2 \\
0.0 \\
15.6 \\
9\end{array}$ & $\begin{array}{l}\text { GL } \\
L \\
L \\
L \\
L \\
L H \\
L\end{array}$ & $\begin{array}{r}1 . \\
0.5 \\
0.5 \\
0.5 \\
3.6 \\
24.9 \\
4\end{array}$ & $\begin{array}{r}1.4 \\
1.7 \\
2.5 \\
2.3 \\
2.0 \\
15.4 \\
4\end{array}$ \\
\hline GROSS BETA (TOTAL) & $\begin{array}{l}11 / 20 / 92 \\
06 / 24 / 93\end{array}$ & $\begin{array}{l}\text { NOO1 } \\
\text { N001 }\end{array}$ & $\mathrm{PCI} / \mathrm{L}$ & & $\begin{array}{c}26.7 \\
5\end{array}$ & $L_{L}^{L H}$ & 4.7 & $\begin{array}{l}3.8 \\
4\end{array}$ \\
\hline LEAD & $\begin{array}{l}06 / 25 / 89 \\
12 / 02 / 89 \\
06 / 25 / 90 \\
01 / 29 / 91 \\
06 / 04 / 91 \\
11 / 20 / 92\end{array}$ & $\begin{array}{l}0001 \\
0001 \\
0001 \\
0001 \\
0001 \\
0001\end{array}$ & MG/L & $\begin{array}{l}< \\
< \\
< \\
< \\
<\end{array}$ & $\begin{array}{l}0.01 \\
0.01 \\
0.01 \\
0.01 \\
0.005 \\
0.0015\end{array}$ & $\begin{array}{l}L \\
L \\
J L \\
L \\
L \\
L\end{array}$ & $\begin{array}{l}0.01 \\
0.01 \\
0.01 \\
0.01 \\
0.005 \\
0.0015\end{array}$ & $\begin{array}{l}- \\
: \\
: \\
-\end{array}$ \\
\hline LEAD (TOTAL) & $11 / 20 / 92$ & NoOI & MG/L & $<$ & 0.0015 & $L$ & 0.0015 & $-=$ \\
\hline $\begin{array}{l}\text { PARAMETER VALUE INDIC } \\
\text { DTHER PARAMETER VALUE } \\
\text { F - LON-FLOW SANPLING } \\
\text { - PH > 9, POSSIBLE } \\
\text { - ESTIMATED VALUE } \\
\text { - LESS THAH THREE B } \\
\text { - SPAKE SAMPLE RECO }\end{array}$ & $<$ - LESS & THAN DE & CTION LI & & \multicolumn{4}{|c|}{$\begin{array}{l}\text { SNMPLE ID COOES: } \\
\text { O001 - FILTERED SNMPLE (.45 MICRONS) } \\
\text { NO01 - UNFILTERED SAMPLE }\end{array}$} \\
\hline
\end{tabular}


GROWWHATER QUALITY DATA BY LOCATION

SOWP ReV. O Data

SITE: MONO1 MONLHENT VALLEY

LOCATION: 0663

NORTH COORDINATE: $\quad 59069.6$ FT

EAST COORDINATE: 88593.5 FT

04/21/88 TO $12 / 13 / 94$

REPORT DATE: 06/20/95

FORYATION OF COMPLETION: DECHELLEY MEMBER OF THE CUTLER FORMATION (DC)

HYDRAULIC FLOU RELATIONSHIP: DOWN GRADIENT (D)

\begin{tabular}{|c|c|c|c|c|c|c|c|c|}
\hline PARAMETER MAME & LOG DATE & $\underset{\text { SD }}{\text { SAMPLE }}$ & $\begin{array}{l}\text { UNITS OF } \\
\text { MEASURE }\end{array}$ & PVI & $\begin{array}{l}\text { PARAMETER } \\
\text { VALUE }\end{array}$ & FLAGS & $\begin{array}{l}\text { DETECTION } \\
\text { LIMIT }\end{array}$ & $\begin{array}{l}\text { PARAMETER } \\
\text { UHCERTAINTY }\end{array}$ \\
\hline LERD-210 & $11 / 20 / 92$ & 0001 & $\mathrm{PCI} / \mathrm{L}$ & & 0.7 & $\mathbf{L}$ & 2.0 & 1.2 \\
\hline LEND-210 (TOTAL) & $19 / 20 / 92$ & NOOI & PCI/L & & 6.0 & $L$ & 0.3 & 0.9 \\
\hline MAGRESIUN & $\begin{array}{l}04 / 26 / 88 \\
06 / 25 / 89 \\
12 / 02 / 89 \\
06 / 25 / 90 \\
01 / 29 / 91 \\
06 / 04 / 91 \\
02 / 20 / 92 \\
08 / 15 / 92 \\
06 / 24 / 93 \\
12 / 11 / 93 \\
05 / 14 / 94 \\
12 / 12 / 94 \\
12 / 12 / 94\end{array}$ & $\begin{array}{l}0001 \\
0001 \\
0001 \\
0001 \\
0001 \\
0001 \\
0001 \\
0001 \\
0001 \\
0001 \\
0001 \\
0001 \\
0002\end{array}$ & MG/L & & $\begin{array}{l}5.2 \\
19.0 \\
10.7 \\
13.5 \\
14.0 \\
13.6 \\
12.0 \\
13 \\
11.0 \\
11.8 \\
9.5 \\
13.3 \\
26.2\end{array}$ & $\begin{array}{l}G L \\
L \\
L \\
J L \\
L \\
L \\
L \\
L \\
L \\
L \\
F \\
F\end{array}$ & $\begin{array}{l}0.001 \\
0.001 \\
0.001 \\
0.001 \\
0.001 \\
0.1 \\
0.1 \\
5 \\
0.1 \\
0.1 \\
0.1 \\
0.1 \\
0.1\end{array}$ & $\begin{array}{l}- \\
: \\
: \\
: \\
: \\
- \\
- \\
-\end{array}$ \\
\hline MAGNESIUM (TOTAL) & $\begin{array}{l}06 / 24 / 93 \\
12 / 11 / 93 \\
05 / 14 / 94\end{array}$ & $\begin{array}{l}\text { No01 } \\
\text { NO01 } \\
\text { N001 }\end{array}$ & MG/L & & $\begin{array}{l}10.3 \\
11.4 \\
10.2\end{array}$ & $L$ & $\begin{array}{l}0.1 \\
0.1 \\
0.1\end{array}$ & : \\
\hline MUNGAKESE & $\begin{array}{l}06 / 25 / 89 \\
12 / 02 / 89 \\
06 / 25 / 90 \\
01 / 29 / 91 \\
06 / 04 / 91 \\
02 / 20 / 92 \\
08 / 15 / 92 \\
11 / 20 / 92 \\
06 / 24 / 93 \\
12 / 11 / 93 \\
05 / 14 / 94 \\
12 / 12 / 94 \\
12 / 12 / 94\end{array}$ & $\begin{array}{l}0001 \\
0001 \\
0001 \\
0001 \\
0001 \\
0001 \\
0001 \\
0001 \\
0001 \\
0001 \\
0001 \\
0001 \\
0002\end{array}$ & $M G / L$ & $<$ & $\begin{array}{l}0.03 \\
0.02 \\
0.02 \\
0.03 \\
0.03 \\
0.02 \\
0.1 \\
0.028 \\
0.03 \\
0.03 \\
0.03 \\
0.02 \\
0.13\end{array}$ & $\begin{array}{l}L \\
L \\
J L \\
L \\
L \\
L \\
L \\
L \\
L \\
L \\
F \\
F\end{array}$ & $\begin{array}{l}0.01 \\
0.01 \\
0.01 \\
0.01 \\
0.01 \\
0.01 \\
0.01 \\
0.0015 \\
0.01 \\
0.01 \\
0.01 \\
0.01 \\
0.01\end{array}$ & $\begin{array}{l}- \\
: \\
: \\
: \\
: \\
- \\
: \\
-\end{array}$ \\
\hline MANGANESE (TOTAL) & $\begin{array}{l}11 / 20 / 92 \\
06 / 24 / 93 \\
12 / 11 / 93 \\
05 / 14 / 94\end{array}$ & $\begin{array}{l}\text { No01 } \\
\text { N001 } \\
\text { N001 } \\
\text { N001 }\end{array}$ & $M G / L$ & & $\begin{array}{l}0.022 \\
0.02 \\
0.03 \\
0.03\end{array}$ & $\begin{array}{l}L \\
L\end{array}$ & $\begin{array}{l}0.0015 \\
0.01 \\
0.01 \\
0.01\end{array}$ & : \\
\hline MERCURY & $\begin{array}{l}06 / 25 / 89 \\
12 / 02 / 89 \\
06 / 25 / 90\end{array}$ & $\begin{array}{l}0001 \\
0001 \\
0001\end{array}$ & $M G / L$ & $\begin{array}{l}< \\
< \\
<\end{array}$ & $\begin{array}{l}0.0002 \\
0.0002 \\
0.0002\end{array}$ & $\begin{array}{l}L \\
\text { dL }\end{array}$ & $\begin{array}{l}0.0002 \\
0.0002 \\
0.0002\end{array}$ & $\dot{-}$ \\
\hline MOLYBDENUY & $\begin{array}{l}04 / 26 / 88 \\
06 / 25 / 89 \\
12 / 02 / 89 \\
06 / 25 / 90 \\
01 / 29 / 91 \\
06 / 04 / 91 \\
02 / 20 / 92 \\
08 / 15 / 92\end{array}$ & $\begin{array}{l}0001 \\
0001 \\
0001 \\
0001 \\
0001 \\
0001 \\
0001 \\
0001\end{array}$ & MG/L & $\begin{array}{l}< \\
< \\
< \\
< \\
< \\
<\end{array}$ & $\begin{array}{l}0.01 \\
0.01 \\
0.01 \\
0.01 \\
0.01 \\
0.01 \\
0.01 \\
0.007\end{array}$ & $\begin{array}{l}G L \\
L \\
L \\
J L \\
L \\
L \\
L \\
L\end{array}$ & $\begin{array}{l}0.01 \\
0.01 \\
0.01 \\
0.01 \\
0.01 \\
0.01 \\
0.01 \\
0.007\end{array}$ & $\begin{array}{l}: \\
: \\
: \\
:\end{array}$ \\
\hline
\end{tabular}

PARAMETER VALUE INDICATOR (PVI): < - LESS THAN DETECTION LIMIT

SAMPLE ID COOES:

OTHER PARAMETER VALUE FLAGS:

F - LOW-FLOW SAMPLING/DEDICATED PLMP

0001 - FILTERED SAMPLE (.45 MICRONS)

0002 - FILTERED REPLICATE SAMPLE (.45 MICRONS)

NOO1 - UNFILTERED SAMPLE

G - PH > 9, POSSIBLE GROUT CONTAMIMATION

J - estimated VALUE

:L - LFSS THAN THREE BORE VOLLAES REMOVED BEFORE SAMPLING 
GROUNDHATER QUALITY DATA BY LOCATION

SOMP ReV. 0 Data

SITE: MONOI MONUMENT VALLEY

LOCATION: 0663

NORTH COORDIHATE: 59069.6 FT

EAST COORDINATE: 88593.5 FT

$04 / 21 / 88$ TO $12 / 13 / 94$

REPORT DATE: $06 / 20 / 95$

FORMATION OF COHPLETIOH: DECHELLEY MEMBER OF THE CUTLER FORMATIOH (DC) HYDRAULIC FLOU RELATIOHSHIP: DOWN GRADIENT (D)

\begin{tabular}{|c|c|c|c|c|c|c|c|c|}
\hline PARAMETER RAME & LOG DATE & $\underset{\text { ID }}{\text { SNMPLE }}$ & $\begin{array}{l}\text { UHITS OF } \\
\text { MEASURE }\end{array}$ & PVI & $\begin{array}{l}\text { PARAMETER } \\
\text { VALUE }\end{array}$ & FLAGS & $\begin{array}{l}\text { DETECTION } \\
\text { LIMIT }\end{array}$ & $\begin{array}{l}\text { PARAMETER } \\
\text { UNCERTAINYY }\end{array}$ \\
\hline MOLYBDENUM & $\begin{array}{l}11 / 20 / 92 \\
06 / 24 / 93 \\
12 / 12 / 94 \\
12 / 12 / 94\end{array}$ & $\begin{array}{l}0001 \\
0001 \\
0001 \\
0002\end{array}$ & MG/L & $\begin{array}{l}< \\
<\end{array}$ & $\begin{array}{l}0.0049 \\
0.01 \\
0.01 \\
0.02\end{array}$ & $\begin{array}{l}\text { LN } \\
L \\
F \\
F\end{array}$ & $\begin{array}{l}0.0049 \\
0.01 \\
0.01 \\
0.01\end{array}$ & :- \\
\hline MOLYBOENUM (TOTAL) & $\begin{array}{l}11 / 20 / 92 \\
06 / 24 / 93\end{array}$ & $\begin{array}{l}\text { No01 } \\
\text { No01 }\end{array}$ & MG/L & $\begin{array}{l}< \\
<\end{array}$ & $\begin{array}{l}0.0049 \\
0.01\end{array}$ & $\begin{array}{l}L N \\
L\end{array}$ & $\begin{array}{l}0.0049 \\
0.01\end{array}$ & $\ddot{-}$ \\
\hline NET GROSS ALPHA • & $\begin{array}{l}04 / 26 / 88 \\
01 / 29 / 91 \\
06 / 04 / 91 \\
02 / 20 / 92 \\
08 / 15 / 92 \\
11 / 20 / 92 \\
06 / 24 / 93\end{array}$ & $\begin{array}{l}0001 \\
0001 \\
0001 \\
0001 \\
0001 \\
0001 \\
0001\end{array}$ & PCI/L & & $\begin{array}{r}0.52 \\
-0.10 \\
3.66 \\
0.71 \\
-0.34 \\
1.66 \\
0.66\end{array}$ & & $\begin{array}{l}- \\
- \\
- \\
- \\
-\end{array}$ & $\begin{array}{l}- \\
- \\
- \\
- \\
-\end{array}$ \\
\hline HET GROSS ALPHA (TOTAL) ** & $\begin{array}{l}11 / 20 / 92 \\
06 / 24 / 93\end{array}$ & $\begin{array}{l}\text { No01 } \\
\text { N001 }\end{array}$ & $\mathrm{PCI} / \mathrm{L}$ & & $\begin{array}{r}-0.69 \\
5.66\end{array}$ & & - & - \\
\hline HICKEL & $\begin{array}{l}06 / 25 / 89 \\
12 / 02 / 89 \\
06 / 25 / 90 \\
01 / 29 / 91 \\
06 / 04 / 91\end{array}$ & $\begin{array}{l}0001 \\
0001 \\
0001 \\
0001 \\
0001\end{array}$ & $M G / L$ & $\begin{array}{l}< \\
< \\
< \\
< \\
<\end{array}$ & $\begin{array}{l}0.04 \\
0.04 \\
0.04 \\
0.04 \\
0.04\end{array}$ & $\begin{array}{l}L \\
L \\
L \\
L\end{array}$ & $\begin{array}{l}0.04 \\
0.04 \\
0.04 \\
0.04 \\
0.04\end{array}$ & $\begin{array}{l}- \\
- \\
- \\
-\end{array}$ \\
\hline HITRATE & $\begin{array}{l}04 / 26 / 88 \\
06 / 25 / 89 \\
12 / 02 / 89 \\
06 / 25 / 90 \\
01 / 29 / 91 \\
06 / 04 / 91 \\
02 / 20 / 92 \\
08 / 15 / 92 \\
11 / 20 / 92 \\
12 / 11 / 93\end{array}$ & $\begin{array}{l}0001 \\
0001 \\
0001 \\
0001 \\
0001 \\
0001 \\
0001 \\
0001 \\
0001 \\
0001\end{array}$ & MG/L & $\begin{array}{l}< \\
< \\
< \\
<\end{array}$ & $\begin{array}{l}0.4 \\
4.8 \\
3.5 \\
0.1 \\
1 . \\
3.1 \\
1.0 \\
3.1 \\
0.20 \\
1\end{array}$ & $\begin{array}{l}\text { GJL } \\
L \\
L \\
J L \\
L \\
L \\
L \\
L \\
L \\
L\end{array}$ & $\begin{array}{l}1 . \\
1 . \\
1 . \\
0.1 \\
1 . \\
1 . \\
1.0 \\
0.075 \\
0.044 \\
1\end{array}$ & $\begin{array}{l}- \\
: \\
: \\
- \\
- \\
-\end{array}$ \\
\hline MITRATE (TOTAL) & $\begin{array}{l}05 / 18 / 94 \\
12 / 12 / 94 \\
12 / 12 / 94\end{array}$ & $\begin{array}{l}\text { No01 } \\
\text { N001 } \\
\text { N002 }\end{array}$ & MG/L & $<$ & $\begin{array}{l}1 \\
1 \\
2\end{array}$ & $\begin{array}{l}\ell \\
\text { FHJN } \\
\text { FHJN }\end{array}$ & $\begin{array}{l}1 \\
1 \\
1\end{array}$ & : \\
\hline MITRITE AND NITRATE & $\begin{array}{l}01 / 29 / 99 \\
06 / 04 / 91\end{array}$ & $\begin{array}{l}0001 \\
0001\end{array}$ & $M G / L$ & $<$ & $\begin{array}{l}1.70 \\
0.70\end{array}$ & $\stackrel{\mathrm{JL}}{\mathrm{L}}$ & $\begin{array}{l}1.05 \\
0.05\end{array}$ & $\dot{-}$ \\
\hline PH & $\begin{array}{l}04 / 26 / 88 \\
06 / 25 / 89 \\
12 / 02 / 89 \\
06 / 25 / 90 \\
01 / 29 / 91\end{array}$ & $\begin{array}{l}0001 \\
0001 \\
0001 \\
0001 \\
0001\end{array}$ & su & & $\begin{array}{l}9.31 \\
8.42 \\
8.48 \\
8.29 \\
8.29\end{array}$ & $\begin{array}{l}G L \\
L \\
L \\
L \\
L\end{array}$ & $\begin{array}{l}: \\
: \\
-\end{array}$ & $\begin{array}{l}- \\
- \\
-\end{array}$ \\
\hline
\end{tabular}

- MET gROSS ALPHA (GROSS ALPHA - URANIUHA) HITH 1 MG URANIUM = 686 PCI

* MET GROSS ALPHA (TOTAL) (TOTAL GROSS ALPHA - TOTAL URANIUH)

PARAMETER VALUE IHDICATOR (PVI): < - LESS THAN DETECTIOH LIHIT

OTHER PARAMETER VALUE FLAGS:

F - LON-FLOW SNMPLING/DEDICATED PUMP

G - PH > 9, POSSIBLE GROUT CONTAMINATION

H - HOLD TIME EXPIRED, VALUE SUSPECT

J- ESTIMATED VALUE

L - LESS THAH THREE BORE VOLUHES REHOVED BEFORE SAMPLING

H - SPIKE SAMPLE RECOVERY HOT HITHIN CONTROL LIMITS
SAMPLE ID COOES:

0001 - FILTERED SAMPLE (.45 MICRONS)

0002 - FILTERED REPLICATE SAMPLE (.45 MICRONS)

NOO1 - UAFILTERED SAMPLE

NOO2 - UAFILTERED REPLICATE SAMPLE 
GROUHDWATER QUALITY DATA BY LOCATION

SOMP Rev. O Data

SITE: MONO1 MONUNENT VALLEY

LOCATION: 0663

MORTH COORDINATE: 59069.6 FT

EAST COORDINATE: 88593.5 FT

$04 / 21 / 88$ TO $12 / 13 / 94$

REPORT DATE: 06/20/95

FORMATION OF COMPLETION: DECHELLEY MEMBER OF THE CUTLER FORMATION (DC) HYDRAULIC FLOW RELATIONSHIP: DOWN GRADIENT (D)

\begin{tabular}{|c|c|c|c|c|c|c|c|c|}
\hline PARAMETER MNME & LOG DATE & $\underset{\text { ID }}{\text { SAMPLE }}$ & $\begin{array}{l}\text { UNITS OF } \\
\text { MEASURE }\end{array}$ & PVI & $\begin{array}{l}\text { PARAMETER } \\
\text { VALUE }\end{array}$ & FLAGS & $\begin{array}{l}\text { DETECTION } \\
\text { LIMIT }\end{array}$ & $\begin{array}{l}\text { PARAMETER } \\
\text { UNCERTAINTY }\end{array}$ \\
\hline PH & $\begin{array}{l}06 / 04 / 91 \\
02 / 20 / 92 \\
08 / 15 / 92 \\
11 / 20 / 92 \\
06 / 24 / 93 \\
12 / 11 / 93 \\
05 / 14 / 94 \\
12 / 12 / 94\end{array}$ & $\begin{array}{l}0009 \\
0001 \\
0009 \\
0009 \\
\text { NOO } \\
\text { NOO1 } \\
\text { NO01 } \\
\text { NO01 }\end{array}$ & SU & & $\begin{array}{l}8.13 \\
8.15 \\
8.03 \\
8.20 \\
8.30 \\
8.20 \\
8.27 \\
8.06\end{array}$ & $\begin{array}{l}L \\
L \\
L \\
L \\
L \\
L \\
F\end{array}$ & 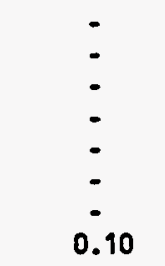 & $\begin{array}{l}- \\
- \\
- \\
- \\
-\end{array}$ \\
\hline PHOSPHATE & $\begin{array}{l}06 / 25 / 89 \\
12 / 02 / 89 \\
06 / 25 / 90\end{array}$ & $\begin{array}{l}0001 \\
0001 \\
0001\end{array}$ & MG/L & $\begin{array}{l}< \\
< \\
<\end{array}$ & $\begin{array}{l}0.1 \\
0.1 \\
0.1\end{array}$ & $\begin{array}{l}L \\
\mathrm{~J}\end{array}$ & $\begin{array}{l}0.1 \\
0.1 \\
0.1\end{array}$ & : \\
\hline PHOSPHATE (TOTAL) & $06 / 24 / 93$ & N001 & $M G / L$ & $<$ & 0.1 & $\mathbf{L}$ & 0.1 & $\because$ \\
\hline POTASSIU:H & $\begin{array}{l}04 / 26 / 88 \\
06 / 25 / 89 \\
12 / 02 / 89 \\
06 / 25 / 90 \\
01 / 29 / 91 \\
06 / 04 / 91 \\
02 / 20 / 92 \\
08 / 15 / 92 \\
06 / 24 / 93 \\
12 / 11 / 93 \\
05 / 14 / 94 \\
12 / 12 / 94 \\
12 / 12 / 94\end{array}$ & $\begin{array}{l}0001 \\
0001 \\
0001 \\
0001 \\
0001 \\
0001 \\
0001 \\
0001 \\
0001 \\
0001 \\
0001 \\
0001 \\
0002\end{array}$ & MG/L & & $\begin{array}{l}2.49 \\
3.0 \\
2.4 \\
2.4 \\
2.6 \\
2.8 \\
2.89 \\
3.0 \\
3.1 \\
2.5 \\
2.4 \\
2.8 \\
3.2\end{array}$ & $\begin{array}{l}G L \\
L \\
L \\
J L \\
L \\
L \\
L \\
L \\
L \\
L \\
F \\
F\end{array}$ & $\begin{array}{l}0.01 \\
0.01 \\
0.01 \\
0.01 \\
0.01 \\
0.01 \\
0.01 \\
0.575 \\
0.1 \\
0.1 \\
0.1 \\
0.1 \\
0.1\end{array}$ & $\begin{array}{l}- \\
: \\
: \\
: \\
: \\
: \\
- \\
-\end{array}$ \\
\hline POTASSIUM (TOTAL) & $\begin{array}{l}06 / 24 / 93 \\
12 / 11 / 93 \\
05 / 14 / 94\end{array}$ & $\begin{array}{l}\text { No01 } \\
\text { N001 } \\
\text { NO01 }\end{array}$ & $M G / L$ & & $\begin{array}{l}2.7 \\
2.4 \\
2.9\end{array}$ & $L$ & $\begin{array}{l}0.1 \\
0.1 \\
0.1\end{array}$ & $\begin{array}{l}- \\
-\end{array}$ \\
\hline RADIUN-226 & $\begin{array}{l}06 / 25 / 89 \\
12 / 02 / 89 \\
06 / 25 / 90 \\
01 / 29 / 91 \\
06 / 04 / 91 \\
02 / 20 / 92 \\
08 / 15 / 92 \\
11 / 20 / 92\end{array}$ & $\begin{array}{l}0001 \\
0001 \\
0001 \\
0001 \\
0001 \\
0001 \\
0001 \\
0001\end{array}$ & $\mathrm{PCl} / \mathrm{L}$ & & $\begin{array}{l}0.2 \\
0.0 \\
0.2 \\
0.2 \\
0.2 \\
0.0 \\
0.3 \\
0.8\end{array}$ & $\begin{array}{l}L \\
L \\
J L \\
L \\
L \\
L \\
L \\
L \\
L\end{array}$ & $\begin{array}{l}1 . \\
1 . \\
1 . \\
1.0 \\
1.0 \\
0.5 \\
0.3\end{array}$ & $\begin{array}{l}0.2 \\
0.1 \\
0.2 \\
0.2 \\
0.3 \\
0.2 \\
0.1 \\
0.3\end{array}$ \\
\hline RADIUA-226 (TOTAL) & $11 / 20 / 92$ & NOOI & PCI/L & & 0.2 & $\mathbf{L}$ & 0.3 & 0.2 \\
\hline RADIUH-226 + RADIUH-228 & $\begin{array}{l}06 / 25 / 89 \\
12 / 02 / 89 \\
06 / 25 / 90 \\
01 / 29 / 91 \\
06 / 04 / 91 \\
02 / 20 / 92 \\
08 / 15 / 92 \\
11 / 20 / 92\end{array}$ & $\begin{array}{l}0001 \\
0001 \\
0001 \\
0001 \\
0001 \\
0001 \\
0001 \\
0001\end{array}$ & $\mathrm{PCl} / \mathrm{L}$ & & $\begin{array}{l}0.50 \\
2.10 \\
0.80 \\
1.10 \\
2.30 \\
0.60 \\
0.70 \\
1.00\end{array}$ & & $\begin{array}{l}- \\
: \\
- \\
: \\
-\end{array}$ & $\begin{array}{l}- \\
: \\
: \\
: \\
-\end{array}$ \\
\hline RADIUH-226 + RADIUN-228 (TOTAL) & $11 / 20 / 92$ & NOOI & PCI/L & & 3.30 & & - & - \\
\hline
\end{tabular}

PARAMETER VALUE INDICATOR (PVI): < - LESS THAN DETECTION LIMIT

SAMPLE IO COOES

OTHER PARAMETER VALUE FLAGS:

F - LOU-FLON SAMPLING/DEDICATED PUMP

0001 - FILTERED SAMPLE (.45 MICROHS)

0002 - FILTERED REPLICATE SAMPLE (.45 HICRONS)

G - PH > 9, POSSIBLE GROUT CONTAHINATION

NOOI - UAFILTERED SNMPLE

J - est IMATEd VALUE

l - LESS THAK THREE BORE VOLUMES REMOVED BEFORE SAMPLIMG 
GROUHDHATER QUALITY DATA BY LOCATION

SOWP ReV. O Data

SITE: MONO1 MONLMENT VALLEY

LOCATION: 0663

MORTH COOROIHATE: 59069.6 FT

EAST COORDIHATE: $\quad 88593.5$ FT

$04 / 21 / 88$ TO $12 / 13 / 94$

REPORT OATE: 06/20/95

FORMATION OF COMPLETION: DECHELLEY MEMBER OF THE CUTLER FORMATION, (DC) HYDRAULIC FLOW RELATIOHSHIP: DOWN GRADIENT (O)

\begin{tabular}{|c|c|c|c|c|c|c|c|c|}
\hline PARNMETER MAME & LOG DATE & $\underset{\text { ID }}{\text { SNMPLE }}$ & $\begin{array}{l}\text { UNITS OF } \\
\text { MEASURE }\end{array}$ & PVI & $\begin{array}{l}\text { PARAMETER } \\
\text { VALUE }\end{array}$ & FLAGS & $\begin{array}{l}\text { DETECTION } \\
\text { LIMIT }\end{array}$ & $\begin{array}{l}\text { PARANETER } \\
\text { UNCERTAINTY }\end{array}$ \\
\hline RADIUN-228 & $\begin{array}{l}06 / 25 / 89 \\
12 / 02 / 89 \\
06 / 25 / 90 \\
01 / 29 / 91 \\
06 / 04 / 91 \\
02 / 20 / 92 \\
08 / 15 / 92 \\
11 / 20 / 92\end{array}$ & $\begin{array}{l}0001 \\
0001 \\
0001 \\
0001 \\
0001 \\
0001 \\
0001 \\
0001\end{array}$ & $\mathrm{PCI} / \mathrm{L}$ & & $\begin{array}{l}0.3 \\
2.1 \\
0.6 \\
0.9 \\
2.9 \\
0.6 \\
0.4 \\
0.2\end{array}$ & $\begin{array}{l}L \\
L \\
J L \\
L \\
J L \\
L \\
L \\
L\end{array}$ & $\begin{array}{l}9 . \\
9 . \\
1 . \\
9 . \\
9 . \\
9.0 \\
1.7 \\
3.9\end{array}$ & $\begin{array}{l}0.7 \\
3.5 \\
0.8 \\
0.7 \\
2.2 \\
1.5 \\
0.9 \\
2.3\end{array}$ \\
\hline RADIUH-228 (TOTAL) & $11 / 20 / 92$ & N001 & $\mathrm{PCI} / \mathrm{L}$ & & 3.1 & $\mathbf{L}$ & 2.1 & 1.5 \\
\hline REDOX POTENTIAL & $\begin{array}{l}11 / 20 / 92 \\
06 / 24 / 93 \\
12 / 11 / 93 \\
05 / 14 / 94 \\
12 / 12 / 94\end{array}$ & $\begin{array}{l}\text { O001 } \\
\text { HOO1 } \\
\text { HOO1 } \\
\text { HOO1 } \\
\text { HOO1 }\end{array}$ & mVOLTS & & $\begin{array}{l}164.5 \\
282 \\
91 \\
-72 \\
352\end{array}$ & $\begin{array}{l}L \\
L \\
L \\
F\end{array}$ & $\begin{array}{l}- \\
- \\
-\end{array}$ & $\begin{array}{l}- \\
- \\
-\end{array}$ \\
\hline SELENIUM & $\begin{array}{l}04 / 26 / 88 \\
06 / 25 / 89 \\
12 / 02 / 89 \\
06 / 25 / 90 \\
01 / 29 / 91 \\
06 / 04 / 91 \\
02 / 20 / 92 \\
08 / 15 / 92 \\
11 / 20 / 92\end{array}$ & $\begin{array}{l}0001 \\
0001 \\
0001 \\
0001 \\
0001 \\
0001 \\
0001 \\
0001 \\
0001\end{array}$ & $M G / L$ & $\begin{array}{l}< \\
< \\
< \\
< \\
< \\
< \\
< \\
< \\
<\end{array}$ & $\begin{array}{l}0.005 \\
0.005 \\
0.005 \\
0.005 \\
0.005 \\
0.005 \\
0.005 \\
0.005 \\
0.0015\end{array}$ & $\begin{array}{l}G L \\
L \\
L \\
J L \\
L \\
J L \\
L \\
U L \\
L H\end{array}$ & $\begin{array}{l}0.005 \\
0.005 \\
0.005 \\
0.005 \\
0.005 \\
0.005 \\
0.005 \\
0.005 \\
0.0015\end{array}$ & $\begin{array}{l}- \\
: \\
: \\
- \\
-\end{array}$ \\
\hline SELENIUM (TOTAL) & $11 / 20 / 92$ & HOOI & MG/L & $<$ & 0.0015 & $L$ & 0.0015 & - \\
\hline SILICA - SIO2 & $\begin{array}{l}06 / 25 / 89 \\
12 / 02 / 89 \\
06 / 25 / 90 \\
01 / 29 / 99 \\
06 / 04 / 99 \\
12 / 11 / 93 \\
12 / 12 / 94 \\
12 / 12 / 94\end{array}$ & $\begin{array}{l}0001 \\
0001 \\
0001 \\
0001 \\
0001 \\
0001 \\
0001 \\
0002\end{array}$ & $M G / L$ & & $\begin{array}{l}15 . \\
15.4 \\
13 . \\
12 . \\
12.8 \\
14.3 \\
11.5 \\
14.3\end{array}$ & $\begin{array}{l}L \\
L \\
J L \\
L \\
L \\
L \\
F \\
F\end{array}$ & $\begin{array}{l}2 . \\
2 . \\
2 . \\
2 . \\
0.1 \\
0.1 \\
0.1 \\
0.1\end{array}$ & $\begin{array}{l}- \\
\vdots \\
: \\
\vdots \\
-\end{array}$ \\
\hline SILICA - SIO2 (TOTAL) & $\begin{array}{l}06 / 24 / 93 \\
12 / 19 / 93\end{array}$ & $\begin{array}{l}\text { No01 } \\
\text { No01 }\end{array}$ & MG/L & & $\begin{array}{l}14.3 \\
14.0\end{array}$ & $\begin{array}{l}H L \\
L\end{array}$ & $\begin{array}{l}0.5 \\
0.9\end{array}$ & $\dot{-}$ \\
\hline SILVER & $\begin{array}{l}06 / 25 / 89 \\
12 / 02 / 89 \\
06 / 25 / 90 \\
01 / 29 / 91 \\
06 / 04 / 91\end{array}$ & $\begin{array}{l}0009 \\
0009 \\
0001 \\
0009 \\
0001\end{array}$ & $M G / L$ & $\begin{array}{l}< \\
< \\
< \\
< \\
<\end{array}$ & $\begin{array}{l}0.01 \\
0.01 \\
0.01 \\
0.01 \\
0.01\end{array}$ & $\begin{array}{l}L \\
L \\
J L \\
L \\
L\end{array}$ & $\begin{array}{l}0.01 \\
0.01 \\
0.01 \\
0.01 \\
0.01\end{array}$ & : \\
\hline Sooluy & $\begin{array}{l}04 / 26 / 88 \\
06 / 25 / 89 \\
12 / 02 / 89\end{array}$ & $\begin{array}{l}0001 \\
0001 \\
0001\end{array}$ & HG/L & & $\begin{array}{l}75.2 \\
77.6 \\
75 .\end{array}$ & $\begin{array}{l}G L \\
L \\
L\end{array}$ & $\begin{array}{l}0.002 \\
0.002 \\
0.002\end{array}$ & - \\
\hline
\end{tabular}

PARAMETER VALUE IMDICATOR (PVI): < - LESS THAN DETECTION LIMIT

SNMPLE IO COOES:

OTHER PARAMETER VALUE FLAGS:

OOO1 - FILTERED SAMPLE (.45 MICRONS)

F - LOH-FLOH SAMPLIMG/DEDICATED PLMP

OOO2 - FILTERED REPLICATE SAMPLE (.45 MICROHS)

G - PH > 9, POSSIBLE GROUT CONTAMIHATION

MOO1 - UNFILTERED SNMPLE

H - hOLD TIME EXPIRED, VALUE SUSPECT

J - ESTIMKTED VALUE

L - LESS THAH THREE BORE VOLUMES REMOVED BEFORE SAMPLIHG

W - SPIKE SAMPLE RECOVERY NOT HITHIK CONTROL LIMITS

$W$ - POST-DIGEST SPIKE OUT OF CNTR LIM HHILE SAMP ABS < 50\% SPIKE 
GROUNDHATER QUALITY DATA BY LOCATION

SOWP ReV. O Dato

SITE: MONO1 MONLNENT VALLEY

LOCATION: 0663

NORTH COORDINATE: 59069.6 FT

EAST COORDINATE: $\quad 88593.5$ FT

$04 / 21 / 88$ TO $12 / 13 / 94$

REPORT DATE: $06 / 20 / 95$

FORMATION OF COHPLETION: DECHELLEY MEMBER OF THE CUTLER FORMATIOH (DC) HYDRAULIC FLON RELATIOHSHIP: DOWN GRADIENT (D)

\begin{tabular}{|c|c|c|c|c|c|c|c|c|}
\hline PARAMETER MAME & LOG DATE & $\underset{10}{\text { SAMPLE }}$ & $\begin{array}{l}\text { UKITS OF } \\
\text { MEASURE }\end{array}$ & PV? & $\begin{array}{l}\text { PARAMETER } \\
\text { VALUE }\end{array}$ & FLAGS & $\begin{array}{l}\text { DETECTION } \\
\text { LIMIT }\end{array}$ & $\begin{array}{l}\text { PARAMETER } \\
\text { UHCERTAIHTY }\end{array}$ \\
\hline scoriun & $\begin{array}{l}06 / 25 / 90 \\
01 / 29 / 91 \\
06 / 04 / 91 \\
02 / 20 / 92 \\
08 / 15 / 92 \\
06 / 24 / 93 \\
12 / 11 / 93 \\
05 / 14 / 94 \\
12 / 12 / 94 \\
12 / 12 / 94\end{array}$ & $\begin{array}{l}0001 \\
0001 \\
0001 \\
0001 \\
0001 \\
0001 \\
0001 \\
0001 \\
0001 \\
0002\end{array}$ & MG/L & & $\begin{array}{l}76.7 \\
78.5 \\
80 . \\
75 . \\
75 \\
74 \\
74 \\
69 \\
73.9 \\
78.7\end{array}$ & $\begin{array}{l}J L \\
L \\
L \\
L \\
L \\
L \\
L \\
F \\
F\end{array}$ & $\begin{array}{l}0.002 \\
0.002 \\
1 . \\
1 . \\
5 \\
1 \\
1 \\
1 \\
1 \\
1\end{array}$ & $\begin{array}{l}- \\
: \\
- \\
- \\
- \\
-\end{array}$ \\
\hline SOOIUN (TOTAL) & $\begin{array}{l}06 / 24 / 93 \\
12 / 11 / 93 \\
05 / 14 / 94\end{array}$ & $\begin{array}{l}\text { NOOI } \\
\text { NOOI } \\
\text { NOOI }\end{array}$ & $M G / L$ & & $\begin{array}{l}70 \\
73 \\
74\end{array}$ & $\begin{array}{l}L \\
L\end{array}$ & $\begin{array}{l}1 \\
1 \\
1\end{array}$ & $\dot{-}$ \\
\hline SPECIFIC CONDUCTAKCE & $\begin{array}{l}04 / 26 / 88 \\
06 / 25 / 89 \\
12 / 02 / 89 \\
06 / 25 / 90 \\
01 / 29 / 91 \\
06 / 04 / 91 \\
02 / 20 / 92 \\
08 / 15 / 92 \\
11 / 20 / 92 \\
06 / 24 / 93 \\
12 / 11 / 93 \\
05 / 14 / 94 \\
12 / 12 / 94\end{array}$ & $\begin{array}{l}0001 \\
0001 \\
0001 \\
0001 \\
0001 \\
0001 \\
0001 \\
0001 \\
0001 \\
\text { N001 } \\
\text { N001 } \\
\text { N001 } \\
\text { N001 }\end{array}$ & UHHO/CM & & $\begin{array}{l}300 . \\
360 . \\
380 . \\
405 . \\
498 . \\
502 . \\
404 \\
413 \\
380 \\
408 \\
396 \\
437 \\
346\end{array}$ & $\begin{array}{l}G L \\
L \\
L \\
L \\
L \\
L \\
L \\
L \\
L \\
L \\
L \\
\text { FJ }\end{array}$ & $\begin{array}{l}- \\
: \\
: \\
: \\
: \\
: \\
: \\
1\end{array}$ & $\begin{array}{l}- \\
: \\
: \\
: \\
: \\
: \\
- \\
-\end{array}$ \\
\hline STRONTIUNA & $\begin{array}{l}06 / 25 / 90 \\
01 / 29 / 91 \\
06 / 04 / 91 \\
02 / 20 / 92 \\
08 / 15 / 92 \\
11 / 20 / 92 \\
06 / 24 / 93 \\
12 / 11 / 93 \\
05 / 14 / 94 \\
12 / 12 / 94 \\
12 / 12 / 94\end{array}$ & $\begin{array}{l}0001 \\
0001 \\
0001 \\
0001 \\
0001 \\
0001 \\
0001 \\
0001 \\
0001 \\
0001 \\
0002\end{array}$ & MG/L & & $\begin{array}{l}0.53 \\
0.5 \\
0.54 \\
0.52 \\
0.57 \\
0.61 \\
0.50 \\
0.54 \\
0.46 \\
0.57 \\
0.26\end{array}$ & $\begin{array}{l} \pm J L \\
L \\
L \\
L \\
L \\
L \\
L \\
L \\
F J \\
F J\end{array}$ & $\begin{array}{l}0.1 \\
0.1 \\
0.01 \\
0.01 \\
0.2 \\
0.00035 \\
0.01 \\
0.01 \\
0.01 \\
0.01 \\
0.01\end{array}$ & $\begin{array}{l}- \\
: \\
- \\
- \\
- \\
-\end{array}$ \\
\hline STRONTIUA (TOTAL) & $\begin{array}{l}11 / 20 / 92 \\
06 / 24 / 93 \\
12 / 11 / 93 \\
05 / 14 / 94\end{array}$ & $\begin{array}{l}\text { NoO1 } \\
\text { No01 } \\
\text { NOO1 } \\
\text { NO01 }\end{array}$ & $M G / L$ & & $\begin{array}{l}0.62 \\
0.47 \\
0.52 \\
0.49\end{array}$ & $\begin{array}{l}L \\
L\end{array}$ & $\begin{array}{l}0.00035 \\
0.01 \\
.0 .01 \\
0.01\end{array}$ & $\begin{array}{l}: \\
: \\
:\end{array}$ \\
\hline SULFATE & $\begin{array}{l}04 / 26 / 88 \\
06 / 25 / 89 \\
12 / 02 / 89 \\
06 / 25 / 90 \\
01 / 29 / 91 \\
06 / 04 / 91 \\
02 / 20 / 92\end{array}$ & $\begin{array}{l}0001 \\
0001 \\
0001 \\
0001 \\
0001 \\
0001 \\
0001\end{array}$ & MG/L & & $\begin{array}{l}53 . \\
46 . \\
48 . \\
40.7 \\
45.3 \\
44 . \\
38 .\end{array}$ & $\begin{array}{l}\text { GL } \\
L \\
L \\
d L \\
L \\
L \\
L\end{array}$ & $\begin{array}{c}0.1 \\
0.1 \\
0.1 \\
0.1 \\
0.1 \\
0.1 \\
10 .\end{array}$ & $\begin{array}{l}- \\
- \\
- \\
-\end{array}$ \\
\hline
\end{tabular}

PARAMETER YALUE INDICATOR (PVI): < - LESS THAN DETECTION LIMIT

\section{OTHER PARAMETER VALUE FLAGS:}

- DUPLICATE ANALYSIS MOT HITHIN CONTROL LIMITS

F - LON-FLON SAMPLING/DEDICATED PUMP

G - PH > 9. POSSIBLE GROUT CONTAMINATION

J. ESTIMATED VALUE

L - LESS tHAH THREE BORE VOLUHES REHOVED BEFORE SMPLING
SAMPLE ID COOES:

0001 - FILTERED SNMPLE (.45 MICRONS)

ODO2 - FILTERED REPLICATE SNMPLE (.45 MICRONS) NOO1 - UHFILTERED SAMPLE 
GROUHDHATER QUALITY DATA BY LOCATION

SOMP ReV. 0 Data

SITE: MOWO1 HOWUMENT VALLEY

LOCATION: 0663

MORTH COORDIHATE: 59069.6 FT

EAST COOROINATE: 88593.5 FT

$04 / 21 / 88$ TO $12 / 13 / 94$

REPORT OATE: 06/20/95

FORMATION OF COMPLETION: DECHELLEY MEMBER OF THE CUTLER FORMATION (DC) HYDRAULIC FLON RELATIONSHIP: DOWH GRADIENT (D)

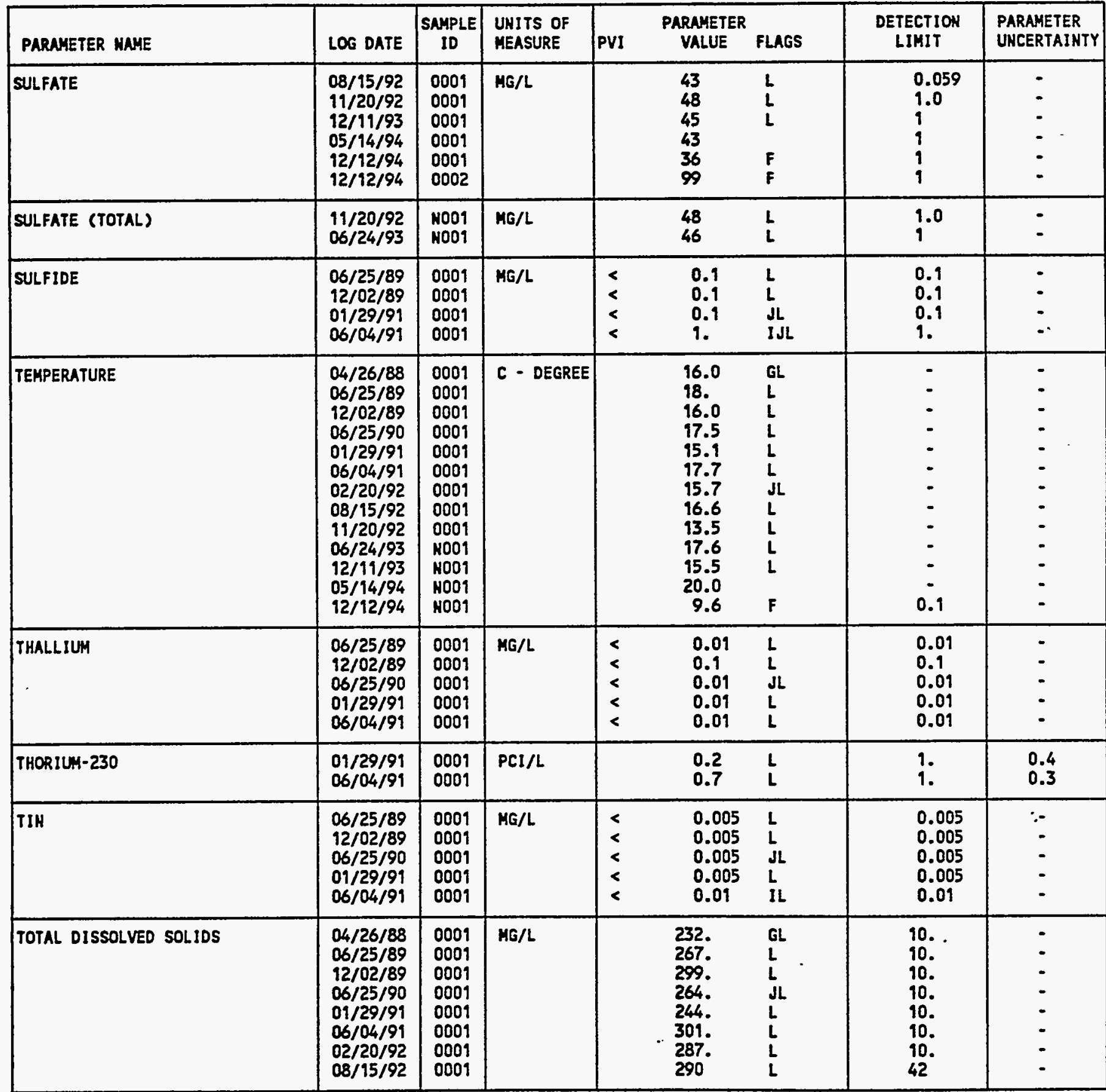

PARAMETER VALUE IMDICATOR (PVI): < - LESS THAN DETECTION LIMIT

SNMPLE ID COOES:

OTHER PARAMETER VALUE FLAGS:

F - LON-FLOW SAMPLING/DEDICATED PUMP

ODO1 - FILTERED SAMPLE (.45 MICROHS)

G - PH $>9$, POSSIBLE GROUT COHTAMINATION

0002 - FILTERED REPLICATE SAMPLE (.45 MICRONS)

1 - IMCREASED DETECTION LIMIT OUE TO REQUIRED DILUTION

J - ESTIMATED VALUE

L - LESS THAN THREE BORE VOLUMES REMOVED BEFORE SMMPLIHG 
GROUNDHATER QUALITY DATA BY LOCATION

SOWP ReV. O Data

SITE: MOHO9 MONUMENT VALLEY

LOCATION: 0663

MORTH COORDINATE: 59069.6 FT

EAST COORDINATE: 88593.5 FT

$04 / 21 / 88$ TO $12 / 13 / 94$

REPORT DATE: $06 / 20 / 95$

FORHATION OF COMPLETION: DECHELLEY MEMBER OF THE CUTLER FORMATION (DC) HYDRAULIC FLON RELATIONSHIP: DOWN GRADIENT (D)

\begin{tabular}{|c|c|c|c|c|c|c|c|c|}
\hline PARAMETER NAME & LOG DATE & $\underset{\text { SAMPLE }}{\text { SAMPL }}$ & $\begin{array}{l}\text { UHITS OF } \\
\text { MEASURE }\end{array}$ & PVI & $\begin{array}{l}\text { PARAMETER } \\
\text { VALUE }\end{array}$ & FLAGS & $\begin{array}{l}\text { DETECTION } \\
\text { LIMIT }\end{array}$ & $\begin{array}{l}\text { PARAMETER } \\
\text { UNCERTAINTY }\end{array}$ \\
\hline TOTAL DISSOLVED SOLIDS & $\begin{array}{l}12 / 11 / 93 \\
05 / 14 / 94 \\
12 / 12 / 94 \\
12 / 12 / 94\end{array}$ & $\begin{array}{l}0001 \\
0001 \\
0001 \\
0002\end{array}$ & MG/L & & $\begin{array}{l}300 \\
280 \\
266 \\
342\end{array}$ & $\begin{array}{l}L \\
F \\
F\end{array}$ & $\begin{array}{l}10 \\
10 \\
10 \\
10\end{array}$ & - \\
\hline TOTAL DISSOLVED SOLIDS (TOTAL) & $06 / 24 / 93$ & N001 & MG/L & & 290 & $L$ & 10 & - \\
\hline TOTAL ORGANIC CARBOH & $\begin{array}{l}04 / 26 / 88 \\
01 / 29 / 91 \\
06 / 04 / 91 \\
02 / 20 / 92 \\
08 / 15 / 92\end{array}$ & $\begin{array}{l}0001 \\
0001 \\
0001 \\
0001 \\
0001\end{array}$ & MG/L & $\begin{array}{l}< \\
< \\
< \\
<\end{array}$ & $\begin{array}{l}25.0 \\
1 . \\
1 . \\
1.0 \\
1.0\end{array}$ & $\begin{array}{l}G L \\
J L \\
L \\
L \\
L\end{array}$ & $\begin{array}{l}1 . \\
1 . \\
1 . \\
1.0\end{array}$ & $\begin{array}{l}- \\
- \\
- \\
-\end{array}$ \\
\hline TURBIDITY & $\begin{array}{l}05 / 14 / 94 \\
12 / 12 / 94\end{array}$ & $\begin{array}{l}\text { N001 } \\
\text { N001 }\end{array}$ & NTU & & $\begin{array}{l}1 \\
7\end{array}$ & $\mathbf{F}$ & $i^{-}$ & - \\
\hline URAKIUM & $\begin{array}{l}04 / 26 / 88 \\
06 / 25 / 89 \\
12 / 02 / 89 \\
06 / 25 / 90 \\
01 / 29 / 91 \\
06 / 04 / 91 \\
02 / 20 / 92 \\
08 / 15 / 92 \\
11 / 20 / 92 \\
06 / 24 / 93 \\
12 / 11 / 93 \\
05 / 14 / 94 \\
12 / 12 / 94 \\
12 / 12 / 94\end{array}$ & $\begin{array}{l}0001 \\
0001 \\
0001 \\
0001 \\
0001 \\
0001 \\
0001 \\
0001 \\
0001 \\
0001 \\
0001 \\
0001 \\
0001 \\
0002\end{array}$ & MG/L & $\begin{array}{l}< \\
< \\
< \\
< \\
< \\
< \\
< \\
< \\
< \\
< \\
<\end{array}$ & $\begin{array}{l}0.0007 \\
0.0003 \\
0.002 \\
0.0005 \\
0.0003 \\
0.001 \\
0.001 \\
0.001 \\
0.001 \\
0.001 \\
0.001 \\
0.001 \\
0.001 \\
0.001\end{array}$ & $\begin{array}{l}\text { GJL } \\
J L \\
J L \\
J L \\
L \\
L \\
L \\
L \\
L \\
L \\
L \\
F \\
F\end{array}$ & $\begin{array}{l}0.003 \\
0.003 \\
0.003 \\
0.003 \\
0.0003 \\
0.001 \\
0.001 \\
0.001 \\
0.001 \\
0.001 \\
0.001 \\
0.001 \\
0.001 \\
0.001\end{array}$ & $\begin{array}{l}- \\
: \\
- \\
- \\
: \\
- \\
- \\
- \\
-\end{array}$ \\
\hline URAKIU: (TOTAL) & $\begin{array}{l}11 / 20 / 92 \\
06 / 24 / 93 \\
12 / 99 / 93 \\
05 / 14 / 94\end{array}$ & $\begin{array}{l}\text { NOO1 } \\
\text { N001 } \\
\text { NO01 } \\
\text { N001 }\end{array}$ & MG/L & $\begin{array}{l}< \\
<\end{array}$ & $\begin{array}{l}0.001 \\
0.001 \\
0.001 \\
0.001\end{array}$ & $\begin{array}{l}L \\
L \\
L\end{array}$ & $\begin{array}{l}0.001 \\
0.001 \\
0.001 \\
0.001\end{array}$ & $:$ \\
\hline VANADIUA & $\begin{array}{l}06 / 25 / 89 \\
12 / 02 / 89 \\
06 / 25 / 90 \\
01 / 29 / 91 \\
06 / 04 / 91 \\
02 / 20 / 92 \\
08 / 15 / 92 \\
11 / 20 / 92 \\
06 / 24 / 93 \\
12 / 11 / 93 \\
05 / 14 / 94 \\
12 / 12 / 94 \\
12 / 12 / 94\end{array}$ & $\begin{array}{l}0001 \\
0001 \\
0001 \\
0001 \\
0001 \\
0001 \\
0001 \\
0001 \\
0001 \\
0001 \\
0001 \\
0001 \\
0002\end{array}$ & $\begin{array}{c}\text { MG/L } \\
.\end{array}$ & $\begin{array}{l}< \\
< \\
< \\
< \\
< \\
< \\
< \\
< \\
< \\
< \\
< \\
< \\
<\end{array}$ & $\begin{array}{l}0.01 \\
0.01 \\
0.01 \\
0.01 \\
0.01 \\
0.01 \\
0.05 \\
0.0019 \\
0.01 \\
0.01 \\
0.01 \\
0.01 \\
0.01\end{array}$ & $\begin{array}{l}L \\
L \\
J L \\
L \\
L \\
L \\
L \\
L \\
L \\
L \\
F \\
F\end{array}$ & $\begin{array}{l}0.01 \\
0.01 \\
0.01 \\
0.01 \\
0.01 \\
0.01 \\
0.05 \\
0.0019 \\
0.01 \\
0.01 \\
0.01 \\
0.01 \\
0.01\end{array}$ & $\begin{array}{l}- \\
\dot{-} \\
- \\
- \\
- \\
- \\
- \\
-\end{array}$ \\
\hline VANADIUA (TOTAL) & $\begin{array}{l}11 / 20 / 92 \\
06 / 24 / 93\end{array}$ & $\begin{array}{l}\text { N001 } \\
\text { N001 }\end{array}$ & $M G / L$ & $<$ & $\begin{array}{l}0.0019 \\
0.01\end{array}$ & $L$ & $\begin{array}{l}0.0019 \\
0.01\end{array}$ & - \\
\hline
\end{tabular}

PARAMETER VALUE IMDICATOR (PVI): < - LESS THAM DETECTION LIMIT

SAMPLE ID COOES:

0001 - FILTERED SNMPLE (.45 MICRONS)

OTHER PARAMETER VALUE FLAGS:

* - DUPLICATE AMALYSIS MDT UITHIH CONTROL LIMITS

0002 - FILTERED REPLICATE SAMPLE (.45 MICRONS)

F - LON-FLON SNMPLING/DEDICATED PUMP

NOO1 - UHFILTERED SAMPLE

G - PH > 9, POSSIBLE GROUT CONTAHIMATION

- ESTIMLTED VALUE

- L - LESS THAN THREE BORE VOLLAES REMOVED BEFORE SAMPLING 
GROUHDWATER QUALITY DATA BY LOCATION

SOWP ReV. 0 Data

SITE: MONO1 MOHUNEHT VALLEY

LOCATIOH: 0663

MORTH COOROINATE: 59069.6 FT

EAST COORDIKATE: 88593.5 FT

$04 / 21 / 88$ TO $12 / 13 / 94$

REPORT DATE: 06/20/95

FORMATION OF COMPLETION: DECHELLEY MEMBER OF THE CUTLER FORMATION (DC)

HYDRAULIC FLON RELATIOHSHIP: DOWN GRADIENT (D)

\begin{tabular}{|c|c|c|c|c|c|c|c|c|}
\hline PARAMETER MAME & LOG DATE & $\begin{array}{c}\text { SAMPLE } \\
10\end{array}$ & $\begin{array}{l}\text { UWITS OF } \\
\text { MEASURE }\end{array}$ & PVI & $\begin{array}{l}\text { PARAMETER } \\
\text { VALUE }\end{array}$ & FLAGS & $\begin{array}{l}\text { DETECTION } \\
\text { LIMIT }\end{array}$ & $\begin{array}{l}\text { PARAMETER } \\
\text { UHCERTAINTY }\end{array}$ \\
\hline VAHADIUA (TOTAL) & $\begin{array}{l}12 / 11 / 93 \\
05 / 14 / 94\end{array}$ & $\begin{array}{l}\text { NOO1 } \\
\text { N001 }\end{array}$ & $M G / L$ & $<$ & $\begin{array}{l}0.01 \\
0.01\end{array}$ & $\mathbf{L}$ & $\begin{array}{l}0.01 \\
0.01\end{array}$ & $\dot{-}$ \\
\hline ZINC & $\begin{array}{l}06 / 25 / 89 \\
12 / 02 / 89 \\
06 / 25 / 90 \\
01 / 29 / 91 \\
06 / 04 / 91 \\
02 / 20 / 92 \\
08 / 15 / 92 \\
11 / 20 / 92 \\
06 / 24 / 93\end{array}$ & $\begin{array}{l}0001 \\
0001 \\
0001 \\
0001 \\
0001 \\
0001 \\
0001 \\
0001 \\
0001\end{array}$ & $M G / L$ & $\begin{array}{l}< \\
< \\
< \\
< \\
< \\
< \\
< \\
<\end{array}$ & $\begin{array}{l}0.005 \\
0.005 \\
0.040 \\
0.005 \\
0.005 \\
0.005 \\
0.02 \\
0.0010 \\
0.005\end{array}$ & $\begin{array}{l}L \\
L \\
J L \\
L \\
L \\
L \\
L \\
L \\
J L\end{array}$ & $\begin{array}{l}0.005 \\
0.005 \\
0.005 \\
0.005 \\
0.005 \\
0.005 \\
0.02 \\
0.0010 \\
0.005\end{array}$ & $\begin{array}{l}- \\
- \\
- \\
- \\
- \\
-\end{array}$ \\
\hline ZINC (TOTAL) & $\begin{array}{l}11 / 20 / 92 \\
06 / 24 / 93\end{array}$ & $\begin{array}{l}\text { NOOI } \\
\text { HOOI }\end{array}$ & MG/L & & $\begin{array}{l}0.0070 \\
0.007\end{array}$ & $\frac{L}{J L}$ & $\begin{array}{l}0.0010 \\
0.005\end{array}$ & $\dot{-}$ \\
\hline
\end{tabular}

PARAMETER VALUE IHDICATOR (PVI): < - LESS THAN DETECTION LIMIT

SAMPLE ID COOES:

OTHER PARAMETER VALUE FLAGS:

0001 - FILTERED SAMPLE (.45 MICRONS)

J - ESTIMATED VALUE

HOO1 - UNFILTERED SAMPLE

L - LESS thaN three bORE VOLUMES REMOVED BEFORE SAMPLING 
GROUMDHATER QUALITY DATA BY LOCATION

SOMP ReV. O Data

SITE: MONOY MONUMENT VALLEY

LOCATION: 0664

MORTH COORDINATE: 61255.9 FT

EAST COORDINATE: 89537.0 FT

$04 / 21 / 88$ TO $12 / 13 / 94$

REPORT DATE: 06/20/95

FORMATION OF CONPLETION: DECHELLEY MEMBER OF THE CUTLER FORMATION (DC) HYDRAULIC FLON RELATIOHSHIP: DOWH GRADIENT (D)

\begin{tabular}{|c|c|c|c|c|c|c|c|c|}
\hline PARAMETER MAME & LOG DATE & $\begin{array}{c}\text { SNMPLE } \\
\text { ID }\end{array}$ & $\begin{array}{l}\text { UNITS OF } \\
\text { MEASURE }\end{array}$ & PVI & $\begin{array}{l}\text { PARAMETER } \\
\text { VALUE }\end{array}$ & FLAGS & $\begin{array}{l}\text { DETECTIOW } \\
\text { LIMIT }\end{array}$ & $\begin{array}{l}\text { PARAMETER } \\
\text { UHCERTAIHTY }\end{array}$ \\
\hline ALKALIMITY & $\begin{array}{l}06 / 28 / 93 \\
05 / 12 / 94 \\
12 / 13 / 94\end{array}$ & $\begin{array}{l}\text { No09 } \\
\text { NO01 } \\
\text { N001 }\end{array}$ & MG/L CACO3 & & $\begin{array}{l}210 \\
208 \\
253\end{array}$ & $\mathbf{F}$ & $10^{-}$ & 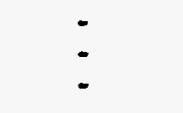 \\
\hline AMHOHIUM (TOTAL) & $\begin{array}{l}06 / 28 / 93 \\
05 / 14 / 94 \\
12 / 13 / 94\end{array}$ & $\begin{array}{l}\text { NO01 } \\
\text { NO01 } \\
\text { N001 }\end{array}$ & MG/L & $<$ & $\begin{array}{l}0.1 \\
0.1 \\
0.35\end{array}$ & $\mathbf{F}$ & $\begin{array}{l}0.9 \\
0.1 \\
0.1\end{array}$ & : \\
\hline ARSEKIC & $06 / 28 / 93$ & 0001 & MG/L & $<$ & 0.005 & & 0.005 & - \\
\hline ARSEKIC (TOTAL) & $06 / 28 / 93$ & N001 & $M G / L$ & $<$ & 0.005 & & 0.005 & - \\
\hline BARIUM & $06 / 28 / 93$ & 0001 & MG/L & & 0.2 & & 0.1 & - \\
\hline BARIUH (TOTAL) & $06 / 28 / 93$ & N001 & $M G / L$ & & 0.2 & & 0.1 & - \\
\hline CALCIUM & $\begin{array}{l}06 / 28 / 93 \\
05 / 12 / 94 \\
12 / 13 / 94\end{array}$ & $\begin{array}{l}0001 \\
0001 \\
0001\end{array}$ & $M G / L$ & & $\begin{array}{r}14.2 \\
12.8 \\
5.3\end{array}$ & $\mathrm{~F}$ & $\begin{array}{l}0.5 \\
0.5 \\
0.5\end{array}$ & : \\
\hline CALCIUM (TOTAL) & $\begin{array}{l}06 / 28 / 93 \\
05 / 12 / 94\end{array}$ & $\begin{array}{l}\text { N001 } \\
\text { HOOI }\end{array}$ & $M G / L$ & & $\begin{array}{l}13.6 \\
14.9\end{array}$ & & $\begin{array}{l}0.5 \\
0.5\end{array}$ & - \\
\hline CHLORIDE & $\begin{array}{l}05 / 12 / 94 \\
12 / 13 / 94\end{array}$ & $\begin{array}{l}0001 \\
0001\end{array}$ & $M G / L$ & & $\begin{array}{l}8.7 \\
6\end{array}$ & $\mathbf{F}$ & $\begin{array}{l}0.5 \\
0.5\end{array}$ & $:$ \\
\hline CHLORIDE (TOTAL) & $06 / 28 / 93$ & N001 & MG/L & & 9.0 & & 0.5 & - \\
\hline DISSOLVED OXYGEN & $\begin{array}{l}06 / 28 / 93 \\
05 / 12 / 94 \\
12 / 13 / 94\end{array}$ & $\begin{array}{l}\text { NOO1 } \\
\text { N001 } \\
\text { N001 }\end{array}$ & MG/L & $<$ & $\begin{array}{l}0.1 \\
0.08 \\
0.02\end{array}$ & $\mathbf{F}$ & $\begin{array}{c}- \\
0.02\end{array}$ & : \\
\hline GROSS ALPHA & $06 / 29 / 93$ & 0001 & $\mathrm{PCI} / \mathrm{L}$ & & 9 & & 3 & 5 \\
\hline GROSS ALPHA (TOTAL) & $06 / 28 / 93$ & NOOI & PCI/L & & 11 & & 3 & 6 \\
\hline GROSS BETA & $06 / 29 / 93$ & 0001. & $\mathrm{PCI} / \mathrm{L}$ & & 7 & & 4 & 4 \\
\hline GROSS BETA (TOTAL) & $06 / 28 / 93$ & N001 & $P C I / L$ & & 7 & & 4 & 4 \\
\hline IRON & $06 / 28 / 93$ & 0001. & MG/L & & 0.05 & $J$ & 0.03 & - \\
\hline IRON (TOTAL) & $06 / 28 / 93$ & N001 & MG/L & & 0.13 & J & 0.03 & $\cdot$ \\
\hline MAGHESIUA & $\begin{array}{l}06 / 28 / 93 \\
05 / 12 / 94 \\
12 / 13 / 94\end{array}$ & $\begin{array}{l}0009 \\
0009 \\
0001\end{array}$ & MG/L & & $\begin{array}{c}16.6 \\
14.8 \\
4\end{array}$ & $\mathbf{F}$ & $\begin{array}{l}0.1 \\
0.1 \\
0.1\end{array}$ & $:$ \\
\hline MAGKESIUA (TOTAL) & $\begin{array}{l}06 / 28 / 93 \\
05 / 12 / 94\end{array}$ & $\begin{array}{l}\text { N001 } \\
\text { N001 }\end{array}$ & MG/L & & $\begin{array}{l}16.5 \\
17.0\end{array}$ & & $\begin{array}{l}0.1 \\
0.1\end{array}$ & : \\
\hline MANGAHESE & $\begin{array}{l}06 / 28 / 93 \\
05 / 12 / 94 \\
12 / 13 / 94\end{array}$ & $\begin{array}{l}0001 \\
0001 \\
0001\end{array}$ & $M G / L$ & $<$ & $\begin{array}{l}0.03 \\
0.02 \\
0.01\end{array}$ & $\mathbf{F}$ & $\begin{array}{l}0.01 \\
0.01 \\
0.01\end{array}$ & $:$ \\
\hline MANGAKESE (TOTAL) & $06 / 28 / 93$ & N001 & MG/L & & 0.03 & & 0.01 & - \\
\hline
\end{tabular}

PARAMETER VALUE INDICATOR (PVI): < - LESS THAN DETECTION LIMIT

SAMPLE ID COOES:

0001 - FILTERED SAMPLE (.45 MICRONS)

OTHER PARAMETER VALUE FLAGS:

NO01 - UNFILTERED SAMPLE

F - LOU-FLOA SAMPLIHG/DEDICATED PUMP

J. estimated value 
GROUNDWATER OUALITY DATA BY LOCATION

SOMP Rev. O Data

SITE: MONOI MONUMENT VALLEY

LOCATION: 0664

MORTH COORDIHATE: 61255.9 FT

EAST COORDIHATE: 89537.0 FT

$04 / 21 / 88$ TO $12 / 13 / 94$

REPORT DATE: $06 / 20 / 95$

FORHATION OF CONPLETION: OECHELLEY MEMBER OF THE CUTLER FORMATION (DC) HYDRAULIC FLOU RELATIONSHIP: DOWN GRADIENT (D)

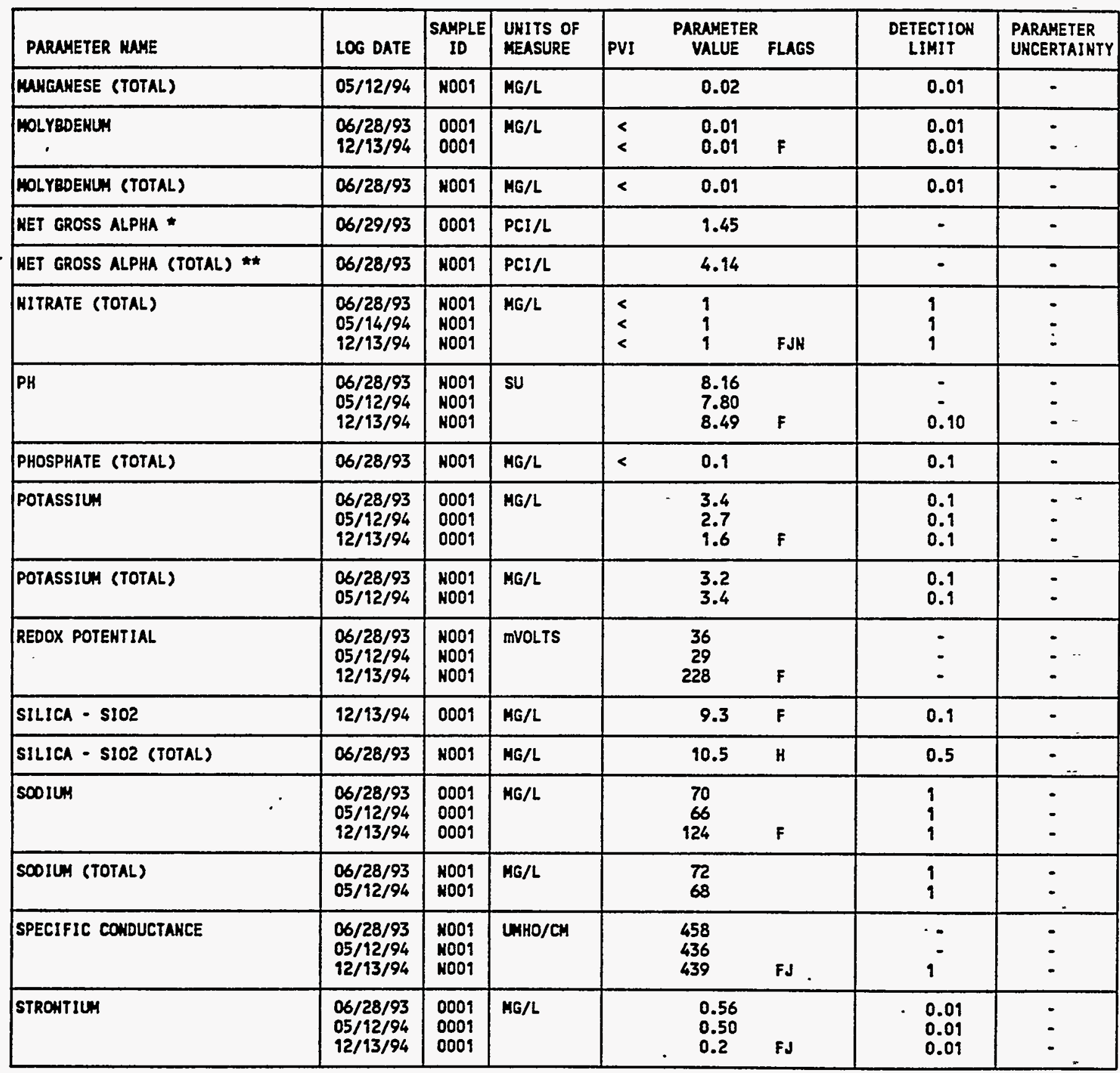

- MET GROSS ALPHA (GROSS ALPHA - URANIUM) WITH 1 MG URANIUH $=686$ PCI

- MET GROSS ALPHA (TOTAL) (TOTAL GROSS ALPHA - TOTAL URAHILH)

PARAMETER VALUE INOICATOR (PVI): < - LESS THAN DETECTION LIMIT

SAMPLE ID COOES:

OTHER PARAMETER VALUE FLAGS:

OOO1 - FILTERED SNMPLE (.45 MICRONS)

F - LON-FLOW SAMPLING/DEDICATED PUNP

NOO1 - UNFILTERED SNMPLE

H - HOLD TIME EXPIRED, VALLE SUSPECT

$J$ - ESTIMATED VALUE

H - SPIKE SAMPLE RECOVERY NOT MITHIN CONTROL LIMITS 
GROUNDWATER QUALITY DATA BY LOCATION

SOUP ReV. $O$ Dato

SITE: MONO1 MONUMENT VALLEY

LOCATION: 0664

MORTH COORDIHATE: 61255.9 FT

EAST COORDIKATE: 89537.0 FT

04/21/88 TO $12 / 13 / 94$

REPORT DATE: $06 / 20 / 95$

FORMATION OF COMPLETION: DECHELLEY MEMBER OF THE CUTLER FORMATION (DC) HYDRAULIC FLON RELATIOWSHIP: DONN GRADIENT (D)

\begin{tabular}{|c|c|c|c|c|c|c|c|c|}
\hline PARNAETER MAME & LOG DATE & $\underset{10}{\text { SAMPLE }}$ & $\begin{array}{l}\text { UNITS OF } \\
\text { MEASURE }\end{array}$ & PVI & $\begin{array}{l}\text { PARAMETER } \\
\text { VALUE }\end{array}$ & FLAGS & $\begin{array}{l}\text { DETECTION } \\
\text { LIMIT }\end{array}$ & $\begin{array}{l}\text { PARAMETER } \\
\text { UHCERTAINTY }\end{array}$ \\
\hline STRONTIU: (TOTAL) & $\begin{array}{l}06 / 28 / 93 \\
05 / 12 / 94\end{array}$ & $\begin{array}{l}\text { No01 } \\
\text { N001 }\end{array}$ & MG/L & & $\begin{array}{l}0.55 \\
0.57\end{array}$ & & $\begin{array}{l}0.01 \\
0.01\end{array}$ & - \\
\hline SULFATE & $\begin{array}{l}05 / 12 / 94 \\
12 / 13 / 94\end{array}$ & $\begin{array}{l}0001 \\
0001\end{array}$ & MG/L & & $\begin{array}{l}39 \\
15\end{array}$ & $\mathbf{F}$ & 1 & - \\
\hline SULFATE (TOTAL) & $06 / 28 / 93$ & no01 & $M G / L$ & & 41 & & 1 & - \\
\hline TEMPERATURE & $\begin{array}{l}06 / 28 / 93 \\
05 / 12 / 94 \\
12 / 13 / 94\end{array}$ & $\begin{array}{l}\text { N001 } \\
\text { N001 } \\
\text { N001 }\end{array}$ & C - DEGREE & & $\begin{array}{l}20.7 \\
17.5 \\
13.9\end{array}$ & $\mathbf{F}$ & $\overline{0}$ & : \\
\hline TOTAL DISSOLVED SOLIDS & $\begin{array}{l}05 / 12 / 94 \\
12 / 13 / 94\end{array}$ & $\begin{array}{l}0001 \\
0001\end{array}$ & MG/L & & $\begin{array}{l}300 \\
314\end{array}$ & $\mathbf{F}$ & $\begin{array}{l}10 \\
10\end{array}$ & $\because$ \\
\hline TOTAL DISSOLVED SOLIDS (TOTAL) & $06 / 28 / 93$ & N001 & ME/L & & 280 & H & 10 & $\cdot$ \\
\hline TURBIDITY & $\begin{array}{l}05 / 12 / 94 \\
12 / 13 / 94\end{array}$ & $\begin{array}{l}\text { H001 } \\
\text { N001 }\end{array}$ & NTU & $<$ & $\begin{array}{l}0 \\
1\end{array}$ & $\mathbf{F}$ & 1 & - \\
\hline URAKIL:M & $\begin{array}{l}06 / 29 / 93 \\
05 / 12 / 94 \\
12 / 13 / 94\end{array}$ & $\begin{array}{l}0001 \\
0001 \\
0001\end{array}$ & $M G / L$ & $<$ & $\begin{array}{l}0.011 \\
0.011 \\
0.001\end{array}$ & $\mathbf{F}$ & $\begin{array}{l}0.001 \\
0.001 \\
0.001\end{array}$ & : \\
\hline URAKIUN (TOTAL) & $\begin{array}{l}06 / 28 / 93 \\
05 / 12 / 94\end{array}$ & $\begin{array}{l}\text { No01 } \\
\text { Ho01 }\end{array}$ & MG/L & & $\begin{array}{l}0.01 \\
0.012\end{array}$ & & $\begin{array}{l}0.001 \\
0.001\end{array}$ & $\dot{-}$ \\
\hline VAHADIUM & $\begin{array}{l}06 / 28 / 93 \\
05 / 12 / 94 \\
12 / 13 / 94\end{array}$ & $\begin{array}{l}0001 \\
0001 \\
0001\end{array}$ & MG/L & $\begin{array}{l}< \\
< \\
<\end{array}$ & $\begin{array}{l}0.01 \\
0.01 \\
0.01\end{array}$ & $\mathbf{F}$ & $\begin{array}{l}0.01 \\
0.01 \\
0.01\end{array}$ & : \\
\hline $\begin{array}{l}\text { VANADIUM (TOTAL) } \\
\text {.. }\end{array}$ & $\begin{array}{l}06 / 28 / 93 \\
05 / 12 / 94\end{array}$ & $\begin{array}{l}\text { N001 } \\
\text { N001 }\end{array}$ & MG/L & $<$ & $\begin{array}{l}0.01 \\
0.01\end{array}$ & & $\begin{array}{l}0.01 \\
0.01\end{array}$ & - \\
\hline ZINC & $06 / 28 / 93$ & 0001 & $M G / L$ & & 0.022 & J & 0.005 & - \\
\hline ZINC (TOTAL) & $06 / 28 / 93$ & NoO1. & $M G / L$ & & 0.030 & J & 0.005 & - \\
\hline
\end{tabular}

PARAMETER VALUE IMDICATOR (PVI): < - LESS THAN DETECTION LIMIT

SAMPLE IO COOES:

OOO1 - FILTERED SAMPLE (.45 MICRONS)

OTHER PARAMETER VALUE FLAGS:

NOO - UNFILTERED SAMPLE

F - LON-FLOU SAMPLIMG/DEDICATED PUMP

H - HOLD TIME EXPIRED, VALUE SUSPECT

J - estiMMTED VALUE 
GROUHDHATER OUALITY DATA BY LOCATION

SOMP Rev. O Data

SITE: MOHOY MONUHENT VALLEY

LOCATION: 0668

MORTH COORDINATE: 60179.4 FT

EAST COORDINATE: 88287.0 FT

$04 / 21 / 88$ TO $12 / 13 / 94$

REPORT DATE: $06 / 20 / 95$

FORHATION OF COMPLETION: DECHELLEY MEMBER OF THE CUTLER FORMATION (DC) HYDRAULIC FLOW RELATIOHSHIP: DOWN GRADIENT (D)

\begin{tabular}{|c|c|c|c|c|c|c|c|c|}
\hline PARAMETER MAME & LOG DATE & $\begin{array}{c}\text { SAMPLEE } \\
\text { ID }\end{array}$ & $\begin{array}{l}\text { UHITS OF } \\
\text { MEASURE }\end{array}$ & PVI & $\begin{array}{l}\text { PARAMETER } \\
\text { VALUE }\end{array}$ & FLAGS & $\begin{array}{l}\text { DETECTION } \\
\text { LIMIT }\end{array}$ & $\begin{array}{l}\text { PARAMETER } \\
\text { UNCERTAIHTY }\end{array}$ \\
\hline ALKALIHITY & $\begin{array}{l}06 / 25 / 89 \\
06 / 29 / 93 \\
05 / 91 / 94\end{array}$ & $\begin{array}{l}0001 \\
\text { NO01 } \\
\text { NO01 }\end{array}$ & $\mathrm{MG} / \mathrm{LACO}$ & & $\begin{array}{l}178 . \\
186 \\
215\end{array}$ & $L$ & : & : \\
\hline ALUNIHUM & $06 / 25 / 89$ & 0001 & MG/L & $<$ & 0.1 & $\mathbf{L}$ & 0.1 & - \\
\hline Am:HIUM & $06 / 25 / 89$ & 0001 & $M G / L$ & $<$ & 0.1 & $\mathbf{L}$ & 0.1 & - \\
\hline NHONIUM (TOTAL) & $\begin{array}{l}06 / 29 / 93 \\
05 / 14 / 94\end{array}$ & $\begin{array}{l}\text { NoO1 } \\
\text { HOO1 }\end{array}$ & MG/L & $<$ & $\begin{array}{l}0.9 \\
0.1\end{array}$ & & $\begin{array}{l}0.9 \\
0.1\end{array}$ & - \\
\hline AHTIMOHY & $06 / 25 / 89$ & 0001 & MG/L & $<$ & 0.003 & $\mathbf{L}$ & 0.003 & - \\
\hline ARSENIC & $\begin{array}{l}06 / 25 / 89 \\
06 / 29 / 93\end{array}$ & $\begin{array}{l}0001 \\
0001\end{array}$ & $M G / L$ & $<$ & $\begin{array}{l}0.09 \\
0.015\end{array}$ & $\mathbf{L}$ & $\begin{array}{l}0.01 \\
0.005\end{array}$ & - \\
\hline ARSEHIC (TOTAL) & $06 / 29 / 93$ & N001 & $M G / L$ & & 0.016 & & 0.005 & - \\
\hline BARIUM & $\begin{array}{l}06 / 25 / 89 \\
06 / 29 / 93\end{array}$ & $\begin{array}{l}0001 \\
0001\end{array}$ & $M G / L$ & $<$ & $\begin{array}{l}0.1 \\
0.1\end{array}$ & $L$ & $\begin{array}{l}0.1 \\
0.1\end{array}$ & : \\
\hline BARIUH (TOTAL) & $06 / 29 / 93$ & N001 & MG/L & & 0.1 & & 0.1 & - \\
\hline BERYLLIUM & $06 / 25 / 89$ & 0001 & MG/L & $<$ & 0.01 & $L$ & 0.01 & $\cdot$ \\
\hline BOROH & $06 / 25 / 89$ & 0001 & MG/L & $<$ & 0.1 & $l$ & 0.1 & - \\
\hline CADMIUH & $06 / 25 / 89$ & 0001 & $M G / L$ & $<$ & 0.001 & $L$ & 0.001 & - \\
\hline CALCIUM & $\begin{array}{l}06 / 25 / 89 \\
06 / 29 / 93 \\
05 / 11 / 94\end{array}$ & $\begin{array}{l}0001 \\
0001 \\
0001\end{array}$ & $M G / L$ & & $\begin{array}{l}6.04 \\
13.3 \\
14.3\end{array}$ & $L$ & $\begin{array}{l}0.01 \\
0.5 \\
0.5\end{array}$ & : \\
\hline CALCIUM (TOTAL) & $\begin{array}{l}06 / 29 / 93 \\
05 / 11 / 94\end{array}$ & $\begin{array}{l}\text { NO01 } \\
\text { NO01 }\end{array}$ & $M G / L$ & & $\begin{array}{l}12.4 \\
34.0\end{array}$ & & $\begin{array}{l}0.5 \\
0.5\end{array}$ & - \\
\hline CHLORIDE & $\begin{array}{l}06 / 25 / 89 \\
05 / 11 / 94\end{array}$ & $\begin{array}{l}0001 \\
0001\end{array}$ & - MG/L & . & $\begin{array}{l}6.1 \\
7.4\end{array}$ & $L$ & $\begin{array}{l}1 . \\
0.5\end{array}$ & : \\
\hline CHLORIDE (TOTAL) & $06 / 29 / 93$ & HOO1 & MG/L & & 5.7 & & 0.5 & - \\
\hline ChromiLm & $06 / 25 / 89$ & 0001 & MG/L & $<$ & 0.01 & $L$ & 0.01 & - \\
\hline COBALT & $06 / 25 / 89$ & 0001 & $M G / L$ & $<$ & 0.05 & $L$ & 0.05 & - \\
\hline COPPER & $06 / 25 / 89$ & 0001 & MG/L & & 0.02 & $L$ & 0.02 & - \\
\hline CYANIDE & $06 / 25 / 89$ & 0001 & $M G / L$ & $<$ & 0.09 & $L$ & 0.01 & - \\
\hline DISSOLVED OXYGEH & $\begin{array}{l}06 / 29 / 93 \\
05 / 19 / 94\end{array}$ & $\begin{array}{l}\text { N001 } \\
\text { N001 }\end{array}$ & MG/L & & $\begin{array}{l}3.2 \\
1.8\end{array}$ & & - & $\because$ \\
\hline FLUORIDE & $06 / 25 / 89$ & 0001 & MG/L & & 0.3 & $L$ & 0.1 & - \\
\hline CROSS ALPHA & $06 / 29 / 93$ & 0001 & $\mathrm{PCI} / \mathrm{L}$ & & 6 & & 2 & 5 \\
\hline GROSS ALPHA (TOTAL) & $06 / 29 / 93$ & NO01 & PCI/L & & 0 & & 2 & 4 \\
\hline
\end{tabular}

PARAMETER VALUE INDICATOR (PVI): < - LESS THAN DETECTION LIMIT

OTHER PARAMETER VALUE FLAGS:

SNAPLE ID COOES:

ODO - FILTERED SAMPLE (.45 MICRONS)

L - LESS THAN THREE BORE VOLUMES REMOVED BEFORE SMMLIHG

HOOI - UNFILTERED SAMPLE 
GROUNDHATER GUALITY DATA BY LOCATION

SOWP ReV. O Data

SITE: MONOI MONLWENT VALLEY

LOCATION: 0668

NORTH COORDINATE: 60179.4 FT

EAST COORDINATE: 88287.0 FT

$04 / 21 / 88$ TO $12 / 13 / 94$

REPORT DATE: $06 / 20 / 95$

FORMATION OF COMPLETION: DECHELLEY MEMBER OF THE CUTLER FORMATIOH (DC)

HYDRAULIC FLON RELATIONSHIP: DONN GRADIENT (D)

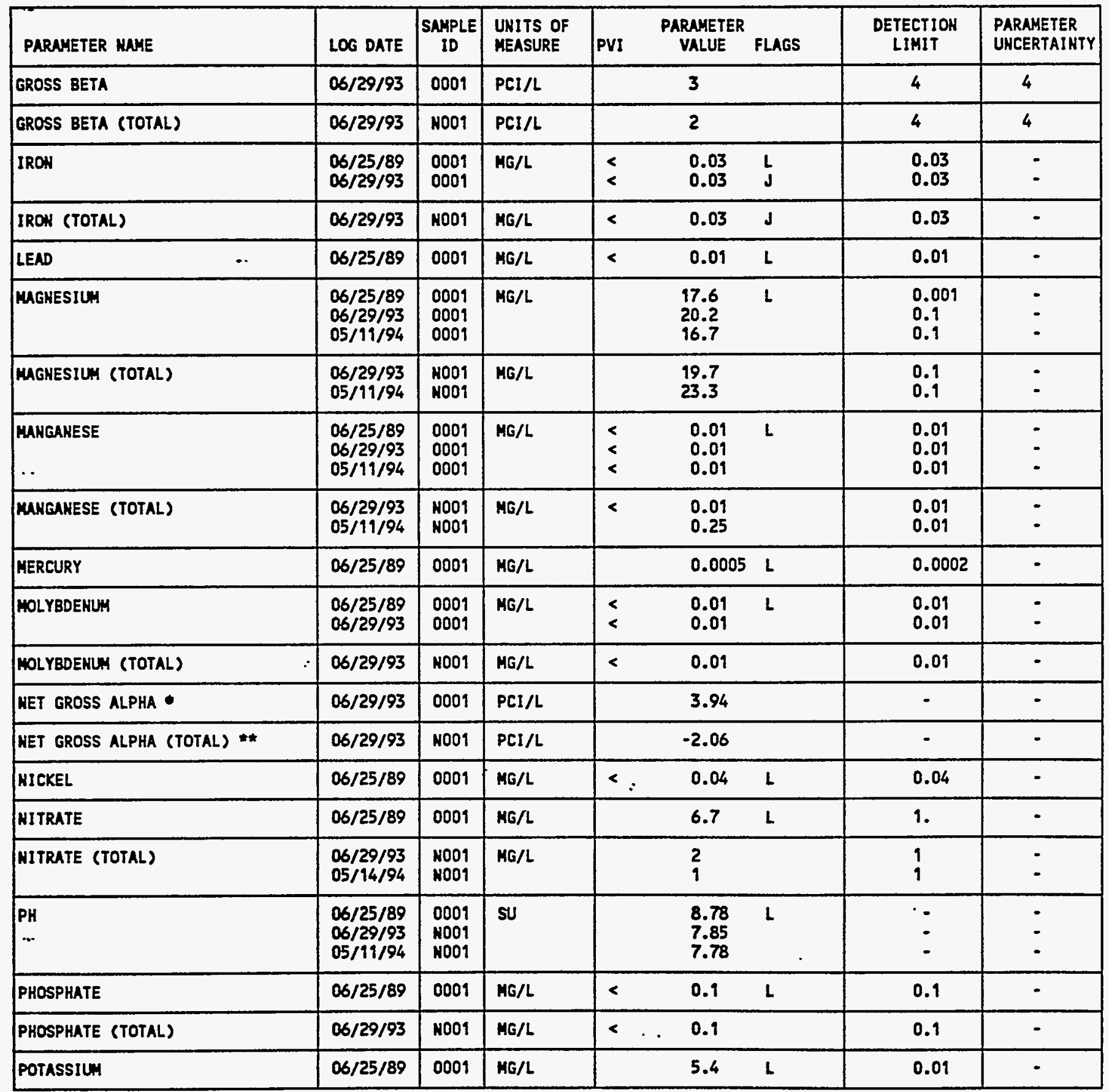

- MET GROSS ALPHA (GROSS ALPHA - URANIUH) HITH I MG URAMIUH = 686 PCI

* MET GROSS ALPHA (TOTAL) (TOTAL GROSS ALPHA - TOTAL URANIUM)

PARAMETER VALUE INDICATOR (PVI): < - LESS THAH DETECTION LIMIT

SAMPLE ID COOES:

OTHER PARAMETER VALUE FLAGS:

OOO - FILTERED SAMPLE (.45 MICROHS)

J - ESTIMATED VALUE

MOO1 - UNFILTERED SAMPLE

- L - LESS THAN THREE BORE VOLUHES REMOVED BEFORE SAMPLIHG 
GROUMDHATER QUALITY DATA BY LOCATIOH

SOLP REV. 0 Data

SITE: MONO M MAMMENT VALLEY

LOCATION: 0668

MORTH COORDIHATE: 60179.4 FT

EAST COORDIHATE: 88287.0 FT

04/21/88 TO $12 / 13 / 94$

REPORT DATE: $06 / 20 / 95$

FORMATION OF COAPLETIOH: DECHELLEY MEMBER OF THE CUTLER FORMATION (DC) HYDRAULIC FLON RELATIOHSHIP: DOWN GRADIENT (D)

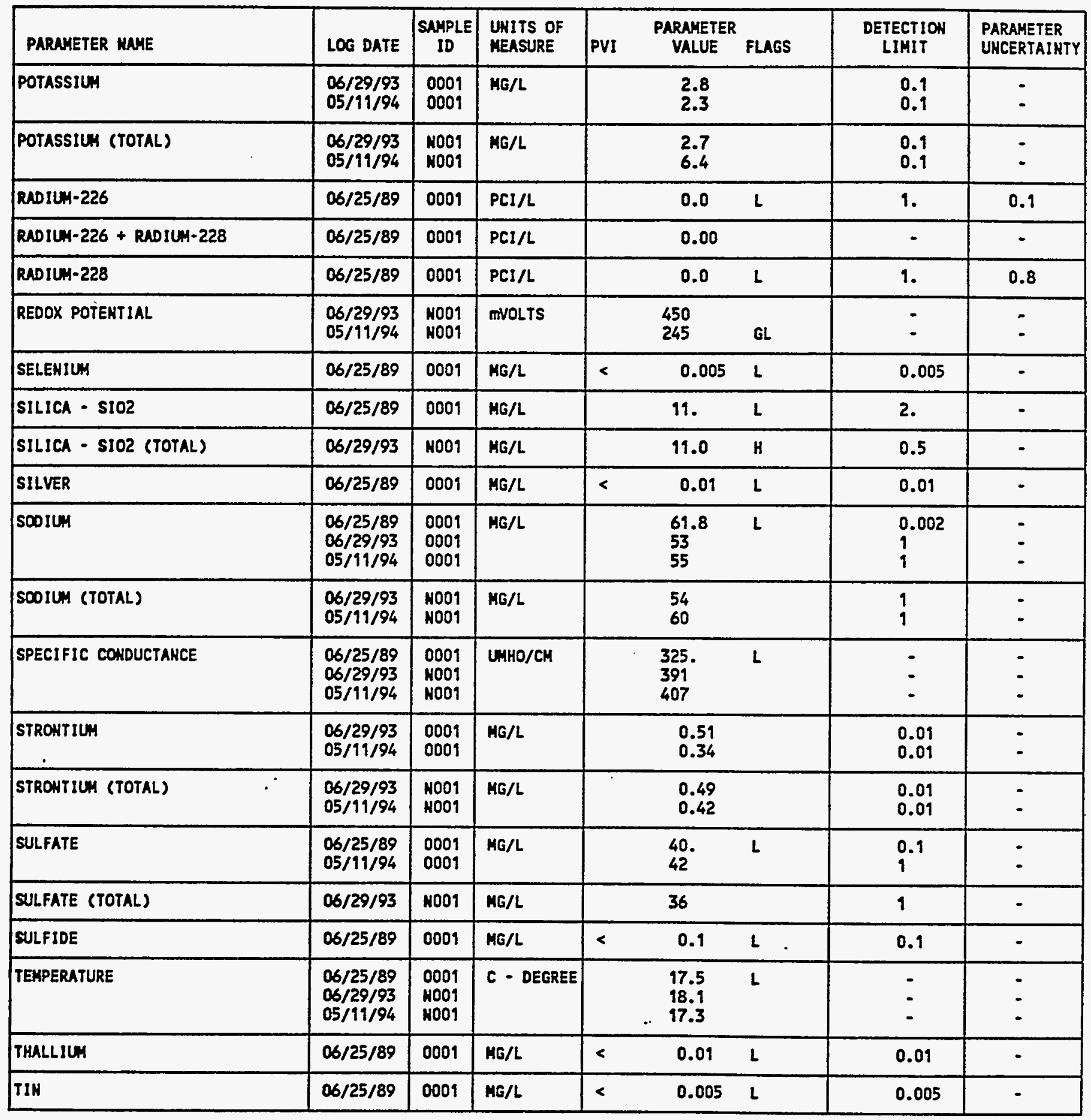

PARAMETER VALUE INDICATOR (PVI): < - LESS THAN DETECTION LIMIT

SAMPLE ID COOES:

OTHER PARAMETER VALUE FLAGS:

0001 - FILTERED SAMPLE (.45 MICROWS)

G - PH > 9, POSSIBLE GROUT CONTAMIMATION

NOOI - UMFILTERED SAMPLE

H - HOLD TIME EXPIRED, VALUE SUSPECT

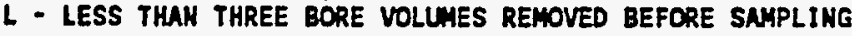


GROWDWATER QUALITY DATA BY LOCATION

SOWP ReV. O Data

SITE: MONO1 MONUMENT VALLEY

LOCATION: $0 S 68$

NORTH COORDINATE: 60171.4 FT

EAST COORDINATE: 88287.0 FT

04/21/88 TO $12 / 13 / 94$

REPORT DATE: 06/20/95

FORHATION OF COMPLETION: DECHELLEY MEMBER OF THE CUTLER FORMATION (DC) HYDRAULIC FLOU RELATIOHSHIP: DOWN GRADIENT (D)

\begin{tabular}{|c|c|c|c|c|c|c|c|c|}
\hline PARAMETER MAME & LOG DATE & $\begin{array}{c}\text { SAMPLE } \\
\text { ID }\end{array}$ & $\begin{array}{l}\text { UNITS OF } \\
\text { MEASURE }\end{array}$ & PVI & $\begin{array}{l}\text { PARAMETER } \\
\text { VALUE }\end{array}$ & FLAGS & $\begin{array}{l}\text { DETECTION } \\
\text { LIMIT }\end{array}$ & $\begin{array}{l}\text { PARAMETER } \\
\text { UHCERTAINTY }\end{array}$ \\
\hline TOTAL DISSOLVED SOLIOS & $\begin{array}{l}06 / 25 / 89 \\
05 / 11 / 94\end{array}$ & $\begin{array}{l}0001 \\
0001\end{array}$ & $M G / L$ & & $270^{2.36}$ & JL & $\begin{array}{l}10 . \\
10\end{array}$ & - \\
\hline TOTAL OISSOLVED SOLIDS (TOTAL) & $06 / 29 / 93$ & NOO1 & MG/L & & 240 & H & 10 & - \\
\hline TURBIDITY & $05 / 11 / 94$ & N001 & NTU & & 213 & & - & - \\
\hline URAKIUN & $\begin{array}{l}06 / 25 / 89 \\
06 / 29 / 93 \\
05 / 11 / 94\end{array}$ & $\begin{array}{l}0001 \\
0001 \\
0001\end{array}$ & $M G / L$ & & $\begin{array}{l}0.0017 \\
0.003 \\
0.003\end{array}$ & JL & $\begin{array}{l}0.003 \\
0.001 \\
0.001\end{array}$ & $\dot{-}$ \\
\hline URANIUH (TOTAL) & $\begin{array}{l}06 / 29 / 93 \\
05 / 11 / 94\end{array}$ & $\begin{array}{l}\text { NOO1 } \\
\text { N001 }\end{array}$ & MG/L & & $\begin{array}{l}0.003 \\
0.004\end{array}$ & & $\begin{array}{l}0.001 \\
0.001\end{array}$ & - \\
\hline VAKAOIUM & $\begin{array}{l}06 / 25 / 89 \\
06 / 29 / 93 \\
05 / 11 / 94\end{array}$ & $\begin{array}{l}0001 \\
0001 \\
0001\end{array}$ & $M G / L$ & $<$ & $\begin{array}{l}0.01 \\
0.09 \\
0.01\end{array}$ & $\mathbf{L}$ & $\begin{array}{l}0.01 \\
0.01 \\
0.01\end{array}$ & : \\
\hline VAKADIUM (TOTAL) & $\begin{array}{l}06 / 29 / 93 \\
05 / 19 / 94\end{array}$ & $\begin{array}{l}\text { NOO1 } \\
\text { N001 }\end{array}$ & $M G / L$ & $<$ & $\begin{array}{l}0.01 \\
0.09\end{array}$ & & $\begin{array}{l}0.01 \\
0.01\end{array}$ & - \\
\hline ZINC & $\begin{array}{l}06 / 25 / 89 \\
06 / 29 / 93\end{array}$ & $\begin{array}{l}0001 \\
0001\end{array}$ & $M G / L$ & & $\begin{array}{l}0.014 \\
0.040\end{array}$ & L & $\begin{array}{l}0.005 \\
0.005\end{array}$ & $\dot{-}$ \\
\hline ZINC (TOTAL) & $06 / 29 / 93$ & NOO1 & MG/L & & 0.017 & J & 0.005 & - \\
\hline
\end{tabular}

PARAMETER VALUE IHDICATOR (PVI): < - LESS THAN DETECTIOH LIMIT

SAMPLE IO COOES:

OTHER PARAMETER VALUE FLAGS:

0009 - FILTERED SAMPLE (.45 MICRONS)

H - HOLD TIME EXPIRED, VALUE SUSPECT

NOO1 - UHFILTERED SAMPLE

J - ESTIMATED VALUE

L - LESS THAN THREE BORE VOLUMES REMOVED BEFORE SAMPLING 
GROUNDUATER QUALITY DATA BY LOCATION

SOWP ReV. O Data

SITE: HONOI HONUWENT VALLEY

LOCATIOH: OS69

MORTH COORDIMATE: 60946.3 FT

EAST COORDINATE: 88247.8 FT

$04 / 21 / 88$ TO $12 / 93 / 94$

REPORT DATE: 06/20/95

FORMATION OF COAPLETION: ALLUVIUM (AL)

HYDRAULIC FLOW RELATIOHSHIP: DOWN GRADIENT (D)

\begin{tabular}{|c|c|c|c|c|c|c|c|c|}
\hline PARAMETER MAME & LOG DATE & $\underset{\text { ID }}{\text { SAMPLE }}$ & $\begin{array}{l}\text { UHITS OF } \\
\text { MEASURE }\end{array}$ & PVI & $\begin{array}{l}\text { PARAMETER } \\
\text { VALUE }\end{array}$ & FLAGS & $\begin{array}{l}\text { DETECTION } \\
\text { LIMIT }\end{array}$ & $\begin{array}{l}\text { PARAMETER } \\
\text { UNCERTAINTY }\end{array}$ \\
\hline ALKALIHITY & $\begin{array}{l}06 / 25 / 89 \\
02 / 24 / 92 \\
06 / 29 / 93 \\
05 / 12 / 94\end{array}$ & $\begin{array}{l}0001 \\
0001 \\
\text { N001 } \\
\text { N001 }\end{array}$ & $\mathrm{MG} / \mathrm{L} \quad \mathrm{CACO} 3$ & & $\begin{array}{l}246 . \\
218 \\
204 \\
202\end{array}$ & $\begin{array}{l}L \\
L\end{array}$ & $\dot{-}$ & : \\
\hline ALUHINUM & $\begin{array}{l}06 / 25 / 89 \\
02 / 24 / 92\end{array}$ & $\begin{array}{l}0001 \\
0001\end{array}$ & MG/L & $<$ & $\begin{array}{l}0.1 \\
0.05\end{array}$ & $\mathbf{L}$ & $\begin{array}{l}0.1 \\
0.05\end{array}$ & $\dot{-}$ \\
\hline AmiOHILM & $\begin{array}{l}06 / 25 / 89 \\
02 / 24 / 92\end{array}$ & $\begin{array}{l}0001 \\
0001\end{array}$ & MG/L & $<$ & $\begin{array}{l}0.1 \\
0.4\end{array}$ & l & $\begin{array}{l}0.1 \\
0.1\end{array}$ & $\dot{-}$ \\
\hline AMHOHILN (TOTAL) & $\begin{array}{l}06 / 29 / 93 \\
05 / 14 / 94\end{array}$ & $\begin{array}{l}\text { No01 } \\
\text { N001 }\end{array}$ & MG/L & & $\begin{array}{l}0.2 \\
0.8\end{array}$ & $\mathbf{L}$ & $\begin{array}{l}0.1 \\
0.1\end{array}$ & $\dot{-}$ \\
\hline AKTIMONY & $\begin{array}{l}06 / 25 / 89 \\
02 / 24 / 92\end{array}$ & $\begin{array}{l}0001 \\
0001\end{array}$ & MG/L & $<$ & $\begin{array}{l}0.003 \\
0.003\end{array}$ & J & $\begin{array}{l}0.003 \\
0.003\end{array}$ & - \\
\hline ARSENIC & $\begin{array}{l}06 / 25 / 89 \\
02 / 24 / 92 \\
06 / 29 / 93\end{array}$ & $\begin{array}{l}0001 \\
0001 \\
0001\end{array}$ & MG/L & $\begin{array}{l}< \\
< \\
<\end{array}$ & $\begin{array}{l}0.01 \\
0.01 \\
0.005\end{array}$ & $\begin{array}{l}L \\
L\end{array}$ & $\begin{array}{l}0.01 \\
0.01 \\
0.005\end{array}$ & : \\
\hline ARSEHIC (TOTAL) & $06 / 29 / 93$ & NOOI & MG/L & $<$ & 0.005 & $\iota$ & 0.005 & - \\
\hline BARIUN & $\begin{array}{l}06 / 25 / 89 \\
02 / 24 / 92 \\
06 / 29 / 93\end{array}$ & $\begin{array}{l}0001 \\
0001 \\
0001\end{array}$ & $M G / L$ & $<$ & $\begin{array}{l}0.1 \\
0.02 \\
0.1\end{array}$ & $\begin{array}{l}L \\
L\end{array}$ & $\begin{array}{l}0.1 \\
0.01 \\
0.1\end{array}$ & : \\
\hline BARIUH (TOTAL) & $06 / 29 / 93$ & NOO१ & MG/L & $<$ & 0.1 & 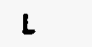 & 0.1 & - \\
\hline BERYLLIUM & $06 / 25 / 89$ & 0001 & ME/L & $<$ & 0.01 & & 0.01 & - \\
\hline BOROH & $06 / 25 / 89$ & 0009 & MG/L & $<$ & 0.1 & & 0.1 & - \\
\hline CADHIUH & $\begin{array}{l}06 / 25 / 89 \\
02 / 24 / 92\end{array}$ & $\begin{array}{l}0001 \\
0001\end{array}$ & $M G / L$ & $<$ & $\begin{array}{l}0.001 \\
0.0005\end{array}$ & $L$ & $\begin{array}{l}0.001 \\
0.0005\end{array}$ & $\dot{-}$ \\
\hline CALCIUH & $\begin{array}{l}06 / 25 / 89 \\
02 / 24 / 92 \\
06 / 29 / 93 \\
05 / 12 / 94\end{array}$ & $\begin{array}{l}0001 \\
0001 \\
0001 \\
0001\end{array}$ & $M G / L$ & & $\begin{array}{l}82.7 \\
80.8 \\
75.4 \\
52.9\end{array}$ & $L$ & $\begin{array}{l}0.01 \\
0.5 \\
0.5 \\
0.5\end{array}$ & - \\
\hline CALCIUH (TOTAL) & $\begin{array}{l}06 / 29 / 93 \\
05 / 12 / 94\end{array}$ & $\begin{array}{l}\text { NOOI } \\
\text { HOOI }\end{array}$ & MG/L & & $\begin{array}{l}71.9 \\
62.3\end{array}$ & L & $\begin{array}{l}0.5 \\
0.5\end{array}$ & - \\
\hline CHLORIDE & $\begin{array}{l}06 / 25 / 89 \\
02 / 24 / 92 \\
05 / 12 / 94\end{array}$ & $\begin{array}{l}0001 \\
0001 \\
0001\end{array}$ & MG/L & & $\begin{array}{l}16 . \\
12.2 \\
10.0\end{array}$ & $L$ & $\begin{array}{l}1.5 \\
0.5 \\
0.5\end{array}$ & $\div$ \\
\hline CHLORIDE (TOTAL) & $06 / 29 / 93$ & N001 & $M G / L$ & & 15.0 & $L$ & 0.5 & - \\
\hline CHROMIUH & $\begin{array}{l}06 / 25 / 89 \\
02 / 24 / 92\end{array}$ & $\begin{array}{l}0001 \\
0001\end{array}$ & $M G / L$ & $<$ & $\begin{array}{l}0.01 \\
0.01\end{array}$ & $\mathbf{L}$ & $\begin{array}{l}0.01 \\
0.01\end{array}$ & - \\
\hline COBALT & $06 / 25 / 89$ & 0001 & MG/L & $<$ & 0.05 & & 0.05 & - \\
\hline COPPER & $06 / 25 / 89$ & 0001 & MG/L & $<$ & 0.02 & & 0.02 & - \\
\hline
\end{tabular}

PARAMETER VALUE IHDICATOR (PVI): < - LESS THAN DETECTION LIMIT

SAMPLE IO COOES:

0001 - FILTERED SAMPLE (.45 MICROHS)

OTHER PARAMETER VALUE FLAGS:

HODI - UNFILTERED SAMPLE

J - ESTIMATED VALUE

L - LESS THAH THREE BORE VOLUMES REMOVED BEFORE SAMPLING 
GROUNDWATER QUALITY DATA BY LOCATION

SOMP REV. $O$ Data

SITE: HOHO1 MONLWENT VALLEY

LOCATIOH: 0669

MORTH COORDIMATE: 60146.3 FT

EAST COORDIHATE: 88247.8 FT

$04 / 21 / 88$ TO $12 / 13 / 94$

REPORT DATE: $06 / 20 / 95$

FORHATION OF COMPLETION: ALLUVIUM (AL)

HYORAULIC FLOW RELATIOHSHIP: DOWN GRADIENT (D)

\begin{tabular}{|c|c|c|c|c|c|c|c|c|}
\hline PARAMETER MAHE & LOG DATE & $\begin{array}{c}\text { SAMPLE } \\
\text { ID }\end{array}$ & $\begin{array}{l}\text { UNITS OF } \\
\text { MEASURE }\end{array}$ & PVI & $\begin{array}{l}\text { PARAMETER } \\
\text { VALUE }\end{array}$ & FLAGS & $\begin{array}{l}\text { DETECTION } \\
\text { LIMIT }\end{array}$ & $\begin{array}{l}\text { PARAMETER } \\
\text { UNCERTAINTY }\end{array}$ \\
\hline CYAHIDE & $06 / 25 / 89$ & 0001 & MG/L & $<$ & 0.01 & & 0.01 & - \\
\hline FLUORIDE & $06 / 25 / 89$ & 0001 & $M G / L$ & & 0.2 & & 0.1 & - \\
\hline GROSS ALPHA & $\begin{array}{l}02 / 24 / 92 \\
06 / 29 / 93\end{array}$ & $\begin{array}{l}0001 \\
0001\end{array}$ & $\mathrm{PCI} / \mathrm{L}$ & & $10^{1.2}$ & $\begin{array}{l}L \\
L\end{array}$ & $\frac{1.0}{5}$ & $\begin{array}{l}6.1 \\
9\end{array}$ \\
\hline GROSS ALPHA (TOTAL) & $06 / 29 / 93$ & NOO1 & $\mathrm{PCl} / \mathrm{L}$ & & 9 & \llcorner & 5 & 9 \\
\hline GROSS BETA & $\begin{array}{l}02 / 24 / 92 \\
06 / 29 / 93\end{array}$ & $\begin{array}{l}0001 \\
0001\end{array}$ & PCI/L & & $\begin{array}{l}7.2 \\
2\end{array}$ & $\begin{array}{l}L \\
L\end{array}$ & $\begin{array}{l}0.5 \\
5\end{array}$ & $\begin{array}{l}4.3 \\
4\end{array}$ \\
\hline GROSS BETA (TOTAL) & $06 / 29 / 93$ & N001 & PCI/L & & 4 & \llcorner & 5 & 4 \\
\hline IROW & $\begin{array}{l}06 / 25 / 89 \\
02 / 24 / 92 \\
06 / 29 / 93\end{array}$ & $\begin{array}{l}0001 \\
0001 \\
0001\end{array}$ & $M G / L$ & $\begin{array}{l}< \\
< \\
<\end{array}$ & $\begin{array}{l}0.03 \\
0.03 \\
0.03\end{array}$ & $\begin{array}{l}L \\
J L\end{array}$ & $\begin{array}{l}0.03 \\
0.03 \\
0.03\end{array}$ & - \\
\hline IRON (TOTAL) & $06 / 29 / 93$ & N001 & $M G / L$ & & 0.29 & JL & 0.03 & $=$ \\
\hline LEAD & $06 / 25 / 89$ & 0001 & $M G / L$ & $<$ & 0.01 & & 0.09 & - \\
\hline MGKESIUM & $\begin{array}{l}06 / 25 / 89 \\
02 / 24 / 92 \\
06 / 29 / 93 \\
05 / 92 / 94\end{array}$ & $\begin{array}{l}0001 \\
0001 \\
0001 \\
0001\end{array}$ & MG/L & & $\begin{array}{l}71.0 \\
62.2 \\
54.3 \\
37.0\end{array}$ & $\begin{array}{l}L \\
L\end{array}$ & $\begin{array}{l}0.001 \\
0.5 \\
0.1 \\
0.1\end{array}$ & : \\
\hline MAGHESIUM (TOTAL) & $\begin{array}{l}06 / 29 / 93 \\
05 / 12 / 94\end{array}$ & $\begin{array}{l}\text { NoO9 } \\
\text { HOOI }\end{array}$ & MG/L & & $\begin{array}{l}55.4 \\
42.4\end{array}$ & 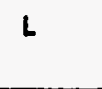 & $\begin{array}{l}0.1 \\
0.1\end{array}$ & - \\
\hline MANGAHESE & $\begin{array}{l}06 / 25 / 89 \\
02 / 24 / 92 \\
06 / 29 / 93 \\
05 / 12 / 94\end{array}$ & $\begin{array}{l}0001 \\
0001 \\
0001 \\
0001\end{array}$ & $M G / L$ & $\begin{array}{l}< \\
< \\
<\end{array}$ & $\begin{array}{l}0.01 \\
0.01 \\
0.01 \\
0.01\end{array}$ & $L$ & $\begin{array}{l}0.01 \\
0.01 \\
0.01 \\
0.01\end{array}$ & $\begin{array}{l}- \\
-\end{array}$ \\
\hline MAHGANESE (TOTAL) & $\begin{array}{l}06 / 29 / 93 \\
05 / 12 / 94\end{array}$ & $\begin{array}{l}\text { NoOI } \\
\text { NOOI. }\end{array}$ & $M G / L$ & & $\begin{array}{l}0.01 \\
0.05\end{array}$ & $L$ & $\begin{array}{l}0.01 \\
0.01\end{array}$ & - \\
\hline MERCURY & $06 / 25 / 89$ & 0001 & $M G / L$ & $<$ & 0.0002 & & 0.0002 & - \\
\hline MOLYBDENUM & $\begin{array}{l}06 / 25 / 89 \\
02 / 24 / 92 \\
06 / 29 / 93\end{array}$ & $\begin{array}{l}0001 \\
0001 \\
0001\end{array}$ & $M G / L$ & $\begin{array}{l}< \\
< \\
<\end{array}$ & $\begin{array}{l}0.01 \\
0.01 \\
0.01\end{array}$ & $\begin{array}{l}L \\
L\end{array}$ & $\begin{array}{l}0.01 \\
0.01 \\
0.01\end{array}$ & - \\
\hline MOLLYBDENUA (TOTAL) & $06 / 29 / 93$ & No01 & MG/L & $<$ & 0.01 & $L$ & 0.01 & - \\
\hline NET GROSS ALPHA • & $\begin{array}{l}02 / 24 / 92 \\
06 / 29 / 93\end{array}$ & $\begin{array}{l}0001 \\
0001\end{array}$ & $\mathrm{PCI} / \mathrm{L}$ & & $\begin{array}{r}-4.97 \\
3.14\end{array}$ & & $\overline{-}$ & - \\
\hline MET GROSS ALPHA (TOTAL) ** & $06 / 29 / 93$ & NoO1 & PCI/L & & 2.14 & & - & - \\
\hline HICKEL & $06 / 25 / 89$ & 0001 & MG/L & $<$ & 0.04 & & 0.04 & - \\
\hline
\end{tabular}

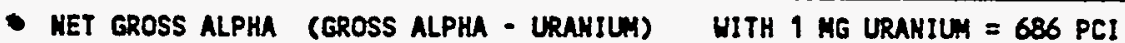

* WET GROSS ALPHA (TOTAL) (TOTAL GROSS ALPHA - TOTAL URANIUN)

PARAMETER VALUE IndICATOR (PVI): < - LESS THAN DETECTION LIMIT

OTHER PARAMETER VALUE fLAGS:

J - ESTIMATED VALUE

l - LESS THAN THREE BORE VOLUMES REMOVED BEFORE SAMPLIMG
SNMPLE ID COOES:

OOD1 - FILTERED SAMPLE (.45 MICRONS)

NOO१ - UNFILTERED SAMPLE 
GROUHDHATER QUALITY DATA BY LOCATION

SOUP Rev. O Data

SITE: MOHOI MONUMENT VALLEY

LOCATION: 0669

MORTH COORDIHATE: 60146.3 FT

EAST COORDIHATE: $\quad 88247.8$ FT

$04 / 21 / 88$ TO $12 / 13 / 94$

REPORT DATE: 06/20/95

FORMATION OF COMPLETION: ALLUVIUH (AL)

HYDRAULIC FLON RELATIONSKIP: DOWN GRADIENT (D)

\begin{tabular}{|c|c|c|c|c|c|c|c|c|}
\hline PARAMETER MAME & LOG DATE & $\underset{\text { SNMPLE }}{\text { SN }}$ & $\begin{array}{l}\text { UNITS OF } \\
\text { MEASURE }\end{array}$ & PVI & $\begin{array}{l}\text { PARANETER } \\
\text { VALUE }\end{array}$ & FLAGS & $\begin{array}{l}\text { DETECTION } \\
\text { LIMIT }\end{array}$ & $\begin{array}{l}\text { PARAMETER } \\
\text { UNCERTAINTY }\end{array}$ \\
\hline MITRATE & $\begin{array}{l}06 / 25 / 89 \\
02 / 24 / 92\end{array}$ & $\begin{array}{l}0001 \\
0001\end{array}$ & MG/L & & $\begin{array}{l}43 . \\
33.5\end{array}$ & \llcorner & $\begin{array}{l}1.0 \\
2.0\end{array}$ & - \\
\hline NITRATE (TOTAL) & $\begin{array}{l}06 / 29 / 93 \\
05 / 14 / 94\end{array}$ & $\begin{array}{l}\text { HOOI } \\
\text { HOOI }\end{array}$ & MG/L & & $\begin{array}{r}3 \\
28\end{array}$ & $\iota$ & 9 & - \\
\hline PH & $\begin{array}{l}06 / 25 / 89 \\
02 / 24 / 92 \\
06 / 29 / 93 \\
05 / 12 / 94\end{array}$ & $\begin{array}{l}0001 \\
0001 \\
\text { No1 } \\
\text { N001 }\end{array}$ & su & & $\begin{array}{l}7.42 \\
7.61 \\
7.18 \\
7.67\end{array}$ & $\begin{array}{l}L \\
L\end{array}$ & $\begin{array}{l}- \\
-\end{array}$ & : \\
\hline PHOSPHATE & $06 / 25 / 89$ & 0009 & MG/L & $<$ & 0.9 & & 0.1 & - \\
\hline PHOSPHATE (TOTAL) & $06 / 29 / 93$ & NoOI & MG/L & & 0.2 & $\mathbf{L}$ & 0.1 & - \\
\hline POTASSIUH & $\begin{array}{l}06 / 25 / 89 \\
02 / 24 / 92 \\
06 / 29 / 93 \\
05 / 12 / 94\end{array}$ & $\begin{array}{l}0001 \\
0001 \\
0001 \\
0001\end{array}$ & MG/L & & $\begin{array}{l}2.2 \\
2.93 \\
3.0 \\
2.3\end{array}$ & $\begin{array}{l}L \\
L\end{array}$ & $\begin{array}{l}0.01 \\
0.01 \\
0.1 \\
0.1\end{array}$ & : \\
\hline POTASSIUM (TOTAL) & $\begin{array}{l}06 / 29 / 93 \\
05 / 12 / 94\end{array}$ & $\begin{array}{l}\text { NOO1 } \\
\text { H001 }\end{array}$ & $M G / L$ & & $\begin{array}{l}3.1 \\
3.1\end{array}$ & $L$ & $\begin{array}{l}0.1 \\
0.1\end{array}$ & - \\
\hline RADIUA-226 & $\begin{array}{l}06 / 25 / 89 \\
02 / 24 / 92\end{array}$ & $\begin{array}{l}0009 \\
0001\end{array}$ & PCI/L & & $\begin{array}{l}0.2 \\
0.0\end{array}$ & $\mathbf{L}$ & 1.0 & $\begin{array}{l}0.2 \\
0.2\end{array}$ \\
\hline RADILH-226 + RADIUH-228 & $\begin{array}{l}06 / 25 / 89 \\
02 / 24 / 92\end{array}$ & $\begin{array}{l}0001 \\
0001\end{array}$ & PCI/L & & $\begin{array}{l}0.20 \\
1.60\end{array}$ & & - & - \\
\hline RADIUA4-228 & $\begin{array}{l}06 / 25 / 89 \\
02 / 24 / 92\end{array}$ & $\begin{array}{l}0001 \\
0001\end{array}$ & PCI/L & & $\begin{array}{l}0.0 \\
1.6\end{array}$ & $L$ & 9.0 & $\begin{array}{l}0.8 \\
1.0\end{array}$ \\
\hline REDOX POTENTIAL & $\begin{array}{l}06 / 29 / 93 \\
05 / 12 / 94\end{array}$ & $\begin{array}{l}\text { N001 } \\
\text { nO01 }\end{array}$ & mNOLTS & & $\begin{array}{l}410 \\
308\end{array}$ & GL & - & $\dot{-}$ \\
\hline SELEMIUY & $\begin{array}{l}06 / 25 / 89 \\
02 / 24 / 92\end{array}$ & $\begin{array}{l}0001 \\
0001\end{array}$ & MG/L & & $\begin{array}{l}0.009 \\
0.007\end{array}$ & $L$ & $\begin{array}{l}0.005 \\
0.005\end{array}$ & - \\
\hline SILICA - SIOZ & $06 / 25 / 89$ & 0001 & $M G / L$ & & 15. & & 2. & $\because$ \\
\hline SILICA - SIO2 (TOTAL) & $06 / 29 / 93$ & HOO1 & $\mathrm{HG} / \mathrm{L}$ & & 15.0 & HL & 0.5 & - \\
\hline SILVER & $06 / 25 / 89$ & 0001 & $M G / L$ & $<$ & 0.01 & & 0.01 & - \\
\hline sooluh & $\begin{array}{l}06 / 25 / 89 \\
02 / 24 / 92 \\
06 / 29 / 93 \\
05 / 12 / 94\end{array}$ & $\begin{array}{l}0001 \\
0001 \\
0001 \\
0001\end{array}$ & MG/L & & $\begin{array}{l}49.5 \\
44 . \\
44 \\
37\end{array}$ & $\begin{array}{l}\mathbf{L} \\
\mathbf{L}\end{array}$ & $\begin{array}{l}0.002 \\
1 . \\
i\end{array}$ & : \\
\hline SOOIUN (TOTAL) & $\begin{array}{l}06 / 29 / 93 \\
05 / 12 / 94\end{array}$ & $\begin{array}{l}\text { N001 } \\
\text { N001 }\end{array}$ & $M G / L$ & & $\begin{array}{r}.43 \\
\\
\end{array} 42$ & $L$ & 1 & $\dot{-}$ \\
\hline SPECIFIC CONDUCTANCE & $\begin{array}{l}06 / 25 / 89 \\
02 / 24 / 92\end{array}$ & $\begin{array}{l}0001 \\
0001\end{array}$ & LHHO/CH & & $\begin{array}{l}760 \\
685\end{array}$ & $\mathbf{L}$ & - & - \\
\hline
\end{tabular}

PARAMETER VALUE IMOICATOR (PVI): < - LESS THAH DETECTION LIMIT

SAMPLE ID COOES:

0001 - FILTERED SAMPLE (.45 MICRONS)

OTHER PARAMETER VALUE FLAGS:

NOD1 - UNFILTERED SAMPLE

G - PH > 9, POSSIBLE GROUT CONTAMINATION

H - hOLD TIME EXPIRED, VALUE SUSPECT

L - LESS THAN THREE BÓRE VOLUMES REMOVED BEFORE SAMPLIHG 
GROWNDHTER QUALITY DATA BY LOCATION

SOWP Rev. O Data

SITE: MONOT MONLHENT VALLEY

LOCATION: O669

MORTH COORDIHATE: 60146.3 FT

EAST COOROIHATE: 88247.8 FT

04/21/88 TO $12 / 13 / 94$

REPORT DATE: 06/20/95

FORMATION OF COMPLETION: ALLUVIUA (AL)

HYDRAULIC FLON RELATIONSHIP: DOWN GRADIENT (D)

\begin{tabular}{|c|c|c|c|c|c|c|c|c|}
\hline PARAMETER NAME & LOG DATE & $\begin{array}{l}\text { SNMPLE } \\
\text { I0 }\end{array}$ & $\begin{array}{l}\text { UNITS OF } \\
\text { MEASURE }\end{array}$ & PVI & $\begin{array}{l}\text { PARAMETER } \\
\text { VALUE }\end{array}$ & FLAGS & $\begin{array}{l}\text { DETECTION } \\
\text { LIMIT }\end{array}$ & $\begin{array}{l}\text { PARAMETER } \\
\text { UNCERTAINTY }\end{array}$ \\
\hline SPECIFIC CONDUCTAKCE & $\begin{array}{l}06 / 29 / 93 \\
05 / 12 / 94\end{array}$ & $\begin{array}{l}\text { NO01 } \\
\text { N001 }\end{array}$ & UNHO/CM & & $\begin{array}{l}927 \\
824\end{array}$ & $\mathbf{L}$ & : & $\dot{-}$ \\
\hline STRONTIUA & $\begin{array}{l}02 / 24 / 92 \\
06 / 29 / 93 \\
05 / 12 / 94\end{array}$ & $\begin{array}{l}0001 \\
0001 \\
0001\end{array}$ & MG/L & & $\begin{array}{l}0.58 \\
0.57 \\
0.42\end{array}$ & $L$ & $\begin{array}{l}0.01 \\
0.01 \\
0.01\end{array}$ & : \\
\hline STRONTIUA (TOTAL) & $\begin{array}{l}06 / 29 / 93 \\
05 / 12 / 94\end{array}$ & $\begin{array}{l}\text { NOOI } \\
\text { NOOI }\end{array}$ & MG/L & & $\begin{array}{l}0.57 \\
0.50\end{array}$ & 1 & $\begin{array}{l}0.01 \\
0.01\end{array}$ & - \\
\hline SULFATE & $\begin{array}{l}06 / 25 / 89 \\
02 / 24 / 92 \\
05 / 12 / 94\end{array}$ & $\begin{array}{l}0001 \\
0001 \\
0001\end{array}$ & $M G / L$ & & $\begin{array}{l}365 . \\
266 \\
182\end{array}$ & $L$ & $\begin{array}{c}0.1 \\
30 . \\
1\end{array}$ & : \\
\hline SULFATE (TOTAL) & $06 / 29 / 93$ & NOOI & $M G / L$ & & 232 & \llcorner & 1 & - \\
\hline SULFIDE & $06 / 25 / 89$ & 0009 & MG/L & $<$ & 0.1 & & 0.1 & - \\
\hline TEMPERATURE & $\begin{array}{l}06 / 25 / 89 \\
02 / 24 / 92 \\
06 / 29 / 93 \\
05 / 12 / 94\end{array}$ & $\begin{array}{l}0001 \\
0001 \\
\text { HOO1 } \\
\text { N001 }\end{array}$ & C - DEGREE & & $\begin{array}{l}16.9 \\
12.7 \\
24.6 \\
20.9\end{array}$ & $L$ & : & : \\
\hline THALLIUY & $06 / 25 / 89$ & 0001 & $M G / L$ & $<$ & 0.01 & & 0.01 & - \\
\hline TIN & $06 / 25 / 89$ & 0001 & MG/L & $<$ & 0.005 & & 0.005 & - \\
\hline TOTAL DISSOLVED SOLIDS & $\begin{array}{l}06 / 25 / 89 \\
02 / 24 / 92 \\
05 / 12 / 94\end{array}$ & $\begin{array}{l}0001 \\
0001 \\
0001\end{array}$ & MG/L & & $\begin{array}{l}786 \\
647 \\
500\end{array}$ & $\mathbf{L}$ & $\begin{array}{l}10 . \\
10 . \\
10\end{array}$ & : \\
\hline TOTAL DISSOLVED SOLIDS (TOTAL) & $06 / 29 / 93$ & NOOI & MG/L & & 600 & HL & 10 & - \\
\hline TOTAL ORGAMIC CARBON & $02 / 24 / 92$ & 0001 & MG/L & $<$ & 9. & $L$ & 1. & - \\
\hline URANIUM & $\begin{array}{l}06 / 25 / 89 \\
02 / 24 / 92 \\
06 / 29 / 93 \\
05 / 12 / 94\end{array}$ & $\begin{array}{l}0001 \\
0001 \\
0001 \\
0001\end{array}$ & MG/L & • & $\begin{array}{l}0.012 \\
0.009 \\
0.01 \\
0.009\end{array}$ & $L$ & $\begin{array}{l}0.003 \\
0.001 \\
0.001 \\
0.001\end{array}$ & $\begin{array}{l}- \\
- \\
.\end{array}$ \\
\hline URAKIUY (TOTAL) & $\begin{array}{l}06 / 29 / 93 \\
05 / 12 / 94\end{array}$ & $\begin{array}{l}\text { NoO1 } \\
\text { N001 }\end{array}$ & MG/L & & $\begin{array}{l}0.09 \\
0.009\end{array}$ & $\mathbf{L}$ & $\begin{array}{l}0.001 \\
0.001\end{array}$ & $\dot{-}$ \\
\hline VANADIUM & $\begin{array}{l}06 / 25 / 89 \\
02 / 24 / 92 \\
06 / 29 / 93 \\
05 / 12 / 94\end{array}$ & $\begin{array}{l}0001 \\
0001 \\
0001 \\
0001\end{array}$ & MG/L & & $\begin{array}{l}0.08 \\
0.06 \\
0.05 \\
0.04\end{array}$ & $L$ & $\begin{array}{l}0.01 \\
0.01 \\
0.01 \\
0.01\end{array}$ & $\begin{array}{l}- \\
- \\
-\end{array}$ \\
\hline VAMADIUA (TOTAL) & $\begin{array}{l}06 / 29 / 93 \\
05 / 12 / 94\end{array}$ & $\begin{array}{l}\text { No0? } \\
\text { N001 }\end{array}$ & $M G / L$ & & $\begin{array}{l}0.05 \\
0.05\end{array}$ & $L$ & $\begin{array}{l}0.01 \\
0.01\end{array}$ & $\dot{-}$ \\
\hline ZINC & $\begin{array}{l}06 / 25 / 89 \\
02 / 24 / 92 \\
06 / 29 / 93\end{array}$ & $\begin{array}{l}0001 \\
0001 \\
0001\end{array}$ & $M G / L$ & $<$ & $\begin{array}{l}0.005 \\
0.005 \\
0.022\end{array}$ & JL & $\begin{array}{l}0.005 \\
0.005 \\
0.005\end{array}$ & - \\
\hline
\end{tabular}

PARAMETER VALUE IMDICATOR (PVI): < - LESS THAN DETECTION LIMIT

SAMPLE IO COOES:

0001 - FILTERED SAMPLE (.45 MICROHS)

OTHER PARAMETER VALUE FLAGS:

NOOI - UNFILTERED SAMPLE

H - HOLD TIME EXPIRED, VALUE SUSPECT

J - estimateo VALUE

- L - LESS tHAN THREE BORE VOLLATES REMOVEd BEFORE SAMPLING 
APPENDIX D

WELL POINTS WORK PLAN 

TO: $\quad$ JMCBee

THROUGH: AHolm Clll

FROM:

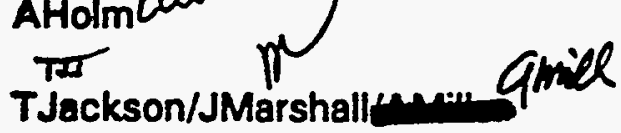

DATE: $\quad$ May 3, 1995

SUBJECT: Work Plan for Installation of Shallow Wells, Monument Valley UMTRA Site

\section{PURPOSE}

To determine the source of uranium, chloride, sulfate, and TDS (indicator parameters) along Cane Valley Wash in the vicinity of the Monument Valley Uranium Mill Tailings Remedial Action (UMTRA) Project site. The work will also aid in determining the nature of the ground water and surface water interactions along Cane Valley Wash. Additionally, if the depth to water is not too great, the downgradient extent of the ground water contaminant plume (between wells 650 and 653 ) will be investigated.

\section{SCOPE OF WORK}

All work will be performed and documented in accordance with applicable standard operating procedures and quality assurance/quality control standards. There will be two phases of work consisting of field activities and office activities.

This plan is a modification to the work plan dated April 14, 1994. The earlier version specified well points that would be installed using hand augers and/or a backhoe and frontend loader. The methods and materials presented in this plan are the same, except that a loader or backhoe will not be used. A hand-held power auger may be used if needed. The reason for the modification to the plan is to minimize damage to the ground surface. Evaluating the extent of the plume between wells 650 and 653 has been added to the plan also.

Installation of the following shallow wells is proposod:

- Installation of 12 shallow wells adjacent to Cane Valley Wash. Wells will be installed approximately a) 50 feet east, b) 50 feet west, and c) 100 to 150 feet west of sampling locations 623 (upgradient), 624 (downgradient), 627 (downgradient), and a location upgradient (south) of 623, near well 658. 
The well configurations will result in transects approximately perpendicular to the wash. Each transect extends toward the west further than an easterly direction because the potential source of ground water contamination is toward the west. Figure 1 shows existing sampling locations, Figure 2 is a schematic of the proposed well configurations, and Figure 3 is a well construction schematic.

- Installation of 4 shallow wells in Cane Valley Wash at sampling locations 623, 624, 627, and the additional upgradient location near well 658. The purpose of these wells is to assess possible changes to water quality due to the sampling methods. Because surface water is occasionally absent in the wash, samples from the surface locations have sometimes been taken by hand-digging a hole at the location and collecting the ground water that flows into the hole. The additional purpose of these three wells is to obtain water-level information in the wash to assess horizontal and vertical gradients.

- Installation of 3 shallow wells between wells 650 and 653 to evaluate water shallow water quality. The limiting factor will be the depth to water and the maximum depth the well points can be driven. The depth to water may be as little as $\mathbf{1 0}$ feet or great as $\mathbf{3 0}$ feet below land surface.

The following research activities (i.e. office activities) are proposed:

- Records from surface assessment and remediation will be obtained. These will include R-Track, vicinity property completion reports, surface and ground water quality analyses, surface water flow measurements, and surface water discharge records.

- The records will be reviewed and evaluated to determine potential sources of the indicator parameters, surface water and ground water interactions, and surface water flow rates.

\section{SITE CONDITIONS}

Depth to the water table nearby the wash is anticipated to be within 5 feet of land surface, based on field observations during surface water sampling and comparing depth to water measurements and ground surface elevations (Figure 4). Therefore, the total depth of the wells 50 feet east and west of the wash will be about 10 feet below grade. Depth to water up to 150 feet west of the wash is anticipated to be within 10 feet of land surface, therefore the maximum depth of those wells should be 15 feet below grade. Depth to water between wells 650 and 653 may range between 15 and 30 feet below grade.

Well logs from monitor wells from wells close to the wash $1605,651,652,653$, and 6541, show that the materials from land surface to at least 30 feet below land surface are medium- to fine-grained sand, with some clay and silt. Therefore, the proposed installation methods are appropriate. 


\section{SHALLOW WELL CONSTRUCTION AND INSTALLATION METHODS}

Each shallow well will consist of 5 feet of PVC or steel well screen and PVC or steel pipe, 1.5- or 2-inches in diameter. The smaller diameter screen and pipe will be easier to install to greater depths and will still allow the use of a bailer for well development and sampling.

The wells will be installed to a total depth of approximately 5 feet below the water table. Well construction materials and tools (including rental of a level, tripod, and rod for surveyingl will be supplied through Jacobs Engineering Group by a local Albuquerque vendor(s) prior to mobilization to the site.

Wells will be installed in an augured or dug hole and then driven into the soils to the total depth. The wells will be driven by hand with a sliding hammer (similar to a fence post driver).

The upper 3 to 5 feet of borehole annulus (i.e. the portion that was dug or augured) will be filled with bentonite pellets or chips to create a surface seal. The bentonite will be hydrated with potable water in accordance with applicable SOPs. Within one day following placement of the bentonite, a protective, lockable steel casing (4- to 6-inch diameter) and a concrete pad (2-feet by 2 -feet by 4 -inches thick) will be installed at the well head. The protective steel casing will be approximately 6-feet long, and placed so that 3 feet are above grade and 3 feet are below grade. The wells will be secured at all times using locks with the same key as the other site wells.

\section{WELL DEVELOPMENT}

The wells will be developed by bailing and surging with the bailer until the discharge is clear. A hand operated pump may also be used if the wells will produce a sufficient volume of water. Discharge of $\mathbf{2 0}$ gallons per well or less is anticipated.

The amount of water removed and approximate well yields will be recorded. Water will be discharged to ground surface nearby each well. Specific conductance and $\mathrm{pH}$ measurements will be taken to evaluate water quality during development. Well development and discharge of water to the land surface will be discontinued if project guidelines for the disposal of investigative-derived wastes are exceeded. Exceedance of the guidelines is not anticipated.

\section{WATER SAMPLIMG AND ANALYSIS}

Filtered and non-filtered water samples will be taken from each newly installed shallow well and surface water sampling location. Field analyses will be performed for $\mathrm{pH}$, temperature, turbidity, and alkalinity. Laboratory analyses will be performed for the following indicator parameters: uranium, nitrate, chloride, sulfate, TDS, strontium, and vanadium. 


\section{SITE RESTORATION}

The installation methods were chosen to minimize site disturbance, therefore very little site disturbance is anticipated. However, areas of disturbed soil will be raked smooth by hand, all trash will be collected immediately, and established roadways and paths will be used whenever possible.

\section{FIFID DOCUMENTATION}

- The well points and surface water sampling locations will be photographed and located on a map relative to nearby, established landmarks (e.g. existing wells). The elevations of the top-of-casing for each well point and the surface water elevations will be surveyed by the TAC field representatives. Horizontal control is anticipated to be within a 20 foot radius; vertical control is anticipated to be within 0.1 feet.

- Water-level measurements at each transect (well points and surface water elevations), and from each existing monitor well will be recorded on several occasions.

\section{PERMITTING}

Permits and access agreements for well point installation will be obtained as part of this scope of work. Well point locations are being reviewed to confirm that archeological surveys have been performed and that the work can be implemented. Based on the attached map (Figure 5), it appears that archeological surveys may be required in some of the proposed work areas. However, Figure 5 does not include all surveys that may have been performed to support surface cleanup activities.

\section{SCHEDULE}

Records research and planning the field work can be initiated immediately. The field work described above linstallation and sampling of the well points and water-level measurements from the entire monitor well network), can be performed as soon as approvals are received.

The field work is anticipated to require two TAC personnel for 15 days, including obtaining equipment, supplies, and mobilization/demobilization.

\section{ANTI-CONTAMINATION MEASURES}

Before being taken onto the site, all well installation materials will be cleaned with potable water and laboratory soap, and then rinsed with potable water. The cleaned equipment will then be wrapped in plastic. Materials will be cleaned at the sample preparation facility prior to departing for the site. Extra water will be taken to the field for additional cleaning, as required. 


\section{HEALTH AND SAFETY}

Personnel involved with well point installation will have the following equipment:

- Hard hat

- Steel-toed boots

- Work gloves

- Safety Glasses with Side Shields

The following items will be on-site:

- First-Aid kit

- Fire extinguisher

- Drinking Water

\section{DATA INTERPRETATION METHODS}

Data will be evaluated in the following manner:

- Surface assessment and remediation records will be reviewed for potential sources of indicator parameters in Cane Valley Wash.

- Water levels along each transect will be evaluated to determine the direction of ground water flow (i.e., if ground water is flowing toward the wash, away from the wash, or under the wash).

- Water-level measurements from the entire monitoring system will be evaluated to determine the direction of ground water flow within the area of the site. Piezometric surface maps, which will include the new shallow wells, will be constructed from water levels taken during the field effort.

- Results from the water quality analyses will be examined to determine the source of chloride, sulfate, and uranium in the downgradient locations in the wash.

AMAI

Attachments

cc: RBennett LPinkel

REdge RSaar

JGibb JSanchez

DMetzler Document Control 


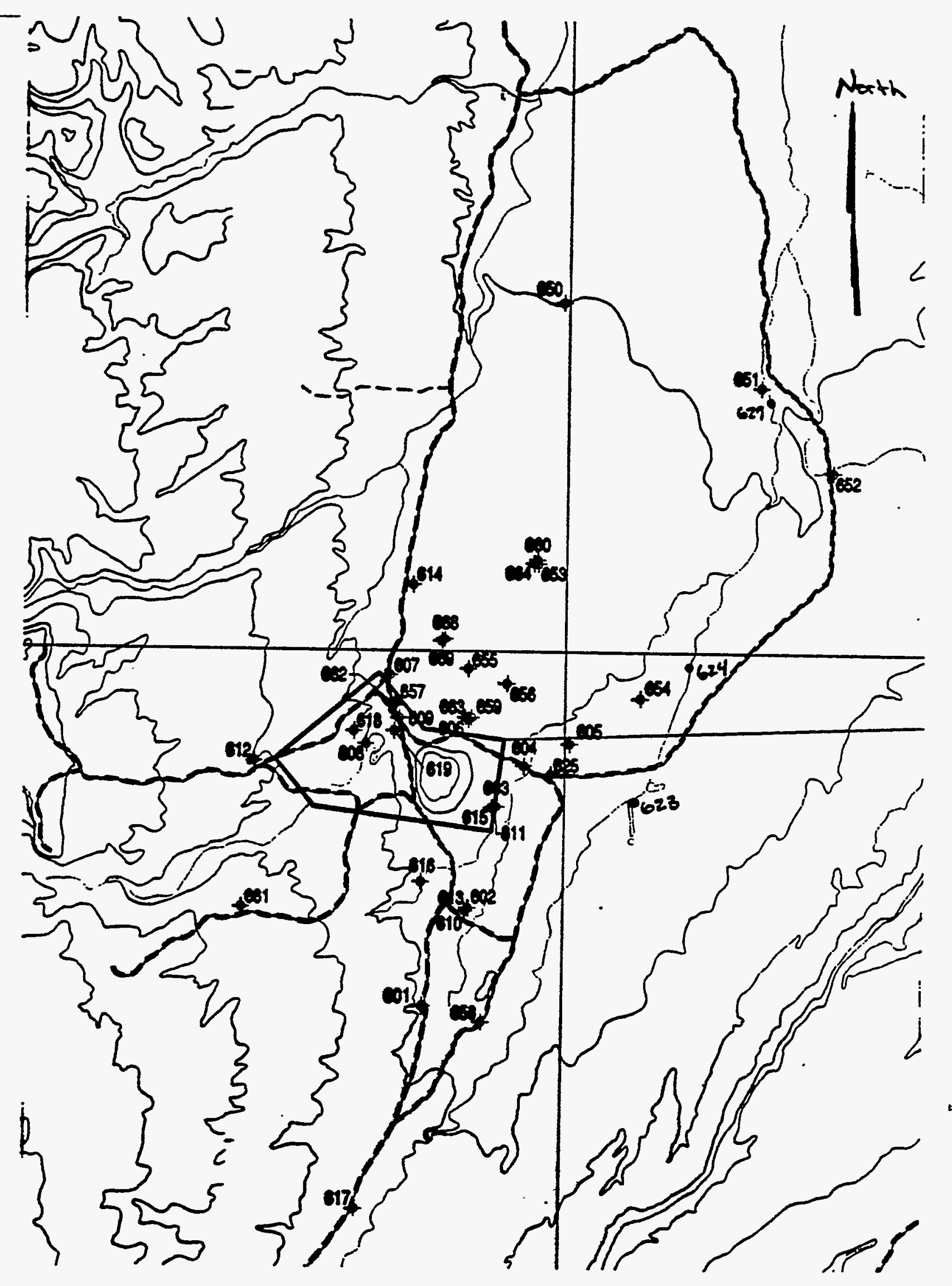

Fiende 1 . MONUMENT VALLEY UMTRA STTE (f Cane Valley, Arizona)

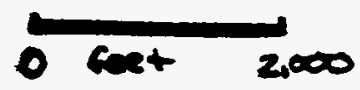


$\therefore x=3 / 8 / 94$

sunset Figure 2. Schematic Diagram no. I

$\therefore$ or.Avere ano.Amm of Proposed Well Point Locations so e no. At od in cane valley Wash

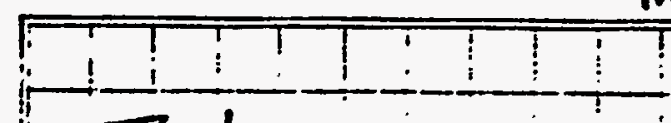

Tar Vire:
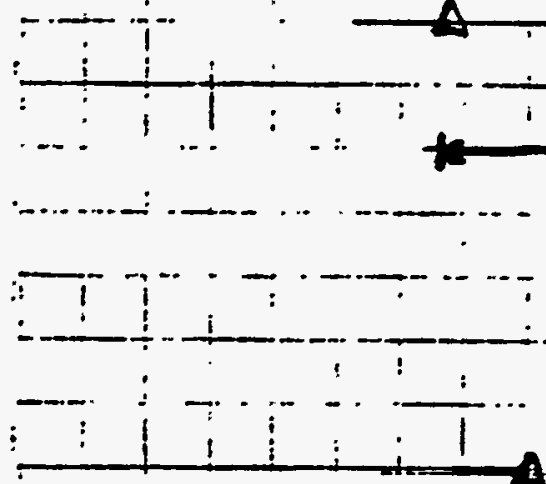

627

$\rightarrow$
$-$
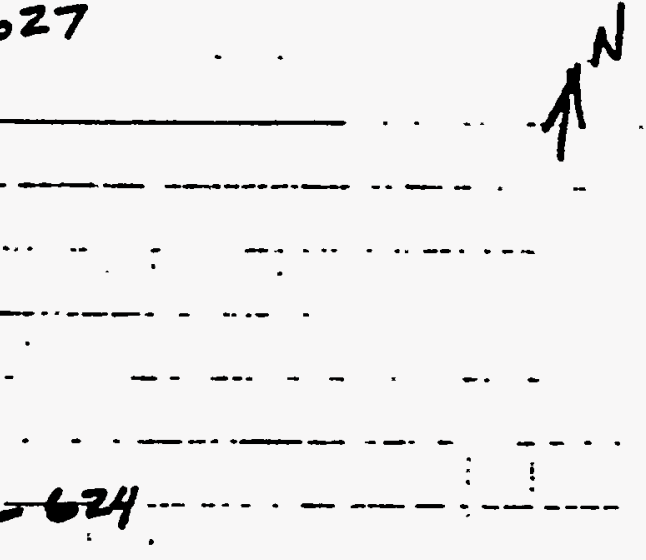

LEGEND.

- sur face sampling

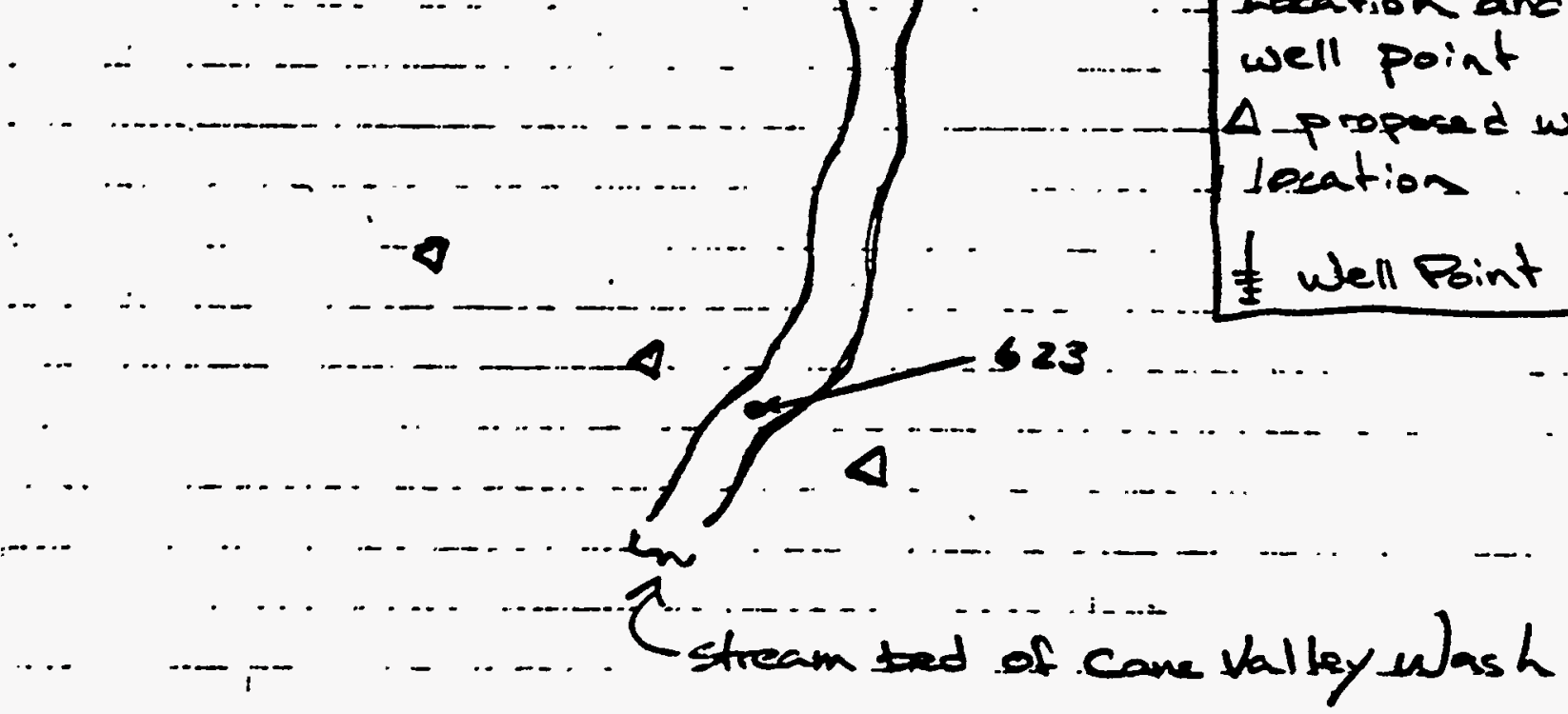

SIDE VIEW:

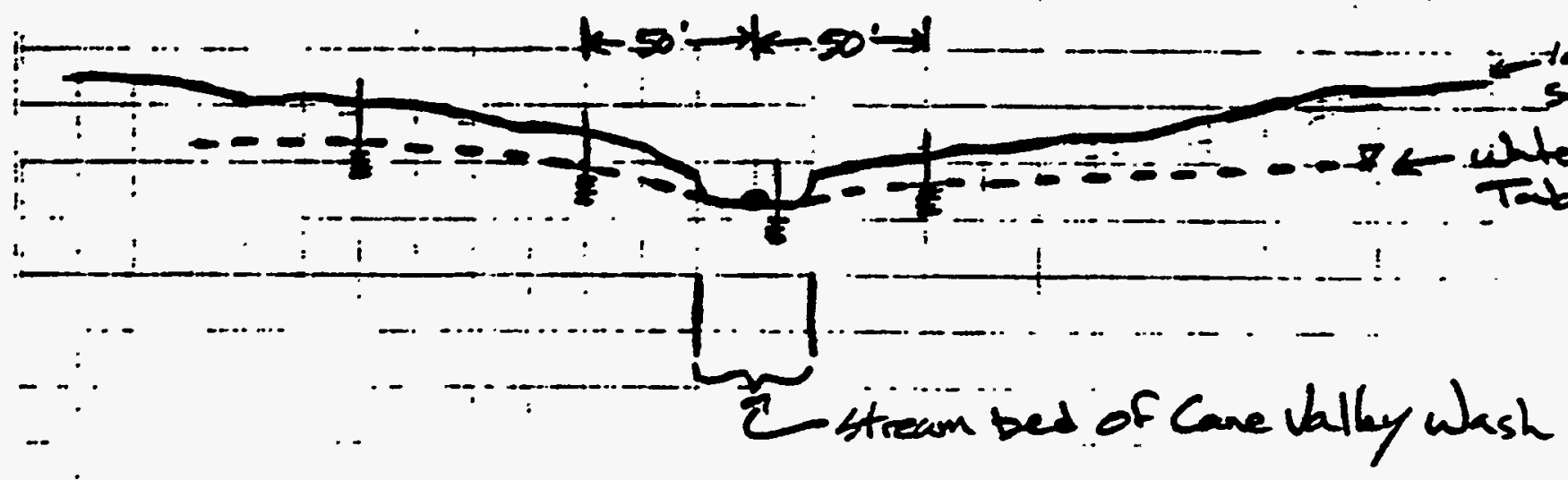

$\ldots-. .$.

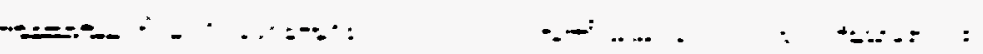


Figure 3. Well construction schematic

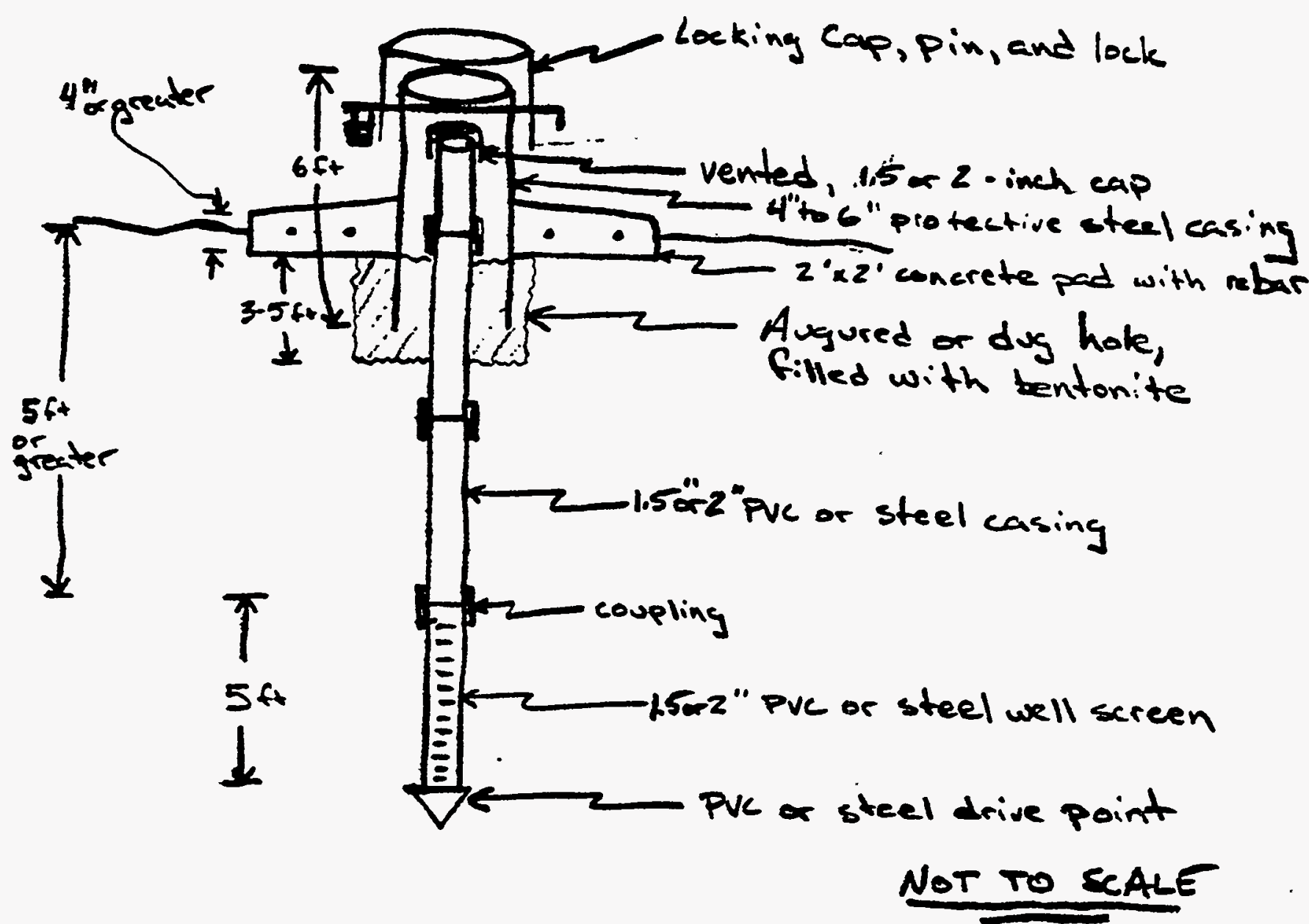

$\stackrel{i}{i}$ 


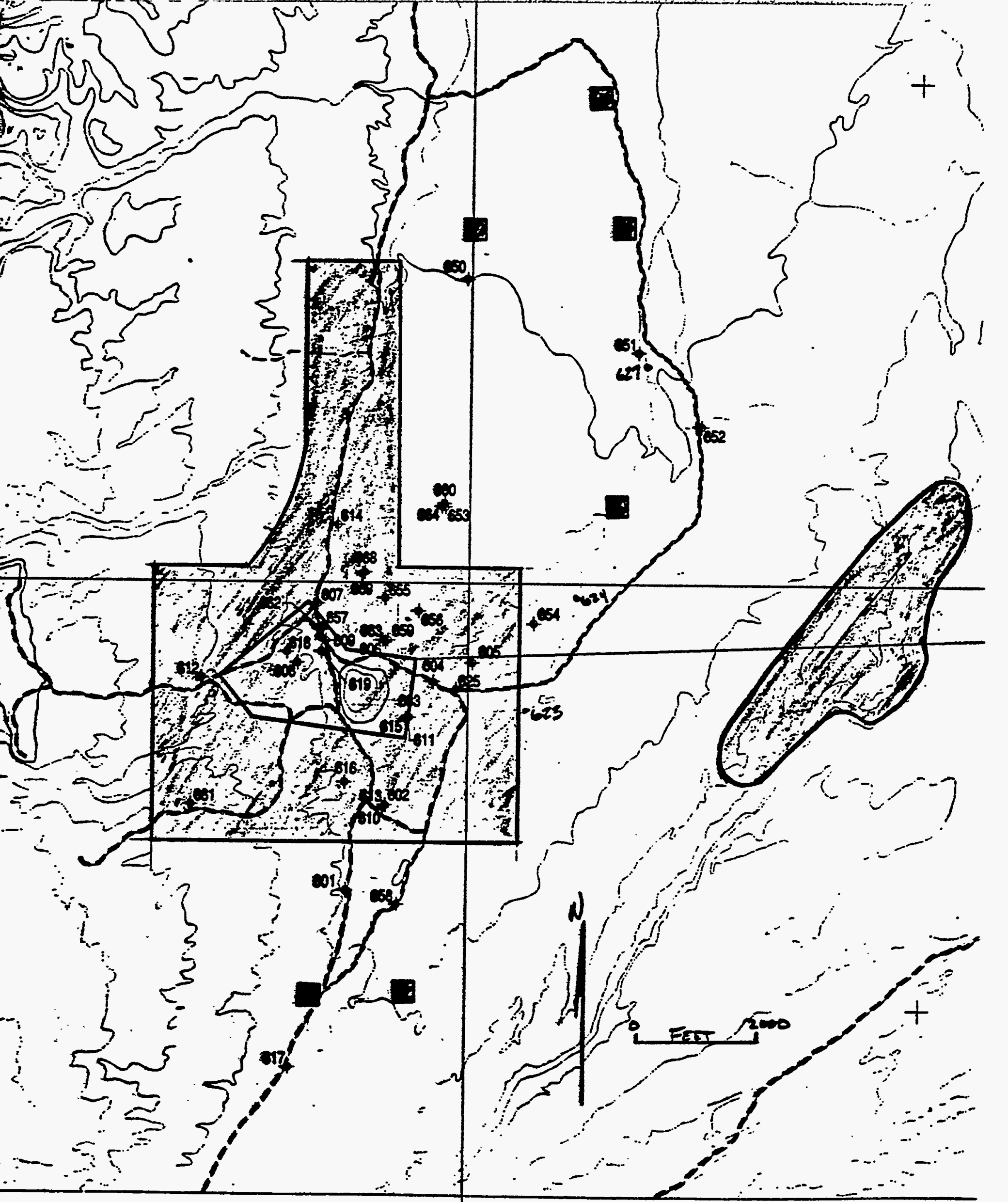

Figure 5. Areas with Archeologieal Clearance", Monument Valley UMTRA Project site 